2

GEM-TN-S3-262

gem

\title{
Technical Design Report
}

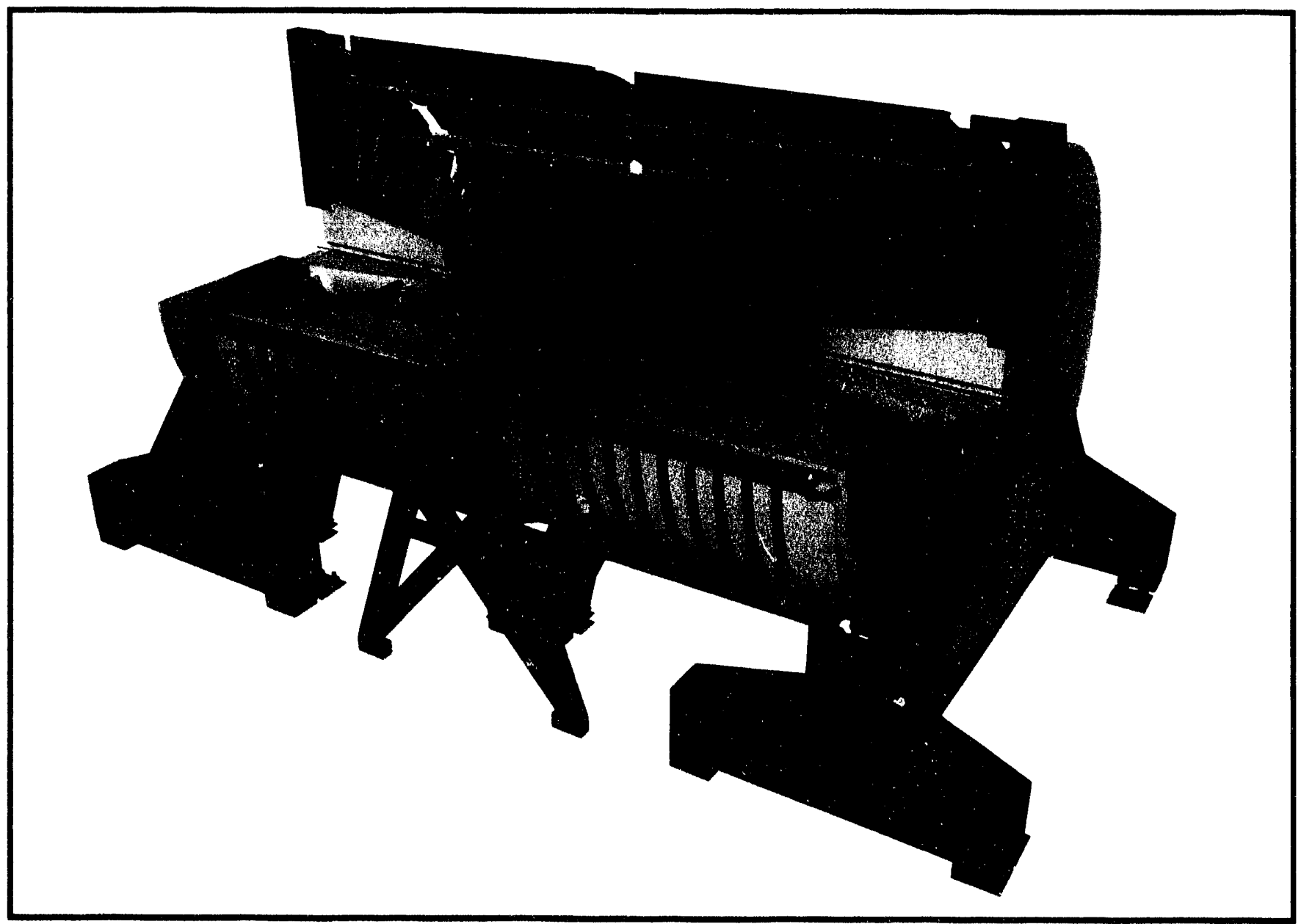

April 30, 1993 


\title{
GEM
}

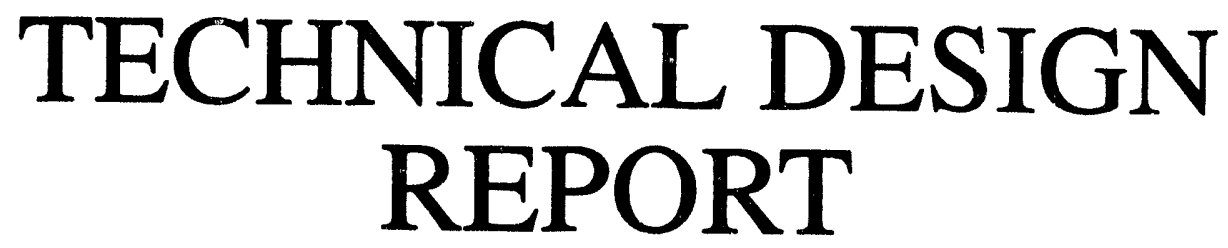

\author{
Submitted by \\ Gammas, Electrons, and MuONS \\ Collaboration
}

to the

Superconducting Super Colllider

LABORATORY*

July 31, 1993

\author{
TECENE
}

FEB 221994

OSTI

Contact persons:

Barry Barish

Division of Physics, Mathematics

and Astronomy

256-48 HEP

California Institute of Technology

Pasedena, California 91125
William Willis

Nevis Laboratory

Columbia University

P.O. Box 137

Irvington-on-Hudson

New York 10533 *Operated by the Universities Research Association, Inc. for the U.S. Department of Energy under Contract
No. DE-AC35-89ER40486.

This research is supported by the U.S. Dept. of Energy under Contract 
This version includes corrections through 15 June 1993.

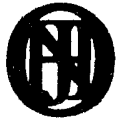




\section{ACKNOWLEDGEMENT}

We thank the many people at the SSC Laboratory who dedicated themselves to the successful completion of the GEM TDR. Very special thanks go to those staff members who led the production with incomparable professionalism, skill, and stamina.

To Andy Alcorn, Elbert Banzon, Kathy Barker, Dianne Compton, Tom Coyne, Holly Durden, Ed Engebretsen, Linda Fowler, Rebecca Harder, Mary Ann Hill, Bill Kendall, Dee Dee Kennedy, Bryan Lakey, Sylvia Lee, Maxine McCormick, Wanda Rice, Cindy Thomas, Karen Voors, and Sue Weaver: the Collaboration is grateful for your invaluable contributions in producing our TDR, and for your gracious and imperturbable demeanor in the face of physicists' most "creative" demands. 


\section{GEM COLLABORATORS LIST}

Adelphi University: W.C. Lefmann, R.V. Steiner

Institute of Nuclear Physics of the Albarian Academy of Sciences, Albania: B. Cico. A. Dodbibaj, N. Domi, P. Fuga, L. Karcanaj, A. Kasneci, M. Kedhi, S. Koji, A. Minxhozi. B. Nevruzaj, D. Rexha, P. Skendo, J. Thereska, F. Ylli, S. Zaganjori, D. Zeneli, J. Zoto

Polytechnical Institute of Tirana, Albania: B. Taullahu

University of Arizona: G.E. Forden, K.A. Johns, P. Loch, L. Shaver, J. Steinberg, J. P. Rutherfoord, M.A. Shupe, C. Zeitnitz

Beijing Glass Research Institute (BGRI), Beijing, China: G. Chen, Z.Z. Dai, X.Y. Dong, Y.C.Gao, T.Z. Li, G.T. Liu, Z.Q. Liu, S.Q. Man, S.X. Ren, Z.L. Su, Y.T. Wang, O. Wen, H. Xiao, H.Z. Yang, F.C. Zhang, F.Y. Zhang, J.Q. Zhang, J. Zhang, Y.N. Zheng

Institute of High Energy Physics (IHEP), Beijing, China: Y.B. Chen, X.Z. Cui, W.X. Gu, Y.F. Gu, Y.N. Guo, Y.Y. Guo, T. Hu, Y.Z. Huang, C.H. Jiang, Y.Y. Jiang, Y.F. Lai, J. Li, Z.G. Li, J.F. Lin, Z.A. Liu, C.S. Mao, Z.P. Mao, Q. Ouyang, H.Y. Sheng, M. Wang, Y.Y. Wang, D.M. Xi, J.W. Xi, Y.G. Xie, R.S. Xu, Y.L. Xu, C.S. Yu, B.Y. Zhang, C.C. Zhang, C.D. Zhang, D.H. Zhang, J.Q. Zhang, Q.J. Zhang, Y. Zhang, D.X. Zhao, J.W. Zhao, Z.M. Zhu, B.A. Zhuang

Byelorussian Research and Production Association of Powder Metallurgy, Minsk, Belarus: S. Barai, N. Naumovich, O. Roman, V. Shelekhina, A. Shevchionok, R. Stefanovich

Institute of Heat and Mass Exchange of Academy of Science of Belarus, Minsk, Belarus: V. Kolpaschikov

National Scientific and Educational Center of Particle and High Energy Physics, Minsk, Belarus M. Baturitsky, S. Degtjarev, O. Dvornikov', I. Emeljanchik, A. Kurilin, A. Litomin, V. Mikhailov, V. Shuljak, N. Shumeiko, A. Solin, A. Soroko, V. Stepanets ${ }^{2}$

Scientific Research Institute of Nuclear Problems, Belarussia State University, Minsk, Belarus:

V. Baryshevsky, A. Fjodorov, M. Korzhik, A. Lobko

Bhabha Atomic Research Center, India: G. Govindarrdan, S.S. Kapoor, G.P. Srivastava

Bochvar Institute for Inorganic Materials, Moscow, Russia: V. Ya Filkin, A.D. Nikulin, E.I. Plashkin, G.K. Zelensky

Boston University: S.P. Ahlen, E. Booth, R. Carey, S. Dye, M. Golden ${ }^{13}$, E. Hazen, D. Higby, T. Johnson, K.D. Lane, A. Marin, J.P. Miller, D. Osborne, B.L. Roberts, J.T. Shank, J.L. Stone, L.R. Sulak, G. Varner, D. Warner, J.S. Whitaker, W.A. Worstell, B. Zhou

Brookhaven National Laboratory: M.S. Atiya, S. Bellavia, I.-H. Chiang, M. Citterio, B. Gibbard, H.A. Gordon, J.S. Haggerty, S. Kahn, H.W. Kraner, D. Lissauer, L.S. Littenberg, H. Ma, D. Makowiecki, S. McCorkle, M.J. Murtagh, P. O'Connor, V.A. Polychronakos, S. Protopopescu, V. Radeka, D.C. Rahm, S. Rescia, L. Rogers, N.P. Samios, J. Sondericker, D. Stephani, I. Stumer, H. Takai, M.J. Tannenbaum, S.P. Yamin, B. Yu

Brown University: M. Widgoff

Bucharest University, Romania: C. Besliu

California Institute of Technology: B.C. Barish, D. Burke, G. Gratta, D. Kirkby, G. Liu, W. Lu, D.A. Ma, R. Mount, S. Mrenna, H. B. Newman, S. Shevchenko, ${ }^{3}$ X. Shi, H. Yamamoto, R.Y. Zhu

University of California, San Diego: J. G. Branson, W. Brower, H.E.G. Kobrak, R. Masek, H.P. Paar, M. Sivertz, B. Swanson

Carnegie Mellon University: R.M. Edelstein, A. Engler, T. Ferguson, R.W. Kraemer, D.M. Potter, M. Procario, J.R. Russ, R.B. Sutton, H. Vogel

Changwon National University, Korea: C.H. Hahn

Charles University, Czech Republic: I. Wilhelm 
University of Science and Technology of China (USTC), IEefei, China: Z.H. Bian, M.F. Cai,Y.M. Fang, L.Y. Hao, R.D. Han, X.S. Lin, B.A. Liu, W. Mei, Q.C. Shi, H.M. Wang, Z.M. Wang, S.L. Wu, S.L. Xing. J.H. Xu, K.Z. Xu, X.L. Xu, B.X. Yang, B.J. Ye, X.Q. Yu, J. Zhang, Y.Z. Zhou

Chonnam National University, Korea: H.I. Jang, J.Y. Kim, I.T. Lim

Choongnam National University, Korea: H.Y. Lee

Chosun University, Korea: B.N. Park

CINVESTAV, Mexico: G. Herrera Corral, H. Castilla Valdez

Columbia University: E. Aprile, Y. Au, A. Bolotnikov, T. Bolton, A. Caldwell, D. Chen, H. Cunitz,

J. Dodd, A. Gara, Y. Ho, W. Lee, M. Leltchouk, E. Mannel, M. Moulsen, R. Mukherjee, J. Parsons, P.C.

Rowson, M. Shaevitz, M. Seman, W. Sippach, E. Stem, W. Willis, L. Zhang

Institute of Electrical Engineering, Czech Republic: M. Polak

Institute of Nuclear Physics, Academy of Sciences, Czech Republic: A. Kugler, R. Mach

Instituie of Physics, Academy of Sciences, Czech Republic: P. Chraska, J. Hladky

Czech Technical University, Czech Republic: V. Bouda, V. Haulena, J. John, P. Kocourek, L. Musilek, J. Navratil, L. Pina, S. Pospisil, J. Tolar, V. Sochor, B. Sopko, J. Stecha, J.J. Venkrbec,

University of Delhi, South Campus, India: V. S. Bhasin, R. Gang, S.K. Jha, V. Kapoor, N. Parashar, R.K. Shivpuri, S. Soni, K.N. Tripathi

Dongsin University, Korea: M.Y. Pac

Draper Laboratories: H. Baker, P. Sebelius, E.C. Berk, M.J. Furey, R.E. Gustavson, M. Hansberry, T.P. Hinds, T.W. Lee, F.E. Nimblett, J.A. Paradiso, E.M. Womble

Drexel University: C. Lane

Fairfield University: D. Winn

Universidad de Guanajuato, Mexico: A. Gonzales, A. Morelos, G. Moreno, L. Villasenor

Gunsan National University, Korea: J.Y. Ryu

Gyeongsang National University, Korea: S.K. Choi, K.S. Chung, I.G. Park, J.S. Song

Harvard University: E. Carlson, M. Golden, E.H. Simmons

University of Houston: K. Lau, B.W. Mayes, L. Pinsky, J. Pyrlik, R. Weinstein

Indiana University: E.D. Alyea, C. Bower, M. Gephart, R.M. Heinz, S. Mufson, J. Musser, S. Nutter, J. Pitts

University of Iowa: N. Akchurin, A. Bravar, D. Kadmas, J. Langland, E. R. McCliment, Y. Meurice, C. Newsom, F. Olchowski, Y. Onel, M. Reno, V. Rodgers

Jackson State University: F. Lott, H. Zhang

KAIST, Korea: J. K. Kim

Kangnung National University, Korea: K.S. Kang, D.W. Kim

Kangwon National University, Korea: S.K. Nam

University Karlovy, Czech Republic: V. Malat

High Energy Physics Institute of Kazakh Academy of Sciences (HEPI), Kazakhstan: E. Boos, E. Khusainov, A. Kushulun, I. Kouchin, N. Nurgozhin, N. Pokrovsky, A. Temiraliev, B. Zhautykov Korea University, Korea: J.S. Kang, C.O. Kim, S.K. Park, K.S. Sim

Kurchatov Institute of Atomic Energy, Moscow, Russta: S.T. Belyaev, Yu. Dubovik, S.L. Fokin, M.S. Ippolitov, A.L. Lebedev, V.I. Manko, N. Martovetsky, G.M. Mgerbrishvili, E. Monitch,

A.S. Nianine, R.I. Scherbachev, A.A. Vasiliev, M.A. Vasiliev

Kyungpook National University, Korea: D.H. Kim, S.Y. Noh, D. Son 
Kyungsung University, Korea: Y.M. Park

Lawrence Livermore National Laboratory: E. Ables, O. Alford, F.C. BelseI, J. Berkey, R.M. Bionta. J.M. Bowers, D. Bupp, A.K. Chargin, O. Clamp, J. Clements, G.A. Deis. O.D. Fackler, D. Garner. F. Holdener, J. Horvath, W. Hsu, C.V. Johnson III, J.R. Heim, M. Haro, N. Lau, D.M. Makowiecki, R. Martin, G.J. Mauger, K. Miller, T. Moore, D. Ng. G. Oberst, H. Olson, L. Pedrotti, R. Pico, S. Pratuch, K.M. Skulina, J. Swan, K.A. Van Bibber, R. Warren, T.J. Wenaus, C. Williams, J. Williams, V. Williamson, S. Wineman, W. Wong, D. Wright, C.R. Wuest, R. Yamamoto, T. Yokota

Los Alamos National Laboratory: R. Barber, J.G. Boissenvain, M.L. Brooks, M. Cafferty, B.J. Cooke, K.R. Fuller, S.F. Hahn, J.E. Hinckley, C.S. Johnson, J.S. Kapustinsky, W.W. Kinnison. D.M. Lee, R.A. Martin, G.B. Mills, D.I. Montoya, R.E. Prael, G.H. Sanders, W.E. Sondheim, B.G. Smith, R.L. Smith, R. Stevens, T.C. Thompson, J.R. VanAnne, L.S. Waters, B. Weinstein

Louisiana State University: R. Imlay, H.-J. Kim, C. Lyndon, R. McNeil, W. Metcalf Martin Marietta Astronautics: T. Adams, K. Barnstable, J. Coulon, N.J. DiGiacomo, B. Easom, R. Humphreys, L. Mason, G. Velasquez

Martin Marietta Y-12 Oak Ridge Laboratory: J. Bohanan, B. Bowden, V. Galyon, J. Gertsen, J. Heck, N. Howell, J. King. S. Robinson, J. Rollins

Massachusetts Institute of Technology: P. Burrows, W. Busza, R.T. Camille Jr., Y.H. Chang, M. Chaniotakis, G.H. East, J. Feng, J.I. Friedman, E.D. Hafen, P. Haridas, J. Kelsey, H. Kendall, A. Korytov, ${ }^{4}$ P. Marston, J.V. Minervini, D.B. Montgomery, R.L. Myatt, L.S. Osborne, Z. Piek, I.R.D. Pillsbury, J. Pisera. A. Pless, S. Pourahimi, L. Rosenson, B. Smith, P. Sphicas, J.D. Sullivan, K. Sumorok, F.E. Taylor, R. Verdier, R. Vieira, B. Wadsworth, E. Wyslovch Memphis State University: D.W. Jones, D.R. Franceschetti, S. Jahan Michigan State University: M. Abolins, R. Brock, C. Bromberg, J. Huston, J. Linnemann, R. Miller, B. Pope, H. Weerts

University of Michigan: S. Nutter, S. McKee, G. Tarle

University of Mississippi: K. Bhatt, L.N. Bolen, M.L. Booke, L.M. Cremaldi, K. Hendrix, B. Moore, J.J. Reidy, E. Tarkovsky, ${ }^{3}$ J.R. Weinstein

Lebedev Physical Institute, Moscow, Russia: A. Chikanian, A. Shmeleva, V. Tikhomirov Moscow Engineering Physics Institute, Russia: A. Medvedev, M. Potekhin, V. Staroseltsev, A. Sumarokov, V. Tcherniatin, A. Vanyashin, S. Voloshin

Moscow State University, Russia: A.A. Arodzero, G.L. Bashindzhagyan, P.F. Ermolov, Yu.V. Fisyak, D. Karmanov, V. Kramarenko, E. Kuznetsov, A.N. Larichev, A. Leflat, M.M. Merkin, E.K. Shabalina, N.B. Sinev, N.A. Sotnikova, A.G. Voronin, V.Yu. Zhukov, S.A. Zotkin

Institute for Nuclear Research Moscow, Russia: V.N. Bolotov, O. Goncharenko, A. Proskuryakov, B. Semenov, V. Suchov, V. Urazmetov

Nanjing University, China: T.Y. Chen, D.T. Gao, G.X. Gao, M. Qi, D.X. Xi, N.G. Yao, Z.W. Zhang State University of New York (SUNY) at Albany: M.S. Alam, I.J. Kim, Z.C. Ling, B. Nemati, J. O'Neill, H. Severini, C.R. Sun

National Teacher's University, Korea: S.N. Kim

State University of New York (SUNY) at Stony Brook: R. Engelmann, C.K. Jung, M.D. Marx, R.L. McCarthy, M.M. Mohammadi, M. Rijsenbeek, A.H. Sanjari, C. Yanagisawa

Institute for Assembling Technology, NIKIMT, Moscow, Russia: I.A. Bachelis, O.K. Drujilovsky, A.A. Kurkumeli

Northwestern University: D. Buchholz, B. Gobbi

Institute of Nuclear Physics, Novosibirsk, Russia: V. Aulchenko, S. Klimenko, G. Kolachev, M. Leltchouk, 5 L. Leontiev, V. Malishev, A. Maslennikov, A. Onuchin, V. Panin, S. Peleganchuk, S. Pivovarov, V. Sidorov, V. Tayursky, Yu. Tikhonov 
Oak Ridge National Laboratory: M.L. Bauer, H.R. Brashear, C.L. Britton, Jr., S. Chae, L.G. Clonts, H.O. Cohn, C. Eberle, Yu.A. Kamyshkov, E.F. Kennedy, F. Plasil, K.F. Read, M.J. Rennich,

M.L. Simpson, R. A. Todd, A.L. Wintenberg, C.C. Wynn, K.G. Young

University of Oregon: A. Arodzero, J.E. Brau, R.E. Frey, K. Furuno, D. Gao, R. Kollipara, N. Sinev, D.M. Strom, $X$. Yang

Panjab University, India: S.B. Beri, V. Bhatnagar, S. Chopra, J.M. Kohli, J.B. Singh, P.M. Sood

Peking University, Beijing, China: C.X. Lai, H.T. Liu, S.J. Xia, Y.L. Ye

University of Pittsburgh: W.E. Cleland, M. Clemen, E. Engels Jr., J. Rabel, P. Shepard, V.J. Sonnadara

Princeton University: P.P. Denes, M.M. Ito, D.R. Marlow, E.J. Prebys, R.L. Wixted

Central Institute for Structural Materials, "Prometei", St. Petersburg, Russia: V.V. Chizhikov, V.I. Kondakov

University of Rochester: J. Dunlea, G. Fanourakis, T. Ferbel, G. Ginther, C. Lirakis, F. Lobkowicz, P. Slattery, M. Zielinski

Institute of Atomic Physics, Bucharest, Romania: A. Aculai, C. Blaj, H. Bozdoc, L. Butacu, M. Ciobanu, A. Dorobantu, D. Ighicianu, G. Pascovici, V. Popa, G. Radulescu, D. Spanu, C. Stan-Sion, V. Valeanu, K. Zimmer

Rutgers University: P. Jacques, M. Kalelkar, R.J. Plano, P. Stamer

Institute of High Energy Physics, Serpukhov, Russia: N.N. Fedyakin, R.N. Krasnokutsky, R.S. Shuvalov, V.V. Sushkov

Institute of Theoretical and Experimental Physics, Moscow, Russia: V. Balagura, S. Bojarinov, S. Burov, V. Chudakov, M. Danilov, A. Droutskoi, Yu. Efremenko, V. Gavrilov, Yu. Gershtein,

A. Golutvin, A. Gordeev, Yu. Kamyshkov, I. Korolko, S. Kuleshov, L. Laptin, V. Morgunov, P. Murat, A. Nikitin, D. Onoprienko, A. Ostapchuk, V. Popov, F. Ratnikov, V. Rusinov, A. Savin, S. Shevchenko, V. Shibaev, K. Shmakov, A. Smimov, V. Stolin, E. Tarkovsky, I. Tikhomirov, V. Tchistilin, A. Zhokin

Joint Institute for Nuclear Research, Dubna, Russia: A.V. Bannikov, L.S. Barabash, S.A. Baranov, D.A. Belosludtsev, Yu.E. Bonyushkin, V.N. Bychkov, A.S. Chvyrov, A.P. Dergunov, Yu.V. Ershov,

V.N. Frolov, I. A. Golutvin, N.V. Gorbunov, Yu.A. Gornushkin, A.B. Ivanov, V.D. Kalagin, A.G. Karev, V.Yu. Karzhavin, M.Yu. Kazarinov, S.V. Khabarov, V.S. Khabarov, B.A. Khomenko, N.N. Khovansky, Yu.T. Kiryushin, O.N. Klimov, V.D. Kondrashov, A.V. Korytov, V.M. Kotov, Z.V. Krumshtein, P.A. Kulinich, V.N. Lysiakov, A.L. Lyubin, A.V. Makhankov, V.L. Malyshev, I.M. Melnichenko, Y.P. Merekov, S.A. Movchan, A.A. Nozdrin, A.G. Olshevsky, V.V. Perelygin, V.D. Peshekhonov, Yu.P. Petukhov, D. Pose, T. Predo, V.P. Rashevsky, Yu. V. Sedykh, S.Yu. Selyunin, S.V. Sergeev, L.M. Smimov, D. A. Smolin, L.G. Tkachev, V.V. Tokmenin, L.S. Vertogradov, Y.B. Viktorov, A.V. Vishnevsky, V.S. Yamburenko, V.E. Zhiltsov

Universidad San Francisco de Quito, Ecuador: S. Gangotena, B. Hoeneisen, C. Marin University of Sao Paulo, Brazil: O.P. Eboli, C.O. Escobar, P. Gouffon, M. Luksys, S.F. Novaes Seongkyunkwan University, Korea: Y.I. Choi Seoul National Teacher's College, Korea: D.G. Ku, E.H. Lee

Seoul National University, Korea: S. K. Kim, J.W. Kim, S.S. Myung

Shanghai Institute of Ceramics (SIC), Shanghai, China: X.L. Fang, P.X. Gu, J.K. Guo, G.Q. Hu, S.K. Hua, P.J. Li, D.Z. Shen, E.W. Shi, W.T. Su, Z.Y. Wei, Y.Y. Xie, L. Xu, Z.L. Xue, D.S. Yan, Z.W. Yin, X.L. Yuan, G.M. Zhao, Y.L. Zhao, W.Z. Zhong, R.M. Zhou

Shanghai Institute of Nuclear Research (SINR), Shanghai, China: X.P. Li, F.H. Yu, W.H. Zheng Sookmyeong Women's University, Korea: J.N. Park

University of South Carolina: C. Rosenfeld, J.R. Wilson

Southern Methodist University: F. Olness, T. Skwarnicki, R. Stroynowski, V. Teplitz 
St. Petersburg Nuclear Physics Institute, Russia: V.M. Andreev, V. Astashin, N. Bondar, A. Denisov, A. Golyash, V. Gratchev, M. Guriev, O. Fedin, N. Isaev, M. Ishmukhametov, V. Ivochkin, S. Kalentarova. O. Kiselev, A. Krivshich, L. Lapina, P. Levtchenko, V. Maleev, M. Nesvizhevskaya, S. Patrichev,

Yu. Platonov, O. Prokofiev, V. Razmislovich, N. Sagidova, V.M. Samsonov, V. Sarantsev, A. Schetkovski. D. Seliverstov, A. Sergeev, V. Sknar, V. Scorobogatov, A. Smirrov, E. Spiridenkov, V. Suvorov. I. Tkatch. G. Velichko, S.Volkov, Yu. Volkov, A. Vorobyov, Yu. Zhelamkov

Superconducting Super Collider Laboratory: N. Baggett, Y. Bonyushkin, ${ }^{4}$ M. Bowden, G.R. Chapman. Z. Chen, L.R. Cormell, D. Crosetto, N. Danesh, G. Deis, ${ }^{6}$ P. Dingus, M. Diwan, H. Fenker, Y. Fisyak, ${ }^{7}$ K. Freeman, M. Gamble, V. Glebov, N. Gober, M. Harris, J. Hilgart, C. Johnson, ${ }^{6}$ V. Kapoor, N. Lau, ${ }^{6}$ N. Martovetsky, ${ }^{8}$ M. Marx, ${ }^{9}$ A.W. Maschke, W.K. McFarlane, R. Meinke, C. Milner, G. Mitselmakher, N. Mokhov, A. Morelos ${ }^{12}$, K. Morgan, F. Paige, B. Parker, P. Reardon, L. Parlier, L.A. Roberts, E. Sabin, G.H. Sanders, ${ }^{11}$ B. Scipioni, I. Sheer, R. Shypit, R. Soundranayagam, F. Stocker, K. Swarts, J. Thomas, H. Uijterwaal, A. Vanyashin, ${ }^{10}$ W. Wisniewski, J. Womersley, R. Woolley, G. Word, J.C. Wu, G.P. Yost, E. Zimmer-Nixdorf

Syracuse University: G.C. Fox, W. Furmanski, T. Haupt

Institute of Physics, Academia Sinica, Taiwan: J. Antos, H.-Y. Chao, Y.C. Chen, M.T. Cheng, C.N. Chiou, T.L. Chu, C.L. Ho, M. Huang, S.C. Lee, A. Sumarokov, P.K. Teng, M.J. Wang, P. Yeh

Tbilisi State University, Georgia: J. Bagaturia, V. Djordjadze, M. Khaindrava, G.V. Melitauri, D. Mzavia, T. Sakhelashvili, R.G. Shanidze, A. Tavkhelidze, Z. Tsamalaidze

Tel Aviv University, Israel: G. Bella, O. Benary, R. Heifetz, Y. Oren

University of Tennessee: S. Berridge, W.M. Bugg, Y.C. Du, R. Kroeger, A. Weidemann

Texas A\&M: C. Gagliardi, G. Glass, Y. Lu, R. Tribble, R.C. Webb

University of Texas, Austin: C.C. Allen, G.W. Hoffmann, K. Lang, M.R. Marcin, J.L. Ritchie

University of Tirana, Albania: L. Aliko, P. Berzhani, A. Borici, E. Cabej, O. Ciftja, Edlira Ciu, A. Dede, M. Dede, B. Duka, A. Fuga, A. Gashi, D. Gjiknodi, Z. Gionai, B. Guda, E. Hysengegasi, M. Ifiti,

K. Islami, F. Klosi, M. Kugo, V. Laska, G. Leka, R. Maksim, R. Mejdani, I. Mele, Z. Mulaj, N. Nosi,

M. Panariti, K. Pance, I. Prifti, R. Sharko, M. Spiro, H. Sykja, N. Thomo, M. Teska, F. Vila, D. Xhuglini, I. Xhunga, E. Xhuvani

Tongji University, Shanghai, China: L.Y. Chen, J. Du, M. Gu, J. Wang, L.M. Wang, X. Wu, K.H. Xiang INFN, Trieste, Italy: A. Bravar, R. Giacomich, A. Penzo, P. Schiavon, A. Vacchi

Tsinghua University, Beijing, China: M. Cao, H.Y. Chen, Z.M. Chen, J.K. Deng, W.H. Gao, K.J. Kang, Y.P. Kuang, D.C. Li, Y.X. Li, L.S. Liu, Y.Q. Liu, W.Z. Luo, D.H. Nan, W.D. Ni, Y.G. Qian, J.L. Ren, R.C. Shang, K. Shen, B.X. Shi, K.R. Shi, J. Wang. J.J. Wang, J.M. Wang, Q. Wang, Y.M. Wang,

K.L. Wen, S.D. Xu, Y.P. Yi, G.Z. Yu, Y. Zhao, H.Y. Zhou, S.J. Zhu

Vanderbilt University: G. Bashindzhagyan, ${ }^{7}$ R.S. Panvini, T.W. Reeves, S. Rousakov, J.P. Venuti

Variable Energy Cyclotron Center, India: R. Bhandari, B. Simha

Vassar College: C. Schwartz

University of Washington: T. Burnett, V. Cook, D. Forbush, J. Franklin, P.M. Mockett, J. Rothberg, F. Toevs, S. Wasserbach

University of Wisconsin: J. Pfotenhauer

Wonkwang University, Korea: S.Y. Bahk

Yale University: R.K. Adair, C. Baltay, B. Barakat, R. Ben-David, W. Emmet, H. Kasha, S.L. Manly, D. Pilon, S. Sen, J. Sinnott, E. Wolin

York College: S. R. Borenstein 
Note: Collaboration Council Representatives are indicated in bold face type.

1. Research and Production Association "Diamant"

2. Research and Production Association "Integral"

3. Also in ITEP, Moscow.

4. Also in JINR, Dubna.

5. Also in Columbia University.

6. Also in LLNL.

7. Also in Moscow State University.

8. Also in Kurchatov Institute, Moscow

9. Also in SUNY at Stony Brook

10. Also in Moscow Engineering Physics Institute

11. Also in LANL

12. Also in Universidad de Guanajuato, Mexico

13. Also in Harvard University. 


\section{CONTENTS}

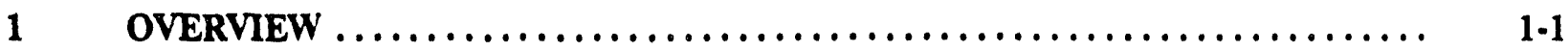

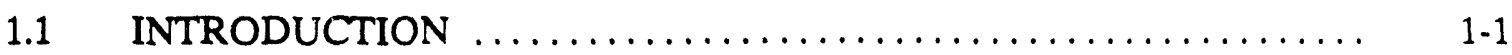

1.2 PHYSICS AND THE GEM DESIGN $\ldots \ldots \ldots \ldots \ldots \ldots \ldots \ldots \ldots \ldots, 1-2$

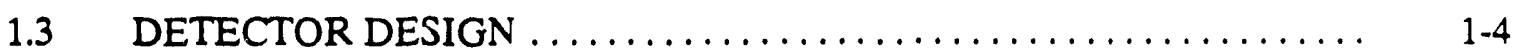

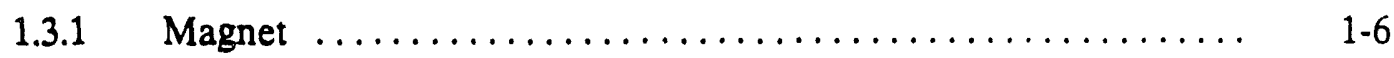

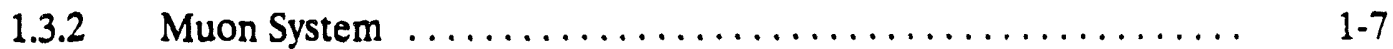

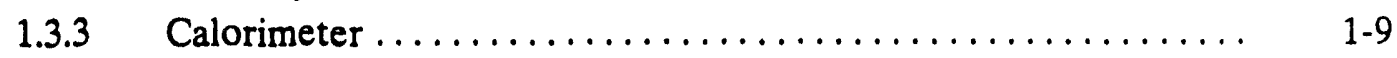

1.3.4 Central Tracker ............................... $1-11$

1.3.5 Electronics/Data Acquisition $\ldots \ldots \ldots \ldots \ldots \ldots \ldots \ldots \ldots, 1-13$

1.4 GEM PROJECT $\ldots \ldots \ldots \ldots \ldots \ldots \ldots \ldots \ldots \ldots \ldots \ldots \ldots \ldots \ldots, 1-13$

1.4.1 Assembly, Access, and Maintenance $\ldots \ldots \ldots \ldots \ldots \ldots \ldots \ldots . \ldots \ldots$

1.4.2 Detector Integration ............................ $1-14$

1.4.3 Upgrades ................................... $1-16$

1.4.4 Commissioning and Initial Operation $\ldots \ldots \ldots \ldots \ldots \ldots \ldots . \quad 1-16$

1.5 GEM COLLABORATION AND ORGANIZATION ............. 1-16

2 PHYSICS PERFORMANCE OF THE GEM DETECTOR $\ldots \ldots \ldots \ldots \ldots \ldots \ldots$ 2-1

$2.1 \quad$ INTRODUCTION $\ldots \ldots \ldots \ldots \ldots \ldots \ldots \ldots \ldots \ldots \ldots \ldots \ldots \ldots \ldots \ldots \ldots \ldots, 2-1$

2.2 MODELING THE GEM DETECTOR $\ldots \ldots \ldots \ldots \ldots \ldots \ldots \ldots \ldots, 2-3$

2.2.1 Event Generation $\ldots \ldots \ldots \ldots \ldots \ldots \ldots \ldots \ldots \ldots \ldots \ldots \ldots \ldots \ldots, 2-3$

2.2.2 Central Tracker $\ldots \ldots \ldots \ldots \ldots \ldots \ldots \ldots \ldots \ldots \ldots \ldots \ldots \ldots, 2-4$

2.2.3 Calorimeter ................................... $2-5$

2.2.4 Muon System .............................. $2-7$

2.2.5 Definition and Measurement of Photons, Electrons and Muons .. $2-8$

2.2.6 Definition and Measurement of Jets ..................... 2-9

2.2.7 Trigger Simulation ............................. $2-9$

2.3 STANDARD MODEL HIGGS PHYSICS $\ldots \ldots \ldots \ldots \ldots \ldots \ldots \ldots, 2-11$

2.3.1 Introduction ............................... $2-11$

2.3.2 $H \rightarrow \gamma \gamma$ Search for $80 \mathrm{GeV}<M_{H}<160 \mathrm{GeV} \ldots \ldots \ldots \ldots \ldots . .2-14$

2.3.3 $H(\bar{t} / W) \rightarrow y \gamma \ell X$ Search for $80 \mathrm{GeV}<M_{H}<140 \mathrm{GeV} \ldots \ldots \ldots . \quad 2-19$

2.3.4 $H \rightarrow Z Z^{*} \rightarrow \ell^{+} \ell-\ell+\ell-$ Search for $140 \mathrm{GeV}<M_{H}<2 M_{Z} \ldots . \quad 2-21$

2.3.5 $H \rightarrow Z Z \rightarrow \ell^{+} \ell^{-} \ell^{+} \ell-$ Search for $2 M_{Z}<M_{H}<800 \mathrm{GeV} \ldots \ldots \quad 2-22$

2.3.6 $H \rightarrow \mathrm{ZZ} \rightarrow \ell^{+} \ell^{-} \nu \bar{\nu}$ Search for $M_{H}=800 \mathrm{GeV} \ldots \ldots \ldots \ldots \ldots .2-23$

2.3.7 $H \rightarrow Z Z \rightarrow \ell^{+} \ell^{-} j j$ Search for $M_{H}=800 \mathrm{GeV} \ldots \ldots \ldots \ldots \ldots . .2-25$

2.4 HEAVY FLAVOR PHYSICS $\ldots \ldots \ldots \ldots \ldots \ldots \ldots \ldots \ldots \ldots \ldots \ldots \ldots \ldots \ldots \ldots, 2-26$

2.4.1 Heavy Top-Quark Detection and Mass Measurement via the $M_{\ell \mu}$ Distribution $\ldots \ldots \ldots \ldots \ldots \ldots \ldots \ldots \ldots \ldots \ldots . \quad 2-27$

2.4.2 Top-Quark Detection and Mass Measurement via the $M_{\mathrm{iij}}$ Distribution .......................... $\quad 2-29$

2.4.3 Search for a Charged Scalar in the Decay of a Heavy Top Quark $\ldots \ldots \ldots \ldots \ldots \ldots \ldots \ldots \ldots \ldots \ldots . \quad 2-32$ 
2.5 JET PHYSICS AT LARGE TRANSVERSE MOMENTUM ......... $\quad 2.35$

2.6 HIGH MASS PHYSICS AT ULTRAHIGH LUMINOSITY .......... 2.38

2.6.1 Precision Studies of New Heavy $Z^{\prime}$ Gauge Bosons .......... 2-39

2.6.2 Studies of Quark-Lepton Substructure in Drell-Yan Processes ... 2-44

2.6.3 High Mass and Luminosity Physics Studies of $\ell^{ \pm} v_{\ell}$ Modes ...... 2-46

2.7 SUPERSYMMETRY AND $\boldsymbol{F}_{T}$ SIGNATURES $\ldots \ldots \ldots \ldots \ldots \ldots \ldots, 2-48$

2.7.1 $F_{T}$ Signature for Gluinos and Squarks $\ldots \ldots \ldots \ldots \ldots \ldots \ldots \ldots . \quad 2-49$

$2.7 .2 \quad$ Leptonic Signatures ........................... $2-52$

2.7.3 SUSY Parameter Determination $\ldots \ldots \ldots \ldots \ldots \ldots \ldots \ldots \ldots . \quad 2-53$

2.8 CONCLUSIONS $\ldots \ldots \ldots \ldots \ldots \ldots \ldots \ldots \ldots \ldots \ldots \ldots \ldots \ldots \ldots \ldots, 2-53$

REFERENCES $\ldots \ldots \ldots \ldots \ldots \ldots \ldots \ldots \ldots \ldots \ldots \ldots \ldots \ldots \ldots \ldots, 2-57$

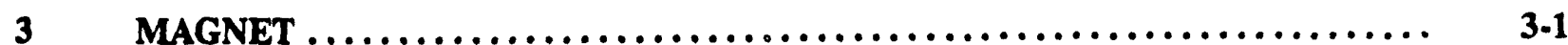

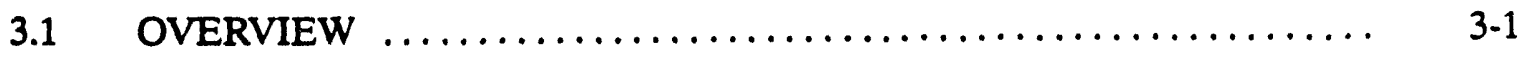

3.1.1 Introduction ................................ $3-1$

3.1.2 General Requirements for the GEM Magnet ............. 3-3

3.1.3 Trade-off, Risk, and Optimization Studies $\ldots \ldots \ldots \ldots \ldots \ldots \ldots . \quad 3-4$

3.1.4 Magnet Description and Rationale $\ldots \ldots \ldots \ldots \ldots \ldots \ldots \ldots . .3 .6$

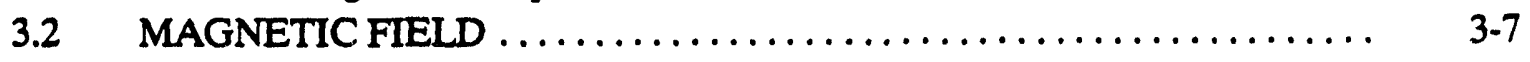

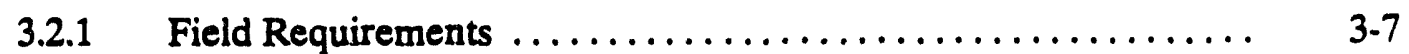

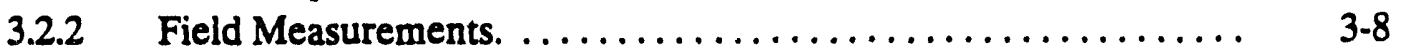

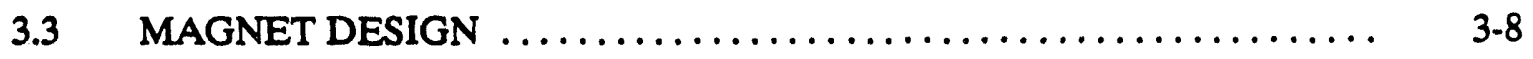

3.3.1 General Approach ............................... 3.8

3.3.2 Magnet System Interfaces ......................... 3.9

3.3.3 Coil Design .................................... 3

3.3.4 Conductor and Coil Joints .......................... 3-14

3.3.5 Forward Field Shapers $\ldots \ldots \ldots \ldots \ldots \ldots \ldots \ldots \ldots \ldots \ldots, 3-18$

3.3.6 Central Detector Support ......................... 3-19

3.4 FABRICATION, ASSEMBLY AND INSTALLATION ............ 3.21

3.4.1 Introduction $\ldots \ldots \ldots \ldots \ldots \ldots \ldots \ldots \ldots \ldots \ldots \ldots \ldots \ldots, \quad 3-21$

3.4.2 Work to Date $\ldots \ldots \ldots \ldots \ldots \ldots \ldots \ldots \ldots \ldots \ldots \ldots \ldots \ldots, \quad 3-21$

3.4.3 Conductor and Coil Joints ...........................

3.4 .4 Coil Winding $\ldots \ldots \ldots \ldots \ldots \ldots \ldots \ldots \ldots \ldots \ldots \ldots \ldots, \quad 3-22$

3.4.5 Magnet Assembly and Installation $\ldots \ldots \ldots \ldots \ldots \ldots \ldots \ldots \ldots, \quad 3-22$

$3.5 \quad$ SUPPORTING SYSTEMS $\ldots \ldots \ldots \ldots \ldots \ldots \ldots \ldots \ldots \ldots \ldots \ldots \ldots, \quad 3-22$

3.5.1 Introduction $\ldots \ldots \ldots \ldots \ldots \ldots \ldots \ldots \ldots \ldots \ldots \ldots \ldots, \quad 3-22$

3.5.2 Liquid Helium Supply System ..................... 3-23

3.5.3 Liquid Nitrogen System ........................ $3-25$

3.5.4 Power Supply and Protection Systems ................. $3-25$

3.5.5 Vacuum System ................................ 3.27

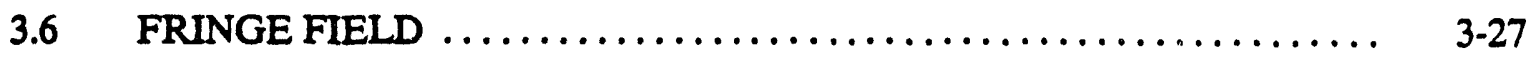

3.6.1 Introduction $\ldots \ldots \ldots \ldots \ldots \ldots \ldots \ldots \ldots \ldots \ldots \ldots \ldots \ldots \ldots \ldots \ldots, 3-27$

3.6.2 Field in the Experimental Hall and Electronic Rack Room .... 3-27 
3.6.3 Field at the Surface $\ldots \ldots \ldots \ldots \ldots \ldots \ldots \ldots \ldots \ldots \ldots \ldots, 3.28$

3.6.4 Interface with the Accelerator $\ldots \ldots \ldots \ldots \ldots \ldots \ldots \ldots \ldots .3 .28$

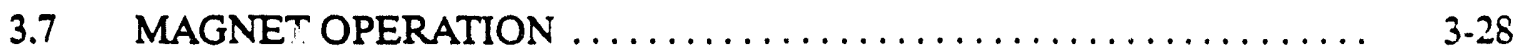

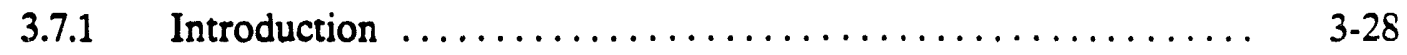

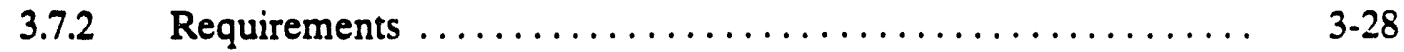

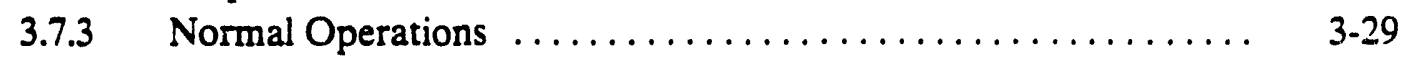

3.7.4 Off-Normal Operations . ...................... 3-29

3.7.5 Reliability, Availability, and Maintainability Analysis ........ 3-30

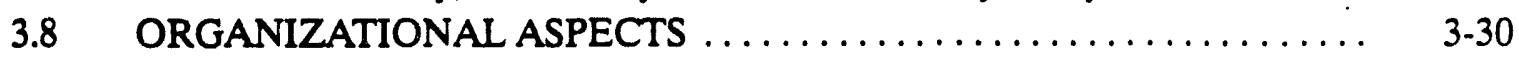

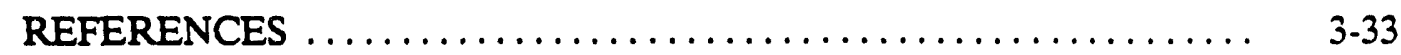

$4 \quad$ MUON $\ldots \ldots \ldots \ldots \ldots \ldots \ldots \ldots \ldots \ldots \ldots \ldots \ldots \ldots \ldots \ldots \ldots \ldots \ldots \ldots \ldots \ldots \ldots, 4.1$

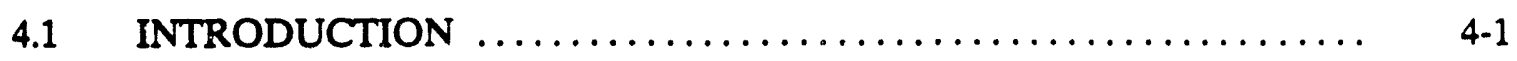

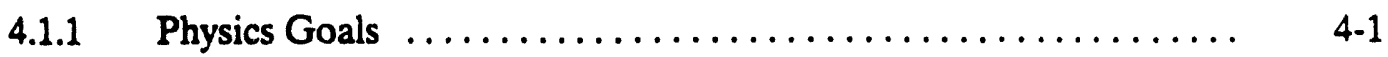

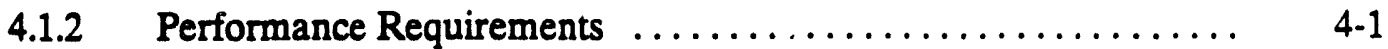

4.1.3 Design Considerations ........................ 4-2

4.1.4 Muon Spectrometer Overview ................... 4-6

4.1.5 System Design Studies ....................... 4-13

4.1.6 Detector Parameters ........................ 4-13

4.2 DETECTOR SIMULATION AND PERFORMANCE $\ldots \ldots \ldots \ldots \ldots \ldots$ 4-13

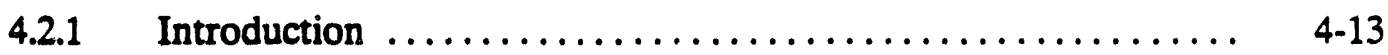

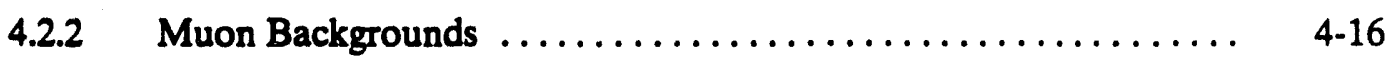

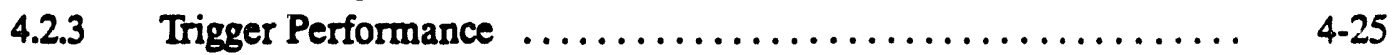

4.2.4 Pattem Recognition ........................... 4-26

4.2.5 Momentum Resolution ...................... 4-30

4.2.6 Performance for Representative Physics Processes .......... 4-33

4.2.7 Performance Verification and Monitoring .............. 4-35

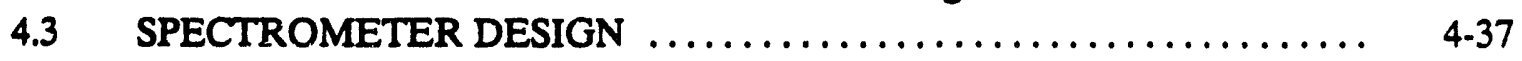

4.3.1 Magnetic Field Description and Specifications ............ 4-37

4.3.2 Chambers ............................. $4-40$

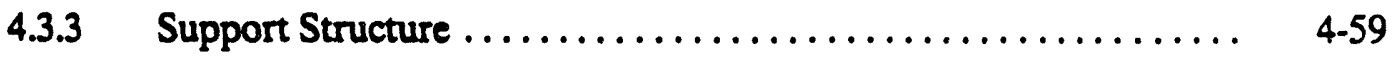

4.3.4 Alignment ............................... 4-66

4.3.5 Muon Electronics ........................... 4-81

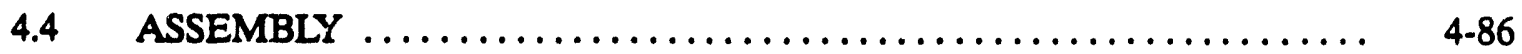

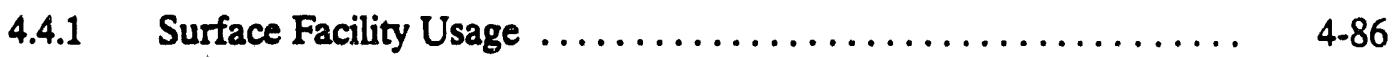

4.4.2 Barrel Region Assembly ........................ 4-87

4.4.3 Endcap Region Assembly ....................... 4.90

4.4.4 CSC Mounting, Positioning, and Alignment ............ 4-91

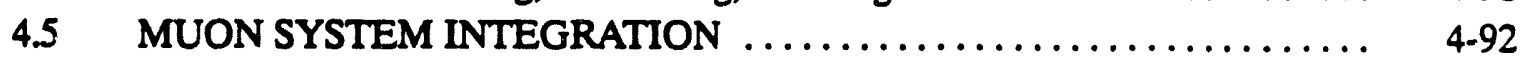

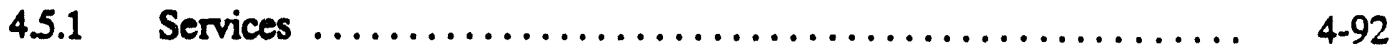

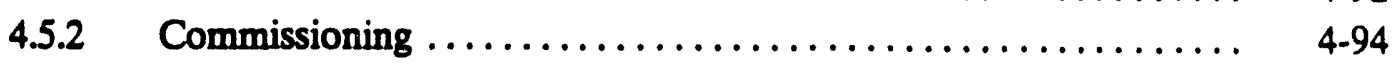

4.5.3 Access and Maintenance ...................... 4-95

4.6 R\&D ENGINEERING AND MANUFACTURING PLANS ........ 4.96

4.6.1 R\&D Engineering Plan ..................... 4-96 


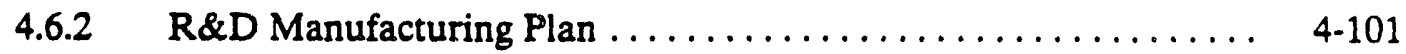

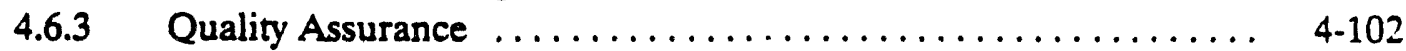

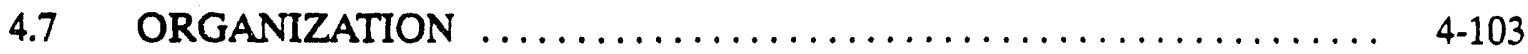

4.7.1 Organization and Institutional Responsibilities .......... 4-103

REFERENCES ................................... 4-105

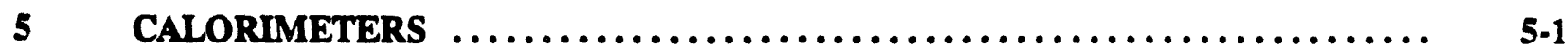

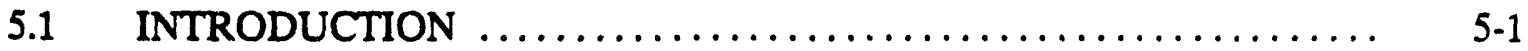

5.1.1 Physics Goals .............................. 5-1

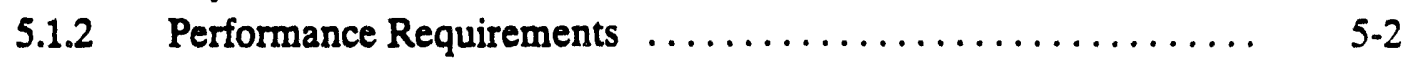

5.1.3 Design Considerations ......................... $5-3$

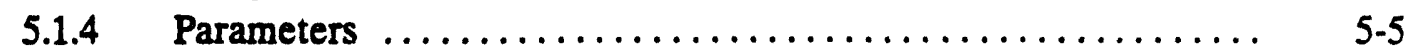

5.2 CALORIMETER SIMULATIONS AND EXPECTED PERFORMANCE 5-5

5.2.1 Electromagnetic Calorimeter ................... $5-12$

5.2.2 Absolute Calibration of the Calorimeter ............... 5-18

5.2.3 Hadronic Calorimetry ......................... 5-19

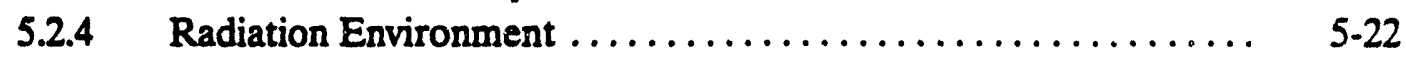

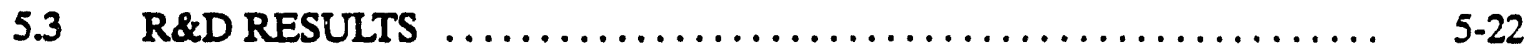

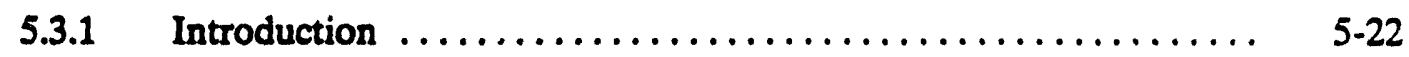

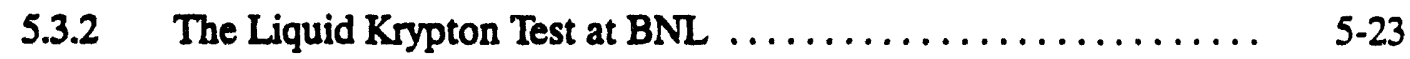

5.3.3 RD3 Results .............................. $5-25$

$5.3 .4 \quad$ Other Results .............................. $5-26$

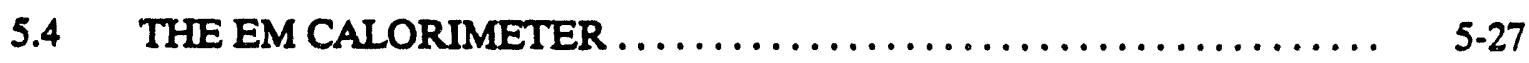

$5.4 .1 \quad$ Barrel EM Calorimeter ......................... 5-27

$5.4 .2 \quad$ Endcap EM Calorimeter ......................... 5-32

5.4.3 Ionization Calorimeter Readout Chain ............... 5-34

5.4 .4 Ionization Calorimeter Calibration System ............. 5-36

5.4.5 Ionization Calorimeter Noise Considerations ............. 5-38

5.5 THE HADRONIC CALORIMETER ................... $5-40$

5.5.1 Inner Barrel Hadronic Modules ................... 5.40

5.5 .2 Endcap Hadronic Calorimeter .................... $5-41$

5.5.3 Scintillating Barrel Calorimeter ...................... 5.45

5.5.4 Phototube Readout and Calibration System ............. $5-50$

5.5.5 Passive Absorber ............................. 5-51

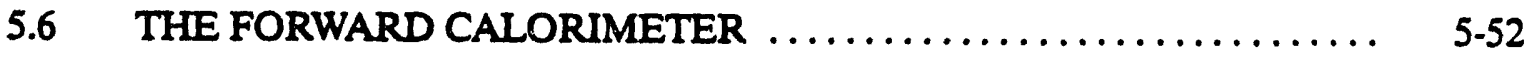

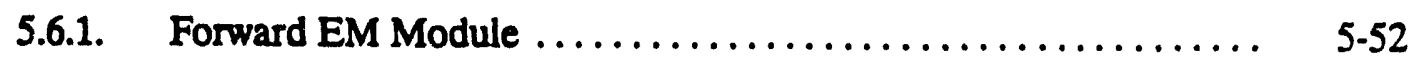

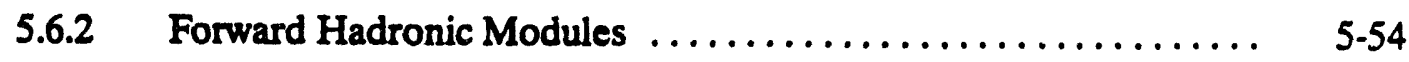

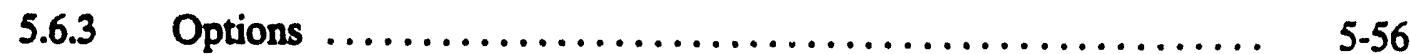

5.7 THE CALORIMETER RESEARCH AND DEVELOPMENT

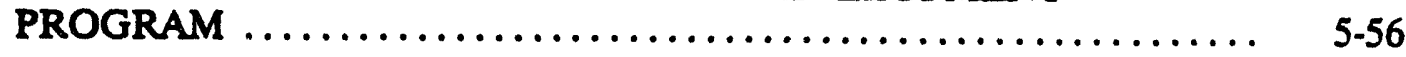

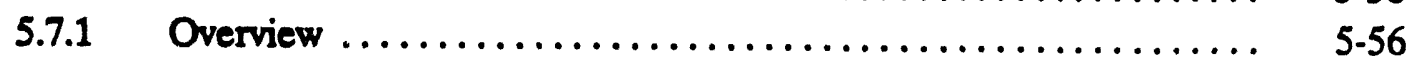

5.7 .2 The BNL R\&D Program at the AGS ................ 5-56

5.7.3 The CERN RD3 R\&D Program ................... 5.56

5.7.4 The FNAL Test Beam Program in MWEST ............ 5-57 
5.7.5 The SSCL Test Beam Program at the MEB ............ 5.59

5.7 .6 Other R\&D Tests ............................ $5-59$

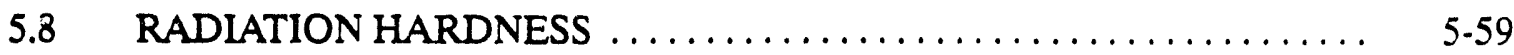

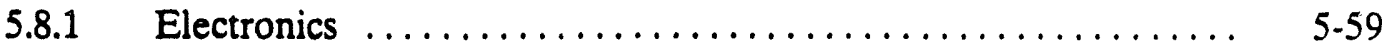

$5.8 .2 \quad$ Materials .................................. $5-60$

5.9 SERVICES: CABLES, CRYOGENICS, VALVES AND VESSELS . . . . . 5-61

5.9.1 Cables, Feedthroughs and Interconnections ............ 5-61

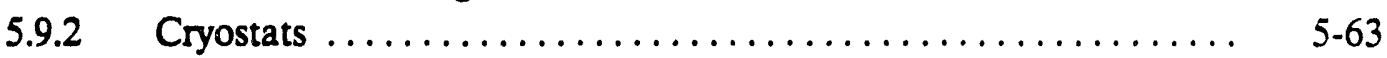

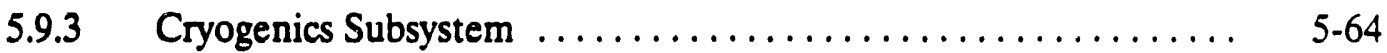

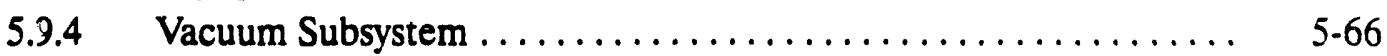

5.9.5 Instrumentation, Power and Operations $\ldots \ldots \ldots \ldots \ldots \ldots \ldots . . \ldots \ldots 6$

5.10 CALORIMETER ASSEMBLY ........................ 5.67

5.10 .1 Inner Barrel Calorimeter Assembly ................ 5-67

5.10 .2 Scintillating Barrel Calorimeter Assembly .............. 5.69

5.10 .3 Endcap Calorimeter Assembly ................... 5-69

5.10.4 Forward Hadronic Calorimeter Assembly .............. $\quad 5-70$

5.10 .5 Passive Absorber Assembly . . . . . . . . . . . . . . . . . . . . . $\quad 5-70$

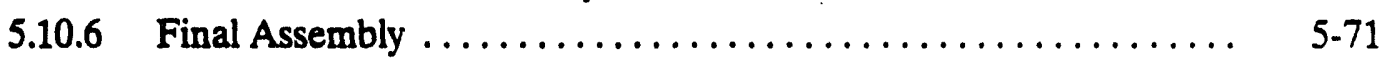

5.11 ENGINEERING AND MANUFACT JRING PLANS .......... $5-71$

5.11 .1 Engineering Process ........................... $5-71$

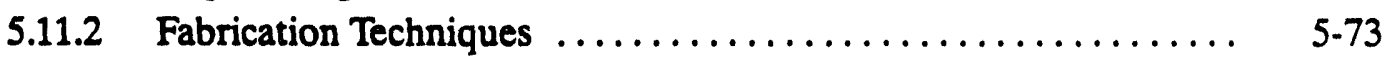

5.11 .3 Production Locations ........................... 5. 577

5.12 SCHEDULE AND RESPONSIBILITIES $\ldots \ldots \ldots \ldots \ldots \ldots \ldots \ldots \ldots \ldots \ldots$

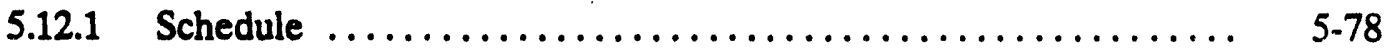

5.12 .2 Responsibilities ........................... $5-80$

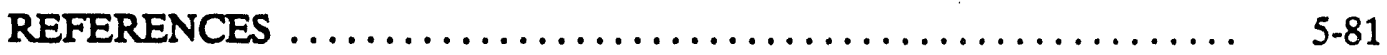

6 CENTRAL TRACKER $\ldots \ldots \ldots \ldots \ldots \ldots \ldots \ldots \ldots \ldots \ldots \ldots \ldots \ldots \ldots \ldots \ldots \ldots \ldots \ldots$

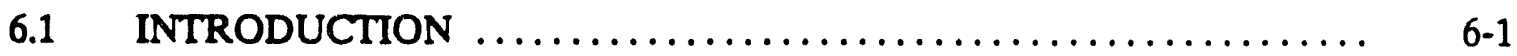

6.1.1 Physics Goals of the Central Tracker ................. 6-1

6.1.2 Design Considerations ......................... 6-1

6.1.3 Detector Configuration .......................... $6-3$

6.1.4 Rationale for Choice of Detector Configuration ............ 6-4

6.1.5 Performance Parameters ........................ 6.5. 6

6.2 DETECTOR SIMULATION AND PERFORMANCE $\ldots \ldots \ldots \ldots \ldots \ldots .6$.

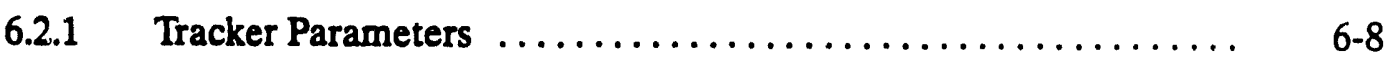

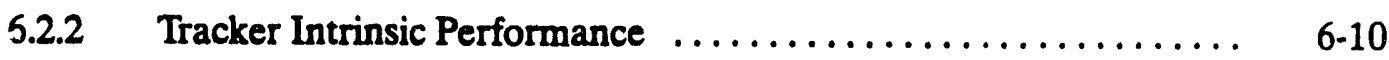

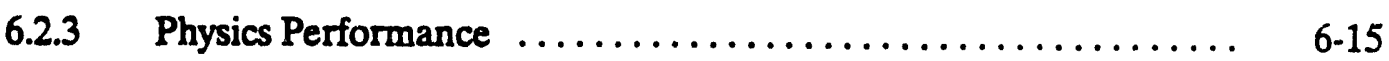

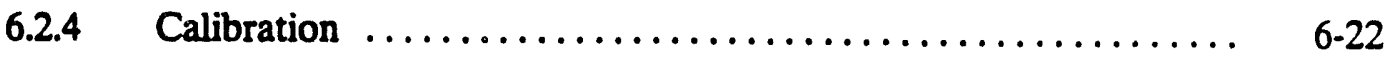

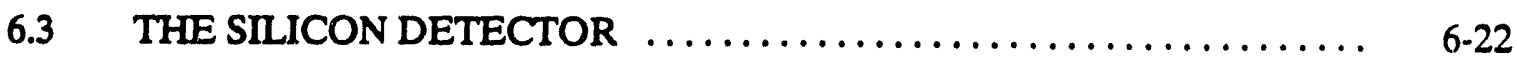

6.3.1 Design Considerations ........................ 6-22

6.3.2 Silicon Tracking System Mechanical Design ............... 6-25

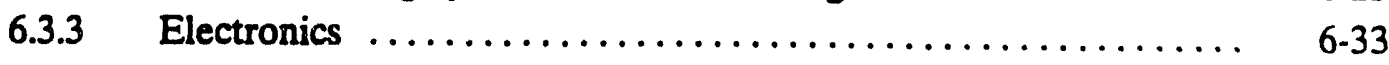


6.4 INTERPOLATING PAD CHAMBER OUTER TRACKER . . . . . . . $6-34$

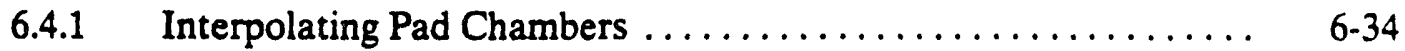

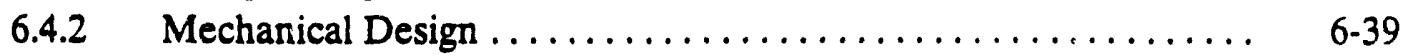

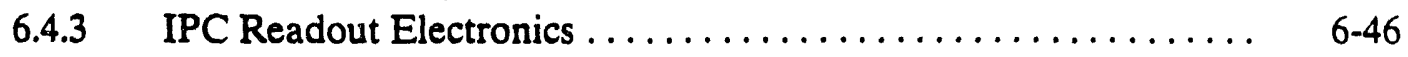

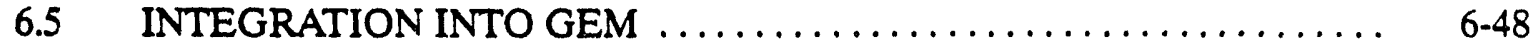

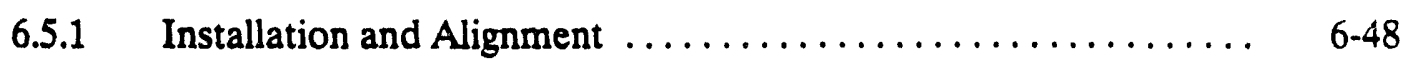

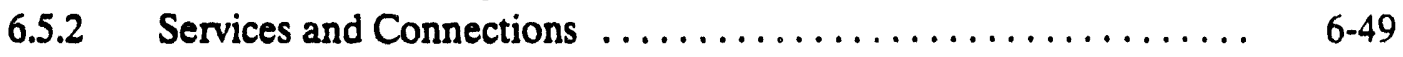

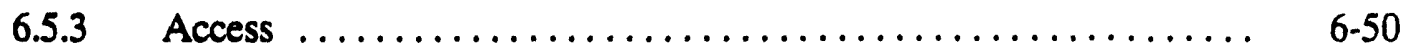

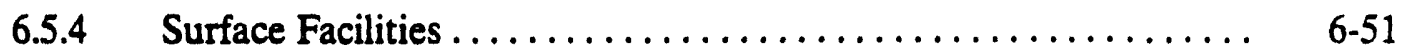

6.5.5 Safety and Environmental Impact $\ldots \ldots \ldots \ldots \ldots \ldots \ldots \ldots \ldots 6 . \ldots \ldots \ldots$

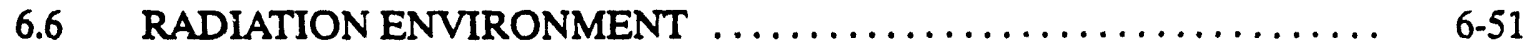

6.6.1 Radiation Dose Due to Charged Particles ............. 6-51

6.6.2 Neutral Particle Backgrounds .................... 6-52

6.6.3 Lifetime Expectancy of the Central Tracker ............... 6-53

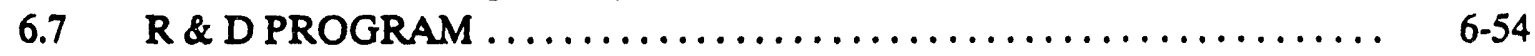

$6.7 .1 \quad$ Silicon R\&D ..................................... $6-54$

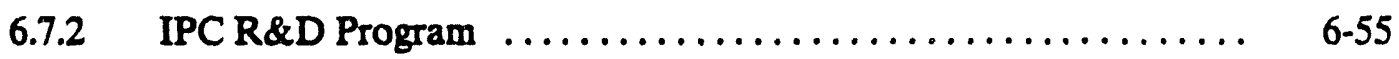

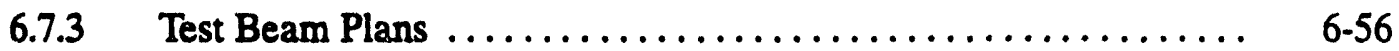

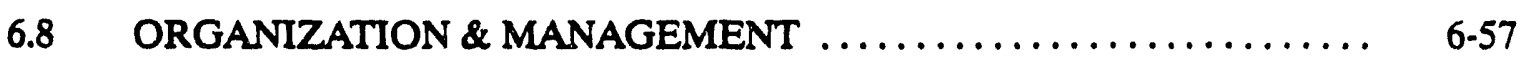

6.8.1 The Central Tracker Group ..................... 6-57

6.8.2 Personal and Institurional Responsibilities ............... 6-58

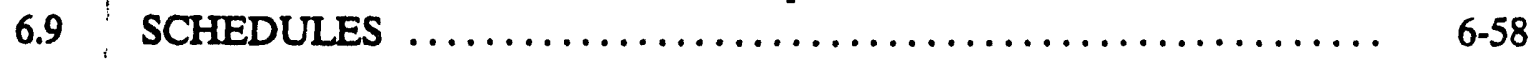

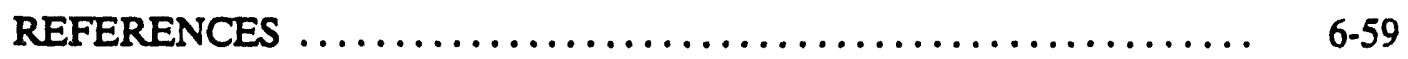

7 ELECTRONICS, TRIGGER, AND DATA ACQUISITION .............. $7-1$

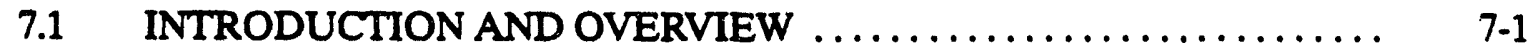

7.1.1 General Requirements and Philosophy ................ $7-1$

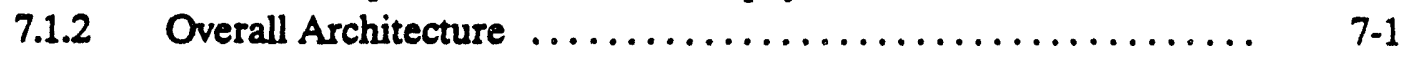

7.1.3 Front-End Subsystem Summaries $\ldots \ldots \ldots \ldots \ldots \ldots \ldots \ldots \ldots . \ldots \ldots$

7.1.4 Overview of Trigger System ...................... $7-4$

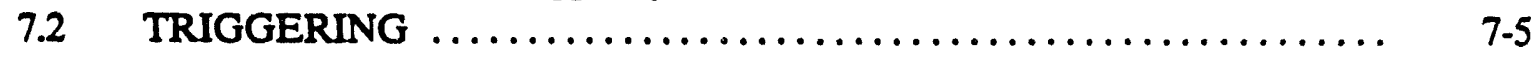

7.2.1 Calorimeter Level 1 Trigger $\ldots \ldots \ldots \ldots \ldots \ldots \ldots \ldots \ldots \ldots \ldots$ 7-6

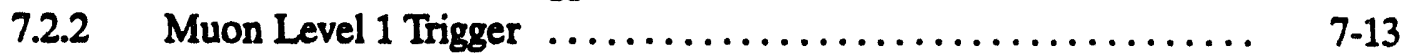

7.2.3 Level 1 Global Trigger ......................... 7-19

7.2.4 Rates and Efficiencies of Level $1 \ldots \ldots \ldots \ldots \ldots \ldots \ldots \ldots . \ldots \ldots$ 7-21

7.2.5 Level 2 Trigger . ........................... 7-29

7.3 DAQ, LEVEL 2 ARCHITECTURE, AND EVENT BUILDING ...... $7-30$

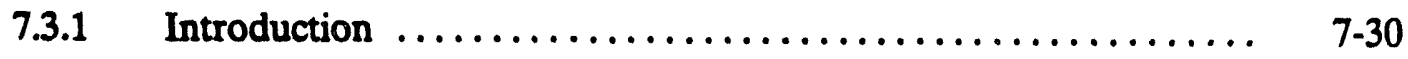

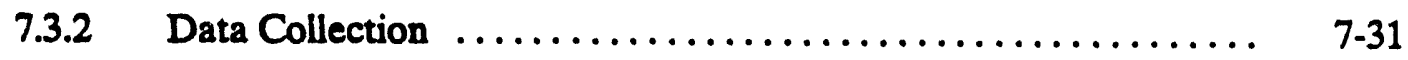

7.3.3 Event Data Collection Modules .................. 7-32

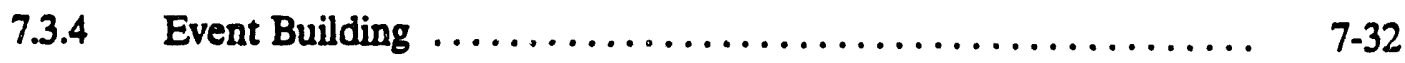

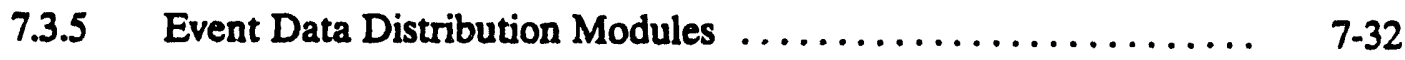

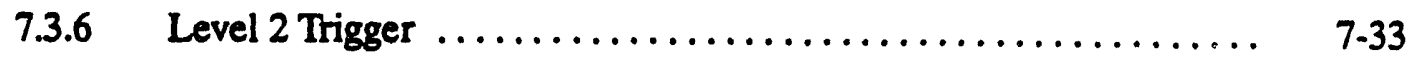

7.3.7 Level 3 Trigger and Mass Storage $\ldots \ldots \ldots \ldots \ldots \ldots \ldots \ldots \ldots \ldots$ 


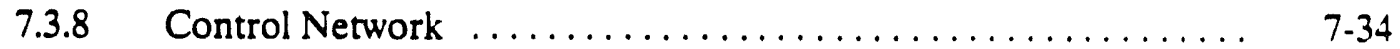

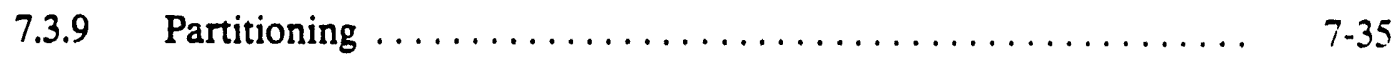

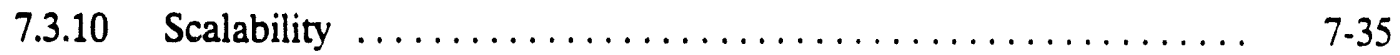

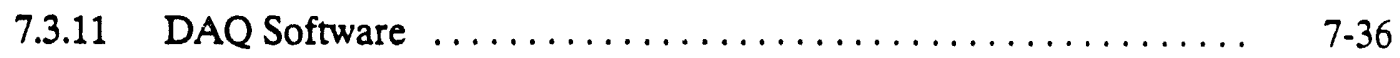

7.3.12 Simulation Studies of the DAQ System $\ldots \ldots \ldots \ldots \ldots \ldots \ldots .7 .36$

7.4 SILICON MICROS ${ }^{\top}$ RIP DETECTOR FRONT-END $\ldots \ldots \ldots \ldots \ldots \ldots \quad 7-37$

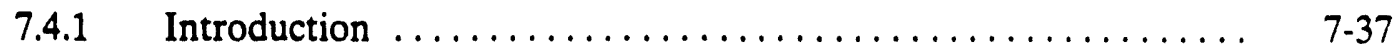

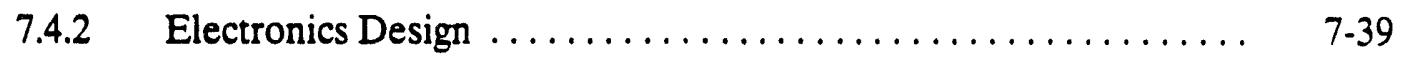

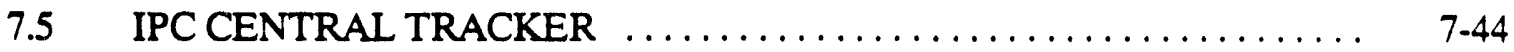

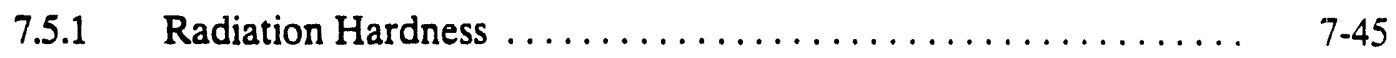

7.5.2 IPC Readout Electronics Architecture $\ldots \ldots \ldots \ldots \ldots \ldots \ldots . \quad 7.45$

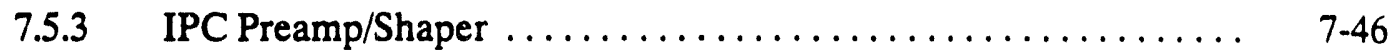

7.5.4 IPC Switched-Capacitor Array $\ldots \ldots \ldots \ldots \ldots \ldots \ldots \ldots \ldots, \quad 7-47$

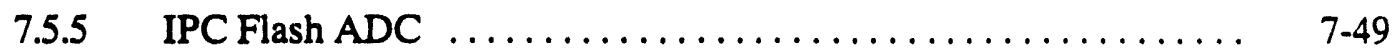

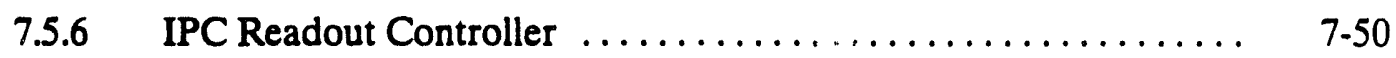

7.5.7 IPC Readout Link ............................ $7-51$

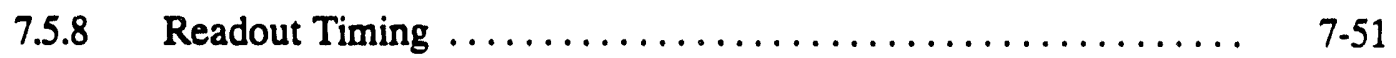

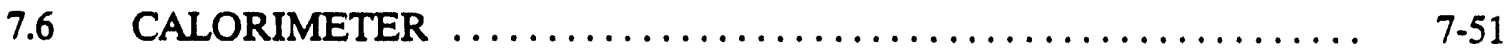

7.6.1 Overview and Requirements of the Readout $\ldots \ldots \ldots \ldots \ldots \ldots$. $7-51$

7.6.2 Front-End Electronics ........................... $\quad 7.52$

7.6.3 Calorimeter Readout Electronics ................... 7.52

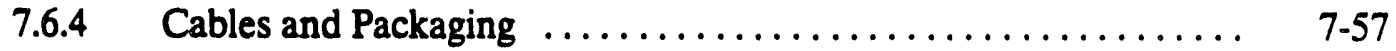

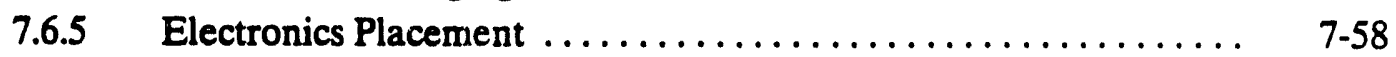

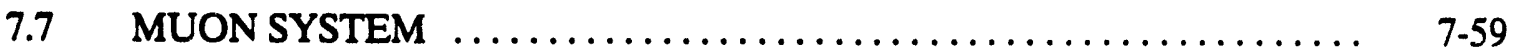

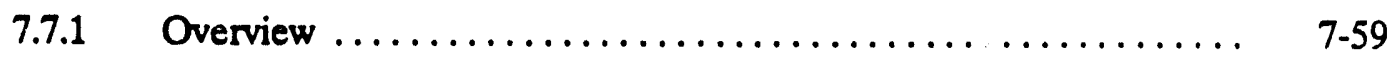

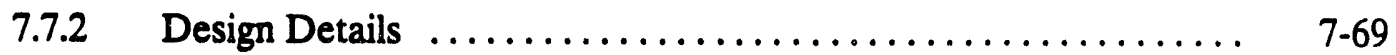

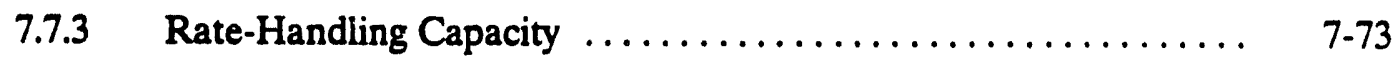

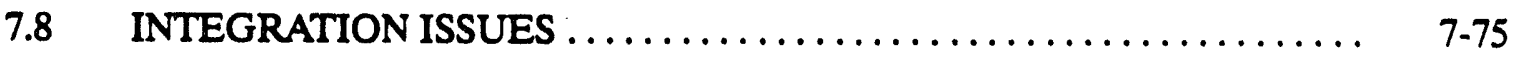

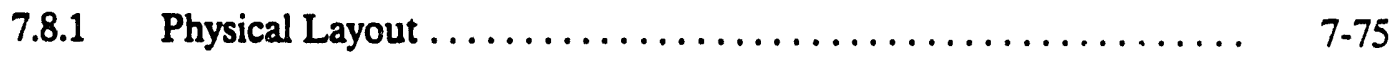

7.8.2 Fiber-Optic Links ............................. $\quad 7.75$

7.8.3 Electronics Rack Design ....................... $\quad 7.78$

7.8.4 Electronics Rack Placement and Services ................. $\quad 7.79$

7.8.5 Magnetic Field Mitigation ......................... $\quad 7-81$

7.9 ELECTRONICS GROUP ORGANIZATION ................. $7-82$

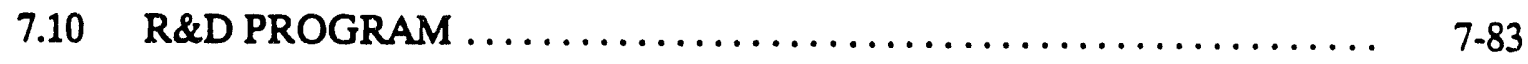

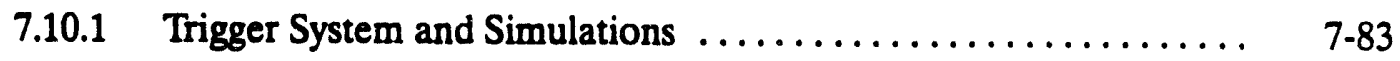

7.10 .2 DAQ System Studies and Development $\ldots \ldots \ldots \ldots \ldots \ldots \ldots \quad 7.83$

7.10.3 Silicon Tracker Electronics R\&D ..................... $7-84$

7.10 .4 IPC Tracker Electronics R\&D $\ldots \ldots \ldots \ldots \ldots \ldots \ldots \ldots \ldots \ldots \ldots \ldots \ldots, 7.84$

7.10 .5 Calorimeter Electronics R\&D ....................... 7.84

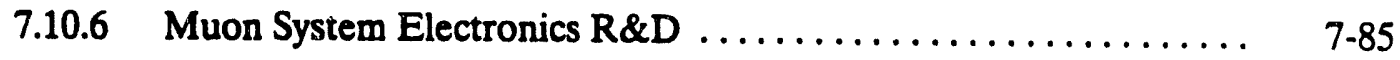

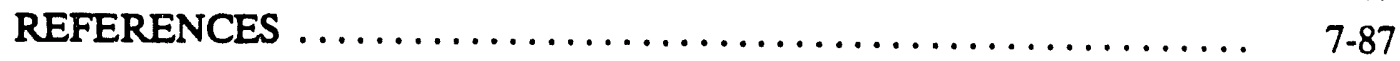


8 COMPUTING, CONTROLS, NETWORKING $\ldots \ldots \ldots \ldots \ldots \ldots \ldots \ldots \ldots, 8, \mathbf{8}$

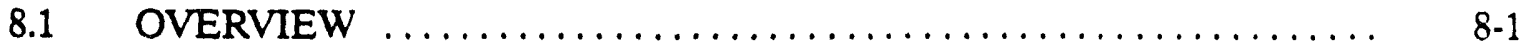

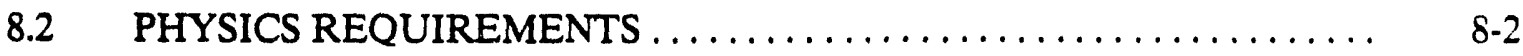

8.3 FUNCTIONAL REQUIREMENTS $\ldots \ldots \ldots \ldots \ldots \ldots \ldots \ldots \ldots \ldots, 8.5$

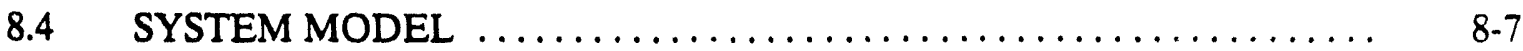

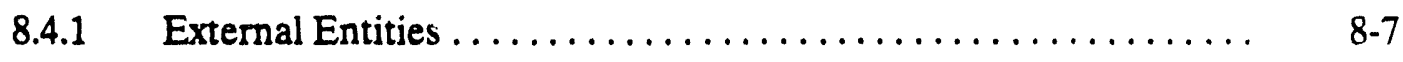

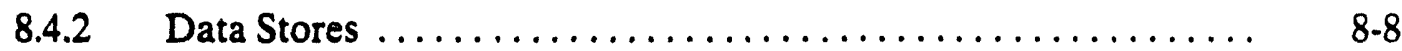

$8.4 .3 \quad$ Global Control System $\ldots \ldots \ldots \ldots \ldots \ldots \ldots \ldots \ldots \ldots . .6 .11$

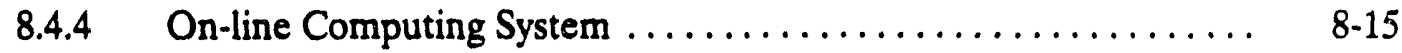

8.4 .5 Off-line Computing System ....................... $8-15$

8.5 SYSTEM DEVELOPMENT $\ldots \ldots \ldots \ldots \ldots \ldots \ldots \ldots \ldots \ldots \ldots \ldots \ldots \ldots, \quad 8-18$

8.5.1 System Development Stages ....................... $8-18$

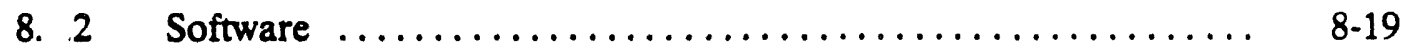

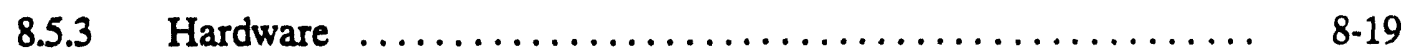

8.5 .4 Testing and Validation $\ldots \ldots \ldots \ldots \ldots \ldots \ldots \ldots \ldots \ldots, \quad 8.20$

8.6 HARDWARE IMPLEMENTATION MODEL $\ldots \ldots \ldots \ldots \ldots \ldots \ldots, \quad 8-20$

8.6 .1 Overview $\ldots \ldots \ldots \ldots \ldots \ldots \ldots \ldots \ldots \ldots \ldots \ldots \ldots \ldots, \quad 8.20$

8.6 .2 Technology Assumptions $\ldots \ldots \ldots \ldots \ldots \ldots \ldots \ldots \ldots \ldots, \quad 8-22$

8.6 .3 Standards and Integration $\ldots \ldots \ldots \ldots \ldots \ldots \ldots \ldots \ldots \ldots, \quad 8-23$

8.6 .4 Global Control System $\ldots \ldots \ldots \ldots \ldots \ldots \ldots \ldots \ldots \ldots \ldots . \quad 8.23$

8.6 .5 On-line . stem .................................. 8.25

8.6 .6 Off-line System .............................. 8.27

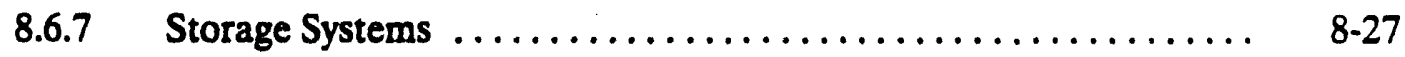

8.6.8 Data Links, Networks, Communications ................ 8.28

8.6.9 Evolution and Optimization ........................ $\quad 8-29$

8.7 SOFTWARE IMPLEMENTATION MODEL $\ldots \ldots \ldots \ldots \ldots \ldots \ldots \ldots, 8.29$

8.7 .1 Standards and Integration ........................ 8.29

8.7 .2 Framework $\ldots \ldots \ldots \ldots \ldots \ldots \ldots \ldots \ldots \ldots \ldots \ldots \ldots, \quad 8.30$

8.7.3 Detector Subsystems $\ldots \ldots \ldots \ldots \ldots \ldots \ldots \ldots \ldots \ldots \ldots . \quad 8.30$

8.7.4 Global Physics Tasks and Validation ................... 8.30

8.7.5 Global Control System $\ldots \ldots \ldots \ldots \ldots \ldots \ldots \ldots \ldots \ldots \ldots . \quad 8.31$

8.7 .6 On-line Computing System $\ldots \ldots \ldots \ldots \ldots \ldots \ldots \ldots \ldots \ldots, \quad 8.31$

8.7 .7 Off-line System $\ldots \ldots \ldots \ldots \ldots \ldots \ldots \ldots \ldots \ldots \ldots \ldots \ldots, 8,31$

8.7.8 Interface Systems $\ldots \ldots \ldots \ldots \ldots \ldots \ldots \ldots \ldots \ldots \ldots \ldots \ldots, 8,31$

8.7.9 Scale of Software Development $\ldots \ldots \ldots \ldots \ldots \ldots \ldots \ldots \ldots .6 .6 .31$

8.7 .10 Quality Assurance $\ldots \ldots \ldots \ldots \ldots \ldots \ldots \ldots \ldots \ldots \ldots ., 8-32$

8.8 DEVELOPMENT PROJECTS $\ldots \ldots \ldots \ldots \ldots \ldots \ldots \ldots \ldots \ldots \ldots, \quad 8.32$

$8.8 .1 \quad$ Detector Simulations ............................. $\quad 8.32$

8.8.2 Global Physics Tasks $\ldots \ldots \ldots \ldots \ldots \ldots \ldots \ldots \ldots \ldots \ldots . \quad 8-34$

8.8.3 Software Framework $\ldots \ldots \ldots \ldots \ldots \ldots \ldots \ldots \ldots \ldots \ldots \ldots, \quad 8.34$

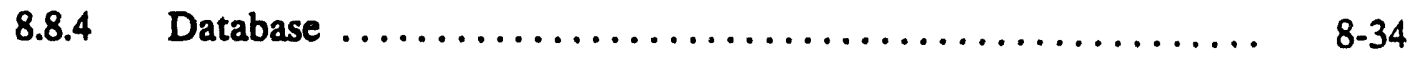

8.8.5 Computing Systems $\ldots \ldots \ldots \ldots \ldots \ldots \ldots \ldots \ldots \ldots \ldots, 8-34$ 


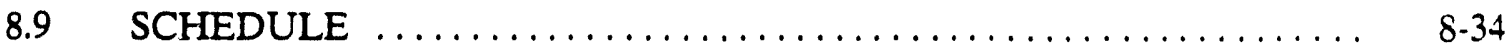

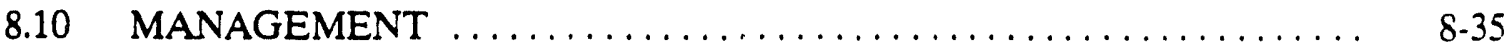

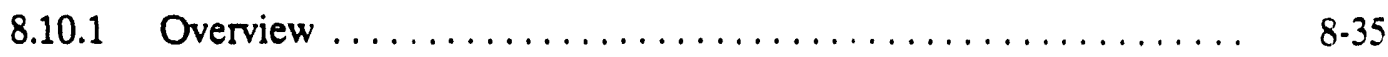

8.10 .2 Review Process $\ldots \ldots \ldots \ldots \ldots \ldots \ldots \ldots \ldots \ldots \ldots \ldots . .6 .35$

8.10.3 Physics Research Computing Department Role ........... $8-35$

8.10 .4 Documentation ............................... $8-36$

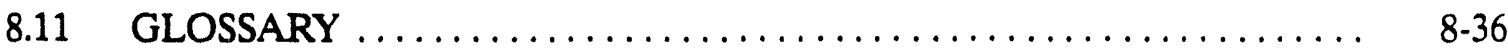

REFERENCES $\ldots \ldots \ldots \ldots \ldots \ldots \ldots \ldots \ldots \ldots \ldots \ldots \ldots \ldots \ldots \ldots \ldots \ldots \ldots, \quad 8-39$

9 FACILITIES, ASSEMBLY, AND INSTALLATION $\ldots \ldots \ldots \ldots \ldots \ldots \ldots \ldots ., 9.1$

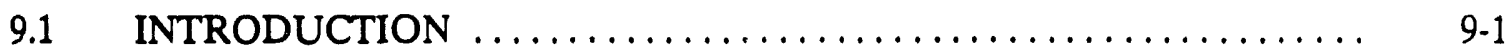

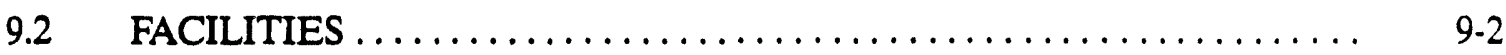

9.2.1 Surface Facilities ............................ 9.3

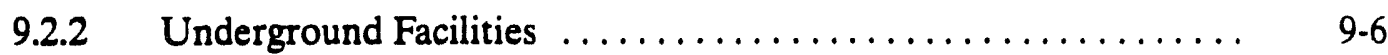

9.2.3 GEFUR $\ldots \ldots \ldots \ldots \ldots \ldots \ldots \ldots \ldots \ldots \ldots \ldots \ldots \ldots \ldots \ldots \ldots \ldots, \quad 9-10$

$9.3 \quad$ ASSEMBLY ......................................... $9-10$

9.3.1 Assembly Facilities and Tools ................... $9-10$

9.3.2 Assembly Strategy and Resources $\ldots \ldots \ldots \ldots \ldots \ldots \ldots \ldots . \quad 9-10$

9.3.3 Assembly Schedule and Contingency Plan $\ldots \ldots \ldots \ldots \ldots \ldots$ 9. $9-11$

9.3.4 Assembly Descriptions ......................... 9-11

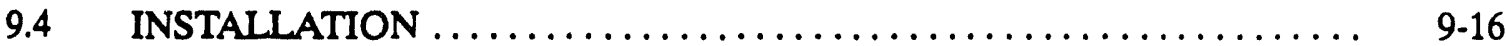

9.4.1 Installation Facilities and Tools ...................... 9-16

9.4.2 Installation Strategy and Resources .................. 9.17

9.4.3 Installation Schedule and Contingency Plan .............. 9-17

9.4.4 Installation Descriptions ......................... 9.18

10 ACCESS; MAINTENANCE, AND OPERATIONS $\ldots \ldots \ldots \ldots \ldots \ldots \ldots \ldots \ldots .10 .1$

10.1 INTRODUCTION $\ldots \ldots \ldots \ldots \ldots \ldots \ldots \ldots \ldots \ldots \ldots \ldots \ldots \ldots \ldots \ldots \ldots, 10.1$

10.1.1 Overview .................................... 10.1

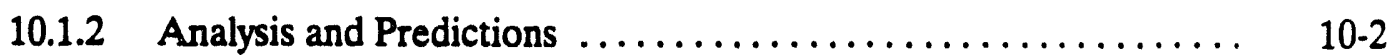

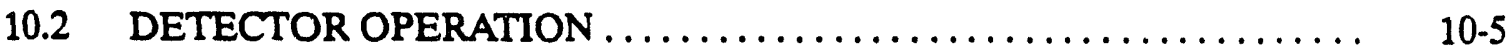

10.2.1 Operational Goals $\ldots \ldots \ldots \ldots \ldots \ldots \ldots \ldots \ldots \ldots \ldots \ldots, 10.5$

10.2.2 Detector Start-up .............................. 10.5

10.3 MAINTENANCE AND ACCESS CONCEPT .................. 10-6

10.3.1 Normal Operations (No Underground Hall Access Permitted) ... 10.9

10.3.2 Short-Term (Restricted Access) . ...................... 10.9

10.3.3 Intermediate-Term (Partial Open Access) . . . . . . . . . . . . 10-10

10.3.4 Long-Term (Full Open Access) .................... 10-11

10.4 SUBSYSTEM MAINTENANCE ACCESS REQUIREMENTS ........ 10-12

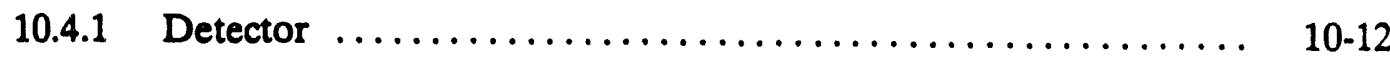

10.4.2 Electronics .................................. 10-13

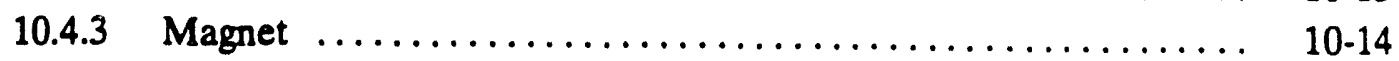

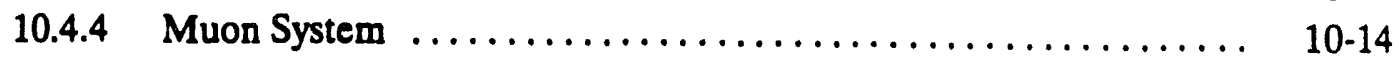

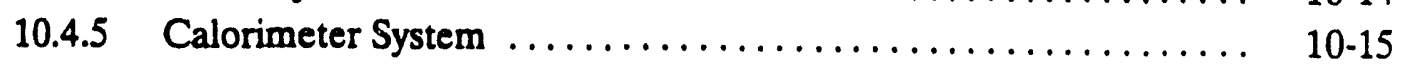


10.4.6 Central Tracker System . . . . . . . . . . . . . . . . . . .

10.4.7 Beamline .................................. 10 15

REFERENCES .............................. 10.17

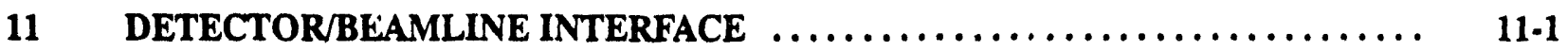

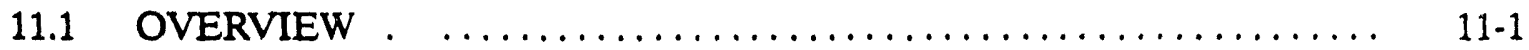

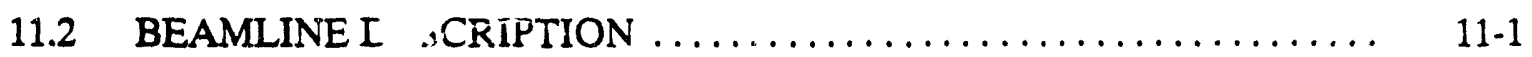

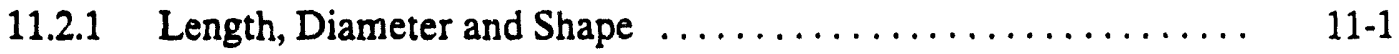

11.2.2 Material and Thickness ......................... 11-3

11.3 PRESSURE REQUIREMENTS IN THE BEAM PIPE
VACUUM SYSTEM $\ldots \ldots \ldots \ldots \ldots \ldots \ldots \ldots \ldots \ldots \ldots \ldots \ldots \ldots \ldots \ldots \ldots \ldots$

11.3.1 Ion Pumps and Non-Evaporable Getter (NEG) Pumps ....... 11-3

11.3.2 Calculated Results ........................... 11-4

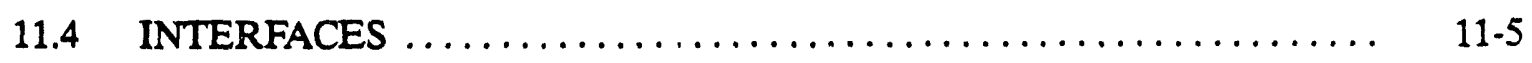

11.4.1 Detector Beamline, Machine Interface ............... 11-5

11.4.2 Beamline,Detector Interface Assembly ............... 11-5

REFERENCES ............................. $11-7$

12 RADIATION ENVIRONMENT AND SHIELDING $\ldots \ldots \ldots \ldots \ldots \ldots \ldots \ldots \ldots \ldots$

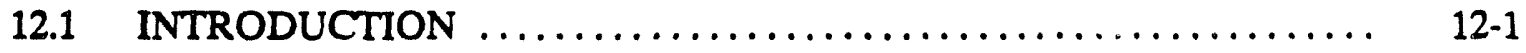

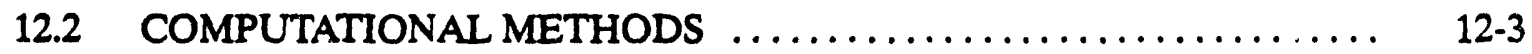

12.3 ENERGY DEPOSITION ............................... $12-4$

12.3.1 Contribution by Protons Lost from the Beam . . . . . . . . . . . $12-4$

12.3.2 Contribution from Residual Gas in the Beam Pipe .......... 12-4

12.3.3 Contribution from Collisions at the Interaction Point ......... 12-5

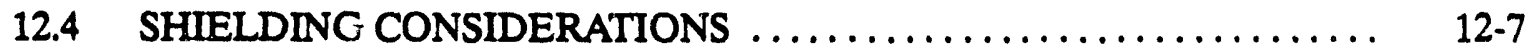

12.4.1 Location and Aperture of the Collimators .............. 12-7

12.4.2 Shielding for the Collimators ..................... 12-7

12.4.3 The Forward Calorimeter ...................... 12-14

12.4.4 Beam Pipe Considerations ...................... 12-15

12.4.5 Summary of Particle Fluxes ..................... 12-16

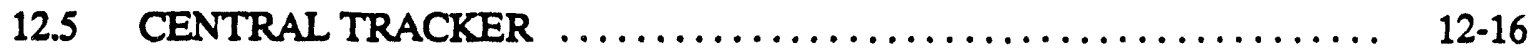

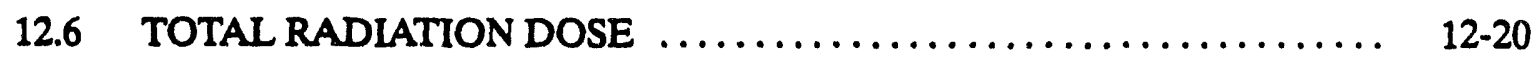

12.6.1 Charged Particle Dose ........................ 12-21

12.6.2 Dose Due to Electromagnetic and Hadronic Showers ........ 12-22

12.6.3 Dose Due to Neutrons ....................... 12-22

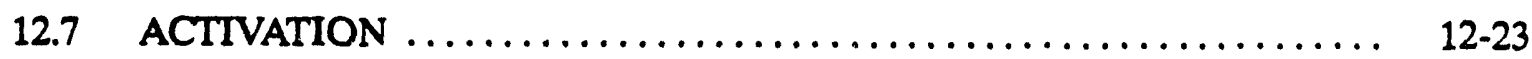

12.7.1 CINDER Calculation of Activation ................. 12-23

12.7.2 Activation Due to Low Energy Neutrons ............... 12-24

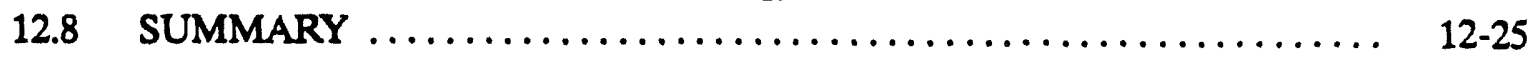

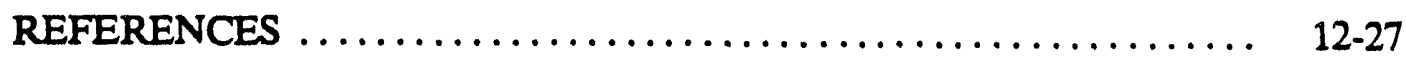

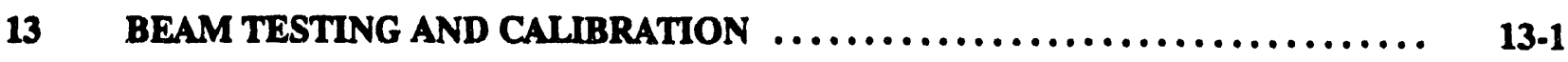

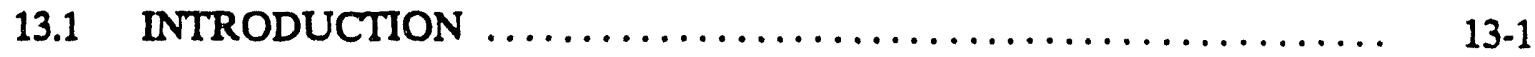


13.2 GOALS OF BEAM TESTING AND CALIBRATION ........... $13-1$

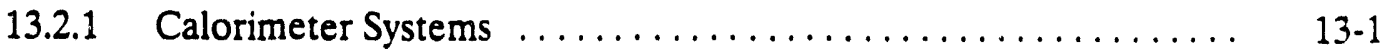

13.2.2 Muon System . . . . . . . . . . .

13.2.3 Central Tracker ............................. 13-2

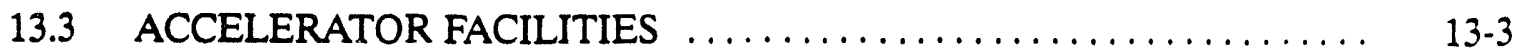

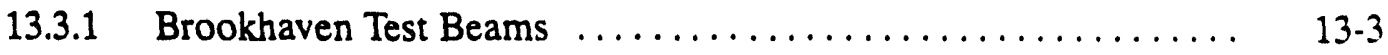

13.3.2 Fermilab Test and Calibration Beams ............... 13-3

13.3.3 SSCL Test and Calibration Beams ................. 13-4

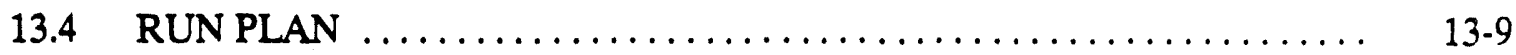

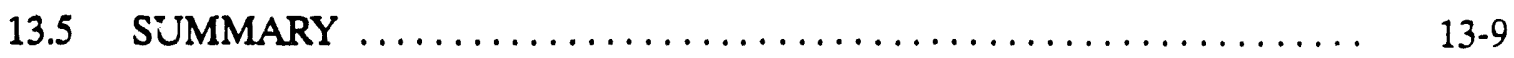

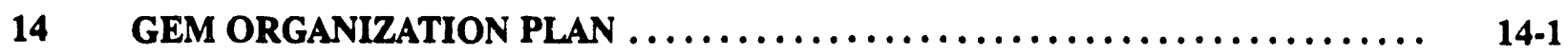

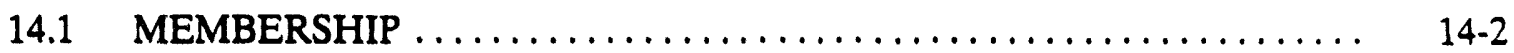

14.2 THE COLLABORATION COUNCIL ..................... $14-2$

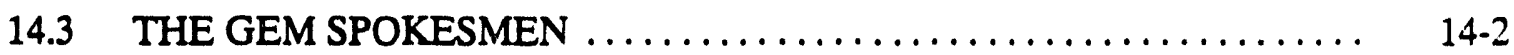

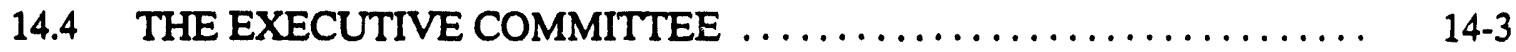

14.5 THE GEM PROJECT MANAGER $\ldots \ldots \ldots \ldots \ldots \ldots \ldots \ldots \ldots \ldots \ldots \ldots \ldots$

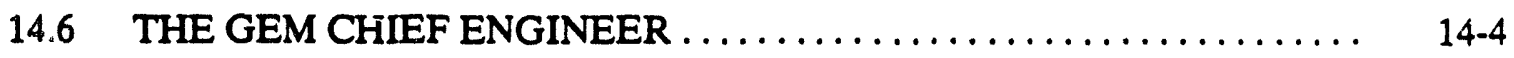

14.7 THE INTERNATIONAL COMMITTEE $\ldots \ldots \ldots \ldots \ldots \ldots \ldots \ldots \ldots \ldots \ldots \ldots$

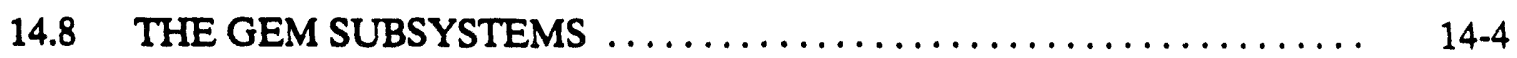

14.9 ADMISSION OF NEW COLLABORATORS INTO GEM ......... $14-5$

14.10 ADOPTION OF THIS ORGANIZATION PLAN .............. 14-5

14.11 AMENDMENTS TO THIS ORGANIZATION PLAN ........... 14-5

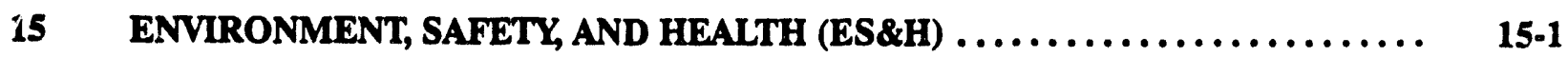

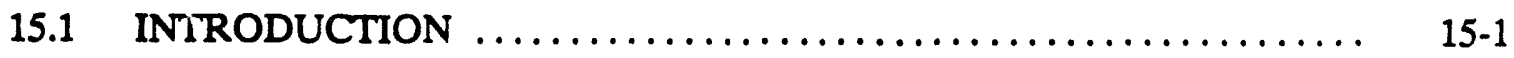

15.2 DETECTOR SAFETY OVERVIEW .................... $15-2$

15.3 MAGNET AND FORWARD FIELD SHAPER ............. 15-2

15.3.1 Safety Overview ............................. 15-2

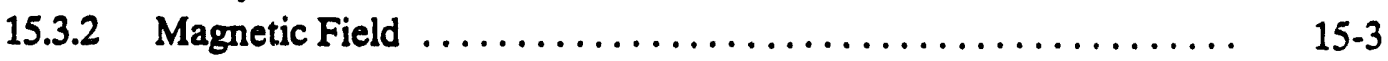

15.3.3 Forward Field Shapers . . . . . . . . . . . . . . . . . . . . 15-4

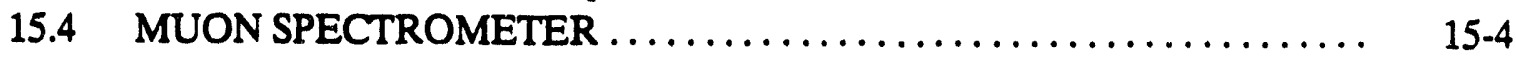

15.4.1 Safety Overview ............................... 15-4

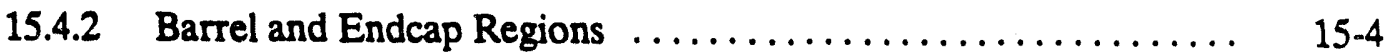

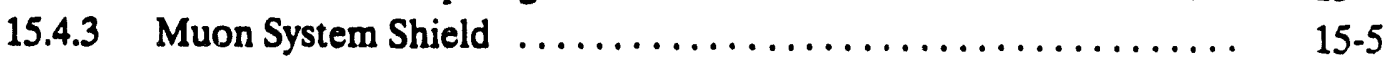

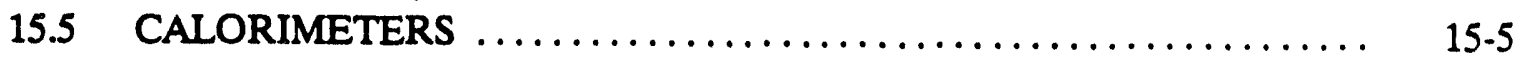

15.5.1 Safety Overview ................................ 15-5

15.5.2 Inner Barrel and Endcap Calorimetry ................. 15-5

15.5.3 Scintillating Barrel Calorimetry ........................ 15-6

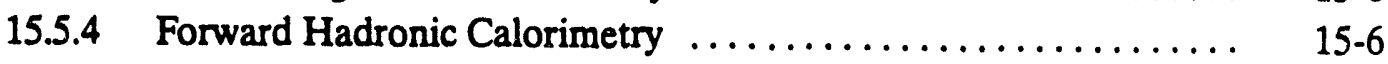

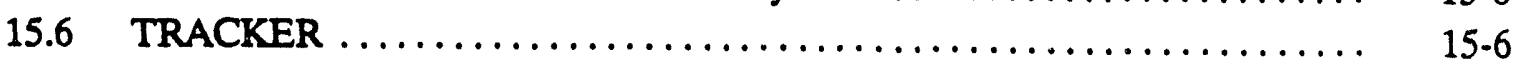

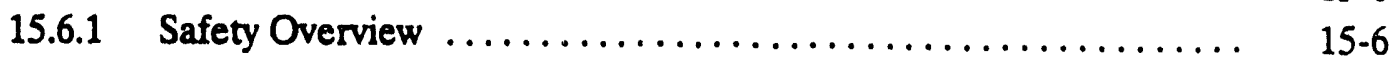

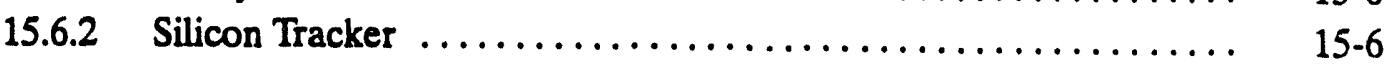

15.6.3 Interpolating Pad Chamber Tracker ................ 15-7 


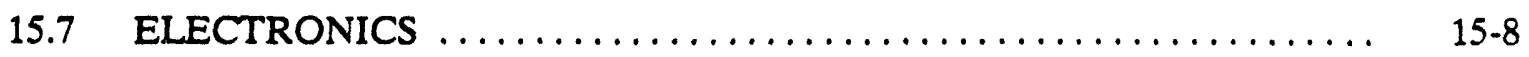

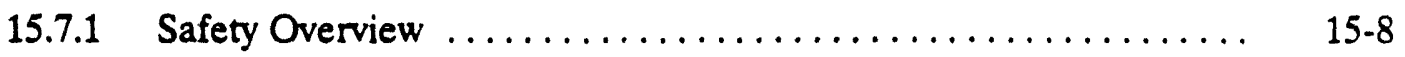

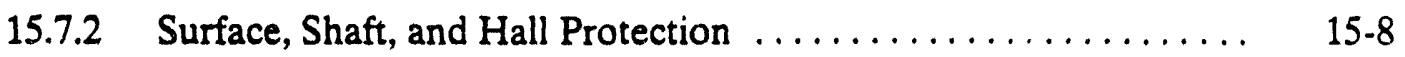

15.7.3 Electronics Rooms .............................. 15.8

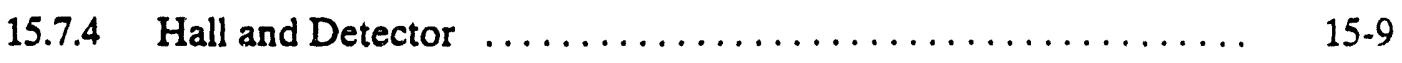

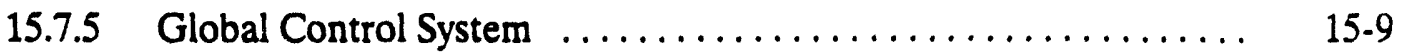

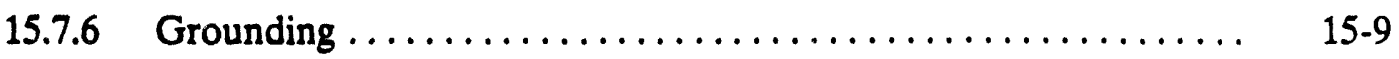

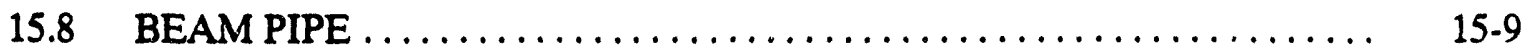

15.9 CENTRAL DETECTOR SUPPORT AND STRUCTURES $\ldots \ldots \ldots \ldots, 15-10$

15.10 ASSEMBLY, INSTALLATION, AND UPGRADES $\ldots \ldots \ldots \ldots \ldots \ldots .15-10$

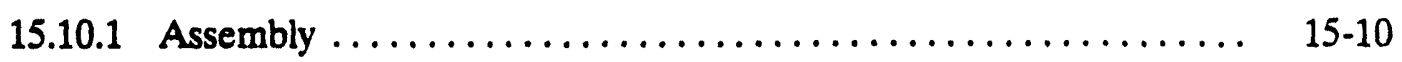

15.10 .2 Installation $\ldots \ldots \ldots \ldots \ldots \ldots \ldots \ldots \ldots \ldots \ldots \ldots \ldots \ldots \ldots, 15-11$

15.11 BEAM TESTING AND CALIBRATION $\ldots \ldots \ldots \ldots \ldots \ldots \ldots \ldots, 15-12$

15.12 UTILITIES ........................................ $15-12$

15.12.1 Water Systems $\ldots \ldots \ldots \ldots \ldots \ldots \ldots \ldots \ldots \ldots \ldots \ldots \ldots \ldots, 15-12$

15.12.2 Heating Ventilation and Air Conditioning (HVAC) System ..... 15-12

15.12.3 Power System ............................... $15-13$

15.12.4 Cryogenic/Gas System ........................... 15-13

15.12 .5 Vacuum System ................................ $15-14$

15.13 RADIATION AND RADIOACTIVE MATERIALS .............. 15-14

15.13.1 Introduction ................................ 15-14

15.13.2 Radiation Work and Controlled Areas ................. 15-14

15.13.3 Work on Activated Components .................... 15-15

15.13.4 Radiation Monitoring ............................ 15-15

15.13.5 Experimental Hall Construction ...................... 15-15

15.13.6 Shielding .................................... 15-15

15.13.7 Air Activation ............................... 15-16

15.13.8 Groundwater Activation .......................... 15-16

15.14 FIRE PROTECTION SYSTEMS $\ldots \ldots \ldots \ldots \ldots \ldots \ldots \ldots \ldots \ldots \ldots, 15-16$

15.14.1 Detection.................................... 15-16

15.14.2 Suppression ................................... $15-16$

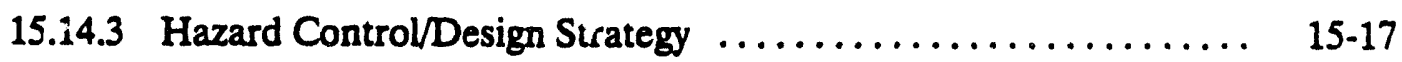

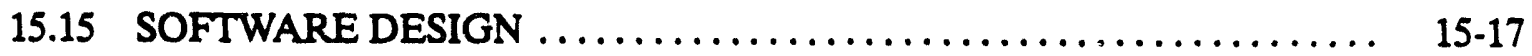

15.16 ENVIRONMENT AND AIR QUALITY $\ldots \ldots \ldots \ldots \ldots \ldots \ldots \ldots, 15-18$

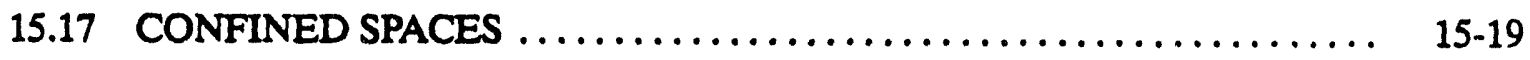

15.18 POTENTIAL FAILURES $\ldots \ldots \ldots \ldots \ldots \ldots \ldots \ldots \ldots \ldots \ldots \ldots, 15-20$

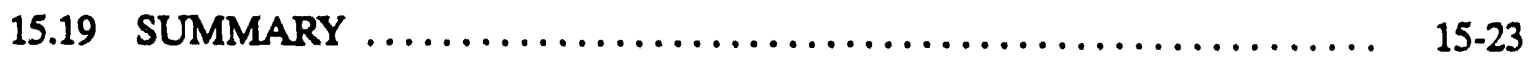

REFERENCES $\ldots \ldots \ldots \ldots \ldots \ldots \ldots \ldots \ldots \ldots \ldots \ldots \ldots \ldots \ldots \ldots, 15-25$

16 COST/SCHEDULE BASELINE SUMMMARY $\ldots \ldots \ldots \ldots \ldots \ldots \ldots \ldots \ldots \ldots . . \ldots, 16-1$

REFERENCES $\ldots \ldots \ldots \ldots \ldots \ldots \ldots \ldots \ldots \ldots \ldots \ldots \ldots \ldots, 16-3$ 


\section{LIST OF FIGURES}

\section{Chapter 1}

1-1. Perspective view of the GEM detector. $\ldots \ldots \ldots \ldots \ldots \ldots \ldots \ldots \ldots \ldots \ldots \ldots \ldots$

1-2. The physics reach of GEM. $\ldots \ldots \ldots \ldots \ldots \ldots \ldots \ldots \ldots \ldots \ldots \ldots \ldots \ldots \ldots \ldots \ldots 1-3$

1-3. The high luminosity performance of GEM. $\ldots \ldots \ldots \ldots \ldots \ldots \ldots \ldots \ldots \ldots \ldots \ldots$

1-4. Vertical section of the GEM detector. $\ldots \ldots \ldots \ldots \ldots \ldots \ldots \ldots \ldots \ldots \ldots \ldots \ldots$

1-5. Quarter view of the detector showing the muon system, including shielding. . . . . $1-8$

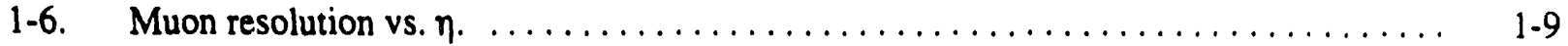

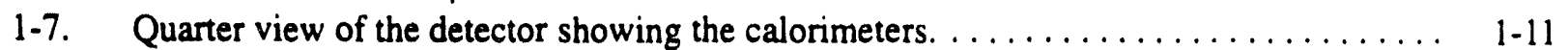

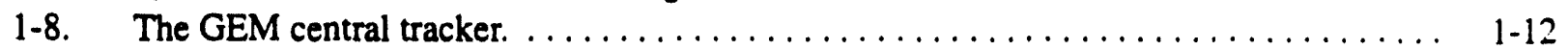

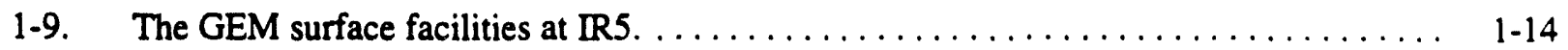

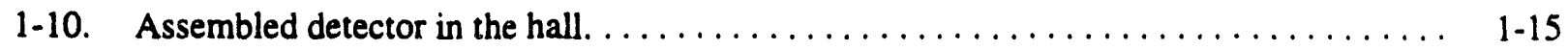

\section{Chapter 2}

2-1. Geometry used in gemfast. ............................ $2-3$

2-2. Central tracker resolution in gemfast for $\mathcal{L}=10^{33} \mathrm{~cm}^{-2} \mathrm{~s}^{-1} . \ldots \ldots \ldots \ldots \ldots 2-4$

2-3. Central tracker resolution in gemfast for $\mathcal{L}=10^{34} \mathrm{~cm}^{-2} \mathrm{~s}^{-1} \ldots \ldots \ldots \ldots \ldots \ldots 2-4$

2-4. Central tracker efficiency in gemfast. . . . . . . . . . . . . . . . . . . $2-5$

2-5. Electron momentum resolution in gemfast and comparison with GEANT result. .... 2-5

2-6. Electromagnetic calorimeter energy for pions in gemfast and comparison with

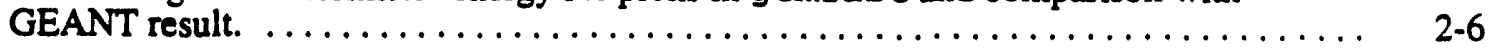

2-7. Electromagnetic calorimeter resolution in gemfast. ................ $2-6$

2-8. Calorimeter pileup distribution in gemfast. $\ldots \ldots \ldots \ldots \ldots \ldots \ldots \ldots \ldots \ldots \ldots$

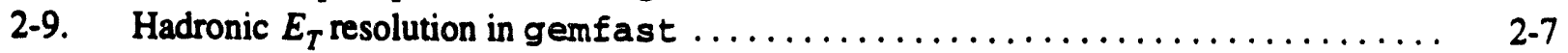

2-10. Muon resolution in gemfast. . . . . . . . . . . . . . . .

2-11. Muon acceptance in gemfast. ........................... $2-8$

2-12. Muon energy loss in gemfast and comparison with GEANT result. . . . . . . . . $2-8$

2-13. Jet correction function in gemfast. ........................ 2.9

2-14. Selected trigger acceptances.in gemfast. $\ldots \ldots \ldots \ldots \ldots \ldots \ldots \ldots \ldots \ldots \ldots \ldots \ldots$ 2-10

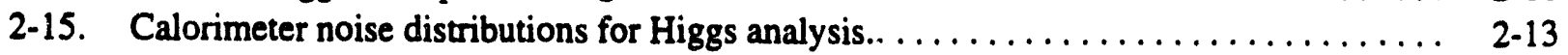

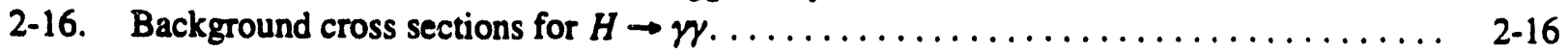

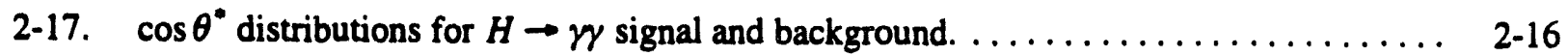

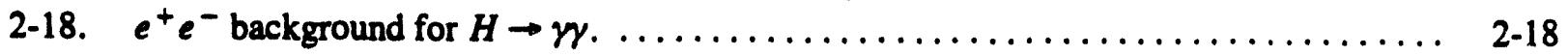

2-19. $H \rightarrow \gamma \gamma$ mass peaks over subtracted background for $10 \mathrm{fb}^{-1} \ldots \ldots \ldots \ldots \ldots \ldots \ldots$ 2-18

2-20. $H \rightarrow \gamma \gamma$ mass peaks over subtracted background for $30 \mathrm{fb}^{-1} \ldots \ldots \ldots \ldots \ldots \ldots . \ldots 2-18$

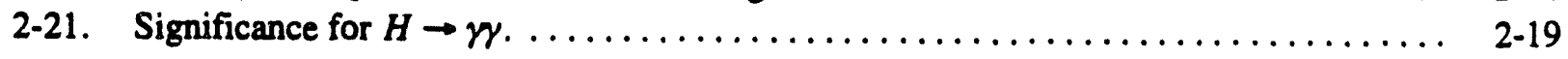

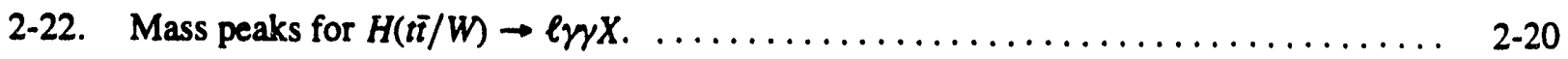

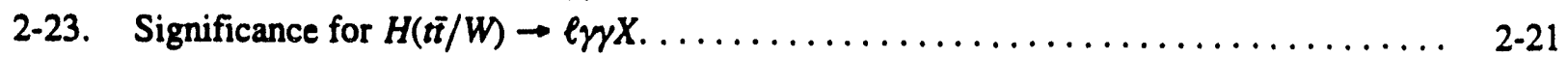

2-24. Higgs mass peaks and background for $H \rightarrow Z Z^{*} \rightarrow \ell^{+} \ell^{-} \ell^{+} \ell^{-} \ldots \ldots \ldots \ldots \ldots \ldots$ 2-22

2-25. Higgs mass peaks and background for $H \rightarrow Z Z \rightarrow \ell^{+} \ell^{-} \ell^{+} \ell-$ with $10 \mathrm{fb}^{-1} \ldots \ldots$ 2-23

2-26. Heavy Higgs mass peaks and background for $H \rightarrow Z Z \rightarrow \ell^{+} \ell^{-} \ell^{+} \ell^{-}$with $30 \mathrm{fb}^{-1}$. . 2-23

2-27. Distributions for $H \rightarrow Z Z \rightarrow \ell^{+} \ell-v \nabla$ and backgrounds. ................. 2-24

2-28. $M_{T}$ distribution for $M_{H}=800 \mathrm{GeV} H \rightarrow Z Z \rightarrow \ell^{+} \ell^{-} \boldsymbol{v} \bar{v} . \ldots \ldots \ldots \ldots \ldots \ldots .2-25$

2-29. Dijet mass distributions for $M_{H}=800 \mathrm{GeV} H \rightarrow Z Z \rightarrow \ell^{+} \ell^{-} j j \ldots \ldots \ldots \ldots \ldots$ 2-26 
2-30. $M_{\ell-\ell-j i j}$ distributions for $M_{H}=800 \mathrm{GeVH} \rightarrow Z Z \rightarrow \ell^{+} \ell^{-}, j j$ signal and

QCD background. ...................................... $2-26$

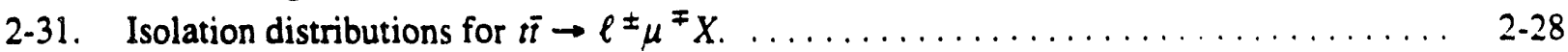

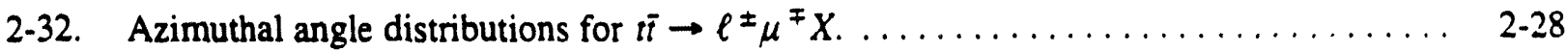

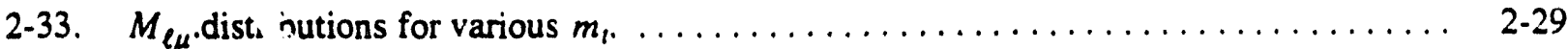

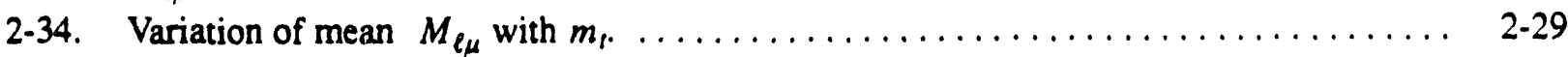

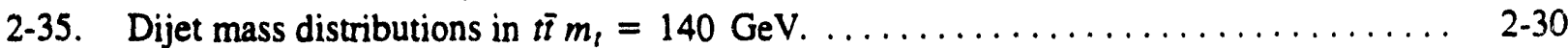

2-36. Trijet mass distribution in $t \bar{t}$ events for $m_{t}=140 \mathrm{GeV} \ldots \ldots \ldots \ldots \ldots \ldots \ldots \ldots . \ldots \ldots$

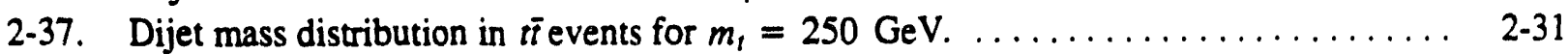

2-38. Trijet mass distribution in $t \bar{t}$ events for $m_{t}=250 \mathrm{GeV} \ldots \ldots \ldots \ldots \ldots \ldots \ldots \ldots . \ldots \ldots$

2-39. Significance of $t \rightarrow H^{+} B, H^{+} \rightarrow \tau^{+} v$ signal for various branching ratios. $\ldots \ldots \ldots .2-34$

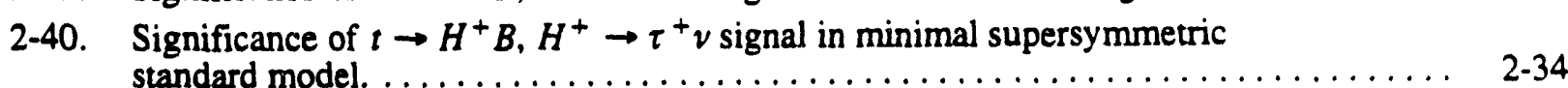

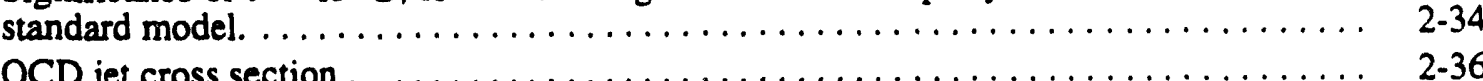

$2-41$.
$2-42$.

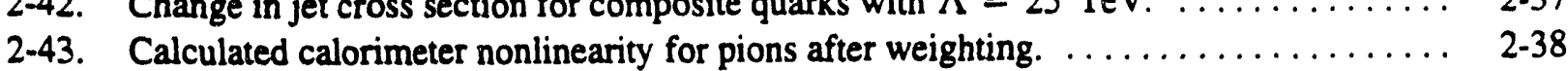

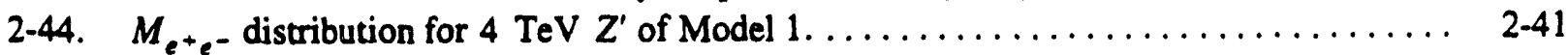

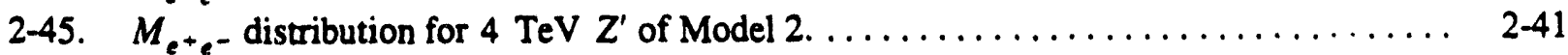

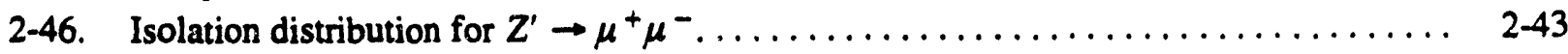

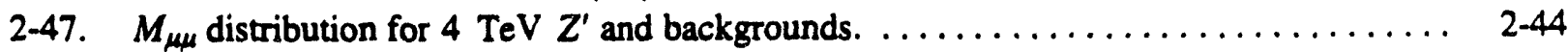

2-48. Reconstructed $\cos \theta^{*}$ distribution for Model $1 Z^{\prime} \ldots \ldots \ldots \ldots \ldots \ldots \ldots \ldots \ldots \ldots$

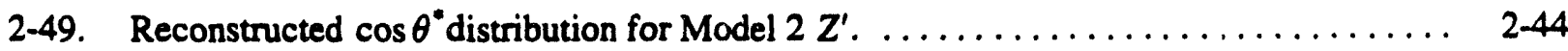

2-50. $M_{\mu^{+} \mu^{-}}$distributions for quark-lepton compositeness. ................ $2-46$

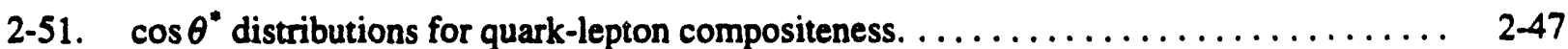

2-52 $\eta$ distributions for quark-lepton compositeness. $\ldots \ldots \ldots \ldots \ldots \ldots \ldots \ldots \ldots \ldots \ldots \ldots$

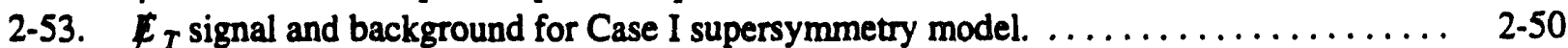

2-54. Ratio of signal to background for Case I supersymmetry model. $\ldots \ldots \ldots \ldots \ldots \ldots \ldots$ 2-51

2-55. $F_{T}$ signal and background for Case II supersymmetry model. $\ldots \ldots \ldots \ldots \ldots \ldots \ldots$ 2-51

2-56. Variation of signal/background with jet multiplicity for supersymmetry models. . . . . 2-51

2-57. $F_{T}$ signal and background for Case III supersymmetry model. $\ldots \ldots \ldots \ldots \ldots \ldots \ldots 2-52$

2-58. Likesign dilepton signal and background for Case III supersymmetry model. . . . . . 2-53

\section{Chapter 3}

3-1. The GEM magnet, showing one half-coil and forward field shaper retracted. ...... 3-2

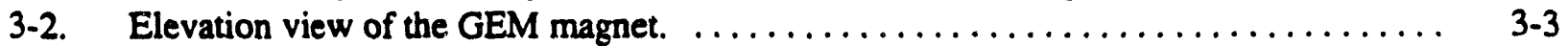

3-3. Cost estimate of the combined magnet and muon system as a function of the magnetic field strength for fixed muon resolution. $\ldots \ldots \ldots \ldots \ldots \ldots \ldots \ldots, 3-5$

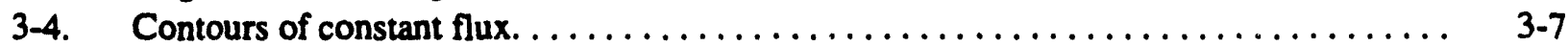

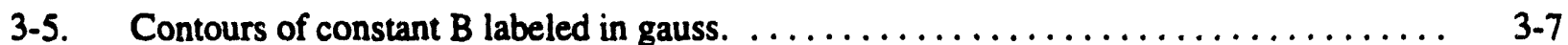

3-6. Contours of constant $B$ for the central region in increments of $40 \mathrm{G} . \ldots \ldots \ldots \ldots . . .3$

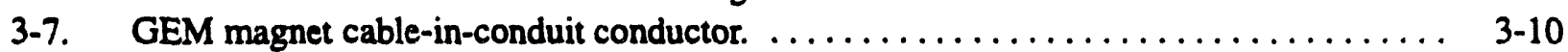

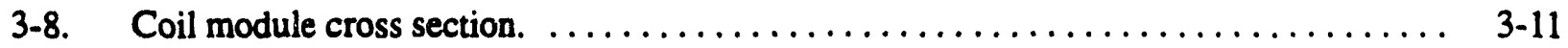

3-9. Cumulative axial pressure within each coil module in pascals. $\ldots \ldots \ldots \ldots \ldots \ldots \ldots, 3-11$

3-10. Hoop stress along the magnet axis in pascals. ...................... $3-11$

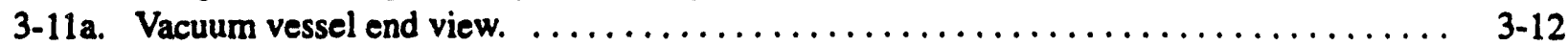

3-11b. Vacuum vessel side view showing the end-rings and periodic ring stiffeners. ...... 3-12 
3-12. Schematic of the axial magnetic orces on restraint rods. $\ldots \ldots \ldots \ldots \ldots \ldots \ldots \ldots$

3-13. Detail of the vacuum vessel support. $\ldots \ldots \ldots \ldots \ldots \ldots \ldots \ldots \ldots \ldots \ldots \ldots \ldots$

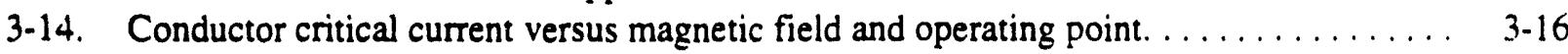

3-15. Hoop stress history during first three load cycles. $\ldots \ldots \ldots \ldots \ldots \ldots \ldots \ldots \ldots \ldots \ldots$

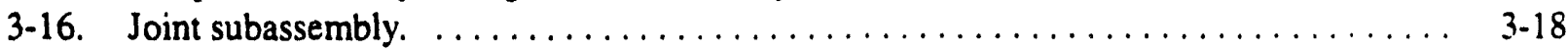

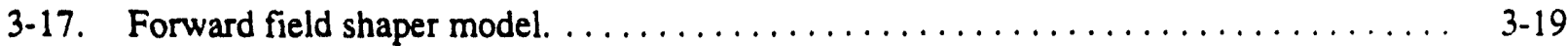

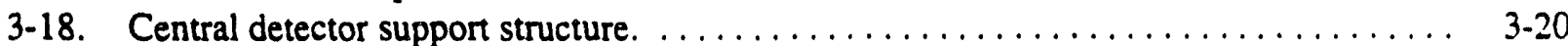

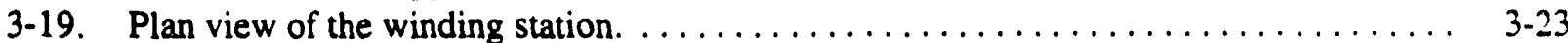

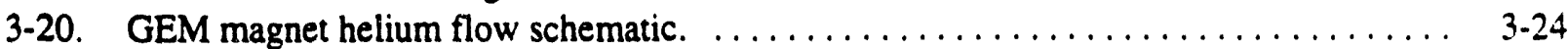

3-21. Major components of the power supply system. $\ldots \ldots \ldots \ldots \ldots \ldots \ldots \ldots \ldots \ldots . \ldots \ldots$

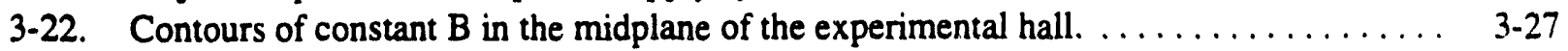

3-23. Contours of constant field on the surface above the detector in gauss. $\ldots \ldots \ldots \ldots \ldots, 3-28$

\section{Chapter 4}

4-1. Overview of the GEM muon system: (a) shown in quadrant view, (b) barrel

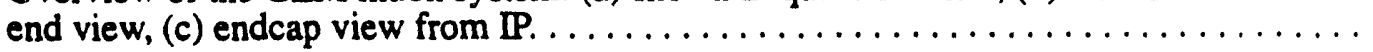

4-2. A quadrant of the muon system showing the magnetic field and the muon detector. ....

4-3. Resolution improvement as a function of number of measurement layers in the middle superlayer for various numbers of measurement layers in the inner (SL1) and outer (SL3) superlayers. . . . . . . . . . . . . . . . . . . . . . . . . . .

4-4. Cutaway model of barrel monolith.

4-5. Alignment contributions to momentum measurement error. $\ldots \ldots \ldots \ldots \ldots \ldots \ldots \ldots$

4-6. The $\delta \phi$ and sagitta concept of the trigger is illustrated for the barrel region. . . . . . . .

4-7. The GEM muon system in the SIGEM simulation for the barrel and

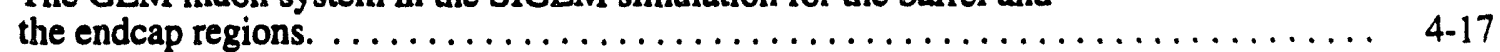

4-8. Event display from SIGEM for a $1 \mathrm{TeV}$ muon in the barrel region . . . . . . . . . . . 4-18

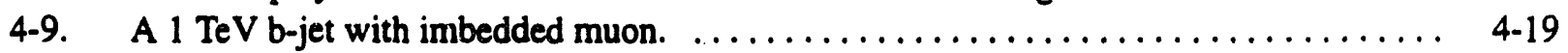

4-10. Charged particle rate versus calorimeter depth. ................... $4-21$

4-11. Charged particle rate versus rapidity in the first muon system superlayer. $\ldots \ldots \ldots \ldots .4-22$

4-12. Charge particle rate exiting the calorimeter versus transverse momentum. . . . . . 4-22

4-13. Neutron detection probability in the RDT filled with $\mathrm{CF}^{4}$ as a function of the threshold for the collected charge for a) thermal neutrons and $b$ ) fast neutrons. ...... 4-23

4-14. Distance between muon and EM debris in the different superlayers

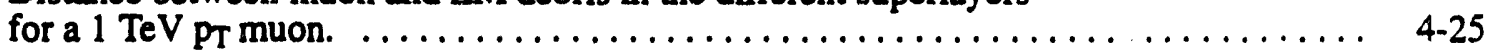

4-15. Trigger efficiency versus muon $\mathrm{pT}_{\mathrm{T}}$ for various thresholds in a) the barrel and $b$ ) the endcap.

4-16. Integrated trigger rate versus $p_{T}$ threshold for $\left.a\right)$ the barrel and $b$ ) the endcap . . . . 4-27

4-17. Probability to have a minimum 3 (solid) or 2 (dashed) good layers out of 6 layers in a superlayer as a function of the single strip occupancy per chamber per event.

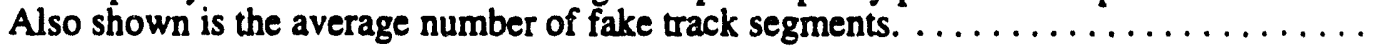

4-18. Probability to have at least 3 good layers in each of the three superlayers versus

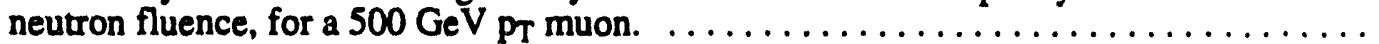

4-19. Transverse momentum resolution for standalone three superlayer system. . . . . . . 4-32

4-20. Transverse momentum resolution for the three superlayers with the inclusion of the central tracker as an independent measurement.

4-21. Transverse momentum resolution for three superlayers with the inclusion of a $200 \mu \mathrm{m}$ vertex constraint.

4-22. Transverse momentum resolution for $1000 \mathrm{GeV}$, with the inclusion of a vertex constraint but without the first superlayer. $\ldots \ldots \ldots \ldots \ldots \ldots \ldots \ldots \ldots \ldots$

4-23. The maximum momentum, for which the probability of determining the charge correctly is $\geq 95 \%$, as a function of $\eta$. 
4-24. Probability to have good track segments in all 3 SL for the muons from the decay

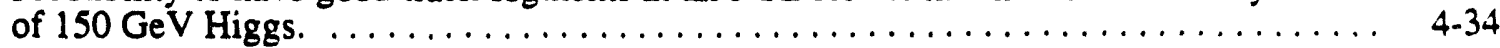

4-25. Probability to have good track segments in all three superlayers for muons from the decay of a $4 \mathrm{TeV} \mathrm{Z}$ as a function of muon rapidity. ................. $4-34$

4-26. Residuals in Houston CSC before (a) and after (b) correcting for systematic effects determined from the data. . . . . . . . . . . . . . .

4-27. a) Magnetic impulse and b) $\beta \lambda^{2}$, as a function of path length for rays at various

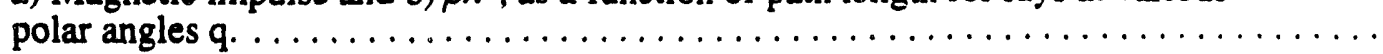

4-28. The axial (a) and radial (b) components of the GEM magnetic field for

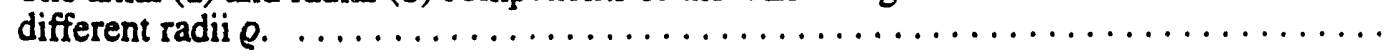

4-29. Schematic diagram of the Cathode Strip Chamber .

4-30. a) Two intermediate strip design and b) equivalent circuit. $\ldots \ldots \ldots \ldots \ldots \ldots \ldots$

4-31. Sketch of the Lorentz effect for barrel.

4-33. Geometric effects in the endcap CSC.

4-34. Endcap CSC resolution a) vs $\phi$ for $\theta=13^{\circ}$; b) vs $\phi$ for $\theta=22^{\circ}$; c) vs $\theta$. B $=0.8 \mathrm{~T} \ldots \ldots$

4-35. The dependence of the spatial resolution as a function of $\phi$ for the $0.5 \mathrm{~m} \times 0.5 \mathrm{~m} \mathrm{BNL}$

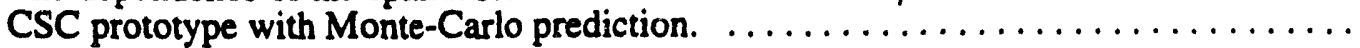

4-36. a) Drift velocities and b) Lorentz angles for $\mathrm{Ar} / \mathrm{CO}^{2} / \mathrm{CF}^{4}$ mixtures. . . . . . . . . .

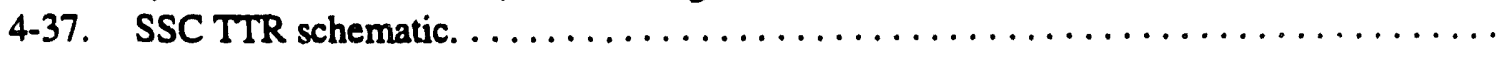

4-38. Cluster charge distribution from single ionizing tracks measured in a CSC. ........

4-39. Single strip fraction of total cluster charge as a function of the strip distance from

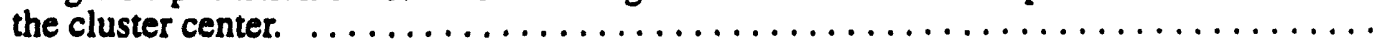

4-40. The single layer spatial resolution: a) for $0.5 \mathrm{~m} \psi 0.5 \mathrm{~m} \mathrm{BNL}$ CSC prototype;

b) for $1.5 \mathrm{~m} \psi 1.0 \mathrm{~m}$ DUBNA CSC prototype; c) for $1 \mathrm{~m} \psi 0.5 \mathrm{~m}$ open-profile

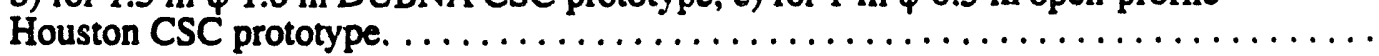

4-41. Efficiency of track-finding algorithm in CSC for a single track hypothesis (unshaded). Shaded region shows efficiency for accepting 2 tracks as

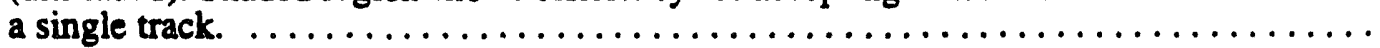

4-42. Measured single track resolution as a function of distance from superimposed

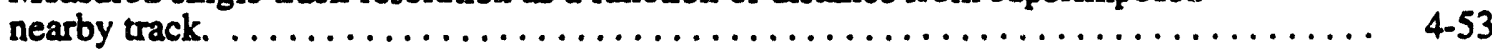

4-43. a) Deviation of strip position from nominal for BNL cathodes;

b) Difference distribution for UH cathodes. . . . . . . .

4-44. OR of four CSC layers showing distribution of stop times. $\ldots \ldots \ldots \ldots \ldots \ldots \ldots \ldots$. 4 -54

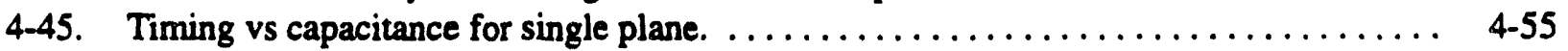

4-46. Cross-section view of CSC showing materials and general structure. . . . . . . . 4-56

4-47. Gain variation in a CSC vs fractional variation of the anode/cathode gap. ........ 4-57

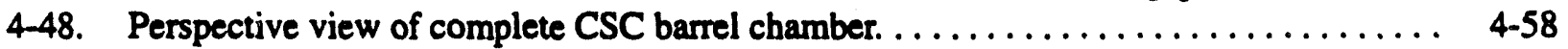

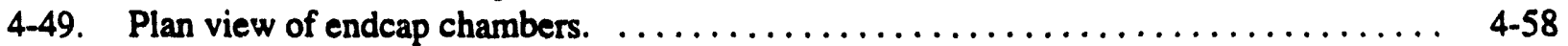

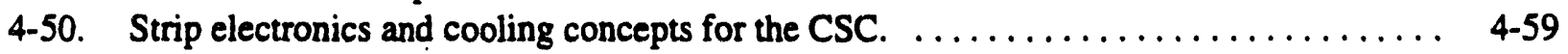

4-51. Service layout for CSC showing gas connection, HV distribution and anode

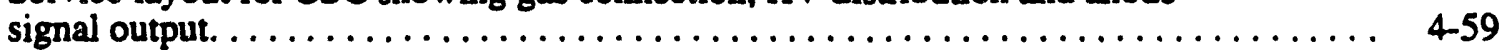

4-52. Comer of a CSC cathode showing relationship of strips, readout connections and

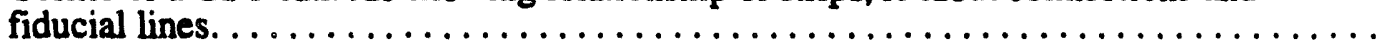

4-53. Top and end views of a CSC package indicating fixturing for internal and

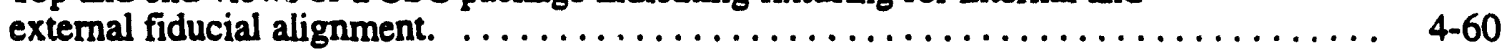

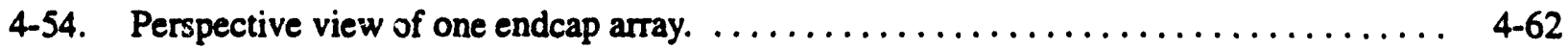

4-55. Side and end views of barrel monolith support on magnet. $\ldots \ldots \ldots \ldots \ldots \ldots \ldots \ldots$ 4-63

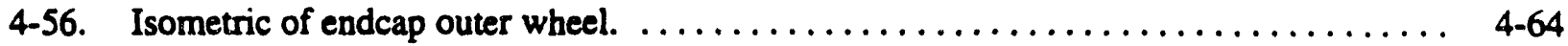

4-57. Local alignment of a projective muon tower. ................... 4 4-68

4-58. Three-point LED/lens/quad-cell straightness monitor as applied at L3. ........ 4-68 
4-59. Sagitta errors in a projective alignment tower before \& after alignment correction. . . . . 4-69

4-60. Maximum allowed local deflections and rotations in an alignment tower. . . . . . . 4 4-70

4-61. Attenuation of statistically distributed alignment errors by monitor correction. . . . . . . 4-71

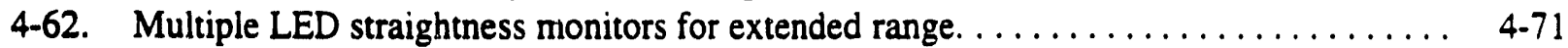

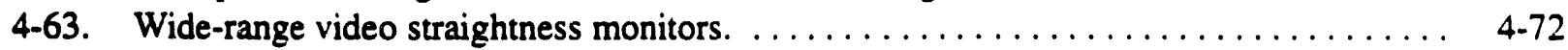

4-64. Conceptual alignment fixtures as mounted on middle superlayer. . . . . . . . . . 4 4-73

4-65. Measured misalignment of chamber layers from cosmic rays at TTR. . . . . . . . 4-74

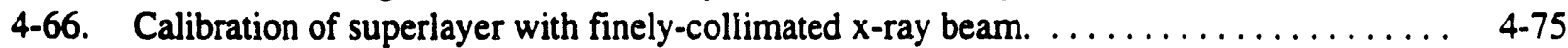

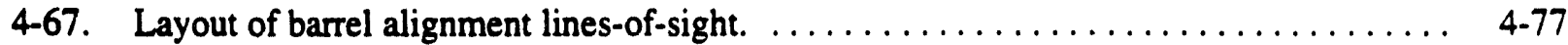

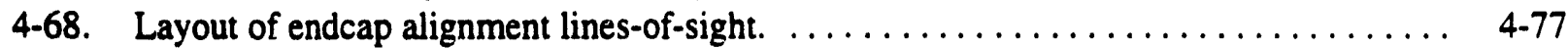

4-69. Alternative schemes for local alignment of the GEM muon barrel. . . . . . . . . . . . . 4-79

4-70. Role of straightness monitor axes in defining muon system global alignment. . . . . . . 4-80

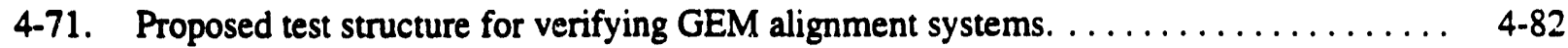

4-72. Schematic of cathode readout. $\ldots \ldots \ldots \ldots \ldots \ldots \ldots \ldots \ldots \ldots \ldots \ldots \ldots \ldots, \ldots \ldots \ldots$

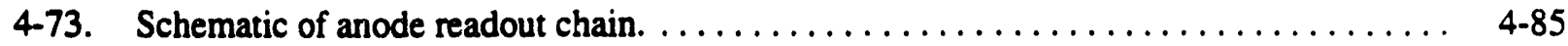

4-74. The local chamber encoders and cables to the off-chamber trigger board. . . . . . . . 4-86

4-75. Rotary test stand for module pre-alignment. $\ldots \ldots \ldots \ldots \ldots \ldots \ldots \ldots \ldots \ldots \ldots$ 4-88

4-76. Barrel module installation fixture. $\ldots \ldots \ldots \ldots \ldots \ldots \ldots \ldots \ldots \ldots \ldots \ldots \ldots \ldots . \ldots \ldots$

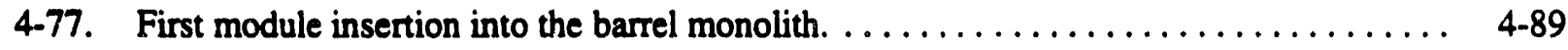

4-78. Assembly of chambers onto an endcap outer wheel. . . . . . . . . . . . . . . . . 4-90

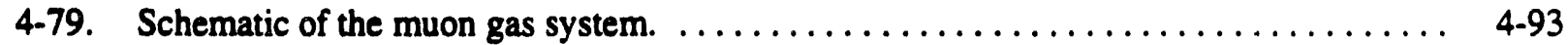

4-80. Schematic of CSC assembly process. Numbers refer to operations requiring

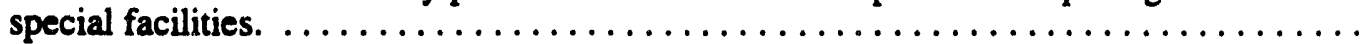

\section{Chapter 5}

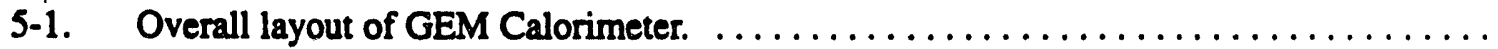

5-2. a) Solid model of calorimeter. b) Number of absorption lengths vs. $\eta$. . . . . . . . .

5-3. EM energy resolution vs. $1 / \sqrt{E}$ for barrel accordion calorimeter. . . . . . . . . . . . .

5-4. Transverse and longitudinal segmentation of the Barrel EM Calorimeter. . . . . . . . .

5-5. Averaged photon shower transverse energy profile. $\ldots \ldots \ldots \ldots \ldots \ldots \ldots \ldots \ldots$

5-6. $\pi^{0}$ rejection vs. $\pi^{0}$ energy for EM barrel accordion calorimeter. $\ldots \ldots \ldots \ldots \ldots \ldots$

5-7. Photon likelihood function for single photons and jets. . . . . . . . . . . . . . .

5-8. Position resolution for the barrel accordion in the $\eta$ and the $\phi$ directions $\ldots \ldots \ldots \ldots$

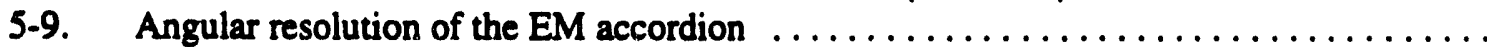

5-10. Invariant mass of two photons from $\mathrm{H}^{0}$ with mass of $80 \mathrm{Gev} . \ldots \ldots \ldots \ldots \ldots \ldots$

5-11. Energy resolution as a function of the radial length of the "massless gap." .........

5-12. Energy resolution as a function of the depth of the EM barrel calorimeter. . . . . . . . .

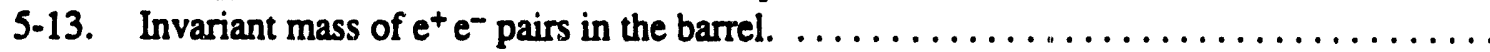

5-14. Relative deviation of invariant mass of $Z^{0} \rightarrow e^{+} e^{-}$pairs vs. $\eta \ldots \ldots \ldots \ldots \ldots \ldots$

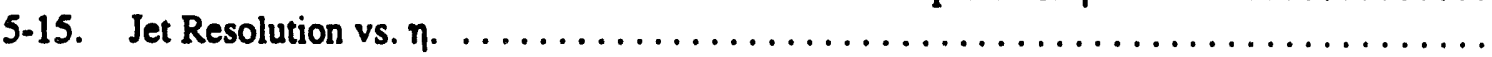

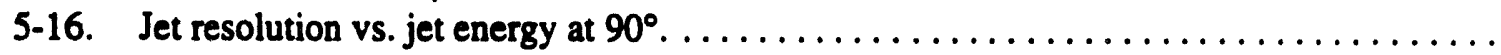

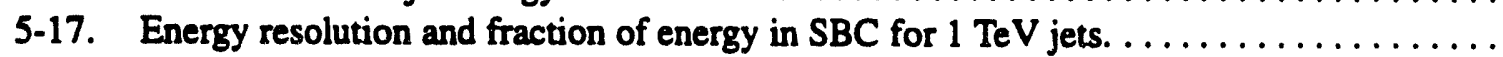

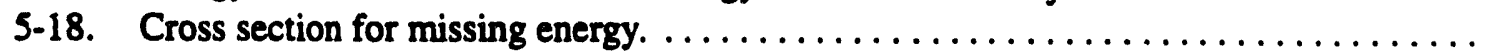

.

5-20. Response of the liquid krypton calorimeter vs. beam energy. . . . . . . . . . . . . 5-24

5-21. Energy resolution for test beam data and from simulation vs. $1 / \sqrt{E} \ldots \ldots \ldots \ldots \ldots \ldots$ 5-25

5-22. Timing resolution of liquid krypton calorimeter. $\ldots \ldots \ldots \ldots \ldots \ldots \ldots \ldots \ldots \ldots . \ldots \ldots$

5-23. Energy resolution obtained by $\mathrm{RD} 3$ collaboration. $\ldots \ldots \ldots \ldots \ldots \ldots \ldots \ldots \ldots .26$ 


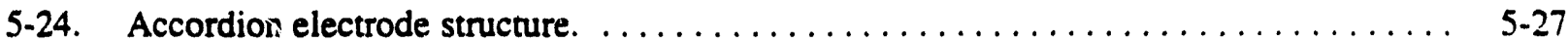

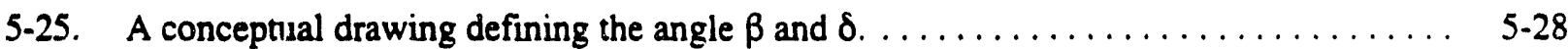

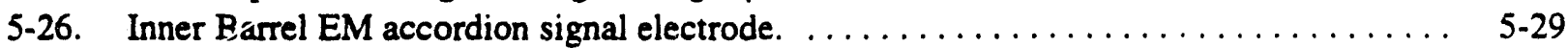

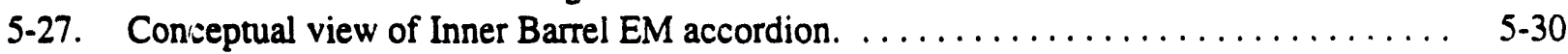

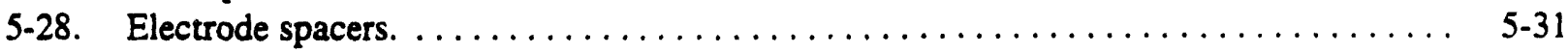

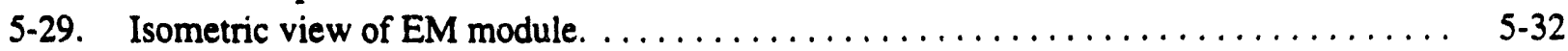

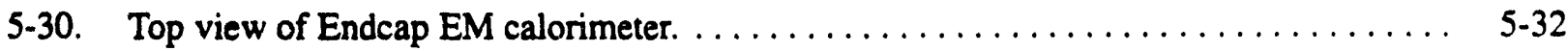

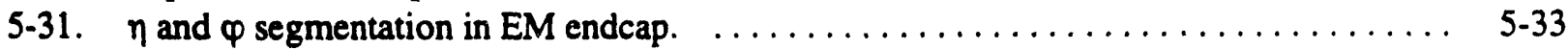

5-32. Calorimeter readout chain. ................................ $5-34$

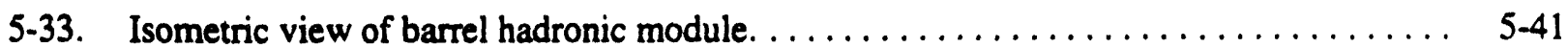

5-34. Unit cell in inner barrel hadronic module. . . . . . . . . . . . . . . . . . . $5-42$

5-35. Typical layer of inner barrel hadrotic module showing tiles. $\ldots \ldots \ldots \ldots \ldots \ldots \ldots, 5-42$

5-36. Electrode and absorber arrangement in the endcap hadron modules. $\ldots \ldots \ldots \ldots \ldots, 5-43$

5-37. Outer Endcap Hadron module. . ............................ $5-44$

5-38. EST capacitance dependence on various parameters. $\ldots \ldots \ldots \ldots \ldots \ldots \ldots \ldots \ldots, 5-45$

5-39. End view of scintillating barrel calorimeter. $\ldots \ldots \ldots \ldots \ldots \ldots \ldots \ldots \ldots \ldots \ldots$

5-40. Assembly of scintillating barrel calorimeter. . . . . . . . . . . . . . . . . . $5-47$

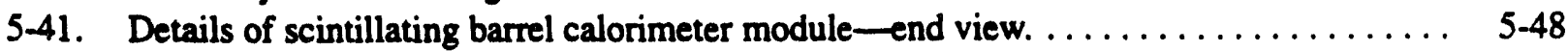

5-42. Detail of tile/fiber arrangement in the readout tower. $\ldots \ldots \ldots \ldots \ldots \ldots \ldots \ldots \ldots .49$

5-43. Details of scintillating barrel calorimeter module - side view. ............. $5-49$

5-44. Distribution of number of photoelectrons per MIP. $\ldots \ldots \ldots \ldots \ldots \ldots \ldots \ldots \ldots, 5-50$

5-45. Mean energy deposited by jets in the scintillating barrel. $\ldots \ldots \ldots \ldots \ldots \ldots \ldots \ldots, 5-50$

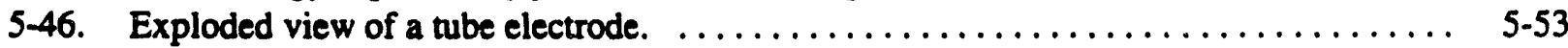

5-47. Close-up of the front face of the EM section of the forward calorimeter. . . . . . . . . 5-53

5-48. Front face of the EM section of the forward calorimeter. $\ldots \ldots \ldots \ldots \ldots \ldots \ldots \ldots$

5-49. A slice along the beamline of the hadronic forward calorimeter section. . . . . . . . 5-55

5-50. Details of the capillaries which hold the liquid scintillator in the hadronic

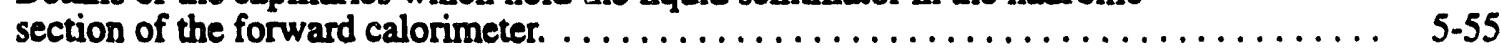

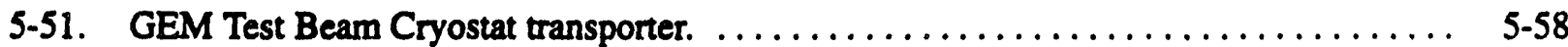

5-52. GEM Test Beam Facility in FNAL's MWEST Hall. $\ldots \ldots \ldots \ldots \ldots \ldots \ldots \ldots \ldots \ldots . \ldots .58$

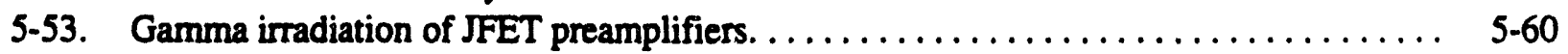

5-54. Equivalent noise charge of JFET preamplifiers after irradiation. $\ldots \ldots \ldots \ldots \ldots \ldots \ldots$ 5-61

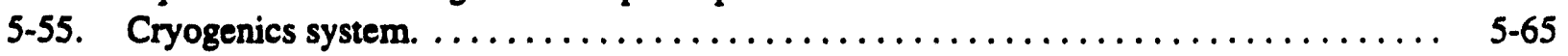

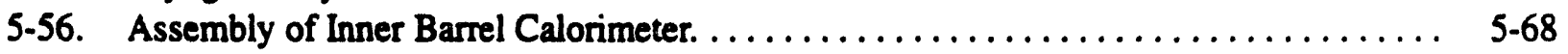

5-57. Assembly of the Endcap Calorimeter. $\ldots \ldots \ldots \ldots \ldots \ldots \ldots \ldots \ldots \ldots \ldots \ldots \ldots$

\section{Chapter 6}

6-1. Schematic layout of the detector elements in the GEM central tracker. . . . . . . . $6-2$

6-2. Schematic layout of the detector elements in the GEM interpolating pad chambers. ..................................... $6-3$

6-3. Impact parameter resolution and 10-yr radiation dose rate as a function of the

6-4. Impact parameter resolution and area of silicon detectors as a function of the

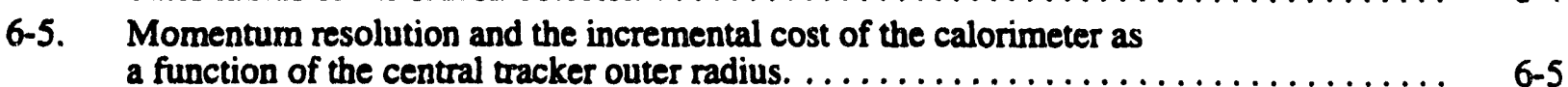

6-6. Material, in units of radiation length, in the central tracker. $\ldots \ldots \ldots \ldots \ldots \ldots \ldots .6 .6 .6$

6-7. Momentum resolution as a function of momentum for the central tracker. . . . . . . . . 6-6

6-8. Momentum resolution for $100 \mathrm{GeV} / \mathrm{c}$ tracks as a function of pseudorapidity $\eta \ldots \ldots 6$ 6-7 
6-9. Momentum for which the sign of the charge of muons and electrons can be

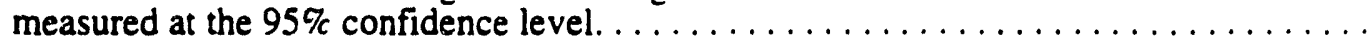

6-10. Resolution in the impact parameter $b$ at the event vertex as a function of

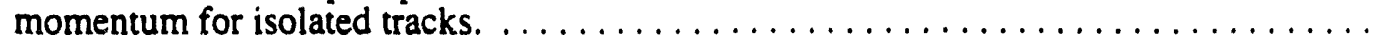

6-11. Resolution in the impact parameter $b$ at the event vertex for isolated

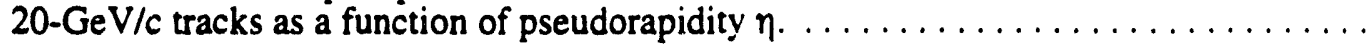

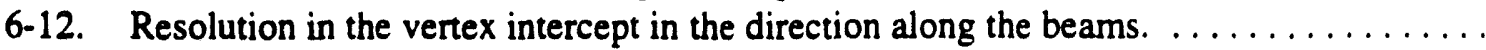

6-13. Measurement-limited momentum resolution at $\eta=0$ as a function of momentum

for the full tracker and for the interpolating pad tracker. .................. $6-10$

6-14. Momentum resolution distributions for 1,10 , and $100 \mathrm{GeV} / \mathrm{c}$ electrons and muons at $\eta=0$.

6-15. The number of detector layers hit by $500-\mathrm{GeV}$ muons passing through the central tracker as a function of $\eta$.

6-16. The fraction of muons from $H \rightarrow Z Z \rightarrow \mu \mu \mu \mu$, $\mu \mu e e$ decays that are found with the tracker pattern recognition program as a function of $\eta$.

6-17. The fraction of electrons from $H \rightarrow Z Z \rightarrow$ eeee, ee $\mu \mu$ decays that are found with the tracker pattern recognition program as a function of $\eta$.

6-18. The single track resolution for simulated tracks traversing an IPC layer as a function of the position across the pad of the incident track.

6-19. Total charge deposited by one, two and three tracks in a layer of IPCs (top figure). The bottom figure shows the total charge averaged over eight layers for one, two

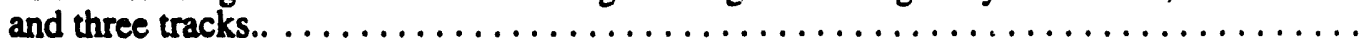

6-20. A simulation of the IPC two track resolution as a function of distance between the two tracks. ........................................ $6-14$

6-21. IPC three track resolution as a function of the distance between the closest two tracks. . . . . . . . . . . . . . . . . . . . . . . . . . . . . . . . . . . . . . .

6-22. (a) The transverse momentum spectrum for $\mathrm{pT}>0.5 \mathrm{GeV}$ isolated charged tracks from standard model Higgs production. (b) The number of these tracks per event from standard model Higgs production.

6-23. (a) The difference between the $\mathrm{z}$ coordinate of the true $\mathrm{H}^{0}$ production vertex and the reconstructed primary vertex (b) Vertex tagging efficiency as a function of the standard model Higgs mass and luminosity.

6-24. The fraction of background electrons rejected vs. the gamma efficiency for identifying $\gamma$ 's in $\mathrm{H}_{0} \rightarrow \gamma \gamma$ events for $\mathrm{MH}=80 \mathrm{GeV}$.

6-25. Distribution in $\mathrm{X}^{2}$ describing the agreement between the muon momentum vectors measured in the muon system and the central tracker.

6-26. The rejection factor for punchthrough hadrons as fake muons as a function of the central tracker momentum resolution.

6-27. Improvement in the momentum measurement of low-energy muons from taking the weighted averages of the central tracker information and the muon system measurement.

6-28. Efficiency for correct sign determination by the central tracker as a function

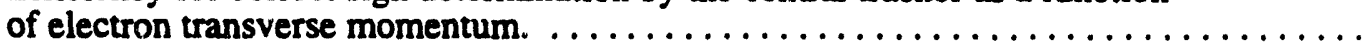

6-29. Transverse momentum distribution for the leptons in $W-W$ scattering $\ldots \ldots \ldots \ldots \ldots$

6-30. Efficiency for reconstructing all three tracks in the three-prong decay of a t-lepton as a function of the transverse momentum of the parent t.. ...................

6-31. Distribution between the minimum and maximum separation at the outermost IPC layer between the three charged tracks from the decays of a $150-\mathrm{GeV} / \mathrm{c}$ t-lepton. . . .

6-32. Remaining position uncertainty after calibration procedure as a function of initial uncertainty in the position of silicon and IPC layers. . . . . . . . . . . . . . .

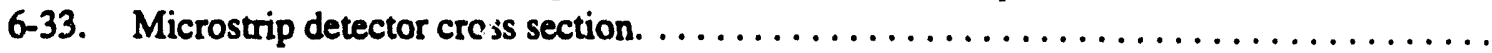

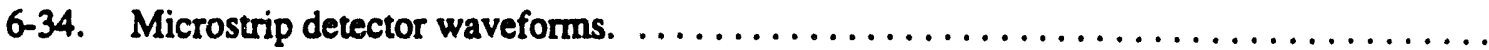

6-35. Dependence of single-hit efficiency for a track on tilt angle. . . . . . . . . . . . . 
6-36. Pulse waveforms for a track passing between strips. $\ldots \ldots \ldots \ldots \ldots \ldots \ldots \ldots \ldots, 6-25$

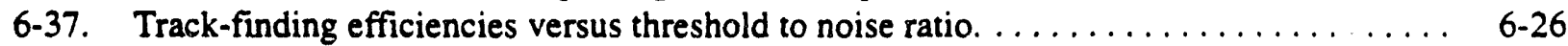

6-38. Track-finding efficiency versus noise level at a threshold to noise ratio of $3: 1 \ldots \ldots \ldots 6-26$

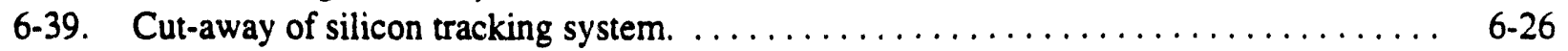

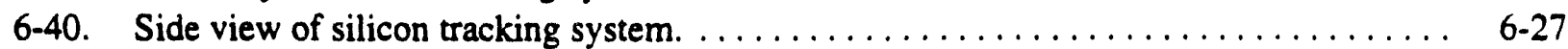

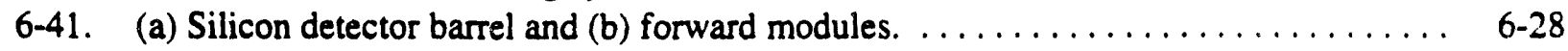

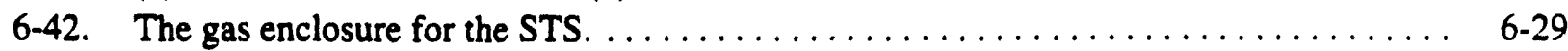

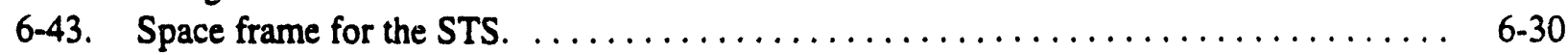

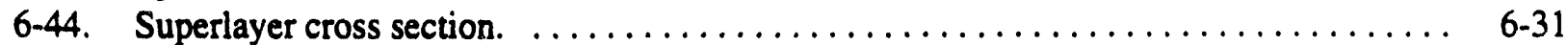

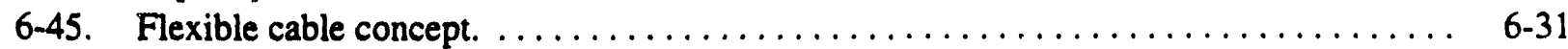

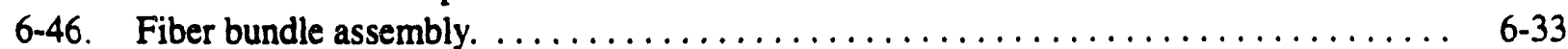

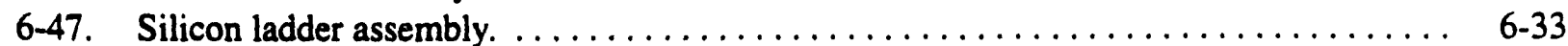

6-48. Silicon tracker electronics system architecture. $\ldots \ldots \ldots \ldots \ldots \ldots \ldots \ldots \ldots \ldots \ldots$. $6 \ldots \ldots$

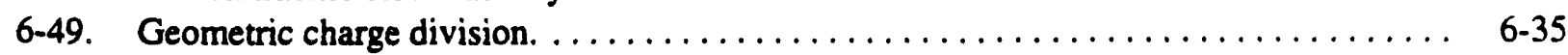

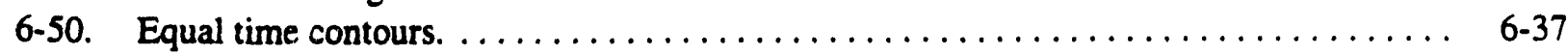

$6-51$. First electron collection time distribution. $\ldots \ldots \ldots \ldots \ldots \ldots \ldots \ldots \ldots \ldots \ldots \ldots \ldots \ldots$

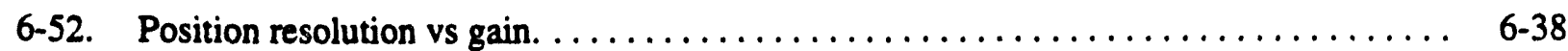

6-53. Distribution of position residuals for $2-\mathrm{KeV} x$ rays, demonstrating a resolution of 44 microns. ................................................. $6-39$

6-54. Central tracker quadrant view. .............................. $6-40$

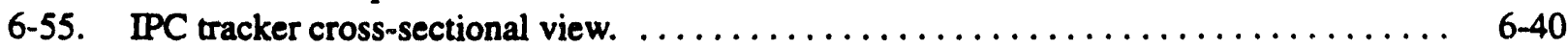

6-56. IPC tracker array, frontal view of endcap. $\ldots \ldots \ldots \ldots \ldots \ldots \ldots \ldots \ldots \ldots \ldots \ldots \ldots \ldots$

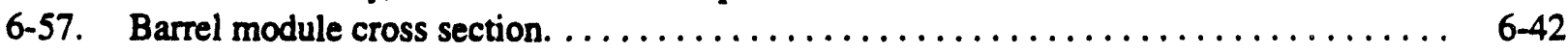

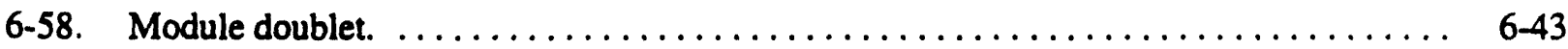

6-59. IPC tracker array, barrel assembly. . . . . . . . . . . . . . . . . . . . . . . 6-45

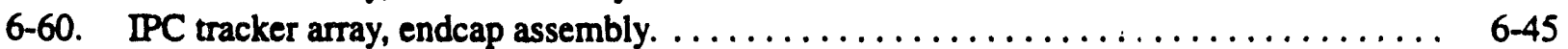

6-61. IPC readout approach. $\ldots \ldots \ldots \ldots \ldots \ldots \ldots \ldots \ldots \ldots \ldots \ldots \ldots \ldots \ldots \ldots, 6 \ldots \ldots$

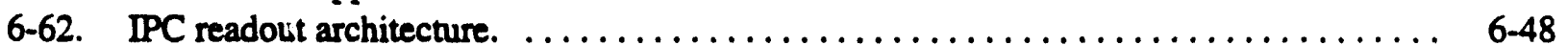

6-63. Zero suppression. .................................... $6-48$

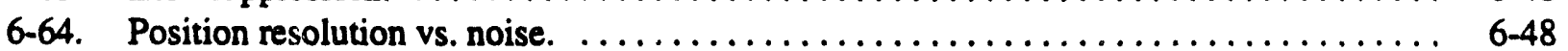

6-65. Position resolution vs time jitter. $\ldots \ldots \ldots \ldots \ldots \ldots \ldots \ldots \ldots \ldots \ldots \ldots \ldots \ldots \ldots \ldots, \ldots \ldots \ldots$

6-66. Position resolution vs. shaping-time variations. . . . . . . . . . . . . . . $6-49$

\section{Chapter 7}

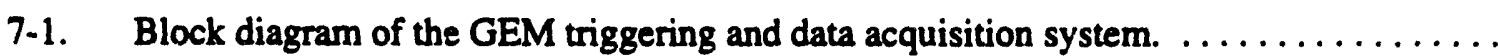

7-2. Schematic depiction of the Level 1 and Level 2 muon trigger algorithms. ..........

7-3. Geometrical configuration of the trigger sums. $\ldots \ldots \ldots \ldots \ldots \ldots \ldots \ldots \ldots$

7-4. Illustration of Level 1 isolation requirement. $\ldots \ldots \ldots \ldots \ldots \ldots \ldots \ldots \ldots \ldots$

7-5. Diagram illustrating the edge counting technique. $\ldots \ldots \ldots \ldots \ldots \ldots \ldots \ldots \ldots$

7-6. a) Connection between the calorimeter trigger towers and the 3D-Flow array after analog summing and digitization by the FADCs. b) Calculation of the superblock

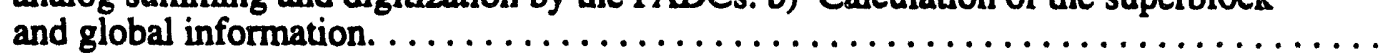

7-7. a) Details of the 3D-Flow programmable processing system crate assembly.

b) The layout and dimensions of the complete digital Level-1 trigger system. . . . . . . $7-14$

7-8. The df method and the sagitta method for muon triggers. $\ldots \ldots \ldots \ldots \ldots \ldots \ldots \ldots$ 7-15

7-9. Muon trigger supertower segmentation for the barrel and the endcap. $\ldots \ldots \ldots \ldots \ldots$ 7-15

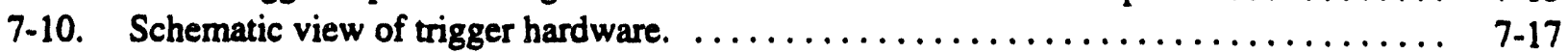

7-11. Superlayer trigger formation using associative, or content-addressable memory. .... 7-18

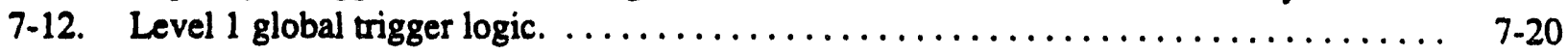


7-13. Efficiency of the $E^{8}$ e/ $\gamma$-trigger for electrons with a $\mathrm{pT}$ of $50 \mathrm{GeV}$.

$7-14$. a) Fraction of events accepted by each of the 12 trigger modes from Table $7-8$ and

b) trigger rates for minimum bias for these triggers.

7-15. a) Trigger efficiency for a single-photon trigger as a function of threshold, a

two-photon trigger, and a single-proton trigger logically ORed with a two-photon trigger

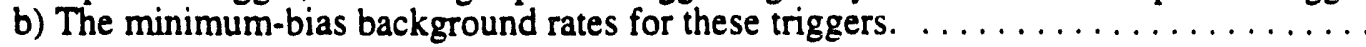

7-16. a) Trigger efficiency for top quark production $\left(m_{t}=140 \mathrm{GeV}\right)$ using a single-jet trigger (circles) and a four-jet trigger (squares) as function of the jet $\mathrm{E}_{\mathrm{T}}$ threshold.

b) Minimum bias backgrounds for these triggers.

7-17. a) Trigger efficiency as a function of the threshold for $500 \mathrm{GeV}$ gluino production. ....

7-18. a) Trigger efficiency for $t$ production using a missing energy trigger combined with various thresholds.

7-19. The trigger rate in the a) barrel and $b$ ) endcap as a function of $\mathrm{pr} . \ldots \ldots \ldots \ldots \ldots$

7-20. Trigger efficiencies in the $a)$ barrel and $b$ ) endcap as a function of $\mathrm{p}_{\mathrm{T}} \ldots \ldots \ldots \ldots \ldots$

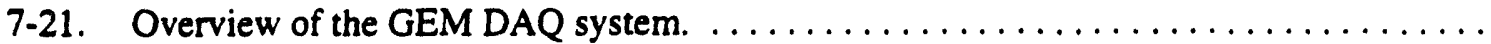

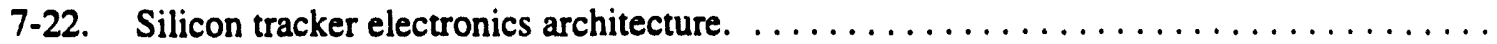

7-23. Silicon tracker amplifier and comparator design used for SPICE simulation. . . . . . . . 7-40

7-24. a) Silicon tracker preamplifier/shaper response to $1,2,4$, and $8 \mathrm{fC}$ input charges.

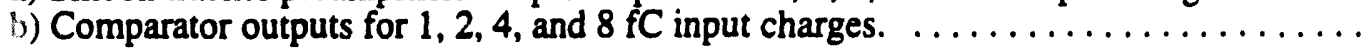

7-25. The processing architecture of the digital data storage on the CMOS-1 chip for

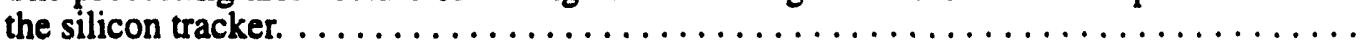

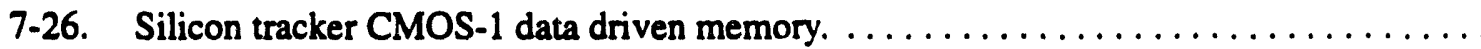

7-27. Silicon tracker MCM unit. Arrangement of VLSI Circuits on MCM and the mounting of the MCM to the ladder assembly for the silicon tracker. .............

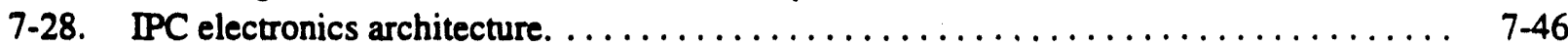

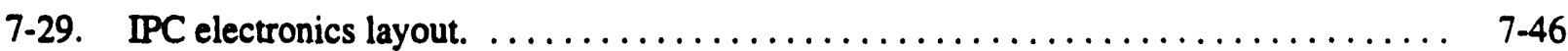

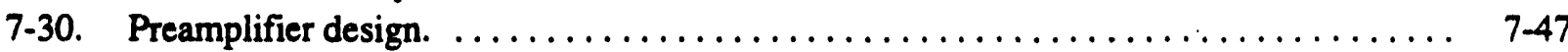

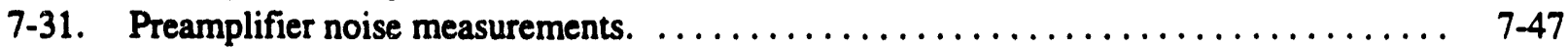

7-32. Voltage-write/charge-read SCA cell. $\ldots \ldots \ldots \ldots \ldots \ldots \ldots \ldots \ldots \ldots \ldots \ldots \ldots \ldots \ldots$

7-33. Voltage-write/voltage-read SCA cell. .......................... $7-49$

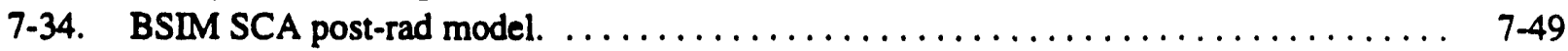

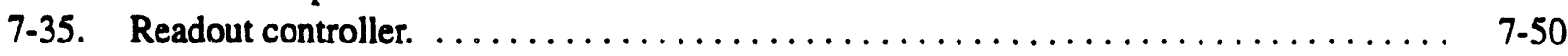

7-36. Schematic depicting the calorimeter readout chain. ................. $7-52$

7-37. Schematic drawing of a calorimeter readout module (CRM) using analog pipelines. . . . 7-54

7-38. Schematic drawing of a switched-capacitor analog memory with simultaneous READ and WRITE operations. ............................ $7-55$

7-39. Schematic drawing of a calorimeter readout module (CRM) using digital pipelines. . . . 7-57

7-40. Schematic representation of the layout of the readout, trigger, and DAQ system for the CSCs. . . . . . . . . . . . . . . . . . . . . . . . . . . . . . . .

7-41. Physical layout of the CSC front-end printed circuit board (FEPCB) $\ldots \ldots \ldots \ldots \ldots 7-62$

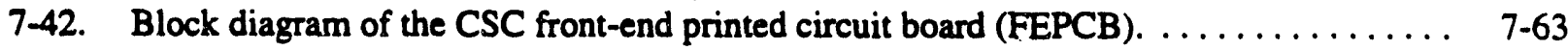

7-43. Block diagram of anode signal processing. $\ldots \ldots \ldots \ldots \ldots \ldots \ldots \ldots \ldots \ldots \ldots$

7-44. Interface of the anode electronics with the CSC readout path. $\ldots \ldots \ldots \ldots \ldots \ldots 7-67$

7-45. Block diagram of the control circuit for ROPCB and the FEPCBs. . . . . . . . . 7-68

7-46. (a) Output waveforms from test chip showing response of preamp and 700-ns semi-gaussian shaper to a 40-fC charge impulse injected through a 2-pF capacitor.

(b) Track and hold response for the preamp 700 -ns shaper test chip. . . . . . . . . . .

7-47. Output voltage versus input charge for the combined preamplifier/shaper. . . . . . . 7-71

7-48. Block diagram of the timing discriminator circuit and typical waveforms. . . . . . . 7-72

7-49. Typical combination of cathode strips corresponding to a valid LCT combination. . . . . 7-72 
7-50. SCA readout amplifier schematic. $\ldots \ldots \ldots \ldots \ldots \ldots \ldots \ldots \ldots \ldots \ldots \ldots \ldots \ldots$

7-51. Block diagram of the multiplexer tree. $\ldots \ldots \ldots \ldots \ldots \ldots \ldots \ldots \ldots \ldots \ldots \ldots .74$

7-52. Schematic representation of how hits appear in the muon readout. . . . . . . . . $7-74$

7-53. View of GEM detector facility showing location of various electronic subsystems. . . . 7-76

7-54. Fiber and cable connections for the GEM electronics system. . . . . . . . . . . . . 7-77

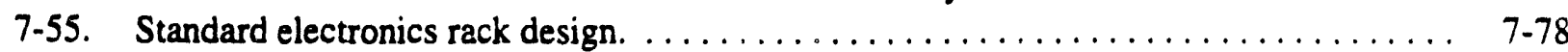

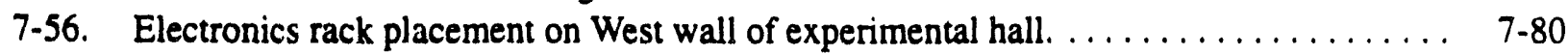

7-57. Electronics room plan view - rack placement. $\ldots \ldots \ldots \ldots \ldots \ldots \ldots \ldots \ldots \ldots \ldots \ldots \ldots$

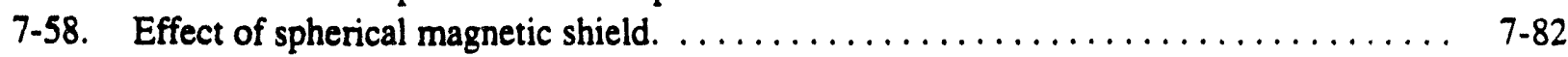

7-59. Effect of magnetic field on transformer primary current. $\ldots \ldots \ldots \ldots \ldots \ldots \ldots \ldots$.

7-60. IPC electronics research and development timeline. $\ldots \ldots \ldots \ldots \ldots \ldots \ldots \ldots \ldots$

\section{Chapter 8}

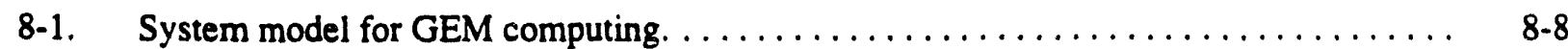

8-2. Model of the GEM GCS, showing major process groups. $\ldots \ldots \ldots \ldots \ldots \ldots \ldots \ldots$

8-3. The GEM off-line system, showing major process groups. ............... 8-16

8-4. Schematic of implementation of GEM computing system. $\ldots \ldots \ldots \ldots \ldots \ldots \ldots \ldots$.

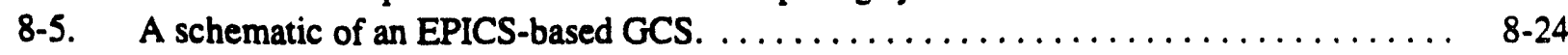

8-6. The structure of gemgen and gemfast as separate tasks. $\ldots \ldots \ldots \ldots \ldots \ldots \ldots \ldots$

\section{Chapter 9}

9-1. Aerial photograph of the ongoing site-clearing and grading activity at the IR5 site. . . 9-2

9-2. Overall IR5 site plan. . . . . . . . . . . . .

9-3. Proposed elevation view of the south assembly building (SAB) $\ldots \ldots \ldots \ldots \ldots \ldots .49$

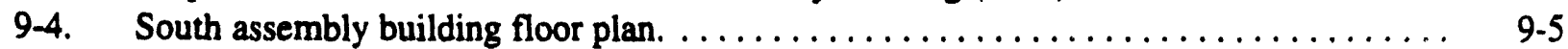

9-5. Assembly of muon system components in the SAB. . . . . . . . . . . . . . 9.6

9-6. Elevation view of north assembly building. $\ldots \ldots \ldots \ldots \ldots \ldots \ldots \ldots \ldots \ldots \ldots, 9.7$

9-7. North assembly building assembly floor layout. $\ldots \ldots \ldots \ldots \ldots \ldots \ldots \ldots \ldots \ldots$ 9-7

9-8. Muon monoliths being assembled in the north assembly building. ........... 9.8

9-9. General configuration of the hall. $\ldots \ldots \ldots \ldots \ldots \ldots \ldots \ldots \ldots \ldots \ldots \ldots . \ldots \ldots$

9-10. Isometric drawing of the underground experimental hall during detector installation. ... 9-19

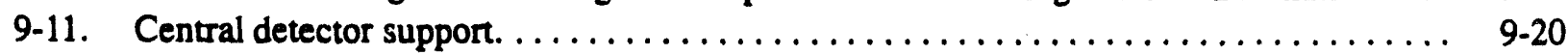

9-12. Scintillation barrel calorimeter installation. . . . . . . . . . . . . . . . . . . .

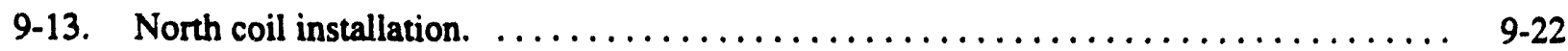

9-14. Installation of the barrel calorimetry system. . . . . . . . . . . . . . . . . .

9-15. South barrel muon installation. ............................. 9-24

9-16. Tracker and noble liquid endcap calorimeter installation. $\ldots \ldots \ldots \ldots \ldots \ldots \ldots \ldots$ 9-26

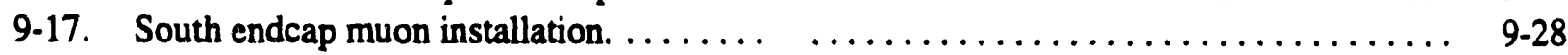

9-18. Cutaway view of the completed GEM detector. . . . . . . . . . . . .

\section{Chapter 10}

10-1. Isometric view: $6-\mathrm{m}$ withdrawal for access to ion pumping station. $\ldots \ldots \ldots \ldots \ldots, 10-10$

10-2. Dimensioned elevation view: 2 -m inner access opening $\ldots \ldots \ldots \ldots \ldots \ldots \ldots \ldots 10-11$

10-3. Isometric view: Full retraction of FFS and magnet vessels. . . . . . . . . . . 10-12

\section{Chapter 11}

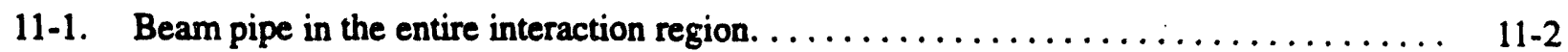

11-2. Beam pipe installed in the central tracker region of GEM. $\ldots \ldots \ldots \ldots \ldots \ldots \ldots \ldots, 11-2$ 
11-3. Pressure distribution in the entire interaction region. $\ldots \ldots \ldots \ldots \ldots \ldots \ldots \ldots \ldots \ldots \quad 11-4$

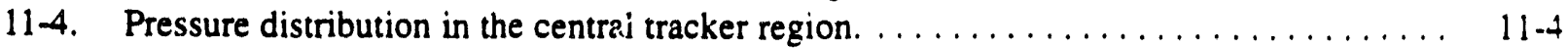

\section{Chapter 12}

12-1. Detector and shielding configuration used in this study.

12-2. Neutron fluence due to local beam loss in the low beta quadrupoles as a function

of distance from the interaction point for a radius of $200 \mathrm{~cm}$ from the beamline. . . . . 12-4

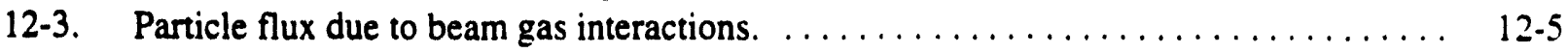

12-4. $\mathrm{dN} / \mathrm{d} \eta$ distribution for charged particles produced by DTUJET for $40 \mathrm{TeV}$ pp collisions. $\quad 12-6$

12-5. $\mathrm{dN} / \mathrm{d}_{\mathrm{T}}$ distribution for charged particles produced by DTUJET for $40 \mathrm{TeV}$ pp collisions. $\quad 12-6$

12-6. Fraction of the energy from the interaction point as a function of pseudorapidity. . . . . 12-6

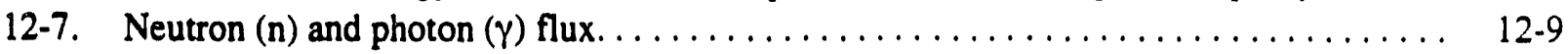

12-8. Neutron flux, from $A$ ) interactions in the collimator, quadrupoles, and beam line elements, $B$ ) interactions in the calorimeter system. . . . . . . . $\ldots \ldots \ldots \ldots 12-11$

12-9. The average $I_{2}$ mber of hadronic interactions in the beam pipe as a function of

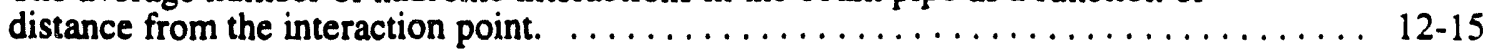

12-10. Neutron $(n)$ and photon $(\gamma)$ flux in the shielded detector. $\ldots \ldots \ldots \ldots \ldots \ldots \ldots \ldots$ 12-17

12-11. The spectra of neutrons and photons in the barrel muon systern of the

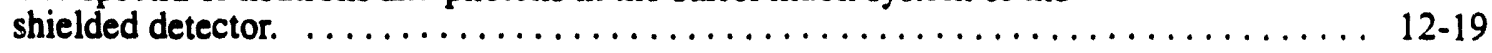

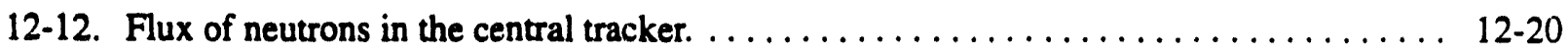

12-13. Flux of photons from $(n, \gamma)$ reactions in the central tracker. $\ldots \ldots \ldots \ldots \ldots \ldots \ldots \ldots$ 12-21

12-14. Neutron and photon energy spectra in the central tracker volume. $\ldots \ldots \ldots \ldots \ldots \ldots 12-21$

12-15. Dose to a silicon device as a function of radius in the central cavity. . . . . . . . . 12-22

12-16. Total dose due to electromagnetic and hadronic showers in the forward calorimeter. . . . 12-22

12-17. Buildup and decay of residual dose rates in the GEM detector. $\ldots \ldots \ldots \ldots \ldots \ldots \ldots$ 12-24

\section{Chapter 13}

13-1. Schematic of the Fermilab MWEST area configured for the GEM test beam program. . .

13-2. SSCL test beam line optics showing the locations of the particle-tagging TRD

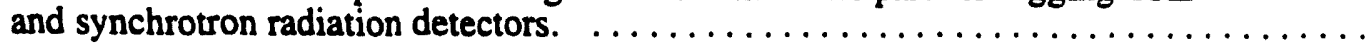

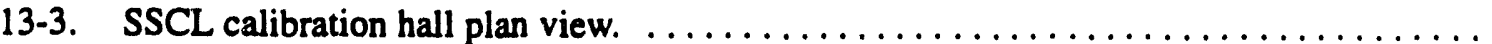

13-4. Secondary beamline energy resolution at the calibration hall. . . . . . . . . . . . .

13-5. Projective view of the calibration hall, showing the tracker, calorimeter, and muon chambers in test position in the beamline.

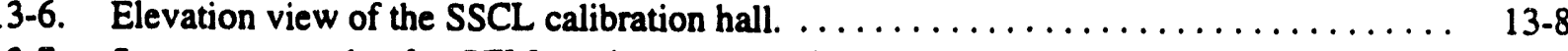

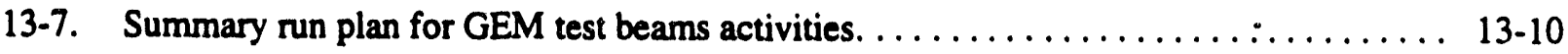

\section{Chapter 14}

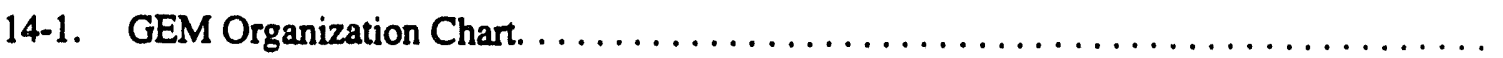

\section{Chapter 16}

16-1. Milestones in the GEM project. 


\section{LIST OF TABLES}

\section{Chapter 1}

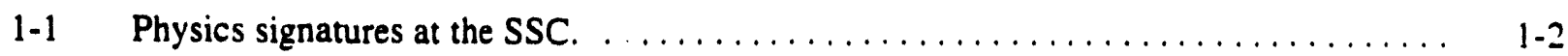

1-2 Top-level specifications for the GEM detector. $\ldots \ldots \ldots \ldots \ldots \ldots \ldots \ldots \ldots \ldots \ldots \quad 1-6$

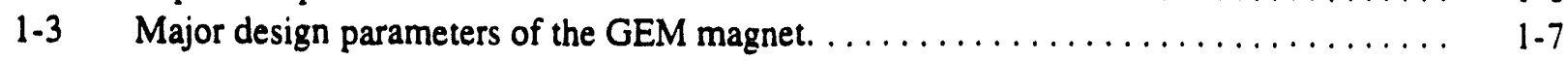

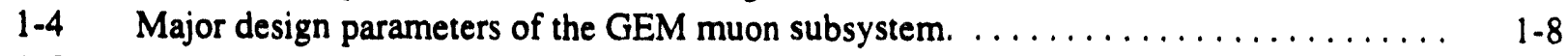

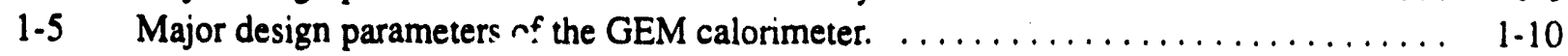

1-6 Major design parameters of the GEM central tracker. ................. 1-12

$1-7$ Trigger system design goals. $\ldots \ldots \ldots \ldots \ldots \ldots \ldots \ldots \ldots \ldots \ldots \ldots \ldots \ldots \ldots$

\section{Chapter 2}

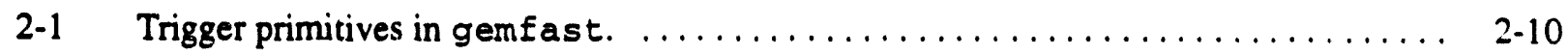

$2-2$ Trigger acceptance for various processes. $\ldots \ldots \ldots \ldots \ldots \ldots \ldots \ldots \ldots \ldots \ldots \ldots$

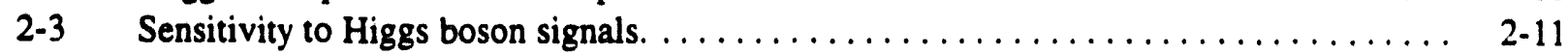

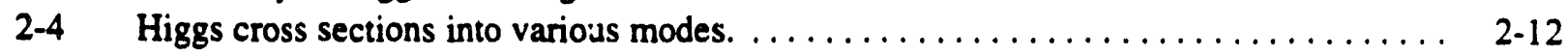

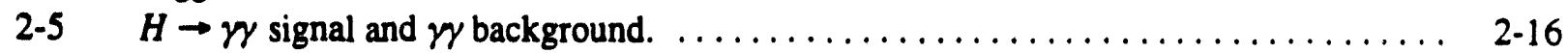

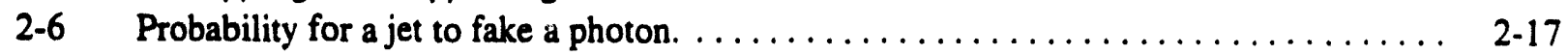

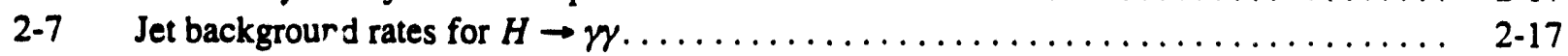

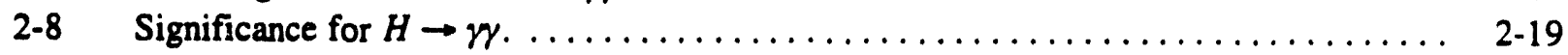

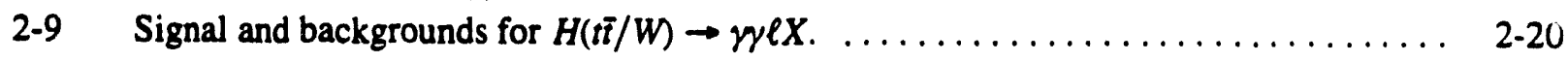

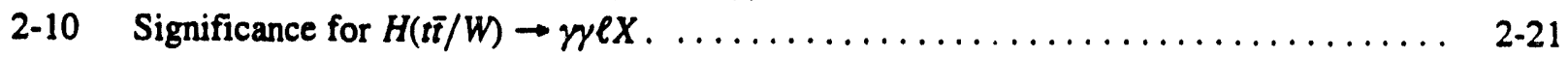

2-11 Signal and background for $H \rightarrow Z Z^{*} \rightarrow \ell^{+} \ell^{-} \ell^{+} \ell^{-} \ldots \ldots \ldots \ldots \ldots \ldots \ldots \ldots \ldots 2-22$

2-12 Signal and background for $H \rightarrow Z Z \rightarrow \ell^{+} \ell^{-} \ell^{+} \ell^{-} \ldots \ldots \ldots \ldots \ldots \ldots \ldots \ldots \ldots .23$

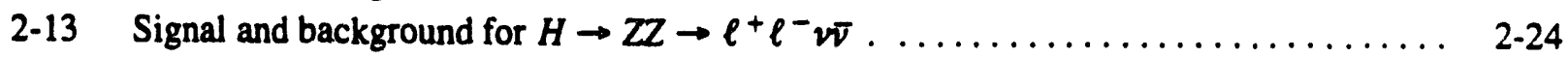

2-14 Signal and background for $H \rightarrow Z Z \rightarrow \ell^{+} \ell^{-} j j \ldots \ldots \ldots \ldots \ldots \ldots \ldots \ldots \ldots \ldots . \ldots \ldots$

2-15 Jet rates for various substructure scales and parton distributions. $\ldots \ldots \ldots \ldots \ldots \ldots$ 2-37

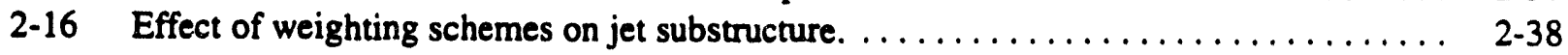

$2-17$ Supersymmetry models. ............................ 2-49

\section{Chapter 3}

3-1 Major magnet design options. ........................... $3-1$

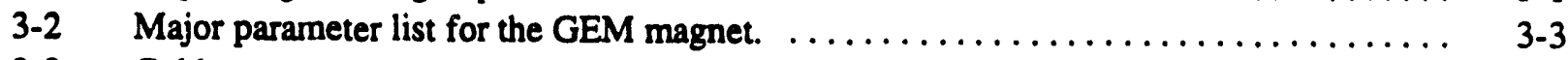

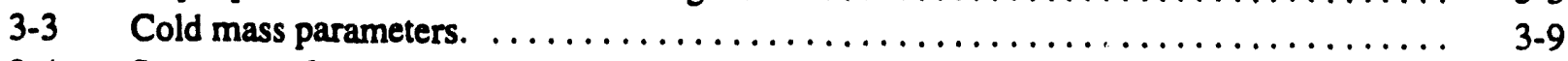

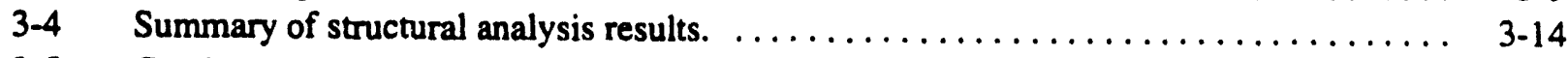

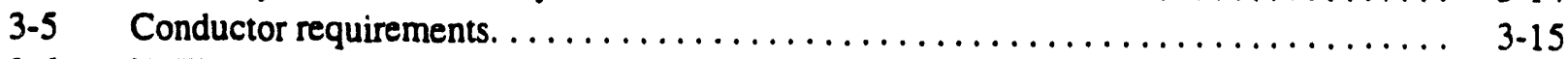

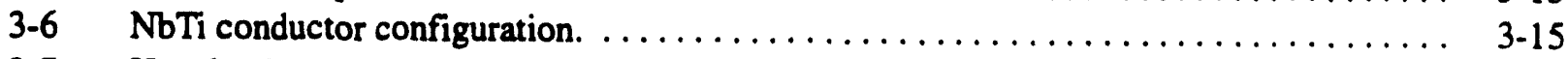

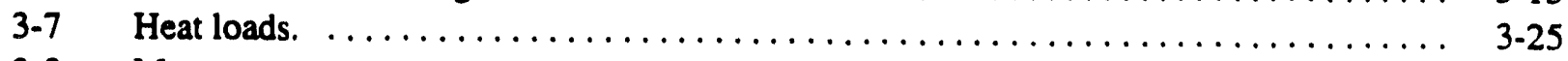

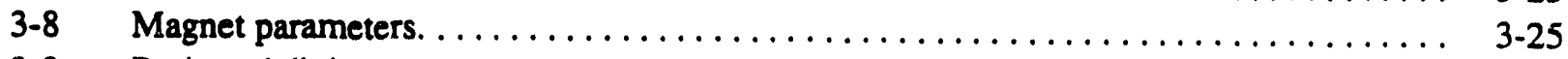

3-9 Projected division of responsibility within magnet project. $\ldots \ldots \ldots \ldots \ldots \ldots \ldots \ldots$ 3-31

Chapter 4

4-1 Contribution to momentum measurement error. . . . . . . . . . . . . . . . $4-11$

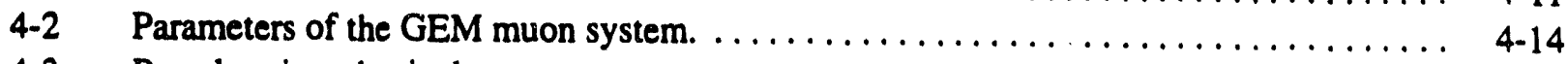

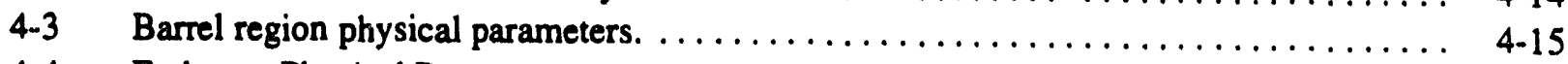

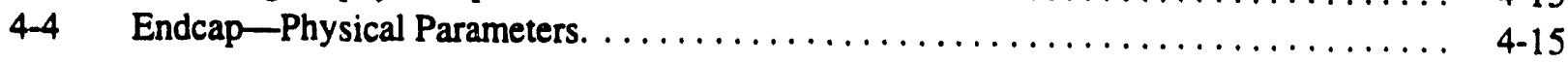


4-5 System Weights (kg).

4-6 CSC single and 4 strip occupancy at $10^{34} \mathrm{~cm}^{-2} \mathrm{~s}^{-1}$ luminosity from particles exiting the calorimeter in two rapidity intervals as a function of calorimeter depth. . . . . . . . . 4-21

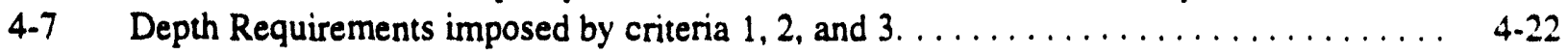

4-8 Detection efficiency of CSCs for thermal and fast neutrons and photons. . . . . . . . 4-24

4-9 Single strip occupancy in the CSCs due to uncorrelated neutrons and gammas

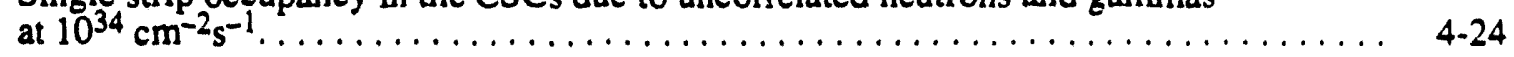

4-10 Probability for having good track segments for isolated muons in:

a) all three superlayers and b) middle and outer superlayers only. . . . . . . . . 4-29

4-11 Probability of having good track segments in a) all three superlayers and b) the middle and outer superlayers only, for muons in b-jets compared with isolated muons. .

4-12 For Higgs events where all 4 muons have $|\eta|<2.46$, the probability to have 1) all 4 muons with good track segments in all 3 superlayers, or 2) 4 muons with

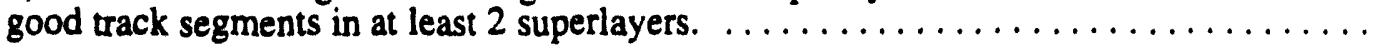

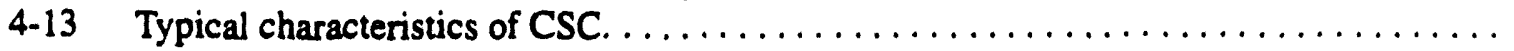

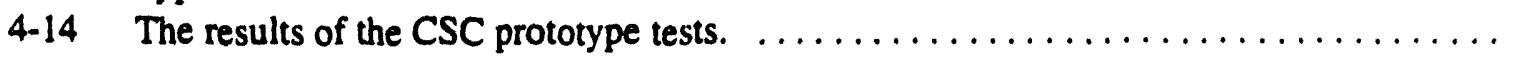

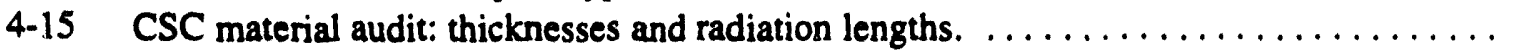

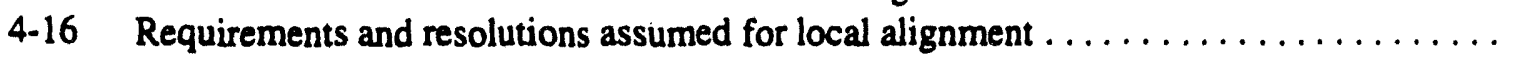

4-17 Requirements and resolutions assumed for global alignment $\ldots \ldots \ldots \ldots \ldots \ldots \ldots$

4-18 Local alignment monitors needed for GEM muon system . . . . . . . . . . . . . .

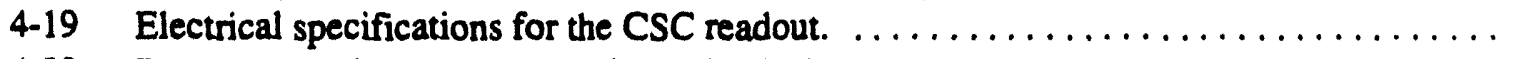

4-20 Barrel and endcap cathode readout, physical layout and channel count. . . . . . . . .

4-21 GEM muon group management organization. . . . . . . . . . . . . . . . . . . .

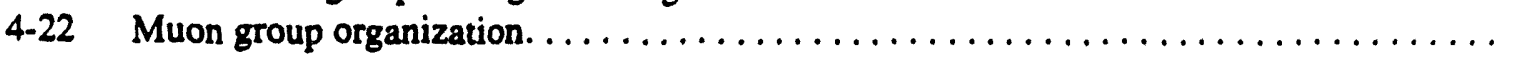

$4-53$

4-56

4.67

4-67

4-78

4-82

4-87

4-104

4-104

\section{chapter 5}

5-1 Relative mass resolution for $H^{0} \rightarrow y \gamma$ as a function of a and $b . \ldots \ldots \ldots \ldots \ldots$

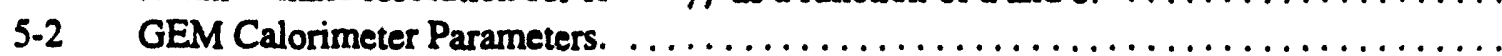

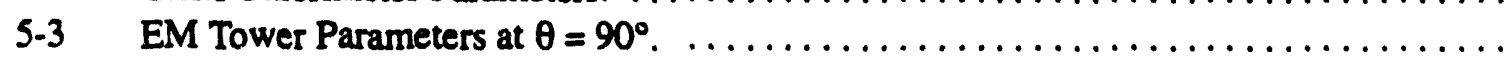

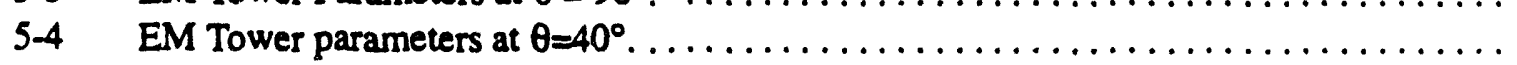

5-34

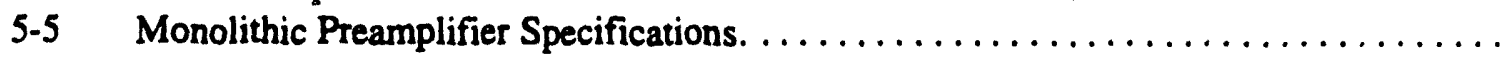

5-6 Calibration Goals and Means. . . . . . . . . . . . . . . . . . . . . . . .

5-7 Estimated Pileup Noise In The Liquid Calorimeter. . . . . . . . . . . . . . . .

5-35

5-35

5-37

5-8 Hadron Tower Parameters at $\theta=90^{\circ} .(\Delta \eta \times \Delta \varphi=0.08 \times 0.08) \ldots \ldots \ldots \ldots \ldots \ldots$

5-9 Hadron Tower Parameters at $\theta=50^{\circ} .(\Delta \eta \times \Delta \theta=0.08 \times 0.08) \ldots \ldots \ldots \ldots \ldots \ldots$

5-10 Characteristics and Parameters of the Scintillator Barrel Calorimeter. ........... $5-48$

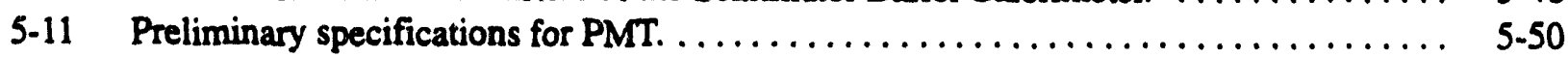

5-12 Variation of selected parameters across the face of the forward calorimeter ........ 5-54

$5-13$ Responsibilities in the Calorimeter Group. ................... $5-80$

\section{Chapter 6}

6-1 Design parameters for the central tracker. $\ldots \ldots \ldots \ldots \ldots \ldots \ldots \ldots \ldots \ldots . \ldots \ldots$

6-2 Central tracker resolutions and alignment stabilities assumed in the simulations. . . . . 6-6

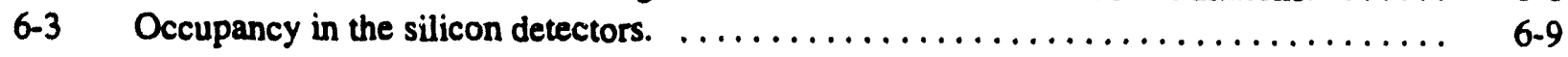

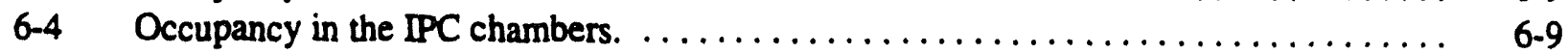

6-5 Probabilities of misidentifying $\gamma, \pi 0, \pi+$, and jets as electrons. . . . . . . . . . $6-17$

6-6 Design goals for the silicon subsystem. .......................... $6-23$

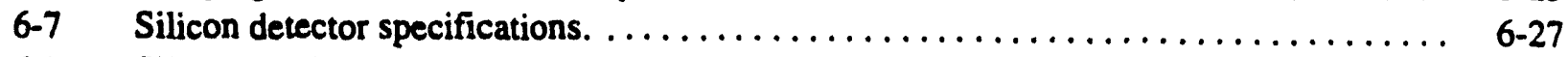

6-8 Silicon mechanical positioning tolerances. . . . . . . . . . . . . . . . . . . $6-29$ 


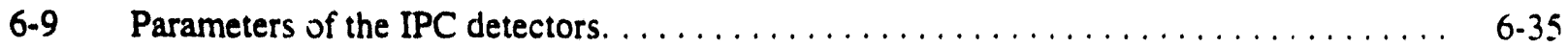

6-10 Parameters of an interpolating pad chamber. ..................... 6-36

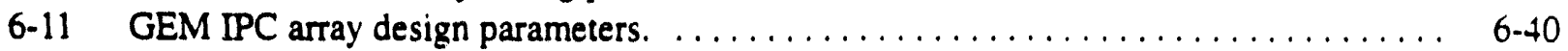

6-12 GEM IPC locations (middle of anode wire plane) $\ldots \ldots \ldots \ldots \ldots \ldots \ldots \ldots \ldots, 6.40$

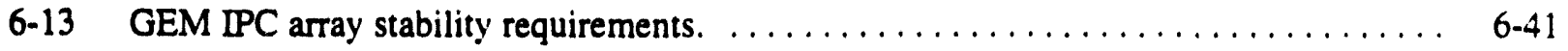

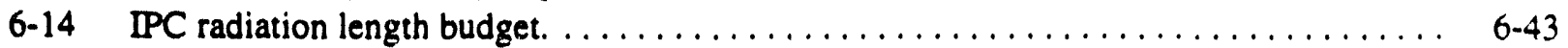

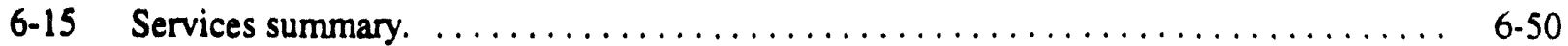

6-16 Neutron fluences $\left(\mathrm{cm}^{-2} \mathrm{SSCY}^{-1}\right), \ldots \ldots \ldots \ldots \ldots \ldots \ldots \ldots \ldots \ldots \ldots \ldots \ldots \ldots \ldots \ldots \ldots$

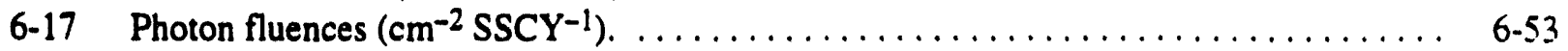

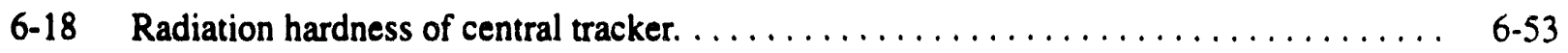

6-19 GEM central tracker silicon test beam needs $1993-1995 \ldots \ldots \ldots \ldots \ldots \ldots \ldots \ldots 6$. $\ldots$. $\ldots 6$

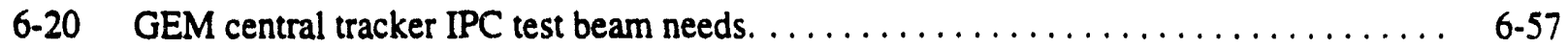

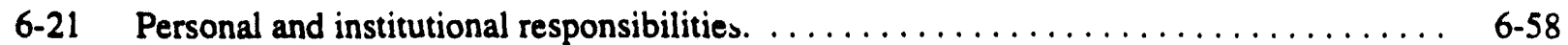

\section{Chapter 7}

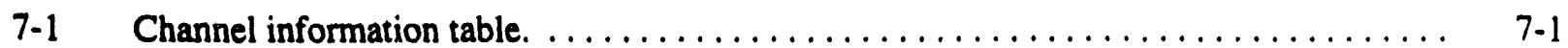

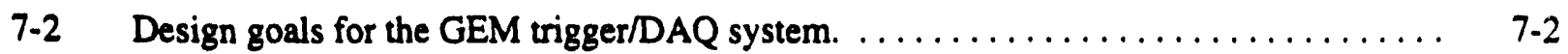

7-3 Calorimetry information available for the Level 1 trigger system. $\ldots \ldots \ldots \ldots \ldots \ldots$. $7-4$

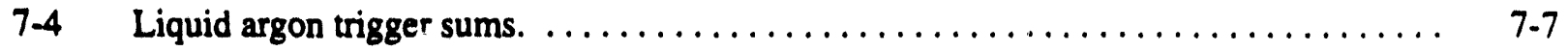

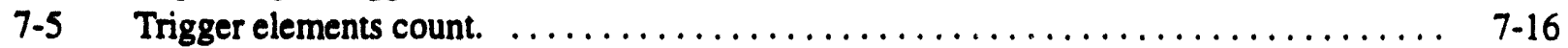

7-6 The number of patterns necessary to implement a minimum $\mathrm{p}_{\mathrm{T}}$ cut. $\ldots \ldots \ldots \ldots \ldots \ldots$ 7-19

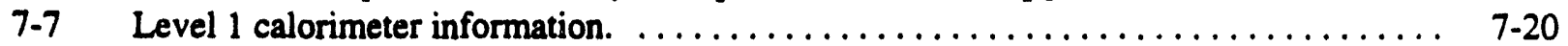

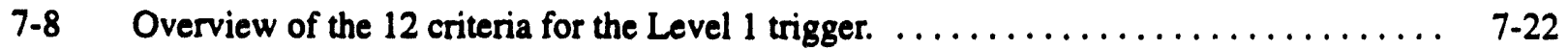

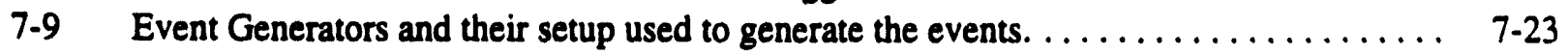

7-10 Efficiency for various physics processes of the Level 1 trigger criteria. . . . . . . . . 7-24

7-11 Numbers of channels and front-end boards for the GEM subsystems, number of bytes per event and data volume at a level 1 trigger rate of $100 \mathrm{kHz} . \ldots \ldots \ldots \ldots .7-31$

7-12 GEM silicon tracker parameters. ............................ $7-37$

7-13 Nominal SSC operational parameters for the GEM silicon tracker. . . . . . . . . 7-37

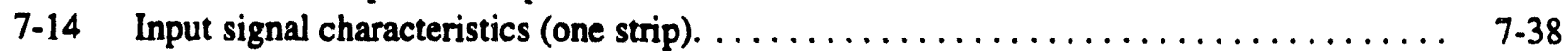

7-15 Performance goals for the silicon electronics analog chip. ............... $7-39$

7-16 ENC as a function of linear strip resistance and capacitance. $\ldots \ldots \ldots \ldots \ldots \ldots \ldots 7-4$

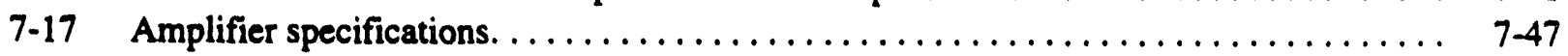

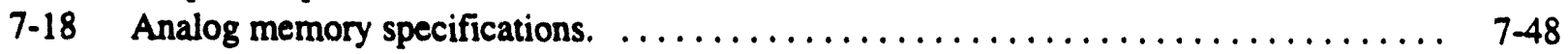

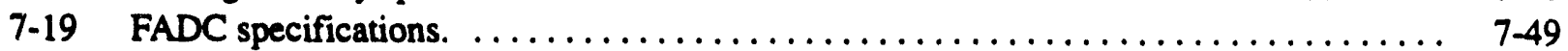

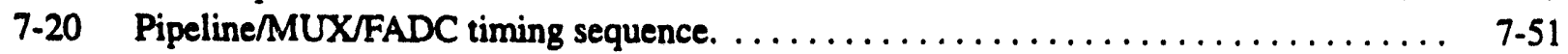

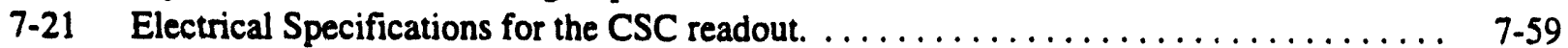

7-22 Barrel cathode readout physical layout and channel count $\ldots \ldots \ldots \ldots \ldots \ldots \ldots \ldots, 7-60$

7-23 Endcap cathode readout channel count. ......................... $7-61$

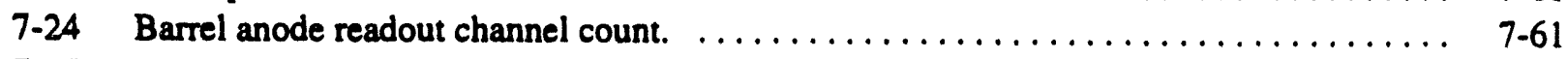

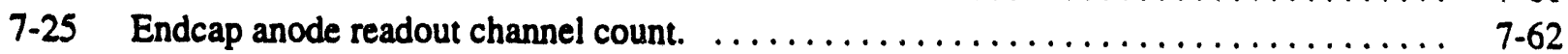

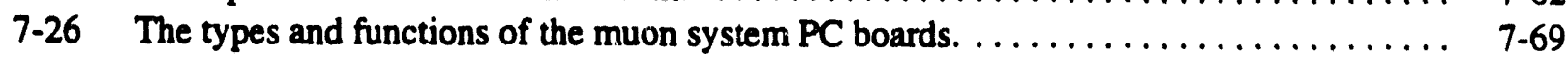

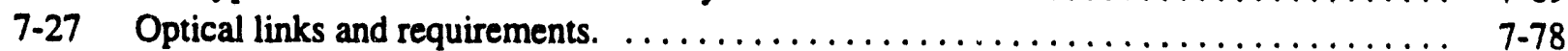

\section{Chapter 8}

8-1 Examples of physics processes (or possible processes) to be recorded in the

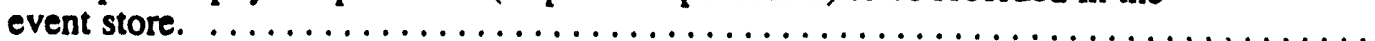

8-2 Overall model for expected rates. $\ldots \ldots \ldots \ldots \ldots \ldots \ldots \ldots \ldots \ldots \ldots \ldots \ldots, 8 \ldots \ldots$ 


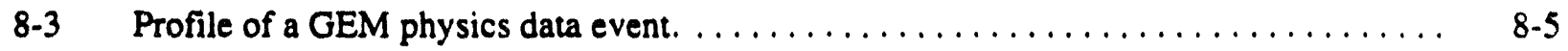

8-4 Anticipated 1-yr event samples, including simulation output. $\ldots \ldots \ldots \ldots \ldots \ldots \ldots$ 8-9

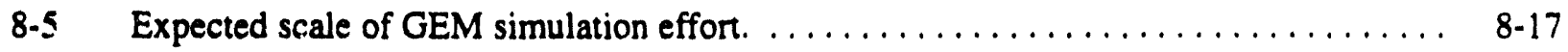

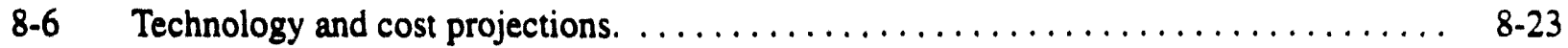

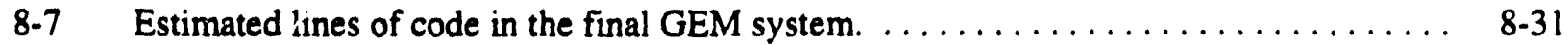

8-8 Estimated manpower profile for software development, in FTE by year. . . . . . . . 8 8-32

\section{Chapter 10}

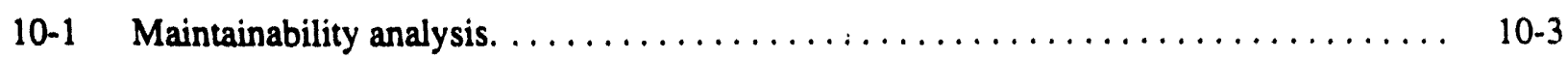

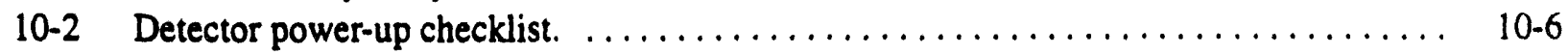

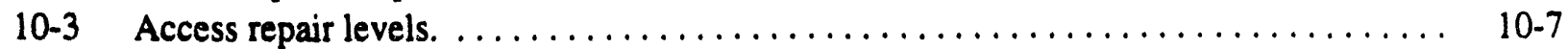

\section{Chapter 11}

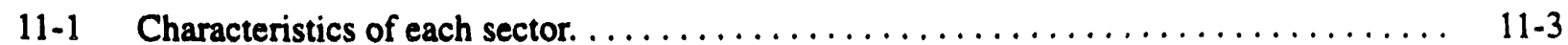

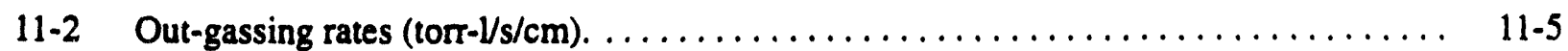

\section{Chapter 12}

12-1 Ratio of the particle flux caused by beam gas interactions to the flux from pp collisions, at selected locations in the detector. . . . . . . . . . . . . . . . . . .

12-2 Fraction of the total energy emitted from the interaction point intercepted by various detector components. ................................ $12-6$

12-3 Power dissipated in the first low beta quadrupole and the expected lifetime of the coil insulation at standard luminosity as functions of the collimator aperture. . . . . 12-7

12-4 Neutron fluxes for two different configurations of shielding around the collimator. . . . 12-14

12-5 Categorization of neutrons of all energies injected into the endcap muon chambers

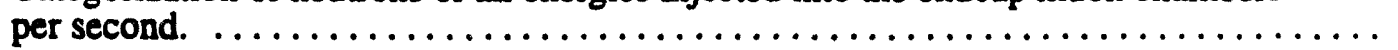

12-6 Maximum dose and neutron flux expected in detector systems for one year of

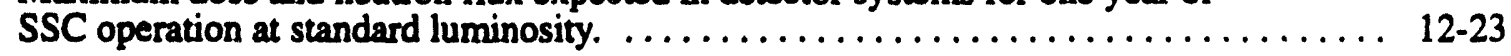

12-7 Maximum dose rates on contact for different regions of the detector from residual activity.

12-8 List of isotopes with high neutrons cross sections for activation. . . . . . . . . 12-25

\section{Chapter 13}

13-1 GEM requirements for the SSCL test beams. . . . . . . . . . . . . . . . .

\section{Chapter 15}

15-1 Radiation Limits and Design Goals.

\section{Chapter 16}

16-1 GEM detector system cost estimate summary, April 93. 


\section{PREFACE}

The theoretical elements of the standard $S U(3) \otimes S U(2) \otimes U(1)$ gauge model of strong and electroweak interactions have been in place for more than 20 years. ${ }^{1,2}$ In all this time, the standard model has withstood extremely stringent experimental tests. ${ }^{3}$ Down to at least $10^{-16} \mathrm{~cm}$, the basic constituents of matter are spin- $\frac{1}{2}$ quarks and leptons. These interact via the exchange of spin-one gauge bosons: the massless gluons of QCD and the massless photon and massive $W^{ \pm}$and $Z^{0}$ bosons of electroweak interactions. There are six flavors each of quarks and leptons-identical except for mass, charge and color-grouped into three generations. All the fermions have been found except for the top quark and the tau neutrino. 4.5 If the number of quark-lepton generations is equal to the number $N_{v}$ of light neutrinos, then there are no more than these three. The evidence for this comes from precision measurements of the $Z^{0}$ at LEP, which give $N_{v}=2.99 \pm 0.04$ in the standard model. ${ }^{5}$

The unbroken nature of the QCD gauge interaction and its ground state makes quarks and gluons almost noninteracting at small distances, while it confines them at large distances into color-singlet hadrons. Even though gauge bosons necessarily begin without mass, interactions can make them heavy. This is what happens to the $W^{ \pm}$and $Z^{0}$ bosons: electroweak gauge symmetry is spontaneously broken, a phenomenon known as the "Higgs mechanism". 6 Finally, fermions in the standard model also must start out massless. To make quarks and leptons massive, new forces beyond the $S U(3) \otimes S U(2) \otimes U(1)$ gauge interactions are required. These additional interactions explicitly break the fermions' flavor symmetry and communicate electroweak symmetry breaking to them.

Despite this great body of knowledge, the interactions underlying electroweak and flavor symmetry breakdowns remain unknown. The most important element still missing from this description of particle interactions is directly connected to electroweak symmetry breaking. This may be a single new particle-the "Higgs boson"; several such bosons; a replication of all the known particles; an infinite tower of new resonances; or something still unimagined. It is also unknown whether the new interactions required for flavor symmetry breaking need additional new particles for their implementation. Until the new dynamics are known, it seems impossible to make further progress in understanding elementary particle physics.

One very important aspect of electroweak symmetry breaking is known: its characteristic energy scale of $1 \mathrm{TeV}$. This scale is set by the decay constant of the three Goldstone bosons transformed via the Higgs mechanism into the longitudinal components, $W_{L}^{ \pm}$and $Z_{L}^{0}$, of the weak gauge bosons:

$$
F_{\pi}=2^{\frac{1}{4}} G_{F}^{-\frac{1}{2}}=246 \mathrm{GeV} \text {. }
$$

New physics must occur near this energy scale. New particles produced in parton scattering processes at this energy may appear as fairly distinct resonances in weak gauge boson or fermion-antifermion final states, or only as relatively featureless enhancements of $W_{L}$ and $Z_{L}$ boson production or of missing energy. Whatever form they take, it is the energy scale of $1 \mathrm{TeV}$ and the size of typical QCD and electroweak cross sections at this energy, $\sigma \simeq 1 \mathrm{nb}-1 \mathrm{fb}$, that determine the energy and luminosity requirements of the SSC: $\sqrt{s}=40 \mathrm{TeV}$ and $\mathcal{L}=10^{33}-10^{34} \mathrm{~cm}^{-2} \mathrm{~s}^{-1}$ ?

The energy scale of flavor symmetry breaking is not known. It may lie anywhere from just above the weak scale, $1 \mathrm{TeV}$, up to the Planck scale, $10^{16} \mathrm{TeV}$. There is the possibility that the SSC will shed light on the flavor problem, but no guarantee. Several examples accessible at the SSC are introduced below and discussed in Chapter 2. Their production cross sections range from quite large $(\sim 1-10 \mathrm{nb})$ to very small $(-1-10 \mathrm{fb})$. Finding the rare processes will require the full SSC energy and the maximum possible luminosity. 
Several scenarios have been proposed for electroweak and flavor symmetries, and their breaking:?

- Standard Higgs models, containing one or more elementary Higgs boson multiplets. These are generally complex weak doublets. The minimal model has one doublet, with a single neutral boson $H^{0}$.

- Supersymmetry. In the minimal supersymmetric standard model there are two Higgs doublets, and every known particle has a superpartner.

- Models of dynamical electroweak and flavor symmetry breaking. The most studied proposal is technicolor-plus-extended-technicolor, with one doublet or one family of technifermions.

- Composite models, in which quarks and leptons are built of more fundamental constituents.

None of these proposals is fully satisfactory. In elementary Higgs boson models, whether supersymmetric or not, there is no explanation of why electroweak symmetry breaking occurs and why it has the scale $F_{\pi}$. In non-supersymmetric models, the Higgs boson's mass, $M_{H}$, and its vacuum expectation value, $\nu=F_{\pi}$, are unstable against radiative corrections. There is no natural reason why these two parameters should be very much less than the energy scale at which the essential physics of the model changes, e.g., a unification scale or the Planck scale. ${ }^{8}$ This radiative instability may be cured because standard model interactions look supersymmetric down to about $1 \mathrm{TeV}$, where soft supersymmetry breaking effects become important. This is the motivation for supersymmetry at the electroweak scale.9,10 A further problem is that elementary Higgs boson models are known to be "trivial", i.e., they cannot make sense as interacting field theories with the cutoff taken to infinity. ${ }^{11}$ This means that elementary Higgs models are effective theories, meaningful only below some cutoff $\Lambda$ at which new physics sets in. Obviously, $\Lambda$ must be somewhat greater than $M_{H}$ for the effective theory to make sense. For a modest separation of these energies, $M_{H}<$ few $\times \Lambda$, both perturbative and lattice gauge calculations give $M_{H} \leq 650 \mathrm{GeV}$ in the minimal one-doublet model with $F_{\pi}$ fixed at $246 \mathrm{GeV} .12$ Finally, elementary Higgs models provide no clue to the meaning of flavor symmetry and the origin of its breaking. The flavor-symmetry breaking Yukawa couplings of the Higgs boson to fermions are arbitrary free parameters.

Despite these apparent problems, the standard Higgs boson, $H^{0}$, charged Higgses, $H^{ \pm}$, and the supersymmetric partners of all the known particles may exist and must be sought. However, if something like the standard $H^{0}$ is found and is heavier than about $700 \mathrm{GeV}$, experiments must have the capacity to discover the additional, unspecified new physics that surely exists in the same energy region, of order $1 \mathrm{TeV}$.

Dynamical theories of electroweak and flavor symmetries-technicolor and extended technicolor-address these shortcomings of the elementary Higgs boson models. ${ }^{13}$ However, they do so at the heavy price of introducing flavor-changing neutral currents that are too large, and pseudo-Goldstone bosons (technipions) that are too light. ${ }^{14}$ These difficulties have been mitigated, but only by invoking an unfamiliar strong dynamics. ${ }^{15,16}$ Further, it is difficult to build realistic models; most simple technicolor models appear to be in conflict with precision tests of the electroweak interactions. ${ }^{3}$ Realistic models of composite quarks and leptons are similarly difficult to construct. ${ }^{17}$ Therefore, no compelling models of dynamical electroweak and flavor symmetry breaking exist. Nevertheless, model-independent phenomenological programs do exist for testing such models at the SSC. ${ }^{7}$

These difficulties have led to the widespread belief that none of the familiar descriptions of electroweak and flavor symmetry breaking is entirely correct. This is, in fact, the most exciting aspect of SSC physics. We know that there is new physics in the TeV energy regime and that the SSC can reach it. We do not know exactly what form it will take. The models are invaluable because they furnish a wide range of predicted signals and backgrounds. These provide a testing ground to guide the design, and to help ensure the discovery potential, of large detectors such as GEM. An overview of the GEM detector is presented in Chapter 1 and GEM's capabilities for a representative sample of processes involving photons, electrons, muons, jets and missing energy are examined in Chapter.2. Whatever physics lies in the $\mathrm{TeV}$ region, its signals and backgrounds are bound to be similar to some of those studied there. 


\section{REFERENCES}

1. S. L. Glashow, Nucl. Phys. 22, 579 (1961); S. Weinberg, Phys. Rev. Lett. 19, 1264 (1967); A. Salam, in Proceedings of the 8th Nobel Symposium on Elementary Particle Theory, Relativistic Groups and Analyticity, edited by N. Svartholm (Almquist and Wiksells, Stockholm. 1968), p. 367; H. Fritzsch. M. Gell-Mann, H. Leutwyler, Phys. Lett. 47B, 365, (1973); D. Gross and F. Wilczek, Phys. Rev. Lett. 30. 1343 (1973); H.D. Politzer, Phys. Rev. Lett. 30, 1346 (1973).

2. R. Cahn and G. Goldhaber, The Experimental Foundations of Particle Physics, (Camoridge University Press, 1989).

3. For a recent review, see P. Langacker, M.-X. Luo and A.K. Mann, Rev. Mod. Phys. 64, 87 (1992).

4. The $95 \%$ confidence-level limit on the top-quark mass, assuming it decays in the standard way, $t \rightarrow W^{\prime}-b$, is $m_{1}>91 \mathrm{GeV}$. The limit obtained without assuming dominance of the standard decay mode is $m_{t}>55 \mathrm{GeV}$. While the tau-neutrino has not yet been directly observed, there is little doubt that it exists and that it and the $\tau^{-}$form a standard lepton doublet. The $95 \%$ limit on its mass is $m_{\nu_{\tau}}<35 \mathrm{MeV}$. See Reference 5.

5. Particle Data Group, K. Hikasa et al., Phys. Rev. D45. S1 (1992).

6. P.W. Anderson, Phys. Rev. 110, 827 (1958); ibid., 130, 439 (1963); Y. Nambu, Phys. Rev. 117, 648 (1959); J. Schwinger, Phys. Rev. 125, 397 (1962); P. Higgs, Phys. Rev. Lett. 12, 132 (1964); F. Englert and R. Brout, Phys. Rev. Lett. 13, 321 (1964); G.S. Guralnik, C.R. Hagen and T.W.B. Kibble, Phys. Rev. Lett. 13, 585 (1964).

7. E. Eichten, I. Hinchliffe, K. Lane and C. Quigg, Rev. Mod. Phys. 56, 579 (1984).

8. K.G. Wilson, unpublished; quoted in L. Susskind, Phys. Rev. D20, 2619 (1979); G. t' Hooft, in Recent Developments in Gauge Theories, edited by G. t' Hooft, et al. (Plenum, New York, 1980).

9. S. Dimopoulos and H. Georgi, Nucl. Phys. B193, 153 (1981); A.H. Chamseddine, R. Arnowitt and P. Nath, Phys. Rev. Lett. 49, 970 (1982); L.J. Hall, J. Lykken and S. Weinberg, Phys. Rev. D27, 2359 (1983).

10. For reviews of supersymmetry and its phenomenology, see H.E. Haber and G.L. Kane, Phys. Rept. 117, 75 (1985); S. Dawson, E. Eichten and C. Quigg, Phys. Rev. D31, 1581 (1985).

11. See, for example, R. Dashen and H. Neuberger, Phys. Rev. Lett. 50, 1897 (1983); J. Kuti, L. Lin and Y. Shen, Phys. Rev. Lett. 61, 678 (1988); A. Hasenfratz, et al., Phys. Lett. B199, 531 (1987); G. Bhanot and K. Bitar, Phys. Rev. Lett. 61, 798 (1988).

12. This is not a pressing issue in the minimal supersymmetric standard model because the Higgs masses are relatively low and, so, the cutoff $\Lambda$ may be very high indeed.

13. S. Weinberg, Phys. Rev. D13, 974 (1976); ibid., D19, 1277 (1979); L. Susskind, Phys. Rev. D20, 2619 (1979); S. Dimopoulous and L. Susskind, Nucl. Phys. B155, 237 (1979); E. Eichten and K. Lane, Phys. Lett. 90B, 125 (1980).

14. E. Eichten and K. Lane in Ref. 13; J. Ellis, M. Gaillard, D. Nanopoulos and P. Sikivie, Nucl. Phys. B182, 529 (1981).

15. B. Holdom, Phys. Rev. D24, 1441 (1981); Phys. Lett. B150, 301 (1985); T. Appelquist, D. Karabali and L.C.R. Wijewardhana, Phys. Rev. Lett. 57, 957 (1986); T. Appelquist and L.C.R. Wijewardhana, Phys. Rev. D36, 568 (1987); K. Yamawaki, M. Bando and K. Matumoto, Phys. Rev. Lett. 56, 1335 (1986); T. Akiba and T. Yanagida, Phys. Lett. B169, 432 (1986).

16. T. Appelquist, T. Takeuchi, M.B. Einhom, L.C.R. Wijewardhana, Phys. Lett. B220, 223 (1989); T. Takeuchi, Phys. Rev. D40, 2697 (1989); V.A. Miransky and K. Yamawaki, Mod. Phys. Lett. A4, 129 (1989); R.S. Chivukula, A.G. Cohen and K. Lane, Nucl. Phys. B343, 554 (1990).

17. See G. $t$ ' Hooft in Reference 8. 


\section{OVERVIEW}

\subsection{INTRODUCTION}

The GEM collaboration was formed in June 1991 to develop a major detector for the SSC. The primary physics objectives of GEM are those central to the motivation for the SSC: to study high $p_{T}$ physics-exemplified by the search for Higgs bosons - and to search for new physics beyond the standard model.

We present in this Technical Design Report (TDR) a detector with broad capabilities for the discovery and subsequent study of electroweak symmetry breaking, the origin of mass and flavor, and other physics requiring precise measurements of gammas, electrons, and muons-hence the name, GEM. (See Figure 1-1.) In addition, as a design goal, we have taken care to provide the robustness needed to do the physics that requires high luminosity. Finally, good coverage and hermeticity allow the detection of missing transverse energy, $E_{T}$.

The GEM design emphasizes clean identification and high resolution measurement of the primary physics signatures for the high $p_{T}$ physics summarized in Table 1-1. Our approach is to make precise energy measurements that maximize the sensitivity to rare narrow resonances, to detect the elementary interaction products (quarks, leptons, and photons), and to builo in the features required to reduce backgrounds. The design of the GEM detector is based on the following principles:

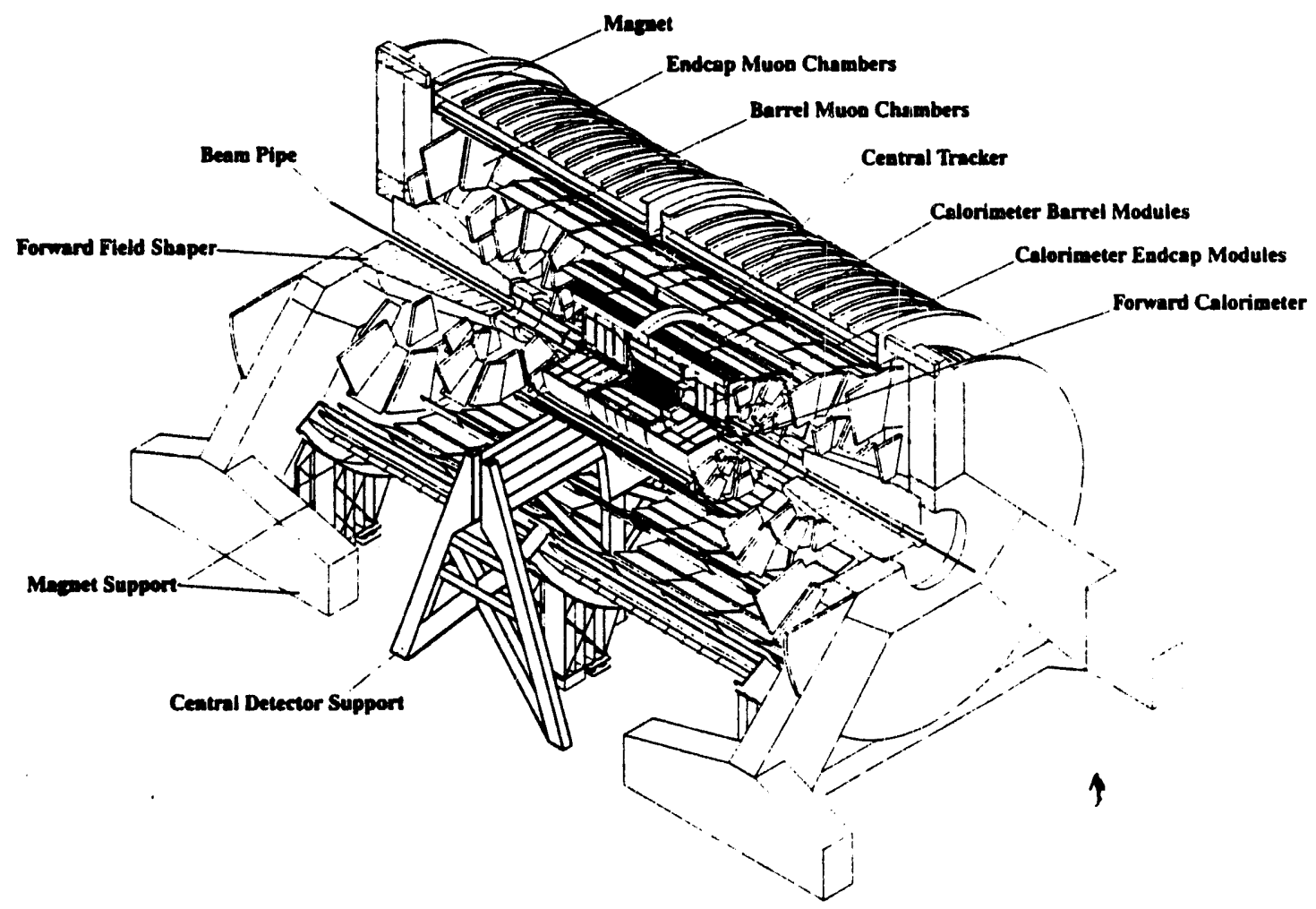

FIG. 1-1. Perspective view of the GEM detector. 
Table 1-1. Physics signatures at the SSC.

\begin{tabular}{|c|c|}
\hline Physics & Signatures \\
\hline Standard $H^{\circ}$ & $\begin{array}{l}\gamma \gamma_{1}(\pi M) H^{0} \rightarrow \gamma \gamma \ell=X \\
Z^{*} \rightarrow \ell^{-} \ell^{-} \ell^{+} \ell^{-} \\
Z Z \rightarrow \ell^{+} \ell^{-} \ell^{+} \ell^{-}, \ell^{-} \ell^{-} j j, \\
\\
\ell^{+} \ell^{-} \nu \tau\end{array}$ \\
\hline Extended $\mathrm{H}^{\circ}, h^{\circ}, \mathrm{H}^{*}$ & $\begin{array}{l}\text { Same as above } \\
t \rightarrow H^{+} b \\
h^{0} \cdot H^{P} \rightarrow \tau^{+} \tau^{-}\end{array}$ \\
\hline $\begin{array}{l}\text { Heavy } Q \bar{Q} \\
Z^{\prime}, W^{\prime}\end{array}$ & $\begin{array}{l}W=q \rightarrow \text { jets }+ \text { isolated } \ell^{ \pm} \\
\ell^{+} \ell^{-}, \ell^{+}+\ell_{T}\end{array}$ \\
\hline Technicolor & $\begin{aligned} e_{T} & \rightarrow j j, W Z\left(\rightarrow l^{ \pm} j \theta t s\right) \\
& \rightarrow \pi_{T} \pi_{T} \\
\pi_{T} & \rightarrow \text { heavy } 7 t \text { dijets }\end{aligned}$ \\
\hline $\begin{array}{l}\text { Supersymmetry } \\
q \text { substructure }\end{array}$ & $\begin{array}{l}\tilde{F}_{T} \text {, jots, } \ell^{ \pm} \ell^{ \pm} \text {, multi-leptons } \\
\text { high-mass dijets }\end{array}$ \\
\hline $\begin{array}{l}q / \ell \text { substructure } \\
\text { None of the above }\end{array}$ & $\begin{array}{l}\text { high-mass dileptons, } \boldsymbol{F}_{T} \\
\text { All of the above }\end{array}$ \\
\hline
\end{tabular}

- Very precise electromagnetic calorimetry without a magnet coil in front of it. This will provide the best measurements of gamma and electron energies, to allow the reconstruction of the mass of narrow states with good resolution.

- A precise $4 \pi$ muon spectrometer in a large superconducting solenoidal magnet, allowing measurement of the momenta of high energy muons with a minimum of multiple scattering. The muon system operates in a quiet environment, shielded by the thick calorimeter.

- Hermetic hadronic calorimetry for the measurement of jets and the reconstruction of missing energy.

- Central tracking in a magnetic field with sufficiently low occupancy to operate reliably at the highest luminosities that can be anticipated at the SSC $\left(10^{34} \mathrm{~cm}^{-2} \mathrm{~s}^{-1}\right)$. The central tracker can be compact, allowing for a compact calorimeter and a large muon tracking volume.
All technology decisions for the GEM detector have now been made, with the relatively small exception of the forward calorimeter hadronic section. The technologies chosen will provide good performance even at the highest luminosities at the SSC. Our reliance on the calorimetry and the muon system to provide the precise gamma, electron, and muon momentum measurements, and thus to allow precise mass reconstruction, further ensures undiminished performance at the highest luminosities available.

The GEM detector has been designed to the cost goal of \$500M (FY90). A careful cost estimate has been carried out for the GEM baseline design described in this TDR, and is presented in detail in the "GEM Summary Cost and Schedule Book." The projected completion date is in 1999 . We have been able to meet our cost goal without any permanent sacrifice in the required performance of the detector. However, a number of items were removed from the baseline design that can be added as upgrades. Significant further cost reductions would seriously compromise the physics performance of the detector. Careful physics simulation studies have demonstrated that the current baseline design is cost-optimized to do the physics for which the SSC is being built. The physics reach of the GEM design for topics of major interest to the SSC is summarized in Figure 1-2.

\subsection{PHYSICS AND THE GEM DESIGN}

One of the primary goals of GEM is to provide complete coverage for Higgs physics from $80-800 \mathrm{GeV}$. The lower mass represents the limit of the LEP II reach, while the upper mass represents the highest value for which the basic idea of the Higgs mechanism makes sense theoretically. As a high-precision lepton and photon detector, GEM has a discovery potential illustrated by its ability to detect Higgs particles in the challenging "intermediate mass" range between 80 and $180 \mathrm{GeV}$. In particular, the distinctive $\gamma \gamma$ decay mode will allow GEM to explore the gap between $80 \mathrm{GeV}$ and $140 \mathrm{GeV}$. The production of the Higgs boson in association with a $\bar{t}$ pair provides important confirmation of the $\gamma \gamma$ signal. 


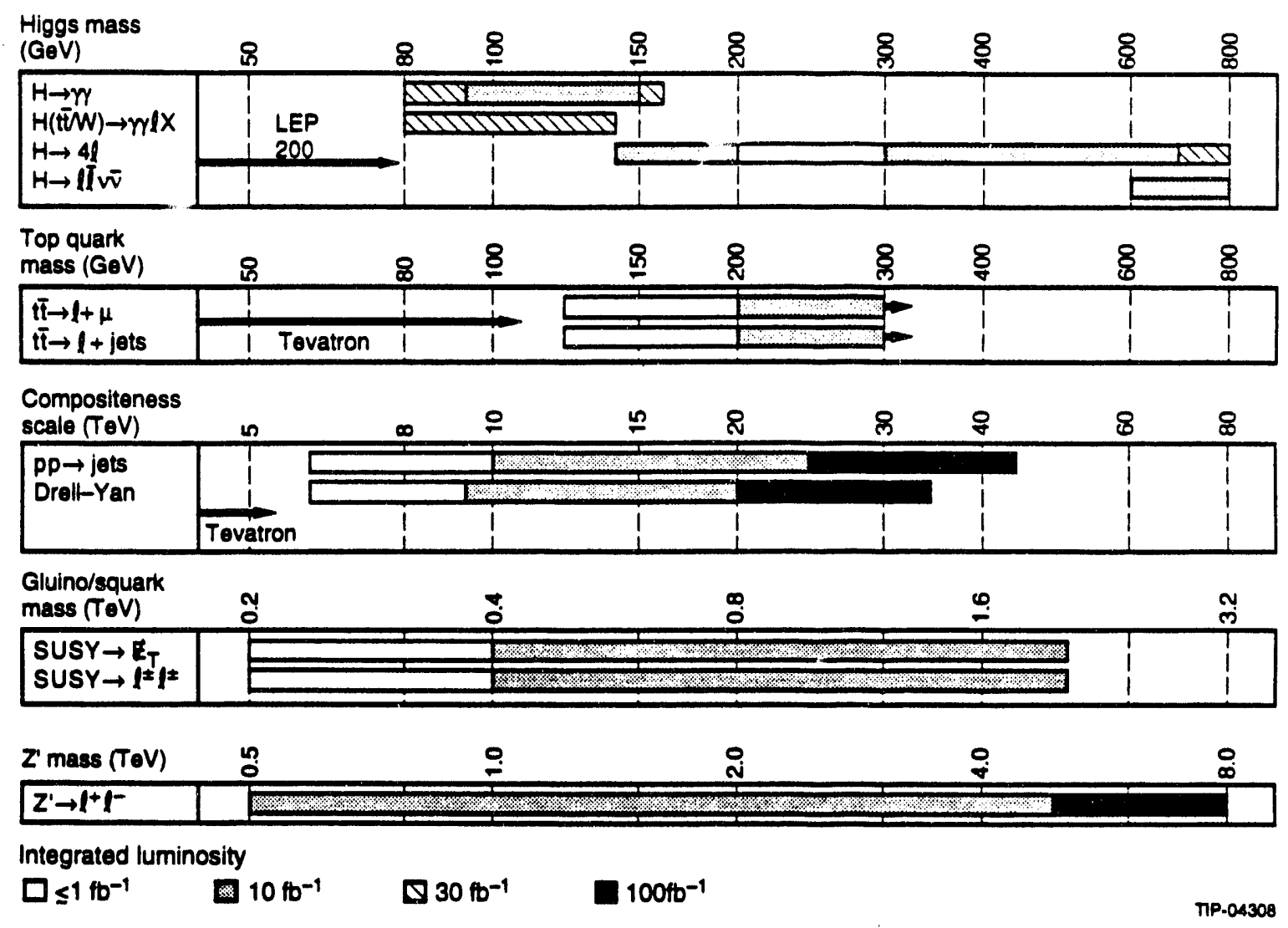

FIG. 1-2. The physics reach of GEM.

$H^{0} \rightarrow \gamma \gamma$ detection places stringent requirements on the overall detector design, especially the design of the electromagnetic calorimeter. In the $80-140 \mathrm{GeV}$ mass range, the $\gamma \gamma$ invariant mass must be measured with high precision and good background rejection in order to detect the signal above the background. In the context of the minimal standard model, the production cross-section is 160 to $260 \mathrm{fb}$, as compared to an irreducible direct $\gamma \gamma$ background that is more than 1000 times larger, in addition to QCD jet background. A Higgs boson signal can still be detected, due to its narrow decay width ( 5 to $10 \mathrm{MeV}$ ), but only if the resolution is sufficiently high and background rejection is good enough. For GEM, this stringent set of requirements has motivated the use of a liquid krypton fine-sampling electromagnetic calorimeter. In this crucial energy region, both precise resolution for the stochastic term $(\leq 6 \% / \sqrt{E}$ in the barrel; $\leq 8 \% / \sqrt{E}$ in the endcap) and good control of the systematic term $(\leq 0.4 \%)$ are required. Much care has been given to the development of a calorimeter design that meets these requirements for GEM. In order to reduce backgrounds, the GEM design includes longitudinal sampling and good pointing ability $(40-50 \mathrm{mrad} / \sqrt{\mathrm{E}}$ $+0.5 \mathrm{mrad}$ ) in the calorimeter. The combination yields a signal/background ratio sufficient for the discovery of the Higgs boson at design luminosity, and for the exploration of the Higgs sector at luminosities up to 10 times higher.

Of similar difficulty is the detection of a Higgs boson in the next higher mass region, approximately $140-180 \mathrm{GeV}$, where the best modes are $H^{0} \rightarrow$ $\mathrm{ZZ}^{*} \rightarrow l+r l+r$. Since this region is below threshold for producing two real $Z s$, the rate is low, again making the detection difficult. In GEM, we plan to measure all modes $-e^{+} e^{-} e^{+} e^{-}, \mu^{+} \mu^{-} \mu^{+} \mu^{-}$, $e^{+} e^{-} \mu^{+} \mu^{-}$-with good acceptance and resolution.

For a standard model Higgs boson mass between $200-800 \mathrm{GeV}$, the signature of four isolated leptons from two $Z$ decays is very clean and straightforward to detect. However, as the mass increases, the rates fall and the Higgs broadens. At the highest mass $(\sim 800 \mathrm{GeV})$, where the rates are lowest, it is necessary either to run at higher 
luminosities or to add the complementary modes $l^{+} \Gamma$ jet jet and $l+r v \bar{v}$, to be able to discover the Higgs boson in one year at the standard luminosity $\left(\mathcal{L}=10^{33} \mathrm{~cm}^{-2} \mathrm{~s}^{-1}\right)$.

The considerations necessary to make a detector robust at high luminosity - choice of technologies, segmentation, ability to withstand radiation, and integrated shielding from backgrounds-are all important. We have paid particular attention to these points, and we expect GEM, without major upgrades, to have important capabilities up to the highest luminosities $\left(\ell=10^{34} \mathrm{~cm}^{-2} \mathrm{~s}^{-1}\right)$ expected at the SSC. This ability is demonstrated in Figure 1-3, which shows the signal expected in one year at this luminosity for $Z^{\prime} \rightarrow \mathrm{e}^{+} \mathrm{e}^{-}$at a mass of $4 \mathrm{TeV}$. Note the rapidly falling Drell-Yan background, the signal with good resolution, and the small residual background under the signal peak. The observed width is model-dependent and our mass resolution of $0.3 \%$ allows us to distinguish among models. It should also be noted that in GEM, the couplings of the $Z^{\prime}$ to fermions can be probed by high luminosity studies of angular distributions of the muons from $Z^{\prime} \rightarrow \mu^{+} \mu^{-}$. Our muon system's unique ability to measure multi-TeV muons with negligible charge confusion will allow us to study the couplings of the heaviest $Z^{\prime}$ that can be produced at the SSC.

If supersymmetry exists-for example, as in the minimal supersymmetric extension to the standard model - then the detection and study of supersymmetric Higgs bosons is likely to be more difficult than in the simple examples given above. The highest possible lepton and photon resolution will be needed to maximize the detector's ability to discover the supersymmetric Higgs boson within the first few years of running at the SSC.

These examples are characteristic of areas where the physics motivation of GEM determines the design. More generally, GEM is designed to aim for all the physics goals listed in Table 1-1. Our philosophy is to cover this wide range of physics with the idea that whether or not any of these specific ideas prove true, GEM's capabilities will provide us with the tools needed to discover and explore whatever unknown physics may exist at the SSC.

A complementary strength of the GEM design, with a compact inner tracker, modular calorimeter, and large volume muon system, is its adaptability to major advances in physics (or particle detection technology) that may occur in the course of the SSC experimental program. Although the GEM design is optimized to cover the broad range of new physics scenarios and signatures that are currently envisioned, progress in our understanding may lead to new requirements for higher performance in the long term. Replacement of an inner detector subsystem or extension of the muon system's lever arm outside the magnet coil could then be implemented at moderate cost, in order to extend the physics reach in specific directions. This adaptability ensures that GEM will be able to continue to do front-line physics for many years, well beyond the first phase of the SSC program.

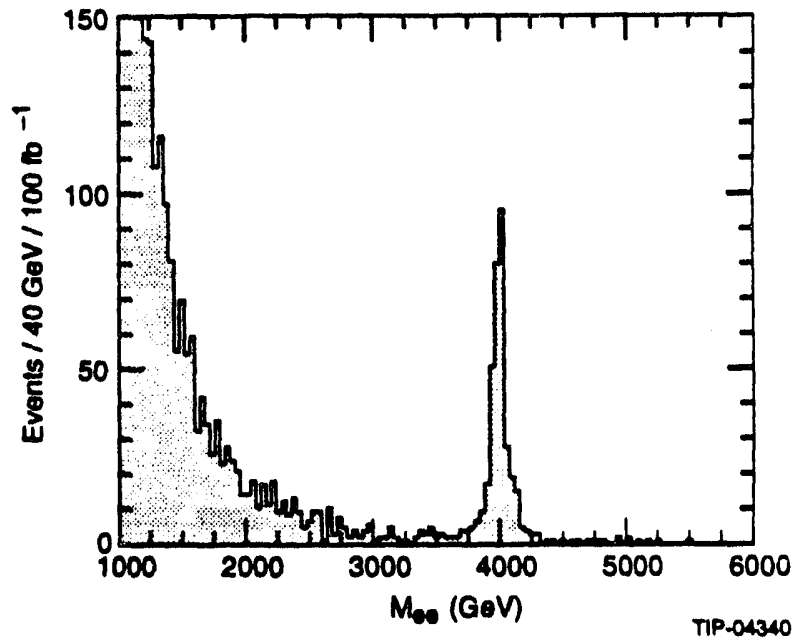

FIG. 1-3. The high luminosity $\left(\ell=10^{34} \mathrm{~cm}^{-2} \mathrm{~s}^{-1}\right)$ performance of GEM for detecting a 4 TeV Z' $\rightarrow e^{+} e^{-}$in one year of running.

\subsection{DETECTOR DESIGN}

In the design of GEM we have extensively used simulation techniques to set requirements and to evaluate performance of the proposed detector. We have used full GEANT simulations in detailed studies and design of our subsystems, and in this TDR we present these studies in the subsystem chapters. In physics simulation studies, for efficient use of the available computer resources, we have used either parametrized studies or, where necessary, hybrids of full and parametrized simulations. In Chapter 2, we evaluate and present the physics performance for a wide variety of processes using these tools, with an emphasis on the parametrized program gemfast, which simulates detector performance well. This approach is founded in a broad 
range of specific full-simulation studies, as described in the various subsystem chapters.

A vertical cross-section view of the large, 0.8-T magnet, with the detector elements placed inside, is shown in Figure 1-4. The main elements are a compact central tracker and hermetic calorimetry for precision electromagnetic measurements of electrons and photons, plus the detection of jet energy and $E_{T}$ with good resolution. The region outside the calorimeters provides a large volume, well-shielded from the interaction point, where accurate muon momentum measurements are made. The top-level specifications for the GEM detector are given in Table 1-2. The detector approach described here is complementary to the SDC detector, going beyond its physics reach in specific areas, while maintaining an important degree of overlap in the two detectors' capabilities. The SDC detector features a large tracker, while GEM emphasizes precise measurement of gammas, electrons, and muons, plus unique capabilities at high luminosity.

The detailed optimization of our design is determined by the physics requirements, the practical ability to meet the necessary performance specifications, and cost/schedule constraints. We have paid particular attention to detector integration issues such as radiation shielding and the interface between the beamline and the inner radius of the detector. The detector design results from a detailed research and development and engineering design phase that has led to the choice of detector technologies and to their application in an integrated system that is optimized for physics discovery. The techn!cal choices are based on overall detector performance, a philosophy of simplicity and uniformity of design, reliability and ease of calibration, flexibility in the means of access and installation, and the issues of cost and schedule. In making the major technology choices, we have used a process of comparative review (often including outside experts), open discussions at GEM Collaboration Council meetings, technical documentation through GEM internal notes, discussion and recommendations by the GEM Executive Committee, and finally decisions by the spokesmen.

Detailed descriptions of the detector subsystems, including technical features, implementation and integration issues, and studies of expected performance are given in the following chapters. In all discussions of assembly, cost, and schedule, the TDR is based on the SSC baseline completion date of 1999. We describe briefly some of the key features of each major subsystem below.

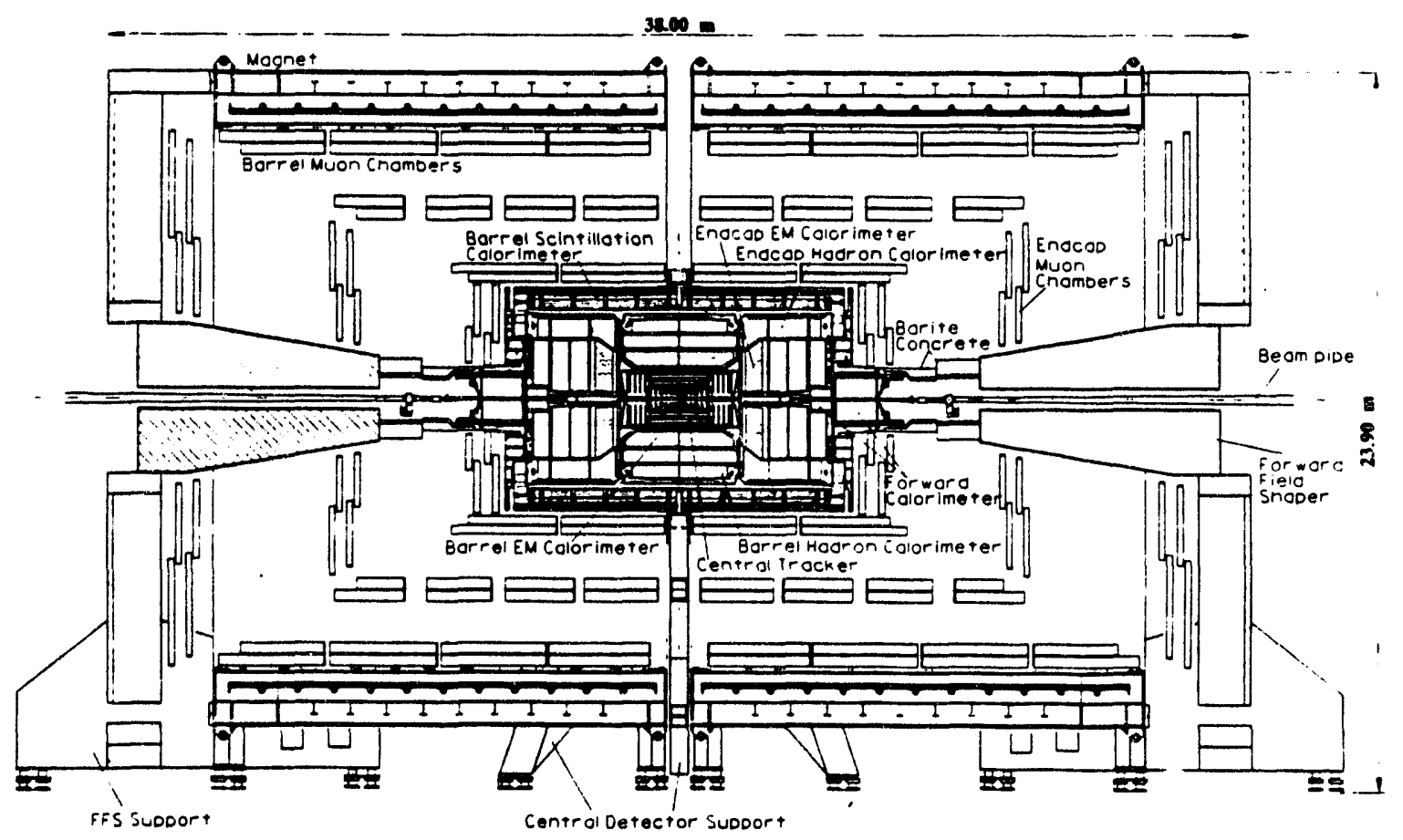

FIG. 1-4. Vertical section of the $G_{L} A$ detector. 
Table 1-2. Top-level specifications for the GEM detector.

\begin{tabular}{ll}
\hline Magnet & \\
Central field & $0.8 \mathrm{~T}$ \\
Inner diameter & $18 \mathrm{~m}$ \\
Length & $31 \mathrm{~m}$ \\
Muon system & \\
Coverage & $0.1<|\eta|<2.5$ \\
$\Delta p_{T} / P_{T}$ at $|\eta|=0, p_{T}=500 \mathrm{GeV}$ & $5 \%$ \\
$\Delta p_{T} / P_{T}$ at $|\eta|=2.5, p_{T}=500 \mathrm{GeV}$ & $12 \%$ \\
Charge separation ( $\eta=0)$ & $p \leq 6.5 \mathrm{TeV}$ at $95 \% \mathrm{C} . \mathrm{L}$. \\
Electromagnetic calorimeter & \\
Coverage & $|\eta|<3$ \\
Energy resolution & $6-8 \% / \sqrt{E} \oplus 0.4 \%$ \\
Position resolution & $4.4 \mathrm{~mm} / \sqrt{E}$ \\
Pointing resolution & $40-50 \mathrm{mrad} / \sqrt{E}+0.5 \mathrm{mrad}$ \\
Hadronic calorir ter & $\Delta p / p=3.5 \%$ \\
Coverage & $|\eta|<5.5$ \\
Jet resolution & $60 \% / \sqrt{E} \oplus 4 \%$ \\
Tracker & \\
Coverage & $|\eta|<2.5$ \\
Charge separation at $95 \%$ C.L. ( $\eta=0$ ) & $p \leq 600 \mathrm{GeV}$ \\
Momentum resolution & \\
at high momenta (measurement limited) & \\
at low momenta (multiple scattering limited) & \\
\hline
\end{tabular}

\subsubsection{Magnet}

GEM employs a very large superconducting solenoid that surrounds the detector elements. In the forward region we employ field shaping iron poles. The magnet design is optimized for field, radius, and length, with a nominal field of $0.8 \mathrm{~T}$, an inner diameter of $18 \mathrm{~m}$, and a length of $30.8 \mathrm{~m}$, as described in Chapter 3. The major design parameters for the magnet are given in Table 1-3.

The very large size of the GEM magnet dictates the choice of a superconducting solenoidal coil design. In addition, cost and risk considerations have led to a conservative design with a single-layer winding, using a niobium-titanium superconductor with a large stability margin. Savings in cost and installation time have been achieved by selecting a design with no return yoke.
The magnet provides a nearly uniform axial field in the region of the central tracker, allowing measurements of the momentum of emerging charged particles from the interactions. This allows sensitivity to same-sign electron and same-sign muon final states, including gluinos over a wide range of parameter space. In the volume of detector outside the calorimeters, the magnet provides a 0.8-T field for muon momentum measurements.

Another feature of the magnet system is the pair of conical forward field shapers, one at each end of the solenoid. The field shapers introduce a radial component to the forward field by concentration of the field lines, enabling the muon system to meet the momentum resolution requirement in the forward direction. The final element in the magnet system is 
the stainless steel central detector support (CDS) that supports the calorimeters and the central tracker.

Table 1.3. Major design parameters ol the GEM magnet.

\begin{tabular}{ll}
\hline Magnet: & \\
Central field & $0.80 \mathrm{~T}$ \\
Inductance & $1.98 \mathrm{H}$ \\
Operating current & $50.2 \mathrm{kA}$ \\
Stored energy & $2.5 \mathrm{GJ}$ \\
Axial force on conductor (each half) & $52 \mathrm{MN}$ \\
Mean radius of windings & $9.5 \mathrm{~m}$ \\
Length of cold mass (each half) & $14.25 \mathrm{~m}$ \\
Total mass of magnet (each half) & $1300 \mathrm{Mg}$ \\
\hline Forward Field Shaper (FFS): & \\
\hline FFS cone minimum Z & $10 \mathrm{~m}$ \\
FFS cone maximum Z & $18 \mathrm{~m}$ \\
FFS cone inner radius (minimum) & $0.350 \mathrm{~m}$ \\
FFS cone outer radius (maximum) & $2.5 \mathrm{~m}$ \\
\hline Total mass of FFS (each) & $899 \mathrm{Mg}$ \\
\hline
\end{tabular}

Th- coil will be manufactured in two halves on the surface, lowered into the underground hall and mounted on each side of the CDS. The coil halves are designed to be movable along the beamline, which is important for installation and detector access. The field shapers are separate assemblies, also movable along the beam axis.

The principal challenge for the magnet is associated with its size; the superconducting coil design is conservative and carries little technical risk. Because of its size, the magnet must be constructed at the site. A "request for proposals" for construction of the magnet has been issued, propos- als have been received. and the proposal evaluationis under way. The scheduled completion during 1996 requires early availability of surface facilities, where the coils will be wound, and of the underground experimental hall, where the magnet will be assembled.

\subsubsection{Muon System}

Precise muon measurements, robust to high luminosity, are a primary goal of GEM. Muons provide signatures for a wide range of possible important new physics. Our design provides excellent muon information up to the kinematic limit of the SSC. At the top end of this range, the ability to operate at high luminosity and to determine the charge of multi- $\mathrm{TeV}$ muons is essential for heavy $Z^{\prime} \rightarrow \mu^{+} \mu^{-}$studies. High-resolution measurements of muon momentum are required to search for $H^{0} \rightarrow Z^{*} \rightarrow \mu^{+} \mu^{-} \mu^{+} \mu^{-}$in the difficult region from 140-180 GeV. Good coverage of muons for $|\eta|<2.5$ is especially important for low rate processes such as $H^{0} \rightarrow Z Z^{*} \rightarrow \mu^{+} \mu^{-} \mu^{+} \mu^{-}$. Robustness comes into play again for $H^{0} \rightarrow \mu^{+} \mu^{-} \mu^{+} \mu^{-}$at high mass (e.g., $800 \mathrm{GeV}$ ) and for the search for quark-lepton substructure.

To perform well for this range of physics, the GEM muon system is designed to be precise: $\Delta p_{T} / p_{T}$ $=5 \%(12 \%)$ at $\eta=0(2.5)$ for $p_{T}=500 \mathrm{GeV}$. In addition, it is shielded very well from background sources, both by the thick hermetic calorimeter and by other shielding. This enables it to be sufficiently robust to operate at the highest luminosities ( $L=$ $10^{34} \mathrm{~cm}^{-2} \mathrm{~s}^{-1}$ ) attainable at the SSC. The major design parameters of the muon system are given in Table 1-4.

Muons are identified by their penetration through the calorimeter system (Figure 1-5). Muon momentum is measured using the sagitta method in three superlayers between the calorimeter and the magnet. The resolution in the sagitta measurement varies as $B L^{2}$, where $B$ is the magnetic field strength and $L$ is the lever arm of the measurement. 
Table 1-4. Major design parameters of the GEM muon subsystem.

\section{Coverage:}

Barrel region:

$\left(29.23^{\circ}<\theta<84.3^{\circ}\right)$

Endcap region:

$\left(9.75^{\circ}<\theta<27.71^{\circ}\right)$

Number of sectors in $\phi$

48

Lever arm:

Barrel $\quad>4.2 \mathrm{~m}$

Endcap $\quad>8.6 \mathrm{~m}$

Chamber parameters:

\begin{tabular}{|c|c|}
\hline Single-layer resolution & $75 \mu \mathrm{m}$ (RMS) \\
\hline Timing resolution & $3.5 \mathrm{~ns}$ \\
\hline Beam-crossing tag efficiency & $>99 \%$ \\
\hline Internal chamber alignment & $50 \mu \mathrm{m}$ \\
\hline $\begin{array}{l}\text { Superlayer-to-superlayer } \\
\text { alignment }\end{array}$ & $25 \mu \mathrm{m}$ \\
\hline Radiation length/chamber layer & $1.1 \%$ \\
\hline $\begin{array}{l}\text { No. of chamber planes per } \\
\text { supertayer (SL1:SL2:SL3) }\end{array}$ & $\begin{array}{l}\text { 6:6:6 barrel } \\
\text { 8:6:6 endcap }\end{array}$ \\
\hline
\end{tabular}

The muon momentum resolution is determined at high momenta by the spatial measurement errors (both inherent and due to misalignment), and at low momenta by the multiple scattering in the middle layer of chambers and energy-loss fluctuations in the calorimeter. It is therefore crucial to have high accuracy in position measurements, minimum scattering material, and the best possible measurement of muon energy loss in the calorimeter. Our studies of the effects of the muon resolution on the ability to detect Higgs boson decays through the signature $H^{0} \rightarrow Z Z^{*} \rightarrow 4 l$, indicate that the middle layer must be less than $10 \%$ of a radiation length in order not to degrade the measurement. For very high momentum (e.g., from $Z^{\prime}$ decay at the highest mass, $\sim 8 \mathrm{TeV}$, that is accessible at the SSC) the most demanding problem is sign selection for each muon. This requirement demands single layer resolutions of $75 \mu \mathrm{m}$ and alignment between superlayers of $25 \mu \mathrm{m}$.

Another consideration that affects the design of the muon system is chamber occupancy. To keep the rates in the muon region at tolerable levels for luminosities above $10^{34} \mathrm{~cm}^{-2} \mathrm{~s}^{-1}$. we employ a thick ( $211 \lambda$ at $\eta=0$, increasing in the forward direction), nearly hermetic calorimeter system with a design

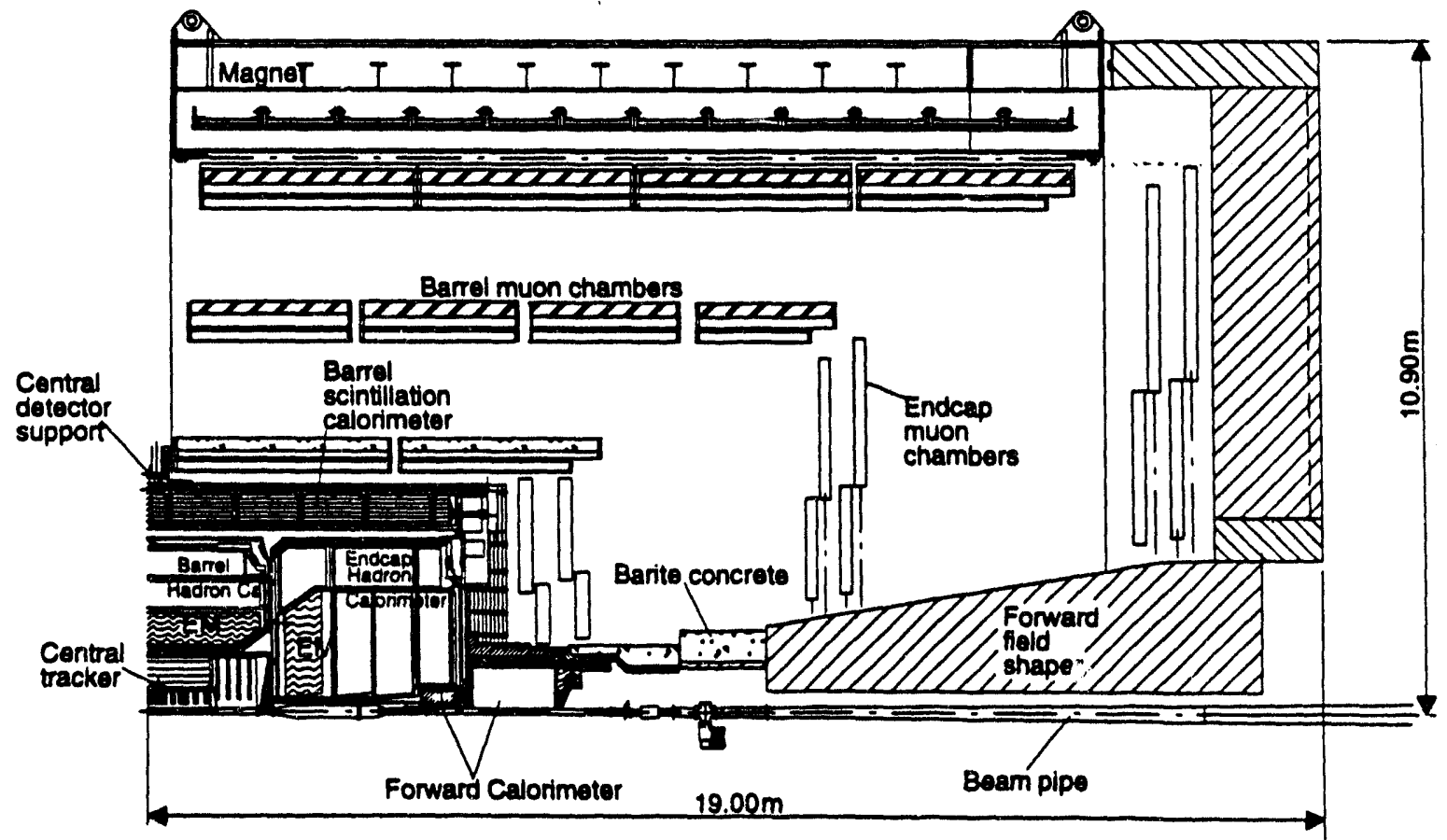

FIG. 1-5. Quarter view of the detector showing the muon system, including shielding. 
for the forward direction that keeps the background contained within the calorimeter volume. The thickness is chosen such that the rate from punchthrough hadrons is significantly below that from in-flight decay muons.

A notable design feature of the muon system is the use of a $0.2-\mathrm{m}$ open space outside the calorimeter, before the first muon superlayer, to bend away charged particles arising from electromagnetic showers initiated by high-momentum muons. The clear space leads to higher reconstruction efficiency for $\mathrm{TeV}$ muons than in systems using chambers interleaved with iron.

A very important element is to provide a carefully designed shield to reduce the large neutron and photon backgrounds that result when particles emerging at large $\eta$ from the interaction region strike the low- $\beta$ quadrupoles, the forward field shapers, the forward calorimeters, and the beam pipe, and create electromagnetic and hadronic showers. It is noteworthy that the compact, close-in design of GEM's forward calorimeter system makes an exceptionally effective shielding configuration possible at moderate cost. A full discussion of these points is given in Chapter 12.

The choice of technology for the GEM muon spectrometer was based on an intensive research and development program. We considered a variety of systems using pressurized and unpressurized drift tubes, resistive plate chambers, and cathode strip chambers (CSCs). The first consideration was to obtain the required spatial resolution, which was achieved with all technologies. Other important criteria included the determination of the $z$ coordinate, triggering, and occupancy. We have selerted the CSCs because they meet all the requirements in a single technology and can be applied in both the endcaps and barrel. The technology choice was made recently, and, although we present here a complete and consistent muon system design that meets our design specifications, we expect the system to be further optimized for minimum material and maximum coverage. This will improve the performance and discovery ability for $H^{0} \rightarrow Z^{*} \rightarrow$ $\mu^{+} \mu^{-} \mu^{+} \mu^{-}$and $\mu^{+} \mu^{-} e^{+} e^{-}$.

Figure 1-6 shows the muon momentum resolution versus pseudorapidity for the baseline design as a function of transverse momentum, resulting from the baseline GEM muon system and magnet. As shown in the figure, this design provides $5 \%$ resolution at $\eta=0$ for muons with $p_{T}=500 \mathrm{GeV}$ and $12 \%$ resolution at $\eta=2.5$.

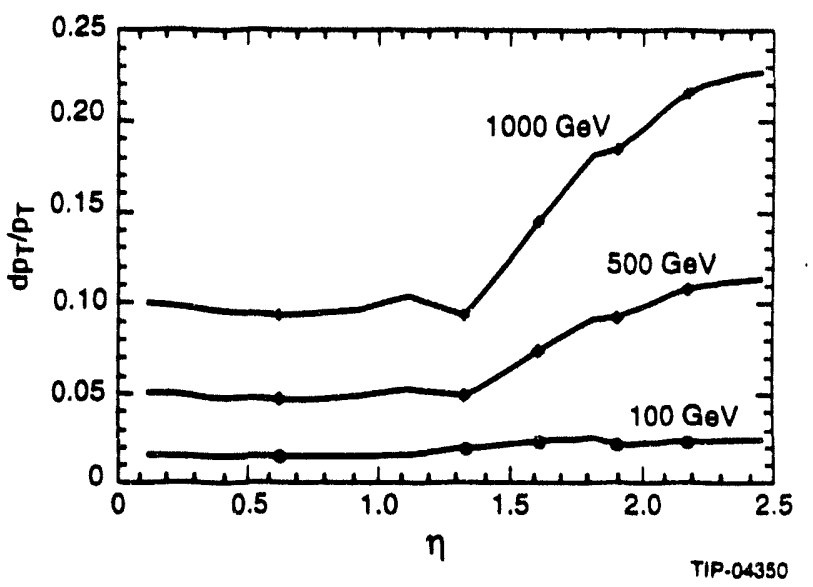

FIG. 1-6. Muon resolution vs. $\eta$.

\subsubsection{Calorimeter}

The major design parameters of the GEM calorimetry subsystem are given in Table 1-5. One of the principal goals of GEM is to achieve the best possible electromagnetic resolution and background rejection. These ambitious goals are motivated by the search for new physics, such as narrow resonances leading tc inulti-photon and/or multi-electron final states, as well as the search for Higgs particles. We also require good resolution for hadron jets and $E_{T}$. The general layout for the GEM calorimeter is shown in Figure 1-7.

The resolution of an electromagnetic calorimeter can be parametrized as $\sigma / E=a \% / \sqrt{E} \oplus b \%$, where $a$ is the stochastic term and $b$ the systematic term, and the terms are added in quadrature. For both the $H \rightarrow \gamma \gamma$ reaction and the $H \rightarrow Z Z^{*} \rightarrow 4$ l reaction, the typical particle energy is less than $100 \mathrm{GeV}$; at such energies, minimizing both terms is important to obtaining the required resolution.

For physics at higher energies $\left(e . g ., Z^{\prime} \rightarrow e^{+} e^{-}\right)$ the control of the systematic term is the most important factor. In addition, for small cross-section signals, good background rejection abilities and robustness at high luminosity are essential.

For the difficult intermediate mass Higgs boson, $80<\mathrm{M}<140 \mathrm{GeV}$, the primary signature is the decay $H^{0} \rightarrow \gamma \gamma$. Another important function of the GEM electromagnetic calorimeter is to provide 
sufficient resolution and background rejection to allow detection of $H^{0} \rightarrow Z Z^{*} \rightarrow e^{+} e^{-} e^{+} e^{-}$and $H^{0} \rightarrow$ $Z Z^{*} \rightarrow e^{+} e^{-} \mu^{+} \mu-*$ The low rate for these reactions makes it important to be able to detect all of the $4 \ell$ decay channels.

After rigorous $\mathrm{R} \& \mathrm{D}$ studies in which a $\mathrm{BaF}_{2}$ crystal calorimeter was compared with a noble liquid sampling calorimeter, we have selected a liquid accordion electromagnetic calorimeter. The noble liquid option with krypton in the barrel and argon in the endcap has been chosen because of its ability to achieve the required resolution, longitudinal segmentation and pointing ability, its intrinsic radiation resistance, its ease of calibration, and the extensive experience that has been acquired with large liquid-argon systems. The accordion geometry provides good hermeticity and allows for faster readout than parallel-plate calorimetry because of lower inductance and capacitance. Results from a prototype accordion calorimeter tested at BNL, with somewhat thicker plates than in the final GEM design, yield an electron energy resolution of $6.7 \% / \sqrt{E}$ and a very small systematic term. All aspects of its performance are well reproduced by our simulations. It is thus expected that this technology choice will provide a system with good intrinsic resolution and a well-controlled systematic term in the electromagnetic resolution. The design goal for the GEM system is $\sigma / E=6 \% / \sqrt{E} \oplus 0.4 \%$ for the barrel and $\sigma / E=8 \% / \sqrt{E} \oplus 0.4 \%$ for the endcap, where the electron and photon energies are higher.

The performance of the electromagnetic calorimeter in GEM is the most demanding, but the hadron calorimeter also plays an important role. It determines jet energies with a resolution of $\sigma / E=$ $60 \% / \sqrt{E} \oplus 4 \%$. The hadron calorimeter is very nearly hermetic because it is used (in conjunction with the forward calorimeter) to measure $E_{T}$.

Three alternatives for hadron calorimetry in the barrel were studied: an integrated noble liquid hadronic section, a sampling scintillator-based calorimeter, and a hybrid system. The integrated calorimeter is the most costly and requires a cryostat too large to manufacture off-site and transport over the road. The scintillator calorimeter involves a difficult problem of bringing the services out of the electromagnetic krypton calorimeter, has a transition region near shower maximum with thick cryostat
Table 1.5. Major design parameters of the GEM calorimeter.

\begin{tabular}{|c|c|}
\hline \multicolumn{2}{|l|}{ EM energy resolution } \\
\hline Barrel & $6 \% /, E \oplus 0.4 \%$ \\
\hline Endcap & $8 \% / v \bar{E} \oplus 0.4 \%$ \\
\hline EM position resolution & $4.4 \mathrm{~mm} /, \bar{E}$ \\
\hline \multicolumn{2}{|l|}{ EM pointing resolution } \\
\hline Barrel & $40 \mathrm{mrad} /, i \bar{E}+0.5 \mathrm{mrad}$ \\
\hline Endcap & $50 \mathrm{mrad} / \mathrm{v} \bar{E}+0.5 \mathrm{mrad}$ \\
\hline EM coverage & $\ln k 3$ \\
\hline Hadron coverage & $|\eta|<5.5$ \\
\hline Jet resolution & $30 \% / \sqrt{E} \oplus 4 \%$ \\
\hline \multicolumn{2}{|l|}{$\begin{array}{l}\text { Number of absorption } \\
\text { lengths }\end{array}$} \\
\hline at $\eta=0$ & $=11 \lambda$ \\
\hline at $\eta=3.0$ & $\begin{array}{l}12 \lambda \text { instrumented, }>16 \lambda \\
\text { total }\end{array}$ \\
\hline \multicolumn{2}{|l|}{$\begin{array}{l}\text { Lateral segmentation } \\
(\eta, \phi)\end{array}$} \\
\hline EM & $0.026 \times 0.026$ \\
\hline$H A D$ & $0.08 \times 0.08$ \\
\hline \multicolumn{2}{|l|}{$\begin{array}{l}\text { Longitudinal } \\
\text { segmentation }\end{array}$} \\
\hline Liquid barrel & $3 E M+3 H A D$ \\
\hline Endcap & $3 E M+4 H A D$ \\
\hline \multicolumn{2}{|l|}{ Scintillating barrel section: } \\
\hline $\begin{array}{l}\text { Lateral readout } \\
\text { segmentation }(\eta, \phi)\end{array}$ & $0.16 \times 0.16$ \\
\hline $\begin{array}{l}\text { Longitudinal } \\
\text { segmentation }\end{array}$ & 1 layer \\
\hline \multicolumn{2}{|l|}{ Forward section: } \\
\hline $\begin{array}{l}\text { Lateral segmentation } \\
(\eta, \phi)\end{array}$ & $0.2 \times 0.2$ \\
\hline Pr resolution for jets & $\Delta$ Pr $/$ pr $\leq 10 \%$ \\
\hline $\begin{array}{l}\text { Instrumented } \\
\text { absorption lengths }\end{array}$ & $11.4 \lambda$ \\
\hline Total weight & $2814 \mathrm{Mg}$ \\
\hline
\end{tabular}

walls, and also has radiation damage concerns. Finally, the hybrid system that we have chosen 


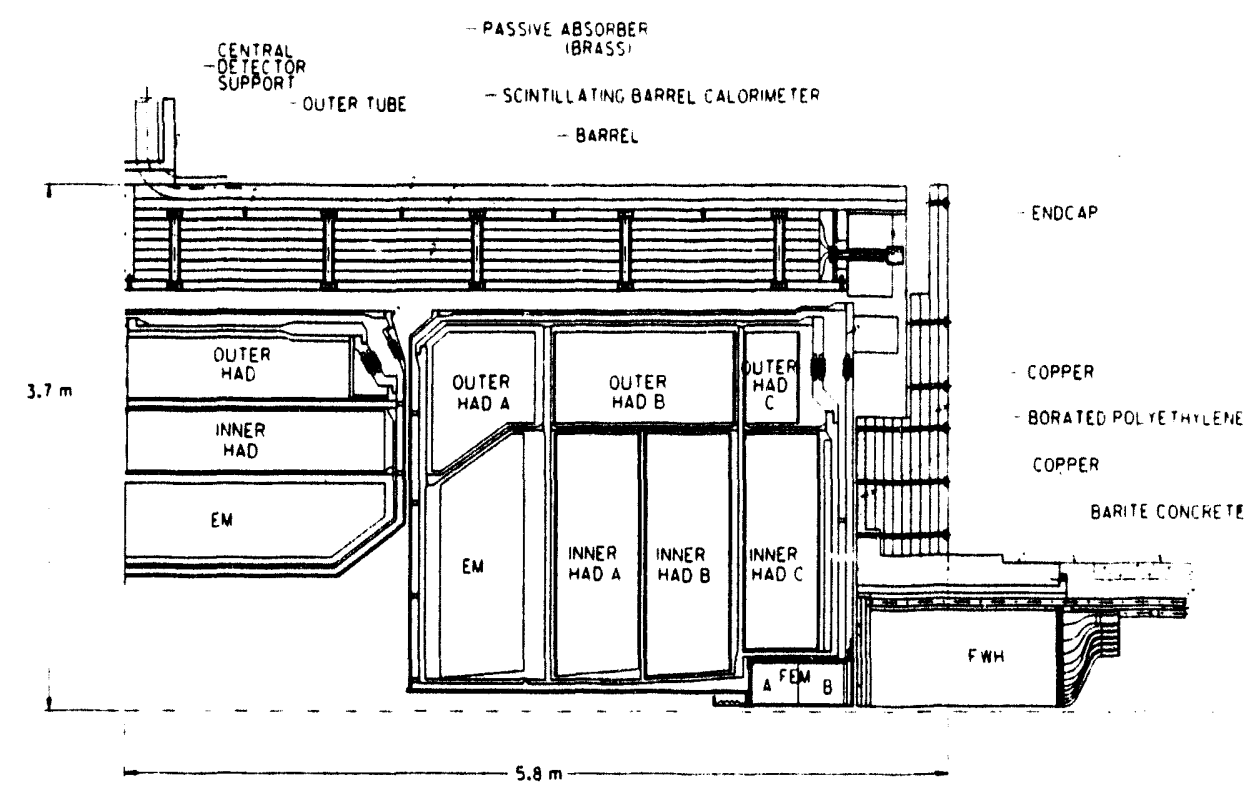

FIG. 1-7. Quarter view of the detector showing the calorimeters.

performs the hadron calorimetry primarily in the noble liquid (in the first $\sim 6 \lambda$ ), then is followed by a relatively inexpensive copper/scintillator calorimeter that provides the necessary shielding for the muon system and calorimetry information for late-developing showers.

The primary function of GEM's forward calorimeters is to measure high-momentum particles near the beam pipe. Together with the barrel and endcap calorimeters, they determine $E_{T}$ down to the level of irreducible background from standard sources of neutrinos. The design goal is to provide $E_{T}$ signatures for massive gluinos and squarks, or other new particles, whose signatures may include jets with measured $E_{T} \geq 75 \mathrm{GeV}$ and electrons with measured $E_{T} \geq 20 \mathrm{GeV}$. In order to achieve these goals, the forward calorimeter must cover the region $|\eta| \leq 5.5$, be sufficiently dense to fully contain hadronic showers, be sufficiently fast to cope with the high-density particle flux in this region, and be radiation-hard. The baseline design we have adopted has a first section consisting of a specially designed liquid-argon calorimeter, followed by a second hadronic section consisting of a liquid-scintillatorcapillary and tungsten calorimeter. The calorimeter is optimized to include good spatial information in the first section and sufficient transverse hadron shower containment in the second section. It also serves the prosaic but important function of helping to shield the muon system.

\subsubsection{Central Tracker}

The purpose of the central tracker in GEM is two-fold: to support the primary GEM goals of measuring gammas, electrons, and muons at high $p_{T}$, and to provide pattern recognition capabilities and vertex resolution for studies involving $b, t$, and $\tau$ physics. The primary goals must be met at high luminosity, $\mathcal{L}=10^{34} \mathrm{~cm}^{-2} \mathrm{~s}^{-1}$, while the secondary tasks need be accomplished only at the standard luminosity of $10^{33} \mathrm{~cm}^{-2} \mathrm{~s}^{-1}$.

The support of GEM's primary physics goals imposes a series of requirements on the central tracker system. These include good separation of gammas and electrons by finding a charged track and measuring the electron sign up to $600 \mathrm{GeV}$. The former requirement is essential to the search for $H^{0}$ $\rightarrow \gamma \gamma$ and to background rejection in $Z^{\prime} \rightarrow e^{+} e^{-}$; the latter, for the gluino search using the signature of same-sign leptons. Another important role for the tracker is to measure the position of the primary vertex, which is crucial for pileup background separation, especially at high luminosities, and for measuring the Higgs boson mass. The tracker must serve as an aid in particle identification (electronhadron separation and muon identification) by providing consistency checks with the other subsys- 
tems. It is also important for background rejection by enabling track isolation cuts to be made. Physics involving $b, t$, and $\tau$ decays requires full pattern recognition capability, including secondary vertex finding and tracking at low momenta. We have incorporated as much of this capability as is practical within the scope of the GEM central tracker.

A variety of technologies were considered for the central tracker. Our design incorporates two technologies. For the inner section of the tracker we considered silicon pixels and long-drift silicon, as well as silicon microstrips. The silicon microstrip technology was chosen because it is more mature and gives the required fine segmentation and radiation resistance. For the outer section, straw tubes and scintillating fibers were considered, as well as interpolating pad chambers (IPCs). IPCs were chosen due to their low occupancy, their correlation of coordinates on a track to provide "near"-three-dimensional space points, their highluminosity capability, and their demonstrated operational resolution of $50 \mu \mathrm{m}$. The major design parameters of the GEM central tracker are given in Table 1-6.

The central tracker is $1.8 \mathrm{~m}$ in diameter by $3.5 \mathrm{~m}$ long, surrounding the interaction point. The tracker size was determined by a combination of factors: placing the calorimeter at a distance sufficieni :o allow $\pi^{0}$ rejection by shower shape analysis, minimizing the calorimeter cost, maximizing the tracker resolution, and preserving sign-selection ability to high momenta. The layout of the tracker system is shown in Figure 1-8.

Table 1-6. Major design parameters of the GEM central tracker.

\begin{tabular}{|c|c|}
\hline Rapidity coverage & $|\eta| \leq 2.5$ \\
\hline $\begin{array}{l}\text { Occupancy } \\
\text { at } \mathcal{L}=10^{33} \mathrm{~cm}^{-2} \mathrm{~s}^{-1} \\
\text { at } \mathcal{L}=10^{34} \mathrm{~cm}^{-2} \mathrm{~s}^{-1}\end{array}$ & $\begin{array}{l}\leq 1 \% \\
\leq 10 \%\end{array}$ \\
\hline Charge separation at $95 \%$ C.L. $(\eta=0)$ & $p \leq 600 \mathrm{G \theta V}$ \\
\hline Momentum resolution & \\
\hline $\begin{array}{l}\text { at high momenta } \\
\text { (measurement limited) }\end{array}$ & $\Delta p / p^{2}=1.2 \times 10^{-3}(\mathrm{GeV})^{-1}$ \\
\hline $\begin{array}{l}\text { at low momenta } \\
\text { (multiple scattering } \\
\text { limited) }\end{array}$ & $\Delta p / p=3.5 \%$ \\
\hline $\begin{array}{l}\text { Vertex resolution } \\
\text { along beam direction } \\
\text { impact parameter }\end{array}$ & $\begin{array}{l}\Delta z \cong 1 \mathrm{~mm} \\
\Delta b \cong 25 \mu \mathrm{m} \text { above } 10 \mathrm{GeV}\end{array}$ \\
\hline
\end{tabular}

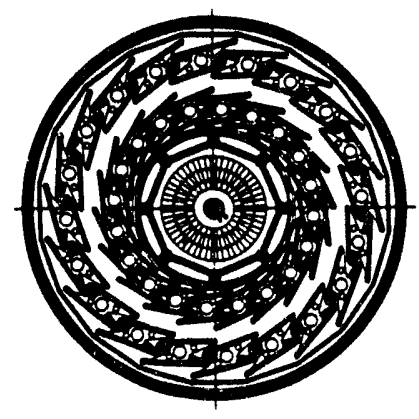

SECTION A-A

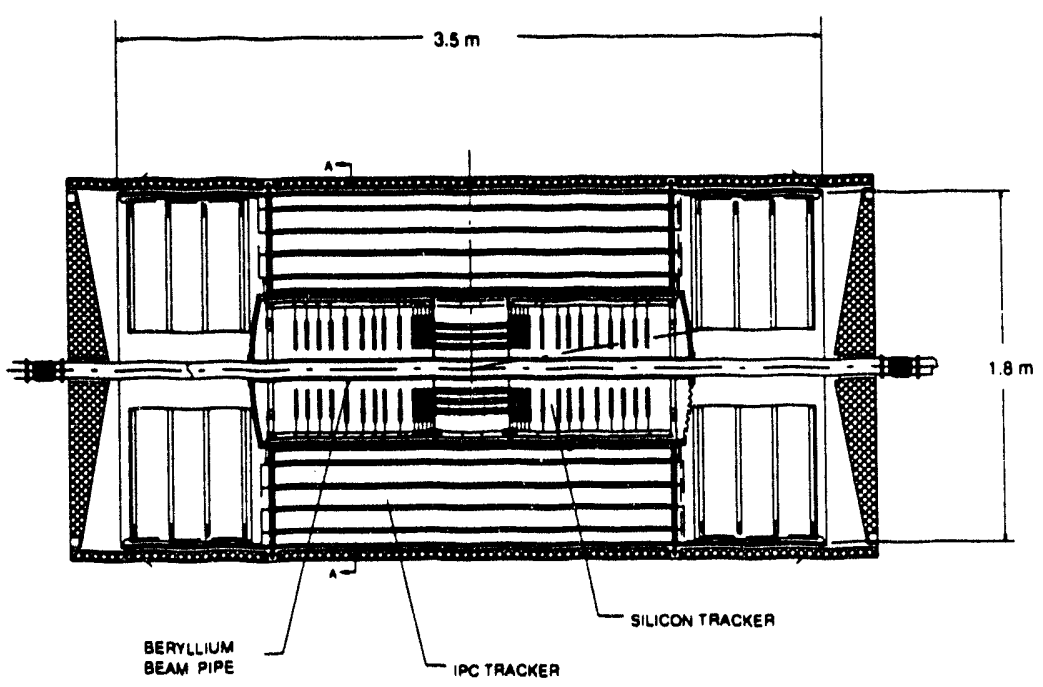

FIG. 1-8. The GEM central tracker. 


\subsubsection{Electronics/Data Acquisition}

Triggering and data acquisition in GEM will follow a three-level strategy to provide a system without deadtime that provides as much information as possible at each trigger level. It is designed for luminosities up to $10^{33} \mathrm{~cm}^{-2} \mathrm{~s}^{-1}$, with provision for improving its efficiency at higher luminosities with modest upgrades. The trigger system design goals are given in Table 1-7.

Table 1-7. Trigger system design goals.

\begin{tabular}{ll}
\hline Level 1 & \\
Rate in & $60 \mathrm{MHz}$ \\
Rate out & $10 \mathrm{kHz}$ \\
Latency & $2 \mu \mathrm{s}$ \\
Level 2 & \\
Rate in & $100 \mathrm{kHz}$ \\
Rate out & $300 \mathrm{~Hz}$ \\
Latency & $\leq 500 \mathrm{~ms}$ \\
Level 3 & \\
Rate in & $3 \mathrm{kHz}$ \\
Rate out & $100 \mathrm{~Hz}$ \\
\hline
\end{tabular}

The GEM trigger and data acquisition architecture consists of a synchronous and pipelined Level 1, an asynchronous Level 2 (possibly with special purpose hardware), and a Level 3 processor ranch. In the data acquisition system, full granularity data is available at Levels 2 and 3. Level 1 is designed to handle up to $60 \mathrm{MHz}$ input rate, with an output rate of $10 \mathrm{kHz}$. Level 2 is designed to handle an average input rate up to $100 \mathrm{kHz}$, with an output rate of $300 \mathrm{~Hz}$. Finally, Level 3 accepts $3 \mathrm{kHz}$, with an output rate of $100 \mathrm{~Hz}$. It should be noted that the Level 2 trigger is implemented as a "virtual Level 2," using the processor ranch with access to the full event data.

The individual subsystems impose special conditions on the electronics. The inner silicon tracker is a digital system that needs radiation-hard electronics, and much of the electronics is integrated on the detectors. The IPC system also must be radiation-hard. It uses an analog readout, requiring $1 \%$ precision on 400000 channels. The Level 1 trigger results in the digitization of the data stored on the tracker, which are then zero-suppressed and collected through a fiber-optic link. The calorimeter electronics of 128000 channels requires wide dynamic range and excellent timing to identify the beam crossing. Finally, the muon cathode strip chambers use chamber-mounted front-end electronics and low-cost, custom integrated circuits due to the large number of channels $\left(\approx 10^{6}\right)$.

\subsection{GEM PROJECT}

\subsubsection{Assembly, Access, and Maintenance}

The GEM detector will be located at interaction region 5 (IR5), which includes a large underground detector hall and associated surface facilities for manufacturing, assembly, operations, offices, and utilities.

The underground hall is $30 \mathrm{~m}$ wide, $100 \mathrm{~m}$ long and $41 \mathrm{~m}$ high, with two large installation shafts, an electronics shaft, and a utility shaft. It is equipped with two $75 / 20-\mathrm{Mg}$ bridge cranes for general use and for handling some detector components. In order to handle the massive assembled subsystems, the floor will be equipped with heavy duty rails and other equipment. This transport system will be used for detector assembly, which will be done mostly in pre-assembled large units, and for detector access and maintenance.

The size and general configuration of the hall has been determined from the parameters of the detector, its installation and maintenance requirements, provision for adequate shielding, and the requirements for the local accelerator systems.

The two installation shafts will be used to lower the magnet halves and assembled detector subsystems from the surface into the experimental hall. The principal consideration that establishes the requirements for the surface facilities is the need to manufacture the large GEM superconducting magnet on-site.

Figure 1-9 shows the GEM surface facilities at IR5. The main features are two large assembly buildings, each connected through heavy load paths to the two installation shafts. Detector subsystems will be assembled in these buildings and lowered into the hall for final detector assembly.

A detailed schedule for assembly is given in Chapter 9, based on the availability of components 


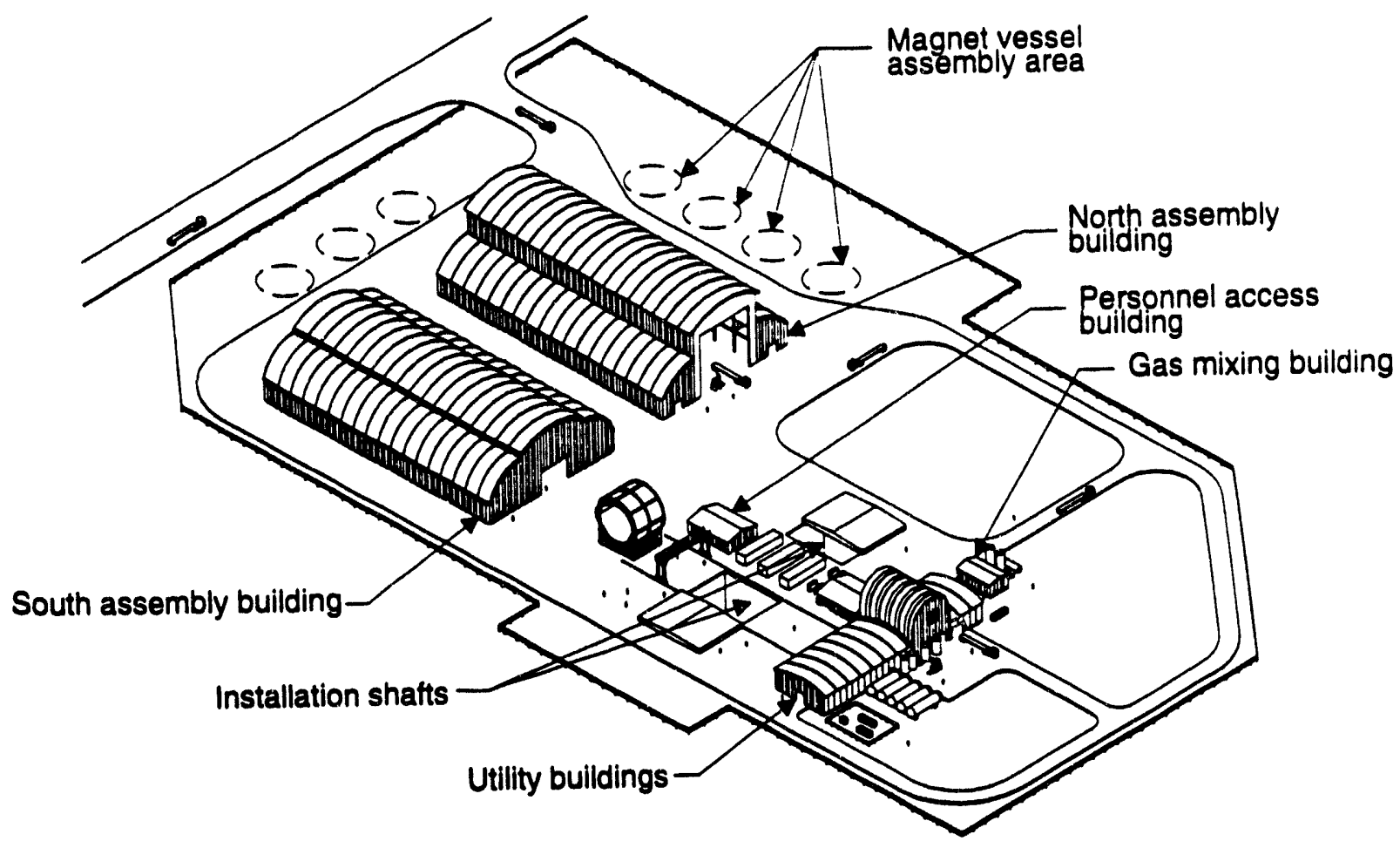

FIG. 1-9. The GEM surface facilities at IR5.

and efficient use of the surface assembly space. We note that the symmetrical nature of the detector and its assembly around a fixed central detector support, the two installation shafts, and the large multi-purpose assembly space offer considerable flexibility in installation scenarios.

A view of the assembled detector in the hall is shown in Figure 1-10. All detector components can be accessed and maintained. For access, we have incorporated the capability to open up the detector along the beam line, pulling back the magnet halves against the far walls of the underground hall for major access. For detector maintenance, we have developed a seven-level scheme (Chapter 10) of access, determined by access restrictions (beam on/off), location in the detector hall, and extent of disassembly required. We have placed critical components in locations where short-term access is possible and have ensured that all components can be maintained within an annual 3-month shutdown period. In addition, we have paid attention to the feasibility of either upgrading or replacing subsys- tems as needed for the long-range evolution of GEM.

\subsubsection{Detector Integration}

Detector integration has received much attention in GEM. The primary integration issue is to select the parameters of the detector to make a coherent, optimized design. The parameters of the detector presented here have gone through careful trade-off studies for cost, performance, and consistency with the main priorities of GEM. High-level integration issues, such as decisions on the transition between two detector systems, support, services, and access, are decided after meetings between the detector groups, engineering meetings, and final discussions in the Executive Committee (see Chapter 14).

Most integration issues are addressed in appropriate subsystem chapters of the TDR; some have been addressed separately. The detector/beamline interface (Chapter 11) involves several issues: attaining the desired vacuum; minimizing secondary 


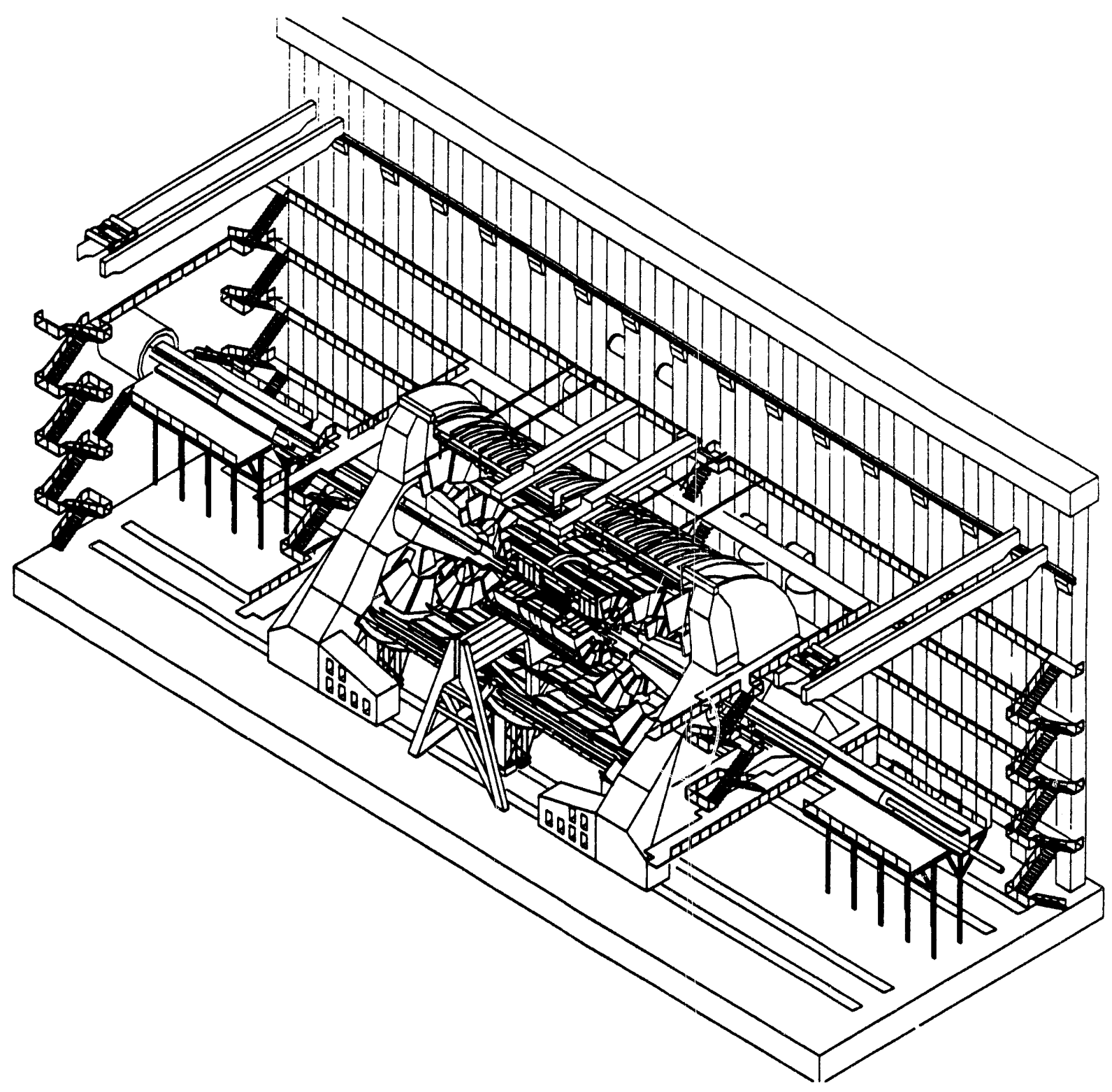

FIG. 1-10. Assembled detector in the hall.

interactions in the beam pipe, associated pumps, and related equipment; and facilitating assembly and access. Forward calorimetry places severe requirements on the beam pipe design. We have determined that the best location for the forward calorimeter is contiguous to the endcap calorimeter. This location is much better than further downstream from the $\mathbb{P}$, because it is far easier to shield as a neutron source for the muon detectors and because the calorimeter is considerably smaller and less expensive. The main problem is to make the beam pipe small enough to permit the required $\eta$-coverage for $E_{T}$ studies. We have designed a beam pipe that begins with an $8-\mathrm{cm}$ diameter in the region of the forward calorimeter, and then is flared so that it lies in the shadow of the calorimeter.

A second important integration issue involves radiation shielding (Chapter 12 ). We have carefully considered sources of background in the detector. A well-shielded entrance to the interaction hall prevents radiation from beam-gas interactions from entering the hall. The primary source is from products of fp collisions at the interaction point. At 
SSC luminosities the neutron and photon fluences could be very large. We have taken great care to reduce them to a tolerable level.

The GEM detector is hermetic for $|\eta|<5.5$ and thick enough to reduce the flux in the muon system. Beyond $|\eta|=6$, we have taken care to minimize material, with the beam pipe shielded by the calorimeter, allowing the scattered particles to strike the collimator at the face of the final low $\beta$ quads. These quads are placed far downstream and are well shielded from the detector. The practical realization of the shielding presented reduces the $n$, $\gamma$, and charged particle fluxes to a manageable level (see Chapter 12) in all regions of the detector up to the highest luminosity expected at the SSC.

\subsubsection{Upgrades}

An example of a deferred item that can be implemented as an upgrade is an extension of the field shaper. It was shortened by $1.5 \mathrm{~m}$ in the baseline design, saving several million dollars. Restoring the extra iron would improve muon resolution by about $10 \%$ at $\eta=2.5$. Another example is to add more powerful Level 1 and Level 2 trigger processors, for which provision has been made in the current isesign.

In addition to these deferred items, these are several other improvements that can be implemented as future upgrades to enhance the performance of the detector. The muon resolution can be significantly improved by the addition of a set of muon chambers outside the magnet, where there is enough room for this purpose. The calorimeter resolution could be improved by using krypton in the endcaps, or possibly by using a xenon-krypton mixture throughout. The performance of the central tracker can be improved at high luminosity $\left(\ell=10^{34} \mathrm{~cm}^{-2}\right.$ $\mathrm{sec}^{-1}$ ) by replacing the silicon microstrip inner detector (which probably cannot tolerate the radiation levels at this high luminosity) with a more radiation-resistant detector based on silicon pixels or gallium arsenide.

\subsubsection{Commissioning and Initial Operation}

The physics simulations presented in this TDR are based on the baseline detector at $\mathcal{L}=10^{33} \mathrm{~cm}^{-2} \mathrm{~s}^{-1}$, except for Section 2.6 , where we address the physics capabilities at $L=10^{34} \mathrm{~cm}^{-2} \mathrm{~s}^{-1}$ (a particular strength of GEM). Initial turn-on scenarios at SSCL may involve a period of running at lower than the design luminosity. It is worth noting the physics potential for such early running, beyond its value for detector commissioning.

In particular, integrated luminosities up to $10^{37} \mathrm{~cm}^{-2}$ (perhaps early shakedown running) can be used for elastic and total cross sections, structure function and jet fragmentation studies, $B$-physics, a precise $W$ boson mass determination, and $t$-quark physics. The GEM detector could address these items and do very useful commissioning work with $W$ and $Z$ events. For integrated luminosities reaching $10^{38} \mathrm{~cm}^{-2}$, detailed studies of $t$-quark decay rates and properties and early searches for light gluinos, techni-rhos, and other new strongly produced particles can begin in a significant way.

At the next step in integrated luminosity, to the level of $10^{39} \mathrm{~cm}^{-2}$, exploration for a Higgs boson can begin over much of the mass range, as well as significant particle searches. Perhaps as important, running with full capability will be crucial to having a detector well understood and capable of the full set of physics goals when integrated luminosities of $10^{40} \mathrm{~cm}^{-2}$ and evertually $10^{41}$ are reached.

\subsection{GEM COLLABORATION AND ORGANIZATION}

The GEM collaboration consists of 1026 collaborators from 118 institutions in 17 countries. We have a broad and deeply talented group committed to developing a powerful detector for the SSC. This international collaboration has been working closely together on the extensive R\&D and engineering program that was needed to design the detector described in this TDR. The collaboration has operated, since its inception, with a temporary organization, which is evolving into a more permanent one with the submittal of this TDR.

The collaboration has worked systematically to develop a plan that is well-suited for implementing GEM as we move past the TDR into the project. We have recently approved the general organization plan presented in Chapter 14. The plan represents an evolution of our successful interim structure. It is founded on democratic principles and is built around an active group of institutional representatives (the Collaboration Council) who discuss and approve all major decisions and appointments. The International Committee ensures that all participating countries 
function effectively within the collaboration, and an Executive Committee advises the spokesmen and project manager on scientific, technical, and managerial decisions as the detector is constructed and operated. We are organized into subsystem groups, each with its own organization, and the entire collaboration is directed by the spokesmen.

The scientific collaboration is integrated into a project organization, responsible for coordinating the overall engineering, budgets, cost, and schedule for GEM. A draft project management plan for GEM has been submitted, and the management team is being put in place on the time scale of the TDR.

Responsibilities for individual groups are being developed and matched to project needs for implementation of GEM. We will be developing memoranda of understanding (MOUs) with each institution during the coming year. We are paying special attention to defining appropriate roles in GEM for all international collaborators, U.S. universities, and laboratories. 


\section{PHYSICS PERFORMANCE OF THE GEM DETECTOR}

\subsection{INTRODUCTION}

The primary goal of the GEM experiment is to explore the $\mathrm{TeV}$ energy region opened up by the Superconducting Super Collider. ${ }^{1,2,3}$ As discussed in the Preface, this region holds the physics of electroweak symmetry breaking, though the precise nature of this phenomenon remains unknown. It is hoped that it also contains the allied physics of flavor symmetry and its breaking, whose origins and mass scale are not understood. Beyond electroweak and flavor physics, there are numerous speculations on what may lie within the SSC energy region. They range from new, heavy fermions, either sequential or nonsequential, to extensions of the standard electroweak gauge group, and even to extended structures associated with electroweak symmetry breaking. Experiments have provided no guidance to whether these or other new physics reasonably may be expected in the TeV region. To achieve GEM's goals, therefore, the detector is designed to be sensitive to the widest possible range of new physics signals.

The new physics of the past 25 years has, almost invariably, been heralded by the appearance of isolated, high-energy leptons. The same is expected to be true for the physics of electroweak and flavor symmetry, as well as for the many proposed extensions of the standard gauge structure. This expectation underlies the principal GEM design concepts: (1) High-precision electromagnetic calorimetry augmented by inner tracking and hadronic calorimetry for excellent single photon and electron identification; (2) Precise muon identification and momentum measurement in a large, open magnetic field outside the calorimeters. The EM calorimetry gives GEM the high energy and spatial resolutions needed to discover the Higgs boson in such processes as $H^{0} \rightarrow \gamma \gamma$. Every effort is made in GEM to maintain the precision measurement of electromagnetic-four-vectors at ultrahigh luminosity, $\mathscr{L}=10^{34} \mathrm{~cm}^{-2} \mathrm{~s}^{-1}$. The muon system design naturally provides the robustness necessary for operations at the highest SSC luminosities. Thus,
GEM will be able to extend its reach for isolated leptons with transverse momenta up to the practical SSC limit, approximately $5 \mathrm{TeV}$. These aspects of GEM define a superb physics program and, at the same time, ensure significant complementary strengths to the SDC detector. ${ }^{4}$

This chapter describes the performance of the GEM detector for several scenarios of electroweak, flavor and new gauge-interaction physics that may be accessible at the SSC. Emphasis is placed on realistic, in-depth simulations of representative processes that demonstrate GEM's strengths, particularly those involving GEM's superior electromagnetic calorimeter and muon system, and GEM's robustness for physics studies at ultrahigh luminosity. We have not attempted to survey all possible new physics which might be discovered at the SSC. The simulations reflect the baseline design of March 1993. There have been some design changes since then, but they are minor insofar as simulations of physics performance are concerned. The GEM design will continue to evolve in response to simulation results and engineering work. The simulations described here are the realistic performance of the current baseline, not the hoped-for performance of the final design.

All of the electroweak and flavor physics discussed in the Preface is at sufficiently high mass or transverse momentum that perturbative QCD and electroweak interactions can be used to describe production of the signatures and their standard model backgrounds. Therefore, we have used event generators such as ISAJET ${ }^{5}$ and PYTHIA / JETSET ${ }^{6}$ to generate complete signal and background events. Unless otherwise noted, the generation of signal and background events in this chapter used the EHLQ Set 1 parton distribution functions ${ }^{7}$ - the default in the current versions of ISAJET and PYTHIA. Also, we generally assume $m_{t}=140 \mathrm{GeV}$ for the mass of the top quark. ${ }^{8.9}$

In principle, the full detector simulation programs GEANT $^{10}$ or CALOR89 11 could be used to 
compute GEM's response to the signal and background for any process. Unfortunately, these programs are very slow for complex events at high energy. It is impractical to use them to simulate the more than $10^{6}$ events often needed to determine a rare signal's background arising from a combination of relatively likely processes. Consequently, two different types of simulations of GEM have been done for this Technical Design Report. Detailed simulations, based on GEANT, of each of the individual detector systems have been performed for single particles or for limited numbers of complete events. These simulations are described in the appropriate subsystem chapters, since they are intimately related to design of the hardware. There is also an overall GEANT simulation program, sigem, 12 which has been used for studies of the muon system performance, described in Chapter 4. The results of these detailed studies have been parameterized and incorporated in gemfast, ${ }^{13}$ a fast simulation program for GEM that is used for determining the performance of the detector for physics processes. For example, the parameterization may be of an energy or momentum resolution or of a muon reconstruction efficiency in the presence of other particles in the event of interest. A description of gemfast is given in Section 2.2 below.

Most of the results presented in this chapter are based on gemfast. Where necessary, hybrid simulations of gemfast and full GEANT have been used. For example, in the study of $H^{0} \rightarrow \gamma \gamma$ in Section 2.3, detailed electromagnetic shower shape studies for real photons and and jets faking photons were carried out with GEANT. Apart from examples such as this, gemfast describes the performance of GEM quite accurately. Following the description of how the GEM detector was modeled in Section 2.2. the rest of the chapter is organized as follows:

- Section 2.3 presents an in-depth study of the search for the Higgs boson of the minimal onedoublet standard model. The signals, backgrounds and discovery potentials for $M_{H}=80-800 \mathrm{GeV}$ are discussed. Depending on the Higgs mass, the modes studied were $H^{0} \rightarrow \gamma \gamma ; t \bar{t} H^{0} \rightarrow \ell^{ \pm}+\gamma \gamma ; H^{0} \rightarrow Z Z^{*}, Z Z \rightarrow$ 4 charged leptons; $H^{0} \rightarrow \mathrm{ZZ} \rightarrow \ell^{+} \ell^{-} \bar{v}$; and $H^{0} \rightarrow \mathrm{ZZ} \rightarrow \ell^{+} \ell^{-}$jet jet.
- Flavor physics involving top-quarks is discussed in Section 2.4. We describe the mass measurement of a heavy top-quark in the standard decay mode $t \rightarrow W^{+} b$ using two methods: $t \rightarrow$ isolated $\ell^{+}$plus non-isolated $\mu^{-}$. and $t \rightarrow 3$ jets. We also discuss the discovery of a charged Higgs boson in the nonstandard decay mode $t \rightarrow \mathrm{H}^{+} b$, followed by $\mathrm{H}^{+} \rightarrow \tau^{+} \nu_{\tau}$.

- Jet physics is discussed in Section 2.5. We discuss the determination of the jet energy scale, using as a physics context the search for quark substructure in high- $E_{T}$ jets. Other jet studies are carried out in Sections $2.3\left(H^{0} \rightarrow Z^{0} Z^{0} \rightarrow \ell^{+} \ell^{-}\right.$jet jet) and $2.4\left(t \rightarrow W^{+} b \rightarrow 3\right.$ jets $)$.

- Section 2.6 is devoted to studies of high-massscale physics at ultrahigh luminosity, with special attention paid to difficulties of experimentation at $\mathcal{L}=10^{34} \mathrm{~cm}^{-2} \mathrm{~s}^{-1}$. These physics studies include precision investigations of the properties of a very massive $Z^{\prime}$ boson in its $e^{+} e^{-}$and $\mu^{+} \mu^{-}$ decay channels, and the character of quark-lepton substructure contact interactions via the process $\bar{q} q \rightarrow \mu^{+} \mu^{-}$. We also describe studies of the properties of heavy $W^{\prime}$-bosons and of substructure via $\ell^{ \pm} v_{\ell}$ modes.

- Physics with missing transverse energy $\left(\boldsymbol{F}_{T}\right)$ signatures is discussed in Section 2.7 using supersymmetry as a paradigm. The $\boldsymbol{E}_{T}$ distribution is calculated for GEM, including the effects of transition regions and dead material. The $E_{T}$ signature is studied for a range of gluino and squark masses. In addition, the likesign dilepton signature for gluino production is investigated.

- Section 2.8 contains a summary of GEM's reach for the physics processes considered in the preceding sections and a discussion of further optimization of baseline design features motivated by our simulatic

For all these processes, the performance of the GEM design as of March 1993 has been determined realistically. This is an important step in optimizing the design. Results are given below for a variety of integrated luminosities: $10 \mathrm{fb}^{-1}$ and $30 \mathrm{fb}^{-1}$, which should be ohtained in one to three years of steady operation at the design peak luminosity of 
$10^{33} \mathrm{~cm}^{-2} \mathrm{~s}^{-1} ;$ and $100 \mathrm{fb}^{-1}$, which should be obtained in about one year at a peak luminosity of $10^{34} \mathrm{~cm}^{-2} \mathrm{~s}^{-1}$.

\subsection{MODELING THE GEM DETECTOR}

The physics goals of the GEM experiment and the main features of the detector itself were presented in the Preface and in Chapter 1. In this section we describe how we model the detector to simulate its response to physics signals and backgrounds. The studies of the physics performance of the GEM detector have been based primarily on gemfast, ${ }^{13}$ a fast parameterized simulation of GEM. It is an outgrowth of the FAST1 simulation, ${ }^{3}$ but it is much more sophisticated. The parameterizations in gem$f$ ast are based, in turn, on detailed GEANT simulations of the performance of individual components of the detector. This section describes gemfas $t$, displays some parameterizations used in it, and briefly discusses $\gamma, e, \mu$, and jet reconstruction.

The key to a fast detector simulation is to use a very simple geometry and to parameterize the response of each detector component in a simple way. The geometry used in gemfast is a set of concentric cylinders, one each for the central tracker (CT), electromagnetic calorimeter (EC), hadronic calorimeter $(\mathrm{HC})$, scintillator calorimeter (SC), forward calorimeter (FC), and muon system (MU). The geometry is shown in Figure 2-1. The density of the material in the cylinders representing the calorimeters is varied with $\eta$ so as to match the detector thickness in the true design. Differences in radiation and absorption lengths for various materials used in the calorimeters are also taken into account. This is not correct in detail, but it is a good approximation. Particles are tracked through each successive volume on straight lines for neutral particles or on helices in a uniform magnetic field, $B=0.8 \mathrm{~T}$, for charged ones. Since only the point at which a particle enters a given detector is needed, particles need not be tracked in the muon system, the only region where the field is nonuniform. Of course this nonuniformity is taken into account in calculating the resolution of the muon system.

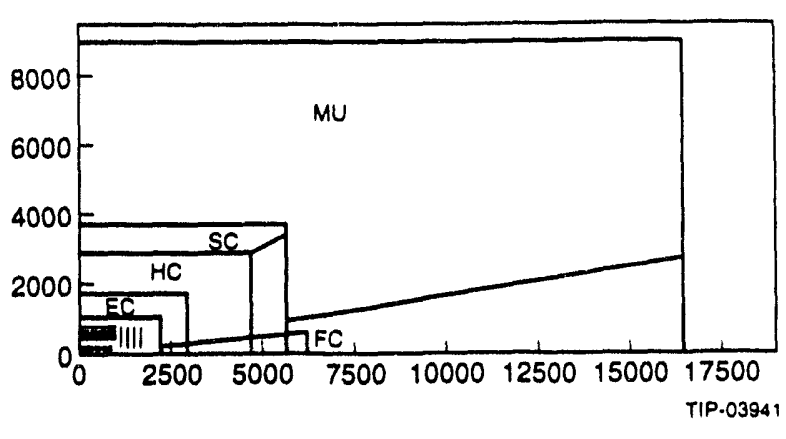

FIG. 2-1. Geometry used in gemfast. The detector is approximated by a set of concentric cylinders. Particles are tracked through the central tracker and calorimeters assuming a uniform magnetic field.

Once a particle enters a given detector system. its energy resolution, angular resolution, and detection efficiency are calculated based on parameterizations of full GEANT-based simulations of the single particle response. This simple single-particle approach is not adequate for the central trarker reconstruction efficiency, which is sensitive to the presence of other tracks in the same event and to pileup, and which therefore has been investigated separately. Simulation of the barrel and endcap region calorimeters is more sophisticated in gemfast and involves calculation of energy in each calorimetric tower. Development of electromagnetic and hadronic showers in transverse and longitudinal directions, including fluctuations, is modeled. The Level 1 trigger response is also simulated. Unstable particles are allowed to decay anywhere in the detector using code adapted from the GEANT package.

\subsubsection{Event Generation}

The first step in simulating a process is to generate the events of interest. This is handled by gemgen, ${ }^{14}$ which so far incorporates ISAJET, ${ }^{5}$ PYTHIA, ${ }^{6}$ and a single particle gun. Both ISAJET and PYTHIA contain parton cross sections for a wide variety of processes, leading-log production of additional QCD jets to give the correct event structure, and phenomenological models for fragmentation of quarks and gluons into hadrons. Both have been widely used in analysis of data at the $S \bar{p} p S$ Collider and the Tevatron. The single particle gun generates a single particle at a given $p_{T}, \eta$, and $\phi$ or a single quark or gluon fragmented with PYTHIA. Other generators will be added as the need arises. 
The gemfast detector simulation is interfaced to the event generators in a flexible way that allows adding a signal event and a Poisson-distributed number of minimum bias events in the same bunch crossing. The same or different generators can be used for each sample. The vertex position of each event is generated according to the expected width, $\sigma_{z}=5 \mathrm{~cm}$.

The same approach could be used to describe pileup from out-of-time bunches. However, this is impractical because of the computing time required to generate minimum bias events over the tails on the sensitive time of the detector. Instead, the effects of out-of-time pileup events have been taken into account for each of the detector systems as an additional noise or inefficiency.

\subsubsection{Central Tracker}

The single particle momentum and vertex position resolutions of the central tracker have been calculated as described in Section 6.2.2. They are based on full GEANT simulations including the magnetic field, detector geometry, chamber positions and resolutions, material distribution including supports and cables, geometrical acceptance, silicon detector efficiency, and distribution of the interaction vertex. The interpolating pad chamber (IPC) efficiency is not included but is known to be high. The effect of out-of-time pileup was included as an additional inefficiency due to the detector deadtime. Both the silicon strips and the IPCs are included for a luminosity of $10^{33} \mathrm{~cm}^{-2} \mathrm{~s}^{-1}$; the silicon detector is assumed to be removed for $10^{34} \mathrm{~cm}^{-2} \mathrm{~s}^{-1}$. Fig. ures 2-2 and 2-3 show the resulting parameterization of the mean muon or pion resolution vs. $p_{T}$ and $\eta$ used in gemfast for $10^{33} \mathrm{~cm}^{-2} \mathrm{~s}^{-1}$ and $10^{34} \mathrm{~cm}^{-2} \mathrm{~s}^{-1}$, respectively, assuming a vertex constraint. The impact parameter resolution for tracks is also parameterized, allowing modeling of vertex reconstruction on an event-by-event basis.

Figure 2-4 shows the isolated track reconstruction efficiency at $10^{33} \mathrm{~cm}^{-2} \mathrm{~s}^{-1}$ in gemfast. The calculation is based on having at least ten good hits on a high- $p_{T}$ track. This number of hits is calculated in gemfast using the positions of the chambers and the actual origin of the track. It is difficult to parameterize the reconstruction efficiency for non-isolated tracks, so only tracks that have
$p_{T}>1 \mathrm{GeV}$ and are isolated at the generator level in $\Delta \eta \times \Delta \phi$, corresponding to three pads in the IPCs, are used in the simulations described here. A detailed simulation of reconstruction efficiency for a few specific processes is described in Section 6.2.2.

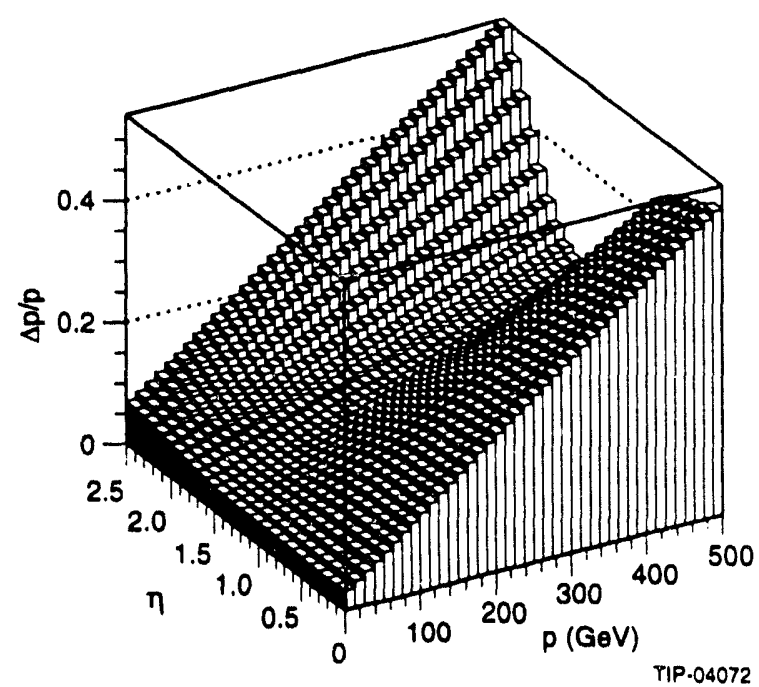

FIG. 2-2. Parameterization of vertex-constrained central tracker resolution in gemfast for muons or charged hadrons vs. $p$ T and $\eta$ for $\mathcal{L}=10^{33} \mathrm{~cm}^{-2} \mathrm{~s}^{-1}$. Both the silicon tracker and the interpolating pad chambers are used.

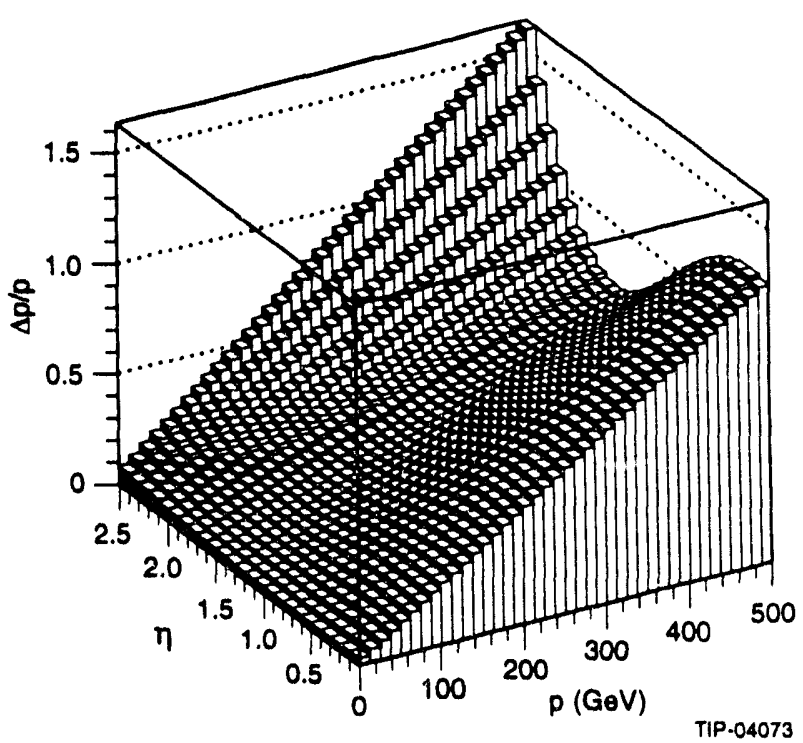

FIG. 2-3. Parameterization of vertex-constrained central tracker resolution in gemfast for muons or charged hadrons vs. $p T$ and $\eta$ for $\&=10^{34} \mathrm{~cm}^{-2} \mathrm{~s}^{-1}$. The silicon tracker is assumed to be removed. 


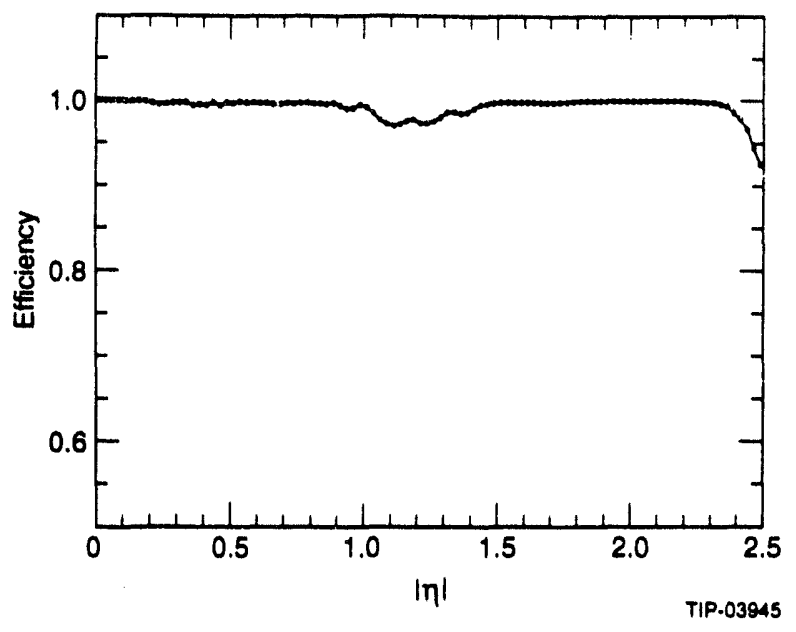

FIG. 2-4. Parameterization used in gemfast of central tracker efficiency for isolated tracks with $p_{T}>1 \mathrm{GeV}$ at $10^{33} \mathrm{~cm}^{-2} \mathrm{~s}^{-1}$. Other tracks are not used in the fast simulations.

The momentum resolution for electrons has been treated separately, taking into account the emission of bremsstrahlung photons caused by the material in the tracker. The latter are assumed to be emitted nearly parallel to the electron and to hit the same calorimeter cell, so that the calorimeter energy resolution is not degraded. Figure $2-5$ shows the electron morsentum resolutions at a particular value of $p_{T}$ and $\eta$ from the full GEANT simulation and from the corresponding parameterization used in gemfast. The parameterization fits the GEANT data well, including the bremsstrahlung tails. These are important for electron sign determination, which can be done up to $p_{T} \leqslant 600 \mathrm{GeV}$ (see Section 6.2.3).

Material in the central tracker is parameterized as a function of $\eta$ and $\phi$ after each layer of the silicon or IPCs, and this is used to convert photons at appropriate space points. Secondary $e^{+} e^{-}$pairs are generated using code adopted from the GEANT package.

\subsubsection{Calorimeter}

The parameterized response of the central calorimeter, which covers $|\eta|<3$, includes shower profiles and energy resolutions. Longitudinal and transverse electromagnetic and hadronic shower profiles are generated using GFLASH 1.3, which was originally developed to describe the $\mathrm{H} 1$ liquid argon calorimeter. ${ }^{15}$ It has been modified to work
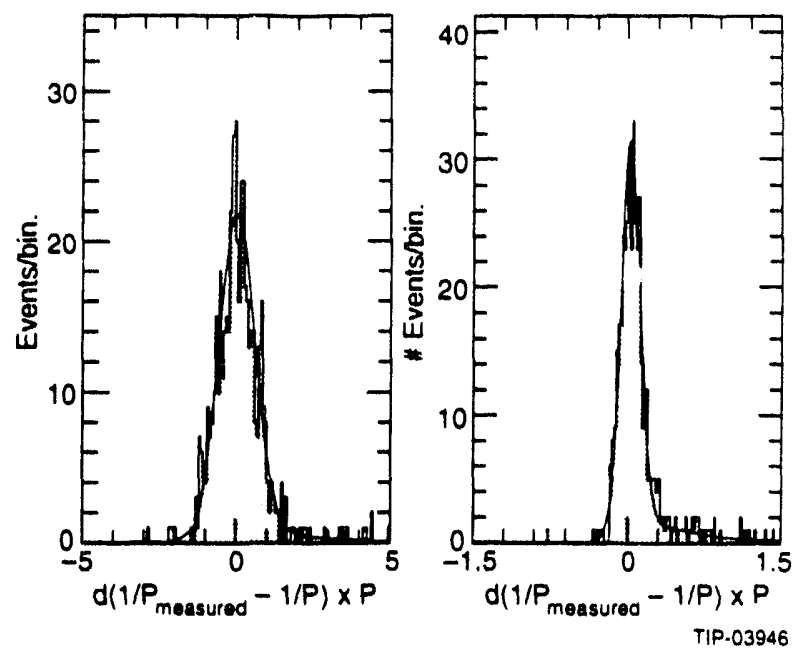

FIG. 2-5. Calculated momentum resolution function for electrons in the central tracker at $p_{T}=525 \mathrm{GeV}$ for $0<|\eta|<0.2$ (left plot), and $p_{T}=10 \mathrm{GeV}$ for $2.2 \leq \eta \leq 2.4$ (right plot). The histogram shows the GEANT simulation, and the smooth curve is the parameterization used in gemfast.

outside the GEANT geometry environment. GFLASH incorporates correlated fluctuations of shower profile parameters, hadronic shower fluctuations into early $\pi^{0} \mathrm{~s}$, transverse profile variations with depth, and shower development along the true direction of incidence as determined by tracking through the central tracker region. It gives a good description of the shapes of both electromagnetic and hadronic showers in uniform regions of the calorimeter. ${ }^{15}$ Figure 2-6 illustrates one such agreement, that for the electromagnetic fraction of hadronic showers. GFLASH is used in gemfast to distribute shower energies among calorimeter towers. The electromagnetic, liquid-hadronic, and scintillator tail-catcher towers are modeled separately, but further longitudinal segmentation is neglected for faster execution.

The segmentation of the calorimeter is varied realistically with $\eta$. The simulated endcap segmentation is in $\eta-\phi$ rather than in $x-y$, but this should have no effect on physics performance. The EC and HC segmentations are $\Delta \eta \times \Delta \phi=0.026 \times 0.026$ and $0.08 \times 0.08$ in the middle of the barrel. Since the segmentation is approximately constant in units of radiation or absorption lengths, it is about a factor of six coarser at the small-angle edge of the endcap than in the middle of the barrel. 


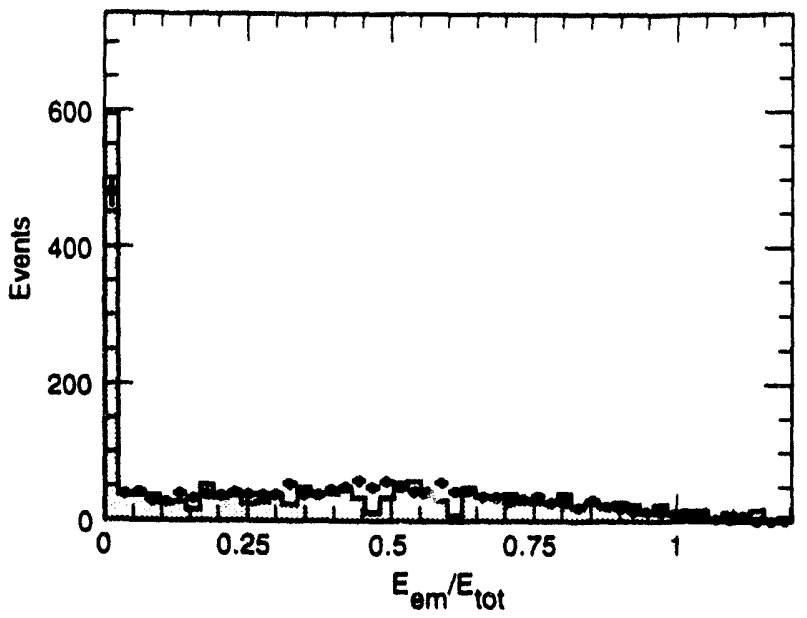

FIG. 2-6. Ratio of electromagnetic to total energy for $100 \mathrm{GeV}$ pions. The histogram shows the result of a full GEANT simulation, and the points show the GFLASH-based parameterization used in gemfast.

The energy resolution of the electromagnetic calorimeter has been calculated using a GEANT simulation including an extremely detailed geometry and very low cutoffs, as described in Section 5.2.1. A similar simulation gave good agreement with test beam data for the-non-projective accordion (see Section 5.3). The resolution has been parameterized in the form

$$
\frac{\Delta E}{E}=\frac{a(\eta)}{\sqrt{E}} \oplus b(\eta)
$$

with the parameters tuned to give the correct resolution as a function of $E$ and $\eta \mathrm{frr}$ the $5 \times 5$ sum of towers used to obtain optimal resolution for isolated electrons and photons. Typically, the stochastic term (a) is about $6 \%$ in the barrel and about $8 \%$ in the endcap, and the constant $b$-term is about $0.4 \%$. The resulting effective single particle resolution is shown in Figure 2-7. Pileup and noise are not included in the energy resolution but are added separately.

The pointing resolution of the electromagnetic calorimeter for photons, calculated using the same detailed GEANT simulation, is described in Section 5.2.1. It is parameterized as

$$
\Delta \theta=\frac{a_{\theta}(\eta)}{\sqrt{E}}+b_{\theta}(\eta)
$$

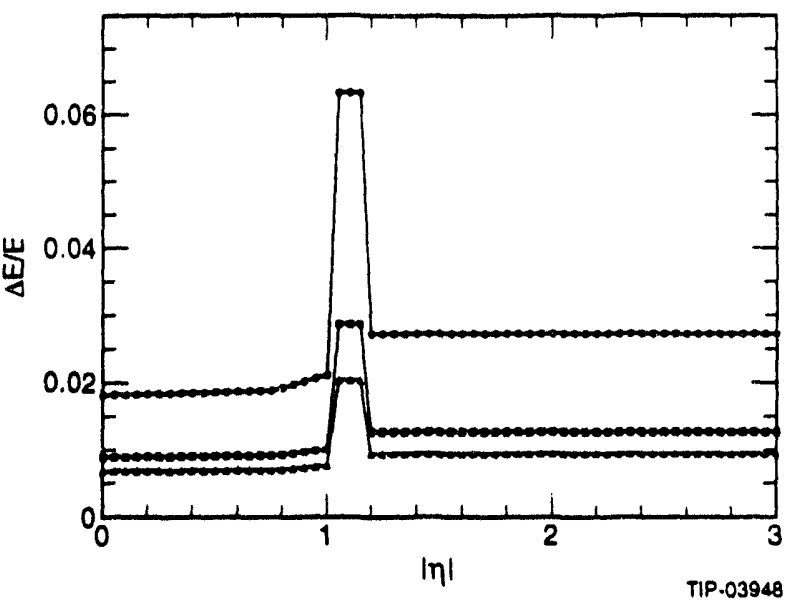

FIG. 2-7. Parameterization of the electromagnetic calorimeter energy resolution in gem $f$ ast for $E=10 \mathrm{GeV}$ (upper), $50 \mathrm{GeV}$ (middle) and $100 \mathrm{GeV}$ (lower). This is tuned to give correct $5 \times 5$ cluster resolution.

for low to moderate energies, where $a_{\theta}$ is about $40 \mathrm{mrad}$ in the barrel and $50 \mathrm{mrad}$ in the endcap and $b_{\theta}(\eta)$ is about $0.5 \mathrm{mrad}$. At the highest energies, $\Delta \theta \approx 2 \mathrm{mrad}$.

Thermal noise is added to each tower depending on $\eta$. In-time pileup events are added explicitly. To determine the effects of out-of-time pileup, a Poisson-distributed number of minimum bias events is generated for each of the 56 preceding and 13 following bunch crossings and simulated with gemfast. The calorimeter response for each bunch crossing is weighted with a response function that takes into account the intrinsic calorimeter response and the shaping circuit in the electronics, using shaping times of $40 \mathrm{~ns}$ for the electromagnetic calorimeter and 100 ns for the hadronic calorimeter. The sum for each cell is calculated to provide a snapshot of the response to pileup. This ensures that all the longitudinal and transverse correlations among cells, caused either by individual shower shapes or by jets, are preserved. One of these snapshots is then superimposed on the response from the signal and-in-time pileup events. As can be seen from Figure 2-8, this approach gives larger fluctuations and a smaller-half-width than the equivalent (enveloping) gaussian noise. ${ }^{16}$ For the analyses described here a sample of $10^{4}$ such snapshots has typically been used. 


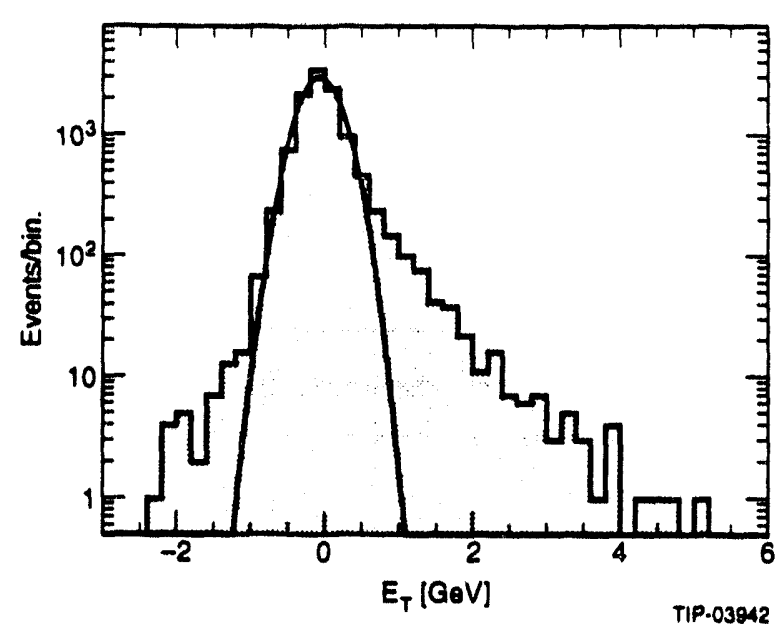

FIG. 2-8. The histogram shows the distribution of pileup, including out-of-time events, in gemfast for a $0.16 \times 0.16$ electromagnetic trigger tower.

The GEM calorimeters have an intrinsic $e / h \neq 1$, so reconstructing a jet energy as a simple sum of the observed energies in the various parts would give a large constant term in the resolution. This effect can be reduced by using an iterative weighting procedure. ${ }^{17,18}$ To save execution time this weighting is not implemented in gemfast. Instead, the sampling and constant terms in the single hadron resolution have been tuned to reproduce the jet energy resolution of the detailed GEANT simulations that included the weighting (see Section 5.2.3). This gives a resolution for isolated jets

$$
\left.\frac{\Delta E}{E}\right|_{\text {jet }}=\frac{0.6}{\sqrt{E}} \oplus 0.04
$$

For many cases the effects of the clustering algorithm used to define jets are comparable to those of energy resolution.

The forward calorimeter covers $3<$ $|\eta|<5.5$, with full measurement capability to $|\eta| \simeq 5.0$. It has been used in the physics studies described here only to determine the missing energy, $F_{T}$. For this, detailed simulation of the response of individual cells is not needed. Rather, the energy and direction of each particle hitting the forward calorimeter is smeared according to a parameterization derived from a full mixture-level GEANT simulation; see Section 5.2.3. The simulation includes all the effects of dead material and shower spreading across calorimeter boundaries.
The resulting $E_{T}$ resolution as parameterized in gemfast is shown in Figure 2-9.

The statistics in the GEANT simulation were not sufficient to study potential nongaussian tails. These are modeled in gemfast by adding a second gaussian with a small amplitude and a larger width. Test beam data for single hadrons from 50 to $100 \mathrm{GeV}$ in the $\mathrm{D} \emptyset$ liquid argon calorimeter show a tail composed of roughly $1 \%$ of the events with a standard deviation two to three times larger than the gaussian calorimeter resolution. ${ }^{19}$ This tail is slightly larger than that seen for $1 \mathrm{TeV}$ jets in GEANT studies of jet resolution using energy-dependent weighting. A similar tail has been assumed for GEM. Unless otherwise specified, this parameterization is the basis of all calculations of $\boldsymbol{E}_{T}$.

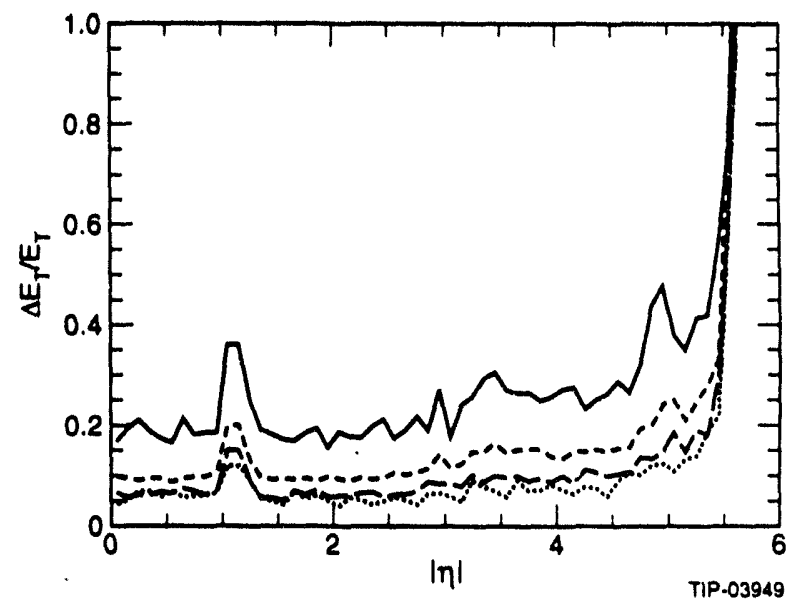

FIG. 2-9. Calorimetric $E_{T}$ resolutions for hadrons vs. $\eta$ for $E=10 \mathrm{GeV}$ (solid line), $50 \mathrm{GeV}$ (dashed line), $200 \mathrm{GeV}$ (dotted line), and $500 \mathrm{GeV}$ (dash-dotted line) used in gemfast for the calculation of $E_{T}$. Most of the fluctuations are caused by limited statistics in the GEANT simulation.

\subsubsection{Muon System}

The muon momentum resolution has been calculated by a full GEANT simulation including a detailed model of the detector and its support structures. The calculation includes chamber resolutions and alignment errors, the calculated shape of the magnetic field, the number of CSC planes in the measurement, and multiple scattering from the chambers and their supports. It is discussed further in Section 4.2.5 and described at length elsewhere. $^{20}$ The resulting parameterization of the resolution is shown in Figure 2-10. The jumps in the 
curves come from the transitions between various sets of chambers in the barrel and endcap. The flattening in the resolution at $\eta \approx 2.5$ is the effect of the forward field shapers.

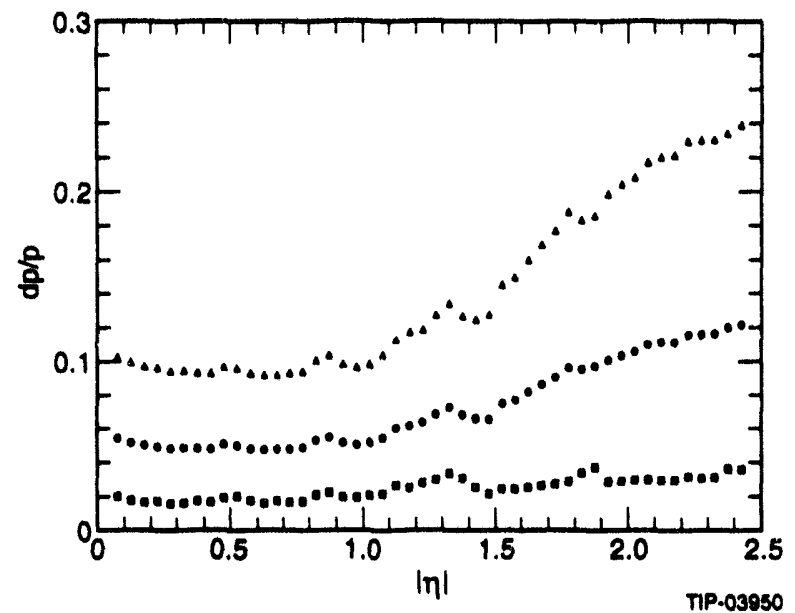

FIG. 2-10. Parameterization of muon resolution vs. $\eta$ for $p_{T}=100 \mathrm{GeV}$ (squares), $500 \mathrm{GeV}$ (circles), and $1 \mathrm{ToV}$ (triangles) in gemfast.

The geometrical acceptance for muons has been calculated similarly, requiring that the muon pass through at least three chambers in each superlayer. It is shown in Figure 2-11 and is essentially independent of muon energy. The coverage of the region $|\eta|<2.46$ is $83 \%$. The losses due to the spoiling of hits by $\delta$ rays and other electromagnetic interactions have also been simulated by GEANT, parameterized as a function of muon energy, and included in gemfast (see Sections 4.2.2 and 4.2.4).

The muon energy loss in the calorimeter has been calculated, including all the proce 'ses modeled in GEANT, and has been parameterized with a simple analytic function. The full calculation and the parameterization are shown in Figure 2-12. The lost energy is added to the appropriate cells of the calorimeter so that energy losses large compared tu the noise can be reconstructed in gemfast. As is discussed in Section 4.2.5, the best algorithm is to use the measured energy if it is greater than a factor $k$ times the most probable value, and the truncated mean value otherwise.

\subsubsection{Definition and Measurement of Photons, Electrons and Muons}

Electrons and photons are identified using a combination of the electromagnetic and hadronic

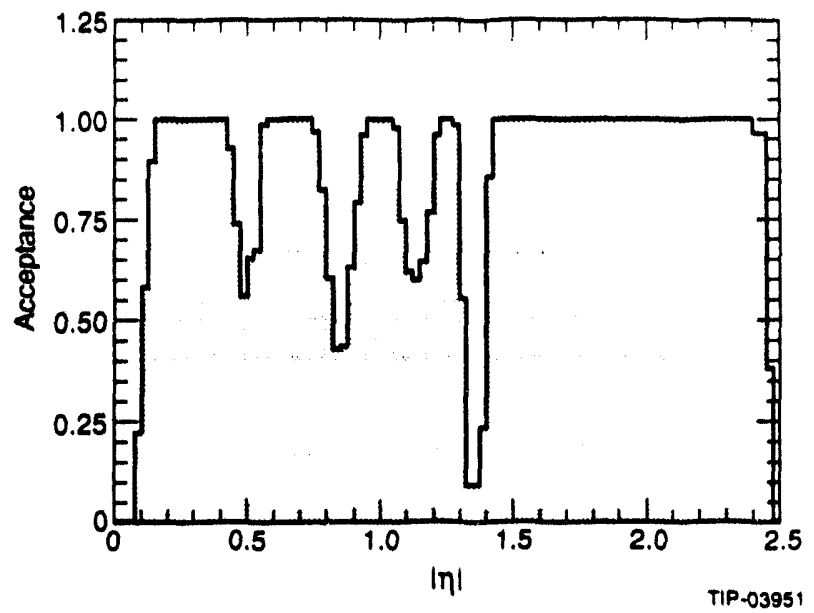

FIG. 2-11. Parameterization of muon acceptance vs. $\eta$ in gemfast. Because of binning of the GEANT data, the gaps in the barrel are wider than in reality but have nonzero acceptance. The average acceptance is correct.

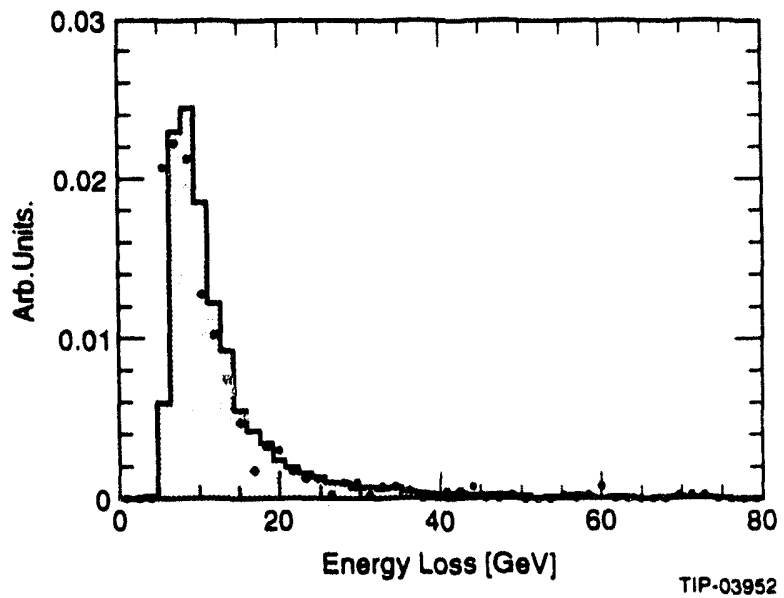

FIG. 2-12. Muon energy loss in calorimeter for $E_{\mu}=500 \mathrm{GeV}$ (solid curve) and gemfast parameterization (dots). Note that the tail at large energy loss is fit well by the parameterization.

calorimeters and the central tracker. A $5 \times 5$ tower cluster with $E_{T}>5 \mathrm{GeV}$ in the electromagnetic calorimeter is accepted as an electron or photon candidate if it has

$$
\frac{E_{3 \times 3}}{E_{5 \times 5}}>0.9
$$

(where $E_{N \times N}$ is the energy in the $N \times N$ EC towers centered on the most energetic one) and if the hadronic energy behind this cluster has less than $10 \%$ of its energy. Generally an isolation cut on the total energy in a larger cone, $R=0.2-0.4$, is also made. 
For maximum rejection of jet backgrounds, especially those to $H^{0} \rightarrow \gamma \gamma$, a cut is made using the likelihood function for the detailed shape of the shower to be consistent with a single photon. Since this level of detail is not included in gemfast, it requires a separate GEANT simulation of the photons that hit the cluster (see Sections 2.3.1 and 2.3.2).

Photons and electrons are separated by trackcluster matching. A good charged particle track must have at least six good hits and a distance of closest approach to the interaction point in the $r-\phi$ plane of less than $5 \mathrm{~mm}$. If there is no charged track within the $5 \times 5$ electromagnetic cluster, it is a photon candidate. For electrons, the energy measured in the electromagnetic calorimeter must match the momentum measured in the central tracker within $3 \sigma$ of the combined resolution. The overall identification efficiencies, both for photons and electrons, are greater than $90 \%$ from a few $\mathrm{GeV}$ up to several $\mathrm{TeV}$.

Muons are identified and measured in the muon chamber system outside the hadron calorimeters. Generally a muon track is required to have at least three good hits in each of the three muon superlayers. The $\chi^{2}$ of the fit must satisfy a loose cut in order to reject the background random hits. In addition, the transverse momentum of the identified muon is required to be greater than $5 \mathrm{GeV}$. Muon identification and reconstruction is well modeled in gemfast. For example, the $Z^{\prime} \rightarrow \mu^{+} \mu^{-}$acceptance obtained from gemfast described in Section 2.6.1 is essentially identical to that obtained from the full simulation of the same process described in Section 4.2.6.

\subsubsection{Definition and Measurement of Jets}

Jets are defined using a simple fixed-cone algorithm implemented in gemfast. The first step is to find the tower (using hadronic calorimeter segmentation) with the maximum $E_{T}$, which must exceed $5 \mathrm{GeV}$. All towers with $E_{T}>0.1 \mathrm{GeV}$ and with centers within a fixed radius $R=$ $\sqrt{(\Delta \eta)^{2}+(\Delta \phi)^{2}}$ (generally $0.3-0.9$ ) of this seed tower are included in the jet and marked. The unmarked tower of highest $E_{T}$ is taken as the next jet seed, and the process is continued until no seed towers remain. The whole event is then iteratively reclustered, using the centroids of the jets as the centers, until the tower lists for each jet are stable.
The algorithm makes no special provisions for towers within the clustering radius of two seed towers. This algorithm was carefully tested and found to give good performance. 21

A function was developed to correct the observed jet energy as a function of $\eta, p_{T}$ and the jet clustering cone size. Single jets were generated with PYTHIA. simulated with gemfast, and clustered. The factor by which the observed energy must be multiplied to obtain the true energy was then determined. Figure 2-13 shows the results vs. $\eta$ for various values of the jet $p_{T}$ with $R=0.4$. As expected, the correction is small, but it is comparable to the jet energy resolution. The average contributions from the underlying event and from the-in-time overlapping minimum bias events were subtracted from the jet $E_{T}$. For a cone size of 0.7 , the underlying event contributes about $3 \mathrm{GeV}$ to a jet at $\eta=0.0$, and about $2 \mathrm{GeV}$ at $\eta=2.5$. Each overlapping minimum bias event contributes about half as much.

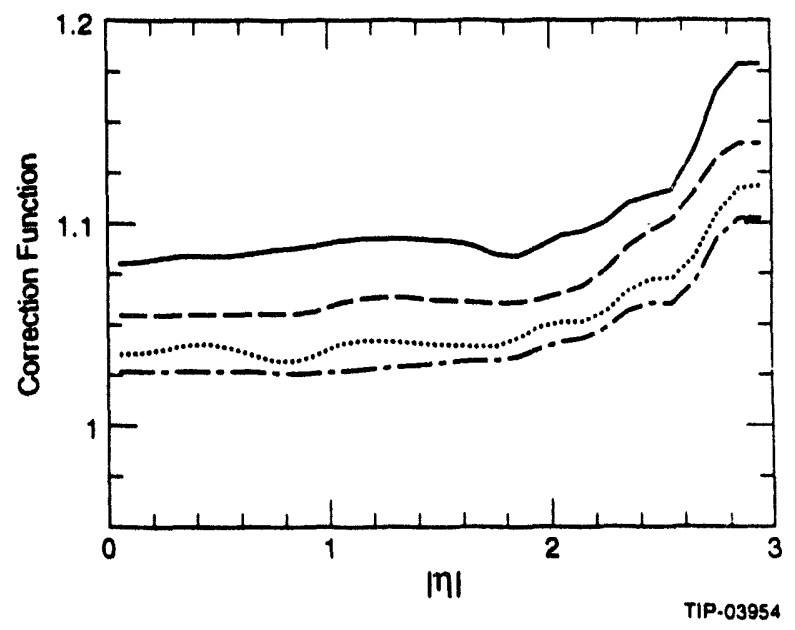

FIG. 2-13. Jet correction function vs. $\eta$ for $p_{T}=100$, 200,500 , and $1000 \mathrm{GeV}$ from top to bottom for a clustering radius $R=0.4$.

\subsubsection{Trigger Simulation}

The Level 1 trigger efficiency is simulated in gemfast and included in the analyses described below. The Level 2 and Level 3 triggers are assumed to be included, to a first approximation, in the cuts made in the analysis.

While the trigger primitives are fixed by the hardware, the thresholds and combinations used will depend on experience and on physics goals. An 
initial set of trigger thresholds from Section 7.2.4 is shown in Table 2-1. The $E_{x}$ triggers include an isolation cut on the surrounding towers and on the hadronic energy behind the cluster. Both the "digital" and the "analog" options described in Section 7.2.2 have been implemented. The $J_{x}$ triggers sum the electromagnetic and hadronic sections in towers reasonably matched to the size of a QCD jet. Both options are implemented in gemfast using the normal calorimeter simulation with sharp thresholds and the fixed trigger towers that will be used in the hardware trigger. The $M_{x}$ thresholds are nominal values at which the acceptance is $84 \%$ and include the coarse resolution of the trigger roads. 22 The missing energy simulation uses the full sum of the calorimeter. Figure 2-14 shows the acceptance for two typical triggers as an example.

Table 2.1. Primitives used in the GEM trigger. The highest threshold can be used alone; the rest can be used in conjunction with other triggers.

\begin{tabular}{|c|c|c|}
\hline Name & Definition & Thresholds \\
\hline$E_{x}$ & $\begin{array}{l}\text { EM cluster with } \\
E_{T}>\times \mathrm{GeV} \text { in } \\
0.16 \times 0.16 \text {, iso- } \\
\text { lated in both EM and } \\
\text { HAD calorimeters. }\end{array}$ & $E_{8}, E_{16}, E_{50}, E_{80}$ \\
\hline$M_{x}$ & $\begin{array}{l}\text { Muon with } \\
P r>\times \mathrm{G} \theta \mathrm{V} .\end{array}$ & $M_{10}, M_{20}, M_{30}, M_{40}$ \\
\hline$J_{x}$ & $\begin{array}{l}\text { Jet cluster with } \\
E_{T}>\times \mathrm{GeV}_{\text {in }} \\
0.48 \times 0.48\end{array}$ & $J_{16}, J_{50}, J_{80}, J_{200}$ \\
\hline$E_{x}$ & $\begin{array}{l}\text { Missing energy } \\
F_{T}>x \mathrm{GeV} \\
\text { summing whole } \\
\text { calorimeter. }\end{array}$ & $\boldsymbol{F}_{50}, \boldsymbol{E}_{100}$ \\
\hline
\end{tabular}

No attempt has been made to simulate the behavior of the actual trigger hardware in gemfast beyond imposing the resolutions and threshold described here. The total Level 1 rate is sensitive to the details of how the trigger is implemented, but this has been taken into account in the design of the trigger system discussed in Chapter 7.

While the highest thresholds in Table 2-1 have acceptable rates by themselves, the lower thresholds can only be used either with prescaling or in combinations. The twelve combinations listed in Section 7.2.4 appear to be sufficient to select all the physics processes so far considered for GEM. In particular they provide triggers with good efficiency for low-mass Higgs bosons and the other (relatively) low-mass processes listed in Table 2-2. The most difficult trigger is that for inclusive $H \rightarrow \gamma \gamma$ at the low end of the mass range; it is discussed in Section 7.2.4. Some low- $p_{T}$ processes such as jets
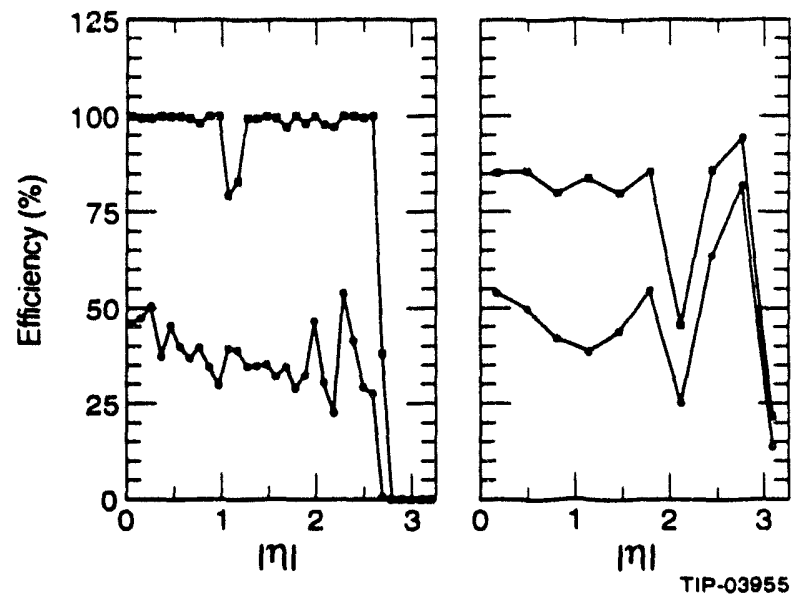

FIG. 2-14. Left plot: efficiency of the $E_{8}$ trigger as a function of $\eta$ for photons with $p_{T}=10 \mathrm{GeV}$ (lower) and $50 \mathrm{GeV}$ (upper) for the analog Level 1 trigger. The dip at $\eta \approx 1$ is caused by the barrel/endcap transition. Right plot: efficiency of a $J_{25}$ trigger for single jets with $p_{T}=50 \mathrm{GeV}$ (lower) and $100 \mathrm{GeV}$ (upper). The dip at $\eta=2.1$ is caused by a change in calorimeter cell size.

Table 2-2. Trigger efficiencies for a variety of processes using the triggers shown in Table 2-1 with standard logical notation. The triggers listed are the principal ones of those given in Section 7.2.4, but the efficiency is for the sum of all combinations. The efficiency for $H \rightarrow \ell^{+} \ell^{-} \ell^{+} \ell^{-}$at $140 \mathrm{GeV}$ could be increased about $5 \%$ by adding an $E_{16} \wedge M_{10}$ trigger. The last four processes are useful mainly for calibration.

\begin{tabular}{|c|c|c|c|}
\hline Process & Mass & Trigger & Efficiency \\
\hline$H \rightarrow \gamma \gamma$ & $80 \mathrm{GeV}$ & $2 E_{16} \vee E_{50}$ & $78.7 \%$ \\
\hline$H \rightarrow y$ & $140 \mathrm{GeV}$ & $2 E_{16} \vee E_{50}$ & $94.7 \%$ \\
\hline$\pi H \rightarrow y \gamma \ell X$ & $80 \mathrm{GeV}$ & $2 E_{16}$ & $94.4 \%$ \\
\hline$H \rightarrow \ell^{+} \ell^{-}-\ell^{+} \ell^{-}$ & $140 \mathrm{GeV}$ & $2 M_{10} \vee 2 E_{16}$ & $81.8 \%$ \\
\hline$H \rightarrow \ell^{+} \ell^{-} \ell^{+} \ell^{-}$ & $400 \mathrm{GeV}$ & $2 M_{10} \vee 2 E_{16}$ & $99.8 \%$ \\
\hline$H \rightarrow \ell^{+} \ell-i j$ & $800 \mathrm{GeV}$ & $2 M_{10} \vee 2 E_{16}$ & $99.9 \%$ \\
\hline$A \rightarrow \ell v b x$ & $140 \mathrm{GeV}$ & $E_{50} \vee M_{30}$ & $75.3 \%$ \\
\hline$\grave{g} \tilde{g}$ & $500 \mathrm{GeV}$ & $3 J_{80} \wedge \mathbb{F}_{100}$ & $99.9 \%$ \\
\hline$W \rightarrow e v$ & - & $E_{16} \wedge E_{50}$ & $15.8 \%$ \\
\hline$W \rightarrow \mu \nu$ & - & $M_{10} \wedge E_{50}$ & $48.7 \%$ \\
\hline$Z \rightarrow \theta \theta$ & - & $2 E_{16}$ & $80.3 \%$ \\
\hline$Z \rightarrow \mu \mu$ & - & $2 M_{10}$ & $86.9 \%$ \\
\hline
\end{tabular}


have such large cross sections that they must be prescaled. No attempt has been made to implement an efficient trigger on the inclusive- $b$ cross section to study $b$-physics, although this might be of interest during the low-luminosity initial period. Level 1 triggers on the higher mass processes which have been considered are generally easier than those listed in Table 2-2. The total Level 1 trigger rate with all of these triggers is calculated in Section 7.2.4 and found to be less than the design goal of $100 \mathrm{KHz}$.

\subsection{STANDARD MODEL HIGGS PHYSICS}

This section describes searches for the minimal, one-doublet, standard model Higgs boson with the GEM detector in the mass range between 80 and $800 \mathrm{GeV}$. This covers the interval between lower limits from LEP (which have excluded the mass range below $\sim 60 \mathrm{GeV}, 23$ and should reach 80-90 GeV at LEP 201); see Ref. 24) and the triviality bounds discusset in the Preface. ${ }^{25}$ The details of these Higgs boson search studies are presented in Ref. 26. Higgs bosons in extensions of the standard model such as the minimal supersymmetric model ${ }^{27}$ generally have similar signatures, albeit with different cross sections.

\subsubsection{Introduction}

The dominant decay modes of the standard model Higgs boson are $W^{+} W^{-}$and $Z Z$ for $M_{i}>2 M_{W}$ and heavy fermion pairs for $M_{H}<2 M_{W}$. The latter, and the dominant decays of
$W$ 's and $Z$ s into jets, all have large backgrounds, so it is necessary to rely on rare decays. The cleanest channel is $H \rightarrow Z Z / Z Z^{*} \rightarrow \ell^{+} \ell^{-} \ell^{+} \ell^{-}(\ell=e$, $\mu$ ), which has four isolated high- $p_{T}$ leptons in the final state. This channel has an inadequate rate at the lowest and highest ends of the mass range. Hence, the search may be divided into three mass regions:

- A Higgs boson of intermediate mass ( $80 \mathrm{GeV}<M_{H}<2 M_{Z}$ ) will be searched for through its decays $H \rightarrow \gamma \gamma$ and $H \rightarrow Z^{*} \rightarrow$ $\ell^{+} \ell^{-} \ell^{+} \ell^{-}$. Both direct $H \rightarrow \gamma \gamma$ and leptonassociated production $(t \bar{t} / W) H \rightarrow \gamma \gamma \ell X$ will be used.

- A heavy Higgs $\left(2 M_{Z}<M_{H}<600 \mathrm{GeV}\right)$ will be searched for through the channel $H \rightarrow Z Z \rightarrow \ell^{+} \ell^{-} \ell^{+} \ell^{-}$.

- A very heavy Higgs $\left(M_{H} \approx 800 \mathrm{GeV}\right)$ will also be searched for in $\ell^{+} \ell^{-} \ell^{+} \ell^{-}$and in the channels $H \rightarrow Z Z \rightarrow \ell^{+} \ell^{-} \nu \bar{v}$ and $H \rightarrow Z Z \rightarrow$ $\ell^{+} \ell^{-} j j$.

The Monte Carlo event generator used in this study was PYTHIA 5.6 and JETSET $7.3,^{6}$ and the top quark mass was assumed to be $140 \mathrm{GeV}$. The study was carried out for the SSC design luminosity $\mathscr{L}=10^{33} \mathrm{~cm}^{-2} \mathrm{sec}^{-1}$.

Table 2-3 summarizes the significance with which GEM could discover the Higgs through the channels listed above. (The significance is based on

Table 2-3. Sensitivity of the GEM detector to standard model Higgs boson signals.

\begin{tabular}{|c|c|c|c|c|c|c|c|}
\hline $\begin{array}{c}M_{H} \\
(\mathrm{G} \otimes \mathrm{V})\end{array}$ & $\int \begin{array}{l}2 d t \\
\left(t b^{-1}\right)\end{array}$ & $r \gamma$ & $\gamma \gamma \ell$ & $\ell+\ell-\ell+\ell-$ & $\ell+\ell-i j$ & $\ell+\ell-v$ & Combined \\
\hline 80 & 30 & 3.90 & 4.50 & & & & $6.3 \sigma$ \\
\hline 90 & 30 & 4.90 & 4.90 & & & & $7.2 \sigma$ \\
\hline 100 & 10 & 4.60 & $2.9 \sigma$ & & & & 5.80 \\
\hline 120 & 10 & $7.8 \sigma$ & $2.7 \sigma$ & & & & 8.50 \\
\hline 140 & 10 & 9.00 & 2.30 & $11 \sigma$ & & & 150 \\
\hline 150 & 10 & 7.30 & & 130 & & & 150 \\
\hline 160 & 10 & 3.20 & & $8.1 \sigma$ & & & 8.90 \\
\hline 170 & 10 & & & $5.7 \sigma$ & & & $5.7 \sigma$ \\
\hline 180 & 10 & & & 100 & & & 100 \\
\hline 200 & 10 & & & 380 & & & 380 \\
\hline 400 & 10 & & & $28 \sigma$ & & & $28 \sigma$ \\
\hline 600 & 10 & & & $9.7 \sigma$ & & & $9.7 \sigma$ \\
\hline 800 & 10 & & & $4.2 \sigma^{\circ}$ & $1.00^{\circ}$ & $4.20^{\circ}$ & $6.60^{\circ}$ \\
\hline
\end{tabular}

Estimated systematic errors. 
Poisson statistics translated to Gaussian confidence levels, as defined in Section 2.3.1.) This demonstrates the sensitivity of the GEM detector at greater than the $5 \sigma$ level for the whole range of Higgs masses considered. The signal can be seen with an integrated luminosity of $10 \mathrm{fb}^{-1}$, except for $M_{H}<100 \mathrm{GeV}$ where 20 to $30 \mathrm{fb}^{-1}$ are needed.

The most difficult part of the Higgs spectrum is the low end (between 80 and $100 \mathrm{GeV}$ ) and the vicinity close to $Z Z$ threshold, $170-180 \mathrm{GeV}$. GEM's sensitivity to a Higgs with $M_{H}$ below $100 \mathrm{GeV}$, in both the usual two-photon decay and the two photon with associated lepton channels, is unique. It provides the confirmation of a Higgs signal in a second decay channel necessary for a credible discovery claim in this mass region.

\section{Higgs Production Cross Section}

Over the entire Higgs mass range the dominant production mechanism at the SSC is gluon fusion, although vector-boson rusion is also important. The Higgs can also be produced associated with a vector boson or a top quark pair ( $t i$ ) which provides an isolated lepton tag.

In the standard model, a Higgs boson with mass $M_{H}>2 M_{Z}$ has a width which grows like $M_{H}^{3}$, but it remains fairly narrow for $M_{H}<600 \mathrm{GeV}$. Table 2-4 shows the Higgs width $\left(\Gamma_{H}\right)$ and the lowest order Higgs production cross section multiplied by specific decay branching ratio for the five modes studied in this section. These cross sections were calculated by using PYTHIA 5.6 with the EHLQ-1 parton distribution function, and a top quark mass of $140 \mathrm{GeV}$. Calculations of these cross sections using the more modern HMRS ${ }^{28}$ and CTEQ $^{29}$ distributions differ from EHLQ-1 by less than $20 \% .^{30}$ Higher order QCD corrections are not included in these numbers.

\section{$\gamma / e / \mu$ Identification}

The physics signatures of Higgs decays all involve identification of isolated photons, electrons and muons. Since the Higgs cross sections are small and the potential backgrounds are large, this identifi-
Table 2-4. Lowest order Higgs production cross section $x$ decay branching ratio (fb) for the modes considered in this section.

\begin{tabular}{|c|c|c|c|c|c|c|}
\hline $\begin{array}{c}M_{H} \\
(\mathrm{GeV})\end{array}$ & $\begin{array}{c}\Gamma_{H} \\
(\mathrm{GeV})\end{array}$ & $\sigma_{t(x) 1}$ & $\sigma_{(H(t) r)}$ & $\sigma_{\text {Heeel }}$ & $\sigma_{H(t)}$ & $\sigma_{\text {He(w) }}$ \\
\hline 80 & .0030 & 124 & 8.4 & & & \\
\hline 90 & .0034 & 144 & 8.0 & & & \\
\hline 100 & .0037 & 169 & 7.8 & & & \\
\hline 120 & .0049 & 211 & 6.9 & & & \\
\hline 140 & .0097 & 180 & 4.2 & 26 & & \\
\hline 150 & .019 & 128 & 2.6 & 32 & & \\
\hline 160 & .097 & 52.6 & 0.87 & 15 & & \\
\hline 170 & .380 & & & 9.2 & & \\
\hline 180 & .620 & & & 22 & & \\
\hline 200 & 1.4 & & & 85 & & \\
\hline 400 & 30 & & & 56 & & \\
\hline 600 & 112 & & & 16 & & \\
\hline 800 & 266 & & & 5.3 & 110 & 32 \\
\hline
\end{tabular}

cation requires particular care. First, an isolation cut is applied, removing most of the jet background. Then, a detailed identification algorithm is used.

The isolation cut for selecting electron and photon candidates was done using gemfast and requiring

$$
\sum_{R} E_{T}-E_{T}^{\gamma / e}<E_{T}^{\text {mean }}+E_{T}^{\text {cut }}
$$

where the sum is over calorimeter towers in a cone of radius $R$. Generally, $R=0.45$ was used for two-body final states and $R=0.30-0.35$ for fourbodies. The transverse energy $E_{T}^{\gamma / e}$ of the $\gamma$ or $e$ candidate was found by summing the energy deposited in $5 \times 5$ cells in the electromagnetic calorimeter; $E_{T}^{\text {mean }}$ is the mean transverse energy from $\mathrm{pi}^{\prime}$ up and noise; and $E_{T}^{\mathrm{cut}}$ is the isolation threshold imposed.

The $E_{T}^{\text {cut }}$ value was determined from the distribution of the thermal and pile-up noise ${ }^{31}$ as described in Section 2.2.3. Figure 2-15a shows the total noise in an $\eta-\phi$ cone of radius $R=0.45$ and 
a Gaussian fit, which has a width of $3.4 \mathrm{GeV}$. This is reduced to $1.7 \mathrm{GeV}$ if only those cells with $\mid E_{T}>0.5 \mathrm{GeV}$ are summed, as shown in Figure 2-15b. Doing this shifts the $E_{T}^{\text {mean }}$ from 0.22 to $1.5 \mathrm{GeV}$, and reduces the signal loss from 10,13 and $19 \%$ to $5.4,7.6$ and $11 \%$ for an $E_{T}^{\text {cut }}$ value of 5 , 4 and $3 \mathrm{GeV}$, respectively. A $4 \mathrm{GeV}$ cut appears to be optimal.

For photons, there must be no reconstructed charged track in the $5 \times 5$ cells. The central tracker can reject $95 \%$ of the electrons while keeping $96 \%$ of the photons. ${ }^{32}$ For electrons, there must be exactly one charged track in the central $3 \times 3$ cells. Also, the energy and shower position measured in the EM calorimeter must match the momentum and track position measured in the central tracker. The background to $H \rightarrow \gamma \gamma$ from misidentified $Z \rightarrow e^{+} e^{-}$is potentially large for $M_{H} \approx M_{Z}$ and is discussed in Section 2.3.2.
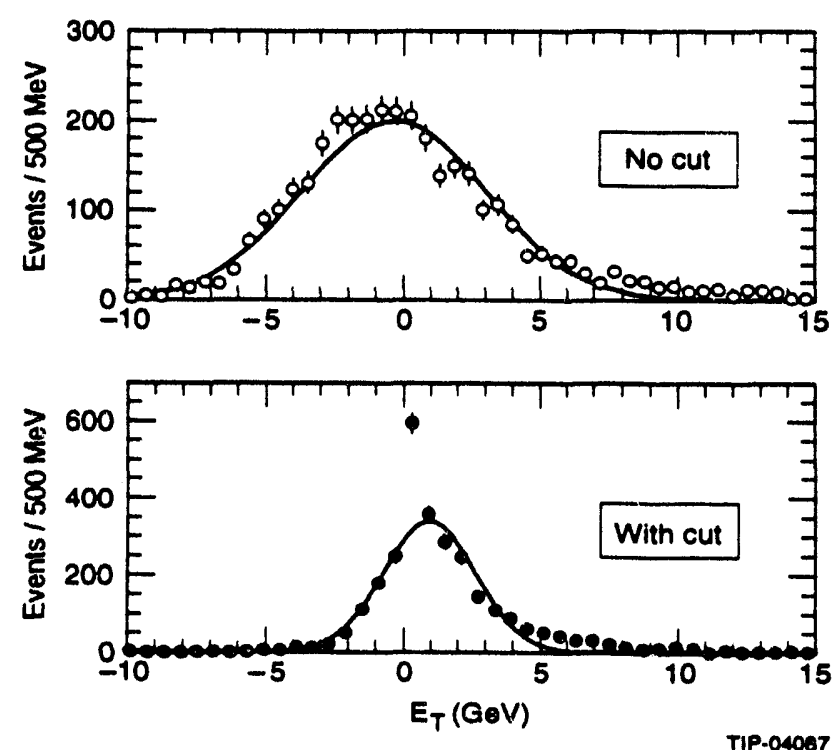

FIG. 2-15. Distributions of the sum of thermal and pile-up noise in a $R=0.45$ cone, with gaussian fits. The open and black dots show the noise measured by using a simple sum and by summing only cells with $\left|E_{T}\right|>0.5 \mathrm{GeV}$ respectively.

A more complete understanding of the capability of GEM to identify isolated photon and electron candidates based on their shower shape requires a detailed GEANT simulation. A sufficiently detailed geometry so far is available for only three $11 \times 11$ cell regions of the barrel. ${ }^{33}$ Therefore, the photons hitting the $5 \times 5$ cluster are rotated to the nearest of these regions, and the full simulation is run. Shower shape cuts (including lateral and longitudinal shower distributions), information from the $\theta$ strips of the first segment of the EM calorimeter, and energy in the hadronic calorimeter are combined in a likelihood function and used to select single electromagnetic showers. Typically this rejects $75 \%$ of the jet background while keeping $90 \%$ of the single photons. The probability of a QCD jet faking an isolated electron, $\mathscr{R}(e / \mathrm{jet})$, was determined to be approximately $10^{-5}$ by using detailed GEANT simulation. 33,34 For channels needing very good resolution, such as $H \rightarrow \gamma \gamma$ and $H \rightarrow Z^{*} \rightarrow e^{+} e^{-} e^{+} e^{-}$, photons and electrons were required not to be in the region of degraded resolution between the barrel and the endcap, $1.01<|\eta|<1.16$. The overall photon identification sfficiency, including this geometrical loss and other cuts, is between $80 \%$ and $85 \%$. The electron identification efficiency is $85 \%-90 \%$. For signals such as $H \rightarrow Z Z \rightarrow e^{+} e^{-} e^{+} e^{-}$for $M_{H}>2 M_{Z}$, one electron was allowed to hit this transition region.

Muons were simulated using gemfast. They are relatively free of jet background and so require less detailed identification. An isolation cut was made,

$$
\sum_{R} E_{T}-\Delta E<E_{T}^{\text {mean }}+E_{T}^{\text {cut }},
$$

where $\Delta E$ is the energy loss of a muon in the calorimeter, calculated as explained in Section 2.2.4 and Figure 2-12. The isolation cone radius was taken to be 0.35 for intermediate mass Higgs searches and 0.3 for heavier masses. This eliminates secondary decay muons in jets and punchthrough. The angle and momentum of the track measured in the muon system is then matched to that in the central tracker. The muon identification efficiency is around $80 \%$ for muons within $|\eta|<2.5$, which includes factors of $85 \%$ from the geometrical acceptance and $95 \%$ from the muon track reconstruction and identification efficiency; see Section 4.2 and Ref. 20.

\section{Significance of Higgs Signals}

Significance is used to indicate how well a Higgs signal can be identified in the presence of background. As in Gaussian statistics, a probability of $1-1.35 \times 10^{-3}$ is expressed as a significance 
of $3 \sigma, 1-2.85 \times 10^{-7}$ is expressed as $5 \sigma$, and so on. In this section, a $5 \sigma$ significance is generally regarded as the minimum for discovery.

For high statistics, the significance of a peak can be simply estimated by $S=N_{S} / \sqrt{N_{B}}$, where $N_{S}$ and $N_{B}$ are the expected number of signal and background events in a mass interval $M_{H} \pm 2 \Delta M_{H}$. Better mass resolution, $\Delta M_{H}$, or better acceptance are equivalent to a shorter discovery time. The significance obtained by summing the signal within $\pm 2 \Delta M_{H}$ is very close to summing only $\pm \Delta M_{H}$, if the reconstructed mass is an approximately gaussian distribution.

For low statistics, the usual formula for the significance is not correct. Since the probability of observing $n$ events with an expectation value of $\lambda$ follows Poisson statistics, $P_{n}(\lambda)=\lambda^{n} e^{-\lambda} / n !$, the probability that the signal fluctuates down to zero is not negligible for a small $\lambda$. The probability of missing the signal or having a fake signal caused by a background fluctuation is given by

$$
P_{\text {low }}=\sum_{n=0}^{\infty}\left[P_{n}\left(N_{B}+N_{S}\right) \times \sum_{m=n}^{\infty} P_{m}\left(N_{B}\right)\right] \text {. }
$$

This can be converted to a significance $S_{\text {low. }}$. If an apparent signal is observed, then the probability of its having fluctuated to zero is not relevant. The significance is given by the probability that the background fluctuates up to the signal:

$$
P_{\text {high }}=\sum_{m=N_{s}+N_{b}}^{\infty} P_{m}\left(N_{B}\right) \text {. }
$$

Since the simulated $N_{S}+N_{B}$ is not in general an integer, a linear interpolation is performed between the two integers closest to it. The probability $P_{\text {high }}$ can be converted to a significance $S_{\text {high }}$. When many bins are considered, some are likely to fluctuate upward by considerable amounts, so at least $S_{\text {high }}=5 \sigma$ is needed for discovery. The same safety margin is not needed to avoid missing a signal. The difference between these definitions is substantial. For $N_{S}=5$ and $N_{B}=1, N_{S} / \sqrt{N_{B}}=5$, while Eqs. (2.3.3) and (2.3.4) give $1.9 \sigma$ and $3.2 \sigma$ respectively.

\subsection{2 $H \rightarrow \gamma \gamma$ Search for $80 \mathrm{GeV}<M_{\mathrm{H}}<$ $160 \mathrm{GeV}$}

Precision electromagnetic energy are essential for the $H \rightarrow \gamma \gamma$ search because of the small production cross section (60 to $200 \mathrm{fb}$ ), the narrow decay width ( 3 to $100 \mathrm{GeV}$ ) of the Higgs boson between 80 and $160 \mathrm{GeV}$, and the large QCD $\gamma \gamma$ background. There is a potentially much larger background from QCD jets fragmenting into electromagnetic particles, which can be reduced using isolation cuts and a shower shape analysis made possible by the fine segmentation of the calorimeter. In PYTHIA, jets can radiate prompt photons as an option. This is properly regarded as an approximation to part of the higher order QCD corrections to the $\gamma \gamma$ background. Since these corrections are explicitly known for both the Higgs signal and the $q \bar{q} \rightarrow y \gamma$ background, the prompt photon radiation has been turned off, and the PYTHIA signal and background cross sections have been scaled to the higher order results.

To accept the Higgs signal and reduce the background, events were selected using the following cuts:

1. $\mid \eta^{\gamma \mid}<2.5$ and $p_{T}^{\gamma}>20 \mathrm{GeV}$.

2. Photon isolation cut with $R=0.45$ and $E_{T}^{\text {cul }}=4 \mathrm{GeV}$, and veto on $1.01<|\eta|<1.16$ as described in Section 2.3.1.

3. Photon identification based on detailed shower shape.

4. Electron rejection, as described below.

5. $\left|\cos \theta^{*}\right|<0.7$, where $\theta^{*}$ is the polar angle of photon in the center of mass system of two photons.

Cut 1 simply ensures that the photons are in the overall acceptance of the detector and are triggered on. Cuts 2 and 3 reduce the large potential backgrounds from misidentified QCD $\gamma$-jet and jet-jet events well below the $\gamma \gamma$ continuum. Cut 4 , which is only important for $M_{H} \approx M_{Z}$, removes the background from misidentified electrons. Finally, cut 5 reduces the real and fake $\gamma \gamma$ backgrounds. This leaves the $H \rightarrow \gamma \gamma$ signal as a narrow bump in the $M_{\gamma \gamma}$ distribution. 


\section{Two-Photon Mass Resolution}

The significance of a $H \rightarrow \gamma \gamma$ mass peak is directly related to the $\gamma \gamma$ mass resolution $\Delta M_{\gamma \gamma}$. This is given by

$$
\frac{\Delta M_{r r}}{M_{r r}}=\frac{1}{2}\left[\left(\frac{\Delta E_{1}}{E_{1}}\right)^{2}+\left(\frac{\Delta E_{2}}{E_{2}}\right)^{2}+\left(\cot \frac{\theta}{2} \Delta \theta\right)^{2}\right]^{1 / 2} \text {. }
$$

where $E_{1}$ and $E_{2}$ are the energies of two photons and $\theta$ is the opening angle between them. It is clear from this equation that uncertainties in both energy and direction measurement would degrade the Higgs mass resolution.

The EM energy resolution is improved for the region $|\eta|>1.9$ by using a $3 \times 3$ rather than a $5 \times 5$ cell sum and a faster shaping time, as discussed in Section 5.4.3. Since gemfast does not incorporate this refinement, the Higgs mass was calculated using the events passing the selection cuts with a separately parametrized energy resolution:

$$
\frac{\Delta E}{E}=\frac{a}{\sqrt{E}} \oplus b \oplus \frac{c}{E},
$$

where $a, b$ and $c$ represent sampling, constant and noise contributions respectively. Here, $a=6 \%$ for the barrel and $8.5 \%$ for the endcaps and $b=0.4 \%$.

The noise term $c=\sqrt{\sigma_{t}^{2}+\sigma_{p}^{2}}$ is the sum of the thermal noise, $\sigma_{t}\left(E_{T}=100 \mathrm{MeV}\right.$ for the barrel and $E=175 \mathrm{MeV}$ for the endcap), and the pile-up noise, $\sigma_{p} \quad\left(E_{T}=120 \mathrm{MeV}\right.$ for $|\eta|<1.4$ and $E_{T}=120+366(|\eta|-1.4) \mathrm{MeV}$ for $\left.|\eta|>1.4\right)$. The effect of different possible values of $a$ and $b$ is discussed in Ref. 34 and Section 5.1.2.

The angular resolution in Eq. (2.3.5) is determined by both the position resolution in the EM calorimeter and the precision of the vertex determination. GEM's calorimeter position resolution, $\Delta x=4.4 \mathrm{~mm} / \sqrt{E}$, has a negligible effect on the mass resolution. ${ }^{34}$ The vertex position for a single event is well determined by the central tracker. The only issue, therefore, is how well the correct vertex can be selected in the presence of an average of 1.6 additional minimum bias events at the standard SSC luminosity plus events from previous bunch crossings.
Two approaches are possible. The first is to use the difference in event topology resulting from the fact that Higgs production is a harder process than most of the minimum bias events and hence radiates more gluons. This leads to a higher multiplicity and a higher average $p_{T}$. Selecting the vertex with the highest $p_{T}$-weighted charged multiplicity gives the correct Higgs vertex with $95 \%$ probability at the standard SSC luminosity. The algorithm for this is discussed in Section 6.2.3.

An independent approach is to use the pointing provided by the longitudinal segmentation of the calorimeter; see Section 5.2.1. This provides an angular resolution of

$$
\Delta \theta=\frac{d}{\sqrt{E}}+0.5 \mathrm{mrad},
$$

where $d=40 \mathrm{mrad}$ for the barrel and $50 \mathrm{mrad}$ for the endcaps. (This parametrization is valid for the energy region used in intermediate mass Higgs searches.) Using this pointing alone without any information from the central tracker degrades the mass resolution by only $20 \%$. Using the pointung and then selecting the closest central tracker vertex gives a vertex within $5 \mathrm{~mm} 87 \%$ of the time at standard luminosity. This degrades to about $65 \%$ at $3 \times 10^{33} \mathrm{~cm}^{-2} \mathrm{sec}^{-1}$. A combination of these two methods improve the vertex-finding efficiency to $97 \% .{ }^{26} \mathrm{~A}$ vertex finding efficiency of $95 \%$ was assumed for this analysis.

\section{$H \rightarrow y \gamma$ Signal and Direct $\gamma \gamma$ Background}

The $H \rightarrow \gamma \gamma$ signal and the $q \bar{q} \rightarrow \gamma \gamma$ and $g g \rightarrow \gamma \gamma$ backgrounds were generated with PYTHIA. The next-to-leading-log (NLL) corrections to the signal cross section have been calculated, ${ }^{35}$ and give an enhancement factor $K \approx 1.5$. The lowest order PYTHIA cross sections listed in Table 2-4 have been rescaled appropriately.

The NLL correction to the $q \bar{q} \rightarrow \gamma$ background has also been calculated. ${ }^{36}$ Figure 2-16 shows the NLL direct photon background cross section after cut 1 and cut 2 at the parton level without detailed photon identification. The Born, box diagram, Born plus box $(B+B)$, and leading log (LL) contributions are also shown, where both $\mathrm{LL}$ and NLL include contributions of single and double bremsstrahlung. Higher order corrections to 
$g g \rightarrow \gamma \gamma$ have not been calculated and are not included. Since this process contributes around $1 / 3$ of the NLL cross section, a $K$ factor of 1.5 for it would increase the total NLL cross section by $16 \%$ and reduce the final significance by $8 \%$. The $\gamma \gamma$ background from PYTHIA has been rescaled by the factor $N L L /(B+B)$.

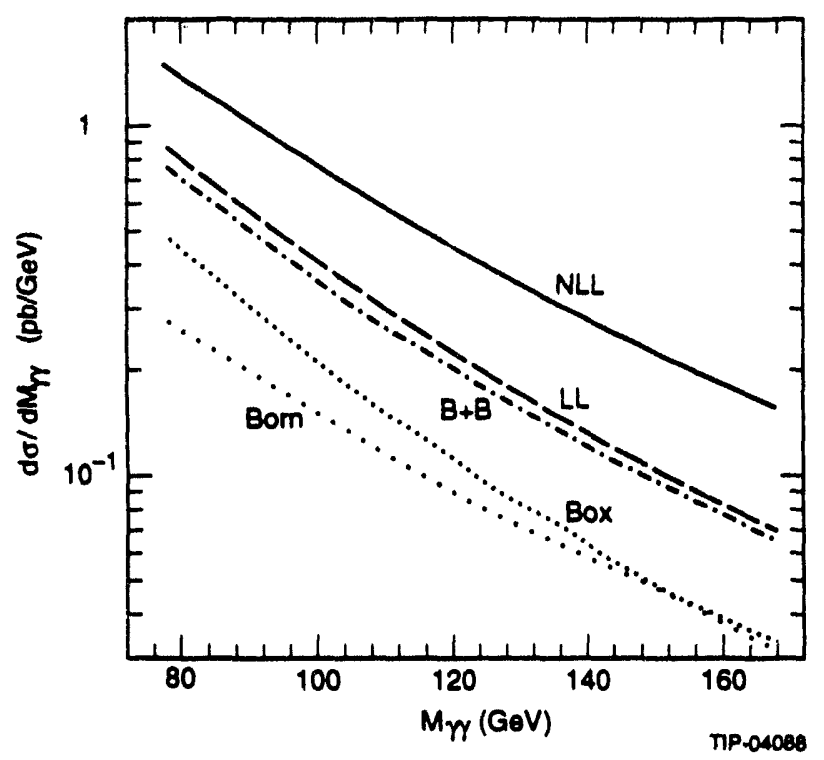

FIG. 2-16. Cross section of direct photon background calculated to next-to-leading-log order is shown as a function of $\gamma \gamma$ invariant mass after the cuts described in the text. Also shown in the figure are cross sections of Born, box diagram, Bom + box $(B+B)$ and leading log calculations.

After cuts 1 to 4 , the cut on $\cos \theta^{*}$ provides an additional rejection for both direct photon and jet background, and thus improves the significance. Figure 2-17 shows the $\cos \theta^{*}$ distribution for an $80 \mathrm{GeV}$ Higgs, NLL direct photon background and the $\gamma$-jet and jet-jet backgrounds. The improvement in significance is optimized by a cut $\left|\cos \theta^{*}\right|<0.7$, for which an improvement of $15 \%$ is obtained. The trigger efficiency for events passing these selection cuts is $98.8 \%$. This is included in all signal and background rates.

Table 2-5 lists the Higgs production cross section $\left(\sigma_{H}\right)$ and the cross sections after cuts $1-4$ and 1-5. It also lists the rate of direct photon $H \rightarrow$ rybackground after cuts $1-2,1-4$, and 1-5. The signals are small compared to the background but still statistically significant.

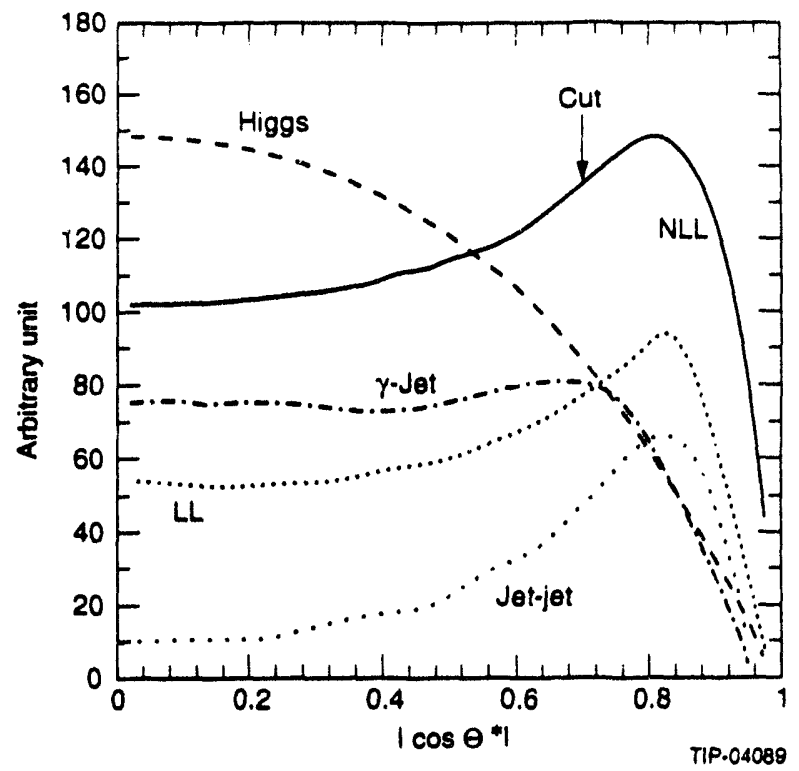

FIG. 2-17. $\left|\cos \theta^{\circ}\right|$ distribution for an $80 \mathrm{GeV}$ Higgs, NLL direct photon background, $\gamma$-jet and jet-jet background. The cut used in this analysis is $\left|\cos \theta^{*}\right|<0.7$.

Table 2-5. Signal and direct photon background for $H \rightarrow \gamma \gamma$. The Higgs mass resolution is $0.66-1.0 \mathrm{GeV}$.

\begin{tabular}{|c|c|c|c|c|c|c|c|}
\hline \multicolumn{8}{|c|}{$H \rightarrow \gamma \gamma$ Signal $(\mathrm{fb})$} \\
\hline $\begin{array}{l}\mathrm{MH} \\
\text { (GoV) }\end{array}$ & 80 & 90 & 100 & 120 & 140 & 150 & 160 \\
\hline$\sigma_{H}$ & 169 & 199 & 233 & 291 & 255 & 182 & 71.2 \\
\hline $\begin{array}{l}\text { After } \\
\text { cuns } 1-4\end{array}$ & 47 & 45 & 74 & 96 & 88 & 64 & 25 \\
\hline $\begin{array}{l}\text { Afts? } \\
\text { cuts i-5 }\end{array}$ & 38 & 35 & 57 & 74 & 66 & 48 & 19 \\
\hline \multicolumn{8}{|c|}{ Direct Photon Background Rate ( $\mathrm{fb} / \mathrm{GeV}$ ) } \\
\hline $\begin{array}{l}M / v) \\
(\mathrm{GeV})\end{array}$ & 80 & 90 & 100 & 120 & 140 & 150 & 160 \\
\hline $\begin{array}{l}\text { After } \\
\text { cuts 1-2 }\end{array}$ & 1840 & 1360 & 1020 & 600 & 370 & 300 & 240 \\
\hline $\begin{array}{l}\text { After } \\
\text { cuts } 1-4\end{array}$ & 1010 & 560 & 560 & 330 & 210 & 160 & 130 \\
\hline $\begin{array}{l}\text { After } \\
\text { cuts } 1-5\end{array}$ & 650 & 330 & 310 & 170 & 100 & $\pi$ & 63 \\
\hline
\end{tabular}

Jet-Jet and $\boldsymbol{\gamma}$-Jet Backgrounds to $\boldsymbol{H} \rightarrow \boldsymbol{\gamma} \gamma$

QCD jets produce photons from $\pi^{0}$ and $\eta$ decays. These sometimes appear isolated and give additional backgrounds to $H \rightarrow \gamma \gamma$. Both $\gamma$-jet events 
giving $\gamma$ " $\gamma$ " and jet-jet events producing " $\gamma$ " must be considered. Thus, the jet background to $H \rightarrow \gamma \gamma$ is

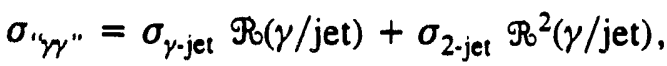

where $\mathscr{R}_{b}(\gamma / \mathrm{jet})$ is the probability for a jet to fake a photon.

The probability $\mathscr{R}(y / j e t)$ was determined using a combination of gemfast for selecting isolated events and GEANT for simulating the shower shape cut, as described in Section 2.3.1. QED bremsstrahlung is not included, since it is properly included in the higher order QCD cross sections. Since $\operatorname{Bo}(\gamma / j \mathrm{jet})$ is different for quark and gluon jets, ${ }^{34}$ separate samples of about $10^{6}$ events each for $\gamma q$ and $\gamma g$ were used to study jet rejection as a function of $M_{\gamma \gamma}$.

Table 2-6 shows $\mathscr{B}(\gamma /$ jet $)$ for quark and gluon jets at several $M_{\gamma^{\prime \prime}} y^{\prime}$. The shower shape analysis made possible by the fine sampling of the GEM calorimeter improves the rejection by about a factor of 4 per photon over isolation alone. Since the jet-jet cross section is about $10^{8}$ times the $\gamma \gamma$ one, it is not possible to generate enough jet-jet events to simulate the background directly. Instead, $g g, q g$ and $q q$ events were generated at the parton level, and the probabilities in Table 2-6 were used to calculate the background.
Table 2-6. Probability of jet faking a photon, $\Re_{0}(\gamma / j \in \ell)$ $\left(10^{-4}\right)$, obtained from GEANT simulation.

\begin{tabular}{cccccc}
\hline \hline$M_{\text { " } \gamma \text { " (GeV) }}$ & 80 & 100 & 120 & 140 & 160 \\
\hline \multicolumn{5}{c}{ Quark Jet } \\
\hline After Isolation & 20 & 18 & 15 & 13 & 12 \\
After $\gamma$ ID & 4.1 & 3.9 & 3.7 & 3.6 & 3.4 \\
\hline \multicolumn{7}{c}{ Gluon Jet } \\
\hline After Isolation & 5.9 & 5.3 & 4.8 & 4.3 & 3.9 \\
After $\gamma$ ID & 1.2 & 1.1 & 1.0 & 0.91 & 0.83 \\
\hline \hline
\end{tabular}

Table 2-7 lists the cross sections for the jet backgrounds after cut 1 at the parton level with a $K$-factor of 1.5 , after cuts $1-2,1-3$ and after all cuts $1-5$. The $y$-gluon background is reduced to a negligible level compared to the NLL direct photon background. The 2 jet background (which is dominated by gluon jets) is reduced to around $15 \%$ of the NLL direct photon background at $80 \mathrm{GeV}$. The $\gamma q$ background, however, remains $40 \%$ of the NLL direct photon backgruund there. Since a factor of two increase in all the jet backgrounds reduces the significance by only $16 \%$, GEM's searches in the $H \rightarrow \gamma \gamma$ channel are not very sensitive to the $Q C D$ jet rejection.

Table 2-7. Jet background rates for $H \rightarrow \gamma \gamma$.

\begin{tabular}{|c|c|c|c|c|c|c|c|}
\hline$M_{r}(\mathrm{GeV})$ & 80 & 90 & 100 & 120 & 140 & 150 & 160 \\
\hline \multicolumn{8}{|c|}{$q g \rightarrow \gamma q$} \\
\hline After cut $1(\mathrm{pb} / \mathrm{GeV})$ & 1170 & 880 & 660 & 370 & 210 & 160 & 120 \\
\hline After cuts $1-2(\mathrm{fb} / \mathrm{GeV})$ & 1590 & 830 & 780 & 380 & 190 & 130 & 91 \\
\hline After cuts $1-3(\mathrm{fb} / \mathrm{GeV})$ & 320 & 180 & 170 & 92 & 50 & 36 & 27 \\
\hline After cuts 1-5 (fb/GeV) & 260 & 140 & 130 & 65 & 32 & 23 & 16 \\
\hline \multicolumn{8}{|c|}{$q \bar{q} \rightarrow \gamma g$} \\
\hline After cut 1 (pb/GeV) & 53 & 41 & 32 & 20 & 12 & 9.2 & 7.2 \\
\hline After cuts $1-2(\mathrm{fb} / \mathrm{GeV})$ & 21 & 11 & 11 & 6.1 & 3.4 & 2.5 & 1.8 \\
\hline After cuts $1-3$ (fb/GeV) & 4.3 & 2.4 & 2.4 & 1.3 & 0.7 & 0.5 & 0.4 \\
\hline After cuts $1-5$ (fb/GeV) & 3.2 & 1.7 & 1.6 & 0.8 & 0.4 & 0.3 & 0.2 \\
\hline \multicolumn{8}{|c|}{2 jets } \\
\hline After cut $1(\mu \mathrm{b} / \mathrm{GeV})$ & 8.3 & 6.8 & 5.6 & 3.8 & 2.5 & 2.1 & 1.7 \\
\hline After cuts $1-2(\mathrm{pb} / \mathrm{GeV})$ & 4.4 & 2.4 & 2.3 & 1.2 & 0.6 & 0.5 & 0.3 \\
\hline After cuts $1-3$ (tb/GeV) & 190 & 110 & 110 & 60 & 34 & 25 & 19 \\
\hline After cuts $1-5(f b / G \otimes V)$ & 95 & 46 & 39 & 16 & 6.8 & 4.4 & 2.9 \\
\hline \multicolumn{8}{|c|}{ Sum of All Jet Backgrounds } \\
\hline Total (fb/GeV) & 360 & 190 & 170 & 82 & 40 & 28 & 19 \\
\hline
\end{tabular}




\section{Drell-Yan Background for $\boldsymbol{H} \rightarrow \boldsymbol{\gamma}$}

Drell-Yan $e^{+} e^{-}$pairs can give a background for $H \rightarrow \gamma \gamma$ if the central tracker fails to identify both charged tracks. Figure 2-18 slows the invariant mass distribution of Drell-Yan $e^{+} e^{-}$after event selection cuts and the lowest order direct photon background. The Drell-Yan cross section is about 300 times larger than the direct photon background at the Z-mass peak, but comparable elsewhere. A hit level GEANT simulation described in Section 6.2.3 was performed ${ }^{32}$ for the central tracker to estimate the rate of electrons faking photons, $\mathscr{P}_{0}(\gamma / e)$. The photon acceptance was found to be $83 \%$ for $\mathscr{R}(\gamma / e)=0.005$ and $96 \%$ for $\mathscr{R}(\gamma / e)=$ 0.05 . The stronger rejection factor was used near the $Z$ and the weaker one elsewhere, as indicated in Figure 2-18. Studies are in progress to determine whether a more favorable acceptance to rejection ratio can be achieved.

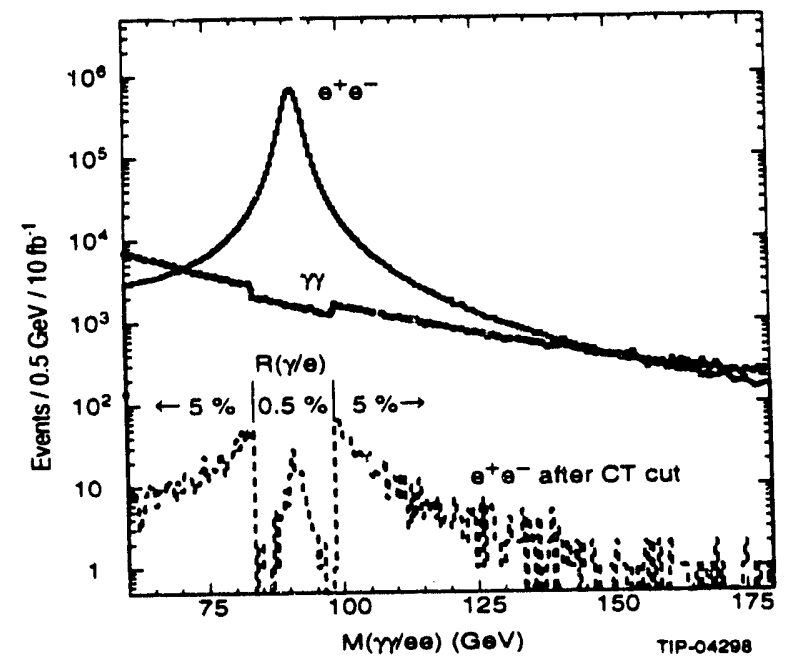

FIG. 2-18. Drell-Yan $\theta^{+} \theta^{-}$cross section after event selection cuts (dashed) and lowest order direct $\gamma \gamma$ cross section (solid). The dots show the rate of fake photon pairs from Drell-Yan electron pairs using $\mathscr{R}_{0}(\gamma / \theta)=0.005$ near the $Z$ and 0.05 elsewhere.

\section{$H \rightarrow$ ry Significance}

Figure 2-19 shows the reconstructed $H \rightarrow \gamma \gamma$ mass peaks after subtracting the background, for an integrated luminosity of $10 \mathrm{fb}^{-1}$, and $M_{H}=80$, $100,120,140$ and $160 \mathrm{GeV}$. Figure 2-20 shows the corresponding peaks after $30 \mathrm{fb}^{-1}$. Table 2-8 summarizes the reconstructed widths, accepted cross sections, total backgrounds, and resulting significances using a $\pm 2 \Delta M_{H}$ mass bin.

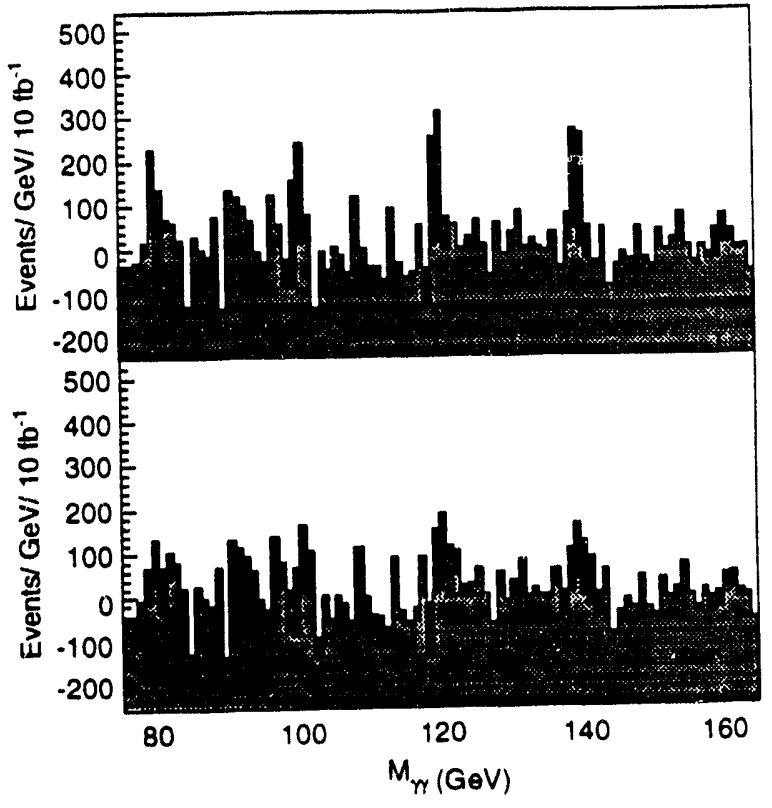

FIG. 2-19. Higgs mass peaks over subtracted background, obtained with $10 \mathrm{fb}^{-1}$, for $\mathrm{H} \rightarrow \mathrm{\gamma r}$ searches with $M_{H}=80,100,120,140$ and $160 \mathrm{GeV}$. The top corresponds to $a=6 / 8.5 \%$ in the barrel/endcaps and $b=0.4 \%$, and the bottom to $a=14,17 \%$ in the barrel/endcaps and $b=1 \%$. The GEM photon identification algorithm is used in both cases; see Section 2.3.1.

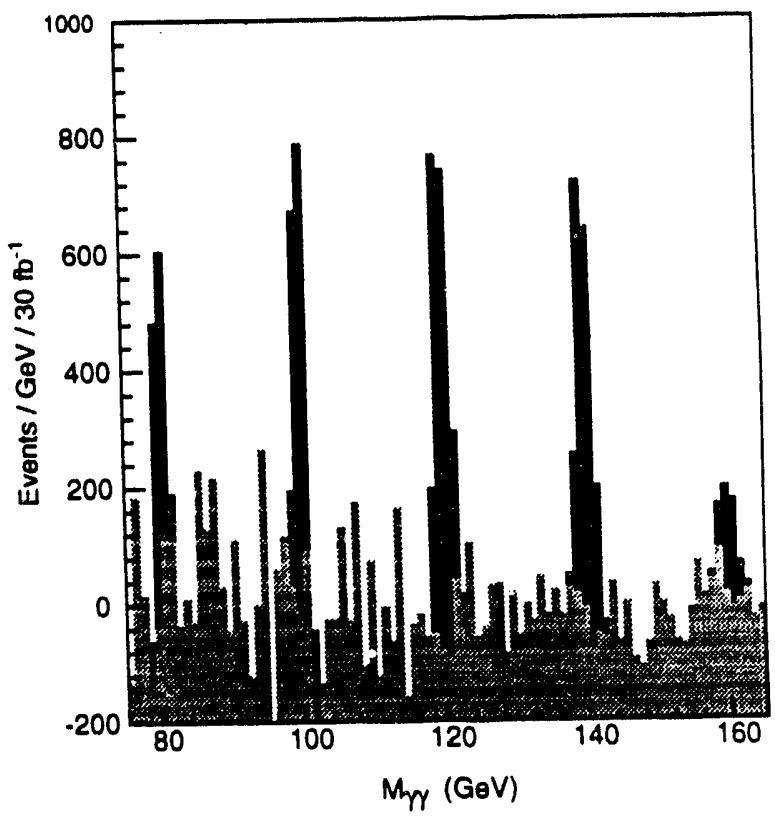

FIG. 2-20. Higgs mass peaks over subtracted background, obtained with $30 \mathrm{fb}^{-1}$, for $H \rightarrow \gamma \gamma$ searches with $M_{H}=80,100,120,140$ and $160 \mathrm{GeV}$ for the GEM detector. 
Table 2-8. Significance for $H \rightarrow \gamma \gamma$; the cross section includes all acceptances.

\begin{tabular}{|c|c|c|c|c|c|c|c|}
\hline $\begin{array}{l}\mathrm{MH}_{\mathrm{H}} \\
\text { (GeV) }\end{array}$ & 80 & 90 & 100 & 120 & 140 & 150 & 160 \\
\hline $\begin{array}{l}\Delta M_{H} \\
(\mathrm{GeV})\end{array}$ & 0.66 & 0.69 & 0.79 & 0.84 & 0.91 & 0.93 & 0.99 \\
\hline$\sigma_{H}(\mathrm{fb})$ & 38 & 35 & 57 & 74 & 66 & 48 & 19 \\
\hline $\begin{array}{l}\text { Beck- } \\
\text { ground } \\
\text { (fo/GeV) }\end{array}$ & 1010 & 520 & 480 & 250 & 140 & 110 & 82 \\
\hline \multicolumn{8}{|c|}{ GEM Significance } \\
\hline $10 \mathrm{fo}^{-1}$ & 2.2 & 2.8 & 4.6 & 7.8 & 9.0 & 7.3 & 3.2 \\
\hline $30 \mathrm{fD}^{-1}$ & 3.9 & 4.9 & 7.9 & 14 & 16 & 13 & 5.5 \\
\hline
\end{tabular}

Figure 2-21 compares the significance as a function of integrated luminosity for various masses for the GEM detector with that for a calorimeter with degraded energy resolution but the same good jet rejection. The upper edge of the bands corresponds to the significance listed in Table 2-8, while the lower edge of the band is the significance obtained if the $\gamma$-jet background is increased by a factor of two, the jet-jet background by a factor of four. With

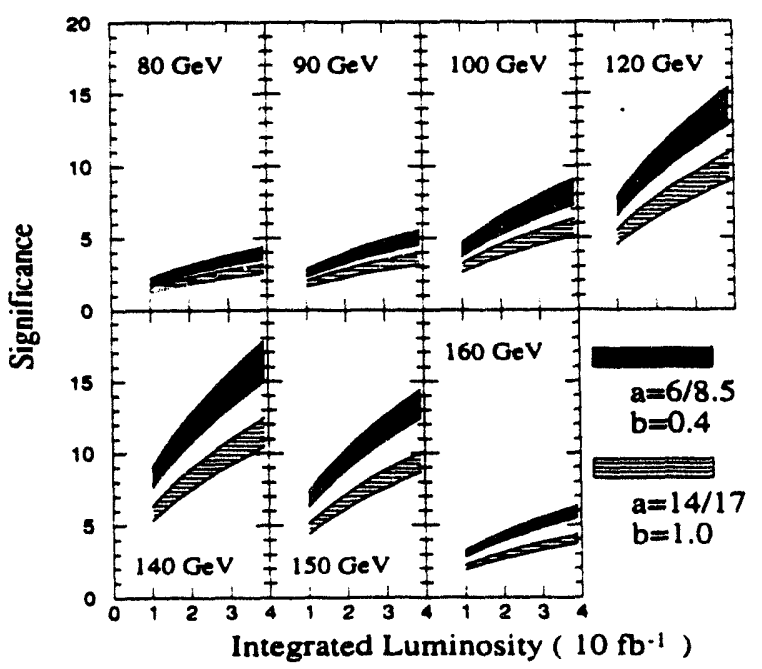

FIG. 2-21. The significance obtainable in $H \rightarrow \gamma \gamma$ searches is shown as function of integrated luminosity for Higgs masses of $80,90,100,120,140,150$ and $160 \mathrm{GeV}$. The dark bands correspond to $a=6 / 8.5 \%$ in the barrel/endcaps and $b=0.4 \%$. The cross-hatched bands correspond to $a=14 / 17 \%$ in the barreVendcaps and $b=1 \%$. The GEM photon identification algorithm is used in both cases; see Section 2.3.1.
$10 \mathrm{fb}^{-1}$, GEM could discover a Higgs boson, using this mode alone, for $110 \leq M_{H} \leqslant 150 \mathrm{GeV}$. With $30 \mathrm{fb}^{-1}$, using this mode alone, it could extend the discovery reach down to about $90 \mathrm{GeV}$ and up to $160 \mathrm{GeV}$. While the heavier masses can also be found in the $Z Z^{*}$ mode, the $\gamma \gamma$ branching ratio is important to distinguish the minimal standard model from nonminimal ones.

\subsection{3 $H(t \bar{t} / W) \rightarrow \gamma_{\gamma} \ell X$ Search for $80 \mathrm{GeV}<M_{H}<140 \mathrm{GeV}$}

The processes $H t \bar{t} \rightarrow \gamma \gamma \ell X$ and $H W \rightarrow \gamma \gamma \ell X$ are complementary to the inclusive $H \rightarrow \gamma \gamma$, providing essential confirmation. ${ }^{37}$ As shown in Table 2-4, the signal cross section for the sum of these two channels is of the order of few fb, but the isolated lepton tag and photon identification cuts leads to smaller backgrounds, so that the signal-tobackground ratio is large. In these associated production channels, most of the effects of detector resolutions on the reconstructed mass resolution, discussed in Section 2.3.2, remain applicable. The main differences with the discussion of $H^{0} \rightarrow \gamma \gamma$ are: (1) With a charged lepton in the final state, the central tracker is able to determine the Higgs vertex more precisely and efficiently. (2) A good understanding of the rate and shape of the background is even more important here because the signal rate is so small.

\section{Signal and Background}

The main backgrounds to $H(t \bar{t} / W) \rightarrow \gamma \gamma \ell X$ searches are:

1. $i \bar{t} \gamma \gamma$ or $b \bar{b} \gamma y \rightarrow \ell \gamma \gamma$.

2. $q \bar{q}^{\prime} \rightarrow W \gamma \gamma \rightarrow \ell \gamma \gamma$, where both photons are radiated from quarks.

3. $q \bar{q} \rightarrow W \gamma \rightarrow \ell \gamma \gamma$, where one photon is radiated from the outgoing charged lepton.

4. $q \bar{q} / g g \rightarrow Z \gamma \rightarrow \ell^{+} \ell^{-} \gamma \gamma$, where the second photon is radiated from the outgoing charged lepton.

5. $q \bar{q} / g g \rightarrow Z \gamma \rightarrow e^{\prime \prime} \gamma " \gamma$, where the fake photon is from misidentification of the electron.

6. $t \bar{i} \rightarrow " \gamma \gamma$ " $X$, where both photons are fakes arising from jet fragmentation.

Since all the higher order QCD corrections to the signal and backgrounds have not been computed, lead- 
ing order cross sections are used for this entire analysis. While $t \bar{t}$ is initially very much larger, all the backgrounds turn out to be comparable after the cuts described below.

Not all of these background processes are included in PYTHIA and, so, they were calculated using a combination of generators. For processes 1 and 2 , the initial hard scattering was generated using PAPAGENO 3.6. ${ }^{38}$ Initial and final state parton radiation, hadronization and decays were then generated using PYTHIA. All the final state photon radiation for processes 3,4 and 5 was generated using PYTHIA with the QED radiation option turned on. Since the process $g g \rightarrow Z \gamma$ is not available in either PYTHIA or PAPAGENO, $q \bar{q} \rightarrow Z \gamma$ was increased by $20 \% .^{39}$

The $t \bar{t}$ background (process 6) was generated with PYTHIA with QED radiation turned on. The study was carried out wiih $2.6 \times 10^{6}$ events. Only 3 events survived all cuts, none of them containing two radiated photons. The bbyy background was also simulated, and it is small compared to the $t \bar{t}$ background after isolation cuts.

The following cuts were made to reject the backgrounds:

1. $\left|\eta_{\ell}\right|<2.5$ and $p_{T}^{\ell}>20 \mathrm{GeV}$.

2. $\left|\eta_{\gamma}\right|<2.5$ and $p_{T}^{\gamma}>20 \mathrm{GeV}$.

3. Photon and lepton isolation with $E_{T}^{\text {cut }}=5 \mathrm{GeV}$ and $R=0.45$ and 0.3 for photon and lepton respectively.

4. Photon and lepton identification by shower shape and track matching.

5. $p_{r}^{\gamma \gamma}>40 \mathrm{GeV}$.

The first two cuts ensured that the lepton and photons could be detected. Cuts 3 and 4 identify the photons and lepton and reject jet backgrounds. Finally, cut 5 helps reject the backgrounds, especially $t \bar{t}$ listed above. The $40 \mathrm{GeV}$ cut applied to the $p_{T}$ of the $\gamma \gamma$ pair reduces the background by a factor of 2 to 3 while losing only $20 \%$ of the signal. The trigger efficiency for events passing these selection cuts is $99 \%$.

Table 2-9 lists the Higgs production cross section $\left(\sigma_{H}\right)$ and the cross sections after event selection cuts $1-4$ and 1-5 together with the corresponding background rates at various masses. The largest backgrounds are the fake $\gamma \gamma$ pairs from $t \bar{t}$ production and the QED radiation $Z \gamma \rightarrow \ell^{+} \ell^{-} \gamma \gamma$. A Z-mass veto improves the rejection of the latter background, but it does not improve the significance. Figure 2-22 shows the $\gamma \gamma$ invariant mass spectrum collected in $30 \mathrm{fb}^{-1}$ with Higgs signals of $80,100,120$ and $140 \mathrm{GeV}$ superimposed on the sum of all backgrounds.

Table 2-9. Signal and background for $H(i \pi / W) \rightarrow y_{\gamma} \ell X$.

\begin{tabular}{|c|c|c|c|c|c|}
\hline \multicolumn{6}{|c|}{$H(t \tilde{H} / m) \rightarrow \gamma_{\gamma} \ell X$ Signal $(\mathrm{fb})$} \\
\hline$M_{H}(\mathrm{GeV})$ & 80 & 90 & 100 & 120 & 140 \\
\hline$\sigma_{H}$ & 8.4 & 8.0 & 7.8 & 6.9 & 4.2 \\
\hline After cuts $1-4$ & 0.95 & 1.0 & 1.0 & 0.82 & 0.61 \\
\hline Atter cuts 1-5 & 0.74 & 0.82 & 0.86 & 0.74 & 0.58 \\
\hline \multicolumn{6}{|c|}{ Background Rates after cuts $1-5$ (fb/GeV) } \\
\hline$M_{r}(\mathrm{GeV})$ & 80 & 90 & 100 & 120 & 140 \\
\hline$\pi \gamma$ & .019 & .018 & .018 & .017 & .016 \\
\hline Wry & .010 & .010 & .009 & .008 & .008 \\
\hline$w_{\gamma} \rightarrow e_{r \gamma}$ & 027 & .025 & .023 & .020 & .016 \\
\hline$z y \rightarrow \ell^{+} \ell^{-} \gamma \gamma$ & .068 & .068 & .064 & .046 & .027 \\
\hline$Z \gamma \rightarrow \theta^{\prime \prime} \gamma^{\prime \prime} \gamma$ & .031 & .024 & .019 & .012 & .008 \\
\hline if & .044 & .044 & .041 & .029 & .016 \\
\hline Total & .20 & 19 & 17 & .13 & .09 \\
\hline
\end{tabular}

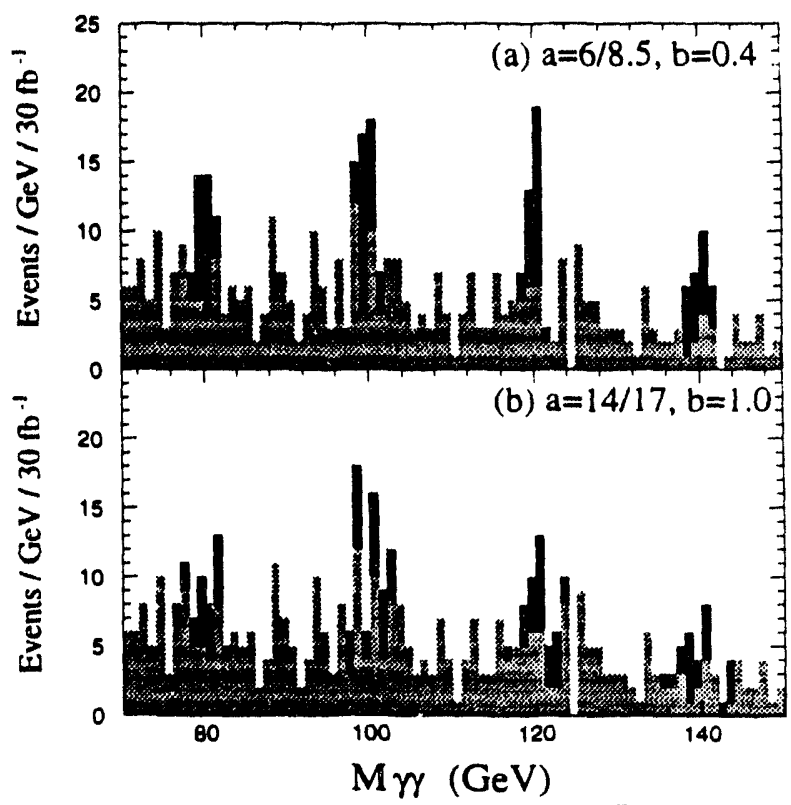

FIG. 2-22. Two-photon mass peaks for $H(H \bar{t} / W) \rightarrow$ errX (black area) over sum of all backgrounds (gray area), for an integrated luminosity of $30 \mathrm{fb}^{-1}$ and $M_{H}=$ $80,100,120$ and $140 \mathrm{GeV}$. 


\section{$H(t \bar{t} / W) \rightarrow \gamma \gamma \ell X$ Significance}

Table 2-10 lists the significance calculated according to Eq. (2.3.4) for the $H(t \bar{t} / W) \rightarrow \gamma \gamma \ell X$ search with integrated luminosity of 10 and $30 \mathrm{fb}^{-1}$. Although the numbers of signal and background events are small, this process provides higher significance than the inclusive $H \rightarrow \gamma \gamma$ at very low masses.

Table 2-10. Significance for $H(t) / W \rightarrow \ell_{y y} X$ for various masses and integrated luminosities. Signal and background are integrated over $\pm 2 \Delta M_{H}$.

\begin{tabular}{lccccc}
\hline$M_{H}(\mathrm{GeV})$ & 80 & 90 & 100 & 120 & 140 \\
\hline$\Delta M_{H}(\mathrm{GeV})$ & 0.66 & 0.69 & 0.77 & 0.84 & 0.91 \\
$\sigma_{H}(\mathrm{fb})$ & 0.74 & 0.82 & 0.86 & 0.74 & 0.58 \\
$\begin{array}{l}\text { Background } \\
\text { (fb/GeV) }\end{array}$ & 0.20 & 0.19 & 0.17 & 0.13 & 0.09 \\
\hline \multicolumn{6}{c}{} \\
\hline $10 \mathrm{fb}^{-1}$ & 2.5 & 2.8 & 2.9 & 2.7 & 2.3 \\
$30 \mathrm{fb}^{-1}$ & 4.5 & 4.9 & 5.1 & 4.8 & 4.3 \\
\hline
\end{tabular}

Figure 2-23 compares the significance in these channels as a function of integrated luminosity for various masses for the GEM detector with that for a calorimeter with degraded energy resolution but the same jet rejection. The upper edge of the band corresponds to the significance listed in Table 2-10. The lower edge of the band corresponds to a doubling of the background with no change in the signal. No $K$ factors are included in these results. Since there are so few signal events in these channels, GEM's high mass resolution is essential. The combination of this channel and the inclusive $H \rightarrow \gamma \gamma$ provides a much more robust search for low mass Higgs bosons than either one alone.

\subsection{4 $H \rightarrow Z^{*} \rightarrow \ell^{+} \ell^{-} \ell^{+} \ell^{-}$Search for $140 \mathrm{GeV}<M_{H}<2 M_{Z}$}

The $H \rightarrow Z Z \rightarrow \ell^{+} \ell^{-} \ell^{+} \ell^{-}$decay provides the cleanest Higgs signal at the SSC. Because of the four isolated leptons in the final state, most of the QCD background can be rejected by an isolation cut. For a Higgs mass larger than $2 M_{Z}$, both $\ell^{+} \ell^{-}$pairs have an invariant mass of $M_{Z}$, so the $Z$ mass constraint can also be used to reject background. If the Higgs mass is less than $2 M_{Z}$, one (or both) $Z$-bosons may be off the mass shell. In either case, high resolution of the EM calorimeter and the muon

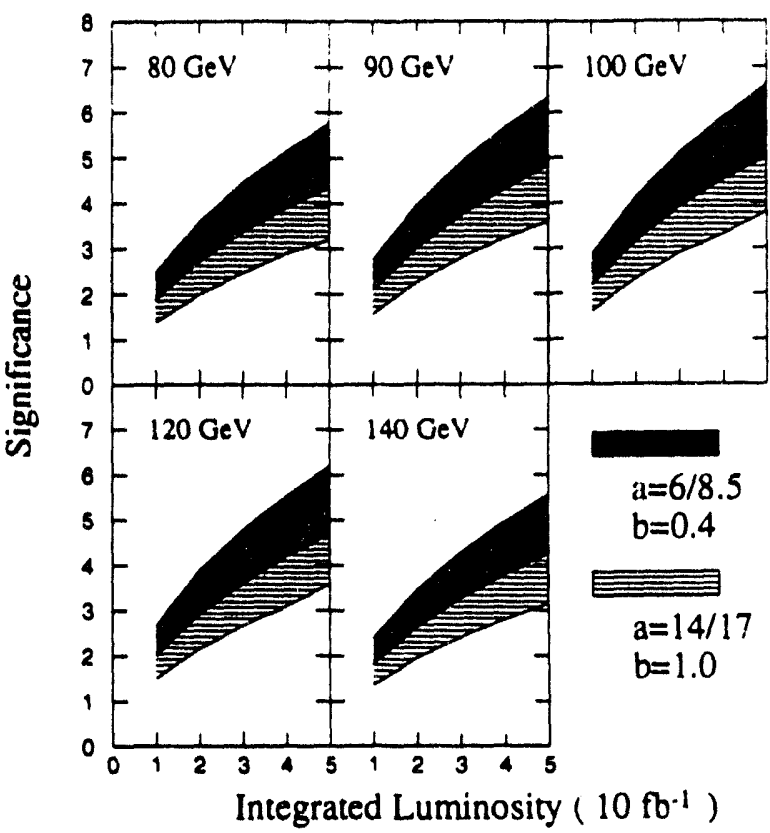

FIG. 2-23. The significance of $H(t \pi / W) \rightarrow \ell_{y} X$ search as a function of the integrated luminosity for $M_{H}=80$, 100,120 and $140 \mathrm{GeV}$ for the GEM detector and a more conventional sampling calorimeter with $a=14 / 17 \%$ and $b=1 \%$. The upper edge of the bands corresponds to the analysis presented here, while the lower edge corresponds to a doubling the background.

system are important for rejecting backgrounds and precise reconstruction of the Higgs mass peak.

The background processes considered are:

- $\mathrm{ZZ} / \mathrm{ZZ}^{*} \rightarrow \ell^{+} \ell^{-} \ell^{+} \ell^{-}$.

- $Q \bar{Q} Z \rightarrow \ell^{+} \ell^{-} \ell^{+} \ell^{-}+X$, where $Q=b$ or $t$.

- $t \bar{t} \rightarrow W^{+} b W^{-}-\bar{b}$, in which the two $W$-bosons decay semileptonically and the $b$-jets fake an isolated lepton.

The $Z Z / Z Z^{*} \rightarrow \ell^{+} \ell^{-} \ell^{+} \ell^{-}$background is irreducible. Since the cross section of $g g \rightarrow Z Z^{*}$ is not yet available in either PYTHIA or ISAJET, its contribution was accounted for by multiplying the contribution of $q \bar{q} \rightarrow Z Z^{*}$ by $1.65 .^{39}$

The following cuts were used to reject the other backgrounds:

1. $\left|\eta^{\ell}\right|<2.5$ and $p_{T}^{\ell}>10 \mathrm{GeV}$. For electrons, the region $1.01<|\eta|<1.16$ was excluded.

2. Lepton isolation with $R=0.35$ and $E_{T}^{\text {cut }}=$ $5 \mathrm{GeV}$; see Section 2.3.1.

3. Lepton identification and track matching. 
4. $10 \mathrm{GeV} \leq M_{\ell \ell}^{(n)} \leq 100 \mathrm{GeV}$ and $70 \mathrm{GeV} \leq$ $M_{\ell \ell}^{(h)} \leq 100 \mathrm{GeV}$ to suppress the continuum background, where $M_{\ell \ell}^{(h)}$ and $M_{\ell \ell}^{(h)}$ are the low and high invariant masses of the two $\ell^{+} \ell^{-}$pairs.

The isolation, $p_{T}$, and mass cuts help reject the heavy flavor background. The trigger efficiency for events passing these selection cuts is higher than $98 \%$ for the four-electron mode and $99 \%$ for the other two modes.

Table 2-11 shows the cross section after event selections $\left(\sigma_{\text {accep }}\right)$ and the corresponding background rate for the various $H \rightarrow \ell^{+} \ell^{-} \ell^{+} \ell^{-}$ channels. The integrated luminosity is $10 \mathrm{fb}^{-1}$. While the mass resolution is quite good in the four-electron channel, it is not as good in the four-muon channel. The reason for this degraded resolution is multiple scattering of the relatively low-energy muons in the second superlayer. As discussed in Sections 4.1.5 and 2.8, studies are underway with the goal of reducing the material in the muon system to a level that will give roughly the same resolutions in the $4 \mu$ and $4 e$ decay channels of the intermediate mass Higgs.

Table 2-11. Signal and Background for $H \rightarrow Z^{\circ} \rightarrow$ $\mu^{+} \mu^{-} \mu^{+} \mu^{-}$and $H \rightarrow Z^{*} \rightarrow \ell^{+} \ell^{-} \ell^{+} \ell^{-}$for an integrated luminosity of $10 \mathrm{fb}^{-1}$. The signal cross section after cuts is $\sigma_{\text {accep. }}$

\begin{tabular}{|c|c|c|c|c|c|}
\hline$M_{H}(\mathrm{GeV})$ & 140 & 150 & 160 & 170 & 180 \\
\hline \multicolumn{6}{|c|}{$H \rightarrow \theta^{+} \theta^{-} e^{+} \theta^{-}$} \\
\hline$\Delta M_{H}(\mathrm{GeV})$ & 1.05 & 1.06 & 1.13 & 1.23 & 1.33 \\
\hline$\sigma_{\mathrm{accep}}$ & 1.2 & 1.7 & .86 & .62 & 1.6 \\
\hline $\begin{array}{l}\text { Background } \\
\text { (fb/GeV) }\end{array}$ & .025 & .025 & .025 & .025 & .040 \\
\hline \multicolumn{6}{|c|}{$H \rightarrow \mu^{+} \mu^{-} \mu^{+} \mu^{-}$} \\
\hline$\Delta M_{H}(\mathrm{GeV})$ & 1.59 & 1.62 & 1.73 & 1.84 & 2.22 \\
\hline$\sigma_{\text {accep }}(\mathrm{fb})$ & .81 & 1.1 & .56 & .36 & .92 \\
\hline $\begin{array}{l}\text { Background } \\
\text { (fb/GeV) }\end{array}$ & .016 & .016 & .016 & .016 & .026 \\
\hline \multicolumn{6}{|c|}{$H \rightarrow \theta^{-} \theta^{-} \mu^{+} \mu^{-}$} \\
\hline$\Delta M_{H}$ & 1.36 & 1.46 & 1.56 & 1.71 & 1.77 \\
\hline$\sigma_{\mathrm{accep}}(\mathrm{fb})$ & 1.9 & 2.6 & 1.4 & 0.89 & 2.4 \\
\hline $\begin{array}{l}\text { Background } \\
\text { (fb/GeV) }\end{array}$ & .038 & .038 & .038 & .038 & .062 \\
\hline \multicolumn{6}{|c|}{$H \rightarrow \ell^{-} \ell^{-} \ell^{+} \ell^{-}$} \\
\hline Significance & 11 & 13 & 8.1 & 5.7 & 10 \\
\hline
\end{tabular}

The significance for all channels combined is also listed in Table 2-11. Using all the four-lepton channels. GEM can discover a Higgs boson in this mass range with an integrated luminosity of $10 \mathrm{fb}^{-1}$. The most difficult mass $15170 \mathrm{GeV}$ where the signal is only 5.7o. Figure $2-24$ shows the $e^{+} e^{-} e^{+} e^{-}, \mu^{+} \mu^{-} \mu^{+} \mu^{-}$, and $\ell^{+} \ell^{-} \ell^{+} \ell^{-}$invariant mass spectra collected in $30 \mathrm{fb}^{-1}$ for $M_{H}=140,150,160,170$ and $180 \mathrm{GeV}$ superimposed on the sum of all backgrounds. The increased integrated luminosity makes even the peak at $170 \mathrm{GeV}$ unambiguous.

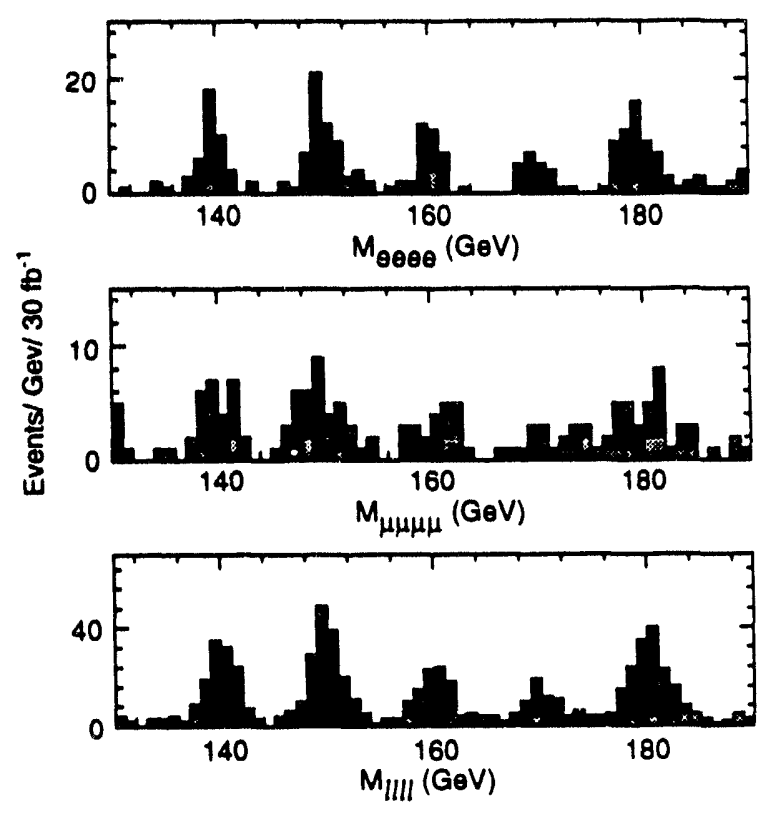

FIG. 2-24. Higgs mass peaks for $H \rightarrow Z Z^{\circ} \rightarrow$ $\ell-\ell-\ell+\ell-$ over the sum of all backgrounds plotted for an integrated luminosity of $30 \mathrm{fb}^{-1}$, and $M_{H}=140,150,160$ and $170 \mathrm{GeV}$. (a) Four electrons. (b) Four muons. (c) All leptons.

\subsection{5 $H \rightarrow Z Z \rightarrow \ell^{+} \ell^{-}-\ell^{+} \ell^{-}$Search for $2 M_{Z}<M_{H}<800 \mathrm{GeV}$}

For a heavy Higgs decaying into $\ell^{+} \ell^{-} \ell^{+} \ell^{-}$ the signal and the $Z Z$ background were generated with PYTHIA. All other backgrounds are negligible. The event selection cuts were taken to be:

1. $\left|\eta^{\ell}\right|<2.5$ and $p_{T}^{\ell}>10 \mathrm{GeV}$.

2. Lepton isolation with $R=0.3$ and $E_{T}^{\text {cut }}=$ $5 \mathrm{GeV}$.

3. Lepton identification and track matching.

4. At least one $Z$ with $p_{T}>\frac{1}{4} \sqrt{M_{Z Z}^{2}-4 M_{Z}^{2}}$

5. $\left|M_{\ell \ell}-M_{Z}\right|<10 \mathrm{GeV}$ for both lepton pairs. 
Cuts $1,2,3$, and 5 are self-explanatory. If the transverse momentum of the $Z Z$ system is neglected, then cut 4 requires $\sin \theta^{*}>0.5$ and so reduces the more peripheral $q \bar{q} \rightarrow Z Z$ background. The trigger efficiency for events passing these selection cuts is higher than $99 \%$.

Table 2-12 shows the production cross section $\left(\sigma_{H}\right)$ and the cross section after event selection cuts $\left(\sigma_{\text {accep }}\right)$ for $M_{H}=200,400,600$ and $800 \mathrm{GeV}$. The rate of the $Z Z$ background is also listed in the table. The combined significance is also listed for an integrated luminosity of $10 \mathrm{fb}^{-1}$. For such a data sample, discovery of heavy Higgs bosons in the $\ell^{+} \ell-\ell^{+} \ell-$ modes will be possible for all masses below about $700 \mathrm{GeV}$. For the very heavy $800 \mathrm{GeV}$ Higgs boson, the low rate can be increased with the muon acceptance improvements discussed in Chapter 4 . In any case, one would seek confirmation of these four-lepton signals in other channels, as we discuss next. We also expect that GEM's capabilities at ultrahigh luminosity (see Section 2.6) will permit the discovery of a very heavy Higgs in the 4-lepton channel in about one year with a data sample of order $100 \mathrm{fb}^{-1}$. This study is underway.

Table 2-12. Signal and Background for $H \rightarrow Z Z \rightarrow$ $\ell^{+} \ell^{-} \ell^{+} \ell^{-}$for an integrated luminosity of $10 \mathrm{fb}^{-1}$.

\begin{tabular}{|c|c|c|c|c|}
\hline$M_{H}(\mathrm{GeV})$ & 200 & 400 & 600 & 800 \\
\hline \multicolumn{5}{|c|}{ Signal (tb) } \\
\hline$\sigma_{H}$ & 85 & 56 & 16 & 5.3 \\
\hline $\begin{array}{l}\text { Mass Bin } \\
(\mathrm{GeV})\end{array}$ & \pm 4.7 & $350-450$ & $500-800$ & $600-1200$ \\
\hline$\sigma_{\text {accep }}$ & 21 & 14 & 4.3 & 1.5 \\
\hline \multicolumn{5}{|c|}{ Background (fb) } \\
\hline $2 Z$ & 3.0 & 2.3 & $1.0^{\circ}$ & .6 \\
\hline \multicolumn{5}{|c|}{ Significance } \\
\hline Significance & 38 & 28 & 9.7 & 4.7 \\
\hline
\end{tabular}

Figure 2-25 shows the $\ell^{+} \ell^{-} \ell^{+} \ell^{-}$invariant mass spectra collected in $10 \mathrm{fb}^{-1}$ for $M_{H}=200$, 400,600 and $800 \mathrm{GeV}$ superimposed on the background. Figure 2-26 shows the spectra collected in $30 \mathrm{fb}^{-1}$ for $M_{H}=600$ and $800 \mathrm{GeV}$. The statistical significance is $4.7 \sigma$ in $10 \mathrm{fb}^{-1}$. There is also a systematic uncertainty caused by lack of knowledge of the shape of the background. This is discussed at the end of the following subsection.
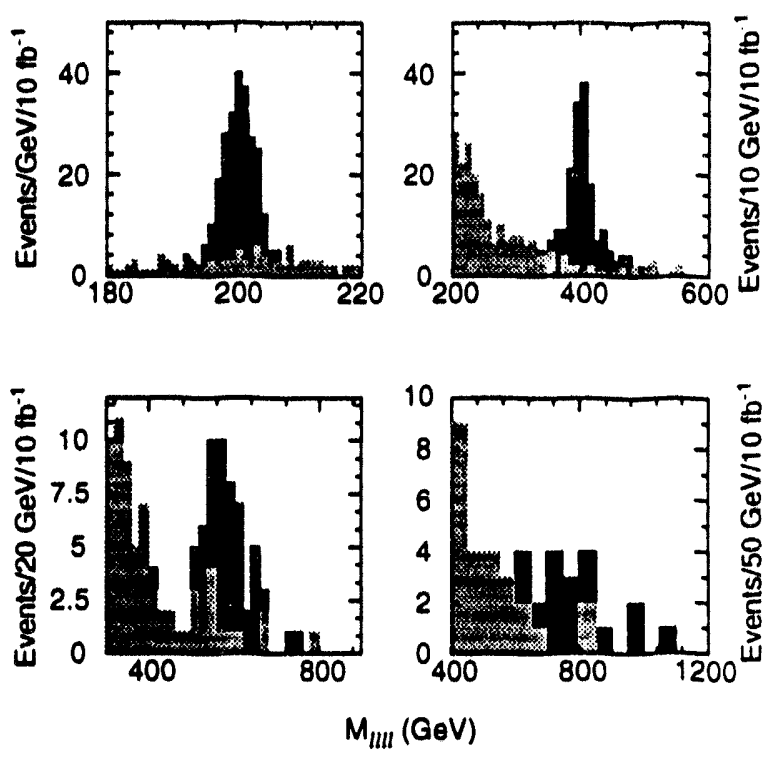

FIG. 2-25. Higgs mass peaks and background for $H \rightarrow Z Z \rightarrow \ell+\ell-\ell-\ell-$ for an integrated luminosity of $10 \mathrm{fb}^{-1}$. (a) $M_{H}=200 \mathrm{GeV}$. (b) $400 \mathrm{GeV}$. (c) $600 \mathrm{GeV}$.

(d) $800 \mathrm{GeV}$.

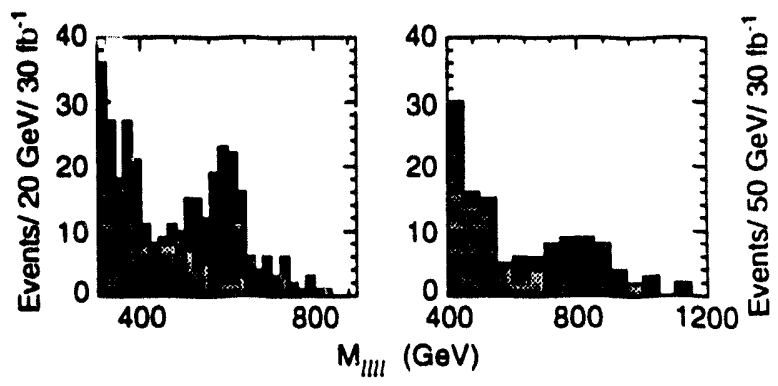

FIG. 2-26. Higgs mass peaks and background for $H \rightarrow Z Z \rightarrow \ell^{+} \ell^{-} \ell^{+} \ell^{-}$for an integrated luminosity of $30 \mathrm{fb}^{-1}$ using gaussian statistics to fluctuate the number of events. (a) $M_{H}=600 \mathrm{GeV}$. (b) $800 \mathrm{GeV}$.

\subsection{6 $H \rightarrow Z Z \rightarrow \ell^{+} \ell^{-}-v \bar{\nu}$ Search for $M_{H}=800 \mathrm{GeV}$}

The branching ratio for $H \rightarrow Z Z \rightarrow \ell^{+} \ell^{-} v \bar{v}$ is six times that for $H \rightarrow Z Z \rightarrow \ell^{+} \ell^{-} \ell^{+} \ell^{-}$. Since one $Z$ decays to $v \bar{w}$, the signature is a high- $p_{T}$ $Z \rightarrow \ell^{+} \ell^{-}$plus missing energy, and the mass of the Higgs boson cannot be reconstructed. The total signal cross section is $32 \mathrm{fb}$.

The following backgrounds were considered: 
- $q q / 88 \rightarrow Z Z$; the cross section is $73 \mathrm{fb}$ for $p_{T}^{Z}>150 \mathrm{GeV}$.

- $q \bar{q} \rightarrow Z_{g}$ and $q g \rightarrow Z q$, with $Z \rightarrow \ell^{+} \ell^{-} ;$the cross section is $66 \mathrm{pb}$ for $p_{T}^{Z}>150 \mathrm{GeV}$ before the $\boldsymbol{E}_{T}$ cut below.

- $q \bar{q}, 88 \rightarrow t \bar{f}$, with $t \rightarrow b \ell v ;$ the cross section is $380 \mathrm{pb}$ before cuts.

Events with an $e^{+} e^{-}$or $\mu^{+} \mu^{-}$pair were selected as follows:

1. $\left|\eta^{\ell}\right|<2.5$ and $p_{T}^{\ell}>20 \mathrm{GeV}$ for each lepton.

2. Lepton isolation with $R=0.3$ and $E_{T}^{\text {cut }}=$ $5 \mathrm{GeV}$.

3. Lepton identification and track matching.

4. $\mid M_{\text {el }}-M_{Z} d<10 \mathrm{GeV}$.

5. $E_{T}^{Z}>250 \mathrm{GeV}$.

6. $E_{T}>250 \mathrm{GeV}$.

These cuts were derived from the distributions shown in Figure 2-27. The trigger efficiency for events passing these selection cuts is higher than $99 \%$. Table 2-13 lists the cross sections for Higgs production after cuts $1-4,1-5$, and $1-6$, for the signal and background in the $e^{+} e^{-} \nu \bar{V}$ and $\mu^{+} \mu^{-} \boldsymbol{\nu} \bar{\nu}$ channels.

Figure 2-28 shows the reconstructed transverse mass, $M_{T}^{2}=2 E_{T}^{Z} E_{T}(1-\cos \Delta \phi)$, where $E_{T}^{Z}$ is the transverse energy of the $Z$ and $\Delta \phi$ is the azimuthal angle between the direction of the $Z$-boson and the $\boldsymbol{F}_{T}$ vector. This distribution is not sensitive to the degradation of the $F_{T}$ resolution that results from adding to the calorimeter response a $1 \%$ nongaussian tail with twice the normal width (see Section 2.2.3). There are 105 signal events over a total background of 91 for an integrated luminosity of $10 \mathrm{fb}^{-1}$. Since the signal and background distributions are similarly shaped and the signal to background ratio is only $1.15: 1$, this cannot be regarded as a convincing discovery channel. A knowledge of the $Z Z$ continuum background to $-25 \%$ would serve to give a $5 \sigma$ systematic-limited significance. This knowledge of the $Z Z$ background should be achievable by comparison with $W Z$ and $Z+$ jets production. The statistical $4.7 \sigma$ significance of the four-lepton signal is reduced to $4.2 \sigma$ by the same background uncertainty. Combining the $\ell \ell \ell \ell$ and $\ell \ell \bar{v}$ channels with gaussian statistics gives a 6.60 signal for $10 \mathrm{fb}^{-1}$
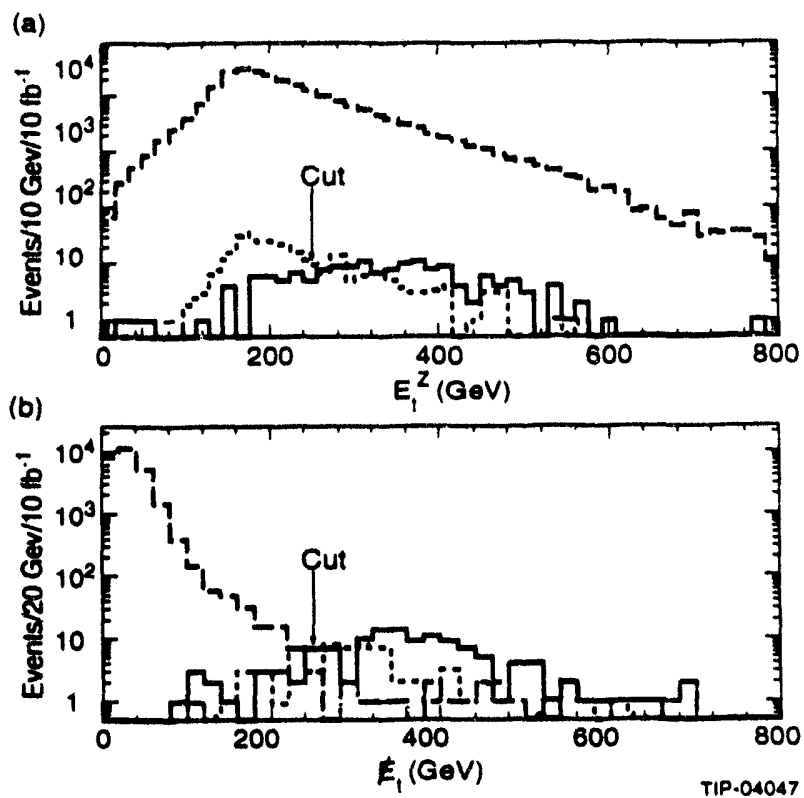

FIG. 2-27. Distributions for an $800 \mathrm{GeV} H \rightarrow \ell^{+} \ell^{-} v \nabla$ signal (solid), $Z Z$ background (dots), and $Z+$ jets background (dash) for $10 \mathrm{fb}^{-1}$. The $f$ background is small. (a) Transverse energy of the reconstructed $Z$. (b) Missing transverse energy $\left(\Psi_{T}\right)$.

Table 2-13. Signal and Background (fb) for $H \rightarrow$ $\ell+\ell-v \nabla$

\begin{tabular}{lcccc}
\hline \multicolumn{5}{c}{$e^{-} e^{-\nu \nu}$ Channel } \\
\hline & Signal & $Z Z$ & $Z+j e t s$ & $t f$ \\
\hline$\sigma_{H}$ & 16.0 & 36.7 & $3.3 \times 10^{4}$ & $2.0 \times 10^{5}$ \\
After cuts 1-4 & 9.5 & 13.8 & $1.2 \times 10^{4}$ & 53.5 \\
After cuts 1-5 & 7.4 & 4.2 & $3.1 \times 10^{3}$ & 0.36 \\
After cuts 1-6 & 6.2 & 3.2 & 2.2 & 0.0 \\
\hline & $\mu^{-} \mu^{-} \nu \bar{V}$ Channel & \\
\hline & Signal & $Z Z$ & $Z+j e t s$ & $t 7$ \\
\hline After cuts 1-4 & 6.5 & 9.4 & $8.1 \times 10^{3}$ & 36.2 \\
After cuts 1-5 & 5.0 & 2.9 & $2.1 \times 10^{3}$ & 0.24 \\
After cuts 1-6 & 4.2 & 2.2 & 1.5 & 0.0 \\
\hline \hline
\end{tabular}




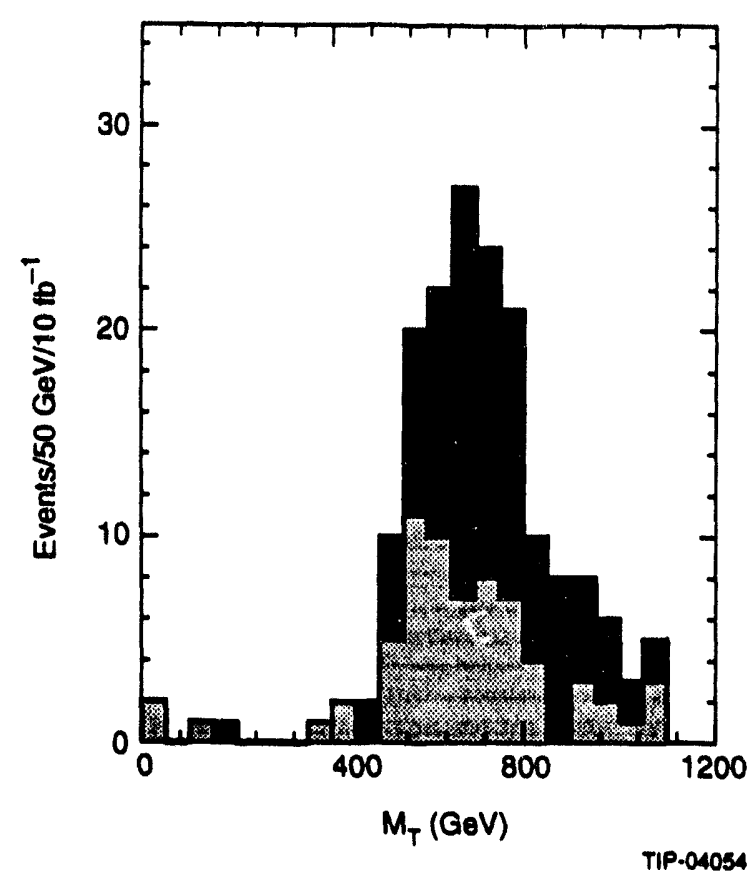

FIG. 2-28. Transverse mass $M_{T}$ of an $800 \mathrm{GeV}$ Higgs signal for $H \rightarrow Z Z \rightarrow \ell^{+} \ell^{-} \nu \nu$ (black area) and sum of all backgrounds (gray area), obtained for an integrated luminosity of $10 \mathrm{fb}^{-1}$.

\subsection{7 $H \rightarrow \mathrm{ZZ} \rightarrow \ell^{+} \ell^{-}-j j$ Search for $M_{H}=800 \mathrm{GeV}$}

The branching ratio for $H \rightarrow Z Z \rightarrow \ell^{+} \ell^{-} j j$ for $\ell=e$ and $\mu$ is approximately 20 times higher than that into all the $\ell^{+} \ell^{-} \ell^{+} \ell^{-}$modes. For $M_{H}=800 \mathrm{GeV}$, the cross section is $110 \mathrm{fb}$. The signal-to-background ratio, however, is much worse than in the four-lepton channel because of the large background from $Z+$ jets production. In addition, since the width of an $800 \mathrm{GeV}$ Higgs is $270 \mathrm{GeV}$, the signal will be seen only as a broad excess of $Z Z$ pairs over the background.

The following backgrounds were studied in this analysis:

- $Z W$ or $Z Z$ with one $Z \rightarrow e^{+} e^{-}$or $\mu \mu$.

- $Z+$ jets with $Z \rightarrow e^{+} e^{-}$or $\mu^{+} \mu^{-}$.

- if with $t \rightarrow b W$ and $W \rightarrow e v$ or $\mu \nu$.

The irreducible $Z Z$ background has a production cross section of $130 \mathrm{fb}$, for $p_{T}^{Z}>200 \mathrm{GeV}$. The $Z+$ jets background has a much larger rate of $27 \mathrm{pb}$ for $p_{T}^{Z}>200 \mathrm{GeV}$. The Z-mass constraint and high $p_{T}$ of leptons and jets was used to reduce this background. The possibility of tagging forward jets and vetoing central jets to enhance the signal-tonoise was studied and found not to be effective. The cross section for the $t \bar{t}$ background with semileptonIc decays to $e^{+} e^{-}+X$ and $\mu^{+} \mu^{-}+X$ is $380 \mathrm{pb}$ before the $Z$-mass constraint is imposed. A sample of $1.5 \times 10^{6}$ events was generated for this background. This background was largely eliminated by $p_{T}$ and isolation cuts and the Z-mass constraint.

Leptons were selected as follows:

1. $\left|\eta^{\ell}\right|<2.5$ and $p_{T}^{\ell}>70 \mathrm{GeV}$ for each lepton.

2. Lepton isolation with $R=0.3$ and $E_{T}^{\text {cut }}=$ $5 \mathrm{GeV}$.

3. Lepton identification and track matching.

4. $\left|M_{\ell \ell}-M_{Z}\right|<10 \mathrm{GeV}$.

5. $p_{T}^{\ell \ell}>230 \mathrm{GeV}$.

The hadronically-decaying Z-boson was reconstructed using the following algorithm:

1. Find all jets at $|\eta|<2.5$ using a large clustering cone with $R=0.9$.

2. Find all jets with a small cone, $R=0.3$, and match these narrow jets with those found using the larger cone radius.

3. Require the highest- $p_{T}$ jet found with $R=0.9$ to have $p_{T}>250 \mathrm{GeV}$ and to be composed of two jets found with $R=0.3$, each having $p_{T}>80 \mathrm{GeV}$.

4. Reconstruct the mass $M_{j j}$ of highest- $p_{T}$ dijet with $R=0.9$, and require that $\left|M_{i j}-M_{z}\right|<$ $15 \mathrm{GeV}$.

The trigger efficiency for events passing all these selection cuts is higher than $99 \%$.

Figure 2-29 shows the reconstructed dijet mass $M_{j j}$ for the signal and backgrounds, and the dijet mass cut is indicated. Note that the background has been multiplied by 0.2 . The mass resolution is about $9 \mathrm{GeV}$. Table 2-14 shows the cross sections for signal and backgrounds after the lepton and jet cuts for $600<M_{\ell \ell j j}<1000 \mathrm{GeV}$.

The invariant mass $M_{\ell \ell_{j j}}$ is shown in Figure 2-30. The signal and background events passing all these cuts are 110 and 430 respectively for $10 \mathrm{fb}^{-1}$. The signal and background have a very similar shape, with no recognizable peak in the mass spectrum. Since the signal is $25 \%$ of the background, 
the latter must be known to better than $5 \%$ to achieve a $5 \sigma$ significance. Thus, this mode cannot be regarded as a discovery channel for the Higgs. It may be used to provide further confirmation of the $\ell^{+} \ell^{-} \ell^{+} \ell^{-}$and $\ell^{+} \ell^{-} \bar{w}$ signals.

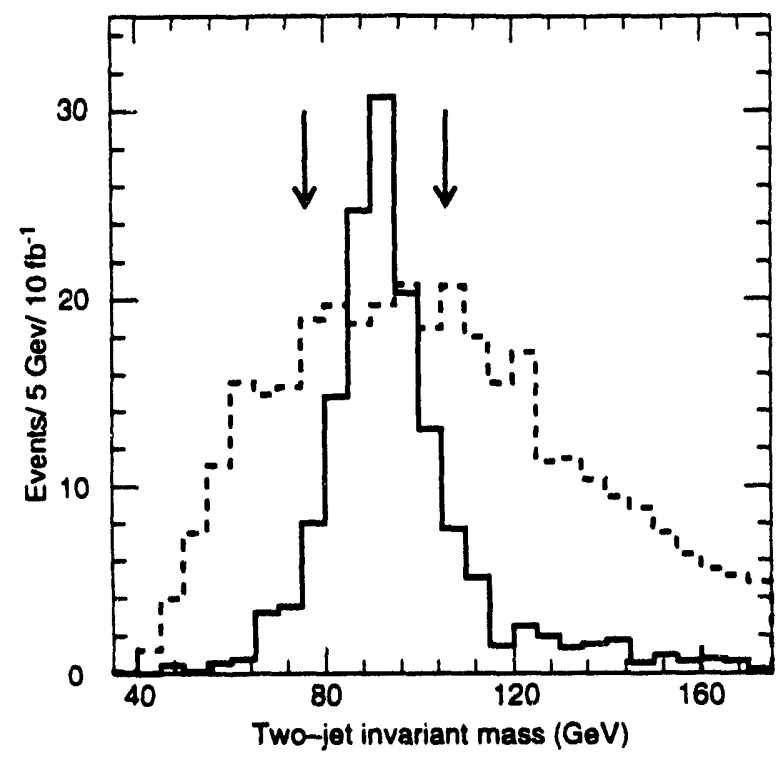

FIG. 2-29. Dijet invariant mass distribution for $H \rightarrow Z Z \rightarrow \ell^{-} \ell^{-} j j$ for $M H=800 \mathrm{GoV}$ (solid) and sum of all backgrounds (dashed), obtained for an integrated luminosity of $10 \mathrm{fb}^{-1}$. The background is multipiled by a factor of 0.2. to facilitate comparison of the shapes.

Table 2-14. Signal and background cross sections ( $f$ for $H \rightarrow Z Z \rightarrow \ell^{+} \ell^{-} j j$ for $10 \mathrm{fb}^{-1}$. The invariant me $M_{\ell e l j}$ signal region is 600 to $1000 \mathrm{GeV}$.

\begin{tabular}{lcccc}
\hline \hline Cut & Higgs & $t$ & $Z+$ jets & $\begin{array}{c}Z W / \\
Z Z\end{array}$ \\
\hline After lepton cuts & 41 & 130 & 3240 & 20 \\
After jet cuts & 12 & 0.5 & 65 & 1.5 \\
Within & 11 & 0.3 & 42 & 0.8 \\
$600<M_{\ell \ell j}<1000$ & & & & \\
\hline \hline
\end{tabular}

\subsection{HEAVY FLAVOR PHYSICS}

As we have emphasized, the characteristic energy of electroweak symmetry breaking lies in the SSC's domain, but the flavor symmetry scale may be near $1 \mathrm{TeV}$ or much higher. Nevertheless, searching for the origin of flavor symmetry and its breakdown at the SSC is as important as it is for electroweak symmetry breaking.

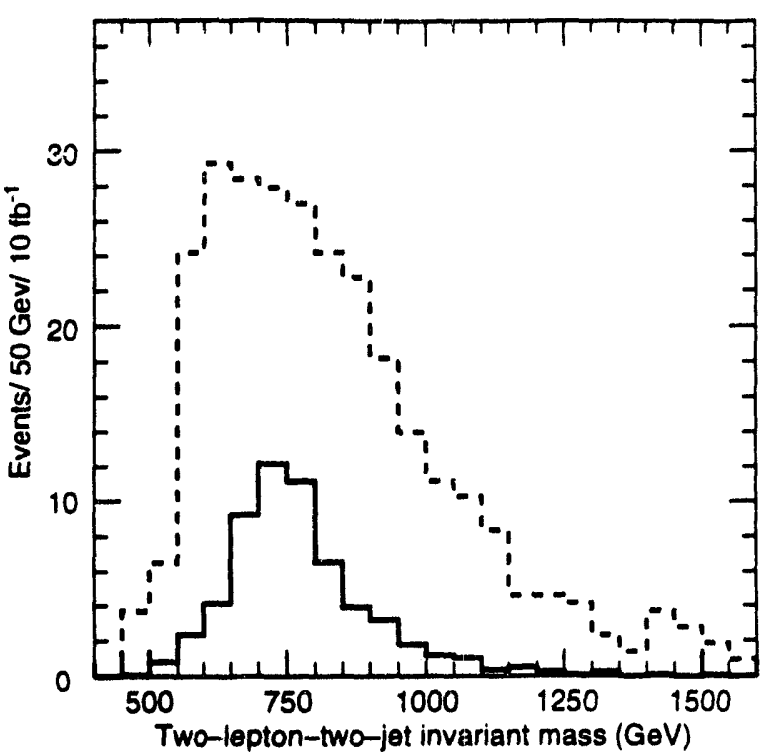

FIG. 2.30. Invariant mass distribution $M_{e \ell j}$ for an $800 \mathrm{GeV} H \rightarrow Z Z \rightarrow \ell^{+} \ell^{-} j j$ signal (solid) and sum of all backgrounds (dashed), obtained for an integrated luminosity of $10 \mathrm{fb}^{-1}$.

The top quark's very large mass, $m_{t}>$ $91 \mathrm{GeV},{ }^{8}$ is a dramatic example of flavor symmetry breaking. Thus, if other manifestations of flavor physics are accessible at SSC energies, the top quark will be an important key to finding them. If the top quark is so heavy that it is not accessible at the Tevatron Collider, it will be necessary to discover it the SSC. If charged scalar bosons, $H^{ \pm}$, exist, the

ays $t \rightarrow H^{+} b$ or $H^{+} \rightarrow t \bar{b}$ are expected to be jortant decay modes. As another example, many technicolor models contain a color-octet, spin-zero "technieta" boson, $\eta_{T}$, with mass in the range $300-500 \mathrm{GeV}$. The $\eta_{T}$ would be copiously produced via gluon fusion and is expected to decay into it pairs. ${ }^{40,7}$ At the same time, $t$-quark production processes can be serious backgrounds to new physics. A particularly difficult case was encountered in Section 2.3.3, where it was seen that isolated-photon backgrounds arising in it production were an important background to the $\bar{t} t H^{0} \rightarrow \ell^{ \pm} \gamma \gamma$ signal for production of an intermediate mass Higgs boson. Thus, it is essential to know as much as possible about the rate and other characterictics of $t$-quark production. Finally, the fact that $t$-production gives a pure sample of $W$-bosons, with $M_{W}$ and $m_{t}$ accurately known, will be useful for calibrating the calorimeters for jet energy measurements. For these reasons, top-quark physics is an essential part of the GEM physics 
program. This section discusses the following aspects of top physics:

1. Discovery and measurement of the mass of heavy top quarks $\left(m_{t} \approx 250 \mathrm{GeV}\right)$ pair-produced in the $\mathrm{QCD}$ processes $g g, \bar{q} q \rightarrow t \bar{t}$ and decaying via the standard mode $t \rightarrow W^{+} b$. In this case, one $t$-quark is tagged via an isolated electron or muon from $W$-decay plus a non-isolated muon, buried in a hadron jet, from the decay $b \rightarrow c \mu \bar{\nu}_{\mu}$. The top-quark mass is determined from the invariant mass distribution, $M_{\ell \mu}$, of the isolated lepton and the non-isolated muon. 3.41

2. Discovery and mass measurement for top quarks with the mass of $140 \mathrm{GeV}$ generally used in this report and with $m_{t}=250 \mathrm{GeV}$. Again, the standard $t \rightarrow W^{+} b$ decay mode is assumed and the $W$-boson and $t$-quark are observed in their hadronic decay modes, $W \rightarrow 2$ jets and $t \rightarrow 3$ jets. ${ }^{42}$

3. Discovery and study of a charged scalar $\mathrm{H}^{+}$produced in the decay of a heavy top quark, $t \rightarrow H^{+} b$. The scalar will be assumed to decay as $H^{+} \rightarrow \tau^{+} \nu_{\tau} \rightarrow$ one or three prongs, demonstrating GEM's ability to detect $\tau$-leptons in interesting physical contexts. ${ }^{43}$ For this search, one $t$-quark will be tagged in the standard mode $t \rightarrow W^{+} b \rightarrow$ isolated lepton.

\subsubsection{Heavy Top-Quark Detection and Mass Measurement via the $M_{\ell \mu}$ Distribution}

For this analysis, ISAJET 6.36 with the EHLQ1 distribution functions was used, to generate $60 \mathrm{~K} \bar{t}$ events, each, for $m_{t}=200,230,250,270$ and $300 \mathrm{GeV}$. The $t$-quarks were generated with $50 \mathrm{GeV}<p_{T}<1000 \mathrm{GeV}$. The $W^{ \pm}$were required to decay to $\ell^{ \pm} v_{\ell}(\ell=e, \mu)$. The $b$ from $t$-decay was forced to decay to $c \mu-\bar{v}_{\mu}$, while the $\bar{b}$ from $\bar{t}$ was allowed to decay into all possible channels. The signal events are those with one isolated electron, one isolated muon and one nonisolated muon. The isolated leptons were required to have opposite charges. Choosing isolated leptons of different flavors eliminates backgrounds involving $Z^{0} \rightarrow e^{+} e^{-}, \mu^{+} \mu^{-}$. The signal cross sections (assuming $B(W \rightarrow \ell v)=B\left(b \rightarrow \mu^{-}+X\right)=1 / 9$ ) for $m_{t}=200,250$ and $300 \mathrm{GeV}$ were found to be $19.9 \mathrm{pb}, 8.2 \mathrm{pb}$ and $4.0 \mathrm{pb}$, respectively. Monte Carlo event numbers were scaled to correspond to data taken in one year at nominal SSC luminosity; $10 \mathrm{fb}^{-1} \cdot 41$

The signal cross sections are much larger than all backgrounds, as discussed below. To optımize the separation between isolated leptons from $W$-decay and non-isolated muons from $b$-decay, the following cuts were imposed to select isolated electrons:

$$
\begin{aligned}
& \left|\eta_{e}\right|<2.4 ; \\
& \sum_{R=0.2} E_{T}(\mathrm{EC})>40 \mathrm{GeV} \\
& \sum_{R=0.4} E_{T}-\sum_{R=0.2} E_{T}(\mathrm{EC})<10 \mathrm{GeV} \\
& 0.8<E_{T}(R=0.2) / p_{T}(C T)<1.2 .
\end{aligned}
$$

The cuts for isolated muons were:

$$
\begin{aligned}
& \left|\eta_{\mu}\right|<2.4 \\
& p_{T}>40 \mathrm{GeV} \\
& \sum_{R=0.4} E_{T}-\sum_{R=0.1} E_{T}<2 \mathrm{GeV} .
\end{aligned}
$$

Non-isolated muons were required to satisfy:

$$
\begin{aligned}
& \left|\eta_{\mu}\right|<2.4 ; \\
& p_{T}>20 \mathrm{GeV} ; \\
& \sum_{R=0.4} E_{T}-\sum_{R=0.1} E_{T}>10 \mathrm{GeV} .
\end{aligned}
$$

Here, $E_{T}$ is the transverse energy in a cell of the full calorimeter; $E_{T}(\mathrm{EC})$ is the transverse energy in $\mathrm{EM}$ calorimeter cells; $p_{T}(\mathrm{CT})$ is the electron momentum as measured in the tracker. As usual, the sums are over $\eta-\phi$ cones of radius $R$.

The choice $R=0.2$ for the cone defining the electron energy is not critical. The electron energy measurement does not need to be very precise for this analysis. The $M_{e \mu}$ and $M_{\mu \mu}$ distributions turn out to be very similar for this choice of isolation parameters and not very sensitive to moderate changes. For the relatively low- $p_{T}$ muons in the top-quark decays, the muon energy may be c'srrected simply by adding an amount equal to the average energy loss for a given momentum and rapidity. 
Figure 2-31 shows the distributions of the isolation variable $\sum_{R=0.4} E_{T}-\sum_{R=0.1} E_{T}$ for muons, from $W$-bosons and $b$-quarks, in the production and decay of $250 \mathrm{GeV} t$-quarks. The cuts in Eqs. (2.4.2) and (2.4.3) clearly separate these two samples. For a top-mass of $250 \mathrm{GeV}$, the isolation cut $(2.4 .2)$ retained $78 \%$ of the muons from $W$-decay while rejecting, $99 \%$ of the muons from $b$-decay. The non-isolation cut in (2.4.3) accepted $89 \%$ of $b \rightarrow \mu+X$ and rejected $91 \%$ of $W \rightarrow \mu v_{\mu}$.

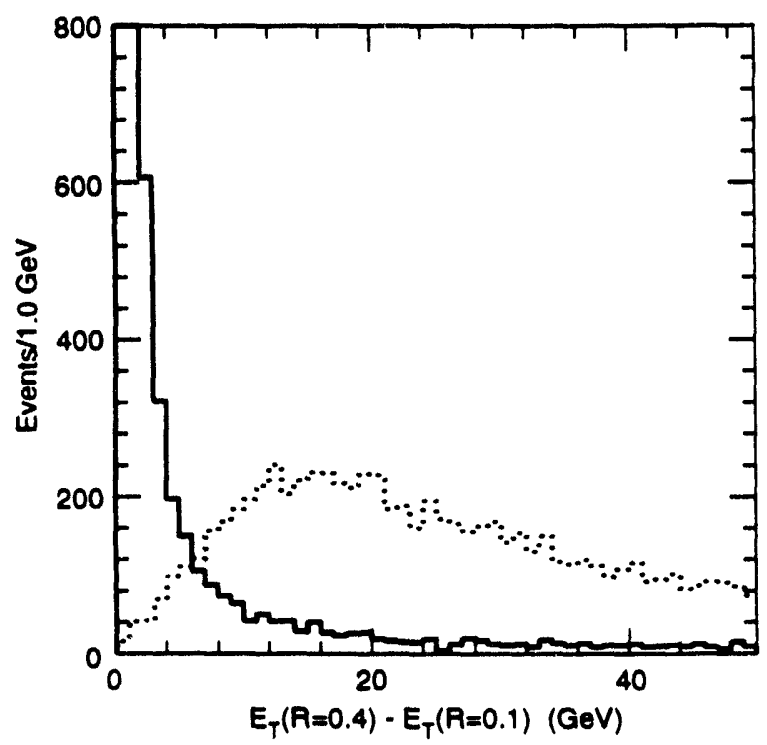

FIG. 2-31. Distribution of $\sum_{R=0.4} E_{T}-\sum_{R-0.1} E_{T}$ for muons from $W$-bosons (solid) and b-quarks (dotted) arising from the decay of $250 \mathrm{GeV}$-quarks. Arbitrary units are used on the vertical scale.

There was a $30 \%$ contamination of the $t \bar{t} \rightarrow e_{\text {isol }}^{ \pm} \mu_{\text {isol }}^{\mp}+\mu_{\text {non-isol }}$ signal due to non-isolated muons in the decay of $c$-quarks which, in tum, came from the decay of the "wrong" t-quark or from $g \rightarrow c \bar{c}$. This background was reduced to the $15 \%$ level by selecting events in which the isolated lepton and non-isolated muon of opposite sign are close to each other and, hence, more likely to be from the decay of the same t-quark. Figure 2-32 shows the azimuthal separation, $\delta \phi(\ell \mu)$, for $250 \mathrm{GeV}$ top-quark production. Events were kept that satisfied $\delta \phi(\ell \mu)<90^{\circ}$. The acceptance of this cut was found to be $67 \%, 56 \%$ and $49 \%$ for $m_{t}=200,250$ and $300 \mathrm{GeV}$.

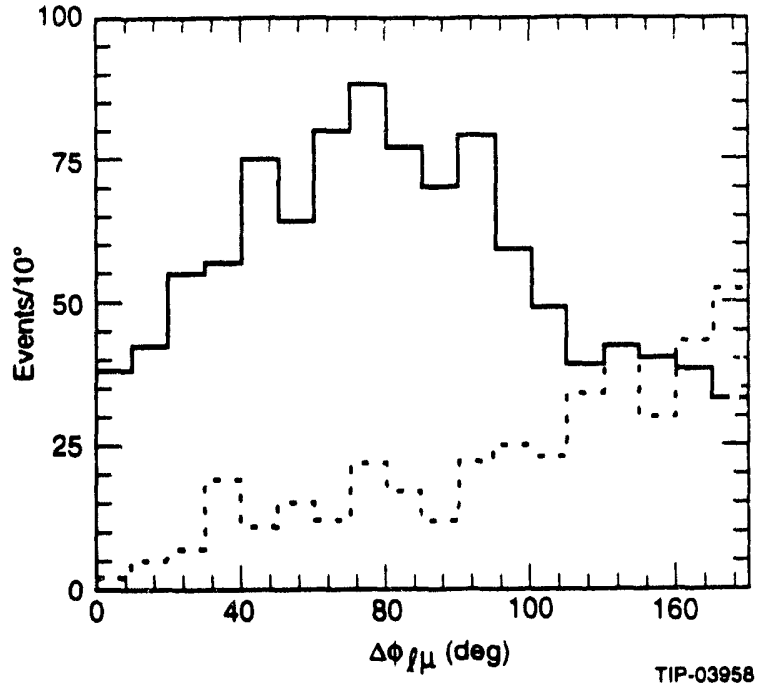

FIG. 2-32. Azimuthal separation, $\delta \phi(\ell \mu)$, for nonisolated muons occurring in the decays of $250 \mathrm{GeV}$ top quarks. Muons are from b-decay (solid) and c-decay (dotted). Arbitrary units are used on the vertical scale.

After trigger and reconstruction efficiencies, including those for non-isolated muons from $t$-decay, ${ }^{44}$ the overall acceptance for the $t \bar{t} \rightarrow$ $e_{\text {isol }}^{ \pm} \mu_{\text {non-isol }}^{\mp}$ events ranged from $1.1 \%$ for $m_{t}=200 \mathrm{GeV}$ to $1.8 \%$ for $m_{t}=300 \mathrm{GeV}$. The range of acceptance for the $\mu_{\text {isol }}^{ \pm} \mu_{\text {non-isol }}^{\mp}$ events was $1.1 \%$ to $1.7 \%$. These efficiencies are low because the analysis was designed to obtain a very clean sample. The total number of $\ell_{\text {isol }}^{ \pm} \mu_{\text {non-isol }}^{\mp}$ events expected with an integrated luminosity of $10 \mathrm{fb}^{-1}$ is 4500 . 2400 and 1400 for $m_{t}=200,250$ and $300 \mathrm{GeV}$.

Backgrounds to the $t \bar{t} \rightarrow e_{\text {isol }}^{ \pm} \mu_{\text {isol }}^{\mp} \mu_{\text {non-isol }}$ signal from production of $W^{ \pm}+$jets and $Z^{0}+$ jets were considered. The most important contributions come from $b$-quark jets and $Z^{0} \rightarrow \tau^{+} \tau^{-} \rightarrow e^{ \pm}+\mu^{\mp}+\boldsymbol{C}_{T}$. After the selections descrived above, the $W^{ \pm}+$jets background was found to be less than $9 \%$ of the signal for the $250 \mathrm{GeV}$ top quark; the $Z^{0}+$ jets background was negligible. ${ }^{41}$

The shapes of the $M_{e \mu}$ and $M_{\mu \mu}$ distributions are nearly identical. For $m_{t}=250 \mathrm{GeV}$, the average values of these distributions are $75.1 \mathrm{GeV}$ and $75.6 \mathrm{GeV}$, respectively. Thus, adding them together to form the $M_{\ell \mu}=M_{e \mu}+M_{\mu \mu}$ distribution adds statistical weight to the $m_{t}$ deter ination without introducing significant systematic error. The $M_{\ell \mu}$ distributions are plotted in Figure 2-33. 
The average value of the $M_{\ell \mu}$ distributions are 62.4. 75.4 and $88.0 \mathrm{GeV}$ for $m_{t}=200,250$ and $300 \mathrm{GeV}$. The mean value of $M_{\ell \mu}$ is plotted against $m_{t}$ in Figure 2-34. Note the very linear relationship. A statistical error of $2.3 \mathrm{GeV}$ on the mass of a $250 \mathrm{GeV}$ top quark is expected for $10 \mathrm{fb}^{-1}$ of data.

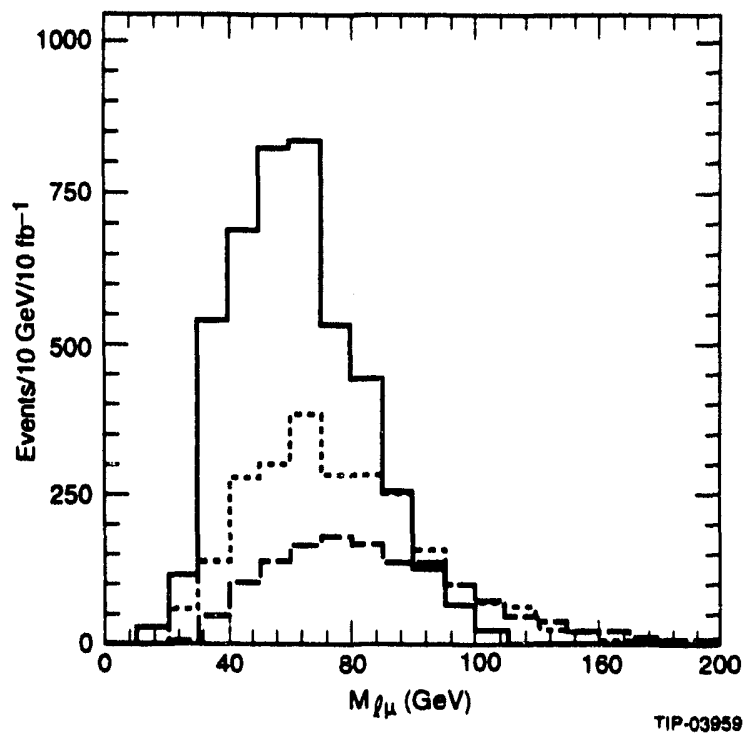

FIG. 2-33. $\quad M_{\ell \mu}=M_{e \mu}+M_{\mu \mu}$ distribution for an isolated lepton and a nonisolated muon for $m_{f}=200$ (solid), 250 (dotted), and $300 \mathrm{GeV}$ (dashed).

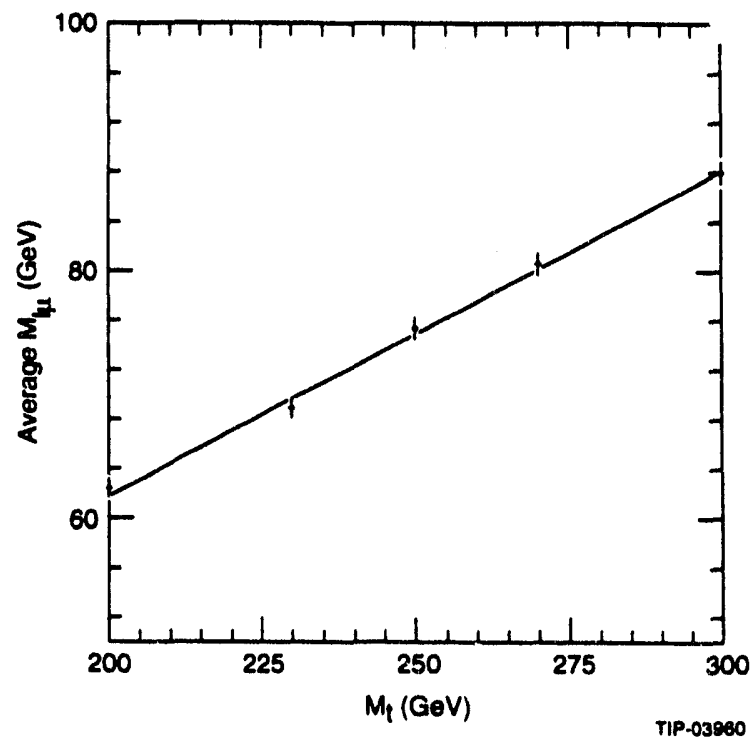

FIG. 2-34. Mean value of $M_{\ell \mu}$ vs. $m_{t}$

The systematic error in this method of determining $m_{l}$ arises from imperfect knowledge of the $b$-quark fragmentation function and of the $p_{T}$ distribution of the $t$-quarks. These affect the momen- tum distributions of the non-isolated muon and the isolated lepton, respectively. The effect of heavyquark fragmentation was studied by varying the parameter $\varepsilon$ in the Peterson fragmentation function from its nominal value of $\varepsilon=0.006 .^{9}$ The range considered, $1 \sigma=0.002$, led to a variation of $2.3 \mathrm{GeV}$ in the mass of a $250 \mathrm{GeV} t$-quark. A measure of the systematic error due to the $p_{T}(t)$-distribution was obtained by varying the amount of initial state radiation in $\bar{t} t$ production. This resulted in a $3.4 \mathrm{GeV}$ change in the mass determined for the $250 \mathrm{GeV} t$-quark. Finally, next-to-leading-order QCD corrections to the $\bar{t} t$ cross section increase the magnitude of the cross section by about $50 \%$, but do not significantly change the shape. Still higher-order corrections are expected to amount to $\pm 15 \%$. Thus, radiative corrections are not a major source of systematic error. We conclude that, with an integrated luminosity of $10 \mathrm{fb}^{-1}$, we shall be able to use the $M_{\ell \mu}$ method to determine the mass of a $250 \mathrm{GeV}$ top quark to within an error of $\pm 2.3 \mathrm{GeV}$ (statistical) $\pm 4.1 \mathrm{GeV}$ (systematic)

\subsubsection{Top-Quark Detection and Mass Measurement via the $M_{\mathrm{ij}}$ Distribution}

The most direct measurement of the top-quark mass comes from nonleptonic $t$-decay into three jets. This is also the most precise measurement if systematic uncertainties associated with jet definition and energy measurement are under control. Furthermore, it is important for flavor physics spectroscopy to observe the top quark in nonleptonic decay modes. An example of this, the $\eta_{T}$, will be discussed below.

ISAJET was used to generate $300 \mathrm{~K} i t$ events for $m_{t}=140$ and for $250 \mathrm{GeV} .{ }^{42}$ As above, top quarks were generated with $50 \mathrm{GeV}<$ $p_{T}<1000 \mathrm{GeV}$. Events were selected in which one $W$ decayed to an electron or muon while the other $W$ decayed nonleptonically. The physics background to $\bar{t} t \rightarrow \ell_{\text {isol }}^{ \pm}+$jets comes mainly from production of $W+$ jets. After the $p_{T}$-cuts described below, this background was found not to be important. Thus, a non-isolated muon tag was not required. The ISAJET cross sections for these events are $4.1 \mathrm{nb}$ and $0.44 \mathrm{nb}$ for the two values of $m_{t}$. The isolated leptons were required to satisfy the cuts in Eqs. (2.4.1) and (2.4.2). 
To eliminate combinatorial backgrounds in the multijet mass distributions due to picking up the wrong $b$-quark (or its products), the $t$-quark and individual jets were forced to be at high $p_{T}$ and jets were selected in the hemisphere opposite the isolated lepton. Consequently, the three jets from the decays of $t$ tend to be close, and a cone of small radius $R=0.4$ was used to define jets. Individual jets were required to have $p_{T}>30 \mathrm{GeV}$ for the $m_{t}=140 \mathrm{GeV}$ case and $p_{T}>50 \mathrm{GeV}$ for the $250 \mathrm{GeV}$ case. In both cases, jets were required to satisfy $\delta \phi(\ell$ jet $)>90^{\circ}$. Finally, the three highest- $p_{T}$ jets satisfying these constraints were required to have $\mid \vec{p}_{T}(3$ jets $) \mid>200 \mathrm{GeV}(300 \mathrm{GeV})$ for $m_{t}=140 \mathrm{GeV}(250 \mathrm{GeV})$. See Ref. 42 for the basis of these cut values. The combined geometrical acceptance and efficiency of these cuts was found to be $0.70 \%$ and $1.0 \%$ for $m_{t}=140$ and $250 \mathrm{GeV}$. The number of events of $\ell^{ \pm}+$jets obtained per $10 \mathrm{fb}^{-1}$, taking into account trigger and reconstruction efficiencies and these acceptances, were $240 \mathrm{~K}$ and $40 \mathrm{~K}$ for $m_{t}=140$ and $250 \mathrm{GeV}$, respectively. The $W+$ jets background was found to be negligibly small for the $140 \mathrm{GeV}$ case. It contributed $15 \%$ to the three-jet mass spectrum for $250 \mathrm{GeV}$, but this had no important effect on the determination of $m_{t}$.

Consider first the case of $m_{t}=140 \mathrm{GeV}$. The $W \rightarrow 2$ jets and $t \rightarrow W b \rightarrow 3$ jets mass distributions, $M_{\mathrm{jj}}$ and $M_{\mathrm{jij}}$, are shown in Figures 2-35 and 2-36. For these plots, the trijet search region was $110 \mathrm{GeV}<M_{\mathrm{iji}}<170 \mathrm{GeV}$. At least one dijet pair was required to satisfy $\left|M_{\mathrm{jj}}-M_{W}\right|<20 \mathrm{GeV}$. All dijet pairs passing the cuts appear in Figure $2-35(a)$. The fitted $W$-mass peak is at $75.8 \mathrm{GeV}$ in this figure. The resolution on this mass is $13.9 \mathrm{GeV}$. The large combinatorial background in this figure is a consequence of the kinematics of this case. For $m_{t}=140 \mathrm{GeV}$ and $M_{W}=80 \mathrm{GeV}$, the three jets tend to be roughly equidistant in $\eta-\phi$ space. The background comes from picking up the $b$-jet from the same or, less frequently, the other $t$-quark decay. The signal-to-noise ratio in the $W$-peak region is about $2: 1$.
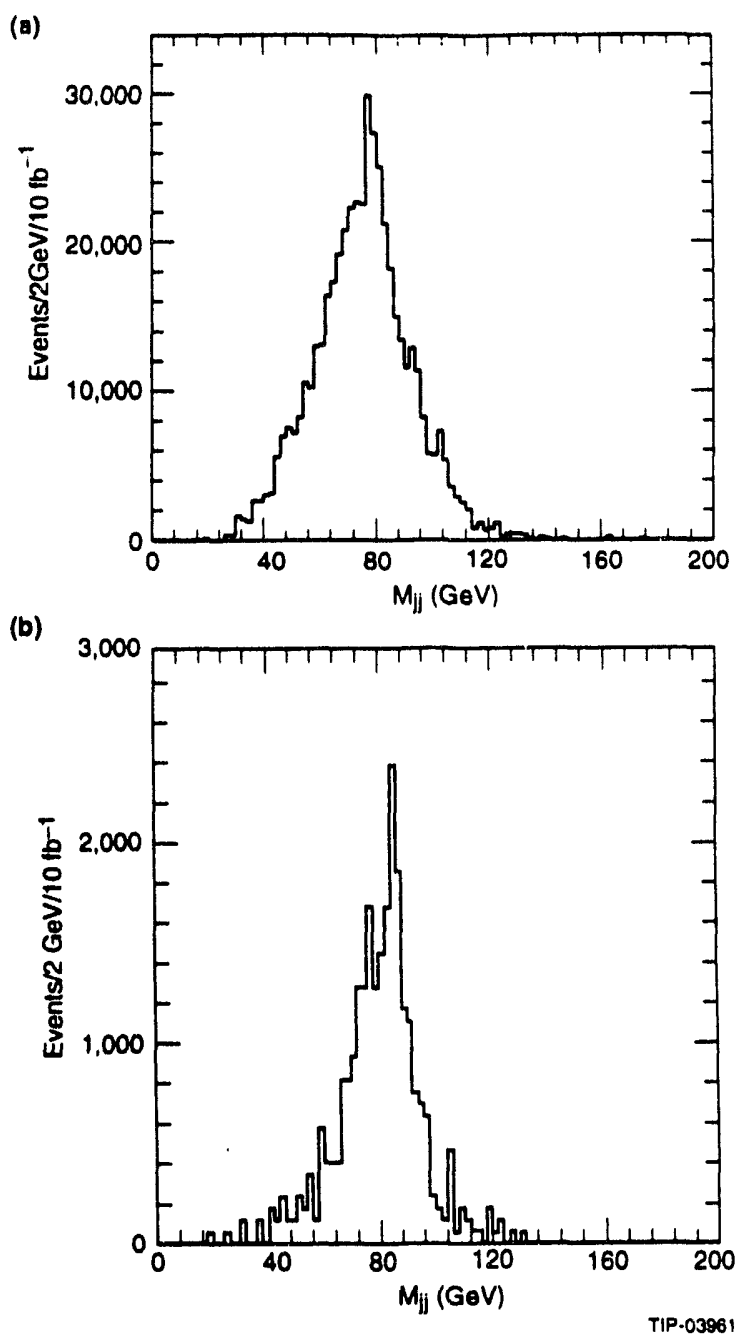

FIG. 2-35. Dijet mass clistribution in $t^{7}$ events with $m_{q}=140 \mathrm{GeV}$. (a) No b-lagging was assumed.

(b) b-tagging was assumed with $20 \%$ efficiency.

This signal-to-noise can be improved considerably by requiring that one of the jets passing the above cuts be tagged as a $b$-jet by the tracker. Figure 2-35 (b) shows the $M_{\mathrm{jj}}$ distribution for events with an identified $b$-jet which is then excluded from the dijet mass. The $b$-tag efficiency for this plot was assumed to be $20 \%$. (See Section 6.2 .3 for a discussion of $b$-tagging with the GEM central tracker.) The $W$ signal-to-noise ratio is improved to $3.9: 1$. The fitted $W$-mass peak in this plot is at $76.9 \mathrm{GeV}$ with a resolution of $8.4 \mathrm{GeV}$. We emphasize that this $b$-tag is not required for determining $m_{t}$. This is seen in Figure 2-36, where the 
top-quark peak appears clearly above little combinatorial background. The mass was determined to be $138.2 \mathrm{GeV}$ with a resolution of $8.1 \mathrm{GeV}$.

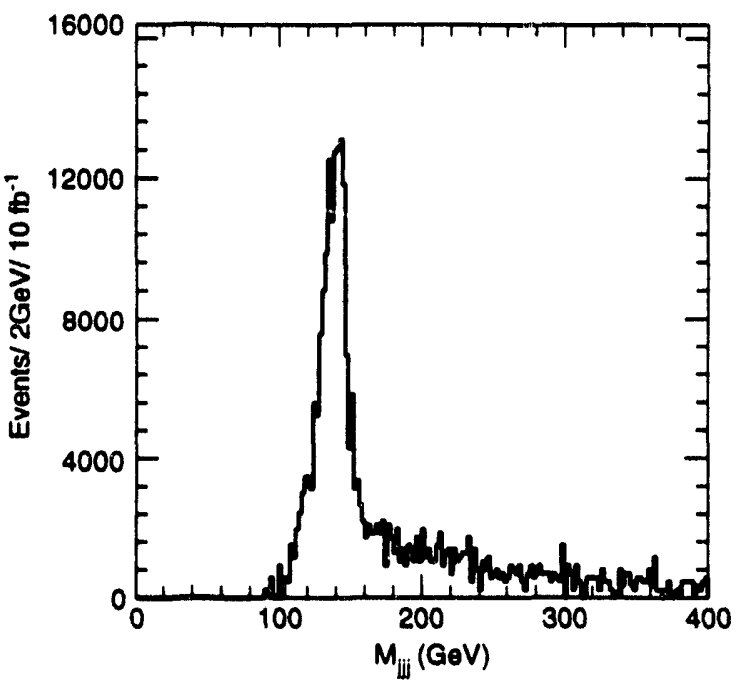

FIG. 2-36. Trijet mass distribution in $f$ events with $m_{l}=140 \mathrm{GeV}$, without b-tagging.

The jet energy correction procedure described in Section 2.2.6 was applied to this data. It was found not to improve the results significantly. For example, the peak of the corrected dijet distribution occurred at $82 \mathrm{GeV}$ while the top-quark mass changed to $142 \mathrm{GeV}$. This result may be due to the fact that, while the correction function was determined for single, isolated jets, the jets here are close together. The jet correction procedure is still under study.

The dijet and trijet mass distributions are shown in Figures 2-37 and 2-38 for $m_{t}=250 \mathrm{GeV}$. The search region was $200 \mathrm{GeV}<M_{\mathrm{jij}}<300 \mathrm{GeV}$. As above, at least one dijet was required to have invariant mass within $20 \mathrm{GeV}$ of $M_{W}$ and the invariant mass of the two closest jets was plotted. There was no need for a $b$-tag to sharpen the dijet mass distribution since, for such a heavy top-quark, the two jets from the $W$ are closer to each other than either is to the $b$-jet. Thus, the combinatorial background under the $W$-peak is mucin smaller if one selects the closest two jets. The fitted $W$-mass was found to be $79.8 \mathrm{GeV}$, with a resolution of $7.1 \mathrm{GeV}$. The top-quark mass was determined to be $247.4 \mathrm{GeV}$ with a resolution of $14.7 \mathrm{GeV}$.

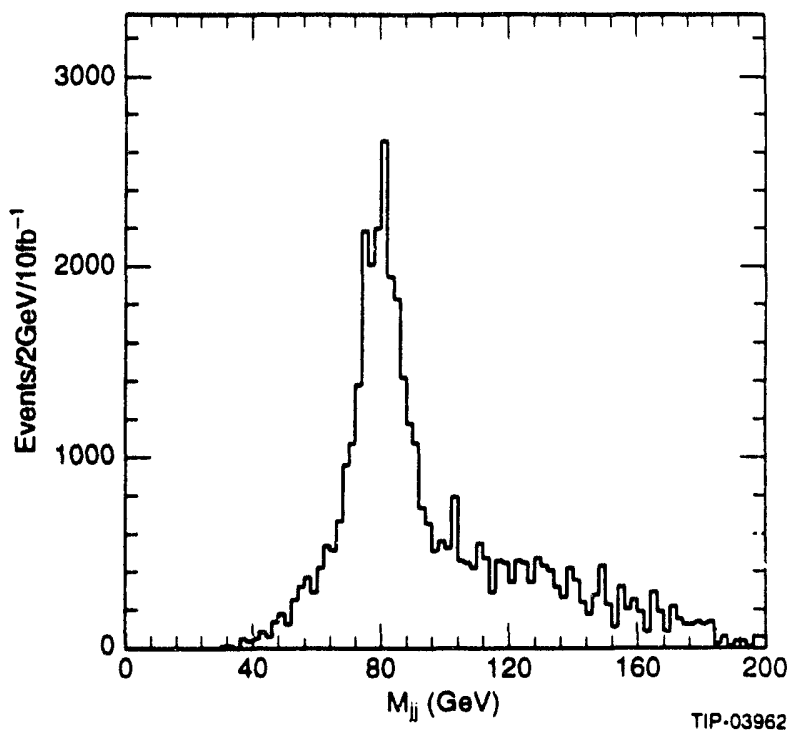

FIG. 2-37. Dijet mass distribution in ti events with $m_{1}=250 \mathrm{GeV}$.

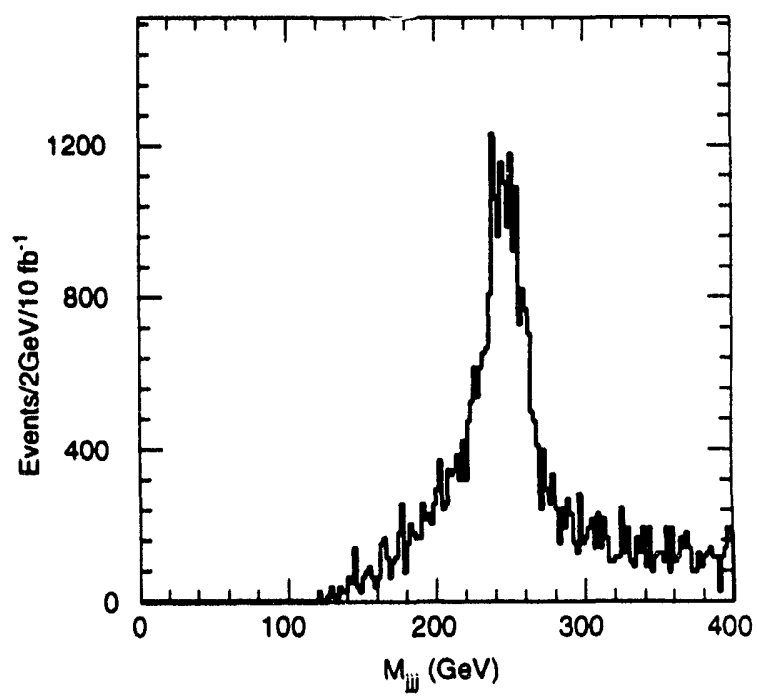

FIG. 2-38. Trijet mass distribution in $t \mathbb{f}$ events with $m_{1}=250 \mathrm{GeV}$.

The statistical uncertainty on the top-quark mass for $10 \mathrm{fb}^{-1}$ of data is approximately $0.03 \mathrm{GeV}(0.11 \mathrm{GeV})$ for $m_{t}=140(250) \mathrm{GeV}$. Systematics dominate the error in the $m_{l}$ measurement. The largest effect is the uncertainty in the jet energy measurement. More detailed studies of the calorimeter-beam tests as well as simulationswill be required to yield adequate correction functions. It will be helpful that the $t \rightarrow W b$ process is self-calibrating: The position and width of the $W$ peak will help calibrate the energies of jets in $t$-decay and determine the systematic uncertainty in $m_{t}$. 
The top quark will be a signal of flavor physics. One example is the $\eta_{T}$ boson, a spin-zero color-octet meson occurring in many technicolor models. ${ }^{40.7}$ In modern models, ${ }^{45}$ the $\eta_{T}$ is expected to have a mass in the range $300-500 \mathrm{GeV} .{ }^{46}$ It is produced in $p p$ collisions via gluon fusion with a cross section of order $0.1-1.0 \mathrm{nb}$, depending on the mass and the number of technicolors. ${ }^{47}$ It is expected to decay predominantly to $t \bar{t}$ with a width of order 10-100 GeV, again model-dependent.

In a search for the $\eta_{T}$, one would tag $t \bar{t}$ production in the $\ell_{\text {isol }}^{ \pm}+$jets mode and look for an enhancement in the $t \bar{t}$ invariant mass distribution. This invariant mass can be determined up to a quadratic ambiguity by assuming that the missing- $E_{T}$ is due to $W^{ \pm} \rightarrow \ell^{ \pm} v_{\ell}$. The resolution on this invariant mass for $M_{t f} \approx 400 \mathrm{GeV}$ is about $15 \mathrm{GeV}$ plus the contribution from the $\boldsymbol{E}_{T}$ resolution. For a $30 \mathrm{GeV}$ wide $\eta_{T}$, and assuming that the $M_{t f}$ resolution is $30 \mathrm{GeV}$, the $\eta_{T}$ appears as an enhancement $75 \mathrm{GeV}$ wide. The underlying $t \bar{t}$ cross section in such a region is about $5,3,1 \mathrm{nb}$ for $M_{\eta_{T}}=300,400,500 \mathrm{GeV}$. The feasibility of discovering the $\eta_{T}$ over such a background is under study.

\subsubsection{Search for a Charged Scalar in the Decay of a Heavy Top Quark}

Charged color-singlet scalar bosons, $H^{ \pm}$, occur in standard multi-Higgs-doublet models, in al! supersymmetric extensions of the standard model and, typically, as technipions in models of dynamical electroweak symmetry breaking. Generally, their couplings are Higgs-like. That is, $H^{ \pm}$tend to decay to the heaviest fermion pairs kinematically accessible. Since $H^{ \pm}$are color-singlets, they are copiously produced only if there is a heavier quark which can decay into them.

We study discovery of an $H^{ \pm}$in the decay products of a heavy top-quark. The masses $m_{t}=250 \mathrm{GeV}$ and $M_{H}^{+}=150 \mathrm{GeV}$ were used for the discussion that follows. At the end, results for other mass combinations will be described. It was assumed that both $t \rightarrow W^{+} b$ and $t \rightarrow H^{+} b$ decays are allowed. The charged scalar was assumed to decay into $c \bar{s}$ and $\tau^{+} v_{\tau}$. The various branching ratios for $t$ - and $H^{+}$-decay are model-dependent.
In the absence of experimental support for any particular model, it is appropriate to assume only that $B\left(t \rightarrow W^{+} b\right)+B\left(t \rightarrow H^{+} b\right)=1$ and $B\left(H^{+} \rightarrow c \bar{s}\right)+B\left(H^{+} \rightarrow \tau^{+} \nu_{\tau}\right)=1$, and to study the reach of the detector for $H^{ \pm}$as a function of the branching ratios. For comparison with other simulations, ${ }^{4,48}$ we also present results in terms of a specific model, the two-Higgs-doublet model occurring, e.g., in the minimal supersymmetric extension of the standard model (see Ref. 27).

Only the decay mode $H^{+} \rightarrow \tau^{+} \nu_{\tau}$ is considered here. ${ }^{43}$ This case demonstrates GEM's capacity to detect and use $\tau$-leptons in a physically plausible situation. For the decay of a heavy $t$-quark into $H^{+}$, it should be straightforward to discover $\mathrm{H}^{+}$and measure its mass in the hadronic decay mode, $\mathrm{H}^{+} \rightarrow \mathrm{cs}$. The analysis would proceed along the lines of the study of $t \rightarrow W^{+} b \rightarrow 3$ jets in the previous section. The most interesting issue is the limit that can be set on the branching ratio $B\left(H^{+} \rightarrow c \vec{s}\right)$ as a function of $B\left(t \rightarrow H^{+} b\right)$. This hadronic $H^{+}$-decay is under investigation. ${ }^{49}$

The presence of $t \rightarrow H^{+} b$, followed by $H^{+} \rightarrow \tau^{+} \nu_{\tau}$, is signalled by a breakdown of lepton universality expected if only $t \rightarrow W^{+} b$ were allowed (also see Refs. 4 and 48). In addition to the $\pi^{+} \nu_{\tau}$ decay mode used as a $\tau$-tag in those references, we employed all hadronic $\tau$-decay modes. The performance of the GEM central tracker $\tau$-lepton identification is described in Sections 6.2.2 and 6.2.3.

ISAJET 6.50 was used to generate $15 \mathrm{~K}$ each of $t \bar{t} \rightarrow W^{+} W^{-} b \bar{b}$ and $t \bar{t} \rightarrow W H^{ \pm} b \bar{b}$ events. Signal events were selected by requiring an isolated electron or muon and a tau-lepton candidate. The criteria for an isolated $\mu^{ \pm}$were taken to be:

$$
\begin{aligned}
& \left|\eta_{\mu}\right|<2.4 ; \\
& p_{T}>20 \mathrm{GeV} \\
& \sum_{R=0.4} E_{T}-\sum_{R=0.1} E_{T}<2 \mathrm{GeV} .
\end{aligned}
$$

Muons were required to be fully reconstructed according to the efficiency parameterized in gemfast. For an isolated $e^{ \pm}$, the criteria 


$$
\begin{aligned}
& \sum_{R=0.3}^{\left|\eta_{e}\right|<2.4 ;} E_{T}>50 \mathrm{GeV} ; \\
& \sum_{R=0.2} E_{T}(\mathrm{EC})>40 \mathrm{GeV} ; \\
& \sum_{R=0.4} E_{T}-\sum_{R=0.2} E_{T}(\mathrm{EC})<10 \mathrm{GeV} ; \\
& 0.8<E_{T}(R=0.2) / p_{T}(\mathrm{CT})<2.0 .
\end{aligned}
$$

were used.

The ISAJET decay table was updated to include all major $\tau$-decay modes. Hadronic modes account for $64.5 \%$ of all decays while the $\pi^{+}$-mode is only $12 \%$. For analysis of the two-body decays, it is important to account properly for the $\tau$-polarization occurring in $W^{ \pm}$ind $H^{ \pm}$decays. The decay $W^{ \pm} \rightarrow \tau^{ \pm} \nu_{\tau}$ conserves chirality (the same as helicity for a high-energy $\tau$ ), while $H^{ \pm} \rightarrow \tau^{ \pm} v_{\tau}$ maximally violates it. Then, for example, the $\pi^{+}$ occurring in $\tau^{+} \rightarrow \pi^{+} \bar{\nu}_{\tau}$ tends to follow the $H^{+}$direction of motion and so has higher $p_{T}$ than $\pi^{+}$from $W^{+} \rightarrow \tau^{+} v_{\tau}$ decays. This effect is enhanced here because $M_{H^{+}}$is larger than $M_{W}$. The polarization correlations were implemented in ISAJET for two-body $\tau$-decays. The $\tau$-polarization effects are small for the three-prong decays and require no special simulation. ${ }^{50}$

Isolated $\tau$-candidates were selected by requiring:

$$
\begin{aligned}
& \left|\eta_{\tau}\right|<2.4 ; \\
& N_{\mathrm{ch}}=1 \text { or } 3 ; \\
& \sum_{R=0.3} E_{T}>50 \mathrm{GeV} ; \\
& \delta \phi(\ell \tau)>100^{\circ} ; \\
& \sum_{R=0.4} E_{T}-\sum_{R=0.2} E_{T}<10 \mathrm{GeV} .
\end{aligned}
$$

For the charge multiplicity cut, tracks were required to have $p_{T}>1 \mathrm{GeV}$ and to lie within a cone of $R=0.1$ around the calorimeter jet axis. The leading track in this cone was required to have $p_{T}>15 \mathrm{GeV}$. Electrons from $\tau$ decays were rejected if they passed the criteria in Eq. (2.4.5). Muons from $\tau$-decays are predominantly isolated and are thus rejected by the absence 0 , their significant activity in the calonmeter. The central tracker was not used to tag $\tau$-leptons by their displaced vertices. If it were, it would further enhance the significance of the nonuniversality signal.

In addition, we required a $b$-jet tagged by the central tracker. The $b$-jet criteria were $\left|\eta_{b}\right|<2.4$, $\delta \phi(\ell b)<100^{\circ}$, and scalur $E_{T}>30 \mathrm{GeV}$. A tagging efficiency $\varepsilon(b$-tag $)=20 \%$ was assumed in this study; see Section 6.2.3. Alternatively, we can tag $b$-jets by a non-isolated muon. In this case the signal significances discussed below decrease somewhat. 43

The statistical significance of the $H^{+} \rightarrow \tau^{+} \nu_{\tau}$ signal is characterized by the number of standard deviations by which the number of $\tau$-events exceeds the expectation from lepton universality:

$N_{\sigma}=\frac{N\left(W H^{ \pm} \rightarrow \ell_{\text {isol }} \tau\right)}{\sqrt{N\left(W W \rightarrow \ell_{\text {isol }} \tau\right)+N\left(W H^{ \pm} \rightarrow \ell_{\text {isol }} \tau\right)}}$.

Here, the event numbers are given by

$$
\begin{aligned}
& N\left(W W \rightarrow \ell_{\text {isol }} \tau\right)= \\
& 2 N_{\tilde{t}} B^{2}\left(t \rightarrow W^{+} b\right) \\
& \times B\left(W \rightarrow \ell \nu_{\ell}\right) B\left(W \rightarrow \tau v_{\tau}\right) B(\tau \rightarrow \text { hadrons }) \\
& \times \varepsilon(W \rightarrow \ell) \varepsilon(W \rightarrow \tau) \\
& \times \varepsilon(b \text {-tag }) \varepsilon(t \text {-tag }) \varepsilon(t \text {-trigger }) ; \\
& N\left(W H^{ \pm} \rightarrow \ell_{\text {isol }} \tau\right)= \\
& 2 N_{\bar{n}} B\left(\tau \rightarrow W^{+} b\right) B\left(t \rightarrow H^{+} b\right) \\
& \quad \times B\left(W \rightarrow \ell \nu_{\ell}\right) B\left(H^{+} \rightarrow \tau \nu_{\tau}\right) B(\tau \rightarrow \text { hadrons }) \\
& \quad \times \varepsilon(W \rightarrow \ell) \varepsilon\left(H^{+} \rightarrow \tau\right) \\
& \quad \times \varepsilon(b \text {-tag }) \varepsilon(t \text {-tag }) \varepsilon(t \text {-trigger }) .
\end{aligned}
$$

For a $250 \mathrm{GeV}$ top quark, the number of events produced with an integrated luminosity of $10 \mathrm{fb}^{-1}$ is $N_{\overline{i t}}=1.5 \times 10^{7}$. The $\varepsilon$-quantities are efficiencies, discussed next.

The efficiencies $\varepsilon(W \rightarrow \tau)$ and $\varepsilon\left(H^{+} \rightarrow \tau\right)$ are the ratios of the numbers of true $\tau$-leptons passing the above cuts to the correct number of generated $\tau$-leptons, including $\tau \rightarrow e$ events misidentified as 
hadronic $\tau$-decays. For $m_{t}=250 \mathrm{GeV}$ and $M_{H}^{+}=150 \mathrm{GeV}$, the $r$-efficiencies were found to be $\varepsilon(W \rightarrow \tau)=9.8 \%$ and $\varepsilon\left(H^{+} \rightarrow \tau\right)=14.7 \%$. The efficiency for finding $W \rightarrow \ell v_{\ell}$ events was found to be $36 \%$ for electrons and $45 \%$ for muons. The average value $\varepsilon(W \rightarrow \ell)=40 \%$ was used. The top-tagging efficiency, $\varepsilon(t$-tag), is the number of top events remaining after the tau-selections, Eq. (2.4.6), divided by the number of generated top events times $\varepsilon(W \rightarrow \ell)$. The top-tagging efficiency was $69 \%$. The efficiency for triggering on top quarks at Level 1 using was found to be $93 \% .{ }^{43}$

The backgrounds to the $\bar{t} \rightarrow W^{\mp} H^{ \pm} \rightarrow$ $\ell^{\mp}+\tau^{ \pm}+X$ signal come from (1) $t \bar{t} \rightarrow W W b \bar{b}$ and $W H^{ \pm} b \bar{b}$ events in which $W^{ \pm}$and $H^{ \pm}$decay to two jets and these fake a $\tau ;(2) W+\bar{Q} Q$ events with $Q=c, b$; and (3) $\bar{b} b$ production. To study the first background, $40 \mathrm{~K}$ two-jet events were generated with $p_{T}$ in the range $50-1000 \mathrm{GeV}$. All jets found by gemfast with $E_{T}>25 \mathrm{GeV}$ were kept and subjected to the $\tau$-selection criteria above. A rejection factor of $R(\tau / \mathrm{jet})=0.0027$ was found. Since there are two to three jets in the hemisphere opposite the isolated lepton, this background amounts to $8.4 \%$ of the $\bar{t} t \rightarrow W W \rightarrow \ell_{\text {isol }}+\tau$ signal and $1.4 \% \times\left(1-B\left(H^{+} \rightarrow \tau \nu_{\tau}\right) / B\left(H^{+} \rightarrow \tau \nu_{\tau}\right)\right.$ of the $\overline{i t} \rightarrow W H \rightarrow \ell_{\text {isol }}+\tau$ signal. Of course, when $B\left(H^{+} \rightarrow \tau \nu_{\tau}\right)$ is very small, one must rely on the $H^{+} \rightarrow c \bar{s}$ mode to discover the charged scalar. Including these backgrounds reduces $N_{\sigma}$ by $3 \%$. The other backgrounds have been shown to reduce $N_{\sigma}$ by less than $3 \% .^{4,48,43}$

The significance for $10 \mathrm{fb}^{-1}, N_{\sigma}$ is plotted againsi $B\left(H^{+} \rightarrow \tau^{+} \nu_{\tau}\right)$ in Figure 2-39 for $B\left(t \rightarrow H^{+} b\right)=0.01-0.99$. Backgrounds are not included in these plots of $N_{\sigma}$. The $t \rightarrow H^{+} \rightarrow \tau^{+}$ signal can be discovered in GEM $\left(N_{\sigma}>5\right)$ so long as $B\left(t \rightarrow H^{+} b\right) \geq 0.02$ and $B\left(H^{+} \rightarrow \tau^{+} \nu_{\tau}\right) \geqslant$ 0.05 .

We have also computed $N_{\sigma}$ for the two-Higgsdoublet model used in the minimal supersymmetric extension of the standard model. ${ }^{27}$ In this model, $B\left(t \rightarrow H^{+} b\right)$ and $B\left(H^{+} \rightarrow \tau^{+} \nu_{\tau}\right)$ are determined by a single parameter, $\tan \beta$, the ratio of the vacuum expectation values of the two Higgs doublets. The significances were determined for four cases: $\left(m_{t}, M_{H}^{+}\right)=(250,225),(250,150),(150,125)$, and $(150,100)$ to facilitate comparison with Ref. 4 . The significances expected in GEM are shown in Figure 2-40. For all hut the heaviest mass combination, GEM is able to detect a $5 \sigma$ enhancement in $\tau$-production for $\tan \beta>0.4$. For $\left(m_{t}, M_{H}^{+}\right)=$ (250.225), the discovery level covers the range $0.7<\tan \beta<2.0$ and $\tan \beta>20$. By tagging the $\tau$ lepton in all its hadronic modes instead of just $\tau \rightarrow \pi v_{\tau}$, the significances have been increased by a factor of 2.3-5.

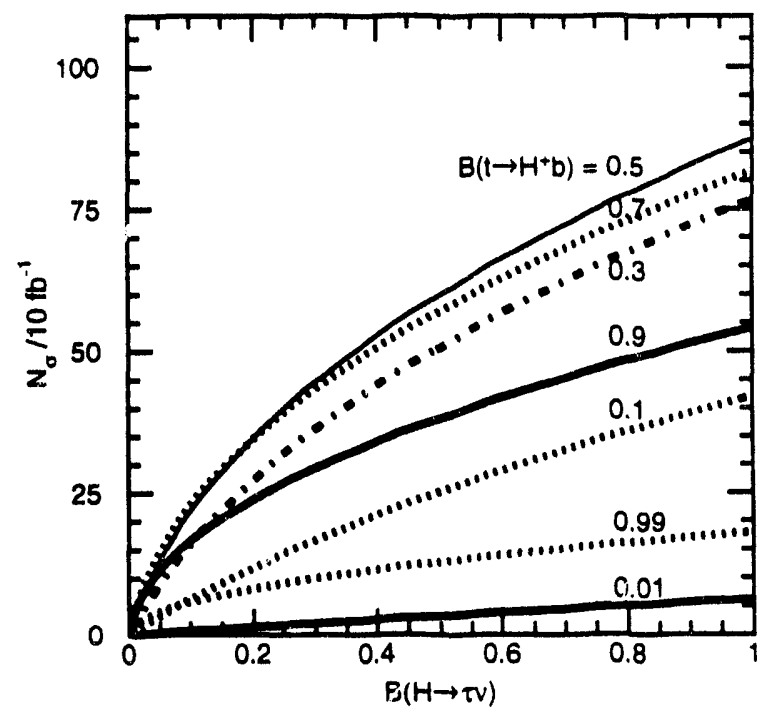

FIG. 2-39. Significance of the $t \rightarrow H^{+} b, H^{-} \rightarrow \tau^{+} v_{\tau}$ signal vs. $B\left(H^{+} \rightarrow \tau^{+} v_{r}\right)$ for various $B\left(t \rightarrow H^{+} b\right)$. Here, $m_{\mathrm{f}}=250 \mathrm{GeV}$ and $M_{H}^{+}=150 \mathrm{GeV}$.

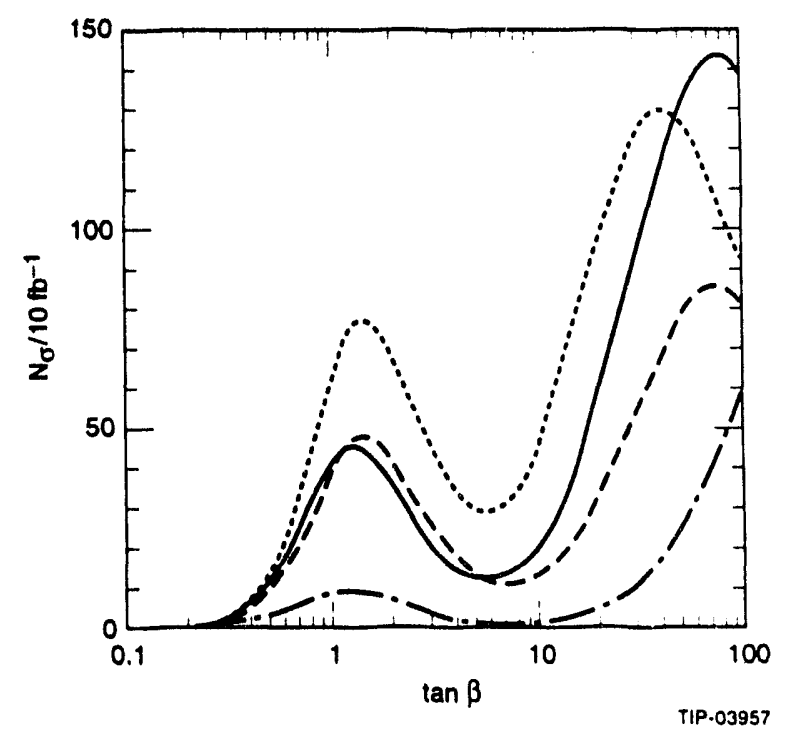

FIG. 2-40. Significance of the $t \rightarrow H^{+} b, H^{+} \rightarrow \tau^{+} v_{z}$ signal vs. $\tan \beta$ for various $m_{t}=250 \mathrm{GeV}$ and $M_{H}^{+}=225 \mathrm{GeV}$ (dash-dotted); 250 and $150 \mathrm{GeV}$ (dashed); 150 and $125 \mathrm{GeV}$ (solid); 150 and $100 \mathrm{GeV}$ (dotted). 


\subsection{JET PHYSICS AT LARGE TRANSVERSE MOMENTUM}

Although the GEM physics program emphasizes precision measurement of photons and leptons up to the highest SSC luminosities, the measurement and use of jets is important to GEM physics and has a high priority. The search for a high mass Higgs boson (Section 2.3.7) and heavy flavor physics accessed through top-quark studies (Section 2.4.2) are two of examples of new physics requiring accurate understanding and measurement of jets. Hadronic jets are their own backgrounds as well as the Uacikgrounds to more exotic processes; $H^{0} \rightarrow \gamma \gamma$ is an outstanding example of this. The errors induced by analysis effects, such as jet definition and clustering algorithms, and by instrumental effects, such as detector resolution, $e / h$ ior the calorimeters, and cracks and dead spaces, must be carefully studied and kept under control. Our approach to these problems was summarized briefly in Section 2.2 and described more fully in Ref. 21. This section discusses the main issues involved in measuring the high- $p_{T}$ jet cross section accurately. The search for quark substructure forms the physics context for this discussion.

If quarks and leptons are composite, with structure at the scale $\Lambda$, the most visible manifestation at subprocess energies $\sqrt{\hat{s}} \ll \Lambda$ is the presence of four-fermion contact interactions, $\mathcal{L}_{\Lambda}$, involving the composite quarks and leptons. ${ }^{51}$ These interactions induce terms in the cross section for dijet and dilepton production that are of order $\pi \hat{s} / \Lambda^{4}$, leading to significant excesses at "low" $\hat{s}$. It is known from experiments at $e^{+} e^{-}$and hadron colliders that $\Lambda \geq 1-2 \mathrm{TeV}$, above the scale of electroweak symmetry breaking. ${ }^{9,52}$ Thus, $\mathscr{L}_{\Lambda}$ must be $S U(3) \otimes S U(2) \otimes U(1)$ invariant, and the compusite quark and lepton fields appearing in it are electroweak, not mass, eigenstates. This raises the possibility that unacceptably large flavor-changing neutral currents will appear in the contact interactions. The most stringent limit on such interactions comes from the allowed magnitude of new effects in the ne" 1 l kaon system. If $|\Delta S|=2$ contact interactions exist, they must have $\Lambda \geqslant 500 \mathrm{TeV}$, well above the reach of the SSC. For the discussion of contact interactions accessible at the SSC, therefore, we shall assume that $\mathscr{L}_{\Lambda}$ is symmetric under interchanges of the three generations of quarks and leptons.

Quark substructure shows up directly as an excess of jets at high $p_{T}$ or $\sqrt{\hat{s}}$. In this section the contact interaction

$$
\mathscr{L}_{\mathrm{qq}}=-\frac{4 \pi}{2 \Lambda^{2}} \bar{Q}_{L a} \gamma^{\mu} Q_{L a} \bar{Q}_{L b} \gamma_{\mu} Q_{L b}
$$

is used as a model to modify QCD-jet production. Here, $Q_{L a}=\left(u_{a}, d_{a}\right)_{L}$ are left-handed quark fields and $a, b=1,2,3$ label the generations. This model for the four-quark contact interaction is essentially the one discussed in Ref. 7 except that, here, all quarks are considered to be composite. PYTHIA 5.6 was used to generate the jet events for $Q C D$ and the quark substructure signal..$^{53}$ Several different choices of parton distribution functions (PDFs) were used and results compared: EHLQ Set $1,{ }^{7}$ the CTEQ Set $1 \mathrm{~L},{ }^{29}$ and Morfin-Tung Set $2 .^{54}$ For all choices, the $\operatorname{sic}_{\mathcal{C}} \mathrm{zal}$ region lies above jet- $E_{T}=4 \mathrm{TeV}$. The study cescribed here was made for an integrated luminosity of $10 \mathrm{fb}^{-1}$. We shall find that GEM can reach a substructure scale of about $25 \mathrm{TeV}$ with this data sample.

The most important issue in searching for quark substructure is to be certain that an observed excess of high- $E_{T}$ jets is not an artifact of the detector nor of the analysis. The jet cross section must be known well enough at low energy that a deviation at high energy is unambiguous. For this, one will normalize the low- $E_{T}$ spectrum to the $\mathrm{QCD}$ expectation to eliminate uncertainties due to luminosity and to parton distribution functions. The jet energy scale must be well understood up to $E_{T}-10 \mathrm{TeV}$. Here, a scheme must be developed that corrects for the calorimeter's lack of compensation.

Figure 2-41 shows the rate for $Q C D$ jet events in which the total scalar transverse energy exceeds some value $\sum E_{T}$. The jets have $|\eta|<3$. A reasonable rate of $1 \mathrm{~Hz}$ is achieved at $\mathcal{L}=10^{33} \mathrm{~cm}^{-2} \mathrm{~s}^{-1}$ with a requirement of scalar $\sum E_{T}>1.2 \mathrm{TeV}$. The events in the current study were generated with a higher $\sum E_{T}$. To simulate a number of events actually expected in one year means to generate them in the signal region, $\sum E_{T} \geqslant 9 \mathrm{TeV}$. A second sample of events was generated with $\sum E_{T}>4.8 \mathrm{TeV}$ to determine the normalization differences that occur among various sets of parton 
distribution functions. In the actual experiment, a normalization sample would also be used to account for uncertainties in the luminosity. These have been approximately $8 \%$ at the Fermilab Tevatron. ${ }^{55}$ In fact, since the cross section at the SSC for jets at the lowest transverse energies is not well-known theoretically, the normalization region would be the middle of the jet- $E_{T}$ spectrum. In contrast, at the Tevatron, the lowest jet-energy bins correspond to jets of much higher $x_{T}$ than at the SSC.

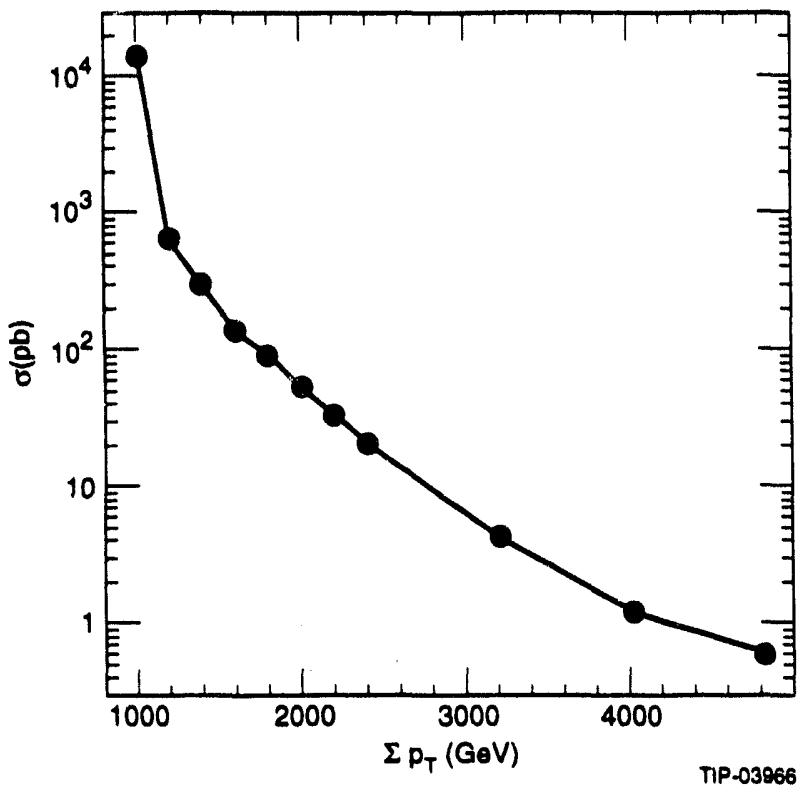

FIG. 2-41. QCD jet cross section for events with total transverse energy greater than the value $\sum E_{T}$. Jets have $|\eta|<3$.

The only kinematic cut imposed on jets is $|\eta|<1.1$. This enhances the roughly isotropic signal relative to the forward-backward peaked QCD background. More than one jet can be taken from a single event. For these central high- $E_{T}$ jets, the triggering efficiency will be close to $100 \%$ (see Section 7.2.2). The discovery criterion adopted for this analysis is an excess of 100 events in an $E_{T}$-region in which the observed cross section is twice as large as the QCD expectation.

As discussed in Section 2.2.6, jet clustering was done with a fixed-cone algorithm, where all cells within a large $\eta-\phi$ radius $R=0.9$ of the jet center were included. Fixed cone algorithms correspond most closely to the cutoffs used in theoretical calculation $c$ and facilitate the comparison of theory to experiment. In the case of the substructure signal, for which no next-to-leading order calculation exists, the large clustering radius was chosen to reduce energy loss out of the clustering cone. Extensive checks of this algorithm were made to ensure that it was efficient, insensitive to detector variations and that it had a well-defined angular resolution. ${ }^{21}$ If the jet-cone radius was decreased to 0.7 , the main effect was to shift the energy scale of the jet- $E_{T}$ spectrum downward by $1.2 \%$.

A full experimental analysis will include the development of a jet energy correction function and an unsmearing procedure for the inclusive jet- $E_{T}$ spectrum (see Section 2.2.6). The correction function will include the effects of the underlying event, energy out of the clustering cone, and detector noise, inefficiencies and nonlinearities. While most of these corrections are reasonably well-understood, most are not very important for high- $E_{T}$ jets. The most important problem is to determine the jet energy scale. Jets whose large $E_{T}$ drifts upward easily produce a false compositeness signal. The jet energy scale will be established for the GEM calorimeter using test beam and collider data as described in Chapter 5 (see Sections 5.2.2, 5.4.4 and 5.7). The energy scale correction will depend on, e.g., the jet rapidity and on the fraction of electromagnetic energy observed in a jet.

The systematic uncertainties that limit the reach in $\Lambda$ come from the parton distribution functions and incomplete knowledge of the jet resolution and the jet energy scale. A number of jet energy reconstruction schemes, particularly weighting algorithms which atten ipt to boost the hadronic part of a shower, have beet ed to improve jet resolution and energy lin y. ${ }^{17,18}$ Since it is difficult to predict how successful the correction scheme will be, we shall estimate the systematic effects due to nongaussian tails in the energy response and to nonlinearity arising from lack of compensation in the calorimetry. Nongaussian tails were modeled in gemfast by adding a second gaussian of $1 \%$ the amplitude and three times the width of the normal gaussian energy resolution by which the calorimeter energies are smeared (see Section 2.2.3). In studying nongaussian effects, we compared the rates of the CTEQ-1L, EHLQ-1 and MT-2 distribution functions.

The inclusive cross sections for jets with $|\eta|<1.1$ are shown in Figure 2-42 for $\Lambda=25$ and 
QCD $(\Lambda=\infty)$. The CTEQ-1L distribution functions were used to generate this figure. Table 2-15 lists the number of jets expected in a nominal SSC year with $E_{T}>E_{T}^{\text {cut }}$. It is clear that a quark substructure signal at the scale $\Lambda=25 \mathrm{TeV}$ could be discovered easily in one year with a detector whose calorimeter is as linear as the one modeled here. The variation in jet rates obtained using the CTEQ-1L, EHLQ-1 and MT-2 distributions are compared in the table. The $\Lambda$ that can be reached according to our discovery criterion varies by about $10-20 \%$, depending on the choice of distributions.

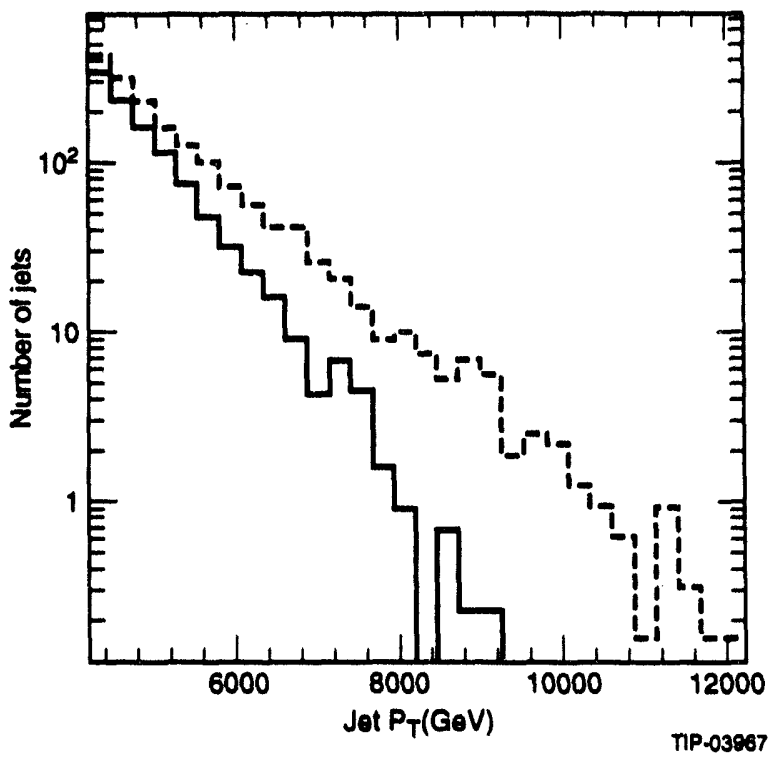

FIG. 2-42. Inclusive jet ET spectrum for substructure at the scale $\Lambda=25 \mathrm{TeV}$ (dashed) and for QCD (solid).

The expected nonlinearity in the charged pion response was modeled using a GEANT simulation of the GEM detector in which the detailed geometry and materials were replaced by simpler volumes filled with an equivalent mixture (see Section 5.2). Realistic $e / h$ values were included in the model. Figure 2-43 shows the charged pion response, averaged over $|\eta|<1.1$, as a function of energy. The relative response has been set to 1.0 at $200 \mathrm{GeV}$. The solid curve is the average relative response for jets in the compositeness sample in which energy-independent weights have been used to calculate the response. Energy-dependent weights were used for the dashed curve. The energy-dependent weighting scheme is more difficult to implement. The naive linear extrapolation to high energies shown here gives noticeable errors in both cases, but mimics the extrapolation from test beam energies that will have to be camied out when calibrating the real detector.

Table 2-15. Number of jets expected with $10 \mathrm{fb}^{-1}$ for various $E_{T}^{\text {cut }}$, substructure scales and parton distributions.

\begin{tabular}{ccccc}
\hline $\begin{array}{c}E_{T}^{\text {eut }} \\
\text { (GeV) }\end{array}$ & 4500 & 5000 & 5500 & 6000 \\
\hline CTEQ-1L Distributions & & & \\
\hline$\Lambda=\infty$ & 704 & 339 & 155 & 74 \\
30 TeV & 905 & 489 & 278 & 164 \\
25 TeV & 1226 & 724 & 447 & 276 \\
\hline EHLQ-1 Distributions & & & \\
\hline$\Lambda=\infty$ & 663 & 326 & 155 & 76 \\
30 TeV & 802 & 439 & 255 & 146 \\
25 TeV & 1106 & 669 & 407 & 251 \\
\hline MT-2 Distributions & & & & \\
\hline$\Lambda=\infty$ & 703 & 326 & 155 & 77 \\
30 TeV & 918 & 498 & 276 & 159 \\
25 TeV & 1328 & 803 & 507 & 309 \\
\hline \hline
\end{tabular}

The effect of the nonlinearity in the two weighting schemes is illustrated in Table 2-16. The CTEQ-1L distribution functions were used to prepare this table. As expected from Figure 2-43 the energy-independent weighting scheme tends to increase the measured $E_{T}$ of the jets, while the energy-dependent scheme reduces jet- $E_{T}$. The effects are small, of order $5 \%$ and the scale $\Lambda=25 \mathrm{TeV}$ is still easily within reach for a one-year data sample. We expect that more detailed, higher-statistics studies will show that the effects of nonlinearity will be smaller using the energy-dependent weighting scheme. However, in this case there will be more theoretical uncertainty because of the greater reliance on the jet fragmentation function to establish the calibration. These studies are still in progress. 


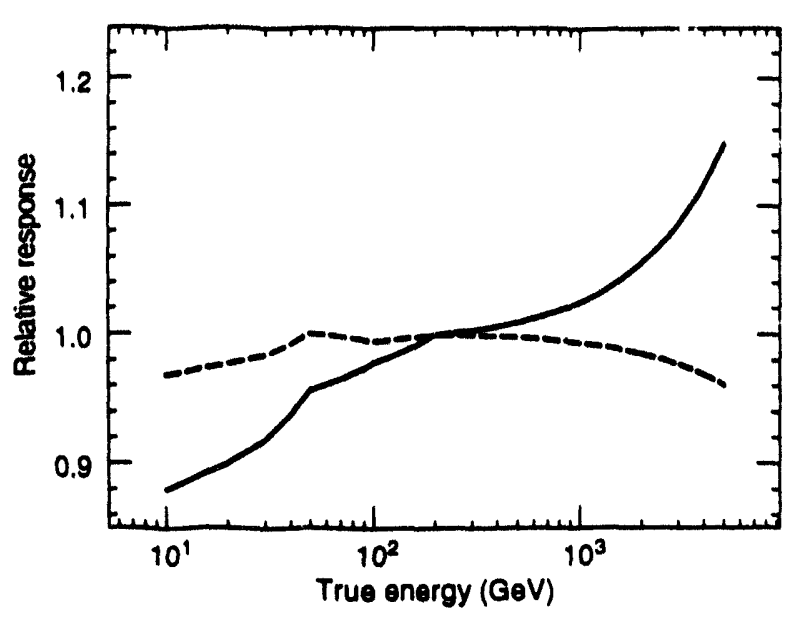

FIG. 2-43. Nonlinearity of calorimeter response to pions after employing corrections with energyindependent weights (solid curve) and energydependent weights (dashed curve).

Table 2-16. Number of jets expected with $10 \mathrm{fb}^{-1}$ in different energy-weighting schemes as described in the text. The CTEQ-1L entries are from Table 2-15.

\begin{tabular}{c}
$\begin{array}{c}E_{Y}^{\text {Uut }} \\
\text { (GeV) }\end{array}$ \\
\hline
\end{tabular}

Finally, we can estimate the reach in the quark scale $\Lambda$ that GEM could attain with a data sample of $100 \mathrm{fb}^{-1}$. We anticipate no special difficulties for high- $E_{T}$ jet measurement associated with operations at $\mathcal{L} \approx 10^{34} \mathrm{~cm}^{-2} \mathrm{~s}^{-1}$. Thus, the reach in $\Lambda$ can be determined from the fact that the subprocess cross section goes as $\hat{s} / \Lambda^{4}$. This yields $\Lambda \approx 45 \mathrm{TeV}$, a factor of 40 greater than the limit set by existing hadron collider data.

\subsection{HIGH MASS PHYSICS AT ULTRAHIGH LUMINOSITY}

The standard integrated luminosity produced in one to two years at the SSC should be sufficient to discover or exclude the signals of much of the collider's initial physics program-electroweak and flavor symmetry breaking. This has been demonstrated elsewhere in this and other documents. ${ }^{4}$ Still, there remain many potential new physics signals which require an integrated luminosity of order $100 \mathrm{fb}^{-1}$. This luminosity can be achieved practically only with extended running at $\mathcal{L} \approx 10^{34} \mathrm{~cm}^{-2} \mathrm{~s}^{-1}$. These new physics opportunities are almost all at very high mass scales, with effective cross sections of order 1-10 fb. Examples include multi-TeV, weakly coupled $Z^{\prime}$ and $W^{\prime}$ bosons, quark-lepton substructure, and very massive technirho vector bosons. In these and most other examples, the new physics is signalled by an excess of well-isolated leptons at high- $p_{T}$. For this reason, the clean identification and precise measurement of high- $p_{T}$ electrons and muons at ultrahigh luminosity are fundamental goals of the GEM design philosophy, 1,2

This section demonstrates the capacity of GEM at ultrahigh luminosity for (1) precision measurements of the masses, widths and chiral couplings of $4 \mathrm{TeV} Z^{\prime}$ bosons via $e^{+} e^{-}$and $\mu^{+} \mu^{-}$final states and (2) for the signatures and properties of quark-lepton substructure at the scale $\Lambda=25 \mathrm{TeV}$ in Drell-Yan production of high-mass dimuons. For both, we show that GEM can distinguish different models with a data sample of $100 \mathrm{fb}^{-1}$. We also find that the reach of the GEM detector is $M_{Z^{\prime}} \approx 8 \mathrm{TeV}$ for $Z^{\prime}$ and $\Lambda=$ $30-35 \mathrm{TeV}$ for quark-lepton substructure. Finally, we briefly describe approaches to studying highmass $W^{\prime \pm} \rightarrow \ell^{ \pm} v_{\ell}$ and quark-lepton substructure in $\bar{q} q^{\prime} \rightarrow \mu^{ \pm} v_{\mu} .56$

The isolated high-energy lepton signals of these and most other new physics processes are relatively free of all physics backgrounds including irreducible ones. For example, a rejection factor of $\mathscr{R}_{0}(e /$ jet $)$ of $O\left(10^{-4}\right)$ reduces the jet backgrounds to $Z^{\prime} \rightarrow e^{+} e^{-}$to the level of a few percent (see Section 2.6.1). Straightforward isolation cuts on muons completely remove the main physics backgrounds to $\bar{q} q \rightarrow \mu^{+} \mu^{-}$(Sections 2.6.1 and 2.6.2). The real difficulties of this experimental program are instrumental and environmental. Robust performance of all the main detector systems, as well as the trigger, at ultrahigh luminosity is the key to success. 


\subsubsection{Precision Studies of New Heavy $Z^{\prime}$ Gauge Bosons}

Extensions of the standard electroweak gauge group, $S U(2) \otimes U(1)$, involve neutral $Z^{\prime}$ and, usually, charged $W^{\prime \pm}$ vector bosons. In the models of interest, the new weak bosons couple to quarks and leptons with strength of $O(e)$. In one year of running at the $S S C$ at $\mathcal{L}=10^{33} \mathrm{~cm}^{-2} \mathrm{~s}^{-1}$, one can reach $M_{Z^{\prime}} \approx 4-6 \mathrm{TeV}$, depending on the $Z^{\prime}$ couplings. ${ }^{7}$ The reach criterion is the detection, in $10^{7}$ seconds, of $10 Z^{\prime} \rightarrow e^{+} e^{-}$events in a narrow $M_{e+e}$ range where none are expected. If such a boson were found, high-statistics studies would be needed to determine its nature. These studies will require extended runs at $10^{34} \mathrm{~cm}^{-2} \mathrm{~s}^{-1}$. This section concentrates on heavy, $4 \mathrm{TeV}, Z^{\prime}$ bosons. At this mass, ultrahigh luminosity generates hundreds of detected events per year in GEM, sufficient for precision studies. Since the couplings of such $Z^{\prime}$ bosons must be flavor-conserving, they may be detected in both modes, $Z^{\prime} \rightarrow e^{+} e^{-}$and $\mu^{+} \mu^{-}$. Very precise measurements of the $Z^{\prime}$ mass and width are made via the $e^{+} e^{-}$decay. The chiral nature of the $Z^{\prime}$ couplings to quarks and leptons is investigated in the $\mu^{+} \mu^{-}$mode by measuring the distribution in the angle $\theta$ between the outgoing $\mu^{-}$and the incoming quark.

The goal of the studies presented here is to determine GEM's ability to distinguish two different $Z$ ' models by the bosons' widths and angular distributions. (Preliminary studies of these two models were presented in Refs. 2 and 3.) The two models considered were:

1. A left-right model, in which $S U(2)_{L} \otimes$ $S U(2)_{R} \otimes U(1)$ breaks down to $S U(2)_{L} \otimes U(1)$. The $S U(2)_{R}$ coupling was tar $n$ to be the same as the $S U(2)_{L}$ one. The extra $Z$-boson of this model is called $Z_{1}$ below.

2. A model in which the grand-unification group $S O(10)$ breaks down to $S U(5) \otimes U(1)$, then to $S U(2)_{L} \otimes U(1)$. Such a model may have an extra $Z^{\prime} \equiv Z_{2}$ well below the unification scale.

The left-and right-handed couplings to quarks and leptons in the two models are given by 57

$$
\begin{array}{cl}
g_{u L}=\beta, & g_{u R}=\beta+\gamma ; \\
g_{d L}=\beta, & g_{d R}=\beta-\gamma ; \\
g_{v L}=-3 \beta, & g_{v R}=-3 \beta+\gamma ; \\
g_{\ell L}=-3 \beta, & g_{\ell R}=-3 \beta-\gamma .
\end{array}
$$

The parameters $\beta$ and $\gamma$ are

$$
\begin{array}{lll}
\beta=0.0528, & \gamma=-0.3630 \quad \text { (Model 1) } \\
\beta=0.0979, & \gamma=-0.1958 & \text { (Model 2). }
\end{array}
$$

In calculating the $Z^{\prime}$ decay widths, it is assumed that there are three generations of quarks and leptons and that right-handed neutrinos exist and are much lighter than $M_{Z^{\prime}}$. If there are no other significant decay modes, the widths are given by

$$
\Gamma\left(Z^{\prime} \rightarrow f_{i} \bar{f}_{i}\right)=\frac{2 a M_{Z^{\prime}} C_{i}}{3 \sin ^{2} 2 \theta_{W}}\left(g_{i L}^{2}+g_{i R}^{2}\right),
$$

where $C_{i}=3$ for quarks and 1 for leptons. The full widths in the two models are $\Gamma\left(Z_{1}\right)=105 \mathrm{GeV}$ and $\Gamma\left(Z_{2}\right)=67.7 \mathrm{GeV}$.

\section{$Z^{\prime} \rightarrow e^{+} e^{-}$}

PYTHIA 5.6 was used to generate 1000 events for each of the two $Z^{\prime}$ models, including full $\gamma / Z / Z^{\prime}$ interference. Except for studies that specifically involve the operation of the tracker, pileup at $\mathscr{L}=10^{34} \mathrm{~cm}^{-2} \mathrm{~s}^{-1}$ was simulated only as increased noise in the calorimeters. Because we are dealing with such high energies, pileup makes a negligible contribution to the isolation cone energies. The total cross sections for $3.75 \mathrm{TeV}<$ $M_{e^{+} e^{-}}<4.25 \mathrm{TeV}$ are $5.44 \mathrm{fb}$ for Model 1 and $4.54 \mathrm{fb}$ for Model 2.58 No rapidity cut was imposed on the generated events. Electron candidates were required to satisfy the following criteria:

- They had $|\eta|<2.46$ so thiat they were within the tracker's coverage. Electrons falling in the transition region between barrel and endcap $(1.01<|\eta|<1.16)$ were excluded because their energies are not as precisely measured.

- They had $E_{T}>250 \mathrm{GeV}$ in a $5 \times 5$ tower in the EC; $98.6 \%$ of the events with $|\eta|<2.46$ passed this cut.

- To reject hadrons, the transverse energy leakage into the first, thin layer of the HC behind the $5 \times 5$ EC tower was required to be less than $10 \%$ of the electron's $E_{T} ; 99.4 \%$ of the 
remaining events passed this selection. If a high energy EC cluster was isolated at this level, any energy in the first layer of the HC behind the cluster was added to the EC energy to improve the resolution.

The geometrical acceptance of the GEM detector for these $e^{+} e^{-}$events is approximately $88 \%$ for both models. The trigger efficiency for these events is close to $100 \%$. Thus, GEM collects $475 Z_{1} \rightarrow e^{+} e^{-}$and $400 Z_{2} \rightarrow e^{+} e^{-}$events in a run of $100 \mathrm{fb}^{-1}$.

Physics backgrounds to the $Z^{\prime} \rightarrow e^{+} e^{-}$signal come from misidentifying jets as electrons in QCD-jet production and in $p p \rightarrow W+$ jets with $W \rightarrow e v$. They may also come from isolated, high-mass $e^{+} e^{-}$pairs from $\bar{t} t$ production. The cross section for QCD jets with $|\eta|<2.46$ and invariant mass $M_{\mathrm{jj}}$ in a bin $500 \mathrm{GeV}$ wide centered on $M_{Z^{\prime}}=4 \mathrm{TeV}$ is $700 \mathrm{pb}$. The corresponding cross section for $W+$ jets $\rightarrow e+$ jets is about $25 \mathrm{fb}$. The rate for $\bar{t} t e^{+} e^{-}+X$ with generated $M_{\hbar}>1.4 \mathrm{TeV}$ and $p_{T}(t, \bar{t})>100 \mathrm{GeV}$ is $1.4 \mathrm{pb}$, for $m_{t}=140 \mathrm{GeV}$. These backgrounds are eliminated by a combination of isolation and $M_{e^{+} e^{-}}$cuts. The calorimetric isolation cut is

$$
I_{e}=\sum_{R=0.5} E_{T}-1.1 \sum_{5 \times 5} E_{T}(\mathrm{EC})<0 .
$$

This cut accepts $97 \%$ of the signal events while providing a jet rejection factor $\mathscr{B}_{(}(e /$ jet $) \approx$ $2 \times 10^{-3}$. Requiring exactly one charged track $^{59}$ with $p_{T}>50 \mathrm{GeV}$ pointing to the EC cluster then gives a rejection $\mathscr{B}_{(}(\mathrm{e} / \mathrm{jet})=4 \times 10^{-4}$. Thus, the dijet background is less than $4 \%$ of the signal, while $W+$ jet is negligible. After the isolation cut, the $\bar{t} t \rightarrow e^{+} e^{-}+X$ background is completely eliminated by requi ing $M_{e^{+} e^{-}}>$ $3.5 \mathrm{TeV}$. (See the discussion below of the $\overline{i t} \rightarrow \mu^{+} \mu^{-}+X$ background to $Z^{\prime} \rightarrow \mu^{+} \mu^{-}$.)

The $e^{+} e^{-}$mass resolution is given by

$$
\begin{array}{r}
\frac{\Delta M_{e^{-e^{-}}}=\frac{1}{2}}{M_{e^{-e^{-}}}}\left[\left(\frac{\Delta E_{1}}{E_{1}}\right)^{2}+\left(\frac{\Delta E_{2}}{E_{2}}\right)^{2}+\right. \\
\left.\left(\cot \frac{\theta_{12}}{2} \Delta \theta_{12}\right)^{2}\right],
\end{array}
$$

where $E_{1,2}$ are the $e^{ \pm}$energies and $\theta_{12}$ is the angle between them. In the $\mathrm{EC}$ energy resolution, only the constant term matters for a $4 \mathrm{TeV} Z^{\prime}$. For the GEM baseline, this is $0.4 \%$.

For the high energies of interest here, the $e^{+}, e^{-}$ directions can be determined to an accuracy of about $2 \mathrm{mrad}$ by using the longitudinal segmentation in the EC. (See Section 5.2.1.) This gives $\Delta \theta_{12}=$ 0.003 . We are investigating whether even greater angular precision may be obtained by using the calorimeter and the tracker in concert to determine the vector from the shower centroid to the event vertex. The shower position is determined to about $1 \mathrm{~mm}$. If the vertex position can be determined to a similar position at $10^{34} \mathrm{~cm}^{-2} \mathrm{~s}^{-1}$, we will achieve $\Delta \theta_{12} \simeq 0.0015$.

In sum, neglecting environmental effects at $\mathscr{L}=10^{34} \mathrm{~cm}^{-2} \mathrm{~s}^{-1}$ that might degrade $M_{\mathrm{e}^{+} \mathrm{e}^{-}}$ resolution, we expect $\Delta M_{c^{+} e^{-}} \cong 0.003 \times M_{Z^{\prime}}$. since $\theta_{12} \geq 60^{\circ}$. This is appreciably less than $\Gamma_{Z^{\prime}} / 2.35$ for the models under consideration. GEM will be able to resolve a $4 \mathrm{TeV} Z^{\prime}$ as narrow as $30 \mathrm{GeV}$, a factor of two better at $\mathscr{L}=10^{34} \mathrm{~cm}^{-2} \mathrm{~s}^{-1}$ than can be achieved by an electromagnetic calorimeter with a constant term of $1 \%$.

Several environmental effects were considered that might affect the $M_{e^{-} e^{-} \text {resolution. }}{ }^{58}$ Pileup noise in the calorimeters is unimportant. Events were simulated without, as well as with, the Gaussian pileup noise turned on. The measured $\Gamma_{Z}$ agreed to $1.3 \%$ and $M_{Z}$ to $0.1 \%$, consistent with statistical fluctuations. The effect of pileup tracks on the performance of the tracker for the purpose of vertex determination was included in the $z$-resolution quoted above. Finally, bremsstrahlung from the passage of the very high energy electrons through the CT material enters the same $5 \times 5 \mathrm{EC}$ tower which defines the electron shower.

Figures 2-44 and 2-45 show the $\mathrm{Me}_{e^{+} e^{-}}$distributions for the two $Z^{\prime}$ models as generated by PYTHIA and as simulated by gemfast. ${ }^{58}$ In the reconstructed distributions, the electron directions were found using the EC shower pointing only. These distributions are fit very well by a Lorentzian. The reconstructed masses determined from this fit are $M_{Z_{1}}=3996 \pm 1.5 \mathrm{GeV}$ and $M_{Z_{2}}=3994 \pm$ $0.9 \mathrm{GeV}$. The widths are $\Gamma_{Z_{1}}=132 \pm 5.0 \mathrm{GeV}$ 
and $\Gamma_{Z_{2}}=99.2 \pm 3.7 \mathrm{GeV}$. Here, the errors are from the fitting routine and include the finite statistics. The reconstructed masses differ from those obtained by fitting the generated distributions by $0.1 \%$. The generated widths are $124 \mathrm{GeV}$ for $Z_{1}$ and $94.2 \mathrm{GeV}$ for $Z_{2}{ }^{60}$ They differ from the reconstructed widths by an amount that corresponds to the detector resolution estimated above. Very similar results were obtained by using the $\mathrm{CT}$ and $\mathrm{EC}$ together to determine the electron directions. This redundancy gives confidence that GEM's precision electron measurement capability will survive at ultrahigh luminosity.

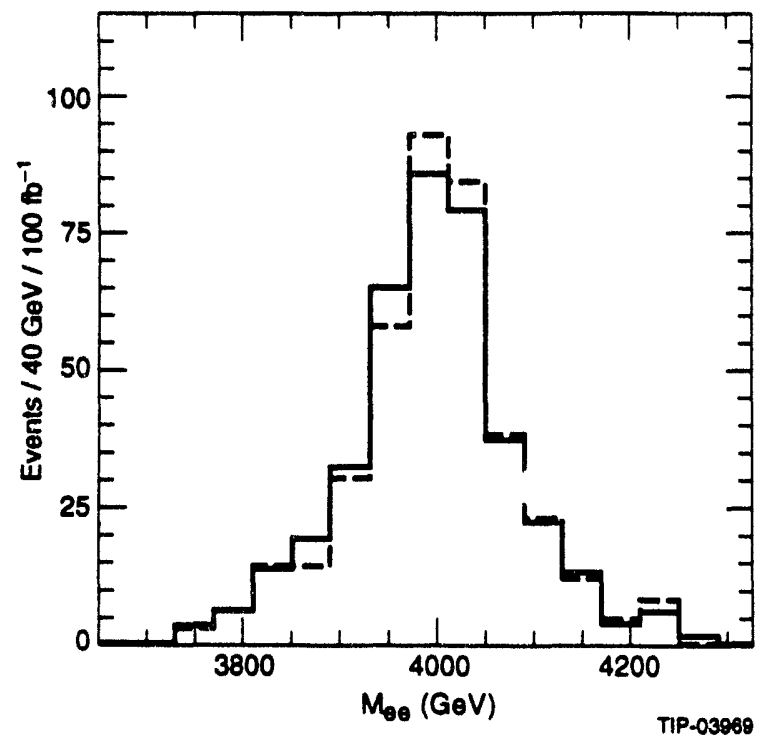

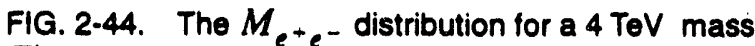
$Z^{\prime}$ of Model 1 as described in the text. Reconstructed (solid) and generated (dashed) distributions are shown.

While the error in $\Gamma_{Z^{\prime}}$ will be dominated by statistics, the main error in $M_{Z}$, is systematic, arising from possible nonlinearity in the EM energy scale in the $\mathrm{TeV}$ region. The energy scale of the EC will be calibrated by test beam data at the highest available energy and determined at higher energies by extrapolation of the data and Monte Carlo simulations (see Chapter 13).

Finally, we estimated the reach of the GEM detector in $M_{Z^{\prime}}$ for an integrated luminosity of $100 \mathrm{fb}^{-1}$. Assuming that ten detected $Z^{\prime} \rightarrow e^{+} e^{-}$events are sufficient for discovery (there is no background after cuts), the reach was found to be about $8 \mathrm{TeV}$ for both models considered. The reach for other models may be more or less by 1-2 TeV. This high mass, then, sets the upper limit of the dynamic range of the GEM electromagnetic calorimeter.

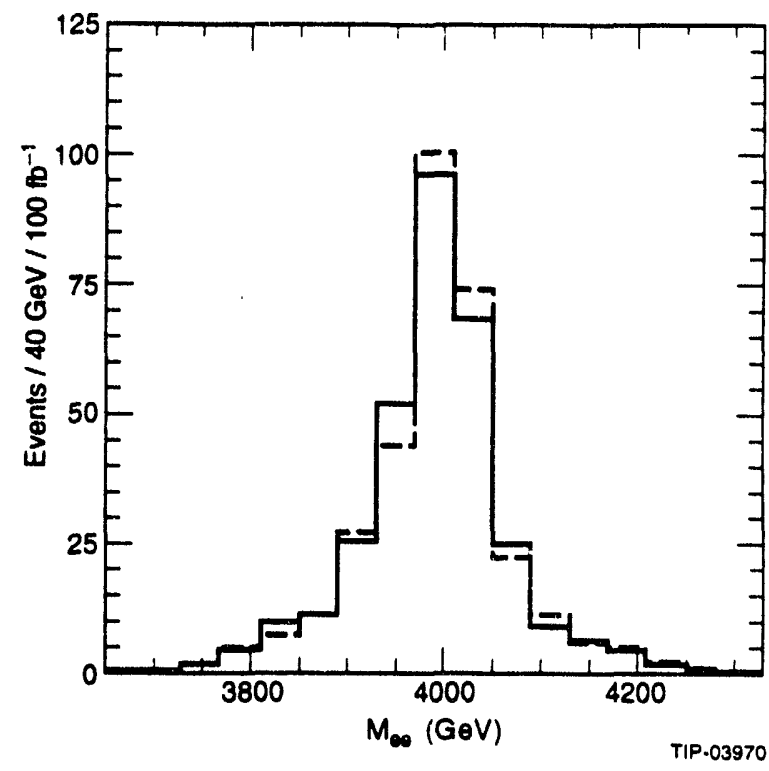

FIG. 2-45. The $M_{e^{+}}$- distribution for a $4 \mathrm{TeV}$ mass $Z^{\prime}$ of Model 2 as described in the text. Reconstructed (solid) and generated (dashed) distributions are shown.

$Z^{\prime} \rightarrow \mu^{+} \mu^{-}$

The chiral couplings of $Z^{\prime}$ bosons to quarks and leptons determine the lepton angular distribution. Thus, the $\cos \theta$ distribution can help distinguish alternative models. For very heavy $Z^{\prime}$ bosons, such as the ones considered here, the angular distributions of the high energy muons can be measured reliably in GEM because their an les and charges can still be determined at ultrahigh luminosity. We imposed the criterion that muon charges must be known with at least $2.5 \sigma$ significance. At lenst $99 \%$ of the signal events that passed the isolation and invariant mass cuts described below have the charges of both muons determined. Events with one mismeasured sign were rejected. The probability of two mismeasured signs is $O\left(10^{-4}\right)$ and no such events are expected in a data sample of $100 \mathrm{fb}^{-1}$. These results are consistent with those of Section 4.2 .5 where a slightly looser, $2 \sigma$, criterion is adopted.

To measure the angular distribution of the $\mu^{-}$ in $Z^{\prime}$ decay, one must determine, in addition to the muons' charges, the motion of the subprocess center-of-mass (c.m.) frame relative to the lab, and the direction of the incoming quark in the lab. The usual procedure is to determine the quark direction 
and, hence, the angle $\theta$ by requiring that the $Z^{\prime}$ be produced at large rapidity, $\eta_{Z^{\prime}} \geq 1 .^{61}$ Only about $20 \%$ of the heavy $Z^{\prime} \rightarrow \mu^{+} \mu^{-}$events pass this cut. Fortunately, this data-limiting criterion is unnecessary. As we will discuss below, the quark direction is well-determined by the muon rapidities, allowing use of the full data sample. ${ }^{62,63}$ This method works just as well for measuring muon angular distributions in continuum Drell-Yan production (see Section 2.6.2).

For dimuon events with $\sqrt{\hat{s}} \geq 2 \mathrm{TeV}$, the $p_{T}$ of the c.m. frame is less than $200 \mathrm{GeV}$ about $70 \%$ of the time. Thus, to a good approximation, one may ignore the transverse motion of the c.m. frame. Its longitudinal motion is then given by the boost rapidity, $\eta_{B}=\frac{1}{2}\left(\eta_{\mu}-+\eta_{\mu}\right)$. Here, of course, $\eta_{B}=\eta_{Z^{\prime}}$. We define the angle $\theta^{*}$ by

$$
\cos \theta^{*}=\operatorname{sgn}\left(\eta_{B}\right) \tanh \left(\frac{\eta_{\mu^{-}}-\eta_{\mu^{*}}}{2}\right) .
$$

For $\sqrt{\hat{s}}>2 \mathrm{TeV}$, the quark is harder than the antiquark, and its direction is given correctly by $\eta_{B}$, at least $75 \%$ of the time. Thus, to the extent that one may also ignore the transverse motion of the c.m. frame, the angles $\theta^{*}$ and $\theta$ are equal $75 \%$ of the time. This is adequate to distinguish the angular distributions of the two $Z^{\prime}$ models considered here, provided enough data is collected.

The $\cos \theta^{*}$ distribution is given in the parton model by (for a muon system with perfect acceptance at $|\eta|<2.46$ )

$$
\begin{aligned}
& \frac{d N}{d\left(\cos \theta^{*}\right)}=\sum_{q} \int_{-\eta_{\max }}^{\eta_{\max }} d \eta_{B} \\
& f_{q}\left(\sqrt{\tau} e^{\eta_{B}}, M_{Z^{\prime}}\right) f_{q}\left(\sqrt{\tau} e^{-\eta_{B}}, M_{Z^{\prime}}\right) \\
& \quad \times\left(\theta\left(\eta_{B}\right) \hat{\mathcal{N}}_{q q}\left(\cos \theta^{*}\right)+\theta\left(-\eta_{B}\right) \hat{\mathcal{N}}_{\overline{q q}}\left(-\cos \theta^{*}\right)\right) \\
& \quad \times \theta\left(2.46-\mid \eta_{\mu}-1\right) \theta\left(2.46-\mid \eta_{\mu}+1\right) .
\end{aligned}
$$

Here, $\tau=M_{Z^{\prime}}^{2} / s=0.01$,

$$
\eta_{\max }=\min (-\ln \sqrt{\tau}, 2.46) \text {. }
$$

and $\theta(x)=1(0)$ if $x>0(x<0)$. The dimensionless $q \bar{q} \rightarrow \ell^{+} \ell^{-}$angular distribution $\hat{\mathcal{N}}_{\overline{q q}}$ is given by

$$
\begin{aligned}
\hat{\aleph}_{\overline{q q}}\left(\cos \theta^{*}\right) & =\left(g_{q L}^{2} g_{\ell L}^{2}+g_{q R}^{2} g_{\ell R}^{2}\right)\left(1+\cos \theta^{*}\right)^{2} \\
& +\left(g_{q L}^{2} g_{\ell R}^{2}+g_{q R}^{2} g_{\ell L}^{2}\right)\left(1-\cos \theta^{*}\right)^{2}
\end{aligned}
$$

The forward-backward asymmetry expected in the parton model is given by

$$
A_{F B}=\frac{N\left(\cos \theta^{*}>0\right)-N\left(\cos \theta^{*}<0\right)}{N\left(\cos \theta^{*}>0\right)+N\left(\cos \theta^{*}<0\right)} .
$$

PYTHIA 5.6 was used to generate 1000 events of $Z^{\prime} \rightarrow \mu^{+} \mu^{-}$with $\left|M_{\mu^{+} \mu^{-}}-M_{Z^{\prime}}\right|<$ $250 \mathrm{GeV}$ for each of the two models, as in the previous subsection. The cross sections in this simulation were found to be $\sigma\left(Z_{1} \rightarrow \mu^{+} \mu^{-}\right)=$ $5.86 \mathrm{fb}$ and $\sigma\left(Z_{2} \rightarrow \mu^{+} \mu^{-}\right)=4.49 \mathrm{fb}$. The geometrical acceptance of the muon system for all events was found to be $63 \%$ for both models; for muons with $|\eta|<2.46$, it is about $70 \%$. For these muons, the trigger was highly efficient. Thus, before any additional losses, a data sample of $100 \mathrm{fb}^{-1}$ yields $375 Z_{1} \rightarrow \mu^{+} \mu^{-}$and $285 Z_{2} \rightarrow \mu^{+} \mu^{-}$ events. A study of the muon system's geometrical acceptance for these $Z^{\prime}$ events was carried out using sigem and is described in Section 4.2.6. The results found there are essentially identical to those obtained here with gemfast.

The only physics backgrounds to the $Z^{\prime}$ signal are continuum Drell-Yan production and $t \bar{i} \rightarrow \mu^{+} \mu^{-}+X$. After momentum smearing is included, the signal region used for angular distribution studies was taken to be $3.2 \mathrm{TeV}<$ $M_{\mu+} \mu^{-}<4.8 \mathrm{TeV}$. The Drell-Yan background was studied by generating $4000 \gamma / Z / Z^{\prime}$ events with $M_{\mu{ }^{+}{ }^{-}}>1.5 \mathrm{TeV}$. Drell-Yan production contributed six background events to the signal region.

The $\bar{t} t \rightarrow \mu^{+} \mu^{-}+X$ background was studied by generating $200 \mathrm{~K}$ events with generated $M_{\bar{i}}>1.4 \mathrm{TeV}$ and $p_{T}(t, \bar{t})>100 \mathrm{GeV}$. The rate for this background is $1.4 \mathrm{pb}$, so the sample corresponds to $140 \mathrm{fb}^{-1}$. (Other potential backgrounds, such as $t W$ and $W W$-production have very much smaller rates.) Detector response to events having $M_{\mu^{+} \mu^{-}}>1 \mathrm{TeV}$ and $\left|\eta_{\mu}\right|<2.6$ at the generator level was simulated with gemfast. There were 2900 such events (for $100 \mathrm{fb}^{-1}$ ), of which 2700 passed the geometrical acceptance simulated in gemfast. This is higher than the 
geometrical acceptance for $Z^{\prime} \rightarrow \mu^{+} \mu^{-}$events because most of the muons from $t$-decay follow their parent's direction, which is fairly close to the beam. These muons are usually accompanied by hadronic debris. Thus, the background can be removed by a combination of isolation and $M_{\mu-\mu}$ - cuts.

The isolation cut was tuned to optimize signal acceptance and background rejection. Figure 2-46 shows the isolation variable

$$
I_{\mu}=\sum_{R=0.7} E_{T}-0.1 p_{T}(\mu)
$$

for signal and background events. Here, the sum is over energy in the full calorimeter and $p_{T}(\mu)$ is the corrected muon momentum. For these studies, the muon momentum was corrected for energy loss in the calorimeter as described in Sections 2.2.4 and 4.2.5. A cut of $I_{\mu}<0$ rejects $96.5 \%$ of the $t \bar{t}$ background while retaining $94 \%$ of the $Z^{\prime}$ events. All these events had identified muon charges. Finally, a cut on the measured dimuon mass of $M_{\mu^{+} \mu^{-}}>3.2 \mathrm{TeV}$ left no $t \bar{t}$ background events.

As discussed in Section 4.2.4, the rate of mismeasured low- $p_{T}$ muons (e.g., ones with their tracks straightened by scattering) is small compared to the real high- $p_{T}$ muon rate. Therefore, the probability of getting two mismeasured high- $p_{T}$ muons with $p_{T}$ balanced to within $300 \mathrm{GeV}$ is wholly negligible.

A potentially serious loss to the $Z^{\prime} \rightarrow \mu^{+} \mu^{-}$ signal comes from muon scattering debris spraying into the muon system and degrading the reconstruction efficiency. This usually affects the first superlayer of the muon system. This effect is discussed in Section 4.2 .4 , and the efficiency was parameterized for use in gemfast. The average reconstruction efficiency for the high-energy $Z^{\prime}$ muons was found to be $85 \%$ per muon. However, it was possible to raise the reconstruction efficiency for $Z^{\prime} \rightarrow \mu^{+} \mu^{-}$ events to $96 \%$ by accepting events with at least one well-reconstructed muon plus one muon with sufficient hits in two unaffected superlayers to determine its angle. 64,65 Since th $p_{T}$ of the poorly-reconstructed muon is expected to match that of the well-reconstructed one to within $300 \mathrm{GeV}$, its momentum can be determined. If necessary to do so, it is reasonable to assume that the charge of the bad muon is opposite that of the good muon. ${ }^{66}$ Finally, the muon reconstruction efficiency and momentum resolution may be significantly enhanced by using the beam line constraint. The knowledge of the beam line relative to the muon system with a precision of about $500 \mu \mathrm{m}$ allows the use of events with no measurable hits in the first superlayer. A goal of the GEM muon design is to know the beam position to within $200 \mu \mathrm{m}$ (see Section 4.1.4). We have not used this constraint in the analysis.

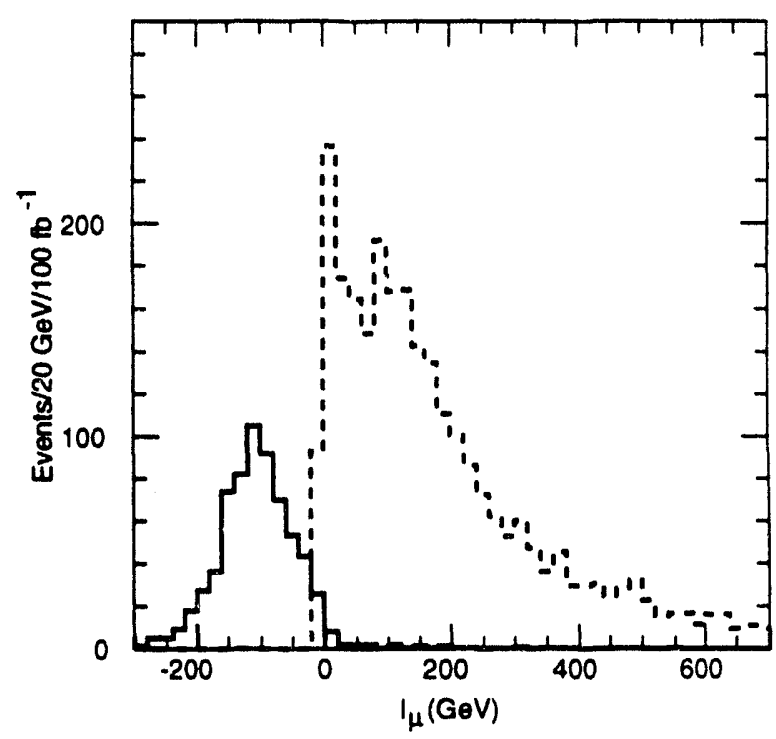

FIG. 2.46. Isolation variable $I_{\mu}=\sum_{R}=0.7 E_{T}-0.1 p_{T}^{\mu}$ for $Z_{1} \rightarrow \mu^{+} \mu^{-}$(solid) and $\bar{t} \rightarrow \mu^{+} \mu^{-}+X$ (dashed). Events have $M_{\mu+\mu-}>1 \mathrm{TeV}$ and $\left|\eta_{\mu}\right|<2.6$ at the generator level.

In summary, the overall acceptance of the signal (including all produced events) is $60 \%$. There are $350 Z_{1}$ and $270 Z_{2} \rightarrow \mu^{+} \mu^{-}$events detected by GEM with $100 \mathrm{fb}^{-1}$ of data. The Drell-Yan dimuon spectrum for the $Z_{2}$ model is shown in Figure 2-47. This shows all events that had generated invariant mass greater than $1.5 \mathrm{TeV}$ and passed all cuts. For comparison, the distribution of dimuon events from the $\bar{t} t$ background that passed the isolation cut is also shown. The Drell-Yan spectra for the two models were fit with an exponential plus a Gaussian to determine the masses of the $Z^{\prime}$ enhancements. These were found to be $M_{Z_{1}}=4036 \pm 40 \mathrm{GeV}$ and $M_{Z_{2}}=3968 \pm 65 \mathrm{GeV}$. The errors are purely statistical. 


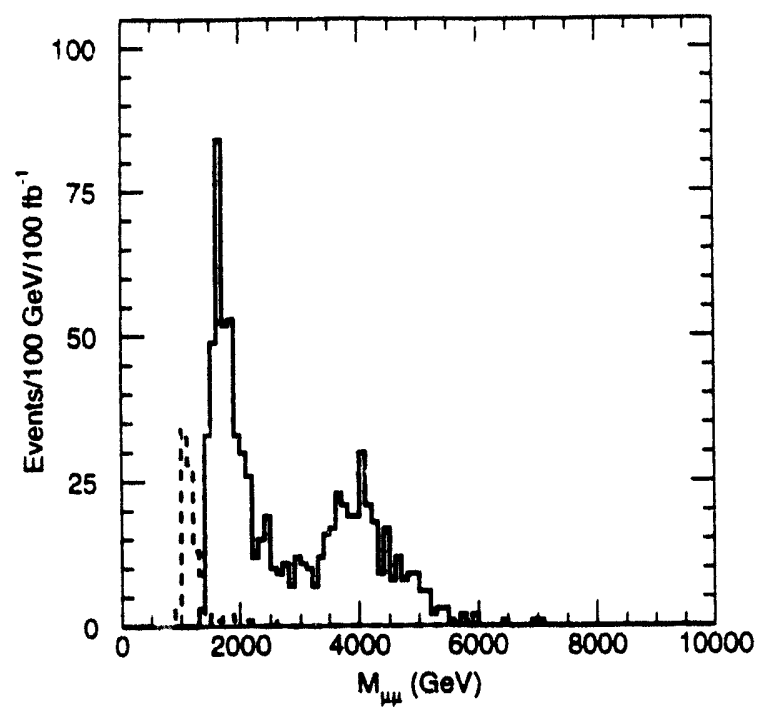

FIG. 2.47. The $M_{\mu+\mu}$ - distribution for the Drell-Yan continuum and $Z_{2} \rightarrow \mu^{+} \mu^{-}$. The $\mathrm{Ht} \rightarrow \mu^{+} \mu^{-}+X$ background before the invariant mass cut is also shown (dashed).

The measured $\cos \theta^{*}$ distributions for Models 1 and 2 are shown in Figures 2-48 and 2-49. Events in the mass range $3.2-4.8 \mathrm{TeV}$ were used for this analysis. There are $319 Z_{1}$ and $257 Z_{2}$ events in this sample; the missing events were lost due to momentum smearing. As noted above, only six Drell-Yan background events contaminate this sample. For comparison, the PYTHIA-generated distributions, corresponding to a perfect detector with GEM's $\eta$-coverage, are shown in these figures. The agreement between theoretical expectation and simulated measurement is quite good. The forwardbackward asymmetries for the two models are:

$$
\begin{aligned}
A_{F B}\left(Z_{1}\right) & =0.113 \pm 0.056(\mathrm{GEM}) \\
& =0.083 \pm 0.051 \text { (PYTHIA) } \\
A_{F B}\left(Z_{2}\right) & =-0.090 \pm 0.062 \text { (GEM) } \\
& =-0.115 \pm 0.058 \text { (PYTHIA) }
\end{aligned}
$$

The PYTHIA and gemfast asymmetries agree well within the statistical errors and the central values of the two models are separated by about $3.5 \sigma$.

\subsubsection{Studies of Quark-Lepton Substructure in Drell-Yan Processes}

The search for quark-lepton substructure may require probing dilepton and dijet masses as high as $10 \mathrm{TeV}$. If a signal is found, the inherent

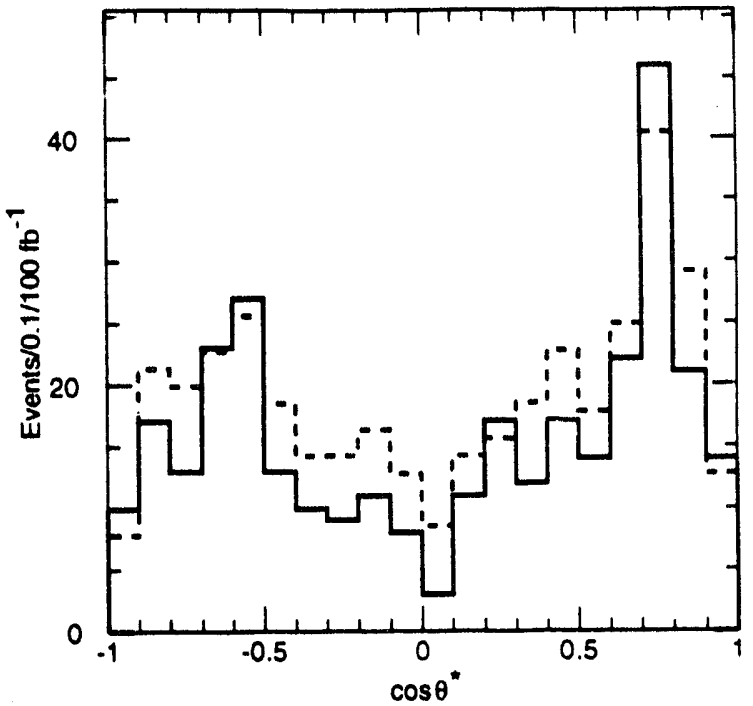

FIG. 2-48. Generated (dashed) and reconstructed (solid) $\cos \theta^{*}$ distributions for $Z_{1} \rightarrow \mu^{-} \mu^{-}$events passing signal selections.

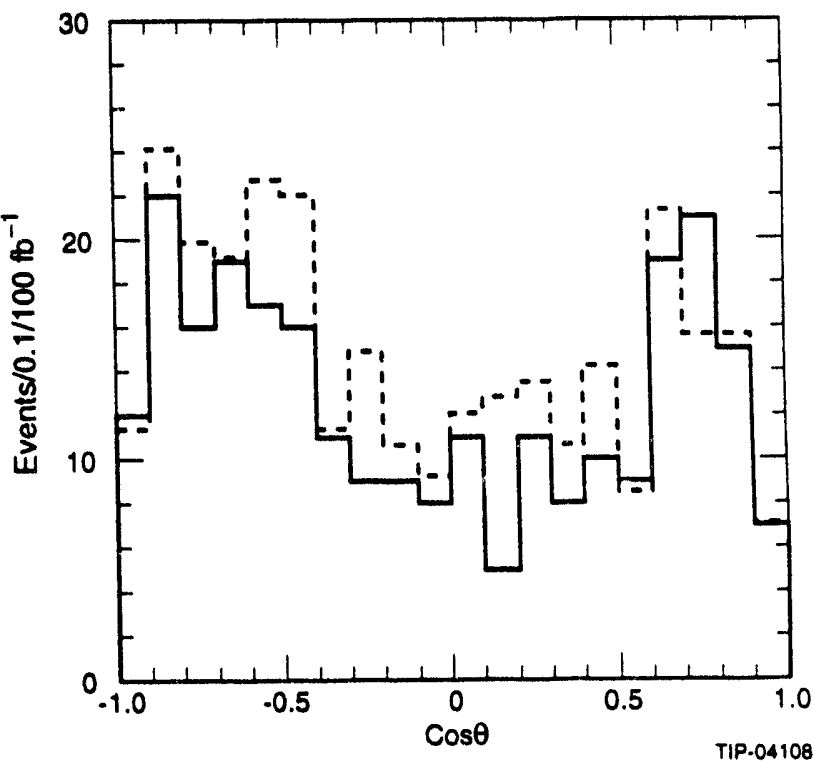

FIG. 2-49. Generated (dashed) and reconstructed (solid) $\cos \theta^{\circ}$ distributions for $Z_{2} \rightarrow \mu^{+} \mu^{-}$events passing signal selections.

precision of lepton measurement at high energies will make the Drell-Yan process a much more incisive tool than high- $E_{T}$ jet production for studying substructure. However, as with the $Z^{\prime}$, hundreds of events at very large energies will be required. As explained in Section 2.5, the contact interactions induced by quark-lepton substructure will be flavorsymmetric if the substructure scale $\Lambda \leqslant 500 \mathrm{TeV}$. Thus, the Drell-Yan processes $p p \rightarrow \ell^{+} \ell^{-}$and 
$\ell^{ \pm} v_{\ell}$ will have equal cross sections for electrons and muons (up to detector-related effects, of course). Signals in $p p \rightarrow \mu^{+} \mu^{-}$are considered here. While it is important to observe the substructure signal in $p p \rightarrow e^{+} e^{-}$as well, the $e^{+} e^{-}$spectrum cannot help distinguish between models because a difference in rate can be compensated for by a change in $\Lambda$. A brief discussion of substructure studies in the $\mu^{ \pm} v_{\mu}$ channel is presented in Section 2.6.3.

Quark-lepton substructure modifies dimuon production in two ways: an excess of events ${ }^{7}$ at high

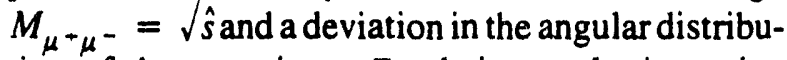
tion of the outgoing $\mu^{-}$relative to the incoming quark. ${ }^{67} \mathrm{GEM}$ 's potential for studies at $10^{34} \mathrm{~cm}^{-2} \mathrm{~s}^{-1}$ of isolated muons at the highest energies, $M_{\mu^{+} \mu^{-}} \leqslant 10 \mathrm{TeV}$, is demonstrated by considering two different chiral forms for the contact interaction $\mathcal{L}_{\Lambda}$ arising from substructure at $\Lambda=25 \mathrm{TeV}$. This scale is well beyond that at which such studies can be done at $10^{33} \mathrm{~cm}^{-2} \mathrm{~s}^{-1}$. The goal is to distinguish these two models by their muon angular distribution. These models received preliminary treatment in Refs. 2 and 3.

In the first model, left-handed quarks and leptons are composite and the contact interaction is the product of two weak-isoscalar currents (the "ISO" model):

$$
\mathscr{L}_{\text {ISO }}=-\frac{4 \pi}{\Lambda^{2}} \bar{Q}_{L a} \gamma^{\mu} Q_{L a} L_{L b} \gamma_{\mu} L_{L b},
$$

where $Q_{L a}=\left(u_{a}, d_{a}\right)_{L}$ and $L_{L a}=\left(\nu_{a}, \ell_{a}\right)_{L}$ are left-handed quark and lepton fields and $a, b=1,2,3$ label generations. This interaction, together with the standard Drell-Yan processes, produces the subprocess cross section

$$
\begin{aligned}
\frac{d \hat{\sigma}\left(q_{i} \bar{q}_{i} \rightarrow \ell-\ell+\right)}{d(\cos \theta)} & =\frac{\pi \alpha^{2}}{24 \hat{s}}\left[A_{i}(\hat{s})(1+\cos \theta)^{2}\right. \\
& \left.+B_{i}(\hat{s})(1-\cos \theta)^{2}\right] .
\end{aligned}
$$

The functions $A_{i}$ and $B_{i}$ were given in Ref. 3. At high energies, $\hat{s}>a \Lambda^{2}, A_{i}(\hat{s})=\left(\hat{s} / a \Lambda^{2}\right)^{2}$ and the angular distribution of $\ell^{-}$relative to $q_{i}$ is approximately $(1+\cos \theta)^{2}$.

The second case involves a helicity nonconserving contact interaction (the "HNC" model) given by
$\mathscr{L}_{\mathrm{HNC}}=-\frac{4 \pi}{\Lambda^{2}} \varepsilon_{i j} \bar{Q}_{L i a} u_{R a} I_{L j b} \epsilon_{K b}+$ h.c., $(2.6 .15)$

where $i, j=1,2$ label indices in an electroweak doublet and $\varepsilon_{12}=-\varepsilon_{21}=1$. This interaction, while theoretically unlikely, is studied here because it generates an angular distribution that becomes isotropic at large $\hat{s}$. The interaction $£_{\mathrm{HNC}}$ affects $u_{i} \bar{u}_{i} \rightarrow \ell^{-} \ell^{+}$only:

$$
\begin{aligned}
\frac{d \hat{\sigma}\left(u_{i} \bar{u}_{i} \rightarrow \ell-\ell+\right)}{d(\cos \theta)} & =\frac{\pi a^{2}}{24 \hat{s}}\left[A_{u}(\hat{s})(1+\cos \theta)^{2}\right. \\
+ & \left.B_{u}(\hat{s})(1-\cos \theta)^{2}\right]+\frac{\pi \hat{s}}{12 \Lambda^{4}} .
\end{aligned}
$$

The functions $A_{u}$ and $B_{u}$ were given in Ref. 3. The contributions of $\mathscr{L}_{\mathrm{HNC}}$ and $\gamma / Z^{0}$ do not interfere because their chiral structures are different.

PYTHIA 5.5 was used to generate 1500 events each of standard Drell-Yan (DY) and DY modified with the ISO and HNC contact interactions with $\Lambda=25 \mathrm{TeV}$. The muons were required to have generated $M_{\mu^{+} \mu^{-}}>2 \mathrm{TeV}$. The cross sections for events in which both muons have $|\eta|<2.46$ are $2.83 \mathrm{fb}, 9.44 \mathrm{fb}$ ald $7.88 \mathrm{fb}$ for the DY, ISO and $\mathrm{HNC}$ cases, respec ively.

Detector response to events was simulated using gemfast with gaussian pileup for $\mathscr{L}=10^{34} \mathrm{~cm}^{-2} \mathrm{~s}^{-1}$. The acceptance was $70 \%$ for events with both muons in $|\eta|<2.46$, consistent with the acceptance of $Z^{\prime} \rightarrow \mu^{+} \mu^{-}$events. Of these, $99.5 \%$ of the standard DY events had the charges of both muons determined. This dropped to $96 \%$ for the ISO and HNC models, reflecting the excess of high-energy muons generated by the contact interactions.

The discussion of backgrounds and muon reconstruction is essentially the same as in the analysis of $Z^{\prime} \rightarrow \mu^{+} \mu^{-}$. The isolation criterion $I_{\mu}<0$ defined in Eq. (2.6.11) and the invariant mass cut $M_{\mu^{+} \mu^{-}}>2.5 \mathrm{TeV}$ eliminated the physics backgrounds. The acceptance of these two cuts for signal events passing previous selections was $41 \%$ for the DY events, $60 \%$ for the ISO events and $66 \%$ for HNC. The higher acceptance in the ISO and HNC cases is due to the excess of high-mass dimuons. Retaining those events in which at least the 
momentum of one muon and the angle of the other are well-me asured, the net reconstruction efficiency was found to be $95 \%$ for the DY and ISO cases and 92\% for the HNC model.

The net acceptances and number of events detected by GEM per $100 \mathrm{fb}^{-1}$ are $23 \%$ and 80 events for DY, $33 \%$ and 360 events for ISO, and $35 \%$ and 315 events for HNC. Figures 2-50 show the $M_{\mu-\mu}$ - distributions for the ISO and HNC detected in GEM per $100 \mathrm{fb}^{-1}$. Also shown are the underlying DY mass distributions. The muon angular distributions were determined as in the $Z^{\prime}$ case. The $\cos \theta^{*}$ distribution for the $\mu^{-}$in the ISO model, compared to DY, is shown in Figure 2-51(a) and for the HNC model in Figure 2-51(b). The DY background was not subtracted from the substructuremodel distributions in these figures. The tendency for a $\left(1+\cos \theta^{*}\right)^{2}$ distribution in the ISO model and a flat one in the HNC model is clear and the two models are very well separated. The forward-backward asymmetries are

$$
\begin{aligned}
A_{F B}(\mathrm{DY}) & =0.295 \pm 0.108 \\
A_{F B}(\mathrm{ISO}) & =0.328 \pm 0.050 \\
A_{F B}(\mathrm{HNC}) & =0.122 \pm 0.056
\end{aligned}
$$

(a)

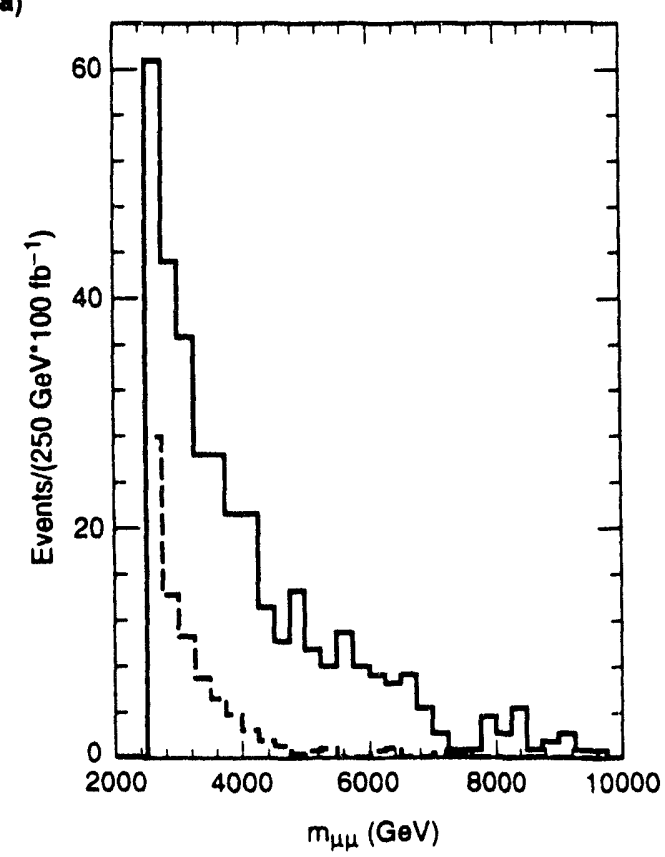

The errors are statistical only. Since the contributions of $\mathcal{L}_{\mathrm{HNC}}$ and Drell-Yan to the HNC events are non-interfering, the Drell-Yan compone ${ }_{i, t}$ can be subtracted. This gives $A_{F B}(\mathrm{HNC})=0.065 \pm$ 0.065 , consistent with an isotropic distribution.

Finally, we estimate the reach in substructure scale $\Lambda$ that GEM could attain with a data sample of $100 \mathrm{fb}^{-1}$. We define the reach as that value of $\Lambda$ which gives $5 \sigma$ over the Drell-Yan expectation, approximately 125 events. Then, since the subprocess cross sections above go as $\hat{s} / \Lambda^{4}$, it is possible to determine the reach by scaling the numbers of events found above. We expect to reach $30-35 \mathrm{TeV}$ ii. one nominal year at ultrahigh luminosity.

\subsubsection{High Mass and Luminosity Physics Studies of $\ell^{ \pm} v_{\ell}$ Modes}

The range of physics that can be studied at ultrahigh luminosity can be greatly extended by searching for isolated, high- $p_{T}$ leptons accompanied by large $\boldsymbol{F}_{T} \approx p_{T}$. Extensions of the standard gauge group generally involve $W^{\prime}$ as well as $Z^{\prime}$ bosons. Their mass and couplings can be determined in high-statistics studies of $W^{\prime \pm} \rightarrow e^{ \pm} v_{e}$ and $\mu^{ \pm} v_{\mu}$, respectively. At $\mathscr{L}=10^{34} \mathrm{~cm}^{-2} \mathrm{~s}^{-1}$, it should be possible to carry out high-statistics studies

(b)

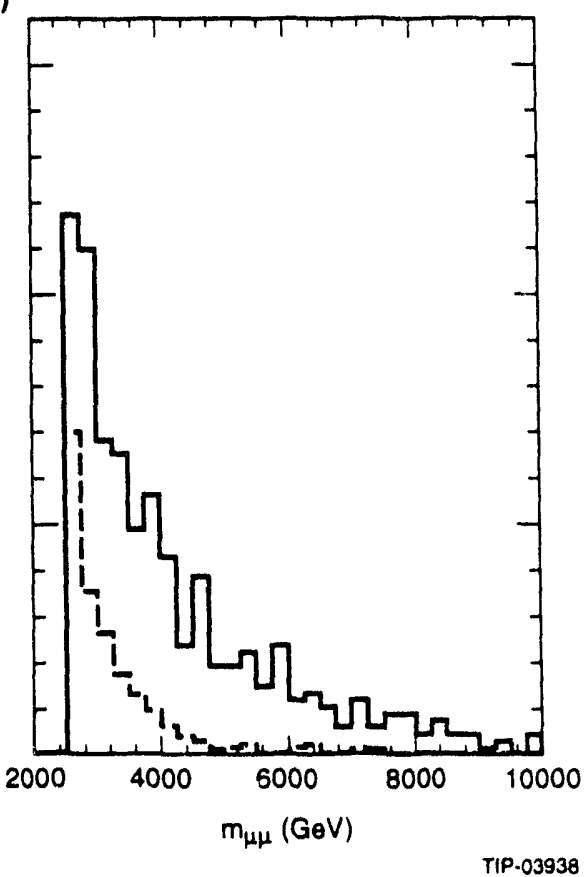

FiG. 2-50. The $M_{\mu-}$ - distributions for the ISO (a) and HNC (b) substructure models defined in the text. The lower dashed histogram is the standard Drell-Yan distribution. 
(a)

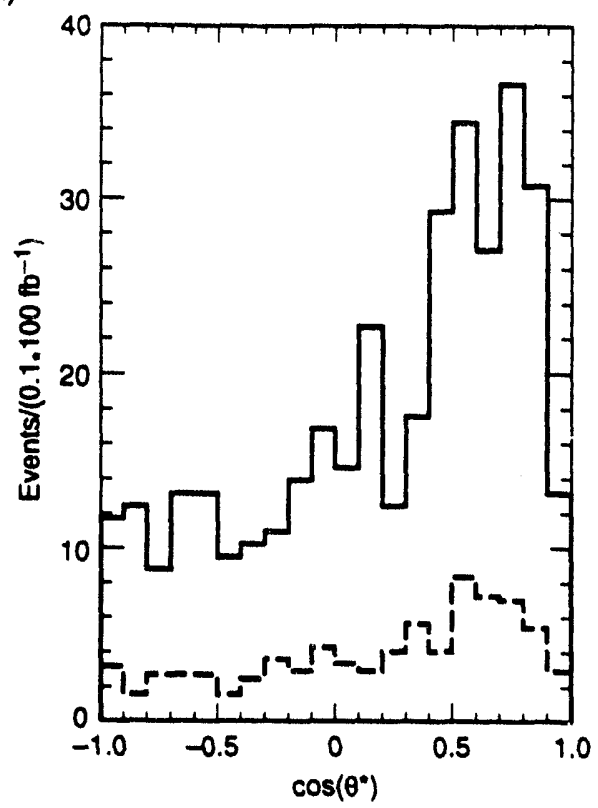

(b)

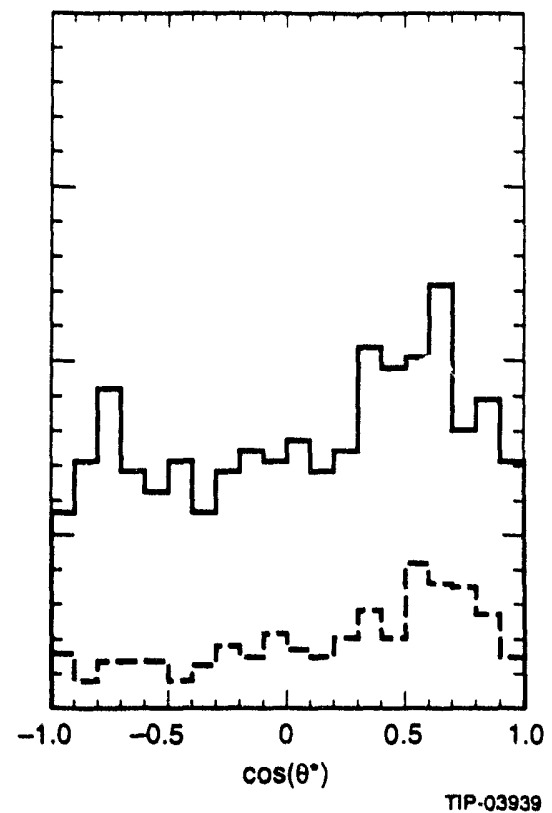

FIG. 2.51. The $\cos \theta^{*}$ distributions for the ISO (a) and HNC dashed histogram is the standard Drell-Yan distribution.

up to $M_{W} \simeq 5 \mathrm{TeV}$ and to reach as high as $10 \mathrm{TeV}$. If contact interactions reflecting an underlying quark lepton substructure exist, they may involve terms of the form $\bar{u} d \bar{\ell} v$ and its conjugate. Much can be learned about the chiral coupling of such interactions by comparing the rapidity distributions of the outgoing $\mu^{+}$and $\mu^{-}$. High-statistics studies should again be possible up to $\Lambda \approx 25 \mathrm{TeV}$. This section contains a brief description of the measurements GEM can perform at $\mathcal{L}=$ $10^{34} \mathrm{~cm}^{-2} \mathrm{~s}^{-1}$ if this new physics exists.

Precise determination of the mass of a $W^{\prime}$-boson should be possible by measuring the $p_{T}$ distribution of the electron in $p p \rightarrow W^{\prime} \rightarrow e v_{e}$. The main issue is how well the Jacobian peak determines $M_{W^{\prime}}$. For high $M_{W^{\prime}}$, there are no significant physics backgrounds. For example, the background jet $+Z^{0}$ where the jet fakes an electron and $Z^{0} \rightarrow \nu \bar{v}$, is removed by the jet rejection $\mathscr{P}_{(}(e / \mathrm{jet})=4 \times 10^{-4}$. The detector-related issues are much the same as in the $Z^{\prime} \rightarrow e^{+} e^{-}$study, except that high precision is not as important here as the resolution on $\boldsymbol{F}_{T} \geqslant 1 \mathrm{TeV}$.

Information on the $W^{\prime}$ couplings to quarks and leptons can be obtained by measuring the angu$\operatorname{lar}(\theta)$ distribution in $W^{\prime \pm} \rightarrow \mu^{ \pm} \nu_{\mu}$ decay. The $\theta$-distribution of $\mu^{-}$relative to the incoming $d$-quark will be the same as that of $\mu^{+}$relative to the incoming $\bar{d}$. Thus, increased statistics can be obtained by adding data from both modes. These angular distributions are the only way in ppcolliders to detect the presence of right-handed neutrinos in the decay of $W^{\prime}$ bosons. To measure them, one proceeds as follows: (1) Select events with $p_{T}(\mu)$ "near" the Jacobian peak found in $W^{\prime} \rightarrow e v_{e}$ and having balancing $\boldsymbol{E}_{T}$. This enhances the signal relative to any Drell-Yan continuum or other background. (2) Measure the muon rapidity $\eta_{\mu}$. (3) Determine the neutrino rapidity, $\eta_{\nu}$, by reconstructing the $W^{\prime}$. For this, assume $\vec{p}_{T}(v)=-\vec{p}_{T}(\mu)$. As noted, this is a good approximation for multi- $\mathrm{TeV}$ $W^{\prime}$ bosons since $p_{T}\left(W^{\prime}\right) \leq 200 \mathrm{GeV} 70 \%$ of the time for $M_{W} \geq 2 \mathrm{TeV}$. The neutrino 4-momentum and $\eta_{\nu}$ are determined up to a quadratic ambiguity by imposing the $W^{\prime}$ mass constraint. One can either select the value that minimizes the $\eta_{W^{\prime}}=\eta_{B}$ or accept each event twice. The analysis of the muon angular $\left(\cos \theta^{*}\right)$ distribution can now be carried exactly as was done for $Z^{\prime}$ and $\bar{q} q \rightarrow \mu^{+} \mu^{-}$. Requiring good hits in all three muon superlayers, the fake high- $p_{T}$ background to the $W^{\prime} \rightarrow \mu+t_{T}$ signal is negligible (see Section 4.2.4). Studies of the analysis procedure are underway. 
Contact interactions of the form $\bar{u} d \bar{\ell} v$ give an excess of muons at high- $p_{T}$. Even though the parton c.m. frame cannot be found in this case, it is still possible to obtain information on the chiral nature of the contact interaction by comparing the rapidity distributions, $\eta_{\mu}-\mid$ and $\eta_{\mu}-\mid$ of muons with $p_{T} \geq 1.5 \mathrm{TeV}$. If, for example, the angular distribution between the incoming $d$-quark and the outgoing $\mu^{-}$is $(1+\cos \theta)^{2}$ in the parton c.m. frame, then $\mid \eta_{\mu}-1$ is pushed to larger values because the $d$-quark is harder than the $\bar{u}$-quark and the $\mu^{-}$tends to be produced forward. Correspondingly, the $\left|\eta_{\mu^{+}}\right|$distribution is squeezed to smaller values. If the angular distribution is flat, as in the HNC model, the two rapidity distributions will be identical. These features are illustrated for the ISO and HNC models in Figures 2-52. These plots were made for the surviving dimuon event samples of Section 2.6.2. The two models are clearly separated by the muons' rapidity distributions. Studies of the event selections and backgrounds are in progress. Finally, concurrent measurements of the angular distributions in the $\mu^{+} \mu^{-}$and $\mu^{ \pm} \nu$ channels should go a long way toward pinning down the chiral structure of the contact interaction.

(a)

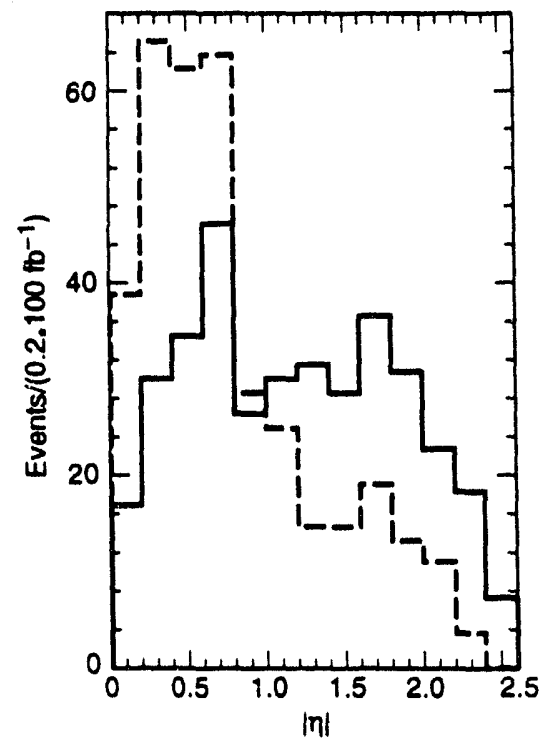

\subsection{SUPERSYMMETRY AND $\ddot{k}_{T}$ SIGNATURES}

Supersymmetry (SUSY) is theoretically attractive because it eliminates the quadratic divergences in the Higgs sector and so allows light elementary Higgs bosons to occur naturally. Its study also provides a good testing ground for many aspects of GEM, including missing energy, jets and leptons. The minimal supersymmetric extension of the standard model 27 or MSSM has two Higgs doublets and superpartners (denoted by a tilde) for all normal particles. In particular there are four neutralinos, $\tilde{\chi}_{i}^{0}$, which are linear combinations of the partners of the photon, $Z$, and neutral Higgs bosons, and two pairs of charginos $\bar{\chi}_{i}^{ \pm}$. If SUSY is broken at the electroweak scale, the masses of all of these particles should be less than about $1 \mathrm{TeV}$. There is a conserved $R$ parity carried by all superparticles, so they must always be produced in pairs and decay to the lightest supersymmetric particle, which is absolutely stable. Only the minimal model with $\tilde{\chi}_{1}^{0}$ being the lightest supersymmetric particle will be considered here. The results demonstrate that in GEM the backgrounds for these signatures are dominated by standard model physics, not by detector effects.

(b)

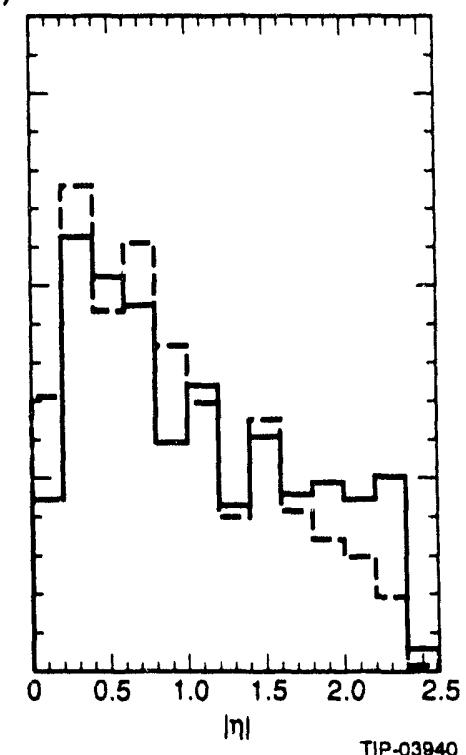

FIG. 2-52. $\quad\left|\eta_{\mu}^{-}\right|$(solid) and $\left|\eta_{\mu}^{+}\right|$(dashed) distributions for the ISO (a) and HNC (b) compositeness modeis defined in the text. 


\subsection{1 $\Psi_{T}$ Signature for Gluinos and Squarks}

Since the lightest supersymmetric particle $\bar{\chi}_{1}^{0}$ is neutral and interacts weakly with matter, it escapes from the detector. Thus, one of the basic signatures for SUSY is missing transverse energy, $\boldsymbol{F}_{T}$, from the $\bar{\chi}_{1}^{0}$ plus multiple jets. A stringent test for GEM's missing energy resolution is to be able to detect, in this mode, gluinos and squarks with masses as light as $300 \mathrm{GeV}$. This is near the limit expected from the Tevatron and is also the mass range expected in some SUSY grand unified models. ${ }^{68,69}$ The MSSM typically produces cascade decays from one supersymmetric particle to another. The events can have many jets and leptons, and the missing energy from the final lightest sup orsymmetric particle $\bar{\chi}_{1}^{0}$ can be small compared to the parent mass. A typical decay sequence for a relatively light squark and gluino with $M_{\dot{q}}>M_{\dot{g}}$ might be

$$
\begin{aligned}
\tilde{u}_{L} & \rightarrow \bar{g} u, \\
\tilde{g} & \rightarrow \bar{\chi}_{1}^{+} \bar{u} d, \\
\tilde{\chi}_{1}^{+} & \rightarrow \bar{\chi}_{1}^{0} e^{+} v .
\end{aligned}
$$

Decay chains can be even more complex for heavier masses. All of these possible decays are included in the version of ISAJET ${ }^{70}$ used for this analysis. ${ }^{71}$

There are a number of other parameters in the MSSM besides the gluino and squark masses, and it is beyond the scope of this study to explore the MSSM parameter space completely. Instead, the representative choices listed in Table 2-17 have been considered. Case I has a light gluino and a heavier squark; it is generally similar to the models of Ref. 68 and to the case considered in previous GEM studies. ${ }^{3}$ Case II has a squark slightly lighter than the gluino and is generally similar to the models of Ref. 69. Since $\bar{g} \rightarrow \bar{q} q$ dominates for $M_{\dot{g}}>M_{\dot{q}}$, the signatures in this case are similar to those for squark pair production. One might think that this case would be more difficult to detect because the events contain just two hard jets from $\tilde{q} \rightarrow \tilde{\chi}_{1}^{0} q$. It is actually easier, because the branching ratios for $\tilde{q}_{L} \rightarrow \tilde{\chi}_{1}^{ \pm} q$ and $\tilde{q}_{L} \rightarrow \tilde{\chi}_{2}^{0} q$ turn out to be large and to provide multijet signatures, and the dominant decay $\tilde{q}_{R} \rightarrow \bar{\chi}_{1}^{0} q$ gives a harder $\tilde{F}_{T}$ distribution. Finally, Case III has all the masses pushed to their highest values if SUSY is to be related to the electroweak scale. It tests the ability of GEM to cover the top of the plausible mass range for weak-scale supersymmetry.

For the three cases samples of $70 \mathrm{~K}, 25 \mathrm{~K}$, and $35 \mathrm{~K}$, respectively, of gluino and squark signal events was generated with a version of ISAJET containing all the MSSM decay modes. ${ }^{70}$ The total production cross sections for all combinations of gluinos and squarks are

$$
\begin{aligned}
\text { Case I : } & \sigma=8.27 \mathrm{nb}, \\
\text { Case II : } & \sigma=7.60 \mathrm{nb}, \\
\text { Case III : } & \sigma=0.81 \mathrm{pb} .
\end{aligned}
$$

The Monte Carlo statistics are therefore small compared to those obtained in $10 \mathrm{fb}^{-1}$ for the first two cases but comparable in the third. This is reflected in the error bars on the plots shown below.

Table 2-17. Choices of MSSM parameters for the three cases considered. These were chosen to have different event topologies and to span the whole mass range. All the squarks and all the sleptons are taken to be degenerate for simplicity. All masses are in GeV. See Ref. 27 for the notation.

\begin{tabular}{cccc}
\hline Parameter & Case I & Case II & Case III \\
\hline$M_{i}$ & 300 & 350 & 2000 \\
$M_{Q}$ & 600 & 325 & 2500 \\
$M_{i}$ & 500 & 200 & 1500 \\
$M_{A}$ & 300 & 300 & 300 \\
$\mu$ & -300 & -300 & -1000 \\
$\tan \beta$ & 2 & 2 & 2 \\
\hline \hline
\end{tabular}

The signal events are characterized by multiple jets and large missing energy. For the lower masses in Cases I and II the dominant standard-model physics background comes from heavy flavors decaying into neutrinos, and the dominant detectorinduced background comes from mismeasuring QCD jets. A total of $1.5 \mathrm{M} Q C D$ jets of all types in twelve $p_{T}$ ranges covering $50<p_{T}<3200 \mathrm{GeV}$ was generated with ISAJET to determine both kinds of backgrounds. For the high masses in Case III, the backgrounds from $W \rightarrow \ell v$ and $Z \rightarrow v \bar{v}$ are also significant. A total of $40 \mathrm{~K} W \rightarrow \ell v$ and $80 \mathrm{~K} Z \rightarrow v \bar{\nu}$ events were generated covering the same $p_{T}$ range.

The detector response to all events was simulated with gemfast. The missing energy was calculated using the single-particle $p_{T}$ resolution of the forward calorimeter determined from GEANT 
plus an additional $1 \%$ nongaussian tail three times as wide as the main peak, as described in Section 2.2.3. The effect of this nongaussian tail is small compared to the effects of angular resolution in the forward calorimeter and of the hole for the beam pipe, so its exact parameterization is not crucial.

In the inclusive $\boldsymbol{E}_{T}$ cross section, the standardmodel physics background is larger than the signal. Furthermore, the detector-induced background from mismeasured jets in the forward region is several times larger than the real background, even for an ideal calorimeter covering $\eta<5.5 .^{72}$ First consider the lower-mass Cases I and II. Since gluinos and squarks are centrally produced with $p_{T} \sim M$, they give multiple jets and "round" events in addition to $F_{T}$. Jets with $p_{T}>75 \mathrm{GeV}$ were found using the gemfast fixed cone algorithm with $R=0.7$. The minimum number of jets, $N_{\text {jet }}$, was varied between two and five. To identify round events, the sphericity in the transverse momentum plane, $S_{T}$, was calculated by summing all calorimeter cells with $E_{T}>0.5 \mathrm{GeV}$ and $|\eta|<3$. A cut on $S_{T}>0.2$ provided good separation of signal and background. After these cuts the signal to background ratio $S / B$ for $E_{T} \sim 250 \mathrm{GeV}$ was about 3 for Case I and about 5 for Case II. The larger $S / B$ for Case II reflects the harder $E_{T}$ spectrum from $\bar{q}_{R}$ decays mentioned earlier.

Semileptonic decays of gluinos and squarks are important; see Section 2.7.2 below. However, a lepton veto further improves the $S / B$ for the $\hat{F}_{T}$ distribution by rejecting $t \bar{t}$ and other standard model backgrounds. Events were vetoed if they contained a muon or an isolated electron. An electron was identified as an isolated electromagnetic cluster in the calorimeter with $p_{T}>20 \mathrm{GeV}$ and $|\eta|<2.5$, matched to a single track in the central tracker with loose matching constraint,

$$
|E / p-1|<\max \left(0.5,3 \sigma_{p}\right) .
$$

Isolated muons with $p_{T}>20 \mathrm{GeV}$ and $\eta<2.5$ were identified using the standard gemfast muon reconstruction. The efficiency of the lepton identification is not crucial for this analysis; even if it were perfect, there still would be background from $\tau$-decays of $b$ and $t$ quarks.

The signal and background $\boldsymbol{E}_{T}$ distributions for Case I with at least five jets and the sphericity and lepton veto cuts described above are shown in Figure 2-53. The $(S+B) / B$ ratio, shown in Figure 2-54, reaches about 8 for $E_{T}=250 \mathrm{GeV}$. Figure $2-54$ also shows the $(S+B) / B$ ratio obtained using $E_{T}$ calculated from the the missing $v$ and $\bar{\chi}_{1}^{0}$ momenta, with the rest of the analysis unchanged. While the GEM calorimeter performance increases the background at low $E_{T}$, it provides reasonable agreement with the perfect detector result in the region for which the ratio is large. The $(S+B) / B$ ratio is larger than that found previously ${ }^{3}$ after similar cuts, partly because both gluinos and squarks are now included, and partly because the description of the decays has been improved and the other MSSM parameters are slightly different. For the same physics assumptions used before, the new simulation gives $(S+B) / B \approx 4$. This is somewhat smaller than found previously, reflecting the larger beam pipe and the more realistic description of the central and endcap calorimeters.

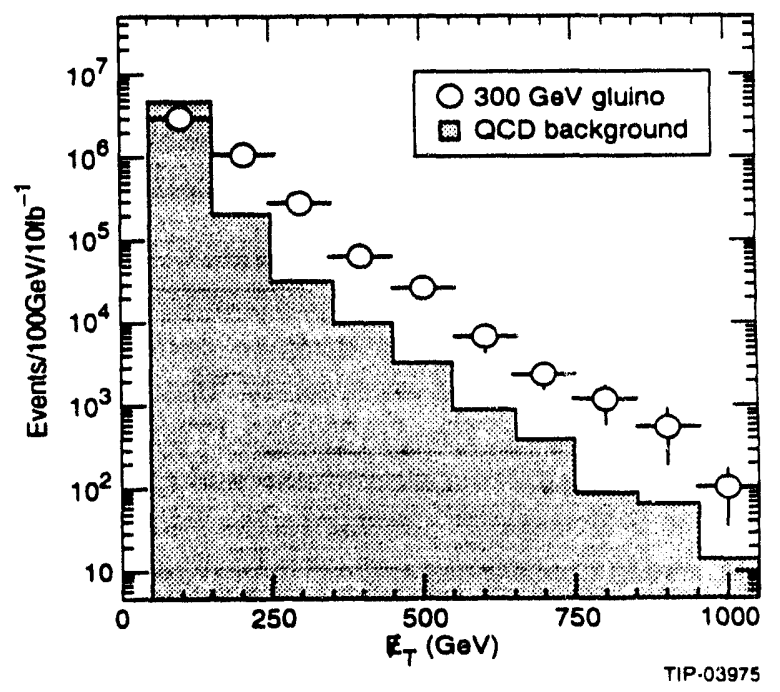

FIG. 2-53. Fignal for Case I MSSM parameters defined in Table 2-17 (open circles) and for QCD background (histogram) after requiring at least 5 jets with $p_{T}>75 \mathrm{GeV}$ and the sphericity and lepton veto cuts described in the text.

Figure 2-55 shows the signal and background for Case II, requiring at least two jets with $p_{T}>$ $75 \mathrm{GeV}$ and the same sphericity and lepton veto cuts. For this case the direct decay $\bar{q}_{R} \rightarrow \bar{\chi}_{1}^{0} q$ dominates and leads to a significantly harder $\boldsymbol{F}_{T}$ spectrum and to lower jet multiplicity. The $(S+B) / B$ ratio is even larger in this case. Fig- 


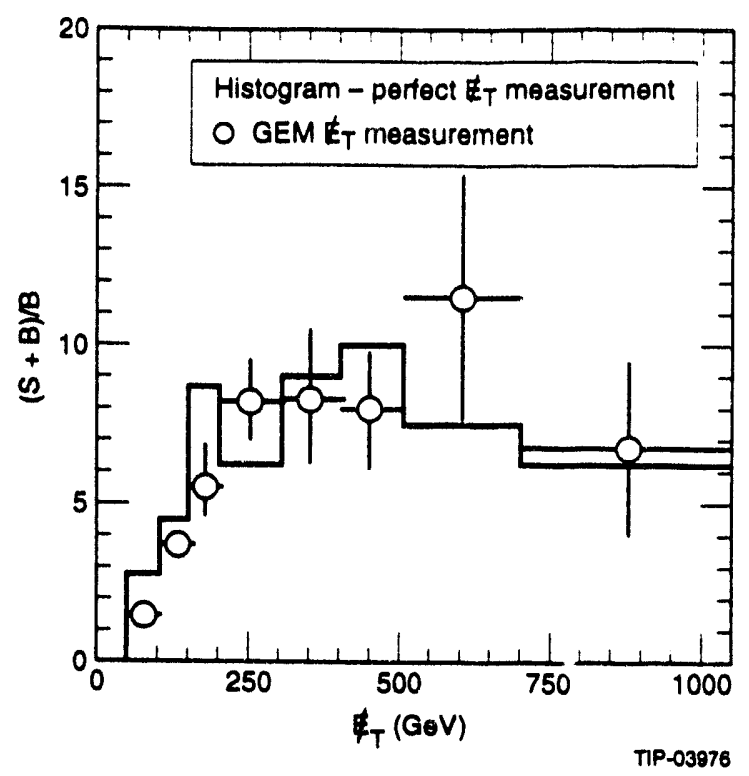

FIG. 2-54. Open circles: Ratio of signal and background curves from Figure 2-53. Histogram: The same ratio for a perfect $E_{T}$ measurement.

ure 2-56 plots the signals for Cases I and II and the standard model background for $\boldsymbol{E}_{T}>250 \mathrm{GeV}$ and $S_{T}>0.2$ vs. the minimum number, $N_{\text {jet }}$, of jets with $p_{T}>75 \mathrm{GeV}$. Both signals and backgrounds are constant for $N_{\text {jet }} \leq 2$. It is impossible to have a large sphericity with only one jet. The signal falls off faster with increasing $N_{\text {jet }}$ for Case II than for Case I because $\tilde{q}_{R} \rightarrow \bar{\chi}_{1}^{0} q$ is dominant and gives a large rate for two jets plus $F_{T}$. Thus, the $N_{\text {jet }}$ dependence provides a handle to distinguish among models.

Given the large number of signal events, the statistical significance of the signals is not an issue. The $t, W$ and $Z$ backgrounds can be checked using isolated lepton samples; the $b$ and $c$ backgrounds can be checked using muons in jets. The $\boldsymbol{E}_{T}$ resolution of the detector can be studied using inclusive data on QCD jets and on $\gamma+$ jets events. While the detector effect is larger than in the less realistic Baseline I design, it is still not the dominant problem. Given all these constraints, the background should be reliably known, so observation of a signal 5-10 times that expected from the standard model should be very convincing. The difficult problem of extracting the masses and other model parameters is briefly discussed in Section 2.7.3.

For heavy gluino and squark masses such as those in Case III, $\boldsymbol{E}_{T}$ is so large that the $\boldsymbol{E}_{T}$ resolution is not important. Figure 2-57 shows the

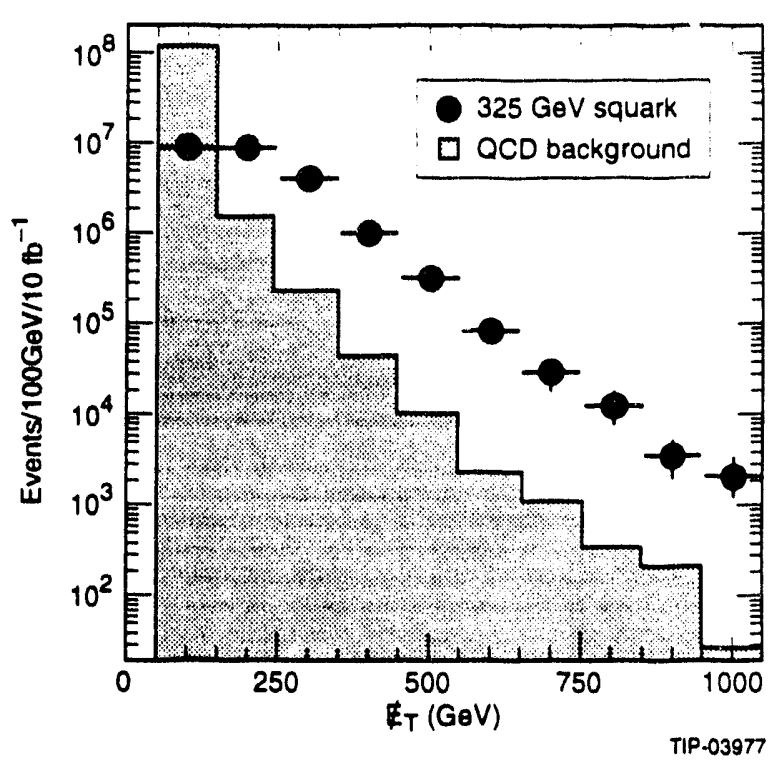

FIG. 2-55. E signal for Case II MSSM parameters defined in Table 2.17 (solid circles) and for QCD background (histogram) after requiring at least 2 jets with $p_{T}>75 \mathrm{GeV}$ and the sphericity and lepton veto cuts described in the text.

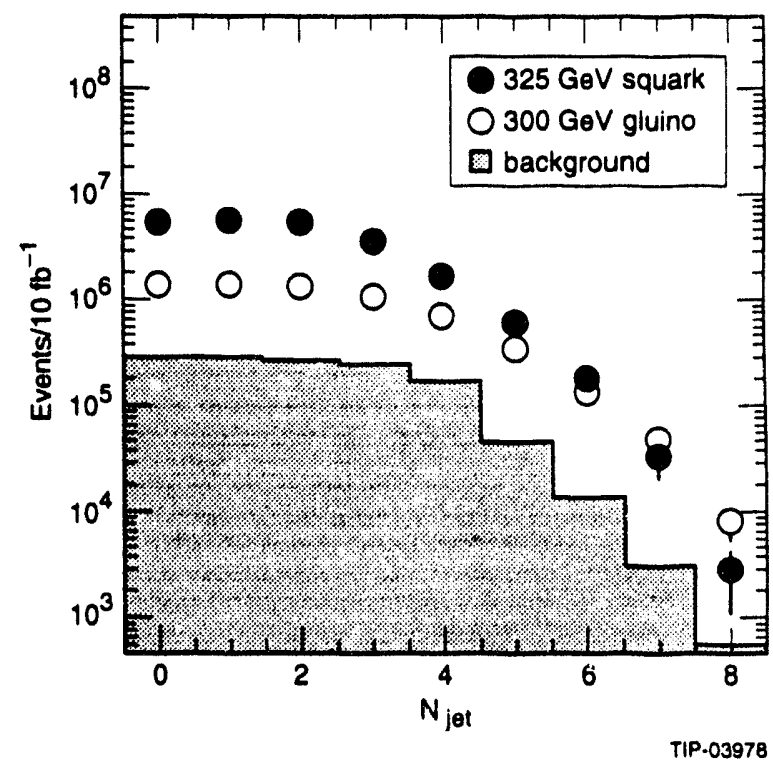

FIG. 2-56. Event numbers with $E_{T}>250 \mathrm{GeV}$ and the sphericity and lepton veto cuts described in the text vs. the minimum number $N_{\text {let }}$ of jets with $p_{T}>75 \mathrm{GeV}$. Open circles: Case I signal. Solid circles: Case II signal. Histogram: QCD background. Case II has more events with low jet multiplicity because $\bar{q}_{R} \rightarrow \tilde{\chi}_{1}^{0} q$ dominates.

signal and background $\boldsymbol{F}_{T}$ distributions for Case III with at least four jets having $p_{r}>300 \mathrm{GeV}$ and with the sphericity and lepton veto cuts identical to those for lighter masses. Heavy flavor backgrounds, detector-induced backgrounds from mismeasured 
$Q C D$ jets, and $W$ and $Z$ backgrounds are included. The QCD background dominates for low $E_{T}$ but falls more rapidly than the $W$ and $Z$ backgrounds, and both must be considered. Since several hundred signal events survive these cuts with large $S / B$, it is evident that GEM could discover SUSY in this channel up to masses of order $2 \mathrm{TeV}$, about the upper limit if SUSY is related to electroweak symmetry breaking. For such heavy masses the ability to run at high luminosity may be important.

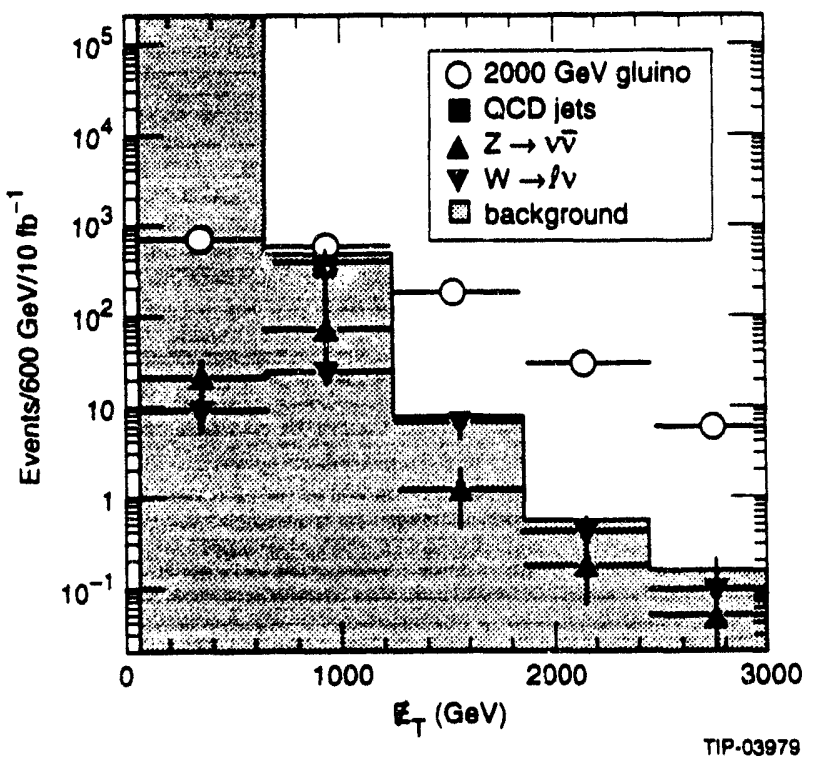

FIG. 2-57. E signal for Case III MSSM parameters defined in Table 2-17 after requiring at least 4 jets with $P_{T}>300 \mathrm{GeV}$ and making the sphericity and lepton veto cuts described in the text. Open circles: Signal. Solid squares: $Q C D$ background. Triangles: $W \rightarrow \ell v$ and $Z \rightarrow v \bar{\nu}$ backgrounds. Histogram: Sum of all backgrounds.

\subsubsection{Leptonic Signatures}

In addition to the $\boldsymbol{F}_{T}$ plus multi-jet signatures described above, there are many other signatures for supersymmetry, including a number involving two or more leptons. ${ }^{73}$ In particular, since the gluino is a self-conjugate Majorana fermion, $\bar{g} \bar{g}$ and $\bar{g} \bar{q}$ pairs can give isolated $\ell^{ \pm} \ell^{ \pm}$pairs. Observing such likesign pairs is essential for establishing the Majorana nature of any gluino signal. It also helps in separating gluinos and squarks. The dominant standard model $\ell^{ \pm} \ell^{ \pm}$background is expected to be from $t \bar{l}$ events in which either a $b \rightarrow \ell X$ lepton appears isolated or an isolated lepton sign is wrongly determined. These backgrounds, calculated previously in Ref. 73, are found to be negligible. For light gluinos, such as those in Cases I and II. the cross sections are so large that one can rely only on the $\mu^{ \pm} \mu^{ \pm}$channel, for which the lepton signs are very well determined in GEM. Therefore, only the issue of measuring the signs of electrons from Case III is addressed here.

The same sample of Case III signal events described in the previous subsection was used for this analysis. While it is possible to enhance the leptonic sample by forcing a particular decay chain, e.g. $\bar{g} \rightarrow \bar{\chi}_{1}^{ \pm} q \bar{q}^{\prime}, \bar{\chi}_{1}^{ \pm} \rightarrow \bar{\chi}_{1}^{0} \ell \pm \nu$, there are many such chains possible, no one of which obviously dominates. It was therefore decided to use the inclusive sample. For the background, only $t \bar{t}$ events, which are expected to dominate, were considered. A total sample of $30 \mathrm{~K} t \bar{t}$ events in twelve bins with $50<p_{T}<3200 \mathrm{GeV}$ were generated, forcing the decays $t \rightarrow e^{+} v_{e} b$ and $\bar{t} \rightarrow \mu^{-} \bar{v}_{\mu} X$. This sample was used to determine the principal detector-induced background, that from misidentification of $e^{ \pm}$signs in the central tracker. From this, the total $\ell^{ \pm} \ell^{ \pm}$background was determined.

Electrons and muons with $p_{T}>50 \mathrm{GeV}$ and $|\eta|<2.5$ were identified using the relatively loose cuts described in the previous subsection. These cuts, optimized for background rejection rather than for signal detection, appear to be adequate to identify this signal. At least two such leptons were required satisfying the isolation criterion

$$
\sum_{R=0.2}^{\prime} E_{T}<0.1 p_{T \ell}+5 \mathrm{GeV} .
$$

Here, the prime on the sum indicates that the lepton itself is not included. This cut effectively rejects ${ }^{73}$ the background from $t \rightarrow \ell^{+} \nu b$ and $\bar{t} \rightarrow \bar{b} X$, $\bar{b} \rightarrow \ell^{+} X$. In addition a missing energy $\hat{c}_{T}>$ $500 \mathrm{GeV}$ and a transverse sphericity $S_{T}>0.2$ were required. After these cuts, the total dilepton rates for the signal and for the $t \bar{t}$ background were comparable, so a very large rejection of unlike-sign pairs is not needed.

Figure 2-58 shows the transverse momentum distribution of the highest $p_{T}$ lepton in like sign dilepton events satisfying the above cuts. The lepton spectrum is soft relative to the gluino mass because the leptons arise from cascade decays. Figure $2-58$ also shows the $t \bar{t} \rightarrow \ell^{+} \nu b \ell^{-} \bar{\nu} \bar{b}$ back- 
ground in which an electron sign is mismeasured by the GEM central tracker. The probability of mismeasurement was determined using the gemfast parameterization of the central tracker electron resolution, including the tail from bremsstrahlung, as described in Section 2.2.2. Muon signs are assumed perfectly determined, an excellent approximation at these momenta. Since the cuts not dependent on the electron sign reduce the background to the order of the signal, and since most of the signal leptons have $p_{T}<600 \mathrm{GeV}$, for which the central tracker determines signs with $95 \%$ reliability, it is not surprising that the background is small compared to the signal. The signal comprises several tens of events per $10 \mathrm{fb}^{-1}$ when both electrons and muons are combined. It would be uncomfortably small if one had to rely on only the $\mu^{ \pm} \mu^{ \pm}$signal, which is a factor of four smaller. Thus, the ability to identify electron signs improves the performance of GEM for this physics.

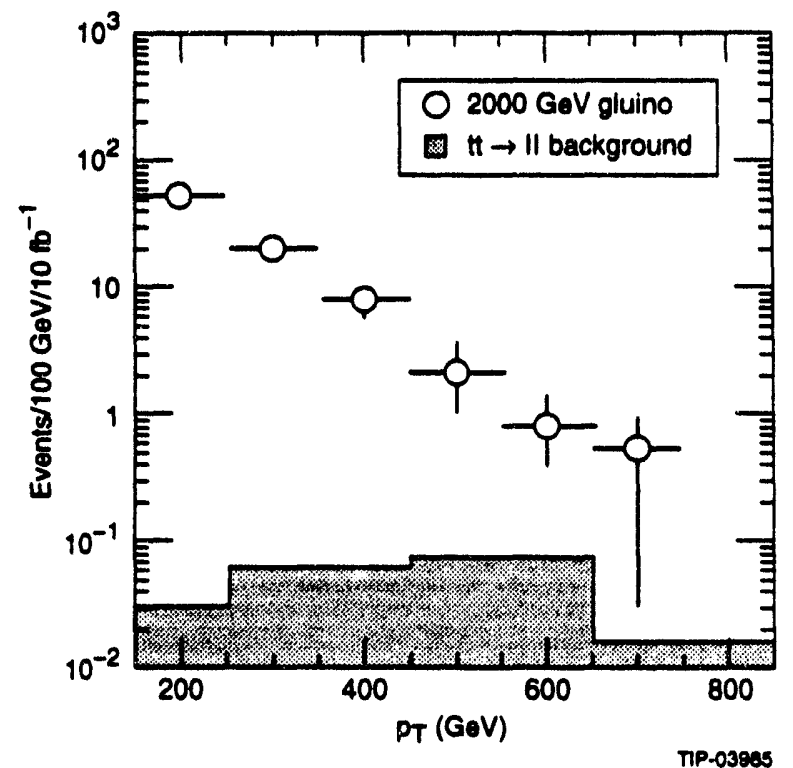

FIG. 2-58. $p_{T}$ distributions for the highest- $p_{T}$ isolated lepton in dilepton events containing two isolated like-sign leptons. Open circles: Signal events generated with Case III MSSM parameters. Histogram: If background from mismeasured electrons in the GEM central tracker.

\subsubsection{SUSY Parameter Determination}

In the MSSM there are at least eight mass parameters $\left(M_{\bar{g}}, M_{\bar{q}}, M_{i_{i}}, M_{i_{R}}, M_{\bar{\ell}_{i}}, M_{\bar{\ell}_{R}}, M_{\bar{v}_{L}}\right.$, and $\left.M_{A}\right)$, two additional parameters related to the Higgs sector $(\mu$ and $\tan \beta)$, and still more parameters related to $i$ decay. Non-minimal SUSY models have even more parameters. Since all supersymmetric particles in the MSSM ultimately decay into the $\tilde{\chi}_{1}^{0}$, which is invisible, it is not possible to reconstruct any masses directly.

The approximate mass scale can be inferred from the $\boldsymbol{E}_{T}$ scale at which the signal deviates from the standard model background, as can be seen by comparing Figures 2-53,2-55, and 2-57. The mean $F_{T}$ for the distribution of the excess of events can be calculated very accurately for low masses because of the high statistics. However, the relationship of this mean to the masses is model dependent. For example, the missing energy is lower and the jet multiplicity is higher if $M_{\dot{q}}>M_{\dot{g}}$ than if $M_{\dot{q}}<M_{\dot{g}}$.

There are a large number of possible signatures to use to determine the parameters. These include the $\boldsymbol{E}_{T}$ cross section with multiple leptons, ${ }^{73}$ multilepton cross sections arising from production of $\bar{\chi}_{1}^{ \pm} \tilde{\chi}_{2}^{0} \rightarrow 3 \ell$ and similar channels, and the observed cross sections or limits for $h, H \rightarrow \gamma \gamma ; h, H \rightarrow 4 \ell$; and $t \rightarrow H^{+} b$. The tools to simulate these signatures have recently been developed, ${ }^{70}$ and the methods to determine all of the MSSM parameters from these signatures are being studied. However, it is clear from the previous two subsections that GEM is capable of observing clean samples of events in the relevant channels.

\subsection{CONCLUSIONS}

In this chapter we have presented in-depth simulations of the GEM detector's response for a selection of electroweak, flavor, and new gauge-interaction physics processes. The examples studied were chosen for their ability to help quantify GEM's capabilities for new physics and to aid in the detector's design and optimization, as well as for their intrinsic physics interest. The simulations were generally carried out using gemfast, a GEANTbased parameterization of the GEM detector. Detailed GEANT simulations were employed in studies where the fast parameterization of GEM was insufficient.

We summarize our principal conclusions:

- GEM is capable of discovering new physics at the SSC. As a result of its high resolution for leptons and photons, it is able to cover a wide range of new physics processes. Most importantly, GEM's ability to measure isolated 
lepton and photon signatures cleanly makes it possible to discover new physics in the $\mathrm{TeV}$ energy region that is unexpected.

- GEM can discover the standard neutral Higgs boson, if it exists, for any mass in the range between the LEP II limit of about $80 \mathrm{GeV}$ up to above the triviality limit of about $700 \mathrm{GeV}$, with an integrated luminosity of $10-30 \mathrm{fb}^{-1}$ (see Table 2-3). The higher integrated luminosity is needed only at the extremes of the mass range of interest: near 80 and near $800 \mathrm{GeV}$. In the intermediate mass region of $80-150 \mathrm{GeV}$, the energy and pointing resolutions of the electromagnetic calorimeter, and the vertex finding and $e / \gamma$ separation capabilities of the central tracker, are essential for detecting the $H^{0} \rightarrow \gamma \gamma$ signal above the backgrounds. If a signal is found, GEM has the important capability of being able to confirm the discovery in the associated production channel, $H^{0}(t \bar{t} / W) \rightarrow \ell^{ \pm} \gamma \gamma$. In the difficult mass region up to $150 \mathrm{GeV}$, detection of the Higgs signals in both of these channels is essential for the discovery. The combined significances for the discovery (in one standard SSC year) of the other intermediate mass Higgs channels, $H^{0} \rightarrow Z^{*} \rightarrow \ell^{+} \ell^{-} \ell^{+} \ell^{-}$, are all high except near the cross section minimum at $M_{H} \approx 170 \mathrm{GeV}$. Near $800 \mathrm{GeV}$, discovery with an integrated luminosity of $10 \mathrm{fb}^{-1}$ requires confirmation of the $\ell^{+} \ell^{-} \ell^{+} \ell^{-}$signal in the $\ell^{+} \ell^{-} \bar{v} v$ and, possibly, the $\ell^{+} \ell^{-}$jet jet channels. Alternatively, a clear signal can be obtained in the $\ell+\ell-\ell+\ell-$ channel within a year at higher luminosity $\left(100 \mathrm{fb}^{-1}\right)$.

- Heavy top quarks can be discovered quickly and their mass can be measured with considerable precision. By measuring the invariant mass of an isolated lepton and a non-isolated muon from $t$-decay, the mass of a 200-300 GeV top quark can be determined to within a few $\mathrm{GeV}$, with an integrated luminosity of $10 \mathrm{fb}^{-1}$. GEM's high identification and reconstruction efficiency for non-isolated muons is central to this measurement. Because the event rate is so high, we may require a second isolated lepton to further purify the sample. Alternatively, a direct $b$-tag by the central tracker can be employed (see Section 6.2.3). The top-mass can also be determined in the decay mode $t \rightarrow 3$ jets. A $b$-tag is not required for the determination of $m_{t}$ via the $M_{\mathrm{iij}}$ distribution. However, because of the kunematics in the decay of a lighter $O(140 \mathrm{GeV}) t$-quark, the tag is useful for obtaining a clean $W \rightarrow$ jet jet decay sample and $M_{W}$ measurement. Systematic errors dominate in this measurement of the top-mass. These can be minimized by using the measuiement of $M_{W}$ as a nearby calibration point.

- The tracker and calorimeters make it possible for GEM to discover a charged scalar in the decays $t \rightarrow H^{+} b, H^{+} \rightarrow \tau^{+} \nu_{\tau}, \tau \rightarrow 1$ or 3 prongs. A large range of $t$ and $H^{+}$branching ratios (alternatively, the parameter $\tan \beta$ occurring in the minimal supersymmetric extension of the standard model) is accessible with a data sample of $10 \mathrm{fb}^{-1}$. Studies in progress on the hadronic decays $\mathrm{H}^{+} \rightarrow c \bar{s}$ are expected to show that GEM can measure the $\mathrm{H}^{+}$mass to within a few $\mathrm{GeV}$.

- The jet energy resolution of $60 \% / \sqrt{E} \oplus 4 \%$ expected for the GEM calorimeter is matched to all physics tasks we envisage. Much more critical will be our knowledge of the jet energy scale and, hence, the $4 \%$ systematic term. Using relatively simple energy weighting schemes to correct for the calorimeter's nonlinearity, a quark substructure scale of $\Lambda \approx 25 \mathrm{TeV}$ can be detected with a data sample of $10 \mathrm{fb}^{-1}$. Verification of this conclusion requires testing the schemes against real data at lower energies.

At the highest SSC luminosity, GEM could probe quark substructure up to a scale of about $45 \mathrm{TeV}$ with a data sample of $100 \mathrm{fb}^{-1}$.

- Operations at the highest luminosity the SSC is expected to deliver, $\mathcal{L} \approx 10^{34} \mathrm{~cm}^{-2} \mathrm{~s}^{-1}$, are essential for realizing the full high-energy potential of the collider and for covering the largest possible mass range in the search for new physics. The GEM design stresses full functionality of its muon and calorimeter systems and sufficient central tracker performance at ultrahigh luminosity. With a data sample of $100 \mathrm{fb}^{-1}$, GEM can distinguish among different $Z^{\prime}$-boson models on the basis 
of the width measured in the $Z^{\prime} \rightarrow e^{+} e^{-}$ decay mode, and angular distributions measured in the $\mu^{+} \mu^{-}$mode. For $M_{Z^{\prime}}=4 \mathrm{TeV}$, the mass resolution is found to be $\Delta M_{e^{+} e^{-}} / M_{e^{-e^{-}}} \simeq 0.3 \%$. Thus, a width as small as $30 \mathrm{GeV}$ can be measured in GEM for a $4 \mathrm{TeV} Z^{\prime}$. Differences in width of $10-15 \mathrm{GeV}$ correspond to $3 \sigma$ separation for the models we considered. GEM's reach in the $e^{+} e^{-}$mode depends on the integrated luminosity, and is about $M_{Z^{\prime}}=8 \mathrm{TeV}$ with $100 \mathrm{fb}^{-1}$. Approximately $95 \%$ of the $Z^{\prime} \rightarrow \mu^{+} \mu^{-}$events within the muon system's geometrical acceptance are reconstructed. The probability of mismeasuring both muon signs is about $10^{-4}$. Thus, with several hundred $Z^{\prime}$ events detected, the angular distributions and forward-backward asymmetries in their decays can be used to further distinguish among the models. A similar method can be applied to detect and study quark-lepton substructure. using even higher-energy muons in the process $\bar{q} q \rightarrow \mu^{+} \mu^{-}$(as was done for two mojels with the scale $\Lambda=25 \mathrm{TeV}$ ). There, events occur all the way up to the effective kinematic limit, $M_{\mu^{+} \mu^{-}} \approx 10 \mathrm{TeV}$. The two models studied were very well-separated by their muon angular distributions. In $100 \mathrm{fb}^{-1}$, the expected reach in the quark-lepton scale is $\Lambda=30-35 \mathrm{TeV}$. Corresponding studies of $W^{\prime}$-bosons and substructure signals involving a high- $p_{T}$ lepton and $\boldsymbol{E}_{T}$ are expected to yield comparable results.

- GEM's calorimetry coverage to $|\eta| \approx 5.0$ ensures that we are able to discover the $\boldsymbol{E}_{T}$ signature of gluinos and squarks that occur in minimal supersymmetry models with masses up to about $2 \mathrm{TeV}$, i.e., above the plausible limit for weak-scale supersymmetry. For masses at the lower end of the discovery range, near $300 \mathrm{GeV}$, there is a large signal of order $10^{6}$ events, with a signal to background ratio of approximately 7 for $t_{T}>250 \mathrm{GeV}$. For $2 \mathrm{TeV}$ gluinos and squarks, there are hundreds of signal events on a much smaller background for $\boldsymbol{E}_{T}>1 \mathrm{TeV}$. In addition, in the likesign dilepton signature for gluino pair production, GEM's muon system and central tracker pro- vide important confirmation of the $\mathbb{E}_{T}$ signature.

The processes studied so far were not intended to represent a full survey of new physics possibilities at the SSC. For some cases that are not explicitly presented here, GEM's performance can be determined by scaling the signal cross sections, leaving the backgrounds unchanged. A case in point is that of the two neutral Higgs bosons, $H^{0}$ and $h^{0}$, which occur in generalizations of the standard model such as the minimal supersymmetric standard model. In other cases, GEM's performance will be studied in new, dedicated studies. An example of this is the production of color-octet technirho vector mesons.

These simulations are currently being developed, together with an improved set of tools for modeling the detector. In an intensely interactive process, this is occurring as the design evolves in response to our simulations. We are developing a package for simulation of the GEM detector which will unify the various approaches described in this chapter (see Section 8.8.1). This package will provide the user the option of choosing from a fast parameterized detector response, a mixture-level GEANT treatment, or a fully-detailed GEANT simulation, separately selectable for each of the subsystems.

The physics goals of GEM have guided its design principles and major technical choices from the outset. Examples are:

1. The requirements for the energy resolution of the electromagnetic calorimeter-both for the stochastic $(-7 \% / \sqrt{E})$ and the constant $(\leq 0.4 \%)$ terms-are set by the discovery potential for $H^{0} \rightarrow \gamma \gamma, \quad H^{0} \rightarrow Z^{*} \rightarrow$ $e^{+} e^{-} e^{+} e^{-}$, and $Z^{\prime} \rightarrow e^{+} e^{-}$.

2. The pointing capability and angular resolution of the electromagnetic calorimeter $((40-50) / \sqrt{E}+0.5 \mathrm{mrad})$ are also motivated by the need to cleanly identify and measure $H^{0} \rightarrow \gamma \gamma$ and $Z^{\prime} \rightarrow e^{+} e^{-}$. The pointing ability of the calorimeter complements the central tracker's determination of the event vertex.

3. The segmentations of the calorimeter systems are dictated by the need to measure EM pro- 
cesses with high precision, and the need to reject hadronic backgrounds. The calorimeter and the tracker combine to provide clean isolation for photons, electrons and muons.

4. The high momentum resolution of the muon system at all rapidities and momenta is needed to. detect and study $Z^{\prime} \rightarrow \mu^{+} \mu^{-}$and $H^{0} \rightarrow$ $Z Z^{*} \rightarrow \mu^{+} \mu^{-} \mu^{+} \mu^{-}$decays with high efficiency.

5. The resolution of the compact central tracker permits the complementary search for supersymmetry in the $\bar{g} \bar{g} \rightarrow \ell^{ \pm} \ell^{ \pm}+X$ channel.

6. The rapidity coverage of the forward calorimeters, extending to $|\eta| \simeq 5.0$, is dictated by the need to suppress backgrounds to the $\boldsymbol{F}_{T}$ signatures for $H^{0} \rightarrow \ell^{+} \ell^{-} \bar{v} v$ decays and for supersymmetry signals.

7. We have designed adequate shielding to suppress neutron and photon backgrounds to low levels, even at $\mathcal{L}=10^{34} \mathrm{~cm}^{-2} \mathrm{~s}^{-1}$. The shielding is necessary to maintain low occupancies in the muon system.

The physics simulations described in this chapter are an essential part of the optimization of GEM. This optimization is an involved process-from simulating the detector design with GEANT and other detailed programs, to parameterizing and encoding the simulations in gemfast, carrying out the simulations of the physics signals and their backgrounds, and, finally, feeding the results of the simulations back into the design. Following the extensive round of simulacions presented in this $T R$, we have initiated a program of design optimization and/or further studies focused on:

1. Improved acceptance for the muon system. This is important both for low-mass, $H^{0} \rightarrow Z Z^{\circ} \rightarrow$ $\mu^{+} \mu^{-} \mu^{+} \mu^{-}$(Section 2.3.4), and high-mass, $Z^{\prime} \rightarrow \mu^{+} \mu^{-}$(Section 2.6.1) processes.

2. Further reduction :- the material in the middle superlayer of the muon system, to improve the resolution in low-mass processes such as $H^{0} \rightarrow Z Z^{*} \rightarrow \mu^{+} \mu^{-} \mu^{+} \mu^{-}$

Based on studies of the further development of the current engineering design, we have set the practical goals of at least a $50 \%$ reduction in the geometrical losses and a $25 \%$ reduction in the overall material in the muon system. We can thus expect an acceptance improvement of about $40 \%$ for $H^{0} \rightarrow Z^{*} \rightarrow \mu^{+} \mu^{-} \mu^{+} \mu^{-}$.

3. A detailed alignment procedure, using muons produced in $p p$ collisions, to determine the $x y$-position of the muon spectrometer with respect to the center of the beam spot to within $200 \mu \mathrm{m}$. This constraint would significantly improve the resolution for high-momentum muons.

4. The detailed calibration strategy for the EM calorimeter. While there is considerable experimental evidence confirming the stability and uniformity of liquid ionization calorimeters, it is essential to ensure that the constant term in the energy resolution is kept small by monitoring physics signals such as $Z \rightarrow e^{+} e^{-}$and by calibration of the electronics. This is important for measurements of $H^{0} \rightarrow \gamma \gamma$ and $Z^{\prime} \rightarrow e^{+} e^{-}$. (See Chapter 5.)

5. A careful program of calibration to ensure that the jet energy scale will be well-understood. This is important for top and other flavor physics, for $H^{0} \rightarrow Z Z \rightarrow \ell^{+} \ell^{-}$jet jet, and for studies of jets at the highest $E_{T}$.

6. Improvement of the $\boldsymbol{E}_{T}$ resolution by use of a smaller beam pipe at the position of the forward calorimeter, to further extend the rapidity coverage. Consideration of the neutron and photon backgrounds (see Chapter 12) and realistic clearances are an integral part of the design process. 


\section{REFERENCES}

1. An Expression of Interest to Construct a Major SSC Detector, SSC EOI-OC20 (July 1, 1991).

2. GEM Letter of Intent, The GEM Collaboration, GEM TN-92-49, SSCL-SR-1184 (November 30, 1991).

3. GEM Responses to the December 1991 PAC Report, GEM TN-92-131, (July 8, 1992).

4. Solenoidal Detector Collaboration Technical Design Report, SDC-92-201, SSCL-SR-1215 (1992).

5. F. E. Paige and S. D. Protopopescu, BNL-38774 (1986).

6. H.-U. Bengtsson and T. Sjostrand, A Manual to the Lund Monte Carlo for Hadronic Processes; T. Sjostrand, The Lund Monte Carlo for Jet Fragmentation and $e^{+} e^{-}$Physics.

7. E. Eichten, .. Hinchliffe, K. Lane and C. Quigg, Rev. Mod. Phys. 56, 579 (1984).

8. The 95\%-confidence-level limit on the top-quark mass, assuming it decays in the standard way, $t \rightarrow W^{+} b$, is $m_{t}>01 \mathrm{GeV}$. The limit obtained without assuming do uinance of the standard decay mode is $m_{t}>55 \mathrm{GeV}$. See Ref. 9.

9. Particle Data Group, K. Hikasa et al., Phys. Rev. D45, S1 (1992).

10. R. Brun, R. Hagelberg, M. Aansroul, J. C. Lassalle, GEANT: Simulation Program for Particle Physics Experiments, CERN-DD/78/2 Rev., (July, 1978).

11. T. A. Gabriel, B. L. Bishop, F. S. Alsmiller, R. G. Alsmiller, and J. Johnson, CALOR89, A Monte Carlo Program Package for the Design and Analysis of Calorimeter Systems, ORNL/TM-11185 (1989).

12. Yu. Fisyak, K. McFarlane and L. Roberts SIGEM - Full GEANT Simulation for the GEM Detector, GEM-TN-92-162 (1992).

13. T. Skwarnicki, GEM Technical Note, in preparation.

14. I. Sheer, GEMGEN - A Generic Monte Carlo Generator Interface Package, GEM TN-93-379 (March 1993).

15. G. Grindhammer, et al., Proc. of the Workshop on Calorimetry for the Supercollider, Tuscaloosa, AL, 1989, p.151, SLAC-PUB5072, 1989 and Nucl. Inst. and Meth. A290, 469 (1990).

16. W. E. Cleland and A. V. Vanyashin, GEM TN93-376, .pril, 1993).

17. K. Borras, et al., The Hl Calorimeter Group, "Study of Software Compensation for Single
Particles and Jets in the H1 Calorimeter", Contributed paper to the XXV International Conference on High Energy Physics, Singapore, 1990.

18. Yu. Efremenko, et al., Simulation Studies for GEM Scintillating Barrel Design, GEM TN-93-349 (1993).

19. M. Shupe, private communication.

20. T. Wenaus, A Detailed Simulation and Performance Parameterization of the GEM Muon Detector, GEM TN 93-297 (April 1993).

21. R. Carey, Jet Studies with the GEM Detector. GEM-TN-93-377 (1993).

22. First Level Trigger in the GEM Muon System, P. Dingus, et al., GEM-TN-93-394 (1993).

23. See, for example, L3 Collaboration, Z. Phys. C57 (1993) 355.

24. ECFA Aachen Workshop on LEP 200, CERN 87-08, June 1987; and LEP 200 Workshop, CERN, September 1992.

25. See, for example, R. Dashen and H. Neuberger, Phys. Rev. Lett. 50, 1897 (1983) J. Kuti, L. Lin and Y. Shen, Phys. Rev. Lett. 61, 678 (1988) A. Hasenfratz, et al. Phys. Lett. B199, 531 (1987) G. Bhanot and K. Bitar, Phys. Rev. Lett. 61, 798 (1988).

26. S. Mrenna, et al., GEM TN-93-373.

27. For reviews, see H. E. Haber and G. L. Kane, Phys. Rept. 117, 75 (1985) S. Dawson, E. Eichten and C. Quigg, Phys. Rev. D31, 1581 (1985).

28. P. N. Harriman, A. D. Martin, R. G. Roberts and W. J. Stirling, Phys. Rev. D42 (1990) 798 and Phys. Lett. B243, 421 (1990).

29. J. Botts, J. Morfin, J. Owens, J. W. Qiu, W. K. Tung and $H$. Weerts, MSUHEP-92-27, Fermilab-Pub-92/371, FSU-HEP-92-1225 and ISU-NP-92-17 (1992).

30. H. Yamamoto, GEM TN-93-374.

31. W. E. Cleland and A. V. Vanyashin, GEM TN93-376, (April, 1993).

32. M. J. Wang and A. Sumarokov, GEM Technical Note, in preparation.

33. H. Ma and M. Leltchouk, GEM TN 92-257 (December, 1992); GEM TN 93-356 (April, 1993).

34. R. Y. Zhu and H. Yamamoto, GEM TN 92-126 (July, 1992).

35. S. Dawson, Nucl. Phys. B359, 283 (1991); A. Djouadi, M. Spira, and P. Zerwas, Phys. Lett. 264B, 440, (1991); S. Dawson and R. Kauffman, Phys. Rev. Lett. 68, 2273 (1992); R. Kauffman, Phys. Rev. D45, 1512 (1992); C. P. Yuan, Phys. Lett. 283B, 395 
(1992); D. Graudenz, M. Spira, and P. Zerwas, Phys. Rev. Lett. 70, 1372 (1993).

36. B. Bailey, J. Owens and J. Ohnemus, Phys. Rev. D46, 2018 (1992).

37. W. J. Marciano and F. Paige, BNL Preprint, BNL-45805; J. F. Gunion, et al., SDC report, SDC-91-00057; R. Kleiss, et al., Phys. Let. B253 (1991) 269; Z. Kunszt, et al., Phys. Let. B271 (1991) 247.

38. I. Hinchliffe, A Monte Carlo Parton Generator, (unpublished).

39. J. J. van der Bij and E. W. N. Glover, Phys. Lett. B206, 701 (1988); E. W. N. Glover and J. J. van der Bij, Nucl. Phys. B321, 561 (1989).

40. S. Dimopoulos, Nucl. Phys. B168, 69 (1980); T. Appelquist and G. Triantaphyllou, Phys. Rev. Lett. 69, 2750 (1992).

41. C. Yanagisawa, Top-quark Detection in the Multileptons Mode with the GEM Detector, GEM. TN-93-371 (1993).

42. C. Yanagisawa, Top-quark Detection in the Multijets Mode with the GEM Detector, GEM TN-93-372 (1993).

43. M. Mohammadi and D. Skrzyniarz, GEM TN-93-363 (1993).

44. The product of geometrical acceptance and reconstruction efficiency for the non-isolated muons is higher than 95\%; see Section 4.2.4.

45. B. Holdom, Phys. Rev. D24, 1441 (1981); Phys. Lett. B150, 301 (1985); T. Appelquist, D. Karabali and L. C. R. Wijewardhana, Phys. Rev. Lett. 57, 957 (1986); T. Appelquist an L. C. R. Wijewardhana, Phys. Rev. D36, 568 (1987); K. Yamawaki, M. Bando and K. Matumoto, Phys. Rev. Lett. 56, 1335 (1986); T. Akiba and T. Yanagida, Phys. Lett. B169, 432 (1986).

46. K. Lane and M. V. Ramana, Phys. Rev. D44, 2678 (1991).

47. We thank B. Balaji for estimating $\sigma\left(p p \rightarrow \eta_{T} \rightarrow \bar{t} t\right)$.

48. R. M. Barnett, R. Cruz, J. F. Gunion and B. Hubbard, Phys. Rev. D47, 1048 (1993).

49. C. Yanagisawa, Study of $t \rightarrow H^{+} b, H^{+} \rightarrow c \bar{s}$, with the GEM Detector, in preparation.

50. M. Mohammadi, Ph.D. Thesis, University of Wisconsin (1987), unpublished.

51. E. J. Eichten, K. D. Lane and M. E. Peskin, Phys. Rev. Lett. 50, 811 (1983).

52. F. Abe et al., The CDF Collaboration, Phys. Rev. Lett. 68, 1104 (1992); Fermilab-PUB91/231-E.

53. We are greatly indebted to T. Sjostrand for embedding the quark and lepton compositeness routines in PYרHIA and for continued help on the event simulations.

54. J. Morfin and W.- K. Tung, Fermilab-Pub90/74. (1990).

55. F. Abe et al., The CDF Collaboration, Phys. Rev. Lett. 68, 1145 (1992).

56. $\mathrm{QCD}$ radiative corrections have not been taken into account in these studies. They are likely to increase the Drell-Yan signal rates. Since all the physics backgrounds are very small after event selections are made, higher-order corrections to them are not expected to change our conclusions. The EHLQ1 distribution functions were used for all simulations in this section.

57. These couplings are related to the PARU(121-128) switches in PYTHIA JETSET by, e.g., $\operatorname{PARU}(121)=2\left(g_{d L}+g_{d R}\right)$ and PARU $(122)=2\left(g_{d L}-g_{d R}\right)$. The parameters $\beta$ and $\gamma$ involve a factor of $\sin \theta_{w}=\sqrt{0.23}$.

58. S. McKee and E. H. Simmons, $Z^{\prime} \rightarrow e^{+} e^{-}$ studies at $10^{34} \mathrm{~cm}^{-2} \mathrm{~s}^{-1}$ with the GEM Detector, GEM PN-93-6 (1993).

59. At $\&=10^{34} \mathrm{~cm}^{-2} \mathrm{~s}^{-1}$, the central iracker has its silicon removed, and tracking is done by eight layers of IPCs. The momentum resolution is degraded by a factor of 2.5 above $p_{T}=$ $20 \mathrm{GeV}$. A benefit is that, with the silicon removed, half the material affecting tracking is gone.

60. The discrepancy between the theoretical widths determined from Eq. (2.6.3) and those from fitting the PYTHIA distributions corresponds to a $0.7 \%$ gaussian noise term that we have not yet understood. Since PYTHIA and gemfast widths agree within the expected detector resolution, this "critter" is not a matter of immediate concern.

61. P. Langacker, R. Robinett and J. Rosner, Proceedings of the 1984 Summer Study on the Design and Utilization of the Superconducting Super Collider, R. Donaldson and J. G. Morfin, editors, p. 812 (1984).

62. This discussion of measuring the muon angular distribution first appeared in Ref. 3.

63. M. Mohammadi and W. Orrick, Studies of $Z^{\prime} \rightarrow \mu^{+} \mu^{-}$at Ultrahigh Luminosity with the GEM Detector, GEM TN-93-364 (1993).

64. A similar result was found for $4 \mathrm{TeV} Z^{\prime}$ events using sigem; see Section 4.2.6.

65. T. Wenaus, A Reconstruction Program for the GEM Muon Detector, GEM TN-93-388 (1993).

66. It may be possible to use a similar trick to recover some of the muons lost in dead regions in the muon system. This would require that the 
muon direction is determined in the tracker or the calorimeter. Studies of the feasibility of this approach are underway.

67. R. S. Chivukula and L. Randall, Phys. Lett. 202B, 429 (1988).

68. R. Arnowitt and P. Nath, Phys. Rev. Lett. 69. 725 (1992).

69. R. Ross and R. Roberts Vucl. Phys. B377, 571, 1992.

70. H. Baer, F. E. Paige, S. D. Protopopescu and X. Tata, in preparation. Production and deciy of $t$ is not yet included.

71. F. E. Paige and A. Vanyashin, GEM TN-93-375 (1993).

72. F. E. Paige and A. Vanyashin, GEM TN-92-70 (1992).

73. H. Baer, X. Tata and J. Woodside, Phy's. Rev. D45, 142 (1992). 


\section{MAGNET}

\subsection{OVERVIEW}

\subsubsection{Introduction}

The GEM magnet provides the magnetic field for charged particle tracking, both near the beam axis in the central tracker and at larger radii in the muon system. The detailed magnetic field requirements were developed by trade-offs between physics needs and technology limits.

To support our physics goals, the magnet tracking systems must meet the following performance requirements:

- Muon momentum resolution in the barrel region: $\Delta p / p=5 \%$ for tracks with $p_{T}=500 \mathrm{GeV} / \mathrm{c}$ at $|m|<1.35$.

- Muon momentum resolution in the forward region: $\Delta p / p<13 \%$ for tracks with $p_{T}=500 \mathrm{GeV} / \mathrm{c}$ at $1.35<|\eta|<2.5$.

- Momentum resolution in the central tracker: $\Delta p / p^{2}=1.15 \times 10^{-3}(\mathrm{GeV} / \mathrm{c})^{-1}$.

- Charge separation in the central tracker at the 95\% confidence level for tracks with momentum below $600 \mathrm{GeV} / \mathrm{c}$.

Our approach to meeting these objectives is to enclose the entire detector in a moderate magnetic field: less than $1 \mathrm{~T}$. Since the momentum resolving power varies linearly with magnetic field strength and quadratically with trajectory path length, maximizing the tracking volume of the muon system in the magnetic field is clearly advantageous. Higher magnetic fields would limit technology options for other detector components and a smaller magnet would degrade the muon momentum resolution. A high-field, small-radius magnet version of GEM was investigated and discarded for these reasons.

Several alternative magnet concepts were considered in the early design stages. These are listed in Table 3-1.
Table 3-1. Major magnet design options.

\begin{tabular}{|c|l|l|}
\hline Option & Comments & \multicolumn{1}{|c|}{$\begin{array}{c}\text { Major } \\
\text { disadvantages }\end{array}$} \\
\hline $\begin{array}{l}\text { Resistive coil } \\
\text { with iron } \\
\text { flux return }\end{array}$ & $\begin{array}{l}\text { Low technical } \\
\text { risk }\end{array}$ & $\begin{array}{l}\text { High construc- } \\
\text { tion cost, } \\
\text { high operat- } \\
\text { ing cost, } \\
\text { schedule } \\
\text { delay }\end{array}$ \\
\hline $\begin{array}{l}\text { Resistive coil } \\
\text { without flux } \\
\text { retum }\end{array}$ & $\begin{array}{l}\text { Low technical } \\
\text { risk, } \\
\text { minimal } \\
\text { construction } \\
\text { cost }\end{array}$ & $\begin{array}{l}\text { High operating } \\
\text { cost, } \\
\text { fringe field }\end{array}$ \\
\hline $\begin{array}{l}\text { Supercon- } \\
\text { ducting coil } \\
\text { with iron } \\
\text { flux retum }\end{array}$ & $\begin{array}{l}\text { Moderate } \\
\text { technical } \\
\text { risk, } \\
\text { lower oper- } \\
\text { ating cost }\end{array}$ & $\begin{array}{l}\text { High construc- } \\
\text { tion cost, } \\
\text { schedule } \\
\text { delay }\end{array}$ \\
\hline $\begin{array}{l}\text { Supercon- } \\
\text { ducting coil } \\
\text { with super- } \\
\text { conducting } \\
\text { shield coil }\end{array}$ & $\begin{array}{l}\text { High perfor- } \\
\text { mance, } \\
\text { high techni- } \\
\text { cal risk, } \\
\text { lower oper- } \\
\text { ating cost }\end{array}$ & $\begin{array}{l}\text { Technical risk, } \\
\text { high } \\
\text { construction } \\
\text { cost, } \\
\text { schedule } \\
\text { delay }\end{array}$ \\
\hline $\begin{array}{l}\text { Supercon- } \\
\text { ducting coil } \\
\text { without iron } \\
\text { flux retum }\end{array}$ & $\begin{array}{l}\text { Low technical } \\
\text { risk, } \\
\text { lower oper- } \\
\text { ating cost }\end{array}$ & Fringe field \\
\hline
\end{tabular}

Given the selection of a large magnet, we concluded that a resistive coil is not a viable option because of the high operating cost. The operating power requirements would have been in excess of $20 \mathrm{MW}$. The size of the magnet dictated that the simplest approaches to design and fabrication issues be adopted wherever possible at reasonable construction and operating costs. These requirements are fulfilled by superconducting technology.

Based on these considerations, we have selected a large superconducting solenoidal coil for the GEM magnet. It is about $31 \mathrm{~m}$ long with an 18-m-diameter inner bore and a field of $0.8 \mathrm{~T}$. The scale of the magnet and the choice of its superconducting technology were set by engineering and cost 
constraints, including practical limits on the size of the detector hall and mechanical constraints in magnet construction, installation, and maintenance. The coil design uses a single-layer winding and no retum yoke. The design has been cost optimized and has low technical risk. It meets GEM requirements for muon resolution and inner tracking in the central rapidity region. However, in a sirple solenoid, the muon momentum resolution in the forward direction, $1.5<\eta<2.5$, was found to be insufficient. Because the resolution in this region is not very sensitive to the radius and length of the solenoid alone, a variety of local forward field shaping options were studied. From among these options, a simple, passive iron field shaper was adopted. The iron forms a truncated cone around the beam axis in the region between 10 and $18 \mathrm{~m}$ from the beam interaction point. It introduces flux concentration and a large field gradient in the region of the forward muon chambers, allowing for substantial improvement of the momentum resolution in that region.

Our present design, shown in Figures 3-1 and 3-2 and described in Table 3-2, fulfills all performance requirements and meets our overall cost and schedule objectives.

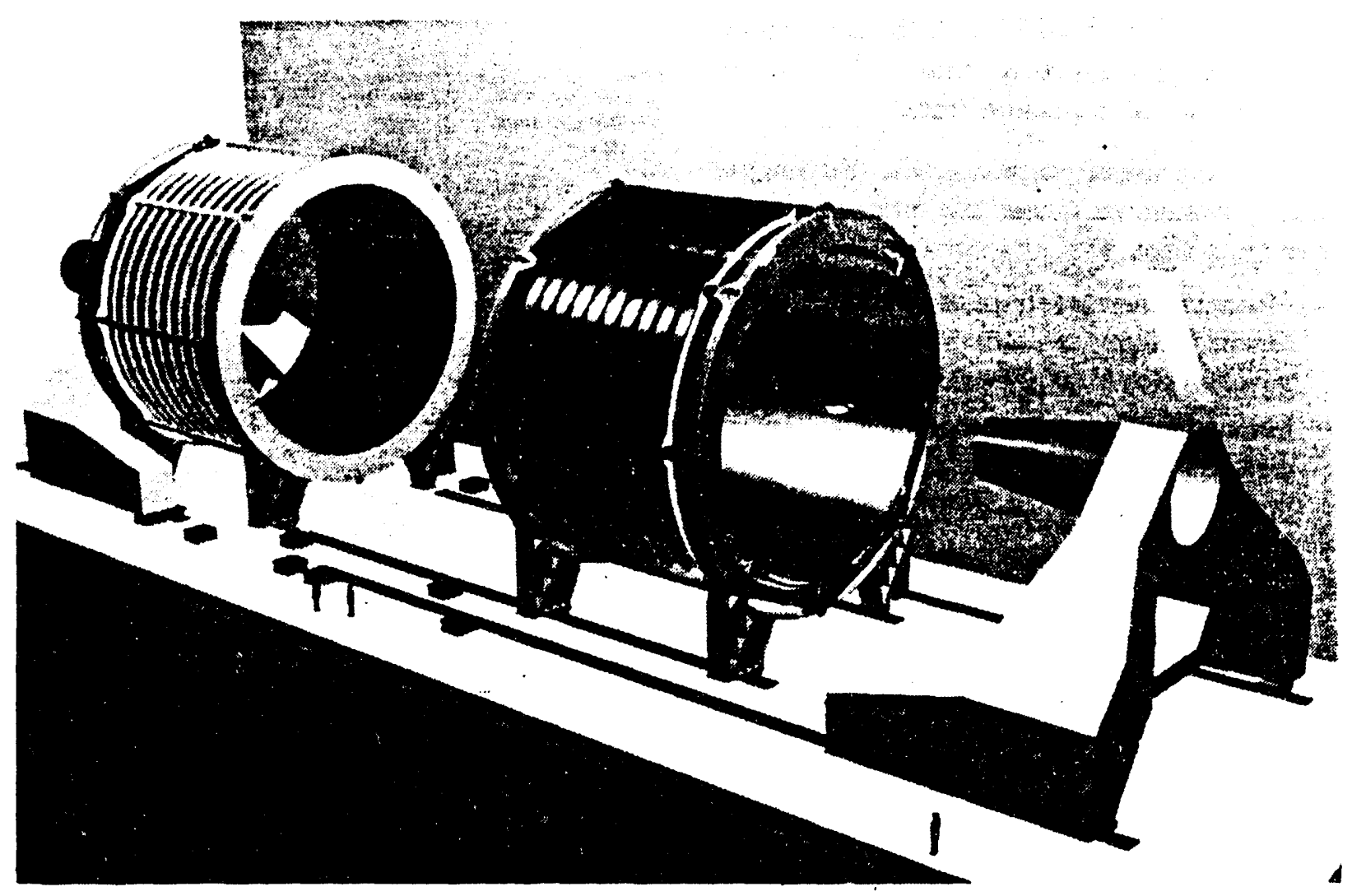

FIG. 3-1. The GEM magnet, showing one half-coil and forward field shaper retracted. 


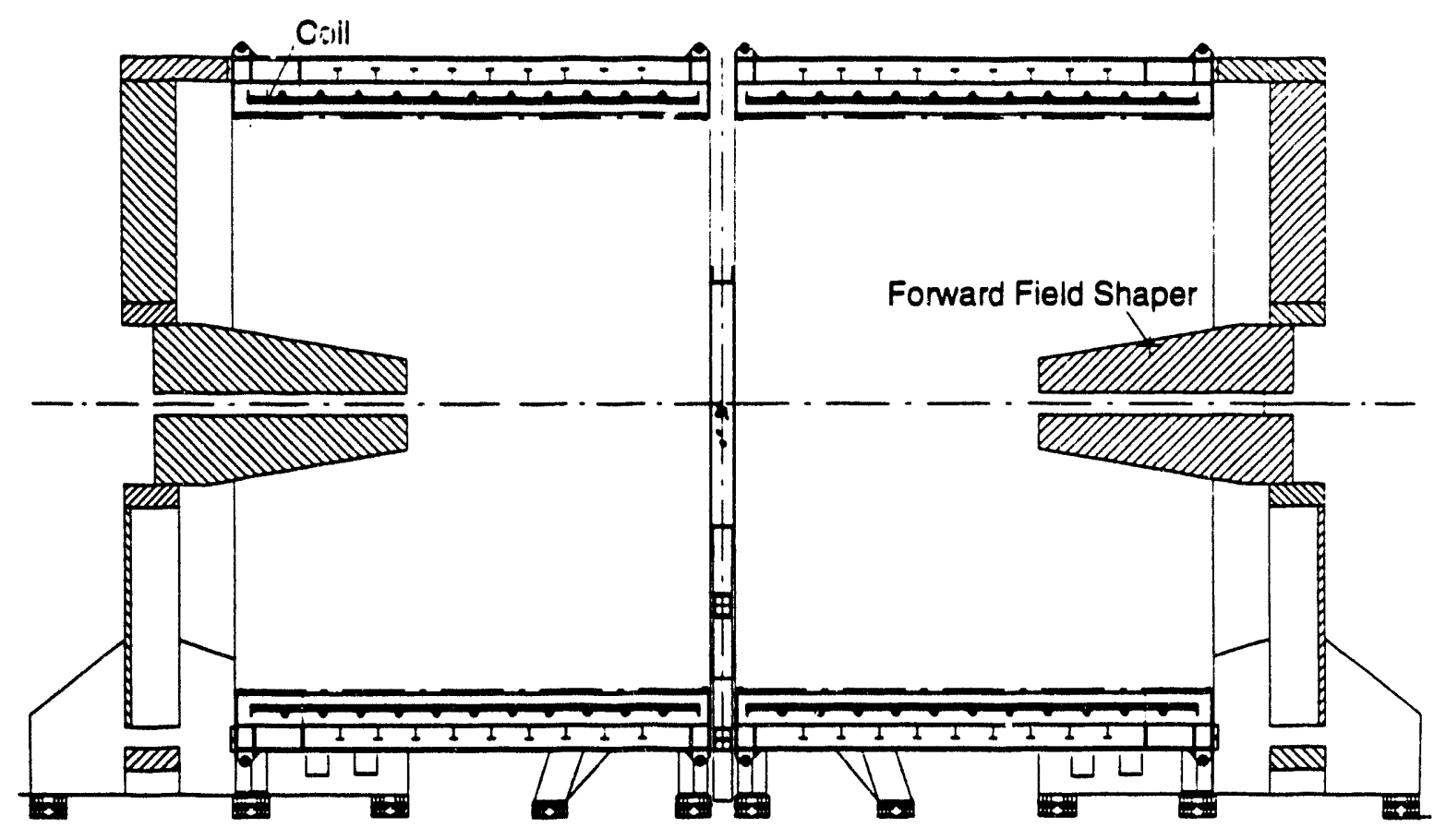

FIG. 3-2. Elevation view of the GEM magnet.

Table 3-2. Major parameter list for the GEM magnet.

\begin{tabular}{|c|c|c|}
\hline Central field & 0.8 & $T$ \\
\hline Mean radius of windings & 9.5 & $m$ \\
\hline $\begin{array}{l}\text { Inner radius of the vacuum } \\
\text { vessel }\end{array}$ & 9.0 & m \\
\hline $\begin{array}{l}\text { Overall outer radius, including } \\
\text { structural ribs }\end{array}$ & 10.9 & $m$ \\
\hline Coil length (per half) & 14.25 & $m$ \\
\hline Number of tums (per half) & 228 & \\
\hline $\begin{array}{l}\text { Central gap between wind- } \\
\text { ings }\end{array}$ & 1.5 & m \\
\hline $\begin{array}{l}\text { Magnet axis height above } \\
\text { floor level at interaction } \\
\text { point. }\end{array}$ & 13.0 & m \\
\hline Operating current & 50.2 & $\mathbf{k A}$ \\
\hline Stored energy & 2.5 & GJ \\
\hline Inductance & 1.98 & $\mathrm{H}$ \\
\hline $\begin{array}{l}\text { Total magnet assembly mass } \\
\text { (per haff) }\end{array}$ & 1500 & $\mathrm{Mg}$ \\
\hline $\begin{array}{l}\text { Forward field shaper mini- } \\
\text { mum z }\end{array}$ & 10.0 & m \\
\hline $\begin{array}{l}\text { Fonward field shaper maxi- } \\
\text { mum } 2\end{array}$ & 18.0 & m \\
\hline $\begin{array}{l}\text { Fonward field shaper included } \\
\text { angle }\end{array}$ & $18.8^{\circ}$ & \\
\hline $\begin{array}{l}\text { Forward field shaper mass } \\
\text { (each) }\end{array}$ & 1265 & $\mathrm{Mg}$ \\
\hline
\end{tabular}

A detailed description of the magnet can be found in the engineering design report. 1

Subsections 3.1.2-3.1.5 below address general requirements, design trade-offs, the magnet option selected, and the forward field shapers (FFS).

\subsubsection{General Requirements for the GEM Magnet}

The momentum resolution of reconstructed tracks results from the interplay of several factors. These include the integral of the magnetic field strength along the trajectory of the particle and the accuracy of the position measurements in the tracking systems. The position measurements are affected by the alignment precision of the tracking chambers and by the precision of knowledge of local magnetic field vectors, which are needed to minimize and correct for Lorentz effects. Furthermore, present plans for the track reconstruction algorithms in the central tracker are based on the assumption of nearly uniform field in the tracker volume. The tracking algorithms and trigger design in the muon system are based on the assumption that the magnetic flux is uniform in the central rapidity region and is well known in the forward region. In both areas, only small deviations from axial symmetry are 
allowed. The choice of cathode strip chamber technology for the muon system together with an adequate understanding of the alignment precision of those chambers (see Chapter 4) impose a requirement that the field integral $\mathrm{BL}^{2}$ along the muon trajectory be known to etter than $0.4 \%$. The precision of knowledge of the field in the central tracker region is even less critical: an uncertainty of less than $0.5 \%$ will not significantly degrade central tracker performance.

The quality of the magnetic field also affects the behavior of the main collider ring, due to a potential coupling of the vertical and horizontal betatron oscillations of the protons in the beam. The radial field component near the beam axis must be very small in order to minimize effects on the accelerator lattice. This requirement imposes alignment and stability criteria on the magnet. A study of the effects of the GEM magnet on SSC beam dynamics ${ }^{2}$ indicates that the uncertainty of the magnet axis alignment must be less than 5 milliradians, which is well within general alignment criteria.

Finally, the SSCL operations cycle imposes a number of general requirements on the GEM magnet. The magnet has to be working during the SSC accelerator cycle of 4500 hours per calendar year. The magnet design assumes an overall lifetime of 20 years and, very conservatively, is designed for 100 thermal cycles, from ambient to operating temperature and back to ambient, and 200 normal charge/discharge cycles.

\subsubsection{Trade-off, Risk, and Optimization Studies}

We conducted numerous trade-off, risk, and optimization studies in developing the overall magnet configuration. In arriving at the present design, the most important of these studies were:

a) optimization of the solenoid field strength, coil radius and length;

b) shielding the fringe field;

c) choice of the approach to meeting the smail angle resolution requirement (FFS);

d) the technical decision on the configuration of the superconducting conductor.

Each of these points is discussed below. a) Field strength, radius, length, and geometr::

The most important and difficult optimization study defined the overall size and field of the magnet. Major considerations taken into account were the muon momentum resolution. muon technology requirements, magnet technology risks, and a combined magnet and muon system cost.

The momentum resolution is defined as:

$$
\Delta p / p=\Delta p_{T / p} p_{T}=\Delta \mathrm{S} / \mathrm{S},
$$

where $p$ is the momentum, $p_{T}=|p| \sin \theta$, and $\theta$ is the polar angle from the beamline. The momentum resolution is computed as a function of angle for a given $p_{T} . S$ is the sagitta, related to the curvature of the track, and $\Delta S$ is the total uncertainty in the sagitta, which includes measurement, multiple scattering, and systematic errors. The uncertainty in resolution depends on the actual trajectory, since path length and angle with respect to the magnetic field vary with trajectory geometry. The sagitta $S$ is given at $90^{\circ}$ by

$$
S=\frac{0.3}{8 p_{T}} \mathrm{BL}^{2},
$$

where $\mathrm{BL}^{2}$ is given by an integral of the field along the trajectory:

$$
\mathrm{BL}^{2}=\left|2 \int_{0}^{L} d s^{\prime} \int_{0}^{s^{\prime}} \mathrm{dx} \times \mathbf{B}\right|
$$

A detailed derivation of the above relations and a discussion of muon resolution are given in Section 4.3.1.

A parametric model of the combined magnet and muon system cost was used to optimize the field strength and size of the magnet. The model showed that for fixed muon transverse momentum resolution, and for fixed muon system alignment criteria, chamber resolution, and number of layers, there is a broad cost minimum at a field of approximately $0.8 \mathrm{~T}$ and an inner radius of $18 \mathrm{~m}$ (see Fig. 3-3). In addition to cost considerations, the field strength is limited by technological risks associated with construction of very large magnets with high magnetic fields. For fields above $0.8 \mathrm{~T}$, the magnet would require multi- 
layer winding and the conductor operational safety margin near the ends of the coil wuald be unacceptably small. Further improvement of the resolution could be achieved by increasing the radius of the magnet. For the magnet alone, the cost increases by about $\$ 2 \mathrm{M}$ for each additional meter of length and about \$10M for each additional $0.5 \mathrm{~m}$ in radius at fixed field strengths of $0.8 \mathrm{~T}$. Although an increase in either radius or length would relax some of the precision requirements imposed on muon chamber technology, a larger radius magnet would require more material for its construction, more superconducting material for the conductor, and a larger surface area of muon chambers. The sizes of the detector hall and magnet construction facilities also impose practical restrictions.

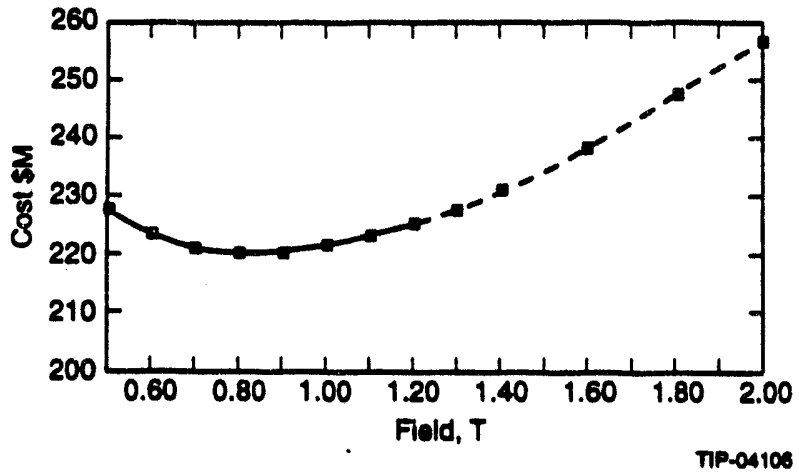

FIG. 3-3. Cost estimate of the combined magnet and muon system as a function of the magnetic field strength for fixed muon resolution.

b) Shielding the magnetic fringe field:

Our early studies ${ }^{3}$ revealed that elimination of a magnetic return yoke would save considerable money and time without introducing any significant safety or health risks or imposing any significant operational challenge. Shielding options were found to be either very expensive, approximately doubling the overall construction cost, or technically risky. Each required a long installation time that would extend the schedule by approximately one year.

Magnets without a flux return to shield the fringe field are common. Large air core electromagnets without flux returns are used in superconducting magnetic energy storage systems, MHD devices, MRI machines, and large magnetic fusion devices such as JET, TFTR, and MFTF. The cost of capturing all the flux for these machines is prohibitive; the manageable alternative is to shield only the sensitive volumes. Experience also comes from ALCATOR at MIT Francis Bitter Magnet Laboratory, LCT (ISMTF) at Oak Ridge National Laboratory, and the 15-ft Bubble Chamber at Fermilab. For GEM, the components of the detector and the electrical, cryogenic, and other services in the experimental hall are either designed to operate in the magnetic fringe field or will be shielded at relatively low cost. The magnetic field on the ground surface also can be managed easily. ${ }^{4}$ The technology to ensure satisfactory operation of equipment is well understood. Calculations of forces and torques that act on ferromagnetic bodies and current-carrying elements are summarized in GEM technical notes. ${ }^{5,6}$ Susceptible components such as vacuum turbopumps and electrical switches located near the detector may require local shielding to operate properly.

Calculated field maps have permitted detailed investigation of these effects, and shielding specifications have been made for the electronics counting house. ${ }^{7}$ While full specification of all shielding requirements has not yet been completed, more than enough has been done to determine that the unshielded magnet presents no major operability problems.

c) Forward Field Shaper:

We studied a variety of options for increasing the bending power for tracks emitted at small angles. These included ${ }^{8}$ forward pinch coils, opposing field coils, and various iron wedges with and without additional windings. We found that the simplest, lowest-risk solution is provided by a conical, passive forward field shaping iron structure-the FFS. By introducing an inward-pointing radial component to the forward field, such iron provides sufficient concentration of the field lines to meet the $13 \%$ momentum resolution requirement.

d) Conductor Choice:

One of the primary considerations governing the design of the GEM magnet is that it must 
operate with a high level of stability and reliability to ensure efficient operation of the experiment. This requires a conservative conductor and coil design with substantial operating margins. Several conductor options were studied in detail. The selection was narrowed down to an indirectly cooled conductor or a cable-in-conduit (CIC) conductor. The CIC conductor chosen ${ }^{9}$ has a large operational safety margin. The operating current is less than $25 \%$ of the critical current and the current-sharing temperature margin is $3.4 \mathrm{~K}$. The design also permits extensive component testing before on-site winding, thus reducing risk of late detection of manufacturing flaws. The selected conductor consists of 450 conventional NbTi:copper superconductor strands, cabled together and surrounded by a 2-cm inner diameter stainless steel conduit filled with supercritical helium at $4.5 \mathrm{~K}$. The helium is in direct contact with superconducting strands. This provides a large heat capacity, allowing the conductor to withstand substantial external heat input without quenching. The stainless steel conduit is surrounded by a rectangular aluminum sheath which provides hot-spot protection in the unlikely event of a quench. This type of conductor has undergone many years of development in magnetic fusion programs and the performance record confirms the analytical predictions of excellent stability.

\subsubsection{Magnet Description and Rationale}

The GEM magnet consists of two (half-length) coils, each $14.25 \mathrm{~m}$ long, separated by a distance of $1.5 \mathrm{~m}$. The decision to split the magnet into two coil halves was dictated by logistics. The magnet is too long to be built and lowered into the hall in one piece. Each coil half is composed of 12 identical segments, each consisting of 19 tums of conductor wound on the inside of a $7.6-\mathrm{cm}$-thick, 1.2-m-long cylindrical aluminum bobbin. The central tracker and both the electromagnetic and hadron calorimeters are supported by a free-standing structure centered between the coils. The gap between the coils will also accommodate signal cables, cryogenics, and electrical services, without significant effect on field uniformity in the central region.
The coils and the two forward field shapers are designed to be mobile along the beam axis. The rails and displacement gear are necessary for initial installation of the magnet components in the experimental hall. We have enhanced their design to provide additional flexibility in planning the detector installation and access scenarios. Indeed, the possibility of separation of the coils from the central detector support system (CDS) during initial installation was found to be a most efficient and cost effective way of assembling large and heavy components of both the barrel and endcap calorimeters. It also allows access to the calorimeters and the central tracker. The two forward field shapers have independent mobile support systems, allowing for their independent separation from the coils and for access to various regions of the muon system.

The large stability margin of the conductor ensures achievable winding and assembly requirements appropriate to the large overall size of the coil. Current plans are for the conductor to be roll-formed on the inside of the bobbin, with conductor segments and joints being prefabricated and tested off-site. The baseline design of the electrical joints between segments is a cryostable, resistive coupling with a separate cooling system. Cooling for the coil structure is provided by a natural-convection liquid helium thermosiphon system connected to the bobbin exterior. The supercritical helium within the conductor conduit provides transient stability and does not remove a significant amount of heat during normal operation. The coils are enclosed in a thermal radiation shield consisting of multilayer insulation and liquid nitrogen cooled panels. These are -enclosed in a cryostat that provides insulating vacuum and structural support. Electrical current from the 20-V, 51-kA power supply is routed to the magnet by air-cooled resistive busses. The system can charge or discharge the magnet in 8 hours. A 5-minute emergency discharge is also possible; it can be automatically initiated by the quench detection system or by other safety interlock systems, and is accomplished by switching a large external dump resistor into the power circuit using large circuit breakers.

Each of the two identical forward field shapers consists of a conical steel mass of approximately $1265 \mathrm{Mg}$, centered on the beam axis, and located at 
$z= \pm 10-18 \mathrm{~m}$. Each FFS is independently mounted at specially reinforced mounting locations on the experimental hall floor. For installation and for maintenance, each assembly can be moved along the beamline to the end of the hall. The support structure of the FFS can also provide support for the forward muon system during installation and for various radiation shielding components. The support structure extends to a maximum $z= \pm 19 \mathrm{~m}$ in order to provide for mechanical stability.

The magnet is on the GEM critical path. Its installation and testing should be completed before underground installation of other subsystems. After testing and preliminary field mapping, the magnet halves will be separated for installation of the calorimeters, muon detectors, and central tracker. Magnet testing must be completed by March 1997 to permit physics operation at SSC turn-on in March 1999.

\subsection{MAGNETIC FIELD}

\subsubsection{Field Requirements}

Knowledge of the magnetic field inside the detector is needed for precise fitting of charged particle trajectories and to correct for Lorentz angle effects in the muon chambers. However, the requirements on field precision imposed by the central tracker and the muon system are not very restrictive. In both systems, the momentum resolution of low momentum tracks is dominated by Coulomb scattering effects; for high momentum tracks, by position measurement resolution. The magnetic field must be known to better than $0.5 \%$ in the central tracker region to preserve the low momentum ( $p_{T}<40 \mathrm{GeV}$ ) resolution. Measurement resolution with the CSC technology selected for the muon system shows little sensitivity to the error on local field knowledge. It requires the field to be known to about $0.09 \mathrm{~T}$, i.e., with approximately $10 \%-30 \%$ precision. The integral of the magnetic field along the trajectory of the muon, however, must be known to better than $0.4 \%$ to achieve required momentum resolution.

Field uniformity facilitates trigger and track reconstruction. Contours of constant flux for the magnetic field are shown in Figure 3-4.

In the central region, the deviations from field uniformity are small: less than $0.3 \%$ in the region of the central tracker and less than $1 \%$ in the barrel muon system. Contours of constant magnetic field are plotted in Figure 3-5 for the whole detector and in Figure 3-6 for the central region.

In the forward region, the field shapers introduce a significant radial field component, which improves momentum resolution and charge separation of forward tracks in the central tracker and the muon system, as discussed in the Section 4.3.1 and Chapter 6.

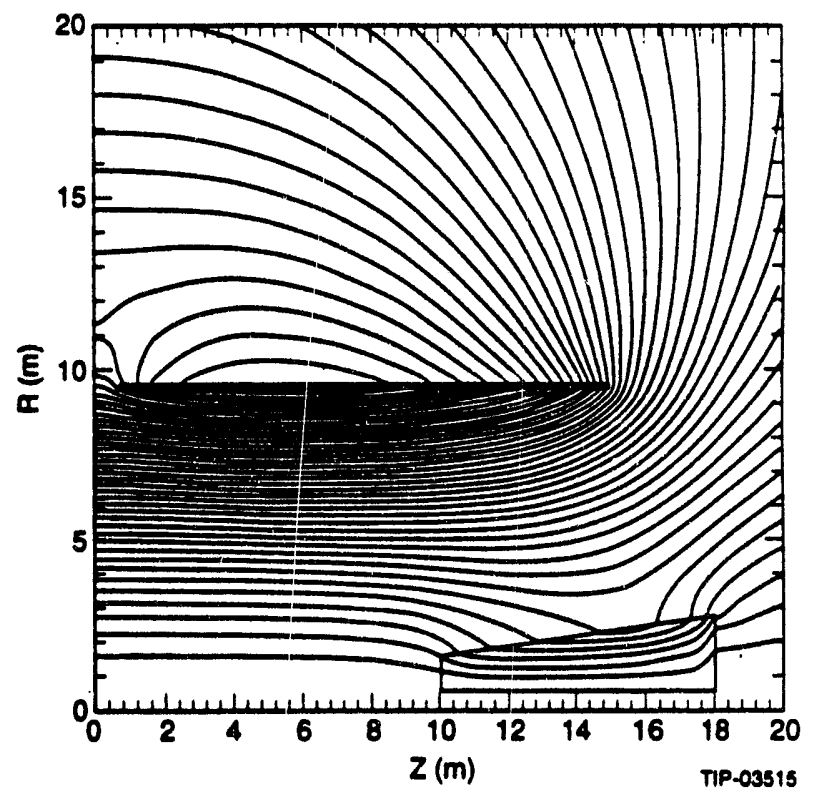

FIG. 3-4. Coritours of constant flux.

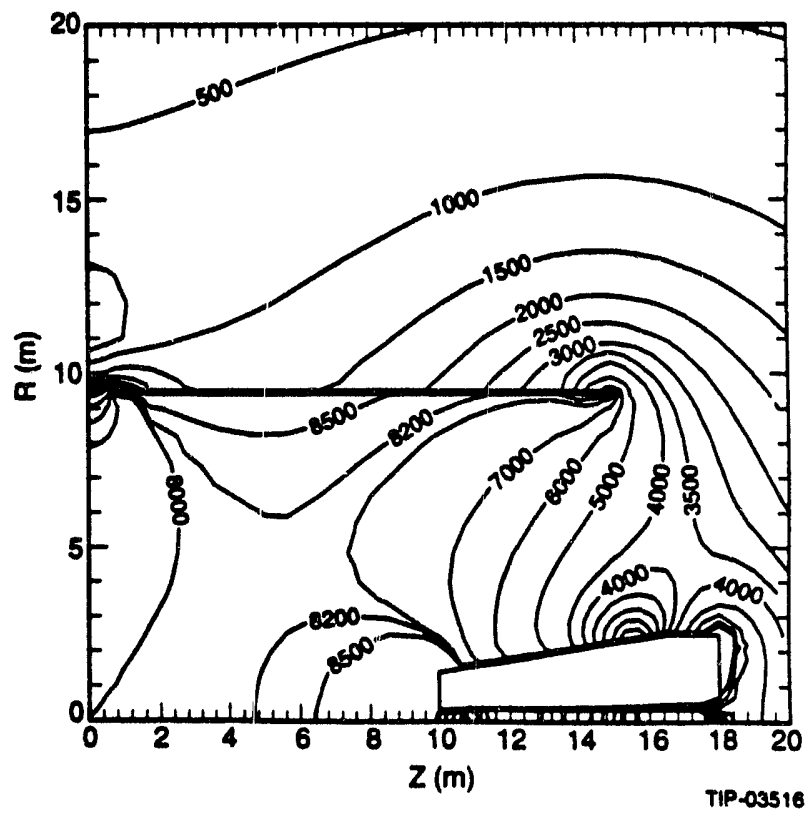

FIG. 3-5. Contours of constant B labeled in gauss. 


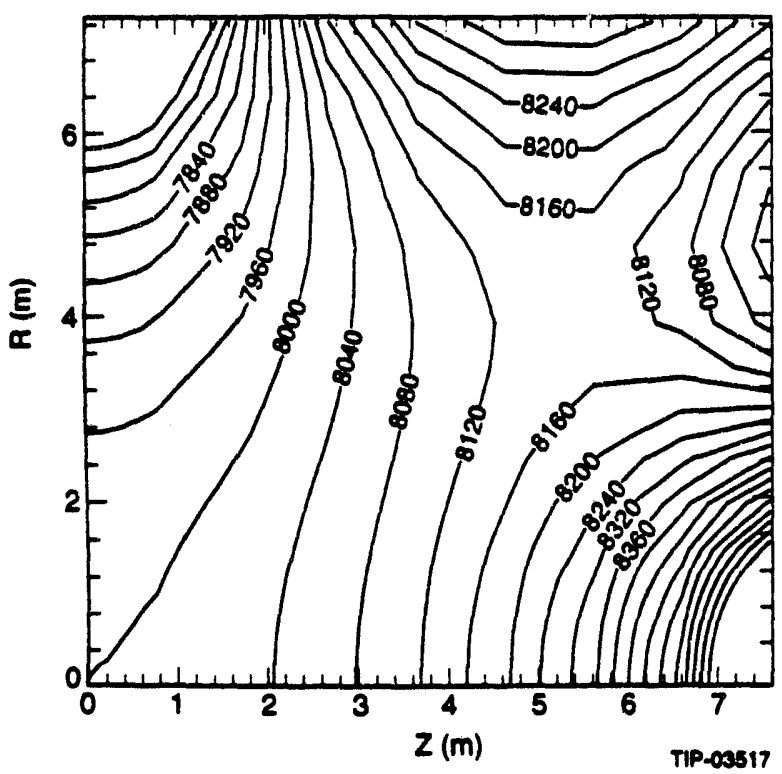

FIG. 3-6. Contours of constant B for the central region in increments of $40 \mathrm{G}$.

\subsubsection{Field Measurements}

The above physics requirements will require knowledge of the field in the central tracker and muon system volumes. This can be accomplished by a combination of Hall probes and magnetoresistors. A field map in the small volume of the central tracker can be obtained by measuring the field on a three-dimensional grid of points spaced a few centimeters apart. This approach has been used in many experiments (e.8., ALEPH, H1, CLEO, SLD), and a number of existing measuring devices can be used. The field in the much larger volume of the muon system can be measured either on a similar grid of space points or by magnetic field probes distributed on outer boundary of the system that encloses no current or iron. In the latter case, the detailed field map is derived from a scalar potential with zero Laplacian, using values of the field measured on the closed boundary. ${ }^{10}$ This technique is also used extensively in field mapping of large magnets for particle physics and plasma-fusion research.

One possible scenario involves placing inexpensive probes (magnetoresistors or Hall probes) on the outer frames of the outer layer of muon chambers and near the FFS. The muon chamber alignment system will then provide precise alignment of the probes. The number and spacing of the probes depend on the local field variation. Very few probes are needed in the central barrel region, where field deviations from uniformity are small. Most of the probes will be needed on the outer boundary of the forward muon chambers, which are situated in a rapidly varying field. Additional probes will be spaced throughout the volume of the detector to provide a cross check on the solution of the summed field model. An extrapolation from the system designed for the L3 magnet yields an estimate of 8200 probes needed for the GEM magnet. An additional constraint in the field determination is provided by its axial symmetry. In order to maintain the symmetry, stainless steel is used in the CDS; magnetic iron is only used in the FFS support. A careful study of the influence of the magnetic materials used for the FFS support ${ }^{11}$ on the symmetry of the field inside the detector shows negligible effects throughout most of the volume. Field deviations from symmetry are maximum in the region of the outer endcap chambers, but reach only $0.03 \mathrm{~T}$ there.

\subsection{MAGNET DESIGN}

\subsubsection{General Approach}

Preliminary magnet design has been underway since January 1992, and we have a self-consistent design that meets the technical, cost, schedule, and facility requirements. An R\&D program supports the few areas where verification or additional design information is desired. The state of the design is summarized in Section 3.4.2. The preliminary design process will be completed soon after the magnet prime contract is awarded in mid-1993. At that time design features will be reviewed and final design will begin.

The GEM coil is similar in concept to thin superconducting solenoids used in other large detectors (DELPHI, ALEPH, VENUS, SLD ) with some design modifications to accommodate its larger size. In each case, the coil winding is held within a thin annular vacuum vessel on long, thin supports. Each cold mass is surrounded by a liquid-nitrogen-cooled thermal shield that, in vacuum, reduces radiation to the cold mass to a manageable level. The windings are restrained within a structural bobbin. Electronic detection and protection systems provide for safe operation. Local control systems provide operator control of the magnet system. 
The larger size of this coil mandates a number of changes from previous designs. The solenoid is separated into independent halves, split at the midplane for easier handling and to provide routes for utilities and data acquisition conjuits. A cablein-conduit conductor, developed for large fusion magnets, is employed. The cable-in-conduit design is more stable against the internal and external energy disturbances predicted for a coil of this size and its stability is less sensitive to fabrication problems expected of more conventional indirectlycooled conductors.

Many GEM coil components are large enough that they must be fabricated at the SSCL IR5 site. Each 14.25-m-long, 525-Mg cold mass is built from twelve 1.2-m-long, 44-Mg segments. Each complete half-solenoid therefore requires eleven stable electrical joints between the twelve segments. These joints are based on a design proven in the US-DPC plasma fusion program. The vessels and the forward field shaper structures also must be erected at the IR5 site from transportable components. While handling and assembly of large components will be time consuming and expensive, a plan that is consistent with the overall cost and schedule objectives has been developed. It is summarized in Chapter 9 and detailed in a GEM report. ${ }^{12}$

\subsubsection{Magnet System Interfaces}

The magnet system has mechanical interfaces with the central tracker, the calorimeters, the central detector support (CDS), the muon system, and the IR5 site facilities. Specific interface control documents are being developed to set the requirements for each of these interfaces.

The muon system dictates magnetic field volume, shape, magnitude, uniformity, stability, and alignment objectives to achieve proper momentum resolution.

The main requirements imposed on the magnet by the interface with the central tracker are modest magnetic field requirements and access for installation and maintenance. The field should be as high as possible and uniform. The magnet must allow access for installation and repair of the tracker and calorimeter components during a long maintenance period. Access requires mobile magnet halves. Calorimeter interface requirements primarily affect the design of the central detector support. However, weight and cabling of the calorimeters and consequent CDS stability lead to the physical separation of the magnet halves at the detector centerline. In addition, the magnet must withstand a liquid argon spill from a calorimeter leak without significant damage. The CDS must allow passage of compression elements between magnet halves to resist the 64-MN net axial force, and must allow room for detector services conduits between the magnet halves.

The magnet makes extensive use of the surface and underground facilities provided at the IR5 site. These include the surface fabrication structures, the heavy equipment movement paths, the experimental hall, and cryogenic, power, and control interfaces.

\subsubsection{Coil Design}

The cold mass of each GEM magnet half consists of modular windings, module-to-module joints, and bobbins and end flanges, which provide radial and axial structural support. Twelve identical coil modules are mechanically and electrically joined to form each of the symmetric coil halves. The mean radius of the windings is $9.5 \mathrm{~m}$, and the active winding length for the 12 modules is $14.25 \mathrm{~m}$. The cross section of a coil is shown together with the vacuum vessel and the forward field shaper in Figure 3-2. Parameters for the cold mass subassemblies and related magnet parameters are summarized in Table 3-3.

Table 3-3. Cold mass parameters.

\begin{tabular}{lrc}
\hline Mean radius of windings & 9.5 & $\mathrm{~m}$ \\
Cold mass length (per half) & 14.25 & $\mathrm{~m}$ \\
Conductor length (total) & 27219 & $\mathrm{~m}$ \\
$\begin{array}{l}\text { Conductor and insulation mass } \\
\text { (per half) }\end{array}$ & 158000 & $\mathrm{~kg}$ \\
Mass of 4 K structure (per half) & 525000 & $\mathrm{~kg}$ \\
Operating current & 50200 & $\mathrm{~A}$ \\
Stored energy & 2.5 & $\mathrm{GJ}$ \\
Inductance & 1.98 & $\mathrm{H}$ \\
Number of turns (per half) & 228 & \\
Peak voltage to ground & 500 & $\mathrm{~V}$ \\
Tum insulation thickness & 1.25 & $\mathrm{~mm}$ \\
Ground insulation thickness & 6 & $\mathrm{~mm}$ \\
\hline
\end{tabular}


The NbTi superconducting cable ${ }^{13}$ is enclosed in conduit and bathed in supercritical helium. Each coil half is joined to the external bus by two vapor-cooled current leads that operate at $50.2 \mathrm{kA}$. Similar leads, rated for 75-kA service, are used at the University of Wisconsin Applied Superconductivity Center High Current Test Laboratory.

A section through the conductor is shown in Figure 3-7. The conductor consists of $450 \mathrm{NbTi-}$ copper superconducting strands that are twisted in a $3 \times 5 \times 5 \times 6$ pattem. The copper-to-superconductor ratio is $3.6: 1$. The cable is compacted inside a stainless steel tube, leaving a helium void fraction of about $37 \%$. The cable-in-conduit is enclosed in an aluminum sheath that provides hot-spot protection for the conductor. The conductor will be assembled at the factory into full-module finished lengths of $1134 \mathrm{~m}$.

The conductor is wrapped with $1.25-\mathrm{mm}$-thick turn insulation. A layer of 6-mm insulation is applied to grounded surfaces. Ground insulation isolates the outer side of the winding from the aluminum bobbin and the inner side from the radiation intercept. The combination of the helium thermosiphon cooled bobbin and inner radiation shield completely surrounds the conductor and shunts heat around it. The helium coolant flows by convection through tubes welded to the outer side of each bobbin.

Each coil segment (see Figure 3-8) is comprised of 19 conductor turns that are wound in a single layer inside the bobbin and then-captured axially by compression flanges. The compression

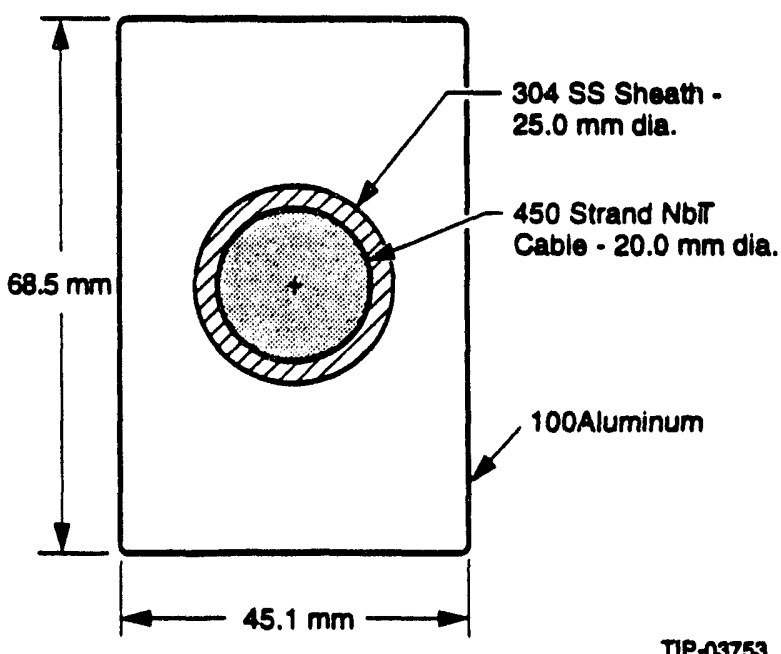

FIG. 3-7. GEM magnet cable-in-conduit conductor. flange bolts are tightened during construction to apply precompression to the coil stack. Precompression is sized to provide an initial axial winding displacement greater than arises in service from electromagnetic compression of the winding. Precompression of the winding stack therefore prevents significant conductor motion when the magnet is powered.

The present plan is to provide three separate helium cooling circuits to the coil modules. The thermosiphon piping attached to the bobbin provides most of the cooling; a low-flow-rate supercritical helium coolant supply to the conductor conduit provides additional transient stability against disturbances; and a separate supercritical helium circuit provides stable coolant supply to the joints. Alternate cooling schemes that might reduce the number of loops and/or simplify the system are being evaluated.

The bobbin is the primary structural element of each cold mass. It restrains the windings against radial magnetic pressure, shares in carrying the cumulative axial load, provides support against gravity, and distributes concentrated loads at the cold mass support points. Each bobbin is fabricated from 76-mm-thick 6061-T6 aluminum plates that are rolled or stretch-formed to a cylinder with a nominal inner radius of $9.54 \mathrm{~m}$. In the baseline design, each bobbin is composed of four arced segments and four tensioning joints, which also act as azimuthal insulating breaks. Insulating breaks are provided in the bobbin to reduce eddy-current heating during fast discharge. Without insulating breaks, the induced currents in the bobbin during emergency discharge would be sufficient to quench the coil. Recharging the magnet would then await cooldown, which would take several days.

The axial compression flange segments are also made from 6061-T6 aluminum. In addition to providing axial pre-stress, the flanges are used to bolt modules together and connect them to the cold mass supports. The bolting rings in the compression flange segments have alternating bolt holes and slots so that, as adjacent flanges are mated, small azimuthal alignment adjustments can be made between coil modules.

Lorentz forces on this solenoid vary slightly from a those on a continuous solenoid due to the gaps 


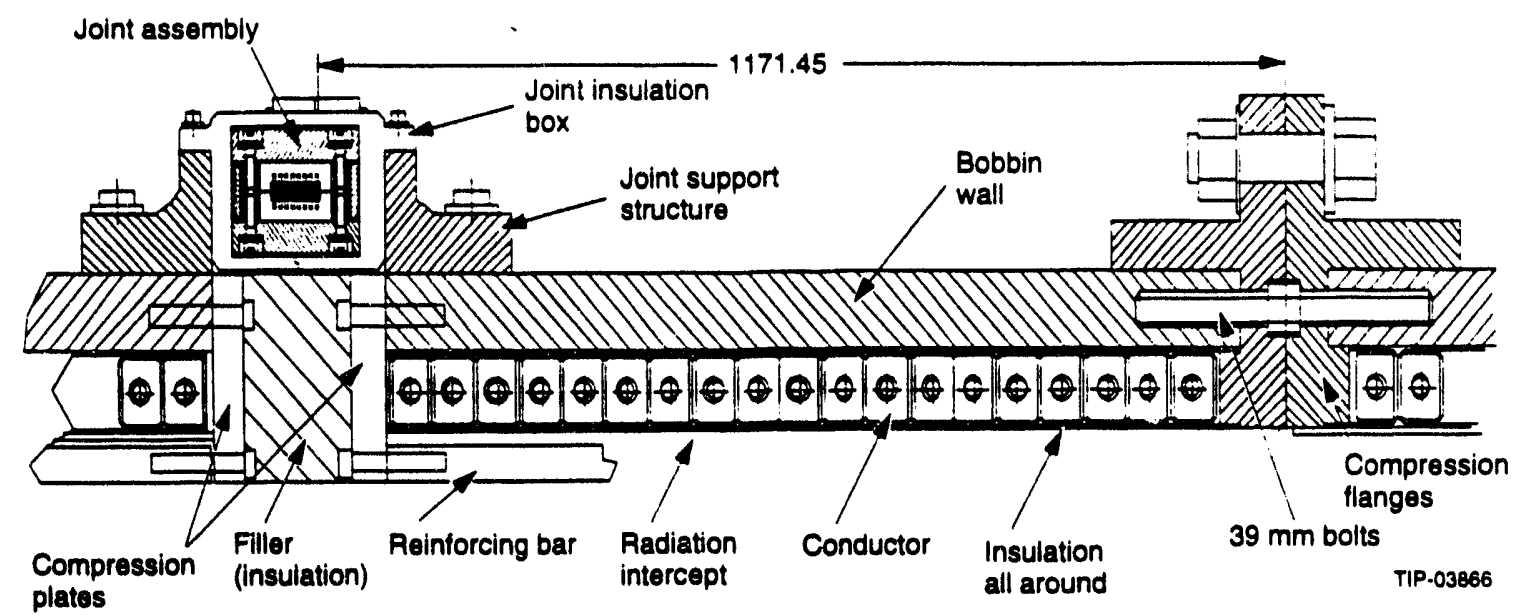

FIG. 3-8. Coil module cross section.

in the windings at module joints and between coil halves. The radial field component on the winding peaks at $1.6 \mathrm{~T}$, at the outermost tum, but is also significant for windings near the magnet midplane due to the $. j-\mathrm{m}$ gap between winding halves. The accumulation of the resulting axial forces within each module is shown in Figure 3-9. The largest axial Lorentz load of $452 \mathrm{kN} / \mathrm{m}$ around the circumference is in the outermost module.

The combined behavior of the cold mass elements has been modeled with a variety of axisymmetric and three-dimensional models. Conductors, bobbins, and flanges all share hoop and axial loads. Slip surfaces at the bobbin/conductor interface, as well as the sheath plasticity, have been considered in the model. The most significant loading is the outward magnetic pressure, which is resisted by bobbin and conductor hoop tension. The stress along the length of the 76-mm-thick bobbin is

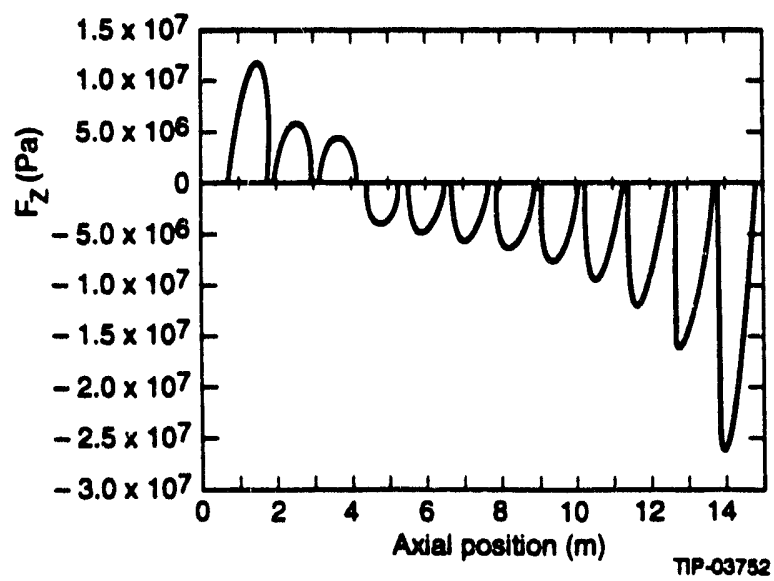

FIG. 3-9. Cumulative axial pressure within each coil module in pascals.

plotted in Figure 3-10. The contribution from the strength of the sheath has been considered in this calculation. The bobbin material yields at $276 \mathrm{MPa}$ at room temperature. When the effect of the local plasticity in the sheath is accounted for, the peak bobbin stress rises to $34 \mathrm{MPa}$. If the bobbin is assumed to bear the entire magnetic pressure, the estimated hoop tension rises to $49 \mathrm{MPa}$, still well below $2 / 3$ yield stress or $1 / 3$ ultimate stress.

The vacuum vessel provides the main structural support and insulating vacuum for the cold mass. ${ }^{14}$ Each of the two identical annular vessels, one for each coil half, weighs approximately $900 \mathrm{Mg}$ and is $15.1 \mathrm{~m}$ long by $21.8 \mathrm{~m}$ in outer diameter (Figure 3-11). They are independently mounted to the floor of the experimental hall, and can be lifted

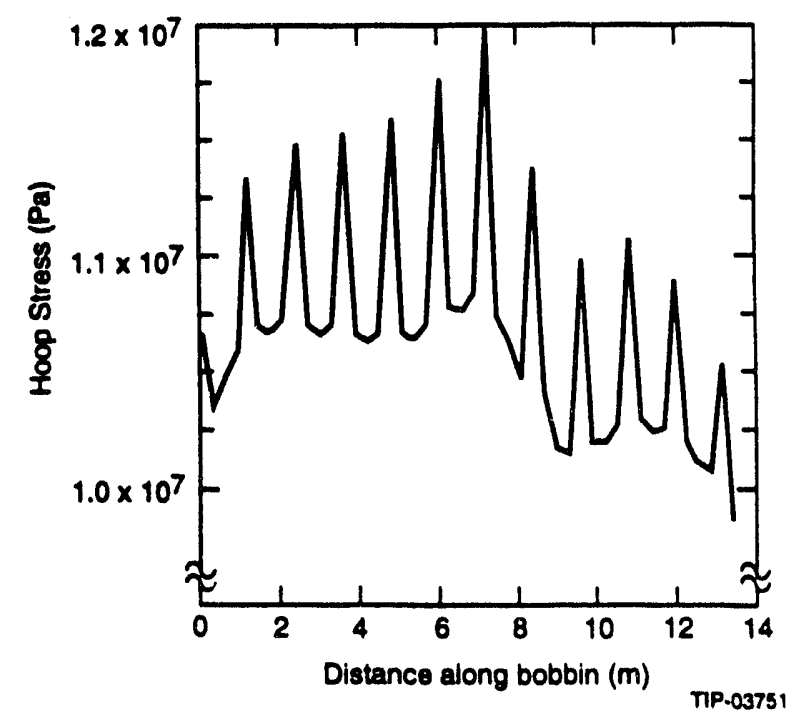

FIG. 3-10. Hoop stress along the magnet axis in pascals. 


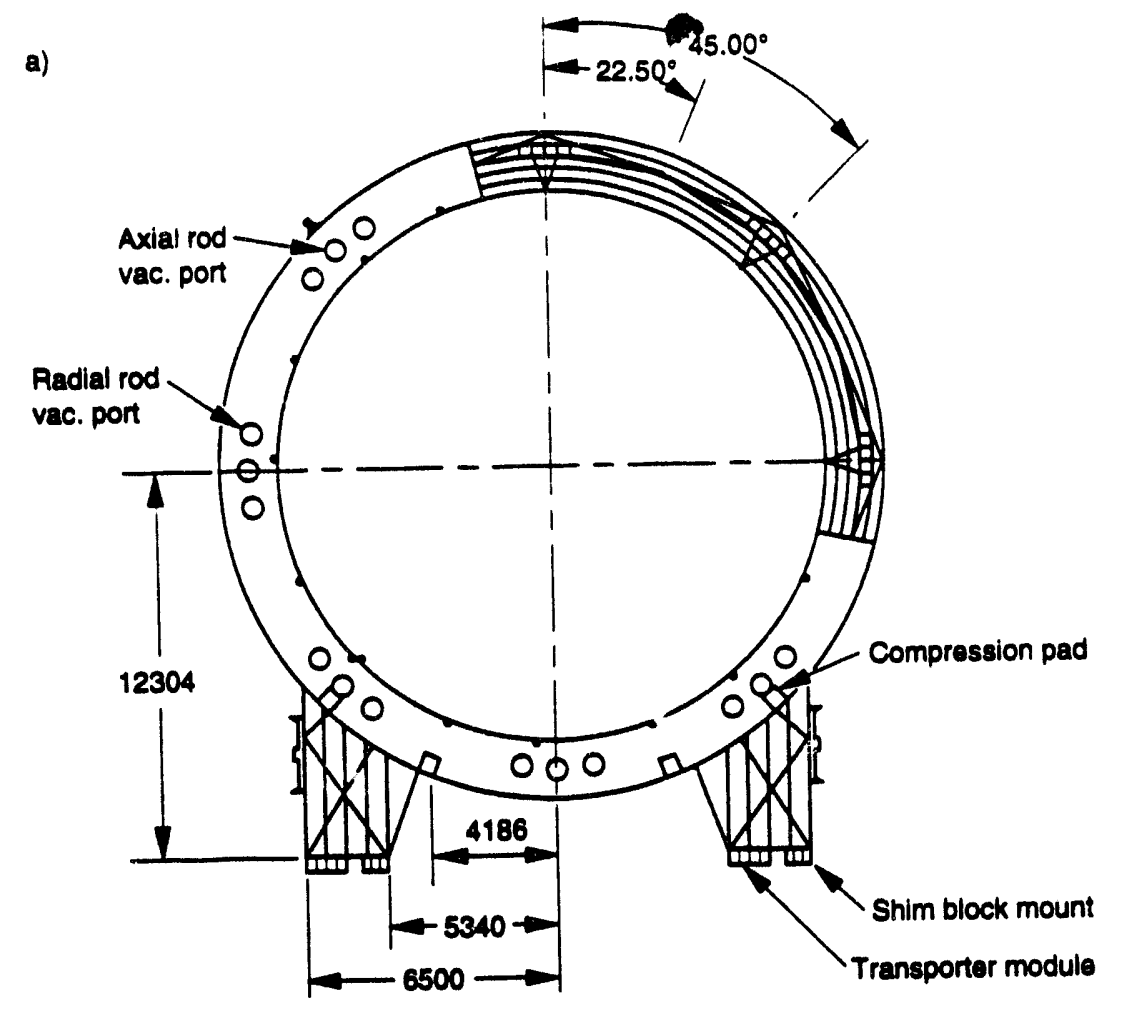

FIG. 3-11a. Vacuum vessel end view.

All dimensions in $\mathrm{mm}$

b)

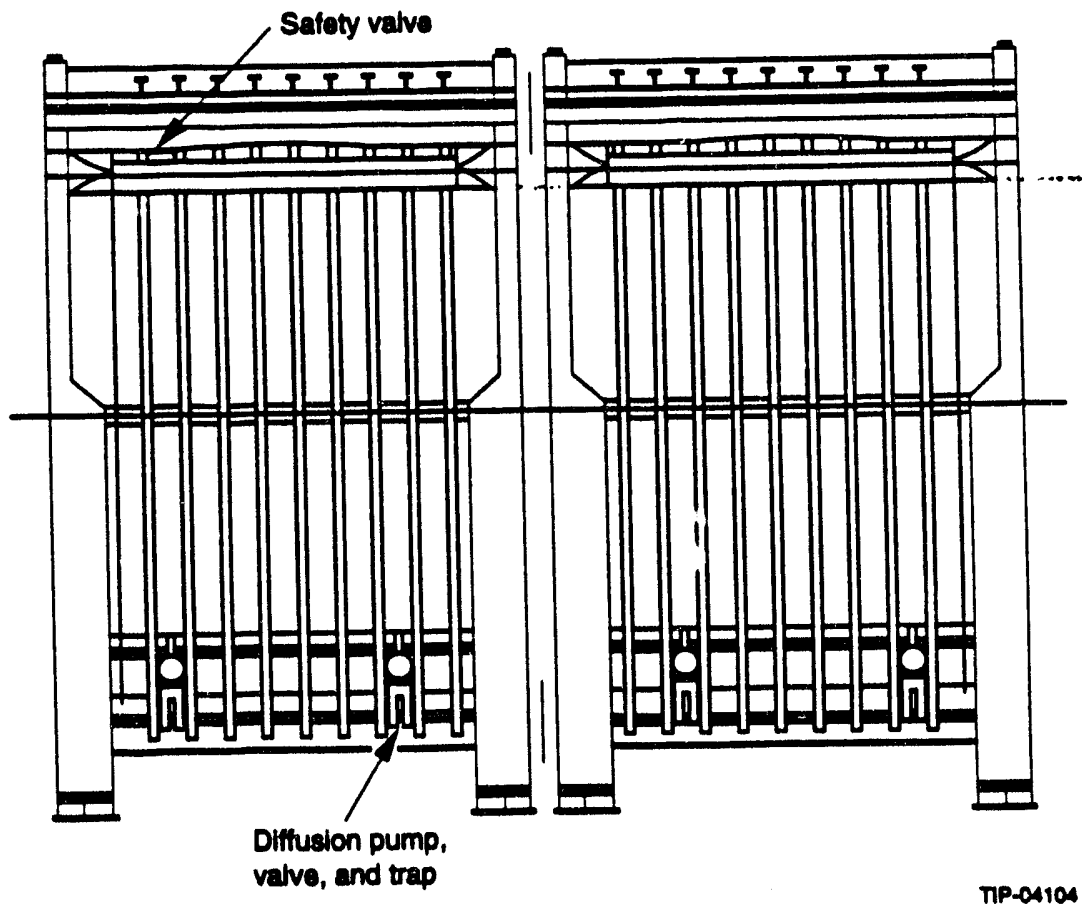

FIG. 3-11b. Vacuum vessel side view showing the end-rings and periodic ring stiffeners. The axial longerons resist the attractive magnetic force between each cold mass and the field shapers. 
off the fixed mounts and transported to the ends of the experimental hall to provide access to components within the detector. Each vacuum vessel car be evacuated independently of the other.

The two vacuum vessels are separated at the detector midplane by the central detector support (CDS). The CDS design allows space for columns that withstand the attractive force between the solenoids without placing stress on the CDS. A total of $64 \mathrm{MN}$ of compression results from the total magnetic forces on the coils and the forward field shapers (Figure 3-12). This force is transmitted from the outer ends of the vessels to the midplane by compression of eight structural columns (longerons) on the outer shell of each ves el. Axial loads from the coil $(52 \mathrm{MN})$ are carried in tension from the mid-point of the cold mass through eight axial flexures that fasten to the outboard vessel end ring at the eight longerons. Axial loads from the FFS (12 MN) bear directly on the outboard end of these longerons through shim blocks.

Feed-through ports are provided in the vessel at various locations for relief valves, vacuum lines, cryogenic lines, vapor-cooled leads, sensor cables, and access for $s$ upport rod tensioning. The selection of the vessel shell material is under review. Type 304 stainless steel, cryogenic (9\%) nickel steel, and low-carbon pressure vessel steel are under consideration. Cost, magnetic and vacuum performance, strength, and fracture safety are the main parameters under study. Currently the plan is to employ stainless steel (type $304 \mathrm{~L}$ ) for all structures that must be non-magnetic to preserve muon system performance. All other structural elements will be constructed from A36 low carbon steel.

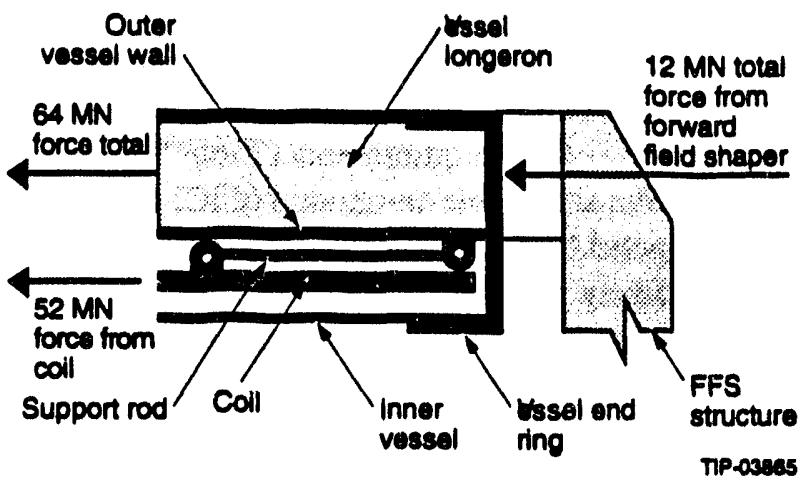

FIG. 3-12. Schematic of the axial magnetic forces on restraint rods.
The cold mass is supported within the vacuum vessel by long, relatively slender rods that attach to the end ring of the vacuum vessel. Radial rods. oriented in a vertical plane at each end of the cold mass, support the weight of the coil. Axial flexures. oriented parallel to the axis of the vessel, transfer the axial forces into the vessel. In the baseline design, a total of eight pairs of radial rods transfer the coil weight to the vessel at each end ring. Figure 3-13 shows the gussets in the vacuum vessel end ring designed to react these loads. The rod ends are fastened to the vessel end ring with rod end nuts on spherical washers, identical to those used on the axial rods. Vacuum ports cap off the rod ends to seal the vessel. Radial rod pairs are pre-tensioned using commercially available hydraulic tensioners. When the proper tension and alignment is achieved in opposing rods, the rod end nuts are tightened, and the hydraulic cylinder is depressurized. The procedure is repeated for each pair of rods on each end of each cold mass.

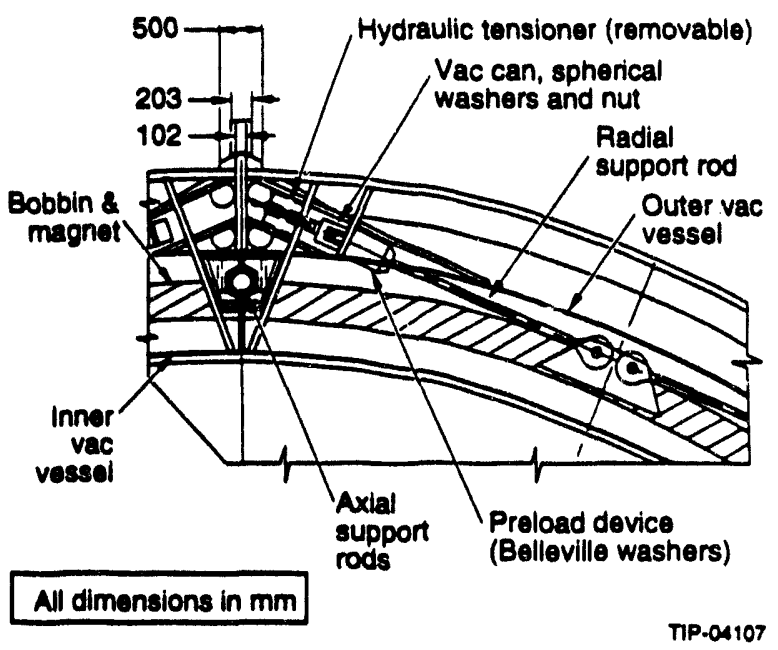

FIG. 3-13. Detail of the vacuum vessel support.

While sufficient structural analysis has been carried out to verify the essential features of the vacuum vessel design, analyses are revisited as required by new design features. Fatigue analysis is not necessary because the vessel is not expected to see more than a few hundred load cycles during its 20 -yr operation. The vacuum vessel is subjected to a complex loading pattern due to the vacuum load, component weights, thermal, magnetic, and possibly seismic forces. Detailed finite element modeling has determined that the stresses and deflections of the vessel and support structures are acceptable (see Table 3-4). 
Table 3-4. Summary of structural analysis results.

\begin{tabular}{|c|c|}
\hline \multicolumn{2}{|l|}{$\begin{array}{l}\text { Sag of vessel end ring due to } \\
\text { gravity: }\end{array}$} \\
\hline 12 o'clock vertical sag & $6.1 \mathrm{~mm}$ \\
\hline $\begin{array}{l}3 \& 9 \text { o'clock horizontal } \\
\text { expansion }\end{array}$ & $6.1 \mathrm{~mm}$ \\
\hline $3 \& 9$ o'clock vertical sag & $1.3 \mathrm{~mm}$ \\
\hline $\begin{array}{l}3 \& 9 \text { o'clock end ring } \\
\text { rotation }\end{array}$ & $4.8 \times 10^{-4} \mathrm{rad}$ \\
\hline \multicolumn{2}{|l|}{$\begin{array}{l}\text { Sag of vessel end ring under } \\
\text { combined loads: }\end{array}$} \\
\hline 12 o'clock vertical sag & $1.0 \mathrm{~mm}$ \\
\hline $\begin{array}{l}3 \& 9 \text { o'clock horizontal } \\
\text { contraction }\end{array}$ & $0.5 \mathrm{~mm}$ \\
\hline 3 \& 9 o'clock vertical sag & $0.3 \mathrm{~mm}$ \\
\hline $3 \& 9$ o'clock Z rotation & $7.4 \times 10^{-5} \mathrm{rad}$ \\
\hline $\begin{array}{l}\text { Maximum radial rod load } \\
\text { (all load cases combined) }\end{array}$ & $2.67 \mathrm{MN}$ \\
\hline $\begin{array}{l}\text { Maximum axial rod load } \\
\text { (magnetic load) }\end{array}$ & $6.5 \mathrm{MN}$ \\
\hline $\begin{array}{l}\text { Maximum rotation on ID of } \\
\text { vessel end ring }\end{array}$ & $8 \times 10^{-4} \mathrm{rad}$ \\
\hline
\end{tabular}

Cold mass supports serve three purposes: to support the weight of a cold mass, to maintain alignment of the coil to the SSC beam axis, and to counteract the axial magnetic force ( $52 \mathrm{MN}$ ). While performing these functions, the supports must also minimize heat to LHe-cooled structures and allow thermal contraction without inducing significant stresses and consequent distortion to the cold mass. The cold mass assembly will contract about $8 \mathrm{~cm}$ in diameter and $6 \mathrm{~cm}$ in length as it cools from room temperature to $4 \mathrm{~K}$. Radial rods are grouped in opposing pairs about the circumference of the coil, spaced at $45^{\circ}$ increments and oriented tangent to the circumference. Eight pairs of rods support each coil end. As the coil cools from $300 \mathrm{~K}$ to $4 \mathrm{~K}$, the radial support rods rotate toward the beam centerline on spherical bearing surfaces. This design preserves the position of the magnetic centerline of the coil relative to the beam centerline of the system.

The function of the axial flexures is to resist magnetic attraction of cold masses. Eight axial flexures are spaced at $45^{\circ}$ intervals about the circumference to transfer the load directly to the eight large compressive members (longerons) on the vacuum vessel. Both the radial and axial supports have $\mathrm{LN}_{2}$ intercepts to reduce $\mathrm{LHe}$ consumption. The LHe heat load from the radial rods is about $60 \mathrm{~W}$; from the axial rods, abuut $35 \mathrm{~W}$. These values compare well with the heat budget provided by the LHe refrigerator system.

The cold mass and supports see a complex combination of loads from gravity, thermal contraction, and magnetic forces. A model has been developed that analyzed the combined effects of these loads on the cold mass with support rods. The maximum stress at the rod attacument points reaches $72 \mathrm{MPa}$. Radial thermal contraction overcomes expansion due to radial magnet loading, resulting in a net shrinkage of $80 \mathrm{~mm}$ in diameter.

Thermal radiation shields enclose the cold mass at four locations. First, 16 shield segments form a cylindrical shell between the outer vacuum vessel and the cold mass. Next, another group of 16 shield segments forms a cylindrical shell between the cold mass and the inner vacuum vessel shell. Finally, shaped elements form radiation shield caps at both ends of the cold mass. These enclosures effectively intercept room temperature radiation to the cold mass. Superinsulation will be applied to each side of the thermal radiation shields. It decreases thermal radiation to both the $\mathrm{LHe}$ and $\mathrm{LN}_{2}$ circuits and reduces refrigerator load oscillation due to vacuum variations. ${ }^{15}$ All $\mathrm{LN}_{2}$ tubing is joined at the outboard end of the magnet. Ports in the end flange of the vacuum vessel will provide access to tube welds for leak finding and repairs.

\subsubsection{Conductor and Coil Joints}

The magnet must be stable and reliable to insure maximum availability of the detector. After careful review of the requirements (Table 3-5) and the state of the art, a cable-in-conduit (CIC) conductor was selected. ${ }^{9}$ This type of conductor has had the benefit of many years of development by the U.S. and international magnetic fusion programs. ${ }^{16,17,18}$ It will simplify the on-site winding and reduce the risk of operating instabilities. It is specifically designed to maximize the use of proven manufacturing processes, tooling and operating experience gained from previous CIC conductors. The chal- 
lenges of manufacturing long lengths of conductor and such a large magnet have been resolved by detailed planning and regular consultation with potential industrial suppliers. ${ }^{19,20}$

Table 3-5. Conductor requirements.

\begin{tabular}{lrl}
\hline Central field & $0.8 \mathrm{~T}$ \\
Nominal field at winding & $1.1 \mathrm{~T}$ \\
Peak field at winding & $1.6 \mathrm{~T}$ \\
Operating temperature & $4.5 \mathrm{~K}$ \\
Operating current & $50.2 \mathrm{KA}$ \\
Maximum hot spot temperature & $100 \mathrm{~K}$ \\
Maximum voltage to ground & $500 \mathrm{~V}$ \\
Number of charge/discharge cycles & 200 & \\
Charging time & $8 \mathrm{hr}$ \\
Maximum number of quenches & 100 & 100 \\
\hline
\end{tabular}

Although the central field of $0.8 \mathrm{~T}$ and the resulting electromagnetic loads are well within the state of the art of superconducting technology, large dimensions of this magnet necessitate on-site coil winding and magnet assembly. To minimize the risk inherent in on-site construction, a robust conductor and coil design are needed.

The conductor is composed of multi-filamentary $\mathrm{NbTi}$-copper composite wires in a multistage cable enclosed by a circular stainless steel conduit. The conduit is then surrounded by a rectangular sheath of aluminum. In this design the stability against fast transients is provided by the local copper in the multi-filamentary strand in contact with supercritical helium. The aluminum sheath provides the low resistance current shunt for hot spot protection during a quench. Key conductor characteristics are shown in Table 3-6. A complete description of the conductor is provided in Refs. 1 and 21 .

The conductor is designed to operate at $50.2 \mathrm{kA}$, generating a central field of $0.8 \mathrm{~T}$. The magnetic field over most of the conductor is approximately $1.1 \mathrm{~T}$, with a peak field of $1.6 \mathrm{~T}$ near
Table 3.6. NbTi conductor configuration.

\begin{tabular}{ll}
\hline Stran diameter & $0.73 \mathrm{~mm}$ \\
Cu:SC ratio & $3.6: 1$ \\
$\begin{array}{l}\text { Cu relative resistivity ratio } \\
\left(Q_{\text {RT }} / Q_{4.5 \mathrm{k}}\right)\end{array}$ & $>150$ \\
Cable strands & 450 \\
$304 \mathrm{~L}$ stainless steel conduit ID/OD & $20 / 25 \mathrm{~mm}$ \\
1100 Aluminum sheath dimensions & $45.1 \times 68.5 \mathrm{~mm}$ \\
Conductor length & $1134 \mathrm{~m}$ \\
\hline
\end{tabular}

the outboard end. The coil is designed to operate with stagnant supercritical helium at a nominal temperature of $4.5 \mathrm{~K}$ and a pressure of $350 \mathrm{kPa}$ within the conductor conduit. In steady-state, the operating heat loads are thermal radiation and conduction. These heat loads are removed continuously by conduction to the aluminum coil bobbin which is, in turn, cooled by the helium thermosiphon. There is no need to have a positive flow of helium through the cable to achieve the required stability.

The nominal operating margins on critical current and current sharing temperature are quite large, e.g., $\mathrm{I} / \mathrm{I}_{\mathrm{c}}=0.225$, with a temperature margin of $3.4 \mathrm{~K}$, as illustrated in Figure 3-14. The superconducting strand requires a critical current carrying capability that is easily achievable by standard materials and wire production methods.

The CIC conductor is very stable against sudden disturbances. CIC conductors are distinguished by the lack of "training" often found in coils made with other conductors. Stability is provided by the high heat capacity of helium in direct contact with a large area of superconducting wires. The superconducting strands are buffered from external disturbance energy by the helium in the conduit. The energy margin is provided by the enthalpy of the helium and the wire between the operating temperature of $4.5 \mathrm{~K}$ and the current sharing temperature. This is dominated by the helium enthalpy, which is 1-2 orders of magnitude greater than that of all the other strand components.

The conductor is also stable in the less likely event of an internal disturbance. Since the nominal charge and discharge time is $8 \mathrm{~h}$, the energy will be 


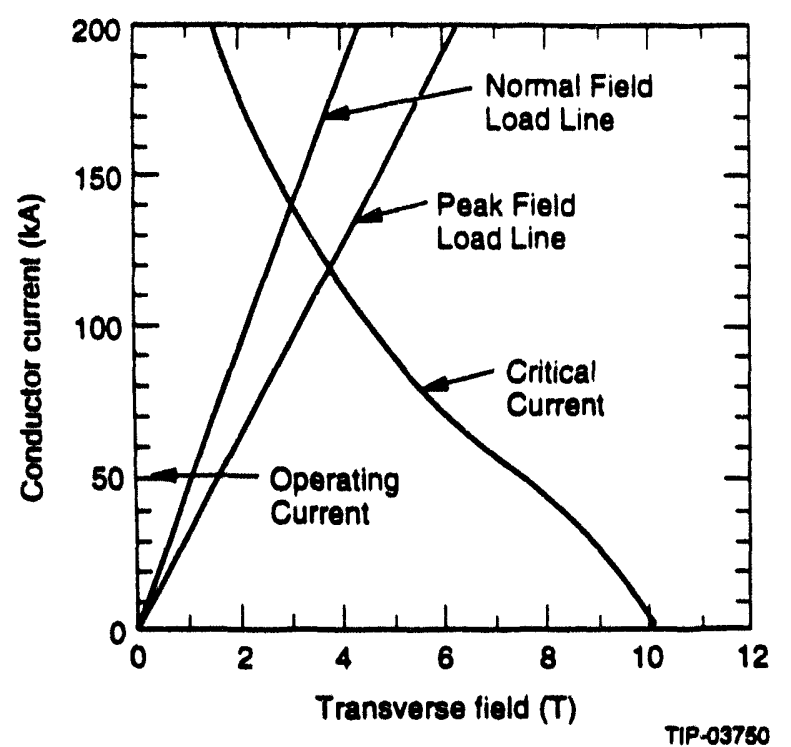

FIG. 3-14. Conductor critical current versus magnetic fieid and operating point.

removed in quasi-steady state by the helium thermosiphon. Another unlikely disturbance source could be sudden wire motion within the cable. Measurements of mechanical hysteresis have been performed at $4.2 \mathrm{~K}$ on a large, similar cable. The results indicate that even if all the energy were deposited suddenly in the cable, the energy margin is at least one order of magnitude greater.

For internal disturbances the stability margin is defined as the largest sudden energy deposition to the conductor from which it can immediately recover the superconducting state. The stability margin is not necessarily equal to the enthalpy margin of the helium because the helium is only effective to the extent that the energy dissipated directly in the cable is transferred to the fluid. This depends on the power balance between heat generation, due primarily to Joule heating in the strand, and the convective heat flux to the supercritical helium. The conductor is designed to ensure that the energy balance is always satisfied, i.e., at normal operating conditions the helium enthalpy is used to maximum advantage. This is accomplished by ensuring that the limiting current is above the operating current. The limiting current is defined as:

$$
I_{\mathrm{lim}}=\sqrt{\frac{h p A_{\mathrm{cu}}\left(T_{c}-T_{b}\right)}{\varrho_{\mathrm{cu}}}},
$$

where

$$
\begin{aligned}
& h=\text { heat transfer coefficient }\left(\mathrm{W} / \mathrm{m}^{2}-\mathrm{K}\right) \\
& p=\text { cooled perimeter }(\mathrm{m}) \\
& A_{\mathrm{cu}}=\text { area of copper stabilizer in cable }\left(\mathrm{m}^{2}\right) \\
& Q_{\mathrm{cu}}=\text { copper stabilizer resistivity at operating } \\
& \quad \text { temperature }(\Omega-\mathrm{m}) \\
& T_{c}=\text { critical temperature }(\mathrm{K}) \\
& T_{b}=\text { initial helium temperature }(\mathrm{K}) .
\end{aligned}
$$

By requiring that the operating current of $50,200 \mathrm{~A}$ is $95 \%$ of the limiting current, the maximum i.sitial helium temperature can be calculated. The resistivity of copper with $R R R=150$, and a field of $1.6 \mathrm{~T}$, is $0.179 \times 10^{-9} \Omega$-m at 4.5 to $5 \mathrm{~K}$. The critical temperature is $8.7 \mathrm{~K}$, the copper area is $147.4 \mathrm{~mm}^{2}$, the effective wetted perimeter is $0.86 \mathrm{~m}$, and the heat transfer coefficient has a value of $-1000 \mathrm{~W} / \mathrm{m}^{2}-\mathrm{K}$. With the above values the maximum initial helium temperature is $4.8 \mathrm{~K}$. However, at $1.1 \mathrm{~T}$ the copper resistivity is $0.156 \times$ $10^{-9} \Omega-m$, which results in a maximum initial temperature of $5.3 \mathrm{~K}$.

The energy margins for the conductor can be calculated for both internal and external disturbances. For external disturbances, the energy margin is a little higher and it is dominated by the enthalpy of helium. With an initial temperature of $4.5 \mathrm{~K}$ and a final temperature of $7.6 \mathrm{~K}$, the energy margin is calculated from the change in enthalpy and it is $300 \mathrm{~J} / \mathrm{m}$. For internal disturbances, a slightly more complicated calculation is required in order to take into account the heat transfer between the copper and the surrounding helium. This calculation gives $250 \mathrm{~J} / \mathrm{m}$ as the margin for internal disturbances.

The energy margins for the coil segments are summarized as follows:

For external disturbances: $300 \mathrm{~J} / \mathrm{m}$

For internal disturbances: $250 \mathrm{~J} / \mathrm{m}$.

For comparison, the energy margins of indirectly cooled, high purity aluminum conductors typical of those used for other large solenoidal detector magnets (e.g., CDF, ALEPH, DELPHI) are between one and two orders of magnitude lnwer.

The specified superconducting strand requires no special development. The requirements on the 
GEM conductor strands are significantly less severe than on those of the SSC collider dipole strands. Production of this wire is straightforward. and thus the full production run should require only a good quality assurance program and acceptance testing.

Given these large stability margins the GEM magnet is stable against quenches for almost all conceivable operating conditions. Allowances must be made, however, for safe removal of the coil energy if an unforeseen event occurs. The coil segments are simultaneously and continuously monitored by redundant compensated voltage taps when the coil is carrying any current. Quenches are detected by quench voltage monitors and triggering circuits. A trigger opens redundant curren interrupters to include a large dump resistor into the DC circuit so that the coil energy can be safely dissipated. The time constant is determined by the $L / R$ time constant of the coil-resistor circuit. The dump resistance of $20 \mathrm{~m} \Omega$ is the largest that is consistent with coil insulation strength, conservatively specified as $500 \mathrm{~V}$ to ground. The resulting time constant for the dump circuit is $99.6 \mathrm{~s}$.

Coil quench calculations have been performed to choose appropriate dimensions of the aluminum sheath for quench protection, and to determine the time-dependent and maximum values of the conductor temperature and helium temperature and pressure. A conservative estimate of the temperature of the hottest region of the coil during a quench provides the basis for the pressure rating of the conduit. It is assumed that the hottest position in the coil is the center of the normal zone where the quench began. Joule heating occurs for the longest time at this location, while no heat is removed by the surrounding structure or the helium stream. It is further assumed that current is shared between the magnet cable and the aluminum sheath. The predicted hot spot temperature is $100 \mathrm{~K}$ for both helium and conductor. The helium pressure will increase, at constant density, to $44.5 \mathrm{MPa}$. These peak values will be reduced in operation by thermal conduction and electromagnetic induction to surrounding structures and most importantly by outflow of helium. Supporting analysis of time-dependent propagation of the quench provides similar results. The conduit is designed and will be tested for these conditions.
While much less strain-sensitive than other conductors, cable-in-conduit conductor must be protected from excessive strain and slippage of components of the winding. Preload of the coil winding and use of thick aluminum bobbin shells reduces strains. Ideally the aluminum sheath should remain in intimate electrical contact with the stainless steel conduit throughout the life of the magnet. Differential contractions produce large contact pressures at cooldown which slightly yield the sheath. Subsequent quench pressurization further strains the sheath; however, analysis shows ${ }^{l}$ that adequate contact pressure remains at $4 \mathrm{~K}$. The local interactions between conduit and sheath have been modeled in an elastic-plastic analysis for a sequence of load steps that includes cooldown, operation, quench, reduction in Lorentz force, and heat-up to room temperature. The model predicts that even after an initial load cycle produces local plastic strain in the sheath, the performance of the conduit, sheath, and bobbin remains adequate. Figure 3-15 shows the hoop stress history of the most strained elements during the first three cycles. The sheath hoop tension increases with the first Lorentz force application, but as the quench pressure is applied, hoop tension is relaxed and compression develops due to the radial compressive stress imposed by the expanding sheath. Compressive stresses remain locked-in for subsequent cycles, and the conductor effectively sheds some of the Lorentz forces to the bobbin. The analysis clearly shows that the local plastic behavior in the sheath stabilizes after one cycle and that the sheath does not continue to accumulate strain.

The baseline joint design provides a low resistance electrical connection between conductor ends on adjacent coil modules. There are eleven segment-to-segment joints and two end-module-tolead joints per magnet half. The joint halves are fabricated, attached to both ends of each length of conductor, and leak tested during conductor fabrication at the factory. This enables a complete length of conductor, with factory pre-terminated ends, to be shipped to the GEM coil winding facility, and virtually eliminates the need to make helium-tight welds to the conductor conduit in the field. During magnet assembly on site, the mating joint halves 


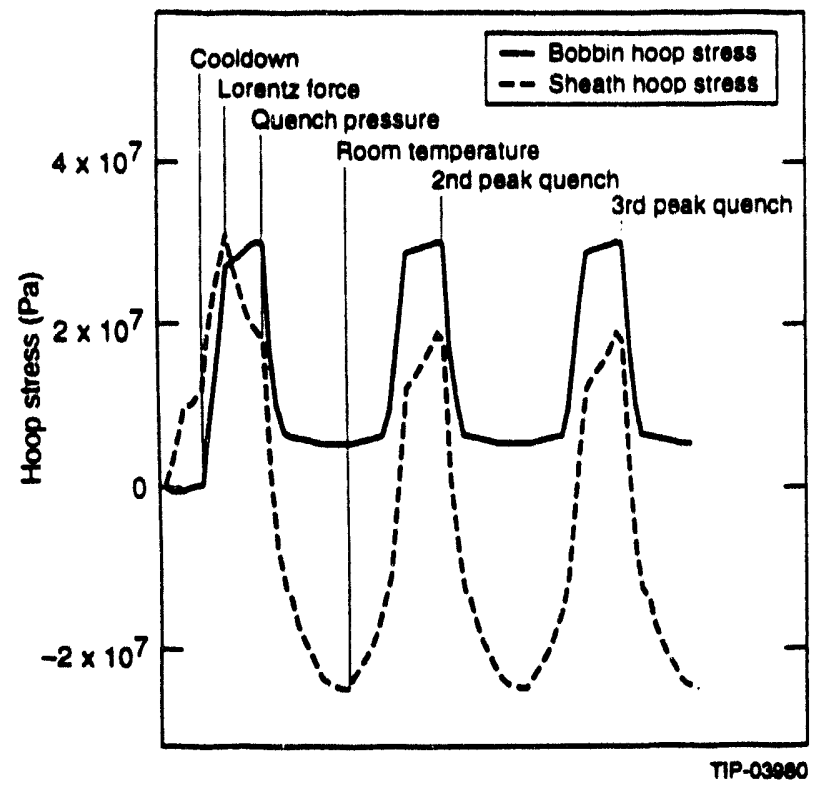

FIG. 3-15. Hoop stress history during first three load cycles. Cooldown, operation quench, and warming represent a single load cycle. Strains stabilize after three cycles.

(each separately sealed) are simply soldered and clamped together. Helium tubing from the cryogenic system is welded to pre-fitted pipe nipples on the joint halves. The supercritical helium is supplied to the conduit of each module conductor at one end of the 1134-m length and it is retumed to its cryogenic system at the other. All fittings are integral to the joint design and are also leak tested at the factory. The joint is depicted in Figure 3-16. A more detailed description is given in the conductor specification. 12 The joints between the coil modules are cryostable. This resistive portion of the coil circuit is not a "weak link," but is actually more stable than the bulk of the coil. This is accomplished by attaching large high conductivity aluminum blocks with integral cooling channels to reduce the Joule heating in the normal joint below the corresponding steady state heat flux capability. The joint lap resistance is expected to be $5 \times 10^{-10} \Omega$, generating the power of $1.25 \mathrm{~W}$ at $50.2 \mathrm{kA}$. This power level is an order of magnitude smaller than the level in joints for which cryostability has been provided. Stability is achieved with a moderate flow of $0.5 \mathrm{~g} / \mathrm{s}$ of supercritical helium at $4.5 \mathrm{~K}$.

\subsubsection{Forward Field Shapers}

The forward field shapers (FFS) are two large truncated steel cones that improve muon momentum

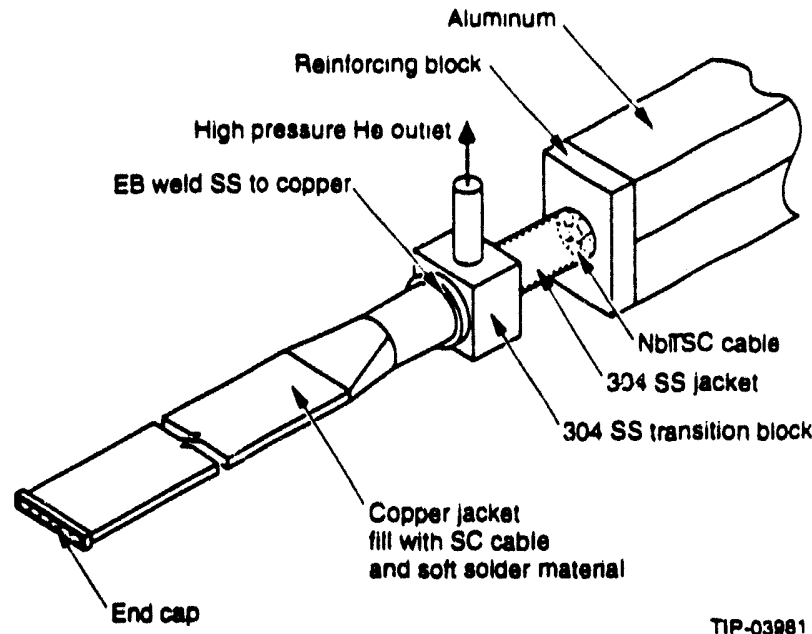

FIG. 3-16. Joint subassembly.

resolution by increasing the radial field component in the endcap region. Each field shaper weighs about $1265 \mathrm{Mg}$ and is centered on the detector axis by a movable support structure that abuts the end of the vacuum vessel. Each FFS assembly, including the support structure, is independently mounted on the experimental hall floor at reinforced mounting locations. The FFS structure will be moved by a transporter similar to those in use on the FNAL DO and CDF detectors. FFS movement is required for initial installation and for regular access to detector arrays within the magnet volume.

The FFS support structure is a large steel-plate weldment, which supports static, magnetic, and seismic loads. The FFS is cantilevered into the interior of the solenoid, putting large moments into the support structure. When retracted from the vessel during maintenance or repair, the field shaper structure is free standing, with no support from the vacuum vessel. The mornent from the cantilevered field shaper is carried by the legs and feet of the support structure, which extend under the vacuum vessel. Once installed, each support structure is supported by four semi-permanent mounts. The function of these steel weldments is to provide demountable structures which can be easily modified or replaced to ensure alignment.

During magnet operation each FFS is attracted toward the center of the detector by a magnetic body force of $12 \mathrm{MN}$. The three arms of the support act as large, deep section box beams to limit FFS movement to less than $2 \mathrm{~cm}$. The body force is transferred by the arms to the vacuum vessel at five bearing 
points. The load is carried by compression of the vessel longerons to the detector midplane where it is balanced by force from the opposite side.

Each pad between the FFS structure and the vessel consists of shims and a cylindrical bearing pad. The bearing pad allows the forward field shaper to deflect under magnetic load without imposing moments or stress concentrations into the end of the vacuum vessel. This gives the field shaper structure a hinged-end condition, which limits end moment loading on the vessel that could potentially misalign the muon system if mounted to its end rings.

Each FFS structure supports the weight of most of the endcap neutron shielding. The shielding has been conservatively estimated to be required at three locations: a nose cap, a layer on the cone surface, and a disk extending between the arms of the structure.

A combination of closed-form analysis and finite element analysis was used to design the forward field shaper and its support structure (Figure 3-17). The analysis included the conical FFS itself and the three-arm structure it attaches to. Structural elements were designed in accordance with the Unified Building Code or American Institute of Steel Construction Code, as applicable. Deflections are predicted to be less than $2 \mathrm{~cm}$ and stresses less than half of the material yield strengths.

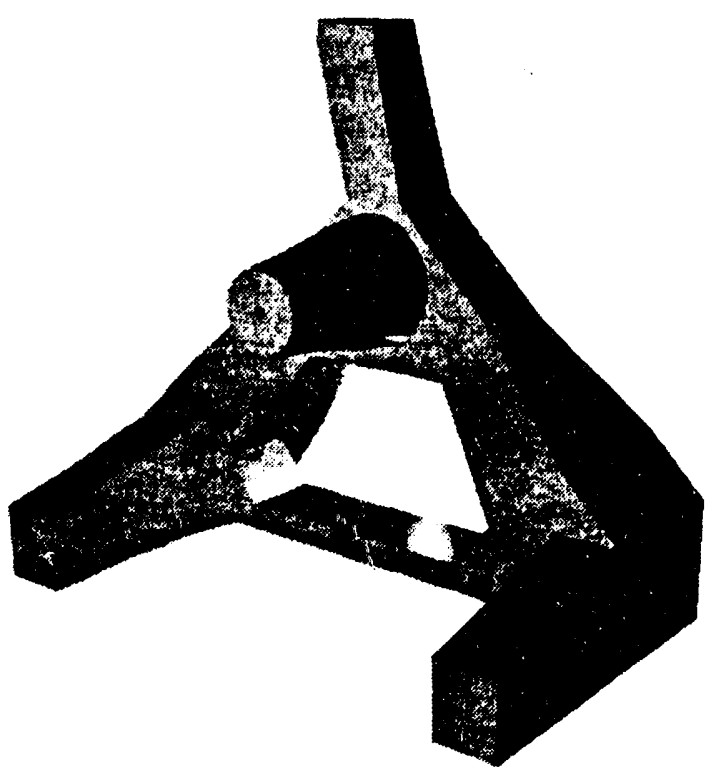

FIG. 3-17. Fonward field shaper model.

\subsubsection{Central Detector Support}

The central detector suppor (CDS) is a welded stainless steel structure designed to support the scintillating barrel calorimeter, the two endcap and barrel noble liquid calorimeters, and the central tracker. The combined weight of the central detectors and their services is approximately $3100 \mathrm{Mg}$. The CDS must fit in the $0.8-\mathrm{m}$ gap between the magnet vacuum vessels and remain clear of the detector hall walls and services transverse to the beam direction. This allows a maximum CDS length of approximately $25 \mathrm{~m}$ transverse to the beam direction. The structure is required to support the calorimeters, in various configurations, independently of the magnet halves but during operation can be shimmed to the magnet to derive some stiffness in the beam direction. The CDS must also be able to readjust the calorimeter position (small transverse displacements and small rotations) to correct for shifts in the facility structure, i.e., floor settlement or floor displacement caused by shifting loads.

The present design is based on conservative criteria, applied to two required loading configurations. The operational mode has all calorimeters installed and the CDS positioned. In this configuration the CDS must withstand the static load with a maximum Von Mises stress of $50 \%$ of the material's yield point stress. A possible maintenance configuration would have one liquid endcap calorimeter removed. Again in this configuration the maximum Von Mises stress was limited to $50 \%$ of the material's yield point stress. In addition to these static load cases the CDS must withstand the unlikely event of an earthquake with minimal damage. The Safe Shutdown Design Response Spectrum for the Comanche Peak steam generating plant in Fort Worth was adopted and applied to the CDS. The maximum acceptable stress for the dynamic scenarios was set at $60 \%$ of the material's ultimate tensile strength.

Figure 3-18 shows the most efficient structure developed from an extensive amount of finite element analysis. The bulk of the dead load is supported by the columns underneath the calorime- 
ters. These are welded structures $0.6 \mathrm{~m} \times 1 \mathrm{~m} \times$ $60 \mathrm{~mm}$ in cross section. A horizontal built up box section beam that attaches to the $A$ frames external to the magnet gives the CDS needed stiffness in the beam direction. This horizontal beam is built from five welded box sections and is designed to give flexural and torsional stiffness to the bartel scintillating calorimeter structure. A rib is added around the outside diameter of the barrel scintillating calorimeter structure to provide attachment points and load paths. Jacks are depicted under the two pedestal columns and the four A-frame legs to position the calorimeters during initial installation and to provide periodic position correction. Shims will be added under the legs to support the load and the jacks will be used when position corrections are necessary. This design is completely independent of the magnet structure in all configurations.

Preliminary finite element analysis results indicate a maximum Von Mises stress of $86.2 \mathrm{MPa}$ for the operational configuration. When one endcap calorimeter is removed (the maintenance configuration) the maximum Von Mises stress is $115 \mathrm{MPa}$. The earthquake results yield a maximum stress of approximately $241 \mathrm{MPa}$. All of these values meet the adopted design criteria for a standard structural stainless steel. More advanced analyses, which include a better model of the barrel scintillating calorimeter and its interface with the CDS, also indicate that the chosen values are conservative.

Because of the asymmetric geometry of the CDS, the entire structure is made from non-magnetic stainless steel. Presently the preferred alloy is $304 \mathrm{~L}$ because of costs, mechanical properties, magnetic properties, and weldability. Standard published mechanical properties for $304 \mathrm{~L}$ are $207 \mathrm{MPa}$ for $0.2 \%$ yield strength and $552 \mathrm{MPa}$ for ultimate tensile strength. However, a recent trip to Russian fabrication facilities revealed their preference for another alloy which is comparable to the US 321

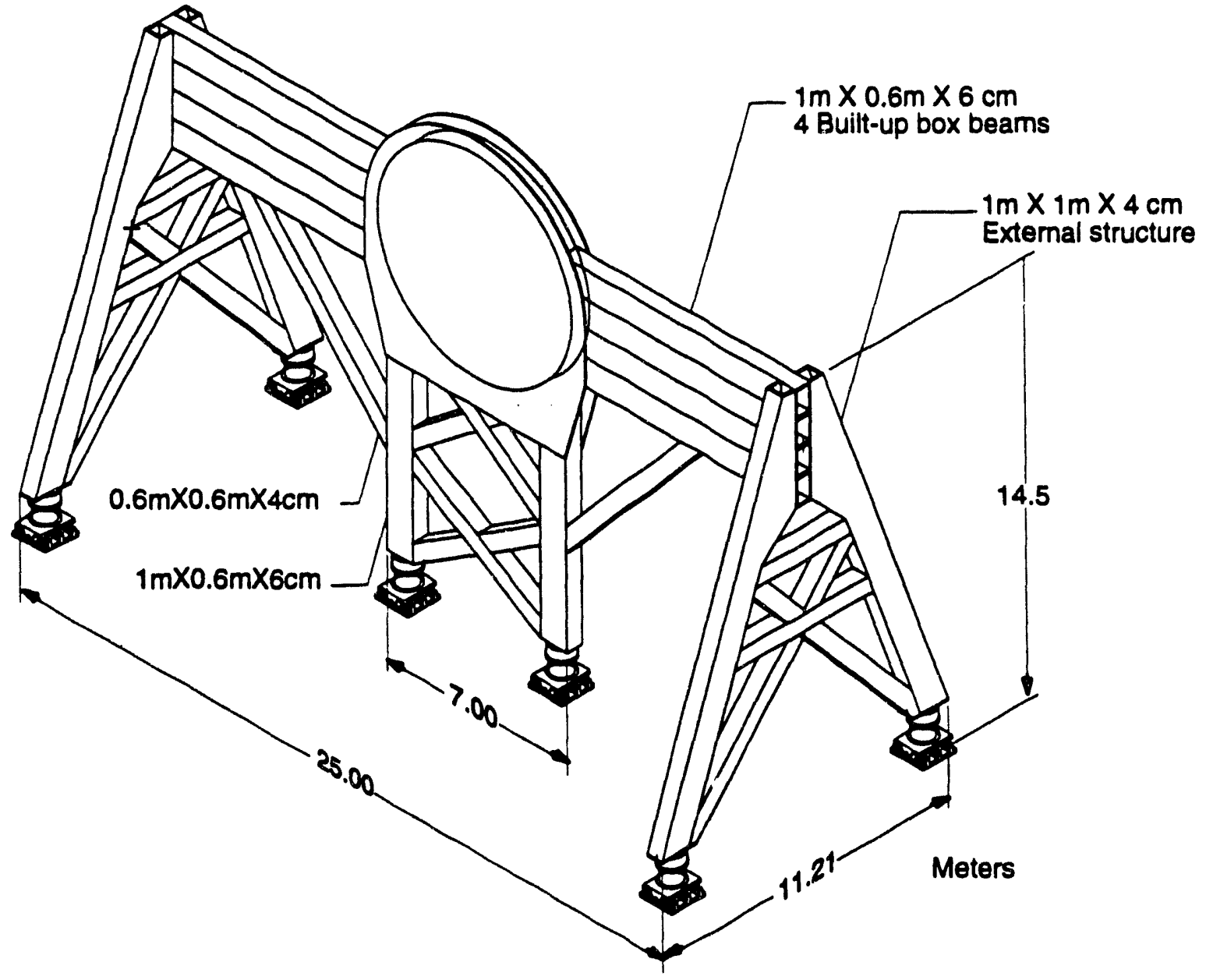

FIG. 3-18. Central detector support structure. 
alloy. This alloy contains $0.40 \%$ titanium which reduces or prevents carbide precipitation during welding or elevated temperature service. It exhibits higher yield point stress, higher ultimate stress; comparable (with 304L) machinability, weldability, and formability characteristics, and a maximum magnetic permeability of 1.004 . The material choice is still under study.

\subsection{FABRICATION, ASSEMBLY AND INSTALLATION}

\subsubsection{Introduction}

The major manufacturing challenge of the magnet system is managing its large size. The preliminary design phase of the magnet was focused on solving the problems associated with the size and weight of components. Plans have been produced and reviewed by potential industrial partners. The key steps-conductor manufacture, coil winding, and magnet assembly - have now been worked out in sufficient detail to ensure their feasibility.

\subsubsection{Work to Date}

Conductor manufacture has been systematically worked out 12,13,14 and evaluated by industry and GEM collaborators. Although the general plan is established, specific elements were identified that required further development and verification. A plan to evaluate prototype conductor performance at the University of Wisconsin's High Current Test Laboratory is being carried out. A winding specialist, Everson Electric Co., is evaluating the tooling requirements and the Martin Marietta Company's Y-12 site, a DOE Integrated Contractor, is building prototype full-scale winding tooling. An MIT/PFC joint development effort is testing subscale and full-scale prototypes. Preliminary specifications $12,13,14$ have been prepared for the conductor and the joint.

A detailed plan for on-site manufacturing, assembly, and test of the magnet has been prepared ${ }^{11}$ and reviewed. ${ }^{22,23}$ A self-consistent work plan including scope, cost estimate, and schedule has been prepared. Fabrication specialists were repeatedly consulted during the development of the vessel and forward field shaper designs and the magnet handling and assembly plans. Specifications were prepared for the vessel and the magnet and proposals for the magnet system are currently being evaluated.
Building the GEM magnet will be a challenge. with drama surpassing past magnet projects because of its "Texas" size, but the task is considered by industrial specialists to be within the state of the art.

\subsubsection{Conductor and Coil Joints}

The 450-strand, $3 \times 5 \times 5 \times 6$ cable is similar to superconducting cables (i.e., the 486-strand Westinghouse LCP cable and the 225-strand United States Demonstration Poloidal Coil (DPC) cable) that have been produced in multiple kilometer lengths. The first operation of the cabling phase is to twist the triplets in the same direction as the strand twist to obtain the best wire lays. The three other cabling operations then proceed in the same twist direction as the triplets. The completed cable is wrapped with a thin stainless steel tape to prevent "bird caging" of the strands and possible interference with the weld arc in the tube mill operation described below. The 1134-m lengths needed for the GEM magnet weigh approximately $1890 \mathrm{~kg}$, and we have located a final-stage machine with at least six 76-cm diameter spools that are capable of holding the 75-strand sub cables.

The baseline plan for fabrication of the GEM conductor conduit uses the same process as proven on the Westinghouse Large Coil Program conductor and more recently for the US-DPC conductor. In this process, the conduit is formed around the cable using a tube mill. The function of the mill is to shape a flat strip into a tube, weld the seam, and compact the tube to final dimensions. Seam welding is a critical process, because the conduit is the primary helium pressure boundary. Therefore, the tube mill operator will be qualified to the standards of the ASME Boiler and Pressure Vessel Code, Section 9. The procedures for this process have been worked out in detail. ${ }^{24}$

After welding, the conductor is rolled to a compaction that yields a nominal cable-space void fraction of $37 \%$. The conductor is then reeled in a single-layer onto a take-up spool with the weld seam in a radially outward position. Each conductor length is pressurized in a proof test to at least $25 \mathrm{MPa}$. For the final leak test, the helium-pressurized conductor reels are immersed in a liquid nitrogen bath.

Methods of extruding the long lengths of aluminum required for the sheath are being eva- 
luated. Discussions with vendors regarding manufacturing of the aluminum sheath and the required take-up spool equipment have identified two candidate designs. Methods for inserting the cable-in-conduit into each of the two candidate sheath designs are also being evaluated.

During joint fabrication, the conductor end is machined to accept the joint components on each end. The conduit is cut away from the superconducting cable and a transition block, prefitted with a pipe nipple for conduit helium supply, is slipped over the cable and welded to the conduit. A length of copper tubing is pulled over the cable and welded to the transition block. The cable is then rolled flat inside the tube and filled with solder. The tube is next soldered into an aluminum block containing the joint cooling grooves. An aluminum cover is then bolted over the transition block to provide sheath continuity. All helium joints are leak tested, and factory preassembly is complete. The joints are designed to be fully cryostable and are based on a similar proven design, part of the US-DPC program, which underwent stringent testing, including ramping at $5 \mathrm{~T} / \mathrm{s}$ without quenching.

\subsubsection{Coil Winding}

The 24 segments will be wound at two winding stations in the south assembly building (SAB) at the IR5 site. The 1134-m-long conductor will be insulated and wound into position within the bobbin at the rate of about one segment per month per station, beginning shortly after the SAB is completed. The challenge at this step will be to master the winding of such large sizes within the tight schedule constraints.

The bobbin will first be assembled on the preparation station adjacent to the two winding stations in the highbay of the SAB. The bobbins are formed of four quadrants held together with an insulated mechanical joint. Each quadrant includes a shell and an upper and lower set of flanges. Ground insulation will be bonded on the inner wall of the bobbin and the completed bobbin will then be transferred by overhead crane to the winding station.

Each winding station is composed of a ring turntable powered by a 20-hp motor (Figure 3-19). It features conductor feed; forming, cleaning, wrapping, and testing stations; an automatic conductor payout spool: and automatic clamps. The bobbin is aligned and secured to the tumtable as the conductor transport spool is placed onto the payout spool. As the conductor is fed from the payout spool, it is first forced through roll forms to achieve the proper radius of curvature. The conductor is then cleaned and wrapped with insulation. The conductor is pushed into place against the bobbin by a tractor drive and held in place during winding by automatic clamps. The top flanges are then installed along with the joint compression plates and the inner radiation shield. After inspection and certification, the finished coil segment is transported to an assembly station in the north assembly building (NAB). Winding is expected to be carried out using two shifts per day to satisfy schedule constraints.

\subsubsection{Magnet Assembly and Installation}

The magnets will be assembled as two independent halves. The assembly sequence is designed to meet the overall program schedule and accommodate the beneficial occupancy dates of assembly areas and the detector hall. A plan has been prepared that describes in detail the assembly steps and the equipment, procedures, and facilities required to assemble and test the magnet at the IR5 site. ${ }^{11}$ The plan is described in Chapter 9.

An important decision was whether to assemble the magnet halves with axis vertical or horizontal. After thorough discussion with vessel and magnet fabricators, qualified rigging specialists and engineers experienced with large special equipment handling, we have decided ou assembly with the axis vertical. The vertical orientation is best for vessel fabrication, coil winding, and assembly, and minimizes the number of special handling fixtures. After the $1500-\mathrm{Mg}$ magnet halves are assembled, they must be rotated to a horizontal position. A number of qualified riggers with experience in rotating large pieces of equipment have stated that $1500 \mathrm{Mg}$ is well within their capacity. Each lift will be carefully engineered and will conform to DOE rigging and handling standards.

\subsection{SUPPORTING SYSTEMS}

\subsubsection{Introduction}

Preliminary design of the cryogenic supply systems and the power and protection systems is well under way. Preliminary specifications have 


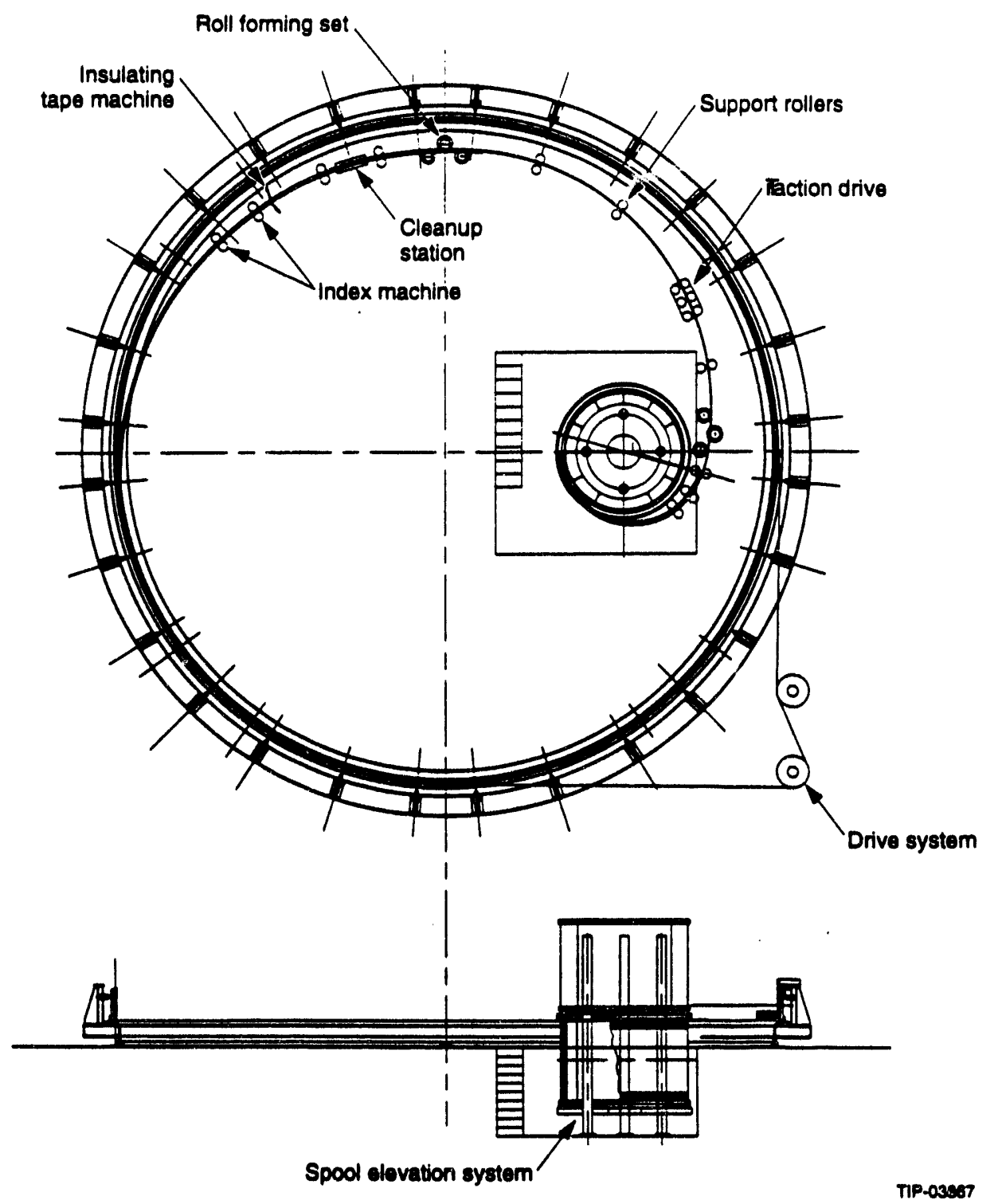

FIG. 3-19. Plan view of the winding station.

been produced for the $r$ wer supply, and layouts of systems within the utility building are complete. A number of important cryogen supply issues are now being resolved, but the design is mature enough to predict successful implementation.

\subsubsection{Liquid Helium Supply System}

The GEM magnet is provided with helium in two circuits - the thermosiphon and the supercritical, pressurized circuit (Figure 3-20). The thermosiphon loop includes a storage dewar located at the surface and supplying the magnet $70 \mathrm{~m}$ below. A second circuit supplies a small amount of supercritical helium, at the temperature of the thermosiphon loop, to the conductor conduit and leads. Despite the large size of the magnet, the steady-state refrigeration requirement (see Table 3-7) can be satisfied by the helium refrigerator used for the SSC Accelerator String System Test ( $2 \mathrm{KW}$ plus $20 \mathrm{~g} / \mathrm{s}$ liquefaction). Because of the very large heat capacity of the GEM magnet, a separate circuit with a large nitrogen heat exchanger will be used in initial cooling to near liquid nitrogen temperature. The nitrogen heat exchanger will be sized to accomplish this cooldown in a few weeks.

The helium refrigerator supplies cold twophase helium to a surface helium storage dewar of approximately 60 000-1 capacity. Liquid helium in the thermosiphon circuit flows from the bottom of 


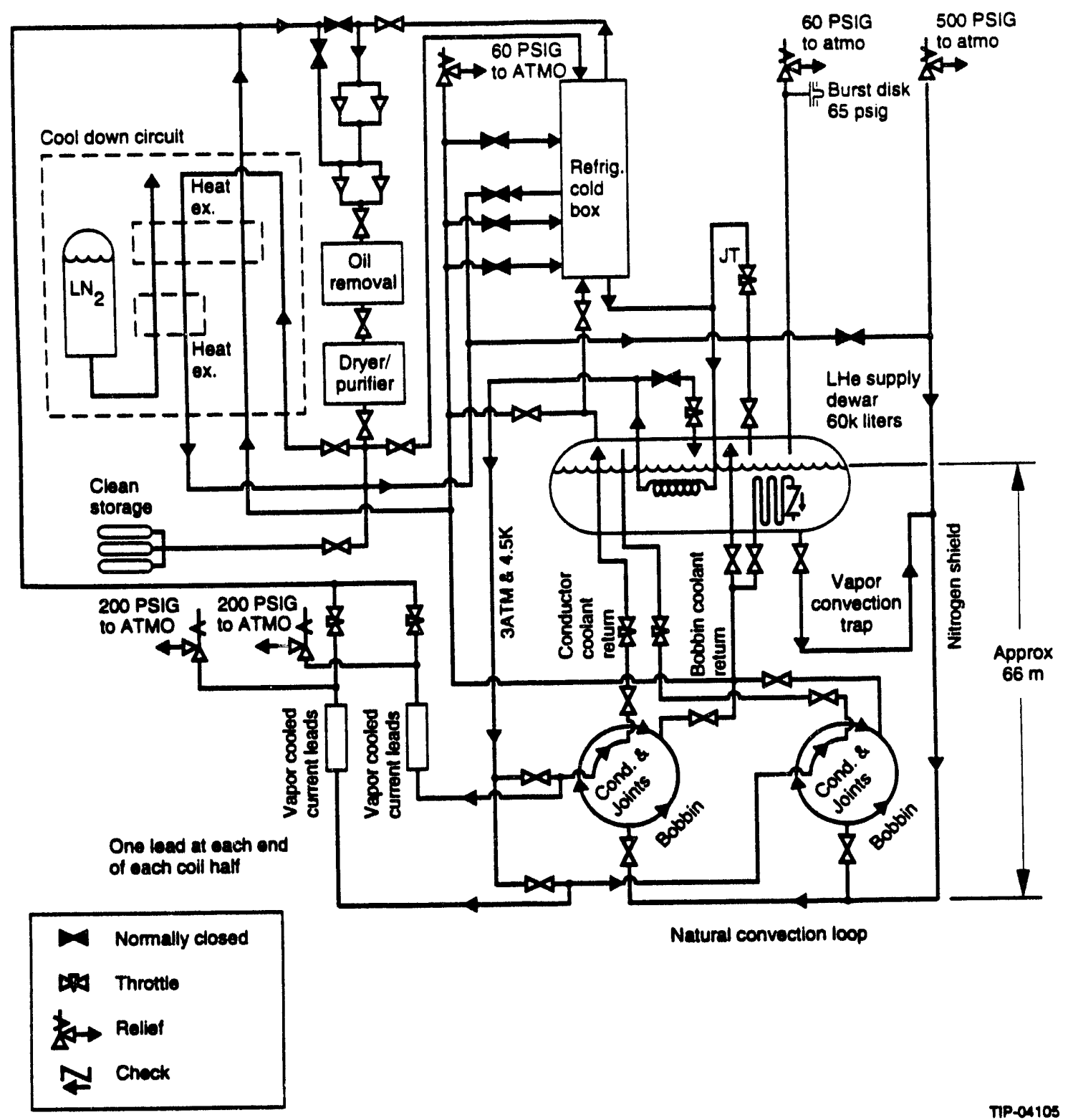

FIG. 3-20. GEM magnet helium flow schematic.

the storage dewar through insulated lines to the bottom of the magnet, approximately $70 \mathrm{~m}$ below.

To provide helium for the interior of the CIC, supercritical helium branches upstream of the JouleThomson valve and flows through a subcooler in the liquid helium storage dewar. This circuit also provides cryogen to the leads.

On cooldown from temperatures in excess of about $85 \mathrm{~K}$, the cooldown circuit with its large nitrogen heat exchanger is used to extract heat from the circulating helium gas. Once a magnet temperature approaching that of liquid nitrogen is achieved, the cooling is switched to the refrigerator with flow initially through just the upper portion. When a magnet temperature of about $20 \mathrm{~K}$ is achieved, the lower two expanders are brought on line and the flow taken through the entire heat exchanger stack.

During a quench, the heating within the magnet vaporizes the helium in the cold mass cooling circuits. To minimize the quantity of vaporized helium, the supply side of the thermosiphon loop is closed at the storage dewar at quench detection and the liquid helium downstream from the shutoff valve is vaporized. There are approximately 7001 of helium in the supply side and 8001 on the return side of the thermosiphon loop. Within 
the bobbin heat exchanger and headers, there are about 400 to 14001 , depending on the number of bobbin heat exchanger tubes. In addition to the helium contained in the thermosiphon system, the helium within the conduit system is returned to the storage dewar on quench. Because of cost constraints, a helium recovery system has not been included in the design and on quench, helium in excess of that which can be contained in the storage vessel is vented to the atmosphere.

Table 3-7. Heat loads.

\begin{tabular}{lr}
$\begin{array}{l}\text { Magnel side wall heating at } 0.18 \mathrm{~W} / \mathrm{m}^{2} \\
\text { (inside and outside) }\end{array}$ & $580 \mathrm{~W}$ \\
$\begin{array}{l}\text { Cold mass supports } \\
\text { Current leads (self-sufficient } 0.125 \mathrm{~g} / \mathrm{s} \mathrm{kA}\end{array}$ & $250 \mathrm{~W}$ \\
pair) & $300 \mathrm{~W}$ \\
Piping & $200 \mathrm{~W}$ \\
Bayonets & $20 \mathrm{~W}$ \\
Supply dewar (0.3\%/day loss) & $150 \mathrm{~W}$ \\
\hline Valves and miscellaneous
\end{tabular}

The helium refrigerator/liquefier is housed in three rooms of the surface level utility building. An acoustically insulated room contains the gas compression system, composed of compressors, oil removal and purification systems, coolers, air compressors, vacuum pump, and support equipment. A second room contains the refrigerator/liquefier cold box module, expander modules, distribution box, dryer/purification station, cool down heat exchanger, vacuum pumps, and regeneration skids. A third room, adjacent to the cold room, serves as the control room, housing equipment to maintain continuous control of the refrigerator/liquefier, heating, cooling water, and HVAC. High and low pressure gaseous helium storage tanks and a liquid helium storage dewar is located adjacent to the building. The liquid helium supply transfer lines are routed from the output of the liquid storage dewar to the utility shaft, down the shaft to the utility tunnel, and into the underground hall to the detector.

\subsubsection{Liquid Nitrogen System}

The liquid nitrogen system is large but simple in concept. A $40000-1$ storage dewar on the surface provides primary storage. It supplies a dewar in the experimental hall that cools the magnet shields and also supplies liquid nitrogen to the precooler in the helium refrigerator/liquefier, the helium purifier and the helium transfer line shields. Recent cost tradeoffs have shown that a nitrogen liquefier is a cost effective method of providing the large amount of liquid nitrogen required.

\subsubsection{Power Supply and Protection Systems}

The functions of the power supply and $\mu$ rotection systems are to supply a controlled current to the magnet, to detect magnet quenches, and to dissipate the energy stored in the magnet during normal discharge and emergency dump situations. While the current and stored energy of the magnet are large compared to those of other superconducting rnagnets, the principles of power and protection are well understood and an adequate supplier base exists to insure timely and successful production of components. The electrical parameters of the magnet are shown in Table 3-8. The power supply system (see Figure 3-21) consists of a 20-V dc, 51-kA power supply, high-current buswork, a magnet discharge resistor, a charge/discharge switch, a quench resistor, redundant dc current interrupters, and local controls. The protection system consists of magnet sensor cabling, instrumentation modules and a local control station.

The power supply provides current with output regulation of $0.5 \%$. While the design of the power supply will be left to the selected supplier, it is common practice to use a multiple-pulse (12 or 24 ) thyristor rectifier design. Primary filtering or phase

Table 3-8. Magnet parameters.

\begin{tabular}{lrl}
\hline Inductance & 1.98 & $\mathrm{H}$ \\
Operating current & 50,200 & $\mathrm{~A}$ \\
Stored energy & 2.5 & $\mathrm{GJ}$ \\
Ground isolation & 500 & $\mathrm{~V}$ \\
Charge/discharge time & 8 & $\mathrm{hrs}$ \\
Emergency dump time constant & 100 & $\mathrm{~s}$ \\
\hline
\end{tabular}


shifting will be required to limit harmonic distortion on the input voltage lines to acceptable levels. The power supply system components will be located inside the utility building. Under normal operating conditions, the principal load on the power supply will be the resistive high current buswork. During a magnet charging cycle, however, the load voltage will increase to about $10 \mathrm{~V}$ due to the drop across the magnet coil. The magnet coil charging time is specified as $8 \mathrm{~h}$ to limit the effects of induced currents in surrounding magnet structures. The voltage between the power supply output terminals is expected to rise to about $960 \mathrm{~V}$ during emergency discharge. The voltage isolation between the terminals must therefore be at least $1 \mathrm{kV}$.

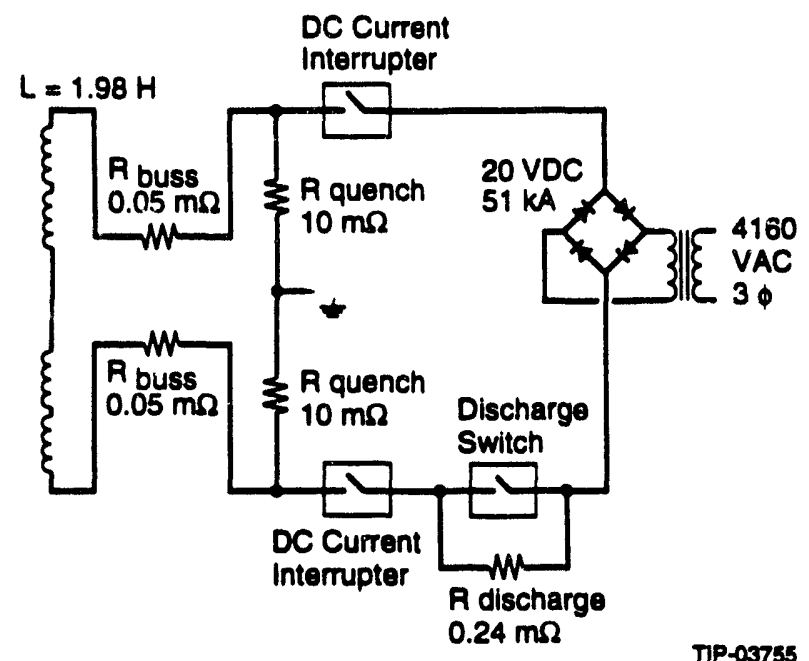

FIG. 3-21. Major components of the power supply system.

The high current buswork will be air-cooled aluminum bars, which are less expensive than water-cooled alternatives. The buswork will be routed through the utility shaft and will consist of six $40 \mathrm{~mm} \times 240 \mathrm{~mm}$ parallel bars. The one-way length of the buswork is approximately $125 \mathrm{~m}$. Under normal operating conditions, $260 \mathrm{~kW}$ will be dissipated in the buswork. Cooling will be provided by $20^{\circ} \mathrm{C}$ air drawn from the experimental hall through the buswork ducting and retumed to the air conditioning system located on the surface, resulting in a buswork temperature rise of approximately $40^{\circ} \mathrm{C}$. The buswork is designed to operate acceptably at higher temperatures by natural convention, if forced cooling is interrupted.

The function of the magnet discharge resistor is to absorb the stored energy of the magnet during a normal discharge cycle. Stainless steel strips arranged in a parallel plate configuration immersed in water discharge the magnet. Its resistance $(0.24 \mathrm{~m} \Omega)$ is selected to ensure that the stored energy is dissipated within the required $8 \mathrm{~h}$. While the added resistance of the discharge resistor in the high- current circuit is necessary during a magnet discharge cycle, it is not desirable to have it in the circuit during magnet charging or normal operation. Consequently, the charge/discharge switch is used to bypass the resistor during these operations to reduce the load on the power supply.

Quick removal of current from the magnet in the event of a quench is critical. Consequently, the power supply system will contain redundant current interrupters, one in each bus leg, to ensure that the magnet current flow is diverted from the coil to the quench resistor. The current interrupters may be either mechanical or a solid state switches. The quench resistor must dissipate $2.5 \mathrm{G} \mathrm{J}$ if a fast discharge occurs. The 100-s energy dump time constant and $1 \mathrm{kV}$ maximum bus-to-bus voltage require a $20-\mathrm{m} \Omega$, low-inductance design. A common approach for this application is a parallel-plate stainless-steel resistor arranged in a series-parallel configuration to produce the desired resistance, inductance, and power density. The requirement for voltage isolation of $500 \mathrm{~V}$ from bus to ground dictates that the quench resistor design include a center tap that can be grounded. This connection is not in the high current loop, so it only needs to be capable of carrying a few hundred amperes for a short time.

The magnet contains sensing elements to monitor normal operating parameters, detect offnormal operating conditions, and initiate actions to protect the magnet. The sensors include voltage taps, strain gauges, and cryogenic lead temperature sensors (CLTS). While the number and location of sensor channels is still being specified, it is expected that there will be 34 voltage taps, 250 strain gauges, and 400 CLTSs. It is likely that the voltage on some or all of the sensor signal leads will rise during an emergency dump of the magnet current. Therefore, the instrumentation modules must have voltage isolation while continuing to provide readout capability. The instrumentation modules will be housed in a rack-mounted system in the utility building. 
Sensor signal cables will be routed between the magnet assembly and the utility building through the utility shaft.

The magnet protection system will include a local control station for monitoring magnet system sensors. The local control station will contain circuitry to detect a magnet quench or other off-normal operation, and to initiate an emergency discharge sequence. Since this is a critical function, all quench detection circuitry will be redundant. The interface from the local control station for remote operation will be via local area network.

\subsubsection{Vacuum System}

The vacuum system for each vessel consists of rough and high vacuum pumping systems. The rough vacuum system, which is shared by the vacuum vessels for the two coil halves, consists of two $4000-C F M$ roots blowers, each backed by a 300-CFM mechanical pump. Mechanical pump oil is isolated from the vacuum lines using refrigerated traps. The high vacuum system for each vessel consists of four 24-in. diffusion pumps backed by a single 300-CFM mechanical pump. These provide adequate margin and cost less than one quarter as much as a turbo pumped system. Refrigerated traps isolate the vessel from contamination by diffusion pump oil. Pump-down calculations show that, after initial clean-up and leak repair, the system should provide insulating vacuum $\left(<10^{-4}\right.$ Torr) after five days of operation. 25

\subsection{FRINGE FIELD}

\subsubsection{Introduction}

Because the magnet has no flux return, the static fringe field in the experimental hall will vary from about $1 \mathrm{~T}$ near the end of the coil windings to about $0.1 \mathrm{~T}$ near the ends of the hall during magnet operations. There will also be a fringe field at the surface directly above the detector. Health, safety, and regulatory considerations of the effects of the fringe field on operations are summarized in Reference 4. Regulatory guidelines for human exposure to static magnetic field allow access to the detector with the magnet on. Full-body exposure for workers in the experimental hall outside the magnet allows up to seven 8-h shifts per week. Access will be per. .aitted, however, only to persons without medical electronic implants such as cardiac pacemakers or metallic prostheses. Sensitive electronic and mechanical equipment will need to be shielded.

\subsubsection{Field in the Experimental Hall and Electronic Rack Room}

The contours of constant field in the experimental hall are shown in Figure 3-22.

The field will generate force densities on magnetizable objects ranging from less than 1 to $20 \mathrm{kN} / \mathrm{m}^{3}$, corresponding to a range of $1-25 \%$ of the object's weight. Calculations of the forces and torques on magnetizable materials in the hall are summarized in Reference 5. They indicate no need for upgrading the civil construction requirements. The crane bridge and movable steel equipment in the hall, however, will need to be secured before energizing the magnet.

The electronic rack room (ERR) is located in a shaft about $20 \mathrm{~m}$ away from the center of the GEM detector. In order to provide unrestricted access for work on the electronics, the ERR will be shielded 7 by placing $297 \mathrm{Mg}$ of iron plate between the walls of the ERR and the shaft. The shielding design takes into account penetrations of the walls for the cable trays and will reduce the stray field to less than $0.005 \mathrm{~T}$ throughout the room.

Whenever practical, components insensitive to the magnetic field will be selected. Equipment sensitive to the field, such as relays, electric motors, and electrically driven valves, will be shielded

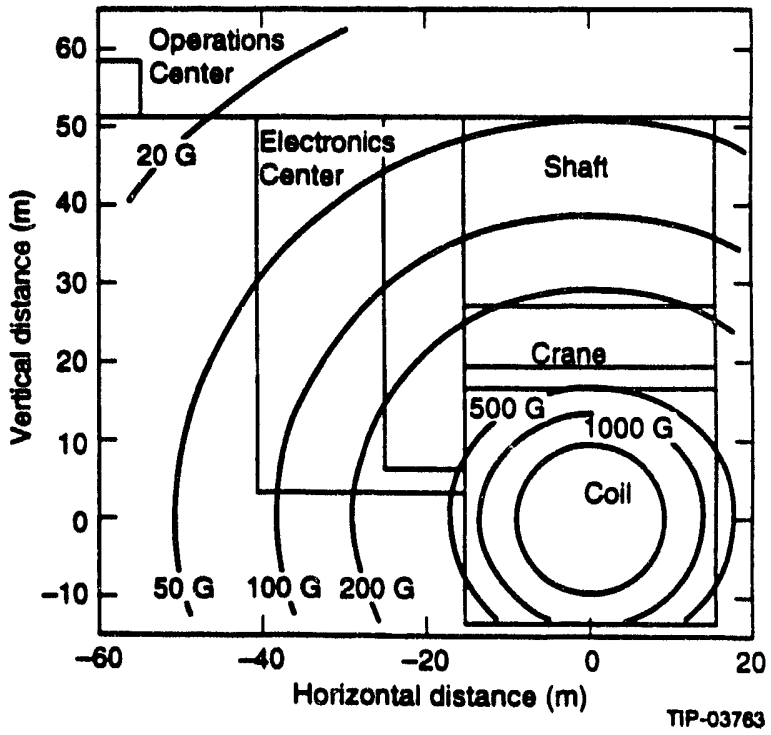

FIG. 3-22. Contours of constant B in the midplane of the experimental hall. 
locally with small iron screens. Turbopumps, which are more sensitive to magnetic field than most equipment and are situated in a 1-T field, can be shielded to $0.003 \mathrm{~T}$ with $4-\mathrm{cm}$-thick, low-carbon iron shields. 26

\subsubsection{Field at the Sur face}

Contours of constant magnetic field on the surface directly above the experimental hall are shown in Figure 3-23.

The field peaks at about $40 \mathrm{G}$ at a point directly above the center of the detector. It drops to $4 \mathrm{G}$ or less at a distance of about $100 \mathrm{~m}$ from this point, and to the level of earth's magnetic field $(0.5 \mathrm{G})$ at the boundary of the east campus site. All equipment in the utility building and the operations center will be in a field of less than $10 \mathrm{G}$. Sensitive computer monitors may require local shielding.

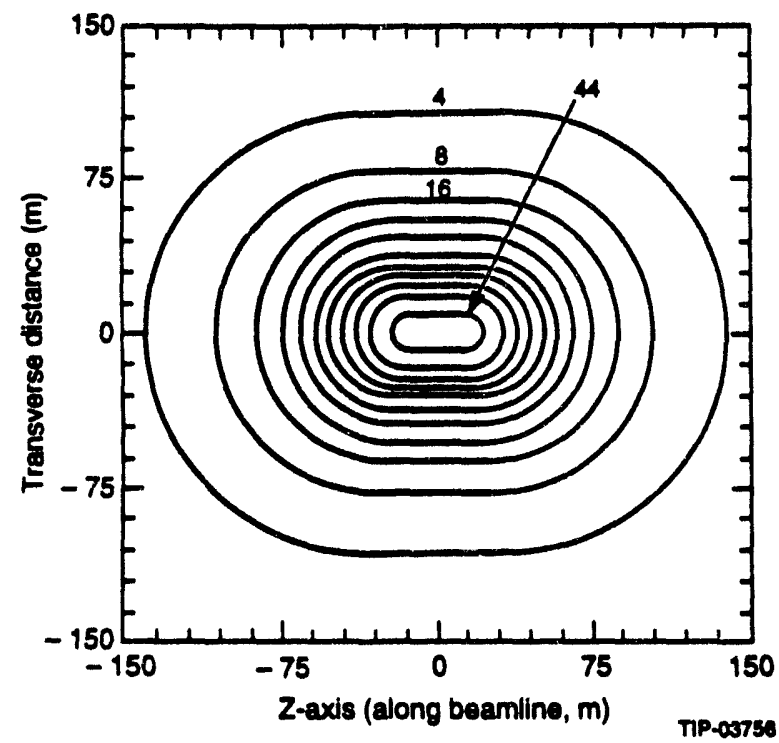

FIG. 3-23. Contours of constant field on the surface above the detector in gauss.

\subsubsection{Interface with the Accelerator}

Axial and radial components of the field act on the collider beam dynamics. The main irregularities in the field along the beam occur in the vicinity of the FFS.

Simulation results show 2 that the coupling of the horizontal and vertical betatron oscillations of the beam induced by the GEM magnet can be corrected with existing skewed quadrupoles both at injection energy and at full energy. The radial component of the field is small in the vicinity of the beam and causes no significant problems. In order to minimize further the range of the fields of the skewed yuadrupoles needed for the corrections of the lattice, it is recommended that the GEM and SDC magnetic fields be anti-parallel. Additional study of the effects of magnet misalignments have been made by the SSCL beam-dynamics group. This study indicated that offsets of the GEM magnet axis by about $\pm 10 \mathrm{~mm}$ and angular misalignment of $\pm 5 \mathrm{mrad}$ are well within the tolerances of the lattice.

\subsection{MAGNET OPERATION}

\subsubsection{Introduction}

Because of its large size and stored energy, time is required to prepare the magnet for physics operation and to respond to off-normal events. The magnet is designed to operate simply and with very high reliability. The conductor is stable and the supporting systems, particularly the cryogenic supply systems, are conservatively designed. Design objectives are to ensure that the magnet is not damaged by operational faults, that magnet-related downtime is minimized or eliminated, and that downtime needed to service other detector subsystems is minimized by providing access where possible. GEM operating plans take advantage of SSC planned and unplanned short shutdowns as well as planned long maintenance periods to allow magnet shutdown for maintenance of detector subsystems.

\subsubsection{Requirements}

The GEM magnet is designed for simple operation. It consists of only three operating subsystems: cryogenics, vacuum, and power/protection, and all three will be run from a single workstation. Each subsystem is designed to operate automatically, with set-points for major parameters (such as DC current) determined by the operator from the GEM global control system. Use of the magnet, over most of its lifetime, will not require the dedicated attention of an operator. However, personnel will be required to monitor periodically and maintain the major elements, particularly the moving parts of the cryogenic and vacuum systems. Additional personnel may be required during planned transients, such as pumpdown, cooldown, charging and discharging, and during recovery from an emergency discharge. 
Access to the underground hall or shafts is not required for normal operation. We have minimized the components located in the underground hall that could fail and interrupt operation. Most valves and controls have been located either in the utility shaft or on the surface, where they can be accessed for inspection and repair at all times, including periods of magnet and collider operations. The few components which must be located in the hall (cryogenic liquid level sensors) will be given adequate redundancy to preclude additional detector downtime. They will be replaced, if required, during the SSC's weekly scheduled maintenance day.

The annual SSC maintenance shutdown (1-3 months) will be used for infrequent activities, such as alignment, that require extended hall access. Based on information from the facility designers, we expect to require realignment of the major magnet components (coil halves and FFSs) no more than once per year. Alignment is corrected by replacing shims under the supports, which requires lifting the components slightly, and will take about a week. The annual shutdown may also be used for access to the other detector subsystems, by moving the magnet halves, as discussed below.

\subsubsection{Normal Operations}

After initial installation, the coil halves must first be evacuated. This is accomplished with a conventional roughing/high-vacuum system, and requires $2-4$ weeks, depending on initial cleanliness of components within the vacuum vessels. Following evacuation, the cold masses will be cooled to operating temperature, $4.5 \mathrm{~K}$, requiring about 4-6 weeks. Cooldown is accomplished by circulating cold helium gas through the magnet, controlling the inlet temperature so that thermal stresses in ti:e components are minimized. In parallel, cold nitrogen gas is circulated through the thermal shields, which are then filled with liquid nitrogen when the temperature falls below saturation. Liquid helium fills the cold mass system when its temperature falls to $4.5 \mathrm{~K}$. After initial cooldown, and before operation begins, an inventory of liquid helium will be accumulated in the above-ground supply dewar, to ensure continued operation even during fault conditions (as discussed below).

In normal operation, the magnet coils will remain under vacuum and at the operating tempera- ture of $4.5 \mathrm{~K}$. Normal operation consists of ramping up the current from zero to approximately $50.2 \mathrm{kA}$ (charging), maintaining it at this value for long operating periods (many months), and then reducing the current back to zero (discharging). Charging and discharging each require $8 \mathrm{~h}$. The cycle allows one shift of field-free access to the detector even for an SSC shutdown as shor as $24 \mathrm{~h}$.

\subsubsection{Off-Normal Operations}

In order to maximize availability, the magnet is designed to operate under a number of off-normal situations, ranging from extended shutdown to loss of site power. A key source of this robustness is a high-reliability cryogenic system that can support continued magnet operation even during outages. The helium and nitrogen systems will be capable of continuing operations for $12 \mathrm{~h}$ during a power outage. This is accomplished primarily by employing a passive (thermosiphon) design for the primary cooling of the $4.5 \mathrm{~K}$ cold mass (no pumps or active elements are required to maintain cooling), ensuring adequate inventory of liquid cryogens at all times, and using a system designed to function on the thermal capacity of the stored liquid, if required. The vacuum system is not a serious concern in this regard, since it can be made fail-safe in the conventional manner: high-vacuum valves on the vacuum vessel fail closed. The power supply system is designed to allow a very slow or a fast, emergency discharge, while maintaining control, monitoring, and safety functions at all times.

\section{Extended Shutdown}

An important scenario for the detector as a whole is an extended (several-month) shutdown, to access internal detector components for maintenance. Access to the interior of the detector is accomplished by moving the magnet halves and/or the FFSs. Movement of the FFSs is a straightforward operation, since there are no connections to break and no services to reconnect. To move the magnet halves, the de busses and cryogenic, vacuum, and instrumentation connections must be broken and essential services reconnected. When retracted for access to the central detectors, the magnet halves are "parked" in a specific location near the ends of the hall, so that cryogenic, vacuum, and limited instrumentation connections can be made. As discussed above, evacuation and cooldown are time-consum- 
ing operations, so we have chosen to keep the magnet cold and under vacuum while it is retracted, to minimize overall downtime. The magnet will not be cooled during movement, however, so after it has been moved back into position, a shor cooldown time will be required prior to operation.

\section{Emergency Discharge}

Emergency discharge, in which the current is rapidly reduced to zero, is a required operating mode for the magnet. Although a quench is highly unlikely, given the stability of the conductor and the conservative magnet design, the capability to remove the current rapidly is a prudent requirement for any superconducting magnet. The rapid-discharge scenario may be used whenever field-free emergency access to the hall is required, as in a fire. An emergency discharge is a programmed event, which can be initiated automatically by the magnet's quench-detection system or by operator command. To minimize the risk of damage to the magnet, we have selected a discharge time of $5 \mathrm{~min}$ (3 time constants). During an emergency discharge, most of the magnet's stored energy is absorbed by an external dump resistor; the rest of the energy is deposited in the cold mass, raising its temperature slightly. The liquid helium supply is temporarily shut off, to avoid vaporizing excess helium. A short downtime for cooldown is required prior to recharging. The magnet is designed to withstand this scenario with no difficulties.

\section{Loss of Site Power}

The magnet is designed to operate through a power outage to the site or to magnet components, with no ill effects. If site power is lost, the magnet's operation will be changed as little as possible until power is restored. As discussed above, the coil will remain superconducting for up to $12 \mathrm{~h}$. The power supply will shut down, but will not interrupt the de current. Instead, the current will slowly diminish, as energy is deposited in the warm dc current busses. Although the forced-air cooling of the busses will be interrupted by the loss of power, they can operate indefinitely using only natural circulation in the long chimney-like duct in which they are enclosed. All controls for the magnet will be on uninterruptable power, so constant monitoring of the magnet will still be possible; we will also maintain the capability to initiate an emergency discharge at any time if required. When site power is restored, the magnet can be discharged and recharged as required.

\section{Cryogenic System Outage}

Refrigerator outages are not uncommon occurrences, so the magnet system is designed to function temporarily without the refrigerator. A redundant compressor is part of the refrigerator design and it can be valved in immediately to replace any other compressor that breaks down. The liquid-helium storage dewar will always contain enough liquid for $12 \mathrm{~h}$ of operation under normal circumstances. During a refrigerator outage, this reserve allows sufficient time to diagnose the problem and to effect repairs on the expanders (the components most likely to fail in operation). If the problem proves more serious, there will be time for a slow magnet discharge, or to import liquid helium as needed to continue operation. The passive nature of the design will minimize or eliminate downtime for this event.

\subsubsection{Reliability, Availability, and Maintainability Analysis}

We have identified the events most likely to affect the operation or safety of the magnet, based on experience, and have developed design approaches that minimize their impact. Further analysis, to be conducted as part of engineering design, will dictate details such as required redundancy, other protective systems, and operational procedures.

\subsection{ORGANIZATIONAL ASPECTS}

Preliminary design of the GEM magnet has been performed by physicists and engineers from the following institutions:

Lawrence Livermore National Laboratory

Los Alamos National Laboratory

Massachusetts Institute of Technology, Plasma Fusion Center

Southern Methodist University

Superconducting Super Collider Laboratory.

The following groups are involved in research and development and verification of the desig-.:

Martin Marietta, Y-12 Oak Ridge Laboratory 
University of Wisconsin.

In addition, extensive consultations have been held with:

Rutherford Appleton Laboratory; Oxford, England

ASEA Brown-Boveri, Baden, Switzerland

Martin Marietta, Denver, Colorado

Lawrence Berkeley Laboratory

JBC Associates, San Diego, California

W. Flick, Austin, Texas.

The magnet steering committee consists of G. Deis, N. Martovetsky, L. Pedrotti, P. Reardon, R. Stroynowski (chair), and B.A. Smith.

P. Reardon is the project manager and G. Deis is the chief engineer of the magnet subsystem.

The GEM magnet project relies heavily on the use of an industrial prime contractor, responsible for most of the final design, procurement, fabrication, assembly, installation, and management, under the management of the existing SSCL/SMU/LLNL/ MIT team. Design and development work to date has been performed by the team and its subcontractors, with the recent addition of a group at the ORNL $Y-12$ plant. who are specialists in production engineering. These groups are pursuing an aggressive preliminary design and development program, to keep the project on schedule despite delays experienced in activating the prime contractor. The RFP for the prime contractor was released to industry on December 22, 1992, with proposals received March 22, 1993. Following receipt and evaluation of these proposals (now in progress), we will determine the most cost- and schedule-effective division of work among the present collaborators, SSCL, the prime contractor, other subcontractors, and foreign sources. Prime contract award is planned for June 1 , 1993. Our present assumption on the breakdown of work is shown in Table 3-9. For each major area of activity, we will identify "cognizant engineers" from within the present magnet team to report to the magnet project manager on technical progress, cost, and schedule. These individuals will be responsible for ensuring that all requirements are met and that activities are proceeding within cost and on schedule. This arrangement will supplement the information obtained from the usual cost/schedule control system, and will provide a very powerful tool for managing the diverse parallel activities.

Table 3-9. Projected division of responsibility within magnet project.

\begin{tabular}{lll}
\hline \multicolumn{1}{c}{ Item } & Responsible Organization & $\begin{array}{c}\text { Cognizant Engineer's } \\
\text { Organization }\end{array}$ \\
\hline Conductor & $\begin{array}{c}\text { Prime contractor } \\
\text { (alternate: Y-12) }\end{array}$ & MIT \\
Cold mass (except conductor) & $\begin{array}{r}\text { Prime contractor } \\
\text { (altemate Y-12) }\end{array}$ & MIT \\
Vacuum vessels & Prime contractor & LLNL \\
Intemal cryostat components & Prime contractor & LLNL \\
Forward field shapers & Foreign source & LLNL \\
Power/protection system & SSCL/PRD/EFD and LLNL & LLNL \\
Cryogenic system & SSCL/PRD/EFD & LLNL \\
Vacuum system & Prime contractor & LLNL \\
Control system & LLNL & LLNL \\
Field measurements & SSCLLLNL & N/A \\
Installationtesting & Prime contractor & SSCL \\
Management & SSCL (overall), with assistance by LLNL, MIT, & N/A \\
\hline
\end{tabular}




\section{REFERENCES}

1. Stroynowski, et al., "GEM Magnet Engineering Design Report," GEM-TN-92-116 Rev. A (1992).

2. T. Garavaglia and T. Sen, SSCL-N-1812 (1993).

3. Stroynowski, "Considerations Leading to the Choice of Open Field Magnet," GEM TN-91-30 (1991).

4. R.P. Woolley, "SSC GEM Magnetic Field Safety and Health Effects," GEM-TN-91-19 (1991) and "Supplemental Environmental Analysis of the GEM Magnet," GEM-IN-92-10 (1992).

5. R. Pillsbury Jr., "Fringe Field Dipole-Dipo!: Force Interactions," GEM-TN-91-35 (1991) and MIT-GEM-EM-06 (1992).

6. R. L. Myatt, "Electromagnetic Forces on Major Iron Components of the SSC GEM Detector," MIT-GEM-EM-11 (1992).

7. R.L Myatt, "Electronic Rack Room Shielding Study," MIT-GEM-EM-10 (1992).

8. GEM Magnet Technical Panel Reports, R. Stefanski ed., GEM-GDT-5 and GEM-GDT-16 (1991).

9. R. Stroynowski, "Magnet Conductor Choice Memorandum," GGT-000004 (1992).

10. H. Wind, Nuclear Instruments and Methods 84 (1970) 117.

11. S.Timofeev, "Axial Symmetry of the GEM Magnetic Field." GEM TN-93-384.

12. "GEM Detector Magnet Preliminary On-site Fabrication, Assembly and Installation Plan," GEM IN-92-27 (1992).

13. "Conductor Specification for the GEM Detector Magnet," GDT-000037 (1993), and "Specification of Quality Assurance and Acceptance Testing Requirements for Manufacturer of the GEM Detector Magnet Conductor," GDT-000037-08 (1993).

14. J. Bowers et al., "Design of the Vacuum Vessel Subsystem for the GEM Detector at the SSC," 5th IISSC Symposium, San Francisco May 6-8, 1993.

15. W.N.. Boroski, et al., "Thermal Performance of Various Multilayer Insulation Systems Below 80K." 4th IISSC Symposium, Ed. Nolte, J., Supercollider 4, Plenum Press, (1992).

16. M. M. Steeves, et al., "Further Progress in the Manufacture of the US-DPC Test Coil," presented at the MT-11 Conference.

17. M. O. Hoenig, "Internally Cooled Cabled Superconductors," Cryogenics, July 1980.
18. P. Marston, et al., Proc. of 2nd Intemational Industrial Symposium on The Supercollider held March 14-16, 1990 in Miami Beach. Florida. Pub. IISSC Supercollider 2, Edited by Michael McAshan, 1990.

19. F. Konig, J Rauch and D. Salathe, "SSC/GEM Detector Conductor Study," ABB Corporate Research, July 1992, HISM 20469.

20. N. N. Martovetsky et al., Conductor Design for the GEM Detector magnet, 5th IISSC Symposium, San Francisco, May 6-8,1993.

21. J. V. Minervini, et al., "Cabie-in-Conduit Conductor Concept for the GEM Detector Magnet," Applied Superconductivity Conference, Chicago (1992).

22. H. Lynch, "Design Review of the GEM Magnet," GEM-IN-92-20 (1992).

23. "Magnet Technical and Management Review," GEM-IN-92-23 (1992).

24. "Specification for the Fabrication of a Stainless Steel Conduit for the GEM Detector Magnet," GDT-000037-04, (1993).

25. G. Chapman et al., "GEM Solenoid Vacuum System Design Study." GEM-TN-93-409 (1993)

26. N. N. Martovetsky, "Shielding in 300-1,000 G Background Field." GEM-TN-92-98 (1992). 


\section{MUON}

\subsection{INTRODUCTION}

The GEM muon system is the largest measurement system in GEM, encompassing a total volume of nearly $5000 \mathrm{~m}^{3}$. Throughout its extremely large measurement volume, the system must measure muon tracks with a precision approaching $55 \mu \mathrm{m}$ in the bend-plane coordinate. This endows the system with the capability of reconstructing the momentum and charge of muons over the entire kinematic range of the SSC. We have developed a system of muon detectors that approaches our goals of a hermetic, precision muon detection system. The performance has been necessarily driven by issues of technology and cost, however, we are confident that our system will meet or exceed our measurement goals.

This chapter is divided into specific discussions covering the implementation of the muon system. We begin by detailing our physics goals, performance requirements, and measurement methods. Short descriptions of our system design, including the effects of magnetic field, calorimeter and central tracker are presented in this section, followed by more detailed presentations in subsequent sections. Practical design considerations are reviewed with respect to chamber technology, chamber support structure, alignment, trigger and system optimization/upgrades. Finally, we discuss our R\&D plans, engineering and manufacturing plans, and muon subsystem organization.

\subsubsection{Physics Goals}

Muons from pp collisions at the SSC provide signatures for a wide range of new physics processes. Some of these processes are expected to br rare, and will require the highest luminosity $(\mathcal{L}=$ $10^{34} \mathrm{~cm}^{-2} \mathrm{~s}^{-1}$ ). The mission of the GEM muon system is to identify these muons, and to provide a precision measurement of their momentum over a wide range.

The muon system is complementary to the electron and photon physics capabilities of the GEM detector, muon trajectories can be measured inside jets, and the sign of the muon charge deternined up to the kinematic limit of the SSC.

The requirements for the GEM muon system are driven by the GEM physics program. Some of the key issues, and the features of the muon system needed to confront them are:

- Higgs Physics: $H^{0} \rightarrow Z Z^{*} \rightarrow \mu^{+} \mu^{-} \mu^{+} \mu^{-}$.

- Coverage with high efficiency for $|\eta|<2.5$.

- Good resolution for low momenta.

- High mass physics: $Z^{\prime} \rightarrow \mu^{+} \mu^{-}, W^{\prime} \rightarrow \mu \nu$, quark-lepton substructure.

- Coverage with high efficiency for $|\eta|<2.5$.

- Good resolution at high momenta for sign selection.

- Clean pattern recognition of high momentum tracks.

- Large suppression of backgrounds at high luminosity.

- Heavy flavor physics.

- Measurements of muons in and near high pT jets.

\subsubsection{Performance Requirements}

To address the anticipated new physics at the SSC, we have designed a muon system to provide the following functionality and performance:

- Muon identification: track identified as a muon candidate if it has penetrated the $11 \lambda$ to $16 \lambda$ calorimeter, consistent with muon ionization.

- Solid angle coverage from polar angles 9.75 to 84 degrees, with the minimum possible acceptance losses due to gaps and dead areas.

- pr trigger: typical threshold range $10 \mathrm{GeV}<$ $p_{T}<50 \mathrm{GeV}$. Level 1 single muon trigger rate: $<1 \mathrm{kHz} @ \ell=10^{33} \mathrm{~cm}^{-2} \mathrm{~s}^{-1}$ for $30 \mathrm{GeV}$ threshold.

- Beam crossing tag: efficiency $>99 \%$.

- Momentum resolution for $500 \mathrm{GeV} p_{T}: \Delta p_{T} / p_{T}$ $=5 \%$ at $|\eta|=0$ and $\Delta p_{T} / p_{T}=12 \%$ at $|\eta|=2.5$. 
- Low chamber occupancy compatible with operation at $\mathcal{L}=10^{34} \mathrm{~cm}^{-2} \mathrm{~s}^{-1}$.

- Charge assignment: correct at $95 \%$ confidence level up to muon momenta ranging from $p_{T}<$ $6.5 \mathrm{TeV}$ at $|\eta|=0$ to $2.8 \mathrm{TeV}$ for $|\eta|=2.5$.

- Knowledge of the beam position with respect to muon system of order $500 \mu \mathrm{m}$, as an additional constraint to improve robustness. $A$ further goal of a $200 \mu \mathrm{m}$ constraint will improve the high momentum resolution and efficiency.

\subsubsection{Design Considerations}

We have designed a system that can function in a stand-alone mode at the highest SSC luminosity, by locating all the muon detectors behind the calorimeter with little reliance on the GEM central tracking system (although tracker information can and will be incorporated when available). Not only is the charged particle background from hadron punchthrough suppressed to levels well below the rate of real muons, but also the neutron and neutron-associated photon backgrounds are reduced to manageable levels by the thick, nearly hermetic GEM calorimeter. The muon system is designed to operate with full efficiency and resolution in the presence of a neutron flux of up to $10^{5} \mathrm{~cm}^{-2} \mathrm{~s}^{-1}$.

The system will provide a beam crossing tag and a first level muon trigger for transverse momentum thresholds ranging from $10 \mathrm{GeV}$ to $50 \mathrm{GeV}$. The chamber system has a multiplicity of measurement layers allowing local track vectors to be determined, which gives a high efficiency for muon track reconstruction even at high energies where muoninduced electromagnetic showers become important. The deleterious effects of these showers are mitigated by providing drift space between the calorimeter and the first muon superlayer, and by minimizing material in the muon system.

The goal of the design process for the muon system is to specify a practical set of parameters that will achieve the baseline performance within reasonable cost constraints. The muon system design concept is based on the sagitta method of reconstructing the muon momentum in a solenoidal magnetic field. In our design, all the muon tracking and triggering elements are located in three superlayers (SL), each containing multiple layers of detectors placed between the calorimeter and the solenoidal magnet coils. In order to provide a muon system with good background suppression up to the highest luminosities and good momentum resolution, we have placed the entire set of chambers behind the calorimeter. As a consequence, in the low momentum region $(<75 \mathrm{GeV}$ ) the momentum resolution is dominated by multiple scattering in the chambers themselves, and by fluctuations of the energy loss in the calorimeter. The reconstruction of low momentum tracks for isolated muons will involve measuring the energy loss in the calorimeter for large energy loss fluctuations, and incorporating tracker information when available. In the high momentum range, spatial resolution and alignment errors are the largest contributors.

\section{Sagitta Method}

Figure 4-1 is an overview of the GEM muon system design. The essential features of the system are the large open solenoid magnet with a shaped field in the forward direction, and the three superlayers of multiple detectors located outside the calorimeter to trigger and reconstruct the muon momentum.

The momentum is determined by means of a three-point measurement of the sagitta. The sagitta is given by:

$$
s=\frac{y_{1}+y_{3}}{2}-y_{2}= \pm \frac{0.3 B L^{2}}{8 p_{T}}
$$

Here the sagitta $s(m)$ is given in terms of the transverse position measurements along the muon trajectory, $y_{i}(\mathrm{~m})$, the magnetic field strength, $B(\mathrm{~T})$, the path length $L(\mathrm{~m})$ normal to the magnetic field, and the momentum transverse to the $B$ field, $p_{T}(\mathrm{GeV})$. The exaci formulae are given in Section 4.3.1.

From the expression above we note that good momentum resolution can be achieved even with a modest magnetic field strength, $B$, for a large enough path length, $L$. In order to improve the momentum resolution in the forward direction, where the solenoid magnet has a diminished bending power, large iron cones ${ }^{1}$ are mounted concentrically with the beam pipe. These iron cones, called forward field shapers (FFS) create a radial magnetic field component, which improves the bending power by a factor of three.

The superlayers of muon chambers are held in rigid space-frames (one for each half of the barrel 


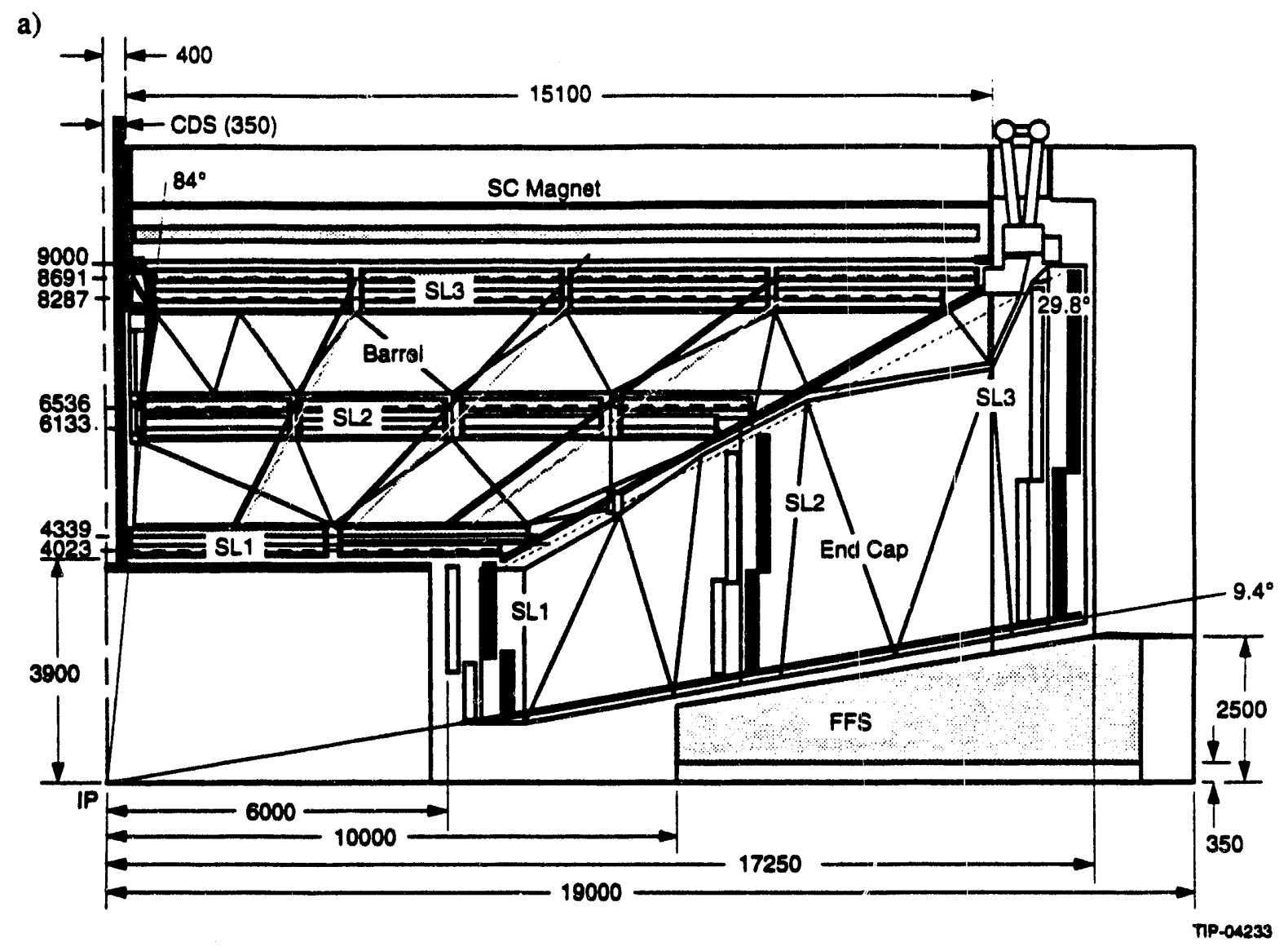

b)

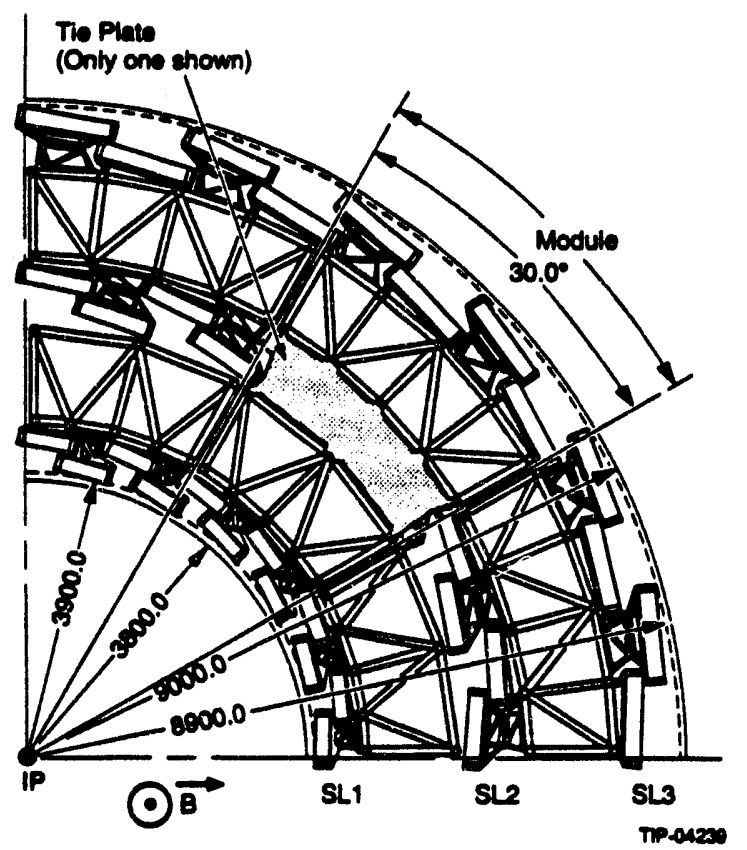

c)

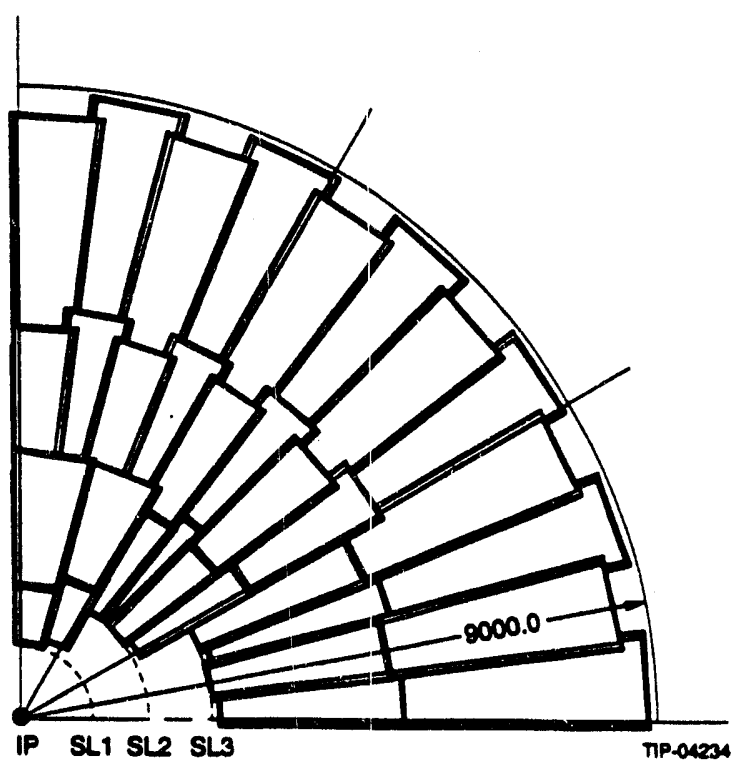

FIG. 4-1. Overview of the GEM muon system: (a) shown in quadrant view, and (b) barrel end view, (c) endcap view from IP. 
and one for each endcap), which are attached to the magnet supports in a manner designed to minimize vibrations. In order to achieve our momentum resolution goal, the space-frames must have sufficient stability so that the muon chambers can be positioned and monitored to a small fraction of the track sagitta. To set the scale for the stability, alignment, and chamber resolution needed in our system, the total sagitta for a $P_{T}=500 \mathrm{GeV}$ track at $\eta=0$ is less than $1100 \mu \mathrm{m}$ for $B=0.8 \mathrm{~T}$ and track length $L=4.3 \mathrm{~m}$. To measure $500 \mathrm{GeV}$ transverse momentum there with an accuracy of $5 \%$ (which corresponds to the capability of sign selecting muons from the heaviest $Z^{\prime}$ producible at the SSC), the total error of the sagitta cannot exceed $55 \mu \mathrm{m}$. This tiny error budget sets the requirements for the muon chamber resolution, alignment, and mechanical stability of the chamber support system.

\section{Magnetic Field}

The defining considerations in the design of the muon system are the choice of the magnetic field strength, shape, and geometrical extent. These parameters must be balanced against the practical limits of magnet cost, the muon tracking chamber resolutions and other associated costs. As noted above there is a premium in choosing a large diameter magnet, because it allows the magnetic field strength to be kept to a modest value while maintaining good momentum resolution. Our magnet design is described in detail in Chapter 3 and Section 4.3.1.

A further magnetic field design consideration is the outside radius of the calorimeter, which determines the inner radius of the muon system. In the GEM detector design the calorimeter has a radius of $3.7 \mathrm{~m}$, with a total of $11 \lambda$ at $\eta=0$. For practical reasons of magnet design the field strength is chosen to be $0.8 \mathrm{~T}$ at the center of the magnet. Given this field value, at least $4 \mathrm{~m}$ of bend path is required in order to obtain the required sagitta in the barrel region at the highest momentum. This bend path requirement determines the outer diameter of the solenoidal field. Similarly, the length of the magnet is determined by the resolution requirements in the forward direction, the size of the calorimeter along the beam axis, and the shaping of the field provided by the FFS. From these considerations we find the lever arm must be almost $9 \mathrm{~m}$. and the minimum magnet half-length is about $15 \mathrm{~m}$.

The flux line profile of the magnetic field is shown in Figure 4-2, superimposed on the chamber layout. Note the radial magnetic field in the forward direction generated by the FFS. This arrangement increases the sagitta, and hence the momentum resolution, in the small angle region.

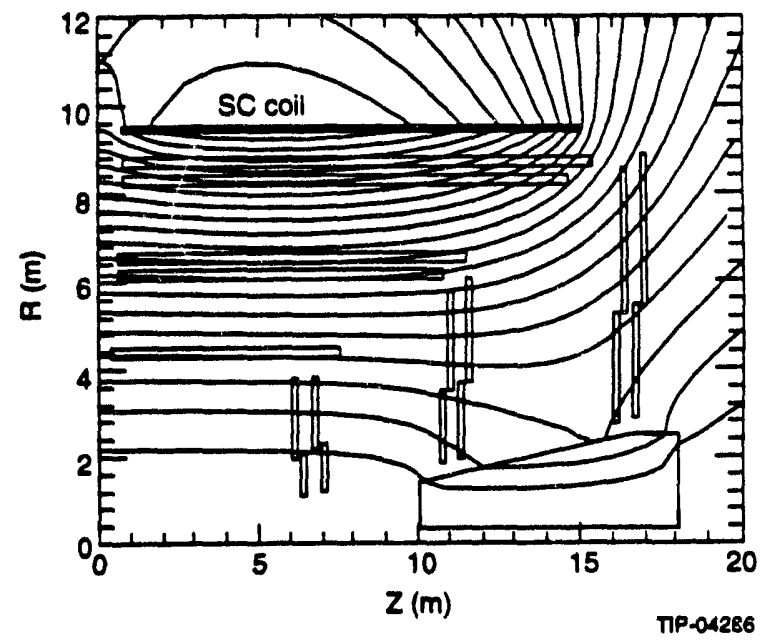

FIG. 4-2. A quadrant of the muon system showing the magnetic field and the muon detector. Note the radial field component generated by the FFS in the forward direction.

\section{Calorimeter Absorber}

The GEM muon system is designed to be independent (i.e., not requiring the central tracker), and is located entirely outside the calorimeter. The major benefit of this design is the capability to measure muon trajectories in air with minimal scattering material, after most of the hadronic debris has been absorbed by the calorimeter.

The backgrounds in the muon system are greatly reduced by the shielding afforded by the calorimeter material, leaving the following residual backgrounds:

- Muon associated background, such as $\delta$-rays and muon-generated showers from the last few radiation lengths of the calorimeter.

- Hadron punchthrough and muons from $\pi / K$ decay in the central tracker and calorimeter volume.

- Neutrons and neutron-induced gammas. This background arises primarily from interactions 
of particles from the $p p$ collisions in the forward calorimeter and the collimator-quadrupole system.

Detailed simulations of these backgrounds are covered in Section 4.2 and in Chapter 12. Here we give a brief summary.

The muon-associated background is reduced by requiring the muon chambers to be separated by at least $20 \mathrm{~cm}$ from the outside boundary of the calorimeter. This provides the space needed for low energy charged particles to curl up, or to separate the background particles from the muon track. Pattern recognition is further enhanced by requiring the number of chamber measurement layers to be larger than that dictated by the resolution performance alone.

In order to have a robust system at the highest luminosity, we require the rate of charged particles penetrating into the muon system from the calorimeter (punchthrough) to be less than half the prompt muons and those muons from $\pi / K$ decay in the central tracker. This leads to a requirement on the minimum thickness of the calorimeter to be greater than that needed for hadron calorimetry alone. Utilizing Monte Carlo studies validated with experimental data, it has been determined that the calorimeter should be at least $11 \lambda$ thick in the barrel region. Also, the occupancy due to charged particles in any readout channel should be no larger than $1 \%$. This demands the calorimeter be at least $16 \lambda$ thick in the forward direction.

The neutron and neutron-induced photon background introduces uncorrelated hits in the muon detectors. However, this background will be reduced to a level well within design tolerances by the placement of special shielding in the forward regions of the detector and beam pipe. Details of the shielding design that mitigates this background are presented in Chapter 12. The radiation and shielding design studies have demonstrated that the muon system can operate with full capability even at $L=$ $10^{34} \mathrm{~cm}^{-2} \mathrm{~s}^{-1}$.

We have also studied the resolution degradation arising from multiple scattering in the chamber material and from energy loss fluctuations as the muons pass through the calorimeter. Both effects are especially important in the low momentum region.
Simulations indicate that momentum resolution at $p_{T} \approx 10 \mathrm{GeV}$ increases to $3.5 \%$. We have the option of including the central tracker information in the reconstruction at low momenta. A detailed discussion of this issue is given in Section 4.2.

\section{Muon Detector Technology}

Several different muon detection technologies were considered prior to the final choice. Each candidate technology was studied, tested and understood during an extensive $R \& D$ program. Tests were performed using the Texas Test Rig (TTR), ${ }^{2}$ a large facility commissioned at the SSC for this purpose. Limited streamer drift tubes (LSDT) ${ }^{3}$ and round drift tubes (RDT) ${ }^{4}$ met the coordinate resolution specifications. Resistive plate chambers $(\mathrm{RPC})^{5}$ and proportional wire chambers (PWC) 6 were found to be capable of providing a trigger and an additional non-bend plane coordinate measurement, in concert with either of these precision drift technologies. Cathode strip chambers (CSC), 7,8 in which the track is determined by interpolation of the charge induced on precision cathode strips, not only met the coordinate resolution specifications but also demonstrated that they could provide the trigger function needed for the muon system. All of these technologies had features that were considered attractive, as well as certain drawbacks.

Our R\&D program gave us confidence that the CSCs offered the most complete and lowest risk solution. We have chosen CSCs because they provide a solution to both the triggering and tracking functions needed for the muon system. The features of this technology are:

- Good single-layer resolution: $75 \mu \mathrm{m}$.

- Flexible configuration: axial strips in barrel, radial in endcaps.

- Non-bend coordinate measurement.

- Good timing resolution for beam cross tagging.

- Precision elements directly accessible for alignment.

- Short electron drift time $<30$ ns.

- All functions in one technology: triggering, timing, and tracking. 


\subsubsection{Muon Spectrometer Overview}

The properties of the cathode strip chambers, as well as the chamber alignment requirements, determine the layout of the chambers in the muon system. Important considerations are the size limitations of the chambers, the resolution dependence on muon incidence angle, and the need to tilt the chambers to compensate for the Lorentz angle.

\section{Chamber Configuration}

Our baseline design calls for a momentum resolution in the barrel region of $5 \%$, for a $p_{T}=$ $500 \mathrm{GeV}$ track. This requirement ensures that the sign of the muons, and hence the forward-backward asymmetry, can be determined unambiguously for a $Z^{\prime}$ with a mass of up to $10 \mathrm{TeV}$ - the heaviest $Z^{\prime}$ that can be produced at the SSC. We have noted that in order to obtain this resolution, the total error of the sagitta must not exceed $55 \mu \mathrm{m}$. To meet this requirement we have designed a robust measurement system, with multiple chamber layers deployed within a given superlayer. In this manner the effective superlayer resolution from random errors is improved by the (statistical) factor $1 / \sqrt{N}$, where $N$ is the number of chamber layers in a given superlayer, and the pattern recognition capability of the system is enhanced. This enables the muon trajectory to be recognized as a set of local vectors that are linked among the different superlayers.

Considerable effort has been expended in the overall performance/cost optimization of the system. We have studied the resolution as a function of the number of measurement layers in each superlayer as illustrated in Figure 4-3, including the effects of intrinsic chamber precision, layer-to-layer alignment, and alignment errors from superlayer-to-superlayer. We have found that our resolution requirements can be met by 6 measurement layers in the middle superlayer and as few as 4 measurement layers in the inner and outer superiayers. Our pattern recognition studies require a minimum of three good measurements (hits) in each superlayer lying within a "road" defined by the intrinsic resolution of the chamber layer. Studies of the efficiency for a good hit in a chamber layer, in the presence of both uncorrelated (e.g., neutron) and correlated (e.g., muon-associated EM shower) backgrounds indicate that the number of layers should be larger than four, to ensure that the system is robust for track-finding.
We have therefore taken the chamber layer-configuration in the superlayers of the barrel (SL1:SL2:SL3) to be 6:6:6, and in the endcaps to be $8: 6: 6$ in consideration of the higher rates and larger background expected there. Thus the number of measuring layers is driven by pattern recognition rather than by momentum resolution.

The optimization of each individual superlayer is driven by the specific requirements of the GEM experiment. The inner superlayer must have the good two-track resolution to help in measuring muons with nearby hadronic and electromagnetic backgrounds. Optimization of resolution allows no compromises in the spatial resolution of the middle superlayer. The outer superlayer has less stringent constraints but is needed to provide lever arm and pattem recognition to isolate the muon track and link it with the inner two superlayers. We have worked to reduce the mechanical cost as well as the number of electronic channels in the outer superlayers, because this superlayer comprises the largest area of chambers.

The spatial resolution of a cathode strip chamber changes as a function of azimuthal angle, and the proper chamber layout is dictated by the need to minimize this source of error. We accomplish this by allowing each chamber to cover only a smail region in azimuthal angle $\left(< \pm 7.5^{\circ}\right.$ in the first superlayer of the endcaps, and $< \pm 3.75^{\circ}$ for the other two endcap layers and everywhere in the bartel).

A related effect is the smearing of resolution due to the altered direction of drifting ionization

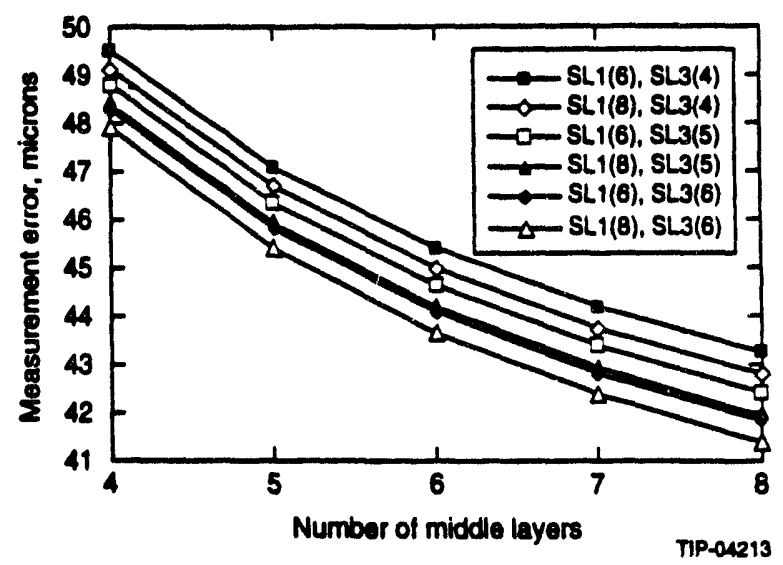

FIG. 4-3. Resolution improvemeitt as a function of number of measurement layers in the middle superlayer for various numbers of measurement layers in the inner (SL1) and outer (SL3) superlayers. 
electrons under the combined effects of electric and magnetic field-the Lorentz effect. In the barrel region the natural solution is to simply rotate the plane of the cathode strip chambers about an axis parallel to the magnetic field vector. For our anticipated gas mixtures and operating conditions, the Lorentz angle is about $8^{\circ}$. Because the resolution of the middle superlayer is critical for achieving the momentum resolution performance we rotate that superlayer by $8^{\circ}$. However, geometrical constraints within the inner and outer superlayers require us to compromise on the optimal Lorentz angle compensation by rotating those planes by only $6^{\circ}$.

Similar considerations apply to the endcap chambers, where the magnetic field is primarily perpendicular to the chamber plane. The axial component of the magnetic field is essentially parallel to the electric field leading to little Lorentz effect. The small component of the electric field perpendicular to the magnetic field can be compensated by orienting the anode wires at an angle with respect to the radial axis of the chambers. The radial magnetic field interacting with the axial electric field is relatively small and the net effect on muon resolution is negligible.

The size limitation of the cathode strip chambers determines the azimuthal partitioning in the outer superlayer. One driving factor is the maximum length of anode wires sustainable (without internal wire supports) before the onset of electrostatic instabilities. In the present design this dimension is limited to about $1.2 \mathrm{~m}$. Therefore our superlayers of chambers are partitioned in 48 chambers, driven by the width limitation of the outer superlayer of chambers. This also happens to be close to the maximum available width of the $\mathrm{Cu}$-clad G10 boards that we are using to produce the cathodes and also to be consistent with azimuthal angle constraint of a chamber. Further, the maximum length of cathode strips determines the partitioning of the system in the polar angle. At the present time the length of the cathode strips is governed by the maximum length of $\mathrm{G} 10$ board that can be manufactured-about $3.5 \mathrm{~m}$. Hence the complete polar angle coverage requires 4 chamber segments for the middle and outer superlayers, and 2 chamber segments for the inner superlayer (for each barrel half). In order to maximize the acceptance and provide continuous alignment registration between sectors, we have chosen to overlap chambers in $\phi$. which also provides the capability to transfer alignment information between $\phi$ sectors using muon tracks. The net result of all these design considerations is illustrated in Figure 4-1.

The operation of the cathode strip chambers requires the precise readout of the charge induced on the cathode strip by avalanches occurring on the anode wire. General considerations indicate that a resolution of about $1 \%$ of the intrinsic strip width can be achieved. This is discussed in detail in Section 4.3. To minimize the overall channel count, the cathode strip widths and the chamber gas gaps increase with increasing superlayer lateral extent: roughly, $0.5 \mathrm{~cm}, 0.7 \mathrm{~cm}$, and $1.0 \mathrm{~cm}$ in each of the three superlayers, respectively. Similarly, the nonbend plane coordinate readout segment size, which is determined by the ganging of groups of anode wires, increases with increasing superlayer extent. These groupings are approximately $5 \mathrm{~cm}, 7.5 \mathrm{~cm}$, and $10 \mathrm{~cm}$, respectively. Again, refer to Section 4.3 for more details.

\section{Support Structure}

The support structure for the barrel is arranged into 12 modules, each of which is subdivided into fourths, making a total of 48 sectors in azimuth. The 12 modules are tied together to form a large monolithic structure, which is mounted on the magnet at stable support points. There is one monolith for each half of the barrel muon system (see Figure 4-4). The endcap muon system is constructed in 3 complete wheel structures that are then tied together to form a monolith. In the barrel the large monolithic structure that is formed from the 12 independent modules is required to minimize the system's response to vibrations. Modules can be loaded with chambers and assembled into the monolithic structures above ground. This procedure is facilitated by surface buildings and underground access shafts large enough to accommodate the GEM nnet halves. Additionally, there is the benefit or Ininimizing the amount of assembly work required in the underground hall. In our design, the modules will be fabricated from aluminum tubes that are bolted, pinned, and welded into truss structures. In order to minimize muon chamber misalignment caused by dynamic deformations of the support siructure, the chamber packages will be 


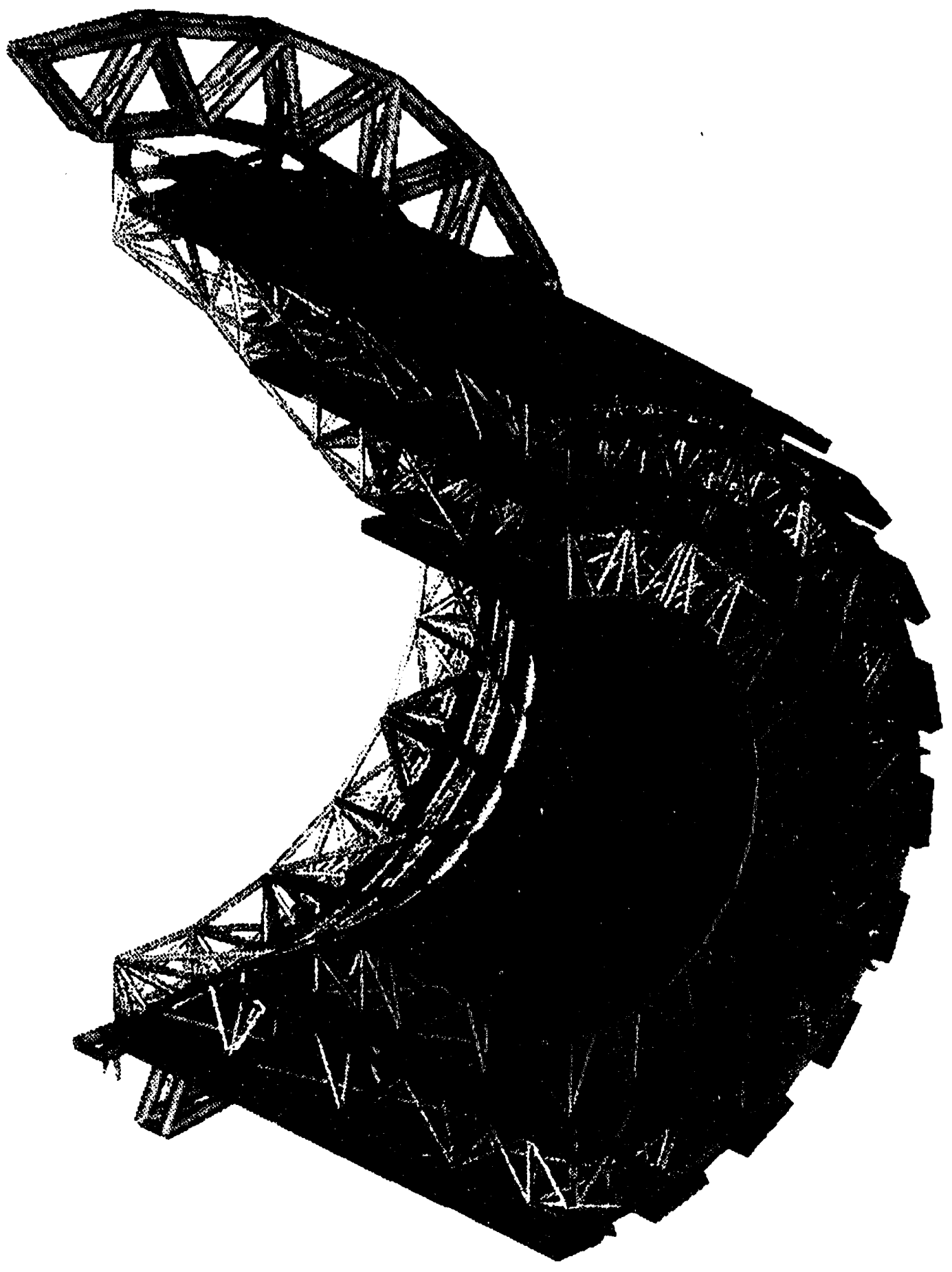

FIG. 4-4. Cutaway model of barrel monolith showing the truss structure and support ring ends. 
kinematically mounted within the modules utilizing 3-point supports. In addition the support structure will have sufficient stability so that location of the chambers is always within the range of our position monitors, thus enabling a precise sagitta error correction. (see Sections 4.3.3, and 4.3.4 for more details.)

\section{Alignment}

The GEM muon system incorporates a muon chamber design allowing the individual chamber packages to be aligned with very high precision. The alignment system specifications are based on the extensive experience gained in similar open-geometry 3-superlayer muon detectors ${ }^{9}$ and on an in-depth analysis of the influence of measurement, scattering, and alignment errors on the muon resolution. As mentioned earlier, our required $5 \% \Delta p_{T} / p_{T}$ at $p_{T}=$ $500 \mathrm{GeV}$ entails maintaining a net sagitta error below $55 \mu \mathrm{m}$.

For the GEM muon detector, the sources of sagitta error can be divided into six contributions: chamber measurement, multiple scattering, strip-tostrip placement, layer-to-layer placement, fiducialization, and superlayer-to-superlayer alignment. These terms are illustrated in Figure 4-5, and a set of corresponding requirements are listed in Table 4-1. The net $55 \mu \mathrm{m}$ goal in sagitta precision is obtained by adding the individual error terms in quadrature, taking into account the radial location of the chambers and the number of chamber layers in each superlayer, as follows:

$$
\begin{gathered}
\sigma_{\mathrm{sug}}^{2}=1.5\left[\frac{\sigma_{\mathrm{ch}}^{2}+\sigma_{\mathrm{L} / \mathrm{L}}^{2}+\sigma_{\mathrm{s} / \mathrm{s}}^{2}}{N_{\mathrm{L}}}+\sigma_{\mathrm{fid}}^{2}\right) \\
+\sigma_{\mathrm{dg}}^{2}+\sigma_{\mathrm{ms}}^{2}
\end{gathered}
$$

This assumes that $\sigma_{\mathrm{ch}}, \sigma_{\mathrm{L} / \mathrm{L}}, \sigma_{\mathrm{s} / \mathrm{s}}$ and $\sigma_{\mathrm{fid}}$ are identical for all layers and $N_{\mathrm{L}}=6$.

The layer-to-layer positioning of CSC strips must be determined to better than $50 \mu \mathrm{m}$, and the deviation in straight-line alignment between the mean superlayer position must be measured to within a $25 \mu \mathrm{m}$ systematic error. To achieve these goals, the alignment technique places the muon

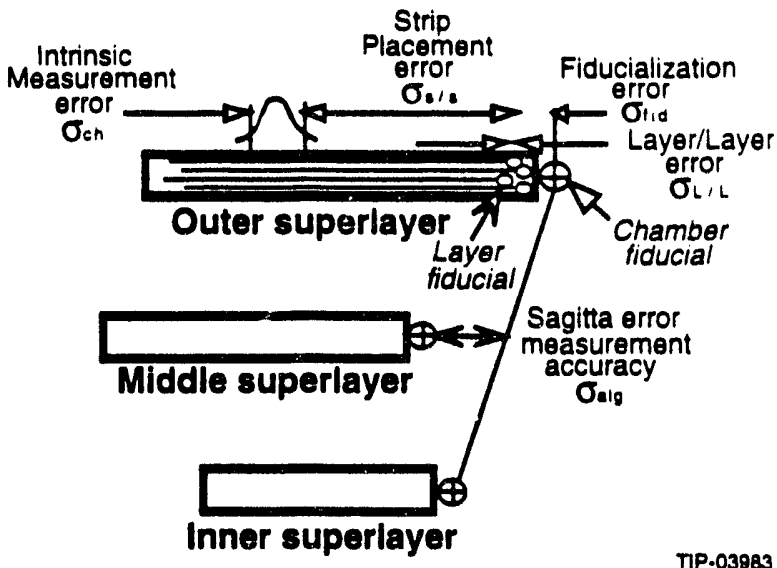

FIG. 4-5. Alignment contributions to momentum measurement error.

Table 4-1. Contribution to momentum measurement error.

\begin{tabular}{lcc}
\multicolumn{1}{c}{ Error Source } & Label & $\begin{array}{c}\text { RMS } \\
(\mu \mathrm{m})\end{array}$ \\
\hline Intrinsic chamber measurement & $\sigma_{\mathrm{ch}}$ & 75 \\
Multiple scattering $(p=500 \mathrm{GeV})$ & $\sigma_{\mathrm{ms}}$ & 5 \\
Strip-to-strip placement & $\sigma_{\mathrm{g} / \mathrm{s}}$ & 17 \\
Layer-to-layer placement & $\sigma_{\mathrm{L}}$ & 50 \\
Fiducialization placement & $\sigma_{\mathrm{fid}}$ & 10 \\
Sagitta error measurement & $\sigma_{\mathrm{alg}}$ & 25 \\
accuracy & & \\
TOTAL & $\sigma_{\mathrm{gag}}$ & 54 \\
\hline
\end{tabular}

chambers within a few millimeters of their nominal position, and interpolates the resulting sagitta corrections to the required accuracy by using three-point optical straightness monitors located along projective paths that link the edges of each chamber in an alignment tower. ${ }^{12}$ These sensor arrays monitor the deviations from ideal projective alignment; the ensuing "false sagitta" imposed on an incident muon track is then estimated and removed. In order to achieve the needed operational stability, the alignment data is periodically monitored to account for thermal and mechanical distortions. Details of our alignment strategy and systems are given in Section 4.3.4.

\section{Beam Line Vertex Constraint}

The knowledge of the transverse beam position relative to the GEM muon systern provides powerful additional track-fitting constraint for the 
muon system. This constraint essentially doubles the available lever arm which quadruples the usable sagitta because of the $L^{2}$ dependence.

We have found that the knowledge of the beamline vertex position, with a precision of about $500 \mu \mathrm{m}$, allows the GEM detector to collect events with no measurement necessary in the first superlayer that otherwise might be lost. In this case the resolution of lower momentum muons is limited by multiple scattering in the calorimeter, and the resolution at the higher momenta is only slightly degraded. All muon detection systems at high energy colliders will be adversely affected by the backgrounds discussed above, which limit the track-finding efficiency. This is particularly true at high muon momentum where muon-associated EM backgrounds emerge from nearby materials, such as the calorimeter. The GEM muon system has been "hardened" to these effects by placing the first superlayer of chambers some distance away from the calorimeter, allowing associated low energy charged particles to become separated from the muon track. Compared to other SSC muon systum designs, additional EM background is much less of a problem because the material near the superlayers has been minimized. In spite of these efforts at mitigation, the efficiency of the first superlayer is still affected by the presence of these backgrounds.

Additionally, if the beam position can be determined to about $200 \mu \mathrm{m}$, then the higher momentum muons can be measured with an improvement of up to a factor of two over the baseline system, i.e., with all superlayers contributing. Thus a beam vertex constraint provides both additional system robustness, and improved resolution capability. The proper establishment of this constraint requires knowledge of the positions of the muon sectors relative to the beam. We expect that this can be accomplished by a combination of methods utilizing survey, beam position monitors, and the use of single muon tracks coupling the vertex to the muon system. It is anticipated that the implementation of a vertex constraint will be an iterative process, requiring full understanding of the systematics. These performance issues are discussed in detail in Section 4.2.

\section{Trigger Concept}

The design of the trigger and beam crossing tag is based on a simultaneous readout of the anode wires and cathode strips of the CSCs. This arrangement will enable both a Level 1 momentum-dependent trigger to be formed, as well as a measurement of the non-bend plane coordinate of the muon track. The CSCs have a time jitter of $<3$ ns (RMS) for 6 layers "OR'd" together, which is sufficiently fast to select the correct beam crossing $99 \%$ of the time.

The Level 1 muon trigger can be based on either a measurement of the bend angle $\delta \phi$, or a fast sagitta determination performed with the resolution of the intrinsic bend plane strip width. The first method does not depend on measurements in the first superlayer, while the second method is independent of the multiple scattering in the calorimeter and reduces the sensitivity to positioning with respect to the vertex. The concept for the Level 1 trigger is shown in Figure 4-6. These two methods can be summarized as:

- A $\delta \phi$ cut utilizing the last two superlayers only. This method identifies the difference in location between the second and third superlayer and extrapolates an effective $p_{T}$ for the candidate muon track assuming it originated at the interaction point.

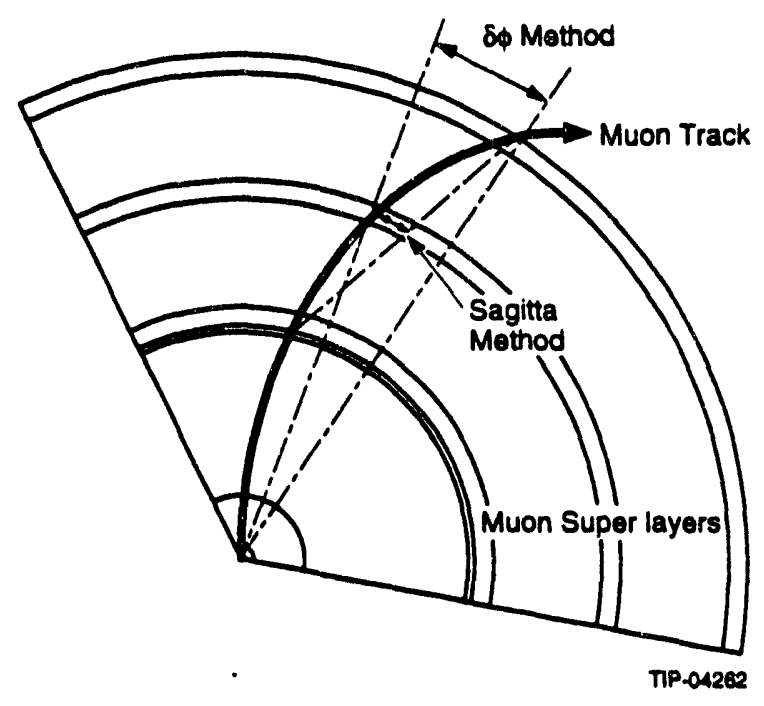

FIG. 4-6. The $\delta \phi$ and sagitta concept of the trigger is illustrated for the barrel region. 
- A sagitta method utilizing all three superlayers. This method forms a line between the first and third superlayers and searches for a hit in the second superlayer within a sagitta distance which determines the muon $p r$.

\subsubsection{System Design Studies}

The muon spectrometer system described in later sections of this chapter is based on a detailed initial engineering design, which uses well-known mechanical design approaches to the production of precision chambers and large stable structures, taking careful account of the cost and schedule. The system as currently designed attempts to meet the performance specifications by taking advantage of the inherent precision of the CSC chamber technology, within the constraints imposed by this technology choice. These technical constraints are: limited CSC cathode panel size and thus chamber size (assuming one cathode panel per chamber enclosure); chamber tilt optimized at a fixed angle for Lorentz angle compensation; projective optical alignment paths outside of the chamber active volume; and chambers designed for stiffness, and thus flatness, where the chamber depth is optimized to provide the maximum practical lever arm within the available radial space.

In many areas the GEM muon system currently meets or exceeds our design specifications. However, in three important areas-coverage. material, and access-we are working to optimize our design as described in Sections 4.3 through 4.6. We have recently completed our first-stage engineering design, along with the resultant understanding of the muon system cost constraints, chamber construction, performance, access, and altemative methods and configurations for achieving our required alignment precision. We are continuing a program to incorporate design improvements into the system to ensure that it will be optimized within cost-constraints.

\subsubsection{Detector Parameters}

The principal parameters of the GEM muon system are summarized in the Tables 4-2 through 4-5.

\subsection{DETECTOR SIMULATION AND PERFORMANCE}

\subsubsection{Introduction}

The muon system is designed to function in a standalone mode providing full track reconstruction, a first level muon trigger with thresholds ranging from $10 \mathrm{GeV}$ to $50 \mathrm{GeV}$, and a beam crossing tag. It can handle random neutron rates up to $10^{5} \mathrm{~cm}^{-2} \mathrm{~s}^{-1}$ without significant degradation in performance. Charged particle rates from hadron punchthrough have been suppressed to levels below the rate of real muons by the thick GEM calorimeter. The use of 6 to 8 layers in a muon chamber superlayer results in high track reconstruction efficiency, even at the highest muon energies where muon-induced showers in the materials of the muon system become substantial. In the following we discuss the main muon backgrounds, the trigger performance, pattern recognition capabilities, and the momentum resolution of the muon system. The performance of the muon system for a representative set of physics benchmarks also is demonstrated. Finally, a discussion of how the performance of the muon system will be verified and monitored is given.

The main simulation tool used to evaluate the performance of the GEM muon system and to guide its design is the SIGEM ${ }^{13}$ simulation program. SIGEM is a GEANT-based program which now includes the CALOR code, which extends the capabilities of GEANT to low energy neutron 
Table 4-2 Parameters of the GEM muon system.

\begin{tabular}{|c|c|}
\hline \multicolumn{2}{|l|}{ Geometry: } \\
\hline Magnetic field at the IP & $0.8 \mathrm{~T}$ \\
\hline Barrel region: $\left(29^{\circ}<\theta<84^{\circ}\right)$ & $0.1<|\eta|<1.3$ \\
\hline Endcap region: $\left(9.75^{\circ}<\theta<28^{\circ}\right)$ & $1.4<|\eta|<2.46$ \\
\hline Number of sectors in $\phi$ & 48 \\
\hline Barrel lever arm & $>4.2 \mathrm{~m}$ \\
\hline Endcap lever arm & $>8.6 \mathrm{~m}$ \\
\hline \multicolumn{2}{|l|}{ Chamber parameters: } \\
\hline \multicolumn{2}{|l|}{ Spatial resolutions: } \\
\hline Single-layer resolution (RMS) & $75 \mu \mathrm{m}$ \\
\hline Nonbend plane resolution & $0.8-1.5 \mathrm{~cm}$ \\
\hline Beam crossing tag eiticiency (6 layers) & $>99 \%$ \\
\hline Intemal chamber alignment & $50 \mu \mathrm{m}$ \\
\hline Superlayer-to-superlayer alignment & $25 \mu \mathrm{m}$ \\
\hline Radiation length/chamber layor & $1.1 \%$ \\
\hline No. of layers per SL (SL1:SL2:SL3) & $\begin{array}{l}\text { 6:6:6 barrel } \\
\text { 8:6:6 endcap }\end{array}$ \\
\hline No. of chambers in barrel & 960 \\
\hline No. of chambers in endcaps & 480 \\
\hline No. of bend plane channels - barrel & 645,120 \\
\hline No. of bend plane channels - endcaps & 276,480 \\
\hline No. of non-bend plane channels - barrel & 221,184 \\
\hline No. of non-bend plane - endcaps & 92,160 \\
\hline
\end{tabular}


Table 4-3. Barrel region physical parameters.

\begin{tabular}{|c|c|c|c|c|c|c|c|}
\hline \multicolumn{8}{|c|}{ Barrel Region } \\
\hline Superlayer & $\begin{array}{l}\text { Chamber } \\
\text { Width } \\
\text { (m) }\end{array}$ & $\begin{array}{l}\text { Chamber } \\
\text { Length } \\
\text { (m) }\end{array}$ & $\begin{array}{c}\text { Chamber } \\
\text { Mass } \\
\text { (kg) }\end{array}$ & $\begin{array}{c}\text { Quantity } \\
\text { (Por Module) }\end{array}$ & $\begin{array}{l}\text { Quantity } \\
\text { (2 Barrels) }\end{array}$ & $\begin{array}{l}\text { Module Mass } \\
(\mathrm{kg})\end{array}$ & $\begin{array}{l}\text { Barrel-Half } \\
(\mathrm{kg})\end{array}$ \\
\hline Outer & 1.390 & 3.540 & 223 & 14 & 336 & 3118 & 37418 \\
\hline Outer & 1.390 & 2.814 & 183 & 2 & 48 & 366 & 4396 \\
\hline Middle & 1.139 & 2.574 & 145 & 4 & 96 & 578 & 6938 \\
\hline Middle & 1.139 & 2.320 & 133 & 6 & 144 & 796 & 9556 \\
\hline Middle & 1.139 & 2.199 & 127 & 4 & 96 & 507 & 6087 \\
\hline Middle & 1.139 & 1.602 & 99 & 2 & 48 & 197 & 2367 \\
\hline Inner & 0.831 & 3.540 & 151 & 6 & 144 & 905 & 10865 \\
\hline Inner & 0.831 & 2.972 & 129 & 2 & 48 & 257 & 3087 \\
\hline TOTALS & & & & 40 & 960 & 6726 & 80716 \\
\hline
\end{tabular}

Table 4-4. Endcap-Physical Parameters.

\begin{tabular}{|c|c|c|c|c|c|}
\hline \multicolumn{6}{|c|}{ Endcap Region } \\
\hline Super Layer & $\begin{array}{c}\text { Chamber Width } \\
\text { (MaxMin) } \\
\text { (m) }\end{array}$ & $\begin{array}{l}\text { Chamber } \\
\text { Length } \\
\text { (m) }\end{array}$ & $\begin{array}{c}\text { Chamber } \\
\text { Mass } \\
(\mathrm{kg})\end{array}$ & $\begin{array}{l}\text { Quantity } \\
\text { (1 Endcap) }\end{array}$ & $\begin{array}{c}\text { Endcap- } \\
\text { each } \\
\text { (kg) }\end{array}$ \\
\hline Outer & 0.87010 .515 & 2.640 & 113 & 48 & 5433 \\
\hline Outer & 1.31010 .840 & 3.530 & 186 & 48 & 8924 \\
\hline Middle & 0.63010 .380 & 1.820 & 66 & 48 & 3164 \\
\hline Middle & 0.90010 .610 & 2.223 & 96 & 48 & 4604 \\
\hline Inner & 0.73010 .435 & 1.154 & 58 & 24 & 1385 \\
\hline Inner & $1.150 / 0.665$ & 1.875 & 113 & 24 & 2705 \\
\hline TOTALS & & & & 240 & 26215 \\
\hline
\end{tabular}


Table. 4-5. System Weights (kg).

\begin{tabular}{lrlr}
\hline \multicolumn{1}{c}{ Barrel } & \multicolumn{2}{c}{ Endcap } \\
\hline Barrel module structure mass & 4274 & Outer wheol structure mass & 3988 \\
CDS support ring mass & 5455 & Outer wheel mass (total) & 18344 \\
FFS support ring mass & 14727 & Middle wheel structure mass (total) & 2133 \\
Barrel-half structure mass & 71465 & Middle wheel mass (total) & 9900 \\
Barrel-half chamber mass & 80716 & Inner wheel structure mass & 1133 \\
Barrel half mass (total) & 152182 & Inner wheel mass (total) & 5224 \\
Barrol region mass (total) & 304364 & Outer/middle structure mass & 4646 \\
& & Middle/inner structure mass & 1650 \\
& & Attachment hardware mass & 494 \\
& & Total structure mass & 14043 \\
& & Total mass endcap-half & 40258 \\
& & Total mass endcap region & 80516 \\
\hline
\end{tabular}

interactions. The GEANT geometry coded in SIGEM includes an accurate description of:

- The central tracker.

- The calorimeters, represented as homogeneous mixtures.

- The magnet coil and the cryostat.

- The beam pipe, forward field shapers, and the collimator low-beta-quadrupole system.

- The muon chamber layout, alignment paths, the chamber frames, the support structures, and the detailed layer structure of the CSC chambers.

The calculated magnetic field map is encoded in SIGEM so that the track reconstruction and momentum measurement can be investigated in detail. Figure 4-7 shows the geometry coded in SIGEM for the barrel and the endcap muon system. Muons are simulated through the GEM detector with the GEANT kinetic energy cutoffs set so that the effect of the muon-induced electromagnetic particles ( $\delta$ rays, bremsstrahlung, and $e^{+} e^{-}$pairs) is properly simulated. Figure 4-8 shows a single $1 \mathrm{TeV}$ muon generated at $\theta=60^{\circ}$ and $\phi=20^{\circ}$. Apparent is the multitude of low momentum electromagnetic particles accompanying the muon as it exits the calorimeter, and those produced as it passes through the material of the muon system. We use SIGEM to evaluate the impact of geometrical acceptance, muon system materials, and support structures on the track reconstruction efficiency. An active effort is continuing to develop an optimized fitting algorithm for use on the muon hits simulated by SIGEM.

\subsubsection{Muon Backgrounds}

\section{Introduction}

Four types of backgrounds can affect the performance of the muon system:

- Hadron punchthrough and decay muons.

- Cosmic ray muons.

- Uncorrelated neutrons and associated photons.

- Muon-induced showers (pairs and muon bremsstrahlung) and delta rays.

To fully realize the performance capabilities of the GEM muon system, careful evaluation of these backgrounds has been performed, and the results used to improve the design. In this section, details of muon background studies are reported. In the following, we justify the design specifications for the GEM detector in light of these backgrounds.

The following specifications were established to assure the robustness of the system: 


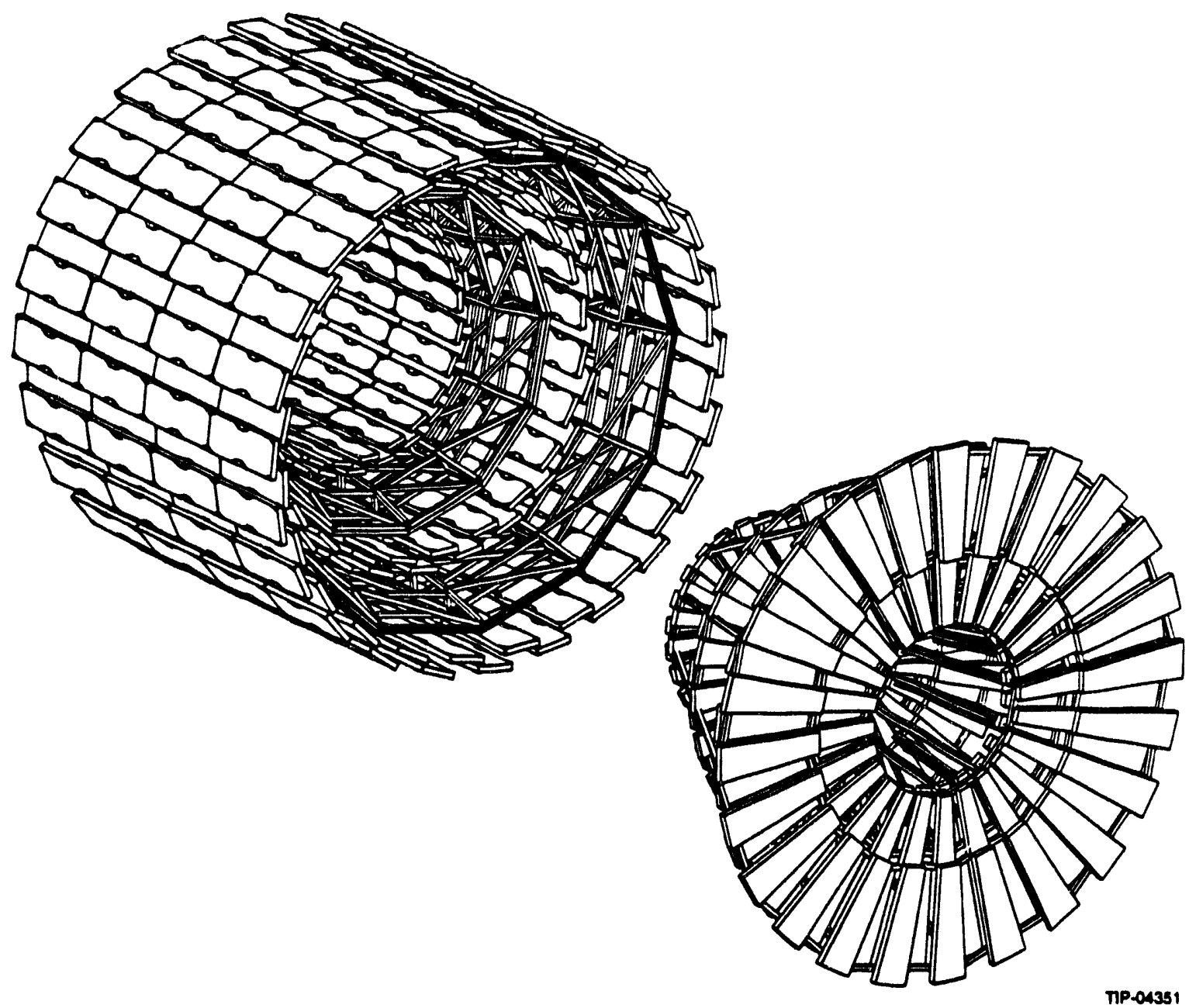

FIG. 4-7. The GEM muon system in the SIGEM simulation for the barrel and the endcap regions.

- The punchthrough rate must be suppressed by a thick calorimeter to levels below that for prompt muons plus muons from $\pi^{ \pm}$and $\mathrm{K}^{ \pm}$ decays in the central tracker.

- The occupancy of 4 contiguous strips from uncorrelated charged particles in a single CSC layer must be $<1 \%$ at the highest SSC luminosity $\left(\ell=10^{34} \mathrm{~cm}^{-2} \mathrm{~s}^{-1}\right)$. This assures a track segment reconstruction efficiency in a superlayer of $>99 \%$, and a low Level 1 muon trigger rate.

- The occupancy of a single strip from uncorrelated neutrons and gammas should be $<3 \%$ at the highest SSC luminosity. This assures a track segment reconstruction efficiency in a superlayer of $>99 \%$ and a fake track segment rate of less than 0.01 per CSC chamber per event.

\section{Hadron Punchthrough, Decay, and the Calorimeter Depth}

The GEANT-based studies have shown that the GEM muon system is robust to hadron punchthrough and decay up to the highest SSC luminosity. The ability to observe muon signals from rare processes at the SSC is uncompromised by hadron punchthrough by virtue of a thick hermetic calorimeter, the fine segmentation, the high rate capability, and the redundancy of the CSCs. The results of comparisons of GEANT simulation and experimental data on hadron punchthrough are reported in Reference 14. The agreement between GEANT and experimental data is good, and an active program is underway to further validate the GEANT code with experimental data. 


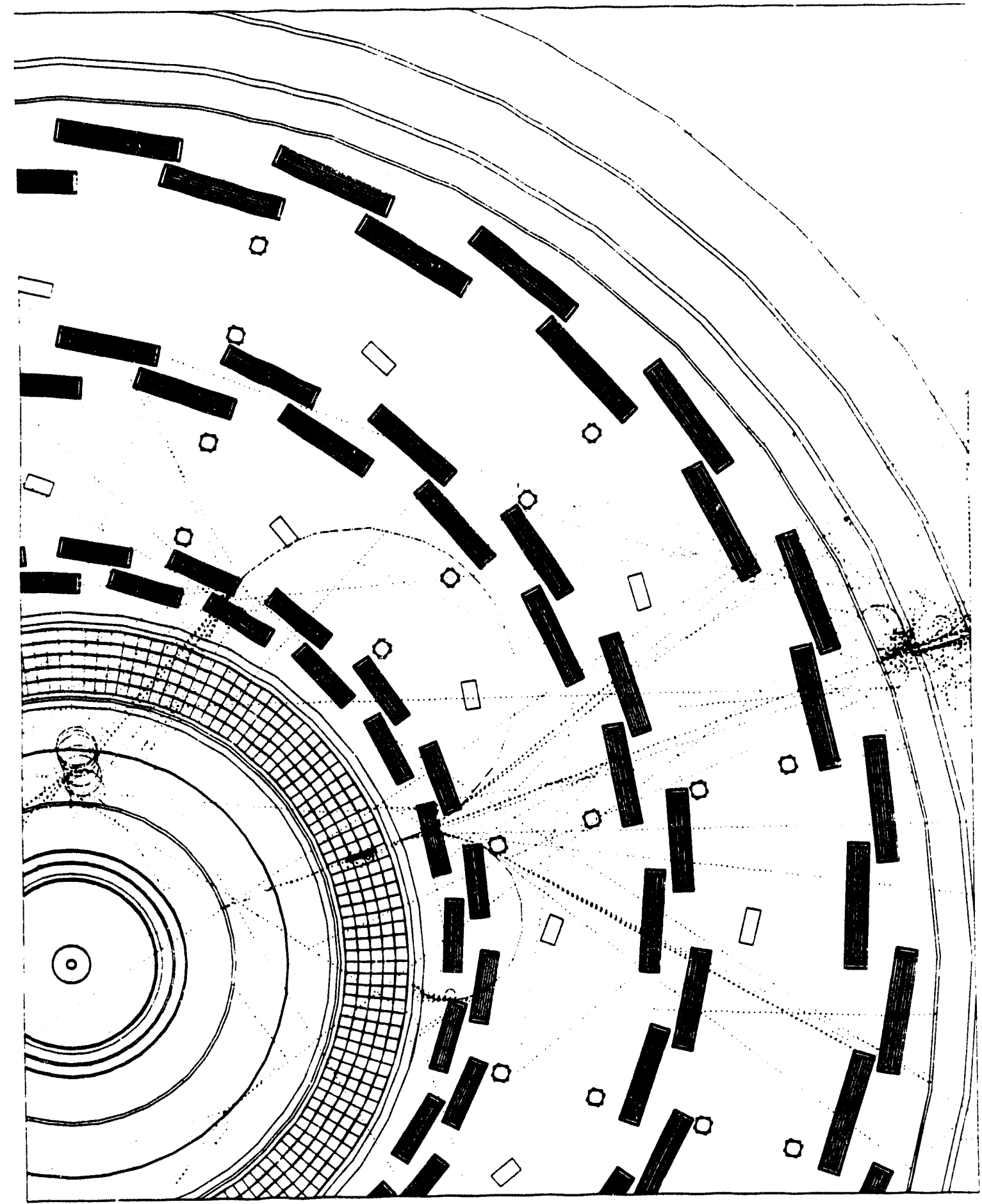

FIG. 4-8. Event display from SIGEM for a 1 ToV muon in the barrel region. The dashed line at $\phi=20^{\circ}$ is the muon. Solid lines are accompanying muon-induced charged particles. Dotted lines are muon-induced gammas. The support structure (not sketched) is included in the simulation, and is responsible for some of the interactions shown. 
To study the correlated hadron punchthrough and its impact on muon pattem recognition, $\bar{t} \bar{t}$ events were generated in which the $b$-mesons from $t$-decays were forced to decay inclusively to muons. GEM is well-suited to search for top with this signature. ${ }^{15}$ The muon from the $b$-decay will be very near the center of the jet, and so this represents a challenging test of the punchthrough background suppression. Figure 4-9 shows a high $p_{T} b$-jet which includes a $480 \mathrm{GeV} \pi$. Only the muon and a few neutrons penetrate the calorimeter. These events will be considered further in Section 4.2.4, where pattern recognition is discussed.

In the GEM detector, all the hadron absorber lies inside the first layer of the muon system. Therefore, the thickness of the hadron absorber sets the scale in cost and size for the muon spectrometer. The depth of the calorimeter in front of the muon system was an issue raised by the PAC in its report on the GEM LOI, and therefore it has been the focus of considerable simulation effort over the past

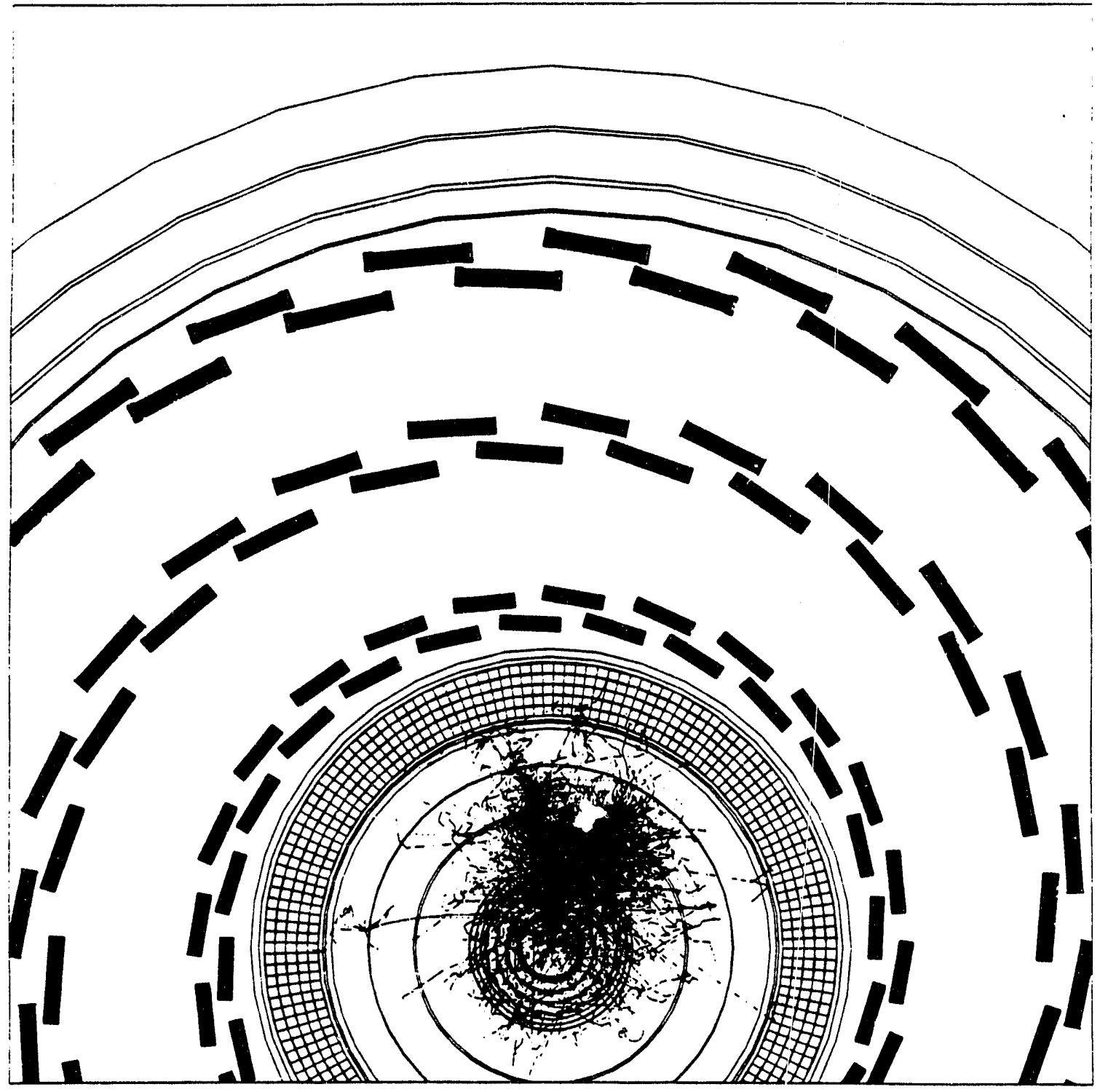

FIG. 4-9. A 1 ToV b-jet with imbedded muon. The dot-dashed lines are neutrons. 
months. Simulation studies of particle rates in the muon spectrometer as a function of calorimeter thickness have been performed ${ }^{16}$ for both the barrel and the endcap regions. Although comparisons of simulated punchthrough with experimental data have shown general agreement, many detailed aspects of the simulated punchthrough have not yet been compared to data. We have thus used a conservative approach in establishing the minimum hadron absorber depth.

For the muon system, a limiting factor will be the rate of particles in the first muon detector layers after the calorimeter. This rate should be sufficiently low that an efficient Level 1 trigger can be formed for muons of interest, (about 10-20 GeV in transverse momentum). Particles entering the muon system consist of prompt (including signal) muons, $\pi / \mathrm{K}$ decay muons, and hadron punchthrough. The first is irreducible and the second is determined by the size of the tracker volume. The third is determined by the depth and composition of the calorimeter. Thus we use the following two criteria to evaluate the calorimeter depth:

1) The overall particle rate exiting the calorimeter from hadron punchthrough should be much less than half the sum of the rates from prompt muons and muons from $\pi / K$ decays.

2) The rate of particles with transverse momentum above a trigger threshold from hadron punchthrough should be much less than that from prompt muons and muons from $\pi / K$ decays.

The first criterion addresses the pattern recognition difficulties brought about by a high charged particle punchthrough rate. The second considers the non-interacting charged hadrons that generate fake muon triggers. Both criteria are independent of the muon system design. However, the muon system design itself imposes some limitations. These can be best expressed as a limit on the charged particle occupancy of the chamber layers. The highest occupancy will be in the inner superlayer immediately following the calorimeter. If we assume that the charged particles entering a muon chamber superlayer are stiff and penetrating tracks, then a $1 \%$ occupancy of charged particles in a 4-strip wide interval (the lateral extent of the charge distribution induced by a charged particle traversing that layer) will translate into a $1 \%$ inefficiency to reconstruct a muon track segment in a superlayer. This is because the charge distribution resulting from a muon and another nearby charged track will fail to reconstruct into a single precise track segment. The inefficiency of muon track reconstruction in all 3 superlayers will be approximately 3 times the 4-strip occupancy of a single superlayer. This has led to a third criterion to gauge the required calorimeter depth:

3) The calorimeter must be thick enough so that the 4-strip occupancy from charged particles in each layer of the first superlayer of the muon system is less than $1 \%$ at $\mathcal{L}=10^{34} \mathrm{~cm}^{-2} \mathrm{~s}^{-1}$.

For the study, TWOJET events were generated via ISAJET ${ }^{17}$ Particle rates outside the calorimeter were determined, including prompt muons, muons from $\pi / \mathrm{K}$ decays, and hadron punchthroughs. The GEANT-based PCHTHR code ${ }^{18}$ was utilized. It produced the 4-vectors for a set of punchthrough particles using tabulated results from simulations of fixed-momentum pions incident on iron. The simulated punchthrough showers were recorded, and probability tables were prepared for the punchthroughs, the shower multiplicity, individual particle types, the momenta, spatial positions, and exiting angles relative to the incident track. The probability tables are incorporated into a single subroutine, which is called for each hadron incident on the calorimeter. We studied calorimeters of thickness $6-16 \lambda$ in the barrel and $10-16 \lambda$ in the endcap.

Figure 4-10 shows, for three rapidity in cervals, the total rate of charged particles exiting the calorimeter as a function of the calorimeter depth (solid curve), the rate from prompt muons and $\pi / \mathrm{K}$ decays in the tracker (large dashed curve), and punchthrough particles (small dashed curve). The figure shows that criterion 1 is satisfied for calorimeter depths greater than $11 \lambda$. Considering only particles with $p_{T}>10 \mathrm{GeV}$, the rate coming from prompt muons dominates for calorimeter depths of $10 \lambda$ and above. Table 4-6 summarizes the single and 4 strip CSC occupancies expected in the two regions of rapidity, corresponding to the highest rate barrel and endcap strips at a luminosity of $10^{34} \mathrm{~cm}^{-2} \mathrm{~s}^{-1}$. 
(e)

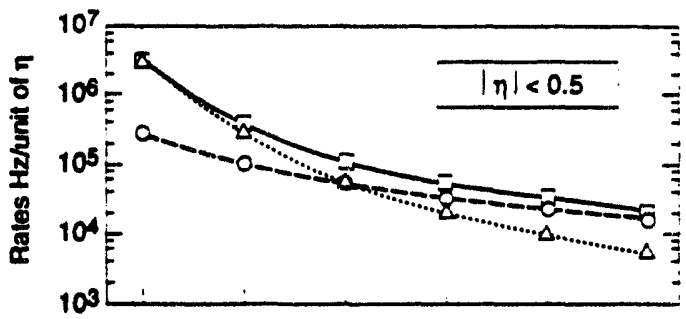

(b)

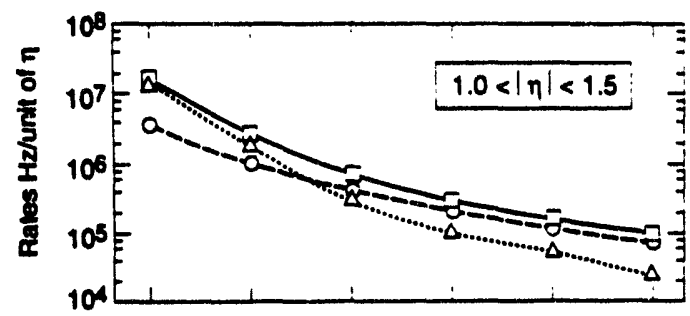

(c)

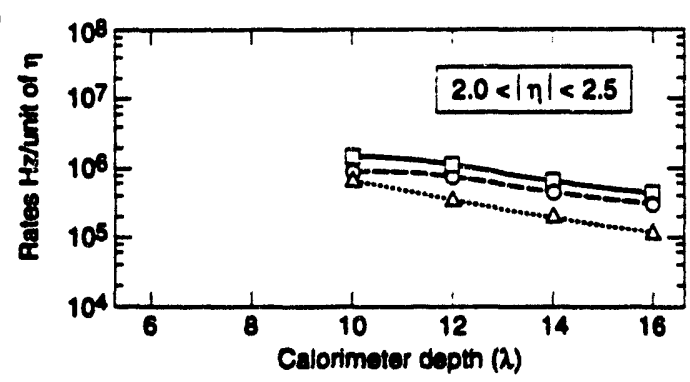

\begin{tabular}{l}
\hline All charged particles \\
O- Red muons (Prompt + decays) \\
$\Delta$ Punchthrough
\end{tabular}

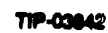

FIG. 410. Charged particle rate versus calorimeter depth for a) $|\eta|<0.5$, b) $1.0<|\eta|<1.5$, and c) $2.0<|\eta|<2.5$ at $10^{33} \mathrm{~cm}^{-2} \mathrm{~s}^{-1}$ luminosity.

The third criterion is satisfied in the barrel region with a calorimeter thickness of $10 \lambda$ or more, while in the far forward region a minimum of $16 \lambda$ of calorimeter depth is required. The rate reduction by increasing the calorimeter depth beyond $16 \lambda$ is small, and it is not cost effective to increase the calorimeter depth beyond that. Instead, the chamber rate capacity will insure its robustness at high luminosities.

Table 4-7 summarizes the calorimeter depth requirements resulting from criteria $1-3$. To satisfy all criteria requires a calorimeter with a minimum depth of $11 \lambda$ over the barrel region, and from $12 \lambda$ to $16 \lambda$ in the endcap region.

The GEM calorimeter is designed to meet these requirements (see Chapter 5). Figure 4-11 shows the rate per unit area at $10^{33} \mathrm{~cm}^{-2} \mathrm{~s}^{-1}$ expected in the first superlayer of the muon system after the GEM calorimeter. We conclude that at $10^{34} \mathrm{~cm}^{-2} \mathrm{~s}^{-1}$ the maximum occupancy from charged particles will be $0.44 \%$ in the barrel region and $1.0 \%$ in the endcap region. This rate will not affect the trigger or patternrecognition at high luminosity. Further sup pressionof punchthrough particles is possible by using the inner tracker, as discussed in Section 6.2.3.4.

Table 4-6. CSC single and 4 strip occupancy at $10^{34} \mathrm{~cm}^{-2} \mathrm{~s}^{-1}$ luminosity from particles exiting the calorimeter in two rapidity intervals as a function of calorimotor dopth. 4 -strip occupancy refers to any hit in a given 4 contiguous strips. The rates shown are for the highest rate strips in each repidity region.

$$
0.1<|\eta|<1.34
$$

\begin{tabular}{llllll}
\hline Calorimeter thickness $(\mathrm{l})$ & 6 & 8 & 10 & 12 & 14 \\
\hline $\begin{array}{l}\text { Rate per strip }(\mathrm{Hz}) \\
\text { Strip size } 350 \mathrm{~cm} \times 0.5 \mathrm{~cm}\end{array}$ & 33000 & 6600 & 2200 & 800 & 480 \\
$\begin{array}{c}\text { Single strip occupancy } \\
300 \text { nsec integration time }\end{array}$ & $2.6 \%$ & $0.40 \%$ & $0.13 \%$ & $0.05 \%$ & $0.03 \%$ \\
\begin{tabular}{l} 
4-strip occupancy \\
\hline
\end{tabular} & $10.4 \%$ & $1.6 \%$ & $0.52 \%$ & $0.20 \%$ & $0.12 \%$ \\
\hline
\end{tabular}

$1.86<\ln \mid<2.46$

\begin{tabular}{lllll}
\hline Calorimeter thickness $(\mathrm{l})$ & 10 & 12 & 14 & 16 \\
\hline $\begin{array}{l}\text { Rate per strip }(\mathrm{Hz}) \\
\text { Strip size } 93 \mathrm{~cm} \times 0.5 \mathrm{~cm}\end{array}$ & 13100 & 10500 & 6640 & 4320 \\
$\begin{array}{l}\text { Sirrigle strip occupancy } \\
300 \text { nsec integration time }\end{array}$ & $0.78 \%$ & $0.64 \%$ & $0.40 \%$ & $0.26 \%$ \\
\begin{tabular}{l} 
4-strip occupancy \\
\hline
\end{tabular} & $3.1 \%$ & $2.6 \%$ & $1.6 \%$ & $1.0 \%$ \\
\hline
\end{tabular}


Table 4-7. Depth Requirements imposed by criteria 1, 2, and 3.

\begin{tabular}{|c|c|c|c|}
\hline \multirow[t]{2}{*}{ Criteria } & \multicolumn{3}{|c|}{ Minimum Depth required } \\
\hline & $0.1<\ln \mid<1.34$ & $1.38<|\eta|<1.86$ & $1.86<\operatorname{l\eta } \mid<2.46$ \\
\hline 1 & 11 & 11 & 11 \\
\hline 2 & 10 & 10 & 10 \\
\hline 3 & 10 & 12 & 16 \\
\hline Overall & 11 & 12 & 16 \\
\hline
\end{tabular}

In general the charged particles exiting the GEM calorimeter are of low transverse momentum. Figure 4-12 shows the $p_{T}$ of these charged particles (in three rapidity intervals) is strongly peaked at low values. This background is further considered in the evaluation of the trigger performance (Section 4.2.3) and the pattern recognition performance (Section 4.2.4).

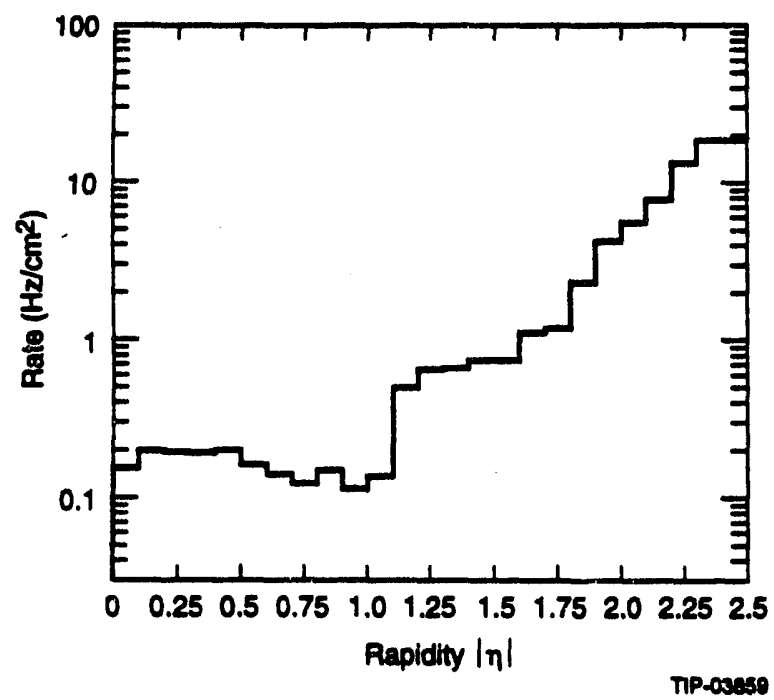

FIG. 4-11. Charged particle rate versus rapidity in the first muon system superlayer.

\section{Cosmic Ray Background}

Cosmic rays will serve as an important calibration and commissioning tool for GEM. The expected cosmic ray flux at the surface above the GEM hall is about $0.02 \mathrm{~cm}^{-2} \mathrm{~s}^{-1}$. The GEM detector is located below the equivalent of $25 \mathrm{~m}$ of soil, and the expected muon energy loss through this material is about $10 \mathrm{GeV}$. Integrating the cosmic ray spectrum ${ }^{19}$ from a lower energy limit of $10 \mathrm{GeV}$ results in an expected flux at the GEM detector of $2 \times 10^{-3}$ $\mathrm{cm}^{-2} \mathrm{~s}^{-1}$. The same cosmic ray spectrum was used by

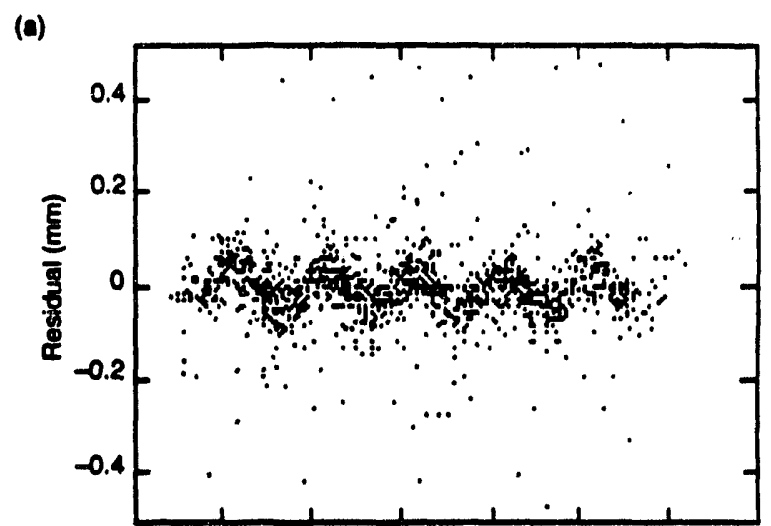

(b)

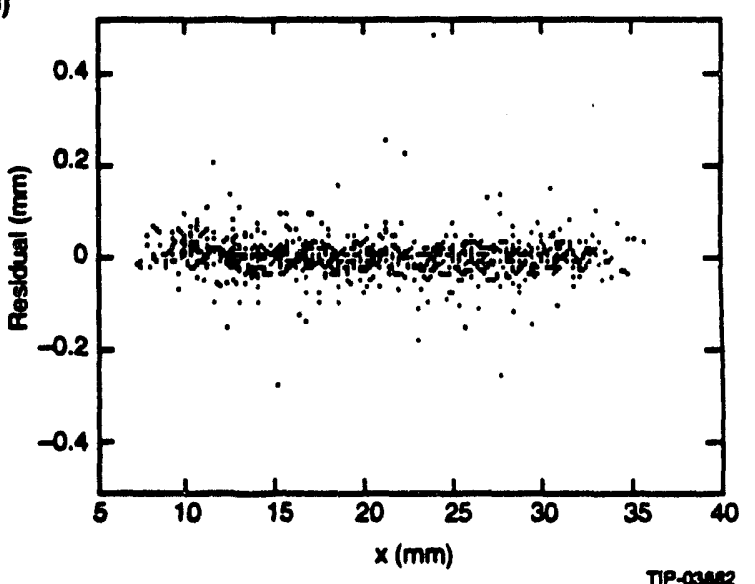

FIG. 4-12. Charge particle rate exiting the calorimeter versus transverse momentum a) $|\eta|<0.5$, b) $1.0<\ln \mid<1.5$, c) $2.0<\ln \mid<2.5$

the $\mathrm{L} 3$ experiment ${ }^{20}$ to estimate the cosmic ray rate at their detector. The contribution to the Level 1 trigger rate from cosmic rays has been calculated, assuming a $10 \mathrm{GeV}$ pr threshold, to be less than $5 \mathrm{~Hz}$. The trigger rate from cosmic rays in the endcap will be negligible. There will be no contribution to the dimuon trigger rate from cosmic ray muons passing near the origin since the time difference between the opposite side hits in the middle superlayer will be more than $40 \mathrm{~ns}$. Each leg of the cosmic 
ray muon will therefore be assigned to a different beam crossing.

\section{Uncorrelated Neutron Background}

As discussed in Chapter 12, considerable effort on shielding design and the detector configuration at large $\eta$ has resulted in an acceptably low flux of neutrons and neutron-associated photons. The low neutron and photon flux, along with the choice of materials to reduce the rate of interactions of these particles in the chambers, will result in a rate of uncorrelated neutron-induced background hits that will not affect the muon trigger rate or the pattern recognition efficiency. The occupancy per readout channel from this type of background is determined by the following factors:

- Neutron/gamma flux through the muon chambers.

- CSC sensitivity to neutrons and gammas.

- Spatial size of one channel.

- Integration (or resolution) time of one channel.

The neutron fluxes and detector sensitivities are functions of the neutron or gamma energies; therefore we integrate the products of these functions over the energy.

The neutron and gamma fluences in the GEM muon system are presented in Chapter 12. The responses of the GEM muon system to neutrons and associated gammas are considered here. Studies of the neutron (and neutron-induced photon) background in the GEM muon chambers are currently. being carried out by a number of institutions using ${ }^{252} \mathrm{Cf}$ sources and neutron beams. ${ }^{21,22,23} \mathrm{~A}$ summary of neutron and gamma detection efficiencies of the GEM muon chambers is presented in Table 4-8.

Elastic scattering of thermal $\left(E_{n}<0.5 e V\right)$ and low energy neutrons $\left(0.5 \mathrm{eV}<E_{n}<100 \mathrm{keV}\right)$ off gas nuclei does not result in ionization. Therefore, the detection efficiency of the CSC to these slow neutrons is due to $(n, \gamma)$ reactions and activation of CSC components (mainly G10 and copper cathodes). The average cross sections of those neutroninduced processes are highest for thermal neutrons. Therefore, for the CSC, the deiection efficiency for thermal neutrons is used as a conservative estimate of background rates due to all neutrons with $E_{n}<$ $100 \mathrm{keV}$. Figure 4-13 shows the efficiency of thermal neutron detection in a proportional chamber filled with $\mathrm{CF}_{4}$ gas as a function of the threshold of the collected charge. It should be noted that neutron capture by a nucleus is usually followed by the emission of several gammas (two-four) of order $1 \mathrm{MeV}$ energy. Each of the gammas can suffer (with low probability) Compton-scattering in the CSC material and gas gaps. These rare events can lead to correlated double or multiple hits in different CSC layers of the same chamber. A dedicated study of CSC component materials from the point of view of neutron or gamma detection efficiency is under way, and we will optimize the design to suppress the multiple hits in the CSC layers. ${ }^{23}$

Neutrons with energy higher than $100 \mathrm{keV}$ can transfer sufficient energy to recoil gas nuclei (C, O, F) to ionize the gas. Hence the elastic neutron scattering dominates the CSC neutron detection
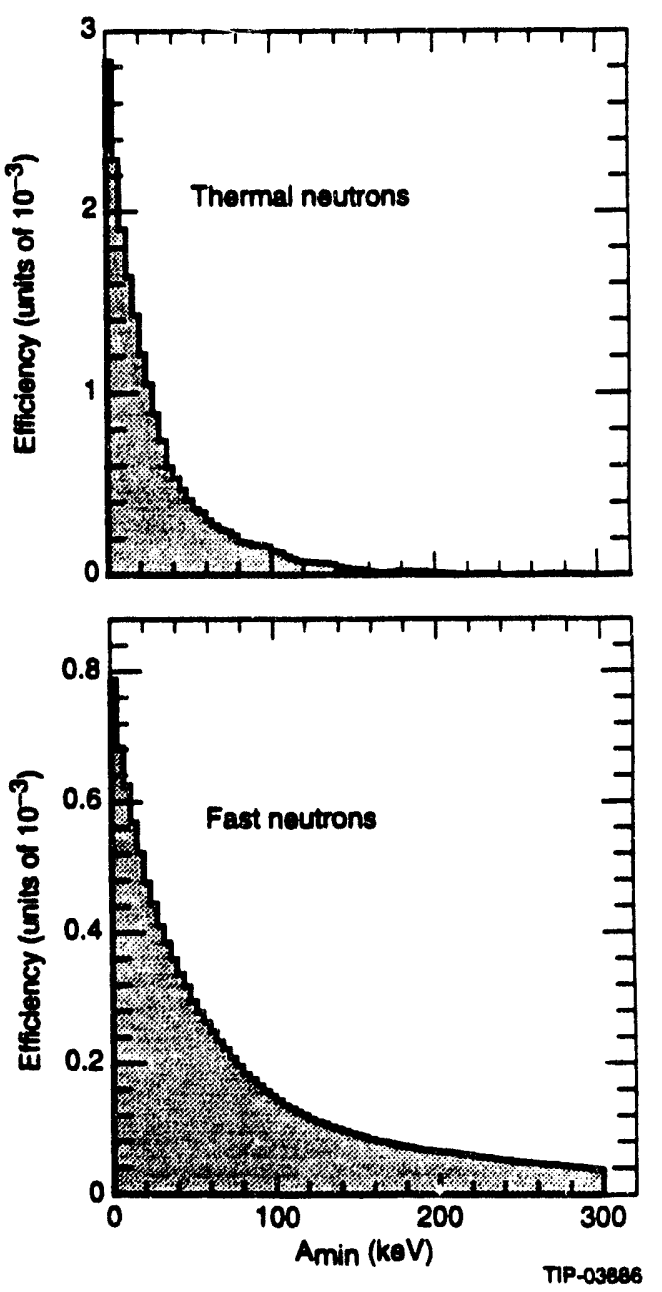

FIG. 4-13. Neutron detection probability in the RDT filled with $\mathrm{CF}_{4}$ as a function of the threshold for the collected charge for a) thermal neutrons and b) fast neutrons. 
efficiency at these energies. The CSC detection efficiency for these neutrons is proportional to the average neutron path length in the gas gap, as reflected in Table 4-8. Figure 4-13b shows the probability of ${ }^{252} \mathrm{Cf}$ fission neutron $\left(<E_{n}\right\rangle=$ $2.3 \mathrm{MeV}$ ) detection in a $2 \mathrm{~cm} \mathrm{CF}_{4}$ gas gap as a function of the threshold of the charge collected in the muon chamber. One can see that neutrons of such energy often give signals of much higher amplitudes than $1 \mathrm{MIP}$ ( $1 \mathrm{MIP}=5 \mathrm{keV}$ in a $1 \mathrm{~cm} \mathrm{CO} / \mathrm{CF}_{4}$ gas mixture). Neutrons of energy higher than $10 \mathrm{MeV}$ are able to knock out protons from CSC cathodes into the gas volume. However, the cross-section for this process is relatively small, and the relative number of these energetic neutrons in the GEM muon system will also be small.

Gammas of energies up to several MeV, due primarily to $(n, y)$ reactions in the GEM components surrounding the muon system, will have a fluence about $30 \%$ of the neutron fluence. However, the CSC detection efficiency for gammas is expected to be about 10 times higher than that for neutrons. ${ }^{22}$
The CSC walls ( $\mathrm{G} 10$ plates covered by $\mathrm{Cu}$ ) are not transparent for photons of energies below 20-30 keV. Near this threshold the photoelectric effect in gas is the main process leading to gamma hits (especially if the gas mixture contains Ar). At energies of several hundred $\mathrm{keV}$, Compton scattering off both CSC gas and electrons in the chamber walls will dominates the gamma detection efficiency. The amplitudes of CSC pulses for gamma hits is then of the same order as that for MIPs. ${ }^{22}$

The neutron and photon fluences (see Chapter 12) were convoluted with the detection efficiencies shown in Table 4-8 to obtain the random hit rates in the muon system. These rates were then used to determine the single strip occupancies in the system by integrating the hit rate over the area of the strip using a \pm 300 ns integration time, as shown in Table 4-9. The strip occupancies are everywhere below the limiting pattern recognition rate of $3 \%$ per strip. The pattern recognition performance corresponding to the occupancies in the table is presented in Section 4.2.4.

Table 4-8. Detection efficiency of CSCs for thermal and fast neutrons and photons.

\begin{tabular}{|c|c|c|c|}
\hline Superia & & $\begin{array}{l}\text { ap thickness } \\
\text { larreVEndcai } \\
\text { (mm) }\end{array}$ & \\
\hline \multicolumn{2}{|c|}{ Inner } & $5 / 5$ & \\
\hline \multicolumn{2}{|c|}{ Middle } & $8 / 7$ & \\
\hline \multicolumn{2}{|c|}{ Outer } & $10 / 10$ & \\
\hline \multicolumn{4}{|c|}{$\begin{array}{l}\text { Table 4-9. Single strip occupancy ( } \pm 300 \text { ns } \\
\text { integration time) in the CSCs due to uncorrelated } \\
\text { neutrons and gammas at } 10^{34} \mathrm{~cm}^{-2} \mathrm{~s}^{-1} \text {. }\end{array}$} \\
\hline \multicolumn{4}{|c|}{ Rapidity Range } \\
\hline Superlayer & $0.10-1.34$ & $1.38-1.86$ & $1.86-2.46$ \\
\hline Inner & $0.6 \%$ & $0.3 \%$ & $0.3 \%$ \\
\hline Middle & $0.6 \%$ & $0.4 \%$ & $0.4 \%$ \\
\hline Outer & $1.1 \%$ & $0.6 \%$ & $0.5 \%$ \\
\hline
\end{tabular}

\section{Muon-Induced Showers}

As the energy of the muon increases above a few hundred $\mathrm{GeV}$, electromagnetic (EM) shower losses (from bremsstrahlung or pair production) increase rapidly and eventually dominate over

Detection efficiency

(in units of 10-3)

$\begin{array}{ccc}\text { Fast } & \text { Gamma } \\ \text { neutrons } & \text { neutrons }\end{array}$

\begin{tabular}{lll}
0.65 & 0.20 & 4.3 \\
0.82 & 0.32 & 5.5 \\
0.98 & 0.40 & 6.5 \\
\hline
\end{tabular}

ionization losses. The amount and character of the muon-induced showers depends on the atomic number and the density of the final layers of the calorimeter preceding the muon system, and the material of the muon system. The GEM muon system design inherently suppresses this background by a combination of the magnetic field and the small amount of material. GEANT simulations of muon-induced showers in the GEM muon system have been performed using the SIGEM simulation program.

In contrast to uncorrelated neutron and cosmic ray backgrounds, muon associated EM background is strongly correlated with muon hits, both in time and space. The EM background is produced by shower leakage from the hadron calorimeter, $\delta$-elec- 
tron production, and muon radiation in the material of the muon system. Shower leakage background is produced by two major mechanisms:

- Photon conversion in the muon chamber.

- Shower electrons (or positrons) exiting the calorimeter with sufficiently high momentum to reach the first superlayer in the vicinity of the muon.

Delta-ray electrons account for the majority of background hits in the second and third superlayers (only about $10 \%$ of the hits arise from the conversion of gammas exiting the calorimeter). Figure 4-14 shows the distance of closest approach to the muon track plotted in units of the SL strip width for the barrel and the endcap from EM background charged particles of momentum higher than $1 \mathrm{MeV}$ in each superlayer. This distribution peaks near the muon because of $\delta$-rays produced in the gas volume and chamber walls of the CSC layer. The distribution also has a tail stretching out to several $\mathrm{cm}$ due to $\delta$-rays and gamma conversions produced in the chamber walls and the calorimeter. Lower kinetic energy cutoffs of $10 \mathrm{keV}$ were used for the pattern recognition studies reported in Section 4.2.4 and 4.2.6.

In the barrel region, the axial magnetic field acts to sweep away much of the electromagnetic debris accompanying the muon as it emerges from the calorimeter. For a $20 \mathrm{~cm}$ clear space before the first superlayer, only $25 \%$ of the charged EM background particles exiting the calorimeter reach the muon system. The EM particles that reach the muon system are swept clear of the muon by the field, allowing a precise measurement of the muon position. On the other hand, in the endcap region, the transverse field is not as strong and does not sweep away the EM debris as efficiently. This is clearly seen by comparing Figure 4-14a (inner barrel superlayer) and Figure 4-14b (inner endcap superlayer). As a result of these simulations and pattern recognition considerations, we have decided to space both the barrel and the endcap superlayers by a minimum of $20 \mathrm{~cm}$ from the material of the calorimeter.

\subsubsection{Trigger Performance}

The design of the muon Level 1 trigger is described in Sections 4.3.5 and 7.2.2. This section describes the performance of the muon trigger in
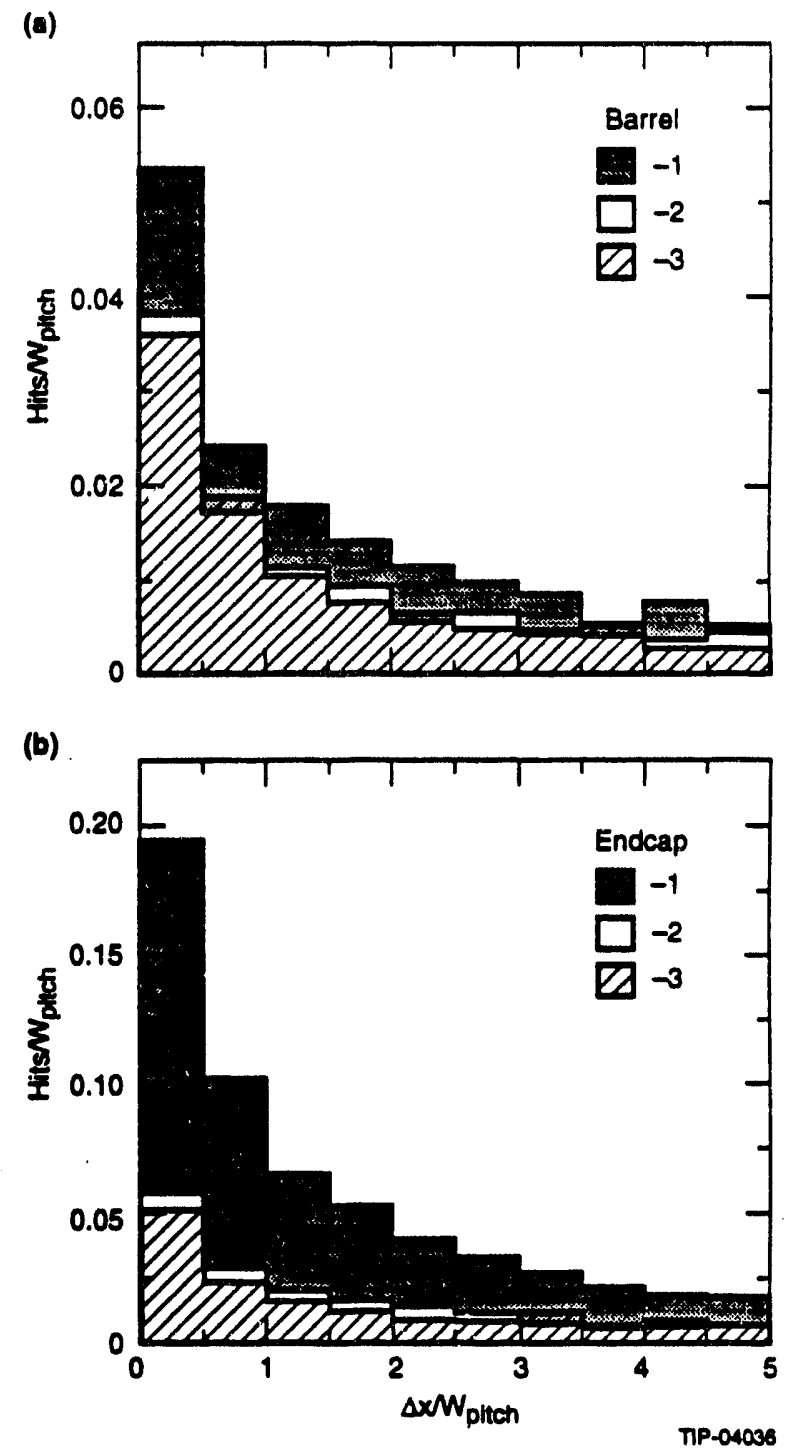

FIG. 4-14. Distance between muon and EM debris in the different superlayers for a $1 \mathrm{TeV}$ pr muon a) for barrel and b) for endcap. The strip width increases with distance from IP.

identifying musns above a certain $p_{T}$ threshold. A GEANT-based hit level Monte Carlo ${ }^{24}$, developed to study the trigger issues, was used to predict the trigger rate for both the barrel and the endcap. For these studies we assumed that the trigger element width is equal to twice the cathode strip pitch in each barrel layer. This translates into $11.42,16.96$, and $21.4 \mathrm{~mm}$ element widths for the three barrel superlayers. The trigger element width in the endcap region was assumed to be a single cathode strip pitch. The simulation was based on electronics that provide single hits per superlayer. The two trigger methods ( $\delta \phi$ and sagitta) under consideration have 
been described previously. The $\delta \phi$ method uses the two outer superlayers and extrapolates an effective $p_{T}$ assuming that the track originated from the interaction point. The sagitta method utilizes all three superlayers and measures the muon $p_{T}$ directly.

We use the sagitta method to evaluate the trigger performance ${ }^{25}$ as a function of the strip number difference. Figure 4-15 shows the trigger threshold num-on for different threshold settings for both the barrel and the endcap. Sharp trigger thresholds can be set for $p T$ values below about $40 \mathrm{GeV}$. Figure 4-16 shows the integrated trigger rate as a function of the $p_{T}$ thresholds in Figure 4-15. This trigger rate was obtained with the hit-level Monte Carlo and includes both muon and punchthrough rates. The curves show the trigger rate from all tracks satisfying the requirements for the sagitta trigger and the rate of real muons above the trigger threshold. The sagitta trigger will operate with single muon $p_{T}$ thresholds of $10 \mathrm{GeV}$ and above at a total trigger rate below $10^{4} \mathrm{~Hz}$ at $10^{33} \mathrm{~cm}^{-2} \mathrm{~s}^{-1}$. The $\delta \phi$ trigger will have comparable rates. ${ }^{26}$ These thresholds are sufficiently low to give high efficiency for the physics processes of interest.

The neutron and gamma fluence in the muon system has been computed to be below $10^{4} \mathrm{~cm}^{-2} \mathrm{~s}^{-1}$ at a luminosity of $10^{33} \mathrm{~cm}^{-2} \mathrm{~s}^{-1}$. The trigger simulation has considered the trigger rate as a function of the neutron rate. The study used the neutron and gamma detection efficiencies given in Table 4-8 and the trigger gate width of $50 \mathrm{~ns}$. The trigger assumes the coincidence of at least 4 out of 6 layers in each superlayer, and hits from the anode signals in the superlayers consistent with a straight track in the non-bend plane. We have determined that the muon trigger works in the presence of neutron rates of an order of magnitude higher than the expected rates.

The identification of the beam crossing responsible for a particular muon trigger is essential for the purposes of data acquisition and event building. We identify the beam crossing locally in each superlayer by using the signals from the six layers of anode wires. The anodes have a maximum drift time of about $30 \mathrm{~ns}$ and the beam crossing can be tagged by using the first arrival.
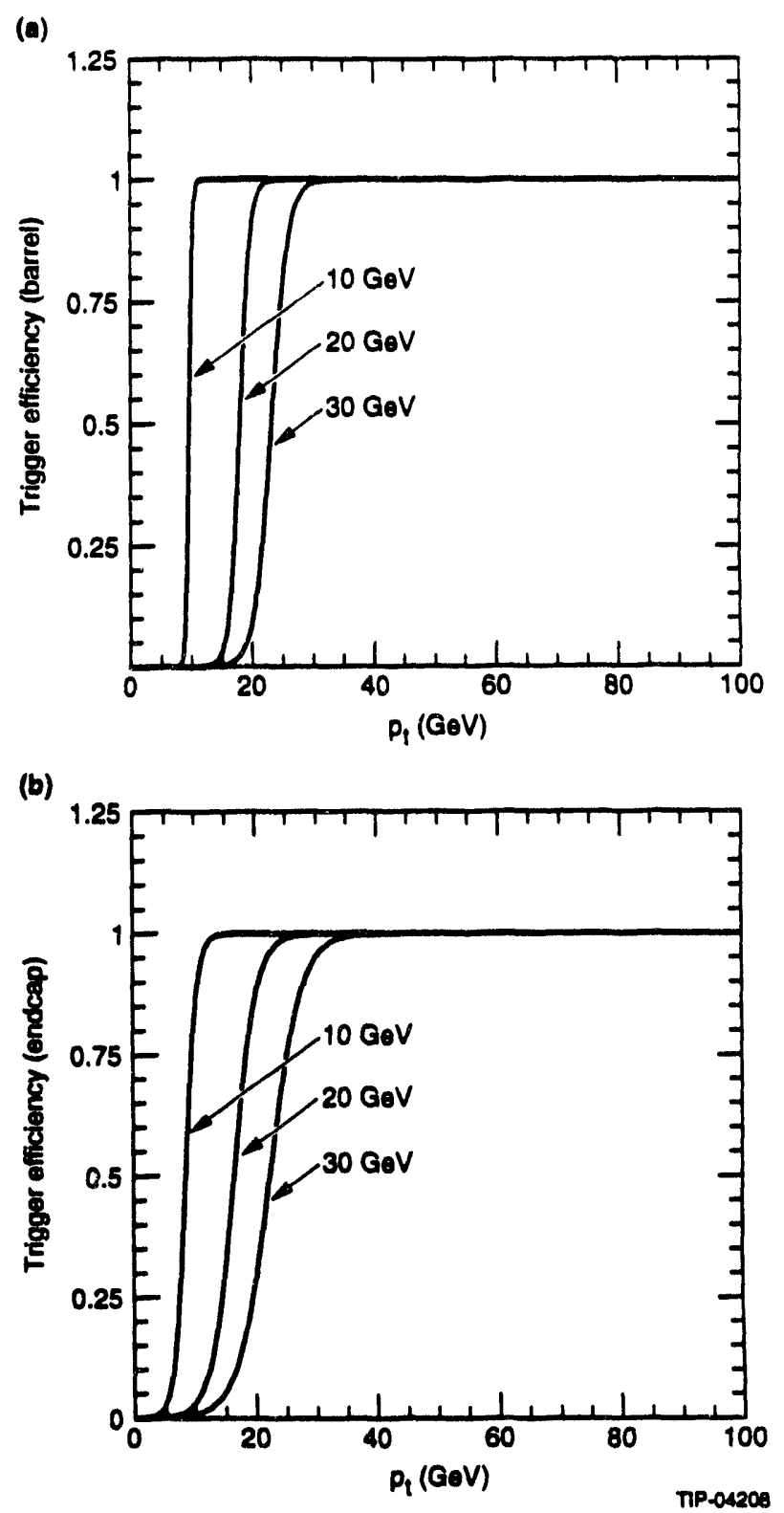

FIG. 4-15. Trigger efficiency versus muon pr for various thresholds in a) the barrel and b) the endcap.

\subsubsection{Pattern Recognition}

Pattern recognition in the GEM muon system must fulfill two tasks:

- Finding the road containing the muon track with high efficiency with a minimum number of wrong track assignments and moderate precision.

- Finding muon hits uncompromised by backgrounds to perform a precise reconstruction of the muon momentum. 
(a)

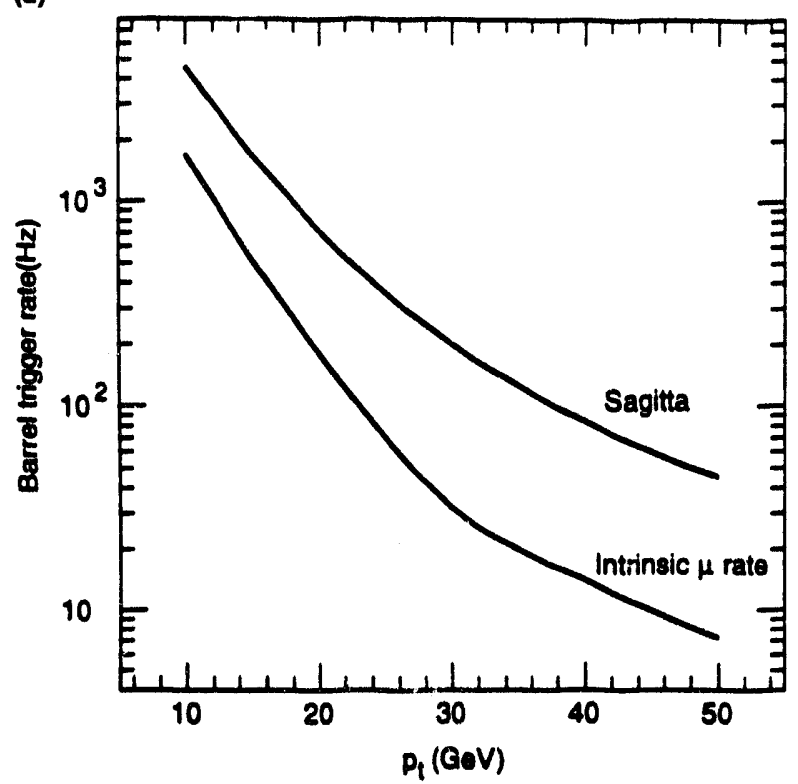

(b)

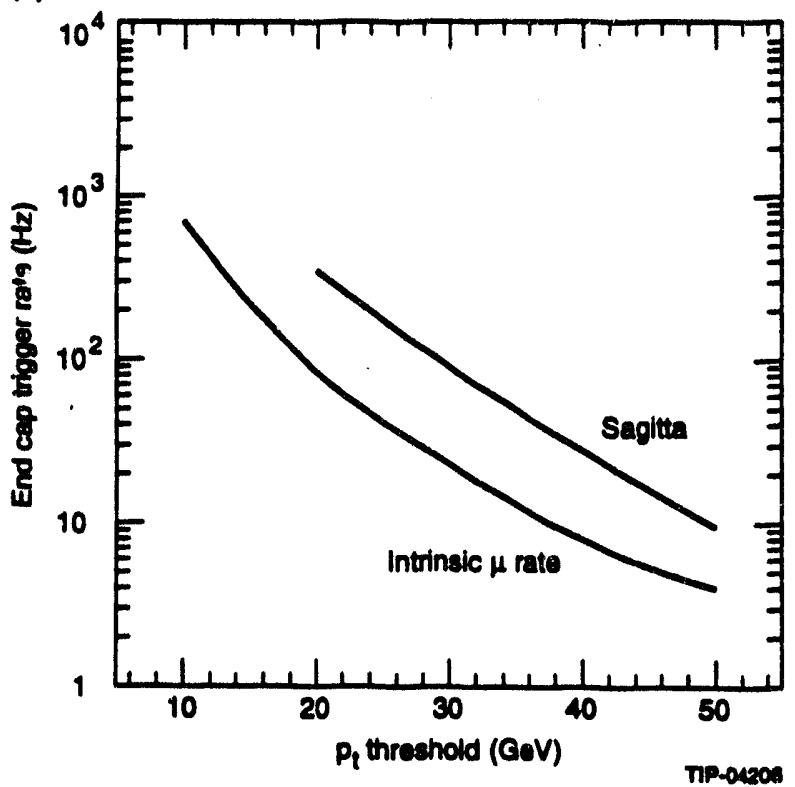

FIG. 4-16. Integrated trigger rate versus pr threshold for a) the barrel and b) the endcap

The efficiency of pattern recognition is more than $90 \%$ in the barrel region for muons of up to $1 \mathrm{TeV} P T$. In the endcap region the efficiency is somewhat less. However, the efficiency for satisfying a minimum 3 out of 6 good layers in the middle and the outer superlayers in the endcap is still $94 \%$ for $1 \mathrm{TeV} p_{T}$ (up to $5.8 \mathrm{TeV}$ energy) muons. Using a vertex constraint, muons of high $p_{T}$ with track segments in at least two superlayers can be fit with reasonable resolution (Section 4.2.5). The pattern recognition performance for selected physics pro- cesses, where the muon system is essential, will be presented in Section 4.2.6. The space-time granularity of the CSC muon system combined with shielding of neutron sources provides for robust recognition of muon road segments in each superlayer as described below.

The general strategy for pattem recognition and track reconstruction in the GEM muon system is as follows:

1. Reconstruction of $\phi$ projection of tracks

a) Selection of good muon hits from the charge distribution on the CSC strips: the definition of a good muon hit in a CSC layer is determined by the two-track resolution of the CSC. The precise position of the muon hit can be measured unambiguously if no extra ionization occurs within $(2 \times w)$ (where $w$ is the strip pitch-see Section 4.3.2) along the anode wire direction. For the barrel muon system, the strips are $5.71 \mathrm{~mm}, 8.48 \mathrm{~mm}$, and $10.7 \mathrm{~mm}$ wide in the inner, middle, and outer superlayers, respectively. In the endcap muon system, the strips are, on the average, $5 \mathrm{~mm}, 7 \mathrm{~mm}$, and $10 \mathrm{~mm}$ wide in the inner, middle, and outer superlayers, respectively. We define a good muon hit as not having any other ionization which occurs in the range $0.2 \mathrm{~mm}<\Delta x<2 \times w$. The conservative approach used to evaluate the GEM muon system pattem recognition assumes that hits from muons that are compromised by extra particles within the loose 2-track separation requirement are unusable. Nevertheless, these hits will be useful for finding the road containing the hits, and will also be useful to some extent in momentum reconstruction.

b) Fitting the muon hits into $\phi$ track segments consistent with a minimum $p_{T}$ from the IP with a loose $\chi^{2}$ constraint to maintain high efficiency: A minimum of 3 good hit layers defines a good track segment in a superlayer. The requirement of a minimum of 3 good hits per superlayer allows position residuals to be used to further suppress the rate of fake track segments from randoms. Figure 4-17 shows the probability for having a minimum of 
3 good hit layers out of a six-layer superlayer as a function of the single strip occupancy. For occupancy less than $3 \%$ per strip, the efficiency for track segments is more than $99 \%$. Also shown in the plot are the average rates of 2-layer and 3-layer tracks from random hits per chamber layer (100 strips wide) per event, as a function of occupancy. The rate of 2-layer fake track segments (loosely pointing to the IP) exceeds that for 3-layer tracks by more than a factor of $10^{3}$ at $3 \%$ occupancy. For this reason the minimum 3 good hit layer track requirement is used to define a good track segment.

c) Fitting $\phi$ track segments from different superlayers: We classify tracks into those having good track segments in all 3 superlayers, those with only 2 good superlayers, and the remaining unmatched single superlayer track segments.

2. Reconstruction of $z / R$ projection of tracks: Track segments in different superlayers are loosely fit to a straight line track through the IP.

3. Assignment of $\phi$ and $z / R$ projections to the same track.

The above procedure gives high efficiency for reconstruction of muon tracks while suppressing the

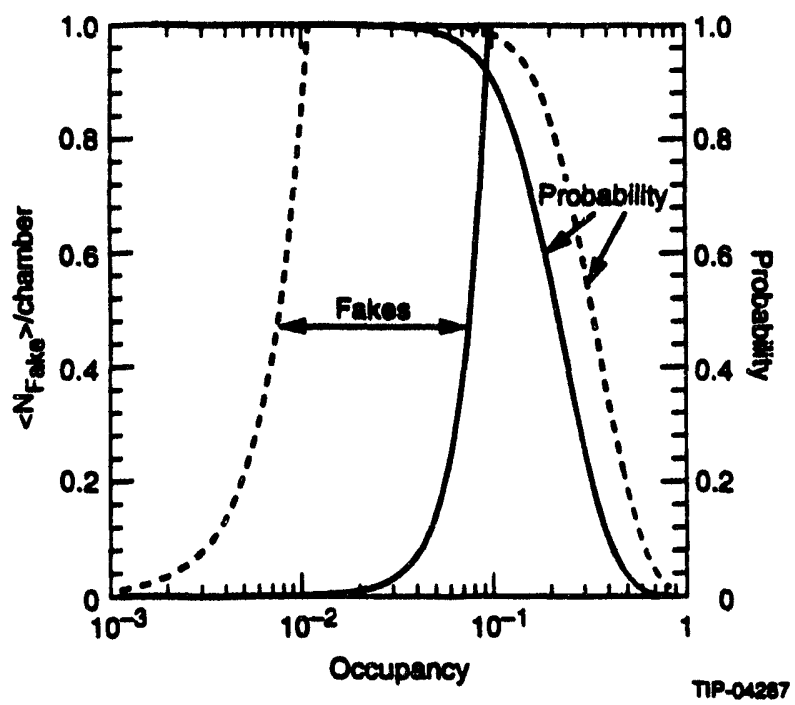

FIG. 4-17. Probability to have a minimum 3 (solid) or 2 (dashed) good layers out of 6 layers in a superlayer as a function of the single strip occupancy per chamber per event. Also shown is the average number of fake track segments. level of fake tracks. We simulate charged particles (prompt muons, decays, punchthrough), the uncorrelated neutron/gamma fluence, as well as the effect of muon-induced showers. We estimate that the reconstruction of high $p_{T}$ fake muon tracks entirely by conspiracy of random hits is less than $10^{-5}$ per $100 \mathrm{fb}^{-1}$. We also estimate the rate of reconstruction of high $p_{T}$ fake muons caused by catastrophic multiple scattering through the muon system or by overlap of a lower $p_{T}$ track and random neutron, gamma, or muon-shower induced hits. The rate depends on the $p_{T}$ of the fake track and the number of superlayers required. For a minimum $1 \mathrm{TeV}$ $(3 \mathrm{TeV}) p_{T}$, the rate of fake tracks with good track segments in all three superlayers is less than $3(0.3)$ per $100 \mathrm{fb}^{-1}$. For the relaxed requirement of good track segments in the middle and outer superlayer only, the rate of fake tracks of minimum $1 \mathrm{TeV}$ $(3 \mathrm{TeV}) p_{T}$ is less than $30(10)$ per $100 \mathrm{fb}^{-1}$. This can be compared to the rate of real high $p T$ muons of minimum $1 \mathrm{TeV}$ of $2 \times 10^{4}$ per $100 \mathrm{fb}^{-1}$ assuming no new muon physics signals. The rate of high $p_{T}$ fake tracks has been compared to the rate of high $p_{T}$ muons in high mass $W^{\prime}$ and $Z^{\prime}$ events (see Chapter 2). It was found to contribute negligible background to these rare physics signals.

In the following studies, the track reconstruction efficiency for a given muon track is defined by the probability to have a minimum of 3 layers with good hits in each of the three superlayers.

\section{Muon-Induced Showers}

In the first superlayer, the muon-induced electromagnetic showers produced in the final layers of the calorimeter provide the major source of background. Simulations were performed with a stay-clear space between the calorimeter and the first superlayer ranging from a few to more than $60 \mathrm{~cm}$ in the barrel region. For a stay-clear space above $20 \mathrm{~cm}$, the probability to have a minimum of 3 good layers in the inner barrel superlayer is constant at $96 \%$ for a $1 \mathrm{TeV} p_{T}$ muon. The probability for the superlayer to have a minimum 3 good layers decreases to $93 \%$ for a minimum $10 \mathrm{~cm}$ stay-clear space. We have thus included a minimum $20 \mathrm{~cm}$ stay-clear zone in the GEM muon system design. Half of the inner barrel superlayer is actually more than $50 \mathrm{~cm}$ away from the calorimeter. In the endcap region the track reconstruction effi- 
ciency also improves as the stay-clear is increased. We also require $20 \mathrm{~cm}$ minimum separation between the inner endcap superlayer and the caloril .eter. The hard muon bremsstrahlung and pair production in the GEM calorimeter and the material of the muon system lead to deterioration of the muon momentum reconstruction for some fraction of the high momentum muons. The efficiency has been evaluated using the full GEANT simulation of the GEM detector provided by the SIGEM program.

The track reconstruction efficiency in various rapidity intervals for different $p_{T}$ values from $20 \mathrm{GeV}$ to $1 \mathrm{TeV}$ is summarized in Table 4-10. The statistical errors in the values given in the table are $1 \%$ or less. The values are for positively charged muons. The efficiency for negatively charged muons is less by $0.5-2 \%$ for $500 \mathrm{GeV}$. It is important to note that Table 4-10 shows the reconstruction efficiency for constant values of $p_{T}$. For $\eta=2.5$, $1 \mathrm{TeV}$ of $p_{T}$ corresponds to $5.8 \mathrm{TeV}$ of energy. The reconstruction efficiency in the endcap region decreases due to both the lower transverse magnetic field acting to sweep out the low momentum EM particles, and the increase in the production of such particles by the more energetic muons.

Energetic muons can be measured with reasonable resolution even if a superlayer is missed. As will be demonstrated in the next section, with a vertex constraint the momentum resolution for high $p T$ muons is degraded by only $20 \%$ with the loss of the innermost superlayer. This is especially important for the endcap region, where the innermost superlayer will have greater background from muon showers emerging from the calorimeter, and from hadron punchthrough and decay. Table $4-10$ also shows the probability for having a minimum 3 good layers in the middle and outer superlayer only. It can be seen that for high $p_{T}$, especially in the endcap region, the probability to have good track segments in the outer 2 superlayers is substantially higher than that for all 3 superlayers.
Table 4-10. Probability for having good track segments for isolated muons in:

a) all three superlayers

\begin{tabular}{llllll}
\hline $\begin{array}{c}\text { Muon } \\
(\mathrm{GeV})\end{array}$ & \multicolumn{7}{c}{ Rapidity Range } \\
\hline & $0.1-$ & $0.50-$ & $0.92-$ & $1.38-$ & $1.86-$ \\
& 0.50 & 0.92 & 1.34 & 1.86 & 2.46 \\
\hline 20 & 0.99 & 0.99 & 0.96 & 0.97 & 0.98 \\
100 & 0.99 & 0.98 & 0.96 & 0.95 & 0.96 \\
500 & 0.97 & 0.98 & 0.94 & 0.89 & 0.89 \\
1000 & 0.95 & 0.93 & 0.90 & 0.84 & 0.82 \\
\hline
\end{tabular}

b) middle and outer superlayers only

\begin{tabular}{lllllll}
$\begin{array}{c}\text { Muon pr } \\
(\mathrm{GeV})\end{array}$ & \multicolumn{7}{c}{ Rapidity Range } \\
\hline & $0.1-$ & $0.50-$ & $0.92-$ & $1.38-$ & $1.86-$ \\
& 0.50 & 0.92 & 1.34 & 1.86 & 2.46 \\
\hline 20 & 0.99 & 0.99 & 0.96 & 0.98 & 0.99 \\
100 & 0.99 & 0.99 & 0.96 & 0.97 & 0.98 \\
500 & 0.99 & 0.99 & 0.96 & 0.95 & 0.97 \\
1000 & 0.97 & 0.95 & 0.93 & 0.94 & 0.96 \\
\hline
\end{tabular}

Uncorrelated Neutron and Gamma Background

Since the GEM muon system must be able to operate at high luminosity it is important to consider the track reconstruction efficiency as a function of the neutron and photon flux, over a wide range. We have studied the efficiency up to $10^{6} \mathrm{~cm}^{-2} \mathrm{~s}^{-1}$, which is more than an order of magnitude greater than the maximum flux expected at $\mathcal{L}=$ $10^{34} \mathrm{~cm}^{-2} \mathrm{~s}^{-1}$, as discussed in Chapter 12. As presented in Section 4.2.2, the occupancy for the CSC detector elements depends not only on the neutron and gamma fluence, but also on the detector area, charge istegration time, and neut $n$ and gamma detection efficiency. It is this occupancy that determines the track reconstruction efficiency as defined by having a minimum of 3 good layers in a superlayer. For our pattern recognition studies, the following conditions were taken: 
- Gamma fluence equal to $30 \%$ of the neutron fluence

- CSC charge integration time of \pm 300 nsec

- Neutron and gamma detection efficiencies per CSC layer according to Table 4-8.

Figure 4-18 shows the probability to have 3 or more good muon hits in each superlayer as a function of the neutron fluence for a $0.5 \mathrm{TeV} p_{T}$ muon in 3 rapidity intervals. Included in the efficiency is the electromagnetic background associated with the muon, and the uncorrelated charged particle background from minimum bias events. These result in an efficiency which is less than $100 \%$ at low neutron fluence. In all three rapidity intervals the efficiency is constant up to about $10^{5} \mathrm{~cm}^{-2} \mathrm{~s}^{-1}$, and then falls off as the occupancy of the strips becomes substantial. At $10^{5} \mathrm{~cm}^{-2} \mathrm{~s}^{-1}$ the single CSC strip occupancy of the outer barrel muon chamber is near $15 \%$. However, as shown in Chapter 12, the expected fluence is much lower in this region at high luminosity.

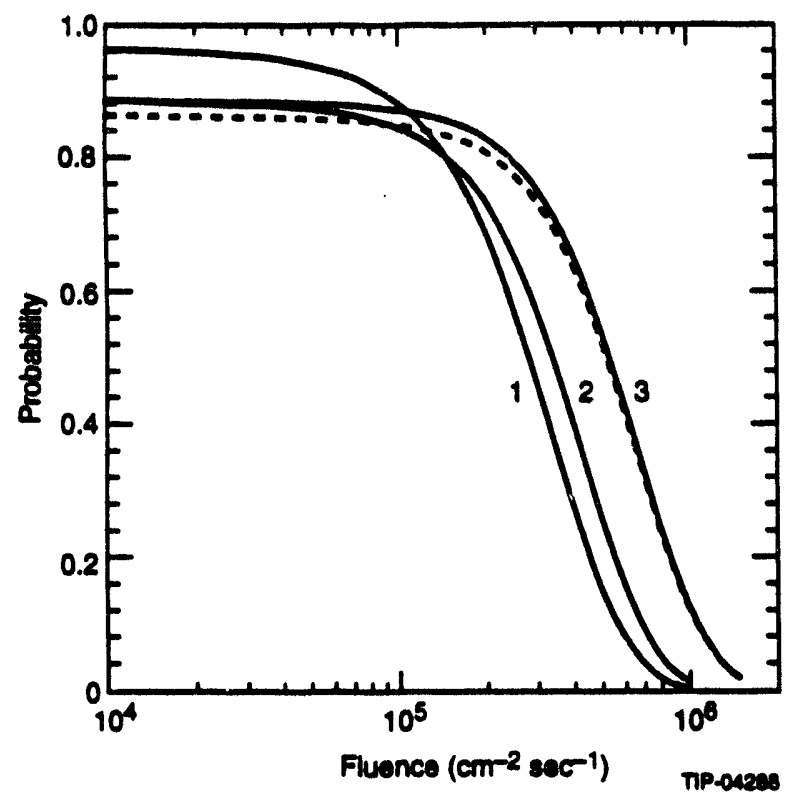

FIG. 4-18. Probability to have at least 3 good layers in each of the three superlayers versus noutron fluence, for a $500 \mathrm{GeV}$ pr muon (including EM background), for three different rapidity intervals: 1) $0.1-1.34$; 2) 1.4-1.85; 3) 1.85-2.45. Dashed curve shows effects of punchthrough at $\ell=10^{34} \mathrm{~cm}^{-2} \mathrm{~g}^{-1}$.
Correlated Hadron Punchthrough Background

An important signature for the discovery of top $^{15}$ is the decay mode $t \rightarrow W+b$, where the $W$ decays to an isolated lepton and the $b$-quark decays to a muon and a jet, plus neutrinos. The muon from the $b$-quark decay is not isolated from the jet. One of the special features of the GEM muon system is its ability to identify and measure muons inside jets without assistance from the inner detectors. This capability was investigated ${ }^{27}$ by considering the pattem recognition performance of the system on muons from the $b$-quark decay. The worst case was considered by requiring the $t$-quark $\left(m_{t}=250 \mathrm{GeV}\right)$ to have a minimum of $200 \mathrm{GeV}$ in transverse momentum. This leads to an energetic (narrow) jet from the $b$ decay with the average angle between the muon and the jet direction of only $1.1^{\circ}$. The muons from the $b$-quark decay have an average energy of $70 \mathrm{GeV}$ and an average $p_{T}$ of $39 \mathrm{GeV}$. Four thousand events of this process generated by PYTHIA were simulated in the detector by SIGEM. Table 4-11 shows the probability for having good track segments (minimum 3 good hit layers) in all three superlayers ar.d for the middle and outer superlayers only. The same rapidity intervals were used as in Table 4-10 for isolated muons. Shown for comparison are the $20 \mathrm{GeV}$ and $100 \mathrm{GeV}$ PT isolated muon probabilities. Despite having an average of 64 particles in the jet from the $b$-quark decay, there is no significant degradation of the track reconstruction efficiency. This is further evidence that the calorimeter depth is sufficient to suppress the hadron punchthrough to a level below that which would adversely affect the performance.

\subsubsection{Momentum Resolution}

We have performed several different calculations of the muon momentum resolution. They are all in agreement with each other. Our results are used in Chapter 2 for representative physics processes. 
Table 4-11. Probability of having good track segments in a) all three superlayers and b) the middle and outer superlayers only, for muons in b-jets compared with isolated muons.

\begin{tabular}{|c|c|c|c|c|c|}
\hline \multirow[b]{2}{*}{ a) All Three Superlayers } & \multicolumn{5}{|c|}{ Rapidity Range } \\
\hline & $0.1-0.5$ & $0.5-0.92$ & $0.92-1.34$ & $1.38-1.86$ & $1.86-2.46$ \\
\hline$b \rightarrow \mu+X:\left\langle p^{\mu} T\right\rangle=39 \mathrm{GeV}$ & 0.99 & 0.99 & 0.98 & 0.97 & 0.96 \\
\hline lsolated $\mu: p_{T}^{\mu}=20 \mathrm{GOV}$ & 0.99 & 0.99 & 0.96 & 0.97 & 0.98 \\
\hline Isolated $\mu: p_{T}^{\mu}=100 \mathrm{GeV}$ & 0.99 & 0.98 & 0.96 & 0.95 & 0.96 \\
\hline $\begin{array}{l}\text { b) Middle and Outer Superlayers } \\
\text { Only }\end{array}$ & $0.1-0.5$ & $0.5-0.92$ & $0.92-1.34$ & $1.38-1.86$ & $1.86-2.46$ \\
\hline$b \rightarrow \mu+X:\left\langle p^{\mu} T\right\rangle=39 \mathrm{GoV}$ & 0.99 & 0.99 & 0.98 & 0.98 & 0.97 \\
\hline Isolated $\mu: p^{\mu} \tau=20 \mathrm{GeV}$ & 0.99 & 0.99 & 0.96 & 0.98 & 0.99 \\
\hline Isolated $\mu: p^{\mu} T=100 \mathrm{GeV}$ & 0.99 & 0.99 & 0.96 & 0.97 & 0.98 \\
\hline
\end{tabular}

One group of simulations ${ }^{28}$ was based on a parametric description of the system and quasi-analytic calculations of the impact of design parameter changes. In this approach a model of the distribution of material in the detector and the shape of the magnetic field is coupled to an analytic track model, and the covariance matrix for the track parameters is calculated. The detector planes are modeled as superlayers with point resolution improving as $1 / \sqrt{N}$ for $N$ layers within a superlayer. Misalignment effects due to relative layer positioning within a superlayer, and relative misalignment between superlayers are added in quadrature. The covariance matrix directly gives the various (correlated) resolutions. It also was used to implement a fast parametric track simulation, by drawing samples of track parameters from the multivariate Gaussian distribution of these quantities. The results obtained in this way are equivalent to those expected from a $\chi^{2}$ fit.

In addition, we have studied the momentum resolution ${ }^{29}$ using a GEANT model of the detector. It simulates individual chambers within superlayers, structural frames, and the support structures. The tracking package of GEANT was used to propagate muons through the magnetic field. It accounts fully for multiple scattering and showering in materials along the trajectory, and determines the intersections of the muon trajectory with the planes of the CSCs. The simulated track positions were used to obtain the momentum resolution, including the effects of the number of measurements along the trajectory, the intrinsic chamber resolution, and alignment uncertainties. The momentum resolution in the exact detector geometry was mapped out and parameterized (along with detector acceptance) as a function of $\eta, \phi$, and $p_{T}$. The parameterization was used for physics studies (see Chapter 2) as part of the gemfast program.

We have also developed hit level Monte Carlo and track fitting programs. Momentum resolution, pattern recognition, reconstruction efficiency, and benchmark physics performance are presently under study using a reconstruction program. ${ }^{30}$ It uses hits generated by the SIGEM simulation; performs pattern recognition, track finding, and ambiguity resolution; fits tracks to momenta and extrapolates them back to the vertex.

In the following, we describe the resolution obtained by the parametric approach. We also discuss performance at high and low momenta and the robustness of the system.

In Figure 4-19 for the baseline (standalone, three superlayer) GEM muon system, we display the transverse momentum resolution as a function of $\eta$ at low and high pr. The resolution for $p_{T} \leq 50 \mathrm{GeV}$ is limited by multiple scattering in the second superlayer and by Landau fluctuations of the unmeasured energy loss in the calorimeter. We add to the momentum measured in the muon system the average momentum loss in the calorimeter unless the calorimeter measurement is greater than 1.5 times the most probable energy loss. In this case the measured energy in the calorimeter is used. We have tuned the factor, 1.5, to minimize the tail and optimize the width of the reconstructed momentum 
(a)

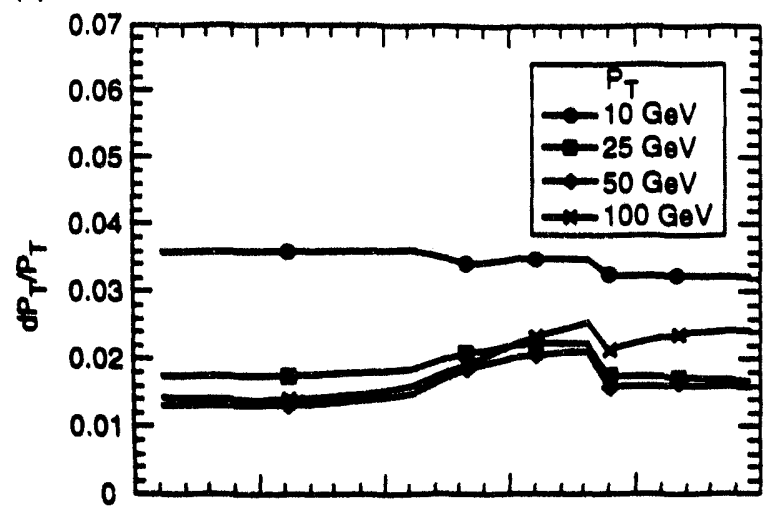

(b)

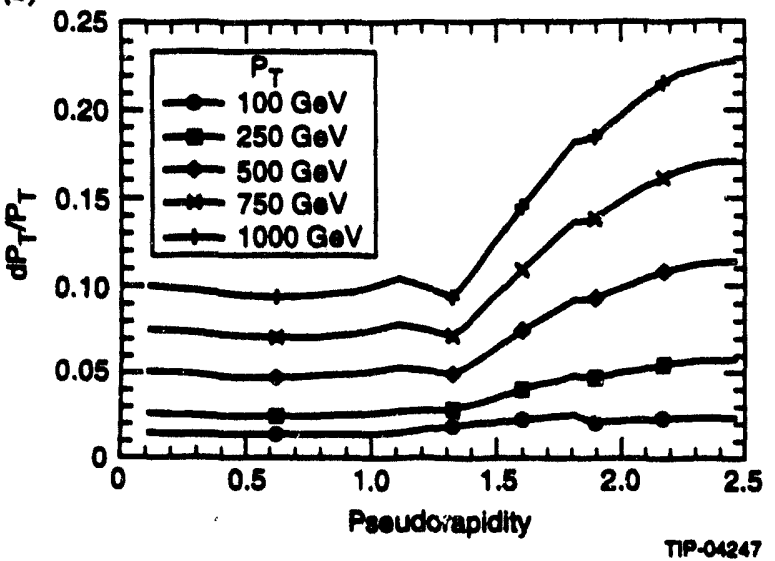

FIG. 419. Transverse momentum resolution for standalone three superlayer system:

a) $10 \leq \mathrm{pr} 100 \mathrm{GoV}$ and

b) $100 \leq$ pr $\leq 1000 \mathrm{GoV}$.

distribution. Inclusion of the central tracker measurement in the determination of the momentum somewhat mitigates the effect of the Landau fluctuations at the lowest energies. In Figure 4-20 we show the low pr resolution, treating the tracker and the muon system momentum determinations as independent measurements. At high $p_{T}$ the resolution is indistinguishable from that obtained with the standalone system.

We expect ${ }^{31}$ to determine the transverse position of the vertex to $\leq 200 \mu \mathrm{m}$ using muon tracks. The availability of the transverse position of the vertex, in addition to the coordinates measured in the CSC superlayers, improves the high energy performance. In Figure 4-21, we show the low and high momentum behavior of the momentum resolution with the inclusion of a vertex constraint. The vertex constraint was added by including a fourth measurement of the transverse coordinate at the origin with an effective measurement error of $200 \mu \mathrm{m}$. The momentum resolution significantly improves over that produced with the baseline system as the $p T$ increases beyond $=500 \mathrm{GeV}$, and leads to an improvement factor of 1.5-2 in the very high momentum region in the barrel. It is noteworthy that

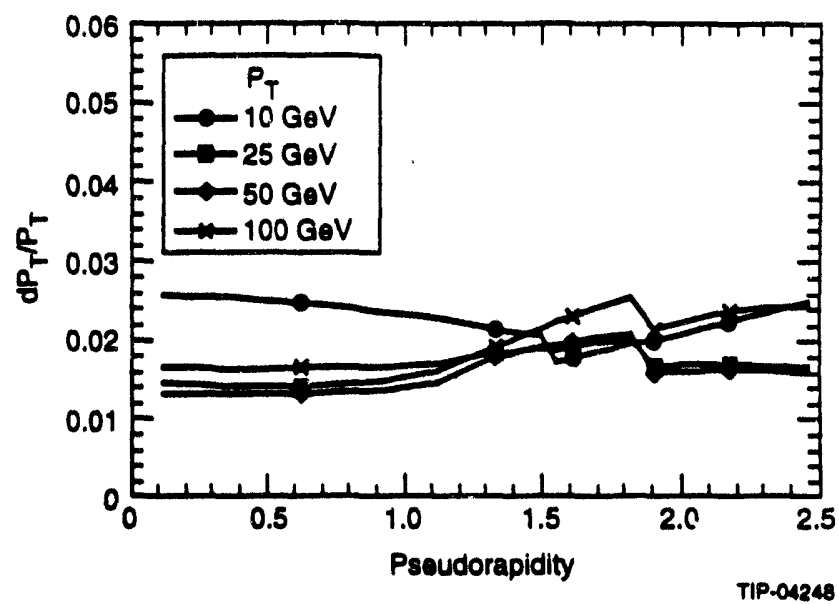

FIG. 4-20. Transverse momentum resolution for the three superlayers with the inclusion of the central tracker as an independent measurement;

$10 \leq$ p. $\leq 100 \mathrm{GeV}$.

(a)

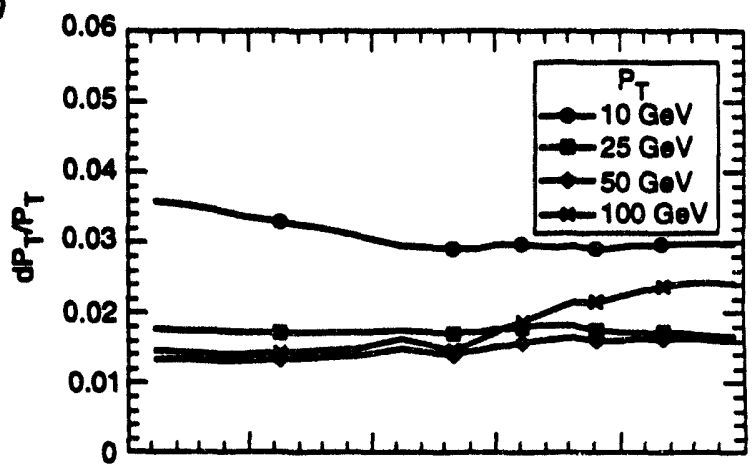

(b)

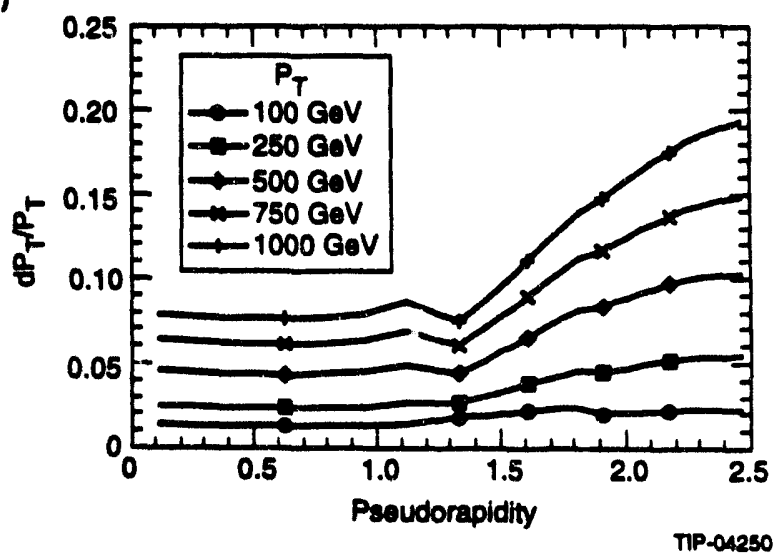

FIG. 4-21. Transverse momentum resolution for three supertayers with the inclusion of a $200 \mu \mathrm{m}$ vertex constraint; a) $10 \leq$ pr $\leq 100 \mathrm{GeV}$.

b) $100 \leq$ pr $\leq 1000 \mathrm{GeV}$. 
as the pseudorapidity increases, the vertex constraint also improves the $p_{T}$ resolution at the lowest transverse momenta, due to the improved angular precision associated with the $F T$ determination at the event vertex.

The vertex constraint may be used to recover those tracks where one of the superlayers does not provide a good track segment (see Table 4-11), increasing system robustness especially at high momenta. In Figure 4-22 we show the pr resolution for the GEM muon system, with the inclusion of the vertex constraint at a progression of values, assuming the loss of the first superlayer. The resolution is generally limited by the multiple scantering in the calorimeter to a fairly constant 8-10\% over a large range of $p r$ and angle. We note, however, that the resolution of the system with the vertex constraint of $200 \mu \mathrm{m}$ and without the first superlayer is comparable with the stand-alone system performance, for transverse momenta $\geq 700-1000 \mathrm{GeV}$.

The charge determination capability of the GEM muon system is excellent. In Figure 4-23 we give the maximum momentum, $p_{\max }$. for which the probability of determining the charge correctly is $\geq 95 \%$, as a function of $\eta$ under the assumption of Gaussian errors in $1 / p$. The baseline system is capable of charge determination for momenta up to $6 \mathrm{TeV}$ at $\eta-0$ and increasing to $15 \mathrm{TeV}$ at $n-2.5$. With a $200 \mu \mathrm{m}$ vertex constraint we ar he kinematic limit over much of the angul.

\subsubsection{Performance for Representative Physics Processes}

This section summarizes the pattern recognition performance of the muon system for physics processes (see Chapter 2). The SIGEM pis zram was used for this study.

$H^{0} \rightarrow \mu^{+} \mu^{-} \mu^{+} \mu^{-}$

One thousand events of each mass were generated with no geometrical cuts. Of these 54,64 , $78 \%$ for the $150,400,800 \mathrm{GeV}$ mass, respectively, have all 4 muons in the rapidity range $|\eta|<2.46$. These events have been analyzed where the muon track segments were found in the different superlayers, using the requirement that a minimum of 3 good muon hit layers define a good track segment. Figure 4-24 shows the probability to have good track segments in all three superlayers as a function of (a)

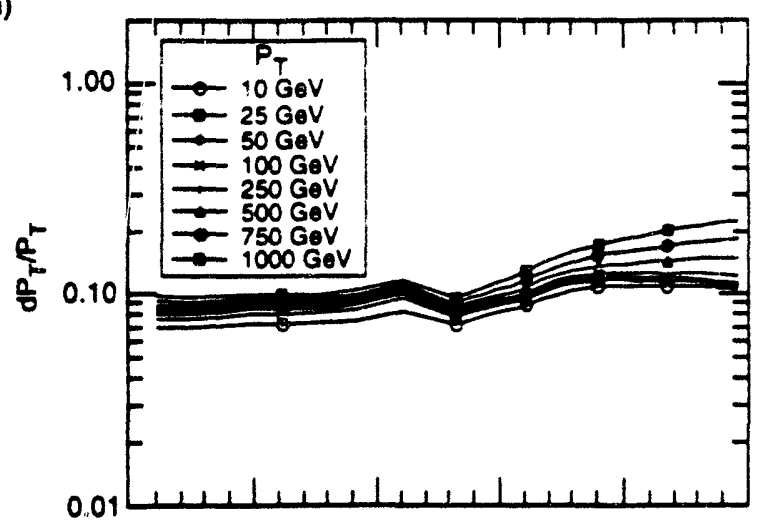

(b)

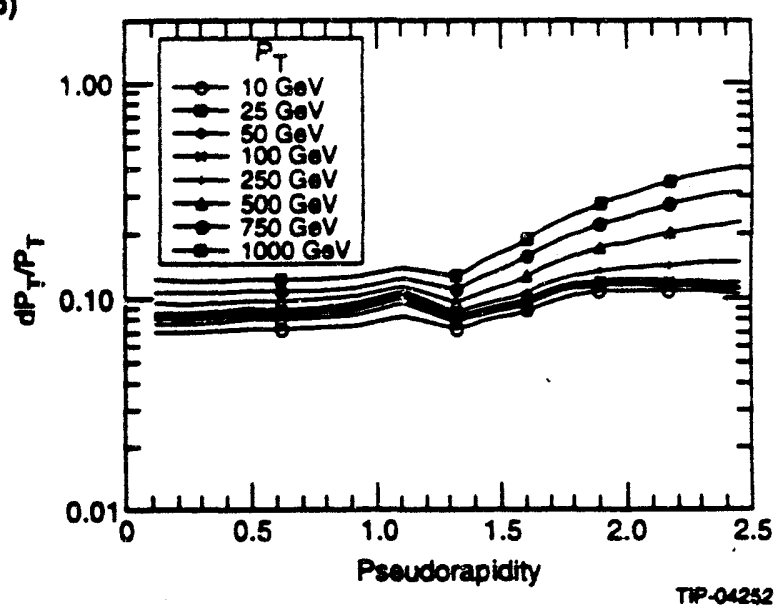

FIG. 422. Transverse momentum resolution for $10 \leq p_{T} \leq 1000 \mathrm{GeV}$, with the inclusion of a vertex constraint but without the first superlayer for a) $200 \mu \mathrm{m}$ and b) $500 \mu \mathrm{m}$ vertex resolution.

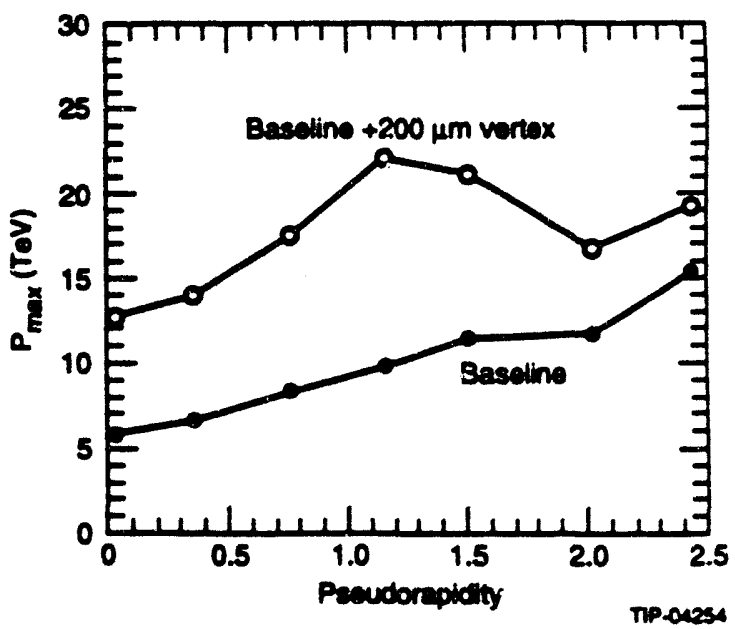

FIG. 423. The maximum momentum, $P_{\text {max. }}$ for which the probability of determining the charge correctly is $295 \%$, as a function of $\eta$ under the assumption of Gaussian errors in 1/p for the baseline systom and with the inclusion of a $200 \mu \mathrm{m}$ vertex constraint. 
rapidity, for muons from a $150 \mathrm{GeV}$ Higgs. The figure shows the effect of the acceptance cracks in the muon system when it is operated in the standalone mode. The acceptance for a sing!e muon with a flat rapidity distribution in the region $|\eta|<2.46$ is $84 \%$. Table $4-12$ shows the probability that all 4 muons have good tracks segments in all superlayers.

For cases where a muon is from a decay of a real $Z$, the constraint on the $Z$ mass can be used. In this case, the second muon need only have its angle measured, and this can be done well with track segments in 2 superlayers. At low momenta 2 superlayers give reasonable resolution utilizing a turning angle measurement, and at high momenta, 2 superlayers with a vertex constraint can give similar resolution to a 3 superlayer measurement (see previous section). Table 4-12 shows the increased probability for the relaxed requirement that 4 muons are well-measured in at least 2 superlayers. The loss of efficiency for Higgs is dominated by geometric acceptance losses. In Higgs events where all 4 muons are detected in the muon system, more than 98\% have at least 3 muons well measured in all 3 superlayers.

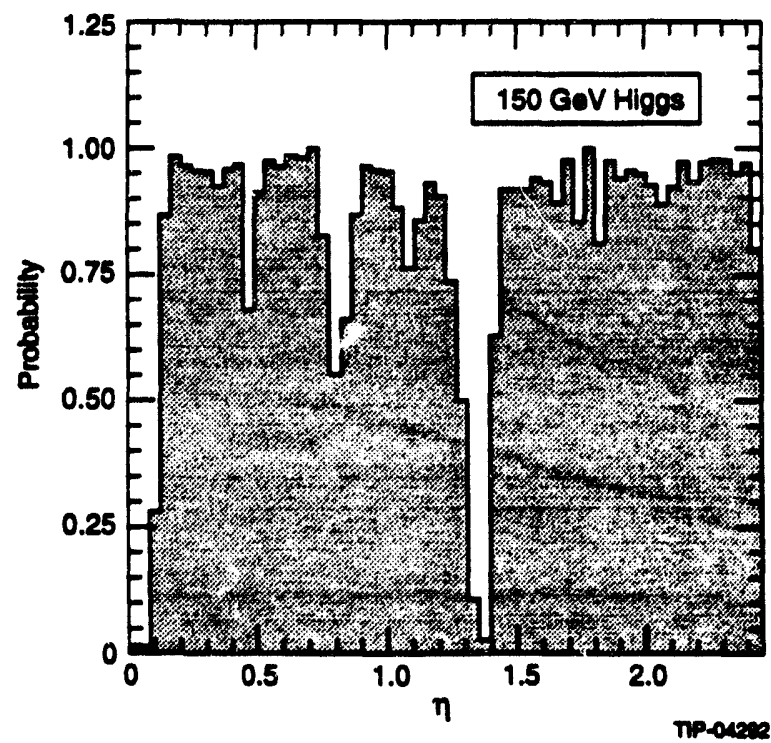

FIG. 4-24. Probability to have good track segments in all $3 \mathrm{SL}$ for the muons from the decay of $150 \mathrm{GeV}$ Higgs. Because the track reconstruction efficiency is very high, this figure clearty shows the effect of the muon system acceptance.
Table 4-12. For Higgs events where all 4 muons have $|\eta|<2.46$, the probability to have 1) all 4 muons with good track segments in all 3 superlayers, or 2) 4 muons with good track segments in at least 2 superlayers.

\begin{tabular}{ccc}
$\begin{array}{c}M_{H} \\
(\mathrm{GeV})\end{array}$ & $\begin{array}{c}4 \text { muons with } \\
\text { all } 3 \\
\text { superlayers }\end{array}$ & $\begin{array}{c}4 \text { muons with } \\
\text { at least } \\
2 \text { superlayers }\end{array}$ \\
\hline 150 & $42.6 \%$ & $54.0 \%$ \\
400 & $45.0 \%$ & $57.7 \%$ \\
800 & $47.4 \%$ & $59.1 \%$ \\
\hline
\end{tabular}

$Z^{\prime} \rightarrow \mu^{+} \mu^{-}$

One thousand $Z^{\prime}$ events of mass $M_{Z}^{\prime}=4 \mathrm{TeV}$ decaying into $\mu^{+} \mu^{-}$were generated by PYTHIA and simulated using SIGEM. 87\% of the events had both muons within the coverage defined by the GEM muon system $(|\eta|<2.46)$. Figure 4-25 shows the efficiency distribution versus rapidity for the generated muons when both muons have rapidity less than 2.46 , with the requirement of a good track segment in all 3 superlayers.

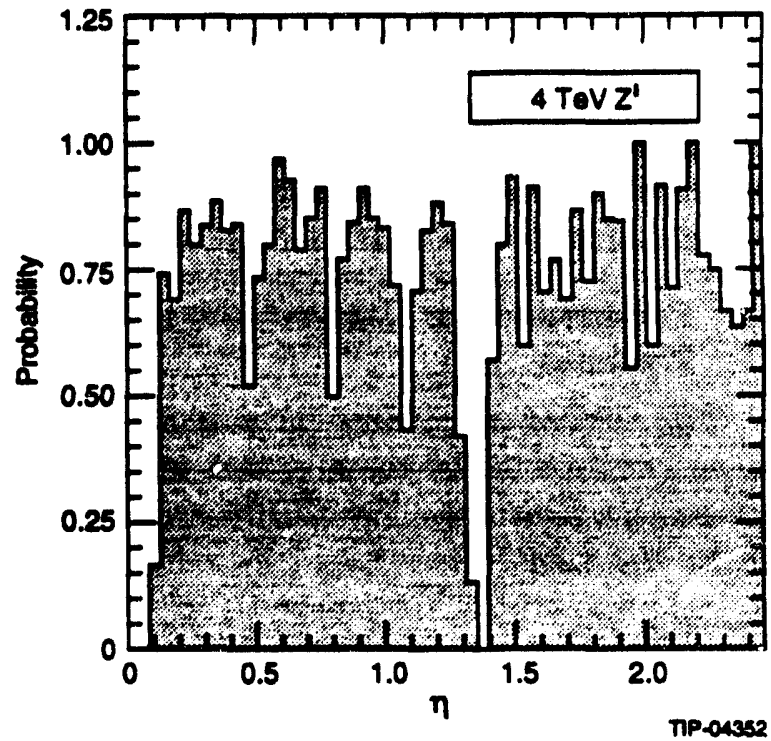

FIG. 425. Probability to have good track segments in all three superlayers for muons from the decay of a $4 \mathrm{ToV} Z^{\prime}$ as a function of inuon rapidity. This figure shows the combined effects of track reconstruction inefficiency and muon system acceptance.

Considering only $Z$ events where both muons have $|\eta|<2.46,59.6 \%$ have both muons with good track segments in all 3 superlayers, and $71.5 \%$ have 
1 muon well-reconstructed in all 3 superlayers plus 1 muon with at least 2 superlayers and $72.6 \%$ have both muons with at least 2 superlayers. This is consistent with what was found using the gemfast simulation and reported in Section 2.6.1. Because the transverse momentum of the muons from a 4 $\mathrm{TeV} Z^{\prime}$ is typically $1 \mathrm{TeV}$ or more, the loss of the first superlayer will only result in a degradation of the momentum resolution of about $20 \%$ if a vertex constraint of $500 \mu \mathrm{m}$ or better is used. About $2 / 3$ of the inefficiencies came from holes within the coverage $|\eta|<2.46$ of the muon system.

\subsubsection{Performance Verification and Monitoring}

The application of experimental data for calibration and verification of the detector performance is a necessary part of maintaining the precision of the system. In some cases, this procedure may be the only available tool for defining essential detector parameters (for example, alignment of the muon superlayers with respect to the interaction point). In other cases, where it does not play the primary role, it is nonetheless vital, in that it will provide a cross-check on the other calibration systems are functioning properly.

We envision verifying and calibrating the following quantities, which essentially determine the muon system performance:

- Intrinsic chamber resolution, including the single plane resolution and any mechanical misalignment within each chamber.

- Alignment of chambers within one projective tower (local) and alignment of the towers with respect to each other, the IP, and the other GEM subdetectors (global).

- The magnetic field using muon tracks to measure known particle masses.

Different sources of particles are to be utilized for these purposes, including prompt muons (both single muons and muonic $Z$ decays), cosmic rays, and beam halo; rates for these processes are specified in Reference 31. Since most of these calculations may be performed on-line, a dedicated software tap into the Level 2 or Level 3 trigger will enable the accum ulation of the required quantities from incident tracks without saturating the data acquisition stream.
The major conclusion of our analysis is that the statistics accumulated in a period of several hours to one month at the standard luminosity-depending on the quantity to be estimated-will always be sufficient to reach the goals specified below. The resolution that is ultimately achieved will be limited by systematics, as discussed below.

\section{Intrinsic Chamber Resolution}

The actual chamber spatial resolu :ion, which is a combination of stochastic and sysiematic terms, will be determined on the basis of data analysis. Contributions of many systematic errors (misalignment of planes; non-linearity, if any; precision of electronics chain calibration; etc.) are correctable.

The actual data obtained with CSC and LSDT/ RDT prototypes at the $T_{T R}{ }^{2}$ have shown that some of these contributions can be brought to a negligible level within a single chamber. Statistics of a few thousand tracks per chamber were sufficient for the reconstruction of plane misalignment and non-linearities to the level of a few microns (see Figure 4-26 and Figure 4-65).

\section{Alignment of Muon Towers}

As was pointed out in Section 4.2.5 and Reference 32, there are two reasons to have the muon system aligned with respect to the IP. First, it gives the capability to perform a precise measurement without the inner superlayer in the barrel. This requires an IP registration of $-500 \mu \mathrm{m}$. Second, a significant improvement of the transverse momentum resolution is attained for $p_{T}>500 \mathrm{GeV}$. This requires about $200 \mu \mathrm{m}$ projective alignment to the IP in the $R \phi$ plane.

In order to obtain the benefit from a vertex alignment of $500 \mu \mathrm{m}$, the superlayer to superlayer postions must be known to $150 \mu \mathrm{m}$. For $200 \mu \mathrm{m}$ vertex alignment the superlayer to superlayer positions must be known to better than $50 \mu \mathrm{m}$.

One of several possible ways to achieve this precision is described below. ${ }^{31}$ The inner superlayer alone provides a precise measurement of the track position $(40 \mu \mathrm{m})$ and inclination ( $0.5 \mathrm{mrad})$. Using these parameters, the track can be extrapolated towards the middle and outer superlayers (this prediction accuracy is on the order of $1 \mathrm{~mm}$ ). Monte Carlo calculation shows that several hours worth of 

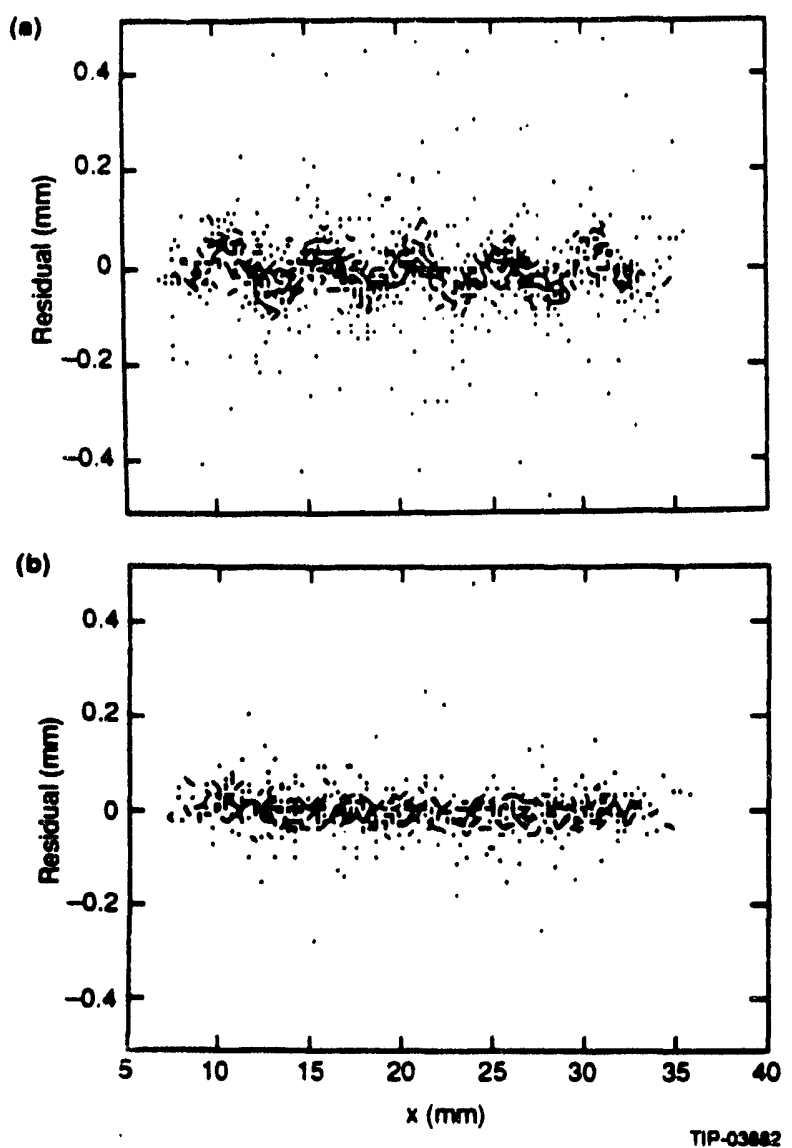

Figure 4-26. Residuals in Houston CSC before (a) and after (b) correcting for systematic effects determined from the data.

statistics at standard luminosity will be sufficient to reach the $50 \mu \mathrm{m}$ goal. The problems of systematic error due to uncertainties in the knowledge of $B L^{2}$ are relaxed considerably by the presence of particles of both signs. The effect of possible systematic errors in the inclination measurements, caused by interlayer misalignment can be removed first, as discussed above.

In another method, ${ }^{31}$ the central tracker (CT) can be used to determine muon track parameters in the muon towers. This will align each muon superlayer to the $C T$, thereby aligning them with respect to the IP and the other superlayers (systematic uncertainties arising from calorimeter inhomogeneity are difficult to predict). This technique, however, requires increased statistics (using tracks of momentum in excess of $50 \mathrm{GeV}$ ). It can be effectively applied to the endcap region at $\mathcal{L}-10^{33} \mathrm{~cm}^{-2} \mathrm{~s}^{-1}$ and to the barrel at $\mathcal{L}=$ $10^{34} \mathrm{~cm}^{-2} \mathrm{~s}^{-1}$. It employs the integrated sagitta distributions for particles of different signs. Anyy superlayer misalignment results in a relative shift of the positive and negative spectra with a value twice the relative superlayer shift. A determination of this shift in each tower will provide the necessary information. ${ }^{33}$

The combination of these essentially different methods (plus using data samples with different $p_{T}$ and fiducial cuts) provides a way of understanding, estimating, and potentially eliminating the systematic errors beyond the level required for attaining global alignment.

The long-term mechanical stability of the GEM muon system and relative subdetector positions is expected to be much better than $200 \mu \mathrm{m}$. For this reason, it will not be necessary to use muon tracks to perform the inter-subsystem and global alignment at frequent intervals. The rapid alignment of the CT with respect to the IP, together with information from beam monitoring systems, will provide the necessary dynamic beam reference. Even if the $\mathbf{I P}$ position changes significantly from run to run, the central tracker and monitors may be used to determine the position of the beam spot, allowing muon track data for alignment to be accumulated over long periods.

\section{P. - 'qtive alignment of different muon towers is neede th for the precise reconstruction of the dimuon mass when each muon is in a different tower, and for the reconstruction of the muon momentum when it crosses the boundary between two neighbor- ing towers. The former requires an angular align- ment with an accuracy comparable to or better than that reconstructed within a single tower. The angular alignment is driven by the much looser accuracy measured in the non-bend direction, $\sim 3 \mathrm{mrad}$.}

The assumed millimeter-level chamber placement tolerances (Table 4-16) are already sufficient to adequately resolve the $3 \mathrm{mrad}$ relative angular alignment. Muon data will thus be used to verify this accuracy; a preliminary analysis shows that the $3 \mathrm{mrad}$ precision can be attained over several hours by analyzing the masses of $Z$ dimuons traversing different towers. 31

Tracks with transverse momenta of $30 \mathrm{GeV}$ or lower will be able to cross the boundary between alignment sectors. At $p_{T}<30 \mathrm{GeV}$, the momentum resolution is on the order of a few percent (limited by 
energy loss in the calorimeter and material in the muon system), which corresponds to $-500 \mu \mathrm{m}$ of error in the sagitta. An alignment accuracy of about $250 \mu \mathrm{m}$ is required between neighboring towers to use these tower-crossing muons. This can be achieved within an hour at standard luminosity, by utilizing high momentum tracks that traverse both neighboring sectors in the $12 \mathrm{~cm}$ overlap regions provided.

\section{Calibration of the Magnetic Field Bending Power}

Preliminary studies indicate that checking the calibration of the magnetic field should also be feasible through the analysis of $Z$ dimuons. ${ }^{31}$ One month's worth of statistics will be sufficient to verify the bending power values in about $1000 \theta$ intervals.

\subsection{SPECTROMETER DESIGN}

\subsubsection{Magnetic Field Description and Specifications}

The magnet design (see Chapter 3) has evolved synergistically with that of the muon system. All aspects of the CSC operation within the magnetic field, materials in the CSC and the support structure, and optimization of the field for the endcap region have been considered during the design process. The ideal magnetic field shape for the GEM detector, with its cylindrical barrel and a radial endcap systems, is not uniform. The physics performance of the system requires a radial component in the forward regions of the system to retain the required momentum resolution for forward going muons. This radial component is provided by the conical iron pole pieces-the forward field shapers (FFS).

The momentum resolution of reconstructed tracks results from the interplay of several contributions. These include the integral of the magnetic field strength along the trajectory of the particle and the resolution of the position measurements in the CSC system. The position measurements are affected by the alignment precision of the CSC and also by the precision of the knowledge of the local magnetic field needed to minimize and correct for Lorentz effects (see Section 4.3.2). Furthermore, the tracking algorithms and trigger design in the muon system are base 1 on the assumption that the magnetic flux is uniform in the central rapidity region and is completely specified in the forward region. In both regions, only small deviations from axial symmetry are allowed.

Field Integral Description of the Magnetic Field

The GEM muon system measures the momentum of a given track by the determination of the sagitta. At low momenta $(<100 \mathrm{GeV}$ ) additional information from the spatial extent of the superlayers improves resolution by contributing independent measurement of the turning angle. An excellent approximation of track displacement for high momentum tracks is given by the magnitude of the second field integral projected along a ray origi-nting at the origin. We begin from first principles and write the standard equation for a charged particle moving in a magnetic field (where vector quantities here are denoted by bold-faced characters):

$$
F=\frac{q v}{c} \times B=\frac{d p}{d t} .
$$

where $F$ is the force on the particle, $q$ is the electric charge, $v$ is the velocity, $B$ is the magnetic field strength, $p$ is the total momentum, and $t$ is time. This equation is also true in the relativistic regime. We can rewrite Equation (1) as:

$$
\frac{d^{2} x}{d s^{2}}=\left(\frac{x}{|p|}\right) \frac{d x}{d s} \times B
$$

where $x=0.3 \mathrm{GeV} \mathrm{T}^{-1}$.

The first integral of the magnetic field is the impulse, proportional to the turning angle:

$$
I_{1}=\int F d t=\int \frac{d p}{d t} d t=\int d p=\Delta p .
$$

Under the approximation that $\rho>L$, where $\rho$ is the radius of curvature of the muon track and $L$ is the path length, we approximate the path integrals above with integrals evaluated along straight rays:

$$
\begin{aligned}
I_{1} & =\int \frac{q v}{c} \times B d t=\frac{q}{c} \int \frac{d x}{d t} \times B d t \\
& =\frac{q}{c} \int d x \times B .
\end{aligned}
$$


The second integral of the magnetic field gives the effective displacement of the curved track from the straight ray:

$$
I_{2}=\int \frac{d^{2} x}{d s^{2}} d s d s^{\prime}=\frac{x}{|p|} \int_{0}^{L} \int_{0}^{s^{\prime}} \frac{d x}{d s} \times B d s d s^{\prime}
$$

For a uniform magnetic field of magnitude $B$, the magnitude of the second integral is given by:

$$
\left|I_{2}\right|=\left(\frac{x}{|p|}\right) \frac{\sin \theta B L^{2}}{2} .
$$

where $L$ is directed along a straight ray and $\theta$ is the angle between $B$ and the muon trajectory. We now define the second integral of the magnetic field for the case of a nonuniform field:

$$
\beta \lambda^{2}(L, \theta)=\left|2 \int_{0}^{L} d s^{\prime} \int_{0}^{s^{\prime}}(d x \times B)\right| .
$$

In this case the total displacement is:

$$
\frac{x}{2|p|} \beta \lambda^{2}(L, \theta) .
$$

The factor of 2 in the denominator in (8) comes about from the condition that $\beta \lambda^{2}=B L^{2}$ when the magnetic field is uniform, and the trajectory is orthogonal to the field. Under the assumption that $p \gg l$, which is correct in our case for all trajectories of interest, the sagitta for a uniform field directed along $z$ is given by:

$$
S(L, \theta)=\frac{1}{4} \frac{x}{2|p|} \beta \lambda^{2}(L, \theta) .
$$

Equation (9) can be rewritten in terms of the transverse momentum $p_{T}$ :

$$
S(L, \theta)=\frac{1}{4} \frac{x \sin \theta}{2 p_{T}} \beta \lambda^{2}(L, \theta) .
$$

For a non-uniform field with a major component along $z$, the sagitta can be written in terms of the path lengths to each separate muon superlayer along the straight rays with respect to the IP:

$$
\begin{aligned}
& S(L, \theta)= \\
& \frac{x}{2(|p|)}\left[\frac{\beta \lambda^{2}\left(L_{1}, \theta\right)+\beta \dot{\lambda}^{2}\left(L_{3}, \theta\right)}{2}-\beta \lambda^{2}\left(L_{3}, \theta\right)\right] .
\end{aligned}
$$

where the path goes from $L_{1}$ to $L_{3}$ and $L_{2}$ is roughly the mid-point. The ray approximations are useful for the detector design, however, the analysis and reconstruction of real muons will utilize the actual fields and trajectories. Figure 4-27a and $b$ show the impulse and the second integral, $\beta \lambda^{2}$, for several values of polar angle, $\theta$, of the ray.
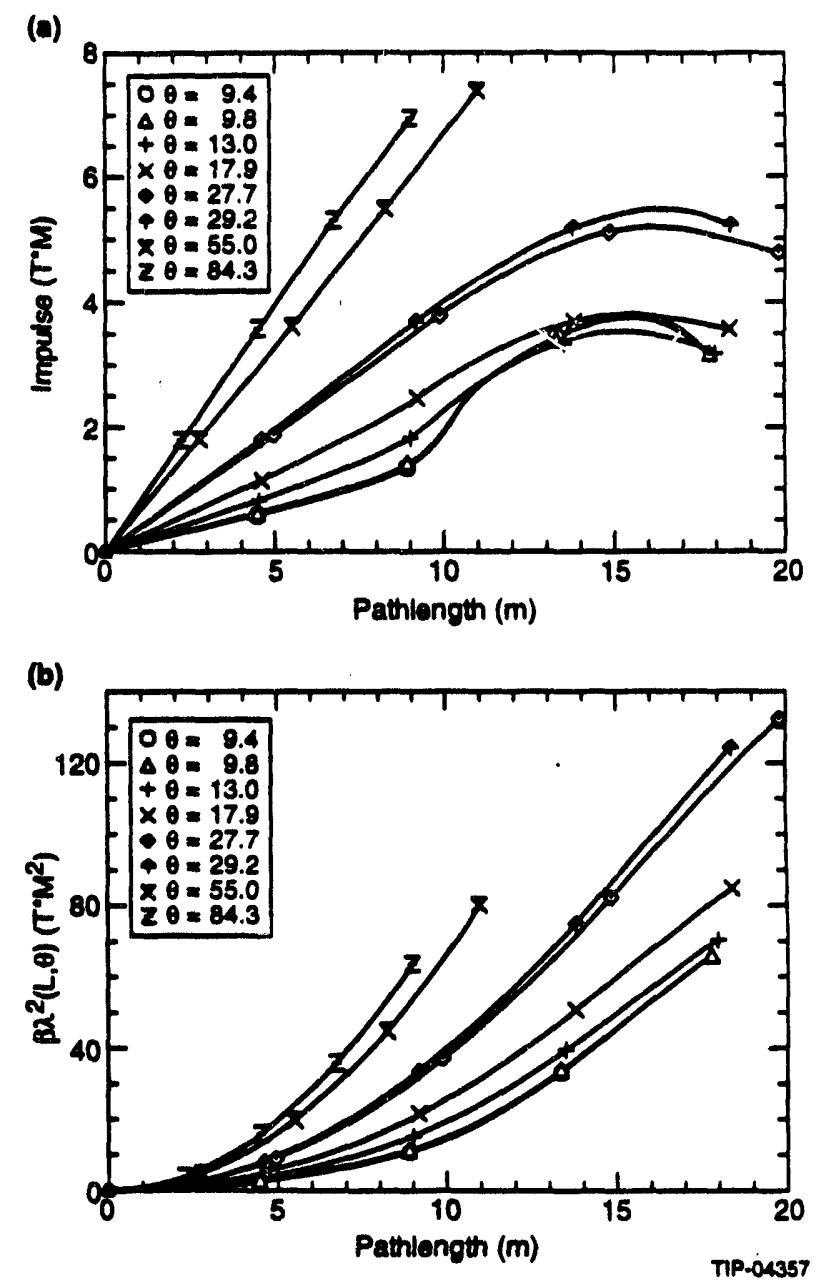

FIG. 4-27. a) Magnetic impulse and b) $\beta \lambda^{2}$, as a function of path length for rays at various polar angles $\theta$.

\section{Field Integral Specification}

In order to achieve the desired momentum resolution we require accurate knowledge of the magnetic field as well as an optimized CSC geometry. There are two major considerations which drive this requirement: 
- Accuracy of reconstructing the sagitta of the muon trajectory

- Validation of the Lorentz angle effect in the muon tracking chambers.

\section{Global Field Knowledge Requirement}

For our global field requirement we specify that any additional systematic error in the momentum resolution contributed by the lack of knowledge of the magnetic field may not exceed $30 \%$ of the minimum resolution. Including the energy-loss fluctuations in the calorimeter, and multiple scattering in the chambers the best transverse momentum resolution of the present GEM muon system is $\Delta p_{T} / p_{T}=1.3 \%$ at $50 \mathrm{GeV}$ in the barrel (see Figure 4-19). Thus we conservatively specify a requirement of knowledge of second integral of the magnetic field corresponding to a measurement of the displacement of a track, $\beta \lambda^{2}$, to better than $0.4 \%$. Note that this same precision is required on the knowledge of any azimuthal nonuniformity anil on the measurement or the reproducibility of the field for successive operation cycles.

\section{Local Field Knowledge Requirement}

The chamber geometry is optimized for a specific Lorentz angle, $\theta_{L}$, as discussed in Section 4.3.2. This means that for a given gas mixture, the tilt angles of the planes in the barrel and of the wires in the endcaps have been calculated assuming a certain value and direction of the magnetic field (e.g., $0.8 \mathrm{~T}$ for the axial field in the barrel). As long as the field is kept to within $10 \%$ of the design value the resolution degradation does not exceed the specified limits.

In the endcaps the field components vary within the area covered by a single chamber. In this case, the geometry is optimized for the value of the field in the center of the chamber. Even if the field has been set exactly at the nominal value there will be some degradation because of the field variation within the module. From the field maps below (Figure 4-28) one can see that the steepest gradient of the radial field is in the area of the middle superlayer. We have estimated the expected resolution at the top, middle, and bottom of the modules of the middle superlayer under the assumption that the geometry has been optimized for the nominal field in the middle of each chamber. In the worst case the degradation due to one field variation across the chambers is within the specified limit of $75 \mu \mathrm{m}$ chamber resolution.

In summary, knowledge of the integral $\beta \lambda^{2}$ is the driving requirement and the field measurements will be focused on evaluating this quantity, particularly in regions of large field gradient and magnitude.

\section{Magnetic Field Map Calculations}

The field maps presented here correspond to the standard configuration for the GEM magnet. For simplicity, no other magnetic structures were included in these calculations. The fields are generated using the two dimensional, axisymmetric, nonlinear, finite element code MITMAP. Figure 4-2 showed an elevation view of one quadrant of the GEM detector volume with equi-spaced lines of constant flux. The superconducting coil, FFS, and muon superlayers are superimposed onto this flux. In general one sees that the flux lines are axial only within a small region of the muon system. The effect of the FFS is evident in the illustration.

In Figure 4-28, the axial component, $B_{Z}$, and radial component, $B_{p}$, of the magnetic field are plotted at the mid-plane of each muon system superlayer. The three curves in each figure correspond to the field strengths for three different radii as a function of distance along the beam axis. These curves indicate the behavior and strength of the magnetic field typical for the barrel region, and the inner and middle endcap superlayer region.

Full three dimensional magnetic fields have also been generated with the ANSYS code. Three dimensional modeling allows an examination of the effects of various axisymmetric and nonaxisymmetric ferromagnetic structures; for example, a soft-iron magnet cryostat, or a soft-iron FFS support structure. Our calculations indicate that the effect of some soft-iron structures are within acceptable limits as described later in this section. In other structures, nonmagnetic materials have been specified in the design based on these calculations.

\section{Field Mapping}

Because of the importance of knowledge of the field to the success of the GEM experiment, and the 
(a)

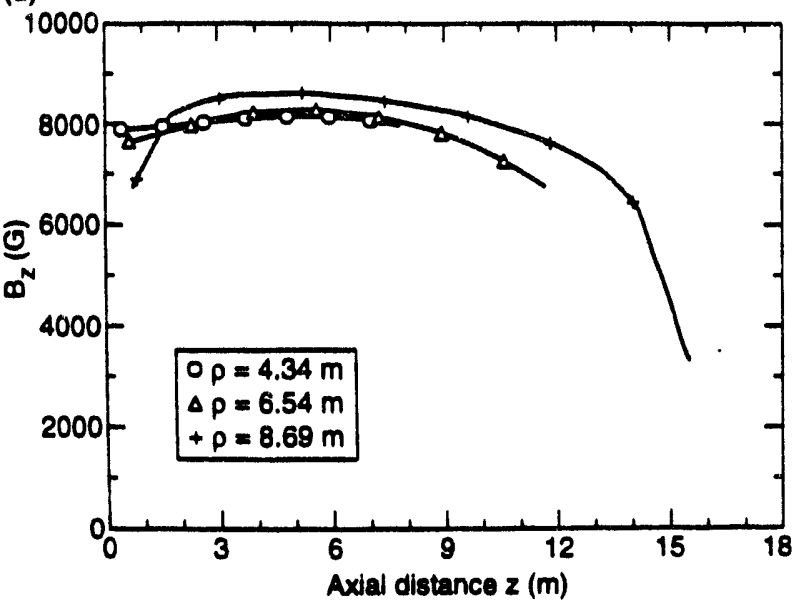

(b)

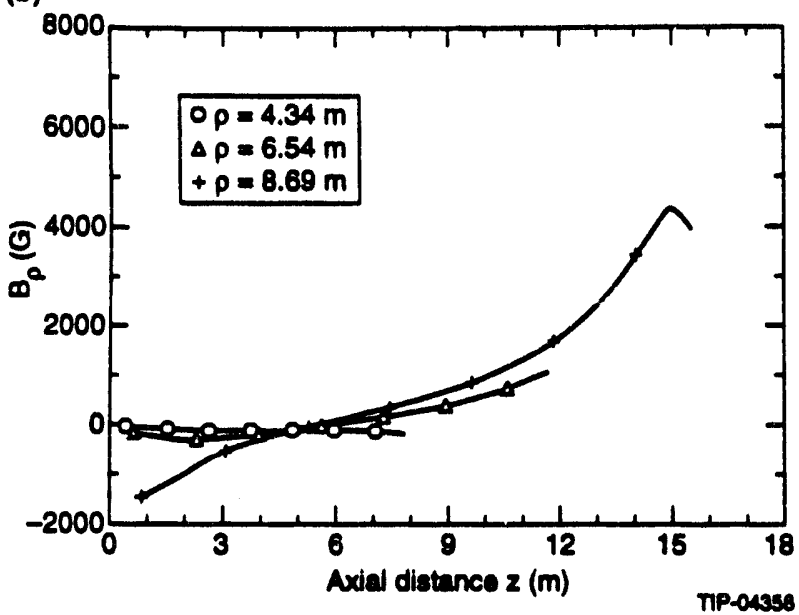

FIG. 4-28. The axial (a) and radial (b) components of the GEM magnetic field for different radil $\rho$.

cost and scheduling impact of direct field mapping, we have developed a concept for mapping the field by using a finite number of measurements and an analytic fit to determine the field in the active detector volume, i.e., a Green's function analysis on a simply-connected surface such as a sector of the muon detector or even the whole barrel; this method has been applied to plasma machines, which have more complex fields than GEM. Several different configurations of probes are being worked out. One possibility is to place sensors on volume surfaces corresponding to the 12-fold detector module arrays, providing an adequate description of the field within each array. The accuracy of measurement needed. the cost and time of measurement with conventional techniques, the necessary field transducers (e.g. search coils, Hall probes, NMR probes, etc.), and their number and spatial distribution for an analytic solution, are under active review.

\subsubsection{Chambers}

The Chamber Technology Choice

We have considered a number of different options in the process of determining our chamber technology. An extensive R\&D program was undertaken to understand the properties of these technologies. Considered were limited streamer drift tubes (LSDTs) and round drift tubes (RDTs), which would have provided the precision track reconstruction for the muon system, and resistive plate chambers (RPCs) and proportional wire chambers (PWCs), which would have been used for triggering and a redundant nonbend plane measurement in concert with the precision drift technology. Also studied were cathode strip chambers (CSCs) based on the proportional chamber concept. All of these technologies had attractive features and drawbacks. Our R\&D program gave us confidence that CSCs offered the most complete and lowest risk solution. The following is a brief description of each of the considered technologies and the reasons for the choice made.

\section{Limited Streamer Drift Chambers}

This technology. was proposed for the tracking function in the barrel region. ${ }^{3}$ The technology uses $2.5 \mathrm{~cm}$ square open profiles, fabricated out of thin Al sheets. A 100 ' $\mathrm{m}$ anode wire is centered in each profile. The chamber is operated in the limited streamer mode; the first (or nearly first) electron to drift to the wire triggers a TDC to determine the drift time. An early prototype of this technology was tested successfully in the $0.5 \mathrm{TeV}$ muon beam at FNAL, where resolutions of about $50 \mu \mathrm{m}$ RMS were measured. Two large scale prototypes were constructed and tested successfully at MIT with cosmic rays, and one chamber was tested at the SSCL Texas Test Rig. Resolutions of better than $100 \mu \mathrm{m}$ were obtained-well within our baseline design goal.

The nonbend plane measurement is accomplished by pickup strips in a cathode plane located above the top open profile in a stack. The strips are orthogonal to the wires; they are wired together in an interdigitated fashion with several electronic channels interlaced. The anode wires are tied together in pairs at one end of the detector, and each wire is read out at the other end. The positions of the hits along the strip lines are determined by the time difference 
with $20 \mathrm{~cm}$ resolution, and the strips then give a refined position measurement with several $\mathrm{cm}$ resolution. This allows the number of nonbend plane channels to be reduced. By correlating the times of the pulses on the wire with the time on the orthogonal strips a 2-dimensional space point along the muon trajectory is achieved. One of the attractive features of this technology is the mechanical isolation of anode wires inside the $\mathrm{Al}$ profiles, minimizing the effect of a broken wire. In addition, the chamber design involves wire supports placed eve $y$ $2 \mathrm{~m}$ along the wire length to minimize the wire gravitational sag.

It was decided that the mechanical design of this technology needed further development. The chamber design had significant material inside walls to hold the wire tension and to support the wire bridges placed every $2 \mathrm{~m}$ along the wire length. Further, a muon system based on LSDTs relied on a relatively new RPC technology for triggering (discussed later). Some effort was invested in forming a stand-alone trigger based on the drift system itself. This looked attractive but was complex and may have not had sufficient resolution to operate in the endcap region at a $p_{T}$ trigger threshold of $50 \mathrm{GeV}$.

\section{Round Drift Tubes}

The RDT is a well-developed technology and variations of it have been in operation in collider experiments for many years. The advocates of this technology originally proposed to employ RDTs as the precision tracking device in the barrel, and further developments of this technology made it an attractive possibility for the endcap application as well. 6 In this option the chambers are deployed in a superlayer 8:10:8 configuration in both the barrel and endcaps. In each superlayer 4 layers of tubes are oriented in a small angle stereo ( $2 U+2 \mathrm{~V}$ at $\pm 4 \mathrm{mr})$ with the remaining tubes parallel (perpendicular) to the axial component of the magnetic field for the barrel (endcap) application. As in the LSDT case, the wires are paired to yield a rough orthogonal coordinate. In addition, the small angle siereo gives $-2.5 \mathrm{~cm}$ resolution in the orthogonal coordinate. A chamber layout was dev sloped with good coverage over most $\theta$ and $\phi$ angles, having only $\epsilon \%$ solid angle loss arising from holes and cracks between chambers used for the projective alignment paths.
Thin walled $(\sim 300 \mu \mathrm{m}) \mathrm{Al}$ tubes, each with a $50 \mu \mathrm{m}$ wire strung concentrically with the axis of the tube, form the basic element of the technology. By a suitable choice of gas mixture and high voltage, the device can be operated in either the limited streamer mode or the proportional mode, permitting considerable latitude in the choice of the operating point. Very rigid and light-weight chambers are fabricated by stacking and gluing tubes together forming honeycomb structures. The wires are supported through precision endplates which are fabricated by numerical control machining techniques. The mechanical design allows the tubes to be pressurized without distorting the precision mechanical alignment of the system. This was shown to improve the spatial resolution by $1 / \sqrt{\mathrm{P}}$, where $\mathrm{P}$ is the gas pressure. Resolutions (RMS) were measured in two full-scale prototypes ( $4 \mathrm{~m}$ long) built by groups at MSU and JINR to be $<100 \mu \mathrm{m}$ at one atmosphere. In one of the prototypes, operating at a pressure of $4.8 \mathrm{~atm}$, a $50 \mu \mathrm{m}$ resolution was measured.

Preliminary engineering and cost estimates of this technology showed that it is cost-effective and practical in terms of system performance. However the RDTs also relied on RPCs for the trigger. Some consideration was given to a stand-alone drift trigger and a PWC system (similar to, but simpler than, the CSC to be described below) as an altemative to the RPC trigger. However it was decided that both of these options were in an immature state at the time that we had to make our decision and therefore both carried an unacceptable risk.

\section{Resistive Plate Chambers}

RPCs have been employed in various experiments since about 1981 . They have many attractive features, such as simplicity, low cost, pulse output with very fast rise time (a few ns) and large amplitude (up to $500 \mathrm{mV}$ into $50 \Omega$ without the need for preamplification), which make them a good choice for the trigger technology of a muon tracking system based on drift technology.5 RPCs can be configured in such a way as to provide both bend and nonbend plane measurements and a beam-cross time-pulse. The device is in principle ideal for the triggering function of the GEM muon system. Addressing a major concern, the RPC R\&D program showed that RPCs could be operated successfully in 
coincidence with LSDTs and RDTs, without affecting sensitive drift chamber electronics in the vicinity.

The RPC is essentially a narrow gap avalanche chamber that generates a streamer that is severely quenched. The quenching is achieved by building the chamber with thin sheets of resistive (semiconducting) plastic to limit the charge which can be supplied to the streamer developed around the primary ionization associated with the muon track. Further quenching is achieved by employing a gas mixture containing a small amount of freon, with a large electronegative component. RPCs are simple devices to construct and operate and have been used in large detector arrays for cosmic ray applications.

For RPC application as a viable muon system trigger, the most critical operating parameters ire the timing jitter and rate capability. To date, RPCs made from Bakelite resistive plates have exhibited slewing and broadening of the time resolution at rates over about 50 to $100 \mathrm{~Hz} / \mathrm{cm}^{2}$. Bakelite RPCs also suffer from poor rate capability because of the high resistivity of the Bakelite used in these devices. Promising R\&D to improve the rate capability was undertaken by the LLNL-MIT RPC group, in which several prototype chambers were constructed with different semiconducting plastic electrode materials. Attractive candidate plastics were found, such as Abstat-M310, which was used to construct a $1.2 \mathrm{~m} \times 2.4 \mathrm{~m}$ prototype chamber that was tested at the Tex.as Test Rig. This plastic has a bulk resistivity of about $6 \times 10^{9} \Omega \mathrm{cm}$, about 20 times less than that of the standard Bakelite of previous RPCs. RPCs built with this plastic exhibit initial rate capabilities of about $15 \mathrm{kHz} / \mathrm{cm}^{2}$. Unfortunately, it was discovered that the bulk resistivity of this plastic increases under the large electric field gradients, degrading the originally attractive high rate capability of the chamber. Other plastics or glasses may have proven to be suitable, but because of the necessity of a timely decision on the muon system technology, the RPC technology option was removed from consideration in the GEM muon system.

\section{Cathode Strip Chambers}

The CSC is a well-established technology with an extensive body of experience. ${ }^{34}$ The CSCs provides the full functionality of the muon system in a single technology: precise measurement of the bend coordinate using induced signals on cathode strips in the axial (radial) directions for the barrel (endcap): fast signals for the momentum trigger: measurement of the nonbend coordinate using azimuthally oriented anode wires (barrel and endcap); and precise timing to provide the SSC beam crossing tag.

The precise measurement of the bend coordinate is made by interpolating among the strip positions according to the induced charges seen on the strips when an ionizing track penetrates the CSC layer. As will be discussed in Section 4.3.5 and Chapter 7, integration of the strip signal with a peaking time of $-300 \mathrm{~ns}$ gives adequate $S / \mathrm{N}$ to allow determination of the position of the traversal of the chamber to the level of $1 \%$ of the strip width. Fast outputs ( $30 \mathrm{~ns}$ peaking time) from the strips are discriminated to provide hodoscopic inputs to the muon momentum trigger.

By reading the anode wires, (or alternatively, by reading signals $\mathrm{fr}^{\mathrm{t}} \mathrm{m}$ coarsely segmented cathode strips oriented parallel to the wires), the required nonbend coordinate resolution can be achieved without introducing additional detector layers. The combination of a fast gas (e.g., $50 \% \mathrm{CO}_{2}-30 \%$ Ar-20\% CF 4 ) and closely spaced anode wires results in a maximum drift time of $<30$ ns. The anode signals will have a 30 ns shaping time for good timing performance. Test results indicate that the earliest arrival of signals from the CSC layers of a given superlayer will identify the correct beam crossing with very high efficiency. The $O R$ of the layers in a chamber will be validated by a tower-wise majority requirement on the layers for high rate capability.

The inherent precision of the CSC comes from the lithographic process used in the etching of the cathode strips. With this technique the strip positions can be determined with a precision of about $15 \mu \mathrm{m}$. Alignment information is obtained via a single transfer from the measurement strips and survey marks photo-etched on the planes to alignment components made visible to the external alignment system. The structure of the cathode planes allows the use of several alignment technologies including 3-point projective alignment and multipoint axial alignment (using, for example, stretched wires with inductive position readout). 
We have pursued an active program of CSC prototyping and development during the past year. Test chambers have been built by six different groups (BNL, BU, UH, PNPI, JNR, ITEP). The chambers have been tested in the cosmic ray Texas Test Rig at the SSCL, at the RD5 muon test beam at CERN, at the $1.7 \mathrm{GeV}$ proton beam at the Gatchina Synchrocyclotron, at the $5 \mathrm{GeV}$ pion beam of the ITIP synchrotron, and by using neutron/gamma radioactive sources. ${ }^{7,8,22,35.36}$ We have stucied the position resolution, timing, rate capability, twotrack resolution, and sensitivity to neutrons and photons. In addition, tests with $x$-rays have been done to study details of chamber design. These results are detailed in the next section and also in Reference 34 . Briefly, the R\&D program established the following CSC performance characteristics:

- Excellent single layer track resolution: a sigma of $\leq 60 \mu \mathrm{m}$ for single muons has been measured with several prototype CSCs at the TTR.

- Good two-track resolution: nominal singletrack resolution is achieved ror each of a pair of tracks separated in the bend direction by 2 or more strips.

- Timing resolution $\sigma \leq 8$ ns per single layer, implying a beam crossing tag efficiency of better than 99\% for 6 good measurements within a CSC.

- Electron drift time less than 30 ns.

- Neutron and gamma sensitivities of CSC are suitable for operating the GEM muon system at luminosity up to $10^{34} \mathrm{~cm}^{-2} \mathrm{~s}^{-1}$.

- Proof-of-concept for CSC components including precision cathode boards, electronics readout systems, and trigger systems.

- All muon system functions-triggering, tracking, and timing-available in a single technology.

The CSC concept is not without its drawbacks, however. There are more chambers and more electronic channels for triggering and tracking, than in the RDT/RPC option. The chamber fabrication and asser.ably procedures are potentially more complex. Optimization of the CSC design to address these concerns will be discussed in greater detail below.
We concluded from our detailed evaluation of muon chamber trigger and tracking technology that the CSC approarh was the best solution and that the risk associated with the RDT-RPC (PWC) technology option was unacceptable. Further R\&D would have been needed to improve the rate capability of the RPC (several promising leads do exist) or to design a PWC system with sufficient time resolution and simplicity to be cost-effective and practical. The "single technology" CSC concept provided an attractive solution to the requirements of the GEM muon system and was therefore selected as the baseline technology.

\section{CSC Parameters and Optimization}

The CSC design development has been guided by principles of simplicity, reliability, and manufacturability, in addition to the capability to deliver the required performance. Chamber dimensions have been set within the dimensional limits of the available materials and within the capacity of industrial vendors to fabricate the cathode planes at the required tolerances. Tolerances are being set and reviewed with the goal of minimizing the cost of the chambers while meeting performance requirements. We have developed a number of design tools for evaluating chamber performance as a function of design parameters, ${ }^{37}$ and we have performed extensive tests of prototype detectors. ${ }^{7,8,35,36,38.39}$ The current baseline design of the GEM muon system using CSCs results from an optimization process where performance is weighed against detector cost and complexity.

The shape and sin Icture of each CSC is adapted to the detector's posit 1 in the muon system. In the barrel, the CSCs a ctangular; the cathode strips run axially (parallel to the $B$ field) and anode wires are oriented azimuthally. In the endcap, the CSC are trapezoidal; the cathode strips run radially and anode wires run approximately azimuthally but are angled to reduce Lorentz effects as described in detail below.

CSCs are arranged in inner (closest to the calorimeter), middle, and outer superlayers in both the barrel and endcap muon systems. Each CSC package consists of six individual detector layers (eight for the inner endcap superlayer) in an integral construction that maintains the internal alignment of each detector layer. This allows use of a combination 
of the independent layer measurements to achieve a spatial resolution improved approximately by the square root of the number of chambers in the superlayer.

The required position resolution of the bend coordinate in the middle superlayer is achieved by combining the independent measurements from the 6 layers in a CSC. For a 6 layer CSC, the single-layer resolution is required to be better than $75 \mu \mathrm{m}$ (random) with $<50 \mu \mathrm{m}$ systematic error within each individual layer.

The CSC "unit cell" is characterized by the anode wire spacing $s$, the anode-to-cathode spacing $d$, and the cathode strip readout spacing $w$. Figure 4-29 illustrates these parameters. Design considerations contributing to the determination of the values for these parameters include: minimizing electronics channel counts, minimizing capacitance (a source of noise), maintaining proper occupancy upper limits, providing good two-track resolution, achieving necessary hodoscopic segmentation for triggering, meeting mechanical constraints, and allowing reasonable mechanical tolerances.

In the GEM muon system the CSC readout pitch varies projectively from $5 \mathrm{~mm}$ to $10 \mathrm{~mm}$, as one moves from inner to outer superlayer. This configuration minimizes the channel count while delivering the required spatial resolution. Good timing performance requires that the wire spacing be no greater than $s=2.5 \mathrm{~mm}$. For mechanical stability and acceptable tolerances, we have chosen the ratio of the readout pitch to anode-cathode spacing to be $w / d=2$. Such a high ratio results in significant $(>50 \%$ ) position encoding differential nonlinearity because the FWHM of the cathode induced charge is about 1.5d. However, work presented in Reference 7 shows that linearity of response can be restored to near optimum with the use of intermedi-

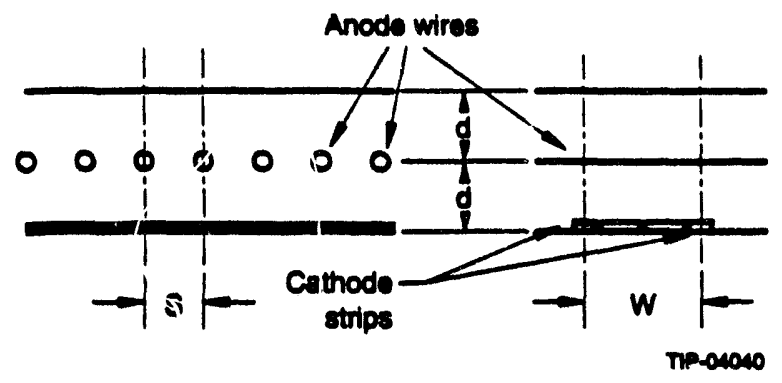

FIG. 429. Schernati: diagram of the Cuthode Strip Chamber ate strips between readout strips. ${ }^{34}$ These intermediate strips provide increased charge sampling frequency and are capacitively coupled to the adjacent readout strips. Such an arrangement with two intermediate strips is shown in Figure 4-30 along with the equivalent electrical circuit. For this capacitive interpolation to work, the interstrip capacitance $C_{1}$ must be much larger than that of a strip to ground, $C_{2}$. In the CSC, this condition is met by maintaining the gap between strips $\leq 400 \mu \mathrm{m}$, resulting in $C_{1} / C_{2}-10$. Further optimization indicates that the intermediate strips should be slightly wider than the readout strips. ${ }^{40,41}$ It is necessary to provide a high resistance path to ground for the intermediate strips to avoid charge buildup. This is provided by a thin strip of resistive epoxy which has a conductivity of $6 \mathrm{M} \Omega$ per square meter and a resistance from strip to strip of $10 \mathrm{M} \Omega$ which is silk-screened on the tips of the strips at the end of the cathode opposite to the amplifiers.

(a)

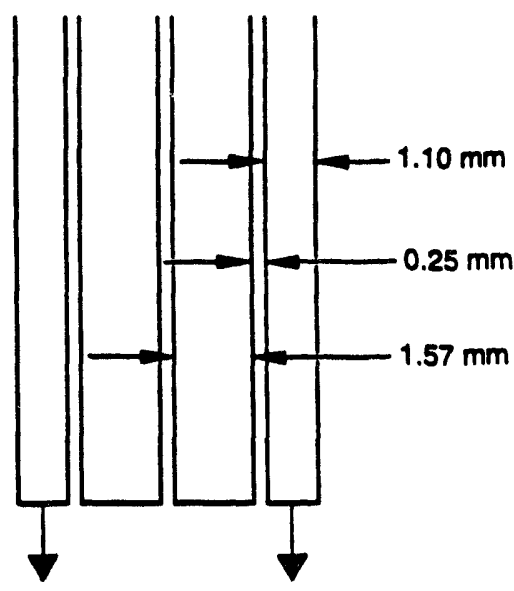

(b)

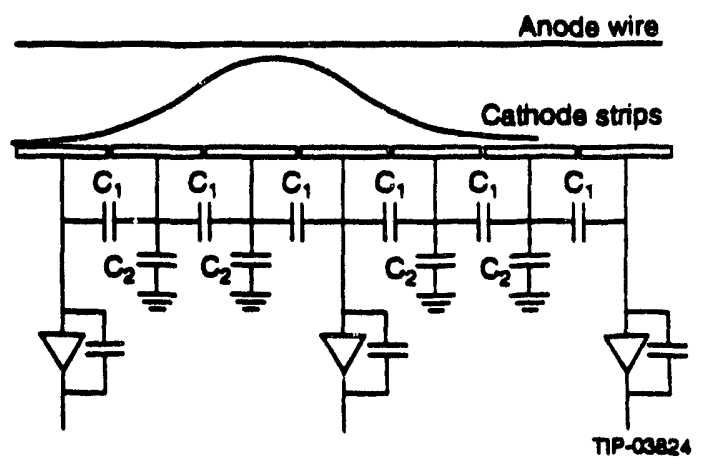

FIG. 4-30. a) Two intermediate strip design and b) equivalent circuit. 
The anode wires run in an azimuthal sense in both the barrel and the endcap chambers. The anode wires are read out to provide measurement of the nonbend coordinate-axial in the barrels, radial in the endcaps-allowing determination of the polar angle of the track. The anode signals are also used to tag the beam crossing.

The anode wires are operated at high voltage and the cathodes are grounded. Groups of wires are ganged to a single readout circuit; in segments of 20 to 40 wires $(5$ to $10 \mathrm{~cm})$, with a width increasing with the distance from the IP. The wire groups in successive planes within a chamber will be staggered to reduce the effective hodoscopic element by a factor of two. The effective RMS resolution in the nonbend coordinate is then $1 /(2 \sqrt{12})$ times the 5 to $10 \mathrm{~cm}$ width of the wire group. This resolution preserves the precision of the momentum measurement and provides sufficiently precise determination of the polar angle so that dimuon mass reconstruction for the $Z$ is limited by the natural width of the $Z$.

An upper limit to the number of wires which can be tied together comes from the degradation of timing performance due to the increased capacitance. Blocking capacitors couple the anode signals to preamplifiers and shaping amplifiers. The shaping amplifiers have a peaking time of $30 \mathrm{~ns}$, which is significantly longer than the signal propagation time along the roughly meter-length wires. This implies that the position dependence of the pulse shape due to reflections, etc., is not important. The shaped pulses are discriminated to identify hit anode channels, and the event time is derived from the OR of discriminated signals from all the layers of a . chamber. Tests show that due to variations in the muon pulse amplitude in a CSC layer, simple threshold discrimination may not be adequate to achieve the required time resolution. A number of alternative schemes (dual threshold, zero crossing, constant fraction, etc.) are under test.

Event reconstruction in a CSC requires the association of the nonbend coordinate from anode wire measurements with the corresponding bend coordinate from cathode strip signals. In events where several particles traverse the same CSC, "ghost" combinatious must be rejected. This can accomplished by measuring the charge of the anode signals in addition to the charge on the cathode strips. In this approach, resolution of multiple hits within a CSC layer is achieved using the charge correlation between anode and cathode measurements (which sense the same proportional chamber signal and so differ only in their noise components). Due to the shorter peaking time and larger capacitance of the anode channels compared to the cathode channels, the charge resolution for the anodes will be several times worse than for the cathodes. However, the Landau distribution of single muon signals is sufficiently broad that the correct combinations can be found with high reliability. There may be pattern recognition techniques that could also resolve the ambiguities without charge measurements, and the issue is currently under study. The conservative (and more expensive) solution-using anode charge measurement-is our baseline design.

\section{Time Resolution}

With appropriate discrimination, the maximum drift time measured for a single gas gap is $<30$ ns. The six layers in a chamber will be combined using a six-f $/ d$ OR majority logic requirement. This provides good efficiency and good timing resolution for genuine tracks while rejecting random backgrounds. The logical combinations will be formed in a way that projects towards the interaction point, further reducing sensitivity to random background hits. Calculations ${ }^{42}$ and preliminary results (see below) indicate that the OR of four measurement layers has an RMS resolution of less than $4 \mathrm{~ns}$, giving $>98 \%$ probability for tagging the correct beam crossing. A six-gap CSC will tag the correct beam crossing with $>99 \%$ probability. To achieve this performance, it will be necessary to compensate for the position of the track in the active area of the chamber. This can be accomplished by the insertion of appropriate delays in the discriminated anode signals. 


\section{Lorentz Effects and Geometric Contributions} to Resolution

After noise contributions, the next most significant contribution to the resolution of the CSCs comes from the related effects of track inclination and of Lorentz drift. These effects are extensively covered in a GEM technical note. ${ }^{37}$ Here we will summarize the main results. The charge interpolation is optimum when the avalanche is formed on a single point along the wire. A finite spatial extent of the anode charge along the wire results in a resolution degradation. Such nonlocal charge deposition can be caused by a number of factors such as diffusion of the drifting electrons, $\delta$-electrons, inclined tracks, and a Lorentz force along the anode wires in the presence of a magnetic field that is not collinear with the electric field of the chambers. The last two effects are by far the most important. We have used both simple geometrical arguments and a Monte Carlo simulation to study these effects in detail.

It should be noted that track inclination and Lorentz effects in the barrel CSC do not result in a systematic shift of the measured coordinate. Rather, the resolution degrades because of fluctuations in the distribution of ionization along the particle's trajectory: when the electron drift path to the anode wires is not parallel to the trajectory, these fluctuations lead to a variation in the charge distribution centroid along the anode wire. Track inclination in the direction along the strips improves performance by increasing the number of ion clusters.

The effect of inclined tracks is minimized by the 48-fold symmetry of the muon system, which limits the maximum azimuthal angle of incidence to $\pm 3.75^{\circ}$. In the barrel region, the Lorentz effer: is compensated by tilting the chambers about the axial magnetic field by an amount equal to the Lorentz angle. The situation is illustrated in Figure 4-31. Geometric considerations and issues of chamber placement favor the choice of a gas with a small Lorentz angle. ine 48-fold symmetry resulted from an optimization process that included chamber dimensions, manufacturing considerations, and structural issues in addition to these performance implications. Figure 4-32 shows the resolution as a function of angles of incidence within a barrel sector, with the GEM magnetic field on and with the chambers tilted by $8^{\circ}$. This Monte Carlo calculation of the resolution included smearing due to Lorentz effects and geometric effects and assumed an intrinsic resolution of $50 \mu \mathrm{m}$.

The Lorentz effect in the endcap CSC is slightly more complicated than in the barrel. There is a significant radial magnetic field component at some chamber positions, although the dominant contribution to the Lorentz drift still comes from the
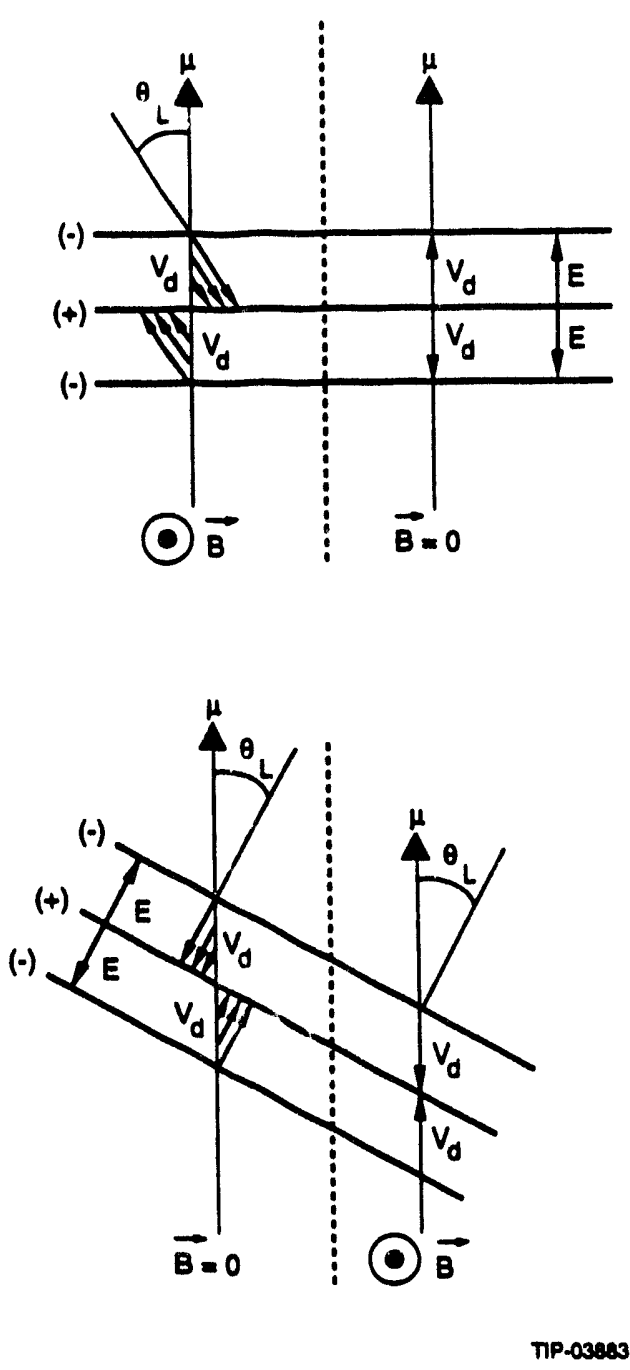

FIG. 4-31. Sketch of the Lorentz effect for barrel. 
axial component of the field, especially for electrons drifting from the outer regions of the cell. Further, there is a geometric effect that is absent in the barrel: the angle of the anode wires with respect to the strips varies across the chamber, as illustrated in Figure 4-33. This is a consequence of the trapezoidal chamber shape and the radial strip orientation. This can cause a systematic shift which is proportional to the product of the tangent of the angle $\phi$ between the strips and the anode wires and the distance between
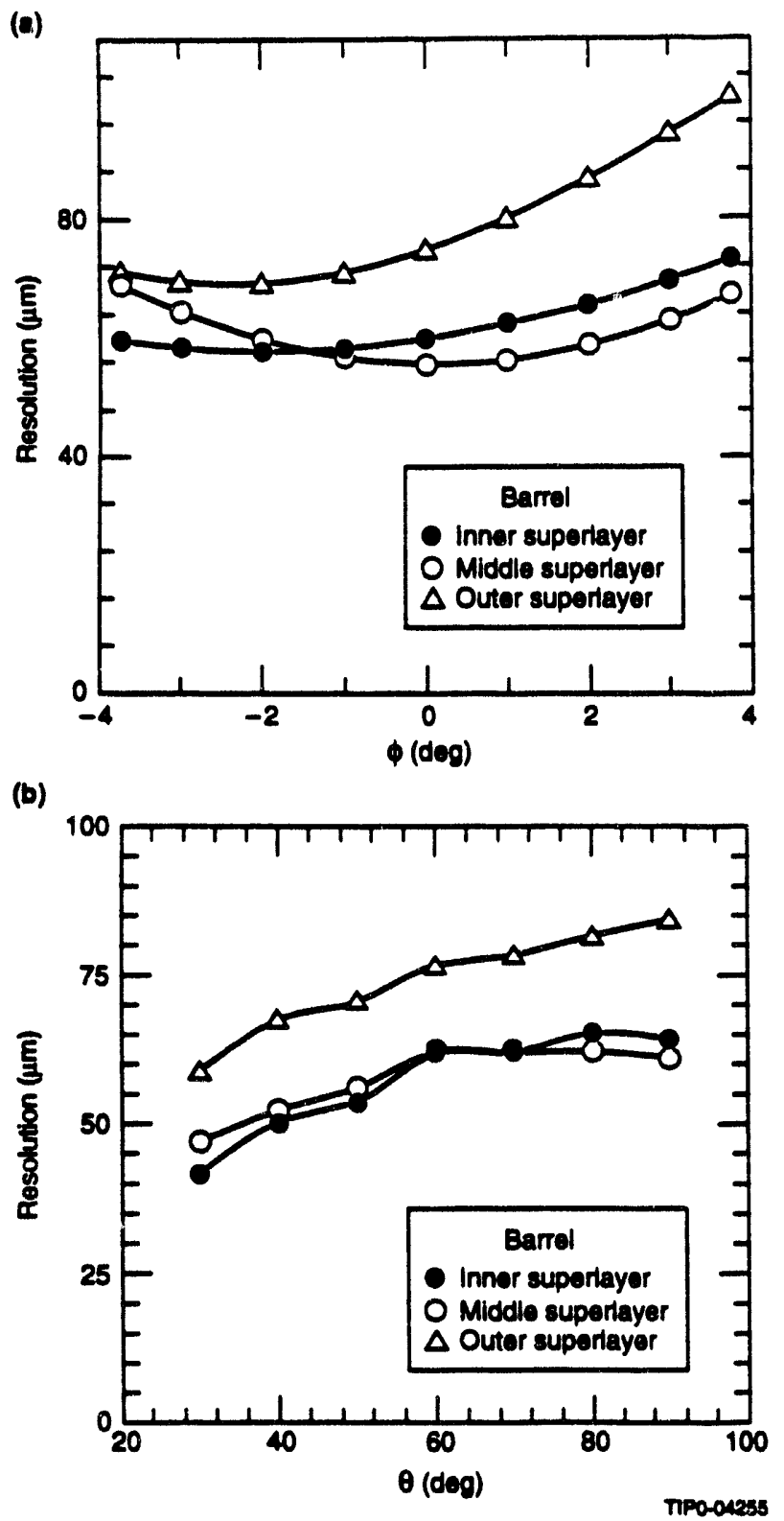

FIG. 4-32. CSC resolution in barrel: a) vs $\phi$ and b) vs $\theta . B=0.8 \mathrm{~T}$. the track and the nearest anode wire. For tracks with a sufficiently large polar angle $\theta$, the primary ionization electrons are collected by two adjacent anodes mitigating this geometric effect.

The design of the endcap chambers has been studied in a Monte Carlo model that incorporates all these effects. The endcap chambers have 24 -fold (a)

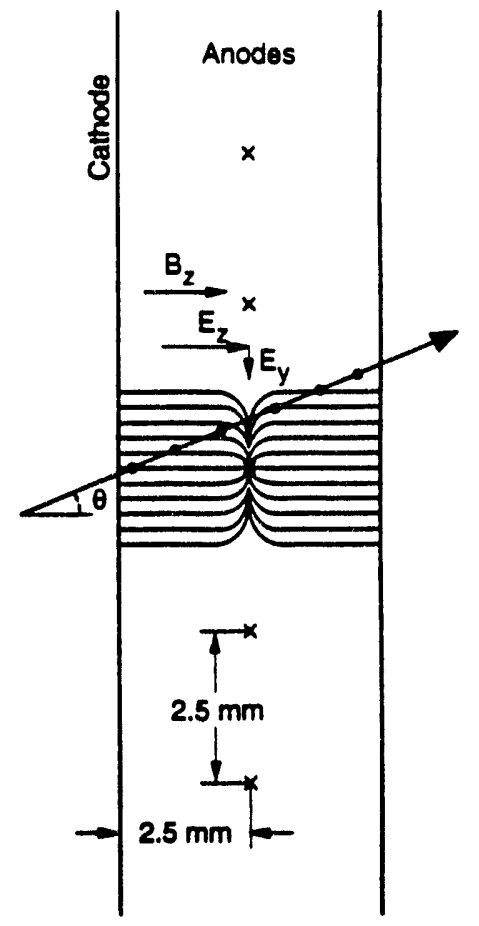

(b)

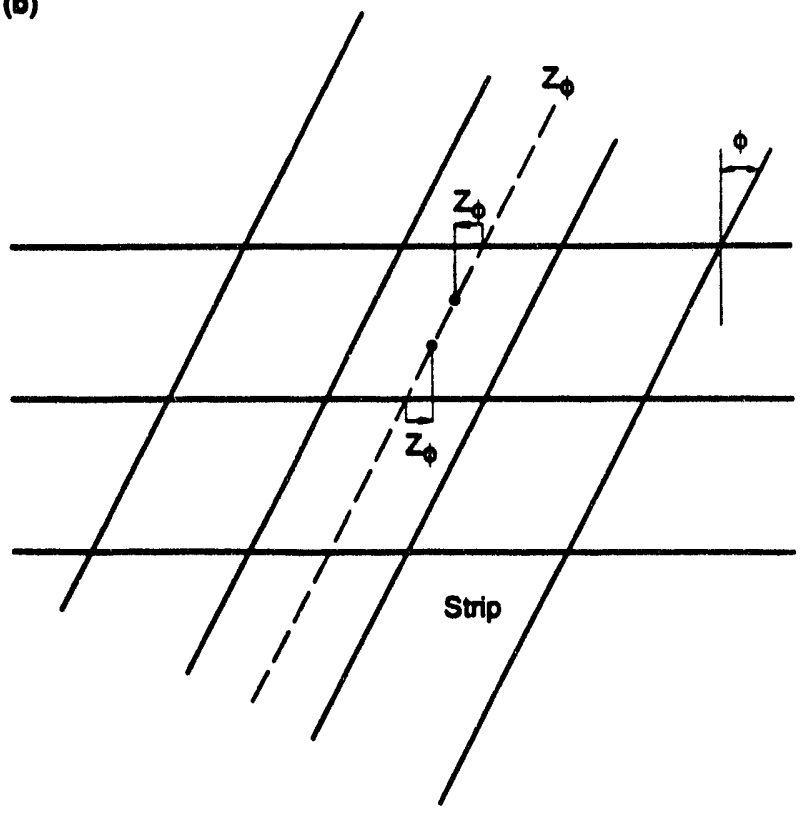

TP-04359

FIG. 4-33. Geometric effects in the endcap CSC. 
symmetry in the first superlayer and 48-fold symmetry in the second and third superlayers. This limits track inclination effects to an acceptable level. Recall that to compensate for the Lorentz effect in the barrel one has to rotate the wires about an axis along the magnetic field by an amount equal to the Lorentz angle. For the barrel this results in "tilting" the modules. The same principle applied to the endcap chamber results in rotating the anode wires about the axial component of the magnetic field in the plane of the anodes. The resulting resolution as a function of track inclination angles as predicted by the Monte Carlo is shown in Figure 4-34.

The predicted resolution of the chambers is well within the requirements set by our system performance goals. Measurements with cosmic rays in prototype chambers in zero magnetic field are in excellent agreement with the Monte Carlo predictions as shown in Figure 4-35. Verification of the Monte Carlo predictions by measurements in a magnetic field will be a key part of our P\&D program for the coming year.

\section{Choice of the Operating Gas}

There are three basic requirements that need to be met by a chamber gas suitable for a CSC-based muon system. These are:

- High drift velocity ( $>60 \mu \mathrm{m} / \mathrm{ns}$ )-for good timing performance,

- Adequate gain with minimum high voltage -for electrostatic stability of the wires,

- Small Lorentz angle $\left(<10^{\circ}\right)$-for good position resolution.

- Nonflammable-for operational safety.

Mixtures of $\mathrm{Ar}, \mathrm{CO}_{2}$, and $\mathrm{CF}_{4}$ have been found that satisfy these requirements. We have measured drift velocities and Lorentz angles for various mixtures of these gases. Figure 4-36 shows these results. ${ }^{7}$ The drift velocities are high and the Lorentz angles are adequately small. We have used these three-component mixes in many of the tests of prototype CSC. The option of adding xenon during alignment studies is also being considered if the development of the $x$-ray alignment system (discussed later) proves to be viable.
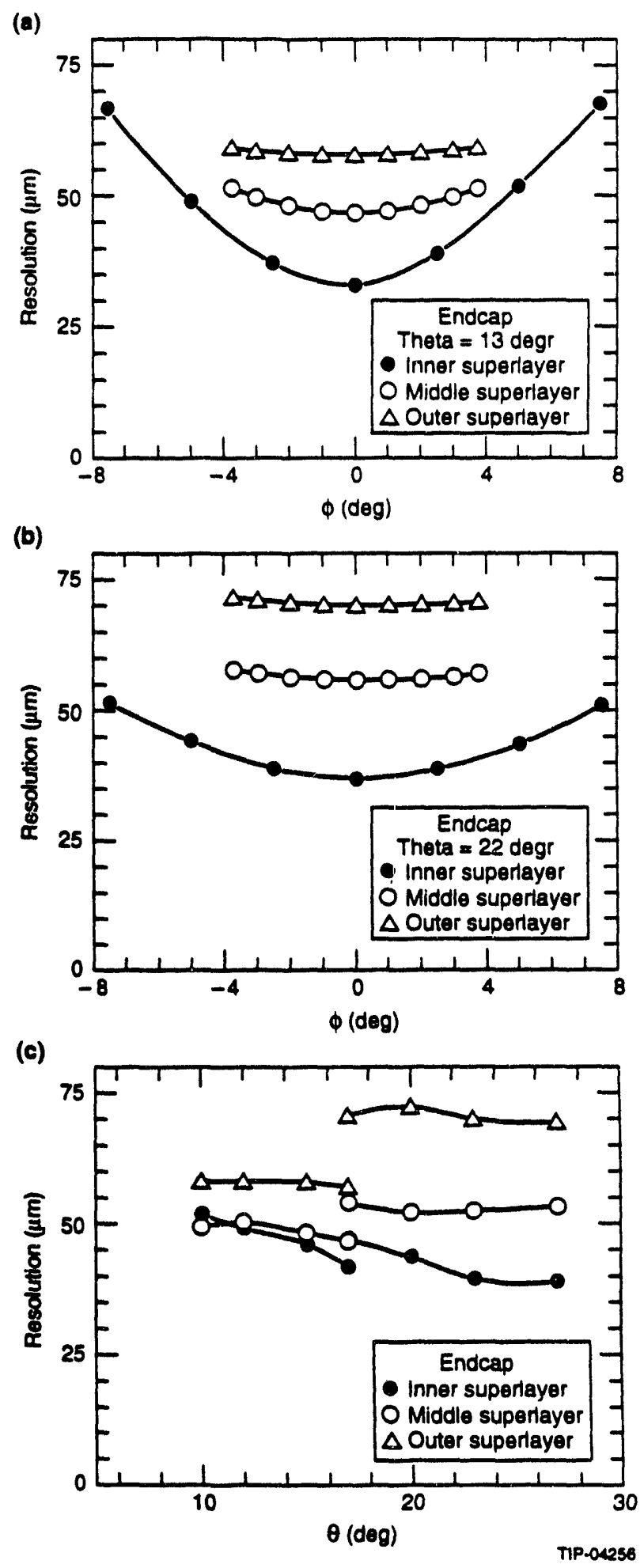

FIG. 4-34. Endcap CSC resolution a) vs $\phi$ for $\theta=13^{\circ}$; b) vs $\phi$ for $\theta=22^{\circ}$; c) vs $\theta . B=0.8 \mathrm{~T}$ 


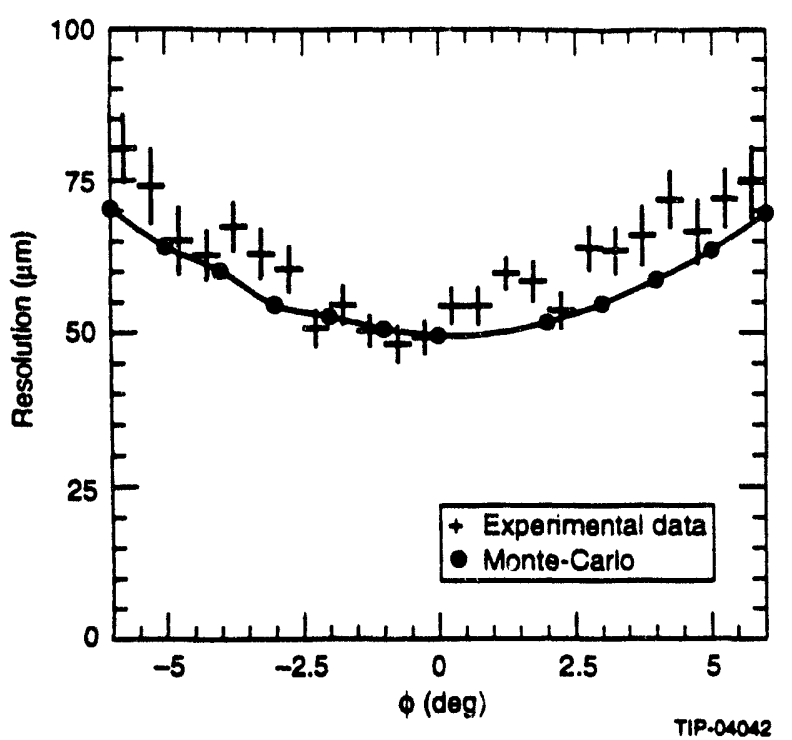

FIG. 4-35. The dependence of the spatial resolution as a function of $\phi$ for the $0.5 \mathrm{~m} \times 0.5 \mathrm{~m}$ BNL CSC prototype with Monte-Carlo prediction.

\section{Calibration}

Knowledge of the relative electronic gains in CSC strip channels at the $1 \%$ level is necessary to achieve the design spatial resolution of $75 \mu \mathrm{m}$. The calibration of the relative electronic gains must be performed in situ-in the electronic environment of the assembled, installed detector. Techniques for achieving the necessary calibration precision have been developed for a number of applications including calorimetry and silicon detector trackers in addition to cathode strip chambers. In our R\&D program, this issue was addressed specifically in the prototype chambers built at BNL. Precision calibration capacitors were built into the multilayer readout printed circuit board. The value of these capacitors was $0.65 \mathrm{pF}$ and their uniformity was measured with a precision bridge to be better than $0.5 \%$. Every fourth capacitor was connected to one of four calibration lines that was terminated in $50 \Omega$ The resulting four calibration lines were individually fed by the same precision pulser via a computer-controlled wide-band router. The trigger and amplitude of the precision pulser were also controlled by computer. At regular intervals, calibration data were taken by stepping the pulser amplitude through 30 values spanning the whole dynamic range in use. (a)

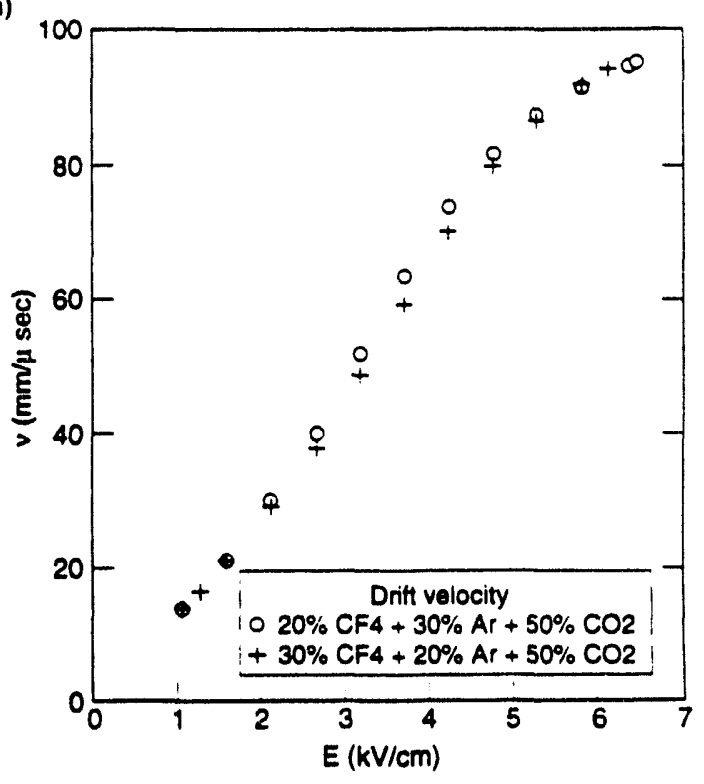

(b)

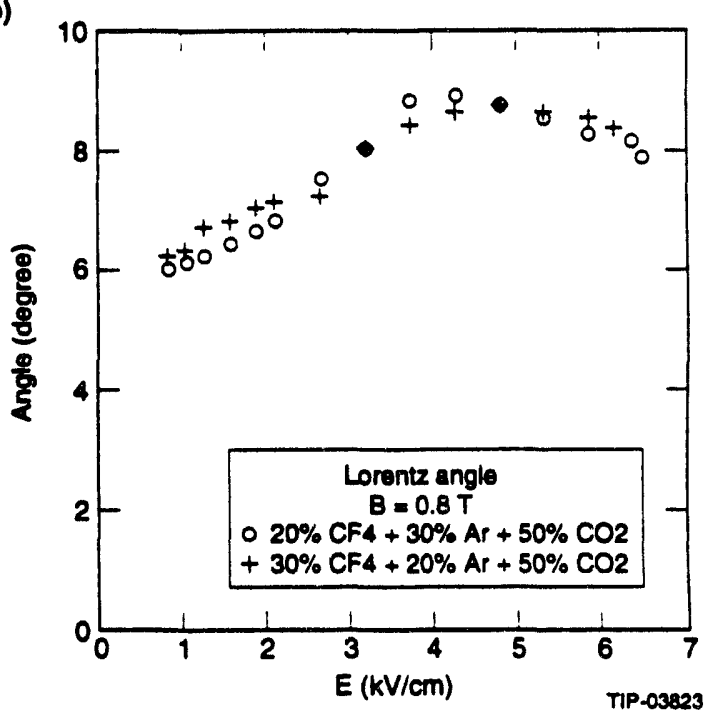

FIG. 4-36. a) Drift velocities and b) Lorentz angles for $\mathrm{Ar} / \mathrm{CO}_{2} / \mathrm{CF}_{4}$ mixtures.

A few hundred events were accumulated for every pulser value and the mean values and RMS deviations calculated for all channels. A third order polynomial was fitted to these values. The fit coefficients were recorded and used to correct the amplitudes during data analysis. To check this calibration procedure, a known pulse was injected in all channels. The values read back were corrected and the distribution of their variations from the nominal value had a relative width of $0.55 \%$. 
It is desirable to recalibrate the electronics frequently to check that the required accuracy is being maintained. However, the readout electronics of several of the prototypes tested at the TTR showed acceptable stability over periods at least as long as 36 hours. Our experience with these detectors gives us confidence that we will be able to achieve the $1 \%$ calibration precision goal.

A collection of physical and operating parameters for the CSC are given in Table 4-13.

\section{Results from the R\&D Program}

We have pursued an active program of CSC prototyping and development during the past year. A number of different test chambers have been evaluated in the cosmic ray Texas Test Rig (TTR) at the SSCL, at the RD5 muon test beam at CERN, and at the $1.7 \mathrm{GeV}$ proton beam at the Gatchina Synchrocyclotron. ${ }^{7.8 .35-39}$ We have also studied sensitivity of a CSC to neutrons, and tests with $x$-rays have been done to study details of chamber design. The detailed results of R\&D on CSCs are presented here.

Table 4-13. Typical characteristics of CSC.

\begin{tabular}{|c|c|c|c|c|}
\hline Parameter & Units & Superlayer 1 & Superlayer 2 & Superlayer 3 \\
\hline Wire spacing, $s$ & $\mathrm{~mm}$ & 2.54 & 2.54 & 2.54 \\
\hline Anode/cathode distance, $h$ & $\mathrm{~mm}$ & 2.54 & 4.0 & 5.0 \\
\hline Readout pitch, w & $\mathrm{mm}$ & 5.71 & 8.48 & 10.7 \\
\hline Gap between strips, $w_{g}$ & $\mathrm{~mm}$ & 0.400 & 0.400 & 0.400 \\
\hline Width of readout strip, $w_{r}$ & $\mathrm{~mm}$ & 1.05 & 1.78 & 2.38 \\
\hline Width of floating strip, & $\mathrm{mm}$ & 1.75 & 2.25 & 3.56 \\
\hline Capacitance of readout node & $\mathrm{pF} / \mathrm{m}$ & 50 & 50 & 50 \\
\hline Capacitance between strips & $\mathrm{pF} / \mathrm{m}$ & 48 & 48 & 48 \\
\hline Resistance between strips (typical) & M $\Omega$ & 20 & 20 & 20 \\
\hline Anode wire radius, $r_{a}$ & $\mathrm{~mm}$ & 0.015 & 0.015 & 0.015 \\
\hline Equivalent cylindrical cell radius, $r_{c}$ & $\mathrm{~mm}$ & 9.35 & 56.9 & 196.1 \\
\hline Wire capacitance per unit length, $C_{0}$ & $\mathrm{pF} / \mathrm{m}$ & 8.68 & 6.79 & 5.90 \\
\hline Operating Voltage (nominal gain) $5 \times 10^{4}$ & v & 2600 & 3400 & 4000 \\
\hline Electric field at either cathode, $E_{c}$ & $\mathrm{~V} / \mathrm{cm}$ & 5000 & 5000 & 5000 \\
\hline Electric field on wire surface, $E_{r}$ & $\mathrm{~V} / \mathrm{cm}$ & $2.7 \times 10^{5}$ & $2.7 \times 10^{5}$ & $2.7 \times 10^{5}$ \\
\hline Positive ion mobility, $\mu^{+}$ & $\mathrm{cm}^{2} / \mathrm{s} N$ & 1.27 & 1.27 & 1.27 \\
\hline Time constant, 6 & ns & 2.11 & 2.09 & 2.10 \\
\hline $\begin{array}{l}\text { Fraction of the avalanche charge collected by an } \\
\text { anode in } 30 \text { ns }\end{array}$ & & 0.21 & 0.17 & 0.14 \\
\hline $\begin{array}{l}\text { Fraction of the avalanche charge collected by an } \\
\text { anode in } 300 \mathrm{~ns}\end{array}$ & & 0.39 & 0.30 & 0.26 \\
\hline $\begin{array}{l}\text { Fraction of the anode charge induced on either } \\
\text { cathode }\end{array}$ & & 0.39 & 0.33 & 0.30 \\
\hline Total ionization (normal track) & ion pairs & 90 & 145 & 180 \\
\hline Minimum wire tension & gr & 50 & 97 & 151 \\
\hline
\end{tabular}


Most of the tests of CSC prototypes (as well as tests of prototypes of competing technologies) were performed at the TTR. The TTR has been built as a part of the GEM muon system R\&D effort. It consists of four planes of scintillator hodoscopes with timing resolution of about $300 \mathrm{ps}$; four planes of $1 \mathrm{~cm}$ pirch Iarocci chambers; and a $1 \mathrm{~m}$ thick stack of steel that absorbed cosmic rays with momentum less than about $1.3 \mathrm{GeV}$. The steel removes the low energy component of the muon spectrum, reducing the effects of multiple scattering. The steel can be magnetized to $15 \mathrm{kG}$ in order to raise the momentum threshold to $10 \mathrm{GeV}$, however this feature was not used in the measurements reported here. A VMEbased data acquisition system developed at the SSCL was used at the TTR. The triggerable volume of the TTR is large, with surface area of $1.2 \mathrm{~m} \times$ $4.5 \mathrm{~m}$ and a height of $3 \mathrm{~m}$, allowing performance studies of as many as six different chambers simultaneously, up to the largest chambers in the system. The TTR test setup for the CSC and RDT prototypes is shown in Figure 4-37.

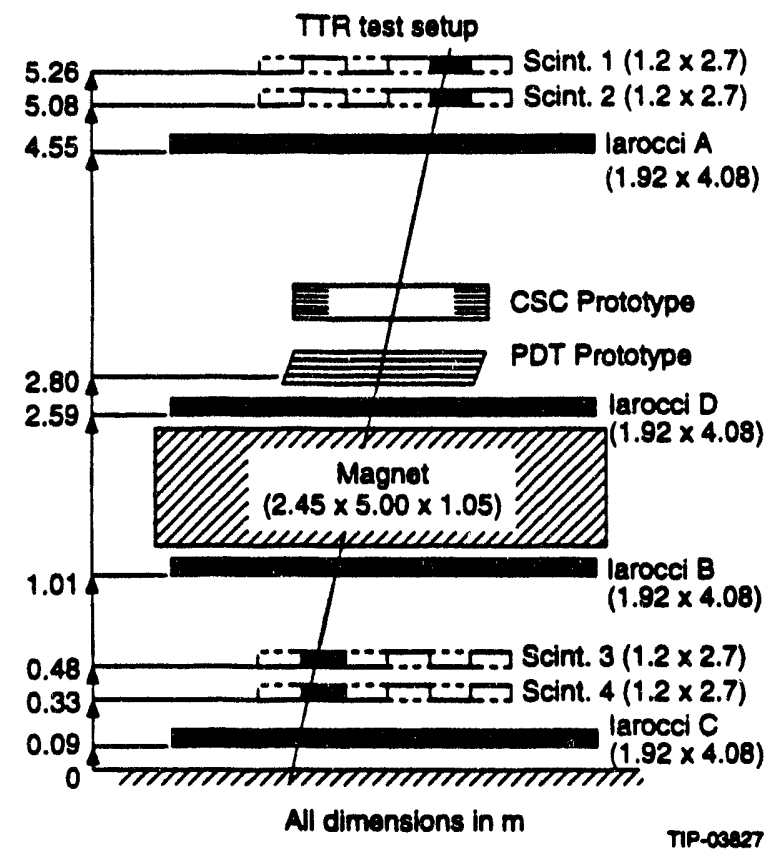

FIG. 4-37. SSC TTR schematic.

\section{Pulse Height Distribution and Efficiency Measurement}

The performance of the various prototype chambers has been extensively studied. Figure 4-38 shows the cluster charge distribution (i.e., sum of all contributing strips) observed using the BNL proto- type CSC at the TTR. The distribution exhibits the characteristic Landau distribution of a properly operating wire chamber. All tested chambers exhibited high efficiency ( $>99 \%$ ), again as expected of wire chambers.

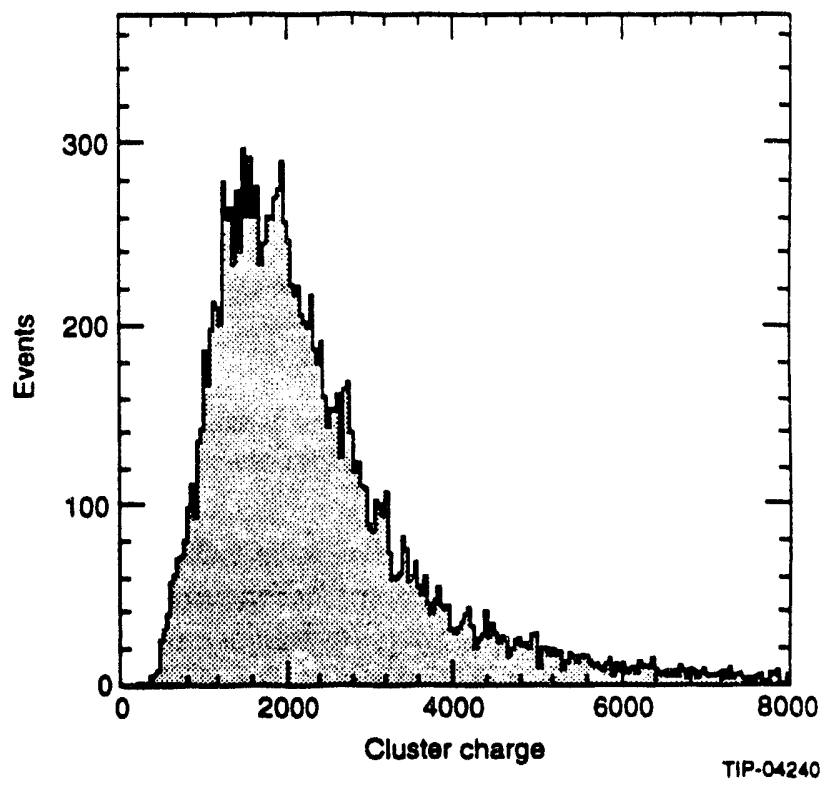

FIG. 4-38. Cluster charge distribution from single ionizing tracks measured in a CSC.

\section{Position Measurement Precision}

The position measurement in a CSC is derived by interpolation of the strip positions according to their induced charges. Figure 4-39 shows a scatterplot of the fraction of total cluster charge induced on one strip versus the distance of the muon hit from the center of the strip. The data were taken with the Dubna CSCs at the TTR. The figure illustrates the potential capability for position determination with a precision that is a small fraction of the strip width.

We have developed several algorithms in order to optimize the resolution of the position determined from the measured charges on the strips. Many results are reported in detail in the references. ${ }^{35-39}$ Here we summarize the performance in Figure 4-40, which shows the spatial resolution obtained for the different prototypes (with different designs, sizes, electronics and gas mixtures). Resolutions for these different CSCs were consistent with the expectations based on the observed noise performance of the electronics systems. The characteristics of the prototype chambers and their measured position resolution are summarized in Table 4-14. Spatial resolutions are given for tracks within $2^{\circ}$ of the normal to 


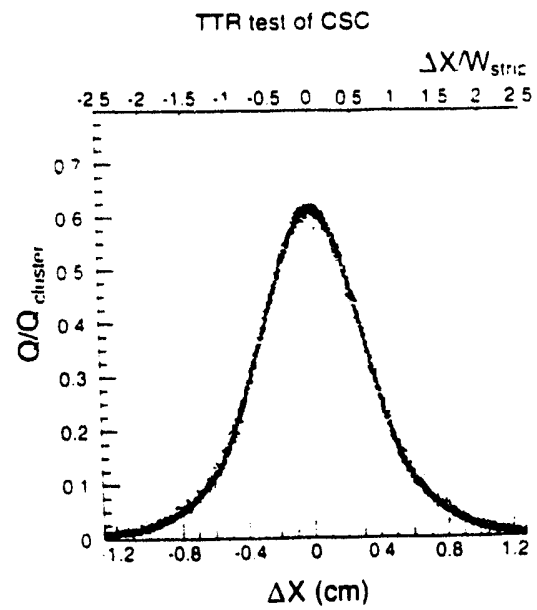

FIG. 4-39. Single strip fraction of total cluster charge as a function of the strip distance from the cluster center.

the cathode plane (in the coordinate orthogonal to the strips) and for tracks within $6^{\circ}$ of the normal. We note that the spatial resolution for tracks within the $\pm 3.8^{\circ}$ range of track inclination to be encountered in the GEM muon system is comfortably less than the $75 \mu \mathrm{m}$ resolution goal.

\section{Position Resolution with Nearby Hits}

As shown in Figure 4-39 a muon will induce significant charge on two to three strips. From this one could anticipate that in cases with several hits in the same CSC, the position resolution for each hit will be the single particle resolution (reported above) when the two hits are separated in the bend coordinate by at least several strip widths, but when the separation of the two hits (projected into the bend coordinate) becomes comparable to the strip width, the position resolution will degrade. However it will still be evident from the width of the induced charge distribution in most cases that there are two hits. When the two-hit separation becomes smaller than the strip width, the event will look like a single hit with large charge deposition and a somewhat broad charge distribution. In the limit that the two hits are very close together the event is equivalent to a single hit with a large charge deposition, and the position resolution of the merged hit will be somewhat better than the average single particle resolution.

These expectations have been borne out by detailed Monte Carlo simulations and by studies using data from prototype chambers. In the experimental studies, two-hit events were constructed by superimposing the measured charges from two
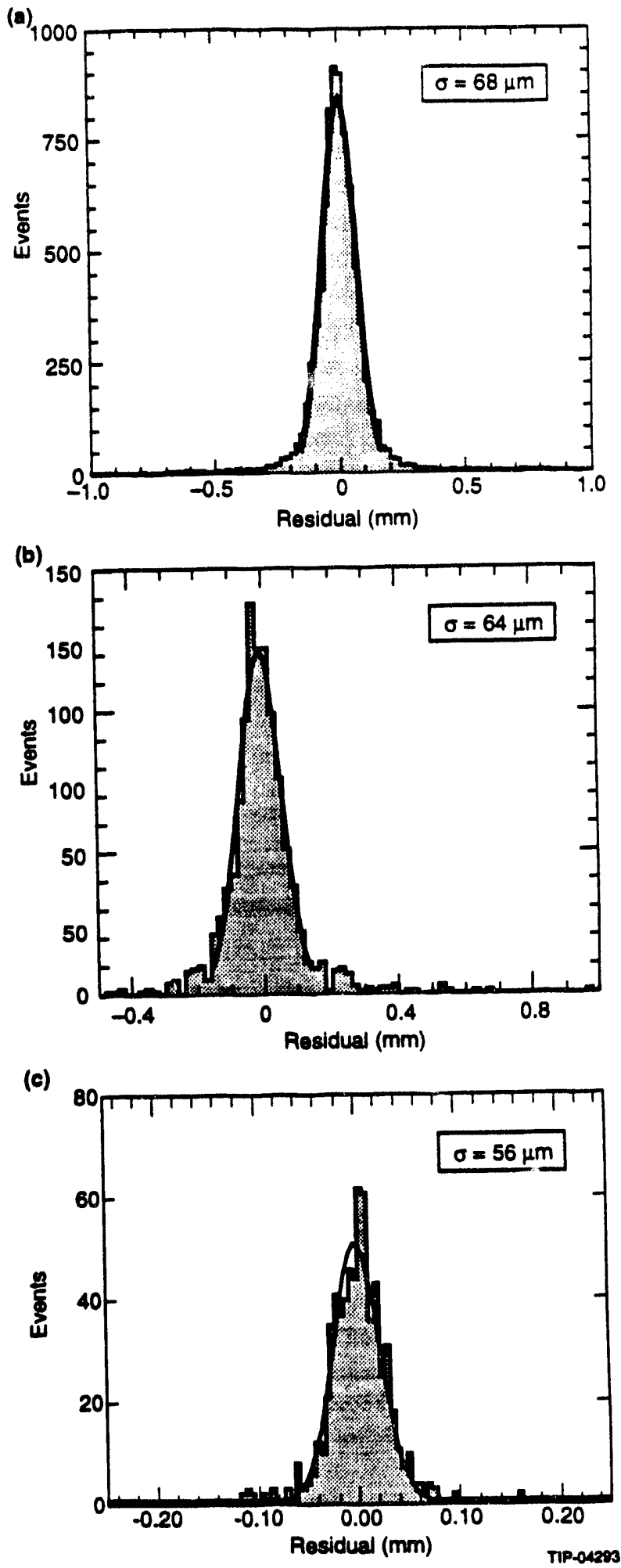

FIG. 4-40. The single layer spatial resolution: a) for $0.5 \mathrm{~m} \times 0.5 \mathrm{~m}$ BNL CSC prototype; b) for $1.5 \mathrm{~m} \times$ $1.0 \mathrm{~m}$ DUBNA CSC prototype; c) for $1 \mathrm{~m} \times 0.5 \mathrm{~m}$ open-profile Houston CSC prototype. 
different muon events. This overestimates the electronic noise that would be characteristic in a single event and so should be a conservative approach to estimating the two particle performance.

Table 4-14. The results of the CSC prototype tests.

\begin{tabular}{lccc}
\hline & Prototype & \multicolumn{2}{c}{$\sigma(\mu \mathrm{m})$} \\
& & $\left|\phi_{\mu}\right|<2^{\circ}$ & $\mid \phi_{\mu}<6^{\circ}$ \\
\hline BNL & $0.5 \times 0.5 \mathrm{~m}^{2}$ & 46 & 66 \\
BNL & $1.8 \times 1.2 \mathrm{~m}^{2}$ & - & $<95$ \\
DUBNA & $1.5 \times 1.0 \mathrm{~m}^{2}$ & 60 & 64 \\
Houston & $1.0 \times 0.5 \mathrm{~m}^{2}$ & 38 & 56 \\
PNPI & $0.5 \times 0.5 \mathrm{~m}^{2}$ & 43 & - \\
\hline
\end{tabular}

These superimposed events were analyzed with both a one-track algorithm and a two-track algorithm, and the algorithm giving the better fit was accepted. Figure 4-41 shows the efficiency of the single track hypothesis algorithm (unshaded) and the efficiency for accepting 2 tracks as a single track (shaded). As expected, the single track algorithm works well for separations down to the strip width. The data points in Figure 4-42 show the resolution versus two-hit separation as determined in this study. Data were taken from a prototype detector with $5 \mathrm{~mm}$ wide strips. The scale at the top of the figure gives the hit separation in strip widths; since the gas gap and strip width increase by the same amount going from the first superlayer to the second and third, the performance in terms of strip widths should apply to all three superlayers. The observed two-particle resolution matches the anticipated requirements in the GEM muon system.

\section{Precision of Etched Cathodes}

The position resolution of the CSC is derived from the lithographic precision of the placement of the strips. We have measured the accuracy of the placement of cathode features on several large cathode planes supplied by commercial vendors. Measured positions of strips and strip edges agreed with the design specifications to typically better than $15 \mu \mathrm{m}$, as illustrated in Figure 4-43: part (a) shows the difference of measured and specified positions of strip edges vs strip position for one of the BNL cathodes, and part (b) shows the difference distribution for one of the UH cathodes. The measurement errors were significant contributors to the widths of

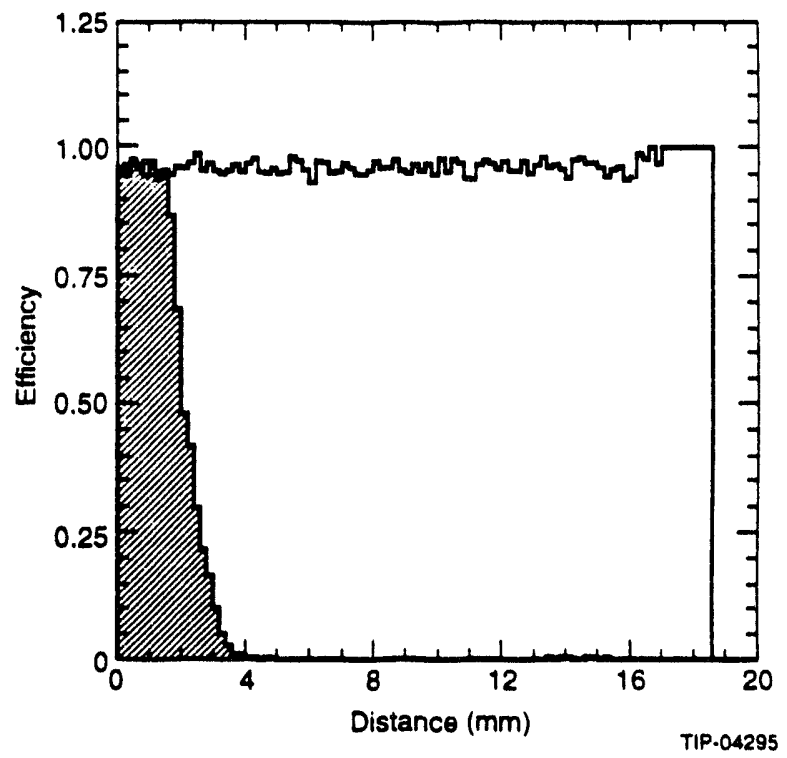

FIG. 4-41. Efficiency of track-finding algorithm in CSC for a single track hypothesis (unshaded). Shaded region shows efficiency for accepting 2 tracks as a single track.

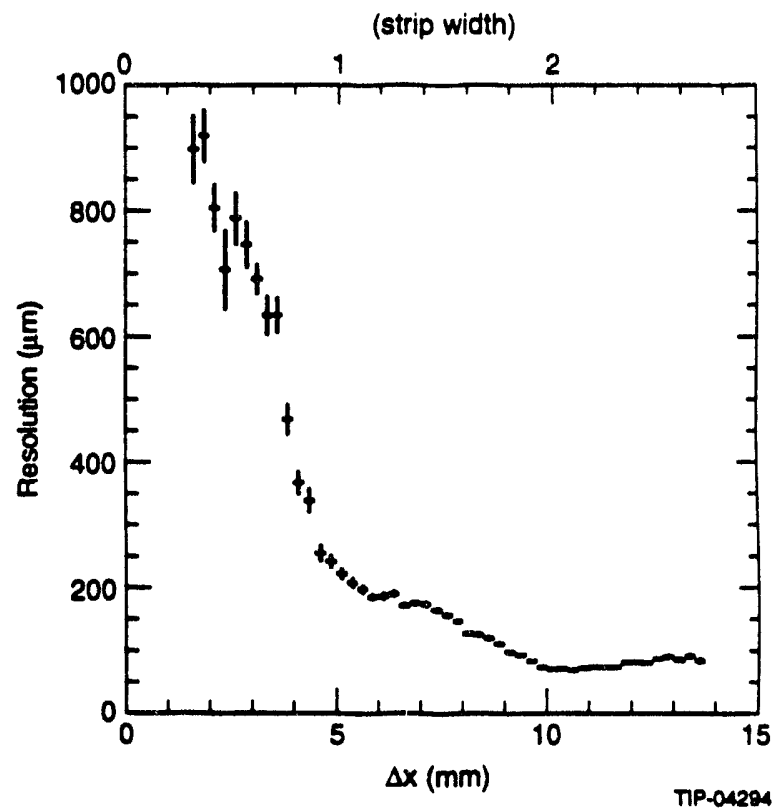

FIG. 4-42. Measured single track resolution as a function of distance from superimposed nearby track.

the distributions, but even so the precision of the etched cathodes is well within the systematic and random error budgets. (see for example Table 4-1).

\section{Anode Wire Readout}

The anode readout requires fast shaping ( $30 \mathrm{ns)}$ in order to provide trigger information and bunch crossing tagging. No amplitude measurements have yet been made on the anode signals in our R\&D program. We propose to include charge measure- 

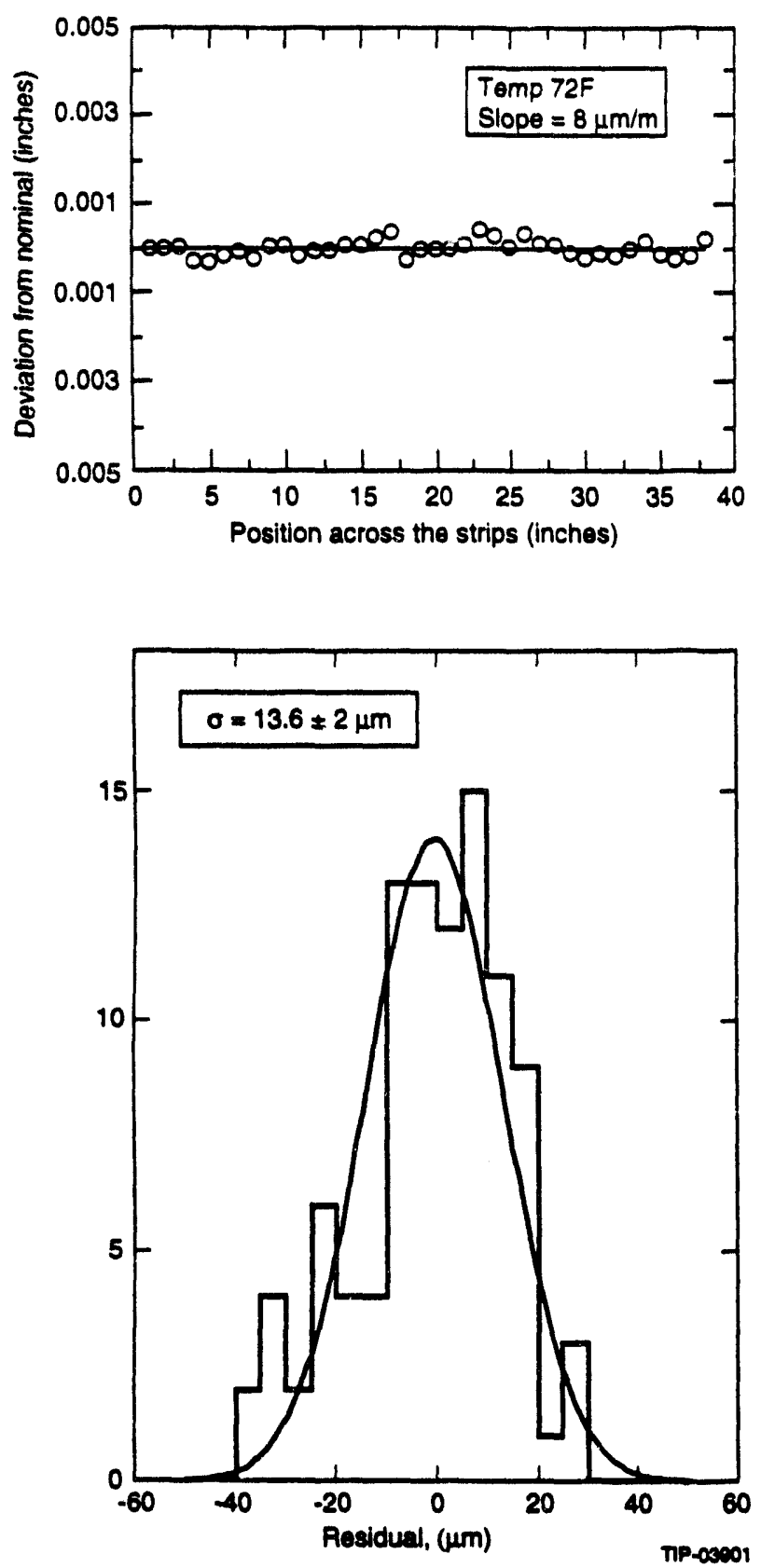

FIG. 4-43. a) Deviation of strip position from nominal for BNL cathodes; b) Difference distribution for UH cathodes.

ment with several percent precision (consistent with the shorter shaping time) for two-particle resolution, as previously discussed.

In our tests the anode wires are connected in groups of 20, providing $5 \mathrm{~cm}$ wide hodoscope elements. We use a bipolar, grounded-base amplifier (BNL Instrumentation Division, 10-354-2) and a 30 ns shaping amplifier (BNL Instrumentation Division, 10-638-01). These are implemented in thin film hybrid technology and are mounted on a printed circuit board on the detector. They are followed by on-board discriminators which provide a fast OR. The CSC is thus self-triggering, not requiring any additional detectors, for tests with sources, cosmic rays, or particle beams. In addition, the discriminator outputs are available for input into TDCs to obtain additional information during the tests, although TDCs will not be used in the final detector. Figure 4-44 shows the timing performance of a CSC measured in a proton beam at PNPI. Constant fraction discriminators were used to determine the stop time relative to beam scintillator counters. The OR of four layers has an RMS resolution of $3.5 \mathrm{~ns}$. This corresponds to better than $98 \%$ efficiency for correct determination of the beam crossing. These results imply an RMS resolution of less than $3 \mathrm{~ns}$ and beam crossing tagging efficiency of $>99 \%$ for a 6-layer CSC as specified in the GEM muon system.

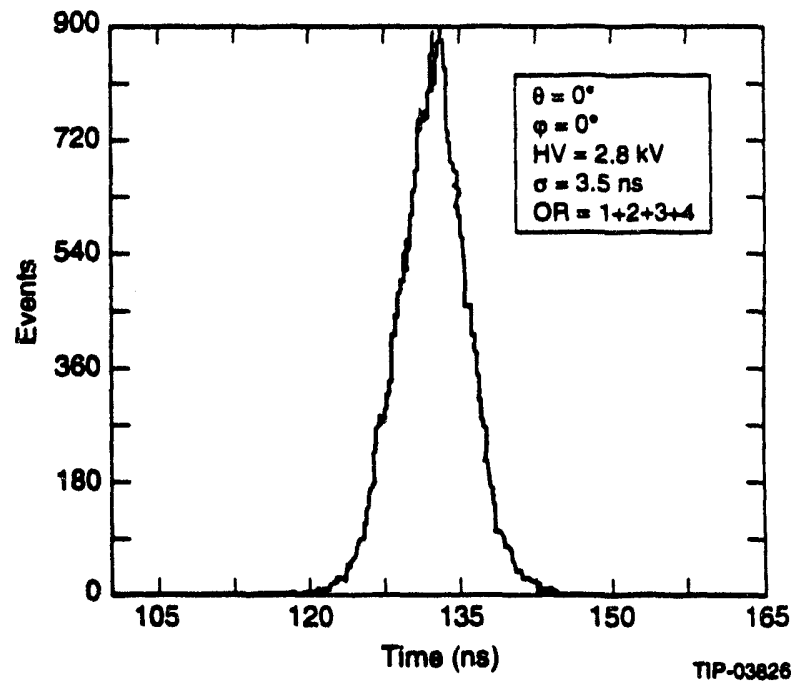

FIG. 4-44. OR of four CSC layers showing distribution of stop times.

Timing measurements have been made with capacitors added to the anode channels to emulate the effect of the largest anticipated wire ganging. Figure 4-45 shows the single-layer timing distribution for cosmic rays for different values of the channel capacitance; the largest capacitance is expected to be approximately $400 \mathrm{pF}$. The degradation of the timing resolution is acceptable.

\section{Chamber Construction}

The position and timing resolution of the CSCs is achieved by constructing chambers with multiple detector layers in a common package. Internal 


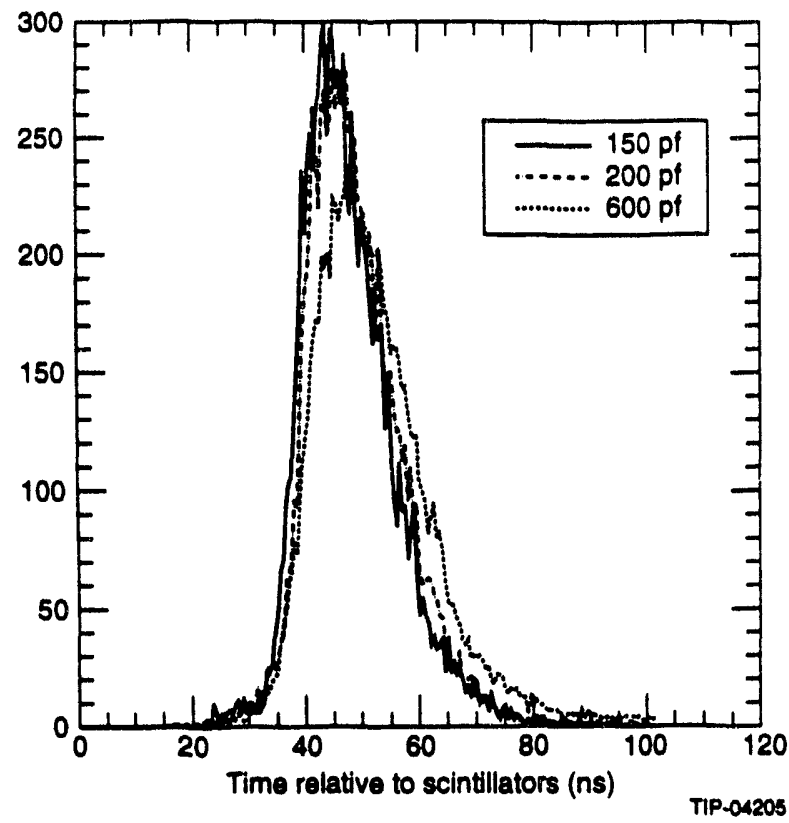

FIG. 4-45. Timing vs capacitance for single plane.

relative alignment of the individual layers is mechanically stable, and the precision of each layer is monitored so that the position and timing resolution of $N$ layers is $1 / \sqrt{N}$ times the single layer resolution. Chambers combine six individual detector layers in all barrel superlayers and in the outer two superlayers of the endcap muon system. For improved pattern recognition and rate capability, the inner superlayer in the endcap muon system consists of eight detector layers. Each chamber has common utilities, control, and DAQ connections. The complete chamber package is the fundamental unit for further alignment, relative to other chambers in the muon system and relative to the detector coordinate system.

Chambers in the barrel are rectangular and the endcaps trapezoidal. The trapezoidal shape improves the efficiency and the sharpness of the momentum trigger threshold due to the radial orientation of the cathode strips. The width (anode direction) of the chambers is limited by several factors. Lorentz effects and angle-dependent resolution have been described earlier. Electrostatic instability of the $30 \mu \mathrm{m}$ diameter tungsten anode wires limits the unsupported wire length to 1.2 to $1.5 \mathrm{~m}$, depending on operating voltage and gas gap. In addition, the size of available cathode materials, also in the range of $1.2 \mathrm{~m}$, and the limitations of technologies for applying the strip pattern provide a practical limit on the width. The length (strip direction) of the CSCs in the barrel is 3 to $4 \mathrm{~m}$, limited by noise from strip capacitance, material availability, and process limitations. In the endcaps, the charged particle flux below $\theta \sim 17^{\circ}$ further limits the strip length in order to keep the occupancies low.

Detector layers can be assembled to form a chamber using a bolted construction (strips on the cathodes can be routed around the bolt holes) with gaskets for the gas seals. To maintain gas gap tolerances and to prevent over-pressure damage, small spacers will be inserted in the gas gaps of the chamber layers. The stack of spacers through the chamber will be fastened by a tensioning rod that constrains the chamber components in the coordinate normal to the cathode planes by tying together the various planes.

It is intended that each CSC package be as independent as possible, to minimize possible electrical noise and external connections. For example, low voltages will be generated and distributed on-chamber using a single power feed. On-chamber processors will handle DAQ, monitoring, and control functions. Trigger primitives will be calculated and transmitted on a serial optical link. The cable plant will consist of about a dozen optical fibers and a single power input. The only other external connections will be for cooling water and gas. Water cooling is required because a six-layer chamber of typical dimensions will dissipate approximately 150 watts.

A conceptual CSC design is shown in cross section in Figure 4-46. This design hus evolved from several generations of prototype chambers and it continues to be developed as we optimize manufacturing and performance. The central requirement of the design is to meet specifications of flatness and rigidity of the chambers. This is accomplished by using panels made by laminating the cathodes onto a lightweight cellular core (e.g., Nomex paper honeycomb). A core thickness of about $2 \mathrm{~cm}$ is foreseen; epoxy-filled side frames will add additional support to the laminated panels to minimize sag. (In the endcaps, gravity-induced sag is not an issue.) 

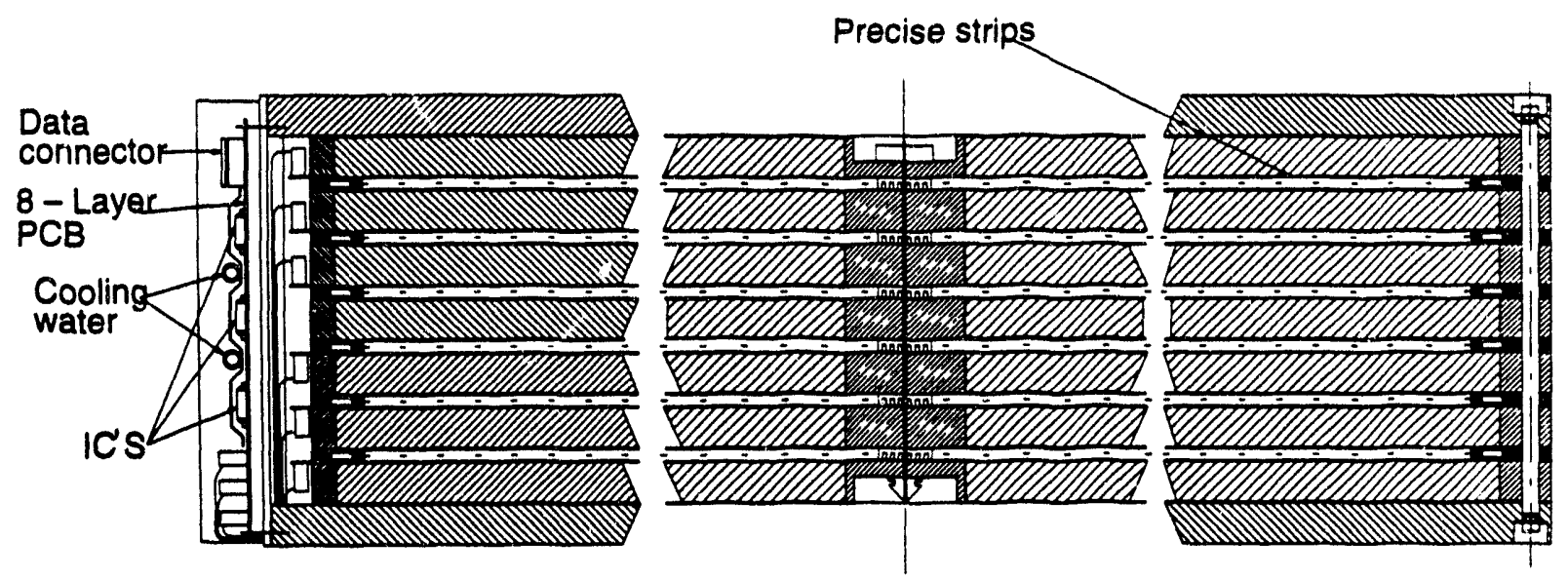

FIG. 4-46. Cross-section view of CSC showing materials and general structure.

An accounting of the nass and radiation lengths in the principal coniponents is given in Table 4-15 for a typical CSC. Each layer in the chamber has an active area surrounded by a frame that is inactive for detecting muons. Chambers are overlapped azimuthally to avoid acceptance losses and muons will occasionally traverse the frames of one chamber as well as the active area of another. For this design, muons traversing only the active area will pass through $6.2 \%$ of a radiation length. A small fraction of the muons pass through the gap frames and the panel edges of an overlapping chamber respectively. The average thickness in radiation lengths in the middle barrel superlayer, including the overlap, is $15 \%$. As part of our R\&D program, we are investigating possible reductions in chamber material, both in the active region and in the frame area.
The chamber design must meet stringent tolerance requirements in a number of areas. The gas gain of the proportional chamber varies with the spacing of the anode wires from the cathode planes. Considerations of electrostatic stability, plateau width for proportional response, and dynamic range of the readout electronics lead to a requirement that the gain variation over a chamber be smaller than roughly $\pm 50 \%$. This in turn places a flatness requirement ',$n$ the cathode planes and a thickness tolerance or the spacers and supports for the wire planes. Fig 1 re $4-47$ shows the measured gain shift versus varation of the anode/cathode spacing in a test chamber; the measurements were made by mechanically displacing one of the cathode planes. From this we conclude that the spacing of the anode wire planes with respect to the cathode planes should be constant within $\pm 150 \mu \mathrm{m}$.

Table 4-15. CSC material audit: thicknesses and radiation lengths.

\begin{tabular}{lclll}
\hline Component & Density $\left(\mathrm{g} / \mathrm{cm}^{3}\right)$ & $\mathrm{cm}$ Xo & Thickness $(\mathrm{cm})$ & Xo \\
\hline NOMEX core & 0.0288 & 1400 & $7 \times 1.9$ & 0.01 \\
Circuit Boards - G10 & 1.7 & 19.4 & $14 \times 0.05$ & 0.0361 \\
Copper cladding & 8.96 & 1.43 & $14 \times 0.0017$ & 0.0166 \\
Epoxy Panel Edges & 1.3 & 31.2 & $7 \times 1.9$ & 0.426 \\
G10 Gap Frames & 1.7 & 19.4 & $6 \times 0.8$ & 0.2474 \\
\hline
\end{tabular}




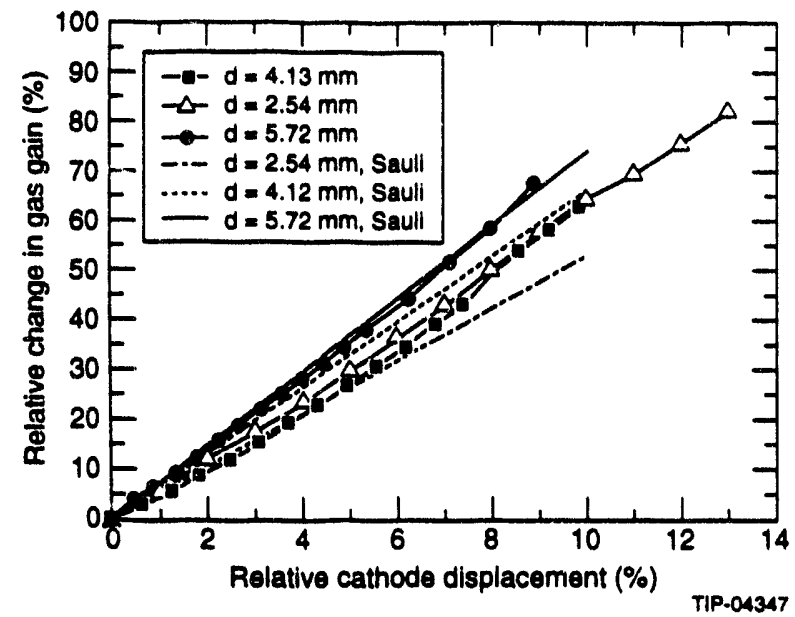

FIG. 4-47. Gain variation in a CSC vs fractional variation of the anode/cathode gap.

Wires are strung on printed circuit frames of precision thickness that are glued to the precision cathode. Tungsten/rhenium wires are tensioned to $\sim 170$ grams, which is $\sim 2 / 3$ of the elastic limit. They are held by glue and solder and the wire pitch is set by the fixtures during construction. The laminated panel of G10 surfaced honeycomb bears the load of the anode wires. The precision frames distribute high voltage and route anode signals to an edge connector. The nonprecision cathode lamination is attached to a frame fabricated with precise moldings produced with pultrusion technology. This frame controls the gas gap and provides access for electrical contact to the nonprecision cathode. Gas distribution is achieved through a manifold built into this frame. This approach provides a precise, low-mass, low-cost structure that does not need extensive machining. It allows accurate control of the important parameters and provides access to the precision cathode all around the perimeter for alignment.

The CSC packages are shown in perspective in Figure 4-48 (barrel chamber) and in plan Figure 4-49 (endcap chamber). The package design is still evolving as we learn from our prototypes and explore commercial manufacturing options. Some conceptual details of construction are shown in Figure 4-50, which depicts the mounting of the strip electronics and the distribution of cooling water, and in Figure 4-51, which depicts the mounting of the anode electronics, the high voltage distribution and chamber services.
The CSC modules will have a centralized electronics package for monitoring and control. This unit will provide regulated low voltages (and possibly high voltages) and will support the calibration, performance monitoring, and data acquisition systems. As shown in Figure 4-48, it will be mounted so as to be compatible with the positioning of the modules in an arrangement that maximizes coverage and resolution. We are studying the relative merits of services at the ends or on the faces of the CSC. This involves comparisons of loss of acceptance vs. loss of resolution due to additional scattering material.

\section{CSC Gas System}

The gas volume of the CSC system is approximately $150 \mathrm{~m}^{3}$, for the barrel and endcaps combined. The gas manifolds and distribution system will increase this volume significantly. The CSCs operate with a small over pressure. For economy, the gas flow will be small. These features will require careful design of the gas manifolds built into the chamber support frames. A conceptual design is shown in Figure 4-51; the gas system is discussed in more detail in Section 4.5.

\section{Cathode Plane Alignment}

In order to align each of the cathode planes in a chamber relative to each other, precision washers will be located at the corner of each cathode plane. Figure 4-52 shows the corner of a CSC cathode indicating the strips and the precision fiducial line. These will allow the planes to be easily referenced to a single precision surface that has been mounted perpendicular to the assembly table surface at the corner of the chamber. Onto this same assembly table other reference surfaces are added and used for the precision location of the projective alignment fiducials. Figure 4-53 shows the orientation of these surfaces relative to the corner reference plane. During assembly the fiducial holders will be pressed up against these surfaces to provide rapid, accurate placement. After proper positioning has been achieved, the holder will be bolted and epoxied into place. The location of these fiducials, using the holder's reference surface, will be optically measured relative to the cathode plane fiducial to validate the placement and to provide information that could be used at a later date to compensate for interlayer placement errors. 


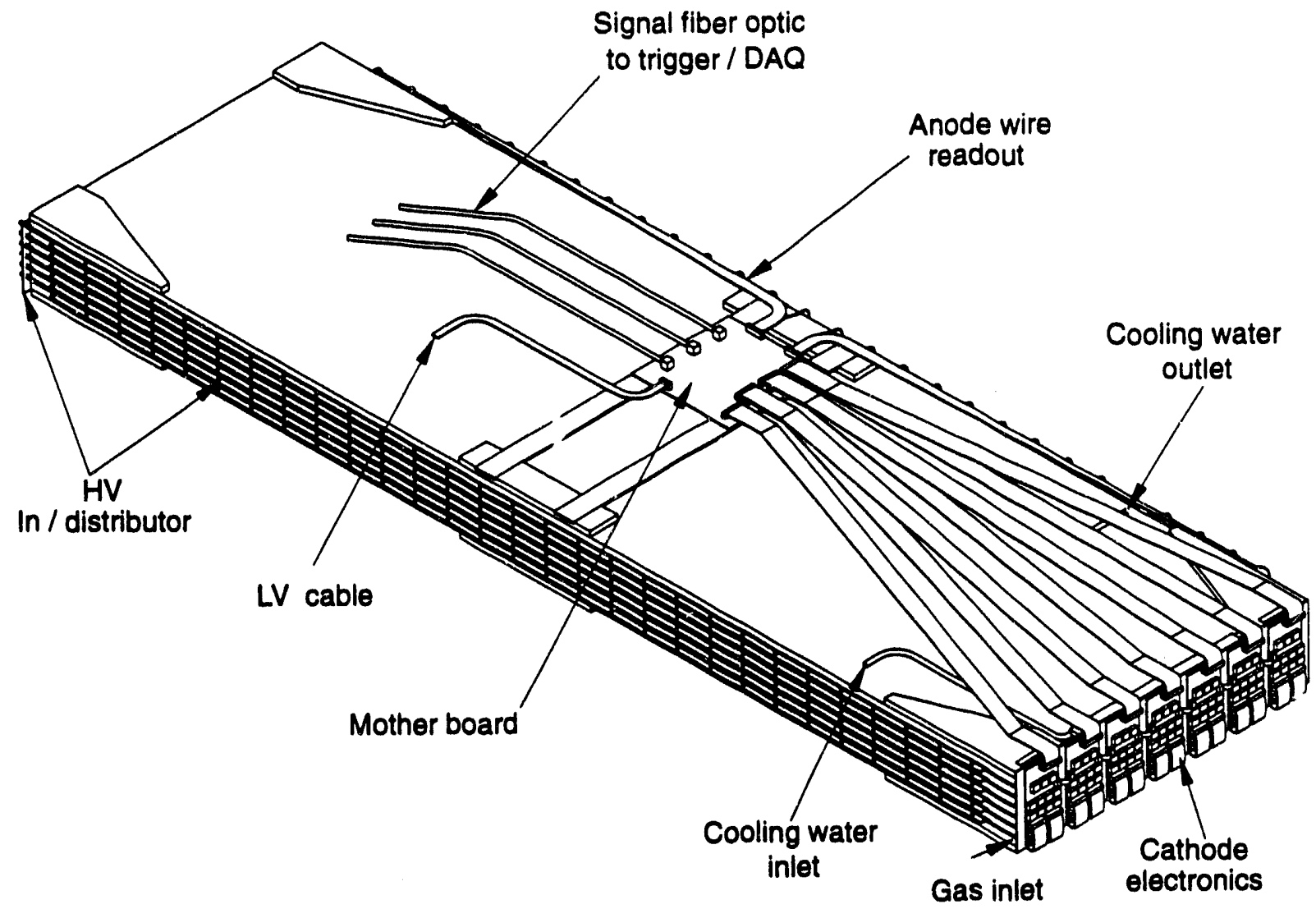

FIG. 4-48. Perspective view of complete CSC barrel chamber.

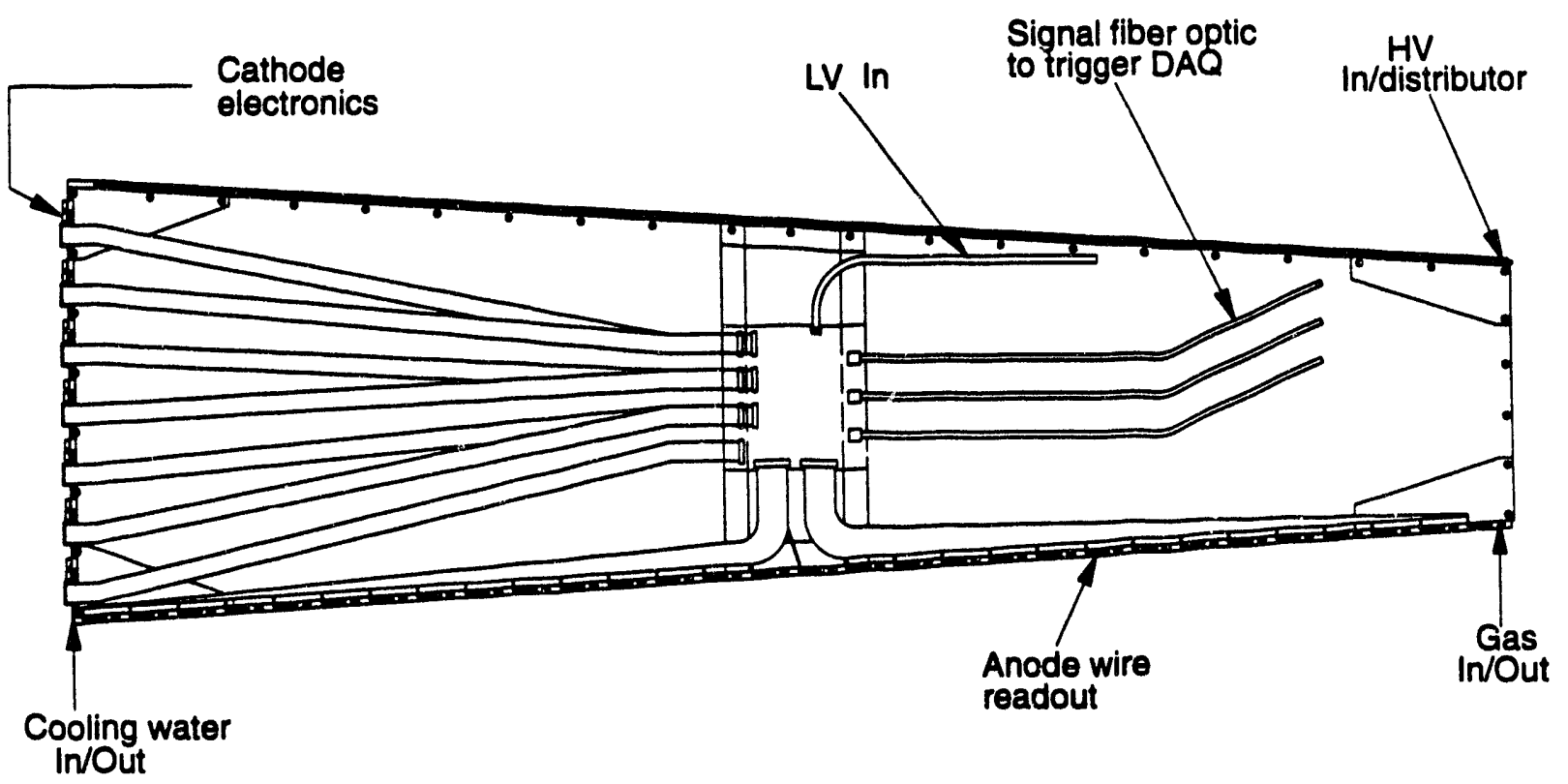

FIG. 4-49. Plan view of endcap chambers. 


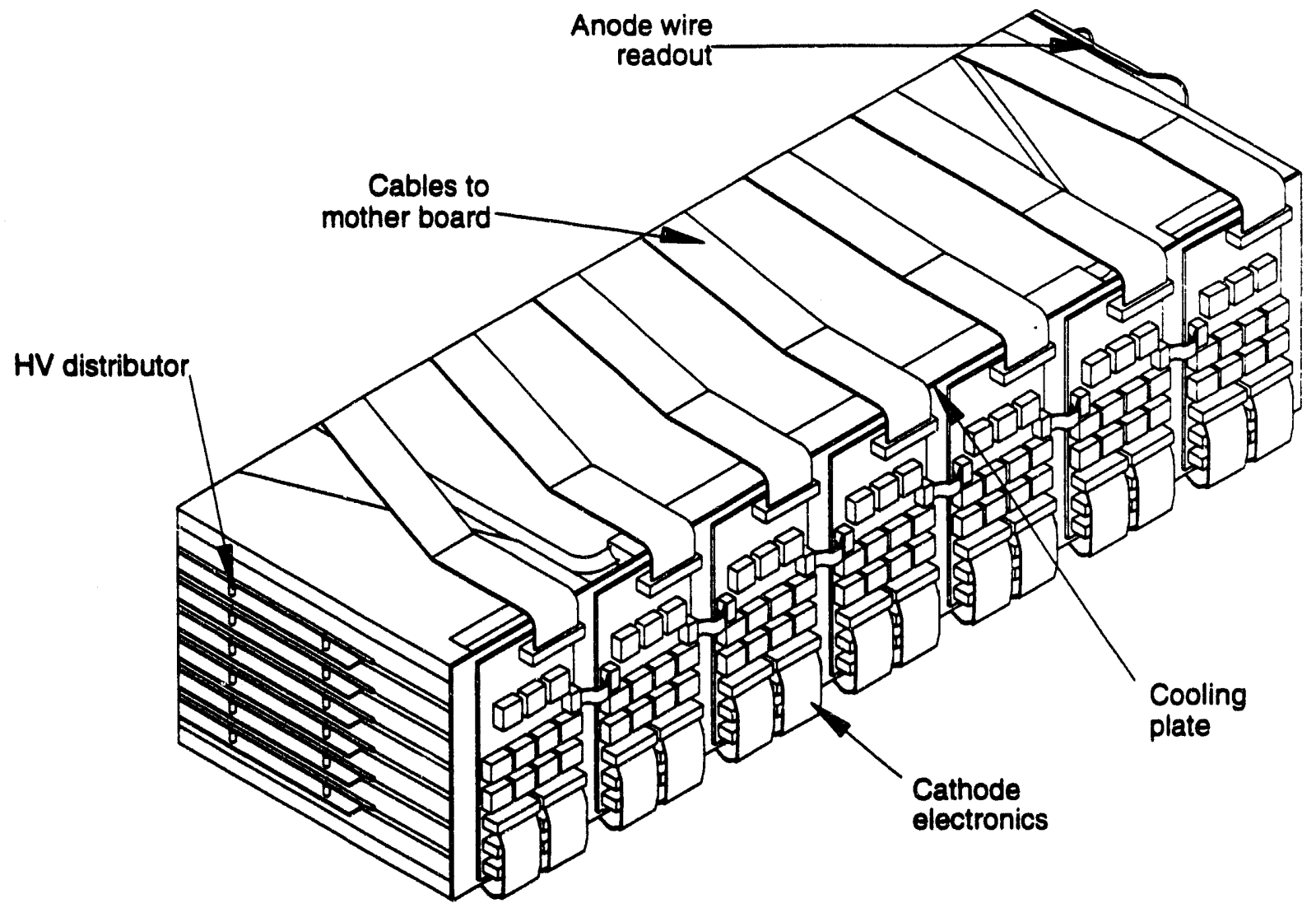

FIG. 4-50. Strip electronics and cooling concepts for the CSC.

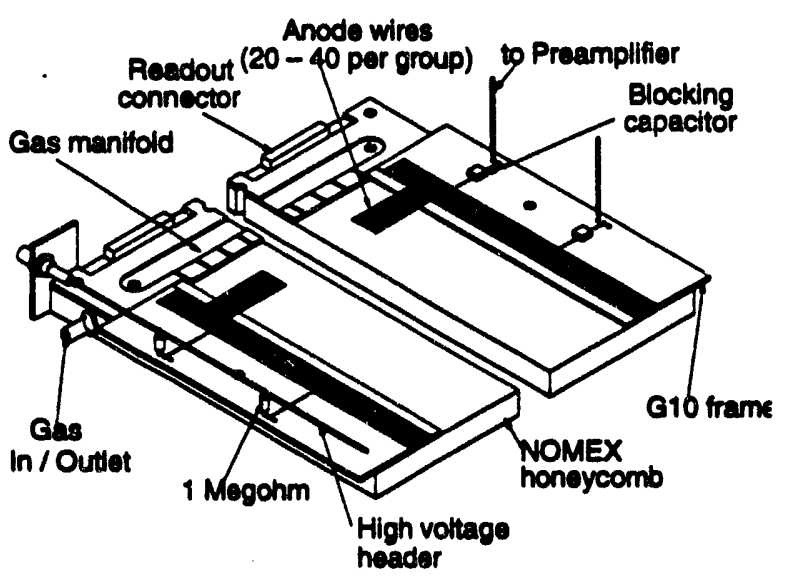

FIG. 4-51. Service layout for CSC showing gas connection, HV distribution and anode signal output.

\subsubsection{Support Structure}

\section{Overview}

The design of the chamber support structures is driven by the chamber construction and layout and by the need for the chamber weights to be carried at

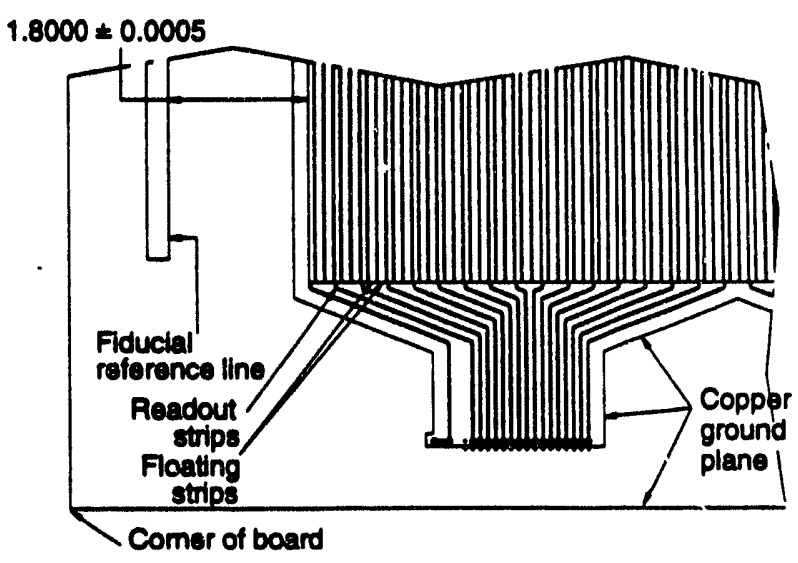

FIG. 4-52. Comer of a CSC cathode showing relationship of strips, readout connections and fiducial lines.

or near structure support nodes. The design has minimized the number of different chamber sizes to minimize chamber production costs. A structural stability of better than $25 \mu \mathrm{m}$ is needed between the respective superlayers of chambers within a muon projective tower. The muon chamber layouts have been designed to maximize the $\theta$ and $\phi$ coverage in 


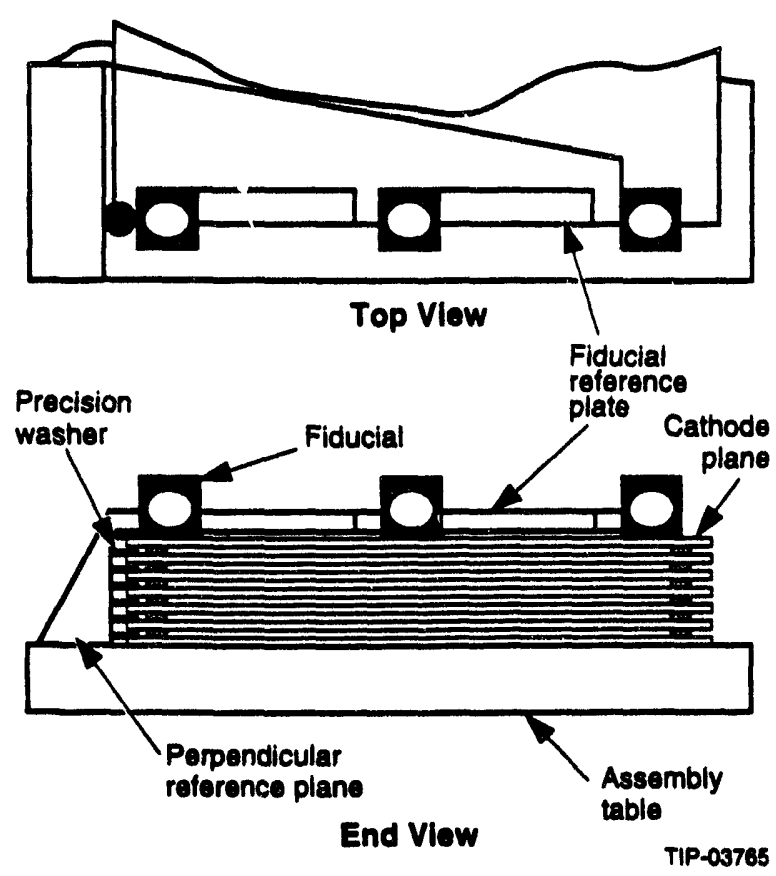

FIG. 4-53. Top and end views of a CSC package indicating fixturing for internal and external fiducial alignment.

conjunction with interface restrictions and projective alignment clearance requirements. The projective alignment lines of sight between the chamber ends requires $50 \mathrm{~mm}$ diameter optical paths. This requirement, in combination with chamber end losses due to frame materials and electronics, dominates the loss of coverage in $\theta$.

The muon system is divided into barrel and endcap regions. The barrel region is bounded by an inside diameter of $7.8 \mathrm{~m}$, an outside diameter of $17.8 \mathrm{~m}$, and the angles from the beam line $(\theta)$ of $29.8^{\circ}$ and $84^{\circ}$. There are $20 \mathrm{~cm}$ stay-clear regions on both the inside and outside diameters of the barrel. The barrel region, including support structure, extends from $z= \pm 0.4 \mathrm{~m}$ along the beam line from the IP to $z= \pm 16.5 \mathrm{~m}$. The endcap region boundaries are defined by the axial limits of $\pm 6.0 \mathrm{~m}$ and $\pm 17.15 \mathrm{~m}$ with projective angular boundaries from the IP of $9.4^{\circ}$ and $29.8^{\circ}$. Figure $4-1 \mathrm{a}$ is a quarter section elevation view illustrating these two regions with the support structure elements in relation to the other GEM subsystems.

The support structure for the barrel region is arranged into 12 identical modules per half for a total of 24 barrel region modules. Each of these modules is then further partitioned by a factor of 4 in order to provide the structure support nodes for the 48 -fold chamber segmentation in the barrel. These modules have chambers which overlap in $\phi$ and thus require an installation procedure which must proceed along the detector $z$-axis. Figure $4-1 \mathrm{~b}$ is an end view illustrating this $\phi$ overlap of all three superlayers of muon chambers as well as the support structure between the chamber superlayers.

The aluminum elements that make up these modules are bolted and pinned together to form an efficient truss structure to support the weight of the muon chambers. The 12 modules per end are merged with two end ring support structures to form a monolithic barrel structure. This monolithic structure is then inserted into the magnet and supported on four mounting points near the bottom of the end support rings, two points per end ring. The end support rings nearest the center of the detector, called the central detector support (CDS) end rings, are supported on the inside diameter of the magnet. The support ring structures at the ends of the magnet, called the forward field shaper (FFS ) end rings, are configured with an inside diameter larger than the magnet free bore to permit the linear insertion of the barrel modules through the FFS support ring. An elevation view of these structural elements is illustrated in Figure 4-1a.

This approach to the barrel structure was selected by an extensive evaluation of a pure monolithic barrel structure versus individual modules in the barrel. Conclusions reached in this study ${ }^{43}$ indicated that a composite design provided the optimum structure for the barrel. The support structure should have a modular character to 1) facilitate assembly of chambers into the structure, and 2) allow for the evaluation of elements of muon system as complete three superlayer assemblies. In addition, it was determined that it is vital to mechanically merge all of these modules into a monolithic structure to 1) improve the response of the structure to random vibrations, and 2) create a uniform and predictable structure deformation. The end support rings, in combination with intermodule attachments, create this desired monolithic barrel region assembly. The disadvantage of this solution is that there is a parallel structure in the adjacent module side frames, which adds a small amount of material in the active region of the muon sectors adjacent to the module side walls. 
Following the attachment and preliminary alignment of the 12 modules to the support end rings at the two points on each end of each module, the modules will be globally aligned and secured to both the end rings and to their respective neighbor modules. The result will be a monolithic structure with desirable performance for vibration modes (higher natural frequencies) and with uniform structural deformations due to subsequent system perturbations. In addition, this barrel region support structure will provide the mounting sites for the endcap muon support structures and also will provide the interface to the magnet for supporting the entire weight of each half of the muon system.

In the endcap region, chambers are overlapped in both $\theta$ and $\phi$. In addition, the endcap chambers are radially merged for regions of equal $\phi$ coverage in the endcap. This results in chamber units of $\phi$ coverage $2 \pi / 24$ for the inner superlayer and $2 \pi / 48$ for the middle and outer superlayers. As a result, the structure required for supporting the endcap muon chambers is quite different from the barrel. The structure proposed for the endcap consists of three wheel-like structures, which support the three superlayers of chambers, and two conical trusses, which tie the three wheel assemblies together. Figure 4-54 illustrates one entire endcap chamber array with its support structure.

The endcap assembly will be designed to use the conical portion of the FFS as an installation fixture. The endcap and FFS assembly will be moved together into the magnet to make the attachments with the barrel region structure. The endcap muon system will then be transferred from the FFS and independently mounted to the barrel region structure. The barrel region structure is attached to the most stable points on the ragnet, which is on or near the magnet legs (Figure 4-55). These mounting sites will provide good access and good isolation from ground motions and magnet turn-on perturbations (compression of magnet cryostat along the $z$-axis). They also reduce the need for realignment of the muon system whenever a magnet half is moved.

Access to the muon system for maintenance is possible in two configurations: 1) when the magnet halves are separated, since they will move together as a single assembly or 2) when the FFS itself is withdrawn from the end of the magnet, exposing the inside conical diameter of the endcap region. With the current barrel region chamber layout and the requirement of independent mounting systems for the chambers, the barrel region structure is quite dense with structural elements. This limits the available space to permit access for maintenance. In addition, the chamber layout in the barrel makes it very difficult for a person to actually reach the chamber in the layer furthest away in a given superlayer. Concepts under consideration to provide access are to either install permanent walkways and footholds in key access areas on the support structure, or provide attachment points for temporary light-weight staging hardware. The temporary hardware could be installed to provide access to regions not covered by the permanent fixtures. Further design concepts to improve access are described later.

\section{Support Structure Design}

\section{Barrel Region Muon Support Structure}

The basic support structure concept first described in the GEM LOI remains the baseline structure design. This is an aluminum truss structure which utilizes pinned and bolted clevis joints as well as welded truss subassemblies to minimize the number of joints. The truss elements are tubular with fittings welded to each end of these tubes. This clevis joint construction minimizes moments placed on the joints due to tensile and compressive loads. This design concept facilitates the remote fabrication of complete truss assemblies, along with their partial disassembly for shipping, and finally reassembly at SSCL. Hardware items used to secure the individual joints consist of nuts, bolts, Belleville washers and Whistler Pins ${ }^{\otimes}$. These items will be fabricated from 18-8 PH stainless steel, a high-strength precipitation hardened alloy that is nonmagnetic, or from beryllium copper alloy. Structure truss elements will nominally be $100 \mathrm{~mm}$ diameter with a $6 \mathrm{~mm}$ wall thickness 6061-T6 aluminum tubing. End fittings will be fabricated using the same alloy and welded, stress relieved, and heat treated for long term stability. Selection of this specific alloy of aluminum was based on low cost, availability, ease of machining, weldability and heat treatability. It is also available in a wide variety of forms, and it exhibits very low, long term creep characteristics. Standard 


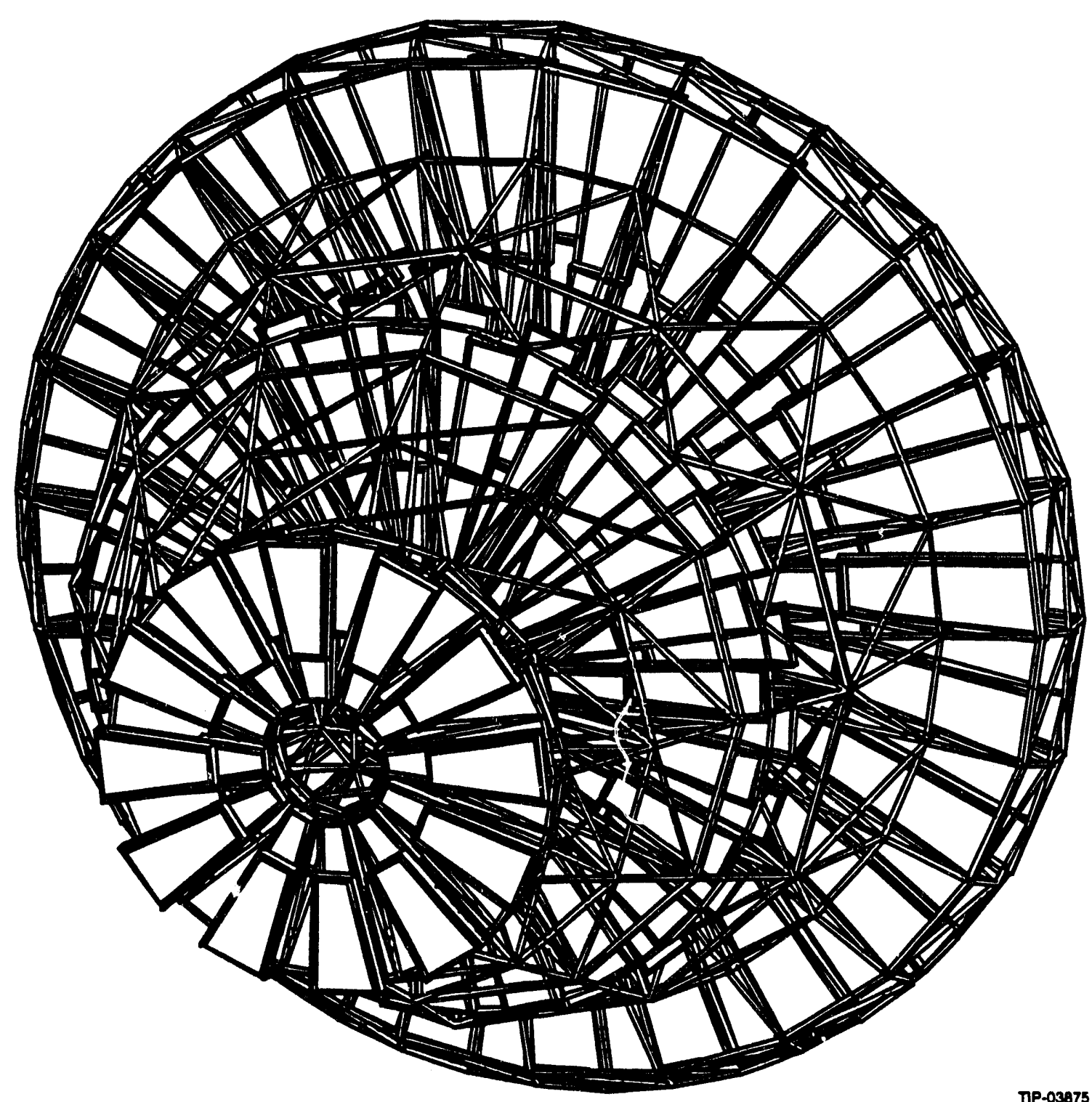

FIG. 4-54. Perspective view of one endcap array.

tubing wall thicknesses for this $100 \mathrm{~mm}$ OD tubing ranges from $3 \mathrm{~mm}$ to $12 \mathrm{~mm}$. This permits design optimization of specific truss elements without affecting the outside interface dimensions of the truss elements.

A side view of the configuration of the truss and the chamber layout for a barrel region module is illustrated in Figure 4-1. Our chamber layout in the barrel, incorporating $\phi$ overlap of the chambers, limits the options for support structure designs. In order to merge the truss structures, which lie stween each two superlayers of chambers, radial tie-plates will be used. The tie-plates join the two truss structures and occupy a portion of the gaps between the middle superlayer chamber ends along the axial direction.

\section{Endcap Region Structures}

The endcap chambers overlap in both $\theta$ and $\phi$. In addition, the chambers covering a prescribed $\phi$ region are physically tied together to permit a projective alignment system in the endcap region. In effect, this creates a single alignment tower covering two $\theta$ regions in the endcap. This configuration allows for placement of the support structure on the inner and outer $\theta$ limits of the region and in the space 

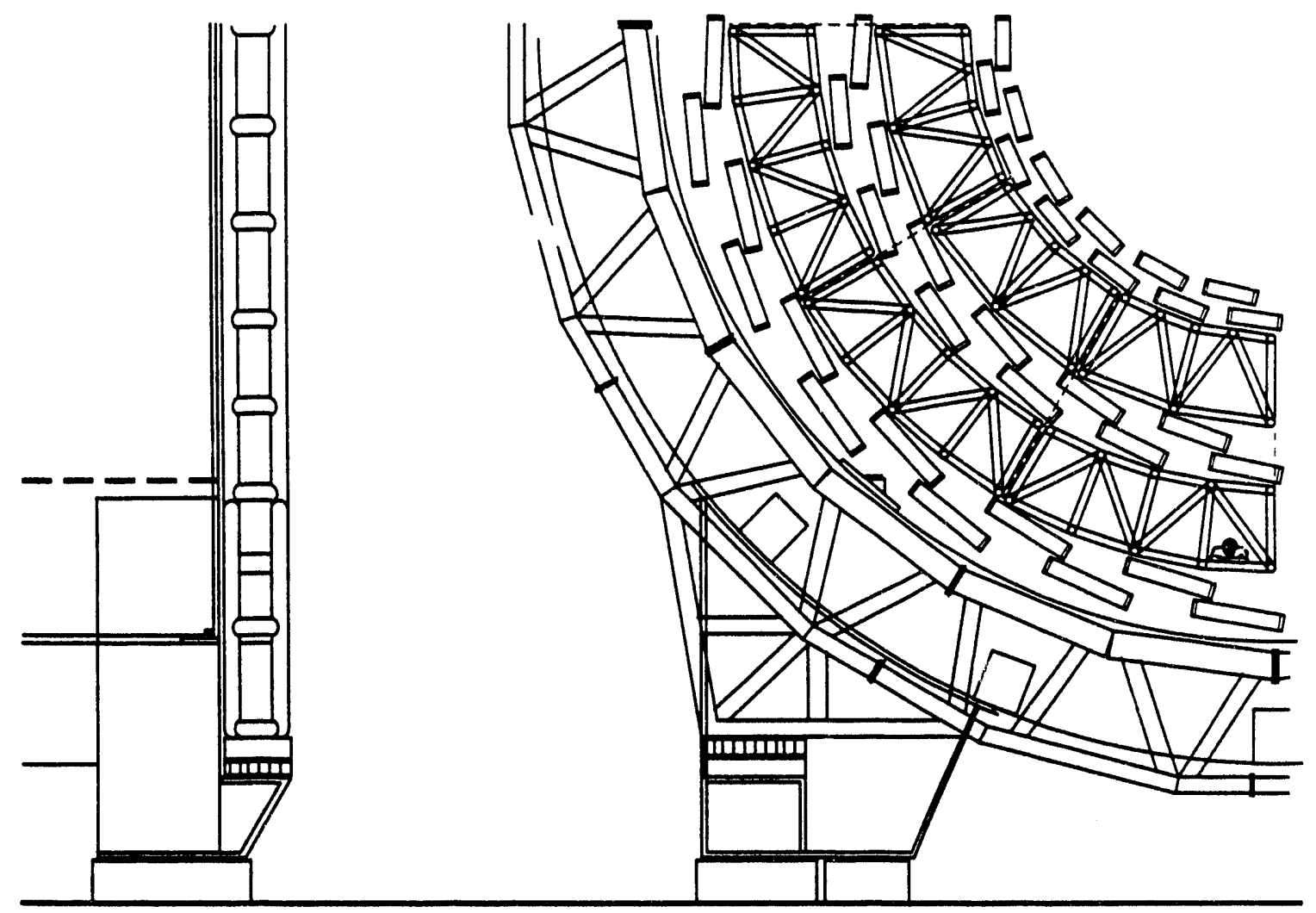

TIP.04236

FIG. 4-55. Side and end views of barrel monolith support on magnet.

between chambers. Given the thickness of the chambers and the desire to maximize coverage, the use of a module concept is no longer a viable solution for the endcap region.

The support structure concept for the endcap region consists of three wheel-like structures, which support the weight of the chambers for each of the three superlayers. Two additional conical truss structures tie the three wheels together into a monolithic structure. The overlap of chambers in $\phi$ is accomplished by mounting adjacent $\phi$ chambers on opposite sides of a given wheel structure in the axial direction. The outer superlayer wheel is illust ted in Figure 4-56. Basic construction techniques used in these support structures will be identical to those described in the barrel region section except that the endcap structure is monolithic by design rather than a modular assembly joined to form a monolithic structure. When assembled with all the chambers and properly aligned, this endcap assembly will be attached to the barrel region structure already mounted inside the GEM magı_c. This will be done utilizing a four- point mounting system capable of orienting the entire endcap into the desired attitude with respect to the IP.

\section{Muon System Coverage \\ Barrel Region $\theta$ Coverage}

We have determined the coverage of the muon system in both $\theta$ and $\phi$ 'or several configurations. The coverage is limited by nonmeasuring (dead) regions of the chambers such as the chamber frames, the space for the projective alignment system optical paths, the space required for support structures, servo-control systems for positioning the chambers, and the necessary clearances between all elements for services such as gas, water and electronics.

From $\theta=90^{\circ}$ to $9.75^{\circ}$ the lost $\eta$ coverage is 0.35 . There is a gap from $\theta=90^{\circ}-84^{\circ}$ associated with the CDS. A portion of this gap is used to house the CDS end support ring structure. It appears possible to cover a significant portion of this particular gap from $88^{\circ}$ to $84^{\circ}$ with at least one set of chambers to prevent a total loss of coverage in this area. Given the current CDS design, some $\phi$ regions 


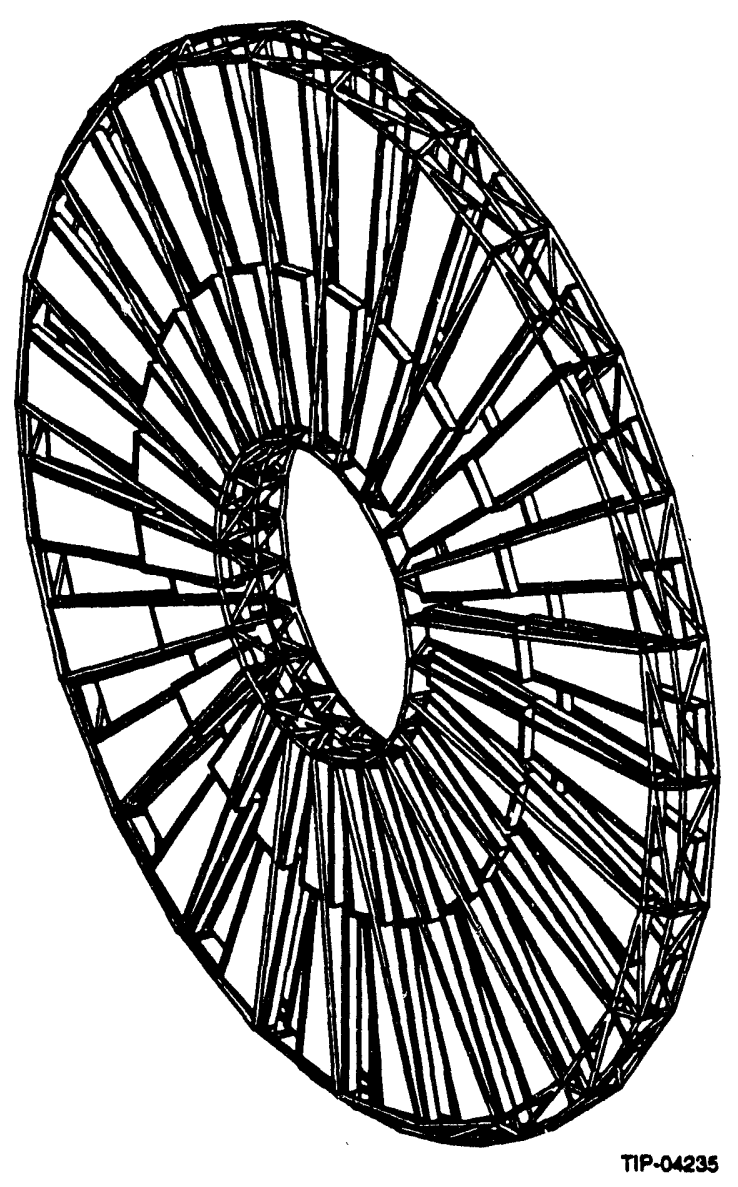

FIG. 4-56. Isometric of endcap outer wheel.

on the top of the detector can be made to completely cover the gap. There are three additional gaps within the barrel, which result from inactive chamber end hardware and the space needed to accommodate the projective alignment system. These gaps, while not desirable, do provide the necessary space for the support structure tie plates, and for the electronics and gas service lines.

The final gap in $\theta$ is the space between the barrel and endcap region. In this gap there are support structure elements for each region, the projective alignment paths for both regions, assembly clearance space between the regions, and space for utilities and services. It is difficult to provide three superlayer coverage for this $\theta$ gap. However, the endcap chamber layout has been configured to provide at least sing: thamber coverage for most of this $\theta$ gap by radially extending the outer chambers of the first superlayer endcap chambers, which are nominally located at $z=6.4$ and $7.0 \mathrm{~m}$. Further improvements in this region may require integration of barrel and end support structures, which will be investigated during final optimization.

\section{Barrel Region $\phi$ Coverage}

Acceptance in $\phi$ for the barrel is $100 \%$ with a minimum of $3 \mathrm{~cm}$ overlap between chambers in a given superlayer, except in the regions where there is no $\theta$ coverage. The muon momentum resolution of certain portions of the $\phi$ coverage is degraded because of multiple scattering caused by the chamber side frames, electronics, and support structure hardware. However overlap in $\phi$ for all chambers provides an opportunity to tie together adjacent chamber towers using muon tracks that pass through these regions. Typically, the $\phi$ overlap is higher than quoted above because only one width chamber is used per superlayer, which is driven by the overlap needed in the outer layer of chambers in a given superlayer.

\section{Endcap Region Coverage}

The endcap detector region is designed to have full $\theta$ and $\phi$ coverage. Edge effects of the chambers in $\phi$ decrease resolution because of multiple scattering in these overlapped areas from passive material in the chamber frames. Edge effects influence the total $\theta$ coverage since there is no overlap in this direction at the outer-most edges of the endcap superlayers. As in the barrel, there is opportunity to relate adjacent alignment towers through the use of coincident muon tracks in the overlap areas.

\section{Structural Analysis}

\section{Barrel Region Analysis}

\section{Mechanical Deformations}

Analyses of the proposed muon structures has been carried out at several levels. Since the barrel structure is made up of twelve modules and two end ring support structures, these individual structures have been evaluated for four different cases:

- On their own merits with simulated loading.

- As a function of angular orientation with respect to gravity for the modules.

- As a partially completed assembly to simulate the problems associated with the assembly process loading.

- As a complete assembly with all connections made to create a monolithic barrel structure. 
Specific analysis performed on these models evaluated deformations, stresses, buckling factors, vibration modes, response to random vibrations, and temperature gradients. The last two studies have been performed on structures similar, but not identical, to the ones we are currently proposing. The work on random vibration responses led us to require that the muon structures be monolithic at final assembly. This minimizes the sagitta errors caused by the deformations induced by random vibrations. Random vibration response calculations for the proposed baseline structures will be performed, but we expect that our preliminary conclusions will be sustained.

These calculations indicate that maximum deflections for the barrel modules, for the case of independent barrel sectors, will be less than $7 \mathrm{~mm}$ in any orientation, and Von Mises stresses less than $5 \mathrm{ksi}$ for $11000 \mathrm{~kg}$ barrel module weights. Specifically, for nodules inserted at the 12 and 3 or 9 o'clock positions, the respective maximum axial stresses are 3 and $4 \mathrm{ksi}$. The gross weight of a barrel region assembly is $152200 \mathrm{~kg}$, which consists of $80700 \mathrm{~kg}$ of chambers and $71500 \mathrm{~kg}$ of structure weight.

Buckling factors for the modules for the worst-case mounting configuration (side-mounted modules) indicate that these elements will fail if gravity loading is increased by a factor of 4.4 . All truss members will be optimized to meet the buckling strengths by using various wall thickness tubing in these locations as required.

\section{Vibration Analysis}

The first 5 modes of vibration for the barrel region modules have natural frequencies in the range of 6.7 to $20 \mathrm{~Hz}$. Specific truss elements within the modules have their lowest natural frequencies around $28 \mathrm{~Hz}$. Without knowing the precise character of the potential input disturbances, it is difficult to make judgments at this time on the possible consequences of these frequencies. However, as a rule, higher frequencies are better. The lowest natural frequency is at $6 \mathrm{~Hz}$ for the module, which is a concern that we are addressing.

\section{Thermal Analysis}

Thermal sensitivity of the barrel region structures has been examined on a similar size monolithic structure. ${ }^{43}$ The effects of uniform temperature changes as well as thermal gradients across the detector were examined. A uniform temperature shift moves the center of the muon system with respect to the IP because the coefficient of thermal expansion is different for the iron magnet components and the aluminum muon structures. This shif: is estimated to be of magnitude $100 \mu \mathrm{m} /{ }^{\circ} \mathrm{C}$. A radial thermal gradient across the diameter of the support structure causes it to deform asymmetrically, resulting in possible sagitta errors. The magnitude of this effect on the monolithic barrel structure is a maximum deformation of $100 \mu \mathrm{m} /{ }^{\circ} \mathrm{C} / \mathrm{m}$, of which only a small portion will affect sagitta measurement. Temperature control of the order of $1^{\circ} \mathrm{C}$ is planned for the muon system volume. The gradients should in fact be much less than the $1^{\circ} \mathrm{C} / \mathrm{m}$. Finally, active straight line monitors on the muon projective alignment towers will indicate any such errors and corrections will be made in the calibration.

\section{Endcap Region Analysis}

An analysis similar to that done for the barrel region is currently under way for the endcap structure. There is a high degree of confidence that we can meet our required design goals based on previous analyses of similar structures.

Maximum deflections for the endcap region truss structures, and for the independent wheel assemblies, described above, are calculated to be less than $3 \mathrm{~mm}$. The axial stresses are less than $4 \mathrm{ksi}$ in the orientation that places the axis of the wheels horizontal with four support points on the inside diameter and two support points on the outside diameter of these wheels. This configuration simulates the assembly loading. Buckling factors for any of the three wheel assemblies is currently at a level of a few $g$ 's. Other orientations were examined for the wheel structure itself under chamber loading. Deflections were calculated to be several centimeters, but the stresses were seen to be low for the case where the wheel axis is vertical. Thus, the wheel can be built flat on the floor and then raised safely to vertical to load the chambers on either side.

Deformation, stress analysis, and modes of vibrations have been examined for an entire endcap assembly assuming the four point mounting system to the barrel region structure. Here, the maximum deformations are $4 \mathrm{~mm}$ and the maximum stress levels are $4 \mathrm{ksi}$. The gross weight of an endcap 
assembly is $40200 \mathrm{~kg}$, which consists of $26200 \mathrm{~kg}$ of chambers and $14000 \mathrm{~kg}$ of structure. An evaluation of structural natural frequencies indicates frequencies from 5.2 to $17.0 \mathrm{~Hz}$ for the first 20 vibration modes of the endcap region assembly. Specific truss elements within the : embly had their lowest natural frequencies arou. $\lrcorner \mathrm{Hz}$.

The thermal sensitivity of the endcap region structures has not yet been examined, however, because the structures have similar diameters and structural detail, similar responses to those seen for the barrel are expected. Given the anticipated thermal environment inside the detector there will be no thermal deformations that cannot be compensated by the planned alignment systems.

\section{Improvement of Solid Angle Coverage}

Several schemes are being considered to improve the solid angle coverage of the muon system. It should be pointed out that all the loss of coverage occurs in the barrel region for $|\theta| \geq 30^{\circ}$ and not in the endcaps, where we have overlap in both $\theta$ and $\phi$.

A straightforward improvement is to cover the region around $|\eta|=0$, where the CDS is located. In the present design of the muon system we have no coverage from $84^{\circ}$ to $90^{\circ}$, corresponding to a $\Delta \eta$ loss of 0.11 units. In this region, chambers can be placed to cover about $64 \%$ of $2 \pi$ in the azimuth.

Another location where some coverage may be gained is in the transition region between the barrel and the endcaps. For each endcap about $2.5^{\circ}$, corresponding to a $\Delta \eta$ of 0.09 , is not covered by three superlayers. This region is more problematic since some gap is inherent to our design of a separately constructed barrel and endcap. Also occupying this space are the projective alignment paths, which must make penetrations in the region for both the barrel and the endcaps. One option that will allow some coverage of this region is to fabricate the support structure as a supermonolith with the barrel and the endcap regions tied together. In so doing, some of the support members in the $30^{\circ}$ region might be eliminated, and those remaining could be reconfigured to introduce some space where chambers could be located. However, a true monolith will be more difficult to construct and will require both the barrel and the endcap chambers to be available when the support structure is assembled.

The gaps between the barrel chambers along the magnet axis ( $z$-direction) are inherent to our design. We believe that the present layout is "conservative," allowing for stay-clears, alignment paths, access to the electronics, and cabling according to standard engineering practice. Nevertheless, we are considering several options to reduce the gaps needed for the alignment system discussed in the following section and illustrated in Figure 4-69.

\section{System Access Improvements}

Several strategies are being considered to improve the access to the detector.

To improve access to chambers internal to the support structure, one option involves making a "super chamber" where two chambers per superlayer of the present projective towers are tied together in a rigid frame. The chambers have to be attached kinematically at the nodes of the support structure. By making an effectively larger chamber the number of nodes of the structure can be reduced approximately $40 \%$. Further reduction of the amount of support structure may be obtained by using the supermonolith option discussed above. We will also investigate integration of the inner and outer barrel trusses, which should reduce material near the middle superiayer and also improve access. These studies are part of a continuous optimization program leading to final design and manufacture.

Access to both the barrel and the endcap regions from the outside may be gained by opening the magnet halves thereby allowing penetration through the central region. Such an access would not be simple and would require the re-establishment of the global alignment of the muon system with respect to the central tracker and the calorimeter. $A$ more attractive possibility is to retract the forward field shaper permitting someone to penetrate the system from the beam axis of the detector.

\subsubsection{Alignment}

The GEM muon system is aligned locally within a specially configured projective tower formed as a part of the CSC chamber layout, as well as globally with respect to the IP. The discussion 
below describes the local alignment between superlayers, which directly impacts the momentum resolution and is quite stringent. The global alignment of the projective towers to the IP, which determines the muon angle and momentum resolution at extremely high energies, where tolerances are much looser is covered at the end of this section. Tables 4-16 and 4-17 summarize local and global alignment and positioning tolerances for the GEM muon system. The meaning and methodology behind these quantities is summarized in the ensuing text.

Table 4-16. Requirements and resolutions assumed for local alignment

\begin{tabular}{|c|c|c|c|}
\hline \multicolumn{4}{|c|}{ Contributing to Momentum Resolution } \\
\hline \multirow{2}{*}{$\begin{array}{l}\text { Relative superlayer positioning } \\
\text { (wrt inner superlayer) }\end{array}$} & Middle superlayer & $\begin{array}{l}\Delta x \\
\Delta y \\
\Delta z \\
R x \\
R y \\
R z\end{array}$ & $\begin{array}{l} \pm 1.5 \mathrm{~mm} \\
\pm 1.5 \mathrm{~mm} \\
\pm 1.5 \mathrm{~mm} \\
\pm 1 \mathrm{mrad} \\
\pm 1 \mathrm{mrad} \\
\pm 3 \mathrm{mrad}\end{array}$ \\
\hline & Outer superlayer & $\begin{array}{l}\Delta x \\
\Delta y \\
\Delta z \\
R x \\
R y \\
R z\end{array}$ & $\begin{array}{l} \pm 3 \mathrm{~mm} \\
\pm 3 \mathrm{~mm} \\
\pm 3 \mathrm{~mm} \\
\pm 1.5 \mathrm{mrad} \\
\pm 1.5 \mathrm{mrad} \\
\pm 5 \mathrm{mrad}\end{array}$ \\
\hline Chamber flatness & All layers & $\begin{array}{l}\text { Random Bumps } \\
\text { Maximum Sag }\end{array}$ & $\begin{array}{l}\sigma=100 \mu \mathrm{m} \\
<200 \mu \mathrm{m}\end{array}$ \\
\hline Monitor positioning & $\begin{array}{l}\text { Inner, outer } \\
\text { middle }\end{array}$ & $\begin{array}{l}\Delta x \\
\Delta x\end{array}$ & $\begin{array}{l} \pm 20 \mu \mathrm{m} \\
\pm 10 \mu \mathrm{m}\end{array}$ \\
\hline Monitor resolution & Measurement error & $\overline{0}$ & $25 \mu \mathrm{m}$ \\
\hline $\begin{array}{l}\text { Line-of-sight } \\
\text { projectivity }\end{array}$ & $\begin{array}{l}\text { From global } \\
\text { alignment }\end{array}$ & $\Delta p$ & $\begin{array}{l} \pm 3 \mathrm{~cm} \\
\pm 3 \mathrm{~cm}\end{array}$ \\
\hline$\theta$-Coordinate Resolution & In each tower & $\overline{\mathbf{\sigma}}$ & $3 \mathrm{mrad}$ \\
\hline \multicolumn{4}{|c|}{ Limits from Other Sources (wrt outer superlayer) } \\
\hline Trigger projectivity & Middle superlayer & $\Delta x$ & $\pm 5 \mathrm{~mm}$ \\
\hline Dynamic range of monitors & $\begin{array}{l}\text { Middle superlayer } \\
\text { Inner superlayer }\end{array}$ & $\begin{array}{l}\Delta x, \Delta z \\
\Delta x, \Delta z\end{array}$ & $\begin{array}{l} \pm 5 \mathrm{~mm} \\
\pm 1 \mathrm{~cm}\end{array}$ \\
\hline
\end{tabular}

Table 4-17. Requirements and resolutions assumed for global alignment

\begin{tabular}{lcc}
\hline Error Source & Consequence & Interpretation \\
\hline & Structural Positioning Accuracy Required \\
Projectivity of Local Alignment & $\Delta \phi, \Delta \theta \leq \pm 3 \mathrm{mrad}$ & LOS pointing to IP from outer layer \\
\hline & Needed Measurement Accuracy \\
Projectivity of Trigger Roads & $\Delta \phi \leq \pm 3 \mathrm{mrad}$ & Strip projectivity to IP from outer layer \\
Muon Angle & $\Delta \phi, \Delta \theta \leq \pm 3 \mathrm{mrad}$ & Tower pointing to IP \\
Inter-Tower & $\Delta \times \leq \pm 250 \mu \mathrm{m}$ & $\phi$ direction; for muons crossing towers \\
Track Linking & $\Delta \mathrm{r} \leq 500 \mu \mathrm{m}$ & Preliminary estimate; $R-\phi$ offset from IP \\
High Mass Momentum Resolution & $\sigma_{\mathrm{p}} \leq 200 \mu \mathrm{m}$ & $\Delta \phi \leq 50 \mu$ rad; outer layer pointing to IP \\
\hline
\end{tabular}




\section{Local Alignment Philosophy}

The GEM muon system measures the trajectory of a muon track in three superlayers. The deviation of these measured points from a straight line (sagitta) determines the track curvature, and hence the muon momentum. If the three superlayers are misaligned along the muon hending direction, a false sagitta will result, leading to errors in the momentum measurement. The bending coordinate misalignment of chamber superlayers must be limited to $\pm 25 \mu \mathrm{m}$ in order to retain the momentum resolution of the GEM muon detector.

To maintain this precision, each tower will be instrumented to dynamically monitor the relative alignment of its composite CSC superlayers, as shown in Figure 4-57. These local alignment systems are based on optical straightness monitors, such as those used at the L3 detector at CERN ${ }^{44}$ (Figure 4-58), which directly measure the deviation of 3 points from a straight line. It has been determined $^{12}$ that superlayer misalignments will produce sagitta errors that may be described to sufficient accuracy by a quadratic in the tangent of the local $\phi$ angle (where $\phi=0$ at the center of the tower) for high-momentum muon tracks originating at the interaction point. By measuring the chamber misalignment in the local projective $\phi$ coordinate at three points across the $\phi$-width of the tower, a quadratic function is determined that can precisely interpolate a sagitta correction. Running 3-point monitor arrays along both sides of a projective tower, ${ }^{12}$ as depicted in Figure 4-57, allows a quadratic interpolation in the $\phi$ coordinate and a linear interpolation in the $\theta$ coordinate of a muon track. This quadratic interpolation can be used to compensate the sagitta errors caused by superlayer misalignments. Only 5 out of 6 monitors are required to track rigid-body displacements and uniform thermal expansion, providing a degree of fault tolerance. If all six monitors are utilized, "torque" errors, where opposite edges of elastic chambers are differentially twisted and/or stretched, are also suppressed by the correction method. 12

Quadratic interpolation has been demonstrated by simulation and analysis. 12,45 The effect of the alignment correction is demonstrated in Figure 4-59. The plot on the top shows the false sagitta injected into a straight-line muon track traversing the first barrel $\theta$-section alignment tower with the superlayers rotated and translated away from their nominal positions as a function of the muon angles $\theta$ and $\phi$. Because of the chamber displacements, the sagitta error ranges up to $4 \mathrm{~mm}$ in this example, with an average error magnitude of $1.1 \mathrm{~mm}$, which is certainly unacceptable. The bottom plot shows the false sagitta after the alignment interpolation correction ${ }^{45}$ has been applied. The surface is now of higher order with the quadratic dependence removed, and error ranges between $\pm 10 \mu \mathrm{m}$, with an average $5 \mu \mathrm{m}$, which is well within our allowed tolerance.

The analysis effort of Reference 46 has examined these post-correction sagitta residuals for random chamber displacements, resulting in the local chamber positioning requirements of Figure 4-60 and Table 4-16. These are the maximum permissible superlayer rotations and translations (both relative to the inner superlaver) over which the sagitta errors are adequately temoved. The error sources quoted for local alignment in Table 4-16 trade off against one another, where tightening one allows another to loosen. The allowed rotations and translations have been selected ${ }^{46}$ to contribute roughly an equal amount of about $10 \mu \mathrm{m}$ in quadrature to the net $25 \mu$ m error budget. In addition,

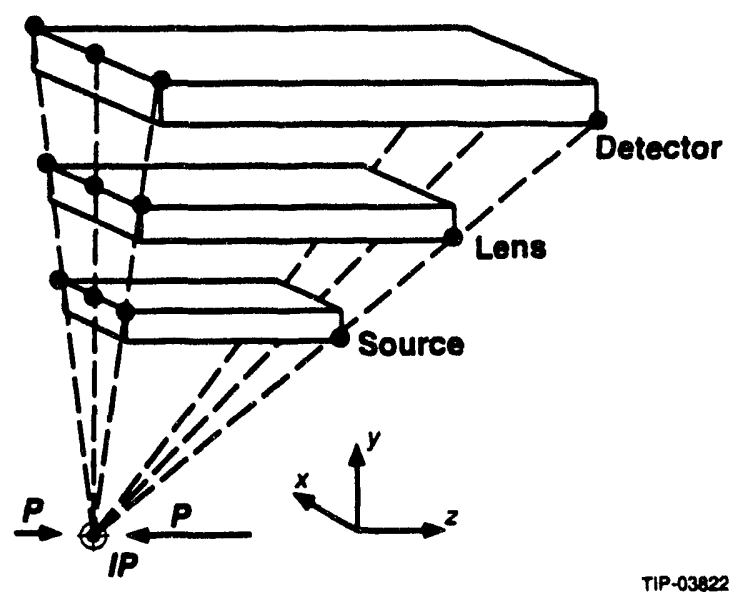

FIG. 4-57. Local alignment of a projective muon tower.

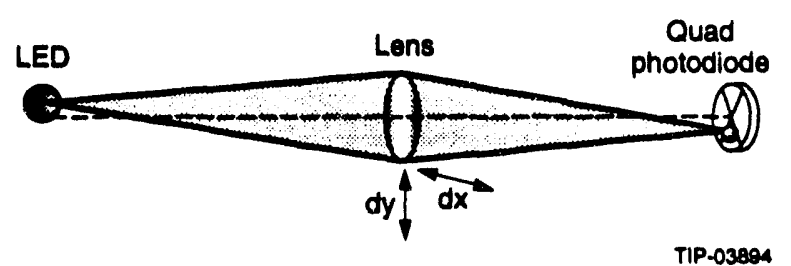

FIG. 4-58. Three-point LED/ens/quad-cell straightness monitor as applied at L3. 
a $z$-coordinate resolution yielding $\sigma_{\theta}=3 \mathrm{mrad}$ has been assumed in this ana $y$ sis. The $\theta$-measurement is neeried for the interpolation between monitor triads, thus this resolution likewise contributes to th: alignment error. Figure 4-61 shows the net residual after alignment correction for straight-line tracks emanating from the interaction diamond (the locus of beam crossings in the region of the IP), assuming simultaneous alignment errors statistically distributed within the limits of Table 4-16. The $\sigma<25 \mu \mathrm{m}$ error budget is shown to be maintained. The stringent $\mu \mathrm{m}$ and $\mu \mathrm{rad}$ positioning requirements that were previously imposed 11,47 are thus removed, provided the straightness monitor axes point toward the IP within a cylinder of $\pm 3 \mathrm{~cm}(z)$, and $\pm 3 \mathrm{~cm}(R)$ (see Figure 4-60). Thus arbitrary deflections up to

(a)

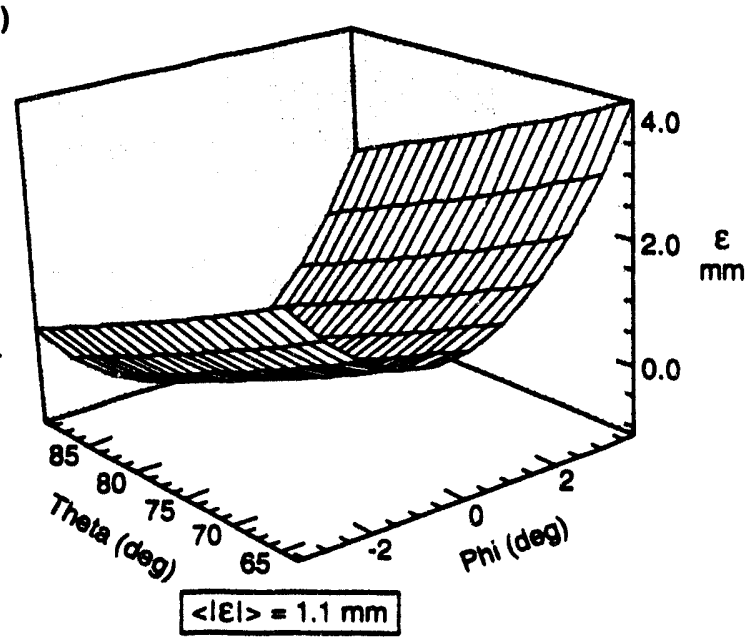

(b)

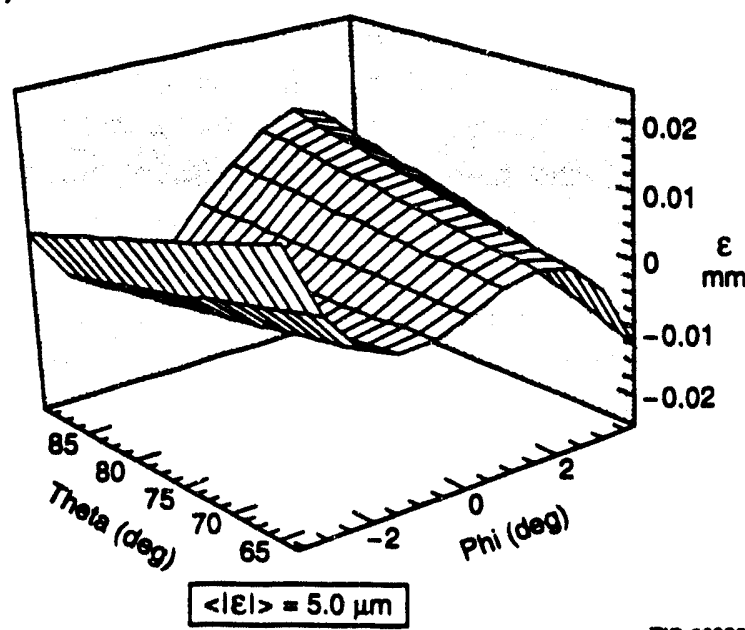

FIG. 4-59. Sagitta errors in a projective alignment tower before \& after alignment correction. several $\mathrm{mm}$ and rotations at the milliradian level may be compensated to produce an average sagitta residual within the $25 \mu \mathrm{m}$ limit for stiff muon tracks coming from the IP. Although only the bendingcoordinate misalignment need be measured to perform this correction, knowledge of the orthogonal (non-bending) coordinate will aid in fault tolerance ${ }^{12}$ and prove useful in resolving positioning ambiguities, thereby increasing the range of sagitta correction. ${ }^{45}$ Analysis of the actual muon data may likewise break the ambiguities in local chamber positioning, significantly expanding the limits of Table 4-16; this is discussed additionally in the description of global alignment. Dual-axis, planar displacement sensors have thus been adopted to monitor the local alignment, as described below.

\section{Hardware Implementation of Local Alignment Monitors}

Although simple LED/lens/quad-cell systems such as depicted in Figure 4-58 have demonstrated high accuracy in detector systems (i.e., below $5 \mu \mathrm{m}^{48,49}$ ) at minimal cost, their useful measurement range is generally restricted to within a millimeter or two. ${ }^{10}$ The projective alignment scheme described earlier enables a wider alignment range to be exploited (as quoted in Figure 4-60), thereby relaxing the positioning requirement. The range of these alignment systems may be extended by replacing the quad-cell by a continuous lateraleffect photodiode ${ }^{10}$ or by inserting a wide-area diffuser over the LED and using a larger quadrant diode. ${ }^{50}$ These techniques, however, can appreciably increase the hardware expense and potentially degrade the alignment resolution beyond the $25 \mu \mathrm{m}$ limit.

An alternative solution is provided by replacing the single source of Figure 4-58 with an array of LEDs or optical fibers. By placing LEDs sufficiently close together such that their measurement range will overlap with adequate defocus and pulsing them individually, precision measurements may be obtained over a range limited only by the size of the LED matrix (Figure 4-62a). By employing local counter electronics, only three logic lines are required to illuminate any LED in the matrix; i.e., one to cycle the counter and address the next LED, one to reset the counter, and one to modulate the LED intensity, thereby filtering out effects of 


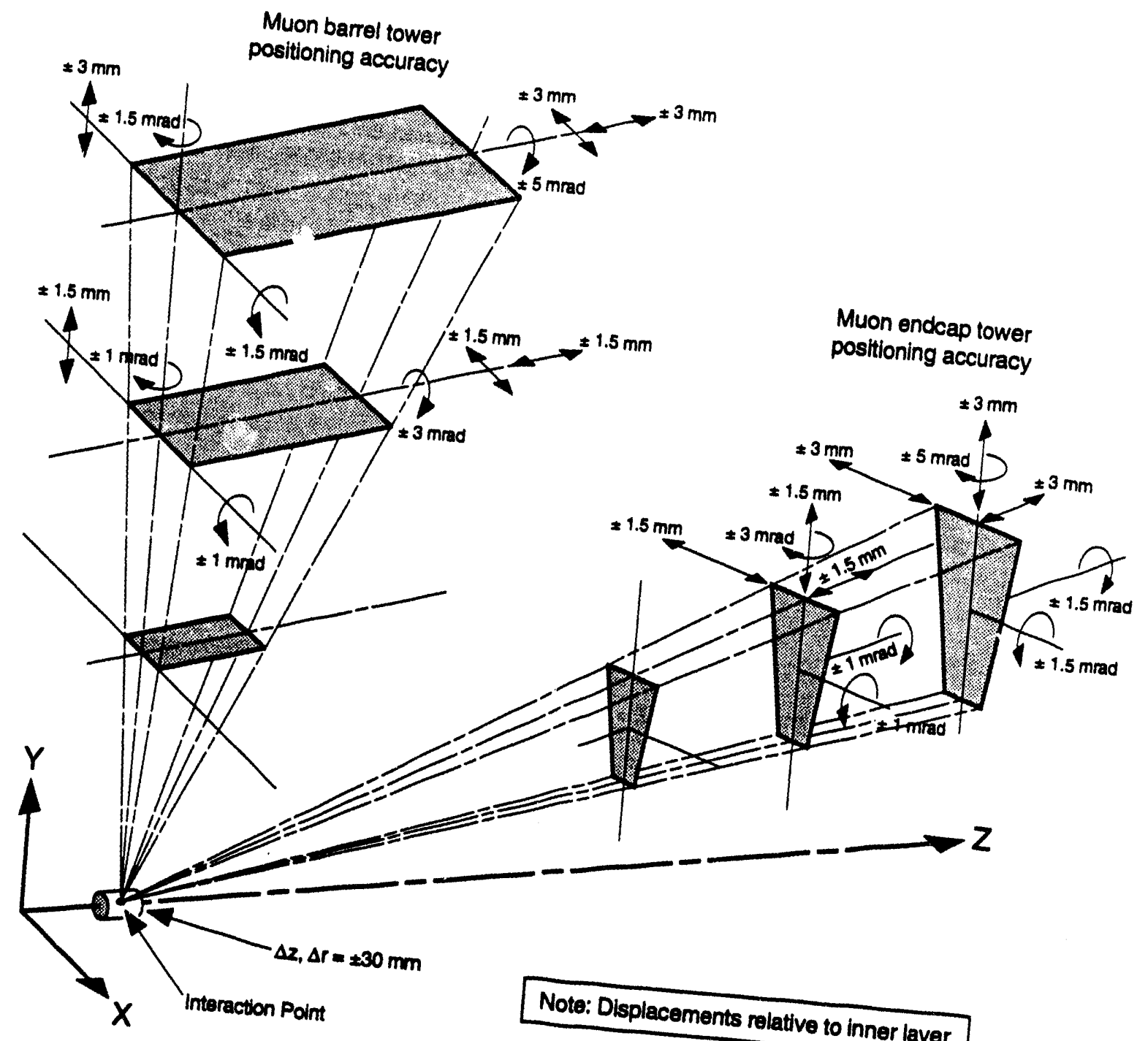

FIG. 4-60. Maxim

cotations in an alignment tower.

arrays are currently being evalue commercial LED in this scheme. ${ }^{51}$ Figure 4-62b from a $5 \times 7$ element 4 -62b shows recent results spacing, manufactured block with a $2.5 \mathrm{~mm} \mathrm{LED}$ Enough defocus was intor alphanumeric displays. and adequately overlap the Here we can see the linear measurement regions. 4 I.EDs leapfrogging to span a prement regions of ment across a full to span a precision measuredisplacement.

Recent advances in imaging technology and the cost and siztronics have dramatically reduced enabling techniques solid-state video cameras, to become competitive as depicted in Figure 4-63

LED/lens/quad-cell schemes of Figs. 4-58 and 4-62. With the video technique, a large precision mask through the lens onto is illuminated and projected focal plane array. correlating the video imagnment is detected by Provided that the portion of the mask template. the camera is unambiguous, the image captured by be precisely located anywhere camera position can mask, yieiding a very wide measuross the projected system is very easy to diagnoasurement range. This gent "watchdog" processingse and verify. Intelliprovides a wealth of inforing of the video frames real-time video can bermation and, if needed, the 2-dimensional barcode bisually analyzed. A to greatly simplify the been developed in order 
taking projections along the $x$ and $y$ axes of the video frames, $x$ and $y$ barcodes can be independently scanned, coarsely positioning the imager relative tthe mask. The centroids of the projected bars may then be fit to the code template to produce a precise position reference. A prototype of this system has been implemented ${ }^{51}$ spanning a 9 meter optical path. The deviation from linearity in a test scan across a $9 \mathrm{~mm}$ section of a barcode mask is given in Figure 4-63c, where it is noted that the residuals

(a)

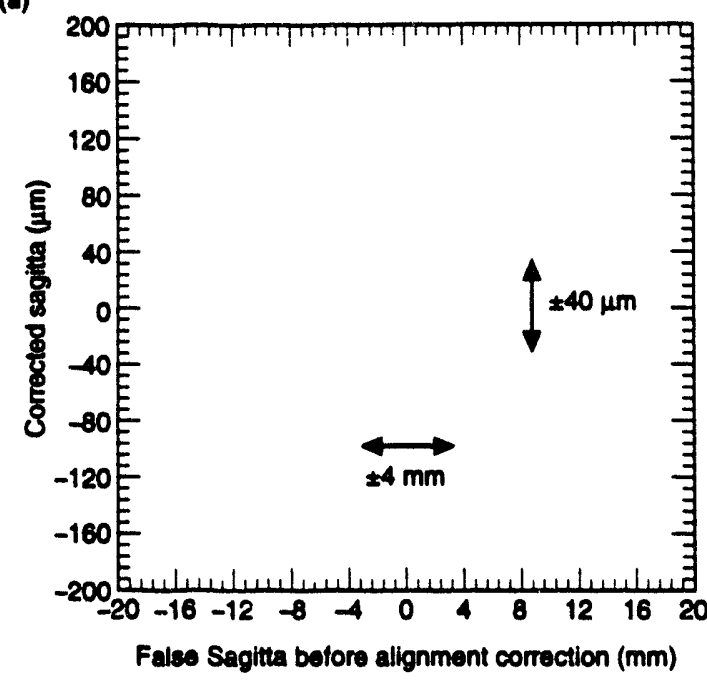

(b)

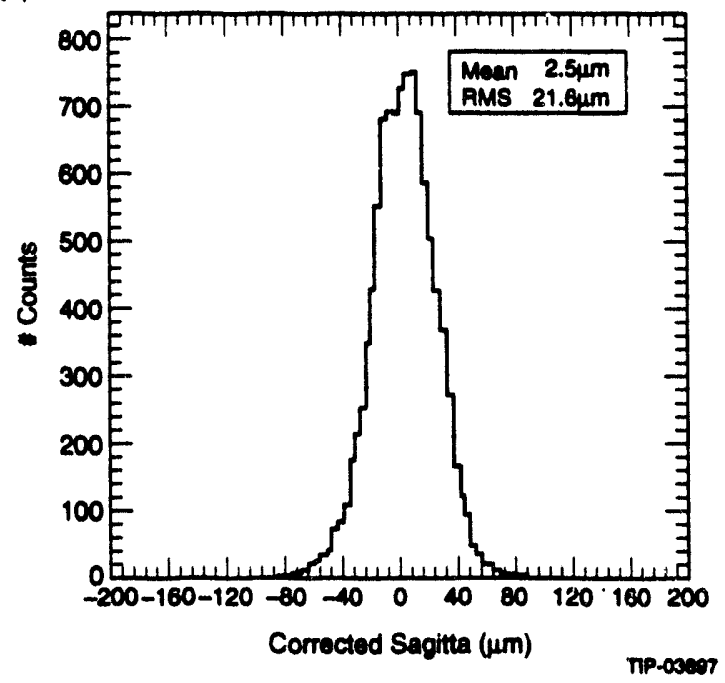

FIG. 4-61. Attenuation of statistically distributed alignment errors by monitor correction. remain within $\pm 4 \mu \mathrm{m}$. Further, very simple refinements promise to glean even superior results. ${ }^{51}$

Figure 4-63b shows the implementation of such a system in the GEM muon detector. Adequate video camera modules are currently available as small (under $3 \mathrm{~cm} \times 5 \mathrm{~cm}$ ) circuit cards, which require only $12 \mathrm{~V}$ power and output composite RS-170 video over a single $75 \Omega$ coaxial cable for less than $\$ 100$ in quantity. This scheme entails minimal cabling, where each camera requires a unipolar power feed and a $75 \Omega$ video line, plus a gate for every LED illuminator. Proper shielding will avoid crosstalk into the chamber signal electronics. All video sources for each muon $\phi$-sector (here assumed to span $1 / 12$ of $2 \pi$; i.e., 96 video feeds in a barrel sector, and 40 video feeds in an endcap sector) are multiplexed and input to a standard frame-grabber, which is managed by a simple processor that

(a)

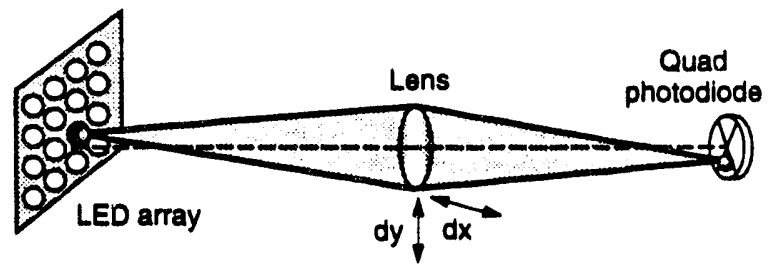

(b)

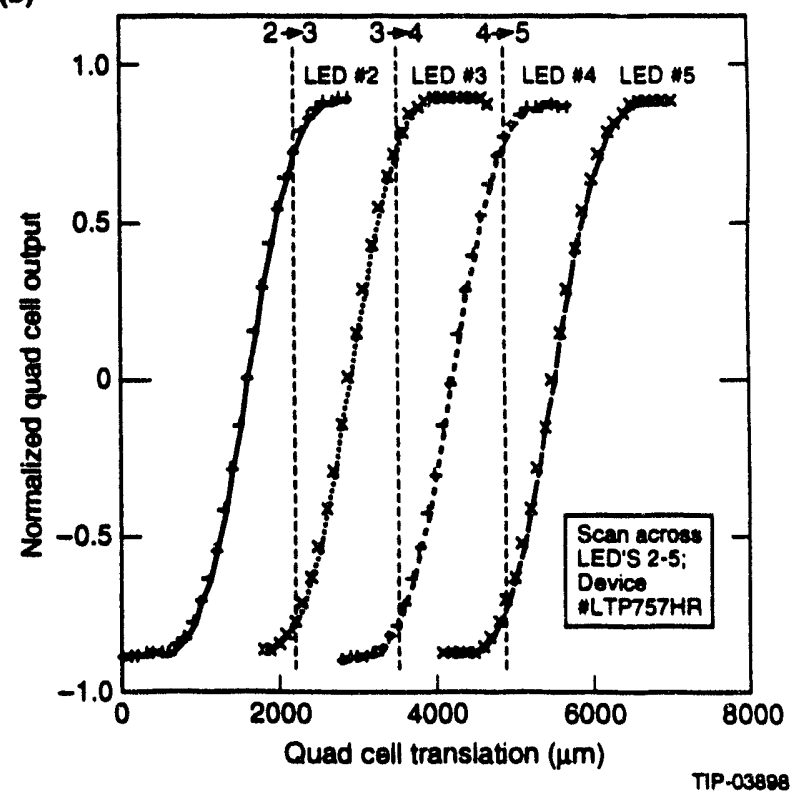

FIG. 4-62. Multiple LED straightness monitors for extended range. 
takes the $x / y$ projections and fits them to the code template. The entire muon system thus can be scanned in under a half-hour. These processors will communicate via a local area network.

The barcode mask will be deposited on glass, and back-illuminated by an IR LED concentrated through a field lens. This system has been demonstrated to produce a very satisfactory image, using an inexpensive video camera card as described above (with a standard RS-170 60 frames/s exposure) in lab tests that employed a $3.8 \mathrm{~cm}$ diameter lens placed at the midpoint of a 9 meter optical path, which is the maximum reach expected at GEM. Successive frames were summed at a $1 \mathrm{~Hz}$ rate for at least 15 seconds in order to average out the effects of atmospheric turbulence. (a)

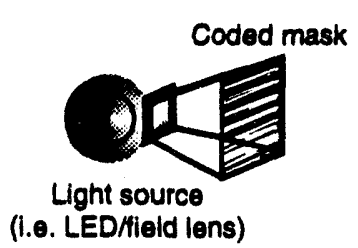

(b)

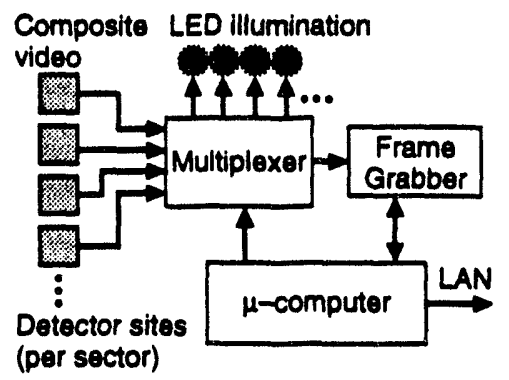

Photodetector (imaging array)

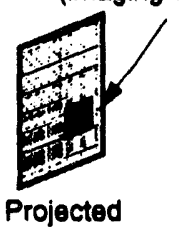
mask image

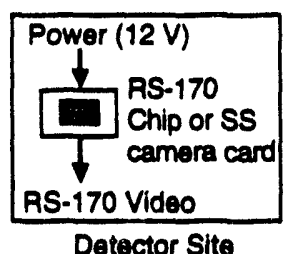

Detector Site (c)

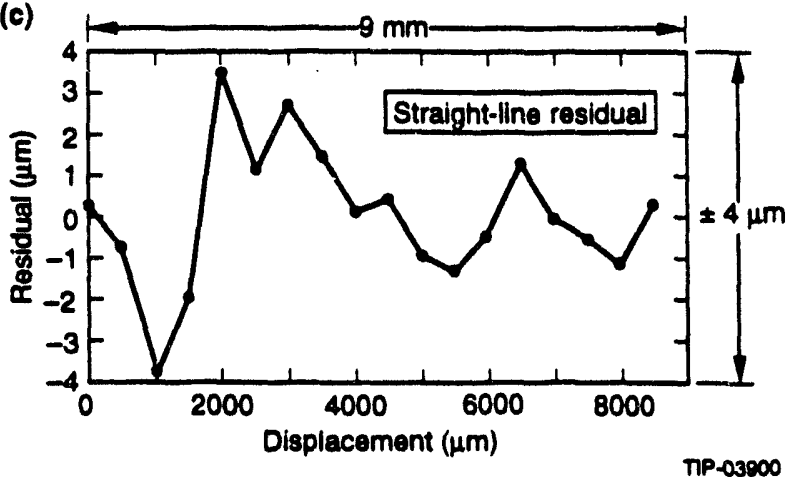

FIG. 4-63. Wide-range video straightness monitors.

The camera card used in our current tests employs a switched MOS photodiode matrix of $324 \times 246$ pixels; studies are underway to insure that such devices will operate properly in the anticipated magnetic and radiation environments. If difficulty is encountered, radiation-tolerant imagers using Charge Injection Devices (CIDs) can be employed. If this proves infeasible, the backup option of multiple LEDs described earlier (Figure 4-62) is also available.

\section{Fixturing and Chamber \\ Fiducialization/Calibration for Local Alignment}

The LED, lens, and photosensor of the straight line monitoring system must be placed relative to the cathode plane fiducial to within $10 \mu \mathrm{m}$ RMS. This includes both the placement of the fiducial in its mechanical holder and the placement of the fiducial assembly onto the muon chamber. Although this requirement is very tight, the relatively small distances ( $1.2 \mathrm{~m}$ maximum), accessibility of the cathode plane fiducial, and manufacturing precision of the fiducial holders makes the tolerance achievable.

Each alignment element (source/lens/detector) will be mounted into a precision holder. These fixtures orient the alignment element at the required angles and provide a precise mechanical reference to the chamber layer on which it is mounted. Previous efforts $^{48,52}$ have centered the components of the straightness monitors to better than $5 \mu \mathrm{m}$ using a fast and simple adjustment technique. This process uses a revolving stage that rotates each optical component about a centerline defined by a source/lens/detector system. The element position is adjusted relative to the holder at multiple angles, until the desired concentricity is seen at the detector (i.e., if the components are all aligned, the detector will measure no position changes with rotation). During this process, a fiducial edge of the holder is maintained against a reference plane, which has been precisely located with respect to the optical line-of-sight. In this fashion, the center of the alignment element has been transferred to the mechanical edge of its holder, which can now be attached to the chamber.

This technique can be readily used to center lenses and quad cells, but it may require modifica- 
tions for imaging arrays, projected masks, and LED blocks. A simple interactive system can be employed to quickly center the arrays; i.e., the imager position can be incrementally adjusted until a reference beam is at the center of the array. The detected position can be displayed in real time on a 2-axis display to greatly speed the procedure. If needed, a similar technique can be developed to center the masks although these may be able to be fabricated with precise glass edges, eliminating the centering procedure. Each LED block may require a calibration scan if the component LEDs have differing illumination profiles, then the holder may be adjusted to center the middle LED (as above), or a reference comer of the block can be precisionmounted to the holder.

The alignment components on each superlayer (either a LED/mask, lens or photo-sensor) will be precisely located relative to the cathode strips on the top or bottom CSC layer. Figure 4-64 shows a schematic depicting conceptual alignment fixtures as mounted on a superlayer. Note that the alignment fixtures are angled both in $\theta$ and $\phi$ to maintain projectivity with the Lorentz-compensated chamber tilt. Any placement error between these fiducials and the strips will contribute to the total measurement accuracy, thus such errors are controlled ${ }^{53}$ to be less than $10 \mu \mathrm{m}$. Also added in quadrature to the intrinsic layer resolution is the bend-coordinate placement accuracy of the cathode planes within a superlayer.

Because of the high chamber precision, the $\phi$-offset of the externally referenced layer is quickly established relative to the superlayer mean by registering incident particles (i.e., $\mathrm{x}$-rays, cosmics, or IP muons); Figure 4-65 shows a result from an array of RDTs tested at the TTR,, 54 which indicates the high accuracy to which the interlayer offsets can be so measured. Figure 4-65a shows the uncorrected hit positions, indicating the wire positioning accuracy. Figure 4-65b depicts these distributions after they have all been shifted to a common mean. Nonetheless, as outlined in Reference 53 and Section 4.3.2, the GEM superlayers will be constructed to maintain a high (i.e., $50 \mu \mathrm{m}$ ) layer-to-layer

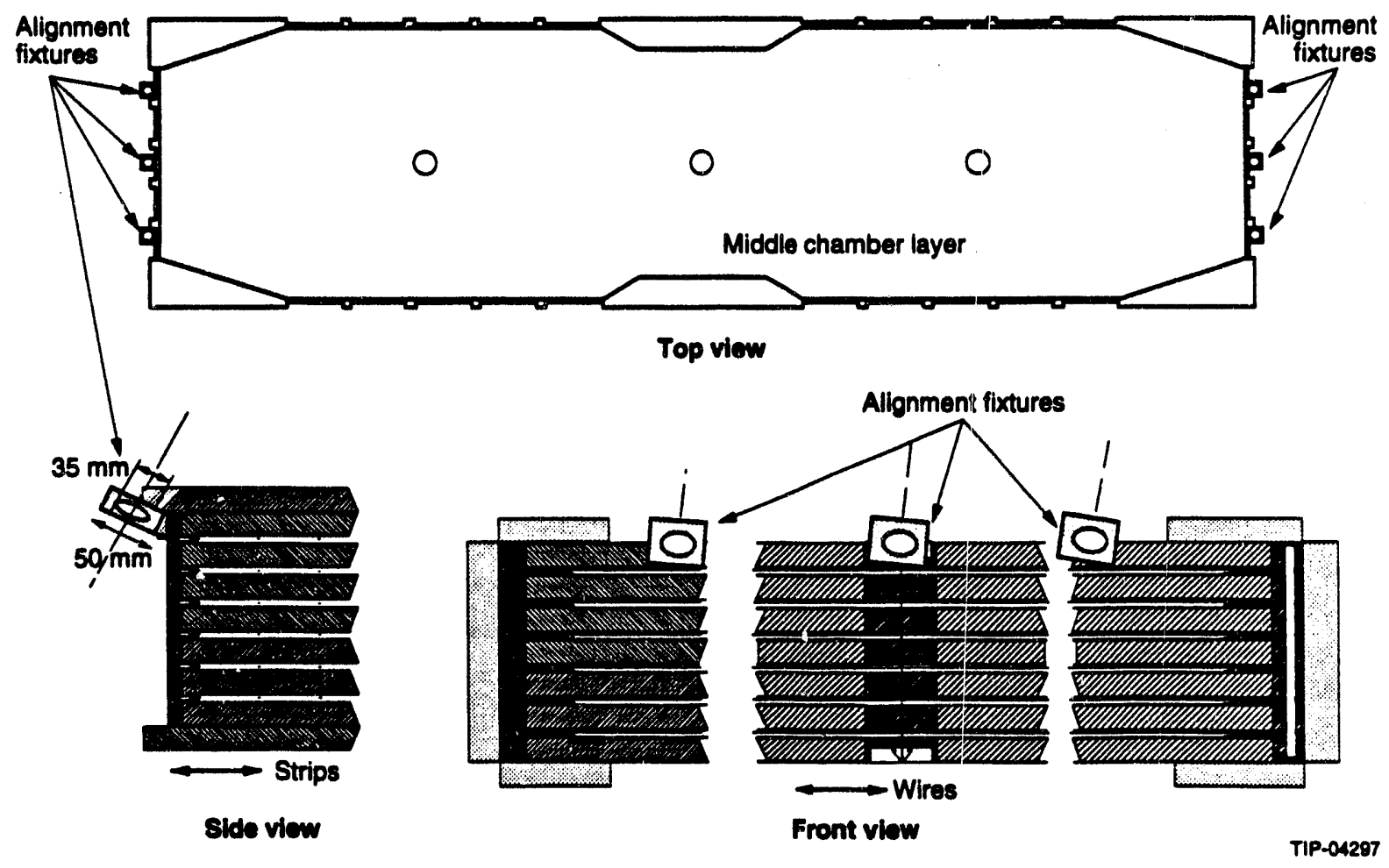

FIG. 4-64. Conceptual alignment fixtures as mounted on middle superlayer. 
placement accuracy in the strip-orthogonal coordinate.

$X$ rays could be used 55 to check intermodule alignment, as depicted in Figure 4-66. In this scheme, a multi-gap CSC is placed on a precision coordinate measuring machine (CMM) table with a finely collimated, mono-energetic $36 \mathrm{keV}$ x-ray beam installed on the CMM three-axis bridge (Figure 4-66c). The CSC gas will be modified to contain an amount of xenon in admixture with the standard argon. Xenon exhibits an X-ray absorption edge at $33.6 \mathrm{keV}$. At these energies, about there is about a $10 \%$ attenuation of the $x$-rays per chamber gas-gap due to the $1 \mathrm{~mm}$ of G10 per gas-gap. Measurements performed at $\mathrm{BNL}^{56}$ show that the position resolution achievable with a wire chamber using $90 \% \mathrm{Xe}$ and $10 \% \mathrm{CH}_{4}$ exhibits a broad minimum about $3 \mathrm{keV}$ wide starting just above the Xe K-edge.

An $x$-ray source consisting of praseodymium on a beryllium window has been constructed (Figure 4-66a), providing $x$-rays with a predominance of $36.03 \mathrm{keV}$ energy. A double crystal monochromator can eliminate the broad bremsstrahlung $x$-ray centinuum and leave a highly monochromatic $x$-ray beam tuned closely to the xenon absorption. Collimators provide for a beam with a $20 \mu \mathrm{m}$ radius and a $50 \mu \mathrm{rad}$ divergence. An $\mathrm{x}$-ray source with these characteristics has been built and demonstrated at PNPI in Russia, ${ }^{55}$ and has achieved a beam radius of $35 \mu \mathrm{m}$ and a divergence less than $50 \mu \mathrm{rad}$. Ionization tracks have recently been measured for the first time using this technique in a single layer CSC, providing a $200 \mu \mathrm{m}$ FWHM distribution of track position for a fixed $x$-ray beam position (Figure 4-66b).

Using the above $x$-ray $C M M$, one can readily simulate the passage of straight line tracks through a chamber stack to verify interchamber cathode plane alignments (per Figure 4-65), as well as general operating characteristics of the chamber. The CMM bridge can provide an $x$-ray pointing accuracy of about $5 \mu \mathrm{m}$ and can be programmed to raster scan a chamber assembly, thereby measuring cathode strip alignments over the entire superlayer. If the CMM head is also able to reference a fiducial on the alignment fixtures, the alignment transfers can be simultaneously verified. (a)

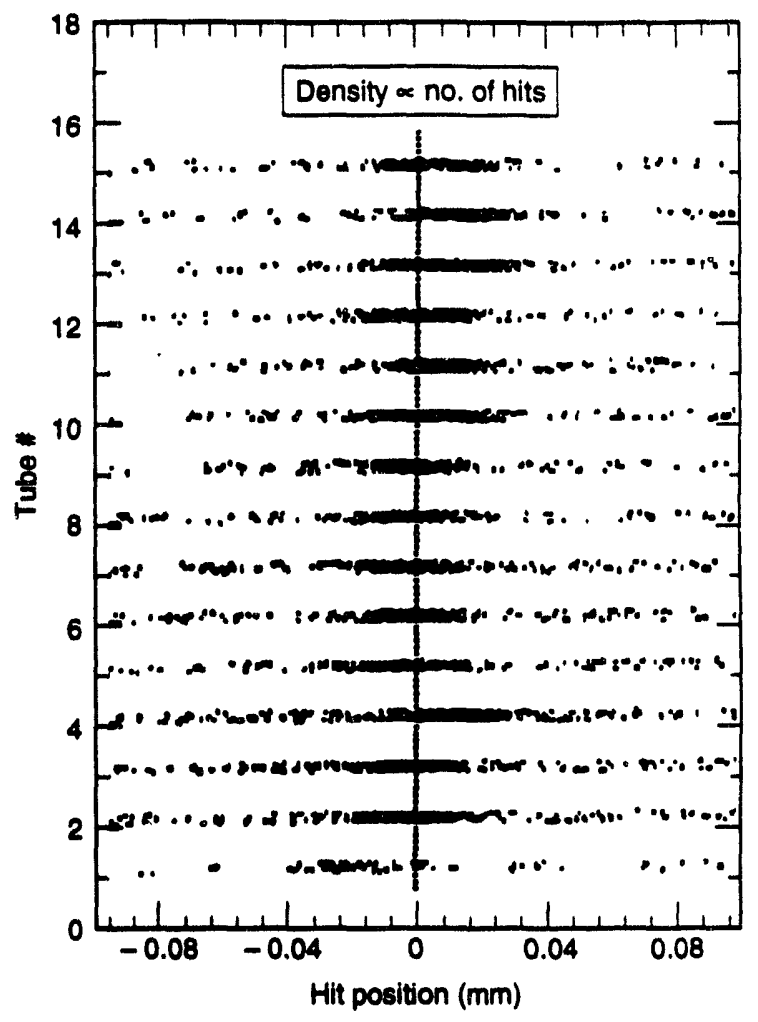

(b)

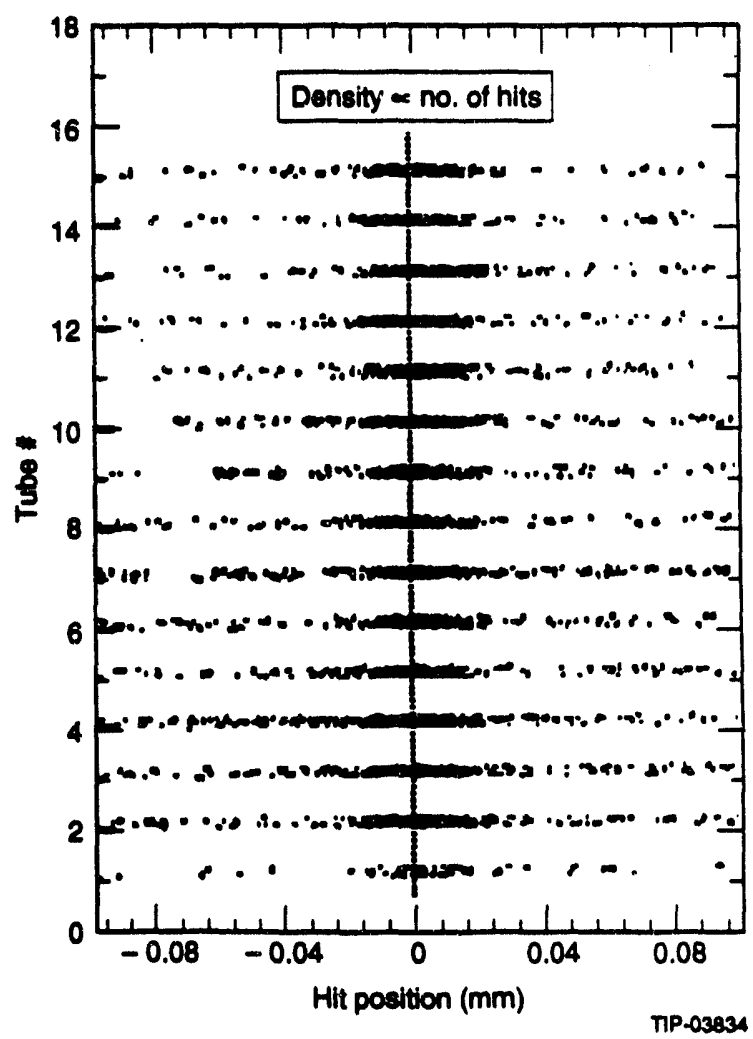

FIG. 4-65. Measured misalignment of chamber layers from cosmic rays at TTR. 
As discussed above and in Section 4.6.3, the alignment transfers between the fixtures and superlayers will be checked with cosmics and/or $\mathrm{x}$-rays during superlayer, sector, and monolitin construction. Attempts are being made to upgrade the intensity of the $x$-ray source of Figure 4-66, enabling

(a)

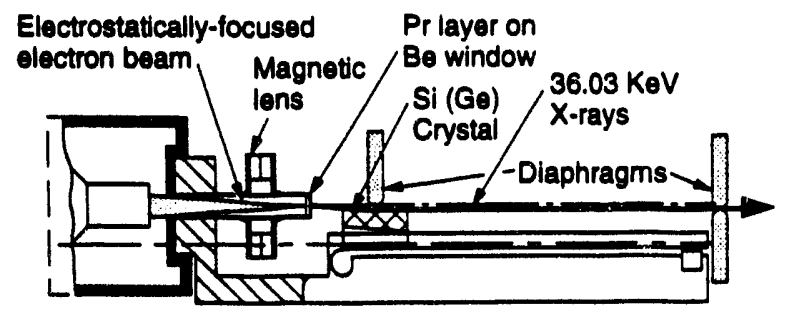

(b)

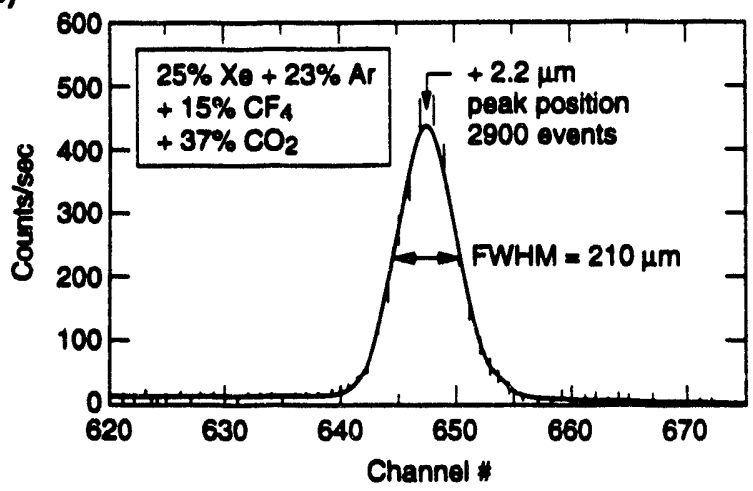

(e)

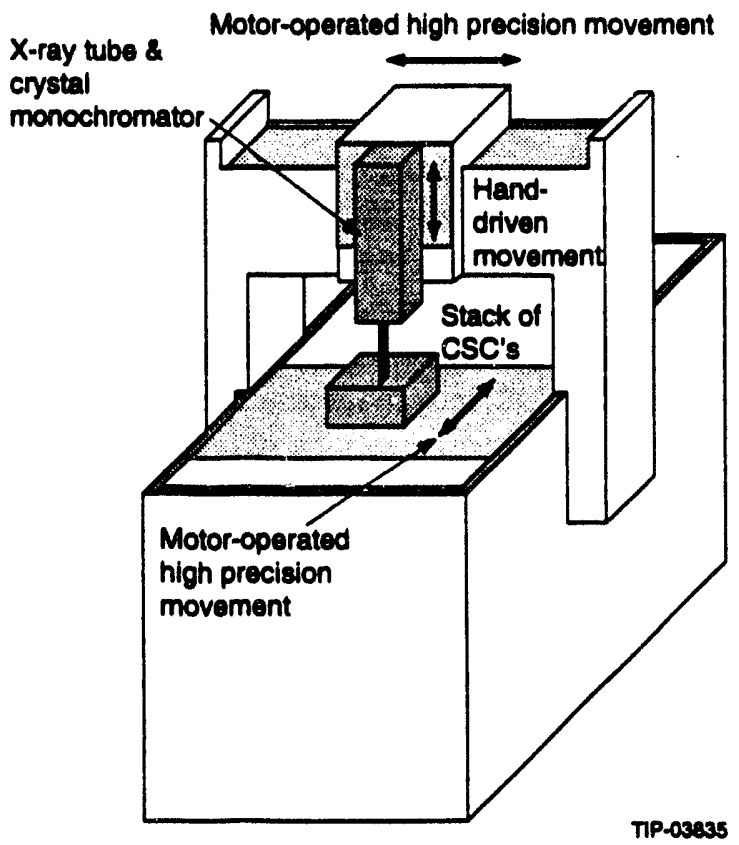

FIG. 4-66. Calibration of superlayer with finely-collimated $x$-ray beam. it to efficiently interrelate all 3 superlayers and thus perform a rapid calibration and verification of alignment after installation. ${ }^{62}$ During SSC operation, the alignment transfer can also be verified by collecting sufficient statistics of muons crossing all superlayers, as discussed in Section 4.2.7 and Refs. 31, 33. Such data can then be correlated with the straightness monitor measurements, and the alignment transfers estimated. Statistics on the order of $10^{5}$ tracks are needed per tower, ${ }^{31}$ which will be obtained in a matter of days at standard luminosity.

\section{Local Alignment Implications of Chamber Deformation, Sag, and Environment}

Depending on barrel superlayer supports and orientation (see Section 4.3.3), the support structure will exhibit some degree of gravitational sag and thermal deformation, primarily in the radial direction (the $y$-direction in Figure 4-57). On the other hand, the chamber packages are very stiff in-plane and the endcap chambers are expected to sag negligibly, since they are parallel to the gravity vector. Since the projective alignment system only monitors the opposing $\theta$-edges of an alignment tower (Figure 4-57), the internal chamber deformation is not measured, and the interpolation method described earlier assumes a planar package, thus errors are injected into the alignment correction. It has been estimated ${ }^{46}$ that we are able to tolerate up to $200 \mu \mathrm{m}$ of peak gravitational sag and $\sigma=100 \mu \mathrm{m}$ of nonuniform deformation across a chamber package before the errors injected into the sagitta reconstruction become significant (Table 4-16). If the sag/deformation can be adequately modeled and predicted, this allotment can be widened.

The consequences of the anticipated thermal environment on the GEM muon system alignment are discussed in Ref. 43 and Section 4.3.3. Tolerances of up to $1^{\circ} \mathrm{C}$ across a chamber are allowed before the alignment becomes significantly degraded. A network of temperature sensors will dynamically monitor the thermal profile across the detector, allowing some measure of analytical deformation correction, and compensating for any steady-state refraction sensitivity of the straightness monitor systems. ${ }^{51}$

Current estimates ${ }^{43}$ of peak chamber deformations including thermal, gravitational, and material flatness considerations range below the stated limits, 
and thus are permissible. If these increase into significance as the chamber design evolves, however, techniques such as proposed by other collaborations 52,57 can be adopted to measure smooth deformations; i.e., adding a pair of axial monitors, such as a set of optical straightness monitors running across the long edges of a chamber. With source and detector at opposite ends, and lens in the center, this configuration provides a 3-point fit to an assumed axial shape. Another strategy involves installing a less-expensive, lower-precision (i.e., circa $100 \mu \mathrm{m}$ ) stretched-wire multipoint monitor along each axial edge of the superlayers as discussed below.

The GEM alignment system cannot compensate for structural dynamics, thus induced vibration of the cathode planes must contribute minimally to the allotted $25 \mu \mathrm{m}$ sagitta error budget. Current estimates of RMS vibration for the GEM muon superlayers are below $5 \mu \mathrm{m}$ (see Section 4.3 .3 and Ref. 43), producing insignificant degradation of the muon alignment.

\section{Implementation of Local Alignment in the GEM Muon System}

The projective alignment systems are required to be integrated into a complex structure that includes densely packed chambers, structural bracing elements, and utility runs, all of which are competing for the same space. The alignment measuring systems must fit within this crowded framework and be integrated in such a way that the impact on the active measurement volume is kept to a minimum. The selected pathways for the alignment system are described below and have been integrated with the chamber design to assure compliance with all other system requirements. Tests ${ }^{51}$ have concluded that a lens of $3.8 \mathrm{~cm}$ aperture will provide better than adequate illumination and resolution over the longest path-lengths implemented in GEM, thus a $5 \mathrm{~cm}$ clearance has been reserved along the alignment lines-of-sight; if necessary, a proportionally smaller diameter opening can be used with the shorter paths (i.e., the low- $\eta$ barrel regions). In order to reduce potential radiation effects on the projective monitors, the light sources will be placed on the inner superlayers and photodetectors (quadcell or imaging array) on the outer superlayers. A short (i.e., $15 \mathrm{~cm}$ ) cardboard tube may be used to collimate the LED light source and detector ele- ments, attenuating contamination from ambient light; tests have shown that these devices perform well with significant airflow across the optical path. 51

\section{Barrel Alignment Paths}

Figure 4-67 shows these proposed local alignment paths for the barrel region. Projective towers are defined in the barrel by the perimeters of chambers in the outer and middle superlayers; alignment systems are deployed in each such tower as depicted in Figure 4-62. Since the barrel chambers are overlapped in $\phi$, a set of projective gaps have been reserved through the $\theta$ breaks in the middle layer to provide an opening for the alignment paths (see Section 4.3.3). Since the inner superlayer has a twice coarser $\theta$-segmentation, some of the alignment paths will originate at the center of the inner chambers, requiring alignment fiducials at these midpoints to be accurately transferred through the cathode plane. The total number of alignment paths in such a barrel sector $\left(\Delta \phi=7.5^{\circ}\right)$ is thus 24 , resulting in 96 alignment paths per assembly module $\left(\Delta \phi=30^{\circ}\right)$, netting 2304 alignment paths for the entire GEM barrel muon system.

The need to maintain these projective lines-ofsight bears considerable impact on the layout and design of the barrel muon system. In order to avoid obscuring an alignment path with an adjacent chamber, interchamber middle-layer gaps are opened along the beam ( $z$ ) direction, and the outer alignment paths are moved away from the chamber edges in $\phi$ where the chambers are overlapped, and thus are drawn closer together. Provided the mounting points of the alignment fixtures are known, the quadratic correction function can still be determined. The projectivity requirement also prevents adjacent alignment paths from sighting the same source. One can envision schemes, however, such as packaging several source fixtures in one unit to simplify mounting. If the video straightness monitors are employed, a "quasi-passive source," such as a barcode pattern etched onto a flat surface, which is illuminated by a flashlamp that fires during the frame integration, could be used.

As discussed in Section 4.2.7, background muons can also be exploited to achieve inter-tower alignment in the areas where the two-chamber 
packages overlap in $\phi$ for each of the $48 \phi$-sectors. An hour of such data at standard luminosity will be more than sufficient to provide enough events in the overlap regions to produce accurate alignment transfers between the chambers. A dedicated software tap into the Level 2 or Level 3 muon trigger to histogram the single muon tracks in this fashion will enable these statistics to be promptly acquired without saturating the data acquisition stream.

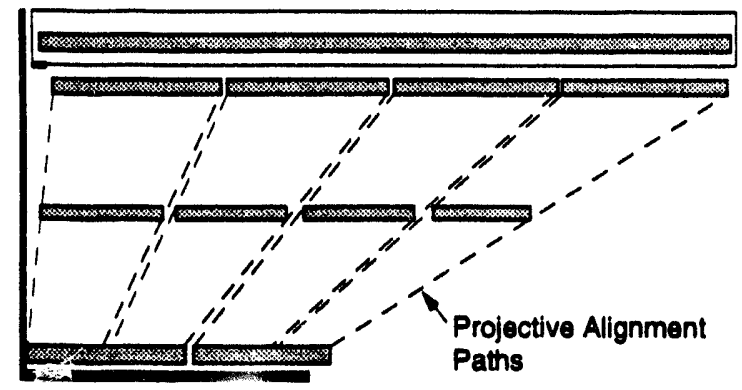

Side Viow $(r \theta)$

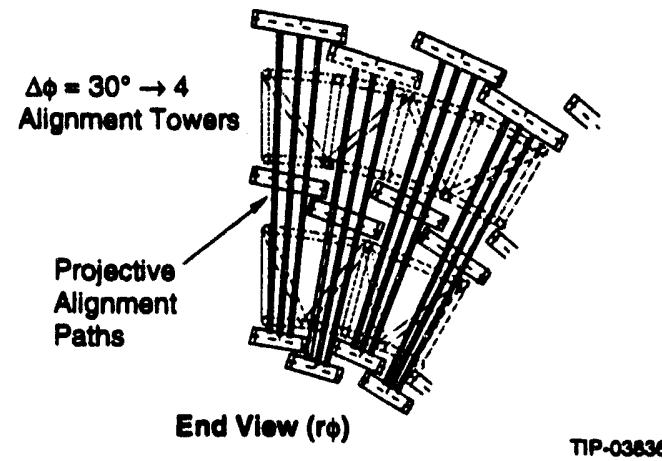

FIG. 4-67. Layout of barrel alignment lines-of-sight.

\section{Endcap Alignment Paths}

As shown in Figure 4-68, each endcap monolith is likewise subdivided into 48 alignment towers (also overlapped in $\phi$ ), again as defined by the chambers in the outer superlayer. Each such endcap tower will have 3 projective alignment paths along its top edge (high $\theta$ ) and two alignment paths along its lower edge (low $\theta$ ). In order to attain complete acceptance coverage, however, the upper and lower endcap chamber packages are overlapped in $\theta$, preventing a line of sight from traversing their inner edges. To overcome this difficulty, the upper and lower sets of chambers are rigidly coupled together at the point of overlap, thereby mechanically transferring the projective alignment measurements between the top and bottom 3-point paths, and forming complete alignment towers.

(a)

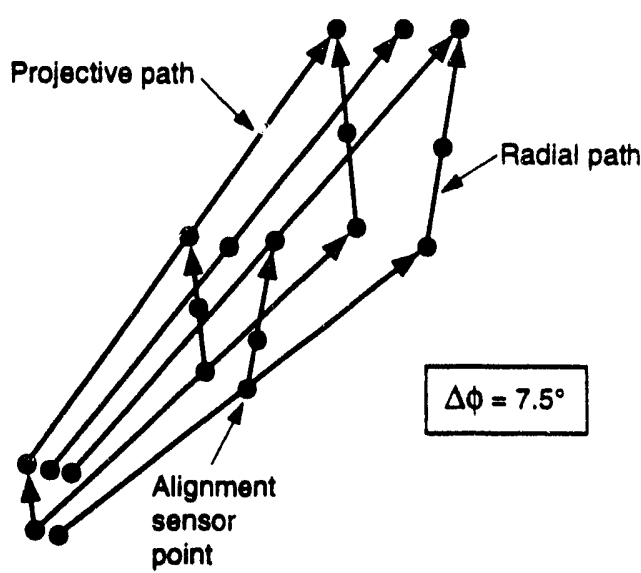

(b)

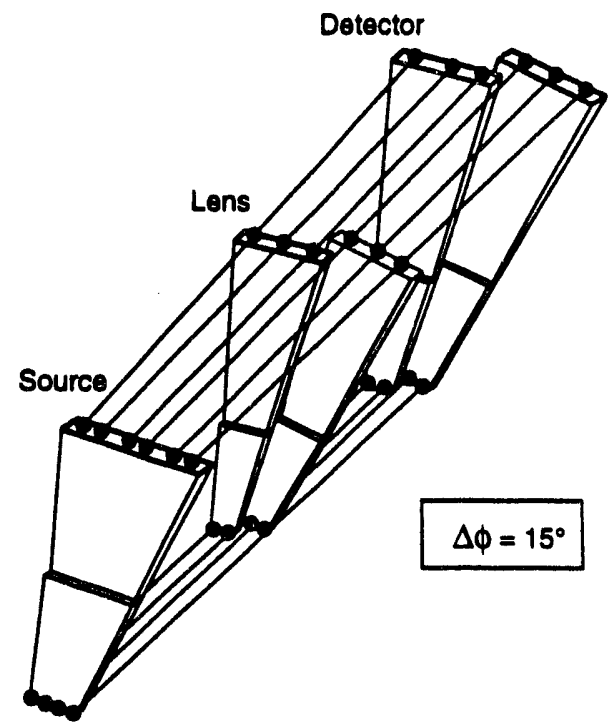

(c)

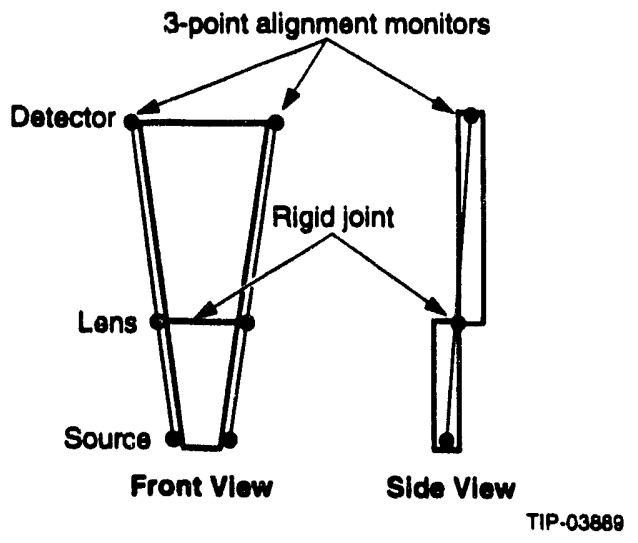

FIG. 4-68. Layout of endcap z'ignment lines-of-sight. 
The link between upper and lower chamber sets can be verified in two fashions. Because they overlap by $5 \mathrm{~cm}$ and are adjacent in $z$, tracks traversing both chamber sets will quickly (i.e., well within an hour at standard luminosity) quantify the bunding-coordinate misalignment to $25 \mu \mathrm{m}$. In addition, we have foreseen a set of radial straightness monitors to be directed along the $\phi$-edges of each linked chamber package, with the LED light source at bottom, lens at the point where the upper/lower chambers are joined, and photodetector at top (Figure 4-68, side views). These will monitor the relative $\phi$ and $z$ deflections of upper and lower endcap segments. The endcap alignment scheme of Figure 4-68 results in 5 projective and 5 radial alignment paths for each alignment tower $\left(\Delta \phi=7.5^{\circ}\right)$, netting 960 3-point alignment devices for the entire endcap muon system.

Note that only two projective alignment paths are prescribed for the smallest $\theta$ edge of the endcap, as opposed to the three implemented elsewhere that are needed to reconstruct the quadratic sagitta error function. Because the projective lines-of-sight are much closer together at small $\theta$, the sagitta errors are much less sensitive to $z$-translations and $y$-rotations there, which the center alignment line effectively determines, thus the need for three alignment paths is relaxed. In addition, the available space is quite limited on these short chamber edges. Regardless, a full 3-point measurement of the quadratic is performed by the three straightness monitors on top, which will be transferred down to the bottom through the radial monitors.

\section{Alignment Path Minimization}

The total number of alignment paths needed for this implementation of the GEM barrel and endcap are summarized in Table 4-18. By structurally linking $\phi$-adjacent pairs of chambers together, ${ }^{53}$ the number of alignment paths can be significantly reduced. A precision endplate assembly would join neighboring chambers to create a single, integral surface whose structural behavior meets the projective alignment criteria. The alignment fixtures would then be mounted onto this plate, and the measurements transferred onto the cathode planes. As discussed earlier, this implies that any deformations that are greater than the passive requirements must be accurately described by the linear and quadratic interpolation functions. We are considering either rigidly coupling the chambers together, or installing a secondary superstructure to which the chambers would be attached. An engineering assessment is in progress to characterize the chamber structural performance. If these concepts appear viable, only 3 (or 4 if the endplate bends significantly) projective alignment paths would be needed per chamber pair, reducing the quantity and density of alignment paths by up to a factor of 2 .

Table 4-18. Local alignment monitors needed for GEM muon system

\begin{tabular}{|c|c|c|c|}
\hline \multicolumn{4}{|c|}{ Number of 3 Point Local Alignment Monitors Needed } \\
\hline GEM Barrel & & GEM Endcaps & \\
\hline $\begin{array}{l}\text { Barrel Tower } \\
\left(\Delta \phi=7.5^{\circ}\right)\end{array}$ & 6 & $\begin{array}{l}\text { Endcap Tower } \\
\left(\Delta \phi=7.5^{\circ}\right)\end{array}$ & 10 \\
\hline $\begin{array}{l}\text { Barrel Sector } \\
\left(\Delta \theta=60^{\circ}\right)\end{array}$ & 24 & $\begin{array}{l}\text { Endcap Sector } \\
\left(\Delta \phi=15^{\circ}\right)\end{array}$ & 20 \\
\hline $\begin{array}{l}\text { Assembly } \\
\text { Module } \\
\left(\Delta \phi=30^{\circ}\right)\end{array}$ & 96 & Single Endcap & 480 \\
\hline Barrel Half & 1152 & & \\
\hline Net GEM Barrel & 2304 & Net GEM Endcaps & 960 \\
\hline
\end{tabular}

\section{Alternative Implementations of Local Alignment for the GEM Muon Barrel}

As illustrated in the discussion of Figure 4-67, middle-layer $\theta$-gaps must be kept between chamber packages to pass the lines-of-sight. Together with the dead regions at the chamber ends, which will be minimized as part of the chamber and electronics prototype R\&D program, significant loss can be accrued in the barrel acceptance particularly at the smaller inclinations. A set of barrel alignment altematives are presented in Figure 4-69 that are und $r$ consideration to close the alignment gaps and ease this problem. In Figure 4-69a, a triad of 3-point optical projective monitors is mounted on either side of a muon sector, and the resulting superlayer-tosuperlayer alignment is transferred between the component chamber packages via a multipoint alignment monitor. A stretched wire could be used as shown, or nested 3-point monitors, such as "fence posts" 52 running along the beam $(z)$ axis. This scheme was introduced in Reference 10, analyzed in Reference 45 , and recently proposed for the CSC barrel. ${ }^{58}$ Several techniques are available for per- 
forming precision alignment to a stretched wire; these include differential capacitors, ${ }^{59,60}$ optical sensors, ${ }^{61}$ and pixelated pickup strips. ${ }^{58}$ Although these techniques have demonstrated (or have the potential for achieving) resolutions surpassing 10 $\mu \mathrm{m}$ at modest cost, the systematics associated with stretched-wire systems (i.e., precise determination of wire tension/sag, susceptibility to vibration and induced noise, possible calibration difficulty), plus their mechanical fragility entail significant risk. A compromise solution may be afforded by Figure 4-69b, where we have eliminated two of the interim projective gaps/paths. The two chambers spaced between the projective paths in each superlayer are linked by four axial 3-point straightness monitor systems; one pair on each side that reference the right and left chamber edges (as in Figure 4-69b), producing an "analytically rigid" chamber pair between the projective lines-of-sight and effectively doubling the length of the alignment tower. Since only 3-point monitors are required, the technology is identical to the baseline of Figure 4-67; no multipoint systems need be developed. Slightly more straightness monitors are needed; i.e., 28 per $30^{\circ}$ assembly module, as opposed to 24 in the all-projective scheme; a modest $17 \%$ increase.

As opposed to the baseline all-projective scheme where each monitor directly measures the local sagitta error, the hybrid alignment approaches of Figure 4-69 transfer the $25 \mu \mathrm{m}$ 3-layer alignment measurement through several systems; first from the 3-point projective monitors to the axial monitors, then from the axial monitors to the chambers, and finally between the 3 superlayers to form the sagitta error. This imposes a significantly stricter alignment measurement accuracy; i.e., if all systems have equal resolution, the axial sensors must attain a bending-coordinate precision under $12 \mu \mathrm{m}$, in contrast to the all-projective specification of $25 \mu \mathrm{m}$, placing increased demand on the alignment hardware.

\section{Global Alignment}

As in the case of local alignment, the global alignment of the GEM muon system, relative to the interaction point (IP) and beamline, separates into two components; positioning requirements, in which the location of the structure must be accurate, and measurement accuracy, in which the GEM (a)

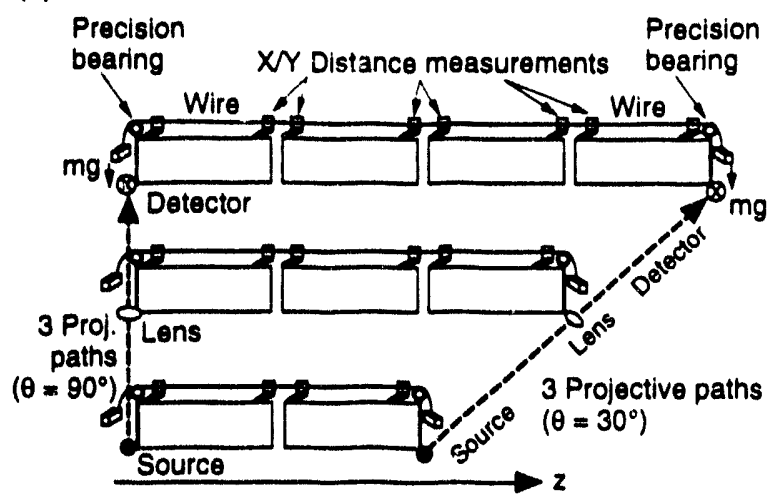

(b)

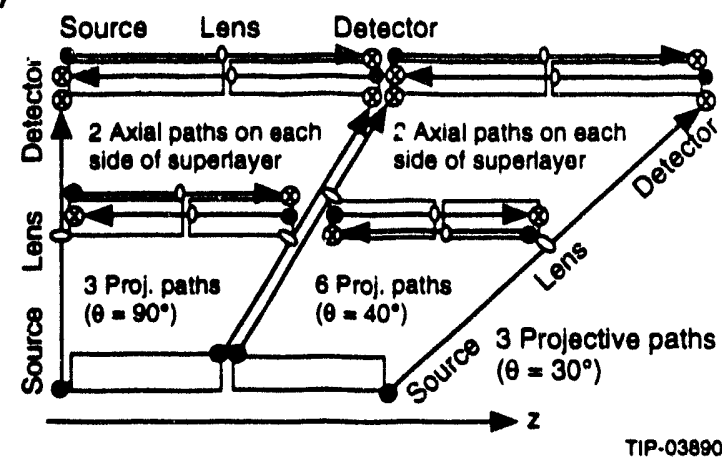

FIG. 4-69. Alternative schemes for local alignment of the GEM muon barrel.

detector position must be known and/or monitored. These requirements are given in Table 4-17 and discussed below.

The first two items in Table 4-17 state limits that relate tower-local systems to the interaction point, and are essentially projectivity requirements. The first item arises from the $\mathrm{r}$ : iction that all alignment lines-of-signt must point to the IP within a cylindrical region of $(\Delta R \leq \pm 3 \mathrm{~cm}, \Delta z \leq \pm 3 \mathrm{~cm})$, in order to adequately measure and compensate sagitta errors in the momentum measurements of projective muon tracks. ${ }^{46}$ This may be interpreted as an angular constraint on the outer chamber layer of $\Delta \theta, \Delta \phi< \pm 6 \mathrm{mrad}$ where the middle and inner layers are slaved through the local requirements, see Figure 4-60 and Table 4-16. The next item in Table 4-17 arises from the trigger system; i.e., where the strips placed in coincidence on the included superlayers must line up projectively for the trigger to be efficient and unbiased. This number represents a limit from the 2-layer $\delta \phi$ trigger option, which is much more restrictive on chamber positioning than the 3-point sagitta trigger discussed in Section 4.3.5. 
Although this is primarily a constraint in the $R-\phi$ plane, producing a tolerance of $\Delta \phi= \pm 3 \mathrm{mrad}$ in tower-to-IP pointing, the trigger will use nonbending information as well, thus will also require a much looser degree of projectivity in the $z$ coordinate already surpassed by the precision employed in pointing the straightness monitors to within $\pm 3 \mathrm{~cm}$ of the IP. The trigger projectivity likewise asserts a local positioning requirement of $\Delta x= \pm 5 \mathrm{~mm}$ between superlayers. This is currently surpassed by the local alignment requirements needed for the sagitta correction, as dictated in Table 4-16.

The gross consequences of Tables 4-16 and 4-17 on the chamber installation and stability are local placement requirements on the order of $\pm 1.5 \mathrm{~mm}$ and global pointing requirements in the vicinity of $\pm 3 \mathrm{mrad}$. These will be attained through surveying techniques, as described in Section 4.4.3 and 4.5.2.

The bottom portion of Tabl 4-17 states positioning measurement resolutions that must be obtained to produce the quoted accuracy of the GEM muon system (see Section 4.2.7). The first such item $\left(\sigma_{\phi}, \sigma_{\theta}=3 \mathrm{mrad}\right)$ limits the error in tower-to-tower and tower-to-beamline pointing, as needed to adequately reconstruct the total muon momentum and multi-muon invariant mass. ${ }^{11,46}$ This is a fairly soft resolution and it is already met by the positioning requirements stated above. The second item states the requirement on transverse $(\phi)$ alignment between towers in order to adequately resolve the momentum of muons that curve across their boundary. This $\pm 250 \mu \mathrm{m}$ requirement will be rapidly met by measuring muons that cross the overlapped regions, as discussed above and in Section 4.2.7. Muon tracks will be linked during reconstruction with corresponding tracks in the central tracker and MIP deposits in the calorimeter; the third item states a preliminary requirement of $\pm 500 \mu \mathrm{m}$ on the knowledge of alignment between the muon system and other GEM subsystems (primarily in $R-\phi$ ). Finally, the last item describes a precision projectivity measurement that is desired in order to improve the momentum resolution at very high momentum (i.e., $\geq 500 \mathrm{GeV}$ ); here the multiple scattering in the calorimeter decreases sufficiently for the beam location in $R-\phi$, where the beam is on the order of $10 \mu \mathrm{m}$ in diameter, to be used as an additional point in the momentum fit. This doubles the effective lever arm and quadruples the resolving power ${ }^{32}$ (see Section 4-2.7), in addition to improving the muon measurement. This concept is illustrated in Figure 4-70; to constrain the IP and thus extend our resolution to higher energies until other factors again limit performance, we mus know the $R \phi$ projectivity of our measured muon tracks to within $\sigma=$ $200 \mu \mathrm{m}^{31}$

The application of 3-point straightness monitors to define alignment towers (per Figure 4-57) allows a very straightforward interpretation of the GEM global alignment problem, as illustrated in Figure 4-70. As discussed carlier, each straightness monitor measures the deviation of the 3 superlayers from a projective line at 3 points on opposite edges of the alignment tower. These measurements are fit to a compensation function that interpolates this correction (Figure 4-59) across the alignment tower; any measured straight-line ray traversing a locally misaligned tower at an arbitrary angle is thus analytically straightened by appropriately combining the measurements of the various straightness monitors. Since there is no measurement or control of the straightness monitor angle except for the loose $\pm 3 \mathrm{~cm}$ projectivity constraint, the muon angle reference will be seen to wander with the $\theta$ and $\phi$ of an incident muon track as it passes from the region dominated by one straightness monitor into the domain of another, which may be canted with a different projectivity error. The error in radial tower positioning ( $\Delta y$ in Figure 4-70) has an insignificant effect on the momentum fit. The major contribution arises from the sagitta errors induced through nonprojectivity.

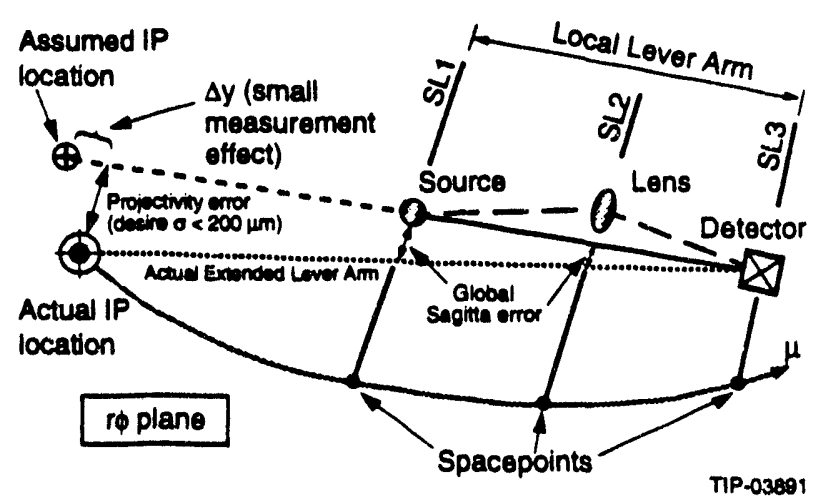

FIG. 4-70. Role of straightness monitor axes in defining muon system global alignment. 
The alignment accuracy needed to link to other detector subsystems and incorporate the vertex constraint are quite restrictive and suggest the application of a dynamic monitoring technique. Although an active multipoint range-monitoring system ${ }^{63}$ such as proposed by SDC ${ }^{64}$ is attractive, the GEM design has very limited viewing access to survey references outside the cryostat or at the beamline. ${ }^{10}$ Other monitoring schemes have been investigated, ${ }^{65}$ but suffer from excessive complication and the limited accessibility of external references.

Another possibility to attain the desired GEM global positioning accuracy is provided by a passive technique of using muons accumulated during SSC runs. There are several ways of implementing this. All methods rely on the fact that the muon measurements are essentially over-determined, allowing the global positioning to be extracted after enough statistics are collected. Section 4.2.7 and Reference 31 discuss both the application of correlated muons from $Z$ decays and the use of the single-particle muon angle information in each superlayer to determine the tower-to-tower and tower-to-IP pointing plus verification and calibration of the mechanical transfers in the local alignment system. As stated in Section 4.2.7, it is estimated that several hours worth of muon data at standard luminosity (with $p T$ above $20 \mathrm{GeV}$ to insure that they are prompt and not contaminated with long lever-arm decays) will adequately determine the most stringent global accuracies demanded in Table 4-17. A dedicated software tap into the Level 2 or Level 3 muon trigger to histogram the appropriate quantities in this fashion will enable these statistics to be promptly acquired without saturating the data acquisition stream. If the muon structure deforms significantly while this data is being collected, the application of distributed sensor information, such as temperature measurements and possibly data from instrumented level systems with a verified structural model should enable these effects to be tracked and compensated in the alignment estimation process. The positioning information estimated from the muon data may also be sufficient to describe the local chamber alignment accurately enough to resolve ambiguities in the straightness monitor measurements, appreciably widening the tolerances quoted in Table 4-16 and loosening the $\pm 3 \mathrm{~cm}$ projectivity requirement discussed above; this is currently under investigation.

It is also necessary that the alignment of the GEM muon system is properly known with respect to the magnetic field, especially in the forward regions where the field is nonuniform. This is attained through a network of magnetic probes, as discussed in Section 4.3.1 and verified with muon data, as outlined in Section 4.2.7.

\section{Proposed Test of Alignment Systems and Concepts}

The test rig sketched in Figure 4-71 has been proposed ${ }^{66}$ in order to experimentally verify the alignment concepts and systems described in this section. The three chambers illustrated here are designed (i.e., sized and positioned) to emulate the first (low $\theta$ ) tower of the GEM barrel. Because instrumented GEM superlayers will not be available initially, these layers will be dummy chambers equipped as shown with 13 sets of optical straightness monitors ( 8 about the perimeter and 5 in the center), which over-determine the anticipated sagitta correction function. As described earlier, the information from sets of straightness monitor triads located at opposite edges of the chamber should be able to predict the sagitta error at all points within the chamber. This prediction will be verified by comparing with the actual measurements made at the other monitor sites. The panels used to support the alignment fixtures will be composed of laminated honeycomb, in order to mimic the actual chamber mechanics as closely as possible. Effects of chamber deformation and sag can be directly studied by introducing deliberate stresses into the structure. Considerable experience and hardware insight will be accrued by installing our straightness monitor systems in a realistic mechanical, thermal, and dynamic environment. The lessons learned will be of significant benefit to the GEM muon system precision.

\subsubsection{Muon Electronics}

\section{Overview}

The Cathode Strip Chambers (CSC) were chosen as the muon measurement technology because they combine, in a single device, the ability to 


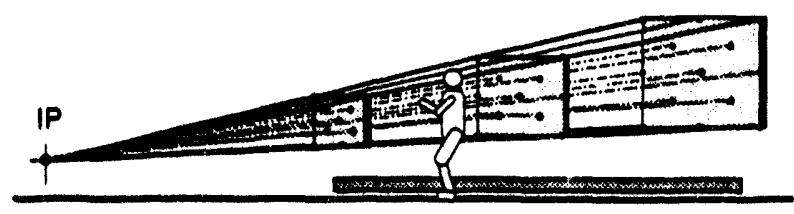

Proposed projective alignment lines of sight

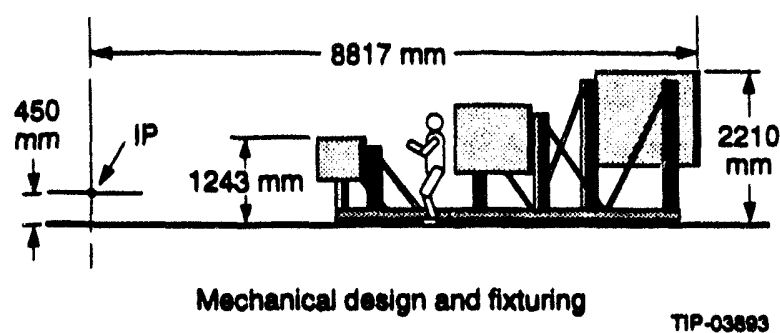

FIG. 4-71. Proposed test structure for verifying GEM alignment systems.

measure both bend and nonbend coordinates, and to provide the signals necessary to form the Level 1 trigger.

The primary challenge of the GEM muon electronics system is to provide a low-noise frontend with a high degree of noise immunity. The noise immunity is essential to allow the system to achieve the desired resolution because the resolution is obtained by interpolation of analog signals. The selected readout and trigger schemes have a high degree of integration and a modest amount of cabling and interconnects. This reduction of complexity is necessary to insure a robust system where the entire front-end readout and a significant fraction of trigger formation reside on-board the chambers. This strategy is also responsible for the reduced cost of readout essential to a chamber scheme with a relatively large number of channels. In addition, the chosen solutions present a conservative approach to instrumentation, with reliance on existing or nearterm technology. This is essential to lower the cost of $R \& D$ and reduce the risk of cost increases throughout the lifetime of the project.

The purpose of this section is to outline the general design and performance aspects of the muon system electronics and trigger, as well as the inventory of components. The details of the electronic implementation of the system components may be found in Sections 7.2.2 and 7.7.
The technical requirements for the muon chamber readout are shown in Table 4-19.

Table 4-19. Electrical specifications for the CSC readout.

\begin{tabular}{|c|c|c|}
\hline $\begin{array}{l}\text { System } \\
\text { Section }\end{array}$ & Parameter & Value \\
\hline \multirow[t]{8}{*}{ Readout path } & $\begin{array}{l}\text { Equivalent input } \\
\text { noise, RMS }\end{array}$ & $<2000 \theta^{-}$ \\
\hline & Pulse peaking time & $300 \mathrm{~ns}$ \\
\hline & Overall system ge 1 & $0.4 \mathrm{fC} /$ Count \\
\hline & Dynamic range & 10 bits \\
\hline & Accuracy & 7 bits \\
\hline & Cross talk & $<-50 \mathrm{db}$ \\
\hline & Readout rate & $100 \mathrm{kHz}$ \\
\hline & Readout latency & $<100 \mu \mathrm{s}$ \\
\hline \multirow[t]{3}{*}{ Trigger path } & Input threshold & $16 \mathrm{fC}$ \\
\hline & Timing jitter, RMS & $<4 \mathrm{~ns}$ \\
\hline & Time walk & $<4 \mathrm{~ns}$ \\
\hline \multirow[t]{4}{*}{ General } & Temperature range & $20 \pm 5^{\circ} \mathrm{C}$ \\
\hline & Power per channel & $<100 \mathrm{~mW}$ \\
\hline & $\begin{array}{l}\text { Radiation hardness: } \\
\text { ionizing }\end{array}$ & $1 \mathrm{krad}$ \\
\hline & $\begin{array}{l}\text { Radiation hardness: } \\
\text { neutrons }\end{array}$ & $10^{14} \mathrm{~cm}^{-2}$ \\
\hline
\end{tabular}

The barrel and endcap muon electronics are identical in design, but the actual implementation of the readout boards are slightly different to account for the differing geometry.

The readout chains for the cathode and anode are implemented separately, but brought together onboard a chamber module. The trigger bend-plane elements are implemented, in an integrated fashion, with the cathode readout. The trigger nonbend plane element and beam crossing time identification are implemented with the anode electronics. The Level 1 (and beyond) digital electronics are implemented outside the detector.

\section{Cathode Readout Chain}

The readout chain for the cathodes must include an amplifier, and two shapers for the measurement (slow) and trigger (fast) signals. In addition, the cathode path must provide for an ADC, and associated analog storage, as well as DAQ readout support. The trigger signals require a discriminator and a logic block for local track segment identification. The schematic for the read- 
out chain is shown in Figure 4-72. A brief explanation of the components of the cathode chains follows. Details can be found in Section 7.7.1.

- The low noise (<2000 electron-equivalent) front-end amplifier. The precision (or azimuthal) coordinate is measured by interpolating the induced charge on the segmented cathode plane. Practical limitations allow cathode segmentation with a minimum of $5 \mathrm{~mm}$, while limitations on chamber operations allow a maximum of $10 \mathrm{~mm}$. To achieve the necessary 75-100 $\mu \mathrm{m}$ resolution, the readout must be capable of $0.5-1 \%$ interpolation. This interpolation must be achieved consistently for every channel. The total number of electrons collected on the cathode is less than $10^{6}$, assuming a gas gain of $5 \times 10^{4}$, a collection efficiency of 0.2 , and about 100 primary ionization electrons. The collection efficiency is the product of several factors. The largest factors are 0.5 due to collection on one cathode, and 0.5 due to the ballistic deficit resulting from collecting only a part of the shaped charge. Thus the 2000 electron-equivalent specification provides a safety factor of at least two.

- The slow shaper for the precision or position measurement. The pulse peaking time for a semi-gaussian shaping is chosen to be $300 \mathrm{~ns}$. This is the shortest practical time commensurate with the $100 \mathrm{pF}$ input capacitance typical for a $3 \mathrm{~m}$ long strip.

- The fast shaper/discriminator for the trigger measurement. This path provides a digital signal for the formation of a $P T$ trigger. The shaper time is to be as short as possible. Current prototypes provide a 30 ns shaping time. The resulting gain, along with the fact that charge is typically distributed over 3 strips, require a discriminator threshold of $16 \mathrm{fC}$.

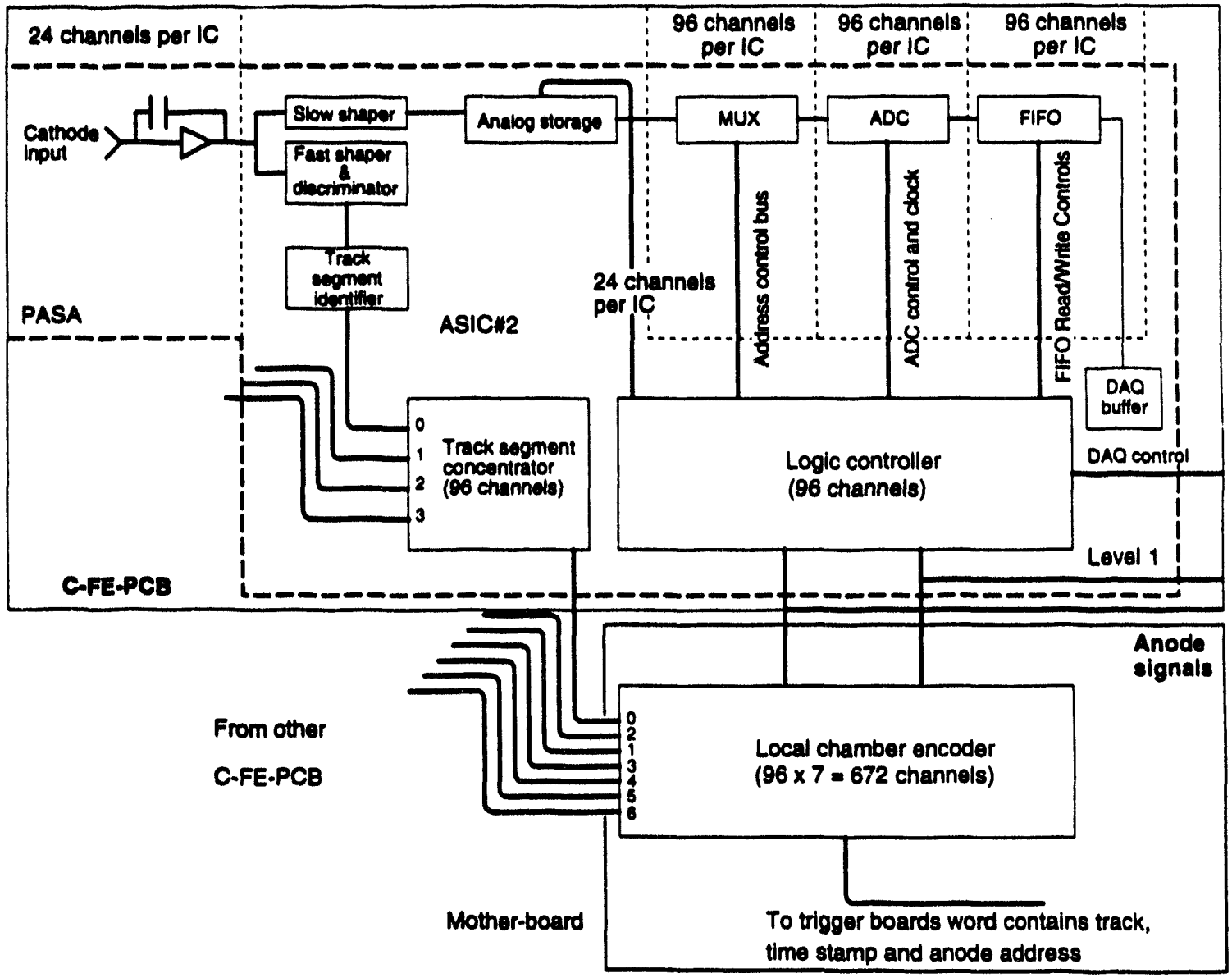

Tip.04241

FIG. 4-72. Schematic of cathode readout. 
- The analog storage unit. This provides for the Level 1 delay and as staging for the ADC to allow multiplexing of several channels onto a single ADC. This storage unit can either be a track-and-hold (T/H) device, or a more elaborate choice such as a switch capacitor array (SCA). The factors governing the choice of storage will be discussed in Chapter 7 .

- The analog multiplexer. This MUX allows the sharing of a single ADC between several channels, specifically 96 channels in the current design.

- The ADC. The ADC allows for charge measurement. The $A D C$ must operate at a $10 \mathrm{MHz}$ sampling rate and have 10 bits of dynamic range. The 10 bits of range results from four design considerations: the $1 \%$ interpolation measurement ( 7 bits), the fluctuations in ionization, the variation in chamber gains, and the variation in amplifier response. Each term adds one additional bit. Tests at the TTR have verified the desirability of this dynamic range. Note that the actual ADC resolution need not exceed 7-8 bits.

- The logic/readout controller. This is a logic state machine controlling the various functions of the cathode measurements and the Level 1 trigger formation. The controller performs many functions, including:

- The control of the MUX addressing and ADC clocking.

- The control of the FIFO which stores the ADC hits to be read out by the DAQ system.

- The readout of the ADC hits by the DAQ. The large area of the muon system and the possible high neutron counting rate could result in a large singles data rate $(-100 \mathrm{Gbyte} / \mathrm{s})$. However if the readout of these signals is made conditional on the existence of a track segment then the rate is significantly lower ( $100 \mathrm{Mbyte} / \mathrm{s}$ ).

- The management of the Level 1 and beam crossing time-stamp signals.

\section{Anode Readout Chain}

The readout chain for the anode must provide an amplifier and constant fraction discriminator, primarily to determine the beam-crossing. The discriminator signals also identify the non-bend coordinate, and thus require DAQ support. The details of the anode readout chain are shown in Figure 4-73 and can also be found in Section 7.7.1.

- The low-noise front-end amplifier. This amplifier is similar to the one described earlier in the cathode chain, but differently optimized for the anode readout. While the summed anode will in general have similar input capacitance to the cathodes, the anodes must be optimized for timing considerations. In addition, the anode readout does not have as strong a constraint on the noise and charge measurement precision.

- The shaper and constant fraction discriminator. The anode signals are processed with a shaping time of $30 \mathrm{nsec}$. The shaped signals are then turned into logic levels with a timing discriminator. This discriminator provides a digital signal for the formation of the beam crossing time from all six measurement layers. Figure 4-44 shows the timing distribution obtained from a four-layer measurement in a test beam. Each layer has a constant fraction discriminator and a first-arrival method is used to obtain the timing. The curve shows that $98 \%$ of the signals are within a single beam crossing. This should improve for a 6-layer arrangement to about $99 \%$.

- The DAQ readout controller. This IC serves to coordinate the readout of the anode channels by the DAQ. This IC also serves to coordinate the transmission of anode and timing signals to the module motherboard for cathode trigger path use.

\section{Level 1 Trigger Chain}

The Level 1 trigger is highly integrated with the cathode and anode readout chains, in order to simplify the design and to reduce system costs. A full discussion of the trigger considerations can be found in 7.2.2. A brief discussion of the elements of the Level 1 trigger follows.

- A track segment identifier of local charged tracks. The discriminator outputs within each grouping of 24 channels ( 6 chamber layers $\times 4$ cathodes) are used to form a local charged track (LCT) segment. These LCT segments require hit patterns corresponding to good tracks through the chambers. Provision is made for 


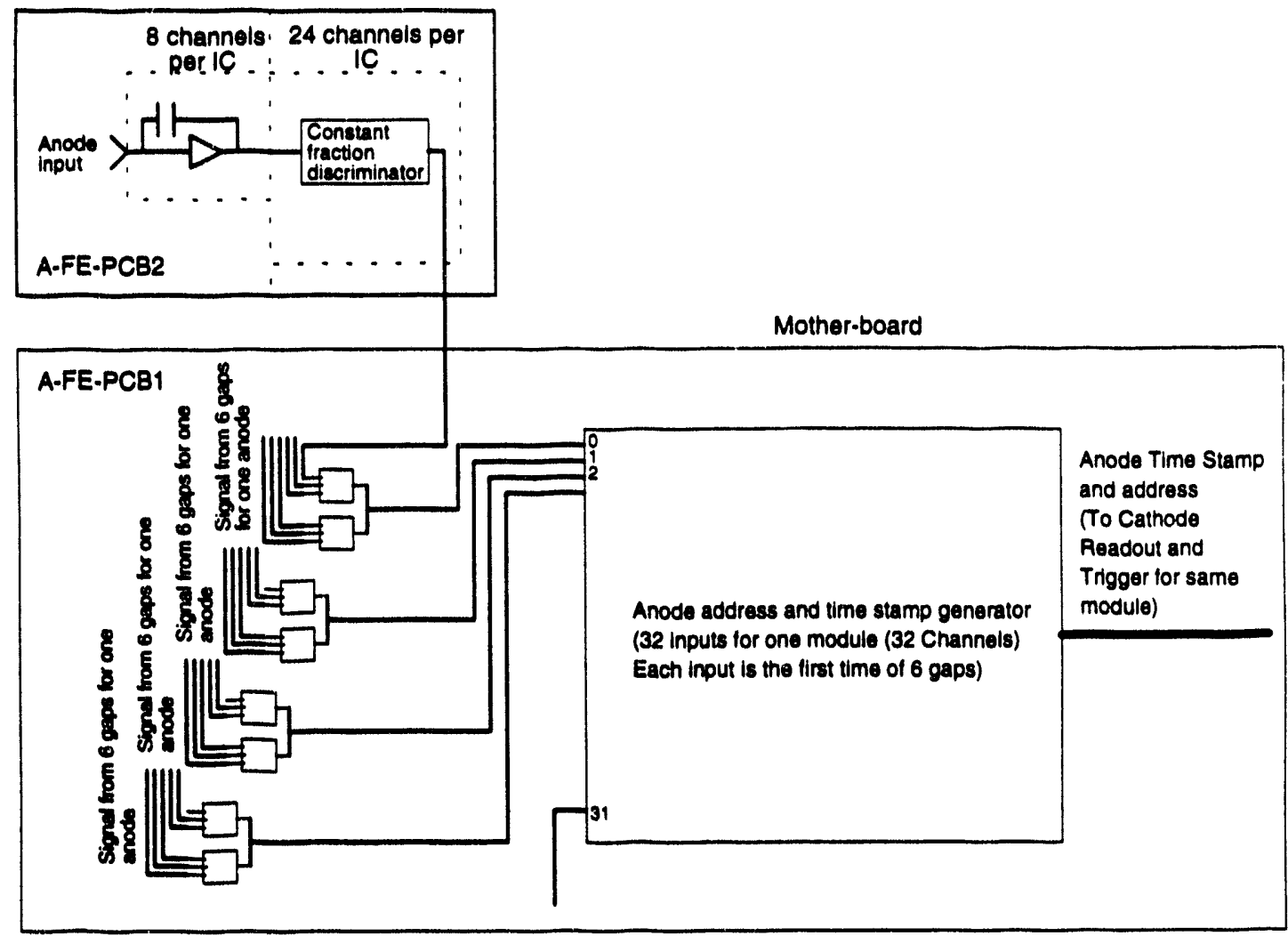

TIP.04242

FIG. 4-73. Schematic of anode readout chain.

tracks that cross group boundaries. The cathode LCT signals correspond to track segments and are formed by imposing a four-out-of-six (or six-of-eight) majority logic requirement on the discriminated outputs of the strips from all six (eight) layers lying within a small angular range.

- A local chamber encoder. This device collects all the local track segments from the track segment identifiers within a single 6 or 8 layer chamber (e.8., 28 in barrel) and outputs a sequential list of single track segments for that module. The device is located on the chamber motherboard. In addition to the track segments, the signals from the local chamber encoder include an identification of the anode grouping responsible for the generated trigger. This information is used to make a geometrical cut that the non-bend hits form a straight line. The output cable also carries the time stamp of the bunch crossing responsible for the generation of the local track segments.
- An off-chamber trigger board. The signals from three superlayers within a single projective segment are brought together to a single location on a board housed in crates located in the electronics room. The trigger is formed by loading the encoded words (LCT addresses) from the three superlayers onto a bus serving the memory cells as shown in Figure 4-74. Each three-cell column in the memory corresponds to an acceptable trigger pattern and any match with the input data signals a valid trigger. Selection of either the $\delta \phi$ or the sagitta method can be made by downloading appropriate cell contents and by requiring either two-out-of-two or three-out-of-three matches within a column. Finally, the "OR" of the accepted patterns is encoded and transmitted to the trigger supervisor. 


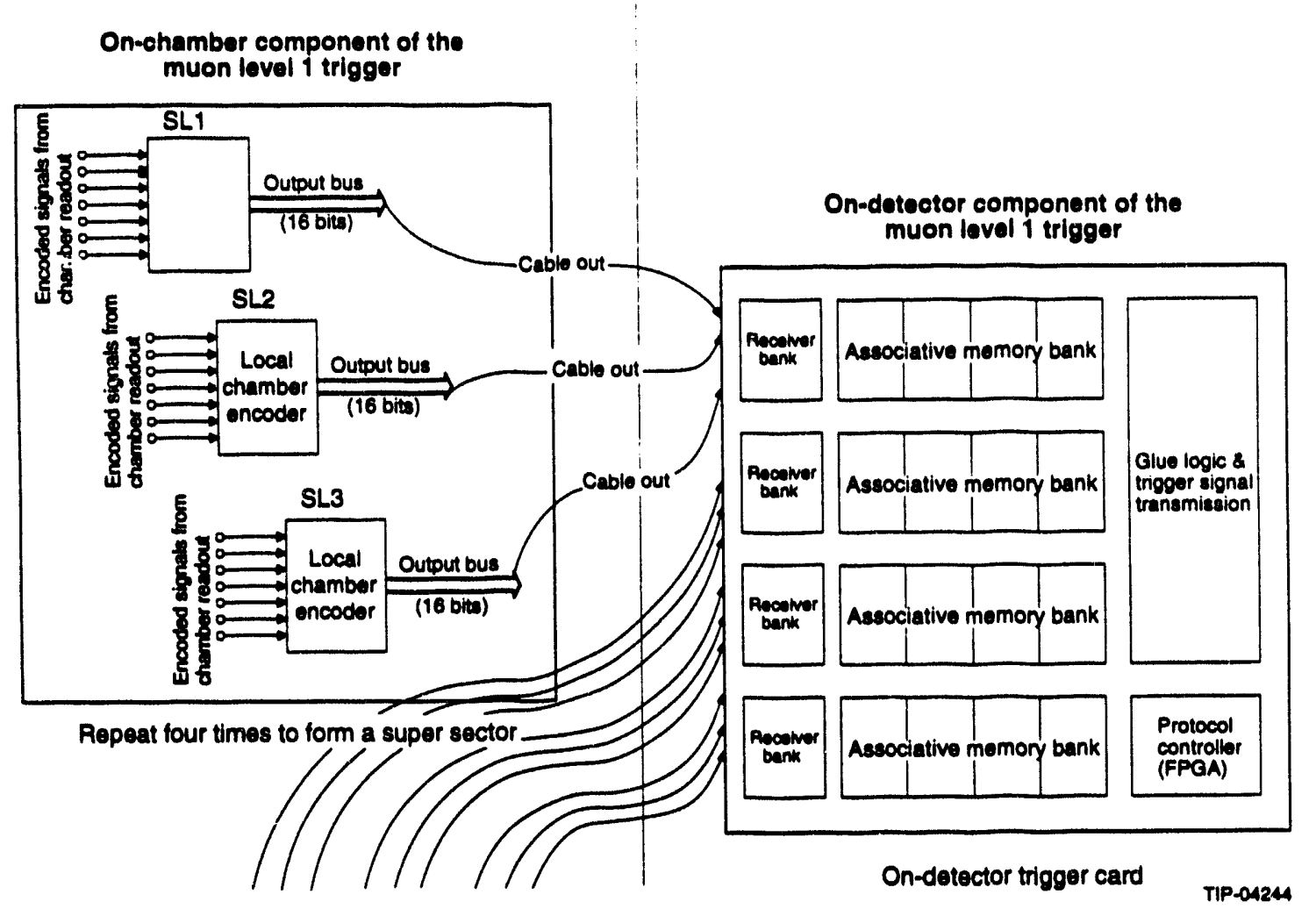

FIG. 4-74. The local chamber encoders and cables to the off-chamber trigger board.

\section{Chamber and Channel Count Inventory}

Table 4-20 summarizes the chamber quantities and cathode and anode channel count for the barrel and endcaps.

\subsection{ASSEMBLY}

The muon system support structure assembly procedures are discussed below. In addition we briefly discuss the facilities for testing and installing CSCs in their support structures, and details of the CSC mounting technique.

\subsubsection{Surface Facility Usage}

There are two dedicated surface facilities located at the IR5 site which will be available for assembly, test, and alignment of the muon system. These facilities are the South and North Assembly
Buildings (SAB and NAB, respectively) and are described in detail in Section 9.2.1. We note here that the facilities are multi-use and include high bays for the assembly of muon prototype module structures, and for the endcap and barrel final assembly. There are also low bays for supply storage, office space, and associated shops. The current assembly plan specifies that additional space will become available as the magnet assembly is completed for receiving, storage, inspection, assembly, and testing of muon system components. Figure 9-5 depicts a later stage of assembly operations when one of these buildings is being used for assembly of muon system components. There will be various cranes available in all surface facilities, to aid in the assembly process and to facilitate the preparation of the assemblies for installation in the detector underground hall. 
Table 4-20. Barrel and endcap cathode readout, physical layout and channel count.

\begin{tabular}{lrrrrrr}
\hline \multicolumn{1}{c}{ Component } & \multicolumn{2}{c}{ Barrel } & & \multicolumn{3}{c}{ Endcap (Each) } \\
\hline $\boldsymbol{\phi}$ & SL1 & \multicolumn{1}{c}{ SL2 } & SL3 & SL1 & SL2 & SL3 \\
$\theta$ - Segments & 48 & 48 & 48 & 24 & 48 & 48 \\
No. of Chambers & 4 & 8 & 8 & 2 & 2 & 2 \\
No. of Cathode Channels & 129,024 & 258,048 & 258,048 & 46,080 & 46,080 & 46,080 \\
No. of Anode Channels & 73,728 & 73,728 & 73,728 & 9216 & 18,432 & 18,432 \\
TOTALS & Cathode Channels: 921,600 Anode Channils: 313,344 & & 96 \\
\hline
\end{tabular}

\subsubsection{Barrel Region Assembly \\ Support Structure Assembly}

The structures that support the CSCs inside GEM are truss structures manufactured from high strength aluminum (6061-T6) pipe sections. The sections will be joined with a combination of welded, pinned, and bolted joints. The discussion of the installation is found in Section 9.4.4.

Muon support structures will be completely fabricated off-site. They will be inspected and evaluated prior to partial disassembly and shipping to the SSCL. Disassembly will be kept to a minimum. Upon arrival at SSCL, the structures will be unpacked, inspected, stored, and reassembled as needed in the SAB. Muon module assembly, as well as chamber testing, will take place in the SAB. Final assembly of the barrel and endcap monolith structures will take place in the NAB. Twenty four barrel modules (twelve for each magnet half), each containing 4 sectors of CSC for a total of 960 barrel chambers, and two endcap three-wheel monoliths containing the 480 endcap CSC, will be assembled.

Utilizing assembly fixtures the entire module will be assembled and all critical dimensions verified. Individual parts may be removed to allow chamber installation to proceed.

\section{CSC Assembly Into Barrel Modules}

The entire barrel region will be assembled and partially aligned in the surface assembly buildings. The assembly process has two major phases: 1) the assembly of the muon chambers into the muon support structures to produce muon barrel modules, and 2) the assembly of the 12 modules per GEM detector half with the CDS and FFS end support rings to produce a barrel region muon assembly (monolith). Two complete assemblies are required for the GEM muon system; mirror images of each other in order to keep the same tilt relationship of chambers with respect to the magnetic field direction.

Insertion of the chambers into the module support structure assemblies will be done from the bottom to the top (outer to inner superlayers) to maximize the use of overhead cranes for handling and positioning of the muon chamber packages. Each superlayer of chambers, with its interface hardware, will be inserted with the aid of an overhead crane lifting fixture.

Following insertion of all chambers, and preliminary alignment of the chambers based on their final orientation in the barrel assembly, there is a final module alignment. This process involves moving the completed module to a rotary stand (shown in Figure 4-75) where the module will be rotated to its planned orientation with respect to the vertical. Alignment systems will be checked to determine and calibrate the offsets of the chambers with respect to their nominal placement. If necessary, adjustments of chambers will be made. Finally, the completed module will be rotated to the storage orientation, outer superlayer down, and will be moved to the barrel module storage area in the North Assembly Building.

\section{Barrel Monolith Assembly}

Each barrel module will be joined to form a monolithic structure. Two monoliths are required, one for each magnet half. Each barrel monolith will be supported by two end rings that are constructed using a simple truss structure. One end ring will be supported within the magnet at the CDS end. The other end ring will be supported from the floor at the forward field shaper (FFS) end. The support rings 


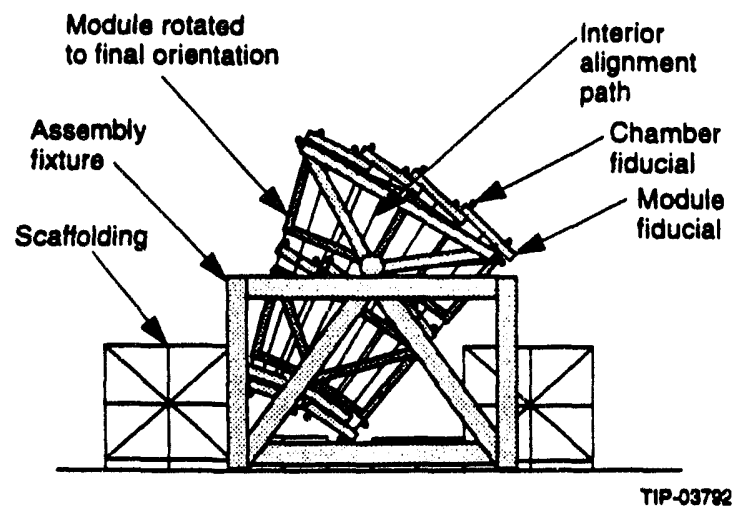

FIG. 4-75. Rotary test stand for module pre-alignment.

will not be stable relative to each other during initial assembly operations, so a special assembly fixture will be used to support the rings and the barrel muon modules while they are being attached to the rings. Since each module must be assembled in the position and orientation that it will have in the detector, special assembly fixtures will be required. The assembly fixture will be set to the necessary height and location for insertion of the barrel module into the ring structure. The module will then be rotated within its frame to its proper azimuthal orientation.

The first assembly operation is the assembly of the two end support rings. The end rings, like the barrel region module structures, will be completely fabricated off-site, with bolted and pinned interfaces between sections to permit subsequent disassembly and accurate reassembly at the SSCL. Upon receipt, the components of the ring assemblies will be inspected and the rings will be reassembled flat on the floor of the NAB. They will then be erected, positioned, and secured with their cylindrical axes horizontal and collinear for the assembly of the 12 muon modules. Both end ring structures will be mounted to base hardware, which will eventually be used as part of the transport fixture that will move the completed muon end assembly to the underground hall.

In order to insert the modules into their final position, attached to the two support rings, it is first necessary to attach a bridging fixture, which contains two installation rails. This structure will be used to translate each of the 12 modules through the inside diameter of the FFS end support ring, to the attachment point on the CDS end support ring. This operation will be repeated 12 times to complete the assembly. Modules are installed from the bottom to the top to maximize usage of the overhead crane. Removal and subsequent repositioning of this module insertion fixture will require an additional fixture for the lower four barrel modules. In addition, the process of inserting a module into the support ring assembly will require two special handling fixtures.

The insertion fixture, shown in Figure 4-76, will consist of a circular structure with a base, capable of moving each module to its proper horizontal location, and a jacking system for the height. Both the base and the jacking system will be equipped with leveling and stabilization systems to allow the position of the module to be fine-tuned for installation into the end rings. The insertion fixture will also be equipped with a set of rails to allow the modules to be inserted from one end and maneuvered into position.

A barrel region module, calibrated for a specific location within the muon system, will be selected and loaded into the circular insertion fixture. This is achieved by placing the module, with the outer superlayer chambers (the largest cham-
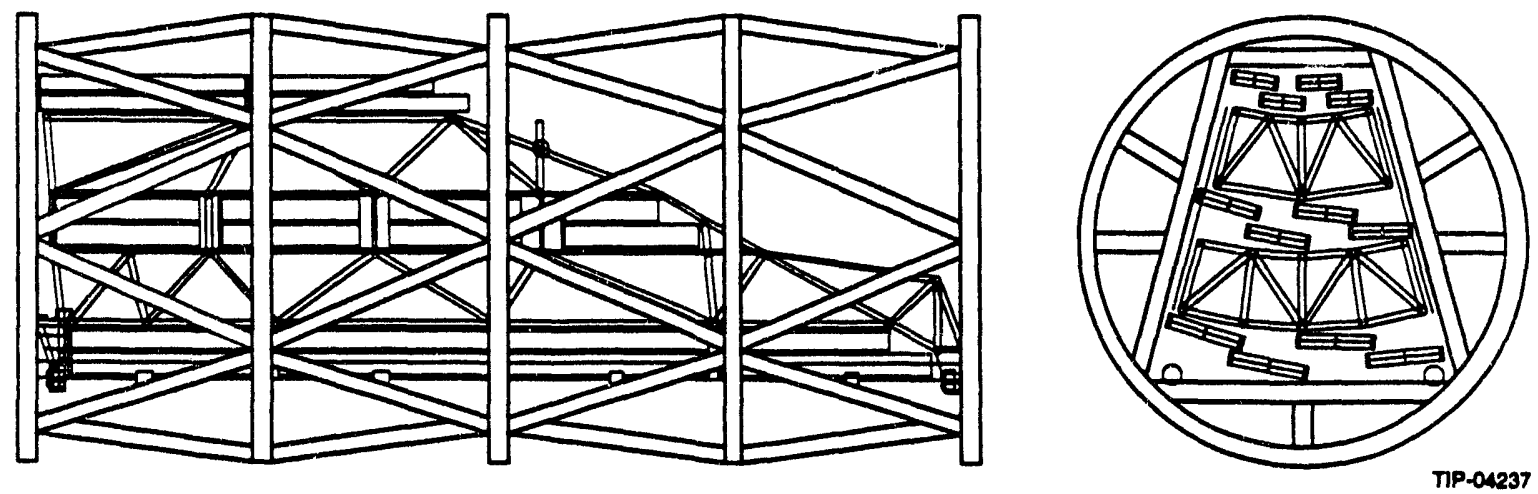

FIG. 476. Barrel module installation fixture. 
bers) facing downward, onto a pair of rails of a small transfer fixture that is matched to a set of rails on the insertion fixture. The module is then rolled into the insertion fixture. Figure 4-77 shows the insertion of the first module.

Following this loading operation, the module is secured, and the module insertion fixture is rotated to its proper orientation and moved by overhead crane to the support staging area. The insertion fixture containing the barrel module is placed on an adjustable fixture located at the top of the support ring. This fixture is a staging assembly with an adjustable stand, to permit accurate alignment of the insertion-fixture rail system with the rail system for the support-ring. Once alignment has been verified between the insertion-fixture, the end rings, and the staging assembly, the module will be transferred from the insertion fixture, through the first end ring, and along the rails into position within the rings. Each end of each muon barrel module will be supported by three connection points, with adjustment hardware to align each module within the rings. The insertion rail system will provide a pivot point for these adjustments, and will also allow for axial adjustment of the entire module.
After the module reaches its proper z-position with respect to the CDS ring, the module weight will be transferred from the rails to a temporary holding and posicioning fixture. The connections to the rails will be disconnected, the module will be properly oriented, and finally fixed to both the CDS and FFS support rings. Final alignment of the individual modules will occur only after all 12 modules have been installed, so that the final pointing errors caused by end ring deformations due to nonuniform loading are minimized.

Hardware that will mate the four load-bearing points between the support rings with an individual module will be inserted and secured, before the load is transferred from the temporary-support to the final-support fixture. Transferring the load in this manner will maintain the prescribed module-pointing alignment requirements. However the process is expected to be iterative, and the hardware will allow adjustments based on the observed results. It is anticipated that the ability to predict the behavior associated with the load transfer will improve during installation, and the number of required iterations during installation will be reduced.

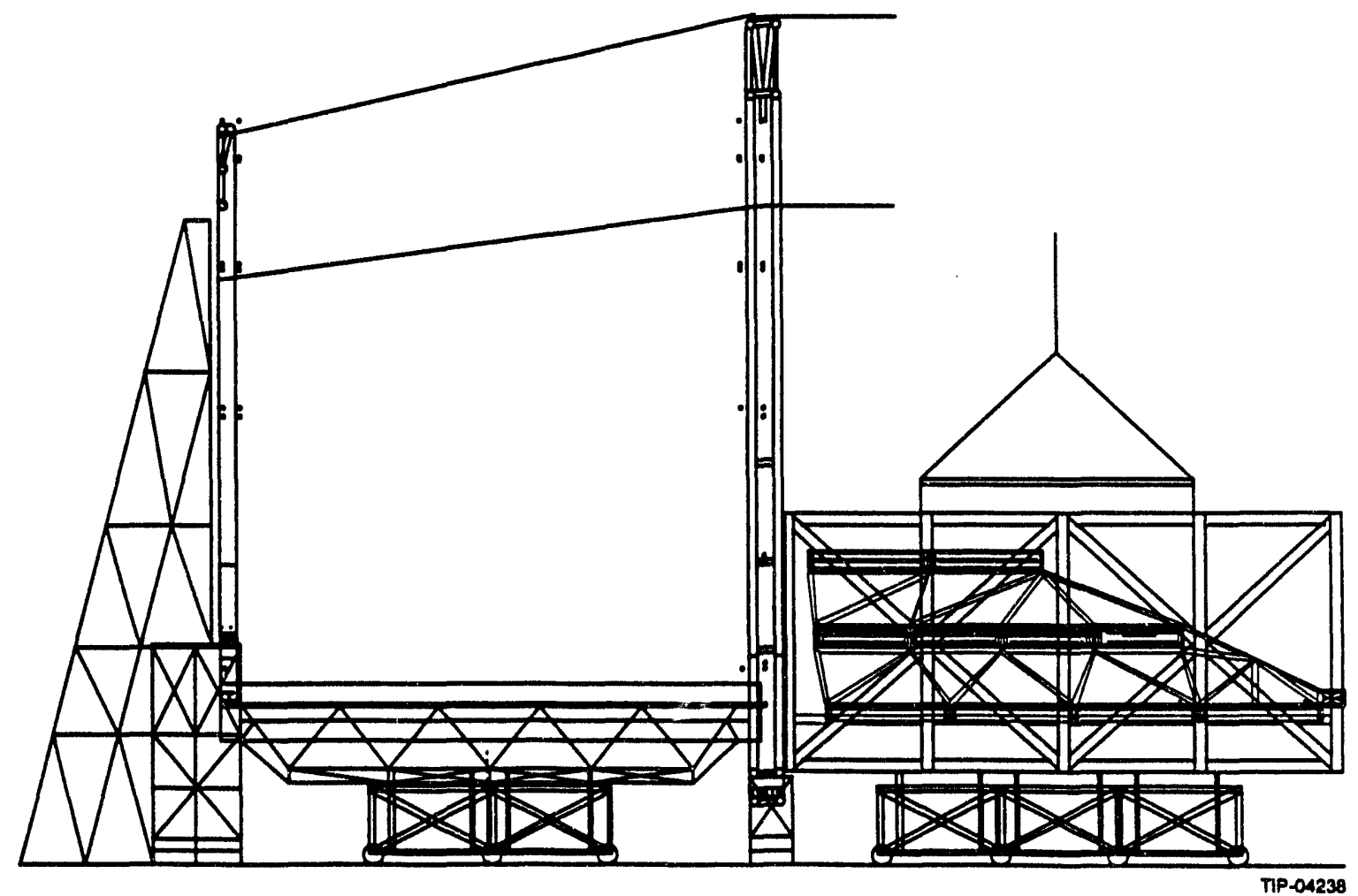

FIG. 4-77. First module insertion into the barrel monolith. 


\section{Barrel Monolith Installation}

The two end support rings for the barrel monolith are built atop structures that will form the end pieces of the muon system transporter. Upon completion of the barrel assembly, the remaining center portion of this transporter will be installed. The barrel region muon system on this transporter will be moved to the appropriate access shaft. A crane will be used to lift this entire assembly over the access shaft, and to lower it to the floor of the underground hall. Here, the muon system is transferred from the transporter to the installation rails, which will be used to translate the muon system through the magnet cryostat. Once the muon system is inside the magnet, it will be fastened into place at attachment points near the CDS and the FFS. This procedure is discussed in detail in Chapter 9.

\subsubsection{Endcap Region Assembly Endcap Chamber Assembly}

The endcap chambers are joined at their edges in $\theta$, in order to maintain full $\theta$ coverage and to create a chamber structure that will behave as a predictable elastic element. This is necessary to provide an alignment system for the endcap region that is projective along the $\theta$ boundaries of an alignment tower and is radial along chamber edges to provide a complete specification of the tower alignment. The radial alignment is only effective if the joined chambers behave in a predictable manner as a rigid body in the radial direction. Conceptually, these chambers will be merged using an additional honeycomb panel structure to supply the continuity between individual chambers.

\section{Endcap Monolith Assembly}

The endcap assembly is made from three wheel-assemblies and two conical connective structures. The wheel-assemblies are rimmed wheels with minimal crossbracing, and radial spokes which provide the mounting sites for the chambers for each of the three endcap superlayers. In order to attain full coverage in $\theta$ and $\phi$, CSCs will be placed in the structure with offsets in the $z$-direction to allow for overlap between each CSC and its two adjacent neighbors. Chambers will be attached on both sides of the radial spokes, to achieve the required $\phi$ overlap. The conical shaped structures add torsional stiffness to the assembly, and tie together the three wheel subassemblies into an endcap monolithic structure.

With the wheel subassemblies separated, there is access to both sides of the wheel structures. This greatly facilitates the installation of CSCs into these structures. The current concept accomplishes this task by utilizing scissors-type lift devices to vomplete the positioning of the chamber (Figure 4-78). The litting device utilizes a fixture, located from above, to support, rotate, and translate each chamber assembly. The chamber assembly is outfitted with special hardware to properly align it with respect to the mounting sites on the support structure. Cham-

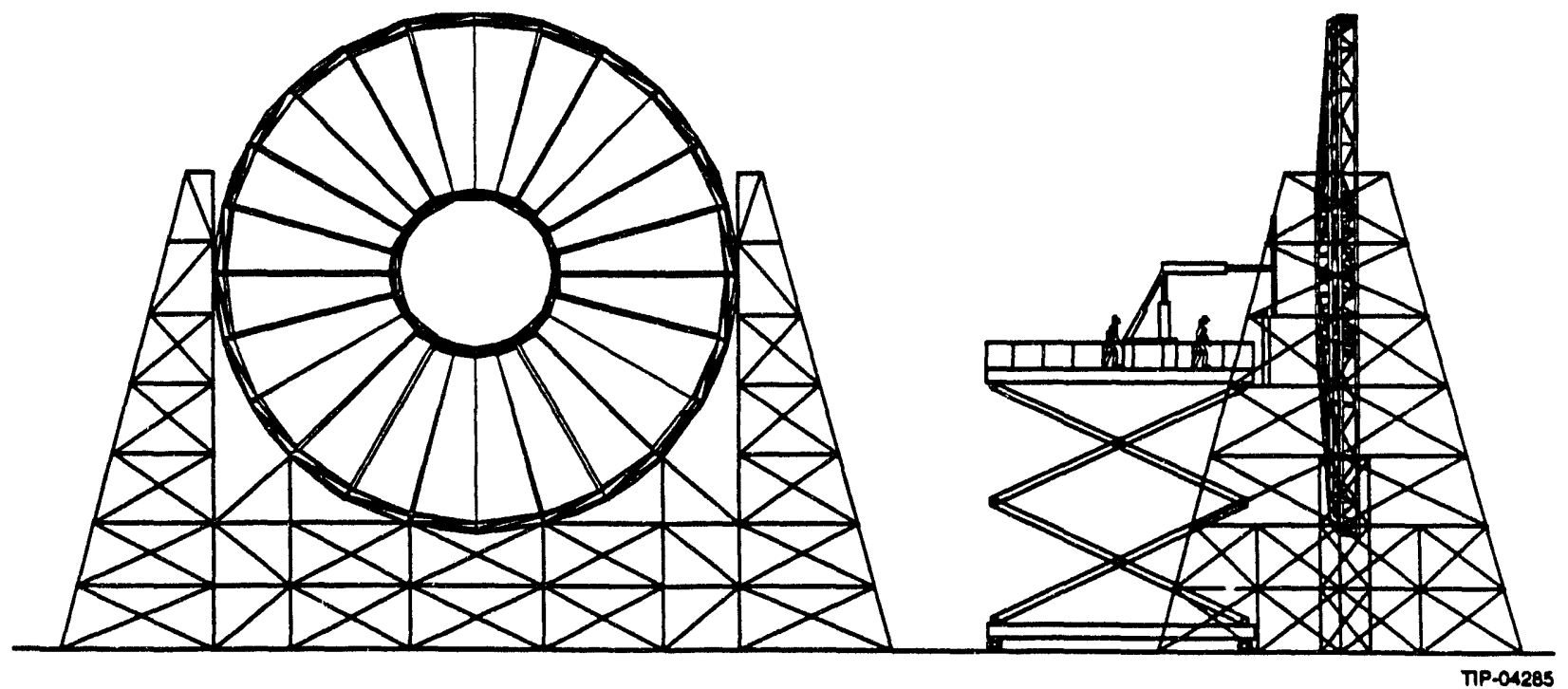

FIG. 4-78. Assembly of chambers onto an endcap outer wheel. 
bers will be installed on opposite sides of the wheel structure in parallel, to minimize unbalanced loads on the support structure. Individual chamber services and superlayer-related service elements will also be attached at this stage.

Once this process is completed, assembly of the endcap monolith will start. The assembly sequence will begin by attaching the largest interconnecting structure to the outer superlayer wheel assembly. This connective structure will be wheeled into place, then jacked into position for attachment of pins and fasteners. The sequence will continue with the other connective structure being attached to the middle superlayer wheel assembly. These two resulting assemblies will then be merged using procedures similar to that for the attachment of the individual assemblies. Finally, the inner superlayer assembly will be attached to the front of the endcap monolith.

Details of transferring loads from temporary fixtures to their adjacent subassemblies have yet to be fully worked out. When finally assembled, the endcap will be supported on its final four weightbearing attachment points, to simulate its mounting to the barrel region. Final alignment of the endcap chambers and the alignment towers will then be completed.

As with the barrel chambers, most of the electronics, gas, and cooling system interconnections will be completed during construction of the endcap monolith. This will minimize the number of connections that have to be made in the experimental hall.

\section{Endcap Monolith Installation}

Each muon endcap monolith will be temporarily mounted to its respective FFS on the surface. The assembly process of merging the FFS and endcap monolith will be done in the NAB. The FFS will then serve as a transport fixture for the muon endcap. Following the final checkout of an endcap monolith, it will be transferred to the FFS where it will be attached to two mounting sites near the ends of the conical surface of the FFS. The entire FFS/monolith assembly will then be moved to the respective access shaft. Here, the assembly will be lifted, translated over the access shaft, and then lowered to the floor of the undergrc $d$ hall. Once set onto the floor mounted transport rails, the FFS will be used as a linear transport fixture to bring the assembly into the magnet. The FFS will be capable of delivering the monoliths at their proper height and orientation at the base of the installation shaft. It will then be pulled to the proper position in the hall, so that the muon endcaps can be joined with the barrel sections. This is discussed in Chapter 9.

\subsubsection{CSC Mounting, Positioning, and Alignment}

The CSC package will be attached to the muon system support structure in such a way that a 3-point kinematic mount is achieved. This system allows motion in all degrees of freedom, in order to provide for precision local and global alignment of the chambers. The mounting scheme consists of multiaxis precision rails with an attachment plate fastened to a swivel joint. The kinematic mount will have three-axis degrees of freedom for one attachment point, two-axis degrees of freedom for the next attachment point, and one degree of freedom for the third attachment point. Each precision rail allows a linear range of about $2 \mathrm{~cm}$.

Alignment of the muon system has been described in detail in Section 4.3. Here we stress the importance of the alignment requirements on the chamber design and the chamber mounting system. Alignment demands for the three superlayers of a muon measurement tower require that we have knowledge of the relative lateral positions of these three measurement layers (radial in the barrel and axial in the endcap) to an accuracy of better than $25 \mu \mathrm{m}$. As result, it is critical that 1) structures, interface hardware, and chambers be stable, 2) chamber positions be initially adjusted to within measurement range of alignment monitoring hardware, 3) there be real-time alignment monitoring hardware, and 4) there be an active servo-controlled positioning system to return the alignment monitor triads to the mid-points of their operating ranges in the event of an excessive excursion.

The 3-point mounting scheme utilizes remote positioning and sensing systems (servo-control) for each 3-point mounted chamber package. The positioning actuators are envisioned to be air-, hydraulic-, or piezoelectric-operated because of the magnetic field, which precludes the use of most electric driven systems. Each actuator will provide the precision mechanisms coupled to optical or mechan- 
ical switching electronics to provide the readout of position. An important aspect of the kinematic 3-point mounting system is that all three positioner systems are coupled together to provide coordinated motion of the chamber package in all degrees of freedom. This coordination implies that the panel will not deform as the individual actuators are driven along their respective axes. The least count of the motion for the positioning/sensing system is not a limitation because of the sagitta correction available from the projective alignment system, as described in Section 4.3. Placement accuracy of the actuator system is therefore only a few millimeters (see Figure 4-60, Section 4.3), to satisfy the alignment requirements for the muon system. The operation of the remote positioning/sensing system will necessarily be closely coupled to the optical alignment system, to allow further verification of the sensor information.

The servo controlled middle superlayer of CSC, additionally, must have hardware to permit the remote adjustment of the chamber's lateral position. This system will be a simple lead screw coupled to a high-ratio gear train driven by a motor which can operate in the magnetic field. Straight-line monitors will provide the position measurement. This system could be run in either closed or open loop modes. If needed, a force-unloading device, to lessen the force on the actuator systems, could be constructed using pneumatic cylinders coupled to a controlled pressure source. The cylinders would apply a constant lateral force appropriate for a given chamber in a specific module. After chambers have been moved to their prescribed location with an allowance for unloading, the pressure to the pneumatic cylinders would release, and the lead-screw system would ensure that no additional chamber motion will occur.

\subsection{MUON SYSTEM INTEGRATION}

\subsubsection{Services}

The services and utilities for the muon chambers include gas and high voltage supplies, as well as DC low voltage, water cooling, and optical fiber systems for the chamber-mounted electronics. The design philosophy for the gas and HV systems is to have central supply units located outside the detector, where easily accessible space is available. The gas and HV from the central units are distributed to chambers via distribution stations, located outside but near the detector. Distribution within a muon chamber is a part of the chamber package design. Hookup points for chamber gas and HV will be located on the chamber support structure. Low voltage power supplies for chamber-mounted electronics, one for each chamber, will be placed on the surface most accessible to personnel for each chamber.

The HV, water and gas distribution schemes are driven by the desire for redundancy, and cost considerations. Each utility line will service the whole or a portion of a chamber. In the event of a chamber failure, that portion of the chamber will be temporarily disabled. The disabled chamber will be repaired or components replaced during regular maintenance. This calls for one gas line, six HV lines for each chamber, and one HV line for each wire plane.

Gas

The total gas volume in the muon CSCs is about $150 \mathrm{~m}^{3}$. The flow rate is determined by many factors, one of which is the leak rate of the chambers. Conservatively, assuming a maximum leak rate of one chamber volume per month, a flow rate of two volume changes per day is sufficient to remove any oxygen that might leak into the chambers. This translates to a recirculating capacity of $300 \mathrm{~m}^{3}$ per day. To allow for a flushing rate at about ten times this normal flow rate, the gas system is designed to have a peak recirculating capacity of $150 \mathrm{~m}^{3}$ per hour.

The final gas mixture has not been chosen, but the prime candidates are $\mathrm{Ar} / \mathrm{CF}_{4} / \mathrm{CO}_{2}$ mixtures. For safety reasons, only nonflammable mixtures are being considered. $\mathrm{CF}_{4}$ was chosen for its speed, Lorentz-angle characteristics, and because $\mathrm{CF}_{4}$ mixtures do not polymerize.

A schematic of the gas system is shown in Figure 4-79. The gas is stored and mixed in a facility located above ground. The gas is piped down the utility shaft to the detector pit, where it is fanned out to 144 channels-one for each muon sector-including 96 in the barrel and 48 in the endcaps. All chambers in a given sector (10 chambers in the barrel, or the endcap) are served in parallel by one supply line with individual pressure regulators. The parallel distribution ensures that the chamber overpressure due to the pressure drops along the exhaust line is well below 


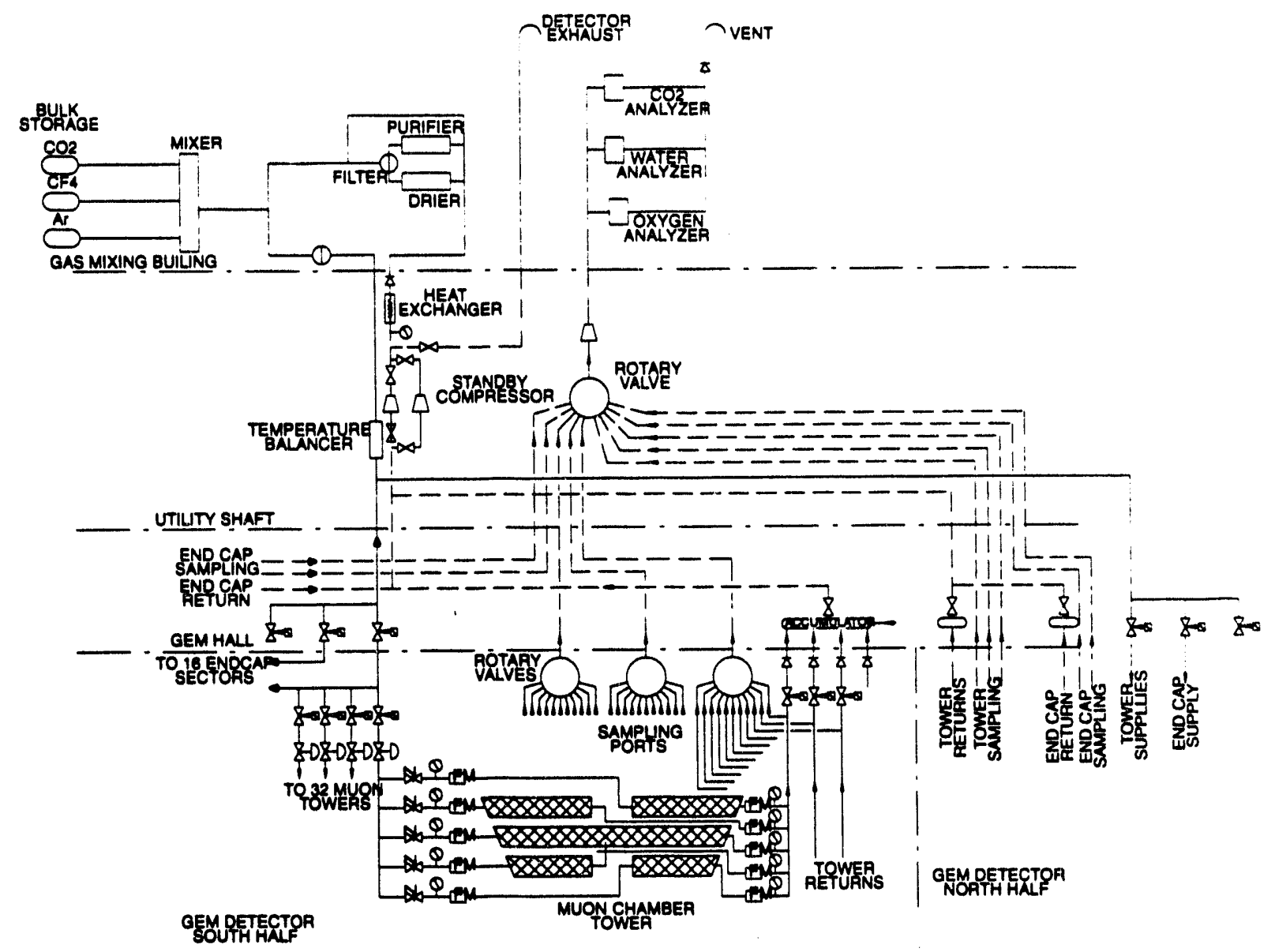

FIG. 4-79. Schematic of the muon gas system.

$0.5 \mathrm{~cm}$ of water. The input pressure and flow rate of each chamber are individually monitored. The returns from all chambers are collected in an exhaust line that returns to the surface mixing building, where the gas is purified and recirculated. Oxygen and water are removed from the gas by standard techniques.

Catastrophic gas leaks are detrimental to the chamber performance and also present safety hazards. Gas sensors will be installed on the chambers to detect leaks. Analog signals from the sensors, which are proportional to the local concentration of the detected gas, will be sent to a monitoring computer where appropriate action will be taken in the event of gas leaks.

\section{Power}

The high voltage system for the GEM muon chambers will be computer controlled. Each CSC anode wire plane (six per 6-gap chamber) will be connected to an independent HV supply. In the event of a wire plane failure caused by a broken wire or a serious gas leak, the HV to that plane will be switched off by the computer by identifying any over current condition. In this scheme, the entire GEM muon system (barrel and the endcaps) requires a total of $8,832 \mathrm{HV}$ channels. The plan is to work with industry to custom design and build an economical HV system for GEM.

The gain of each chamber may vary due to differences in construction and size. It will be desirable to operate each anode plane at a different HV in order to keep the gain constant. The gain also varies according to the temperature $(T)$ and barometric pressure $(P)$, roughly as $T / P$. At the location of the GEM detector, $T / P$ is expected to vary at the level of $1-2 \%$, which results in a typical gain variation of $10-20 \%$. It is desirable to correct for these changes by adjusting the HV to compensate the gain variation using a dynamic feedback loop. ${ }^{67}$ This tech- 
nique has been tried and proven to be effective in a muon chamber system in the SMC experiment at CERN where a daily $T / P$ variation of $\pm 3 \%$ was successfully corrected. The proposed scheme for GEM is to continually measure the gain of a subset of the muon chambers, which are located at different parts of the detector. The information will be used to determine the $\mathrm{HV}$ in order to compensate for the gain variations.

The power supplies providing low voltage to the chambers and integral front-end electronics will be mounted on the outside of the magnet vacuum vessel, as close to the FFS as possible. Magneu. field tolerance will be a criterion in power supply selection, and magnetic field shielding will be included if necessary. Input low power units will be supplied from the cable electronics shaft.

\section{Cooling}

The cooling system for the front-end electronics is mounted on the chambers and will be distributed throughout GEM. There will be pressure, temperature, flow control, and measurement hardware on each sector. There will be about $80 \mathrm{~kW}$ of cooling power available for the barrel and $30 \mathrm{~kW}$ for the endcaps, corresponding to a temperature rise of $4^{\circ} \mathrm{C}$ in the chilled water. Each chamber will require less than 30 liters/hour, which can be met by a standard water cooling system.

\section{Cabling}

The cabling from individual chambers will be tied first into sector bundles and then into monolith bundles. Cables for fast systems that will require short runs will exit the monolith at one end ring in as many locations in azimuth as are necessary to match the electronics crates mounted on the outside of the magnet vessel. All other cables will be brought to single points on either side of the monoliths, and suspended between the monoliths and the walls of the hall on flexible support systems that will allow the magnet halves with the monoliths installed to be moved axially by up to 10 or 15 meters without the need to disconnect the cables. The cables will then be routed to the appropriate room in the electronics shaft or to the surface buildings as required.

\subsubsection{Commissioning}

Thorough testing of the muon system and its individual components at every phase of its construction and assembly is of the utmost importance for the GEM detector. During construction of the individual muon chambers, tests will be made for gas tightness and proper high voltage operation. Each superlayer will be fully equipped with electronics at the construction site and tested with the $x$-ray station, as described in Section 4.3 (Figure 4-66). These tests will not only verify the interchamber alignment, but also will provide a complete map of all superlayer parameters that affect muon system performance (e.g., calibration of the entire electronic readout chain, local gas gain variation, etc.).

The muon chambers will be flushed with dry gas and HV tested on receipt at IR5. Next, they will be filled with an operating gas mixture connected to a data acquisition system and then verified with cosmic rays and $x$-rays. After the barrel superlayers are mounted into an assembly module $\left(\Delta \phi=30^{\circ}\right)$, they will be cabled and once more tested for electronics performance, high voltage stand-off, and gas leaks. The elements of the alignment system will then be activated and verified. The assembly module will be fixed to a revolving stand and oriented at the intended $\phi$ angle. The chambers will then be positioned in the center of the range of the alignment monitoring system, within the tolerances given in Table 4-16. The remote actuators for chamber positioning will be checked to verify performance and to check the response of the alignment monitors. The chambers will again be connected to a data acquisition system, and checked using cosmic rays and the $x$-ray source. During this phase, the fixtureto-chamber alignment transfers can be roughly measured and/or checked using cosmic ray data or more precisely determined with the $\mathrm{x}$-ray beam. This alignment transfer could be achieved by filling the chambers with xenon-doped gas and scanning them with $x$-rays originating from a rotating $x$-ray head at the effective IP location or from a translating/ rotating head at other positions. These techniques provide a source of straight-line tracks, which can be reconstructed and compared with the alignment measurements to correct the transfer errors. ${ }^{68}$

Because of the tilanner in which the endcap systems are constructed (see Section 4.4.3), they are commissioned in a somewhat different fashion. As the chamber packages are installed into each endcap wheel (Figure 4-56), their position is adjusted to the 
required tolerance (Table 4-16). Upon completion of a wheel, the chambers will be resurveyed and realigned, and their performance will be rechecked. After the wheels are joined into endcap monoliths, the chamber performance and alignment will be verified with cosmics and $x$-rays, as discussed above for the barrel.

After the barrel modules and endcap chambers have been installed into their respective monoliths, a similar series of checks will be performed on the operation and calibration of chambers, electronics, gas, and alignment. Upon completion of each monolith, the chamber positions may have drifted and can be trimmed again using the mechanical adjustments, to meet the quoted positioning requirements (Tables 4-16 and 4-17). Cosmic ray data will be taken to verify the chamber performance. The collimated $x$-ray source will be used to recheck local and global alignment transfers.

After the monoliths are lowered into the experimental hall and inserted into each magnet half, all cables and utilities will be connected, the gas system activated sector-by-sector, and the flows balanced and sampled. The high voltage circuits will be selectively activated and tested, and chamber electronics checked. The alignment systems will be activated and their performance verified. Significant distortions of the overall system positioning will be corrected as required using mechanical adjustments and survey systems to bring the chambers back into specification. The alignment system will also be employed to check the proper operation of the remote actuators. Chamber performance will be verified with cosmic rays.

\subsubsection{Access and Maintenance}

The muon system, based on sealed cathode strip chambers, will require minimal maintenance during normal operation. Chambers will be extensively checked and tested during all phases of construction and assembly into the monolith to insure proper operation. The chamber design, consisting of wires stretched across cathode strips, will be susceptible to failures due to wire breakage over the lifetime of the experiment. Wire breakage is expected to be minimized by tensioning the wires to $2 / 3$ of their yield strength, and by bonding to the printed circuit boards using epoxy as well as solder. Experience has shown that after initial tests at the factory locate faulty wires, subsequent breakages are very rare. No attempt will be made to remove or replace broken wires during normal short maintenance periods. The failure of a wire in a chamber will likely result in the loss of that particular chamber gas gap. There are 6 gas gaps per chamber ( 8 in the first endcap superlayer) and 1440 chambers in the muon system for a total of 8832 gas gaps. Clearly, the loss of a single gas gap will have minimal effect on the performance of the system.

For the wire readout electronics, one hybrid amplifier chip is used to read out 40 wires ganged together in each gas layer. The failure of a chip will result in the loss of $10 \mathrm{~cm}$ of wire position readout used for the readout of the coarse $z$-position for that particular gap. The extremely large number of wires in the muon system implies that these losses will have minimal effect on system performance. It should be noted that it will be difficult to replace barrel chambers without transporting the barrel monolith back to the surface facility. A system for replacement of wires in situ will be developed (to be done during annual long shutdowns). Replacement of endcap chambers may be possible under special circumstances, by pulling the endcap assembly clear of the barrel assembly as detailed in Section 10.4.

The other muon chamber subsystems can be accessed and maintained during normal longer term shutdown periods. These include gas and water cooling systems; high and low voltage cables and connectors; cathode strip electronics boards and wire readout chips; chamber motherboards; chamber alignment components, including LEDs, lenses, video cameras, and position actuators plus encoders.

\section{Gas and Water Cooling Systems}

The concept for the gas system has the supply, mixing and pressure regulation systems outside the magnet, either on the surface or in a distribution system located adjacent to the detector in the hall. Gas system components inside the magnet include gas leak sensors, flow monitors, pressure relief valves, tubing and fittings, and sensor and monitor cabling for power and readout. In the event of chamber gas leaks, detectors placed within the muon volume will notify operations control. The gas system is modular enough that leaks should be quickly identified to the sector level. Beyond the sector level, manual leak checking would be required. To prevent leaks from occurring there will be maximal use of welded joints. 
A water cooling system will be implemented for the chamber electronic boards reading out the cathode strips. Again, maximal use of welded joints will be made to prevent leaks from occurring. Shutoff valves will be installed at the board level to aid in replacing faulty boards.

\section{High and Low Voltage Systems}

All high and low voltage power supplies will be located outside the magnet for unimpeded access during beam down time, without removing the FFS or magnet structures. High voltage is distributed to the chamber wires via standard cables (one per chamber layer). Low voltage is distributed to the chamber motherboard where it is fanned out to the strip and wire readout electronics, as part of the ribbon cables and wire hamess assemblies.

\section{Chamber Electronics}

Chamber electronics boards will be located on the chamber edges, and the surfaces most accessible to personnel. The cathode strip readout boards can be mounted either on the edges of the narrow dimension of the chamber, leaving spa ,e between boards for alignment fixturing, or on the surface facing into the support structure volume. In the concept where boards are mounted on the chamber edge, ribbon cables connect the cathode strips to the readout boards and also the readout board to the chamber motherboard. Replacement of a cathode strip board may involve disconnecting water cooling tubes, disconnecting the cathode strip ribbon connectors ( 6 connectors) from the outside of the board, disconnecting the board ribbon connector to the motherboard (which contains low voltage distribution as well as signals), and removing the board from its mount and away from the edge of the chamber. Wire readout electronics are located on the long side of the chamber, and consist of a high voltage distribution network, a series of readout amplifiers, and preamplifier assemblies. The preamplifier assemblies will occasionally need to be replaced by a simple plug-in operation. Motherboards will be located in the central region of the chamber facing the inside of the structure for ease of access. Replacement of a motherboard is aided by the use of ribbon cable connectors for the strip readout boards and wire readout chips, and fiber-optic connectors for the signals out of the board to the higher level electronics located outside the magnet.

\section{Chamber Alignment and Positioning Systems}

Chamber alignment components include LED light sources mounted on the inner superlayer, lenses in the middle superlayer and imaging detectors on the outer superlayer. The 6 light sources per chamber will be powered by low voltage $D C$ distributed from the chamber motherboard. Sources under consideration for the alignment system are discussed in Section 4.3.4, and are standard devices with a high MTBF. Nevertheless, the ability to replace the LEDs is foreseen. Access to LEDs will be at the edges of the short sides of the chambers. The LED is mounted in a precision fixture, which will be inserted into a precision mounting block attached to the CSC. Replacement will involve disconnecting the power and removing the LED from its mounting block using standard tools. No maintenance is expected to be necessary for the lenses mounted in the middle superlayer. The imaging deicctors mounted on the outer superlayer are small chips mounted on boards, providing connections for low voltage power and signal (RS-170 video). A precision fixture will mount the video card onto the chamber, and will enable the replacement of a board in a similar manner to that of the LED fixture. Position actuators will be pneumatically operated, with an encoding read-out system for position monitoring.

\subsection{R\&D ENGINEERING AND MANUFACTURING PLANS}

\subsubsection{R\&D Engineering Plan}

The muon $R \& D$ engineering plan will guide the concept of the GEM muon system into a fully-researched, tested, and engineered design; ready for mass production and ultimately for installation in the GEM magnet. We foresee that the R\&D engincering plan, which at this time is the central activity of the muon group, will follow the life-cycle of the experiment. In these early stages (at the time of submission of the Technical Design Report), the effort will be concentrated on the development of the final chamber, support structure, and alignment designs that can be manufactured economically, and on the optimization and integration of the muon system with the rest of GEM. In the longer term, the plan will focus on mass production of parts of the system and the development of the quality control, installation and commissioning procedures. - inally, the body of knowledge and experience with the 
chambers, alignment, and support structure accumulated through this program will form the basis for the analysis algorithms used in the physics program when the detector is finally commissioned.

The R\&D engineering plan is comprised of two interrelated parts: 1) development of CSC muon detectors, their associated hardware, alignment, servo-positioning systems, electronics, water and gas systems, and integration to the support structure; and 2) support structure engineering and prototyping, including analyses of the stresses and deflections, and a study of the support structure interfaces to the chambers, alignment system, central detector support, magnet, and FFS.

The GEM muon group has assembled a team of engineers and physicists who will participate in the two major parts of the engineering aspects of the plan. Lawrence Livermore National Laboratory (LLNL) will lead the effort for CSC engineering and integration, while Charles Stark Draper Laboratories (CSDL) will lead the effort for the support structure engineering and integration of the support structure to GEM. There will be contributions to all these efforts from SSCL and university groups as well as our collaborators overseas.

The R\&D engineering plan will have four phases: (1) final engineering design and industrialization of the design for mass production of chambers, support structure, electronics, and alignment systems, including final optimization of the system in terms of performance, costs, and integration with the GEM detector; (2) mass production of elements of the system, quality control, and assembly of parts above ground at the SSCL; (3) installation of support structure-chambers-alignment systems in the GEM solenoid; and (4) final testing and commissioning of the detector in preparation for data taking.

\section{Beam Test Facilities}

Cosmic ray tests of muon chamber prototypes are under way at the TTR at the SSCL. These tests, described previously in Section 4.3, have been instrumental in our muon detector technology development and selection process.

Further testing of muon chambers will be carried out in a manner complementary to the TTR using muon beams at various facilities around the world. We expect that these test beams will be available throughout our $R \& D$ and production eras in order to continuously check performance and to optimize our designs. Chapter 13 presents a detailed discussion of test beams that are available to GEM and we will describe here our specific program of testing.

The pre-SSC test beams era will consist mainly of prototype testing and debugging. The SSC era will consist mainly of system tests of calibration and alignment hardware with muon chambers that could subsequently be installed into the detector. There will be some overlap, both in schedule and function.

We anticipate that non-SSC beams will be available to GEM at FNAL, BNL, and as well at CERN through our collaboration with CERN groups. Beams in Russia (at PNPI, ITEP, and JINR) have also been used to test muon chamber prototypes and will be used extensively. The FNAL beams cover a range of energies up to $800 \mathrm{GeV}$ and a wide range of particle types and fluxes. However, they will not be available before the end of 1994, as currently scheduled. BNL test beams are available in 1993 at $9 \mathrm{GeV}$, which is adequate for many purposes.

We also intend to use the RD5 beam at C.ERN. Members of the GEM muon group are members of the RD5 collaboration and have tested a small-scale prototype endcap CSC in that beam. Another opportunity for testing chambers in a high energy muon beam is to use the SMC beam at CERN. Other participants of the GEM muon system are members of the SMC collaboration and they have received permission from the SMC management to test small open profile CSC chambers in the $200 \mathrm{GeV} / \mathrm{c}$ muon beam of that experiment.

\section{Muon Detector Design}

The design of cathode strip chambers (CSC) will quickly evolve from the scientific prototypes built up to this point to the engineering prototypes necessary to provide a fully-developed chamber design for production start-up in mid-FY95. The engineering program will involve the selection of all CSC design criteria from a review of current and planned scientific prototypes, including CSC panel and wire frame fabrication techniques, and precision requirement for system physics performance. Engineering prototypes will incorporate designs for chamber alignment fixturing, mounting and posi- 
tioning systems, and chamber services. Also incorporated into the CSC design will be any necessary external stiffeners, braces or carrier frames for the preservation of chamber mechanical precision. The CSC design program will attempt to provide chambers for the GEM muon system that minimize the number of chambers and alignment paths, and minimize the amount of material in dead regions such as frames, and gaps. Altemative designs for chambers and chamber layouts are actively being considered and evaluated in close coordination with the GEM muon simulation group.

An important element in the first phase of the R\&D Engineering program will be the "industrialization" of the chamber design with attention given to cost reduction of parts and labor. To achieve this goal, we will fabricate a series of small chambers to optimize certain aspects of CSC design (see Section 4.3.2). Also during this period several full-scale prototypes will be constructed and fully instrumented to investigate the mechanical and electrical performance parameters and to certify the overall design prior to mass production.

In the later phases of our plan, the chamber prototyping and testing program will be important in assessing the effect of any changes in the CSC design forced by requirements of mass production. While we intend to keep such changes to a minimum, some changes will likely be unavoidable and will demand full testing to assure performance. Further, any changes in the chamber design could be reflected in their resultant layout in the support structure. For example, increase in chamber sizes would impact the support structure and alignment scheme.

Engineering designs will be validated by the fabrication of prototype chamber packages at SSCL, LLNL, JINR, IHEP, and elsewhere. Validated design concepts will be incorporated into production engineer- ing designs and implemented as quickly as possible to allow for lead times of materials and supplies prior to production. Also included in this program will be prototyping of fabrication and assembly procedures for factory production, and prototyping of tooling, temporary supports and stands, testing hardware, and shipping containers.

Results from our prototype program and R\&D efforts have thus far been encouraging. The goals of our $R \& D$ program of cathode strip chambers for the short term will concentrate on the following points:

- Certify that the cathode and anode configurations envisioned for each superlayer yield the requisite spatial and timing resolutions. This will involve the fabrication of several small prototype chambers and testing them with cosmic rays.

- Evaluate an altemative and potentially less expensive construction of cathode strip chambers. In this scheme wires are strung in open profiles in the manner of larocci ubes. An R\&D program will be undertaken to fabricate a chamber constructed in this way with $5 \mathrm{~mm}$ profiles. Preliminary results with $1 \mathrm{~cm}$ profiles are quite promising.

- Verify the detailed performance of a CSC in a magnetic field. We will build small chambers and test them in various magnetic field configurations to validate the Monte Carlo predictions of the Lorentz drift and geometric contributions to chamber resolutions.

- Develop preproduction full-scale chamber modules. This effort will test construction techniques, internal alignment of layers of the chamber within a superlayer, and the electrical performance in an integrated 6-layer chamber.

- Study different gases and voltages to determine the most satisfactory combination from the standpoints of resolution, safety, dead time, trigger speed, and cost. 


\section{Beam Test Program}

Some of the above testing can be done in the continuously operational TTR. However, the muon system will require additional tests using accelerator beams. We envision tests of the following:

- The GEM muon chambers will operate in a magnetic field, in certain orientations where Lorentz angle effects may have substantial impact. Therefore, we will need to carry out beam tests of the muon chambers in various orientations in a $0.8 \mathrm{~T}$ magnetic field.

- Muon radiation is the dominant source of energy loss above about $200-300 \mathrm{GeV}$ momentum. Pattern recognition problems resulting from this radiation will be studied, preferably in a $0.8 \Upsilon$ magnetic field, simulating the GEM environment.

- Hadron punchthrough is also a problem for pattern recognition. This will be studied using material simulating the calorimeter placed in front of muon chambers. The track curvature induced by the magnetic field has a significant effect on the punchthrough probability and pattern recognition. Therefore, it is necessary to do this testing in a $0.8 \mathrm{~T}$ magnetic field using a hadron beam of the highest available energy.

- Tests of full-scale prototypes, and actual production modules, in the barrel and endcap configurations, are envisioned as an important part of our preproduction program. In the barrel configuration, for example, there will be three superlayers with approximately $5 \mathrm{~m}$ separation between the inner- and outer-most layers. In the endcap, this separation will be even greater. There will be tests of performance and alignment in high energy (to minimize multiple scattering) muon and hadron beams.

Beam testing and calibration is an activity that will continue even after installation of all of the detector components and the start of running. There will be detector upgrades to be developed and tested. It is likely that detailed measurements, such as long term stability tests of alignment systems, will be important for the proper characterization of our system. Therefore, we are planning for long-term use of the SSCL calibration hall.

\section{Chamber Integration}

The design of the chambers and mounting fixtures needed to support them will be developed in conjunction with the design of the support structure. During the course of the design, analysis will be performed to certify that the baseline resolutions are achieved. Short term work will include design of all devices and stands required for fabrication, and testing of chamber mounting and positioning devices. Engineering analysis will be performed to optimize and to confirm the performance of the designs. Interfaces to the chambers and assembly procedures will be verified. The design will be documented in terms of drawings and specifications sufficient for preproduction prototype construction to begin in the later part of FY93.

Chamber engineers will necessarily assist in the design of the support structures for the barrel and endcap. This will include aiding in detailed structural analyses of the fully loaded structure for vibration, thermal effects and alignment stability. Special attention will be devoted to chamber interfaces to the structure, chamber/structure integration in the magnet and alignment system integration.

The integration of the chamber design will concentrate on the following issues:

- Integration of chambers with support structure and alignment. Chamber engineers will design kinematic mounting techniques, perform chamber structural characterization, define environmental specifications and develop requirements and specifications for chamber interfaces. Detailed drawings will be developed by the end of FY93 and prototype hardware will be procured and tested.

- Integration of muon system services and access with other GEM subsystems. Long term plans include integration of all aspects of the muon system with other GEM detector subsystems including specification of mounting interfaces for achieving placement accuracy required by GEM. Services including gas, cooling, cabling for high and low voltage and signals will also be integrated as part of this plan. 
- Development of plans for chamber installation into the truss structure. Chamber installation plans will be developed to ensure placement accuracy within the range of alignment monitors. Prototype structures and chambers must be designed and fabricated to test installation techniques and to assess placement accuracy.

- Prototype support structure/chamber (barrel and endcap) module development. Our program will be to specify chamber and alignment interfaces into the prototype support structures in order to evaluate stability and placement accuracy. Structural analysis will be performed on the prototype structure and compared with measurements. In the longer term design and fabrication of a full-scale prototype or preproduction sector assembly with chambers will be carried out.

\section{Chamber Alignment}

The detailed design of the chamber precision alignment system will be developed and analyzed. Emphasis will be placed on the evaluation of sensors, integration of alignment systems with CSC packages, and the testing of the alignment system. The design will be documented in terms of drawings and specifications so that a prototype of the alignment system can be built, both as a stand-alone sub-scale system and, later, in coordination with the assembly of the prototype muon system sector.

To test the alignment concepts in the short term, an alignment test system (see Section 4.3.4) will be fabricated with a support structure integrated with chamber mock-ups. This system will allow testing of alignment systems prior to the actual availability of real chambers and structural elements. X-ray radiography of wire chambers will be reviewed and adapted, if necessary, to the characterization of chamber wire/pad placements.

At this time there are several attractive alignment schemes which have demonstrated the required level of performance. The most promising of these is the projective alignment scheme, whereby the three superlayers of a given tower of the muon system can be aligned. Certain variants of this system are still under development and our test bed will be utilized to make the final technology choice, as discussed in Section 4.3.4. In the early phase of this work the alignment technologies will be studied independently. Crucial to the success of the muon system as a whole, is the efficient integration of the alignment system with the mechanical construction of the chambers and support structure. Therefore, alignment fixtures will be built into prototype chambers as soon as possible to gain an empirical understanding of the integrated system.

Included in the alignment task is the coordination with the support structure engineering to ensure proper lines of sight for alignment. Local and global alignment pathways and details of paths through chamber gaps will be designed to minimize impact on acceptance. Industrialization for fabrication of alignment sensor and associated hardware will be developed This includes generating conceptual drawing packages for sensors and associated hardware for attachments to chambers/structures. In the longer term we will provide detailed drawings and coordinate with chamber and structure engineering, as well as identify potential industrial (or university) vendors.

\section{Engineering Design of Chamber Services}

Conceptual designs of the chamber support facilities are needed early in the overall planning. Included in this task are specifications of the locations of chamber electronics, cabling, HV and LV power supplies, gas and cooling systems. The following items will be addressed.

- Specification and initiation of conceptual design of $L Y$ and $H V$ distribution and monitoring systems. We will design $\mathrm{LV}$ and $\mathrm{HV}$ distribution systems for the CSC system including alignment systems and other monitors such as gas and water flow monitors. Coordination with chamber and structure engineering will be needed to identify cable paths, attachment points, etc.

- Specification and initiation of conceptual design of gas system, distribution and monitor systems, and gas recovery system. A conceptual design package has been prepared and completed. Concept designs include purity monitors, leak monitors and re circulators. Longer term effort will be needed to fully engineer these systems.

- Coordination of the design of muon system with other engineering plans for the GEM environmental control and monitoring system. 
- Determination of cable volume and routing for all systems. We will work to assess cable systems for the CSC and support systems and identify the proper cabling methods to allow for safety, optimization of chamber coverage and minimization of cable mass for the optimization of muon tracking.

\section{Support Structure Design and Prototype}

It will be necessary to check the design of the support structure and its integration with the chamber and alignment system design. We require an alignment precision ( $25 \mu \mathrm{m}$ superlayer-tosuperlayer) and the structure must be stable, and the resultant false sagitta must be measurable to better than this precision. To verify our designs, we propose to construct a three-superlayer sector with a truss support structure in a mockup of the final support structure sector. This will test the structural support concept and validate the engineering calculations of structure rigidity and deformation. It will also provide a realistic test bed for the alignment and chamber position actuator systems proposed for the final system. We will test this sector using cosmics, $x$ rays, and high energy muon beams. We will gain invaluable experience with the operation of the chambers and alignment, and also glean a measure of the ultimate muon system performance as a whole.

\subsubsection{R\&D Manufacturing Plan}

There are roughly $1500 \mathrm{CSC}$ to construct and test for the complete GEM muon system. The chamber production process will involve the efforts of many manufacturers and factories. The critical components of the chambers-the cathode strip electrodes and the honeycomb panels formed with these electrodes faces-will be manufactured using a number of U.S. industrial manufacturers. The CSC components accepted by quality control, will be provided to several chamber assembly factories. Chamber components, wire, adhesives and fasteners will also be ordered by GEM in the U.S. and provided to the assembly factories. The current plan considers assembly factories in Russia, China, and in the USA.

The different assembly factories will use processes that have been reviewed and standardized as much as possible to ensure the maximum commonality of the final chambers. Each will have performance tests specified and documented before chambers are shipped to the IR5 assembly point.

The fabrication of the chamber support structures will be carried out in a similar manner with U.S. suppliers, as needed, providing materials to overseas and domestic assembly factories.

\section{Chamber Industrialization and Factory}

Studies, analyses, and plans will be initiated to develop a prototype factory for chamber mass production. This will be a joint effort of engineering and physics personnel. An analysis of parts, precision and nonprecision, will be made along with a determination as to whether foreign and/or domestic sources are more appropriate. A review of the most viable industrial methods will be performed. Solicitation from industry for mass production will be initiated. A detailed plan for the transition from prototype to full-scale production at the required rate will be developed. The appropriate system engineering will be applied for the development of the mass production of the chambers.

Layout of chamber production techniques will be studied by analyzing previous production facilities. Special emphasis will be placed on optimizing production sequences to minimize cost and manpower and to maximize throughput potential. As part of this task, a technique for the rapid installation of wire planes into the CSC chamber to the required precision will be developed and implemented in all factories.

Detailed plans and drawings and a prototype demonstration factory will be completed by the end of FY93. Cathode plane assemblies must be accurately registered relative to each other. A technique will be developed to assure this requirement during the assembly process. Complete conceptual designs and detailed designs for chamber integration will be developed by the end of FY93.

We will solicit interest from industry for mass production of chamber components. We are especially interested in specifying the proper methods for production of precision components for the chamber technologies. This specification will involve incorporating new concepts in fabrication of precision parts, developed at muon group institutions and elsewhere, into mass production processes. Identification of industrial vendors will be facilitated by 
experience with suppliers of special materials and parts for precision assemblies. Precision measuring techniques will be developed to verify critical tolerances of as-received components and completed assemblies. Chamber design engineers will work to assure that precision tolerances of the cathode plane can be achieved by commercial vendors. Manufacturing technology, vendor capabilities and QA procedures will be confirmed.

A factory capable of mass production will be commissioned. This will involve developing a design which addresses all tooling, space allocation, storage and shipping requirements. We will work with foreign institutes to initiate a prototype production factory to be built in the U.S., coordinating the supply and distribution of production materials and parts, shipping containers, quality assurance, and inspection. Also, we will develop plans for additional factories to achieve the required production rates. In the event that foreign production is not viable. facilities in the U.S. will be identified that could be utilized on short notice for the production of chambers.

One key to the monitoring of our progress will be production analyses. Development of measurement techniques to confirm compliance of all manufacturing tolerances will be carried out. This includes but is not limited to cathode plane flatness, spacer straightness, wire placement, and interplane registration. Complete conceptual designs and techniques will be implemented on initial chamber component purchases. The production processes will be reviewed and efficiency evaluated in terms of cost and time. It will be necessary to develop a system model which will permit optimization analyses to be performed so that tradeoffs can be identified and evaluated for capital investment alternatives and labor-intensive solutions. We will provide justification that validates our specific production factory design.

\section{Chamber Production Process}

At the factories, the manufactured cathode panels with precision electrodes will be assembled into chambers using well-established multiwire proportional chamber (MWPC) production techniques, with only a few steps requiring precision fixturing. We list below the major steps in the production process for CSC. The conceptual design has been shown in Figures 4-48 to 4-53 (Section 4.3.2).

The major steps in CSC assembly are shown in Figure 4-80. Numbers in parenthesis refer to the following operations which require special facilities.

- Operations "1" requires a temperature controlled room to ensure dimensional stability.

- Operations " 1 " and " 2 " require special jigs and fixtures for accuracy.

- Operation "3" requires that we develop new winding machines, optimized to our application, that can be mass-produced economically.

- Operations "3" and "4" require clean areas because of the exposed electrodes.

- Operation " 5 " requires special jigs and tools and will be carried out in a clean temperature controlled area; this operation defines the chamber accuracy.

Delivery of CSC will begin when the first sector of chambers is completed at the production facilities. Chambers will be packaged in special containers and sealed to prevent moisture contamination. The shipping containers will also be built to prevent shocks from mishandling that could cause wire breakage or alignment shifts. Production chambers from factories overseas will likely be shipped by sea using standard container shipping, or with the option of using air cargo depending on schedules and costs.

\section{Support Structure Fabrication}

Aluminum pipe sections will be fabricated into truss sections at factory locations. The design allows for a maximum of welding into flat structures, with the remaining joints being pinned for alignment and bolted for load carrying ability. The pipe sections with welded end joint features and the welded sections will be heat treated to recover the original T-6 temper. The sector modules will be assembled at the factory to verify all dimensions and fits. They will be then unbolted and packaged for shipping to the final assembly site.

\subsubsection{Quality Assurance}

Quality control and quality assurance requirements and implementation plans will be an integral part of the conceptual design process. It is clear that 


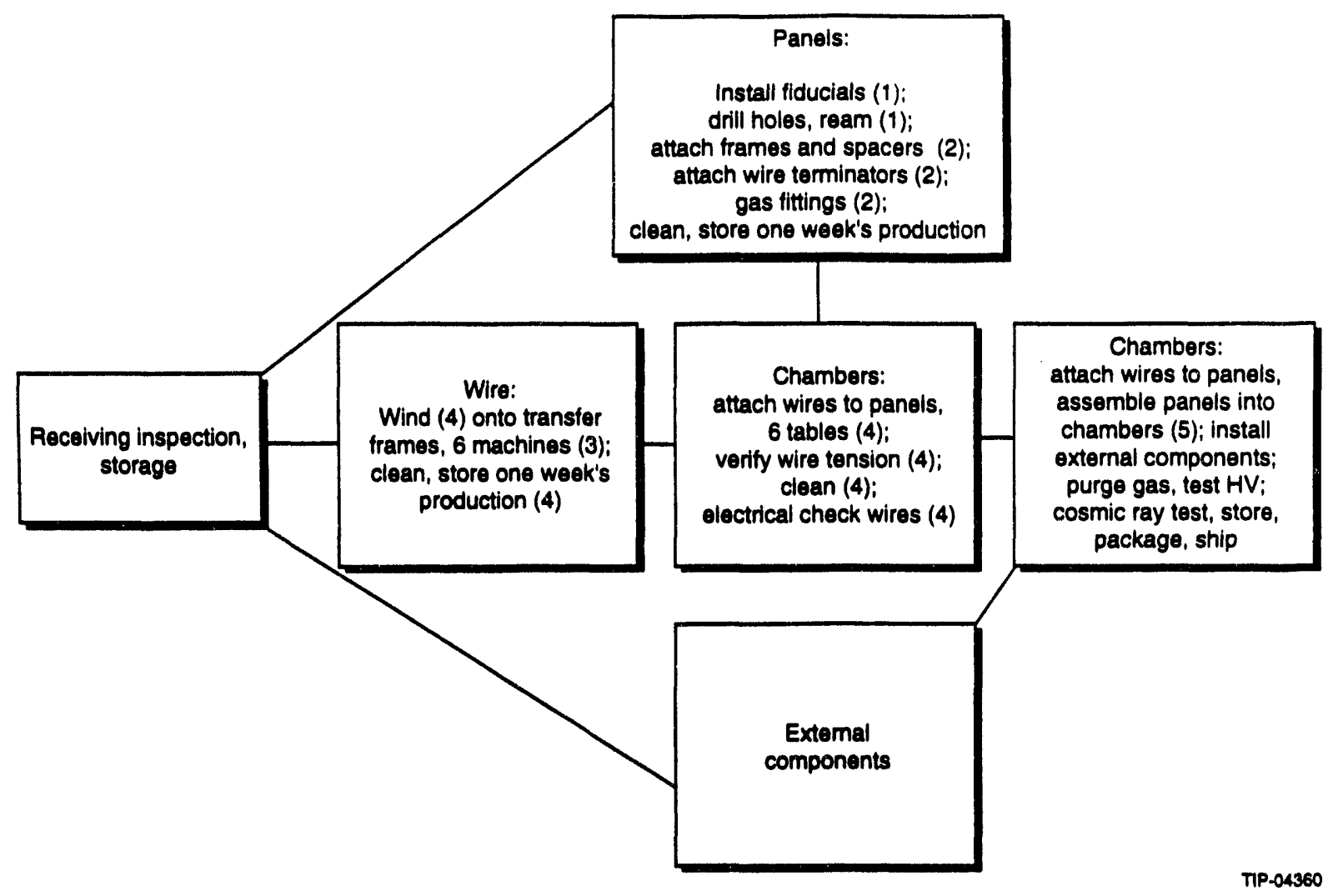

FIG. 480. Schematic of CSC assembly process. Numbers refer to operations requiring special facilities.

independent oversight by both physicists and engineers will be required at the assembly factories and the final assembly locations at the GEM test site. Inspection and oversight at all the commercial manufacturing plants producing critical elements of the chamber will also be undertaken. The precision cathode boards will be measured to ensure that the cathode strips meet the dimensional tolerances and the assembled panels will also be measured to ensure that they meet the flatness and parallelism specifications. The measurement techniques are being developed by our design team. The required measurement sampling rates will be determined statistically. The decision to have factory monitors, or to send sampled items to independent measurement facili- ties, will be made later, after results of prototype parts have been measured. Required documentation and part "traveler" forms will be developed as a result of this effort.

\subsection{ORGANIZATION}

\subsubsection{Organization and Institutional Responsibilities}

The management organization of the Muon Group is indicated in Table 4-21.

The Muon Group Steering Committee provides technical, engineering, and scientific guidance to the Chief Engineer, Project Manager, and Scientific Group Leaders. At present the members of this committee are: 
F. E. Taylor-MIT (Chairperson)
M. Atiya-BNL
H. Baker-CSDL
C. Bromberg-MSU
Y. Guo-IHEP
I. Golutvin-JINR
M. MarX-SUNY-SB and SSCL
R. McNeil-LSU
G. Mitselmakher-SSCL
F. E. Nimblett-CSDL
L. S. Osborne-MIT
V. Polychronakos-BNL
L. Rosenson-MIT
A. Vorobyov-PNPL
S. Whitaker-BU
C. Wuest-LLNL

Table 4-21. GEM muon group management organization.

\begin{tabular}{|c|c|}
\hline Position & Duty \\
\hline $\begin{array}{l}\text { Project Leader } \\
\text { and Deputy }\end{array}$ & Overall coordination \\
\hline Project Manager & $\begin{array}{l}\text { Manages schedules, budgets, } \\
\text { and oversees project }\end{array}$ \\
\hline Project Engineer & $\begin{array}{l}\text { Manages engineering and } \\
\text { coordinates with PM and } \\
\text { Muon Subsystem Leaders }\end{array}$ \\
\hline Steering Committee & $\begin{array}{l}\text { Management, technical, } \\
\text { engineering, scientific } \\
\text { advice }\end{array}$ \\
\hline
\end{tabular}

\section{Personnel and Institutional Responsibilities}

The effort of the Muon Group is divided into a number of different activities, including engineering of the support structure, chamber R\&D, and simulations. In general terms the organization of the key tasks is shown in Table 4-22.
Table 4-22. Muon group organization.

\begin{tabular}{|c|c|}
\hline Task & Institutions \\
\hline $\begin{array}{l}\text { Support structure } \\
\text { engineering }\end{array}$ & CSDL, SSCL, LLNL \\
\hline $\begin{array}{l}\text { Chamber } \\
\text { engineering }\end{array}$ & $\begin{array}{l}\text { LLNL, JINR, SSCL, BU, MIT, } \\
\text { BNL, UH }\end{array}$ \\
\hline Chamber R\&D & $\begin{array}{l}\text { BU, BNL, IHEP, ITEP, JINR, } \\
\text { LLNL, MIT, } \\
\text { MSU, PNPI, SSCL, UH }\end{array}$ \\
\hline $\begin{array}{l}\text { Chamber } \\
\text { performance }\end{array}$ & $\begin{array}{l}\text { BU, SSCL, SUNY-SB, } \\
\text { UH, MIT }\end{array}$ \\
\hline $\begin{array}{l}\text { Chamber } \\
\text { construction }\end{array}$ & $\begin{array}{l}\text { IHEP-China } \\
\text { JINR-Russia } \\
\text { PNPI-Russia } \\
\text { USA }\end{array}$ \\
\hline Alignment system & CSDL, LLNL, MIT, SSCL \\
\hline Trigger & BNL, SUNY-SB \\
\hline Electronics A\&D & BNL, BU, PNPI, PU \\
\hline $\begin{array}{l}\text { Assembly and } \\
\text { installation }\end{array}$ & CSDL, SSCL, LLNL \\
\hline Simulations & All institutions \\
\hline
\end{tabular}

\section{Institutions}

University of Arizona

Boston University

Brookhaven National Laboratory

Brown University

BSU-Minsk

Carnegie Mellon University

Charles Stark Draper Laboratory

University of Houston-SCARF

IHEP-Beijing

ITEP-Moscow

JINR-Dubna

Lawrence Livermore National Laboratory

LeCroy Corporation

Louisiana State University

Massachusetts Institute of Technology

Michigan State University

Moscow State University

Oak Ridge National Laboratory

PNPI-St. Petersburg

State University of New York-Stony Brook

Superconducting Super Collider Laboratory

University of Tennessee

Tsinghua University-Beijing 


\section{REFERENCES}

1. E. Dolgosheina, T. Taylor, W. J. Willis, "Field Shaping by Iron for Muon Measurement at Hadron Colliders," Nucl. Instrum. Methods, A301 (1991) 451.

2. G. Mitselmakher et al., "Muon Meeting -Brookhaven National Laboratory," GEM TN-92-241.

3. R. Sumner et al., "Limited Streamer Drift Tubes for the GEM Muon System, "GEM TN-92-203.

4. C. Bromberg et al., "Muon Tracking System for the GEM Detector Using Pressurized Drift Tubes," GEM TN-92-205.

5. M. Widgoff et al., "Resistive Plate Chamber Technology Review," GEM TN-92-206, Revision A.

6. C. Bromberg et al., "The RDT-RPC Technology Option for GEM," GEM TN-93-288.

7. S. Whitaker et al., "The GEM Muon System Based on Cathode Strip Chambers," GEM TN-92-199.

8. V. Polychronakos et al., "Interpolating Cathode Strip Chambers of the GEM Muon System Prototype Development and Performance," GEM TN-93-301.

9. U. Becker, et al., "Precision Muon Detectors in the TeV Region," Nucl. Instrum. Methods A253, (1986), 15.

10. J. Paradiso, "Some Alignment Concepts for the GEM Muon Array," GEM-TN-92-124.

11. J. Paradiso, "Alignment Requirements for the GEM Muon Detector," GEM TN-92-125.

12. G. Mitselmakher and A. Ostapchuk, "New Approach to Muon System Alignment," GEM TN-92-202.

13. Yu. Fisyak, K. McFarlane, L. Roberts, "SIGEM-Full Geant Simulation for GEM Detector (Status Report)," GEM TN-92-162.

14. R. McNeil, M. Mohammadi, and C. Yanagisawa, "Depth of Calorimeter for Punchthrough Suppression," paper submitted to III International Conference on Calorimetry in High Energy Physics, Corpus Christi, TX (1992).

15. GEM Collaboration, "Expression of Interest to Construct a Major SSC Detector," GEM TN-91-1.

16. R.McNeil, "How Thick Should the GEM Barrel Calorimeter Be?" GEM TN-92-68 Revision A(1992); GEM Collaboration, "Responses to the December 1991 PAC Report," GEM TN-92-131.

17. F. E. Paige and S. D. Protopopescu, "ISAJET," BNL-38774 (1986).

18. R. McNeil, "PCHTHR - A Fast Simulation for Hadron Punchthrough," GEM TN-92-193.

19. A. Dar, "Atmospheric Neutrinus Astrophysical Neutrons, and Proton Decay Experiments, Phy. Rev. Lett. 51, 227 (1983).

20. L3 Collaboration, "High Energy Cosmic Muons and the Calibration of the L3 Electromagnetic Calorimeter," Nucl. Instrum. Methods A275, (1989) 81.

21. A Korytov, et al., "Neutron Sensitivity of LSDT Chambers," GEM TN-92-122.

22. S. Boyarinov, et al., "Neutron and Gamma Sensitivity of Gas Detectors," GEM-TN-93-345.

23. O. L. Fedin, et al., "Differential Sensitivity of the CSC's to Neutrons in Functions of Neutron Energy in the Range $0.1 \mathrm{eV}<\mathrm{E}_{\mathrm{n}}<20 \mathrm{MeV}$," GEM TN-93-352.

24. A. Yamashita, "Monte Carlo Simulation for GEM Muon Chamber Trigger Rate," GEM TN-92-198.

25. M. Atiya and A. Yamashita, "GEM Muon Trigger Studies," GEM TN-93-393.

26. GEM Letter of Intent, GEM TN-92-49.

27. P. Dingus, "Pattern Recognition in the GEM Muon System," GEM TN-93-387; "An Approach to Muon Reconstruction in the GEM Muon System," GEM TN-93-416.

28. L. Rosenson, "Momentum Resolution and Track Reconstruction Quality for the EMPACT Detector," EMPACT 224 (1990).

29. T. Wenaus, "Detailed Simulation and Performance Parameterization of the GEM Muon Detector," GEM TN-93-297.

30. T. Wenaus, "A Reconstruction Program for the GEM Muon System," GEM-TN-388; Yu. Fisyak, "Kalman Filter Approach to the Track Finding and Fitting in the GEM Muon System," GEM TN-93-389.

31. A. "apchuk, "Background Muons for Alignnuent of the GEM Muon System," GEM TN-93-334.

32. L. Rosenson, "Considerations on the Addition of Detector Planes Outside the GEM Magnet and on the Implementation of a Vertex Constraint," GEM TN-92-97. 
33. G. Alexeev et al., "The Possibility of Using Particle Punch-Through for Muon System Alignment," ATLAS Internal Note, MUON-No-018, 1993.

34. E. Mathieson and G. C. Smith, IEEE Transactions on Nuclear Science, Vol. 36, No. 1, February 1989.

35. I. Golutvin et al., "The Results of Tests of the Dubna $1.5 \times 1.0 \mathrm{~m}^{2}$ CSC Prototype at the TTR," GEM-TN-93-330.

36. K. Lau et al. "Test Results of UH CSC Prototype," GEM-TN-93-392.

37. V. Polychronakos and V. Tcherniatine, "The Effect of Inclined Tracks and the Lorentz Angle," GEM TN-92-137.

38. J. Shank, et al., "CSC Tests at RD5," GEM-TN-93-402.

39. S. Boyarinov, et al., "Neutron and Gamma Sensitivity of Gas Detectors," GEM-TN-93-345.

40. B. Yu, G. C. Smith and V. Radeka, "Developments in interpolazing cathode structures for high precision gas proportional detectors," presented at the 1992 IEEE Nuclear Science Symposium, Orlando, Florida, 1992.

41. B. Yu, "Gas Proportional Detectors with Interpolating Cathode Pad Readout for High Track Multiplicities," Ph.D. Thesis, Pittsburgh 1991 (BNL 47055 Informal Report).

42. Yanan Guo, "Timing Calculations for CSC," GEM-TN-93-395.

43. M. Furey, et al., "GEM Muon Support Structure Study," GEM-TN-93-386.

44. Toth, W. E., "Muon Detector Program; Prototype Octant Construction and Evaluation with Production Phase Recommendations," Draper Lab Report CSDL-R-1885, Oct. 1987.

45. J. Paradiso, "Analysis of an Alignment Scheme for the GEM Muon Barrel," GEM-TN-92-150.

46. G. Mitselmakher and A. Ostapchuk, "Alignment Requirements for the GEM Muon System," GEM TN-93-333.

47. G. Mitselmakher and V. Zhukov, "Alignment Requirements to Muon System," GEM-TN-92-120, June, 1992.

48. P. Duinker, et al., "Some Methods for Testing and Optimizing Proportional Wire Chambers," Nucl. Instrum. Methods, A273 (1988) 814.

49. F. Ayer, et al., "The Engineering Development of an Actively Controlled Precise Muon Chamber for the SDC Detector," Proceeding of the IISSC Conference, New Orleans, LA March 1992.
50. W. Post, "A Homogeneous Lightsource for RASNIK," NIKHEF.H report, July/August, 1992.

51. J. Paradiso, D. Goodwin, "Testing and Development of Extended Range Straightness Monitor Systems," GEM TN-93-331.

52. J. Govignon, "SDC Muon Measurement System Conceptual Design of the Alignment System," SDC-92-381.

53. R. Sawicki, "Engineering Elements of the Muon Alignment System," GEM TN-93-348.

54. S. Movchan, "Status of Dubna PDT Prototype," Muon Meeting - Brookhaven National Laboratory, GEM TN-92-241.

55. The Monochromator Collaboration, "X-Ray Test Station for Cathode Strip Muon Chambers at SSCL; Technical Proposal," GEM TN-93-380.

56. G. Smith et al., Brookhaven National Laboratory, Personal Communication, April, 1993.

57. G. van Apeldoom, et al., "Honeycomb Strip Chambers for the ATLAS air core toroid muon detector; Alignment," NIKHEF-H/ATLAS report, October, 1992.

58. A. Korytov, "The Axial + Projective Alignment for Muon Chambers," GEM TN-93-302.

59. E. Ables, "Results of Stretched Wire Capacitive Pickup Tests at LLNL," GEM TN-93-342.

60. I. Wilson, "Alignment Studies for the CERN Linear Collider," CERN SL/92-41.

61. R. Sawicki, E. Bliss, L. Griffith,"Precision Alignment Capabilities at LLNL," GEM-TN-93-339.

62. A. Ostapchuk, V. Scheglesky, "Test of GEM Alignment Scheme with Cathode Strip Chambers and X-Ray Source," GEM TN-92-228.

63. D Payne, R. Parker, R. Bradley, "Rangefinder with Fast Multiple Range Capability," Review of Scientific Instruments, 63, June 6, 1992.

64. J. Govignon, "A Solution to the Global Alignment of the SDC Muon Detector," SDC-92-296.

65. R. Sawicki, "Muon Chamber Alignment Alternati ves," GEM TN-93-338.

66. J. P. Amory, "PASS; Projective Alignment System Simulation," GEM Muon System Alignment Meeting on Jan. 6, 1993, GEM TN-93-335.

67. R. Weinstein, "Need for Dynamic Control of the High Voltage Supply for the GEM Muon Detector," GEM TN-93-273. 


\section{CALORIMETERS}

\subsection{INTRODUCTION}

\subsubsection{Physics Goals}

Calorimetry will play a key role in achieving the goals of the SSC physics program. A major physics obji_.ive of the SSC is to confront the new physics that must emerge in this new energy domain. For example, the source of electroweak symmetry breaking should emerge either from the standard model (SM) Higgs mechanism (with a Higgs boson mass in the range from 80 to $\approx 800 \mathrm{GeV}$ ), from supersymmetry, or from some other mechanism such as technicolor. Calorimeters play an important role in searches for new physics because their energy resolution improves with increasing energy, and they can provide precision measurements not only for hadronic jets, but, most importantly, for electrons and photons.

The precision measurement of photons and electrons is a unique capability of GEM allowing the study of objects like the Higgs in the difficult mass region $80-180 \mathrm{GeV}$. A Higgs boson of mass $<150$ $\mathrm{GeV}$ puts particularly stringent requirements on the electromagnetic energy measurement, because the experimentally most accessible decay mode into two photons is strongly contaminated by an irreducible background of QCD photon pairs, as well as by a potentially overwhelming background from jets, which may imitate single photons by fragmenting into high momentum neutral pions. Electromagnetic energy resolution is the key to suppressing the background under the narrow mass peak of the Higgs boson. Other features which make the GEM calorimeter unique at the SSC are excellent photon pointing, position resolution, and jet rejection. The EM calorimeter will also be crucial for measuring and identifying electrons. We are confident that the GEM EM calorimeter will be the most precise EM calorimeter at the SSC, thereby providing complementarity to the SDC calorimeter.

The GEM hadron calorimeter will be used to measure jets and $F_{T}$ and to help in identifying electrons and photons. Both the EM and hadronic calorimeters will aid in identifying muons, including measuring catastrophic energy loss by individual muons in the calorimeter. The calorimeter design will give the GEM detector excellent discovery potential across a wide range of physics scenarios. A few examples which depend critically on the calorimeter performance are:

- In the context of the standard model, GEM's excellent electromagnetic resolution will permit the discovery of the Higgs boson in the intermediate mass range $(80-140 \mathrm{GeV})$ within one to three standard SSC years $\left(\ell=10^{40} \mathrm{~cm}^{-2}\right)$, by observing the Higgs mass peak in the mode $\mathrm{H}^{0} \rightarrow \gamma \gamma$ or, equally important, by showing that it is not there.

- GEM's electron resolution will offer a unique capability for detecting $\mathrm{H}^{0} \rightarrow \mathrm{ZZ}^{*} \rightarrow e^{+} e^{-} e^{+} e^{-}$ $\left(140<\mathrm{M}_{H}<180\right)$, a mode where even a small number of events in a narrow peak will stand out above the background.

- A heavier SM Higgs $\left(400<\mathrm{M}_{H}<800 \mathrm{GeV}\right)$ would also be discovered by GEM within one standard SSC year, using the calorimeter's energy resolution for jets along with its lepton resolution to tag the $Z^{0}$ s in the decay $\mathrm{H}^{0} \rightarrow l^{+} \Gamma$ jet jet (Section 2.3.7). The $E_{r}$ resolution would also allow GEM to study the complementary discovery channel $\mathrm{H}^{0} \rightarrow I^{+} l^{-} v \bar{v}$ (Section 2.3.8). These channels, combined with the measurement of $l+l-l+l$-final states, would permit the discovery of a heavy SM Higgs.

- In supersymmetric extensions of the SM, GEM's lepton and photon resolution, combined with measurements of $k_{T}$, would allow detection of the supersymmetric Higgs through its 4 lepton decays. In the context of the minimal supersymmetric model (MSSM), for example, we will be able to cover a reasonable fraction of the $m_{A}-\tan \beta$ plane. In this situation, the GEM EM calorimeter design, which combines high resolution with precise photon angle determination-indepen- 
dent of the event vertex and thus independent of the tracker-would have a unique advantage. Prior to the actual Higgs discovery, the measurement of events with large $E_{T}$ within the region $|\eta|<5$ could give early indications of supersymmetry through the decays of gluinos.

- We will search for a $Z^{\prime} \rightarrow e^{+} e^{-}$up to a mass of $8 \mathrm{TeV}$ by running at $10^{34} \mathrm{~cm}^{-1} \mathrm{~s}^{-2}$ (see Section 2.6.1).

- Most importantly, if there is new physics beyond the standard model, good resolution and clean identification of electrons and photons could be vital to the discovery of new narrow resonances, such as a $Z^{\prime}$ or a techni-rho, or quark or lepton compositeness up to the $\mathrm{TeV}$ range.

\subsubsection{Performance Requirements}

From these and the other broad physics goals discussed in Chapter 2, we have derived the performance requirements for the GEM calorimeter.

- EM Energy Resolution: The energy resolution is parametrized as (Section 5.2.1.):

$$
\frac{\sigma}{E}=\frac{a \%}{\sqrt{E}} \oplus b \% \oplus \frac{c}{E}{ }^{1}
$$

For $\mathrm{H}^{0} \rightarrow \gamma \gamma$, we show in Table 5-1 the relative mass resolution as a function of the $E M$ calorimeter energy resolution.

Table 5-1. Relative mass resolution for $\mathrm{H}^{0} \rightarrow \gamma \gamma$ as a function of $a$ and $b$.

\begin{tabular}{cccc}
\hline a(barrellend) & $6 / 8$ & 10 & 15 \\
\hline$b=0.4$ & 1.0 & 1.41 & 2.03 \\
$b=1.0$ & 1.38 & 1.69 & 2.26 \\
$b=2.0$ & 2.25 & 2.45 & 2.87 \\
\hline
\end{tabular}

We take as our goals for EM energy resolution $a=6$ in the barrel and 8 in the endcap and $b=$ 0.4 .

- Time Resolution: We have to determine the time of every $\gamma$, electron or jet candidate within $<4$ ns in order to know its correct bunch crossing. We thus require a fast response time to reduce pileup in the measurement of the electromagnetic and hadronic showers.
- Separation of $\gamma$ and e from Jets: To separate electrons from charged hadrons the most powerful cut is the longitudinal development of the shower. Longitudinal electromagnetic and hadronic segmentation facilitates this. The transverse shower shape and position measurement also aids in jet rejection. For $\gamma /$ jet separation, an additional requirement is to identify multi-photon events where a $\pi^{0}, \eta$ or other leading electromagnetic particle would otherwise fake a single photon. Fine transverse segmentation near the start of the EM shower is required for this purpose.

- Photon pointing: In high luminosity conditions the ability of the calorimeter to determine the vertex to $\sim 5 \mathrm{~mm}$ from the measurement of two electrons or photons is particularly important. The ability to measure the direction of a photon as well as its energy allows us to obtain the effective mass of pairs of photons without an independent determination of the production vertex. This capability will allow us to use narrower roads in which to look for electrons in the Central Tracker, thereby obtaining more robust electron detection at high luminosities.

- Hadronic Energy Resolution: The energy resolution for jets must be sufficient so that the mass resolution for $\mathrm{H}^{0} \rightarrow l^{+} r$ jet jet is not degraded. This is not a very stringent requirement on the hadronic energy resolution, since there are important contributions to the resolution from particles from neighboring jets, and from other unavoidable systematic effects in the jet reconstruction method. Studies have indicated that our design goal for jet energy resolution $a=60$ and $b=4$ satisfies the criteria for detecting the Higgs signal.

- Isolation: In order to isnlate photon and lepton candidates the transverse energy within a surrounding cone of radius $R=\sqrt{(\Delta \eta)^{2}+(\Delta \phi)^{2}} \leqslant 0.5$ is required to be small. The calorimeter must have small thermal and pileup noise for this to be useful.

- $E_{T}$ : Any contribution to the $E_{T}$ from imperfections in the calorimeter must be small compared to the irreducible cross section from standard model neutrino production. In prac- 
tice this means that the coverage must extend to $\eta \geq 5$, that the transitions from one region of the calorimeter to another must have minimum dead material, and that the active depth must be $-10 \lambda$.

- Muons: The calorimeter must be deep enough to reduce punch through to a level less than the irreducible background from hadron decays. The calorimeter will also aid in identifying muons, and will measure the catastrophic energy luss of individual muons. It is beneficial to keep the number of radiation lengths to a minimum in order to reduce the multiple scattering of muons as they traverse the calorimeter.

\subsubsection{Design Considerations}

To meet these goals the GEM calorimeter employs two technologies: liquid ionization for the $\mathrm{EM}$ and inner hadron calorimeters and scintillator for the outer hadron calorimeter. We have chosen liquid ionization calorimetry because of the extensive experience that has been acquired in building and operating large systems of this type, which has resulted in a unique combination of excellent uniformity, time stability, ease of precision calibration, energy resolution, and time determination. As a result of our beam tests, simulation studies, and analysis of the tradeoffs. (see Section 5.2-5.3), we judge that this detector will deliver the besc energy resolution for electrons and photons-our highest priority. For the EM calorimeter we have chosen an accordion electrode structure which allows the extraction of signals without degrading the high frequency components which are vital to fast shaping, and a structure which is also convenient for implementing the longitudinal and transverse segmentation that provide our position and angular resolution. ${ }^{2}$ We have devised a liquid ionization EM calorimeter configuration which incorporates multiphoton rejection as well as supplying position and angular measurement from the same electrode structure used for the high precision energy measurement, thereby avoiding complication and compromised performance associated with an interleaved detector. The corrugated form of the electrodes also provides an inherent rigidity even for thin electrodes and absorbers, which aids in maintaining a mechanically precise and continuous structure.
The bulk of the hadronic energy is also measured in a liquid ionization calorimeter, in order to position the massive supports, services, and feedthroughs well beyond the maximum of the hadronic showers. In the barrel region, the remainder of the hadronic shower is measured in a relatively simple scintillator calorimeter with higher density than allowed in the liquid calorimeter. In this region the requirement of an adequate lever arm for muon momentum measurement puts a high premium on minimizing the outer radius of the calorimeter. The transition between liquid and scintillator techniques is fixed by four criteria which give roughly coincident values: 1 ) events with false missing energy due to energy lost in inactive material at the outside of the liquid calorimeter should not significantly increase the intrinsic missing energy background; 2 ) the diameter of the liquid calorimeter cryostat should allow its transport from a remote fabrication site; 3 ) the pile-up from minimum bias events should be sufficiently reduced by absorption in the liquid calorimeter to allow the use of simple read out and trigger techniques in the scintillator calorimeter; 4) the amount of energy in the scintillator calorimeter should be sufficiently small that very simple instrumentation (coarse sampling) may be used in this large volume, to reduce costs without compromising jet resolution, and such that few percent systematics on uniformity and calibration do not degrade the overall jet resolution.

In the barrel portion of the liquid calorimeter, shower fluctuations are a limiting factor in the resolution for electromagnetic showers, and noise is also a significant issue, particularly in the hadronic section. By the use of liquid krypton in the barrel, we have improved the photon energy resolution by $25 \%$, and the noise and timing performance by 1 factor of two, compared to liquid argon values. These factors are less significant in the endcap calorimeter, where the average energy of photons is higher.

In the endcap it is more cost effective to carry the liquid calorimeter to the end of the active region for three reasons: there is only a linear increase in volume per incremental interaction length; the cost of additional liquid krypton is not incurred; and the muon lever arm is less constrained. Shielding the 
forward muon detector will require some additional absorber, which need not be active.

The liquid calorimeters are not intrinsically compensating - that is, the average response is not the same for hadronic and electromagnetic energy deposited in the calorimeter. Simulations and previous experimental studies have shown that effective compensation can still be achieved by suitably weighting the energies recorded in the spatially segmented contributions to a hadron or jet shower. ${ }^{3}$ We have chosen liquid krypton and lead for the barrel, and liquid argon and copper for the endcap. to achieve similar relative responses for hadronic and electromagnetic energy.

Spatial resolution, particularly for electromagnetic showers, has been a major design consideration for the GEM calorimeter. An EM shower is read out in three longitudinal segments, of three, ten and eleven radiation lengths $\left(\mathrm{X}_{0}\right)$. The longitudinal shower profile improves the rejection of hadrons, allows a very effective correction which essentially eliminates the loss of resolution due to material in front of the calorimeter, and is vital for the methods of shower pointing and neutral pion rejection that we use. The last two sections of the EM calorimeter, containing most of the energy, are read out in projective towers of 0.026 in $\varphi$ and $\eta$, which match the shower size. This segmentation is fine enough to provide good rejection of multi-photons from jets, and even a degree of neutral pion rejection by shower shape analysis, as well as increased hadron rejection. By measuring shower centroids at two different depths, we can determine the shower angle. The shower polar angle precision is further improved by making the segmentation in the $\eta$ direction of the first longitudinal section six times finer. This also substantially improves the neutral pion rejection. In the $\varphi$ direction these strips extend over six towers, or one trigger tower, to limit the channel count.

In the forward direction $(3<\eta<5.5)$ we wish to measure jets for tagging purposes, and we need to measure $E_{T}$ in order to deduce the $E_{T}$. The limiting measurement becomes the direction of energy flow rather than its magnitude. For this reason, the calorimeter design shifts to a very dense axial cylindrical structure which reduces the transverse extent of the hadronic showers. Longitudinal segmentation is found to improve the precision of the energy flow measurement, using the information from the early part of the shower where the transverse size is smaller. Three segments are used, the front two integrated into the liquid calorimeter where segmentation is easy and radiation levels are highest. The third section is integrated into the shielding of the muon system. It is implemented in a "spaghetti" structure with liquid scintillator or quartz fibers, and catches the remainder of the shower where the transverse size is larger. The effect of the beam pipe aperture on the $E_{T}$ resolution has been carefully studied in order to bring the forwand calorimeter as close to the interaction region as possible. This not only minimizes the cost of the detector, but also allows the neutron flux generated in and beyond the forward calorimeter to be isolated most effectively from the muon tracking chambers.

In selecting the technologies used in the GEM calorimeter, we also considered scintillating crystals $\left(\mathrm{BaF}_{2}\right)$, and decided on liquid ionization calorimetry based on comparison of the following properties:

- Energy resolution for liquid ionization EM calorimetry is expected to be dominated by the stochastic term and for the crystal calorimetry by the constant term. If we assume $a=6, b=0.4$, and $c=0.1$ for the former and $a=2$, $b=0.7-1$ and $c=0$ for the latter, the energy resolution becomes equal at a photon energy of 39-100 GeV, the energy resolution for liquid ionization improving at higher energies and for crystals at lower energies. While our reliable knowledge of $\boldsymbol{b}$ in both cases is limited, we have also given weight to the following points.

- Liquid ionization calorimetry makes possible longitudinal segmentation and better transverse segmentation, which result in better pointing resolution and $\pi^{0}$ rejection, and in better $e$ vs. $\pi^{ \pm}$ separation.

- The $\mathrm{BaF}_{2}$ crystals are subject to radiation damage which changes the attenuation length in the crystal, resulting in a variation in the longitudinal uniformity and effective change in gain. The SSC Laboratory set up a panel of experts who were given the mission of determining if crystals could be produced in the near term which are sufficiently radiation resistant. The panel met in early 1992. and assisted in the definition of an R\&D program. In August 1992, the panel concluded that the time required to meet the goals of radiation resistance could not be determined. A system of dynamic 
bleaching of the damage by visible light has been investigated, and would probably be successful. but we do not have enough experience with a large system of this type to be sure that the non-uniformities in time and space would yield a value of $b$ less than the $1 \%$ used in the assumption above.

\subsubsection{Parameters}

Figure 5-1 shows a quarter cross section of the GEM calorimeter. Figure 5-2 shows a solid model of the calorimeter with a ray trace as a function of $\eta$. The major parameters are listed in Table 5-2.

In the remainder of this chapter, we describe the detailed physics performance of the GEM calorimeter. We then outline some recent R\&D results which have led to the design chosen. The current status of the calorimeter will be reported, including the design of the calorimeter modules and of the front end electronics. The readout electronics and trigger are described in Chapter 7. The balance of this chapter discusses the $R \& D$ plan, the radiation hardness of both mechanical and electronic components, and outlines the plans for assembly, engineering, and production, and summarizes the schedule and institutional responsibilities. The installation and integration of the calorimeter into GEM is covered in Chapter 9.

\subsection{CALORIMETER SIMULATIONS AND EXPECTED PERFORMANCE}

The expected calorimeter performance presented here is based on detailed GEANT Monte Carlo simulations. The EM calorimeter simulation used a full GEANT model with the detailed geometry of all the dead material, the accordion absorbers and electrodes described later in this chapter. Both the barrel and endcap designs have been optimized through extensive studies, some of which are shown in this section. Similar Monte Carlo studies for the beam tests at BNL (Section 5.3.2) and for RD3 at CERN (Section 5.3.3) agree well with the data, building confidence in these simulations for the GEM design.

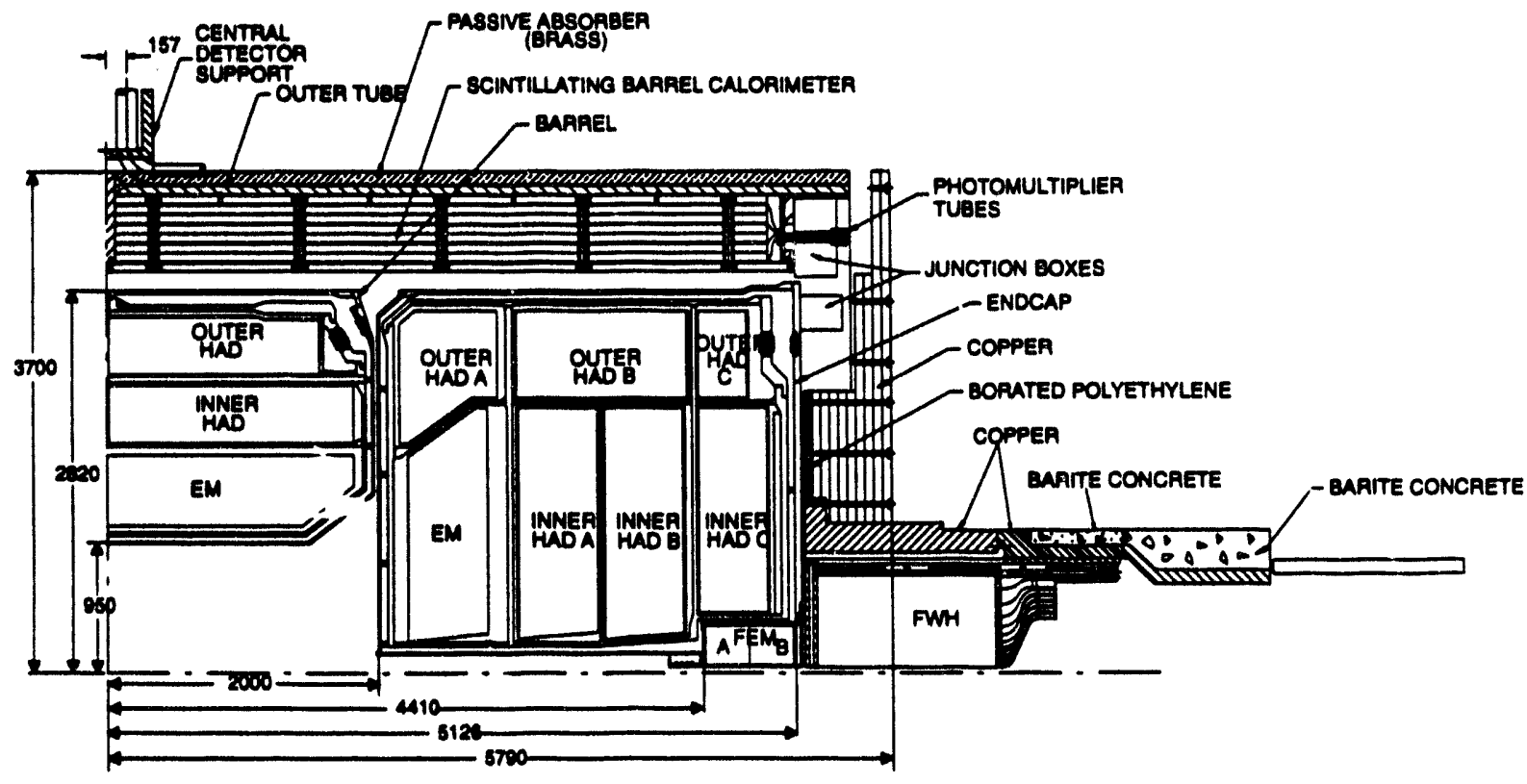

FIG. 5-1. Overall layout of GEM Calorimeter showing the Inner Barrel with LKr, the Outer Scintillating Barrel Calorimeter, and the Endcap with LAr. 

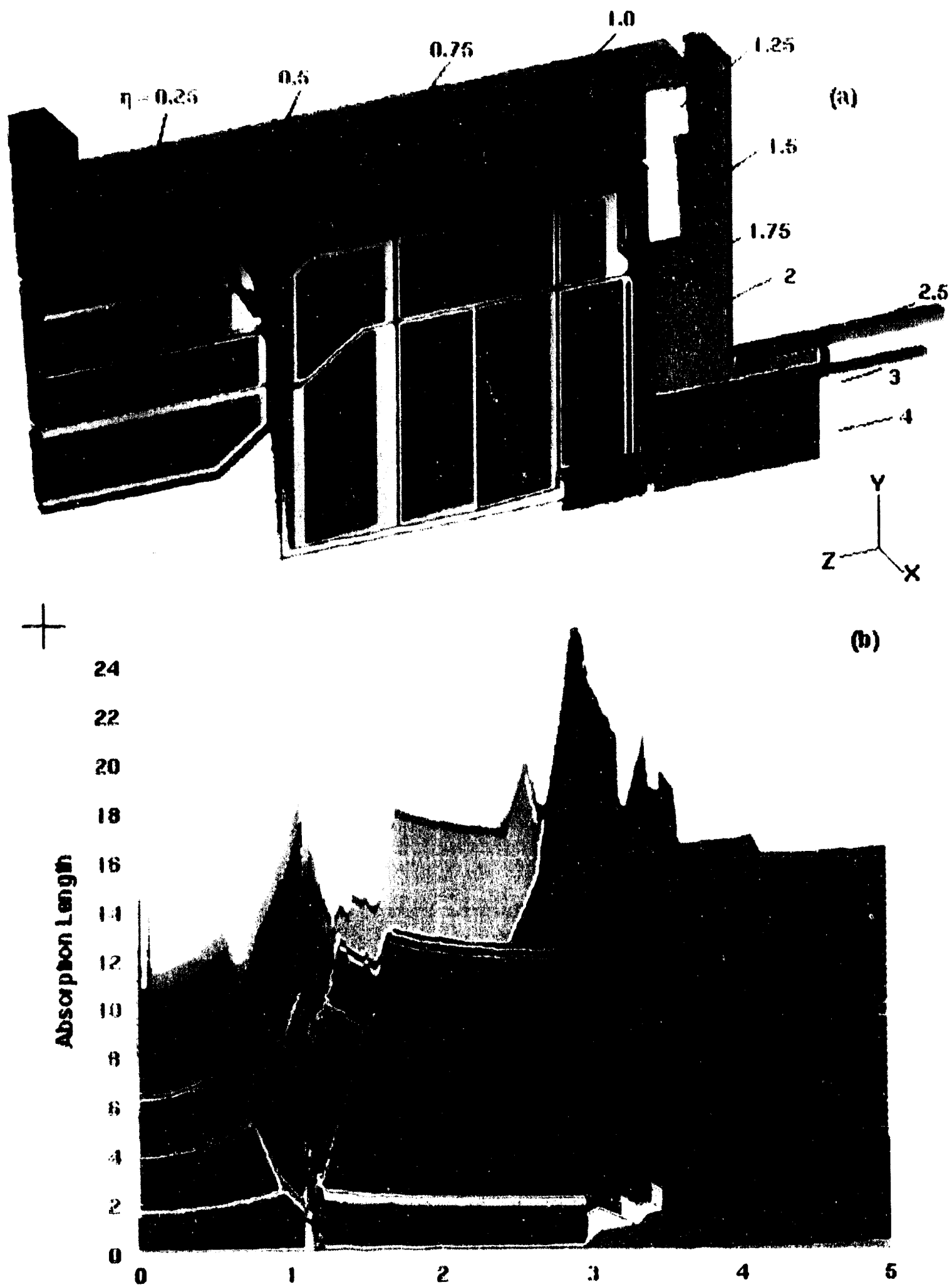

FIG. 5-2. a) Solid model of calorimeter. b) Number of absorption lengths v8. $\eta$. (Colors refer to a). 
Table 5-2. GEM Calorimeter Parameters.

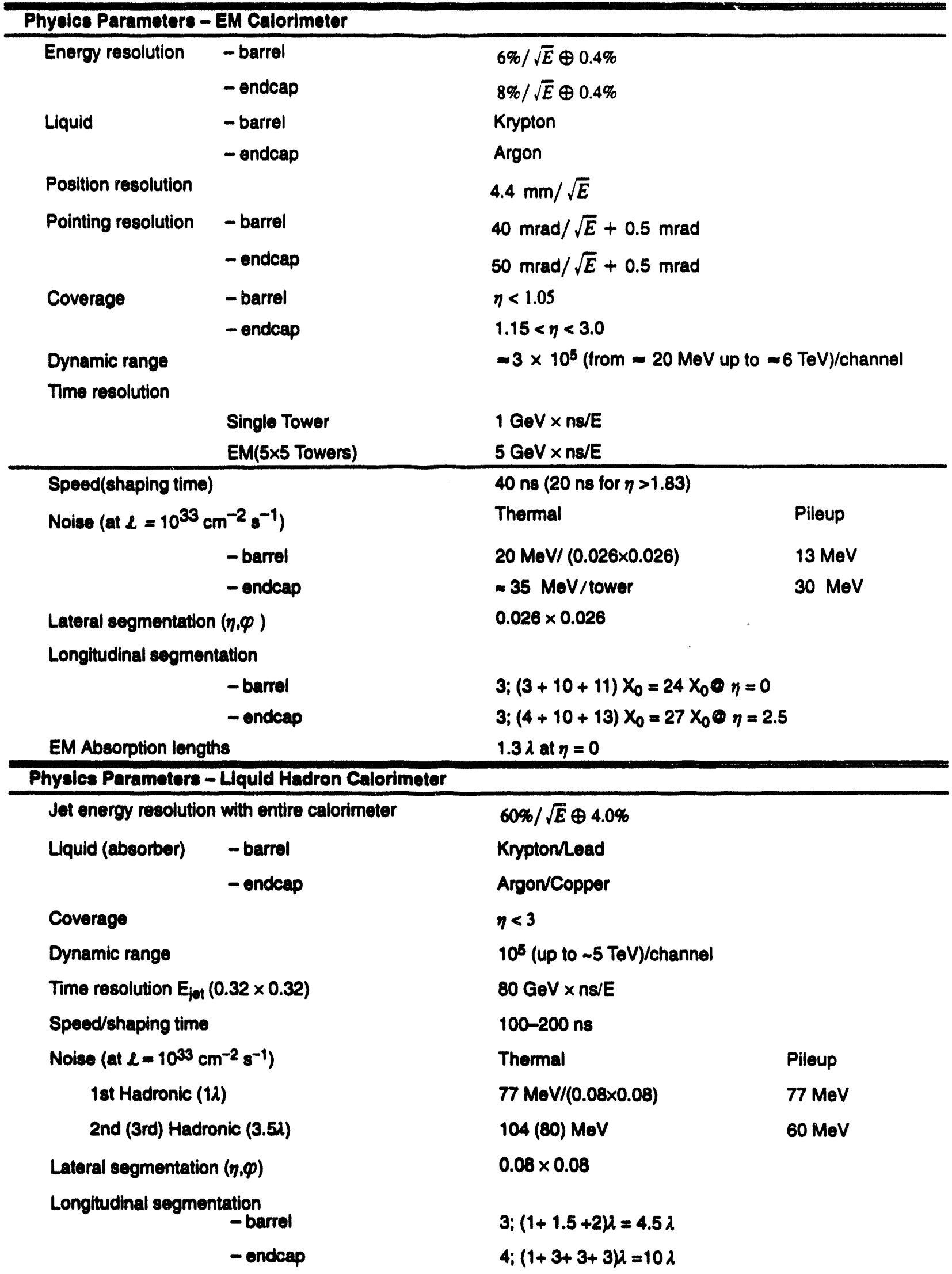


Table 5-2. GEM Calorimeter Parameters. (Cont.)

\begin{tabular}{|c|c|}
\hline $\begin{array}{l}\text { Number of absorption lengths } \\
\begin{aligned} \text { at } \eta & =0 \text { (including EM and scintillating barrel) } \\
\text { at } \eta & =3.0\end{aligned}\end{array}$ & $\begin{array}{l}-11 \\
12 \text { active, }>16 \text { total }\end{array}$ \\
\hline \multicolumn{2}{|c|}{ Physics Parameters - Scintillating Barrel Hadron Calorimeter } \\
\hline Dynamic range & $10^{5}$ \\
\hline Time resolution & $3 \mathrm{~ns}$ \\
\hline Speed/shaping time & 32 ns \\
\hline Noise (at $\mathcal{L}=10^{33} \mathrm{~cm}^{-2} \mathrm{~s}^{-1}$ per readout tower) & $10 \mathrm{MeV}$ thermal, $20 \mathrm{MeV}$ pileup \\
\hline Lateral segmentation $(\eta, \varphi)$ & $0.16 \times 0.16$ \\
\hline Longitudinal segmentation & 1 \\
\hline Absorption length & $4.5 \lambda$ \\
\hline \multicolumn{2}{|l|}{ Physics Parameters - Forward Calorimeter } \\
\hline Pr resolution for jets & $\Delta p_{T} / p_{T}<10 \%$ \\
\hline Fiducial coverage & $3<\eta<5$ \\
\hline Dynamic range & $10^{5}$ \\
\hline Speed/shaping time & 20 ns \\
\hline Lateral segmentation $(\eta, \varphi)$ & $0.2 \times 0.2$ \\
\hline Longitudinal segmentation & 3 \\
\hline Active Absorption Length & $16 \lambda$ \\
\hline \multicolumn{2}{|l|}{ Phyalcal Paramotore - Barrel Llquild Kr } \\
\hline Barrel Dimensions - inner (outer) radius & $950(2820) \mathrm{mm}$ \\
\hline Inner (outer) Radius EM - accordion & $1083(1605) \mathrm{mm}$ \\
\hline Inner (outer) Radius HAD & $1710(2649) \mathrm{mm}$ \\
\hline Length & $4000 \mathrm{~mm}$ \\
\hline \multirow[t]{2}{*}{ Absorber material - EM } & $0.2 \mathrm{~mm}$ SS/0.08 mm prepreg/1 mm Pb \\
\hline & $0.08 \mathrm{~mm}$ prepreg/0.2 mm SS \\
\hline Readout Board - EM & $0.4 \mathrm{~mm}$ (Kapton/Cu) \\
\hline Active liquid thickness - EM & $2 \times 2 \mathrm{~mm}$ \\
\hline Number of Channels - Total EM(Hadron) & $60480(6000)$ \\
\hline Readout Device & JFET preamplifier \\
\hline Hadron absorber thickness - (Pb) & $9 \mathrm{~mm}$ \\
\hline Hadron active liquid thickness - (Kr) & $2 \mathrm{~mm}$ \\
\hline \multicolumn{2}{|l|}{ Physical Parameters - Endcap Llquid Ar } \\
\hline Distance - interaction point to front (rear)face & $2000(5126) \mathrm{mm}$ \\
\hline Absorber material - EM & $0.15 \mathrm{~mm}$ SS/0.08 mm prepreg/1.4 mm Pb \\
\hline
\end{tabular}


Table 5-2. GEM Calorimeter Parameters. (Cont.)

\begin{tabular}{|c|c|}
\hline & $0.08 \mathrm{~mm}$ prepreg/0.15 SS \\
\hline Number of Channels - Endcap EM (Hadron) & $24216(3,500)$ \\
\hline Absorber Thickness - hadron (Cu) & $27 \mathrm{~mm}$ \\
\hline Active liquid thickness - hadron (Ar) & $4 \times 2 \mathrm{~mm}$ \\
\hline \multicolumn{2}{|c|}{ Physlcal Parameters - Barrel Scintillating Calorimeter } \\
\hline Dimensions - Inner (outer) Radius & $2923(3700 \mathrm{~mm})$ \\
\hline Length & $2 \times 4840 \mathrm{~mm}$ \\
\hline Sense Material: ScintillatorMLS (by volume) & $4 \%$ \\
\hline Absorber: $\mathrm{Cu}$ & $91 \%$ (by volume) \\
\hline Readout - Photomultipliers & 640 \\
\hline \multicolumn{2}{|c|}{ Physlcal Paramotors - Forward Liquid Argon Calorimeter } \\
\hline Distance - interaction point to front face & $4476 \mathrm{~mm}$ \\
\hline Dimensions - Inner (Outer) Radius & $55(340 \mathrm{~mm})$ \\
\hline Depth along beam & $402 \mathrm{~mm}$ \\
\hline Absorber & $\mathrm{Cu}$ \\
\hline Channels (each side) & 400 \\
\hline \multicolumn{2}{|l|}{ Luquid Volume } \\
\hline Barrel (krypton) & 23,244 liters \\
\hline Endcap (each) (argon) & 21,394 liters \\
\hline Reserve in head vessel & $3 \times 1,200$ liters \\
\hline Reserve & $3 \times 2,000$ liters \\
\hline Total (krypton/argon) & $27,334 / 44,880$ liters \\
\hline \multicolumn{2}{|l|}{ Total Wolght of Calorimetor Systom } \\
\hline Barrel Weight & $465 \mathrm{Mg}$ \\
\hline Endcap Woight (each) & $389 \mathrm{Mg}$ \\
\hline Fonward argon (each) & $2 \mathrm{Mg}$ \\
\hline Forward scintillating (each) & $47 \mathrm{Mg}$ \\
\hline Liquid - Barrel + Endcaps & $1,243 \mathrm{Mg}$ \\
\hline Passive Absorber $(\mathrm{Cu})+$ forward & $428 \mathrm{Mg}$ \\
\hline Barrel Scintillating Calorimeter & $1,143 \mathrm{Mg}$ \\
\hline Total & $2,814 \mathrm{Mg}$ \\
\hline
\end{tabular}


The response of the entire calorimeter to hadrons and jets has been studied using full GEANTGHEISHA simulations. The detailed geometry of all the dead material (cryostat walls and structural members) has been included.

\subsubsection{Electromagnetic Calorimeter}

We have optimized the performance of the EM calorimeter for the best energy, position, angular resolution and jet rejection possible. The optimization process considers many factors. We have attempted to stay within constraints for space, number of electronic channels and costs.

\section{Energy resolution}

The presence of the irreducible 2-photon background to the $\mathrm{H}^{0} \rightarrow \gamma \gamma$ signal in the $80-140 \mathrm{GeV}$ mass range means that this signal can only be detected as a peak on a large continuum distribution. The narrower the peak, the greater the statistical significance obtained for a given number of events. The requirement that a Higgs be detectable over the entire mass range therefore places strict requirements on the EM energy resolution. The energy resolution may be parametrized as:

$$
\frac{\sigma}{E}=\frac{a \%}{\sqrt{E}} \oplus b \% \oplus \frac{c}{E} .
$$

The sampling term $a$ reflects fluctuations in the fraction of energy measured in the active medium; it may be reduced by increasing the sampling frequency in the calorimeter. The constant term $b$ is due to non-uniformity, calibration errors, energy leakage and instability of response. The noise term $c$ is due to electronics noise and pileup from other events.

The statistical significance for a Higgs discovery (see Section 2.3) varies with the number of signal events, $N_{s}$ and the number of background events, $N_{B}$, as $N_{\mathrm{S}} / \sqrt{\mathrm{N}_{\mathrm{B}}}$, for $N_{S}$ and $N_{B}$ sufficiently large. Since the background decreases with increasing $\gamma \gamma$ invariant mass, $\mathrm{M}_{\mathrm{H}} \sim 80 \mathrm{GeV}$ provides the most severe requirements on resolution and luminosity. In Table 5.1 we show the relative width of the $\mathrm{H}^{0} \rightarrow \gamma \gamma$ as a function of $a$ and $b$. The stochastic term $a$ dominates in the barrel whereas the constant term $b$ is more important in the endcap because of the higher average energy of the photons. This led us to choose krypton in the barrel while using argon in the endcap. The noise term $c$ is kept as low as possible by finely segmenting the detector, and by optimizing the shaping time, so that the thermal and pileup noise are minimized (Section 5.4.5). With our design the noise term does not affect the mass resolution.

The liquid krypton accordion calorimeter prototype tested at BNL in 1992 attained an $a$ of 6.7, and has confirmed the accuracy of our simulation (described in Section 5.3). We have designed the final GEM EM barrel calorimeter with finer sampling to have a resolution averaged over the barrel of $<6 \% / \sqrt{E}$, as shown in Figure 5-3. The choice of liquid ionization technology is motivated in part by the observation that the constant term $b$ is crucial to the performance, and can be at risk in the high radiation environment of the SSC. Stability of response, uniformity from cell to cell, and relative ease of calibration are all strengths of this technique; all of these factors are important in keeping the constant term small.

Our estimate of the constant term $\boldsymbol{b}$ includes the following terms. The accordion structure including $\varphi$ and $\eta$ dependences gives $0.2 \%$. We expect from various bench and system tests that one can achieve $0.1 \%$ in the electronics gain calibration (Section 5.4.4). These added in quadrature are less than our goal of $0.4 \%$.

To maintain the energy resolution at high luminosity requires a fast response from the calorimeter to minimize the effects of pileup. This is

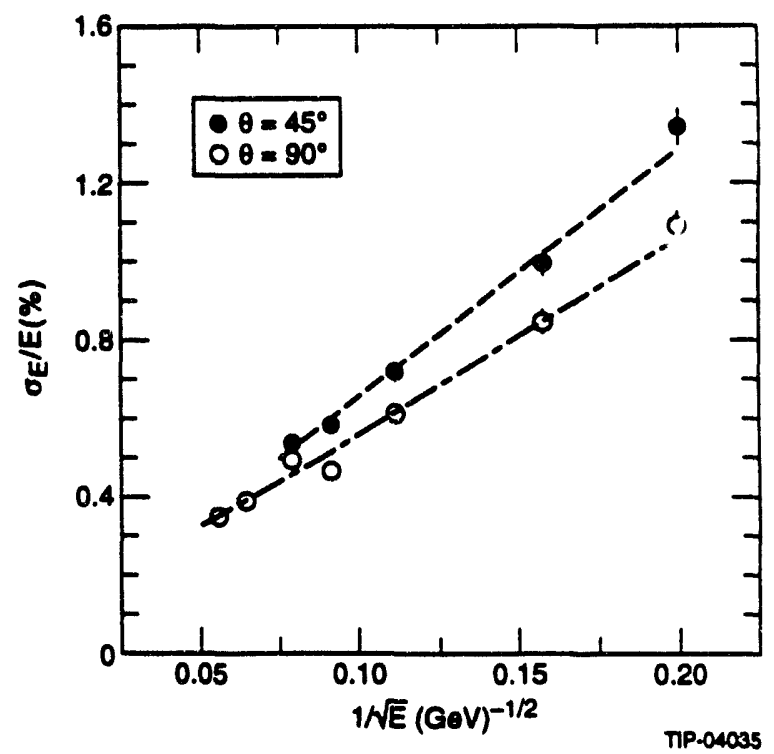

FIG. 5-3. EM energy resolution vs. $1 / \sqrt{E}$ for barrel accordion calorimeter. The lines are the fit results: dot-dashed $(5.2 \pm 0.12) / \sqrt{E} \oplus(0.21 \pm 0.02) \%$, dashed $(6.4 \pm 0.19) / \sqrt{E} \oplus(0.15 \pm 0.08) \%$. 
achieved by shaping the signal to yield a narrow output pulse (see Section 5.4.5). Electrons, photons and jets should also be timed with sufficient precision to determine with which beam crossing they are associated, to reduce background due to overlaps. Section 5.3.3 gives the experimental results which demonstrate that the time resolution is more than sufficient to associate electrons and photons with the correct beam crossing.

\section{Transverse Segmentation and Trigger}

The transverse and longitudinal segmentation of the GEM barrel calorimeter is shown in Figure 5-4. These $6 \times 6(\Delta \eta \times \Delta \phi=0.16 \times 0.16)$ towers also represent an EM trigger tower as discussed further in Section 7.2. There are $2 \times 2(\Delta \eta \times \Delta \phi=$ $0.16 \times 0.16)$ hadron towers behind the $\mathrm{EM}$ trigger tower. The fine strips in the first EM layer match the trigger towers in $\phi$ while providing jet rejection and pointing in $\eta$ as discussed below. This arrangement contains the same number of channels in each longitudinal section.

The transverse segmentation is determined by the need to separate showers. This has been optimized by considering shower shape analysis, pointing, position resolution and energy resolution. For most of these effects we find that the optimal tower size is close to the average Molière radius for the EM calorimeter. The Molière radius is $\approx 39 \mathrm{~mm}$. Fig-

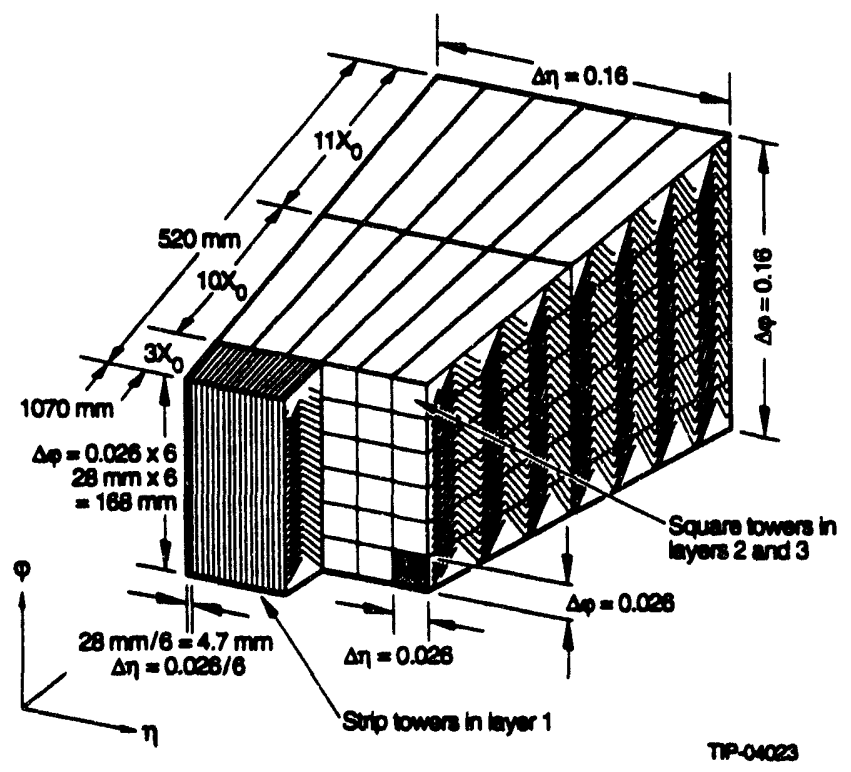

FIG. 5-4. Transverse and longitudinal segmentation of the Inner Barrel EM Calorimeter. ure 5.5 shows the shower energy profile as a function of transverse distance to the photon in the three depth layers. For $\mathrm{M}_{\mathrm{H}}{ }^{0}>150 \mathrm{GeV}$ the most striking Higgs decay mode for discovery is into two $Z^{0}$ bosons (one virtual if $\mathrm{M}_{\mathrm{H}}{ }^{0}<2 \mathrm{M}_{\mathrm{Z}}^{0}$ ), each of which decays leptonically. At higher Higgs masses the $Z^{0} \mathrm{~s}$ are boosted considerably in the detector frame so the electrons into which they decay are relatively close together (a separation in pseudorapidity and $\phi$ of as little as $R=0.3$ for $\mathrm{M}_{\mathrm{H}}=600 \mathrm{GeV}$ ). The requirements derived from the considerations above show that our segmentation is more than adequate for this case.

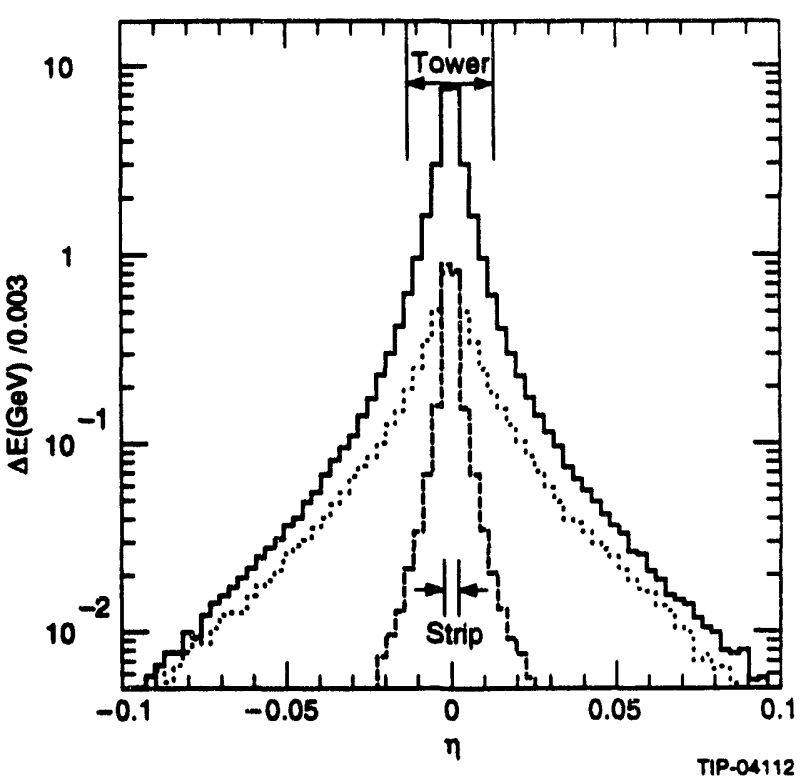

FIG.5-5. Averaged photon shower transverse energy profile as a function of the distance to the photon direction in units of rapidity. The three curves correspond to the different depth layers: layer 1-dashed; layer 2-solid; layer 3-dotted.

\section{Longitudinal Segmentation}

The longitudinal segmentation has been optimized by several considerations. The first layer must be deep enough to allow the photons to start their showers, but not so deep that the showers become too broad. The third layer is used for pointing and so the second layer must be as deep as possible to maximize the distance between the first and third layer, but not so deep as to prevent sufficient energy from reaching the third section to have adequate position resolution. This optimization is energy dependent, and we have chosen the goal of detecting the low mass $\mathrm{H}^{0} \rightarrow \gamma \gamma$. 


\section{Jet Rejection}

The cross sections for QCD two-jet production and jet plus photon production are much larger than that for two-photon production. Even a small fraction of jets faking photons by fragmenting into one or more hard neutral pions will become the dominant background. To reduce the jet backgrounds to a level comparable with the photon background requires a jet rejection (for each jet) of $-10^{4}$. The jet rejection is achieved by three means: a) shower shape analysis, b) identifying additional EM clusters in a cone with radius $R=\sqrt{(\Delta \eta)^{2}+(\Delta \phi)^{2}}-0.5$, and c) identifying additional hadronic clusters in the same cone. For the shower shape analysis we utilize both longitudinal and transverse information. To enhance this analysis the first layer is divided into narrower strips in the $\eta$ direction to give better isolation and shower shape measurements. The strips are one-sixth the width in $\eta$ of the cells behind them $(0.026 / 6)$, and they extend over six cells in the $\phi$ direction to reduce the channel count. A GEANT simulation has been done with such strips yielding the $\pi^{0}$ rejection displayed in Figure 5-6. ${ }^{4} \mathrm{~A}$ photon likelihood function for jets which pass the isolation cuts and single photons is shown in Figure 5-7. These jets were generated with PYTHIA and passed through a GEANT simulation of the detector. This photon likelihood function (see Figure 5-7) uses the signal in the strips as well as the shower size in the towers. A cut on the likelihood at -15 gives $90 \%$ acceptance for photons, and a rejection of the jets by a factor of 5 at $E \cdot=40 \mathrm{GeV}$.

To identify additional electromagnetic showers, we use the fine transverse granularity of the calorimeter, as well as the excellent timing achievable in a liquid krypton electromagnetic calorimeter. ${ }^{5}$ The timing resolution allows bunch crossing identification for energies as low as $200 \mathrm{MeV}$ deposited in an individual tower.

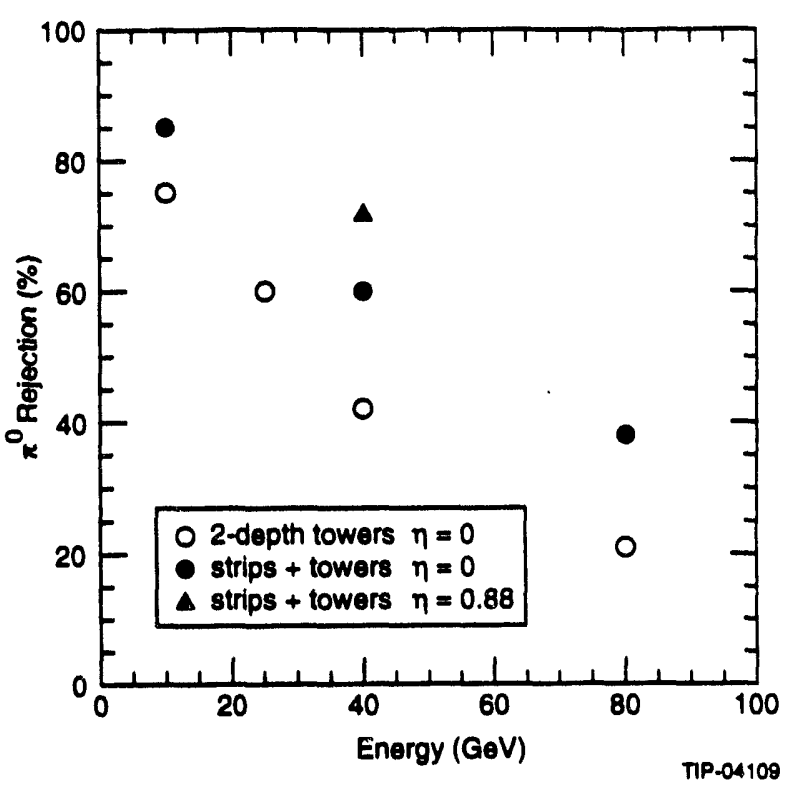

FIG. 5-6. $\pi^{0}$ rejection vs. $\pi^{0}$ energy for EM barrel accordion calorimeter.

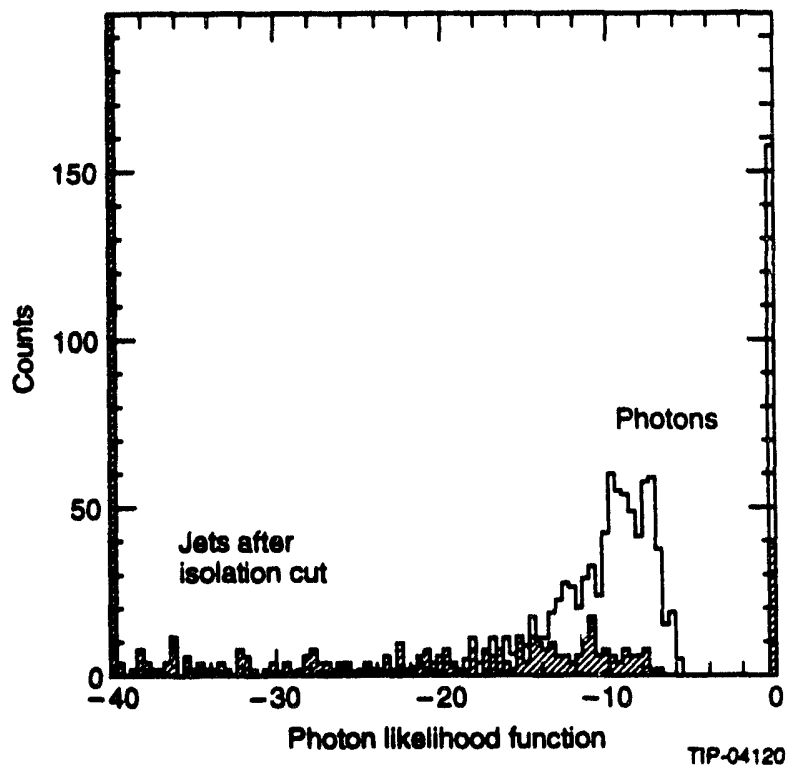

FIG. 5-7. Photon likelihood function for single photons (open histogram) and jets (hatched histogram) after isolation cuts. 


\section{Position Measurement}

The photon direction required for the reconstruction of the invariant mass of the photon pair is determined from the position of the production vertex and the position of the shower in the EM calorimeter. Studies have shown that the photon shower position resolution that we will attain, $\approx 4 \mathrm{~mm} / \sqrt{E(\mathrm{GeV})}$, will not degrade the achievable mass resolution. The accordion design has been demonstrated to be able to achieve such a resolution (see Section 5.3). In Figure 5-8, we show the Monte Carlo results for the position resolution in $\varphi$ and $\theta$ directly as a function of energy. Note that in the $\varphi$ direction we are using EM depth layers 2 and 3 only. In the $\theta$ direction the strips provide a better position resolution.

A major background for electrons is the overlap between a random charged particle and an energetic $\pi^{0}$. The suppression of this background depends on the efficiency for finding a track, and the matching of this track with EM energy in the calorimeter. A position resolution of $<2.5 \mathrm{~mm}$ reduces this background to a satisfactory level.

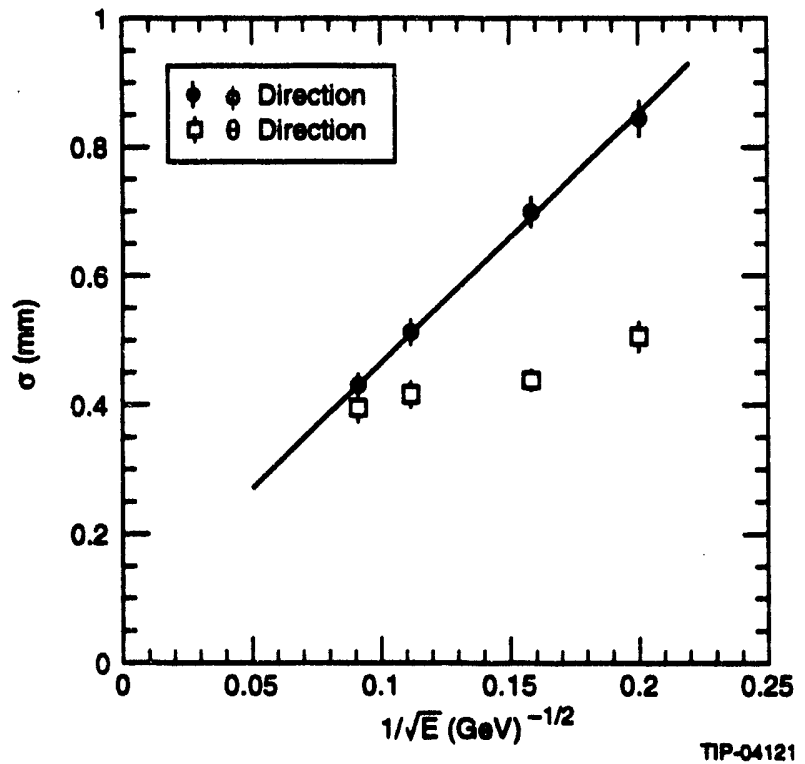

FIG. 5-8. Position resolution for the barrel accordion in the $\theta$ and the $\varphi$ directions.

\section{Pointing Resolution}

At standard luminosity $\left(10^{33} \mathrm{~cm}^{-2} \mathrm{~s}^{-1}\right)$, for typical triggered rare events there are 2.6 vertices per event. Choosing the vertex with the largest multiplicity of high-pr charged tracks selects the correct vertex $85 \%$ of the time. The angular resolution of the calorimeter can resolve ambiguities at standard luminosity, and it is crucial at high luminosity where it will be necessary to associate photons with the vertex by pointing back from the calorimeter. For this purpose, the EM calorimeter must have at least two longitudinal sections to be able to determine the incident photon direction; in fact, three will be used in the $\eta$ direction. For the accordion design an angular resolution of $55 \mathrm{mrad} / \sqrt{\mathrm{E}}+1.8 \mathrm{mrad}$ is obtained from Monte Carlo studies using only the last two layers of calorimeter cells. This result has been confirmed in the 1992 calorimeter beam test. This is improved to $(39 / \sqrt{E}+0.5)$ mrad if the strip information is also used as shown in Figure 5-9.4 In Figure 5-10 we show the effect on the Higgs mass for different assumptions on the precision of the determination of the vertex. As one can see, at high luminosity there is a significant gain from the pointing ability of the calorimeter.

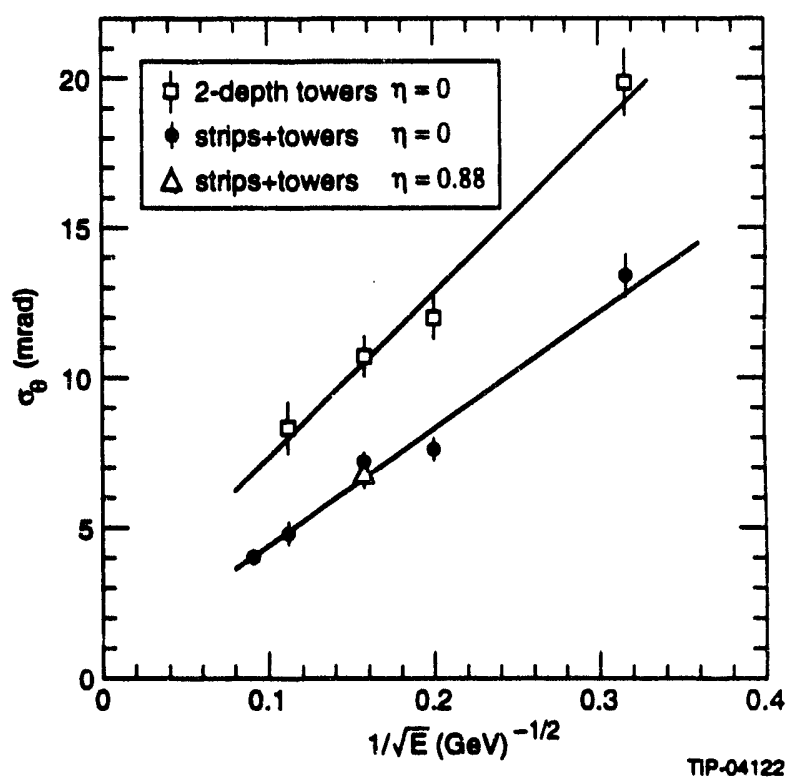

FIG. 5-9. Angular resolution of the EM accordion. Using two towers $\sigma_{\theta}=(55 / \sqrt{E}+1.8)$ mrad. Using the strips and towers, $\sigma_{\theta}=(39 / \sqrt{E}+0.5) \mathrm{mrad}$.

\section{Electron/Photon Discrimination}

In order to detect $\mathrm{H}^{0} \rightarrow 2 \gamma$ where the Higgs mass is close to the $Z^{0}$ mass, it is necessary to reject electron pairs from the photon sample at the level of $10^{-3}$. This may be difficult to achieve simply by searching for reconstructed particles in the Central Tracker. Our strategy is to generate a "road" in the IPC tracker from the space vector of the shower in the EM calorimeter, with the accuracy described in 


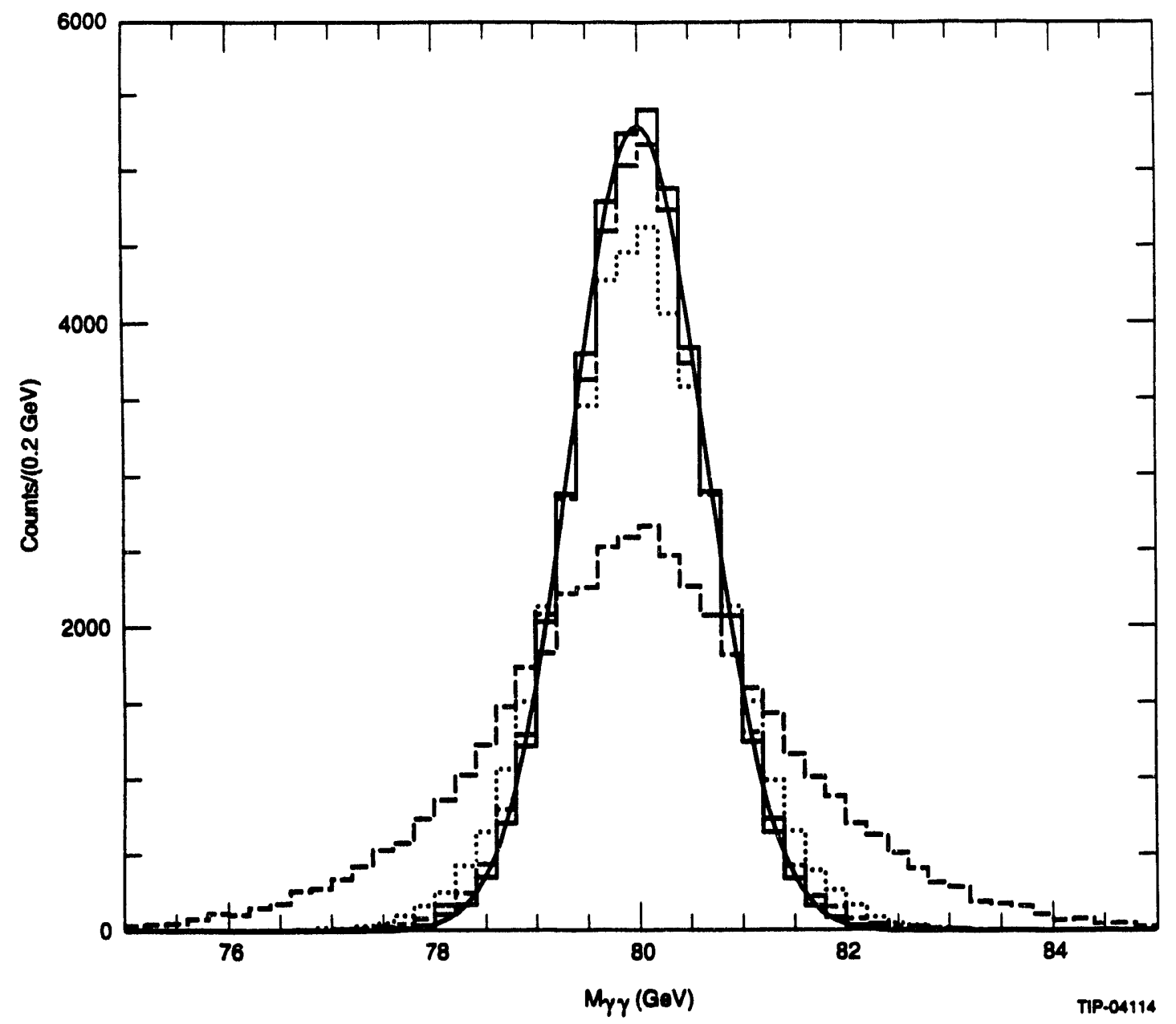

FIG. 5-10. Invariant mass of two photons from $\mathrm{H}^{0}$ with mass of $80 \mathrm{GeV}$. Solid histogram and fitted line are with all resolution and expected noise at standard luminosity $(\sigma=0.65 \mathrm{GeV})$. The long dash-short dash histogram puts in the uncertainty of the vertex as determined using the pointing resolution to find the most probable Higgs vertex, out of all the vertices reconstructed by the tracker $(\sigma=0.69 \mathrm{GeV})$. The dotted histogram uses only the angular resolution of the calorimeter to determine the vertex $(\sigma=0.79 \mathrm{GeV})$. The dashed histogram is obtained when no vertex information is used $(\sigma=1.48 \mathrm{GeV})$.

the previous two sections. The IPC hits near the road are then examined. Conservative criteria are then used to reject as a photon candidate any case which shows charged hits in the IPC which might be consistent with a charged track, in any of the eight IPC planes. True photons may sometimes be rejected, but the probability of an electron passing as a photon is well below the required value.

Similarly, our technique for finding electrons at very high luminosities where straight forward pattern recognition may not be completcly reliable, is again to start with a road given by the EM shower, and to require charged particle hits consistent with this road in at least five IPC planes (see Section 6.2.3).

\section{Massless Gap}

To optimize EM energy resolution one needs to minimize the amount of material in front of the active region of the calorimeter. In our case at $\eta=0$ we have $-1 \mathrm{X}_{0}$ of material before the active calorimeter, this is composed of the tracker, the calorimeter cryostat, a radial distance of $\mathbf{4 0} \mathrm{mm}$ inside the cryostat filled with electronics, cables, G10 spacer and kapton, inactive liquid, etc. We compensate for this by making the front part of the first active section of the calorimeter much less dense by removing the $\mathrm{Pb}$ from the absorber plate and replacing it with $\mathrm{G10}$. This together with the use of the measured longitudinal shower profile in the EM calorimeter compensates for the dead material and 
recovers the optimal calorimeter resolution. In Figure 5-11 we show how the optimal radial length of the "massless gap" is chosen. The optimal length of the massless gap is independent of $\eta$ for the entire barrel.

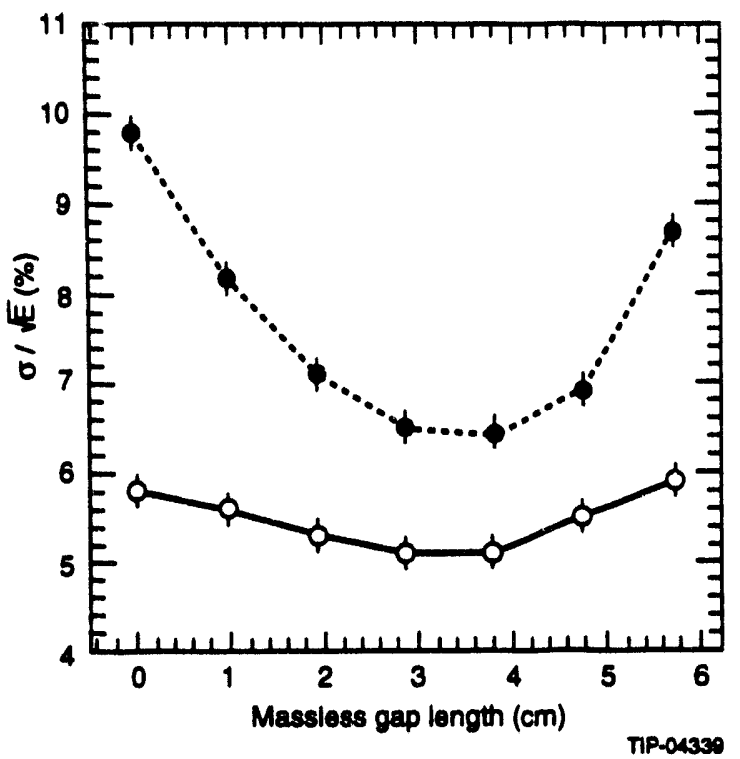

FIG. 5-11. Energy resolution as a function of the radial depth of the "massless gap" in the barrel EM calorimeter for a $25 \mathrm{GoV} \gamma$. Open circles are for $\eta=0$ and closed circles are for $\eta=0.88$.

\section{EM Calorimeter Depth}

An EM section with an active depth of $24 \mathrm{X}_{0}$ is adequate to have negligible rear-end leakage at energies up to about $100 \mathrm{GeV}$, and to maintain the energy resolution and position measurement required for the low mass Higgs search. For electrons of much higher energies (up to 6-8 TeV), such as might be produced by the decay of a high mass new $Z$ boson, significant leakage will occur. To improve the performance we will add the energy measured in the first hadronic layer, which is designed to function as a fourth EM section. In Figure 5-12 we show the energy resolution as a function of the depth of the EM calorimeter for an $80 \mathrm{GeV}$ photon showing how we chose $24 X_{0}$.

\section{Angular Coverage}

The angular coverage of the EM calorimeter must be adequate for low rate processes such as $\mathrm{H}^{0} \rightarrow \mathrm{Z}^{0} \mathrm{Z}^{0} \rightarrow e^{+} e^{-} e^{+} e^{-}$at high Higgs masses. The detection of all four electrons with good efficiency requires losses of less than a few percent per electron

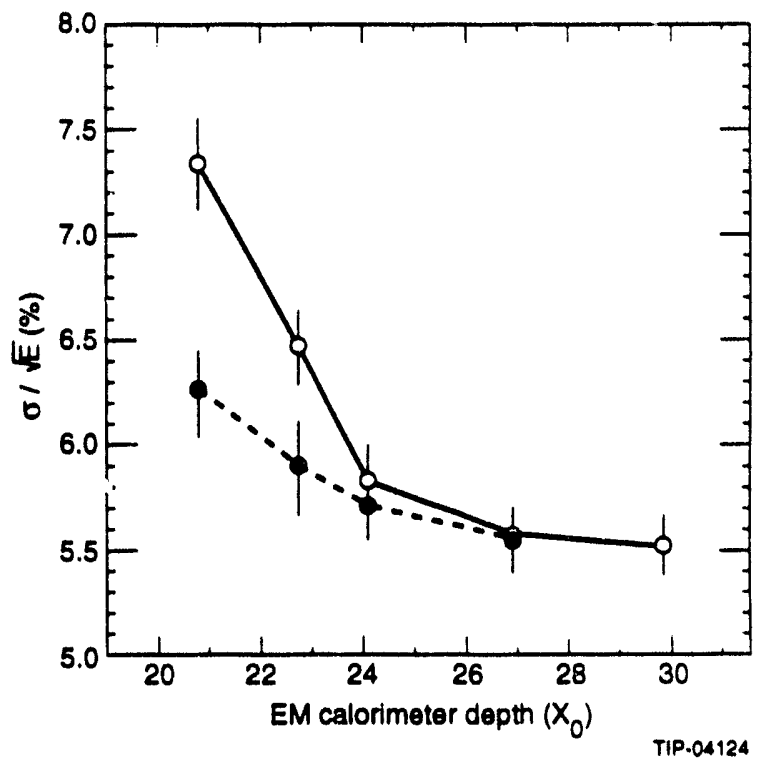

FIG. 5-12. Energy resolution as a function of the depth of the EM barrel calorimeter for an $80 \mathrm{GeV}$ photon. Open circles: energy in the EM calorimeter only, closed circles: including energy in the first hadron section.

and therefore minimal cracks and gaps in acceptance. Azimuthal cracks are completely absent in the accordion design. The pseudo-rapidity coverage for electron detection should extend out to at least 2.5, which in turn demands that the EM calorimeter cover up to about $\eta=3.0$. This provides a test of isolation around the electron candidate, over the full rapidity range.

\section{Dynamic Range and Noise}

The low end of the dynamic range is set by two processes: measurement of energy deposited by a muon passing through the EM calorimeter $(-300 \mathrm{MeV})$ and measurement of showers from low energy electrons $(\sim 10 \mathrm{GeV})$ from $\mathrm{H}^{0} \rightarrow Z^{0} Z^{*}$ where $M_{H^{0}}-150 \mathrm{GeV}$. To determine the position and shower shape (for background rejection) of these low energy electron showers, measurement of the tails of the shower must be performed down to the level of $1 \%$ of the energy, or $100 \mathrm{MeV}$. The noise per tower is less than a few tens of $\mathrm{MeV}$, which gives a reasonable signal to noise ratio for both these processes. The high end of the dynamic range is set by the need to measure electrons of energies up to $\approx 8 \mathrm{TeV}$ from possible new $Z^{\prime}$ bosons. The dynamic range for a single channel may be as high as $\approx 3 \times$ $10^{5}$, from $20 \mathrm{MeV}$ noise to $6 \mathrm{TeV}$ energy deposition. 


\section{Cryostat}

In order to minunize the amount of material in front of the EM calorimeter we have selected aluminum as the material of choice for the cryostat design. A stainless steel vessel would increase the amount of high $Z$ material at $90^{\circ}$ by one radiation length (increasing as $1 / \sin \theta$ ), which would significantly degrade the EM resolution in the barrel.

For the barrel to endcap transition we have selected a projective barrel end. We thus reduce our acceptance for $\gamma \mathrm{s}$ and electrons by a small amount rather than degrade the $\gamma / e$ resolution over a much larger region with a fully cylindrical barrel.

The use of aluminum for both cryostats also minimizes the amount of dead material for jets in the transition between barrel and endcap.

\subsubsection{Absolute Calibration of the Calorimeter}

New levels of precision calibration will be necessary to study the two-photon decay of the Higgs. The calibration methods are summarized in Section 5.4.4 and Table 5-8. An essential part of the calibration process will be an extensive series of test beam measurements using production-line calorimeter modules. Experience in CDF and DO has demonstrated the usefulness of such an exercise, even when other in-situ calibration methods are available. The test beam will be used to map out the calorimeter response as a function of energy, angle, rate and particle species as discussed in Section 5.7. It will also be used to determine position and angular resolution, and to characterize the shower shape. It is worth noting that this requires the determination of many parameters for the EM calorimeter, each of which may be energy and $\eta$-dependent. In principle this can be done by simulation, but it is clearly preferable to verify the results in a beam. For the hadronic section it is necessary to determine the weighting schemes to add the energies in the various calorimeter layers. The test beam should allow electrons, muons and hadrons of energies 5-150 GeV to be directed into the calorimeter along various trajectories, as though coming from the SSC interaction point. For electronic channel calibration the same pulser system should be used as is installed in the full detector, in order to allow absolute charge comparisons. The test beam energy scales will be used to set the trigger thresholds at startup.

\section{Calibration of EM Calorimeter}

We expect that the electronic calibration of the EM calorimeter as installed will be better than $1 \%$. The detector calibration will be further refined in-situ. The first and most crucial stage is to assure the uniformity of response and to map the response over the calorimeter. This must be done to better than $0.2 \%$, small compared to the $0.4 \%$ constant term in order not to dominate the constant term of the energy resolution. The primary determination of energy scale will be done using $\mathrm{Z}^{0}$ boson decays.

The cross section for $Z^{0}$ production at the SSC is estimated to be $64 \mathrm{nb}$ for mass between $86 \mathrm{GeV}$ to $96 \mathrm{GeV}$. Taking into account the $\mathrm{Z}^{0} \rightarrow e^{+} e^{-}$ branching ratio, the $e^{+} e^{-}$pair production cross section is $1.98 \mathrm{nb}$. Using gamfast (see Section 2.2) and the following simple selection criteria,

1. electron $E_{T}>20 \mathrm{GeV}$

2. electron $|\eta|<2.5$, and excluding the barrelendcap transition,

3. additional $E_{T}<5 \mathrm{GeV}$ in a cone of $R=0.45$ around the electron,

the acceptance is $32 \%$. Of all the events accepted, $28 \%$ have both electrons in the barrel, $25 \%$ have both electrons in the endcap, and $47 \%$ have one in the barrel and one in the endcap.

The electronic gains of all channels are equalized with the precision calibration system (see Section 5.4.4), and test beam data provide the map of the response. The $Z^{0}$ s are used to set the absolute energy scales for the barrel and endcap. Two hundred $Z^{0} \mathrm{~s}$ in the barrel and endcap of the calorimeter, which corresponds to less than an hour's data at $10^{33} \mathrm{~cm}^{-2} \mathrm{~s}^{-1}$ luminosity, will calibrate the absolute energy scale of barrel and endcap to better than $0.2 \%$. Figure 5-13 shows the spectrum of $-200 e^{+} e^{-}$pairs. The mean of $\mathrm{M}_{e e}$ is determined to $0.1 \%$ statistically. In addition, the width of the $\mathrm{Z}$ peak provides a check on the resolution performance. The FWHM of the Breit-Wigner fit is about $3.0 \mathrm{GeV}$, with a $2.5 \mathrm{GeV}$ contribution from the $\mathrm{Z}^{0}$ intrinsic width. 


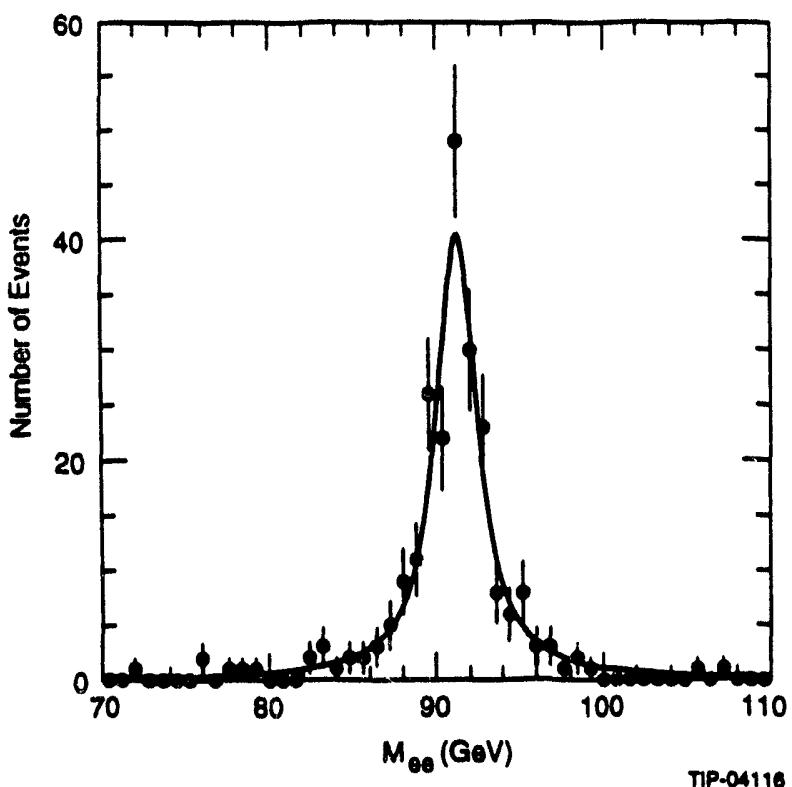

FIG. 5-13. Invariant mass of $\theta^{+} \theta^{-}$pairs in the barrel after -20 minutes of running at nominal SSC luminosity.

We will check the uniformity of the response across the detector, to control effects such as variation of dead material in front of the active calorimeter as a function of $\eta$, channels with different shaping time in the endcaps and change of tower size in the endcaps. The mean position of the $Z^{0}$ mass peak can be plotted as a function of the $\eta$ of one of the electrons integrating over all $\varphi$ angles. Corrections as a function of $\eta$ can then be found. An iterative process can then be used to eliminate any $\eta$ dependence, by applying the correction function. Figure 5-14 shows the results of a simulation of data acquired in 1/3 day. The error bars give a measure of the precision of this procedure. The entire EM calorimeter can be subdivided into smaller sections, for example, 50 divisions in $\eta$ and 40 divisions in $\varphi$. Ten days $\left(300 \mathrm{pb}^{-1)}\right.$ are required to fill each section with at least 200 electrons. When the $Z^{0}$ sample is large enough, it is possible to check the response of each tower. Simulations show that if the response of a particular tower has shifted by $5 \%$, the $Z^{0}$ mass peak for events where one electron hits this tower would shift by $1.7 \%$. With $3000 \mathrm{pb}^{-1}$, we expect 100 electrons in each tower. Therefore, in a few months of running we will confirm the response of each individual tower to about $0.4 \%$. Thereafter, the gains will be monitored by the electronic calibration system with periodic re-calibration using $\mathrm{Z}^{0} \mathrm{~s}$.
No long-term shifts are expected in a liquid ionization system. Nevertheless, in case of unexpected shifts in response, a full calibration analysis using inclusive Upsilons and other copious sources of electrons could be used, to maintain the response to the necessary precision.

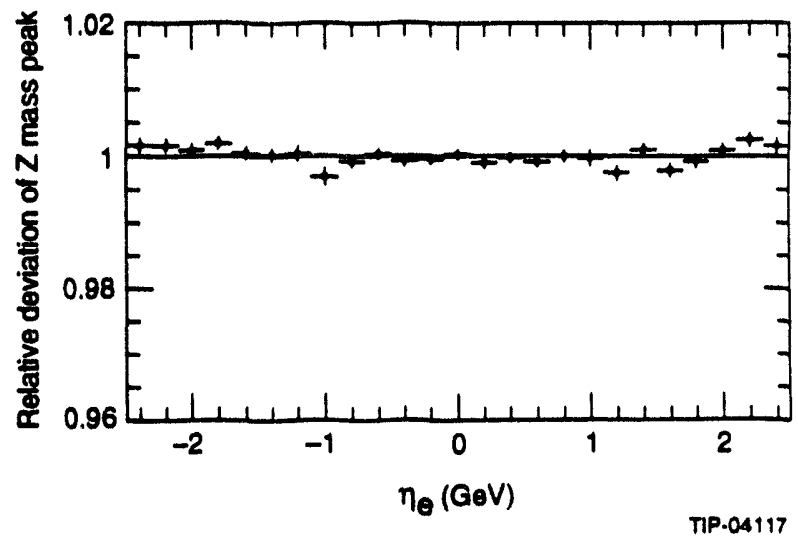

FIG. 5-14. Relative deviation of invariant mass of $Z^{a} \rightarrow \theta^{+} \theta^{-}$pairs vs. rapidity for $10 \mathrm{pb}^{-1}$.

\section{Alignment}

The accuracy of the alignment of the EM calorimeter with respect to the tracker has to be about $10 \mathrm{~mm}$, but the relative positions have to be known to $<1 \mathrm{~mm}$ for electron and photon identification. This can be done in situ using isolated electron tracks from $W$ and $Z^{0}$ decays.

\subsubsection{Hadronic Calorimetry \\ Jet Measurements}

Measurement of the inclusive jet cross section as a function of $E_{T}$ will allow us to test for possible substructure in quarks and gluons. To test $Q C D$, the energy response must be understood over the full energy range. The calorimeter must also have a Gaussian response. Due to the steeply falling $P_{T}$, events in the tail on the high side of the resolution function will dominate the apparent cross section at large pT. Events in the tail on the low side will contribute to $\hat{E}_{T}$

In order to measure the top quark mass in the lepton + jets channel, or to search for a Higgs through the channel $\mathrm{H}^{0} \rightarrow \mathrm{Z}^{0} \mathrm{Z}^{0} \rightarrow l l$ jet jet; we need to reconstruct $Z^{0}$ s by measuring the invariant mass of a two jet system. Another limit on the constant term $b$ in the energy resolution is set if one wishes to reconstruct a high mass $Z^{\prime}$ boson decaying hadroni- 
cally into very high $p T$ jets. A jet energy resolution with a sampling term $a$ of 60 and constant term $b$ of 4 is sufficient.

Clearly, the constraints on hadronic energy resolution are not as stringent as those on the EM performance. However, the stat ity of response of liquid ionization calorimetry is important for long term use of weighting algorithms and for the mapping across the barrel-endcap interface. Figure 5-15 shows the energy resolution for jets from the GEANT simulation. 6 Although all the dead material of the cryostats is included, the transition between barrel and endcap is rather smooth.

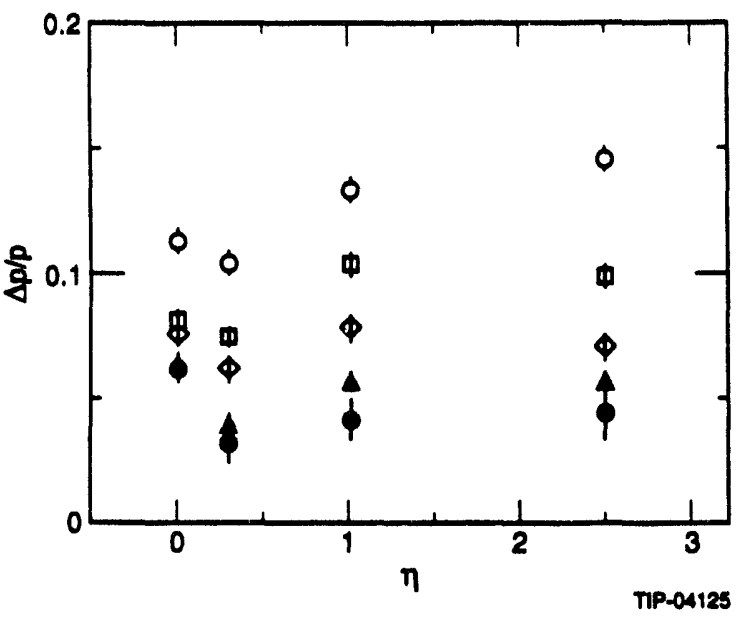

FIG. 5-15. Jet resolution v8. $\eta$. Open circles are for $50 \mathrm{GeV}$ jets; open squares for $100 \mathrm{GeV}$ jets; open diamonds for $200 \mathrm{GeV}$ jets; open triangles for $500 \mathrm{GeV}$ jets; and filled circles for the constant term.

\section{Missing Transverse Energy}

$E_{T}$ is a signature for neutrinos and for possible new, weakly interacting particles. The search for supersymmetry at a relatively low mass scale provides constraints on the $E_{T}$ performance of the detector. Standard model physics gives an irreducible $\hat{E}_{T}$ background, resulting from neutrino production in the decays of heavy quarks and of vector bosons. The detector must not introduce erroneous $E_{T}$ above this background level. To observe a signal from the production of gluinos and quarks of masses $>200 \mathrm{GeV}$, above the background at the SSC, it is necessary to be sensitive to $E_{T}=100 \mathrm{GeV}$. Masses lower than this should have been detected at the Tevatron by the time SSC running begins.

The calorimeter coverage must, therefore, extend to high enough $\eta$ so that undetected forward jets do not create significant $E_{T}$ Jets should be detectable out to $\eta=5$. The forward calorimeter should measure $E_{T}$ with a precision at least $\Delta E_{T} / E_{T}$ $=10 \%$. This is dominated by the precision of the jet angle measurement, which requires that the forward calorimeter be finely segmented. At high rapidities, the jet energies in the forward calorimeters are always large, and the $E_{T}$ measurement is very sensitive to the angular resolution. As a result, the energy resolution is not as important in the forward direction.

While the energy resolution in the barrel and endcap calorimeters is not crucial, it is important that there be no non-Gaussian tails and that cracks, feedthroughs and cryostat walls do not introduce false $E_{T}$ at or above the standard model background level.

\section{Depth of Calorimeter}

A depth of about 9-10 interaction lengths is required to contain jet energy for measurement purposes. This is sufficient to contain $95 \%$ of the energy for $95 \%$ of $1 \mathrm{TeV}$ jets. ${ }^{7}$ A greater depth requirement for the total absorber (active and passive) is set by the need to shield the muon system from punch through and shower debris. The muon system has specified $11 \lambda$ at $\eta=0$ increasing to $16 \lambda$ at $\eta=3$.

\section{Transition from Liquid to Scintillator Calorimeter}

This transition point has been optimized in terms of physics performance, cost and flexibility in construction (see Section 5.1.3). The main physics consideration is to locate the feedthroughs in a deep enough location so that showers would not be significantly affected by the energy lost there. As can be seen in Figure 5-2, there is $-6 \lambda$ in the liquid calorimeter for the $\eta$ region before the barrel feedthroughs, and only $-5 \lambda$ in the liquid hadron calorimeter at the $\eta$ region of the feedthroughs. Figure 5-15 shows that jet energy is rather constant as a function of $\eta$. To study the liquid/scintillator transition, a GEANT simulation was done for hadron jets aimed at various $\eta$ regions, see Figure 5-16. The hadron jet energy resolution is calculated versus jet energy for different numbers of readout layers in the Scintillating Barrel Calorimeter (SBC) for a fixed thickness of absorber. A modest improvement in the 
jet energy resolution is obtained if the scintillating barrel is instrumented, though the nu nber of scintillation layers need not be more than a few. The jet energy distribution for $1 \mathrm{TeV}$ jets is shown in Figure 5-17a. There is a small tail on the low side of the distribution. However, as can be seen in Figure 5-18, the contribution to the $E_{T}$ spectrum, even if the scintillating barrel were uninstrumented, would be an order of magnitude below the "background" from real neutrinos. Figure 5-18 also shows the contribution to the $E_{T}$ from not measuring particles with $\eta>$ 5. These undetected forward jets limit the sensitivity to any anomalous signal $<100 \mathrm{GeV}$ in the $E_{T}$ spectrum. Figure 5-17b shows the energy deposited in the scintillating barrel calorimeter for $1 \mathrm{TeV}$ jets. While the mean of this distribution is on the order of $50 \mathrm{GeV}$, there are many events with significant energy deposited.

Near $\eta=0.8$, where the barrel feedthroughs are located, the scintillating barrel has a larger fraction of the energy of jets and more importance for the $E_{T}$ spectrum. If the transition between liquid and

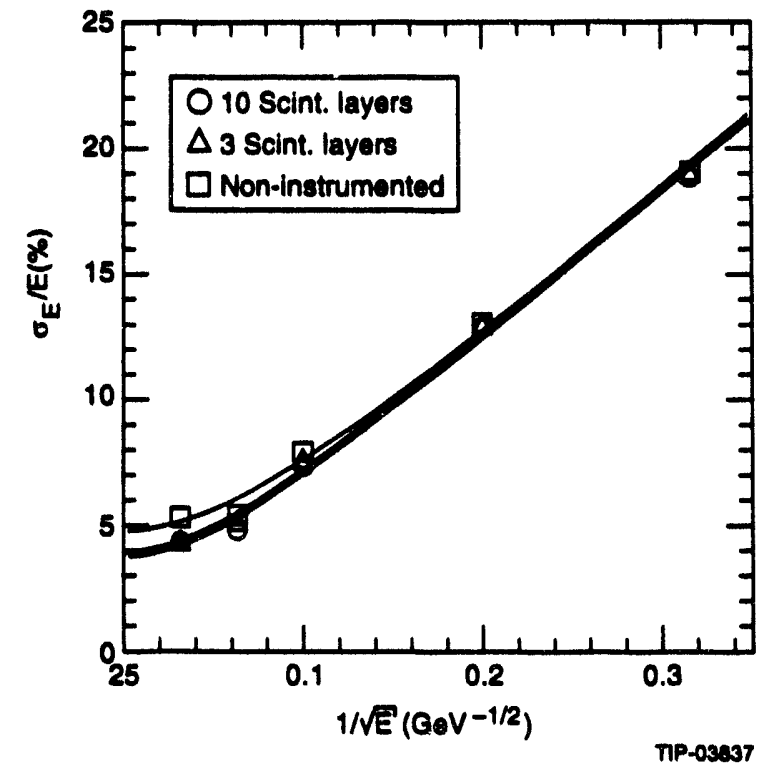

FIG. 5-16. Jet resolution vs. jet energy at $90^{\circ}$ (PYTHIA + GEANT simulation) in the GEM Pb/Kr longitudinally segmented barrel calorimeter including the scintillating barrel calorimeter for different numbers of instrumented layers in the scintillating barrel. Three longitudinal readout segments are assumed in the liquid hadron calorimeter section. The jet energy resolution can be parametrized as: $(59.6 \pm 0.7) / \sqrt{E} \oplus$ $(3.75 \pm 0.13) \%$ for 10 layers of instrumentation; $(60.2 \pm 0.7) / \sqrt{E} \oplus(3.94 \pm 0.13) \%$ for 3 layers of instrumentation; $(59.2 \pm 0.8) / \sqrt{E} \oplus(4.77 \pm 0.14) \%$ for no instrumentation. scintillator were at a smaller $\lambda$ at $90^{\circ}$, this region would be located closer to the peak of the hadron shower and cause problems. A technical consideration is that, if this transition were at a smaller radius, there would be less area for the feedthroughs which are already tightly packed. If the transition were placed further out in $\lambda$ at $90^{\circ}$, the cryostat would become larger and more difficult to transport.

\section{Muons}

The calorimeter must serve as a filter, eliminating hadronic debris through absorption. Punch through should be reduced to a level where it is

(a)

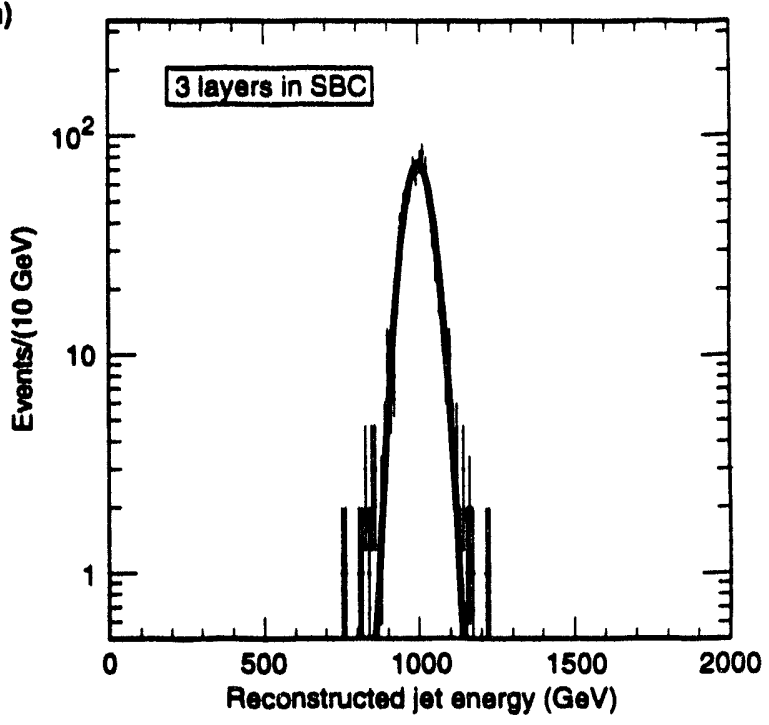

(b)

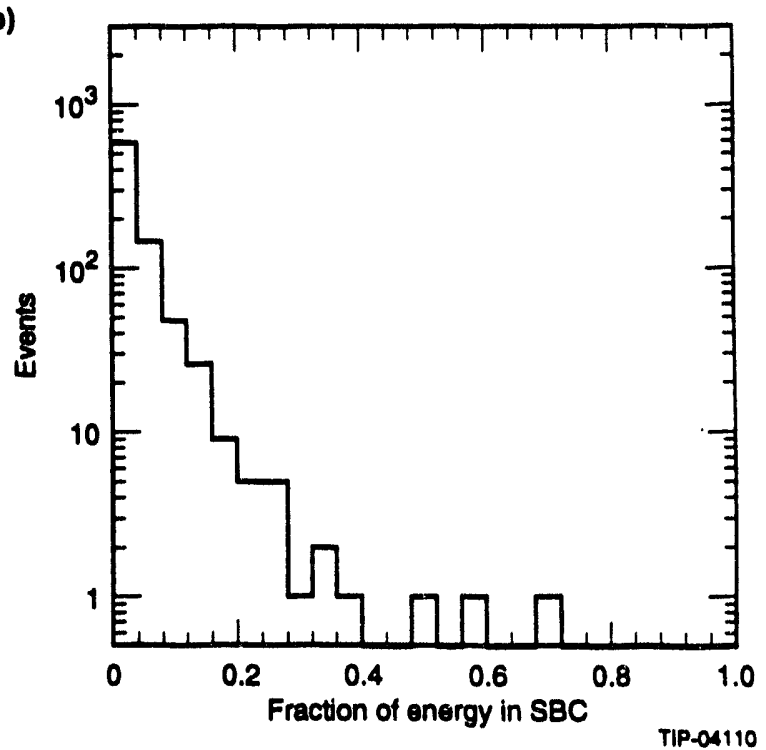

FIG. 5-17. a) Energy resolution for $1 \mathrm{TeV}$ jets for entire barrel calorimeter. b) Fraction of energy in the SBC from $1 \mathrm{TeV}$ jets. 


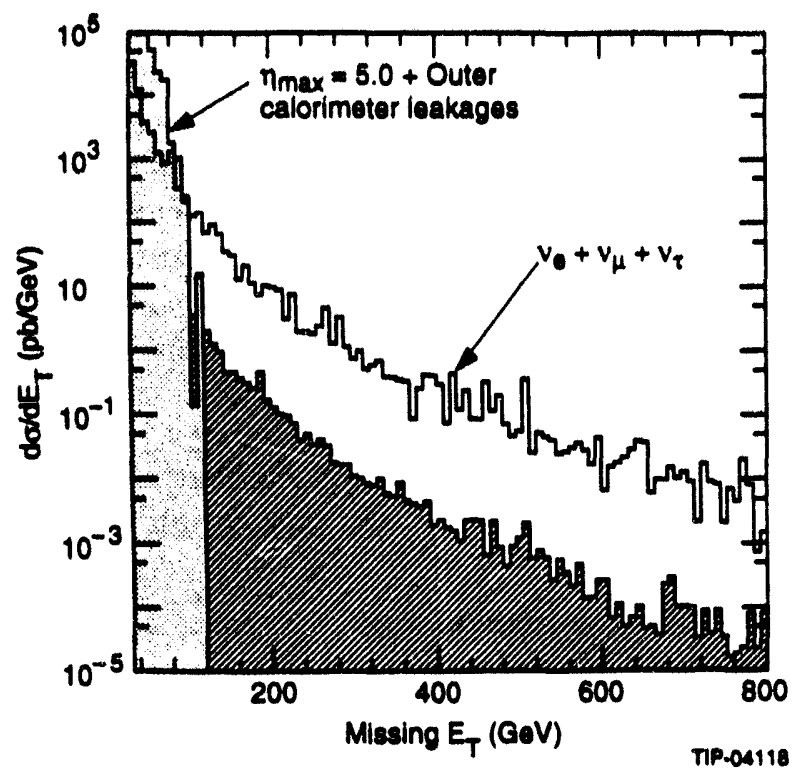

FIG. 5-18. Cross section for missing energy. The cross hatched histogram results from not measuring particles with $\eta>5$ and leakage from the SBC. The hatched histogram would result if in addition the SBC were uninstrumented. The solid histogram is the cross section from neutrino production.

dominated by the prompt and decay muons. Beyond this point, extra thickness does not reduce the hit rate in the muon chambers significantly. This can be achieved with a calorimeter thickness of $\sim 11 \lambda$ at $\eta$ $=0$, increasing to $\sim 16 \lambda$ in the forward direction.

The calorimeter must also measure the electromagnetic energy produced along the muon track, particularly any deposition from catastrophic energy losses. This energy must be added to the reconstructed muon momentum to give the true muon energy at the vertex. This requires that the hadronic calorimeter measure EM energy with a sampling term $a$ of about 35 .

The calorimeter is designed to see the minimum ionizing energy deposition of the muon passing through the calorimeter to help in muon identification. The calorimeter noise must be a few times less than the signal from a MIP to permit this.

\section{Calibration of Hadron Calorimeter}

It is particularly important to map the hadron response actoss the barrel-endcap boundary with an accuracy of $1-2 \%$. The mapping will be performed in the test beam and transferred to the complete GEM detector. In the measurements with collider data isolated low-momentum hadron tracks can be used to set an absolute scale at low energies by requiring a match with the momentum measured in the tracker. At higher energies the hadronic scale can be compared to the previously established EM scale, using events where a jet recoils against an electromagnetic cluster (either a true photon or an electromagnetically fragmenting jet). A Monte Carlo calculation by supplying the fragmentation distributions must be used to translate the test beam energy scale for single hadrons to determine the jet energy scale. Requiring a balance in $E_{T}$ for two jet events allows the calibration to be extended from the barrel to the endcap, and into the forward calorimeter.

\subsubsection{Radiation Environment}

The calorimeter must operate in a high radiation environment without any degradation of its performance. The highest doses are in the inner comers of the endcap calorimeters (a few tens of Mrad after 10 years at nominal luminosity) and close to the beamline in the forward calorimeters (up to $5 \mathrm{Grad}$ after 10 years). This motivates the use of an inherently radiation hard technology such as liquid ionization. The approach to provide radiation hard com.ponents for the calorimeter is described in Section 5.8 and the expected radiation doses in Chapter 12.

\subsection{R\&D RESULTS}

\subsubsection{Introduction}

GEM's decision to rely primarily on liquid ionization calorimetry has been motivated by recent $R \& D$ results as well as by the successful operation of many large scale liquid argon calorimeters, including DO, SLD and H1. The proposed GEM electromagnetic calorimeter is based on the accordion design first introduced by RD3 at CERN. ${ }^{2}$ Their results with liquid argon (LAr) indicate that a resolution of $(9-10) \% / \sqrt{E}$ and a constant term of less than $0.4 \%$ can be obtained with this type of geometry. GEM has established the goal of achieving even better energy resolution. In the summer of 1992 a test was performed at the BNL AGS by the GEM liquid calorimeter group to study the properties of an accordion calorimeter employing a thinner lead absorber and a denser sampling medium-liquid krypton ( $\mathrm{LKr}$ ) - in addition to investigating the test calorimeter's performance in 
LAr. The use of $L K r$ allowed the overall density of the BNL calorimeter to remain about the same as the RD3 device, while significantly increasing the sampling ratio.

\subsubsection{The Liquid Krypton Test at BNL Construction}

The accordion structure, absorber and electrodes, were similar to those used by the RD3 collaboration. The absorber plates were made of lead of thickness $1.3 \mathrm{~mm}$ reinforced with $0.2 \mathrm{~mm}$ thick stainless steel on each side (the stiffness of the composite is determined by the thickness of the stainless steel and by the corrugations, not by the thickness of the lead). The stainless steel foils and the lead plates were first bent and then glued together using prepreg (cloth impregnated with epoxy). The signal electrodes were made of copper coated kapton foils of $0.36 \mathrm{~mm}$ thickness. The copper itself was only $40 \mu \mathrm{m}$ thick and $24.5 \mathrm{~mm}$ wide with an interstrip separation of $0.5 \mathrm{~mm}$. The $\mathrm{LKr}$ gap in this design was $2 \times 2.0 \mathrm{~mm}$. A unit cell included one absorber plate, one signal electrode, and two liquid gaps. The layers that carry the high voltage had distributed film resistors to protect the preamplifiers in case of a discharge. The test module was segmented longitudinally into two sections of $6 \mathrm{X}_{0}$ (front) and $18 \mathrm{X}_{0}$ (back), respectively. The calorimeter tower size was defined to be three readout cells, corresponding to an area of $2.5 \times 2.7 \mathrm{~cm}^{2}$ at the face of the module.

\section{Electronic Readout and Calibration Chain}

The electronic readout chain used in the test was similar to that proposed for GEM. The preamplifiers were located as close as possible to the signal electrodes and operated at cryogenic temperatures. Pre-amplified signals were carried by strip cables to the exterior of the cryostat, where they were amplified and sent to shaping amplifiers. A peaking time of $\mathbf{4 0} \mathrm{ns}$ was chosen for the shapers. This choice was dictated by considerations of electronic noise and pile-up. A high uniformity of energy response was maintained throughout the detector which allowed the formation of precision analog sums over large sections of the calorimeter for trigger purposes. The shaped signals were sent to track and hold units, where the signal peak information was held and sent to ADCs for digitization and recording. Data were also recorded with multiple time samples to better understand the readout proposed for the GEM calorimeter. The timing resolution of the calorimeter was measured using an amplitude independent discriminator applied to the total energy sum.

To attain the desired constant term in the energy resolution, a precise calibration system is necessary. For the BNL test, a scheme similar to the one adopted in our final design was used. ${ }^{8}$ Since it is inherently difficult to accurately distribute to all preamplifiers pulses that simulate those generated in the $\mathbf{L K r}$, pulses are generated in situ by calibration circuitry. A precision clock signal with a sharp rise time (3-4 ns) injects a known amount of DC current into the preamplifiers. This clock signal is distributed from channel to channel for 16 to 32 channels by means of a lumped parameter delay line, where the delay between nodes is known to better than 100 ps. Each node is selected externally which permits the calibration of individual channels. During the test the current sources were controlled with 12 bit accuracy, and the channels were calibrated to better than $0.2 \%$.

\section{Test Run Results}

The test module was placed in the $\mathrm{A} 3$ beamline at the AGS. The beam was defined by means of scintillators, and electrons were identified by Cerenkov counters. A fine grained hodoscope was used to determine the position of incidence on the face of the calorimeter. A set of veto counters was used to reject particles which showered before reaching the detector. Beams of $\mu^{-}, \pi^{-}$and $e^{-}$with $5,10,15$, and $20 \mathrm{GeV} / \mathrm{c}$ momenta were used. The system was also pulsed randomly during data collection to record events that could be used to determine the noise in the system under real operating conditions. The measured noise was approximately $17 \mathrm{MeV}$ per tower; no coherent noise was observed, for sums of up to -150 channels.

The on-line calorimeter performance is displayed in Figure 5-19, which shows the overlap of 110 digitally stored pulses, occurring over an area of $2.5 \times 5.0 \mathrm{~cm}^{2}$, during the $20 \mathrm{GeV} e^{-}$run. The signals shown correspond to an analog total energy sum derived from a $5 \times 5$ tower configuration around the beam. The basic shape of the waveform is determined by the convolution of the shaper impulse response, which has a peaking time of $40 \mathrm{~ns}$, and the 
detector current waveform. This results in a peaking time of $70 \mathrm{~ns}$, and a positive lobe which extends over the electron drift time of $500 \mathrm{~ns}$. The width of the line in Figure 5-19 shows the excellent energy resolution, and the low noise attained prior to any corrections.

Off-line analysis of the data required hits in the beam counters and none in the vetoes. Electrons were identified at this stage from Cerenkov information. The gain of each channel was corrected using the on-line calibration, and $5 \times 5$ towers around the highest energy tower were summed to obtain the calorimeter's response to electrons. Due to the presence of inactive material in front of the calorimeter (estimated to be $1.5 \mathrm{X}_{0}$ ), significant energy loss can occur if a shower starts before the active region. Taking advantage of the longitudinal segmentation, a correction was determined using the data and applied on an event by event basis. The correction is energy dependent, and becomes negligible for events where the shower starts deep in the calorimeter and deposits most of its energy in the back section.

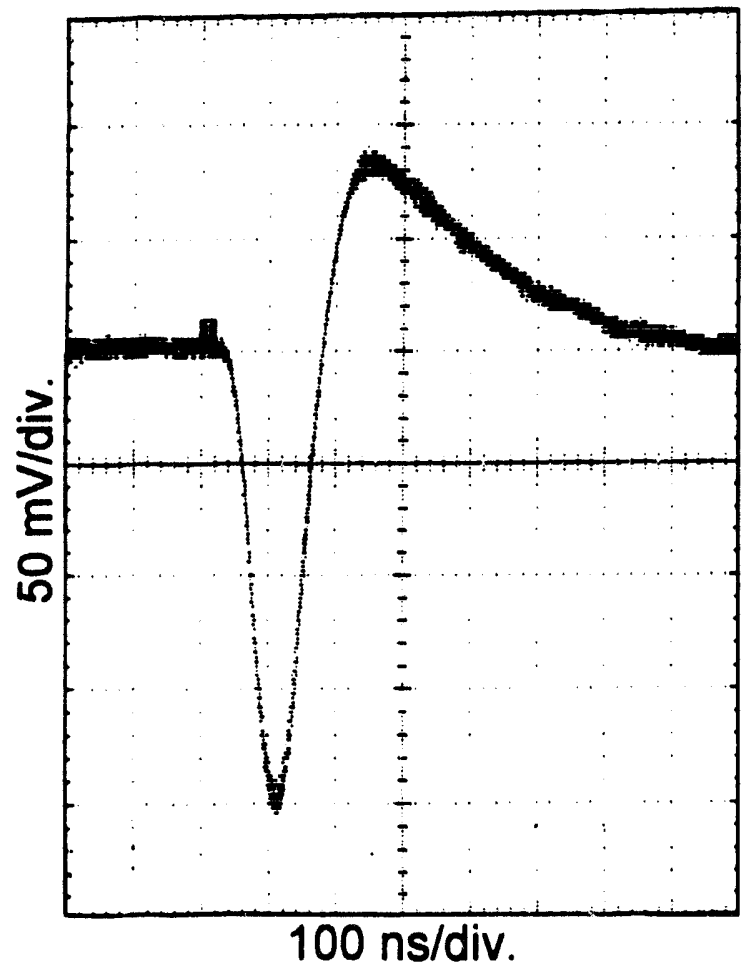

FIG. 5-19. The response of the LKr accordion test calorimeter to $20 \mathrm{GeV}$ electrons. The recording shows 110 traces from an analog sum of $5 \times 5$ towers.
The uniformity of the response vs. position has also been determined. In the $x$ coordinate (strips), the uniformity is very good and no correction is needed. In the $y$ coordinate (accordion bends), a position dependent correction is applied. This correction has a variation of $\pm 0.33 \%$ (rms). To extract the energy resolution, a beam momentum spread of $0.5 \%$, defined by the beam optics, was assumed.

\section{Energy Resolution and Linearity}

The centroid and the rms width of the $e^{-}$energy distributions were obtained from Gaussian fits over the range $\pm 3 \sigma$. The difference between the centroid and the nominal value, normalized to the nominal value, is shown in Figure 5-20 as a function of beam momentum. A maximum deviation of $0.1 \%$ from expectation shows the level of linearity obtained with the test module. The experimental resolution of the calorimeter as a function of energy is displayed in Figure 5-21. It is well represented by the parameterization:

$$
\begin{gathered}
\frac{\Delta E}{E}=(0.00 \pm 0.2) \% \oplus \frac{(6.72 \pm 0.04) \%}{\sqrt{E}} \\
\oplus \frac{0.08 \mathrm{GeV}}{E} .
\end{gathered}
$$

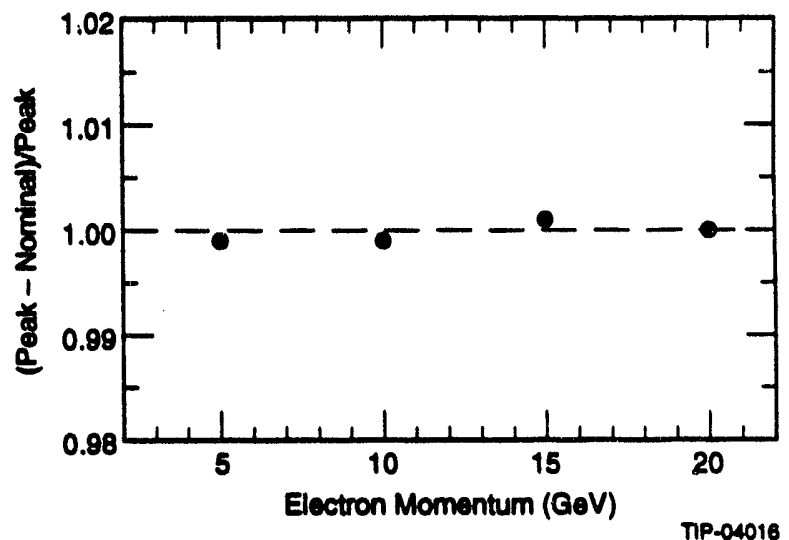

FIG. 5-20. Percentage deviation in the response of the liquid krypton calorimeter vs. beam energy. The error bars are smaller thait the circles.

The sum of $3 \times 3$ towers was also studied. The data show that relative to the sum of $5 \times 5$ towers, the sum of $3 \times 3$ towers is $6 \%$ lower in centroid, and $10 \%$ worse in resolution, in excellent agreement with GEANT simulations.

\section{Timing Performance}

The timing performance of the calorimeter was also investigated at these beam momenta. ${ }^{5}$ The 


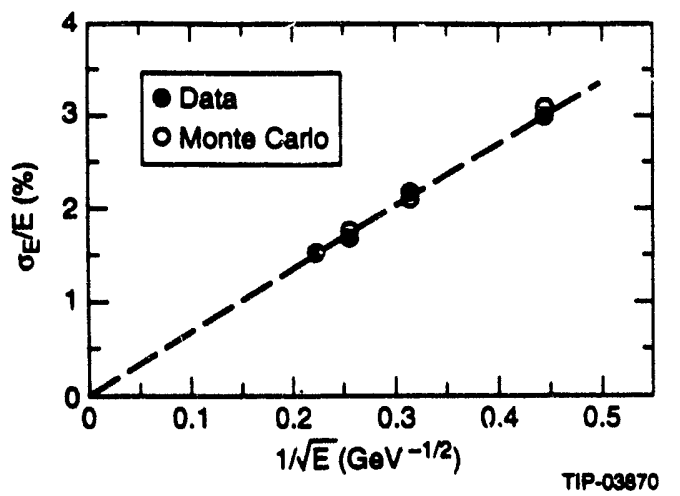

FIG. 5-21. Energy resolution from test beam data and from simulation vs. $1 / \sqrt{ } E$, obtained from the liquid krypton beam test.

timing signal was generated by a constant fraction discriminator using a circuit developed by Turko and Smith. ${ }^{9}$ The on-line timing signal was corrected off-line for the differences in cable length of individual channels contributing to the total energy sum. Corrected and uncorrected timing distributions for the $20 \mathrm{GeV} e^{-}$run are displayed in Figure 5-22(a). After off-line corrections, a resolution of (280 \pm 25 ) ps was obtained for a $20 \mathrm{GeV}$ signal. The energy dependence of the timing resolution is displayed in Figure 5-22(b). A fit to the experimental points which takes into account the convolution between a constant term and an ener "nendent term yields the result:

$$
\sigma_{t}=(0.19 \pm 0.01) \mathrm{ns} \oplus \frac{(4.15 \pm 0 .}{E} \quad \frac{V \cdot \mathrm{ns}}{}
$$

In the test this constant term arises from the timing resolution of the beam counters. The energy dependence can be understood as the contribution of the thermal noise to the total energy $?+1$ ise. ${ }^{10}$ Based on this, we calculate that the timing resolution of the EM calorimeter in the presence of the thermal and pileup noise expected at $\mathcal{L}=10^{33} \mathrm{~cm}^{-2} \mathrm{~s}^{-1}$ will be $9 \mathrm{GeV} \times n s / E$ for $5 \times 5$ towers, corresponding to $\Delta \eta$ $x \Delta \phi=0.13 \times 0.13$. A single tower would have a time resolution of $2 \mathrm{GeV} \times n s / E$.

\section{Conclusions}

The results from the AGS test run demonstrate that an energy resolution of $6.7 \% / \sqrt{E}$ is achievable with a liquid ionization calorimeter, using an accordion structure with $1.3 \mathrm{~mm} \mathrm{~Pb}$ plates and liquid krypton. The measured resolution is close to what is predicted by Monte Cario studies: $6.4 \% / \sqrt{E}$. The tests also demonstrate that high speed ionization (a)

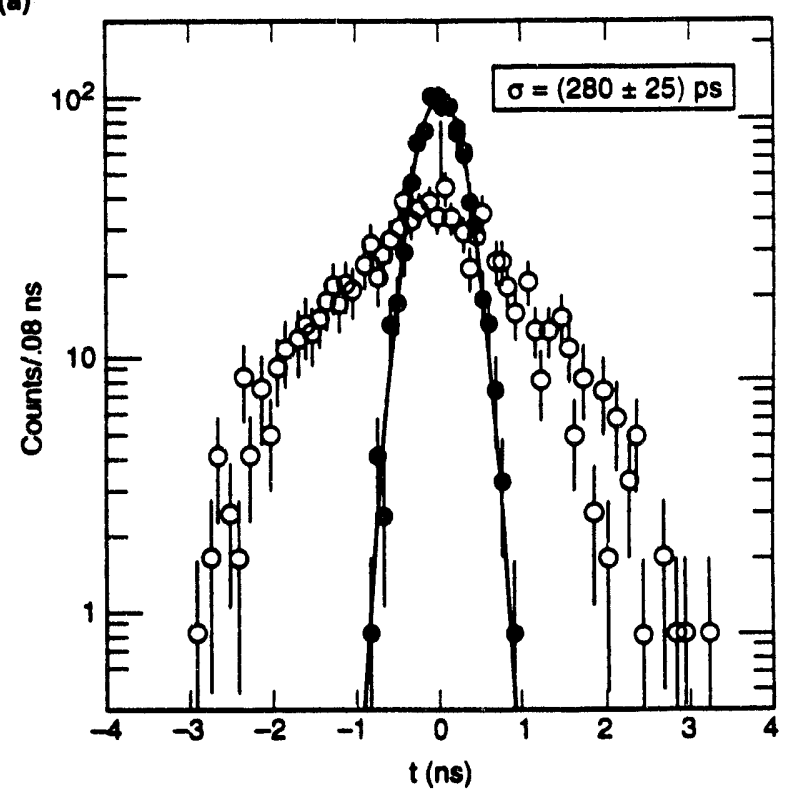

(b)

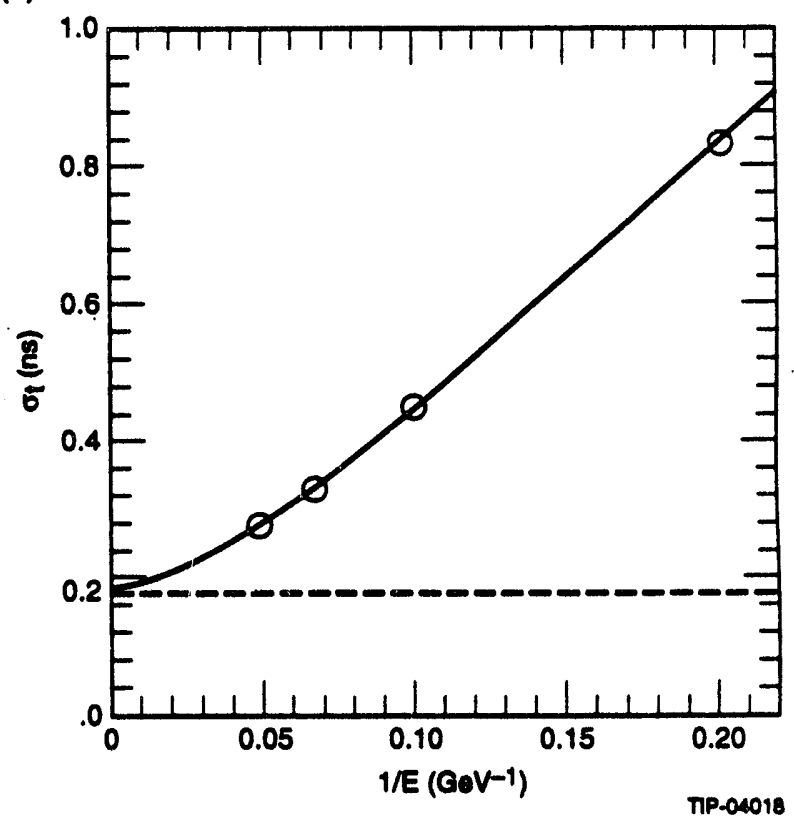

FIG. 5-22. Timing resolution of liquid krypton calorimeter. a) Open circles - raw timing distribution at $20 \mathrm{GeV}$; solid circles - after corrections. b) Energy dependence of timing resolution from test beam data.

calorimetry can be built on a large scale, with very good energy and timing resolution.

\subsubsection{RD3 Results}

The RD3 collaboration has pioneered the concept of an accordion EM calorimeter. We have benefited tremendously from this work, and our participation in this R\&D program. In 1992 the RD3 
collaboration obtained results using a $2 \mathrm{~m}$ long projective EM accordion at the CERN SPS, and found the same energy resolution as with smaller non-projective prototypes. The first large projective prototype using $1.8 \mathrm{~mm} \mathrm{~Pb}$ absorber and $\mathrm{LAr}$ yielded $\sigma / E=0.62 \% 11$ at $287 \mathrm{GeV}$ (see Figure 5-23). Since the energy resolution followed $10 \% / \sqrt{\mathrm{E}}$, the bound on the constant term was $\sim 0.2 \%$. Very good agreement was observed between the behavior predicted for the $2 \mathrm{~m}$ module and the test beam results.

There are also results from an accordion hadron module that used an "electrostatic transformer"(EST) similar to the device proposed for GEM and described in Sections 5.5.1 and 5.5.2. Since this was the first beam test of the EST concept, the transverse profile of the hadron shower was measured, and no broadening due to the presence of the EST was observed. The module was small

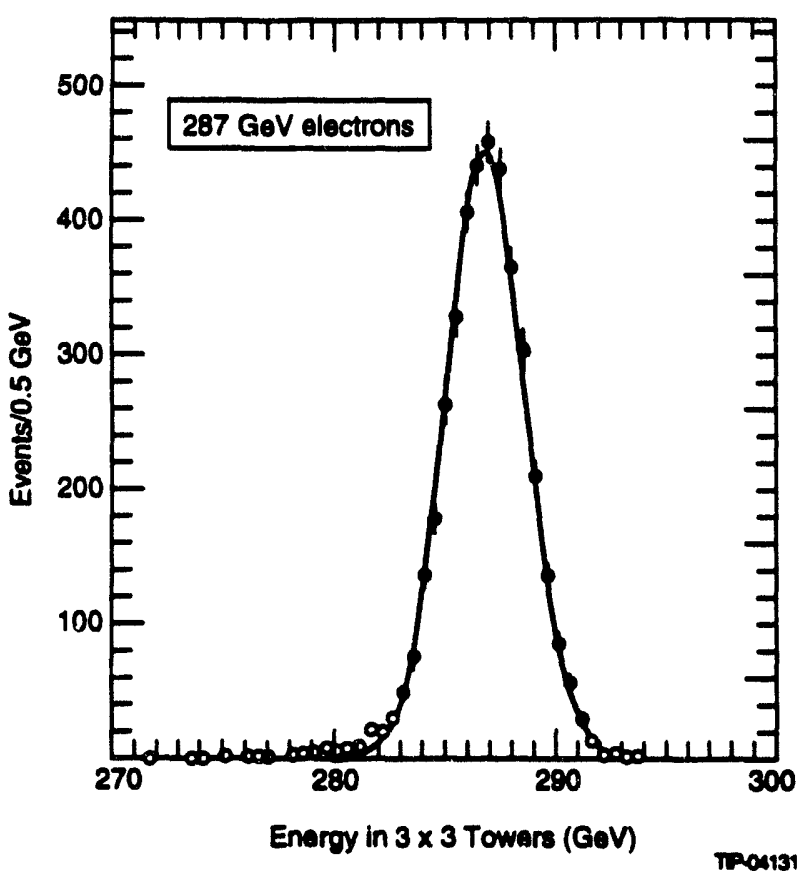

FIG. 5-23. Energy resolution obtained by the RD3 collaboration with a large projective LAr accordion prototype. $\sigma_{E} / E=0.62 \%$. transversely, and only $7 \lambda$ deep, so an energy resolution could not be determined from the data. However, all the characteristics of the data followed closely a GEANT simulation that predicted $\sigma / E=$ $50 \% / \sqrt{E}+2.4 \%$ for jets in a cone of $\mathrm{R}=0.7$, using longitudinal weighting. ${ }^{3}$

\subsubsection{Other Results}

The DO, H1 and SLD collider experiments are currently acquiring data with large liquid ionization calorimeters. The D0 collaboration has constructed a hermetic liquid argon calorimeter for measuring electrons, photons, jets as well as $E_{T}$ at the Fermilab Tevatron Collider. Test beam studies with electrons and pions demonstrated that the design energy resolution $(\sim 15 \% / \sqrt{E}$ for electrons and $45 \% / \sqrt{E}$ for pions) and position resolution $(1.6 \mathrm{~mm} / \sqrt{E}$ for electrons) has been achieved. ${ }^{12}$ The $\mathrm{D} 0$ calorimeter was commissioned in the spring of 1992, and has proved extremely reliable for triggering and detecting jets and electrons.

The SLD calorimeter became operational in 1990. ${ }^{13}$ This $4 \pi$ detector features a lead liquid argon calorimeter (LAC). The LAC is followed by an larocci tube calorimeter that acts much as the copper scintillator calorimeter will do for GEM. There are approximately 40,000 channels in the SLD LAC. Fewer than $1 \%$ of the channels have failed, most due to shorts between the electrodes. The SLD electrode structure involves exposed lead plates and tiles. Lead electrodes may contain conductive slivers on the surface that eventually work loose and cause shorts. The GEM structure in contrast will have no exposed lead, and these sorts of failures should be largely eliminated.

The SLD LAC has remained very stable with an $\mathrm{O}_{2}$ equivalent of $0.8 \mathrm{ppm}$ contamination over three years of operation. The calorimeter has demanded the least attention of all the SLD systems, being monitored by a cryogenics techni- 
cian and requiring very little physicist attention. The barrel electron resolution has been measured using wide angle Bhabhas to be $15 \% / \sqrt{E}$, compared with an approximate Monte Carlo estimate of $12 \% / \sqrt{E}$. In arriving at this estimate, the presence of spacers and electric field effects were ignored. No gain corrections to the towers were included, and only the charge calibration of the amplifiers was used. The $15 \%$ value incorporates the effect of any constant term that might be present.

\subsection{THE EM CALORIMETER}

\subsubsection{Barrel EM Calorimeter}

\section{Accordion Structure}

The barrel accordion electrode structure is shown in Figure 5-24. A basic cell consists of an absorber plate, a liquid krypton gap, a signal electrode and a second liquid krypton gap. The signal electrode is a three layer kapton printed circuit. Signal collection occurs on the outer layers, which are at a high voltage potential. These signals are capacitively coupled to the inner layer of the electrode. The dimensions of the structure are given in Table 5-2. Equal sampling in the accordion, as a function of radius, and full projectivity are achieved by varying the bend angle as well as the length of the arm between bends. There are many possible ways to meet the projective requirement (e.g., by varying the initial bend angle, the length between the bends, etc.). The solution we have chosen is characterized by the parameter $\beta / \delta$ close 104 , as defined in Figure 5-25. This shows that rnost rays from the origin pass through 4.0 cells (absorber, liquid, electrode, liquid) per accordion period. The particular value chosen, $\beta / \delta=3.89$, is closer to optimizing the energy resolution and transverse uniformity of shower response.

The total depth of the EM calorimeter was chosen to obtain excellent energy resolution in the photon energy range corresponding to the Higgs mass range of $80-140 \mathrm{GeV}$. For the $\gamma$ mode, the background decreases faster than the signal with increasing mass, and so energy resolution is most critical in the lowest mass range. At higher energies, there is some leakage of the energy, and a correction

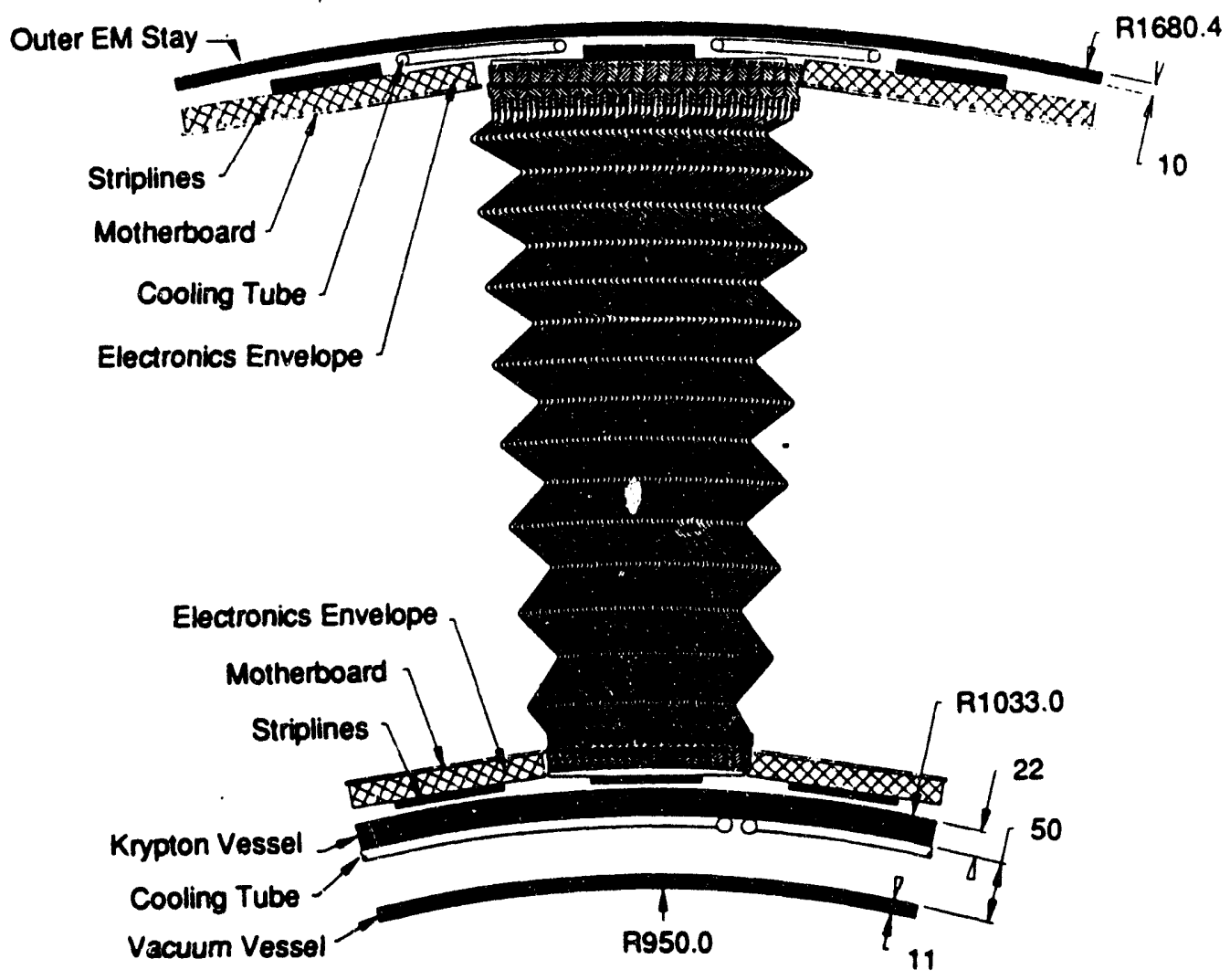

FIG. 5-24. Accordion electrcde structure. A $9^{\circ}$ section is shown with 24 electrode cells. 


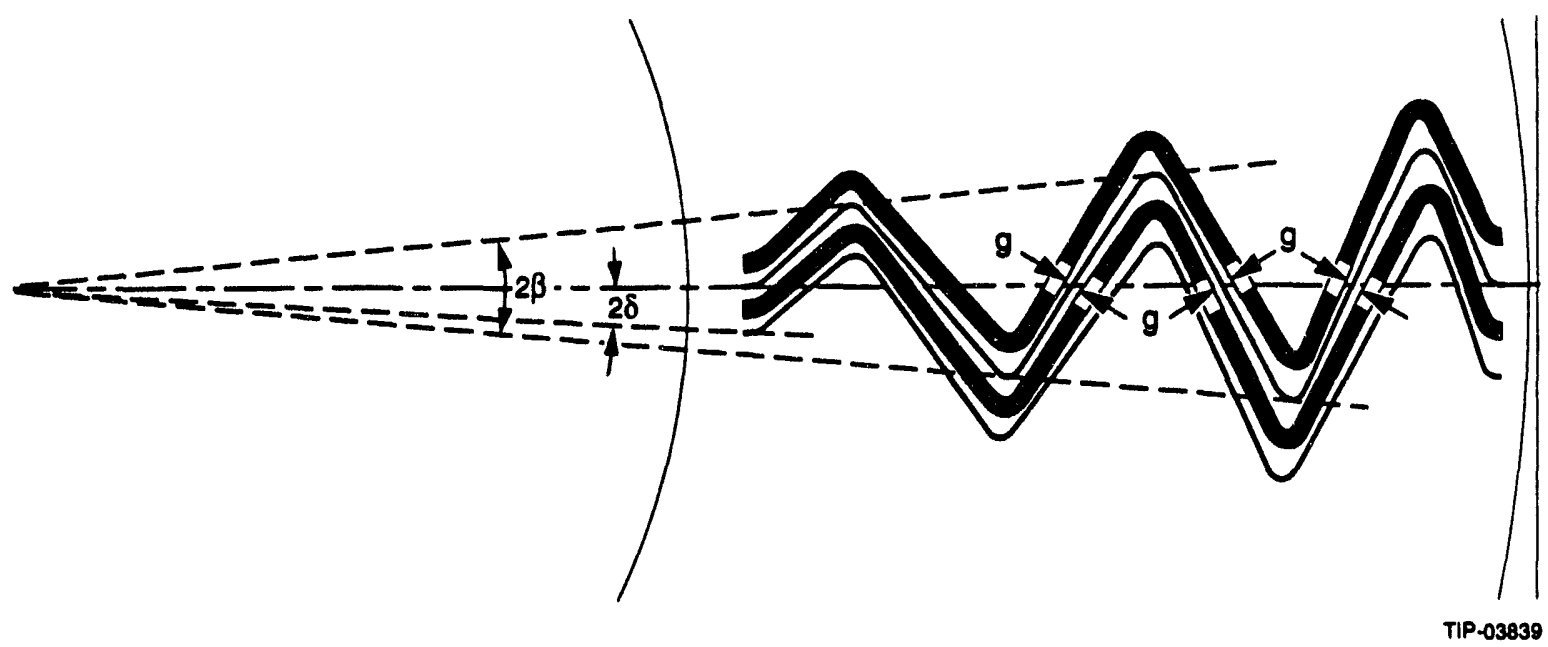

FIG. 5-25. A conceptual drawing defining the angles $\beta$ and $\delta . \beta / \delta$ is the number of cells traversed by a ray from the origin in one accordion period (not to scale).

using energy measured in the hadronic section restores the good energy resolution.

The need to optimize hadron rejection and to achieve the best possible angular resolution for electromagnetic showers determined the three longitudinal subdivisions of the calorimeter. The divisions selected are $3 \mathrm{X}_{0}$ in layer one, $10 \mathrm{X}_{0}$ in layer two, and $11 \mathrm{X}_{0}$ in layer three, for a total of $24 \mathrm{X}_{0}$ at $\eta=0$. This increases as $1 / \sin \theta$ with increasing angle. To improve hadron rejection and pointing, the front section has $4.7 \mathrm{~mm}$ wide strips in $\theta$ (see Figure 5-4). To correct for the energy loss in the material in front of the active calorimeter region, a "massless gap" is incorporated into the first longitudinal section, (the massless gap was discussed in Section 5.2.1 and Figure 5-11). This is accomplished by replacing the lead absorber with G-10 filler in the first $38 \mathrm{~mm}$ of the accordion absorber plate. The resulting structure has 960 absorber plates and signal electrodes in $\varphi$.

\section{Absorber}

The absorber plates are made of $1.0 \mathrm{~mm}$ precision rolled lead, sandwiched between two sheets of $0.2 \mathrm{~mm}$ stainless steel. The stainless steel is required for mechanical strength and provides a better surface than the unclad lead. A layer of $0.08 \mathrm{~mm}$ prepreg adhesive inserted between the lead and stainless steel completes the absorber electrode. The composite electrode is formed into an accordion shape and is then cured in a heated mold.

The stainless steel must be of such quality that it retains its non magnetic properties throughout the manufacturing process. The prepreg should be free of all additives that might poison the krypton. This includes bromides that are added as fire retardants. Radiation damage tests, at cryogenic temperatures, inust confirm the structural integrity of the absorber plates, the absence of bubbles or delamination and the absence of harmful chemical emissions at the expected dosage levels. More details of absorber fabrication are given in a separate document. ${ }^{14}$

\section{Sig 'Electrode}

the signal electrode is a three layer kapton printed circuit board. ${ }^{15}$ Electrical connections to the inner signal layer are made at the ends of the circuit board via plated or printed-through holes. The outer layers are high voltage electrodes made of copper strips connected by high value $(\sim 1 \mathrm{M} \Omega)$ silk screened resistors. The resistors isolate individual channels, as well as limit the current in case of an electrical discharge. The signal charge induced on the electrodes is capacitively coupled to the inner signal layer. The coupling capacitor is provided by the parallel capacitance between the signal layer and the high voltage electrode, where kapton is the dielectric material. Figure 5-26 shows a partial layout of the signal electrode.

\section{Tower Structure}

The transverse granularity of the accordion is determined in the $\varphi$ direction by the number of accordion cells connected to one readout channel, and in the $\eta$ direction by the readout pattern on the signal electrode. Four cells per channel in the $\varphi$ 


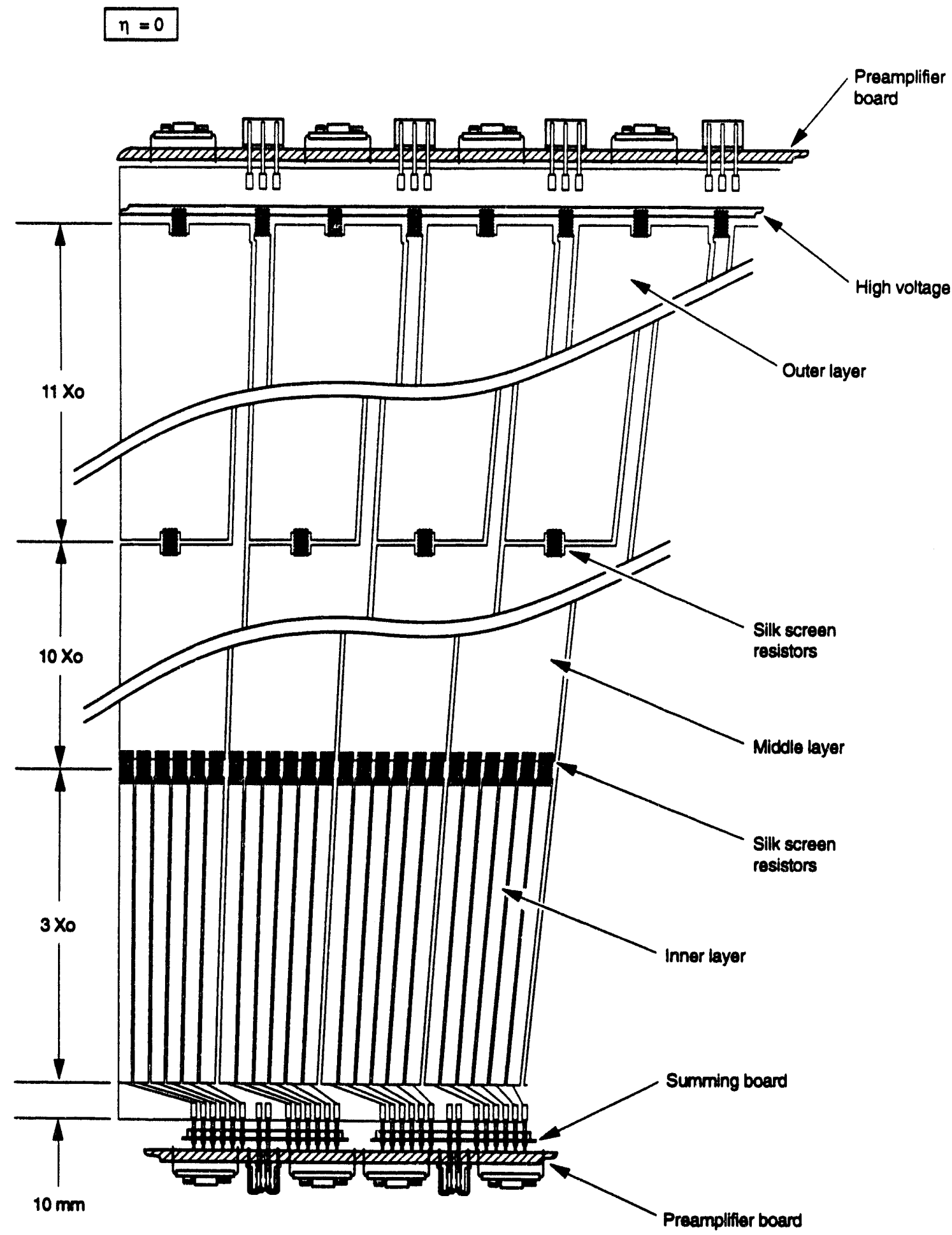

TP-0.4013

FIG. 5-26. Inner Barrel EM accordion signal electrode.

direction will be connected and a constant $\Delta \eta$ interval will be kept in the barrel region. To improve hadron rejection and pointing, the front section of the calorimeter requires finer granularity in $\eta$ than the normal tower segmentation provides. We do this by increasing the number of cells connected in the $\varphi$ direction from four to 24 cells, and by subdividing each electrode strip into six narrower strips 
(Figure 5-4 illustrates this concept.) The number of electronic channels remain the same. Figure 5-27 illustrates the electrode subdivision and connections to the preamplifiers. The narrow strips are first interconnected by a flexible kapton board ("jumper strips"). This simplifies the interconnection of the electrode to the preamplifier board. The connection posts (securely anchored on the signal electrodes) pass through the kapton boards, into the connectors on preamplifier boards, making a connection between the strips and the preamplifier.

\section{Preamplifier Boards}

The preamplifier boards ${ }^{16}$ contain the preamplifier and calibration hybrid circuits. They are attached to the inner and outer projections of the accordion calorimeter via signal and ground connector posts (Figure 5-27). The connector posts are approximately $25 \mathrm{~mm}$ long and penetrate the preamplifier board, mating with connectors located on the component side of the circuit board. The posts act as a lever arm, compensating for the differential

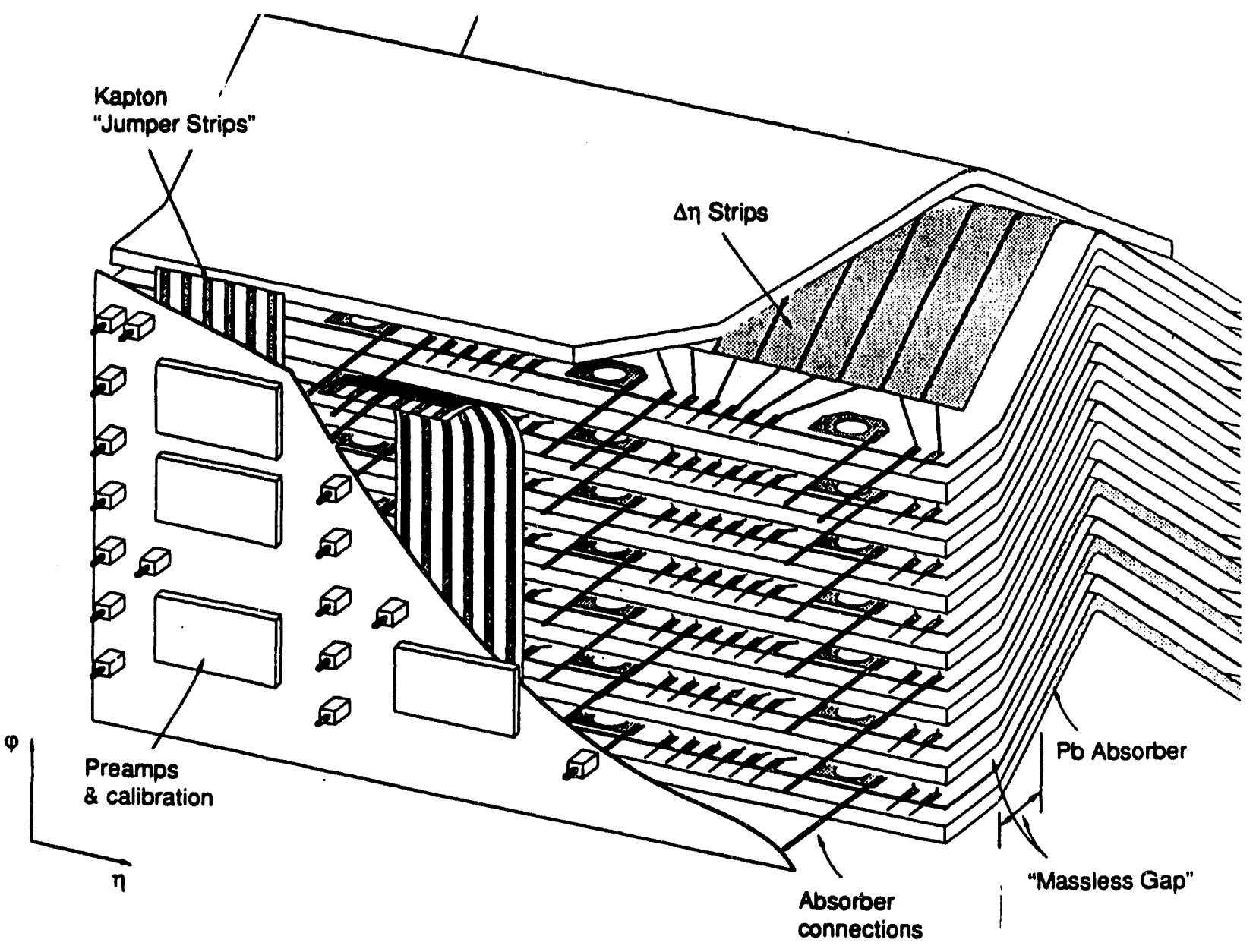

FIG. 5-27. Conceptual view of Inner Barrel EM accordion showing how the strips are connected with jumper strips. 
contraction between the electrode stack and the preamplifier board during the cool down. The inner layer circuit boards contain 36 preamplifier channels, and 36 calibration channels, arranged in a six by six tower matrix (the basic trigger tower). Because the towers are projective, each ring of boards along the $z$ axis is unique. A 36 channel high quality transmission line connects each preamplifier board to a signal feedthrough. ${ }^{17}$ The outer layer circuit boards are similar in design to the inner layer, but contain twice the number of channel because both middle and outer layers are read out on these boards.

\section{Module Assembly and Testing}

The $9^{\circ}$ modules will be assembled horizontally (one $9^{\circ}$ module has 24 electrode cells, $\Delta \varphi=0.16$, six towers) using a temporary structure to facilitate handling. ${ }^{18}$ Each module is self contained. Continuous electrical testing is required during the assembly of a module to guarantee that the module conforms to all electrical specifications. This will be accomplished by measuring DC resistance, electrode capacitance, leakage current, high voltage charge and discharge rates, and maximum sustainable high voltage across an ionization gap. Preamplifier boards and high voltage connections do not cross module boundaries. This permits us to completely assemble and test individual modules before transportation to the SSCL site.

After assembly, the module is immersed in a liquid nitrogen bath, and a complete electrical test is performed at cryogenic temperatures. This includes testing for high voltage breakdown, leakage current, electrical and signal connections, and preamplifier and calibration performance. As the modules are assembled into a barrel, much of the temporary structure that has held the modules together is rernoved, and the modules form a continuous barrel assembly.

The modules are designed in such a way that none of the signal or other electrical connections between the signal electrodes need to be removed during the barrel assembly.

\section{Mechanical Structure}

The EM barrel calorimeter is assembled from 40 separate $9^{\circ}$ modules. The modular concept is used to facilitate handling and to allow for the indepen- dent testing of subsections of the barrel calorimeter. A temporary suppoit structure is provided during assembly. As the modules are assembled into a barrel, much of the temporary support structure is removed and replaced with a permanent structure. ${ }^{19}$ The important components of the assembled structure are spacers and a series of inner and outer straps. These straps or bands hold the inner and outer radii of the barrel in compression. The straps pass through slots in fiberglass spacers, that have been glued along the lengths of each absorber. Figure 5-28 shows an inner spacer. Each spacer defines the ionization gap at the front and back of the calorimeter, and also locks in and accurately positions the signal electrodes. The fiber orientation of the fiberglass spacers has been chosen to compensate for differences in the expansion coefficient of the barrel calorimeter and the aluminum containment vessel. Figure 5-29 shows an assembled module along with the location of the spacers and tension bands. The preamplifier boards are nestled in between the spacers.

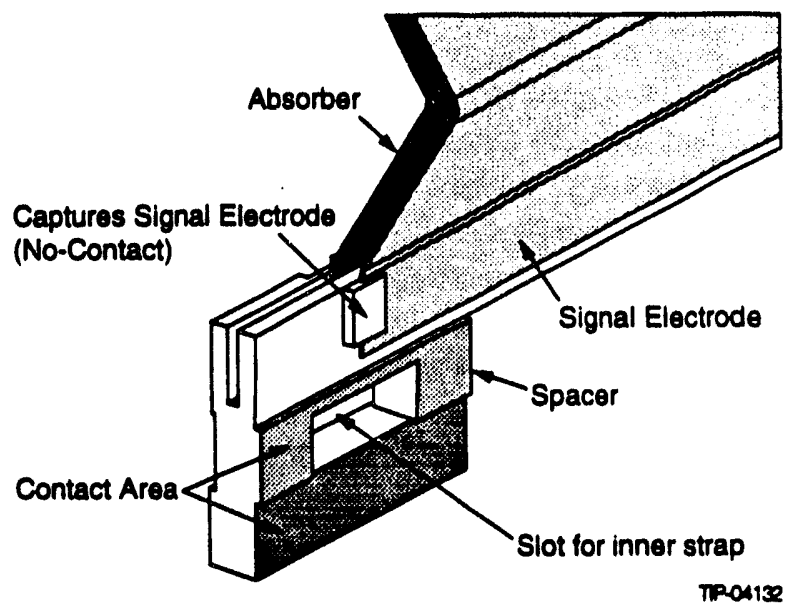

FIG. 5-28. Electrode spacers.

\section{Thermal Contraction Considerations}

The geometry of the signal electrodes and absorber plates is based on the physics performance requirements of the calorimeter at $\mathrm{LKr}$ temperature. This geometry will be different at room temperature. Since manufacture and assembly of the components into modules occurs at room temperature, a proper design must anticipate, and compensate for, both dimensional and structural differences in the constituent components. For example, because of the composite structure of the absorber plate, and the non-symmetric bend angles of the accordion struc- 


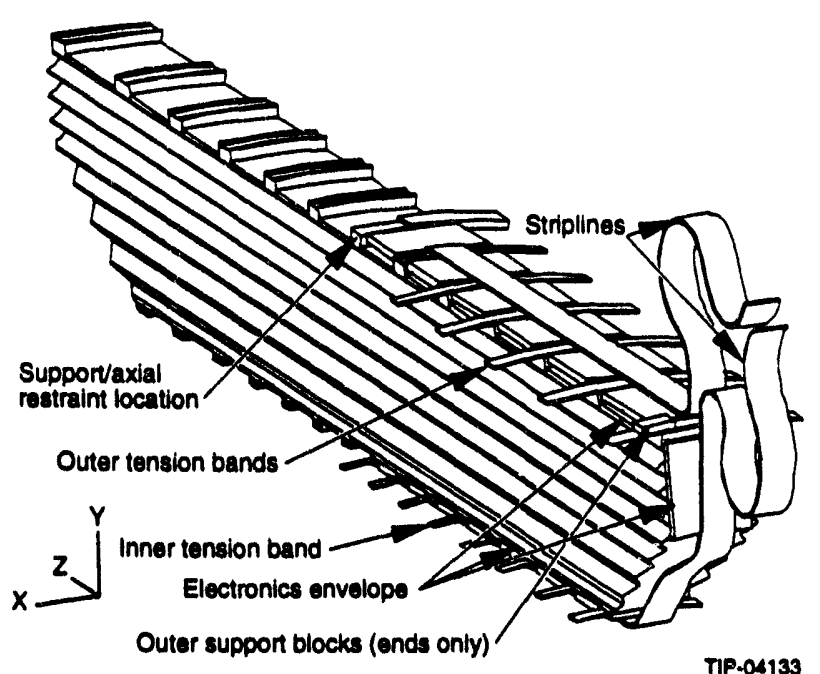

FIG. 5-29. Isometric view of EM module showing bands that hold the assembly in tension, the preamplifier motherboards and cables.

ture, a $3 \mathrm{~mm}$ radial deflection of the absorber plate occurs between room temperature and LKr temperature. This geometry transformation is best analyzed using 3D finite element analysis combined with sample testing.

The signal electrodes have a geometry which corresponds to lines of constant $\eta$ at cryogenic temperatures. Since the signal electrode circuit board is manufactured flat and at room temperatures, the artwork must be designed to produce signal strips with lines of constant $\eta$ in the cold and bent condition. A program of thermal analysis modeling has been undertaken at Martin Marietta Corporation and Brookhaven National Laboratory, to accurately determine the effects of these temperature differences.

\subsubsection{Endcap EM Calorimeter}

The EM endcap section utilizes a similar accordion concept as in the barrel. The endcap is effectively a part of a barrel with a vertical axis. To minimize the space requirements along $z$ while preserving full projectivity, the absorber plates and signal electrodes have incremental steps as shown in Figure 5-30. The absorber plates are vertical, so the mechanical support considerations are simplified compared to the EM barrel. We define $\beta / \delta=2.87$ in the endcap. This leads to smaller towers and minimizes the size of the steps. Since the mean particle energies are higher in the endcap, the contribution of the constant term to the energy resolution will be significant compared to the sampling term. Thus, we are able to use a siightly larger sampling term. This allows us to use argon, which costs less, as the ionization liquid.

Because the energies are larger in the endcap, we must increase the depth of the EM section to $27 \mathrm{X}_{0}$ and use thicker absorber plates. The ionization gap remains at $2 \mathrm{~mm}$. The thickness of the lead in the absorber plates is $1.4 \mathrm{~mm}$, and the stainless steel cladding is $0.15 \mathrm{~mm}$ (the vertical plates have no horizontal sag component, and require less structural support then the barrel.) Also shown in Figure 5-30 is the outline of the mini-modules. The

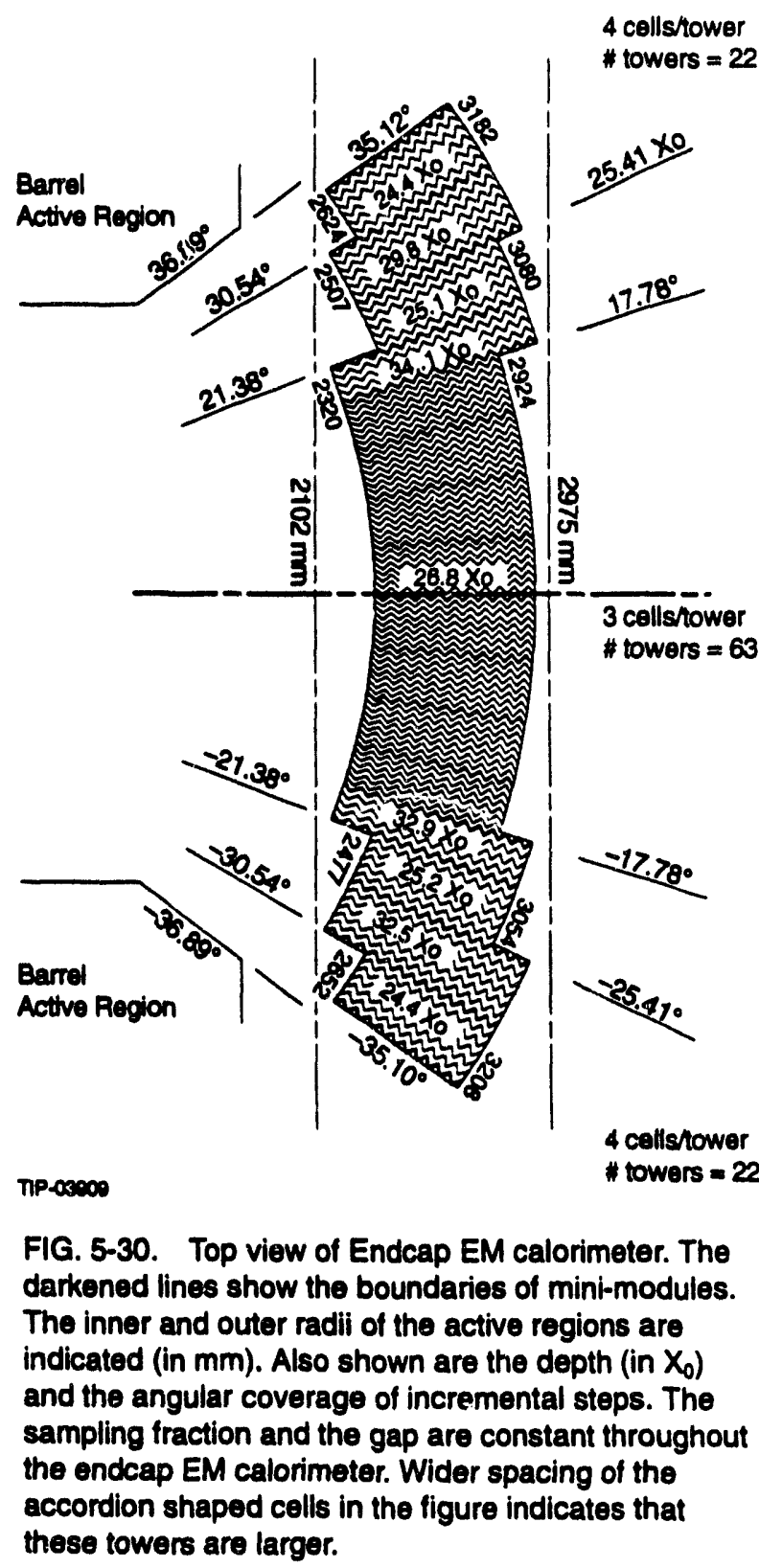


module boundaries are indicated by a darkened line. The number of cells in a module ranges beiween 20-24 cells. The mini-module concept allows us to completely assemble and test a module before it becomes a part of the monolith. As the modules are assembled into the endcap section, the temporary support structure, that has defined the mini-module, is removed and replaced with the conical endcap support shell.

The longitudinal segmentation comprises three sections, $4 \mathrm{X}_{0}$ in the first layer, $10 \mathrm{X}_{0}$ in the second layer, and $13 \mathrm{X}_{0}$ in the third layer. To compensate for energy losses in the vacuum and containment vessels, there will be a $\sim 42 \mathrm{~mm}$ massless gap in the first longitudinal section. The active depth of the EM calorimeter is $603 \mathrm{~mm}$ on the average.

To improve pointing and multi-photon rejection, the first layer of the endcap calorimeter $(\eta \leq 1.86)$ will be instrumented with narrow strips, similar to the EM barrel design.

At larger $\eta$, where strips do not give an additional improvement in $\pi^{\circ}$ rejection or pointing, rectangular towers are used. To approximate a constant $\eta$ segmentation, the tower structures in the second and third layers are also divided into two regions. An outer region where four cells are summed to form a tower, and an inner region, where three cells define a tower. The lateral segmentation in the second and third layers is shown in Figure 5-31. The second and third layers are read out from the rear of the calorimeter.

The higher rates and faster shaping time in the inner region, make it advantageous to use the so called "zero transistor" solution 20 i.e., amplifiers outside the cryostat. This means that there are no active elements, (e.g., preamplifiers) in the region of high radiation.

\footnotetext{
Absorber

The method used to fabricate the endcap absorber plates is analognus to the bartel fabrication technique, although a different set of bending dies and molds must be used for the endcaps. This is because the distance between the interaction point and the beginning of the calorimeter differs between endcap and barrel calorimeter. The bending machine
}

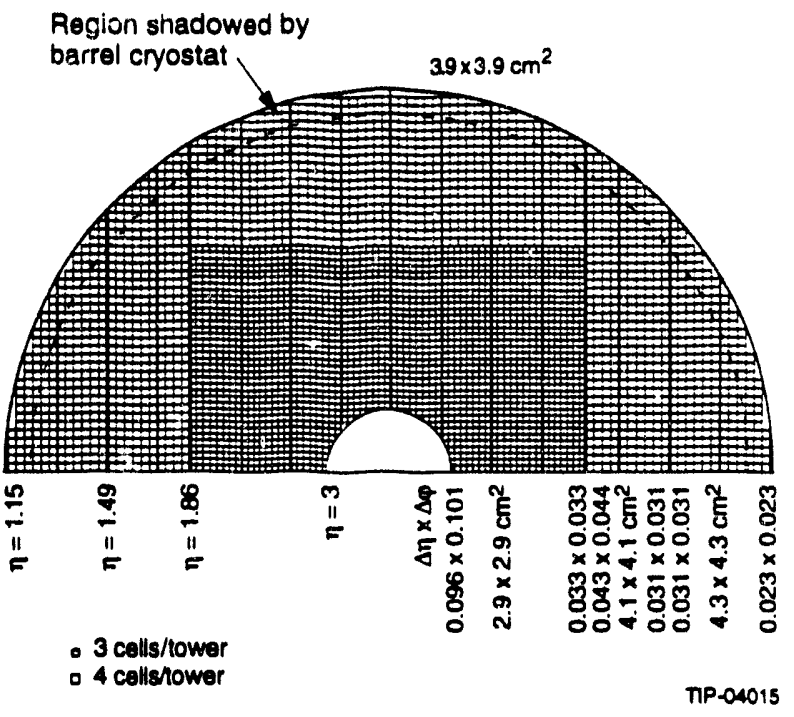

FIG. 5-31, $\quad \eta$ and $\varphi$ segmentation in EM endcap. Again the darkened lines show mini-modules, while heavy dark lines show positions where the inner and outer radius of the active region changes. Near to the beam axis the dimensions of the towers correspond to 3 cells, and far from the axis to 4 cells. The approximate cell dimensions are shown (in $\Delta \eta \times \Delta \varphi$ and in $\mathrm{cm}$ ). The coverage at outer radius ensures that showers which enter endcap from the comer of the barrel cryostat will be fully contained.

will be designed to accommodate either sets of dies. Each endcap requires 366 absorbirr plates.

\section{Signal Electrode}

The endcap design requires approximately 40 different types of signal electrodes. The electrodes differ in their strip geometry and panel size. The thickness, fabrication method and connection scheme are exactly the same as in the barrel calorimeter. Each endcap requires 365 signal electrodes.

\section{Preamplifier and Connection Boards}

Preamplifier boards are used only in the outer region of the endcap. Their design and component density is similar to the EM barrel preamplifier boards. Remote preamplifiers are used in the inner region on all layers. The cells comprising a tower are first interconnected by a flexible kapton printed circuit board. A high quality transmission line transfers the analog information from the board to the feedthroughs and junction boxes, which are located along the outside periphery of the endcap vacuum vessel. The preamplifiers and cable drivers are located in the junction boxes. (See Figure 5-1) 


\section{Module Assembly and Testing}

The endcap modules will be assembled horizontally, using a temporary structure to facilitate handling. Similar in design to the EM barrel, each module is self contained. The testing and assembly of the modules will be similar to that of the barrel. The assembly is encased in a conical shell ${ }^{21}$ which provides the necessary structural support.

The modules are designed such that none of the electrical connections between the stack and the signal electrodes need to be removed during the assembly procedure.

\subsubsection{Ionization Calorimeter Readout Chain}

Figure 5-32 outlines the building blocks of the calorimeter readout chain and their location. Each component will be discussed in the following paragraphs.

\section{Capacitance of the Electromagnetic Tower}

The highly segmented EM calorimeter has "accordion" shaped electrodes to minimize the stray capacitance of the connections. Each capacitance has two components: the gap capacitance between the signal electrode and the absorber, which is proportional to the area of the cell, and the edge capacitance (side between readout electrode and its neighbors) which is proportional to the boundary length. Four cells (eight gaps) are connected in parallel in the second and third sections of the barrel.

Tables 5-3 and 5-4 summarize the dimensions and tower capacitances for two values of $\eta$ in the barrel calorimeter.

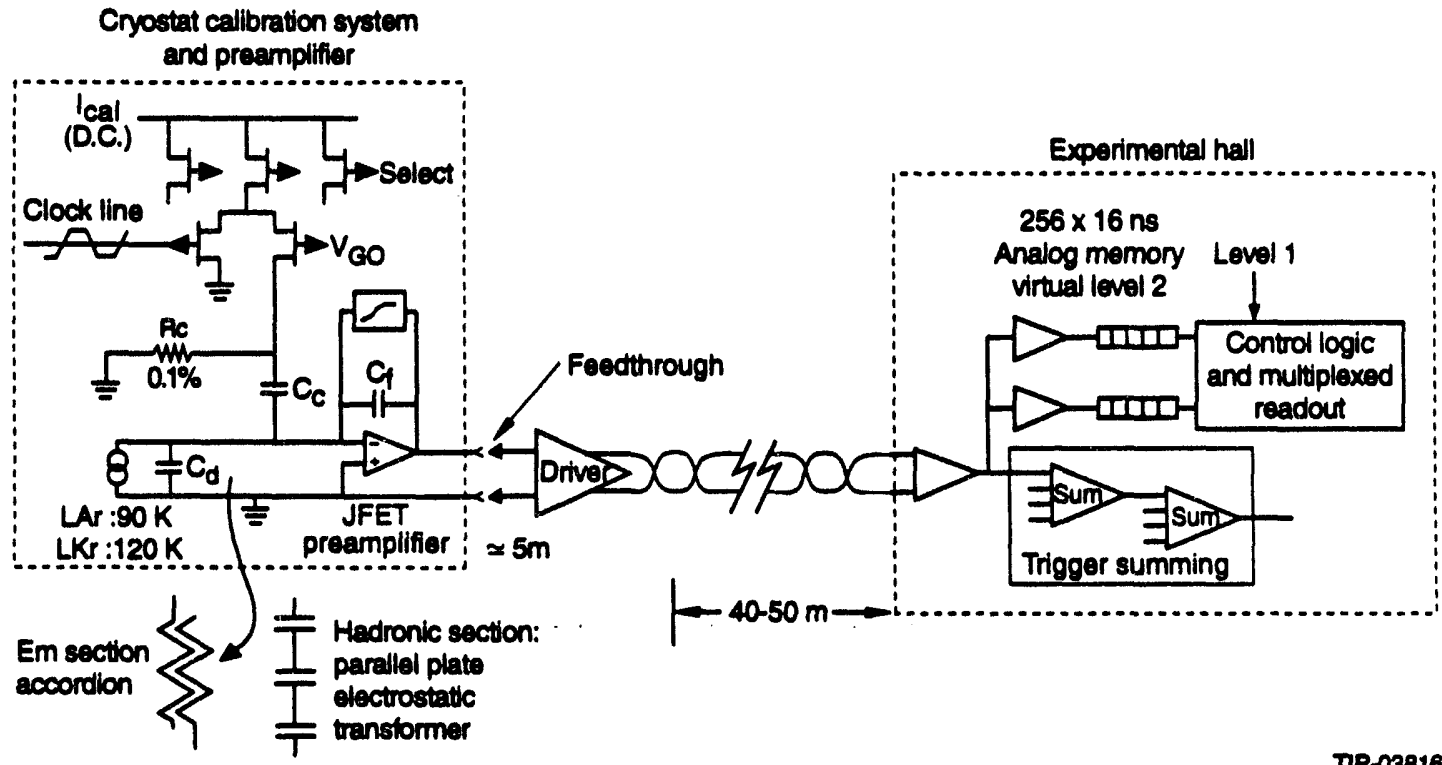

FIG. 5-32. Calorimeter readout chain.

TABLE 5-3. EM Tower Parameters at $\theta=90^{\circ}$.

\begin{tabular}{|c|c|c|c|c|c|c|c|}
\hline Section & $\begin{array}{l}\text { Depth } \\
\left(x_{0}\right)\end{array}$ & $\Delta \varphi$ & $\Delta \eta$ & $\begin{array}{l}\text { Electrode } \\
\text { Length(mm) }\end{array}$ & $\begin{array}{l}\text { Gap Area } \\
\left(\mathrm{cm}^{2}\right)\end{array}$ & $\begin{array}{l}\text { Cap. } \\
\text { (pf) }\end{array}$ & $\begin{array}{l}\sigma_{\text {el }}(\mathrm{MeV}) \\
{[\odot 40 \mathrm{~ns}]}\end{array}$ \\
\hline FRONT & 3 & 0.16 & 0.00436 & 117 & 4.7 & 400 & 7.7 \\
\hline MIDDLE & 10 & 0.026 & 0.026 & 300 & 100 & 650 & 8.4 \\
\hline BACK & 11 & 0.026 & 0.026 & 322 & 126 & 800 & 10. \\
\hline
\end{tabular}


TABLE 5-4. EM Tower parameters at $\theta=40^{\circ}$.

\begin{tabular}{|c|c|c|c|c|c|c|c|}
\hline Section & $\begin{array}{l}\text { Depth } \\
\left(X_{0}\right)\end{array}$ & $\Delta \varphi$ & $\Delta \eta$ & $\begin{array}{l}\text { Electrode } \\
\text { Length (mm) }\end{array}$ & $\begin{array}{l}\text { Gap Area } \\
\left(\mathrm{cm}^{2}\right)\end{array}$ & $\begin{array}{l}\text { Cap. } \\
\text { (pF) }\end{array}$ & $\begin{array}{l}\sigma_{\theta 1}(\mathrm{MeV}) \\
{[\odot 40 \mathrm{~ns}]}\end{array}$ \\
\hline FRONT & 3 & 0.16 & 0.00436 & 117 & 4.7 & 400 & 7.7 \\
\hline MIDDLE & 10 & 0.026 & 0.026 & 300 & 100 & 650 & 8.4 \\
\hline BACK & 25 & 0.026 & 0.026 & 730 & 194 & 1250 & 13. \\
\hline
\end{tabular}

\section{High. Voltage Electrode}

We determined the value of the re istances on the high voltage electrodes by considering the following five factors:

1. Parallel noise from the resistive layer

2. DC voltage drop due to the average signal current

3. Need for discharge protection

4. "Transparency" to the signal which may be smeared out due to diffusive RC effects

5. Minimization of the dead region in case of an accidental short.

\section{Electromagnetic Calorimeter}

The signal electrode, and hence the preamplifier is capacitively coupled to the high voltage electrode via a thin layer of dielectric material, which ensures a large coupling capacitance.

The high voltage electrode is segmented along the length of the accordion strip in areas of sufficiently low capacitance which are connected in series by means of silk screened resistors. It is sound practice to place such a resistor in the middle of an accordion straight region, to prevent cracking which might occur if it were placed at the bend.

\section{Preamplifier}

\section{Barrel and Endcap Calorimeter}

Given the processing times in most of the EM (40 ns peaking time) and hadronic calorimeter (100-200 ns, see Section 5.4.5) at the SSC luminosity, the preamplifier must be situated as close to the signal electrode as possible. This allows for rapid charge transfer between the signal electrode and the preamplifier input, and for optimization of the signal to noise ratio for different experimental conditions. Since the detector capacitance varies from $400 \mathrm{pF}$ in the front $\mathrm{EM}$ sections to $2000 \mathrm{pF}$ in the hadronic section, several versions of the preamplifier with different sizes of the input junction field effect transistor (JFET) are required. In the inner endcap region, shorter shaping (20 ns) and remote preamplifiers will be used.

Table 5-5 summarizes the most important characteristics of the preamplifier. The dimensions of the typical input transistor are $\mathrm{W} / \mathrm{L}=$ $(11400 \mu \mathrm{m}) /(5 \mu \mathrm{m})$, which makes it suitable for detector capacitance $\leq 500 \mathrm{pF}$.

To avoid bubble formation and density variations caused by temperature gradients in LAr or $\mathrm{LKr}$ due to preamplifier heat dissipation, cooling in the vicinity of the preamplifiers boards will be needed.

TABLE 5-5. Monolithic Preamplifier Specifications.

\begin{tabular}{ll}
\hline Monolithic PA Parameter & Value \\
\hline Power Dissipation & $<100 \mathrm{nW}$ \\
Gain Bandwidth Product & $200 \mathrm{MHz}$ \\
Aise Time $\left(\mathrm{C}_{d}=500 \mathrm{pF}\right)$ & $-25 \mathrm{~ns}$ \\
Input Relerred Noise & $0.7 \mathrm{nV} / \sqrt{\mathrm{Hz}}$ \\
Linear Range & $0-200 \mathrm{GeV}$ \\
Acceptable Range & $>2 \mathrm{TeV}$ \\
ENC (40 ns shapino $\bullet \mathrm{C}_{D}=500 \mathrm{pF}$ & $14000 e^{-} \mathrm{rms}$ \\
ENC (100 ns shat $\odot \mathrm{C}_{D}=500 \mathrm{pF}$ & $8700 \mathrm{e}^{-} \mathrm{rms}$ \\
\hline
\end{tabular}

Considerable experience has been gained concerning the reliability of operation of preamplifiers connected to the calorimeter electrodes in LAr and LKr. Quantities of preamplifiers ranging from 400 to 1300 have been operated in four different experiments and tests, with several cooling cycles used over time periods from 1-3 months to 4 years. In these cases there were no more than one dead channel per experiment containing 400 to 576 preamplifiers. Failures occurred entirely during the installation and not during cooling and operation, and they were predominantly due to contacts rather than the preamplifiers. The preamplifiers were fabricated in hybrid circuit technology with JFETs in 
SOT-23 packages. This technology involves several high temperature processing steps and it requires attention throughout the production process because of the need to match the thermal expansion of various components. (During this development work over the last 8-9 years we have also learned about some technologies that do not give satisfactory reliability). In these tests and experiments we have also gained the experience that the electrode designs outlined in this TDR, and described in technical support documents, provide full protection of the preamplifiers against any discharge (high voltage electrodes subdivided into small area by film resistors limit the amount of stored charge). All preamplifiers were tested after a standard burn-in procedure prior to installation. The HELIOS experiment operated over four years, with several (4) cooling cycles and showed no preamplifier failures during the entire experiment among 576 cold preamplifiers. Thus, we expect a failure rate of less than the one in 500 associated with installation rather than the length of operation. The reliability and testing are described in a separate note. ${ }^{22}$

Silicon JFETs used in these preamplifiers are radiation hard as discussed in Section 5.8.1.

\section{Forward Calorimeter Preamplifier}

The expected higher rate in this region imposes a shorter shaping time (peaking time $-20 \mathrm{~ns}$ ) for signal to noise optimization. Higher levels of ionizing radiation and of neutron flux are also expected. For this range of shaping times, a preamplifier located outside the cryostat and connected with a 2-3 $\mathrm{m}$ transmission line will achieve a sufficient signal to noise ratio. Locating the preamplifier away from the electrode has the additional advantage of removing the preamplifier from the high radiation area.

\section{Single-ended To Differential Cable Driver}

The signal processing electronics will be located on several platforms anchored on the wall of the experimental hall, about 40-50 m away. To reject electromagtietic interference and signal crosstalk we plan to use a shielded, double-terminated twisted pair cable.
A junction box located as near to the cryostat as possible will convert the single-ended signal out of the preamplifier to a differential signal, and will provide enough gain to compensate the attenuation due to the termination and the attenuation in the cable itself. A possible location for the junction boxes is at the end of the scintillating fiber calorimeter for the barrel calorimeter, and just next to the feedthroughs for the endcap calorimeter, as seen in Figure 5-1.

Given the low noise gain in the preamplifier (a requirement dictated by the dynamic range) an input noise density of less than $2.5-3 n V / \sqrt{\mathrm{Hz}}$ is required for the cable driver in order to avoid degrading excessively the signal to noise ratio.

\subsubsection{Ionization Calorimeter Calibration System \\ The Calibration Concept}

The purpose of the calibration system is to maintain the lowest possible constant term in the energy resolution and to have an accurate knowledge of the calorimeter response over the whole volume. The performance will be based on the inherent properties of ionization calorimetry, on design efforts to achieve uniformity and stability of response and on carefully defined calibration procedures.

The calibration will be based on 1) a very accurate determination of the readout chain response which can be monitored at any time during the experiment, 2) the mapping of the profile of the response of the calorimeter, and 3 ) the absolute calibration of the charge vs. energy scale. The mapping, once established for the calorimeter configuration and in combination with the monitoring described in (1), would remain constant. The readout chain is designed to have very small gain variations and to require only infrequent calibration runs. Due to the large volume of liquid, the time scale of changes in purity (and hence ionization yield) will be quite long. Monitoring of the ionization yield for $a$ particles provides a very sensitive measure of any change in liquid purity. A liquid recirculation system with an in-the-liquid purifier will be used to ensure stability and uniformity of the ionization yield. 
The methods of calibration, along with the goals and procedures, are listed in Table 5-6. The readout chain calibration is described in some detail in this section. The absolute calibration is described in Section 5.2.2, and the beam tests in Sections 5.7.4 and 5.8.5.

\section{Implementation}

At the fast shaping times required for SSC calorimetry, a precision calibration $(0.2 \%$ relative accuracy) faces some obstacles. First it is extremely difficult to distribute fast pulses (rise time less than a few ns, exponential decay with controlled time constant, precise peak value) in a large system. For this reason it was decided to distribute a precise DC current and to generate a precision calibration pulse in situ at each channel with a dedicated circuit. The only time-varying signal which has to be routed is a fast clock with a rise time <3-4 ns. Many calibration circuits, of which only one will be enabled, would be pulsed at the same time. A "star" configuration of the clock line would slow down the clock edge. This effect may be avoided by daisy chaining the calibration circuits. A delay line embedded in the preamplifier board introduces a fixed and constant delay of about 1 ns between successive channels, which is corrected by advancing the clock signal.
Only one of the calibration circuits driven by a single clock line will be enabled at a time by means of a "select" line with a constant logic level. Since precision calibration requires averaging over several hundred pulses, the select line will be switched at very low rates (order of $\mathrm{kHz}$ or less) and no special requirements are necessary. One select line may drive an unlimited number of circuits, connected to different clock lines. In GEM a practical subdivision may be to drive 36 channels (a trigger tower), with two clock lines: a grand total of about 7000 clock lines and calibration currents will be needed.

\section{Calibration Circuit}

A precisely known and variable calibration current is switched by means of a JFET long tail pair to an RC shaping network as shown schematically in Figure 5-32. An exponentially decaying current pulse, approximating the triangular liquid argon signal is injected into the input of the charge sensitive preamplifier. Since the preamplifier uses $\mathrm{N}$-channel JFETs, having a higher $\mathrm{g}_{\mathrm{m}} / \mathrm{C}$ ratio and lower noise, and is optimized for a positive output swing, the calibration circuit has to employ P-channel devices to inject a negative input current at its fast transition edge. A calibration uniformity better than $0.1 \%$ at currents larger than $100 \mu \mathrm{A}$ has been

Table 5-6. Calibration Goals and Means.

\begin{tabular}{|c|c|c|c|}
\hline Calibration Means & Purpose & $\begin{array}{l}\text { Type of Informe.ion } \\
\text { (Quantity Defined) }\end{array}$ & Time of Calibration \\
\hline $\begin{array}{l}\text { Current injection into front } \\
\text { end at the electrodes }\end{array}$ & $\begin{array}{l}\text { Readout chain - gain } \\
\text { calibration and } \\
\text { monitoring }\end{array}$ & $\begin{array}{c}\text { Absolute charge } \\
\text { calibration; relative } \\
\text { channel-to-channel } \\
\text { gain, time and amplitude } \\
\text { response }\end{array}$ & $\begin{array}{l}\text { All tests and } \\
\text { periodically during } \\
\text { experiment runs }\end{array}$ \\
\hline $\begin{array}{c}\text { e's and } \pi \text { 's } \\
\text { Beam test at FNAL }\end{array}$ & $\begin{array}{c}\text { Mapping of calorimeter } \\
\text { response of EM and hadronic } \\
\text { modules }\end{array}$ & $\begin{array}{l}\text { Response profile } \\
\text { (relative) for a set of } \\
\text { modules including } \\
\text { boundaries in } \varphi \text { for } \\
\text { hadronic modules }\end{array}$ & $\begin{array}{l}\text { Prototype testing in } \\
1995\end{array}$ \\
\hline $\begin{array}{c}\text { e's and } \pi \text { 's } \\
\text { Beam test at SSCL }\end{array}$ & $\begin{array}{l}\text { Mapping of calorimeter } \\
\text { response, mainly vs. } \theta \text { over } \\
\text { the barrel-endcap boundary }\end{array}$ & $\begin{array}{l}\text { Response profile } \\
\text { (relative) for the } \\
\text { boundaries in } \theta\end{array}$ & $\begin{array}{l}\text { SSCL test beam } \\
\text { operation in } 1997\end{array}$ \\
\hline $\begin{array}{l}Z^{0} \text { mass and particles with } \\
\text { measured momentum in } \\
\text { tracker. }\end{array}$ & $\begin{array}{l}\text { Establishing absolute energy } \\
\text { reference points for the barrel } \\
\text { and endcaps }\end{array}$ & Charge vs. energy scale & $\begin{array}{c}\text { Experiment start and } \\
\text { during experimental } \\
\text { runs. }\end{array}$ \\
\hline a-cells in the cryostats & Liquid purity monitoring & $\begin{array}{c}\text { Ionization yield, absolute } \\
\text { charge vs. electric field } \\
\text { for } a \text { 's }\end{array}$ & $\begin{array}{l}\text { Continuously during } \\
\text { the experiment }\end{array}$ \\
\hline
\end{tabular}


obtained in bench tests, and in the BNL accordion test in 1992 as discussed in Section 5.3.7.

The RC network in Figure 5.32 approximates the shape of the early part of the induced signal current (the slope of the triangular current pulse) and it does not affect the magnitude of the injected current. The tolerances of the resistor and the capacitor appear as variations in the injected charge attenuated by $2 t_{d} / t_{m}$, where $t_{d}$ is the electron drift time across the gap and $t_{m}$ is the effective integration time (peaking time) of the electronic chain. In our case $t_{d}=20-40 \mathrm{~ns}$, so that the sensitivity to the tolerances of these two components is attenuated by a factor of $\mathbf{2 0}$ or more. The ionization current from the electrodes and the current injected by the calibration are treated in the same way, and the calibration is independent of the electrode capacitance. The overall system gain does change with the electrode capacitance, but equally for both the signal ionization charge and the calibration charge. This method of current (charge) injection also calibrates the system response (pulse shaping), and, in combination with signal processing using several samples, ${ }^{21}$ it makes the system independent of differences in the pulse shaping from channel to channel due to component tolerances.

The calibration circuit will be built as a monolithic P-channel JFET using the same junction isolated technology developed for the monolithic version of the preamplifier, thus achieving a very good matching of the input devices at no extra cost. With the present technology the preamplifier and the calibration circuit cannot be integrated together; junction isolation allows the use of either $P$ or $\mathrm{N}$-channel devices. It will be mounted on the same hybrid substrate as the preamplifier.

\section{Clock Fan-out and Driver}

Since tolerances in JFET pinch-off voltage cause different circuits to switch at different parts of the clock edge sigral, a slow clock edge degrades the calibration precision. The effect is more imr stant at short shaping times. A clock edge less than about 4 ns must be maintained in the clock distribution. One driver circuit per clock line will be needed. The drivers will be housed in a module in the junction boxes.

\section{Calibration Current Fan-out}

One calibration current per clock line will be necessary. All the calibration currents must be highly stable, with a relative matching better than the precision goal of the calibration system. This is readily achieved with simple current generating circuits. The current generator modules will be housed in the junction boxes.

\subsubsection{Ionization Calorimeter Noise Considerations}

We estimate here various sources of noise and their implication on the shaping time. Pileup or physics noise is caused by the background of high cross section events which continuously deposit energy in the calorimeter cells. We denote by $\rho_{p}^{2}$ the variance of the transverse energy deposited per unit time in a calorimeter cell. Because of the time structure of the machine, the variance in the detected signal due to this type of noise is an incoherent sum of contributions from the previous crossings. Thus

$$
\sigma_{p}^{2}=\rho_{p}^{2} \sum_{i} w_{i}^{2}=\rho_{p}^{2} S_{2} .
$$

The summation is oyer the normalized signal waveform $w_{i}$, evaluated at points spaced by the beam crossing interval of $T_{c}=16 \mathrm{~ns}$. For $(\mathrm{CR})^{?}(\mathrm{RC})^{6}$ filtering with peaking times in the range of $50-100 \mathrm{~ns}$, the approximate value of $S_{2}$ is

$$
S_{2} \sim 2.0 t_{p} \text {. }
$$

where $t_{p}$ is the peaking time. Thus the pileup noise is made up of two factors, the pileup noise density $p_{p}$ coming from the fluctuations in the energy deposition arising from high cross section interactions, and the "pileup sum" $S_{2}$, coming from the choice of shaping function. 
Table 5-7 gives estimated values for the pileup noise for different tower types. The measurement time $t_{m}$ for the EM cells is assumed to be $45 \mathrm{~ns}$. For the hadronic cells, we have used $t_{m}=100$ ns for the front section. The pileup noise for the first hadronic section is estimated on the basis of that occurring for the full calorimeter, since there is a reasonably rapid falloff with depth. Values are for $\mathcal{L}=10^{33} \mathrm{~cm}^{-2} \mathrm{~s}^{-1}$.

Using 2-jet (with $E_{T}>5 \mathrm{GeV}$ ) events produced by the ISAJET event generator, along with the Bock parameterization of the calorimeter response to obtain values for the different calorimeter sections, we have estimated ${ }^{23} \rho_{p}$ and found that it is essentially independent of $\eta$. We therefore work with values averaged over $\eta$. We have found ${ }^{24}$ that $p_{p}$ can be reasonably well parametrized in terms of the area $\Delta \eta \Delta \varphi$, luminosity $\mathcal{L}$, and depth in the calorimeter $l$ (in units of interaction length) by the formula

$$
\rho_{p}=380(\Delta \eta \Delta \phi)^{0.76}\left(\frac{\mathscr{L}}{L_{0}}\right)^{\frac{1}{2}} e^{-0.81} \mathrm{MeV} / \sqrt{n s},
$$

in which $L_{0}=10^{33} \mathrm{~cm}^{-2} \mathrm{~s}^{-1}$. We use this formula to estimate the values of $\rho_{p}$ for tower sizes of interest. We show, in Table 5-7 values for three types of tower sizes: (a) physical EM and HAD towers, which will permit the calculation of $\sigma_{p}$ for individual towers, and (b) $3 \times 3$ blocks of hadronic towers and $3 \times 3$ or $5 \times 5$ blocks of EM towers, which are the approximate sizes that will be needed for physics analysis, and (c) first level trigger towers $(6 \times 6 \mathrm{EM}$ cells, $4 \times 4 \mathrm{HAD}$ cells for the SP sums, and $8 \times 8 \mathrm{HAD}$ cells for the JET sums). The "analysis" tower sizes are used below in the analysis of the hardware shaping time for the EM section, since we wish to optimize this shaping for the final physics analysis. The trigger tower sizes are used to determine the optimal shaping time for the trigger sums.

The optimal hardware shaping time for the individual towers depends primarily on the level of thermal noise $\sigma_{t}$ and pileup noise $\sigma_{p}$. The optimal value for $t_{m}$ is found 24 by minimizing the value of $\sqrt{\sigma_{p}{ }^{2}+\sigma_{t}{ }^{2}}$. By expressing the values for $I_{1}$ and $S_{2}$ in terms of their "form factors" and $t_{m}$, the optimal shaping time $t_{m}^{0}$ is given approximately by:

$$
t_{m}^{0}=1.5 \sqrt{\frac{\rho_{t} t_{d}}{\rho_{\mathrm{p}}}},
$$

in which $\rho_{t}=r_{q} \sigma_{t} / \sqrt{I_{1}}$ is the thermal noise density, $r_{q}$ is the ratio of signal charge to generated charge, and $t_{d}$ is the drift time. To find the optimal EM hardware shaping time, we use the pileup and thermal noise corresponding to the "analysis" towers, defined as a $3 \times 3$ or $5 \times 5$ matrix of cells. The values of $\rho_{p}$ for these towers are shown in Table 5-7. We now estimate the value of optimal shaping time for the EM section.

For the thermal noise we take the values found in the liquid krypton test run 5 of $76 \mathrm{MeV}$ for a $5 \times 5$ sum of both front and back sections. For a $3 \times 3$ sum we expect $\sigma_{t}=(3 / 5) \times 76 \mathrm{MeV}=46 \mathrm{MeV}$. The values of $I_{1}$ and $r_{q}$ for this case are $0.076 \mathrm{~ns}^{-1}$ and 0.072 ,

\begin{tabular}{|c|c|c|c|c|c|}
\hline Section & Tower type & Size & Area & $\rho_{p}(M e V / \sqrt{n s})$ & $\sigma_{p}(\mathrm{MeV})$ \\
\hline EM & unit cell & $1 \times 1$ & $0.027 \times 0.027$ & 1.5 & 13 \\
\hline EM & analyors & $3 \times 3$ & $0.08 \times 0.08$ & 8.2 & 71 \\
\hline EM & analysis & $5 \times 5$ & $0.13 \times 0.13$ & 18.1 & 157 \\
\hline EM & EM sum & $6 \times 6$ & $0.16 \times 0.16$ & 23.4 & 204 \\
\hline EM & SP sum & $12 \times 12$ & $0.32 \times 0.32$ & 67.2 & 585 \\
\hline$H A D_{1}$ & unit coll & $1 \times 1$ & $0.08 \times 0.08$ & 2.5 & 34 \\
\hline$H A D_{1}$ & analysis & $3 \times 3$ & $0.24 \times 0.24$ & 13.3 & 177 \\
\hline$H A D_{1}$ & $\begin{array}{l}\text { SP sum and } \\
\text { EM veto }\end{array}$ & $4 \times 4$ & $0.32 \times 0.32$ & 20.3 & 273 \\
\hline$H A D_{1}$ & JET sum & $8 \times 8$ & $0.64 \times 0.64$ & 58.3 & 785 \\
\hline
\end{tabular}

Table 5-7. Estimated Pileup Noise In The Liquid Calorimeter. 
respectively, giving $\rho_{t}=12.0 \mathrm{MeV} \sqrt{\mathrm{ns}}$ for the $3 \times 3$ or $20 \mathrm{MeV} \sqrt{\mathrm{ns}}$ for the $5 \times 5$ tower. Combining this with the value of $\rho_{p}$ from Table 5-7 and using $t_{d}=$ $600 \mathrm{~ns}$, we find $t_{m}^{0}=45 \mathrm{~ns}$ for the $3 \times 3$ analysis cell and for operation at $\mathcal{L}=10^{33} \mathrm{~cm}^{-2} \mathrm{~s}^{-1}$. From the dependence of $t_{m}$ on $\rho_{p}$, it is easy to see that the optimal hardware shaping time scales like $\mathrm{L}^{-1 / 4}$, so the optimum would move to about $25 \mathrm{~ns}$ at $10^{34}$.

For the hadronic section, we calculate $t_{m}^{0}$ for a single cell, as the noise level must be optimized for the purposes of a hadronic veto for identification of an isolated electromagnetic shower. The value of $p_{t}$ is estimated from the structure of the calorimeter to be approximately $63 \mathrm{MeV} \sqrt{\mathrm{ns}}$ at $\eta=0$. Using the value of $\rho_{p}$ of $2.5 \mathrm{MeV}$ from Table 5-7 and repeating the calculation done for the EM section, we find $r_{m}$ $=187 \mathrm{~ns}$ for operation at $10^{33}$ and $104 \mathrm{~ns}$ for operation at $10^{34}$. For larger towers, $t_{m}^{0}$ decreases as $(\Delta \eta \Delta \phi)^{-0.13}$, so the value of $t_{m}^{0}$ for the $4 \times 4$ hadronic trigger towers is about $25 \%$ lower.

\subsection{THE HADRONIC CALORIMETER}

\subsubsection{Inner Barrel Hadronic Modules}

The barrel hadronic modules are a total of $4.7 \lambda$ deep at $90^{\circ}$. Of this, $4.3 \lambda$ is active volume, while the balance is consumed by supports, electronics motherboards, cables etc. A more detailed discussion is available; 25 here we present only a summary of the design.

A hadronic tower has segmentation in $\Delta \eta \times \Delta \phi$ of $0.08 \times 0.08$. Thus each hadronic tower overlaps nine towers in the EM calorimeter. Mechanically there are two separate modules in depth, each one covering $0.1570 \mathrm{rad}=9^{\circ}$ in $\varphi$, and extending from the center support washer to one end of the barrel. The modules in the second hadronic layer are rotated by $4.5^{\circ}$ relative to the modules in the first layer. Any particle going through the crack between modules in the first layer impinges onto the center of a module in the second layer. There are $\mathbf{8 0}$ modules in each layer. Electrically there are three layers in depth, as discussed below. An isometric view of a module is shown in Figure 5-33.

\section{The Electrostatic Transformer Arrangement; Unit Cell}

The noise/signal ratio in an ionization calorimeter is proportional to the square root of the electrostatic capacitance of such a detector. The noise can be reduced by transforming the capacitance of the detector to match the capacitance of the amplifier. Since our calorimeter is inside the magnetic field of the GEM superconducting coil, we cannot use a conventional magnetic transformer. Instead we use the electrostatic transformer ${ }^{26,27}$ where several readout gaps are placed in series for readout purposes rather than connecting all ionization gaps in parallel as is usually done. This arrangement increases the effect of the cross capacitance between neighboring channels. This effect is roughly proportional to the absorber thickness in each gap. An optimum transformer ratio (number of gaps in series) lies between $3: 1$ and $4: 1$, when one chooses an absorber thickness of 9-10 $\mathrm{mm}$. We have chosen an absorber thickness of $9 \mathrm{~mm}$ and a 3:1 ratio (three gaps in series) in the barrel. Two such arrangements can be naturally set in parallel, and this is the unit we call one cell. It is sketched in Figure 5-34. Each module consists of a total of 6 cells.

\section{Absorber Material}

If one wants to maximize the absorption length per unit of depth, but is not willing to accept esoteric materials such as tungsten, then the natural absorber of choice would be copper. However, copper $(Z=$ $29)$ and krypton $(Z=36)$ are sufficiently similar so that there is little electromagnetic suppression. The value of $e / h$ is estimated to be 1.9. It was therefore decided to use lead as the primary absorber material, but use copper or brass where both absorption and mechanical rigidity are required. Another advantage of brass (or copper) is the fact that during cool down its shrinkage is very similar to that of aluminum, the material selected for the overall cryostat.

\section{Absorber Planes}

There are two different types of absorber material. Every sixth absorber is a ground plane. This is a solid copper (or brass) sheet $9 \mathrm{~mm}$ thick, extending across the whole module. Electrically each ground plane is tied to the signal ground of the readout cable leading to the amplifiers. All other absorber planes are tile planes. A typical tile plane is shown in Figure 5-35. It consists of individual tiles, each corresponding to a single tower, separated by approximately $0.3 \mathrm{~mm}$ gaps. A $0.5 \mathrm{~mm}$ thick copper clad G-10 board which is laminated to each 


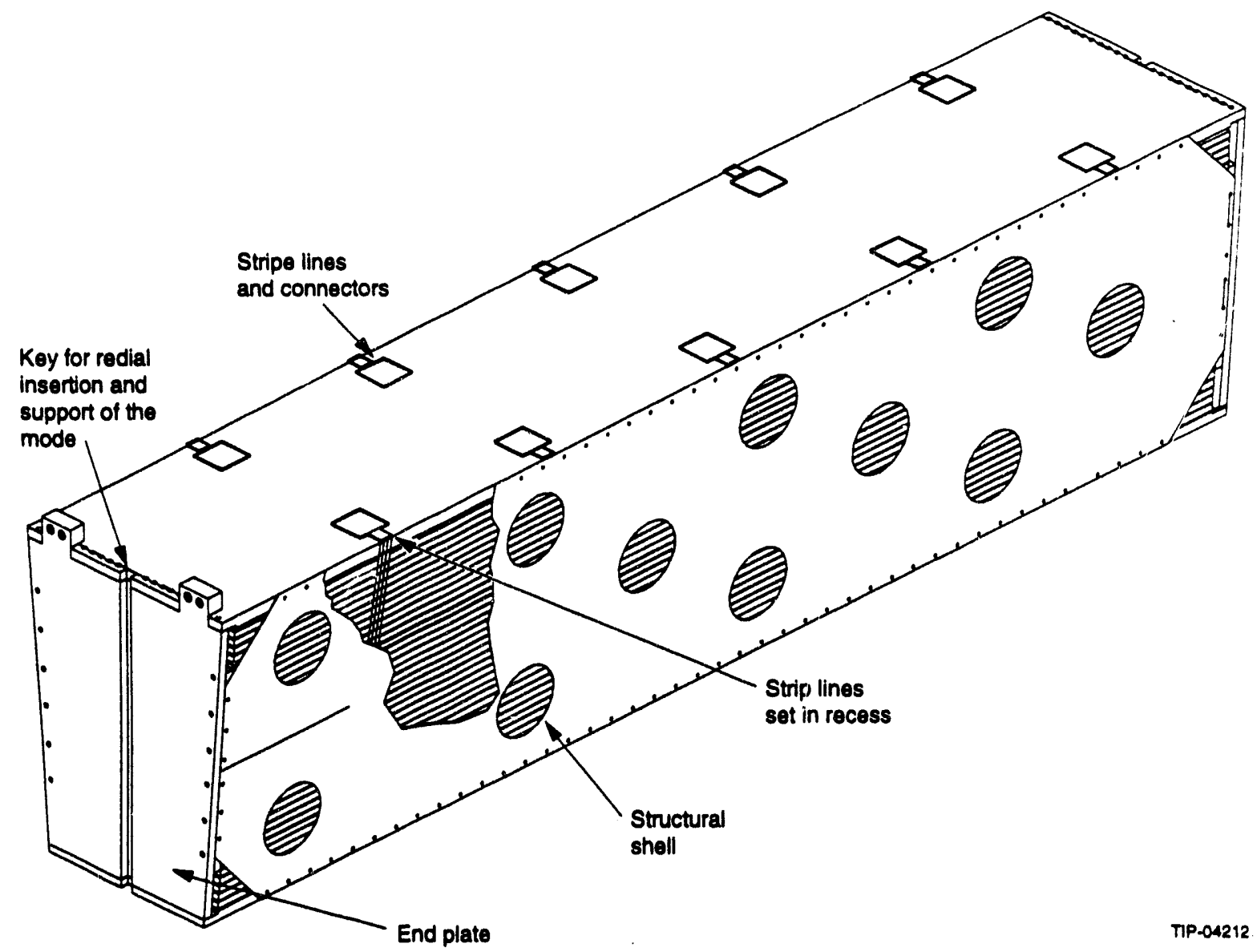

FIG. 5-33. Isometric view of barrel hadronic module.

side of the tiles with the copper facing outwards. The cladding is subdivided into squares of the same size as a single tower, with only about $0.5 \mathrm{~mm}$ between each square. The individual squares are connected by a thin line of resistive ink, providing a resistance of $\sim 100 \mathrm{k} \Omega$ Thus all the pads are independent at signal frequency, but are at the same voltage in DC mode. For most of the tile planes one side of these pad structures is tied resistively to high voltage (HV), the other resistively to ground. In the plane where the tiles are connected to the signal line which feeds the capacitively coupled pulse to the preamps, both are tied resistively to HV. These signal lines (not shown) have an impedance of 5-10 $\Omega$.

\section{Electrical Readout Structure}

High energy electrons or photons will deposit a small fraction of their energy in the first layers of the hadronic calorimeter. One cell corresponds to about 11 radiation lengths. The first two cells of the first hadronic module will be read out separately, and thus provide a "tail catcher" to the electromagnetic calorimeter. The next four cells of the first hadronic modules form the second readout group in the first hadronic module. The second hadronic module $(2 h)$ could either be read out as a single readout group or again subdivided into two sections.

\subsubsection{Endcap Hadronic Calorimeter}

There are six different types of endcap hadronic modules as shown in Figure 5-1. The modules are labeled Inner Had A, B, C and Outer Had A, B and $C$. All the modules are supported from keys mounted on the washers that engage grooves in the module end plates. The radial position is fixed by bolting the modules to the washers using ears or brackets. Slotted holes and Belleville washers are used at one end of the modules to accommodate the differential thermal contraction of the copper module structure and the aluminum support structure. This is very similar to the SLD mounting scheme. The crack between modules averages $1 \mathrm{~mm}$ for the 


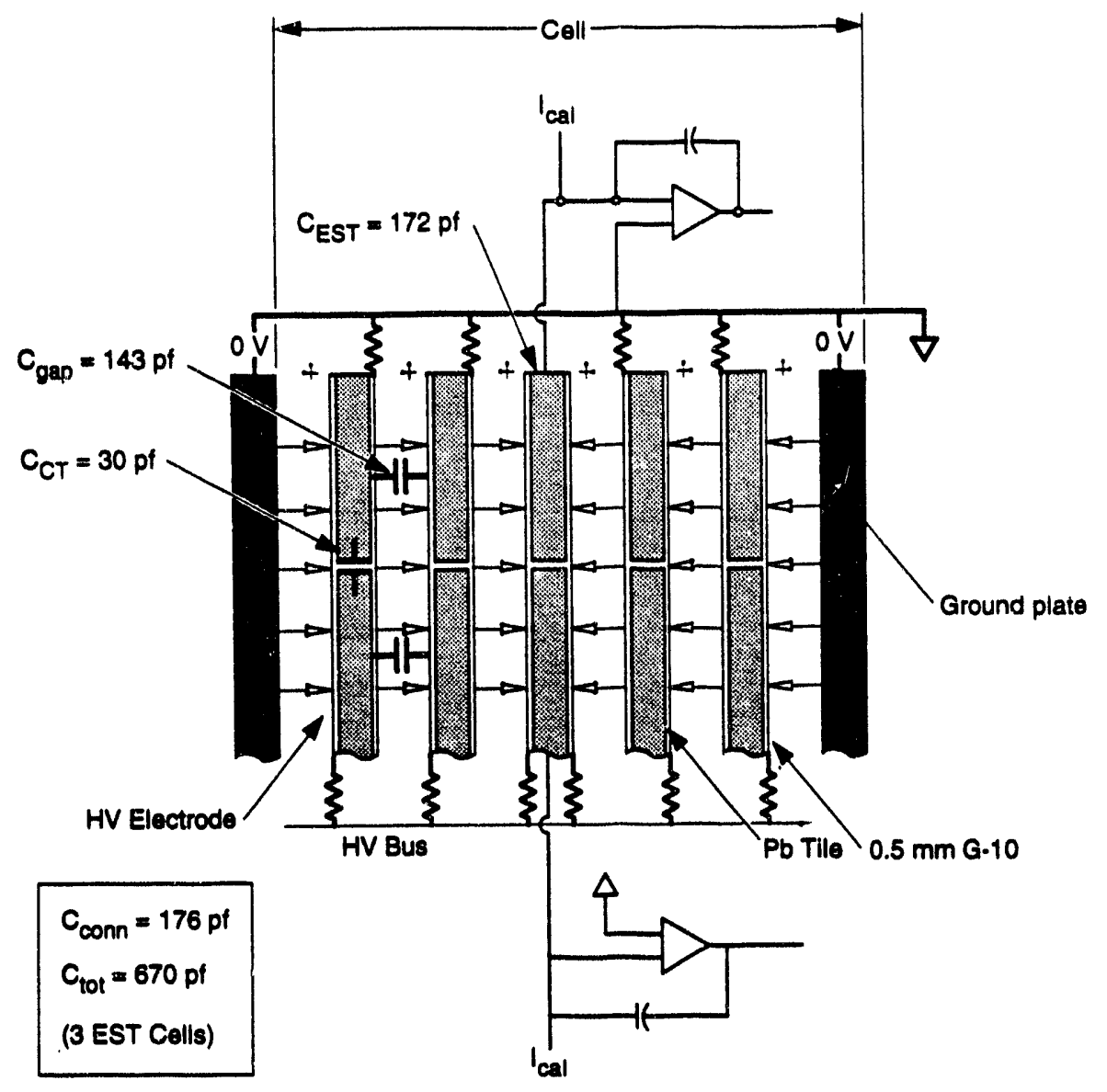

TP.04312

FIG. 5-34. Unit cell in inner barrel hadronic module showing electrostatic transformer.

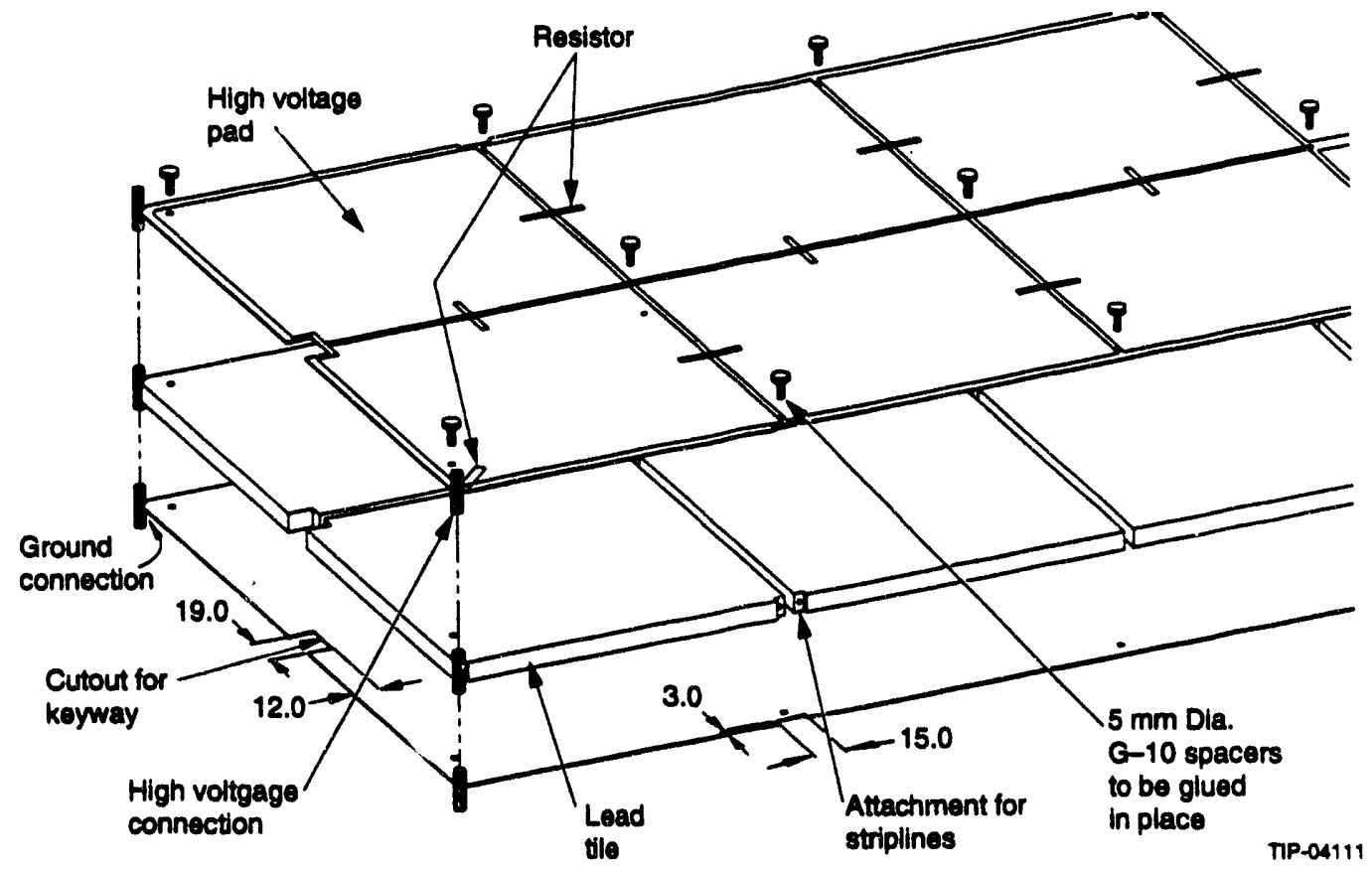

FIG. 5-35. Typical layer of inner barrel hadronic module showing tiles. 
ground plates because of tolerance limits and $4.5 \mathrm{~mm}$ for the tiles because of cross talk limits. The modules will be mounted so that there are no projective cracks. The Inner Had A modules have no relative rotation, the Inner Had B modules have an $18^{\circ}$ rotation and the Inner Had C modules have a $9^{\circ}$ rotation. The Outer Had A modules have no rotation, the Outer Had B modules have a $9^{\circ}$ rotation and the Outer Had $\mathrm{C}$ modules have no rotation. In this way there will be no cracks between sequential modules or between the inner and outer modules. The inner radius of the Inner Had A and B modules is about 230 $\mathrm{mm}$. All plates, electrodes and tiles are oriented perpendicular to the beam axis.

The cell structure in the modules is a sequence of copper ground plates, copper-Kapton electrodes and copper tiles, see Figure 5-36, the standard cell. The basic cell is composed of one ground plate, three electrode layers, a tile layer and three more electrode layers. An additional ground plate is added to the last cell to complete the structure. The copper in the first and last cells is reduced to compensate for the aluminum in the washers and the extra ground plate. The signal is collected from the center tile. The electrodes and tiles provide the segmentation for the modules. The ground plates and tiles are made from $27 \mathrm{~mm}$ copper. The tiles are glued between $0.5 \mathrm{~mm}$ etched $\mathrm{GlO}$ boards to give them structural integrity and also supply the capacitive decoupling from the high voltage. The spaces between the tiles are mostly filled with argon to minimize the capacitive coupling between towers. The argon gaps between the electrodes are $2 \mathrm{~mm}$. The charge collection utilizes the electrostatic transformer technique with a transformer ratio of four. A ratio higher than the barrel is used because the ionization in argon is less than in krypton.

Operating at $2 \mathrm{kV}$, simulations confidently indicate that space charge will not be a problem at a luminosity of $10^{33} \mathrm{~cm}^{-2} \mathrm{~s}^{-1}$ but may be a problem at $10^{34} \mathrm{~cm}^{-2} \mathrm{~s}^{-1}$ at the very largest $\eta$. This matter is under investigation. Once the uncertainty on the positive ion mobility is resolved the appropriate increase to the operating voltage will be made for the largest $\eta$ region if necessary. The allowed luminosity scales as the square of the high voltage. The ground and high voltage pads on the electrodes and tiles are decoupled from the true ground and high voltage by means of resistors. Because of the current due to the flux of particles traversing the detector there is a limit on the allowed magnitude of the resistor to keep the voltage drop from the true value to be less than 1 volt. At $10^{34} \mathrm{~cm}^{-2} \mathrm{~s}^{-1}$ there is about a $1 \mu \mathrm{A}$ current in a layer within a tower cell. Hence a decoupling resistor as large as $1 \mathrm{M} \Omega$ could be safely used. This is about an order of magnitude larger resistance than required so as to not contribute significantly to the amplifier noise.

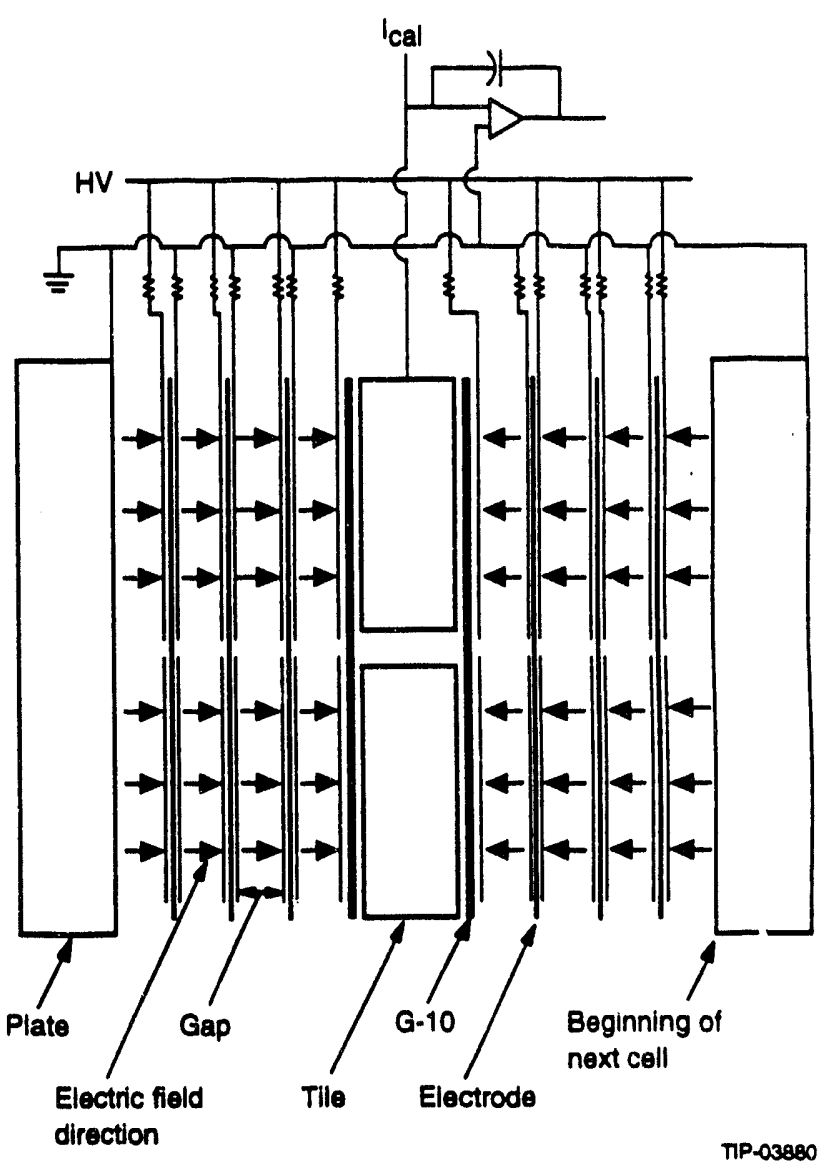

FIG. 5-36. Electrode and absorber arrangement in the endcap hadron modules. 
At the outer radius there are 40 modules of each type. These are segmented into $\Delta \eta \times \Delta \varphi$ towers of $0.08 \times 0.08$ making each module just iwo tiles wide. This structure facilitates the wiring of the signals and high voltage because it can be done on the exposed edges of the tiles. Figure 5-37 shows the structure of module Outer Had B, the largest of the outer modules. The modules each have a top and bottom plate bolted to the ground plates which when analyzed as a Veirendeel Truss arrangement provides the necessary stiffness across the module with a safety factor of about ten. The electrodes and tiles are supported from the ground plates.

There are 20 modules of each of the inner types. These would naturally be four tiles wide with the standard segmentation; however these modules begin at their outer radius with a $\Delta \eta \times \Delta \varphi$ of $0.04 \times$ 0.16 which keeps each tower area constant but allows the wiring to be done at the exposed tile edge as was done for the outer modules. This segmentation does not affect the trigger scheme or the jet spatial resolution. At about half the distance to the

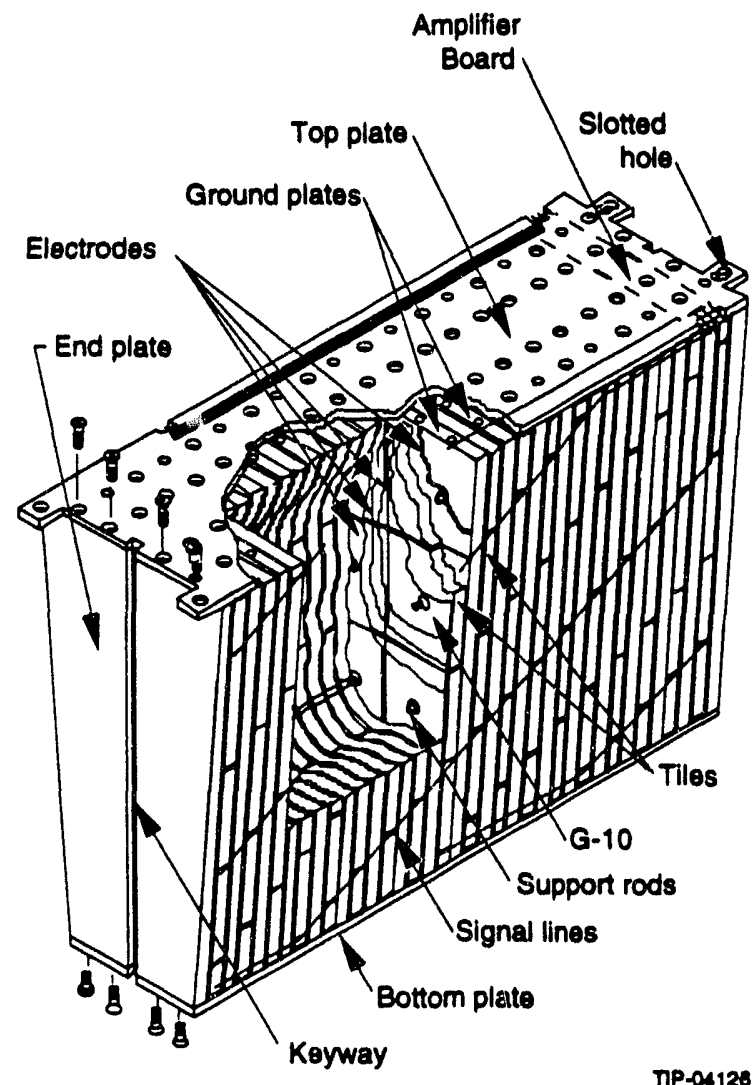

FIG. 5-37. Outer Endcap Hadron module. beamline the segmentation changes to $0.08 \times 0.16$ because the towers are getting smaller.

The Outer Had A module measures the energy of showers that have already developed in the barrel and passed through several unsampled layers of cryostat wall, cables, liquid krypton and liquid argon. This lost energy must be sampled by a massless gap. Although a passive massless gap is possible by reducing the absorber in the first cell of each module we plan to make it an active gap sampled with two layers of argon and a separate amplifier for each tower. The high voltage electrodes for these gaps are made from etched Kapton or G10 boards and are supported from the end plate of the module.

Kapton striplines carry the charge signal to amplifiers mounted on the modules as shown in Figure 5-37. These striplines are connected to the tiles and ground plates by pins soldered to them that plug into sockets pressed into holes drilled in the tiles and ground plates. These striplines pass through notches in the ground plates recessed $2 \mathrm{~mm}$ from the module edge.

We have scaled the data for a liquid argon and iron calorimeter ${ }^{28}$ to determine the rasolution of this liquid argon and copper calorimeter. To be conservative, we assume the sampling fluctuations are dominated by the electromagnetic sampling. The resolution scales to $58 \% / \sqrt{E}$ for the endcap configuration based on the measurement at $10 \mathrm{GeV}$ which gave a value of $52 \% / \sqrt{E}$. These values are obtained without the use of shower weighting techniques.

The first section in Inner Had A will serve as a tail catcher for the endcap EM. This section is two cells deep and adds $7.5 \mathrm{X}_{0}$ for electromagnetic shower absorption. Properly designed and corrected for material between the EM and hadron module, this section maintains the excellent electromagnetic resolution performance at higher energies.

\section{Capacitance of the Hadronic Towers}

The barrel hadronic calorimeter towers encompas: a region of $3 \times 3 \mathrm{EM}$ towers $(\Delta \varphi=0.08 \times$ $\Delta \eta=0.48)$. The hadronic part of the calorimeter has parallel plate tile electrodes. Due to the larger tower size, the additional capacitance from the connections plays a lesser role. In order to properly match the tower capacitance to the preamplifier input 
capacitance, an "electrostatic transformer" (EST), consisting of a series connection of $n_{t r}=3$ gaps is used to reduce the capacitance to (ideally) $C_{g a p} / n_{t r}$. Due to the "crosstalk" capacitance to adjacent towers, though, the actual capacitance is larger than the ideal value. Fig. 5-38 shows the extent of this increase for $n_{t r}=3$ and $n_{t r}=4$ as a function of crosstalk capacitance. For a typical ratio $\mathrm{C}_{\mathrm{gap}} / \mathrm{C}_{\mathrm{CT}}=$ 7 (CT=cross talk) such an increase is approximately a factor of two. Tables 5-8 and 5-9 summarize the dimensions and capacitance levels and electronic noise in the hadronic calorimeter.

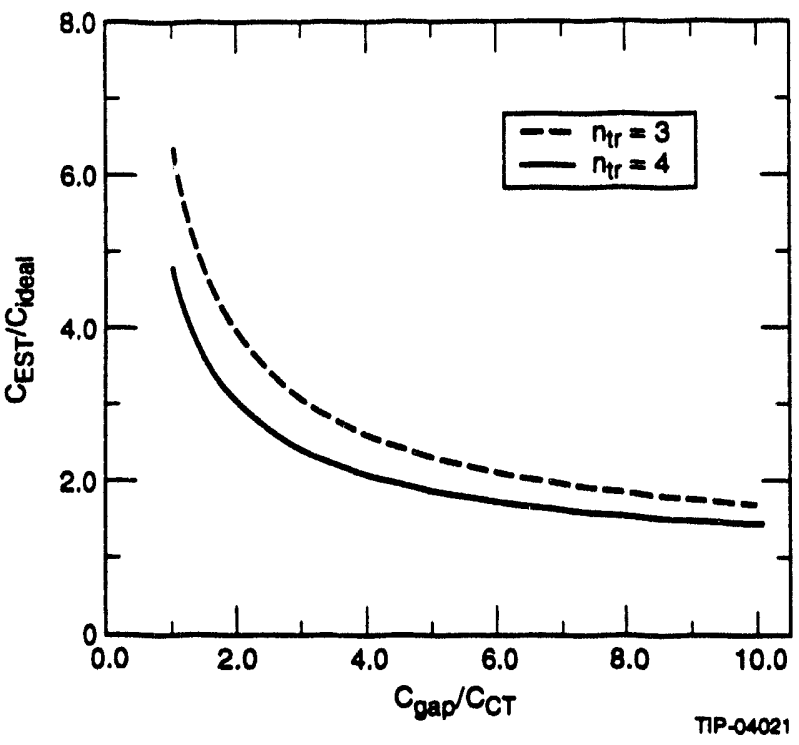

FIG. 5-38. EST capacitance dependence on the transformation ratio $n_{\mathrm{tr}}$, gap capacitance $C_{\text {gap }}$ and tile-to-tile capacitance $\mathrm{C}_{\mathrm{T}}$.

\subsubsection{Scintillating Barrel Calorimeter}

The Scintillating Barre 1 Calorimeter surrounds the liquid calorimeter system (see Figure 5-1), increasing the system active absorption length (up to $11 \lambda$ at $\eta=0$ ) and providing structural support for the three noble liquid cryostats and two endcap passive absorbers with embedded forward hadronic units. The scintillating barrel serves as an outer hadronic calorimeter for the tail of the hadronic jets and improves the jet energy resolution and $E_{T}$ measurements.

The role of the scintillating barrel calorimeter becomes important for the detection of very high $p_{T}$ (5-10 TeV) jet events. The detection system of the scintillating barrel calorimeter can also distinguish minimum ionizing particles above the level of noise and neutron background with a resolution time of about 2-3 ns rms.

The scintillating barrel is mounted in the Central Detector Support (CDS) (Figure 5-1) and is equipped with rails (Figure 5-39) which are used for the installation and support of the liquid system and the passive end-caps. A faceted stainless steel tube (Figure 5-39) provides the foundation for the scintillating barrel. It is attached to the central detector support with bolted joints. The structural support tube will be formed and partially assembled at the manufacturing site. Pieces of the tube small enough to be shipped will be delivered to IR-5 at the SSCL where the complete tube will be welded together in an assembly stand prior to installation of the modules.

TABLE 5-8. Hadron Tower Parameters at $\theta=90^{\circ}(\Delta \eta \times \Delta \varphi=0.08 \times 0.08)$.

\begin{tabular}{lcccccc}
\hline & $\begin{array}{c}\text {-Number of EST } \\
\text { colls }\left(n_{\mathrm{tr}}=3\right)\end{array}$ & $\lambda$ & $\begin{array}{c}\text { Average } \\
\text { Width } \\
(\varphi)(\mathrm{mm})\end{array}$ & $\begin{array}{c}\text { Average } \\
\text { Length } \\
(\theta)(\mathrm{mm})\end{array}$ & Cap. (pF) & $\sigma_{\text {ol }}(\mathrm{M \theta V})$ \\
\hline $\begin{array}{l}\text { Section } \\
\text { FRodron 1 }\end{array}$ & 3 & 1.17 & 140 & 140 & 700 & $77[100 \mathrm{~ns}]$ \\
$\begin{array}{c}\text { Hadron } 1 \\
\text { BACK }\end{array}$ & 4 & 1.56 & 160 & 160 & 1200 & $104[100 \mathrm{~ns}]$ \\
\begin{tabular}{l} 
Hadron 2 \\
\hline
\end{tabular} & 5 & 1.95 & 195 & 195 & 2050 & $80[200 \mathrm{~ns}]$ \\
\hline
\end{tabular}


TABLE 5-9. Hadron Tower Parameters at $\theta=50^{\circ}(\Delta \eta \times \Delta \theta=0.08 \times 0.08)$.

\begin{tabular}{lcccccc}
\hline Section & $\begin{array}{c}\text { Number of EST } \\
\text { cells }\left(n_{t r}=3\right)\end{array}$ & $\lambda$ & $\begin{array}{c}\text { Average Width } \\
(\varphi)(\mathrm{mm})\end{array}$ & $\begin{array}{c}\text { Average } \\
\text { Length } \\
(\theta)(\mathrm{mm})\end{array}$ & Cap. $(\mathrm{pF})$ & $\sigma_{\theta \mathrm{l}}(\mathrm{MeV})$ \\
\hline $\begin{array}{c}\text { Hadron 1 } \\
\text { FRONT }\end{array}$ & 3 & 1.5 & 140 & 180 & 900 & $107[100 \mathrm{~ns}]$ \\
$\begin{array}{c}\text { Hadron 1 } \\
\text { BACK }\end{array}$ & 4 & 2.0 & 160 & 210 & 1400 & $133[100 \mathrm{~ns}]$ \\
Hadron 2* & 3 & 1.5 & 195 & 240 & 1500 & $62[200 \mathrm{~ns}]$ \\
\hline
\end{tabular}

The hadronic section at $\theta=50^{\circ}$ is shorter than at $\theta=0^{\circ}$.

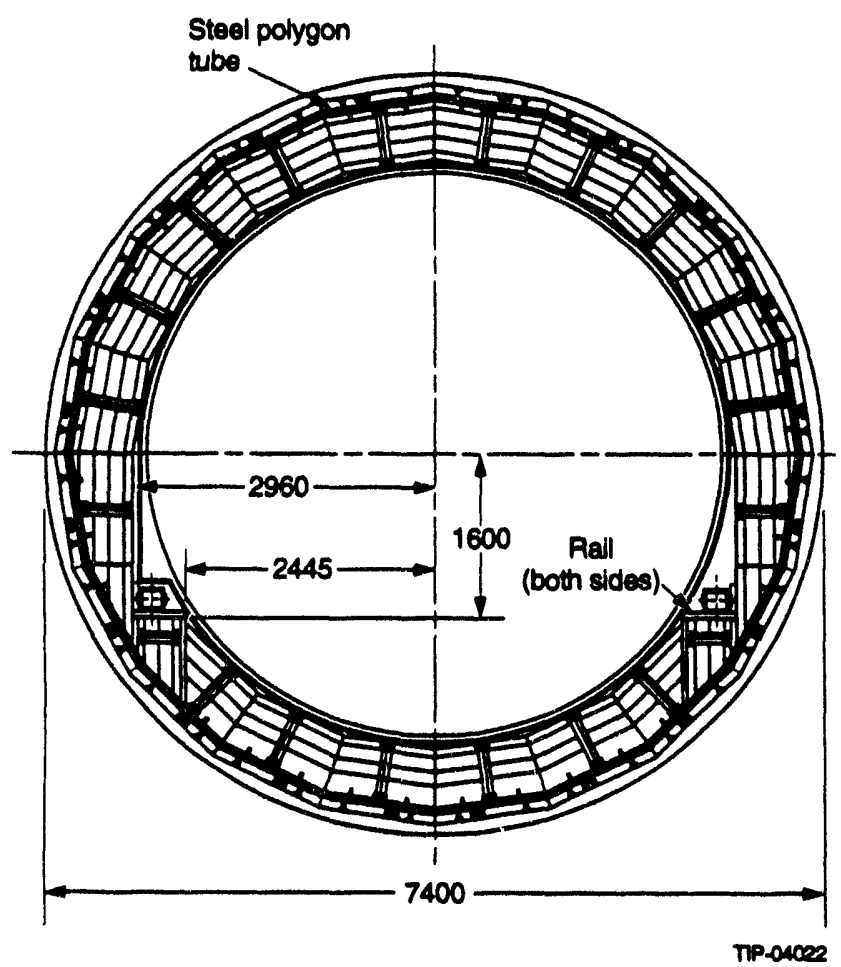

FIG. 5-39. End view of scintillating barrel calorimeter. Rails structure on the inner side of SBC supports barrel and two endcaps of liquid calorimeter

Connection of the support tube to the CDS will be accomplished via flanged extensions which are designed to accommodate the large cabling and piping bundles which will be routed through the CDS. The flanged extensions will be welded to the scintillating barrel and bolted to the CDS in order to reduce the complexity of the assembly operations. A finite element analysis of the scintillating hadron barrel indicates that the constraining criterion is the amount of deflection resulting from the support of the liquid system and passive endcaps. The predicted full load deflection of the system is approximately $5 \mathrm{~mm}$.

Copper (or brass) was chosen as an absorber material to provide maximum absorption length at minimal cost. Modular construction has been used to provide a means of manufacturing the scintillating calorimeter in collaborating countries. Brass absorber plates can be produced in one of several collaborating countries. The plates will be rolled to the correct thickness at the mill then sized to fit the modular assemblies prior to shipment for installation into the modular assemblies. The 44 modules (Figure 5-40) are of a size and weight which can be handled and shipped via conventional means. Since the SBC supports the liquid calorimeters, not all of the 44 modules are identical.

Major characteristics and parameters of the Scintillating Barrel Calorimeter (SBC) are given in the Table 5-10.

\section{Scintillating Barrel Calorimeter Modules}

The modules of the Scintillating Barrel Calorimeter are about $570 \mathrm{~mm}$ thick, $1000 \mathrm{~mm}$ wide, and $5000 \mathrm{~mm}$ long with weight about $24 \mathrm{Mg}$ each (Figure 5-41). The modules have several layers of absorber plate interleaved with four layers of readout tiles. The plates are bolted together. Spacers are used to create gaps for scintillating plates. The completed 44 modules are bolted to the outer structural support tube. The modules are designed to be self-supporting. The primary liquid calorimeter support is provided by the outer structural tube. The scintillating calorimeter is designed to require no welding after installation of the plastic tiles and fibers. 


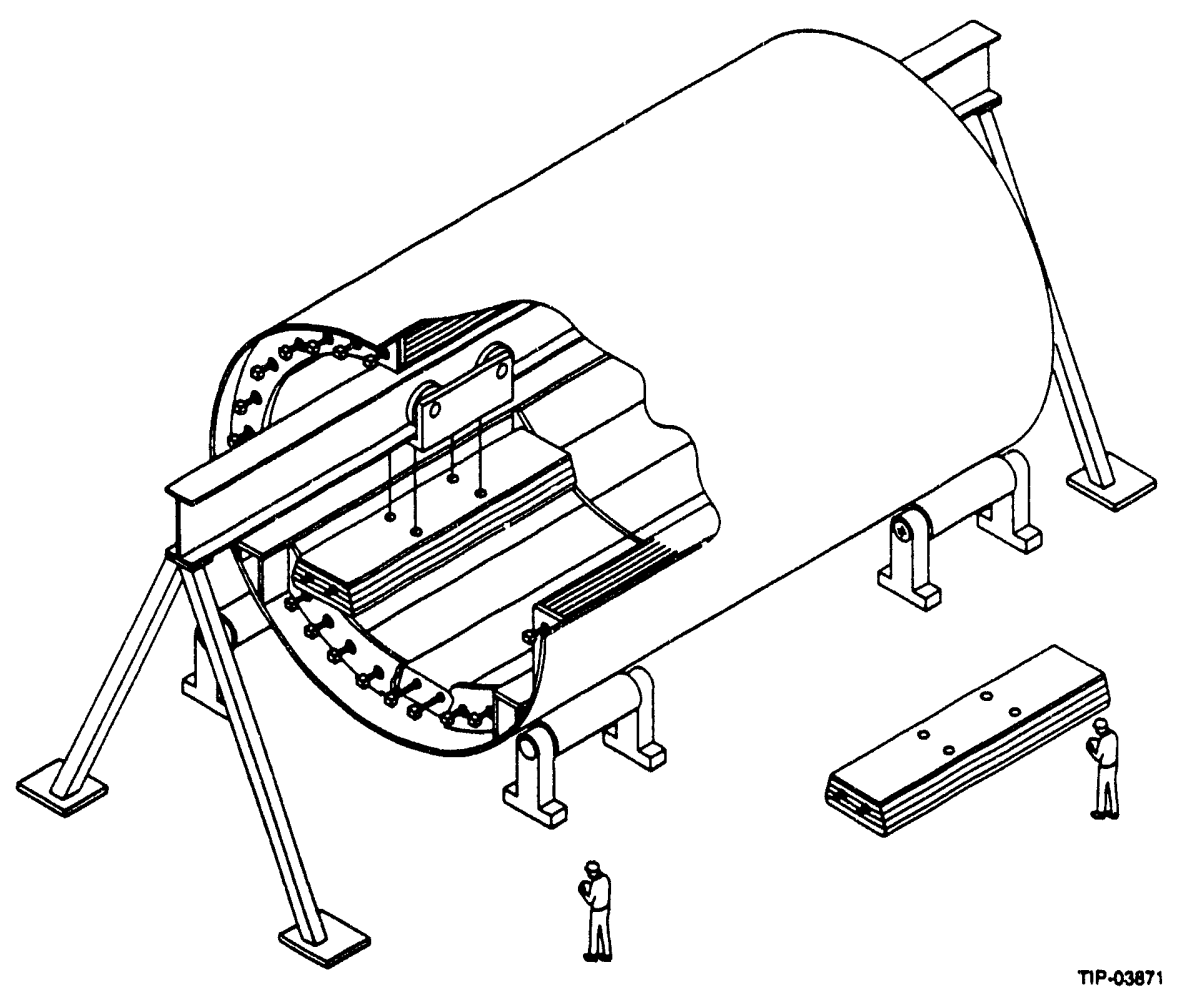

FIG. 5-40. Assembly of scintillating barrel calorimeter. One of 44 modules is shown being assembled. Another module is shown outside the support tube for scale.

The 640 PMT readout assemblies will be mounted in clusters located between the liquid system electronics junction boxes. Each readout assembly is expected to produce approximately 1 to 2 watts of power. Heat resulting from the assemblies will be removed via the same forced water heat-exchanger system required for the noble liquid electronics.

All the utilities and readout cabling for the tracker, noble liquid, forward and scintillating barrel will be routed around the outside of the barrel. The piping and cables will be embedded in grooves in a $100 \mathrm{~mm}$ layer of brass (Figure 5-41). Final placement of the utilities will take place in the experimental hall.

There are four layers of scintillating tiles in the scintillating barrel. The first layer samples the hadronic jets/showers after the dead material of the outer cryostat walls and first absorber layer of the scintillating modules. The other three layers are equally spaced in the absorber $130 \mathrm{~mm}$ apart.

The scintillating layers are made of $6 \mathrm{~mm}$ thick tiles formingprojective towers of size $\Delta \eta \times \Delta \varphi=$ $0.16 \times 0.16$ (matching $2 \times 2$ hadronic towers in the liquid hadron calorimeter). The size of the tiles in the tower is approximately $80 \mathrm{~mm} \times 480 \mathrm{~mm}$ at $90^{\circ}$ and $80 \mathrm{~mm} \times 870 \mathrm{~mm}(\varphi \times \eta)$ at the end of the barrel. Each tile is read out by two wavelength shifting (WLS) fibers embedded in the scintillator as shown in Figure 5-42. The WLS fibers (1 mm diameter) are parallel to the $z$-axis and laid in a $1.1 \mathrm{~mm} \mathrm{U}$-shaped groove. The far end of the WLS fiber is mirrored to increase the collection of light. The WLS fiber is slightly $(100-150 \mathrm{~mm})$ longer than the length of the tile and positioned in the groove such that the exiting non-mirrored end protrudes above the neighboring tile (Figure 5-42). This WLS fiber end is spliced to the clear transport fiber with the aid of a thin plastic sleeve. The scintillating tiles are wrapped in aluminized mylar or other reflecting material. All fiber 
TABLE 5-10. Characteristics and Parameters of the Scintillator Barrel Calorimeter.

\begin{tabular}{|c|c|}
\hline Outer radius of the system & $3700 \mathrm{~mm}$ \\
\hline Inner radius of the system & $2923 \mathrm{~mm}$ \\
\hline$z$-extent of absorber & $\pm 4840 \mathrm{~mm}$ \\
\hline $\begin{array}{l}\text { Number of Absorption } \\
\text { Lengths }\end{array}$ & $4.5 \lambda$ \\
\hline $\begin{array}{l}\text { Total z-extent (including } \\
\text { readout) }\end{array}$ & $\pm 5500 \mathrm{~mm}$ \\
\hline $\begin{array}{l}\text { Number of Mechanical } \\
\text { modules (total) }(\phi \times \eta)\end{array}$ & $44=22 \times 2$ \\
\hline (of the same type) & $(14+2+2+2+2) \times 2$ \\
\hline $\begin{array}{l}\text { Total weight (SS support and } \\
\text { modules) }\end{array}$ & $1143 \mathrm{Mg}$ \\
\hline $\begin{array}{l}\text { Weight of outer SS support } \\
\text { tube }\end{array}$ & $195 \mathrm{Mg}$ \\
\hline $\begin{array}{l}\text { Weight of outer barrel passive } \\
\text { absorber }\end{array}$ & $223 \mathrm{Mg}$ \\
\hline Weight of 44 modules & $948 \mathrm{Mg}$ \\
\hline $\begin{array}{l}\text { Weight of the largest } \\
\text { (smallest) module }\end{array}$ & $24(8) \mathrm{Mg}$ \\
\hline $\begin{array}{l}\text { Thickness of } \\
\text { Scintillator/Copper stack }\end{array}$ & $586 \mathrm{~mm}$ \\
\hline $\begin{array}{l}\text { Thickness of each Copper } \\
\text { layer }\end{array}$ & $64 \mathrm{~mm}$ \\
\hline $\begin{array}{l}\text { Maximum weight of one } \\
\text { Copper layer }\end{array}$ & $3 \mathrm{Mg}$ \\
\hline Number of Scintillator layers & 4 \\
\hline $\begin{array}{l}\text { Gap for scintillator in the } \\
\text { absorber }\end{array}$ & $12 \mathrm{~mm}$ \\
\hline Thickness of scintillator tiles & $6 \mathrm{~mm}$ \\
\hline $\begin{array}{l}\text { Number of scintillator tiles in } \\
\text { one module layer }\end{array}$ & $12(\varphi) \times 8(\eta)$ \\
\hline Tile size & $\begin{array}{l}0.026(\varphi) \times 0.16(\eta), \\
\text { or } \approx 80 \mathrm{~mm}(\varphi) \times \\
400-870 \mathrm{~mm}(\eta)\end{array}$ \\
\hline$\eta$ coverage & $\pm 1.28\left(31^{\circ}-149^{\circ}\right)$ \\
\hline Number of WLS fibers per tile & 2 \\
\hline Average WLS fiber length & $610 \mathrm{~mm}$ \\
\hline WLS fiber diameter & $1 \mathrm{~mm}$ \\
\hline $\begin{array}{l}\text { Total number (length) of } \\
\text { WLS fibers }\end{array}$ & $30,800(18.8 \mathrm{~km})$ \\
\hline $\begin{array}{l}\text { Transport fiber length } \\
\text { (average) }\end{array}$ & $350-4850(2600) \mathrm{mm}$ \\
\hline Transport fiber diameter & $1 \mathrm{~mm}$ \\
\hline
\end{tabular}

TABLE 5-10. Characteristics and Parameters of the Scintillator Barrel Calorimeter. (Cont.)

\begin{tabular}{ll}
\hline $\begin{array}{l}\text { Total number (length) of } \\
\text { transport fibers }\end{array}$ & $30,800(80.2 \mathrm{~km})$ \\
$\begin{array}{l}\text { Total number of readoutPMT } \\
\text { channels }\end{array}$ & 640 \\
$\begin{array}{l}\text { Number of fibers per PMT } \\
\text { Required photomultiplier } \\
\text { tube gain }\end{array}$ & 48 \\
$\begin{array}{l}\text { Expected time resolution for a } \\
\text { single readout tower }\end{array}$ & $3 \mathrm{~ns}$ RMS \\
\hline
\end{tabular}

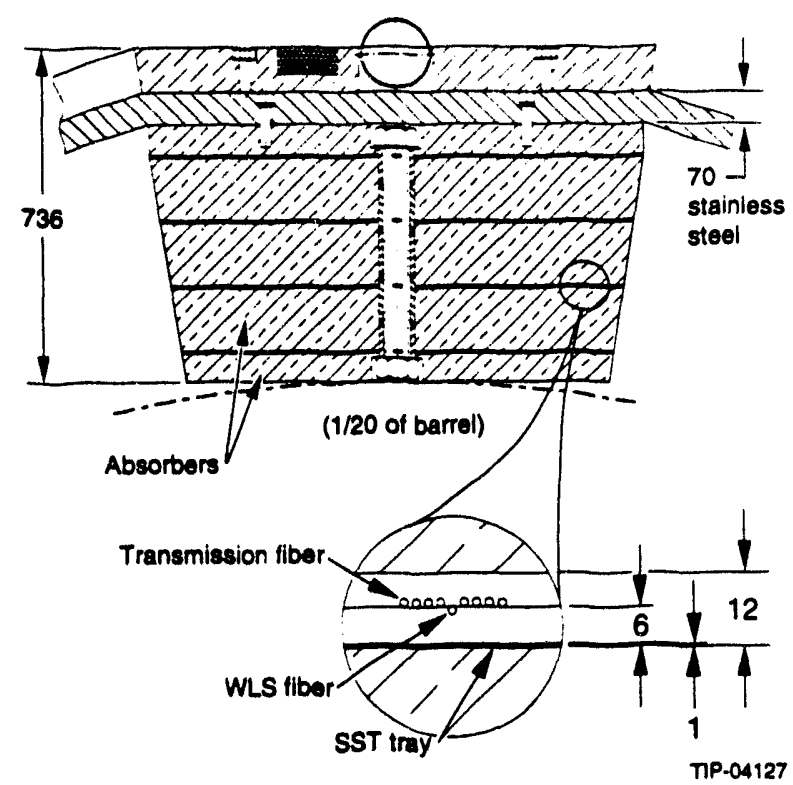

FIG. 5-41. Details of scintillating barrel calorimeter module - end view.

connections and transport fibers are laid and fixed in the $12 \mathrm{~mm}$ gap between absorber plates during the module assembly. The gaps between the absorber plates are maintained by spacers. Eight tiles in $\eta$ by 12 rows in $\varphi$ constitute one scintillator layer in the module. Six tiles constitute each $0.16 \times 0.16$ layer. Fibers from four layers within one $0.16 \times 0.16$ tower, or a total of 48 fibers, are read-out by one $1 / 2$ " photomultiplier tube (see Figure 5-43). Eight photomultipliers are gathered to a common assembly located at the end of the module. Thus, each module has two such assemblies. The total number of channels in the system is 640 . Since the modules are only a half-barrel long, the PMT assemblies are located on one end of each module, i.e., both encis of the barrel. In a possible upgrade, the number of PMT readout channels can be increased by a factor of 6 by ganging together fibers from only one tile per layer 
in all four layers. This will create readout segmentation in the SBC of $\Delta \eta \times \Delta \varphi=0.16 \times 0.026$ which can be useful for muon reconstruction at higher luminosity.

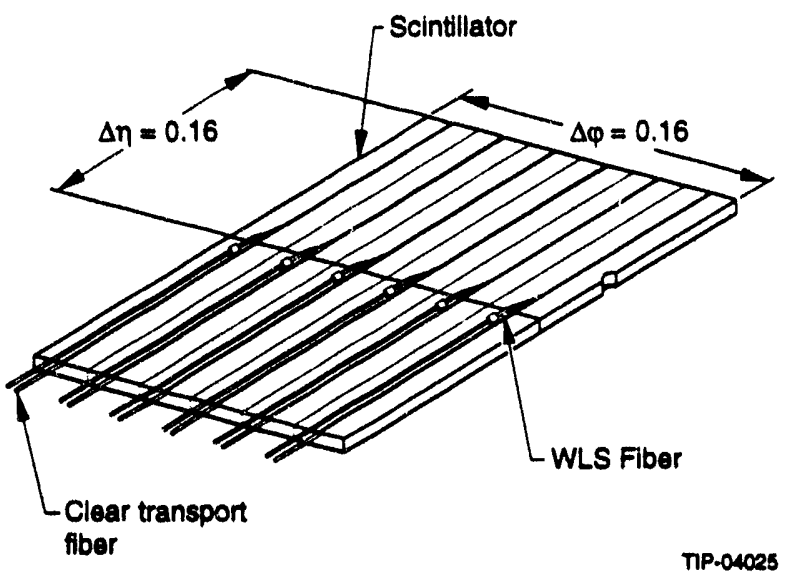

FIG. 5-42. Detail of tile/fiber arrangement in the readout tower.

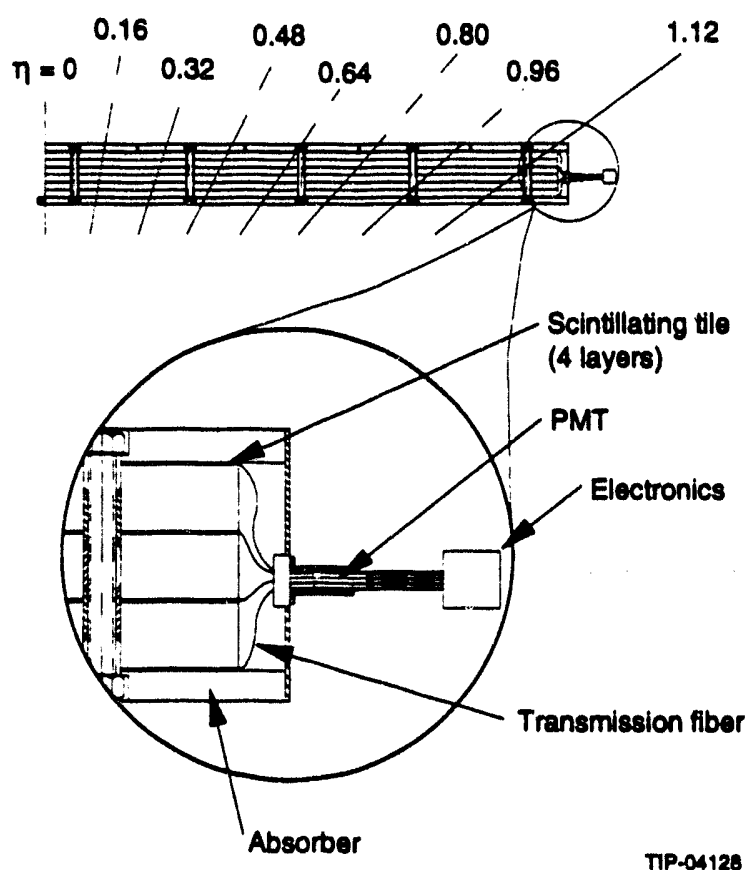

FIG. 5-43. Details of scintillating barrel calorimeter module - side view.

\section{Expected Performance}

The scintillating tile-WLS fiber technique has been extensively explored by the SDC and CDF Collaborations for the last few years. ${ }^{29}$ In the GEM scintillating barrel calorimeter design, the experience and techniques developed by SDC and CDF are used to minimize the amount of R\&D work, cost, and production time. The GEM scintillating barrel
R\&D program emphasizes studies of practical production, schemes of optical assembly, reproducibility of components and assemblies, and long term stability. Calorimeter prototypes will be built in 1993-1994 to test required scintillating tile/fiber performance. Tests of these prototypes will be done with cosmic muons using the TTR at SSCL and at Fermilab. During the Fermilab test, response of the prototype of scintillator calorimeter module will be measured with and without the liquid calorimeter in front of it in order to extend the calibration constants over the whole dynamic range of detected energies in the scintillating barrel calorimeter.

The number of active layers in the scintillating barrel calorimeter, as well as the size of scintillators and number of WLS readout fibers, was optimized by Monte-Carlo simulations. A light transport code LTRANS $^{30}$ has been used. Figure 5-44 shows the simulated response to a $50 \mathrm{GeV}$ muon for one tower (four scintillating samples). The most probable signal is 13.2 p.e. and the distribution has a long tail due to delta electrons, muon radiative energy losses, and Landau fluctuations. For comparison the signal generated by a $1 \mathrm{MeV}$ neutron interacting in a scintillator layer would correspond to channel 1-2 on this histogram. Monte Carlo calculations show that muon detection efficiency within the active volume of the scintillating module is $98 \%$. The $\mathrm{rms}$ time resolution is approximately $2-3 \mathrm{~ns}$. Also non-uniformity of the tile response does not exceed $5 \% \mathrm{rms}$ in the $\varphi$ direction and $10 \%$ in the $\eta$ direction.

Performance of the scintillating barrel section together with the Lead/Kr longitudinally segmented EM and hadronic sections was simulated by PYTHIA + GEANT for high energy hadronic jets and muons. The minimum number of scintillator layers required for readout in the outer barrel was the main concern of these simulations. Jet energy resolution, $E_{T}$ and muon energy loss corrections were studied. ${ }^{31}$ Figure 5-16 shows simulated hadron jet resolution versus jet energy in the GEM calorimeter at $90^{\circ}$ for different numbers of readout layers in the scintillating barrel for a fixed thickness of absorber. A modest improvement in the jet energy resolution is obtained if the scintillating barrel calorimeter is instrumented, though the actual degree of instrumentation ( 3 or 10 scintillator layers) is not of great importance. The mean energy deposited in four layers of the scintillator barrel calorimeter at $90^{\circ}$ for 
hadronic jets simulated by PYTHIA + GEANT is shown in Figure 5-45. For comparison the most probable energy deposition for a $50 \mathrm{GeV}$ muon and the typical level of a recoil proton signal from the interaction of a $1 \mathrm{MeV}$ neutron with the hydrogen in the scintillator are shown.

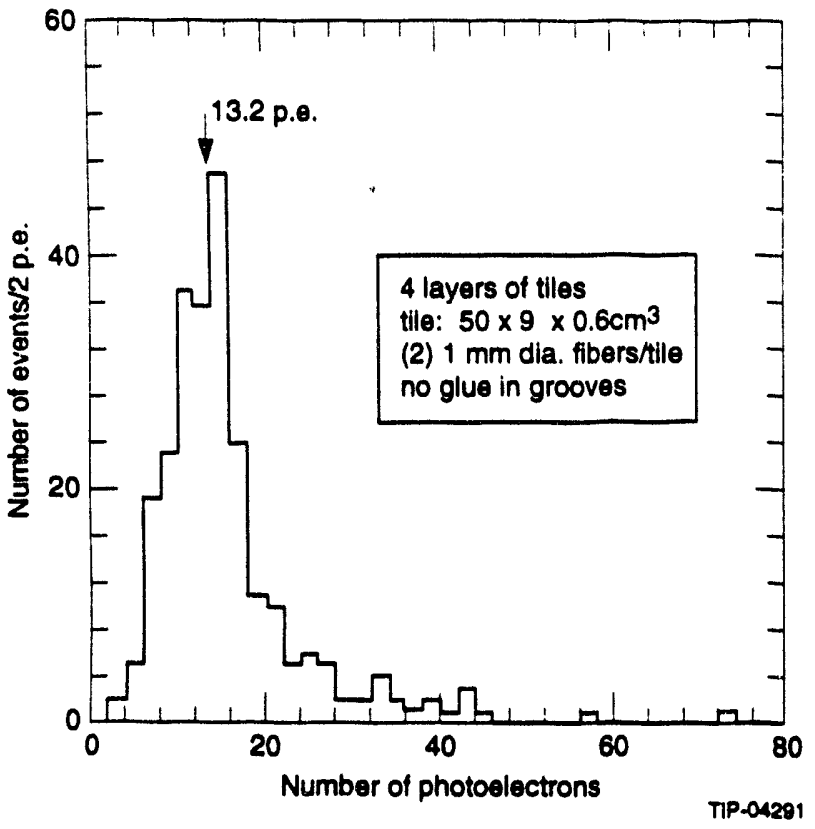

FIG. 5-44. Distribution of number of photoelectrons per MIP (GEANT-LTRANS MC simulation).

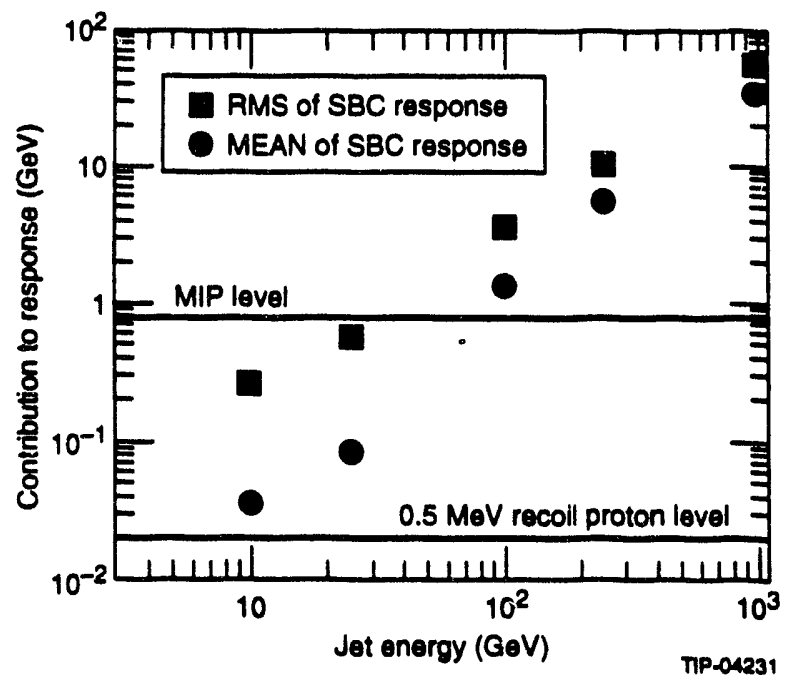

FIG. 5-45. Mean energy deposited by jets in the scintillating barrel behind the liquid calorimeter at $90^{\circ}$ vs. jet initial energy. Energy deposition in the scintillator is corrected for Birk's law. For comparison most probable MIP response and average recoil proton response (protons are originated by $1 \mathrm{MeV}$ neutrons) in one readout tower are shown.

\subsubsection{Phototube Readout and Calibration System}

\section{Readout System}

There are 640 photomultiplier tubes and readout channels in the scintillating barrel calorimeter. Each 48 fibers are read by a single photomultiplier tube. The dynamic range varies from minimum ionizing particles above the level of noise and neutron background, to very rare high energy 5-10 TeV pr jets. There is a plan to evaluate the need to include information from the scintillating barrel calorimeter in the Level 1 trigger.

\section{Photomultiplier Tube}

There are several existing candidate photomultiplier tubes that meet the Scintillating Barrel Calorimeter specifications. About $1 \%$ stability is required of this system, and this is attainable based on the experience of several large scale experiments. Although high magnetic field operation of the PMT still needs to be investigated, there are commercially available PMTs which are advertised to perform well in a field $>1 \mathrm{~T}$. In addition to satisfactory high magnetic field operation, photocathode sensitivity in the green-extended region is highly desirable. The required gain is about $10^{4}$. Table 5-11 summarizes the PMT requirements for the scintillating barrel calorimeter.

Table 5-11. Preliminary specifications for PMT.

\section{Mechanica}

Diameter/Length

End window

Photocathode

Uniformity

Quantum efficiency

Lifetime photo current

Pulsed photo current

Average photo current

Multipller

Gain
$0.5 \mathrm{inch} /-100 \mathrm{~mm}$

Flat face

$$
\text { diameter circle }
$$$$
\leftarrow 10 \% \text { over } 10 \mathrm{~mm}
$$

$>15 \%$ at $520 \mathrm{~nm}$

$>1 \mathrm{mC}$ for $50 \%$ gain loss

$<5 \%$ linearity for $10^{6}$ p.e. in $10 \mathrm{~ns}$

\section{$0.5 \mathrm{nA}$ for uniform} illumination 
Table 5-11. Preliminary specifications for PMT. (Cont.)

\begin{tabular}{|c|c|}
\hline Pulsed current & $\begin{array}{l}25 \mathrm{~mA} \text { in } 10 \mathrm{~ns}<1 \% \\
\text { nonlinearity, } 100 \mathrm{~mA} \text { for } \\
10 \text { ns with }<3 \% \\
\text { nonlinearity }\end{array}$ \\
\hline Max. charge & $200 \mathrm{~mA} \times 10 \mathrm{~ns}=2 \mathrm{nC}$ \\
\hline Rise time $(20 \%-80 \%)$ & $\begin{array}{l}<4 \mathrm{~ns} \text { (10 mA into } 50 \Omega \\
\text { load) }\end{array}$ \\
\hline Fall time $(80 \%-20 \%)$ & $<6 \mathrm{~ns}$ \\
\hline Pulse width (FWHM) & $<8 \mathrm{~ns}$ \\
\hline Width at Baseline $(5 \%)$ & $<12$ ns \\
\hline $\begin{array}{l}\text { Pulse change at high } \\
\text { current }\end{array}$ & $\begin{array}{l}\text { FWHM changes }<20 \% \\
\text { for } 1 \mathrm{~mA} \text { to } 50 \mathrm{~mA} \text {. }\end{array}$ \\
\hline Average anode current & $1 \mu A$ \\
\hline Lifetime & $100 \mathrm{C}$ with $50 \%$ gain loss \\
\hline Anode capacitance & $\begin{array}{l}<10 \text { pf to all other } \\
\text { electrodes combined }\end{array}$ \\
\hline $\begin{array}{l}\text { Inductance of anode and } \\
\text { last dynode }\end{array}$ & $<8 \mathrm{nH}$ \\
\hline \multicolumn{2}{|l|}{ Noise } \\
\hline Single p.e. & $<\underset{\text { peak }}{100 \mathrm{kHz} \text { at } 0.5 \text { of } 1 \text { p.e. }}$ \\
\hline After pulsing & $\begin{array}{l}<0.1 \% / p . e . \text { for after } \\
\text { pulses > } 10 \text { p.e. }\end{array}$ \\
\hline Prepulsing & $\begin{array}{l}\text { Arrival }<5 \text { ns early } \\
\text { amplitude }<0.5 \% \text { of } \\
\text { signal from } \\
\text { photocathode }\end{array}$ \\
\hline \multicolumn{2}{|l|}{ Stabillty } \\
\hline Long term & $<1 \% /$ month \\
\hline Hysteresis & $\begin{array}{l}<1 \% \text { gain shift within } 100 \\
\text { ns after pulse of } 10 \mathrm{~mA} \\
\times 10 \text { ns. }\end{array}$ \\
\hline
\end{tabular}

The Hamamatsu R2490-05, for example, a 2" mesh dynode proximity focusing photomultiplier is stable beyond $1 \mathrm{~T}$ of magnetic field with a gain of $2 \times 10^{5}$ and a rise time of $<3 \mathrm{~ns}$. The fine mesh dynode structure extends the current range up to $1 \mathrm{~A}$, with a $0.1 \%$ deviation, and still remains linear within $2 \%$ at $2 \mathrm{~A}$. The investigations of the conventional PMT characteristics, particularly regarding their operation in high magnetic fields, are continuing. Other photodetectors, however, are also under consideration.

\section{Calibration and Monitoring Systems}

For large PMT-based systems, several tested calibration methods and monitoring techniques are available to attain about $1 \%$ tower-to-tower and module-to-module calibration with good overall stability. In general, these techniques involve the use of radioactive sources and of flashing light sources.

Using a mechanically driven, movable, radioactive source $\left(e . g ., \mathrm{Cs}^{137}\right.$ ) can provide a quick calibration of a large system within a few percent accuracy; this was demonstrated by T 840 in a test run at Fermilab. This technique with longitudinal and transverse scans can be exploited for attenuation length, local radiation damage, uniformity of response and calibration monitoring measurements.

Another method of relative calibration and monitoring can be realized by the use of a flashing light source (nitrogen laser, LED, etc.). Using a nitrogen laser, the short term stability of $0.5 \%$ (days) and a long term stability of $1.4 \%$ ( 2 to 3 years) were achieved for a 600-PMT system. ${ }^{32}$ Similar monitoring studies using LEDs, and LEDs in combination with a radioactive source, report better than one percent stability.

The energy flow measurement is another candidate for calibration and monitoring. One can transfer the absolute calibrations of a few beamtested cells onto the entire detector by measuring the average energy deposited from all $p p$ interactions per cell. This can be done by measuring the PMT DC-current as a function of luminosity once the $\eta$ variation is known.

\subsubsection{Passive Absorber}

The passive absorber provides additional neutron and hadron absorption material for the endcap muon system. It also serves as a foundation for the hadronic forward calorimeter. The passive endcap will be constructed as a single module at the SSCL by bolting copper or brass plates together to form a ring. A structural tube will be positioned inside the plates to support the forward calorimeter.

The passive absorber will be constructed as a stand-alone component which is installed after the completion of the liquid calorimeter system. It will be installed on the same rail system which is used to install the liquid system.

Alignment of the $120 \mathrm{Mg}$ passive endcap and forward system will be accomplished by mechanical jacking between the endcaps and the rails. Alignment will be performed during the installation process with the beam axis as a reference. 
The absorber plates could be manufactured by the same supplier as the barrel scintillating calorimeter. Consequently, the specified plate thickness is assumed to be the same.

\subsection{THE FORWARD CALORIMETER}

The two forward calorimeters for GEM serve multiple purposes. They complete the calorimetric coverage so that the $E_{T}$ of each event can be measured and any can be determined. They detect jets and determine the kinematics of the jets. They act as corks in a bottle with carefully crafted beam holes to protect the muon system from spray and neutrons.

The required precision of the measurement is set by the most demanding physics signature, SUSY. When the SSC turns on, the lower mass scales of possible SUSY physics will have been explored at existing accelerators. If SUSY has not yet been discovered then the scale of $E_{T}$ physics for the SSC will be around $300 \mathrm{GeV}$ and above. ${ }^{33}$ Sufficient precision for the discovery of SUSY requires fiducial calorimetric coverage out to $|\eta|$ of 5.0 with $E_{T}$ resolution for jets of $\Delta \mathrm{E}_{T} / E_{T} \leq 10 \% .33,34$

Physics signatures with forward tagging jets include a very heavy Higgs and $W W$ scattering in the strong coupling regime. Jet measurement in the range $3 \leq|\eta| \leq 5$ covers a large fraction of the phase space for the jets recoiling from radiated Ws. Theorists have not agreed whether such jet tags are effective. Should it turn out they are, GEM will be prepared to exploit them.

The hermetic coverage of the GEM calorimeters helps to shield the muon chambers from hadronic spray and from neutrons. While the forward calorimeters add negligibly to the albedo neutrons in the central tracker volume, they act as very effective collimators to funnel low energy particles down to the region of the final focus quads. The GEM geometry is optimized to make a clean "shadow" behind the calorimeters with as little secondary spray as possible.

The forward calorimeters detect jets and measure their $E_{T}$. Jets up to $|\eta|=5.0$ are measured with adequate precision by requiring that $\gamma^{\prime}$ s (from $\pi^{0}$ decay) and hadrons be measured with $E_{T}$ resolution of better than $10 \%$ for $E_{T}>50 \mathrm{GeV}$. Precision in the measurement requires precision in both the energy measurement and in the angle measurement. Near $|\eta|=3.4$ the limiting precision is in the energy measurement while at $|\eta|=5.0$ the limit is in the angle measurement. The challenge is to design a calorimeter which (1) survives the very high dose rate (see Table 5-12), (2) shields the muon system. (3) has an acceptably small constant term in the energy resolution ( $\leq 7 \%$ ), (4) measures the angles of hadrons (particularly at large $|\eta|$ ) to better than $7 \%(\Delta \eta<0.07)$, and (5) limits the transverse spreading of hadronic showers. Even in the densest absorbers (tungsten) the transverse size of an hadronic shower is significantly larger than the jet cone size at $|\eta|=5$. About $90 \%$ of the energy of an hadronic shower is contained within a cylinder of radius $160 \mathrm{~mm}$ in a tungsten absorber. But the size of a jet with a cone size of $R=0.7$ at the front face of the GEM forward calorimeter at $4476 \mathrm{~mm}$ from the IP (see Table 5-12) is $42 \mathrm{~mm}$ in radius, covering only $7 \%$ of the area of the shower. A less dense absorber material would allow the hadronic shower to spread even more and make jet identification in this region more difficult. Also, in order to obtain fiducial coverage up to $|\eta|=5.0$, the physical coverage must extend to even larger $\eta$. A less dense absorber forces larger physical coverage in order to maintain the same fiducial coverage. 6,35

The location of the GEM forward calorimeters close to the IP is unique. The challenges due to rate, radiation damage, and transverse shower size are severe but this geometry, integrated with the endcap, reduces the cross-over problem at the transition, gives manifestly hermetic coverage, and allows GEM to mitigate the neutron problem.

\subsubsection{Forward EM Module}

Each forward calorimeter is divided longitudinally into three sections. The first is an EM section. It's purpose is to measure the energy and position of $\gamma$ 's from $\pi^{0}$ decay and to make a precision angle measurement of hadronic showers near the beginning of the shower where the transverse spreading is small. We have simulated the position resolution for hadron showers as a function of the depth of the first measurement. The position resolution has a minimum when the first section is $\approx 2.5 \lambda$. The resolution is $30 \%$ better than if the calorimeter were of full depth. This is crucial in obtaining $\Delta \eta / \eta=0.07$. The second "forward EM" section also helps in 
defining the angle for jets. In addition, since the tubes in all the forward calorimeters are parallel to the beams instead of being projective, this threefold longitudinal seqmentation provides a more accurate angular measurement. The EM section is a liquid argon sampling calorimeter chosen because the argon and absorber material are manifestly radiation hard. A novel electrode structure has been employed which gives very fast readout. Figure $5-46$ is an exploded view of one electrode which we call a "tube". It consists of an inner rod of the absorber material. The rod fits loosely within a tube, also made of the absorber material. There is a small gap, of order $100 \mu \mathrm{m}$, between the rod and tube which is maintained by a spiraled quartz fiber. Liquid argon fills this gap and the rod is held at high voltage $(-100$ V) relative to the tube. Tubes are evenly distributed within a matrix of absorber material. The purpose of this unusual electrode scheme is to avoid the positive ion buildup problem, even at the highest luminosities. ${ }^{36}$ The tubes have an ID of $5 \mathrm{~mm}$ and are arrayed hexagonally with nearest neighbor spacing of 7.5 mm center-to-center. The tubes are parallel to the beam so that they run longitudinally through the calorimeter. Tests of the performance of such electrodes as ionization chambers is described elsewhere. ${ }^{37}$ A close-up of the front face of this module in the region of the beam pipe is shown in Figure 5-47.

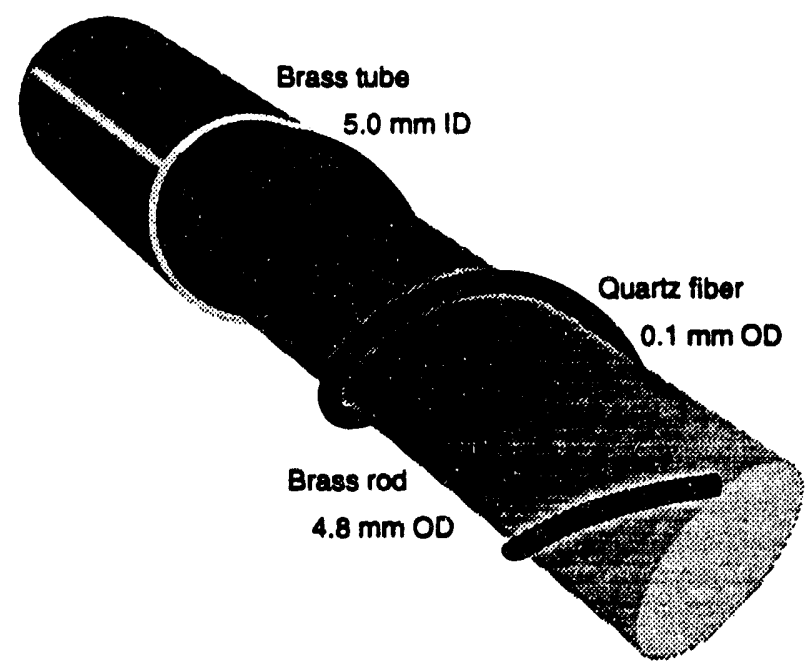

Tip-osces

FIG. 5-46. Exploded view of a tube electrode. The inner rod is held at high voltage while the outer tube is at ground. Liquid argon fills the gap between the rod and tube. A spiraled quartz fiber supports the rod within the tube, maintaining the gap.

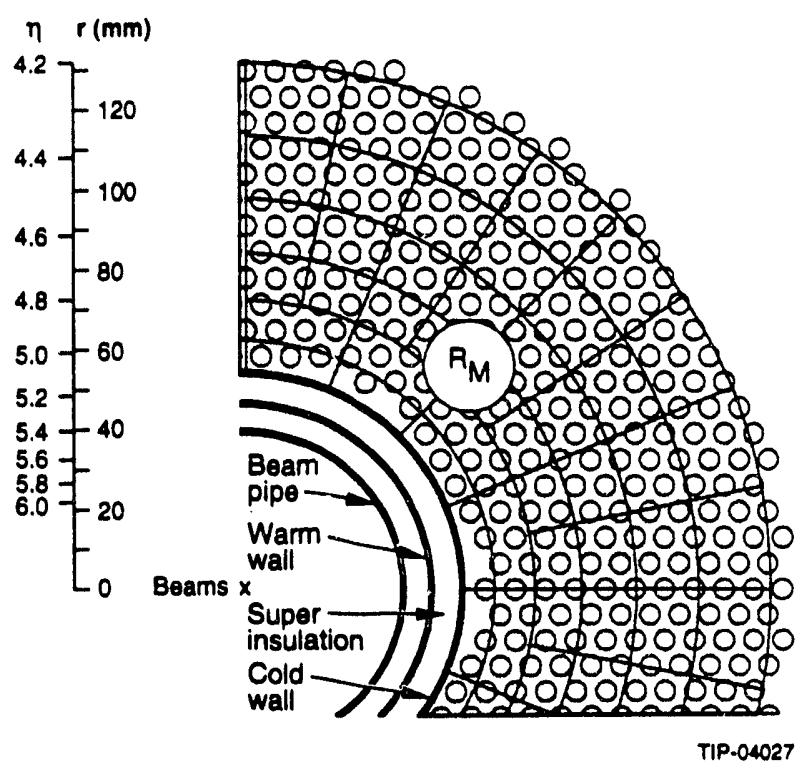

FIG. 5-47. Close-up of the front face of the EM section of the forward calorimeter in the region of the beam tube. $A$ circle with the Moliere radius is indicated.

The absorber material is copper in the EM section. Copper was chosen (rather than a denser absorber material like tungsten) for several reasons. (1) The Molière radius in copper is larger $(11.4 \mathrm{~mm})$ so that showers are sampled more uniformly across the face of the calorimeter. When the transverse EM shower profile is of order or smaller than the nearest neighbor tube spacing then spatial non-uniformities in the sampling can occur. Detailed simulation ${ }^{38}$ of this tube geometry give excellent position and energy resolution, well within our specifications. (2) Because the radiation length is longer, the region of shower maximum is spread longitudinally, relaxing radiation damage problems a bit but specifically reducing the positive ion buildup problem. (3) At luminosities of $10^{34} \mathrm{~cm}^{-2} \mathrm{~s}^{-1}$ the heat deposited in each EM forward calorimeter is 100 watts. The excellent thermal conductivity of copper is such that the temperature at the hottest spot in the EM section is less than $1 \mathrm{~K}$ above the temperature at the outer radius. This is quite acceptable for our cryogenic system. Within a liquid argon gap, heating due to the ohmic resistance to the drifting charges is larger than heating due to shower deposition. The thermal conductivity of liquid argon is low. However the geometry is such that the temperature at the middle of the gap is only milli-Kelvin above the temperature of the copper tubes and rods. Boiling of liquid argon is not a problem. (4) The cost of copper is much 
lower than the cost of tungsten, particularly when machined in the configurations required.

The EM module is $400 \mathrm{~mm}$ deep, about $24 \mathrm{X}_{0}$ and $2.4 \lambda$. The active volume covers down to $550 \mathrm{~mm}$ from the beam centerline, i.e., to $|\eta|=5.1$. This gives adequate fiducial coverage up to $|\eta|=5.0$. The sampling fraction by volume is $3.2 \%$. Three tubes are ganged together in the electronic readout. Low impedance cables carry the collected charge to common base preamps located on the outer periphery of the endcap calorimeter. (See Section 5.4.3). The ganged electrode capacitance is of order $1 \mathrm{nF}$. With a cable impedance near $10 \Omega$ the signal rise-time is $10 \mathrm{~ns}$. The electron drift time in the gap is 20 ns so an effective signal gating time of about two bunch crossings is practical. Table 5-12 gives the physics pileup noise and the electronics noise at a luminosity of $10^{33} \mathrm{~cm}^{-2} \mathrm{~s}^{-1}$ within a jet cone size which contains both the jet and the hadronic shower. After the preamplification, signals are summed in tower arrangements near the beam pipe as shown in Figure 5-47 and overall as in Figure 5-48. The tower segmentation is chosen to give adequate angle resolution for both EM showers and the initial hadronic shower. GEANT simulation of this geometry is used in the GEM physics simulation work discussed elsewhere in his document.

\subsubsection{Forward Hadronic Modules}

The hadronic sections of the forward calorimeter complete the energy and angle measurement for hadronic showers that started in the EM section. The first hadronic module is similar to the EM module. It is a liquid argon sampling calorimeter with tube electrodes. The gap in the tubes is $300 \mu \mathrm{m}$ since the ionization rate is about a factor 10 lower than in the EM module. The center-to-center tube separation is

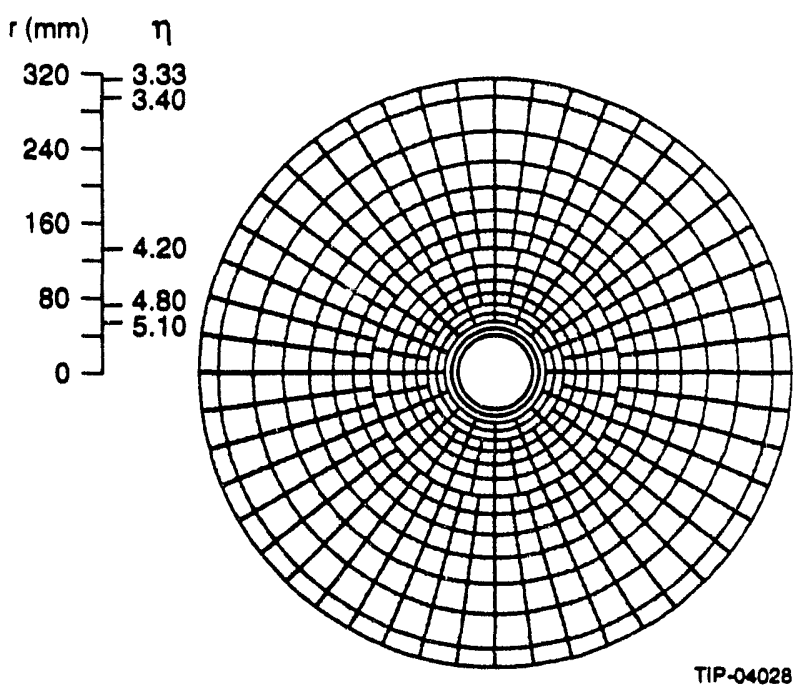

FIG. 5-48. Front face of the EM section of the forward calorimeter showing the readout segmentation. The scale at the left gives the dimensions in $\mathrm{mm}$ and the $\eta$ coverage.

larger in order to form pseudo-projective towers. Brsides providing another longitudinal section this arrangement reduces a source of neutrons leaking out between the endcap cryostat walls.

The last hadronic module is outside of the endcap cryostat. The sensitive material is scintillating liquid which slowly flows through capillaries parallel to the beam. See Figure 5-49. Because of the geometry it is natural to separate this module from the endcap. A separate technology is appropriate because performance demands for this section are dramatically different from the EM module. The peak ionization density and radiation are an order of magnitude lower and the transverse size of showers much larger. So the damage potential is less and the segmentation requirements relaxed. The front face of this hadronic section is located at $5150 \mathrm{~mm}$ from

Table 5-12. Variation of selected parameters across the face of the for ward calorimeter at $10^{33} \mathrm{~cm}^{-2} \mathrm{~s}^{-1}$. Noise values are within a jet cone and within an hadronic shower radius.

\begin{tabular}{cccccc}
\hline$\eta$ & $\begin{array}{c}\mathrm{R}=0.7 \text { cone } \\
(\mathrm{mm})\end{array}$ & $\begin{array}{c}\text { Current Draw } \\
(\mathrm{nA})\end{array}$ & $\begin{array}{c}\text { Physics Pileup } \\
E_{T} \text { Noise } \\
(\mathrm{GoV})\end{array}$ & $\begin{array}{c}\text { Thermal } \\
E_{\text {T Noise }} \\
(\mathrm{GeV})\end{array}$ & $\begin{array}{c}\text { Dose at EM } \\
\text { Shower Max. } \\
(\text { Mrad/SSC yr) }\end{array}$ \\
\hline 3.4 & 205 & 0.03 & 1.1 & 1.7 & 0.9 \\
3.8 & 138 & 0.09 & 1.1 & 0.8 & 2.8 \\
4.2 & 92 & 0.3 & 1.2 & 0.4 & 9.3 \\
4.6 & 62 & 1.0 & 1.8 & 0.3 & 30 \\
5.0 & 42 & 3.3 & 2.7 & 0.2 & 96 \\
\hline
\end{tabular}


the interaction point (IP) and is approximately $1.5 \mathrm{~m}$ in length. With an inside diameter of $84 \mathrm{~mm}$ and an outside diameter of $1434 \mathrm{~mm}$, this hadronic section covers $|\eta|$ from 2.80 to 5.63 at the middle of the active section. Each end will consist of 328 channels. This hadronic forward calorimeter has approximately $14 \%$ sampling fraction by vulume. The active length is approximately $1315 \mathrm{~mm}$ $(115.3 \mathrm{~mm} / \lambda)$ in length and weighs approximately $47 \mathrm{Mg}$ per end. The readout section (readout fibers, light mixer, PMTs and cables) will require an additional $600 \mathrm{~mm}$. The segmentation is chosen to keep the dimensions in the $\eta$ and $\varphi$ directions approximately the same and to match segmentation breaks in the EM section.

The absorber material is tungsten (heavymet) in order to limit the transverse spreading of hadronic showers. This choice allows adequate $E_{T}$ resolution up to $|\eta|=5.0$ where transverse shower leakage down the beam pipe begins to dominate the resolution. The total depth of the three sections is $16 \lambda$. Currently, this forward calorimeter design is based on commercially available $3 \mathrm{~mm}$ OD $(0.15 \mathrm{~mm}$ thick) copper tubes within the tungsten absorber matrix. The inner wall of the copper tubing is lined with $0.1 \mathrm{~mm}$ thick glass tubing to produce total internal reflection (TIR). Each hadronic forward calorimeter will be contained in a stainless steel shell.

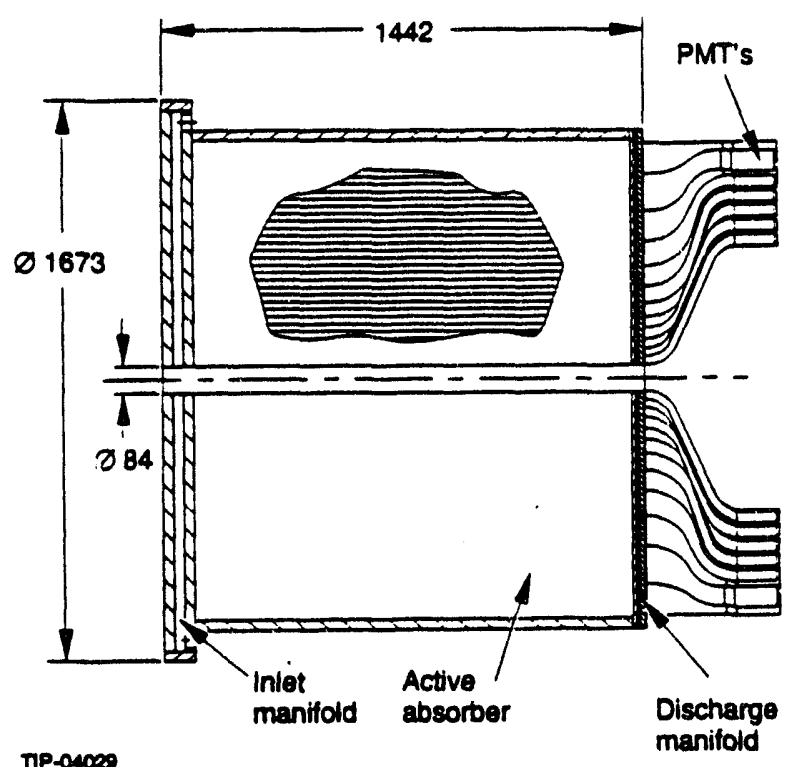

FIG. 5-49. A slice along the beamline of the hadronic forward $c$. imeter section.
The scintillating liquid from each forward calorimeter module will be continuously circulated through a dedicated filtration and purification system to prevent excessive degradation of the liquid due to radiation damage. Each module will have $-n$ inlet manifold at the front end (facing the interaction point) and a discharge manifold at the back end (see Figure 5-50). The filtration and purification systems for the scintillating liquid forward calorimeter will be placed in the utility shaft of the experiment hall. The sensing materials (scintillating liquid) currently being considered for the forward calorimeter have as their primary solvent light mineral oil. Both of the mixtures have approximately the same chemical composition, however one is commercially produced, BC-517 and the other is a custom mix produced for the MACRO experiment. Each dissolves approximately 5-15\% pseudocumine and further wave length shifting chemicals along with an anti-oxidant preservative to make their mixtures.

The scintillation light emitted in the capillaries is collected at the back end of a tower by fused silica quartz fibers across a small gap from the capillaries. Each tube is extended into a separate bore to prevent

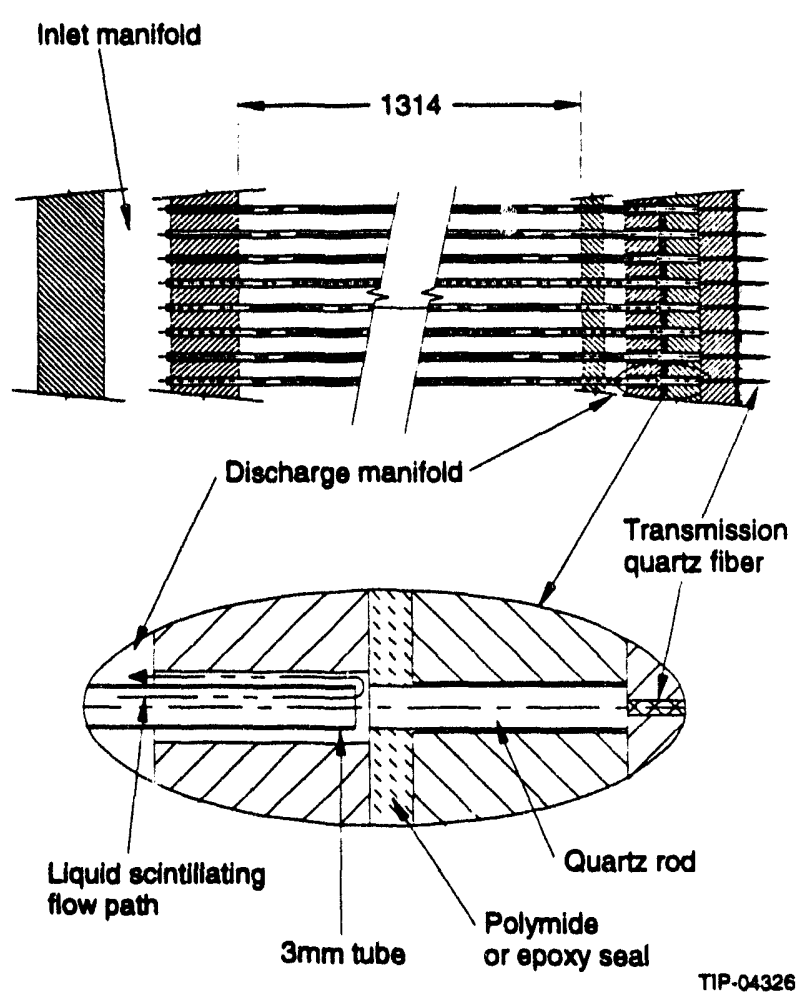

FIG. 5-50. Details of the capillaries which hold the liquid scintillator in the hadronic section of the forward calorimeter. 
light mixing between the adjacent tube positions. One end $(\sim 1 \mathrm{~mm}$ diameter) of the opt $i$ al fiber is aligned to each tube end to collect the scintillation light in the tube. The other ends of the optical fibers for each physics tower are bundled into clusters and connected to the light mixers and/or photomultiplier tubes at the back end of the calorimeter. The readout fiber bundles are designed to allow easy replacement of relatively small sections of radiation damaged fibers without draining the liquid from the forward calorimeter module.

\subsubsection{Options}

As opposed to most other GEM subsystems the technology choices for the forward calorimeter are not made to the same $c$ gree of definition. Final decisions are scheduled for the Fall of 1993. Presented above is the present baseline and possible options are sketched below.

The GEM collaboration is exploring the possibility of an hadronic section which uses quartz fibers as the sensitive medium instead of liquid scintillator capillaries. These fibers would be aligned horizontally within a tungsten matrix and read out -ia photo tubes in much the same geometry as the liquid scintillator version.

An alternative to the baseline is to locate the forward EM module and the inner hadronic modules of the endcap within a separate cryostat at the same $z$ position as the present design. The hadronic forward calorimeter would be attached to this separate cryostat. This option will allow 1) easier installation snugly around the delicate beam pipe since the separate forward calorimeter assembly is now lighter than the combined endcap, 2) the forward calorimeter to be removed for those maintenance operations where personnel exposure to the activated forward calorimeter is a problem, 3 ) access to bellows in the endcap cryostat vacuum wall in case of a vacuum leak, and 4) more flexibility in the design and implementation.

Options include 1) three modules in depth, all liquid argon sampling calorimeters, 2) a monolithic liquid scintillator $\mathrm{c}^{-}$, rimeter including the EM section, 3) a monolinnic quartz fiber calorimeter including the EM section with a modified sampling fraction, and 4) high pressure gas sampling calorimetry.

\subsection{THE CALORIMETER RESEARCH AND DEVELOPMENT PROGRAM}

\subsubsection{Overview}

The GEM cryogenic calorimetry must achieve a level of performance which exceeds that of any previous device of this type. While the design of liquid argon calorimetry is basically well-understood, with many successful devices of this type having been employed in high energy physics both in the United States and Europe, it is nevertheless essential that an ongoing program of calorimeter testing and calibration be conducted in parallel with construction of the production modules, in order to optimize the performance of the GEM calorimeter. The calorimetry choices for GEM were strongly influenced by radiation damage considerations. Our goal is to have a robust system capable of many years of operation at luminosities of $10^{34} \mathrm{~cm}^{-2} \mathrm{~s}^{-1}$. Our choices allow this, but there are many details in the construction that require particular care.

The primary goals of this program are coupled and complementary: (1) to refine the design and fabrication of the GEM calorimeter with the goal of improving the performance of the device ultimately employed by GEM, and (2) to study the actual characteristics of this device in order to optimize its use in GEM. The testing and calibration program makes use of beam time at BNL, CERN, FNAL and the SSCL.

\subsubsection{The BNL R\&D Program at the AGS}

In 1992, GEM tested a small prototype accordion calorimeter at the Brookhaven AGS using both argon and krypton as sampling media as discussed in Section 5.3. It is anticipated that BNL beams will continue to play a significant role in GEMs calorimeter testing program prior to the initiation of full-scale testing at Fermilab. Specifically, there will be a test of the forward EM calorimeter at BNL in 1993.

\subsubsection{The CERN RD3 R\&D Program}

The RD3 calorimeter testing program at CERN is focused primarily on developing calorimetry for use by the ATLAS experiment at the LHC. The cryogenic option being considered by ATLAS employs liquid argon throughout; however, an accordion-type readout is planned. This makes this 
R\&D program especially relevant to GEM, and members of the GEM calorimeter group have participated in RD3 from the beginning.

A sequence of annual beam tests of increasing sophistication have been carried out, starting in 1990 when a non-projective accordion calorimeter with 40 ns shaping time was evaluated. In 1991, the first projective prototype was tested, and the shaping time was reduced to $20 \mathrm{~ns}$. In 1992, a $2 \mathrm{~m}$ long projective electromagnetic calorimeter with accordion readout was exposed to a beam of $\sim 300 \mathrm{GeV} / c$. At the same time, an accordion hadronic calorimeter employing $10 \mathrm{~mm}$ thick iron absorber was tested.

It is anticipated that the RD3 program will continue to play an important role in the GEM calorimeter development during the period prior to initiation of GEM's Fermilab test beam program in late 1994. In particular, in 1993-94, tests of alternate designs for endcap calorimetry are scheduled to be performed at CERN as well as an EM prototype for the forward calorimeter.

\subsubsection{The FNAL Test Beam Program in MWEST}

The highest energy beams in the world are those available at Fermilab, which makes this laboratory an ideal location in which to test and calibrate SSC detector components. As discussed in Chapter 13, GEM proposes to mount a comprehensive test beam program in the Meson West (MWEST) area at Fermilab. The primary goals of the calorimetry component of this program are the following:

- Perform full-scale engineering studies of all calorimeter subsystems: mechanical, electrical and cryogenic;

- Achieve full system integration of the electromagnetic and hadronic cryogenic calorimetry, and of the external (scintillating) calorimetry;

- Carry out a full system test of the calorimeter readout and calibration systems;

- Measure the response of the scintillating calorimetry, and determine the reproducibility of its performance from module to module;
- Study the calorimeter's resolution at the highest available energies;

- Investigate the calorimeter's response in the vicinity of all cracks;

- Study the response for single particles across the barrel to endcap transition region;

- Investigate the calorimeter's response to high energy muons.

To carry out these tests, the MWEST beamline will be instrumented with silicon strip detectors capable of tagging incident particles to a precision of $\pm 0.2 \%$. It will also be equipped with high energy electron identification. A versatile calorimeter transporter will be constructed and mounted on the existing MWEST rail system. An end view of the test cryostat mounted on its transporter is shown in Figure 5-51. The transporter provides for independent horizontal and vertical motion, as well as for rotation and tilting capability. Figure 5-52 displays the MWEST hall in a view which emphasizes the immediate vicinity of its rail system. The test cryostat is shown in several positions-out of the beamline in the orientation used for loading, and in the beam at the two angular extremes required to carry out the full set of beam tests that will be required.

Plans call for the testing of two full-size (4 m long) $9^{\circ}$ electromagnetic wedges, each consisting of three readout layers. The hadronic towers are also full-size, split at $90^{\circ}$ into two $2 \mathrm{~m}$ long modules, as in the final GEM calorimeter. The hadron calorimetry also employs two physical, but three readout, layers. To completely contain hadronic showers, the first physical layer will contain four modules, and the second three, on each side of the $90^{\circ}$ split. The outer modules will be shifted in azimuth by $4.5^{\circ}$ relative to the inner modules to minimize the consequence of cracks. The outer hadronic modules (in radius, relative to the GEM axis) are full size, while those at larger $\eta$ (in the GEM context) are special units truncated to fit within the test cryostat. Behind the test cryostat are two full-size wedges of the external scintillating calorimeter. 


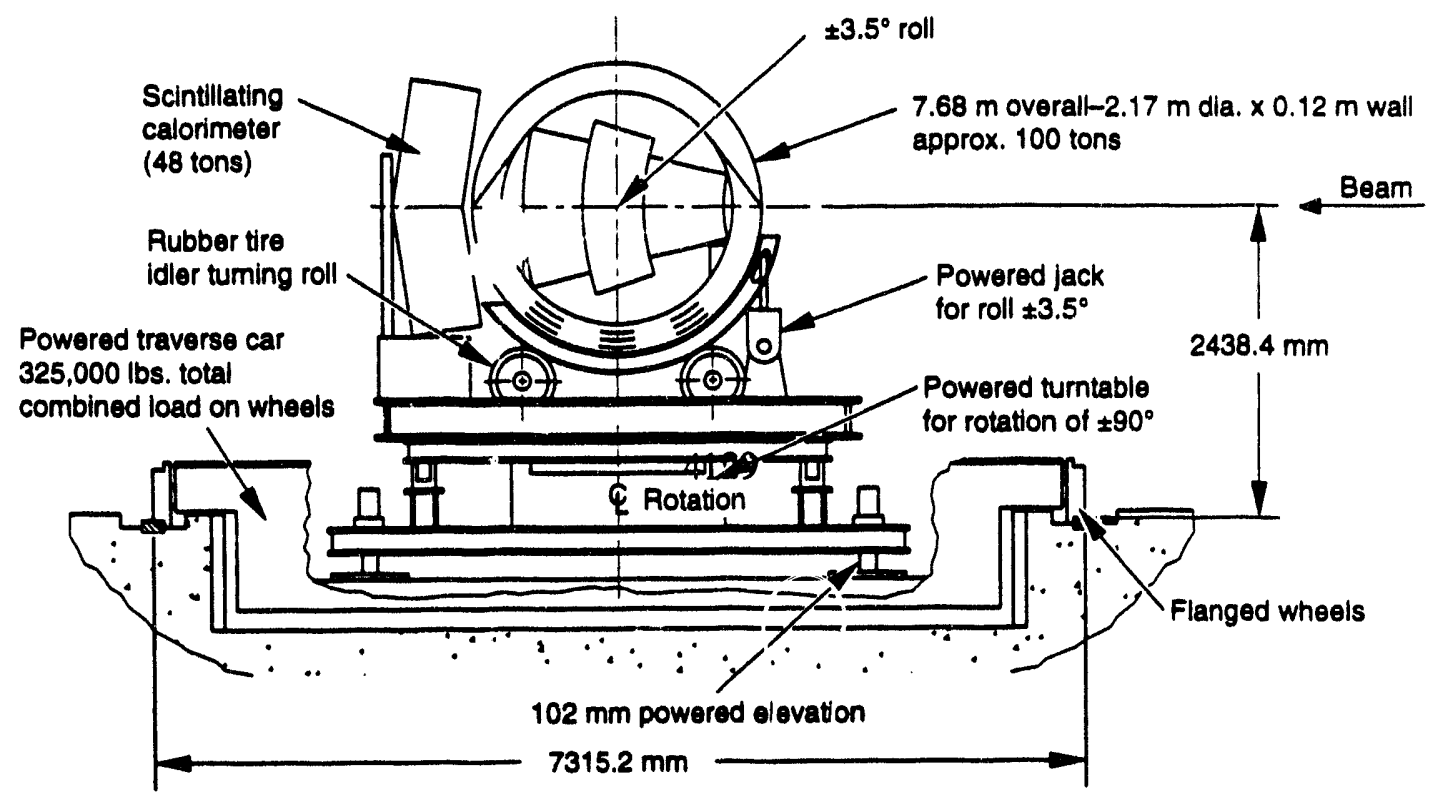

TP-04129

FIG. 5-51. GEM Test Beam Cryostat transporter.

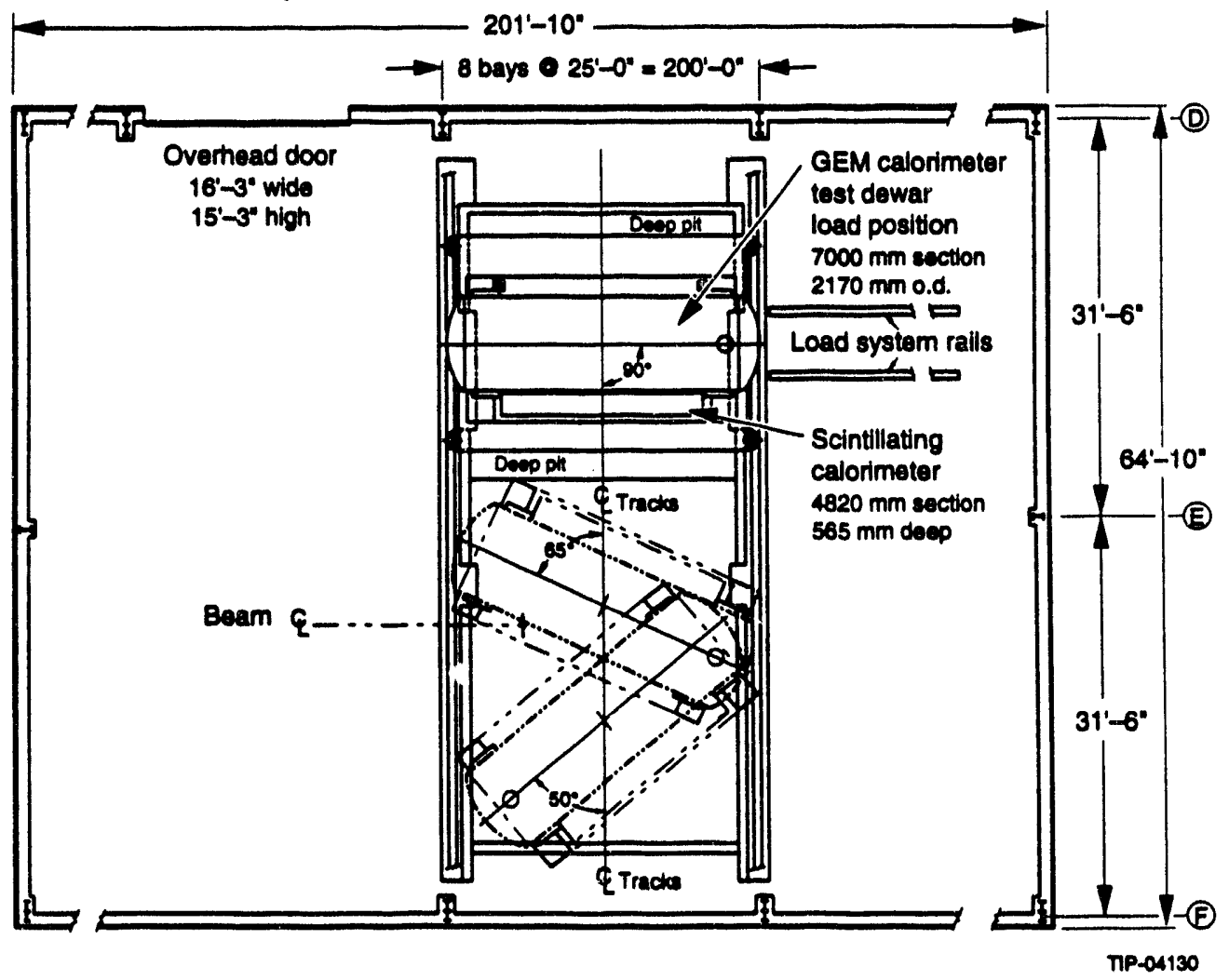

FIG. 5-52. GEM Test Beam Facility in FNAL's MWEST Hall. 
Our strategy allows great flexibility in the mix of modules included in a given load of beam testing. These include starting with only one side of the hadron calorimetry, thus postponing the study of the $90^{\circ}$ crack, or delaying the installation of the endcap prototypes, thus postponing the investigation of the transition region. The test cryostat and transporter are being designed to accommodate multiple loads with minimum down time. MWEST beam time will be available for other GEM test beam purposes during these transition periods. The GEM endcap calorimeter will employ liquid argon. To avoid the expense of building separate test cryostats, plans call for conducting the MWEST tests using first argon and then krypton throughout a single test cryostat. Monte Carlo simulation will be employed to extrapolate the measured beam data to the actual GEM configuration.

\subsubsection{The SSCL Test Beam Program at the MEB}

The Fermilab running schedule calls for fixed target operations commencing in FY 1995. It is clear, however, that the complete program of GEM calorimeter studies cannot be finished in a single run, and that beam tests in 1996 and beyond will be required. Since the present schedule for test beam capability at the SSCL MEB calls for operations staring in late 1996, this facility could in principle provide this follow-on calorimeter testing capability. One complication, however, is that the maximum energy of the MEB-based program is $170 \mathrm{GeV}$, which precludes testing at energies fully characteristic of secondary particles produced in SSC collisions, particularly at the forward rapidities intercepted by the endcap calorimetry. Further complicating long-range calorimeter $R \& D$ planning is the fact that the details of the Fermilab running schedule for FY 1997 and beyond are not presently known. It is, however, certain that there will need to be high energy fixed target running during this period in order to complete the already approved Fermilab physics program, and moreover, once Main Injector operations commence, $120 \mathrm{GeV} / \mathrm{c}$ test beams in parallel with collider operation are planned to supplement the intermittent program of $800-900 \mathrm{GeV}$ fixed target running.

To prepare for all eventualities, the MWEST test cryostat transporter is being designed with the constraints of GEM's area of SSCL's Calibration Hall in mind. Thus, a second device sould be quickly constructed for installation in this area should circumstances warrant setting up a second full-scale calorimeter testing program at the SSCL. Once higher energy test beams from the HEB become available, it is clear that GEM calorimeter studies at the SSCL will be the option of first choice.

One potentially very useful study that could realistically only be carried out at the SSCL is a full-scale $(2 \pi)$ test of the final endcap EM calorimeter, backed up by at least three azimuthal towers from the first layer of the endcap hadronic calorimeter. Such test data would provide a valuable transition between the FNAL endcap prototype results, and performance studies of the actual GEM endcap calorimeter. The practical feasibility of this final beam test will depend on financial and personnel constraints, and on the relative timing of the MEB test beam commissioning and GEM calorimeter installation.

\subsubsection{Other R\&D Tests}

In addition to the test beam work referred to above, there are other active $R \& D$ programs in progress. We give only a few examples. As discussed in Section 5.8.2, all materials used in the calorimeter need to be able to withstand the radiation doses at the places they will occupy as well as not contaminate the liquid ionization media. A recent test by RD3 has irradiated an EM absorber lamination with $10^{15}$ neutrons $/ \mathrm{cm}^{2}$. No adverse effects and no outgassing were observed. ${ }^{39}$ There is another program to develop with vendors the stripline cables and feedthroughs specified in 5.9.2. For the liquid hadron calorimeter, tile lamination techriques are being developed. For the scintillator calorimeter, basic measurements of light yield and uniformity are being made. For the liquid scintillator forward calorimeter, the main task is to find the most stable components for the extreme radiation environment that will be encountered.

\subsection{RADIATION HARDNESS}

\subsubsection{Electronics}

The calorimeters for $\eta<3$ at hadronic collider experiments would be exposed to ionizing radiation levels in excess of $500 \mathrm{kGy}$ (50 Mrad) and neutron 
fluences of up to $5 \times 10^{14} \mathrm{n} \mathrm{cm}^{-2} /$ year of operation at full design luminosity in a limited region near the forward EM(see Chapter 12). The front-end electronics has to withstand not only a cryogenic environment but also such substantial radiation levels.

\section{Gamma Ray Irradiation}

By far the most sensitive parameter potentially affected by the irradiation is the device noise. A promising solution for rad-hard low noise front-end electronics is represented by silicon JFET circuits. Several low noise JFETs built in a monolithic process have been irradiated at room temperature in a power-on condition up to an integrated total dose of $1 \mathrm{MGy}(100 \mathrm{Mrad})$ using a ${ }^{60} \mathrm{Co} \gamma$-ray source. ${ }^{40}$ DC characteristics are hardly affected even after this total dose with the exception of the gate current which increases up to several $\mathrm{nA}$ at room temperatures. This is far below the condition from noise at this current to be less than about $10 \mu \mathrm{A}$. The increase in gate current happens at very low doses $(-10 \mathrm{kGy})$ and increases slowly after that. At cryogenic temperatures this increase is completely recovered. Figure 5-53 compares the noise performance of the cryogenic preamplifier developed for the LAr/LKr GEM calorimeter before and after a $100 \mathrm{Mrad}{ }^{60} \mathrm{Co}$ irradiation. No increase in the equivalent noise charge (ENC) has been measured at cryogenic temperatures (actually the values after irradiation appear lower, due to the fact that different batches have been measured). The increase in ENC measured at room temperature is due to the $1 / f$ noise increase and the gate leakage current. Tests performed on $\mathrm{GaAs}$ based preamplifiers show greater degradation due to ionizing radiation.

\section{Neutron Irradiation}

Neutrons are energetic enough to cause lattice damage in the crystal structure. Silicon JFETs still compare favorably with respect to other devices. In comparative tests, silicon JFET preamplifiers and GaAs preamplifiers have been irradiated up to $\sim 4.6 \times 10^{14} \mathrm{n} \mathrm{cm}^{-2}$ (with a contamination of $23 \mathrm{Mrad}$ of $\gamma$-ray) at room temperature. ${ }^{41}$ Both types of preamplifiers were able to withstand the tests. The silicon JFET ones have shown less noise degradation in the region of interest for SSC calorimetry. Measurements of the equivalent noise charge (ENC) for silicon JFET preamplifiers at the liquid argon temperature $(87 \mathrm{~K})$ after $-4.610^{14} \mathrm{n} \mathrm{cm}^{-2}$ are plotted in Figure 5-54. The ENC increase is about $35 \%$ at $90 \mathrm{~K}$ at $50 \mathrm{~ns}$. For the same parameters, the GaAs preamplifiers exhibit an $80 \%$ increase in ENC. Such neutron dose levels however are an extreme which will be reached only in the high rapidity region. In the forward EM calorimeter where a high neutron flux is expected, we have already planned to locate the preamplifiers in the junction boxes outside of the cryostat (see Figure $5-1$ ) because at the short shaping times ( $20 \mathrm{~ns}$ ) there is less benefit in signal to noise ratio in having the preamp close to the electrode.

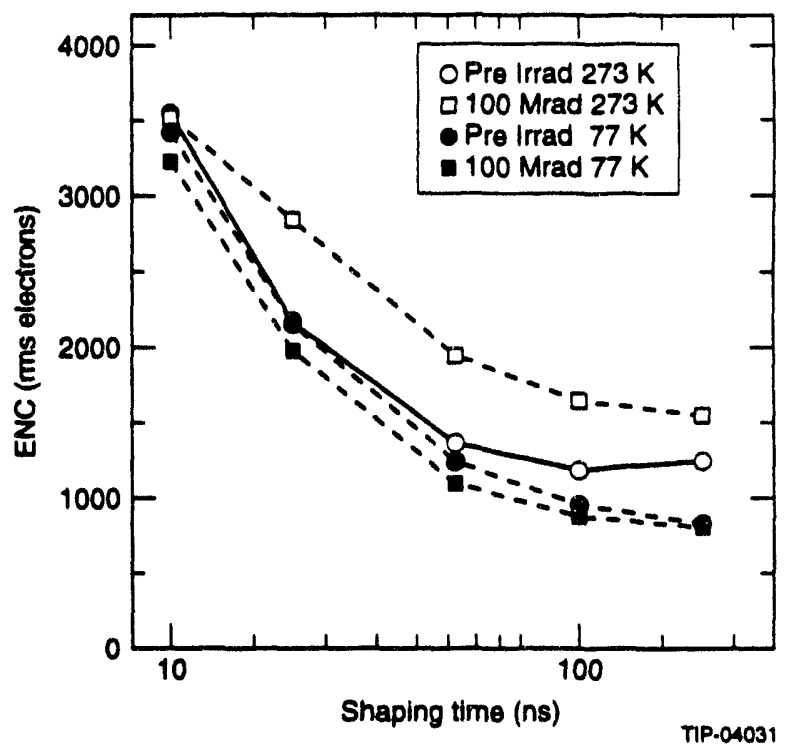

FIG. 5-53. Gamma irradiation of JFET preamplifiers to 1 MGy (100 Mrad). Equivalent noise charge vs. pulse shaping (peaking time) is shown.

\subsubsection{Materials}

With extensive use of liquid argon calorimetric techniques considerable experience has been acquired with "safe" materials which can be used within the calorimeter and do not add contaminants to the liquid which degrade the signal. Such contaminants can either attach electrons or catalyze recombination. Both degrade the signal in different ways. The first has little effect at short shaping times. The GEM calorimeter design benefits from this experience but there are several departures which require $R \& D$ in order to determine with certainty which materials are safe. Because the barrel calorimeter will use liquid krypton and because krypton has a boiling point $\sim 30^{\circ} \mathrm{C}$ above argon and is therefore more susceptible to poisoning than argon, 
(a)

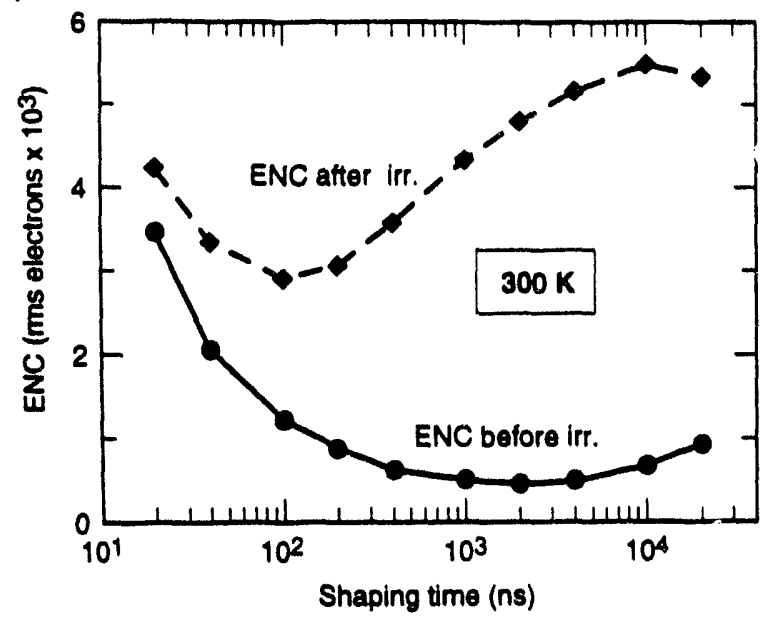

(b)

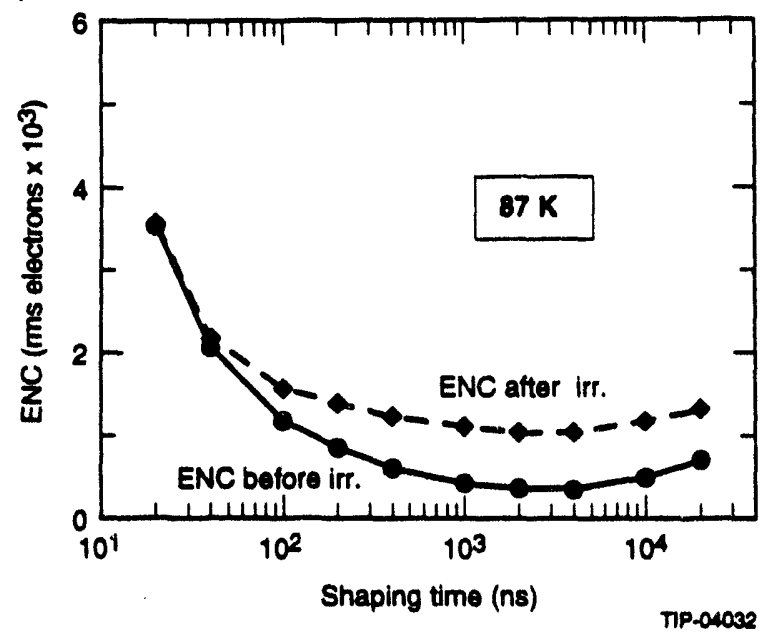

FIG. 5-54. Equivalent noise charge of JFET preamplifiers before and after irradiation with $4.6 \times 10^{14} \mathrm{n} \mathrm{cm}^{-2}$.

we have initiated a program to test a variety of materials in liquid krypton. For example during the test beam work in the summer of ' 92 at BNL where liquid krypton was used in the test, we found that Rohacell foam, which was used as an excluder in liquid argon, caused a mild loss of signal in the krypton which was recovered using our purifier.

The very high doses to which parts of the GEM calorimeter system will be exposed may damage "safe" materials and become a new source of contamination. The collaboration has established centers where contamination studies under extreme radiation will take place. The University of Arizona has acquired a high energy ( $320 \mathrm{kV}$ ) DC x-ray source, and a fast Fourier transform infrared spectrometer; and has built a special cryostat and test calorimeter cells in order to measure the effect of anything released by common calorimeter construction materials under extremes of ionizing radiation in argon and krypton. The setup is nearly complete and testing will start soon thereafter. Our collaborators at Tsinghua University will test contamination of materials exposed to very high neutron fluences. This R\&D work over the next year will firmly establish a list of safe materials which we will use in the affected parts of the calorimeter system.

\subsection{SERVICES: CABLES, CRYOGENICS, VALVES AND VESSELS}

\subsubsection{Cables, Feedthroughs and Interconnections}

\section{Cables Inside The Cryostat}

There are several different types of cable that penetrate the cryostat. ${ }^{42}$ These cables are used to provide power to the active elements inside the cryostat, provide high voltage across the ionizing gap, provide in place calibration and to extract the signals froin the individual towers.

\section{Summing, Cables \\ Hadronic: Sections}

Summing cables are used in the hadronic sections of the barrel and the endcaps to add the signals from the individual tiles to form towers. These cables are low impedance Kapton dielectric striplines with a characteristic impedance of about $7 \Omega$. Figure 5-33 shows a typical hadronic module with the summing cables exposed.

\section{EM Section}

The EM section in both barrel and endcap calorimeters use finely segmented strips to improve pointing and $\pi^{\circ}$ rejection. The sums are formed, using flexible kapton strips, that are situated between the signal electrodes and the preamplifier boards. The connection between signal electrode and summing board uses a standard AMP (crimp type) flat cable connector.

\section{Signal Cables}

The signal cables between the preamplifiers, located on the mother board, and the junction box, located outside the cryostat, are $50 \Omega$ stripline transmission cables. These are three layer flexible printed circuit boards. The outer layers are shield or ground layers and the inner layer carries the signal 
traces. The stripline cable with kapton as a dielectric material represents a high quality low loss transmission line. Kapton is extremely resistant to radiation damage and contains none of the additives that can poison argon or krypton.

\section{Calibration Cables}

Several cables are rc uired for the proposed calibration scheme. These include the bias voltage, the DC current source, the clock signal and the select lines. A brief description of each calibration function follows, along with the cable requirements.

\section{Clock Lines}

The clock lines must be a high quality transmission line. Typically the rise time of the clock pulse which mus: te preserved is two to four nanoseconds. The clock signals will be most likely generated in the junction boxes. A single clock line can drive up to 36 calibration circuits. Each clock signal will drive 18 calibration channels. The clock lines, like the signal lines, will be high quality stripline transmission lines. Care must be taken to insure that the feedthroughs do not introduce any impedance mismatch.

\section{Channel Select}

The channel select lines are TTL levels and toggle at about $1 \mathrm{kHz}$. Since these control lines serve many channels, the total number of calibration lines required for each module is small and may be handled with only a few stripline cables of the type used for signal cables and clock pulses. The logic and cable drivers required for each module will be located in the junction boxes. The most efficient use of cable space would suggest that a series to parallel conversion of the select lines be performed in the junction boxes. This is a technique that we have used many times in the past with good results.

\section{Bias and Current Source}

The bias and current lines carry quiescent sources. The line quality is less critical. The current source will be driven externally by a DAC. These DC signals should not be disturbed by pick up or low frequency line signals. The cable adhesives should be chosen to provide a very high impedance $D C$ path and should not provide a leakage path, which would compromise the integrity of the current source.

\section{Power Cables}

We expect to be able to use the same type of cable for both power and high voltage distribution. The diameter of the inner conductor should be such that a voltage drop of no more than $50 \mathrm{mV}$ occurs under normal operating conditions. This may require more than one cable in parallel, especially on the ground returns. Since prudent design would require a redundant feed for each power and high voltage connection, and noting that the conductivity of copper increases by a factor of five at cryogenic temperatures, staying below the maximum voltage drop requirement is easily achieved. The cable would have a solid core of copper wire covered with an extruded radiation hard dielectric jacket. Cables would pass continuously from warm to cold feedthrough with no interconnect in between.

\section{High Voltage Cables}

High voltage cables are similar to the power cables. The dielectric material must be sized to withstand an operating voltage of between 2 and $3 \mathrm{kV}$ under normal operating conditions.

\section{Feedthrough And Interconnections}

Signal from the preamplifiers located inside the cryostat must be transported to signal processing electronics, which is located outside of the cryostat. Specialized feedthroughs, which transport the signals from the cryogenic containment vessels, through the insulating vacuum walls, and to junction boxes (located along the periphery of the vacuum vessel) must be designed and constructed. The feedthroughs must faithfully transmit analog signals with rise times between 5 and 10 nanoseconds and introduce no reflections or crosstalk between channels. A quality $50 \Omega$ characteristic impedance transmission line is required. Besides providing good signal fidelity, the feedthrough design must maximize the signal density, minimize the heat loss along the cable length, as well as providing an excellent vacuum seal. Not only must the insulating vacuum be maintained, but the seal must prevent oxygen (which would poison the ionization liquid) from migrating into the containment vessel.

Two feedthrough designs are being evaluated at present. ${ }^{17}$ One concept uses glass sealed pins in a metal matrix which connects to $50 \Omega$ flat cable on 
both sides. This proposal is based on a successful feedthrough design used at the SLD detector. The second design uses several continuous flat 50 stripline cables, sealed into a stainless steel flange, using a proprietary process which IBM calls Cyberflex. Both designs incorporate a double feedthrough at the inner cryostat wall. Auxiliary pumping is possible in this region for added security, should a small leak develop during the operation of the detector.

\section{Junction Box}

The junction box contains cable drivers for all signal lines, calibration electronics, narrow range voltage regulation for preamplifiers and filtering for high voltage supplies. In addition, the junction boxes, which are located on the endcaps, contain external preamplifier electronics for EM towers with large rapidity. Stripline signal cables, each with 36 signal channels, connect to 72 channel cable driver boards. The cable driver boards (approximately $228 \mathrm{~mm} \times 254 \mathrm{~mm}$ ) contain 24 hybrids, each with three cable driver channels. To reduce the electromagnetic interference, it is advantageous to add gain and a differential cable driver, to drive the long twisted pair cables that connect the junction box and the analog pipeline. Since the strong magnetic field in this region eliminates the use of transformers, our only solution is the use of active drivers.

\section{Cables Outside The Cryostat}

\section{Signal Cables}

We have had success in earlier calorimeter designs using individually shielded twisted pair cables. Each twisted pair is enclosed in a conductive foil with connections made to a continuous drain wire. The combination, twisted pair cable and shield, is covered with a PVC jacket. The jacket is designed to lay on $2.54 \mathrm{~mm}$ centers. Each twisted pair, shield, drain wire, and jacket assembly, is woven into a flat cable of 36 pairs. Standard IDC terminations are made to commercially available connectors. The drain wires must be individually soldered to connector shields. The balanced line is designed to have a characteristic impedance of $100 \Omega$.

\section{Power Cables}

The cables providing power to the junction box and preamplifier electronics will be sized to minimize the voltage drop along their length. It is assumed that power conversion and pre-regulation will be accomplished in an area where the stray magnetic field is minimal and standard regulation methods can be employed. The final power regulation for the preamplifier power will be done at the junction box, using narrow range regulators.

\section{High Voltage Cables}

High voltage connections between the high voltage power supplies and the junction box are made with RG 59 cable, using standard SHV connectors. It is assumed that passive filtering of the high voltage lines will be performed in the junction box. We recommend the use of standard multiwire proportional chamber high voltage power supplies with current limiting and external monitoring. These supplies are available from a number of sources and packaged as standard NIM modules.

\subsubsection{Cryostats}

\section{Barrel Cryostat}

The inner barrel calorimeter consists of a liquid krypton vessel (liquid vessel) and an outer vacuum vessel (vacuum jackct). An isolating structural support carries the weight of the liquid vessel to the feet of the vacuum jacket and minimizes the thermal heat leak into the liquid vessel. This support is thermally efficient and allows deflections resulting from the thermal expansion of the liquid vessel.

The material selected for construction of the liquid vessel was 5083 aluminum, a material acceptable to the American Society of Mechanical Engineers Boiler and Pressure Vessel Code (ASME Coded). This material was chosen for its cryogenic temperature properties, its ease of welding, its long radiation length characteristic, and economics both in raw material cost and efficiency in construction.

The vessels will be designed using flanged joints, where possible, which will be sealed by welds on final assembly. This has several advantages. The vessels can be fabricated in smaller pieces, as- 
sembled to verify fit and assembly methods/tools at the point of manufacture, easily shipped, and a light weight vessel is obtained.

The design of the vessels will be to the intent of the current ASME Code, Section VIII Division 2. The thermal/structural supports between the vacuum and the liquid vessels will utilize materials selected from the ASME Code but will be designed to the requirements of the American Institute of Steel Construction Ninth Edition of the Steel Construction Manual (AISC Hand Book). Both the liquid and vacuum vessels will be designed to the intent of the ASME Pressure Vessel Code, only the liquid vessel will be a stamped vessel.

The liquid vessel design has to incorporate the ability to carry the structural loads that represent the various phases of assembly as well as its operational loads. Assembly loads will govern the configuration of some vessel elements. For instance the stay between the EM modules and the first layer of fine hadronic modules must act as a segment of an $8 \mathrm{~m}$ simply supported beam carrying the total weight of the liquid vessel, less the outer shell, during the installation of the outer vessel shell. These beam loads will effect the thickness of the stay and govern the bolt configuration at the end of the stay.

\section{Endcap Cryostats}

The design approach for the endcap vessels is similar to the barrel calorimeter with allowances for its unique loads and size. The primary design approach difference being the bolt-in stays outside the EM and inner hadronic modules. The bolt-in stay design reduces the amount of welding on assembly and permits assembly verification at the source of fabrication. The vacuum vessel of the endcap calorimeter cannot carry tension or compressive loads through the inside of the forward EM without requiring that tube to be thick. A bellows will be used to prevent the pressure load from passing through the forward EM. Instead the pressure load will be reacted through thermally efficient bumpers to the liquid vessel heads. Positive pressure in the vacuum vessel will be limited by blow-off ports.

\subsubsection{Cryogenics Subsystem}

\section{General Description of Main Subsystems}

The basic configuration of the liquid krypton and argon facilities are shown in diagram form in
Figure 5-55. The cryogenic subsystem for the GEM calorimeter consists of three subsystems as briefly described below.

\section{Liquid Krypton (Argon) System}

The liquid krypton (argon) system consists of the necessary storage containers, transfer lines, temperature conditioning equipment, valves, and safety devices required to maintain the krypton (argon) in a condition which will allow it to function as the ionizing medium in the barrel (endcap) calorimeter.

\section{Liquid Nitrogen System}

The purpose of liquid nitrogen is to condense and control the temperature of krypton and argon. The endcaps are filled with liquid argon and cooled with liquid nitrogen. From a surface supply vessel, liquid nitrogen is fed to pumps which provide high pressure nitrogen to the interstage cooling system. The interstage cooling system is also used to control the temperature of the argon coolant for the krypton in the barrel calorimeter. In addition, liquid nitrogen is supplied from the surface vessel to the main argon dewar on the surface to recondense boiloff. This circuit also supplies the nitrogen condensers in the $\mathrm{Kr} / \mathrm{Ar}$ head and storage dewars located in the hall. The nitrogen condensers in all dewars allow transfer of krypton and argon as either vapor or liquid.

\section{Description of Liquid Krypton System}

\section{Surface Facilities}

The facility for off-loading incoming liquid krypton has a vacuum and gas purge system for evacuating and filling the transfer lines from the truck to assure that the krypton does not become contaminated during the transfer process. The incoming krypton will be sampled and tested for purity by an in-line analyzer. The surface bulk storage will consist of an insulated cryogenic storage dewar supplied by a vendor.

\section{Transfer Lines}

From the bulk storage dewar, a vacuum insulated transfer line carries the liquid krypton to a storage dewar in the underground hall.

\section{Storage Dewar}

The hall storage dewar has a capacity which is approximately $125 \%$ of the volume of the barrel calorimeter. 


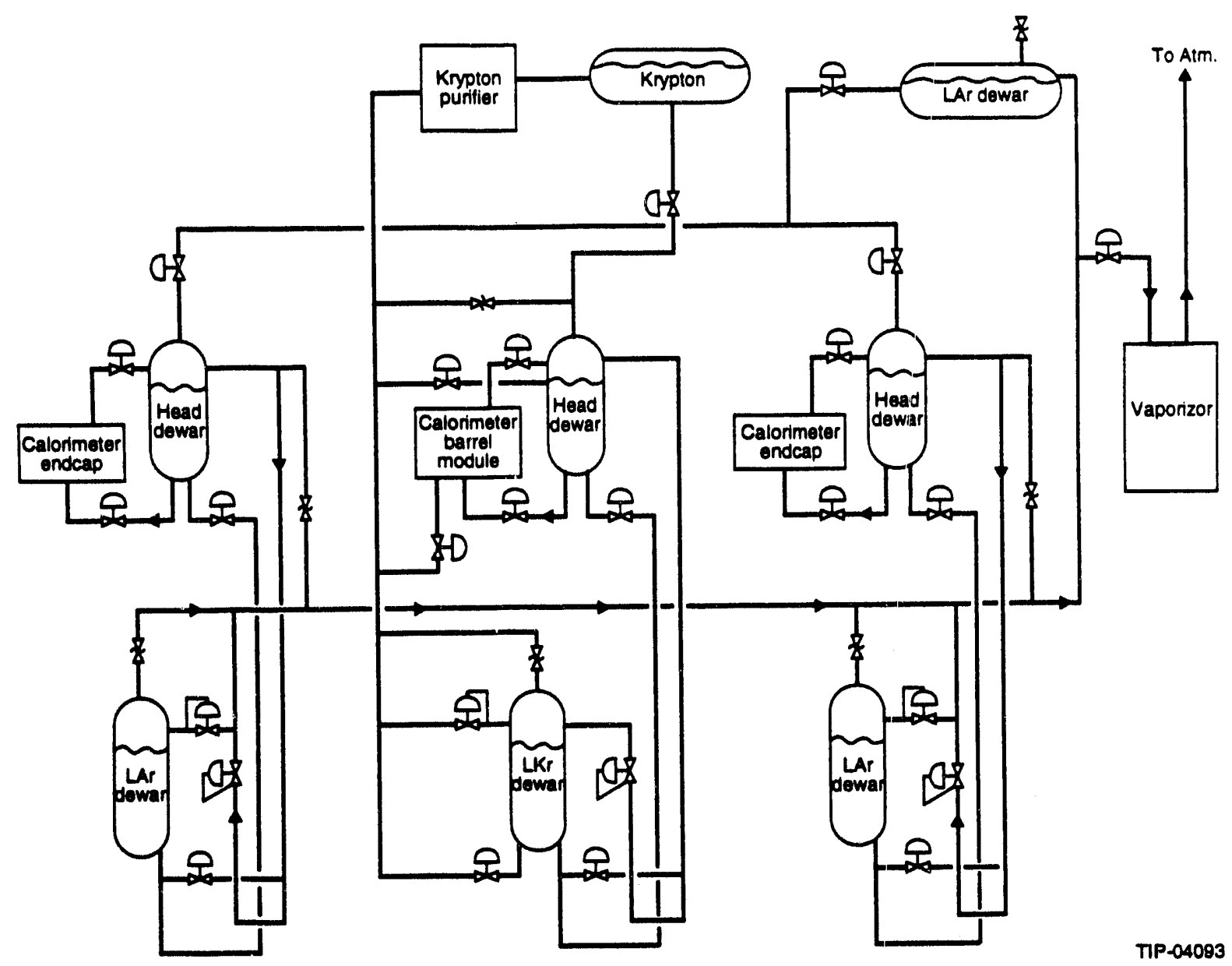

FIG. 5-55. Liquid krypton and argon system schematic.

\section{Local Head Dewars}

The maximum capacity of the head dewar for the barrel calorimeter is approximately 1000 liters. The head dewar acts as an accumulator during normal operation. It provides pressure reduction and acts as an expansion device for liquid during operation.

\section{Krypton Purifier}

The krypton purification system will have both warm and cold purifiers. To insure the removal of all impurities from the feed stream, it is required that the krypton be withdrawn as a liquid.

\section{Description of Liquid Argon System}

\section{Surface Facilities}

The facility for off-loading incoming liquid argon has a vacuum and gas purge system for evacuating and filling the transfer lines from the truck to assure that the argon does not become contaminated during the transfer process. The

incoming argon will be sampled and tested for purity by an in-line analyzer.

\section{Transfer Lines}

From the bulk storage dewar, a vacuum insulated transfer line carries the liquid argon to a storage and head dewars that are located in the underground hall.

\section{Storage Dewar}

The liquid argon is transferred into two storage dewars, each dedicated to one endcap calorimeter. The capacity of the storage dewars is approximately $110 \%$ of the volurne of the endcap calorimeters.

\section{Local Head Dewars}

The capacity of the head dewars for the endcap calorimeters is approximately 1000 liters. The head dewars act as accumulators during normal operation. They provide pressure reduction and act as an expansion devices for liquid during operation. 
Emergency Argon Evacuation System

In case of emergency the liquid argon is evacuated using an air forced flow vaporizer system.

\section{LAr Barrel Cooling Loop}

The liquid krypton in the barrel is subcooled using a cooling loop with liquid argon as the working fluid. The argon is circulated using pumps. The argon temperature is controlled using liquid nitrogen.

\section{Description of Liquid Nitrogen System Surface Facilities}

The incoming liquid nitrogen from the liquefaction plant is routed to a large storage dewar located on the surface. From the surface dewar two lines carry liquid nitrogen to the hall. One high pressure line carries nitrogen to the liquid argon heat exchanger. The liquid nitrogen is vaporized in the heat exchanger and vented to the atmosphere. A second line carries liquid nitrogen from the surface dewar to the condensers using pressure differences between the dewars.

\section{Transfer Lines:}

From the control exchanger, a vacuum insulated transfer lines carry the liquid nitrogen to the liquid argon condensers, liquid krypton condensers, and the calorimeter modules. This transfer system is driven by pumps.

\section{LAr Barrel Cooling}

Liquid nitrogen is used to cool the LAr which is then used to cool the liquid krypton in the barrel.

\section{Liquid Krypton Condensers}

The local head dewar contains a condenser for recondensing the krypton boiloff.

\section{Endcap Calorimeter Modules}

These modules are cooled directly by liquid nitrogen.

\section{Liquid Argon Condensers}

The local head dewars contain condensers for recondensing the argon boiloff. These are cooled directly by liquid nitrogen.

\subsubsection{Vacuum Subsystem}

There are two vacuum subsystems required to support the calorimeter. One provides vacuum to evacuate the insulating spaces around the cryostat and the insulating spaces in the cryogen transfer lines. The other provides vacuum to evacuate the interior of the cryostat prior to filling with argon or krypton.

\section{Insulating Vacuum Subsystem}

The main insulating vacuum system for the three cryostats is relatively simple and can consist of several (for redundancy) roughing and backing pumps. The process is to rough the insulating region down to approximately 30 microns and then switch over to several (for redundancy) turbo-molecular or diffusion pumps in parallel on each module. The main purpose of this system is to produce a vacuum of well below 1 micron in the insulating space. Super insulation will be used to reduce the radiation heat load. Some care should be taken with procedures to eliminate oil backstreaming during the roughing stage. Vacuum is also provided by this system to evacuate the insulating space on the cryogen transfer lines.

\section{Calorimeter Vacuum and Purge Subsystem}

The pump and purge system to be installed to evacuate the modules has much more rigorous requirements on its performance than the insulating vacuum system. Purity of the noble liquid and no backstreaming of oil are the concerns in this instance. The noble liquid is easily contaminated and less than $1 \mathrm{ppm}$ of most electronegative materials will make it unusable. The system needs to have the capacity to pump the systems to a pressure in the range of 1 Torr in a few hours and yet it must be clean enough to keep any contaminants, oil, etc., from backstreaming into the calorimeter. Several $100 \mathrm{cfm}$ high quality mechanical pumps with in-line filtration to reduce oil backstreaming and particulate back flow will suffice.

\subsubsection{Instrumentation, Power and Operations}

\section{Instrumentation}

The instrumentation system will provide the information required to ensure that safety, reliability and experiment control can be maintained throughout the detector lifetime. Sensors will be specified with emphasis on reliability and accuracy consistent with their intended service.

Platinum resistors will be used to monitor cryogenic temperatures in the noble liquid calorime- 
ter structure and modules as well as in the cryogenic system (piping, vessels, etc.). Thermocouples will monitor ambient and high temperatures at locations such as the ambient calorimeter electronics and the getters in the krypton purification system.

Electronic pressure sensors with electrical readout will monitor pressure in the cryostats, and as needed throughout the cryogenic and electronics thermal control systems. Redundancy will be provided on all safety related pressures, which will be transmitted to the alarm and control systems. Mechanical pressure gages will be provided at locations where local readout is useful, e.g., the off-loading station. Vacuum gages will be used to monitor vacuum levels in the cryostat insulating vacuum spaces and in the vacuum pumping systems as necessary.

Level sensors will monitor liquid levels in the cryostats, head control dewars, and all storage vessels.

Strain gages will monitor thermally induced stresses in the modules and structure during cooldown and warm-up.

Flow rate monitors will be provided as needed for all fluid transport systems.

A small dewar with radioactive sources will be provided to monitor the purity of krypton and argon inventory at delivery, and sources will be included in the calorimeter to monitor purity during operation. There will be two purification systems for liquid krypton, one for the cold liquid, and one with getter for the gas phase.

\section{Power}

The calorimeter requires power for operating electronics, instrumentation and the cryogenics system. Approximately $800 \mathrm{~kW}$ of clean power, is required for electronics allocated as follows:

\begin{tabular}{|l|l|}
\hline Preamps, in Kr or Ar (cold) & $12 \mathrm{~kW}$ \\
\hline PMTs and cable drivers & $18 \mathrm{~kW}$ \\
\hline Rack electronics & $600 \mathrm{~kW}$ \\
\hline Power supply losses & $170 \mathrm{~kW}$ \\
\hline
\end{tabular}

Approximately $60 \mathrm{~kW}$ of power is required to operate the instrumentation and cryogenics systems, most of which is consumed by the cryogenic pumps and purifiers.

\section{Operations}

The GEM utility building will contain a control center to be shared by calorimeter and magnet operators. The cryogenic and vacuum systems will be operated from this control center, with a trained operations crew always on duty. Control panels will show process and instrumentation diagrams, with sensor and hardware identifications clearly shown at the proper locations on the panels. In some cases, sensor output may be read directly from the panels. All sensor data and remote process control will be available from interactive computer terminals in the control center.

\subsection{CALORIMETER ASSEMBLY}

The calorimeter is composed of eight major subassemblies: the Inner Barrel, the Scintillating Barrel, two Endcaps, two Forward Hadronic sections, and two Passive Absorbers. Each of these subassemblies is separately assembled and tested. The Forward Hadronic Calorimetry and Passive Absorbers for each end of the Calorimeter are assembled in the surface buildings at IR-5 and installed in the Experiment Hall as a unit. Major components of each subassembly are fabricated at offsite locations, shipped to the SSCL, and assembled into the calorimeter. Components to be fabricated and assembled at offsite locations were chosen to facilitate assembly and minimize the need for specialized fabrication equipment at the SSCL.

\subsubsection{Inner Barrel Calorimeter Assembly}

The Inner Barrel includes thirteen major components which are assembled in a series of eleven operations (Figure 5-56). The assembly procedure begins with the stacking of the electromagnetic (EM) modules on two halves of an assembly stand. These are then closed, forming the barrel EM monolith (Figure 5-56a). The EM outer cylinder structure (Figure 5-56b), which includes the central washer, is then placed over the EM modules (Figure 5-56c) and attached to the suppor tabs on the outer circumference of the EM modules to form the EM module assembly. After routing the signal strip line cables from the modules through the notches in the upper flange of the barrel structure 
assembly, one head plate of the liquid vessel, including half of the liquid vessel inner cylinder, is lowered onto the EM module assembly and bolted to it (Figure 5-56d). The second head plate, with the other half of the liquid vessel inner cylinder, is placed on the rotation stand/assembly support tube. The EM module assembly, lifted by its head plate, is lowered over the rotation stand/assembly support tube (Figure 5-56e). The balance of the strip lines are routed through notches between the lower head plate and EM module assembly. After bolting the second liquid vessel endplate to the EM module assembly, the two halves of the liquid vessel inner cylinder are welded. This entire assembly is rotated so that its axis is horizontal in preparation for placement of the hadronic modules.

The inner layer of hadronic modules are placed in the assembly using an installation tool (module manipulator) which permits each module to be oriented to its correct attitude and slid into position over support keys attached to a head plate and the central washer (Figure 5-56f). These modules are mounted with a gap between the end of the module and the head plate to provide space for the EM and hadronic strip lines to run radially along the head plates between the hadronic module support keys. After the inner layer of hadronic modules are installed, the rotation fixture/support tube is reconfigured: half of the liquid vessel outer cylinder and one of the conical rings, in which the cold feedthroughs are mounted, are positioned on the support tube at each end of the assembly. The conical rings are rolled into position and bolted to the liquid vessel head plates. The strip lines from the modules are connected to the feedthroughs. The hadronic stays and the outer hadronic modules support washers are installed, followed by the outer hadronic modules (Figure 5-56g). After testing all electrical connections, the liquid vessel outer cylinder halves are moved into position and bolted to the central washer and a conical ring. After another reconfiguration of the rotation fixture/support tube, the unit is ready (a)

(b)

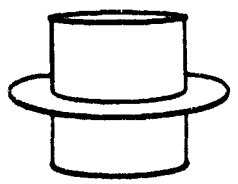

(c)

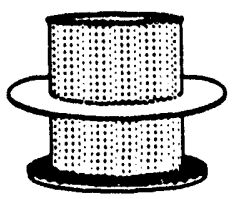

(d)

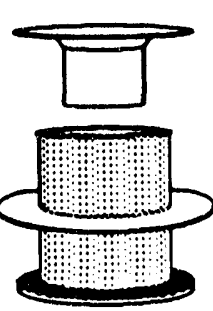

(e)

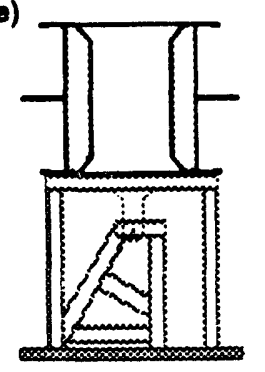

(f)

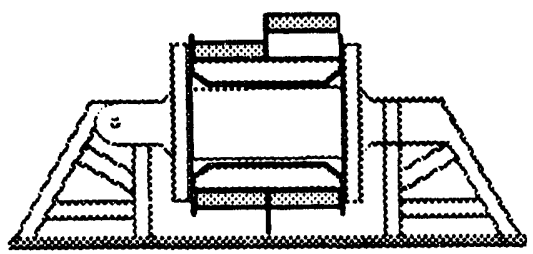

(g)

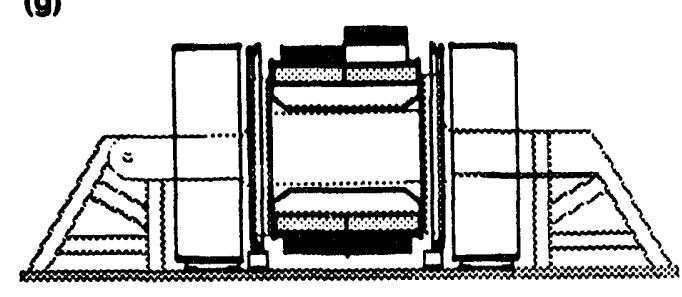

(h)

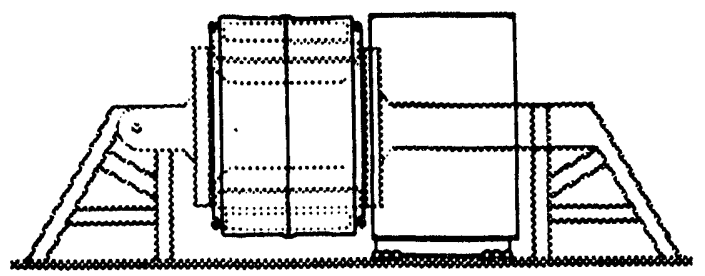

(i)

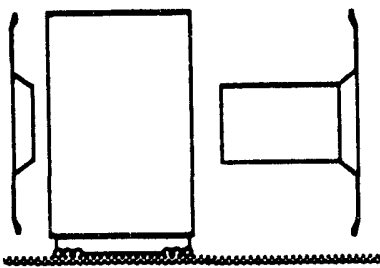

(j)

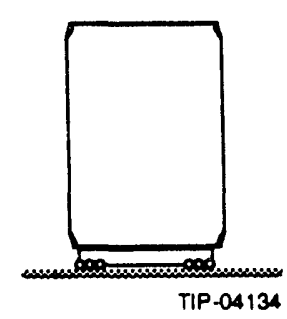

FIG. 5-56. Assembly of Inner Barrel Calorimeter. 
to receive the vacuum vessel outer cylinder (Figure 5-56h).

The vacuum vessel outer cylinder is mounted on a support cradle which provides position adjustments for installation into the scintillating barrel in the Experiment Hall. The vessel is rolled into position and the cold mass support posts are attached to the liquid vessels support bands. These support posts provide the load path from the liquid vessel via the support bands and provide thermal isolation. The load is transferred from the rotation fixture/support tube to the cradle. The rotation fixture is then removed (Figure 5-56i) to permit attachment of the vacuum vessel end plates which are conical rings containing the warm feedthroughs, and vacuum vessel inner cylinder. These major components are moved into close proximity to the assembly to permit the aup lines to be strung through the ports and the warm feedthroughs to be attached to the vacuum vessel. The Inner Barrel Calorimeter assembly is completed with the bolting of the vacuum vessel conical rings to the outer cylinder and the welding of the vacuum vessel inner cylinder to the vacuum end plates (Figure 5-56j). Additional details of the assembly process for the Inner Barrel are contained elsewhere. ${ }^{43}$

\subsubsection{Scintillating Barrel Calorimeter Assembly}

The Scintillating Barrel Calorimeter is assembled in three operations at the SSCL. The sections of the support tube are welded together to form a monolithic structure. This operation will take place with the barrel axis vertical. The barrel tube is then placed in a trunnion cradle with its axis horizontal. The cradle provides both support and rotational capability for module installation. The barrel is rotated so that the location where the module will be placed is at the bottom of the barrel (Figure 5-40). Each of the modules is lowered into position with a special handling fixture and bolted into place. Shims will be used to fill gaps between modules to provide absorber continuity. The shape of the modules near the support rails for the inner barrel calorimeter permits them to be inserted last in this radial insertion process. Readout assemblies in cylindrical tubes and their associated cables are mounted to the exposed end of each module. The Scintillating Barrel will then receive final operation- al testing, including accumulation of a large cosmic ray data sample, in preparation for the installation process. Both the readout installation and testing will be performed in the trunnion fixture to provide safe access to all areas of the assembly.

\subsubsection{Endcap Calorimeter Assembly}

The assembly process for the Endcap Calorimeter is similar to that used for the Inner Barrel (Figure 5-57). The endcap spool piece, which consists of the inner liquid vessel cylinder, the outer head plate, and two washers, is delivered to the SSCL where it is placed on the assembly fixture (Figure 5-57a).

The inner hadronic modules are inserted sequentially, beginning with the module closest to the outer head plate followed by the other hadronic modules in that $\phi$ slice (modules C, B, A) (Figure 5-57b). An installation tool (module manipulator) permits each module to be oriented to its correct attitude, slid into position over support keys attached to the structural washers, and bolted into place. Strip lines are inserted through slots in the washers as the modules are moved into position. The (C, B, A) assembly sequence allows the strip lines to be permanently placed by positioning the strip lines from succeeding modules on top of, and following the same path as, those from preceding modules. After each module is inserted, more of the structure is completed by bolting stays between the structural washers radially outward from each module. After all inner modules have been installed, the liquid vessel feedthrough ring, which had been positioned on the assembly stand earlier, is moved into position, bolted to the outer head plate, and seal welded (Figure 5-57c). The strip lines which have already been installed are connected to the feedthrough. The assembly fixture is then reconfigured to permit installation of the EM monolith which has been assembled within the EM stay. This unit is rotated from the horizontal position to the vertical with a floor fixture and attached to the assembly with bolts through the EM stay. (Figure 5-57d) The EM strip lines are run following the same path as the those for the inner hadron modules and are connected to the feedthrough. The liquid vessel inner head plate is then bolted to the outer side of the EM stay. The strip lines for the outer hadronic modules are first connected to the feedthrol ghs and run to the module 
(a)

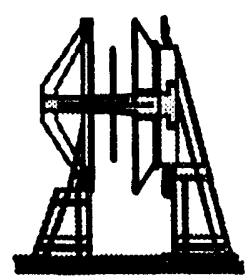

(o)

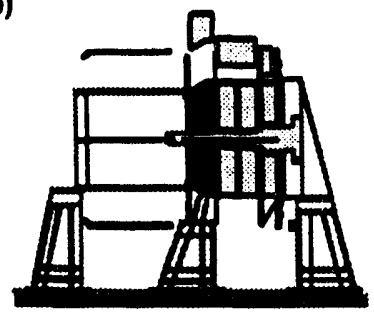

(h)

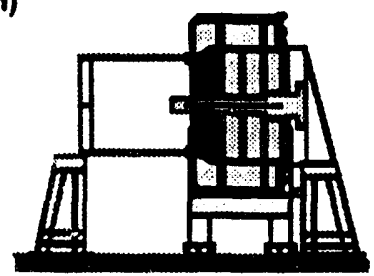

(b)

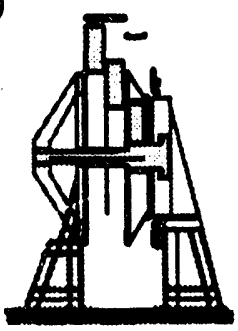

(c)

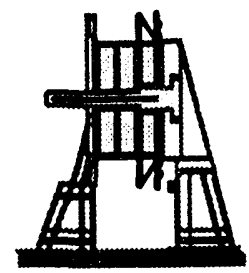

(d) (n)

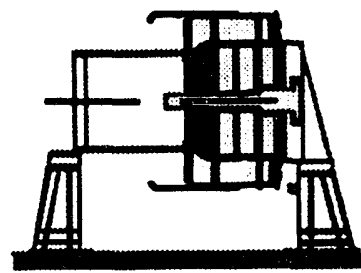

(I)

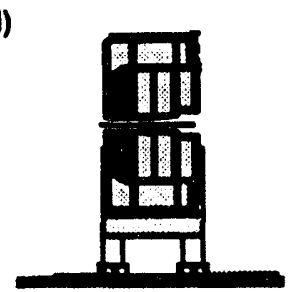

())

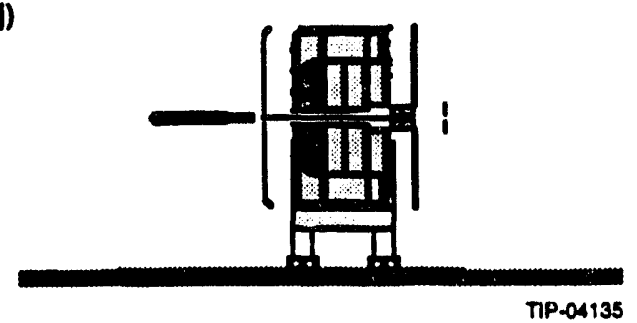

FIG. 5-57. Assembly of the Endcap Calorimeter.

location prior to insertion of the modules. The assembly fixture is then reconfigured to permit installation of the liquid vessel outer cylinder while the outer hadronic modules are being installed (Figure 5-57e). After these modules are inserted and checked, the liquid vessel outer cylinder is moved into position, bolted to the end plates and central washers, and seal welded (Figure 5-57f). Following a leak check of the liquid vessel, the assembly fixture is reconfigured to permit the vacuum vessel outer cylinder to be prepositioned for installation (Figure 5-57g).

\subsubsection{Forward Hadronic Calorimeter Assembly}

The Forward Hadronic Calorimeter (FWH) includes three major sections: scintillator section, readout section, and the liquid handling system. Like the other major components of the Calorimeter, each section of the FWH will be fabricated and partially assembled prior to arriving at the SSCL for assembly completion.
Offsite assembly will include attachment of the inlet and exit manifolds to the scintillator section, installation of the copper passive absorber into the scintillator section, and insertion of this assembly into the structural ring. To preclude shipping damage to the delicate transmission fibers and PMTs, the readout sections will be installed on the active portion of the calorimeter at the SSCL. After functional tests of the integrated assembly, it is inserted into a thermal dome to complete the Forward Hadronic Calorimetry assembly. Upon comnletion of assembly, both units are rotated and stored in the vertical position in anticipation of their insertion into the Passive Absorbers during final assembly.

\subsubsection{Passive Absorber Assembly}

The Passive Absorbers are composed of layers of copper plate which are cut and fitted to a center tube which serves as the support for the forward hadron calorimeter. These components will be completely assembled and inspected at the manufac- 
turing site and disassembled for shipment to the SSCL. The assembly at the SSCL is accomplished with the axis of the Passive Absorber in the vertical position, simplifying the procedure and tooling. The center tube is fixed vertically in the assembly tooling and the copper plates are placed over the tube. The plates are attached to each other with mechanical fasteners, eliminating the need for welding. Each completed Passive Absorber remains on the assembly fixture in this position until the Forward Hadronic Calorimetry has been joined to it.

\subsubsection{Final Assembly}

The Passive Absorbe-s and Forward Hadronic Calorimetry are assembled into one unit to simplify their installation in the Experiment Hall. The Forward Calorimetry units are lifted with the overhead crane, placed in the Passive Absorber central cylinder, and bolted in place. The integrated unit is then rotated to the horizontal position using the Passive Absorber assembly fixture and the overhead crane. The overhead crane is attached to the Passive Absorber lifting rings and the assembly fixture is removed, allowing the device to be placed upon a transportation cradle for movement to the installation shaft.

\subsection{ENGINEERING AND MANUFACTURING PLANS}

\subsubsection{Engineering Process}

The GEM Calorimeter is being designed within the National Laboratories, Universities, and Industry. Direction, coordination, and integration of these engineering organizations will be the responsibility of the GEM Calorimeter Chief Engineer in consultation with the lead physicists.

The design process for the Calorimeter will be controlled through monthly Engineering/Integration meetings and a series of in-depth formal design reviews intended to obtain GEM management and SSCL concurrence with requirements, concepts, preliminary designs, and detailed designs. The three formal design reviews and the objectives of each are as follows:

1. Technical Design Report - Baseline the Calorimeter System Design Concept.
2. Preliminary Design Review - Review Calorimeter requirements and allocation to subsystems and compliance of design concepts with requirements. Baseline the Calorimeter $\mathrm{Re}$ quirements Specification (GDT 000032) and design concept. Authorization to proceed with detailed design.

3. Critical Design Review - Review completed detailed design and confirm readiness for release to fabrication. Authorization to proceed with fabrication.

Baselines established at each of these reviews will be documented and changes will be formally controlled after review to obtain GEM Management and SSCL concurrence and to minimize cost and schedule impacts.

To facilitate the integration process, interfaces between the Calorimeter and other GEM Detector subsystems (External Interfaces) will be documented and controlled through formal Interface Control Documents (ICD) signed by the SSCL and the interfacing design organizations. Interfaces between Calorimeter subsystems (Internal Interfaces) with different design agencies will also be controlled by the ICD process.

Technical risks associated with the Calorimeter will be identified and assessed. Risk reduction Research and Development (R\&D) or Engineering Development activities such as parallel development, development testing, or additional simulations will be considered for mel $\mathrm{m}$ or high risk items identified during the assessment process.

\section{Noble Liquid Calorimeter}

The Noble Liquid Calorimeter includes the Inner Barrel and two Endcaps which are supported by the Scintillating Barrel Calorimetry. The Inner Barrel and the Endcaps both include EM and Hadronic modules housed and mechanically supported within cryostats which include liquid and vacuum vessels. The liquid vessel is supported within the vacuum vessel through stanchions which transmit the entire Inner Barrel and Endcap load to the Scintillating Barrel. During operation, the liquid vessels are filled with noble liquid (krypton or argon) and thermally stabilized using heat intercepts 
on the outer vessel walls, internal cooling for internal preamp heat removal, and heat intercepts at electronic feedthroughs and structural supports.

The liquid vessels, functioning as containers for the noble liquid and structurally supporting the EM and Hadronic modules, will be designed to the intent of the American Society of Mechanical Engineering, Pressure Vessel Code Section VIII Division 2 (ASME Pressure Vessel Code). The material selected for these vessels, 5083 aluminum, minimizes the number of radiation lengths of the vessel walls and maintains vessel structural integrity at both ambient and cryogenic temperatures. Wall thicknesses will be determined by considering internal pressure and deflection requirements during and after assembly. The design pressure for these vessels is the sum of the fluid head pressure, expected liquid surface over pressure, and ASME Code specified relief and burst disc pressure settings. The vacuum vessels, also $5 \mathrm{C83}$ aluminum, will be designed to the intent of the ASME Code for hard vacuum.

Feedthroughs and piping interfaces with the vessels will require bi-metallic fittings. The cryogenic piping external to the vessels will be stainless steel with vacuum jackets to prevent heat gain and condensation. These lines will be designed to the ASME Code for Pressure Piping, B31, an American National Standard.

Supports for the cryogenic vessel will be designed to American Institute of Steel Construction, Manual of Steel Construction, Allowable Stress Design (Steel Handbook). The materials selected for these supports will be from the current revision of Mil HBK 5 with ambient and cryogenic operating temperatures, thermal performance, structural integrity, and cost as major considerations.

As mentioned in Section 5.11.1, R\&D or Engineering Development activities can be used to reduce technical risk. The following are examples of this type of activity:

1. Materials and processes will be selected based upon the results of a R\&D investigation to determines the radiation resistance of epoxies, laminates, films, and electronics. The selected materials and processes will be used to produce
Engineering Development hardware which will be tested to determine material characteristics such as lead alloy creep behavior when bonded between stainless steel layers or three dimensional thermal expansion/contraction characteristics unde - typical structural loads. These test results will be incorporated into the structural analy'sis to produce a design with structural integrity over the life of the calorimeter.

2. Preliminary test data on prototype configurations of the EM module absorber plates show that the radial (aci oss the bends) thermal expansion of these plates is influenced by the bi-metallic effects in the absorber bends, causing the radial expansion to be greater than the simple planar material characteristics. The analytical models have been modified and now accurately match the limited test data. To allow this expansion to occur with minimal radial load build-up in the modules, the outer radius of the module is designed to expand in unison with the aluminum vessel structure while the inner radius of the EM calorimetry is designed to expand radially without loading the absorber plates. The expansion of the spacers between the absorber plates must be consistent with the expansion of the absorber inside radius to satisfy both hoop and radial dimensions simultaneously. As part of the Engineering Development activity, the absorber and its support will be analyzed structurally and thermally using more detailed and accurate modeling techniques.

3. Electronic feedthroughs that penetrate the liquid and vacuum vessel walls require efficient packaging, minimum heat leakage to the liquid vessel, and relative motion between the liquid and vacuum vessel walls. These requirements along with feedthrough locations, strip line dimensions and routing, and cost/schedule are considerations in establishing the feedthrough design. A R\&D task will be performed to select the feedthrough technology that most effectively meets these requirements. Structural, thermal, and sable routing aspects of the selected design concept will be verified in the beam test at Fermilab (see 5.7.4). 


\section{Scintillating Calorimeter}

The scintillating barrel calorimeter will be designed to accomplish two primary functions; to measure hadron energy emanating from the back of the liquid calorimeter and to provide structural support of the liquid calorimeter. The structural requirements for the barrel will be based on deflection rather than stress due to the alignment requirements of the internal detector systems. Stress limits will be determined in accordance with American Institute of Steel Construction, Manual of Steel Construction, Allowable Stress Design (Steel Hand Book). Welding will be in accordance with ASME Boiler and Pressure Vessel Code Standards.

The design of the absorber modules, tile layers and readout assemblies will be based on the physics requirements of the detector. Consequently, engineering will proceed with the oversight of the lead physicists in the collaboration. A major goal of the engineering team will be to develop methods of manufacturing and assembling the components as efficiently as possible. In order to accomplish this it is the goal of the GEM engineers to involve the engineers from the institutes fabricating and assembling detectors components in the final design process.

\subsubsection{Fabrication Techniques EM Production}

The electromagnetic portion of the GEM calorimeter uses an accordion electrode structure in both the barrel and endcap regions. (See Figure 5-1). Although the barrel and endcap electrodes differ dimensionally, the production and assembly techniques of individual modules is similar. A basic cell consists of an absorber plate, a liquid ionization gap, a signal electrode and another liquid ionization gap.

The absorber plate is a composite structure. It is made of precision rolled lead plate sandwiched between stainless steel sheets. The stainless steel provides the structural rigidity, needed to prevent sag and deformity over the length of the absorber plate. An epoxy adhesive is used to glue the stainless steel sheets to the lead.

The signal electrode is a three layer Kapton printed circuit board. Signal collection occurs on the outer layers, which are at a high voltage potential. The signals are capacitively coupled to the inner layer of the electrode. Electrical signal connections are made between the inner layer of the signal electrode and the preamplifier boards, which are mounted in the front and back of the calorimeter, via copper beryllium connection posts.

Two slightly different techniques have been developed to produce the absorber plates. The first method forms the lead - stainless steel package into the accordion shape without using the moisture sensitive prepreg adhesive. The prepreg is temporarily replaced with a filler. After bending, and just prior to the final cure in the mold, the prepreg is inserted between the lead and stainless steel sheets. The composite assembly is then cured. The second technique, ${ }^{14}$ uses a flat lay-up and a prepreg adhesive that has been manufactured at an earlier stage of cure. The various layers that make up the absorber plate are cut slightly oversized and are assembled on a flat plate. The prepreg should be stored in a humidity free environment until used. The composite is vacuum bagged, and placed in an oven at $85^{\circ} \mathrm{C}$ for approximately 15 minutes. The prepreg adhesive takes a set, but is not fully cured. The composite structure is sufficiently well bonded to be handled at room temperatures. Reheating will cause the adhesive to become soft and flex again. The part is considered to be B staged. The B staged technique was developed to easily add a massless gap to the absorber plate. The massless gap usually consists of a number of layers of prepreg ddhesive cloth, which locally, replaces the lead absorber material. Once the lead and stainless have been bent into an accordion shape it becomes extremely difficult to insert and position the prepreg filler. The B stage method, where everything is laid up flat before bending, simplifies the insertion of a massless gap into the absorber plate laminate.

Prior to bending, the B staged absorber plate must be reheated in an oven at $85^{\circ} \mathrm{C}$ for 15 minutes. This softens the glue. It is then placed in the bending machine. ${ }^{44}$ The bending machine consists of a pivot arm with sliding triangular shaped dies. The dies run in tracks orthogonal to the pivot arm. A large press is required to drive the bending machine.

After bending, the absorber plate is placed into a mold, and the composite is cured. The curing cycle is controlled by using hot and cold oil to regulate the temperature of the mold. ${ }^{45}$ The cured absorber plate 
will take the exact shape of the mold. Therefore, care must be taken to manufacturer the mold precisely. The final step in the preparation of the absorber plate will be to trim along the perimeter to the exact outline dimensions. After a thorough inspection, precision machined spacers are glued to the absorbers along each long edge.

The signal electrode is a three layer kapton printed circuit board. ${ }^{15}$ The boards will be produced under contract with a flexible circuit manufacturer. After an incoming inspection and electrical testing, the signal electrode is ready to be bent into the accordion shape. The boards are placed between two aluminum filler sheets and inserted into the bending machine. The filler sheets are required to replicate the absorber thickness. Once formed, the signal electrode, along with filler sheets, is placed into the mold and thermoset.

The absorber plates and signal electrodes, along with the associated electronics, are assembled into modules. ${ }^{18}$ The barrel modules contain 40 cells, while the endcap modules range between 20 cells for the inner modules, and 40 cells for the outer modules. The module designation is only a temporary state required for handling and testing. As the modules are assembled into a barrel or endcaps, much of the temporary structure that has held the modules together is removed and the modules are assembled into a monolith using bands for the barrel modules ${ }^{19}$ and a conical shell for the endcaps. ${ }^{21}$

\section{Hadronic Module Production}

\section{Barrel Module Construction}

Hadronic calorimetry is included in both the Barrel and the Endcap of the GEM calorimeter. The Barrel Hadron Calorimetry Subsystem includes two fine Hadronic Sections. Each section consists of 80 modules, 40 in $\varphi$ and 2 in $z$. Modules are made of layers of absorber material (mostly lead) interspersed with $\mathrm{LKr}$ filled ionization gaps. Most of the absorber material is subdivided into individual tiles.

The readout structure of the hadronic modules uses the Electrostatic Transformer (EST) principle. In an electrostatic transformer, several readout layers are arranged as series capacitances. The basic arrangement, called a single cell of an EST, consists of a ground absorber plate, made from copper or brass, two lead tile absorber plates, a tile sense plate, which is electrically connected to the amplifier, followed by another two tile absorber plates. A $2 \mathrm{~mm}$ $\mathrm{LKr}$ ionization gap separates each plate. The sequence repeats itself for the next cell. Thus one readout cell consists of six layers: ground, tile, tile, sense, tile, tile. However, there must be an extra ground absorber plate at the end of each module. These extra strong back absorbers plates provide the mechanical stability for each module.

An integer number of EST cells are assembled into a hadronic module as shown in Figure 5-34. There are six such cells in the first (inner) hadronic section and six in the second (outer) section.

The individual tile layers (consult Figure 5-35) are produced in a special assembly fixture with built-in heaters. For this both the lead sheets have to be cut into tiles as well as the G-10 sheets prepared by creating the pad structure. The lead will be cut either using a waterjet or by a computer controlled saw; one should note that each layer in the hadron absorber, and each $\eta$ bin, requires a different size tile. Each lead tile is inspected and cleaned, and a connector socket is inserted into each of the tiles in the readout layers.

The copper cladding on the individual G10 sheets is divided into pads either by etching or by using a special machine which removes the copper mechanically. Two such machines exist at Fermilab; they are computerized plotting/scribing tables with a small milling attachment mounted on the mobile head. The choice between the two techniques will be made after a careful cost comparison of the two techniques.

After all the components for a single layer are ready, a thin prepreg layer is mounted on the back of each G-10 sheet; next one tile layer, consisting of two G-10 sheets and the tiles between, is assembled in the assembly fixture. Spacers are added to keep all the pieces aligned with each other. The fixture then compresses the assembly while heating it until the prepreg is cured. After this the resistors between the individual pads are silk-screened on both sides between the pads. Then the individual tile layer is subjected to HV on either of the sides and checked for leakage currents or breakdowns.

The ground absorber sheets-which are the size of a whole module-are also prepared at the 
same time. These also are equipped with sockets for attaching to the readout cable grounds. Next the spacers which define the distance between the tile absorber layers are inserted and epoxied in. Then the module is assembled by stacking the individual layers on top of each other, beginning with the strongback. Several times during the assembly the accumulated mechanical tolerances are checked and if necessary corrected for. After the module is mechanically assembled, all the electrical connections are made. The signal connections to the preamplifier boards are three-layer Kapton printed circuit boards of a constant impedance of $7 \Omega$. They are connected to the individual readout tiles or the ground absorber by pins soldered into throughplated holes on the cables. The ground and HV connections to the pads are made using ordinary insulated wire, since these are high impedance connections.

The motherboards are mounted, and the whole assembly is checked out for shorts. About $1000 \mathrm{~V}$ is applied to the HV connections, and leakage currents are measured. Finally the preamplifiers are mounted into the motherboards and the individual module is checked out again. Finally the skins-the two thin brass sheets on either side of a module-are mounted and pre-stretched, converting the whole assembly into a rigid box.

\section{Endcap Modules Construction}

The plates and tiles are machined from $27 \mathrm{~mm}$ thick hot-rolled copper plates. These can be purchased with $\pm 0.05 \mathrm{~mm}$ thickness tolerance. The top and bottom plates are machined from $16 \mathrm{~mm}$ thick copper plates. The tiles are glued between two $0.5 \mathrm{~mm} \mathrm{G10}$ printed circuit boards with copper on one side. The copper is etched with the tile pattern keeping a $2 \mathrm{~mm}$ gap between the pads and from the board edges. The pads are electrically interconnected using $100 \mathrm{~K} \Omega$ resistive ink connections. The tiles are assembled using $4 \mathrm{~mm}$ spacers between tiles and a jig surrounding them. The injection molded spacers are shaped to allow the free circulation of the liquid argon between them. At present argon is the lowest dielectric material available. Kapton printed circuit boards serve as intermediate electrodes between the ground plates and tiles.

The assembly begins with one of the end plates (first ground plate) lying flat on the assembly table.
The top and bottom plates are bolted in place using a fixturing jig to assure their alignment accuracy. The design calls for $0.1 \mathrm{~mm}$ assembly accuracy. This tolerance could be relaxed if problems arise in holding to this precision. G10 posts are inserted into holes in the end plate. These posts will support the electrodes and tiles between the ground plates as well as align them. Next three Kapton electrodes are placed on the posts. They are kept separate from the ground plate and each other by $2 \mathrm{~mm}$ thick plastic washers that are placed on the posts between each layer. The tile layer is added and then three more Kapton electrode layers. This completes a cell. The second ground plate is lowered in place and bolted to the top and bottom plates. This plate captures the free end of the G10 posts. A new set of G10 posts are added and the process is repeated until the last ground plate is bolted in place. The high-voltage, ground and signal wiring is added to complete the module. The strip lines carrying the signals are connected by means of pins soldered to the lines that plug into sockets secured in the copper tiles. The sockets are driven deep enough to allow the use of long enough pins to provide some flexibility between the signal lines and the tiles and ground plates.

After high-voltage testing, plastic covers are inserted between the ground plates to keep the gaps free of dirt. The module is crated and made ready for shipment to the loading area at the SSCL.

A detailed description of the Barrel Hadronic Module and Endcap production can be found elsewhere. 25,46

\section{Forward Calorimeter}

The 14,000 tube electrodes, which constitute the Forward Calorimeter, will be assembled in a clean room. A special fixture allows easy winding of the quartz fiber spacer onto the inner brass rod, prior to insertion into the electrode tubes. Plugs made of G10 or PEEK, and holding contact springs will be seated in each tube end. This will prevent the fiber ends from unwinding. A high voltage test will be performed immediately, to confirm each assembly's electrical integrity, checking for continuity and electrical breakdown.

The absorber consists of a stack of $25 \mathrm{~mm}$ brass plates. Each plate has a matrix of drilled holes for the insertion of the electrode tubes. The plates are keyed 
to each other by means of dowel pins. Tie rods hold the assembly together. The assembly fits into an outer jacket with front and back end plates. The end plates have precisely positioned reamed holes. These holes accurately locate the electrodes and establish a good electrical contact between the electrode tubes and the end plates. The outer jacket is designed to allow for adequate evacuation of trapped air, and also to attach the assembled module to the endcap cryostat.

Connections between the electrodes and signal cables are made via a flexible Kapton interconnect board. This board is attached to the downstream end of the module. Electrode contacts and cable connectors are soldered to this board. Low impedance stripline cables connect the module to the junction boxes, which are located along the periphery of each endcap cryostat. The Forward Calorimeter will share the same feedthroughs used by the EM and Hadronic Modules.

\section{Cryostat Fabrication and Assembly}

Cryostats are required for both the Inner Barrel and the Endcaps of the GEM Calorimeter. Each cryostat consists of a liquid containment vessel, (liquid krypton or liquid argon) within a vacuum vessel. Besides serving as the cryogenics reservoir for the EM and Hadronic ionization liquids, the containment vessels also provide the structural load path for supporting the EM and Hadronic modules. The vessel is designed to be supported within the vacuum vessel by four insulated support pads, which are located on the lower half of the containment vessel. The vacuum vessel is self supporting.

Major components of the vessels are fabricated offsite, and shipped to the SSCL for assembly. The cryostats are closed upon completion of the Inner Barrel and Endcap calorimetry assembly. The Inner Barrel cryostat includes approximately eight major components. The Endcap cryostat has a similar complexity. Specific cryostat components have been chosen for fabrication off site to facilitate assembly and minimize the need for special equipment at the SSCL. However, several pieces of specialized handling equipment will be required in the assembly operations. A detailed description of the assembly process for each cryostat is contained elsewhere. ${ }^{43,47}$

\section{Scintillating Barrel Calorimeter}

The scintillating calorimeter barrel will be fabricated and assembled using modular subassembly techniques. The four primary modular elements are, the two halves of the structural shell, 40 absorber stacks, 160 tile layers, and 80 readout assemblies.

The structural shell will be made from stainless steel plate, which has been formed on a break press, and welded using vessel welding equipment and techniques. Due to the size of the shells, the final welded assembly operations must take place at the SSCL.

The absorber plates will be machine cut from stock plate, which is ordered to thickness from the mill. The plates will be drilled and spaced with small bars and bolted into stacks. The stacks will be inspected and prepared for the installation of the tile layers.

The tiles are manufactured using conventional shop mills and laser cutters. The assembly of the tile layers will take place in two steps. First, 160 tile layer assemblies, including reflective film and readout fibers, will be prepared. After completion of the absorber stacks, the tile layers will be inserted and the readout fibers will be grouped and aligned for the readout assemblies.

Readout assemblies will be installed on the absorber/tile stacks as the final operation. The subassemblies will be self-contained allowing independent test and evaluation. With the completion of this testing the subassemblies will be installed into the structural shells at the SSCL.

The above procedure is designed to maximize the use of in place facilities away form the SSCL and to reduce the need for new construction on-site.

\section{Passive Endcap}

The passive endcaps have been designed to be bolted assemblies. This will enable the copper plate manufacturer to fabricate and pre-assemble the units at the manufacturing site for inspection. The passive endcaps will be disassembled and shipped in pieces for reassembly at the SSCL. The hadronic forward calorimeters will be installed in the passive endcaps prior to installation in the experimental hall. 


\section{Forward Hadron Calorimeter}

The hadronic forward calorimeters are relatively small self-contained units which are intended to be completely assembled by a single institute away from the SSCL. Virtually all the components, such as capillary tubes, drilled stainless steel plate, and quartz fibers, are available from conventional industrial sources. The exception is the large quantity of tungsten absorber material, which can be provided by only a limited number of countries or companies. The design of the absorber, using small shaped pellets, has been developed with industrial help to permit mass production and easy shipment. It is assumed that the completed forward hadronic calorimeters would be fully assembled at the manufacturing institute or company and tested prior to shipment to the SSCL. After acceptance the units would be packaged and shipped to the beam test site at SSCL.

\section{Quality Control}

Quality assurance activities associated with all aspects of the design and assembly of the GEM calorimeter will be coordinated by the SSCL. A specific quality control plan and procedure will be written in accordance with SSCL guidelines to cover both the total project and individual vendor procurements and work packages. Each responsible institute or company will provide a quality assurance plan for the fabrication of components and the assembly of the modules.

A primary principle of the calorimeter quality control system will be on-site testing and acceptance of all components and assemblies prior to shipment. This procedure insures that companies, institutes and international partners accept full responsibility for the final condition and function of the detector components.

\subsubsection{Production Locations}

The location of the production and assembly of the calorimeter are being negotiated and finalized at the present time. We plan to produce major component of the calorimeter utilizing our overseas collaborators. The final assembly from tested components will be done in the US.

The calorimeter production has been divided into major tasks (described in detail in Section 5.12.1). Each task will be carried out by a designated team of physicists, engineers and industrial collaborators.

\section{The EM Calorimeter Modules}

The major tasks of the EM calorimeter modules are: the construction of the absorber plates, readout electrodes, G10 spacers and mechanical support structure, individual module assembly, and the final assembly into a $2 \pi$ structure.

At present we are investigating the possibility that the EM absorber plates could be produced in China by the Shanghai Aircraft Manufacturing Factory (SMAF) in collaboration with IHEPBeijing.

A prototype consisting of two modules (an $18^{\circ}$ sector) will be built in the US with the participation of engineers from China.

The EM readout electrodes are as large as $4 \mathrm{~m}$ by $1 \mathrm{~m}$ multilayer $\mathrm{Cu} / \mathrm{Kapton}$ printed circuit boards. There are a number of companies in the US and Europe that have this capability and we will use them for the prototype construction as well as the final calorimeter unless we find an overseas collaborator who can produce them. The US will produce the actual $\mathrm{Cu} / \mathrm{Kapton}$ board. The bending and preparation for installation, testing and pins, could be done either in the US or possibly in China by SMAF in collaboration with IHEP Beijing.

The production of the G10 mechanical spacers that act also as part of the mechanical structure require detailed machining. At this stage we are investigating the possibility of molding and/or machining these components.

Assembly of the absorber plates and signal electrodes into mini-modules will be done in the US with assistance from the overseas collaborators. All component testing will have to be done before the assembly.

The assembly of the individual modules into the barrel or endcap will be done at the SSC Lab.

\section{The Hadronic Calorimeter Modules}

The Liquid Calorimeter module production has been divided in a similar way so that major mechanical components can be manufactured either in the US or overseas. We are now investigating the possibility of the components being produced in 
China. From our preliminary discussions it seems that the SMAF has the capacity to produce all the mechanical components needed for the calorimeter.

The assembly of the hadron modules could be done overseas but at this stage we plan to assemble it in the US. Tested modules will then be shipped to the SSC for installation in the cryostat.

\section{Cryostat}

The cryogenics system is now under design. We have designed the calorimeter in such a way that even the largest pieces can be transported from an outside manufacturer to the SSC site. The present plan is for the cryostat to be built by industry either in the US or overseas and we are now investigating the relative advantages of either.

The assembly of the cryostat will be done at the SSC. The feedthroughs are designed so that they can be preassembled and tested before installation in the cryostat.

\section{Cryostat Purification and Cryogenics System}

We plan to fabricate a large fraction of the cryogenic system overseas. At this time we are negotiating with our collaborators in Novosibirsk to define their contribution to the krypton system as well as the cryogenics system.

\section{Krypton Supply}

Our collaborators from BINP (Novosibirsk) are coordinating the procurement of krypton.

\section{Scintillating Barrel Calorimeter}

The modular configuration of the scintillating hadron barrel will permit most of the fabrication activities to be performed away from the SSCL. Several scenarios have been proposed which including U.S. universities, national laboratories and international collaborations; the following arrangement may have some cost and schedule advantages.

The structural shell, readout assemblies and copper absorber modules are fabricated by an international partner. Several countries have expressed interest in these components. Albania and/or Belarus could supply copper plate, possibly formed into modules. Philips Corp. has expressed an interest in providing the PMTs through its subsidiary in Prague. China has designated Tsinghua University in collaboration with the Xian Aero Engine Factory as a potential site for either complete or partial fabrication of the modules for the scintillating barrel. US universities would be involved in the final assembly of the tile/fiber/readout assemblies at the SSCL.

\section{Passive Endcaps}

The passive endcaps are bolted assemblies of copper plate. The country which will provide copper plates is also a good candidate for providing the entire passive endcap assemblies.

\section{Forward Hadronic Calorimeter}

The two forward hadronic calorimeters are relatively compact systems which could be entirely fabricated and assembled away from the SSCL.

China has identified the Xian Aero Engine Factory in collaboration with Tsinghua $U$. as a potential site for either complete or partial fabrication of all the mechanical components for the forward hadron calorimeter. Readout PMTs could be supplied by Philips Corp. of the Czech Republic. The tungsten absorber may be provided by the Powder Metallurgy Institute of Belarus.

\subsection{SCHEDULE AND RESPONSIBILITIES}

\subsubsection{Schedule}

The GEM calorimeter schedule has been crafted to match the global GEM installation schedule while using the resources of the calorimeter group optimally. The schedule is based on a detailed understanding of the procedures used in manufacturing, assembling, and installing the calorimeter components, and takes advantage of experience gained in constructing prototypes for this calorimeter and constructing earlier calorimeters. See Chapter 16 for the important milestones of this schedule.

The construction of the major components of the calorimeter, scintillating barrel, noble liquid barrel, noble liquid endcaps, and forward calorimetry, have been phased. The scintillating barrel, which is the most conventional portion of the calorimeter and which acts as a support device for the noble liquid and forward sections, is scheduled to be installed in the underground hall in July 1996. The noble liquid barrel calorimeter section follows in April 1997. The noble liquid endcaps are installed in May 1998, after installation of the tracker. The 
forward calorimeter installation follows in July 1998. The temporal separation of the barrel and noble liquid sections allows for additional design and prototype work on the less mature endcaps. The expertise developed in the construction of the nobie liquid barrel, as well as much of the tooling, can then be applied most efficiently to the endcap work. This avoids increased cost and dilution of expertise. The phasing of the construction of the sections of the calorimeter provides for a flatter system funding profile and allows more efficient use of the limited surface facilities during assembly.

An intermediate milestone is provided by the availability of the MWEST test beam at Fermilab in Fall 1994. Preparation of pre-production prototype modules for both the scintillating barrel and noble liquid systems, as well as cryostat and transporters, is extremely challenging.

The scintillating barrel section of the calorimeter is now in conceptual design phase. In order to meet the July 1996 installation date in the underground hall, as well as the Fall 1994 test beam date, design of this subsystem must be complete within a year. An ambitious R\&D program to test all components of the readout structure is already underway. Module construction must begin in May 1994. The first production module will be used for the MWEST test beam. The module manufacture is performed by two assembly lines, one for readout structures(scintillator and fibers) and one for the absorber structure. These assembly lines complete their tasks before November 1995. The assembly of the readout system into the absorber structure begins in April 1995, with the last of 44 modules completed in December 1995. Installation of the modules onto the support structure begins in August 1995 and is completed by year's end. The monolith is then cosmic-ray tested and is ready for installation in the underground hall in July 1996 . The early construction and installation dates require quickly settling on where module construction will take place.

The schedule of the liquid barrel section of the calorimeter is driven by the manufacture of pre-production prototypes in time for the Fall 1994 beam tests. Tooling design for absorber and electrode structure production is proceeding in parallel with the design of the electromagnetic and hadronic portions of the calorimeter, which must be completed by June 1994. Production of electromagnetic absorber and electrode accordion plates begins in June 1994. Parts production ends in October 1995. Investigation of time needed for this work indicates that multiple molding and bending presses working in parallel production lines are needed to ensure meeting the schedule. Electromagnetic module assembly begins in April 1995 and is completed by November 1995. Assembly of these modules into a monolith maly begin in May 1995 and will be completed in November 1995. Production of absorber plates and electrodes for the barrel hadronic modules will be completed in June 1995. Module stacking using these parts will be completed in October 1995. Installation of these modules onto the barrel liquid vessel spool piece will be completed in June 1996. The liquid vessel is sealed and leak tested in November 1996. The vacuum vessel is completed, and the barrel is filled with noble liquid while in the surface assembly building in February 1997. Installation in the underground hall begins in April 1997. Cryogenic facilities, which will already have been installed, will then be connected.

The installation of the endcap sections of the noble liquid calorimeter in 1998 allows a full R\&D program for the endcap electromagnetic section. The hadronic module construction has, like the barrel hadronic section, a milestone of participation in the Fall $1994 \mathrm{FNAL}$ test beam program. However, these modules need not be final pre-production prototypes. Farts manufacture for both sections of the endcap can reuse much of the barrel tooling. One of the endcap electromagnetic structures must be completed by early 1996 in order to be calibrated in the SSC test beams. Assembly of the hadronic modules and the electromagnetic monoliths onto the spool pieces of both endcaps begins in the North Assembly Building in January 1997. This procedure, as well as completion of the liquid vessels and the vacuum vessels will occur before February 1998. A two month fill and test with liquid argon follows. Installation in the underground hall occurs in May 1998. 
Detailed schedules for the forward calorimetry and passive absorber sections of the calorimeter have not yet been developed. The passive absorber is primarily a conventional materials acquisition task. Time is available for full test beam studies for the forward calorimetry. Manufacture and installation will be completed in July 1998.

\subsubsection{Responsibilities}

We have organized ourselves as shown in Table 5-13. We have many working groups which are functioning to complete the design and $R \& D$ as well as plans for fabrication, installation, commissioning and extracting the physics. These groups break up the calorimeter into more manageable tasks such as the EM calorimeter, simulation, test beam program, etc.

Table 5-13. Responsibilities in the Calorimeter Group.

\begin{tabular}{ll}
\hline Head - William Willis & \\
\hline $\begin{array}{l}\text { Liquid Calorimetry } \\
\text { Head - Veljko Radeka } \\
\text { Chief Engineer - Lyle Mason }\end{array}$ & $\begin{array}{c}\text { Adelphi, Arizona, BINP (Novosibirsk), BNL, } \\
\text { Columbia, IHEP(Beijing), Martin Marietta, } \\
\text { Pittsburgh, Rochester, SSCL, Stony Brook, Tel Aviv, } \\
\text { Washington }\end{array}$ \\
\hline $\begin{array}{l}\text { Scintillating Calorimetry } \\
\text { Heads - Yuri Kamyshkov, Larry Sulak } \\
\text { Chief Engineer - Mark Rennich }\end{array}$ & $\begin{array}{c}\text { Boston, Fairfield, lowa, Mississippi, ORNL, } \\
\text { SSCL, Texas A\&M }\end{array}$ \\
\hline $\begin{array}{l}\text { Fonward Calorimetry } \\
\text { Head - John Rutherfoord }\end{array}$ & $\begin{array}{c}\text { In collaboration with liquid and scintillator } \\
\text { groups. }\end{array}$ \\
\hline $\begin{array}{l}\text { Integration } \\
\text { Heads - William Wisniewski } \\
\text { Engineer - Greg Velasquez }\end{array}$ & SSCL \\
\hline
\end{tabular}




\section{REFERENCES}

1. $\oplus$ means the terms are added in quadrature.

2. B. Aubert, et al., Nucl. Instrum. Methods, A309, 438 (1991), Nucl. Instrum. Methods, A321, 467 (1992), Nucl. I istrum. Methods, A325, 116 (1993).

3. W. Braunschweig, et al., Nuclear Inst. and Methods, A275, 246(1989). H. Oberlack, "Compensation by Software-Single Particles and Jets in the H1 Calorimeter", The 25th Int. Conf. on HEP, Singapore, 1990.

4. M. Leltchouk, H. Ma, and M. Seman, Monte Carlo Study of the Noble Liquid GEM EM Calorimeter, GEM TN-93-357.

5. O. Benary, et al., Precision timing with Liquid Ionization Calorimeters, Preprint BNL-48159 (1992), and submitted to Nucl. Instrum. Methods.

6. M.A. Shupe, Simulation of Various GEM Forward Calorimeter Options Using a Mixture Level Description of the Detector, GEM-TN-92-243 and GEM TN-93-359.

7. D. Bintinger, Depth of Calorimeter for SSC Experiments, SSC-225, 1989.

8. L.C. Rogers, BNL, private communication.

9. B.T. Turko and R.C. Smith, Proceedings of the 1991 IEEE Nuclear Science Symposium, Nov 2-9, 1991, p.711.

10. W. E. Cleland and E.G. Stern, "Signal Processing for Liquid Calorimeters", to be submitted to Nucl. Instr. and Methods.

11. RD3 Collaboration, Status Report and Further R\&D for EM and Hadronic Calorimetry, CERN/DRDC 93-4, Jan. 1993.

12. S. Abachi et al., Nucl. Instrum. Meth. A324, 53(1993); and $\mathrm{H}$ Aihara et al., Design, Construction, and Performance of the Electromagnetic Module of the DO End Calorimeter, submitted to Nucl. Instrum. Meth. A(1992).

13. D. Axem, et al., The Lead-Liquid Argon Sampling Calorimeter of the SLD Detector, SLAC-PUB-5354 (1992).

14. S. Bellavia, EM Absorber Production, GEM-TN-93-323.

15. D. Makowiecki, EM Electrode Production, GEM-TN-93-322.

16. D. Makowiecki, S. Rescia, and L. Rogers, EM Barrel Pre-amplifier Board Design, GEM-TN-93-328.

17. F. Lobkowicz, et al., Feedthrough Design, GEM-TN-93-315.

18. D. Makowiecki and S. Bellavia, EM Barrel
Module Assembly, GEM-TN-93-326.

19. B. Easom, et al., EM Barrel $2 \pi$ Structure Analysis, GEM-TN-93-320.

20. R.L. Chase, et al. Transmission Line Connections Between Detector and Front End Electronics in Liquid Argon Calorimetry, accepted for publication in Nucl. Instrum. Methods, 1993.

21. B. Easom, et al., EM Endcap Structure, GEM-TN-93-321.

22. S. Rescia \& D. Stephani, Reliability of Preamplifiers, GEM-TN-93-399.

23. W. E. Cleland, E. A. Scholle, and E. G. Stern, "Pileup and thermal noise in a liquid ionization calorimeter" in Symposium on Detector Research and Development for the Superconducting Supercollider, Ft. Worth, Texas, October 15-18, 1990, p. 318.

24. W. E. Cleland and E. G. Stern, "Signal Processing for Liquid Ionization Calorimeters", III International Conference on Calorimetry, Corpus Christi, Sep 29-Oct 2, 1992.

25. F. Lobkowicz, Production Specification of the Barrel Hardonic Modules, GEM TN-93-311.

26. J. Colas, Proceedings of the Workshop on Calorimetry for the Supercollider, Mar. 13-17, U. of Alabama, Tuscaloosa, Alabama, World Scientific, p. 633.

27. J. Colas, M. Pripstein, and W.A. Wenzel, Nucl. Instrum. Methods A294, 583 (1990).

28. C. Fabjan, et al., Nucl. Inst. and Methods, 141, 61(1977).

29. SDC TDR and P. de Barbaro, Nucl. Inst. and Methods, A315, 317(1992).

30. A. Savin, et al.,"LTRANS Code", ITEP (Moscow) Preprint (in preparation).

31. Yu. Efremenko, et al., Simulation studies for GEM Scintillating Barrel Design, GEM-TN 93-349.

32. L. Holm, et al., Nucl. Instrum. Methods $\mathbf{A 2 3 4}$, 517 (1985); M. Bottcher, et al., Nucl. Instrum. Methods 206, 177 (1983); J. Berger, et al., Nucl. Instrum. Methods A279, 343 (1989).

33. F. Paige and A. Vanyashin, Effects of Limited Calorimeter Coverage on Missing $\mathrm{E}_{\mathrm{T}}$, GEM-TN-92-70.

34. M. Shupe and J. Rutherfoord, Progress Report on Design and Simulation Studies for a Tungsten and Liquid Argon Forward Calorimeter for the GEM SSC Detector, GEM-TN-92-52; K. Shmakov Missing $E_{T}$ in Jets Due to Leakage into Scintillator Barrel Calorimeter., GEM TN-93-295; F. Paige, Jet Contribution to Missing $\mathrm{E}_{\mathrm{T}}$ Cross Section, GEM 
PN-91-0001.

35. See also M.A. Shupe "Fast Simulation Using GEANT Mixtures of a Liquid Argon/Tungsten Tube Calorimeter for GEM" III Int. Conf. on Calorimetry, Corpus Christi, Sept.29-Oct.2, 1992.

36. J. Rutherfoord, Ion Loading in Liquid Ionization Calorimetry, GEM TN-91-27.

37. W. Don Carlos, et al, Experimental data on GEM LAC FCAL tube. GEM TN-92-179.

38. P. Loch and S. Savin, private communications.

39. RD3, private communication.

40. M. Citterio, S. Rescia, and V. Radeka, "A Study of Low Noise JFETs Exposed to Large Doses of Gamma Rays and Neutrons," To be published in the Proceedings of the 1992 IEEE NSS 25, 31 October, 1992, Orlando, FL.

41. G. Battistoni, et al., "Results of the Preliminary Exposure of $\mathrm{Si}$ and GaAs Preamplifiers to a High Neutron Fluence and to Gamma Rays," RD3 Internal Note, CERN 1992.

42. H.Takai, Calorimeter Information Flow, GEM-TN-93-319.

43. B. Humphreys, et al., Inner Barrel Calorimetry Assembly Concept., GEM TN-93-309.

44. S. Bellavia, Crocodile Press, GEM-TN-93-324.

45. S. Bellavia, EM Mold Press, GEM-TN-93-325.

46. P. Mockett, Production Requirements for Endcap Hadronic Modules, GEM-TN-93-312.

47. B. Humphreys and B. Wisniewski, Assembly of Liquid Endcap Calorimeter, GEM-TN-93-310. 


\section{CENTRAL TRACKER}

\subsection{INTRODUCTION}

\subsubsection{Physics Goals of the Central Tracker}

The physics goals for the central tracking in GEM can be divided into two categories. The first are those features that are required to support the primary objectives of GEM, namely the detection of gammas, electrons, and muons at high $p_{T}$. Some examples of these are:

- Identify the primary vertex of an event of interest, so that it can be separated from other pileup events in the memory time of the detector.

- Separate electrons and gammas using the presence or absence of a charged track pointing to an electromagnetic shower in the calorimeter.

- Help with electron-hadron separation by providing a momentum measurement that can be compared with the energy deposition in the calorimeter.

- Help with rejection of fake muon backgrounds by matching the muon momentum measured in the central tracker with the momentum measured in the muon chambers.

- Determine the electron sign up to $\sim 600 \mathrm{GeV} / \mathrm{c}$.

- Provide track information for $e, \mu$, or $\gamma$ isolation cuts, and to help with rejection of conversions and Dalitz pairs.

- Separate close together multi-charged particle tracks from single-charged particle tracks, which will be useful in tagging $\tau$-decays and $\gamma$-conversions.

The tracker should be able to fulfill these goals well at the design luminosity of $10^{33} \mathrm{~cm}^{-2} \mathrm{~s}^{-1}$. These capabilities should also survive to luminosities up to $10^{34} \mathrm{~cm}^{-2} \mathrm{sec}^{-1}$. These minimum goals do not require full pattern recognition, but can be met by looking for hits in the tracker in a specific road extrapolated from the calorimete. Ir the muon system.
The physics goals in the second category are more ambitious:

- Full reconstruction of the charged tracks in the event.

- Secondary vertex finding.

- Tracking at low momenta with good resolution.

These features would enhance GEMs ability to address issues such as $b$ and top physics. They are more demanding in that they require pattern recognition capabilities and very good vertex resolution. It is expected that these more ambitious goals can be met at luminosities up to $10^{33} \mathrm{~cm}^{-2} \mathrm{~s}^{-1}$, but probably not much higher. This, however, seems satisfactory since the physics topics requiring these more ambitious features have relatively large cross sections and can thus be studied at luminosities of $10^{33} \mathrm{~cm}^{-2} \mathrm{~s}^{-1}$ or below.

\subsubsection{Design Considerations}

The central tracker of the GEM detector is designed to operate in the 0.8 -T magnetic field of the large GEM superconducting solenoid. The solenoidal field is assumed to be relatively uniform, but small variations $(<5 \%)$ are allowed as long as the fieid is known to $0.5 \%$. This requirement is set by the low momentum resolution limit. Also, since the tracker elements are tilted to account for the Lorentz angle of drifting charge, the field direction is fixed and cannot be reversed. The tracker is compact, with a $90-\mathrm{cm}$ outer radius and a total length of $350 \mathrm{~cm}$. It covers a pseudorapidity range of \pm 2.5 units. The baseline design consists of a silicon microstrip (SM) inner tracker and an interpolating pad chamber (IPC) outer tracker. The geometry of the central tracker in this design is shown in Figures 6-1 and 6-2.

Since the submission of the GEM Expression of Interest, a variety of technologies were considered for use in the GEM central tracker. For the inner tracker, silicon microstrips, silicon pixels, and long-drift length silicon detectors were discussed. 
(a)

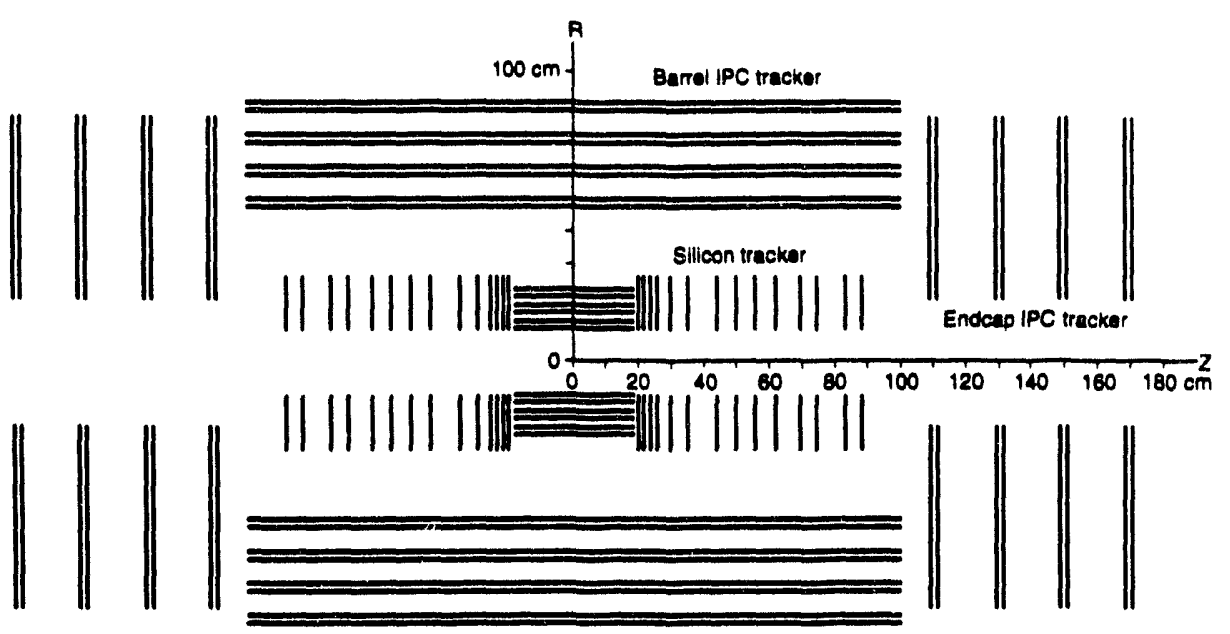

(b)

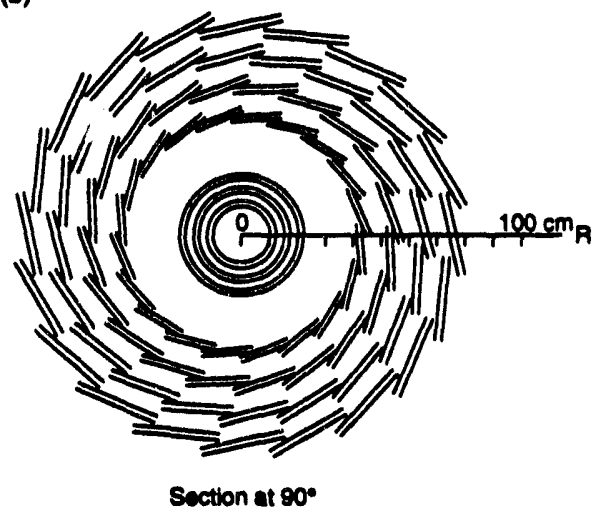

(c)

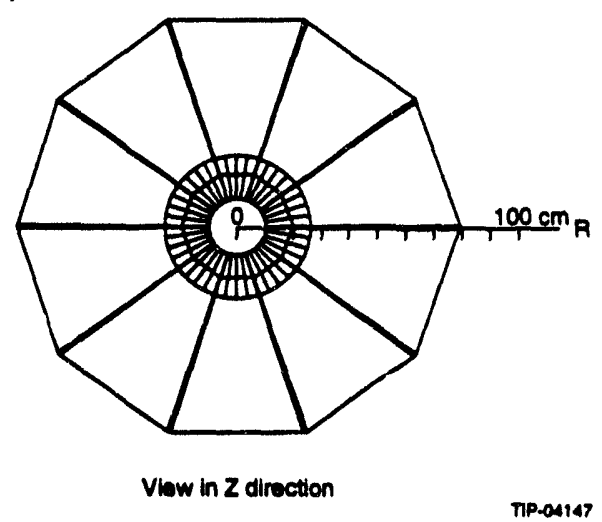

FIG. 6-1. Schematic layout of the detector elements in the GEM central tracker. (a) Side view of the chamber.

(b) End view of barrel section. (c) End view showing forward sectors of IPC and silicon rings.

Silicon pixel and long-drift silicon detectors were considered to be too immature, with large uncertainties of performance, radiation resistance, and costs, to be a sensible choice at this time. Silicon microstrip detectors were chosen as the baseline design for the following reasons:

- The very fine segmentation possible combined with proven high radiation resistance make this detector ideal as the element closest $(\sim 10 \mathrm{~cm})$ to the interaction point.

- Very high spatial resolution allows very precise vertex position and track impact parameter measurement.

- It is a mature technology, which is presently in use in a number of fixed target and collider experiments with relatively well understood performance, radiation resistance and cost properties.

Silicon detectors were considered to be too expensive for the outer tracker. Technologies under serious discussion for the outer tracker were straw tubes, scintillating fibers, and interpolating cathode pad chambers. The interpolating pad chambers were chosen for the baseline design for the following reasons:

- Pad sizes of the order of a few $\mathrm{cm}^{2}$ in area are quite natural. This allows for a low occupancy even at a luminosity of $10^{34} \mathrm{~cm}^{-2} \mathrm{~s}^{-1}$. Thus, this technology is suitable even at the highest luminosities of the SSC. The other technologies would result in considerably higher occupancies. 


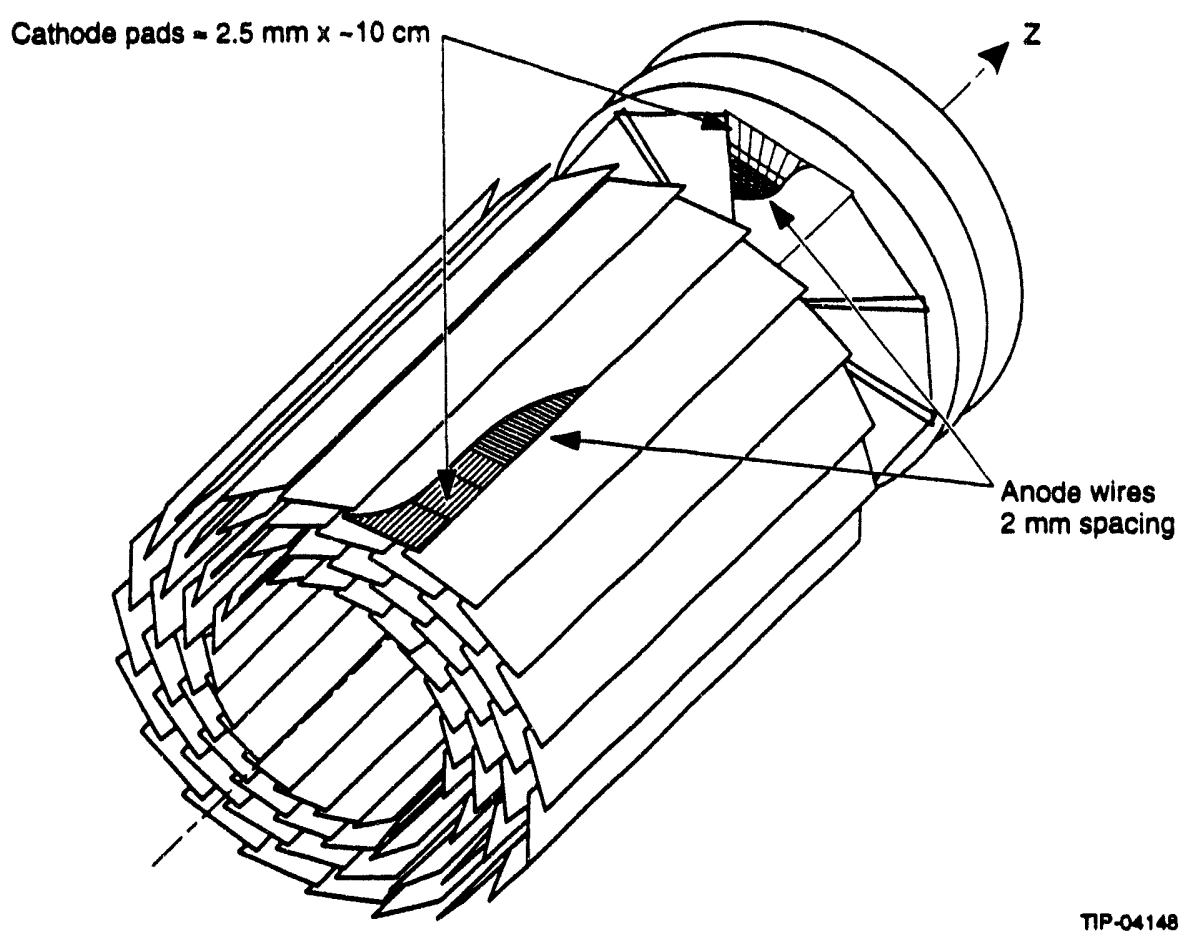

FIG. 6-2. Schematic layout of the detector elements in the GEM interpolating pad chambers.

- The pads, in some approximation, approach three dimensional points, which is quite important for good tracking in the high rate and multiplicity environment of the SSC. The two other technologies produce stereo images i.e., all tracks projected onto a plane, that make pattern recognition more difficult in this environment.

- Interpolating pad chambers are not a new technology; they have been demonstrated to have the resolution needed with chamber sizes similar to those required for the GEM tracker design.

\subsubsection{Detector Configuration}

The silicon microstrip inner tracker consists of six layers of silicon strip detectors. Each layer is composed of two back-to-back, single-sided silicon sensors with $\mathrm{a} \pm 5 \mathrm{mrad}$ stereo angle between the two sensors. Each sensor is $300-\mu \mathrm{m}$ thick with a strip pitch of $50 \mu \mathrm{m}$. Each pair of sensors provides a space point with a resolution of $15 \mu \mathrm{m}$ in the $\mathrm{r}-\phi$ plane and $3 \mathrm{~mm}$ in the $r-z$ projection. The six layers of ladders are organized into three superlayers, each of which provides a track stub to a track-finding algorithm. In the forward region, the silicon sensors are mounted into disks with the strips projecting radially inward toward the beam axis. The silicon tracker is $\sim 200-\mathrm{cm}$ long and extends in radius from 10 to $35 \mathrm{~cm}$. The total area of silicon ladders in the detector is about $7 \mathrm{~m}^{2}$, with about $2.5 \times 10^{6}$ strips to be read out. The readout will be highly multiplexed, with 1280 strips to one fiber-optic readout channel, for a total readout channel count of 1942 .

The outer tracker consists of eight layers of pad chambers, both in the barrel region at radii between 35 and $80 \mathrm{~cm}$, and in the forward region, which extends from 110 to $170 \mathrm{~cm}$ from the interaction point. The eight layers are arranged in four superlayers with two layers each. Each barrel layer will consist of 20 chambers, each covering $18^{\circ}$ in azimuth, with the largest chamber being $30-\mathrm{cm}$ wide $\times 200-\mathrm{cm}$ long. The forward layers will be disks divided into 10 trapezoidal chambers about $60 \mathrm{~cm} \times$ $60 \mathrm{~cm}$ each.

The pad sizes vary around an average of $\Delta \eta \times$ $\Delta \phi=0.0007$, which ensures a low occupancy even at luminosities of $10^{34} \mathrm{~cm}^{-2} \mathrm{~s}^{-1}$, with a total of just under 400000 pads for the entire device. The pads are $2.5-\mathrm{mm}$ wide (in the $\phi$ direction) and a few centimeters long (in the $z$ direction, which is the direction along the beams). With an analog readout we expect to interpolate to obtain a precision in the 
$\phi$ direction of $\sim 50 \mu \mathrm{m}$. To obtain better precision than the pad length in the $z$ direction, the pads will be tilted by $\pm 50 \mathrm{mrad}$ in the two layers of each superlayer, respectively. This stereo angle will provide a $z$ resolution of $\sim 700 \mu \mathrm{m}$, which is good enough to provide a vertex $z$ resolution of $\leq 2 \mathrm{~mm}$ from the IPCs alone, so that this vertex $z$ resolution will be available th the highest luminosities at the SSC.

The wires will not be read out in the IPCs since all of the information comes from the pads. The readout electronics for each pad include a fast front-end amplifier and shaper feeding an ana. o, pipeline that is multiplexed at the output by a factor of 128 , giving a total of 3200 channels.

\subsubsection{Rationale for Choice of Detector Configuration}

The choice of the various parameters that define the configuration of the central tracker can be summarized as follows:

- Inner radius of silicon detector. The impact parameter resolution improves as the inner radius of the silicon detector decreases. However, the radiation dose of the innermost silicon layer increases as $1 / R^{2}$. The resolution and the radiation dose are shown as a function of $R_{\min }$, the inside radius of the silicon detector, in Figure 6-3. A safe lifetime dose that silicon detectors can tolerate is around $5 \mathrm{Mrad}$. This sets the safe lower limit on the inner radius to around $10 \mathrm{~cm}$.

- Outside radius of the silicon detector. Increasing $\mathbf{R}_{\max }$, the outer radius of the silicon detector, improves the impact parameter determination. However, the area of silicon required and therefore the cost $\left(\sim \$ 2 \mathrm{M} / \mathrm{m}^{2}\right.$, including electronics) goes up as $\mathrm{R}^{2}$ max (keeping the same rapidity coverage). These quantities are shown as a function of $R_{\max }$ in Figure 6-4. $R_{\max }$ was chosen to be $22 \mathrm{~cm}$ in the present design. $\mathbf{R}_{\max }$ smaller than this for the chosen number of tracking chambers is not practical mechanically. Increasing $\boldsymbol{R}_{\max }$ beyond this value does not improve the impact parameter resolution very rapidly, but would drive up the cost of the silicon system considerably (see Figure 6-4).
- Silicon microstrip pitch and strip length. The $50-\mu \mathrm{m}$ pitch of the present design is now quite standard for silicon detectors and provides adequate resolution and low occupancy. The silicon strip length was chosen to be $18 \mathrm{~cm}$. This length simplifies the mechanical design; it allows the elimination of cooling rings near the center of the barrel and allows all electronics to be at the ends of the barrel and the outside edges of the forward disk, with all the cooling and cabling on the outer edges as well. Strip lengths longer than $18 \mathrm{~cm}$ would not be practical because of signal-to-noise considerations.

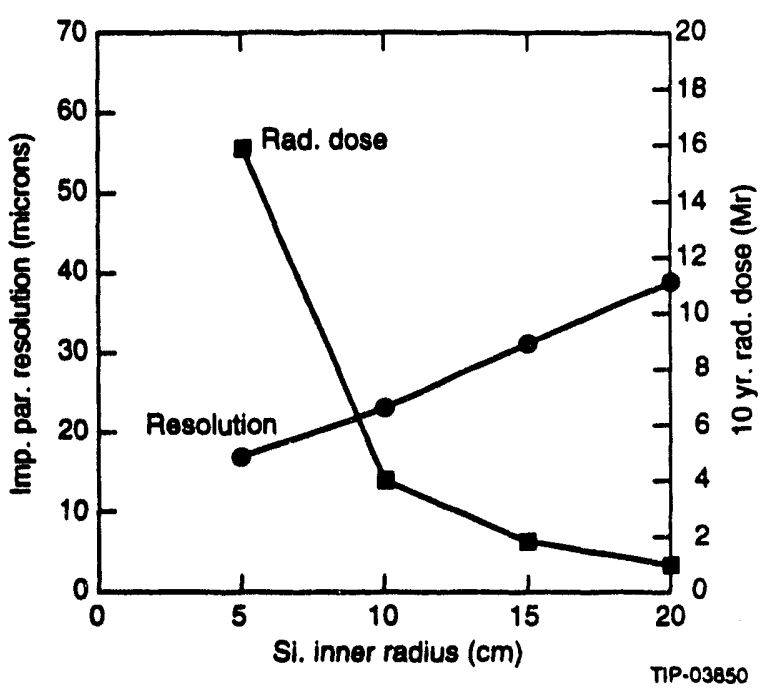

FIG. 6-3. Impact parameter resolution and 10-yr radiation dose rate as a function of the inner radius of the silicon detector.

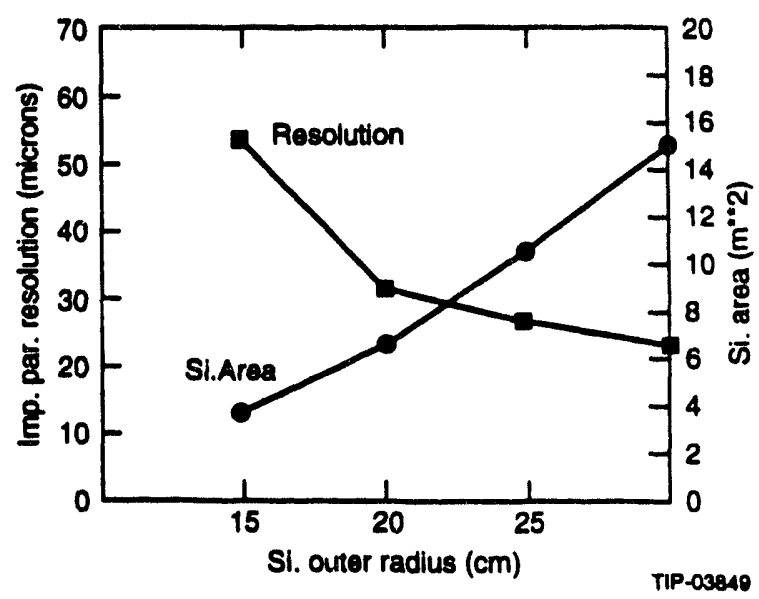

FIG. 6-4. Impact parameter resolution and area of silicon detectors as a function of the outer radius of the silicon detector with the inner radius fixed at $10 \mathrm{~cm}$. 
- Pad size and channel count. Pad size and channel count in the IPC detector are determined by the requirements to keep the occupancy sufficiently low to allow the IPC tracker to operate at luminosities up to $10^{34} \mathrm{~cm}^{-2} \mathrm{~s}^{-1}$. Previous experience with trackers indicates that the performance degrades significantly at occupancies above $10 \%$. The pad sizes in this design, averaging $\Delta \eta \Delta \phi \sim 0.0007$, meet this requirement with some safety margin. These pad sizes lead to a total number of pads in the system in the vicinity of 400000 . Increasing the number of pads beyond this would be desirable, but not necessary, and would increase the cost of the electronics.

- Outer radius of the central tracker. The magnetic field of $0.8 \mathrm{~T}$ is determined by an optimization of the muon system and magnet cost vs. performance. At this fixed field the momentum resolution of the central tracker improves roughly like the square of the outer radius, $R^{2}$ max. From the point of view of the tracker, a larger $R_{\max }$ would be clearly better. If the number of layers and the electronics channel count were kept fixed as $R_{\max }$ increases, the cost of the tracker would not increase very much. The cost constraint comes from the calorimeter cost, which increases very roughly by $\sim \$ 10 \mathrm{M}$ with a $25-\mathrm{cm}$ increase in tracker radius (see Figure 6-5). An inner radius of around $1 \mathrm{~m}$ makes the EM calorimeter tower sizes at the chosen $\eta$ and $\phi$ segmentation convenient from a mechanical point of view as well as appropriate in comparison with the Moliere radius of an EM shower. Thus, the tracker outer radius was set in large part by the optimization of the calorimeter.

- The number of layers of silicon and IPCs was set by the desire to have a reasonable pattern recognition efficiency. Much fewer than the 14 layers of this design would not allow acceptable track reconstruction efficiency in the high rate environment of the SSC. The momentum resolution improves as the square root of the number of layers, and the electrongamma separation also improves with more layers. Increasing the number of layers much over the present design would not be possible

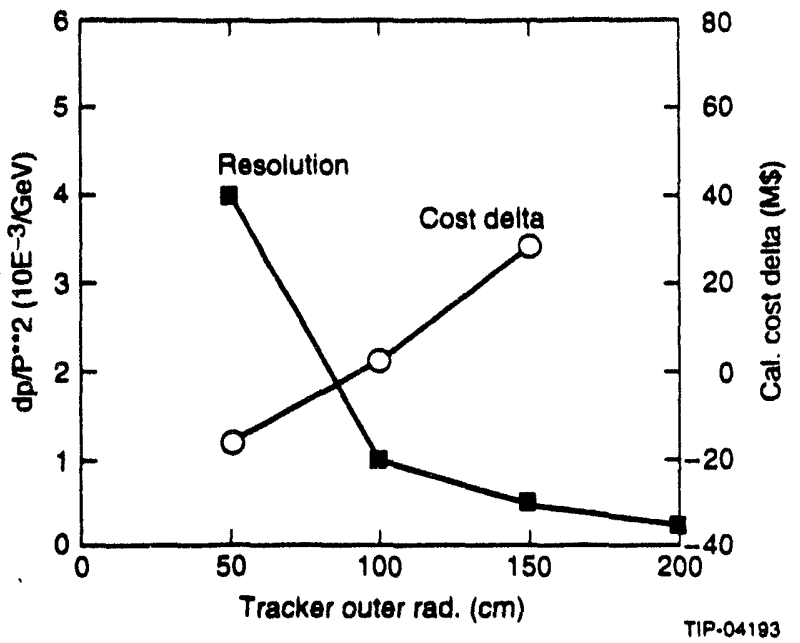

FIG. 6-5. Momentum resolution and the incremental cost of the calorimeter as a function of the central tracker outer radius.

because of both space considerations and the significant increase in cost.

- The stereo angles in the silicon and IPC detectors. Larger stereo angles give better $z$ resolution but also increase the probability of multi-track ambiguities. The present design of $\pm 5 \mathrm{mrad}$ in the silicon and $\pm 50 \mathrm{mrad}$ in the IPC is large enough to provide the required $z$ resolution of around $1 \mathrm{~mm}$ (see Figure 6-12), while keeping the probability of multi-track ambiguities below $\sim 1 \%$ at $\mathcal{L}=10^{33}$ and below $10 \%$ in the IPC at $\mathcal{L}=10^{34}$.

\subsubsection{Performance Parameters}

The design parameters for the central tracker that will satisfy the physics goals outlined above are summarized in Täble 6-1.

A GEANT-based Monte Carlo simulation program is now ready and running with a detailed description of the central tracker. The simulation includes our current best estimate of the amount of material in the silicon, the pad chambers, the support structures, the cooling loops, gas piping, the frontend electronics, and cables. The amount of material, in units of radiation lengths averaged over $\phi$, is shown as a function of $\eta$ in Figure 6-6. The average amount of material before the outermost layer of the tracker at $90^{\circ}$ is less than $15 \%$ of a radiation length. 
Table 6-1. Design parameters for the central tracker.

\begin{tabular}{|c|c|}
\hline Outer radlus & $90 \mathrm{~cm}$ \\
\hline Length & $350 \mathrm{~cm}$ \\
\hline Rapidity coverage & $|\eta| \leq 2.5$ \\
\hline Magnetic field & $0.8 \mathrm{~T}$ \\
\hline $\begin{array}{l}\text { Occupancy } \\
\text { at } \mathcal{L}=10^{33} \mathrm{~cm}^{-2} \mathrm{~s}^{-1} \\
\text { at } \mathcal{L}=10^{34} \mathrm{~cm}^{-2} \mathrm{~s}^{-1}\end{array}$ & $\begin{array}{l}\leq 1 \% \\
\leq 10 \%\end{array}$ \\
\hline Charge separation at $95 \%$ c.l. & $p \leq 600 \mathrm{GeV}$ \\
\hline $\begin{array}{l}\text { Momentum resolution at high } \\
\text { momenta (massurement } \\
\text { limited) }\end{array}$ & $\begin{array}{l}\Delta p / p^{2}= \\
(1.2) \times 10^{-3}(\mathrm{GeV} / \mathrm{c})^{-1}\end{array}$ \\
\hline $\begin{array}{l}\text { Momentum resolution at low } \\
\text { momenta (multiple scattering } \\
\text { limited) }\end{array}$ & $\Delta p / p=3.5 \%$ \\
\hline $\begin{array}{l}\text { Vertex resolution } \\
\text { along beam direction } \\
\text { impact parameter }\end{array}$ & $\begin{array}{l}\delta z \sim 1 \mathrm{~mm} \\
\delta b \sim 25 \mu \mathrm{m} \text { above } \\
10 \mathrm{GeV} / \mathrm{c}\end{array}$ \\
\hline
\end{tabular}

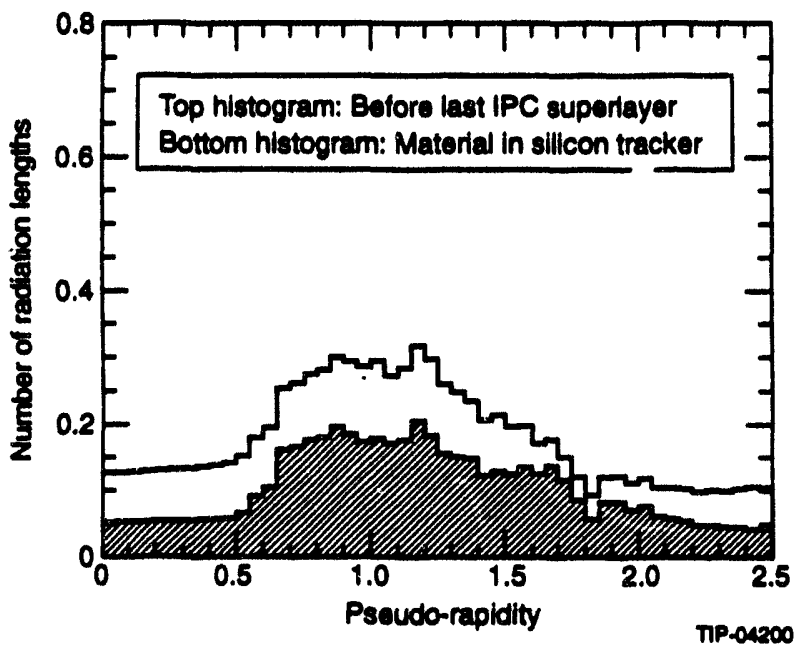

FIG. 6-6. Material, in units of radiation length, in the central tracker. Shaded histogram is the silicon detector only; the open histogram includes the IPC tracker up to the outermost detector layer. The rise in material after pseudorapidity of 0.7 is due to the electronics mounted on the detectors and the cables.

This simulation assumes the chamber resolutions as shown in Table 6-2. With this input, and the amount of material discussed above, the momentum resolution of the tracker, given the $0.8 \mathrm{~T}$ field, was calculated. Figure 6-7 shows the momentum resolution at $90^{\circ}$ as a function of momentum. For this curve it was assumed that a reconstructed primary vertex point from several tracks in each event can be used in the best fit for each track. For the highest momentum, the multiple scattering is negligible, and we see that the measurement error near $90^{\circ}$ is $\Delta p / p \sim 1 \times 10^{-3} \times p(\mathrm{GeV} / \mathrm{c})$. The resolution at low momentum is dominated by multiple scattering, which limits the low momentum resolution near $90^{\circ}$ to $\Delta p / p-3.5 \%$. Great care has been taken to minimize the material in the tracker without compromising the physics goals in the demanding environment of the SSC. (Any further reduction in material without a physics penalty can be found only in a significantly increased central tracker budget, and the relative improvement in the low momentum resolution is small.)

Table 6-2. Central tracker resolutions and aligmnent stabilities assumed in the simulations.

\begin{tabular}{ll}
\hline Silicon microstrips resolutionlayer & $10 \mu \mathrm{m}$ \\
Silicon alignment stability & $10 \mu \mathrm{m}$ \\
Pad chamber resolution & $50 \mu \mathrm{m}$ \\
Pad chamber alignment stability & $25 \mu \mathrm{m}$ \\
\hline
\end{tabular}

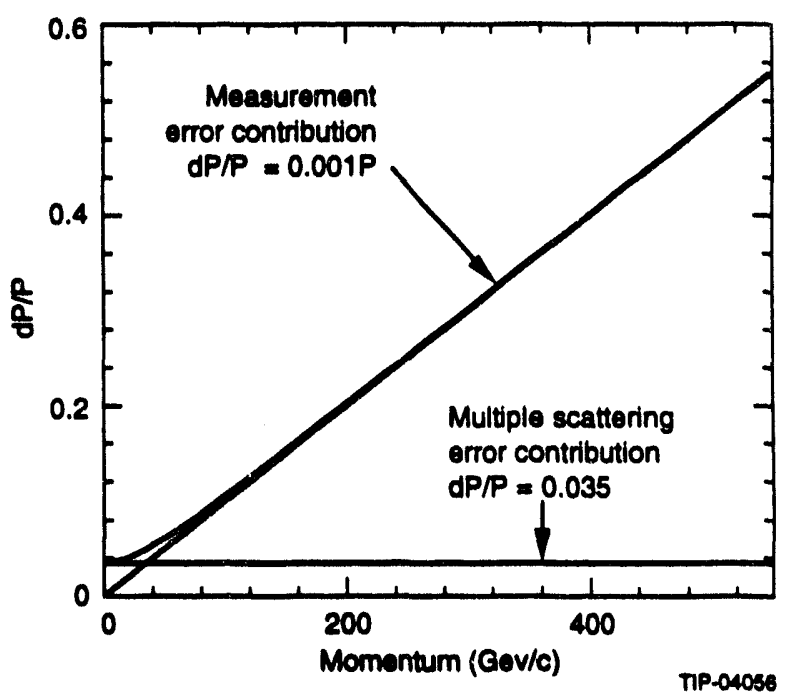

FIG. 6-7. Momentum resolution as a function of momentum for the central tracker. The multiple scattering error dominates at very low momenta.

Figure 6-8 shows the momentum resolution as a function of $\eta$. The momentum for which the sign of a particle can be determined at a $95 \%$ confidence level has been calculated and is shown in Figure 6-9 for both muons and electrons.

Figures 6-10 and 6-11 show the impact parameter resolution as a function of momentum and rapidity, respectively. Figure 6-12 shows the $Z$-reso- 
lution at the vertex for a single track. The upper curve is for the IPCs alone, as will be the case at $10^{34} \mathrm{~cm}^{-2} \mathrm{~s}^{-1}$. The determination of the vertex position will normally use many tracks so that the resolution will be correspondingly better.

The GEANT simulation was also used to estimate the occupancy in various parts of the

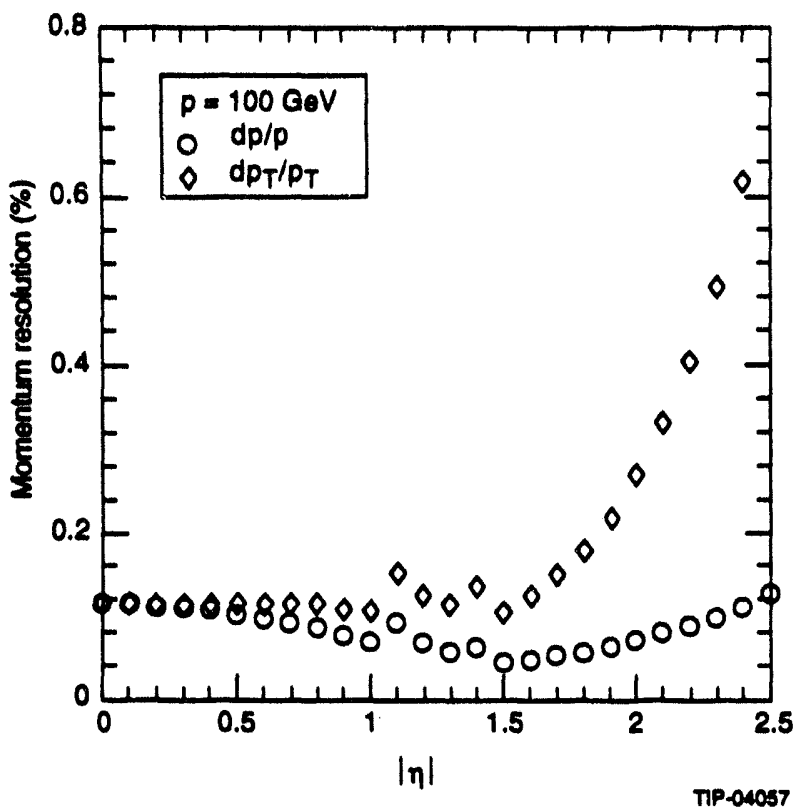

FIG. 6-8. Momentum resolution for $100 \mathrm{GeV} / \mathrm{c}$ tracks as a function of pseudorapidity $\eta$.

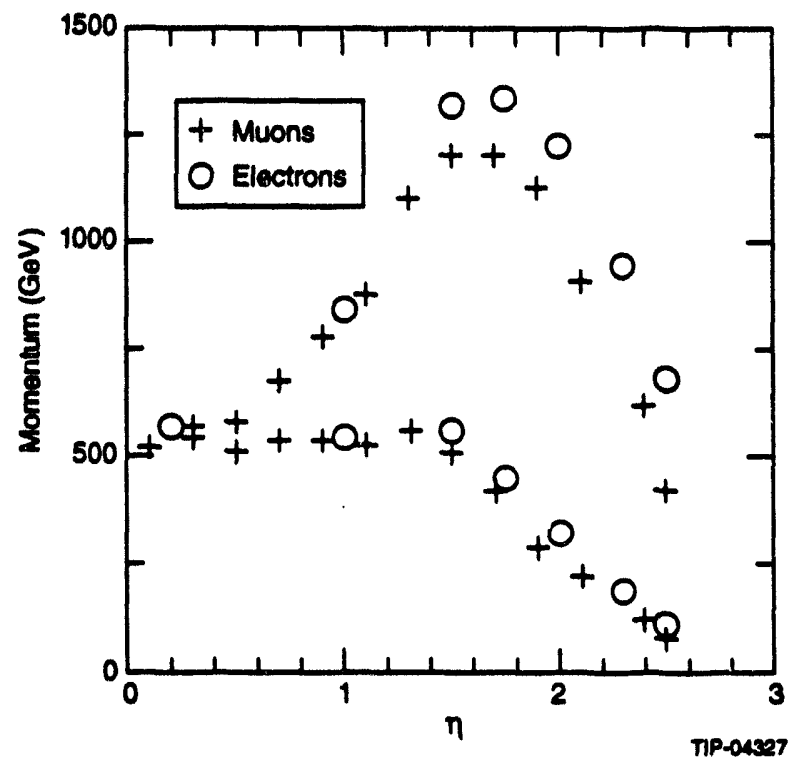

FIG. 6-9. Momentum for which the sign of the charge of muons and electrons can be measured at the $95 \%$ confidence level. The upper curve is for total momentum $p$; the lower curve is for transverse momentum Pr. detector. These calculations included the primary tracks produced in the $p p$ collisions as generated by PYTHIA (which agree quite well with $d n / d \eta d \phi \sim 1$ charged track per event), as well as secondaries produced in interactions, gamma conversions in the material of the tracker, and loopers; i.e., low-energy particles curling up in the magnetic field and producing multiple hits in the tracker. In this calculation, it was assumed that the silicon detector integrates over one crossing only, while the pad

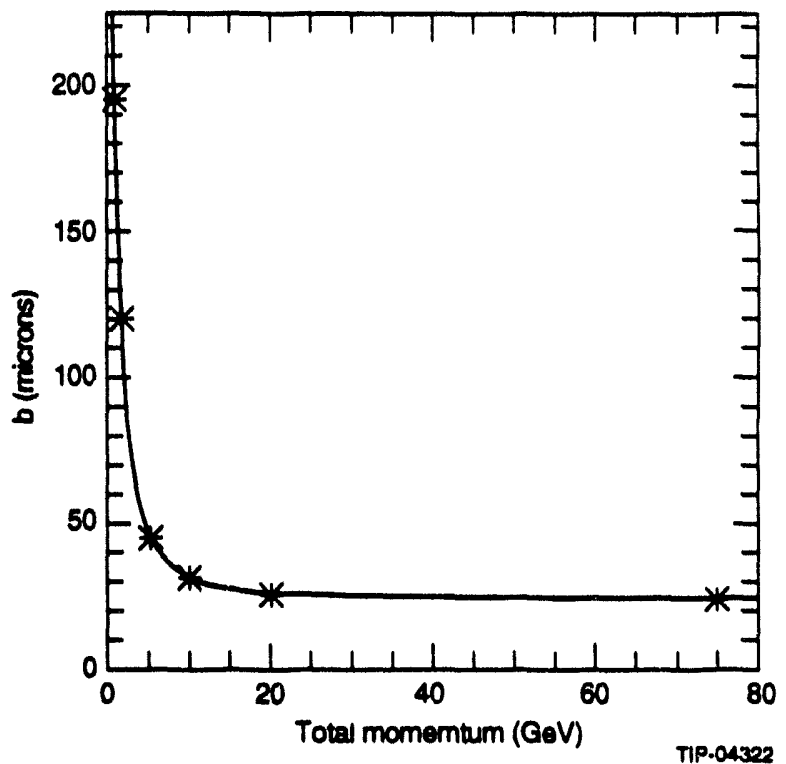

FIG. 6-10. Resolution in the impact parameter $b$ at the event vertex as a function of momentum for isolated tracks.

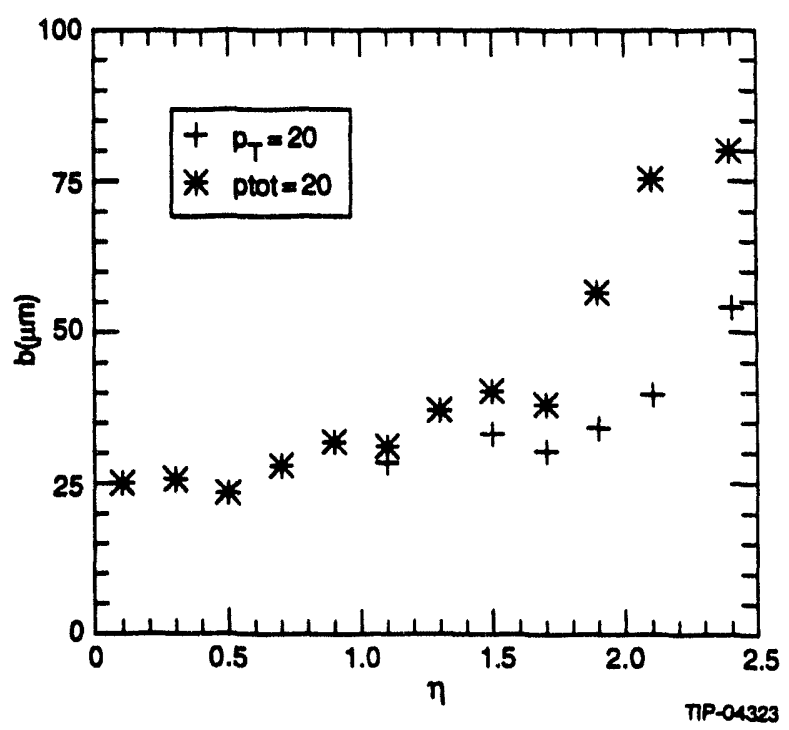

FIG. 6-11. Resolution in the impact parameter $b$ at the event vertex for isolated $20-\mathrm{GeV} / \mathrm{c}$ tracks as a function of pseudorapidity $\eta$. 


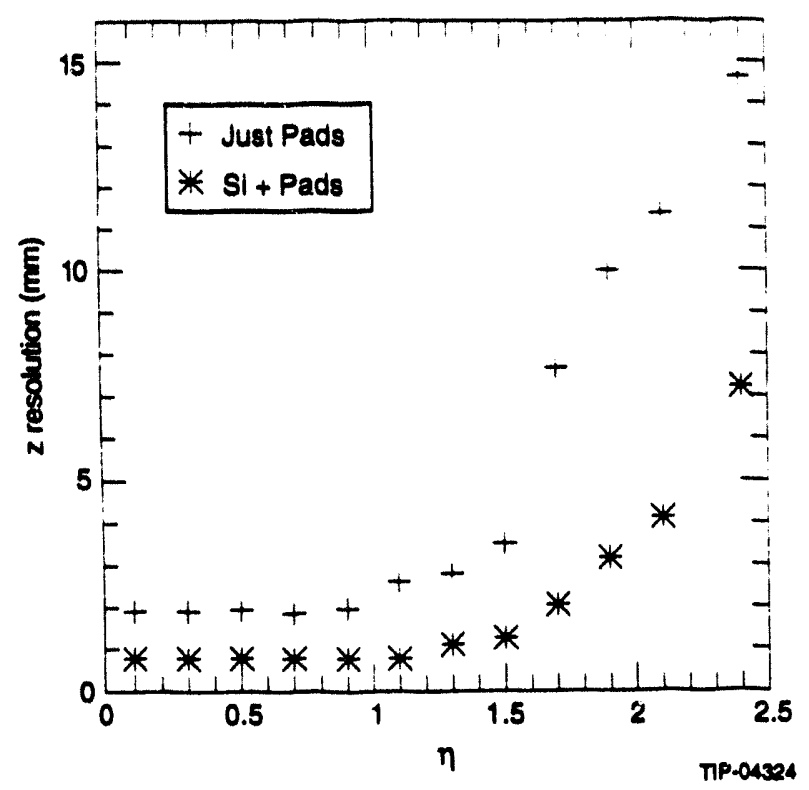

FIG. 6-12. Resolution in the vertex intercept in the direction along the beams (z) for isolated tracks.

detector integrates over two crossings of the beams. Near $90^{\circ}$ the occupancy in the silicon is typically less than $0.1 \%$, and in the pads it is around $0.4 \%$ per pad at a luminosity of $10^{33} \mathrm{~cm}^{-2} \mathrm{~s}^{-1}$ and around $4 \%$ at a luminosity of $10^{34} \mathrm{~cm}^{-2} \mathrm{~s}^{-1}$. We therefore expect the pad detector to perform well even at the highest luminosities of the SSC.

\subsection{DETECTOR SIMULATION AND PERFORMANCE}

\subsubsection{Tracker Parameters}

\section{Position Resolutions}

The technologies chosen for the GEM central tracker (silicon microstrips and interpolating pads) must provide the position resolution necessary to achieve the overall momentum and other track measurement requirements. Silicon microstrip detectors are in widespread use with demonstrated resolutions of about $15 \mu \mathrm{m}$ for normal incidence with $50-\mu \mathrm{m}$ pitch detectors. This level of performance achieves the desired resolutions for momentum measurement and vertex reconstruction, as will be demonstrated in Section 6.2.2. below. This performance is achieved without analog information on charge sharing between strips, although double strip hits for non-normal incidence can reduce the resolu- tion to about $10 \mu \mathrm{m}$. In the GEM central tracker, the microstrip detectors are aligned with their microstrips running approximately axially. The longitudinal position is obtained by a small stereo angle between pairs of strips. The longitudinal resolution $(\delta z)$ of two strips with a half-angle of $\phi$ between the two strips (called the stereo angle) and with a single strip transverse resolution $\sigma$ is

$$
\delta z=\sigma / \sqrt{2} \phi .
$$

The ambiguity of paired strips increases with the stereo angle. Larger stereo angles increase the :ikelihood that a pair of tracks will cross strips with common partners and lead to an ambiguous matching of the strips. Therefore, one cannot arbitrarily increase this angle in the GEM environment. Nevertheless, based on the nominal occupancies in the GEM silicon tracker of roughly 0.001 , a $5-\mathrm{mrad}$ stereo angle, resulting in longitudinal resolution of better than $2 \mathrm{~mm}$ per pair, allows sus acceptably low ambiguity rate of less than a few percent.

The position measurement in the interpolating pad system is achieved through charge division between the cathode pads. In this system, a transverse pad size of $2.5 \mathrm{~mm}$ must be converted into the design goal position resolution of $50 \mu \mathrm{m}$. This is done by measuring the induced charge on three to five adjacent pads with a signal resolution of about $1 \%$ of the total measured cathode signal. The GEM prototype has demonstrated this level of performance as described in Section 6.4.1. The longitudinal position measurement from the interpolating pad system is achieved using the same technique as for the silicon: stereo strips. Since the resolution of the interpolating pad system is inherently worse than silicon, a larger stereo angle is required to achieve millimeter precision. The design calls for a more precise measurement from the outer tracker, since at the highest luminosities $\left(10^{34}\right)$ the silicon will eventually fail, and the IPC system will be required to provide this measurement on its own. A goal of 1-mm per pad layer longitudinal resolution has been set. To achieve this, the pads must operate at a larger stereo angle of $\pm 50 \mathrm{mrad}$. The performance of the pads will be somewhat degraded for tracks that are not of normal incidence. Such issues are under investigation as part of the R\&D program. 


\section{Material Thickness}

The GEM central tracker has been designed with the minimization of material as an important constraint on the overall optimization. Support structures are designed with composite materials, detector configuration choices are made to minimize the paths of services, and other trade-offs are evaluated with material minimization in mind. Such concerns are important as the material within the tracking volume limits the low-energy momentum resolution of the tracker and degrades the GEM high-precision electromagnetic calorimeter performance through the conversion of $\gamma s$ within the tracker and the bremsstrahlung radiation from electrons. The silicon microstrip length choice is an example of a design choice made specifically to address this issue. By increasing the strip lengths to $18 \mathrm{~cm}$ (through bonding of three microstrip detectors together) the inner material in the form of electronics, cabling, and cooling services has been reduced. Figure 6-6 presents the profiles of the material found in the central tracker.

\section{Occupancy}

In the design and optimization of the GEM central tracker, occupancies have been a major concern. The silicon subsystem has inherently small occupancies ( $\approx 0.001$ at $10^{33}$ luminosity) due to the very small cell sizes that result from the $50-\mu \mathrm{m}$ strip pitch. While not characterized by quite such small occupancies, the interpolating pad system does nearly as well due to the small pad sizes and limited region of induced signal. The pads typically achieve occupancies better than $1 \%$ at $10^{33}$ luminosity and are better than $10 \%$ at $10^{34}$. The occupancies have been calculated using a PYTHIA generator with a full GEANT simulation of the material in the tracker. They include the effects of secondary interactions, $\gamma$-conversions and knock-on electrons, and low-energy particles curling up in the magnetic field. Table 6-3 presents the occupancies in the rlicon, and Table $6-4$ shows the pads occupancies.
Table 6-3. Occupancy in the silicon detectors.

\begin{tabular}{|c|c|c|}
\hline \multirow[b]{2}{*}{ Barrel layer } & \multicolumn{2}{|c|}{$\begin{array}{c}\text { Occupancy at } \mathcal{L}=10^{33} \mathrm{~cm}^{-2} \mathrm{~s}^{-1} \\
\text { per strip }(\%)\end{array}$} \\
\hline & $\begin{array}{l}\text { Min. Bias } \\
\text { Background }\end{array}$ & $\begin{array}{l}\text { Min. Bias + } \\
\text { Higgs Event }\end{array}$ \\
\hline 1 & 0.16 & 0.35 \\
\hline 2 & 0.13 & 0.27 \\
\hline 3 & 0.09 & 0.19 \\
\hline 4 & 0.07 & 0.15 \\
\hline 5 & 0.06 & 0.12 \\
\hline 6 & 0.05 & 0.10 \\
\hline Forward layer & & \\
\hline 1 & 0.04 & 0.09 \\
\hline 2 & 0.04 & 0.09 \\
\hline 3 & 0.04 & 0.09 \\
\hline 4 & 0.04 & 0.09 \\
\hline 5 & 0.04 & 0.09 \\
\hline 6 & 0.05 & 0.10 \\
\hline 7 & 0.05 & 0.10 \\
\hline 8 & 0.05 & 0.10 \\
\hline 9 & 0.05 & 0.10 \\
\hline 10 & 0.05 & 0.10 \\
\hline 11 & 0.05 & 0.10 \\
\hline 12 & 0.06 & 0.11 \\
\hline 13 & 0.06 & 0.11 \\
\hline 14 & 0.06 & 0.11 \\
\hline
\end{tabular}

Table 6-4. Occupancy in the IPC chambers.

\begin{tabular}{|c|c|c|}
\hline \multirow{2}{*}{} & \multicolumn{2}{|c|}{ Occupancies per pad (\%) } \\
\cline { 2 - 3 } & $\mathcal{L}=10^{33} \mathrm{~cm}^{-2} \mathrm{~s}^{-1}$ & $\mathcal{L}=10^{34} \mathrm{~cm}^{-2} \mathrm{~s}^{-1}$ \\
\hline 1 & & \\
2 & $0.4-0.6$ & $4-6$ \\
3 & $0.4-0.5$ & $4-5$ \\
4 & $0.4-0.5$ & $4-5$ \\
& $0.3-0.4$ & $3-4$ \\
Forward layer & & \\
1 & $0.6-0.5$ & $6-5$ \\
2 & $0.6-0.5$ & $6-5$ \\
3 & $0.6-0.4$ & $6-4$ \\
4 & $0.5-0.4$ & $5-4$ \\
\hline
\end{tabular}




\subsubsection{Tracker Intrinsic Performance}

\section{Detector Resolutions}

\section{Momentum Resolution}

The GEM central tracker momentum resolution is limited at low momentum by the multiple scattering in the tracker and at higher momenta (above $=30 \mathrm{GeV}$ ) by the measurement errors of the tracking detectors, the magnetic field of the GEM solenoid, and the overall tracker volume. As discussed in Section 6.2.1, the design has been constrained to reduce material in the tracking volume, in order to minimize the multiple scattering effects and to optimize performance at low momentum. A multiple scattering limit of about $3.5 \%$ momentum resolution has been achieved. The measurement-limited resolution is constrained by the $0.8-\mathrm{T}$ field of the GEM solenoid. The tracking volume is constrained by technical and financial constraints on the calorimeter and the muon systems. The tracker radius has been optimized through a thorough review of the trade-offs between the subsystems, and as a result the tracker outer radius has increased somewhat in a final iteration of the GEM design. The result is a configuration that gives a measurementlimited momentum resolution of $0.00115 p_{T}$. Figure 6-13 illustrates these features. This figure presents the momentum resolution at $90^{\circ}(\eta=0)$ as a function of momentum. The points indicate the results of a full GEANT simulation of tracking through the GEM tracking system followed by reconstruction. The solid curve is a parametrization of the resolution in the form

$$
\frac{\sigma}{p}=\sqrt{(0.00115 p)^{2}+(0.035)^{2}} .
$$

The resolution versus pseudorapidity for fixed transverse and total momenta has been presented in Figure 6-8. There we see that the basic performance remains uniform out to about $\eta=1.5$, beyond which the track lengths in the transverse plane are truncated by the end of the tracker volume.

As GEM moves to operation at the highest luminosities, the tracking reconstruction will depend more and more on the pad system alone as the silicon subsystem gradually degrades. The upper curve on Figure 6-13 shows the momentum resolution for the pad system alone compared to the resolution of the full system including silicon.
The pad system alone represents the expected performance at the highest luminosities $\left(\mathcal{L}=10^{34} \mathrm{~cm}^{-2} \mathrm{~s}^{-1}\right)$.

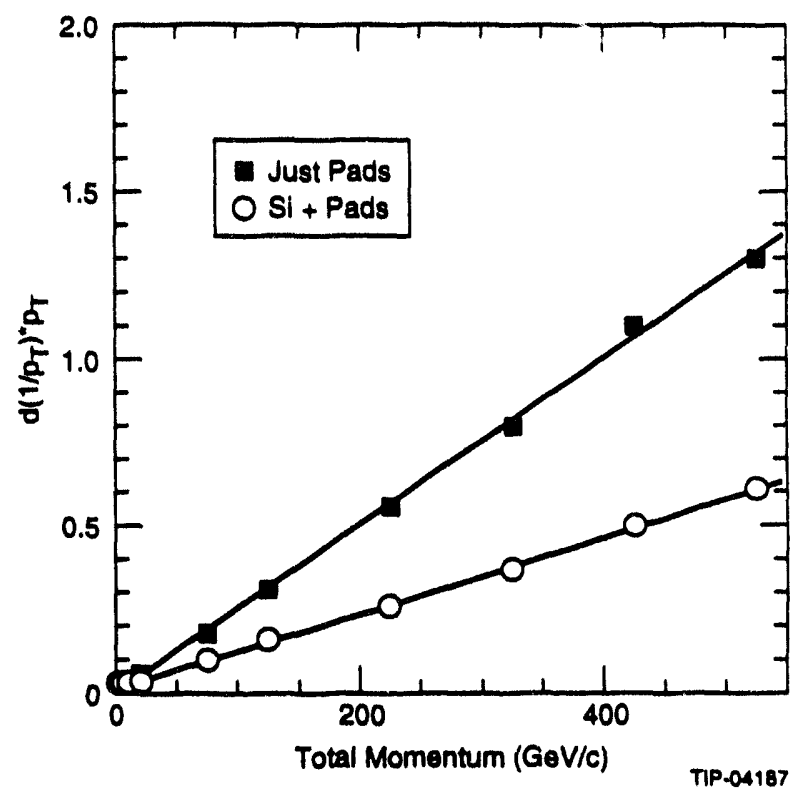

FIG. 6-13. Measurement-limited momentum resolution at $\eta=0$ as a function of momentum for the full tracker (relevant for $10^{33}$ luminosity) and for the interpolating pad tracker (relevant for $10^{34}$ luminosity). The data points are shown with the functions

$\frac{\sigma \text { (full) }}{p}=\sqrt{(0.00115 p)^{2}+(0.035)^{2}}$ and $\frac{\sigma \text { (pads) }}{p}=\sqrt{(0.0025 p)^{2}+(0.035)^{2}}$.

The momentum measurement of electrons is complicated by the loss of energy by the electron through bremsstrahlung as it passes through the tracker. However, the tracker represents about $15 \%$ of a radiation length of material in the barrel and only a few percent of the tracks have secondary electrons produced. The result is reasonably good reconstruction of electron tracks. Furthermore, the effect of the bremsstrahlung is to add curvature to the track, improving the charge determination. Figure 6-14 presents the distribution of reconstructed momenta for muons and electrons at 1,10 , and $100 \mathrm{GeV}$. The peak of the electron distribution is slightly offset to lower momenta due to the bremsstrahlung (this offset can be corrected for) and several percent of the tracks are significantly mismeasured. The one-sided tail on the electron distribution is evident. Nevertheless, the width of the central portion of the electron distribution, neglecting the tail, is very similar to that 
(a)

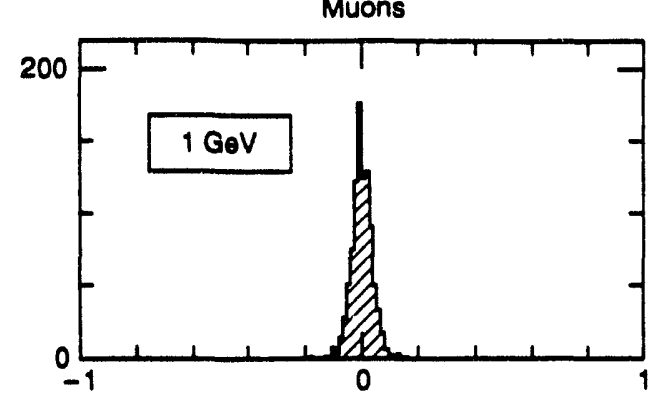

(c)

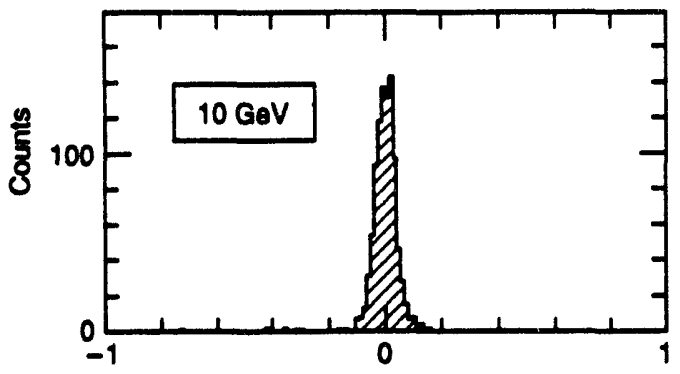

(b)

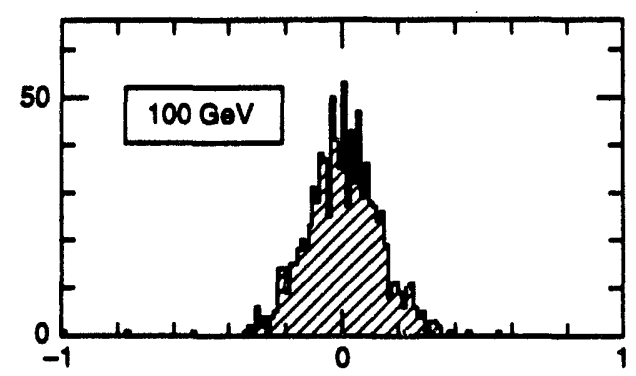

(b)

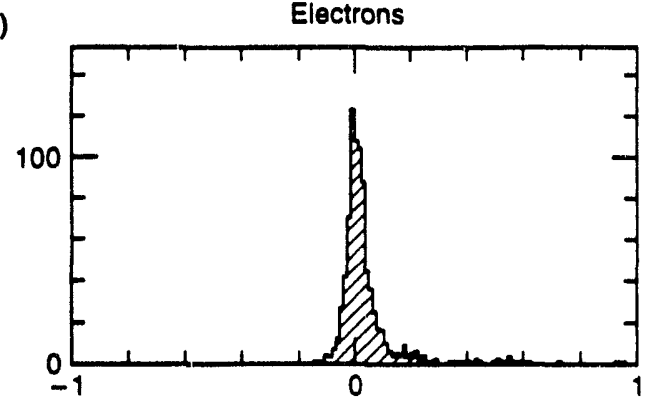

(d)

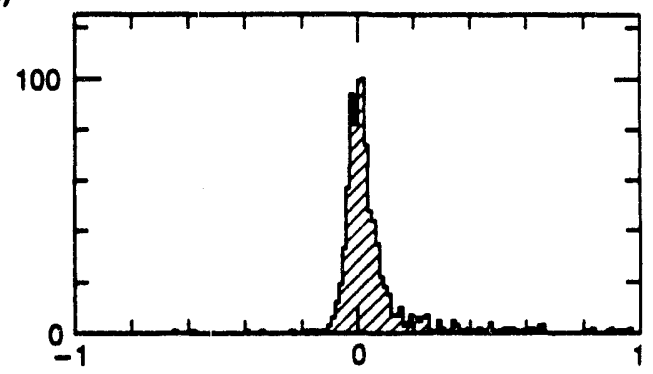

(1)

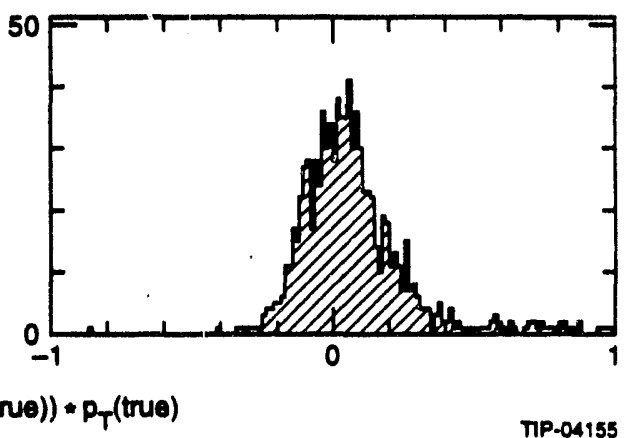

FIG. 6-14. Momentum resolution distributions for 1,10 , and $100 \mathrm{GeV} / \mathrm{c}$ electroris and muons at $\because y=0$.

Bremsstrahlung shifts the electron peak to lower momenta and the asymetric tails show that several percent are significantly mismeasured. The widths of the central region of the distributions arie the same as for the muons.

of the muon distribution and the isolated electron efficiency is $98 \%$.

\section{Impact Parameter Resolution}

The GEM central tracking system provides secondary vertex measurements through detection of tracks that have measurable impact parameters when projected back to the beamline. This capability is important for the heavy-quark physics goals of GEM. Figure 6-10 presented the impact parameter resolution at $90^{\circ}(\eta \approx 0)$ as a function of momentum, and Figure 6-11 showed the resolution versus pseudorapidity for fixed transverse and total momenta. At $90^{\circ}$ the resolution is well described by the function

$$
\sigma_{b}=24 \sqrt{1+\frac{70}{p^{2}}} \mu \mathrm{m}(p \text { in } \mathrm{GeV}) .
$$

\section{Pattern Recognition}

In order to demonstrate the track reconstruction capabilities of the GEM tracking configuration, a prototype pattem recognition algorithm has been developed and applied to GEANT-simulated events. The effects of expected detector inefficiencies and the geometrical dependence on detector coverage have been included. Figure 6-15 shows the number of tracking layers crossed by tracks emerging from the interaction region (with $\sigma=5 \mathrm{~cm}$ along $z$ ) as a function of pseudorapidity $(\eta)$. In the barrel $(\eta<1)$ 
there are typically 14 layers hit (6 double layers in the silicon system and 8 pad layers in the pad system). Due to the detailed geometry of the tracker, in some regions $(\eta \approx 1.2)$ the number is smaller and in some regions $(\eta \approx 2)$ it is larger.

The track-finding efficiency of the detector depends upon such factors as the geometric efficiency, the detector efficiencies, the track density, the deadtime of the detectors, the number of layers and the placement of the layers in the detector, and the pattern recognition algorithm itself. All of these factors have been investigated. The pattem recognition algorithm in present use builds vector trees originating on an inner layer of the tracker. The tree is searched for best $\chi^{2}$ sets of vectors. For the baseline configuration, the track-finding efficiency has been found to exceed $95 \%$ for muons and electrons. Figure 6-16 presents the efficiency for muons from Higgs decay as a function of pseudorapidity. The overall efficiency for these muons with $|\eta|<2.4$ is $97 \%$. Figure $6-17$ presents the efficiency for electrons from Higgs decay. For electrons the overall efficiency with $|\eta|<2.4$ is $97 \%$.

With time, the silicon microstrip detectors will develop increasing levels of electronic noise due to

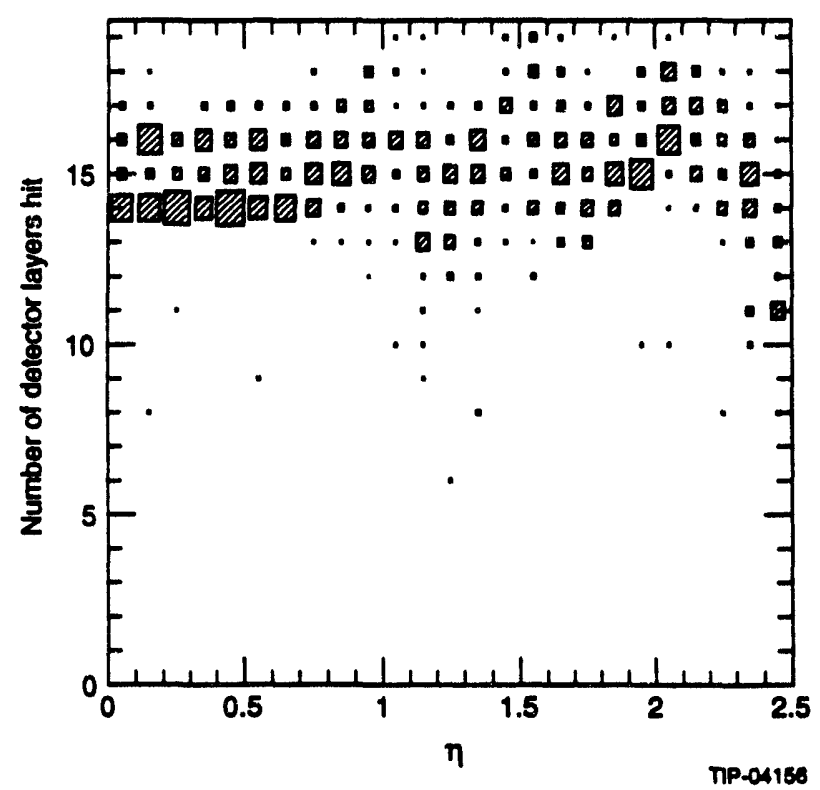

FIG. 6-15. The number of detector layers hit by $500-G e V$ muons passing through the central tracker as a function of $\eta$. Vertex smearing has been included. The box size represents the number of entries. The effects of expected detector inefficiencies and geometrical acceptance have been included. radiation damage to the silicon detectors and electronics. In this case, inefficiencies due to deadtime can become important. A careful study including all of these effects has shown that reconstruction efficiency remains large ( $>95 \%$ ) as long as the hit

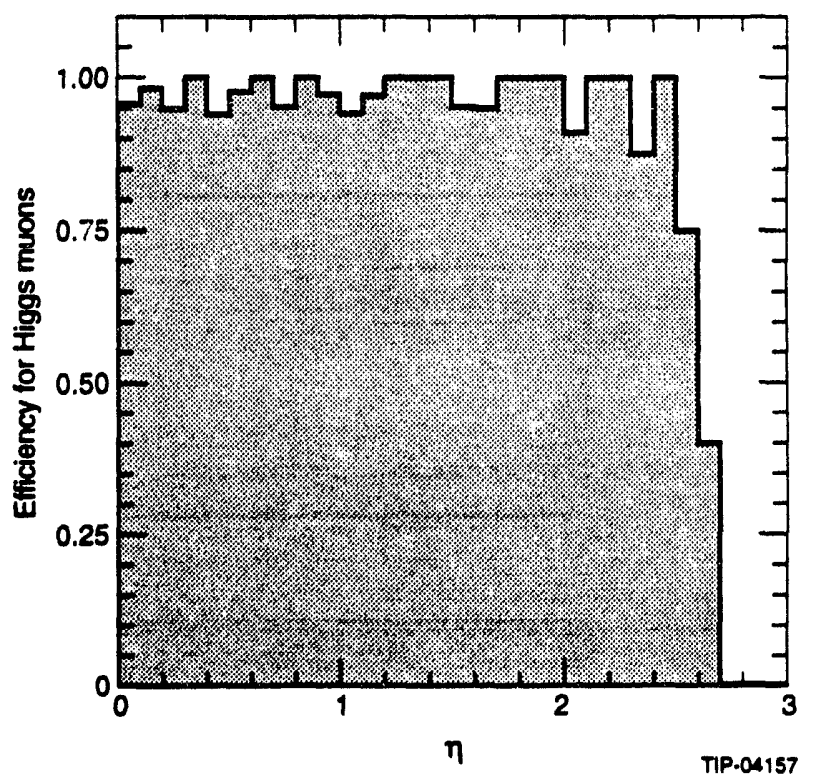

FIG. 6-96. The fraction of muons from $H \rightarrow Z Z \rightarrow$ $\mu \mu \mu \mu, \mu \mu \theta \theta$ decays that are found with the tracker pattern recognition program as a function of $\eta$. The efficiency is relatively constant at $97 \%$ out to $\eta=2$.

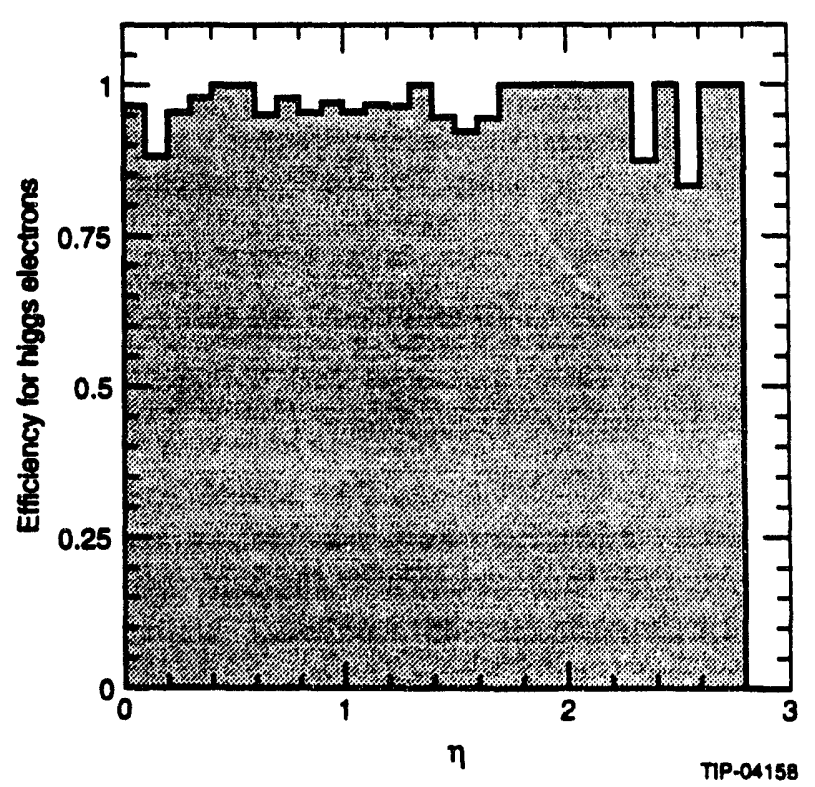

FIG. 6-17. The fraction of electrons from $H \rightarrow Z Z \rightarrow$

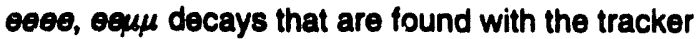
pattem recognition program as a function of $\eta$. The efficiency is very similar to that for muons, remaining constant at about $97 \%$ until $\eta=2$. 
threshold is raised to about three times the noise, for noise levels up to 3000 electrons.

For most of the physics goals of the central tracker, such as $e / \gamma$ or $e$ /hadron separation, full pattern recognition is not required; we need only look for the number of hits in a road extrapolated into the tracker from the calorimeter or the muon system. (See discussion in Section 6.2.3, for example.)

\section{Multitrack Resolution}

The ability of the GEM tracker to do multitrack finding is very important for charge identification of a lepton inside a jet, for reduction of backgrounds in electron identification from $\gamma$-conversion and Dalitz pairs, for eventual total reconstruction of a heavy quark jet, for top reconstruction, for $\tau$-identification, and simply for signal tracks of interest that have a minimum bias track very close to them. Multitrack resolution in the silicon tracker is excellent due to the $50-\mu \mathrm{m}$ pitch of the microstrips. The equivalent figure in the interpolating pad system is $2.5 \mathrm{~mm}$, and multitrack separation becomes a process of disentangling the analog signals that are mixed in neighboring strips. Multitrack resolution in the IPCs has been studied using a simulation program written by Cherniatin and Chikanian at BNL. 1 The program incorporates test data collected using cathode strip chambers running in $\mathrm{CO}_{2} \mathrm{CF}_{4}$.

\section{IPC One Track Resolution}

The single track resolution was estimated ${ }^{2}$ by generating the induced charge from a track passing through a pad at a given position and then fitting the resulting charge distribution to the function best describing charge induced on pads by Mathieson et al., 3,4,5,6

$$
q(x(i))=2 \frac{Q_{t o t}}{\pi} \tan ^{-1}\left[\frac{\sinh \left(\pi \frac{w}{4 l}\right)}{\cosh \left(\pi \frac{x(i)-x(0)}{2 l}\right)}\right]
$$

where $w=$ pad width, $x(i)=$ the distance from the center of the pad, $x(0)=$ the charge centroid, and $l=$ anode/cathode spacing. The resultant resolution is shown in Figure 6-18 as a function of position across the pad.

\section{IPC Two Track Resolution}

When two tracks are separated by more than one pad width in the outer tracker, the pattern is easily recognizable: two peaks are observed in the charge distribution. However, when the tracks cross the same pad, it is possible to recognize them as such only by looking at the average total charge, shown in Figure 6-19, which has been obtained at the SSCL using data taken with the cathode strip chambers with geometry similar to the outer tracker chambers. In the top figure, the total charge for one layer is shown, while in the bottom figure the average over eight layers is displayed. Once the signature of two tracks has been established, it is possible to fit the charge distribution to the Mathieson function for two tracks (essentially adding the charge from another track to the first one) and fitting for three parameters: the two positions and the ratio of the induced charges. The result of the two track resolution is shown in Figure 6-20, where only good fits are selected by a $\chi^{2}<2$ cut. The resulting efficiency is also shown. Tracks separated by more than $0.5 \mathrm{~mm}$ can be clearly resolved.

\section{IPC Three Track Resolution}

For three tracks trossing one pad, it is not possible to resolve the three peaks in the induced charge distribution. However, the total average charge can be used to determine that three tracks are present. As the maximum separation (the distance

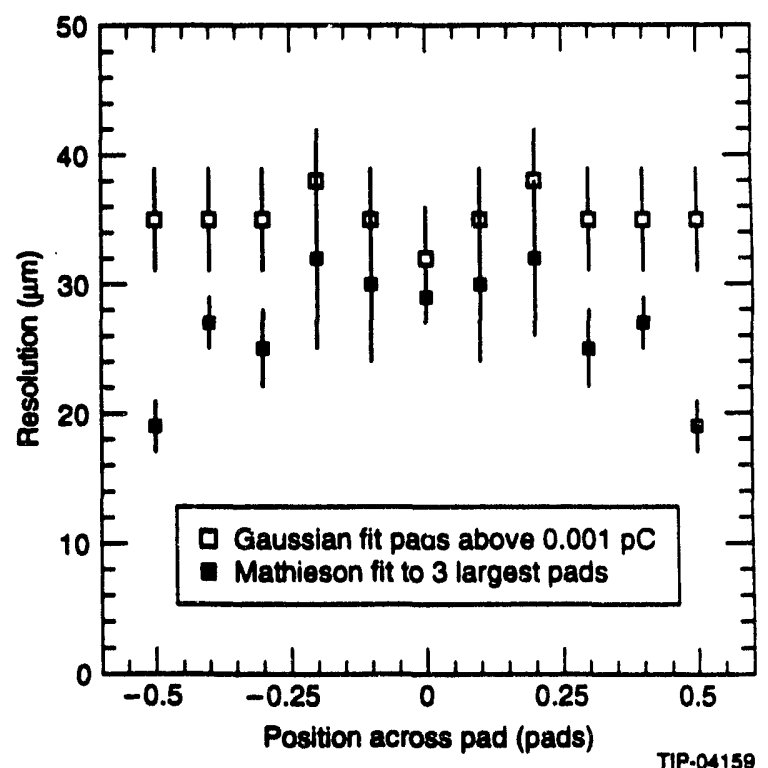

FIG. 6-18. The single track resolution for simulated tracks traversing an IPC layer as a function of the position across the pad of the incident track. Two different functions are fit to the charge distribution. The one due to Mathieson, which better describes the induced charge distribution, gives a better resolution than a Gaussian fit. 
between the two furthest tracks) is increased to two pad widths, three tracks can be resolved, and Figure 6-21 shows the average position resolution of the three tracks as a function of separation between
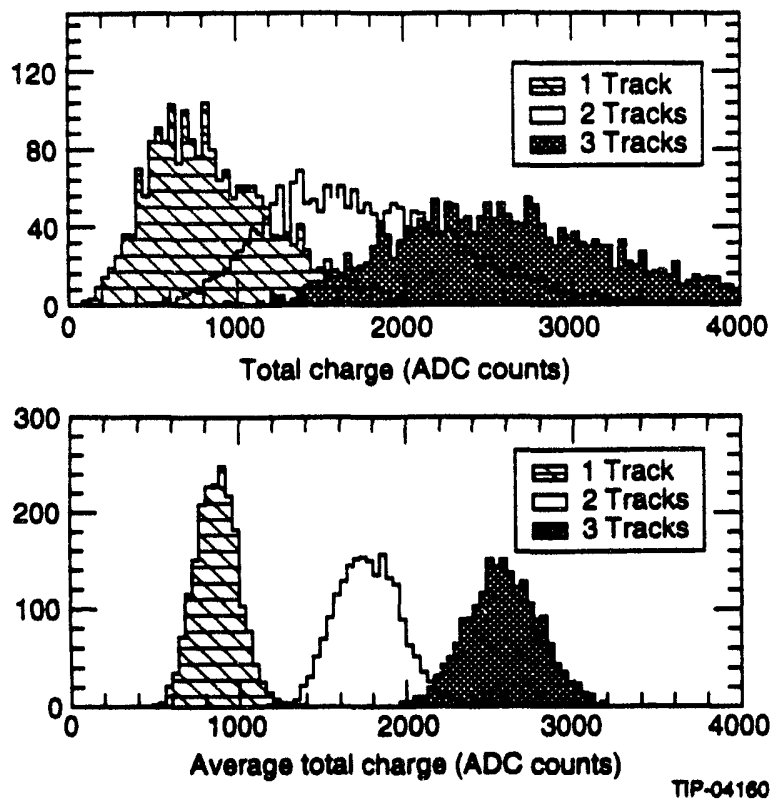

FIG. 6-19. Total charge deposited by one, two and three tracks in a layer of IPCs (top figure). The bottom figure shows the total charge averaged over eight layers for one, two and three tracks. There is a clear separation between one and two tracks with $<0.2 \%$ confusion when making a cut at $1300 \mathrm{ADC}$ counts, and between two and three tracks with $<2 \%$ confusion when making a cut at 2160 ADC counts.

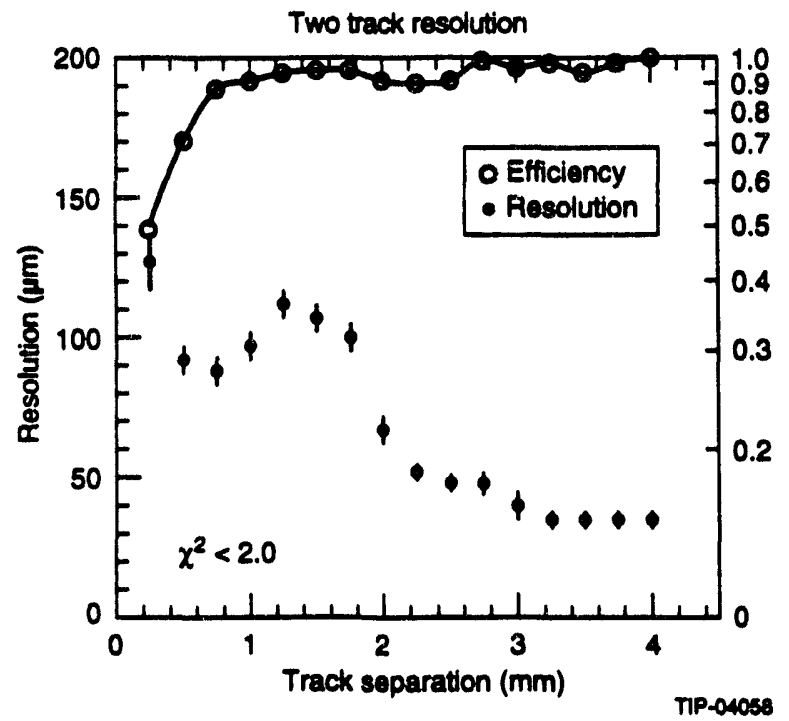

FIG. 6-20. A simulation of the IPC two track resolution as a function of distance between the two tracks as found by fitting two Mathieson functions to the charge distribution. The efficiency for the fit passing a $\chi^{2}$ cut $<2.0$ is also plotted. the two closest tracks for a maximum separation of two pad widths. The resolution is less than $100 \mu \mathrm{m}$ for tracks with $5 \mathrm{~mm}$ or more of maximum separation. A resolution of $250 \mu \mathrm{m}$ can be achieved when the minimum spacing is $>2 \mathrm{~mm}$, or approximately one pad width.

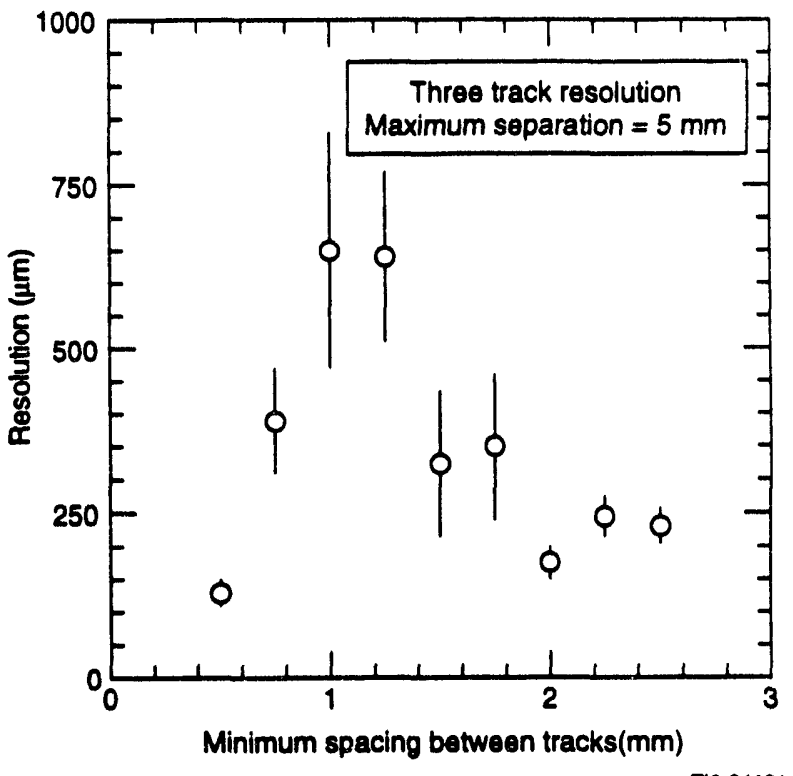

FIG. 6-21. IPC three track resolution as a function of the distance between the closest two tracks (minimum spacing) for a set separation between the farthest two tracks of $5.00 \mathrm{~mm}$. A resolution of $250 \mu \mathrm{m}$ can be achieved when the minimum spacing is $>2 \mathrm{~mm}$, or approximately one pad width.

\section{Vertex $z$ Resolution}

The measurement of the longitudinal position (z) of the primary vertex along the beamline is an important responsibility of the GEM central tracker. Accurate momentum vector reconstruction of neutral showers in the electromagnetic calorimeter depend on this measurement, as well as separation of multiple events in the beam crossing. The longitudinal vertex position resolution from individual tracks at $90^{\circ}(\eta=0)$ is $0.8 \mathrm{~mm}$ for tracks with momenta of $1 \mathrm{GeV}$ or more. It must be realized that the vertex position resolution for an event will be improved from this performance by the many tracks emerging from the vertex, all of which give independent measurements of its position. The vertex position resolution for the pad system alone from single tracks at $90^{\circ}$ was shown in Figure 6-12, along with the full silicon-pad resolution. This plot demonstrates that the GEM tracking system operating at the 
highest luminosities of the SSC, after the silicon subsystem has ceased to contribute to track reconstruction, will still provide good vertex position measurements.

\subsubsection{Physics Performance}

Vertex $z$ Resolution and $\gamma$ Direction Measurements

The measurement of the direction of $\gamma$ momentum vectors by locating the primary vertex is an important function of the GEM central tracker. For example, the search for the decays of a standard model Higgs into two photons relies on a primary vertex reconstruction by the tracker. For this search, the $z$ coordinate of the $\mathrm{H}^{0}$ vertex must be determined to within $5 \mathrm{~mm}$ in order to obtain the mass resolution necessary to identify a Higgs. ${ }^{7}$ The Higgs is produced with a substantial amount of transverse momentum that is balanced by particles recoiling against it. The tracker can count high $p_{T}$ charged particles associated with each interaction in a given time slot and determine the vertex with the highest probability of being the origin of the Higgs. ${ }^{8}$

Figure 6-22(a) shows the $p_{T}$ spectrum for $p_{T}>$ $0.5 \mathrm{GeV}$ isolated charged tracks. Signal and minimum bias interactions are shown separately. In Figure 6-22(b) the multiplicity for both signal and minimum bias interactions are shown. Higgs interactions contain on average many more tracks with higher $p_{T}$ than minimum bias interactions. From these tracks the vertex can be determined. Figure 6-23(a) shows the difference between the $z$ coordinate of the estimated Higgs vertex and the $z$ coordinate of the true Higgs vertex. The percentage of events in which the estimated vertex lies within $5 \mathrm{~mm}$ of the true Higgs vertex is shown in Figure 6-23(b) as a function of the $\mathrm{H}^{0}$ mass and luminosity. These results are not very dependent on the isolation requirement for track selection. The vertex tagging efficiency is approximately $95 \%$ at nominal luminosity and $75-80 \%$ at high luminosity. The observed tagging efficiency is independent of the $\mathrm{H}^{0}$ mass.

\section{Electron-Gamma Identification}

One of the most important functions of the GEM central tracker is the separation of electron and gamma showers in the electromagnetic calorimeter. This function is subdivided into identifying a
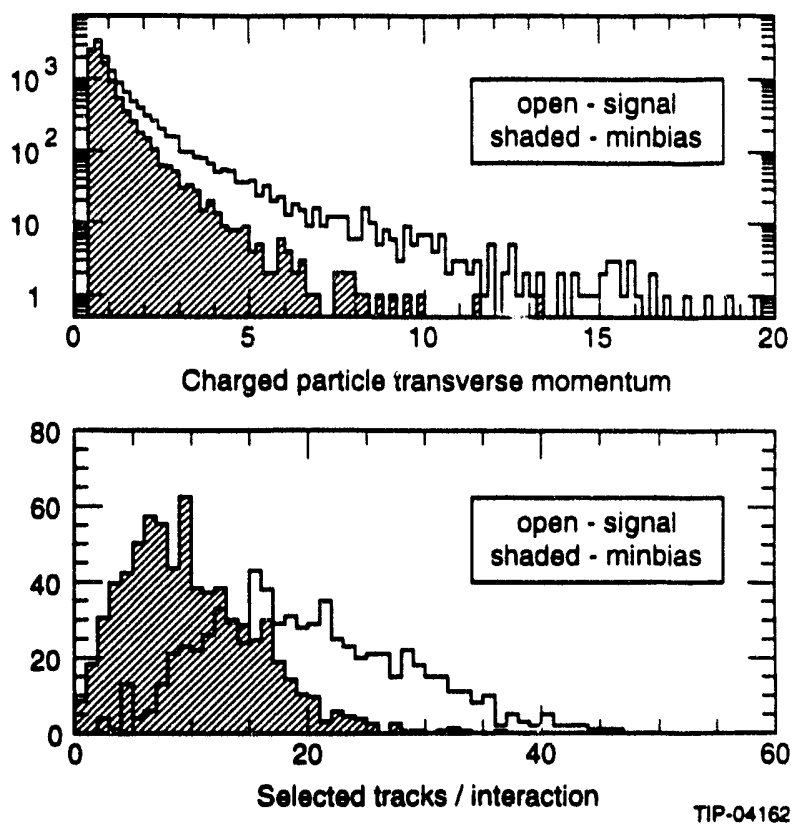

FIG. 6-22. (a) The transverse momentum spectrum for $p_{T}>0.5 \mathrm{GeV}$ isolated charged tracks from standard model Higgs production (open histogram) for $M_{H}=120 \mathrm{GeV}$, and minimum bias events (hashed histogram) produced using the PYTHIA Monte Carlo. (b) The number of these tracks per event from standard model Higgs production (open) and minimum bias events (hashed).
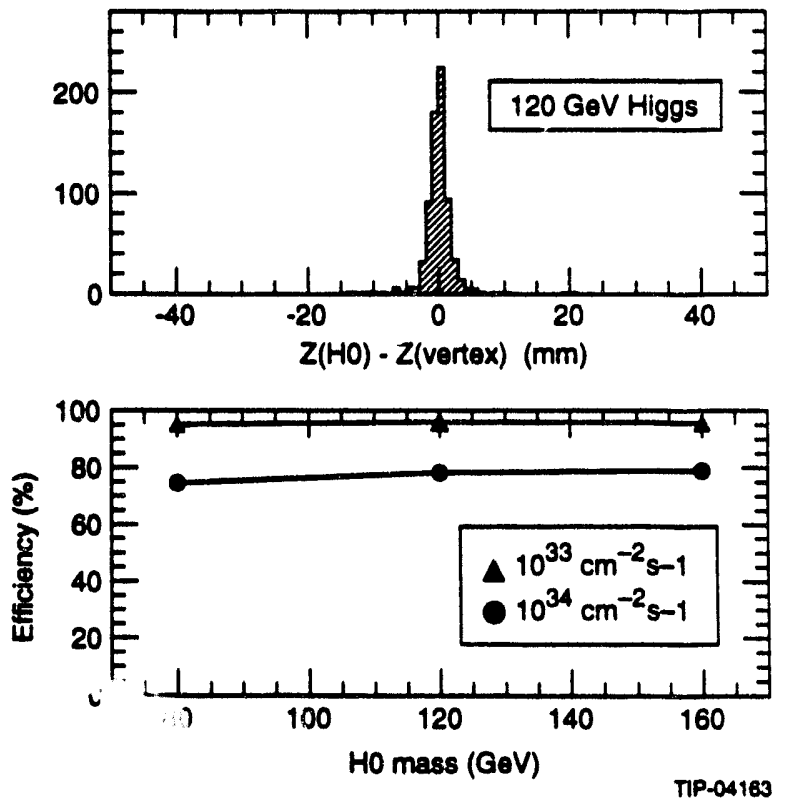

FIG. 6-23. (a) The difference in $\mathrm{mm}$ between the $z$ coordinate of the true $\mathrm{H}^{0}$ production vertex and the reconstructed primary vertex selected by choosing the vertex made up of tracks with the hightest $\Sigma p_{T}$ for a 120-GeV staridard model Higgs. (b) Vertex tagging efficiency as a function of the standard model Higgs mass and luminosity. 
gamma signal and rejecting electrons, and identifying an electron signal and rejecting $\gamma \mathrm{s}$ and $\pi^{0} \mathrm{~s}$. We consider each of these separately in the following discussion.

\section{Identifying signal gammas and rejecting background electrons.}

One example of this situation is the search for $\mathrm{H}^{0} \rightarrow \gamma \gamma$ decays. For Higgs masses near the $\mathrm{Z}^{0}$ mass one of the most severe backgrounds is $Z^{0} \rightarrow e^{+} e^{-}$ decays, with the $e^{ \pm}$showers faking $\gamma \mathrm{s}$. One of the functions of the central tracker in GEM is to distinguish electromagnetic showers induced by gammas and electrons by detecting the absence or presence of a charged track pointing to the shower in the electromagnetic calorimeter.

To study the rejection factors for electrons in the central tracker a sample of $\mathrm{H}^{0} \rightarrow \gamma \gamma$ and $\mathrm{Z}^{0} \rightarrow$ $e^{+} e^{-}$decays was generated in a full GEANT simulation of GEM. Electromagnetic showers were selected by the following calorimeter cuts:

- $E_{T}$ of shower $\geq 20 \mathrm{GeV}$

- $(\mathrm{E}$ in $\mathrm{EM} \mathrm{Cal}) /(\mathrm{E}$ in $\mathrm{EM} \mathrm{Cal}+\mathrm{E}$ in $\mathrm{Had} \mathrm{Cal})$ $\geq 0.95$

- Isolation Cut $\left(\Sigma_{\mathrm{R}} \leq 0.3 E_{T}-E_{\text {shower }}\right) \leq 10 \mathrm{GeV}$

These cuts select showers from $\gamma \mathrm{s}$ and $e s$ with similar efficiency. At this point information from the central tracker was introduced:

- The location of the interaction vertex was found from other charged tracks in the event in the central tracker (see Section 6.2.3) to a precision of $\sim 1 \mathrm{~mm}$. The shower position is measured from the calorimeter to $\sim 1-\mathrm{mm}$ precision.

- A road was defined in the tracker around a straight line joining the event vertex and the shower, with a width of $1.5 \mathrm{~cm}$ in both coordinates transverse to the center line of the road.

- The number of hits in this road was counted (the sum of the silicon and the pad chamber hits) and a cut on this number was used to separate $\gamma s$ and es. A tighter cut (i.e., insisting on a smaller number of hits) improves the rejection of electrons, but results in a lower efficiency for retaining $\gamma \mathrm{s}$.

The result of this study is shown in Figure 6-24, where the fraction of electrons rejected is plotted versus the gamma efficiency. The variable along the c"rve in this figure is the number of hits used in the cut. From this figure we see that as much as $99.5 \%$ of the electrons can be rejected (i.e., a rejection factor of 200) with acceptable $\gamma$ efficiency. This results in a rejection factor of $4 \times 10^{4}$ against $Z^{0} \rightarrow$ $e^{r} e^{-}$.

Identifying an $e^{ \pm}$signal and rejecting a $\gamma$ or $\pi^{0}$ background.

An example of this situation is the search for $\mathrm{H}^{0} \rightarrow \mathrm{ZZ}^{*} \rightarrow e^{+} e^{-} e^{+} e^{-}$or $e^{+} e^{-} \mu^{+} \mu^{-}$decays. Electromagnetic showers from $\gamma \mathrm{s}, \gamma \rightarrow e^{+} e^{-}$conversions and $\pi^{0}$ decays present a severe background that has to be reduced by the central tracker by looking for the presence of a charged track pointing to the shower, and also by detecting the $e^{\mp}$ pair to the $e^{ \pm}$from $\gamma$ conversions, and thus vetoing $\gamma s$ and $\pi^{0}$ s.

To study the rejection capability of the central tracker a sample of $\mathrm{H}^{0} \rightarrow 4$ leptons (with $\mathrm{M}_{\mathrm{H}}=$ $150 \mathrm{GeV}$ ), with background $\gamma \mathrm{s}$ and $\pi^{0} \mathrm{~s}$ from a two-jet event sample, was generated and run through a full GEANT simulation of GEM. Electromagnetic showers were selected using calorimeter cuts similar to those described in the $\mathrm{H}^{0} \rightarrow \gamma \gamma$ study discussed above. Similarly a road in the tracker was defined for each shower. However, to improve the $\gamma$ rejection further, electrons were required to have at least six hits in the road, and the momenta of the electrons were reconstructed in the tracker. A cut was then imposed requiring the agreement between the momentum from the curvature in the tracker and the

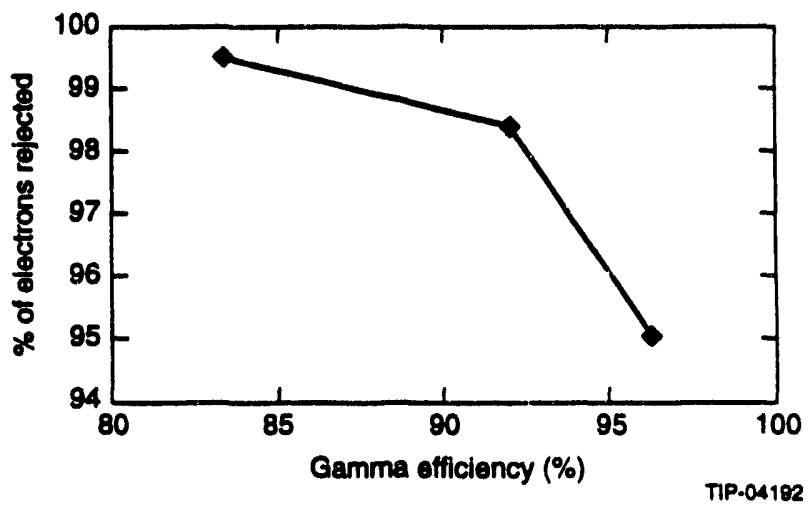

FIG. 6-24. The fraction of background electrons rejected vs. the gamma efficiency for identifying $\gamma$ 's in $H^{\circ} \rightarrow \gamma \gamma$ events for $M_{H}=80 \mathrm{GeV}$. For the tightest cut on the number of hits in the road, a rejection efficiency of $99.5 \%$ can be achieved with a corresponding $\gamma$ efficiency of $83 \%$. 
energy of the shower of the calorimeter. This resulted in a rejection factor of 100 against $\gamma s$ and $\pi^{0} \mathrm{~s}$. Essentially all of the remaining background is due to $\gamma \mathrm{s}$ (or $\gamma \mathrm{s}$ from $\pi^{0} s$ ) that converted to an $e^{+} e^{-}$ pair in the volume of the central tracker or Dalitz pairs from $\pi^{0} \mathrm{~s}$, and the electromagnetic shower was due to an actual $e^{ \pm}$. This background can be further reduced by detecting the partner $e^{\mp}$. If the $e^{+} e^{-}$pair separates by more than a pad width, the tracks can be reconstructed with an efficiency $\sim 97 \%$ (see Figure 6-17). If the $e^{+} e^{-}$pair does not separate and stays within the same pad, we can identify them as multiple tracks with good efficiency (see Figure 6-19). Thus, we should be able to reduce the $\gamma$ and $\pi^{0}$ background by another factor of 10 , obtaining a rejection factor of -1000 or better. (See Table 6-5.) This factor comes in at least squared in $\mathrm{H}^{0} \rightarrow Z Z^{*} \rightarrow e^{+} e^{-} e^{+} e^{-}$or $e^{+} e^{-} \mu^{+} \mu^{-}$-events. We thus believe that the central tracker has the capability of reducing the $\gamma$ and $\pi^{0}$ backgrounds to a manageable level.

Table 6-5. Probabilities of misidentifying $\gamma, \pi^{0}, \pi^{ \pm}$, and jets as electrons in GEM.

\begin{tabular}{ll}
\hline Background & \multicolumn{1}{c}{$\begin{array}{c}\text { Probability of misidentification } \\
\text { as electron }\end{array}$} \\
\hline$\gamma$ & $10^{-3}$ \\
$\pi^{0}$ & $10^{-3}$ \\
$\pi^{ \pm}$ & $8 \times 10^{-4}$ \\
jet (non-isolated) & $2 \times 10^{-4}$ \\
jet (isolated) & $1 \times 10^{-5}$ \\
\hline \hline
\end{tabular}

\section{Electron-Hadron Separation}

The electron identification capability of GEM and the rejection of hadrons depends both on the calorimeter by distribution of the shower energy between the electromagnetic and hadronic calorimeters, and on the central tracker by matching the position of a charged track with the shower position and the comparison of momentum from curvature with the energy in the calorimeter. An example where this capability is essential is in the reduction of QCD jet backgrounds in the search for $\mathrm{H}^{0} \rightarrow$ $Z Z^{*} \rightarrow e^{+} e^{-} e^{+} e^{-}$or $e^{+} e^{-} \mu \mu^{+} \mu^{-}$. The background from jets can be due to jets fragmenting into high-pT $\gamma s$ or $\pi^{0} \mathrm{~s}$, which were discussed in the previous section, or jet fragmentation into high-p (mostly $\pi^{\dagger}$ ) charged hadrons which are the subject of this section.
To study the hadron- and jet-rejection capability of the central tracker combined with the calorimeter, the sample of $\mathrm{H}^{0} \rightarrow 4$ leptons and the sample of two-jet background events discussed in the previous sections have been used. The same calorimeter selection criteria for electromagnetic showers were used, and the same analysis using the central tracker was done, i.e., looking for hits in the road pointing to the shower, matching the position of the charged track to the shower position, and making the $E / p$ comparison. The results of this analysis are shown in Table 6-5. The rejection of charged hadrons and jets are sufficient for the requirements of the Higgs search, as is discussed in more detail in Chapter 2 , Physics Goals and Performance of the GEM Detector.

\section{Muon Identification and Measurement}

The GEM central tracker contributes to the GEM muon performance in a variety of ways.

1. Improvement in muon identification by comparing the momentum measurement in the central tracker with the momentum measurement in the muon system for a muon candidate track. The backgrounds to muons in the muon system (punchthrough backgrounds) consist of i) hadrons that decay into muons in the central tracker volume or in the calorimeter before interacting, and ii) hadrons, typically secondaries produced by hadrons interacting in the calorimeter, which penetrate into the muon system. These backgrounds can be significantly reduced by requiring that the momentum and angles measured for these tracks in the central tracker agree with the momentum and angles measured in the muon system. The muon system does not rely on this capability for its muon identification; however, it provides an additional measurement for verification and efficiency determination.

A quantitative study of this background reduction has been carried out ${ }^{9}$ by generating a sampie of tit events (using $M t=250 \mathrm{GeV}$ )

$$
t+\bar{t} \rightarrow W+b W-\bar{b} \rightarrow \mathrm{e}^{ \pm} \mu^{\mp}+\mu_{\text {incl }}+X,
$$

where the $e^{ \pm}, \mu \mp$ are isolated but the $\mu_{\text {incl }}$ is in a jet from $b$-decay. A significant background to this process comes from two-jet events 


$$
p+p \rightarrow 2 \text { jets } \rightarrow \mathrm{e}^{ \pm} \mu^{\mp}+\mu_{\mathrm{incl}}+X,
$$

where both muons may be punchthrough or decay muons.

The selection criteria used in this study were

- $\left|\eta_{e, \mu}\right| \leq 2.5$

- Isolated $e^{ \pm}$with an isolated $\mu^{\mp}$, both with $p_{T} \geq 30 \mathrm{GeV} / \mathrm{c}$

- A non-isolated muon with $p_{T} \geq 5 \mathrm{GeV} / \mathrm{c}$.

This produced a sample of $t \bar{t}$ events with a $50 \%$ background surviving due to the punchthrough background. For a clear study of this process, the background should be $\leq 10 \%$, requiring a further background reduction by a factor of 5 . To achieve this, the muon candidate tracks were extrapolated into the central tracker, where the correct matching track was found for $90 \%$ of these tracks. A $\chi^{2}$ was formed for these tracks:

$$
\chi^{2}=(\Delta p / \delta p)^{2}+(\Delta \theta / \delta \theta)^{2},
$$

where $\Delta p$ and $\Delta \theta$ are the differences in the momentum and angle between the tracks as measured in the muon system and the central tracker, and $\delta p$ and $\delta \theta$ are the corresponding errors. The $\chi^{2}$ distribution is shown in Figure 6-25.

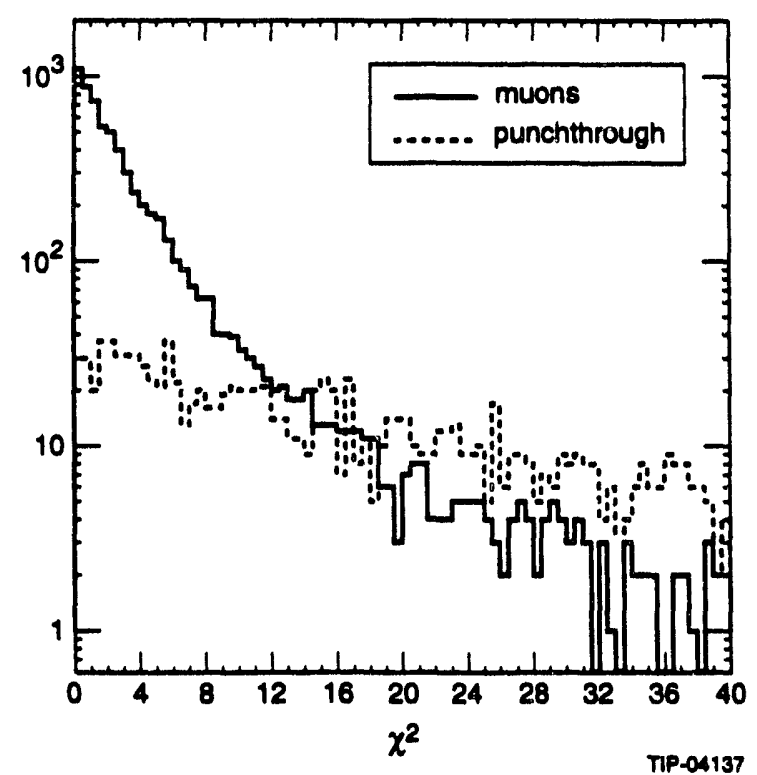

FIG. 6-25. Distribution is $\chi^{2}$ describing the agreement between the muon momentum vectors measured in the muon system and the central tracker. A cut at $\chi^{2}<10$ reduces the punchthrough by a factor of 5 and keeps $95 \%$ of muons.
A cut at $\chi^{2} \leq 10$ keeps $95 \%$ of the true muons but reduces the punchthrough background by a factor of 5 , which results in a clean $t \bar{t}$ signal.

To study the requirements that this background rejection places on the momentum resolution of the central tracker, this analysis was repeated, varying the central tracker momentum resolution $\delta p / p^{2}$ from 0.001 to 0.05 . The resulting rejection factor of fake muons versus $\delta p / p^{2}$ is shown in Figure 6-26. We can see that we do need to be in the vicinity of $\delta p / p^{2}$ $\sim 0.001$ to get the required rejection factor.

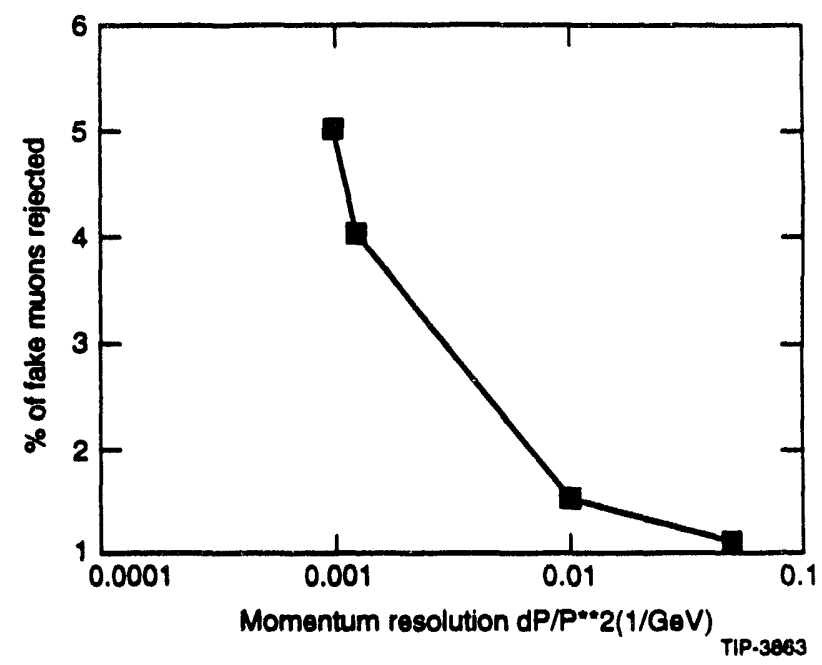

FIG. 6-26. The rejection factor for punchthrough hadrons as fake muons from a $\chi^{2}$ cut on the momentum vector agreement between the central tracker and the muon system as a function of the central tracker momentum resolution.

2. Improvement in muon momentum measurement at high momenta by providing the position of the vertex from the central tracker. A study of this effect ${ }^{10}$ shows that the inclusion of the vertex constraint from the central tracker in the muon momentum analysis will result in an improvement across the whole angular region of 10 to $30 \%$ for muon momenta above $1 \mathrm{TeV}$.

3. Improvement in the muon momentum measurement at low momenta. The muon system momentum resolution degrades significantly below $p_{T} \sim 20 \mathrm{GeV} / \mathrm{c}$ due to fluctuations in the energy loss in the calorimeter; the resolution rises to about $6 \%$ at $10 \mathrm{GeV} / \mathrm{c}$, where the central tracker resolution is $-3.5 \%$. By performing a weighted average an improvement can be achieved, ${ }^{11}$ as is shown in Figure 6-27. 


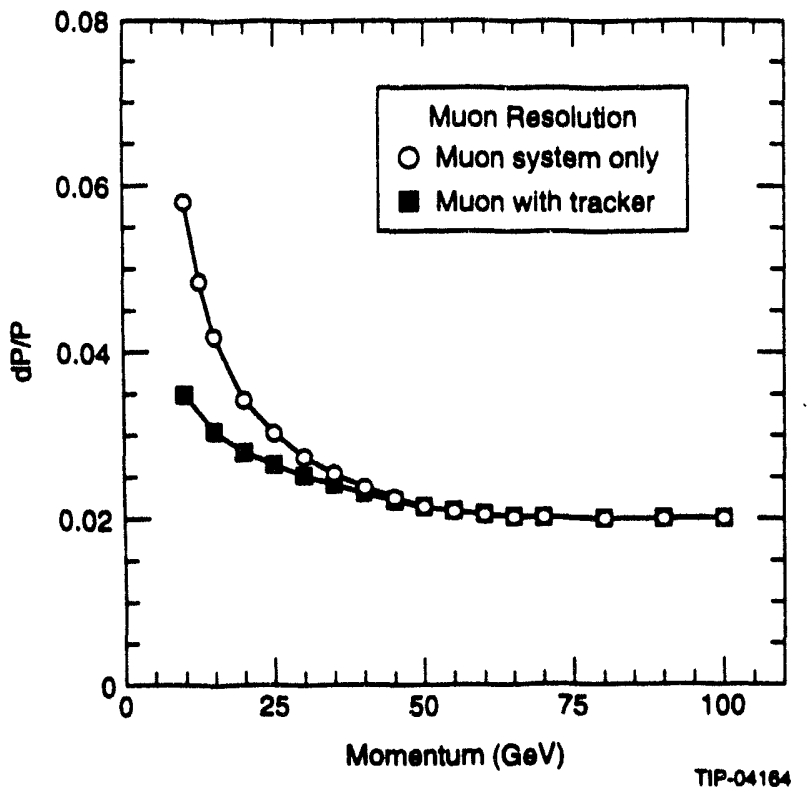

FIG. 6-27. Improvement in the momentum measurement of low-energy muons from taking the weighted averages of the central tracker information and the muon system measurement.

\section{Charge Sign Determination for Electrons}

The GEM detector can measure the charge sign of muons to very high $p_{T}(\approx 6 \mathrm{TeV}(\eta=0), 2.5 \mathrm{TeV}$ $(\eta=2.5)$ ) using the excellent resolution of the muon system. Electron charge sign determination is provided solely by the central tracker. This capability is important, for example, in testing lepton universality in new physics. The intrinsic capability of the GEM central tracker is $95 \%$ charge separation up to $600 \mathrm{GeV} / \mathrm{c}$ transverse momentum. However, electron charge sign determination is affected by the bremsstrahlung of the electron as it passes through the central tracker. In fact the curvature of the track in the tracker increases with energy loss, slightly enhancing the tracker's ability to separate charge. However, the event is not as clean. The confusion from delta rays and converted gammas is a potential problem. For about $95 \%$ of the electron tracks, the bremsstrahlung resulting from the material of the tracker presents no difficulty in measuring the tracks. For a few percent, however, significant confusion results. Figure 6-28 shows the efficiency for correct sign determination by the central tracker as a function of electron transverse momentum. The onset of degradation is evident, but is primarily limited by the tracker radius, magnetic field, and resolution, not bremsstrahlung. Figure 6-9 showed the $95 \%$ confidence level charge separation momenta determined from Figure 6-28 .

One example of a process where electron sign determination up to $600 \mathrm{GeV}$ is important is the identification of like-sign dileptons from gluinos. The use of the tracker in this physics is discussed in Section 2.7.2. Another example of a process where electron sign separation could be important is in the study of $W \cdot W$ scattering. This process could be of crucial importance if no light Higgs boson is found. One manifestation of strong $W \cdot W$ scattering would be an anomalously large cross section for the process

$$
p+p \rightarrow W^{ \pm}+W^{ \pm}+X \rightarrow I^{ \pm}+I^{ \pm}+X .
$$

The isolation of this process depends on detecting the two isolated final state leptons. The background in opposite sign pairs, $W^{+} W^{-} \rightarrow l^{+}+$is much larger, but appears to be manageable for like sign pairs $W^{ \pm} W^{ \pm} \rightarrow I^{ \pm} I^{ \pm}$. The sign of the muon charge is well determined in the muon system. Both electrons and muons could be used if the sign of the electron charge can be measured in the central tracker, which would increase the number of events by a factor of four over the $\mu^{ \pm} \mu^{ \pm}$final state. Since the event rate for this process is very small, this factor of four is crucial in being able to detect it. The $p_{T}$ spectrum of the leptons for this process has been calculated $^{12}$ for various model assumptions for the $W W$ scattering process. These distributions are shown in Figure 6-29. As can be seen from this figure, electron sign determination up to $600 \mathrm{GeV} / \mathrm{c}$ covers most of the distributions for any of the three models.

\section{Help with Isolation Cuts}

Strategies for background reduction in physics measurements have been developed by GEM that rely heavily on isolation cuts in the calorimeter. The GEM central tracker has the capability to provide complementary isolation information. For example, in the search for standard-model Higgs decays into two photons, the principal method for reducing the overwhelming background from jets that fragment a large fraction of their energy into a tight cone of electromagnetic energy is to demand no associated energy in the calorimeter within the isolation cone. Due to noise, however, the threshold on this criteria must be kept above several GeV. Few GeV tracks in the central tracker are easily detected and the.ufore 

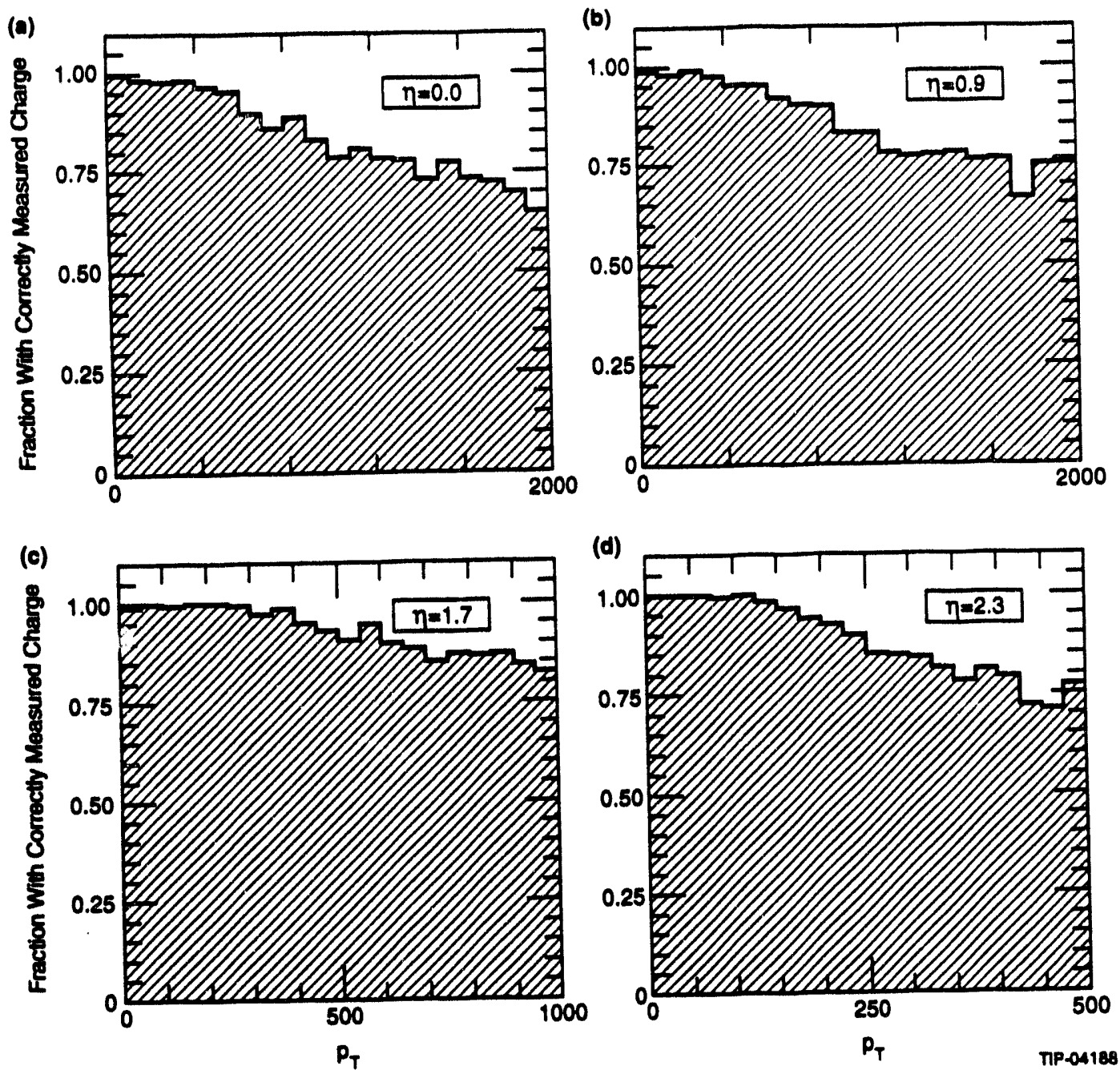

FIG. 6-28. Efficiency for correct sign determination by the central tracker as a function of electron transverse momentum.

allow a tighter cut on the isolation. An additional rejection of jet backgrounds by a factor of at least two over that achieved by the calorimeter isolation cuts alone looks feasible and is under study. ${ }^{13}$

\section{Tau Identification}

Identification of $\tau \mathrm{s}$ is an important goal of the GEM central tracker. Testing lepton universality in any new physics could be an interesting issue. Full exploration of a Higgs will call for measuring its branching ratios, with $\tau$-decays being of significant interest (see Section 2.4.3).

Figure 6-30 shows the GEM central tracker efficiency for measuring three-prong $\tau$-decays as a function of $p r$. Measurements require good separation of the three tracks at the outer pad layer. The 0.8-T magnetic field aids in separation of the tracks, and the very fine segmentation of the silicon

microstrips ensures good track separation in the inner tracker. Good separation at the outer pad layer is defined as having the two tracks with largest separation separated by at least $5 \mathrm{~mm}$ (two pads), and those closest separated by no less than $2.5 \mathrm{~mm}$ (one pad). Figure 6-31 shows the distributions of these two distances for $\tau$ s with $p_{T}=150 \mathrm{GeV}$. These figures show that the separation between the two closest tracks is the most severe requirement, with most losses in Figure 6-30 resulting from failing this criterion.

In the high-pT extreme, where good momentum measurements are not possible due to the overlap referred to above, the central tracker can identify three-prong decays by the very distinct pulse height information as described in Section 6.2.2. Having identified the three-track topology, the total energy can be measured in the hadron calorimeter. 
This measurement technique may be very useful in $Z^{\prime}$ studies, for example.

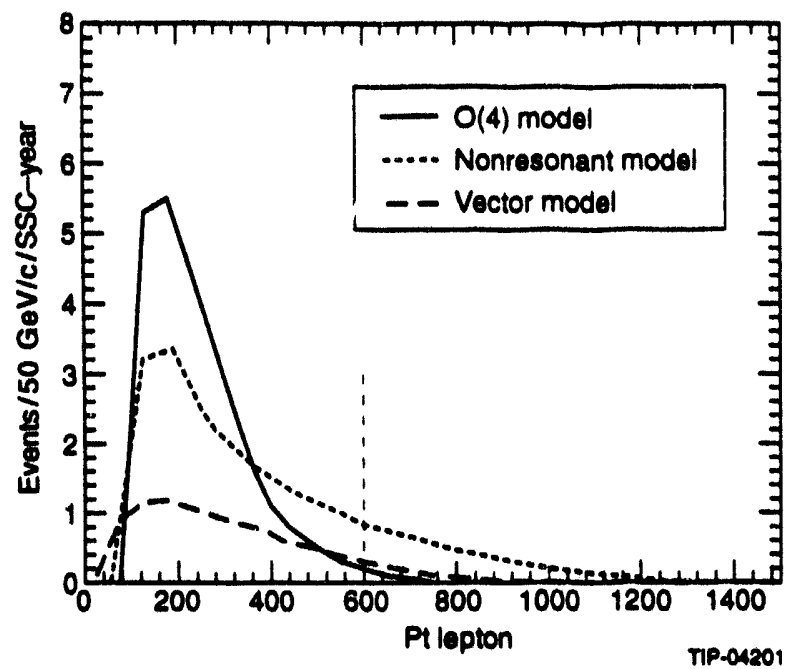

FIG. 6-29. Transverse momentum distribution for the leptons in the $W \cdot W$ scattering $p+p \rightarrow W^{ \pm}+W^{ \pm}+X \rightarrow$ $I^{ \pm}+I^{ \pm}+X$ for various models of $W \cdot W$ scattering. The solid line is the nonperturbative Higgs $O(4)$ model, the dashed line is the Techni-rho model, and the dotted line is the non-resonant model. If a cut is enforced on the $\theta^{-}$of $600 \mathrm{GeV}$ to ensure good $\theta^{-}$-sign determination, a large fraction of the signal is kept.

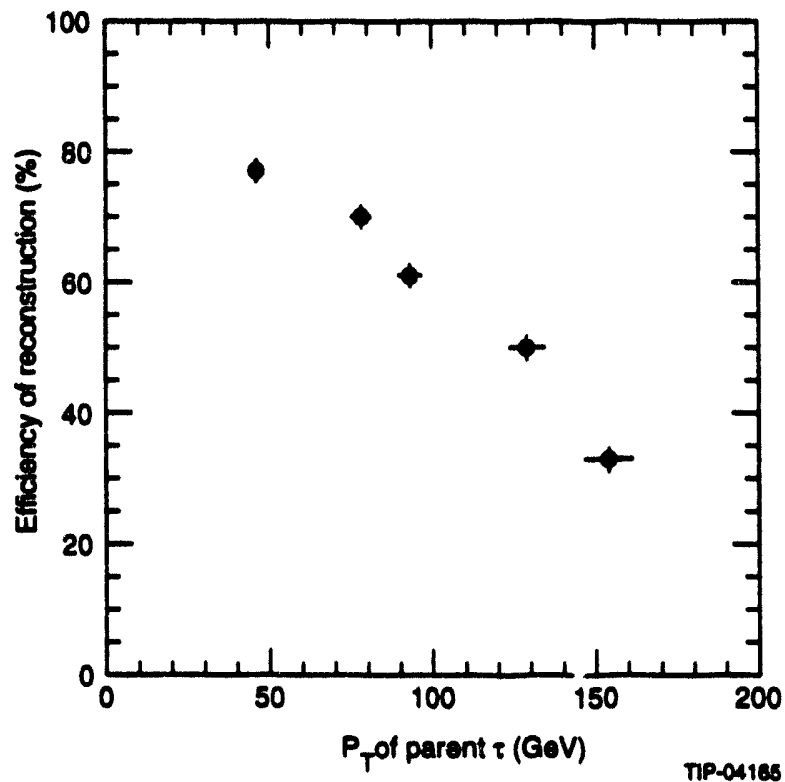

FIG. 6-30. Efficiency for reconstructing all three tracks in the three-prong decay of a $\tau$ lepton as a function of the transverse momentum of the parent $\tau$. As described in Section 6.2.2, in order to reconstruct all three tracks the separation between the two farthest tracks must be $>2$ pads $(5 \mathrm{~mm})$, and the distance between the two closest tracks must be >one pad $(2 \mathrm{~mm})$.
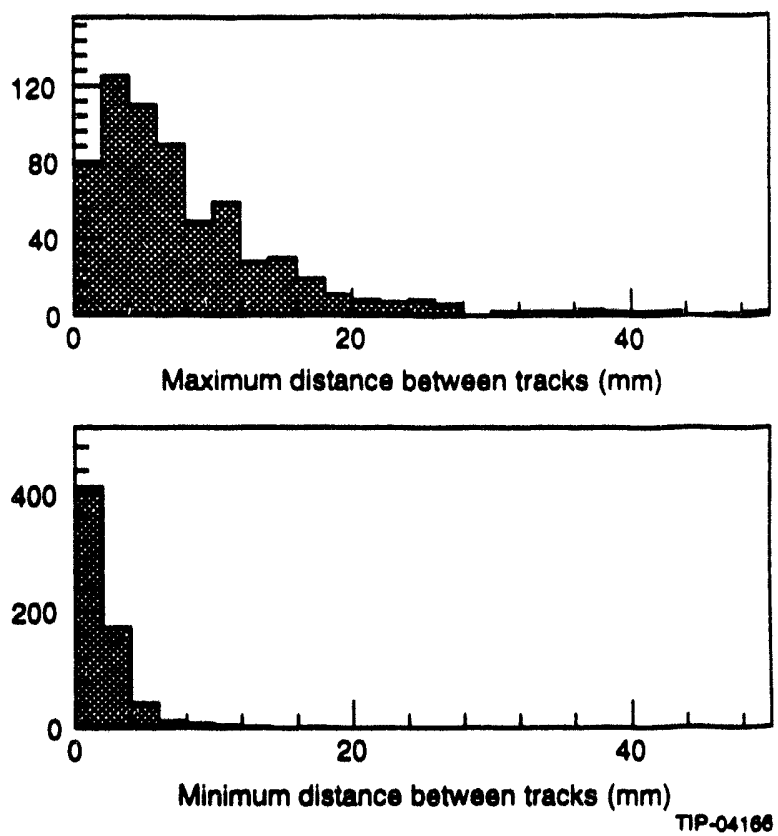

FIG. 6-31. Distribution between the minimum and maximum separation at the outermost IPC layer between the three charyed tracks from the decays of a 150-GeV/c r lepton.

\section{Secondary Vertex (b-tagging)}

While the principal design goals of the GEM central tracker address the new high $p_{T}$ physics expected at the high-energy frontier of the SSC, the enormous rate of heavy-quark production makes it an important subject of study for the tracker. With the very fine impact parameter resolution $(\approx 25 \mu \mathrm{m})$ of the inner silicon subsystem, heavy-quark studies will be done. Even though a fraction of the full phase space is lost in the forward direction, $60 \%$ of the $B$ meson production at the SSC will occur for $|\eta|<$ 2.5. ${ }^{13}$ One of the most difficult obstacles to full utilization of this enormous sample $\left(=10^{12} b \bar{b}\right.$ /year at $10^{33}$ ) is the development of an efficient trigger. The GEM single muon trigger will be rate limited to about $20 \mathrm{GeV} / \mathrm{c}$ at full design luminosity, but early operation at lower luminosities may permit the trigger threshold to be reduced to the $10-\mathrm{GeV} / \mathrm{c}$ range. Multi-muon triggers for $B$ physics must also be investigated.

Interesting decay modes include $B_{d}^{0} \rightarrow J / \psi K_{s}^{0}$. It is possible to construct a scenario in which several hundred of these decays are tagged as $J / \psi \rightarrow \mu^{+} \mu^{-}$ and $K_{s}^{0} \rightarrow \pi^{+} \pi^{-}$, with the $B_{d}$ decay vertex reconstructed. ${ }^{14}$ This scenario could lead to a sensitivity of 
0.15 in $\sin 2 \beta$ where $\beta$ is the usual angle in the $\mathrm{CP}$-violating triangle of the $B$ asymmetry.

The $b$-jet tagging efficiency of the GEM central tracker is high due to the very good impact parameter resolution presented in Figures 6-10 and 6-11. Preliminary studies indicate that the efficiency for tagging the $b$-jet is between 30 and $40 \%$ for $b$-jets from top decay (with top mass of $140 \mathrm{GeV} / \mathrm{c}^{2}$ ). Tagging algorithms have relied on identifying three large impact parameter tracks within a jet cone of $\Delta R=0.5$. Each track is required to have an impact parameter in excess of three times the measurement error for its momentum and rapidity. Backgrounds have been investigated by studying light quark jets with similar $p_{T}$ distributions and are found to fall below a $1 \%$ level of tagging. These results were derived from the full GEANT simulation of the central tracker. Thus, the GEM central tracker can enhance the heavy quark content of jets by a large factor.

\subsubsection{Calibration}

The internal calibration of the central tracker is crucial to achieving the optimum position resolution and maintaining the required stability. This can be done continuously during data taking runs by using isolated muons produced in the pp collisions. A preliminary study has been performed to assess whether the alignment goals are realistic. Hits were generated inside tracking layers which had undergone shifts in their $\phi$ positions of a magnitude expected from the initial alignment errors. High-pT muons whose curvature is known are then used to realign the tracking layers. Using information from the beam spot, which can be measured through an iterative procedure, residual errors of $5 \mu \mathrm{m}$ in the silicon and $20 \mu \mathrm{m}$ in the IPC's can be achieved. Figure 6-32 shows the final calibration error as a function of initial alignment for the silicon and the IPC's.

The main source of muons which can be used for this procedure (prompt and isolated) are from $W \mathrm{~s}$ and $Z s$ and top events. At a luminosity of $10^{33} \mathrm{~cm}^{-2}$ $\mathrm{s}^{-1}$, the cross section for $W \mathrm{~s}$ and $Z s$ predicts about 350 muons/hour $/ 24^{\circ}$ sector in each half of the tracker. For a top mass of $140 \mathrm{GeV}$ and a $20 \%$ branching fraction to muons for one of the two tops in an event, a similar number of muons is obtained from top. The preliminary and conservative study shows that 50 high-pT $\left(p_{T}>30 \mathrm{GeV}\right)$ muons will be sufficient to calibrate each $24^{\circ}$ sector of each half of the central tracker to sufficient accuracy. This number would be obtained in about six minutes running at this luminosity.

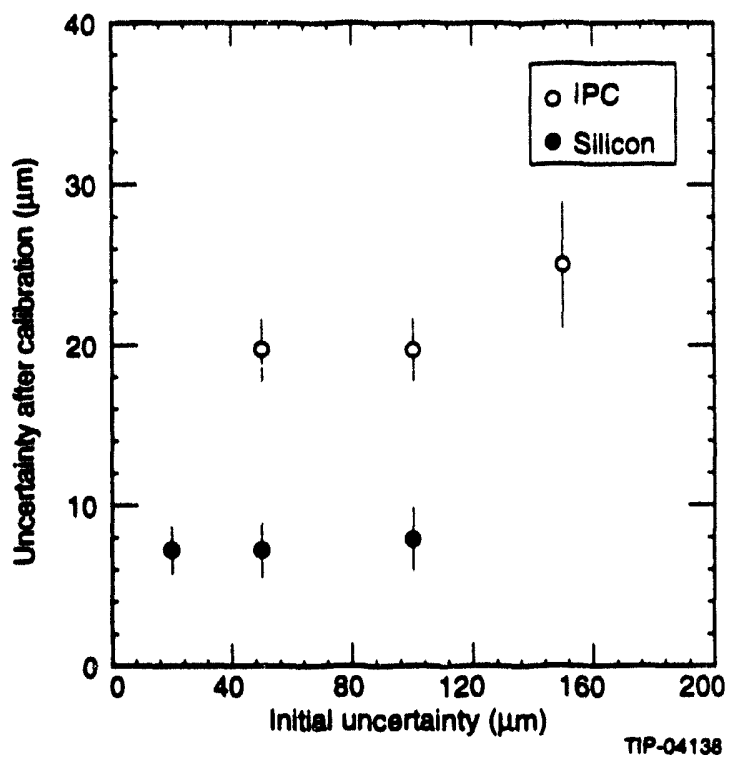

FIG. 6-32. Remaining position uncertainty after calibration procedure as a function of initial uncertainty in the position of silicon and IPC layers.

\subsection{THE SILICON DETECTOR}

\subsubsection{Design Considerations}

\section{Design Parameters}

The GEM central tracker has been divided into two subsystems to provide the different performance demands of the inner volume and the outer volume with different tracking technologies. The design parameters for the inner silicon subsystem of the GEM tracking are set by resolution, occupancy, and speed requirements of the central tracker system. Table 6-6 presents some of the important and relevant design goals. These are the goals specific to the silicon subsystem that are required to achieve the overall tracking goals presented earlier. The critical performance parameter of the tracker is the spatial resolution of $15 \mu \mathrm{m}$ per layer in the azimuthal direction derived jointly from the momentum resolution and impact parameter resolution requirements. 
Table 6-6. Design goals for the silicon subsystem.

\begin{tabular}{ll}
\hline Postion resolution & $\begin{array}{l}15 \mu \mathrm{m} \text { per silicon layer } \\
2 \mathrm{~mm} \text { per silicon layer along } \\
\text { the beam }\end{array}$ \\
& $60 \mathrm{MHz}$ \\
Rate & approx 0.001 at $10^{33}$ \\
Occupancy & $>95 \%$ per silicon layer \\
Efficiency & $<6 \%$ radiation length at $90^{\circ}$ \\
Material thickness & $5-10 \mathrm{yr}$ \\
\hline
\end{tabular}

\section{Choice of Silicon Microstrip for Inner Tracker}

Silicon microstrip detectors are a natural choice for the inner tracking subsystem. The position resolution requirement of $15 \mu \mathrm{m}$ azimuthally is achieved by the very fine segmentation of $50 \mu \mathrm{m}$ pitch microstrips. The longitudinal position resolution (along the beam) is achieved by introducing a stereo angle into the system. The very fine segmentation leads to low occupancy. Silicon microstrip detectors are in broad use in high-energy physics and can now be considered a mature technology. They provide a detector medium that is relatively radiation hard, an important consideration for the choice of the technology for the inner tracking regions where the charged particle dosages will be most hostile. Existing operational experience has demonstrated that a system can be constructed that will withstand many years of running at the high luminosities of the SSC.

We have selected single-sided AC-coupled silicon detectors as opposed to double-sided detectors as the baseline for the tracker design. Singlesided detectors have the advantages of cost, maturity in design, and multiple vendors. In addition, a baseline $18-\mathrm{cm}$ strip places stringent requirements on the capacitance. The $n$-side of the double-sided detector has too high a capacitance to be feasible.

The geometrical configuration of the silicon tracker was based on considerations of cost, radiation damage, tracking efficiency, and physics. The radius of the inner layers of silicon is primarily determined by lifetime limitations due to radiation damage. At $10 \mathrm{~cm}$ our projected lifetime is about $10 \mathrm{yr}$. The silicon tracker outer radius is primarily determined by cost, since the surface area of silicon and the electronic channel count increase rapidly with radius. The number of layers in the barrel section and disks in the forward sections was optimized by investigating the track-finding efficiency for different numbers of hits per track. Our results indicate that to maintain track-finding efficiencies above $95 \%$ the track should intersect at least 6 layers. Inefficiencies on each layer due to dead regions, dead times, or noise can be tolerated by requiring four or more good hits per track. Position information along the beam is accomplished by introducing either a +5 or -5 mrad stereo angle on the two layers. Simplicity of design led us to chose 18-cm ladders.

\section{Microstrip Response}

Optimization of the silicon microstrip detectors for use in the GEM tracking system requires a careful study of induced currents in the microstrips in the specific environment of the Super Collider. The GEM microstrip detectors will be operated in a 0.8-T magnetic field, and tracks will be crossing the detectors at angles in excess of $10^{\circ}$ in the transverse plane. Both effects potentially cause the deposited charge in the detector to be shared by two or more microstrips, thereby reducing the induced current in each strip. Figure 6-33 illustrates the physics of the charge-collection process in the silicon microstrip detectors. Electron-hole pairs are liberated along the charged track according to the Landau distribution, with a most probable rate of 80 pairs $/ \mu \mathrm{m}$. These electrons and holes drift to opposite electrodes with an angle to the normal determined by the Lorentz force and their drift velocities. Induced currents in the microstrips are due to the motion of the holes and electrons in the detector. The goal of the optimization is to maximize the signal in the microstrips by a proper design of the silicon tracker.

To study the dependence of the induced signal on the motion of electrons and holes, a calculation was done using the Santa Cruz code. ${ }^{15}$ This code uses the weighting-field concept based on the reciprocity theorem of electrostatics. ${ }^{16}$ For single-sided microstrip detectors reversed biased in an n-substrate, the microstrips on the $p-n$ junction side are primarily sensitive to the drift of the holes in the detector. Simulations for the GEM configuration show about $80 \%$ of the induced current comes from the drifting holes, and most of the induced current from the electrons is generated by electrons originating near the anode (the n-p junction). Two calculated wave- 
forms from the detector are shown in Figure 6-34. One waveform includes Landau fluctuations and the other assumes charge uniformly distributed through the silicon. The Landau fluctuations will alter the pulse shape and therefore introduce time walk.

To correct for the sharing of charge by the Lorentz drift it is desirable to tilt the silicon detector at the correct Lorentz angle $\left(6^{\circ}\right.$ for electrons, $1.4^{\circ}$ for holes). This can be only partially effective since the individual detectors are wide enough $(3.2 \mathrm{~cm})$ and close enough to the interaction point (IP) (10 to $22 \mathrm{~cm}$ ) that a stiff (high momentum) track from the IP often will cross the detector at a large angle in the

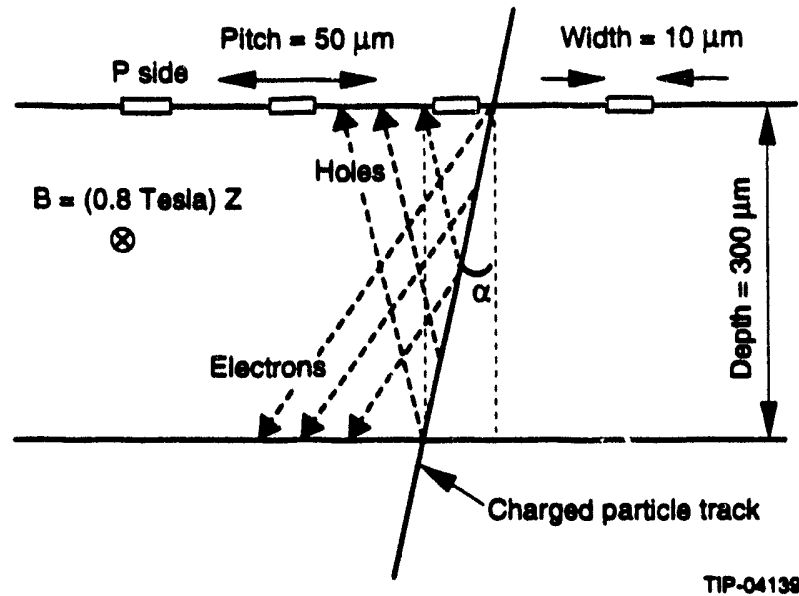

FIG. 6-33. Microstrip detector cross section.

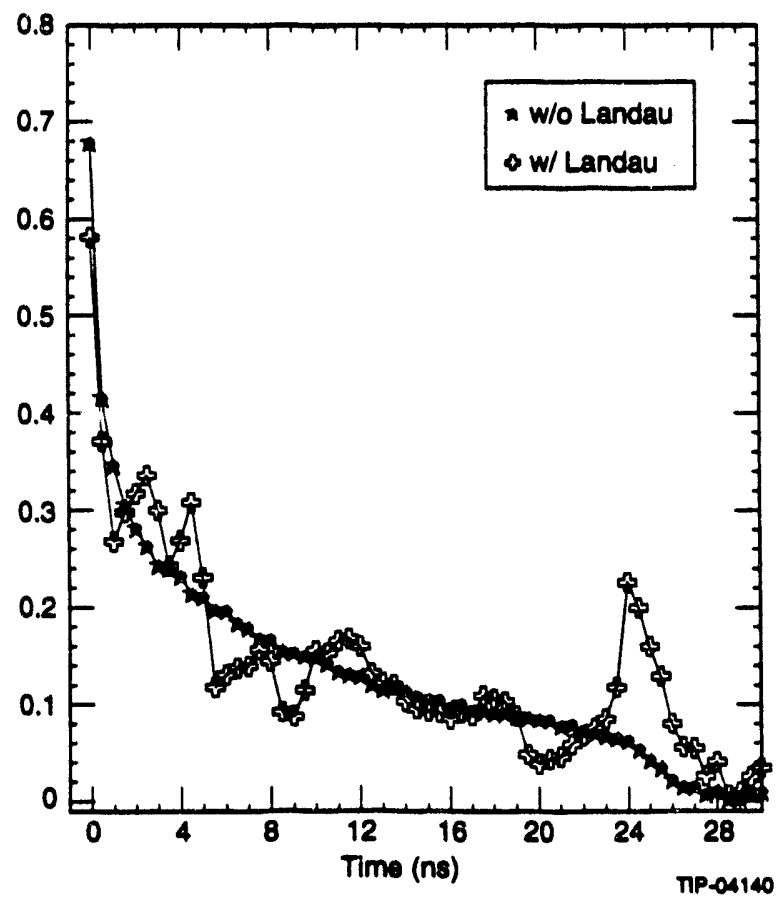

FIG. 6-34. Microstrip detector waveforms. transverse plane. The Santa Cruz code was used to determine the sensitivity of the efficiency on the detector tilt angle. Figure 6-35 shows this dependence. Tilt angles out to $5^{\circ}$ are clearly possible. We have used $5^{\circ}$ in our baseline design since this allows us to take advantage of the existing research in the cooling rings.

An additional important effect resulting from charge sharing between adjacent strips has implications for the timing resolution of the detectors. Figure 6-36 shows the induced wave-form on two adjacent strips for the situation in which a charge track crosses at a slight angle near the interface and induces charge on both strips. Since the induced signal on one strip comes from charge deposited near the $p-n$ junction and on the other strip on the $n$-side away from the $p-n$ junction, there is a time lag in the signal for the strip with charge closer to the n-side. This effect is being carefully considered in the design of the front-end electronics.

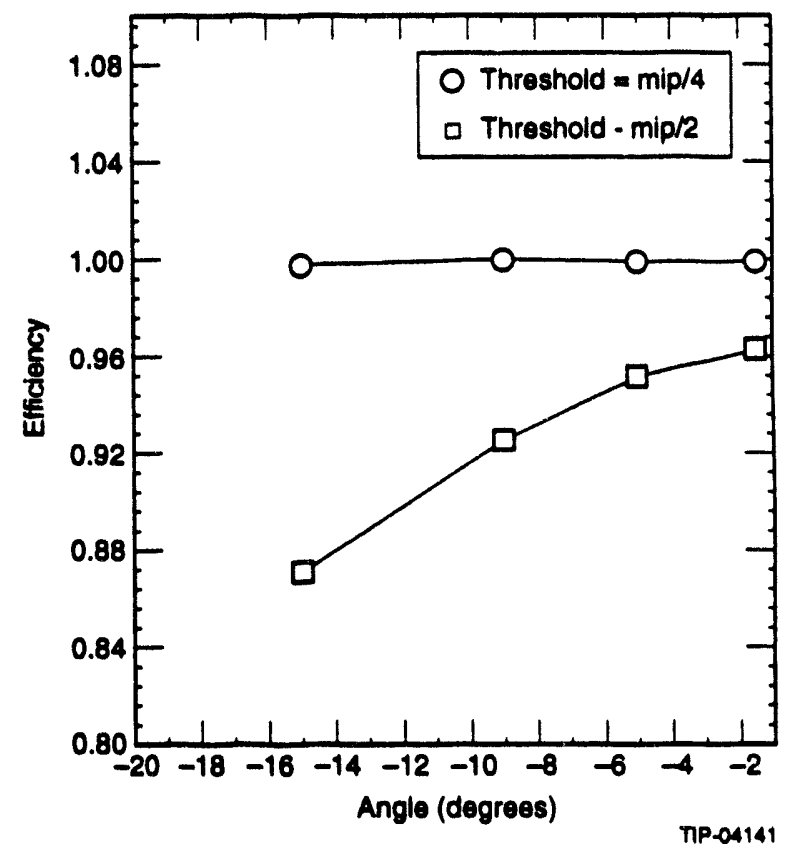

FIG. 6-35. Dependence of single-hit efficiency for a track on tilt angle.

\section{Strip Length Considerations}

It is desirable to make the microstrips as long as possible to minimize channel count and subsystem complexity (and therefore cost). Additionally, long strips make it possible to remove all electronics and associated services from within the barrel of the central tracker and to remove them from the tracking 


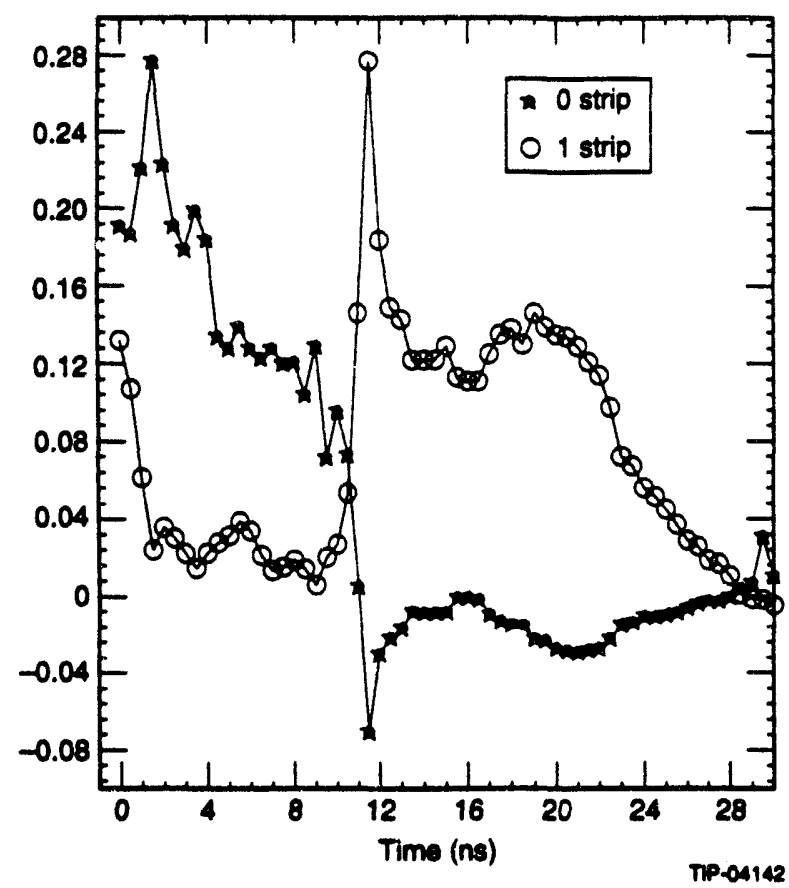

FIG. 6-36. Pulse waveforms for a track passing between strips.

volume in the forward regions. However, increased strip length may degrade performance because of decreased signal-to-noise, causing strip inefficiencies and pattern recognition problems due to increased occupancy.

Ultimately, detector performance depends on strip capacitances and strip length resistances. Strip capacitance drives the front-end electronics noise and must be held small to achieve best signal-tonoise performance. The strip capacitance is dominated by inter-strip capacitance and is weakly dependent on strip width. ${ }^{17}$ Strip widths should be maintained in the $10-$ to $15-\mu \mathrm{m}$ range for $50-\mu \mathrm{m}$ pitch strips. The speed of response of the strip wils be determined by the combined RC time constant of the strip. Inter-strip capacitances typically are $1.2 \mathrm{pF} / \mathrm{cm}$, although recent results indicate these values may be engineered to somewhat lower values. ${ }^{18}$

Strip length resistances are presently under research and development by GEM to improve on the microstrip performance. Since 18-cm microstrip capacitances are less than $22 \mathrm{pF}$, to maintain an RC time constant for the strip of less than $10 \mathrm{~ns}$, the strip resistance should be less than $25 \Omega / \mathrm{cm}$. To accomplish this the present development work involves attempting to increase the strip cross section to a height of about $2 \mu \mathrm{m}$, with a width of 10 to $15 \mu \mathrm{m}$.

The combined effect of capacitance, resistance, and strip length is to introduce dispersion and attenuation into the ideal strip line. Coupled with reduced signals due to radiation damage to the silicon detector and the amplifier, the response of the microstrip will be less than the ideal pulse height, which for minimum ionizing tracks at normal incidence is 24000 electrons. Noise and reduced signals directly lead to strip inefficiencies and therefore track-finding inefficiencies. To understand what limits are placed on the noise level (and also the strip characteristics) by the need to maintain track-finding efficiencies greater than $95 \%$, detailed simulations were performed. The simulations included strip deartimes due to previous hits (10 crossings), increased equivalent noise due to attenuation and radiation damage, noise on the signal, and noise hits due to all physics processes during the crossing of interest as well as the previous crossings. Figure 6-37 shows the track-finding efficiencies for various threshold-to-noise ratios. Clearly, to maintain good track-finding efficiencies a threshold-to-noise level of at least 3:1 is needed. Figure 6-38 shows the track-finding efficiencies for a fixed noise to threshold of 3:1 as a function of noise. For an equivalent noise charge (ENC) greater than 3000 electrons, the track-finding efficiency falls gradually, dropping by $5 \%$ at 4000 electrons. In order to maintain the highest efficiencies, the GEM R\&D efforts on microstrip response are aimed at achieving an ENC of $\leq 2500$ electrons. This yieids a signal-to-noise of about 10 for normally incident, minimum-ionizing particles and somewhat larger for non-normally incident tracks or higher momentum (higher ionizing) tracks.

\subsubsection{Silicon Tracking System Mechanical Design}

The silicon tracking system (STS) baseline design shown in Figure 6-39 contains 10836 silicon strip detectors that are distributed radially and axially about the accelerator interaction region. The STS has an overall length of $2.1 \mathrm{~m}$ and an outside radius of $0.38 \mathrm{~m}$. The silicon microstrip detectors are arranged in the volume in three distinct groupings in the tracker; the central or barrel region and two forward planar regions. The total number of 
detectors in the central region is approximately 2484 , with approximately 4176 wafers in each forward region. The tracker specifications demand that each of these wafers be precisely located relative to one another and relative to the interaction region. It is also imperative that the alignment of the

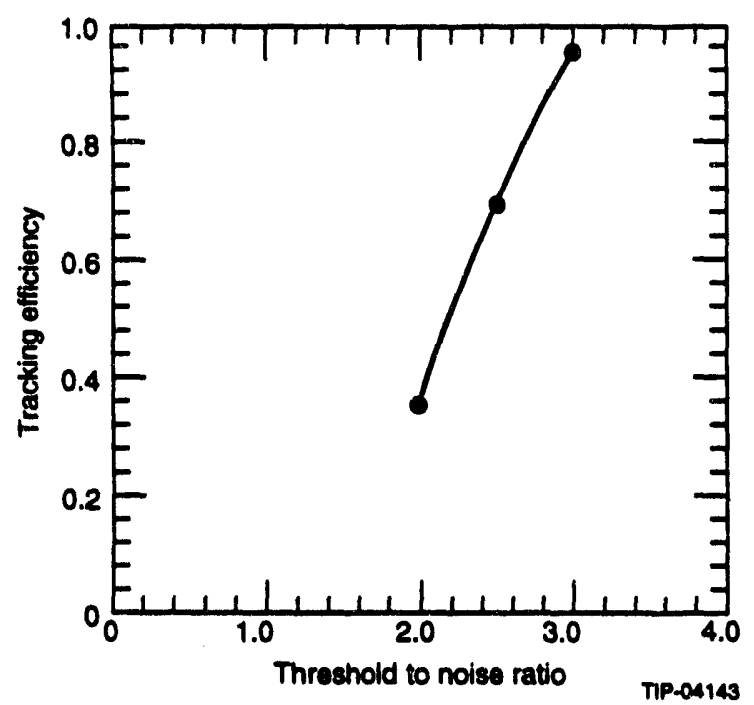

FIG. 6-37. Track-finding efficiencies versus threshold to noise ratio. wafers relative to one another be exceptionally stable over long periods of time. Figure $6-40$ is a schematic of the STS baseline with a few important dimensions. The central region contains six layers of silicon ladder assemblies, extending $180 \mathrm{~mm}$ axially on each side of the interaction region.

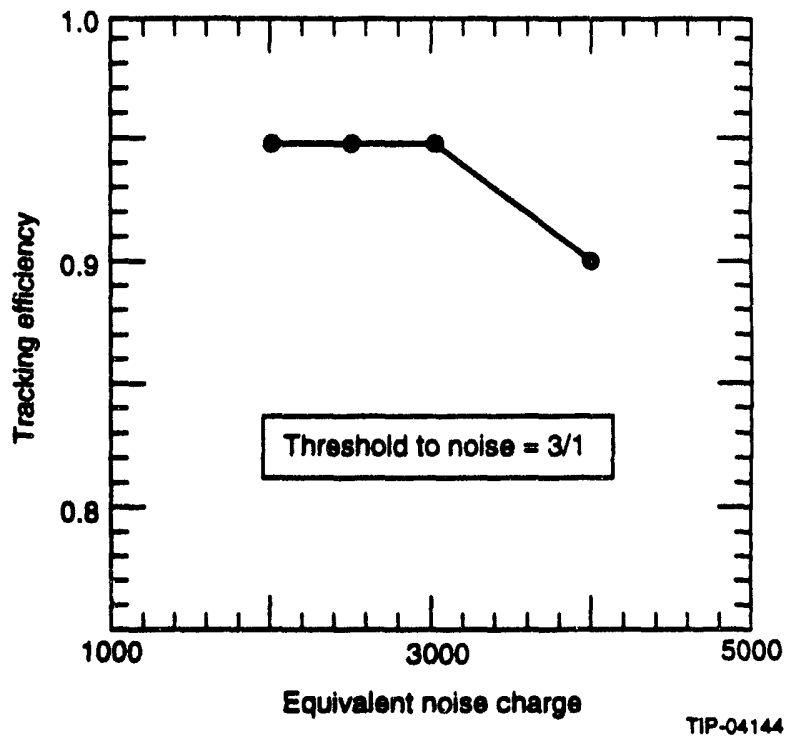

FIG. 6-38. Track-finding efficiency versus noise level at a threshold to noise ratio of $3: 1$.

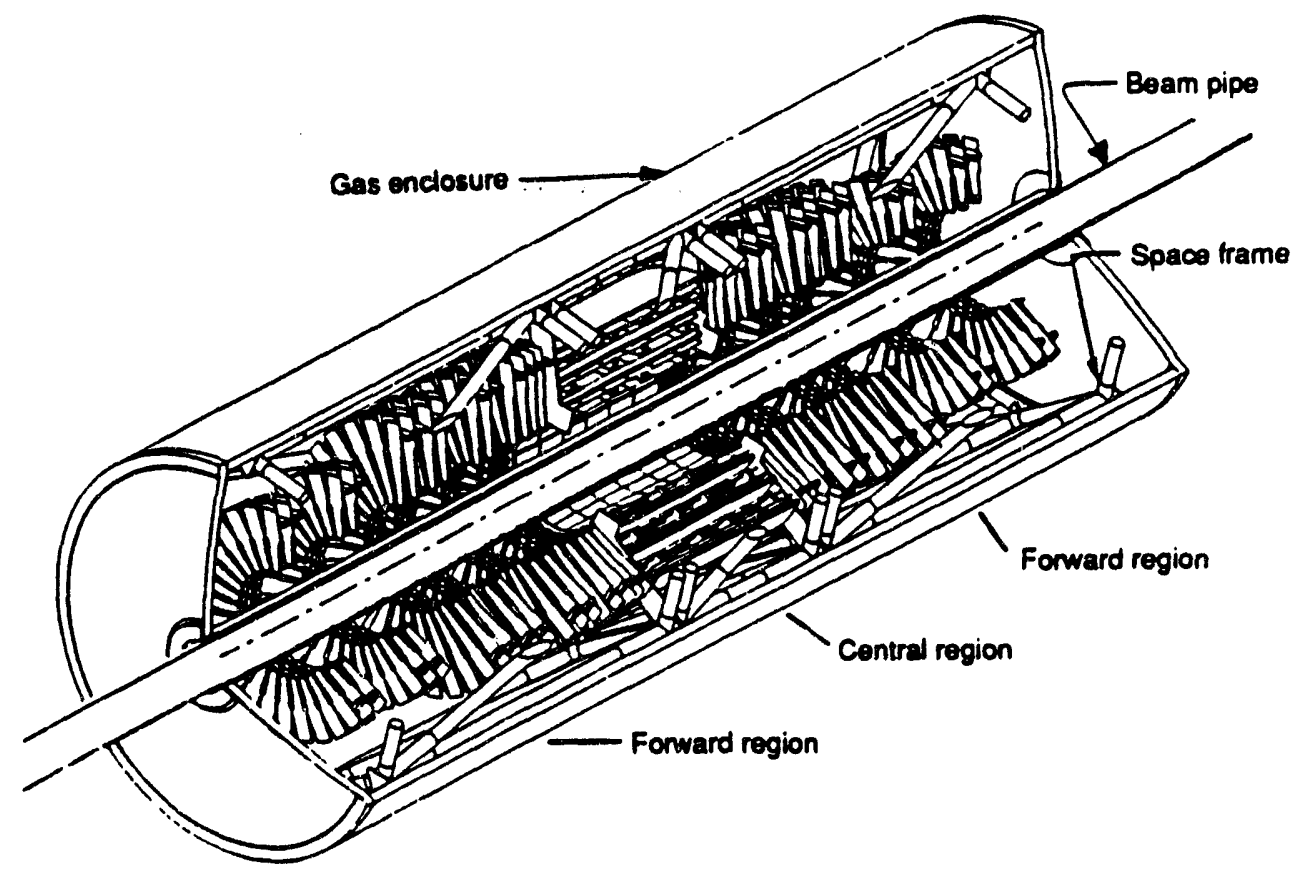

FIG. 6-39. Cut-away of silicon tracking system. 


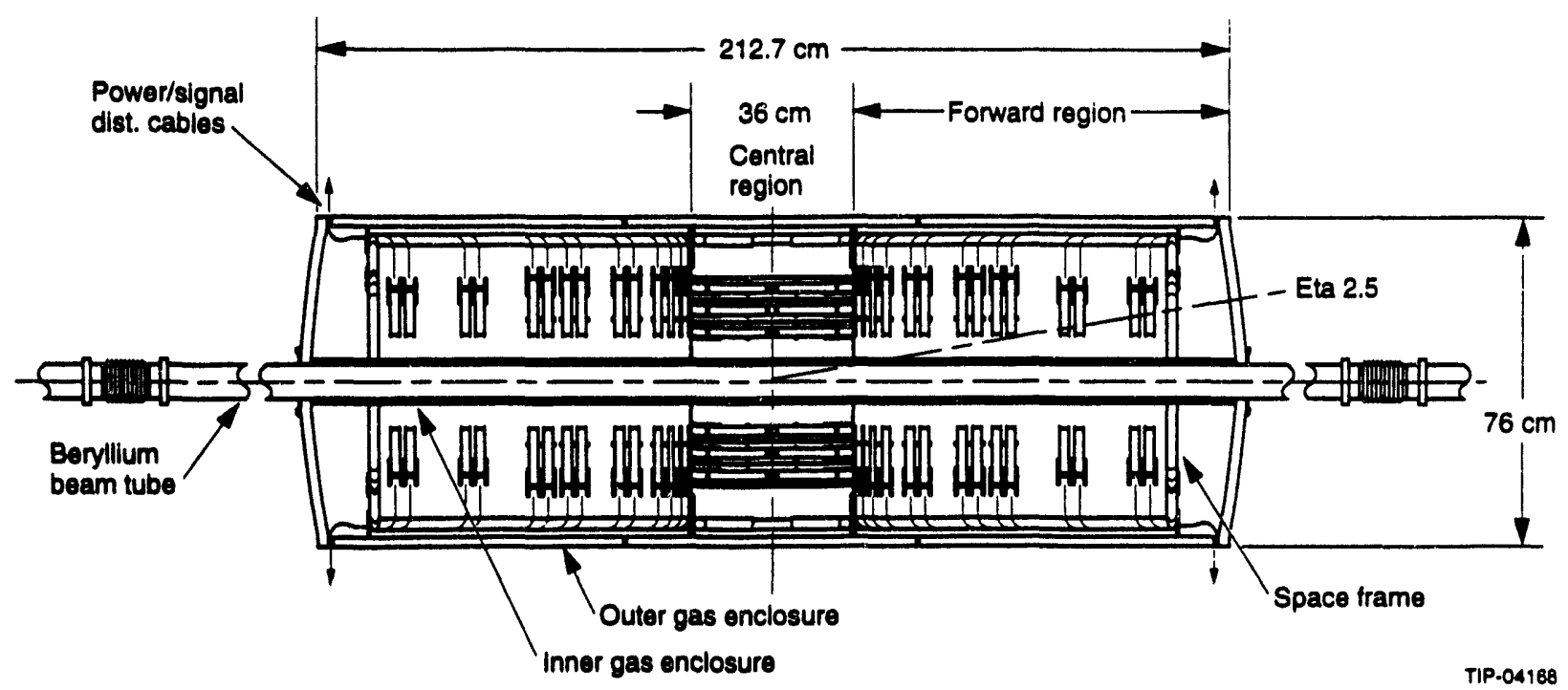

FIG. 6-40. Side view of silicon tracking system.

The baseline silicon detector ladder specifications are shown in Table 6-7. The specifications highlight the differences between the central and forward regions of the silicon tracker.

The radii of the barrel region layers range from a minimum of $100 \mathrm{~mm}$ to a maximum of $221 \mathrm{~mm}$. The two forward regions each contain 14 similar

Table 6-7. Silicon detector specitications.

\begin{tabular}{|c|c|c|}
\hline Item & Central Region & $\begin{array}{l}\text { Forward } \\
\text { Region }\end{array}$ \\
\hline Shape & Rectangular & $\begin{array}{l}\text { Rectangular I } \\
\text { Trapezoidal }\end{array}$ \\
\hline $\begin{array}{l}\text { Active detector } \\
\text { width }\end{array}$ & $3.2 \mathrm{~cm} \max$ & $3.2 \mathrm{~cm} \max$ \\
\hline $\begin{array}{l}\text { Number of } \\
\text { channels }\end{array}$ & $\begin{array}{l}1280 \\
\text { (640 per side) }\end{array}$ & $\begin{array}{l}1280 \\
\text { (640 per side) }\end{array}$ \\
\hline $\begin{array}{l}\text { Number of } \\
\text { wafers }\end{array}$ & 2484 & 8352 \\
\hline Stereo angle & $\begin{array}{l}10 \text { mrad } \\
( \pm 5 \text { per side) }\end{array}$ & $\begin{array}{l}10 \mathrm{mrad} \\
( \pm 5 \text { per side) }\end{array}$ \\
\hline $\begin{array}{l}\text { Module heat } \\
\text { load }\end{array}$ & $2.97 W$ & $2.97 W$ \\
\hline $\begin{array}{l}\text { Number of } \\
\text { modules }\end{array}$ & 414 & 1528 \\
\hline $\begin{array}{l}\text { Module heat } \\
\text { flux }\end{array}$ & $1.3 W / \mathrm{cm}^{2}$ & $1.3 \mathrm{~W} / \mathrm{cm}^{2}$ \\
\hline $\begin{array}{l}\text { Operational } \\
\text { temperature }\end{array}$ & $0^{\circ} \mathrm{C}$ & $0^{\circ} \mathrm{C}$ \\
\hline $\begin{array}{l}\text { Temperature } \\
\text { control }\end{array}$ & $\pm 0.1^{\circ} \mathrm{C}$ & $\pm 0.1^{\circ} \mathrm{C}$ \\
\hline Cant angle & $5^{\circ}$ & $0^{\circ}$ \\
\hline
\end{tabular}

silicon disk layers. The central and forward region layers are positioned so that any track from the interaction region will intercept six layers down to an $\eta$ coverage of 2.5 .

A metal matrix composite space frame supports and positions the central and forward region subassemblies. The entire tracker is sealed in a gas enclosure to contain the butane, which is the coolant used to remove the heat generated from the electronics. The signal cables, power cables, and cooling lines penetrate the enclosure at the ends.

The silicon ladders are bonded to structural rings made from graphite/cyanate ester (G/CE), which provide structural support and an enclosed passage for an internal heat-pipe wick. The detectors in the central region are arranged in concentric cylinders around the beam axis. Each detector ladder is canted at an angle of $5^{\circ}$, as is shown in Figure 6-41(a). The top and bottom surfaces of the cooling ring take on a sawtooth appearance to support the canted detectors. Heat from the electronics flows through the thin-walled composite cooling ring surface and evaporates the cooling fluid in the internal wick.

Each of the two forward regions is each composed of 14 silicon disks. The individual forward ladders are in the shape of trapezoids in order to cover the disk with the minimum overlap. The detector ladders in the forward region are not 
canted. Alternate trapezoids are mounted flat on the front or back of the cooling rings, as depicted in Figure 6-41(b). A more complete description of this geometry is discussed in the section on the Forward Region. Evaporative-butane cooling is used, as in the central region. The cooling/structural rings that support the trapezoidal-shaped modules must not introduce distortions into the silicon ladder assemblies. The module support interface will have built-in compliance to allow for a minor mismatch in the coefficients of thermal expansion (CTE).
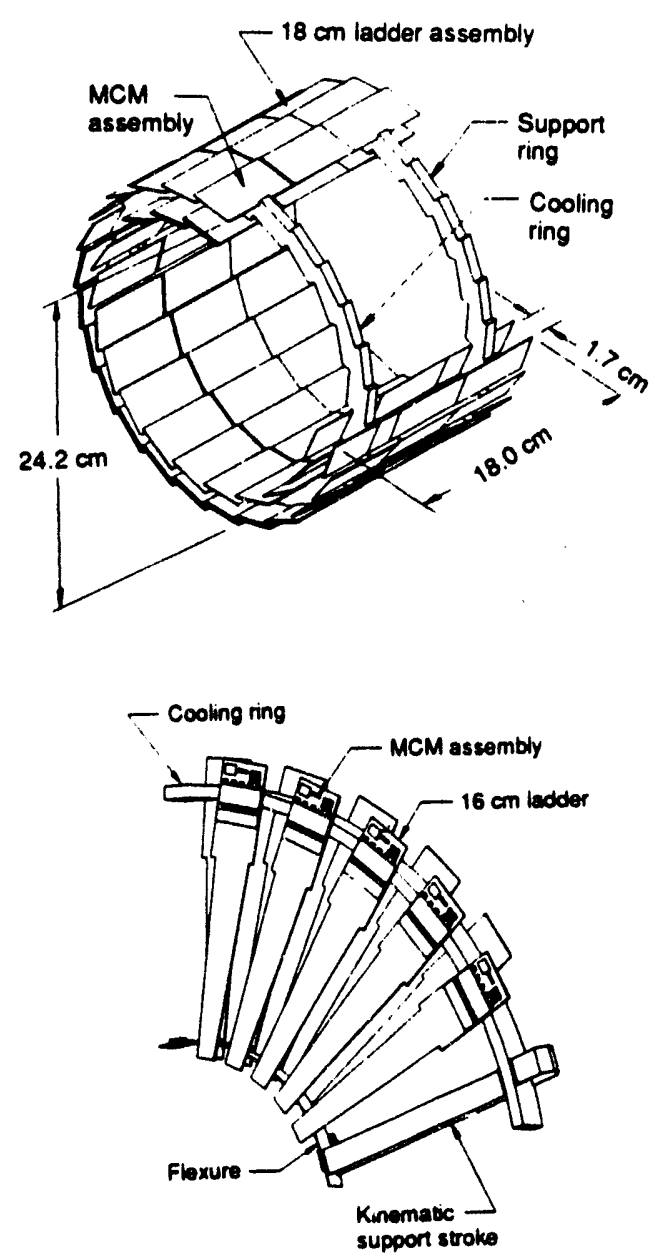

FIG. 6-41. (a) Silicon detector barrel and (b) forward modules.
We have designed a space frame to provide support for both the central and forward regions. We chose a metal matrix composite (MMC) material, tailored for a zero CTE, to eliminate distortions due to thermal and/or radiation effects. The tubular frame will be assembled with joints that are adhesively bonded. The use of adhesives helps eliminate the possibility of hysteresis in mechanically fastened joints that are subject to temperature cycling.

The entire silicon tracking system is sealed in a gas enclosure that is designed to safely contain the butane vapor of the cooling system. Introduction and removal of butane into and out of the containment vessel is accomplished in a manner imposing only slight differential pressures $(0.1 \mathrm{~atm})$ across the containment vessel wall. Any pressure differential excursion outside the 0.3 -atm limit will be automatically vented under controlled conditions.

Kinematic mounts will be used to position the internal space frame with respect to the outer containment vessel wall. Since the butane vapor pressure is controlled by the condenser pressure, large internal pressure excursions are not expected. Slight outside pressure excursions (up to $0.1 \mathrm{~atm}$ ) will result from barometric pressure variations (e.g., Texas thunderstorms). At the points where the space frame connects with the end of the gas enclosure, "hard points" will be embedded into the gas enclosure to minimize deflections due to pressure differentials across the enclosure. Nonetheless, the internal kinematic mounting of the space frame will be configured to ensure that the STS axis does not become misaligned to the outer support structure (OSS) reference frame.

Design concepts chosen to achieve the STS performance goals are presented in the following sections. The design effort has concentrated on addressing the stringent stability, positional accuracy, radiation exposure, and radiation-length limits required for this high-resolution tracking system.

To meet the high-resolution requirements, the individual ladder must meet the mechanical location tolerances shown in Table 6-8. 
Table 6-8. Silicon mechanical positioning tolerances.

\begin{tabular}{lrrr}
\hline \multicolumn{1}{c}{ Silicon Ladder Tolerance } & Circumferential & Radial & \multicolumn{1}{c}{ Longitudinal } \\
\hline Assembly placement tolerance & $25-100 \mu \mathrm{m}$ & $50-200 \mu \mathrm{m}$ & $100-250 \mu \mathrm{m}$ \\
Assembly opsical inspection precision & $1 \mu \mathrm{m}$ & $25 \mu \mathrm{m}$ & $50 \mu \mathrm{m}$ \\
X ray calibration measurement precision & $3 \mu \mathrm{m}$ & $25 \mu \mathrm{m}$ & $50 \mu \mathrm{m}$ \\
Long-term relative stability tolerance & $10 \mu \mathrm{m}$ & $80 \mu \mathrm{m}$ & $250 \mu \mathrm{m}$ \\
\hline
\end{tabular}

The assembly placement tolerance is the requirement for assembling the ladders into a subassembly. Each ladder will then be inspected to the assembly optical inspection precision requirements. The entire tracker assembly will be mapped with a highly collimated $x$ ray source to the $x$ ray calibration measurement precision listed. In order to maintain a high resolution, the tracker subassemblies must meet the long-term relative stability tolerance. Regular recalibrations will be required during the 10-yr operational lifetime of the STS. These calibrations will normally be in situ, utilizing stiff tracks as described in Section 6.2.4.

\section{Material Considerations}

The demanding design requirements for the STS call for the need of specialized high performance materials. ${ }^{19}$ The construction materials must have the following characteristics: high radiation length, high stability, compatibility with butane and radiation, low coefficients of thermal expansion, and insensitivity to moisture. Achieving the design goals will require the application of state-of-the-art material composites with high stiffness-to-weight ratio construction techniques. On the basis of fabrication and affordability, the primary structural candidates are beryllium, graphite/cyanate ester (G/CE) composites, and aluminum metal matrix composite (MMC). Dimensional stability in the G/CE composites can be achieved by using a hydrophobic cyanate-ester binder matrix.

The materials are also required to be compatible in a $100 \%$ butane vapor environment for up to 10 yr. G/CE material sarsjles have been fabricated and saturated in the coolant fluids then tensile tested, indicating no apparent incompatibilities. Similar tests need to be performed using the cyanate-ester binder in a radiation environment similar to the interaction region.

\section{Gas Enclosures and Kinematic Supports}

A gas enclosure system is required to contain the butane vapor and prevent external gases from contaminating the butane coolant. Like the spaceframe, the gas enclosure will be designed for low mass, strength and dimensional stability. The space frame attachments will connect directly to the mounting bulkheads on the gas enclosure. Figure 6-42 shows an enclosure design that meets the radiation length requirements and is capable of withstanding the range of external or internal pressures.

Under operating conditions, the pressure may vary by as much as $0.2 \mathrm{~atm}$. Relief valves and controls will be added to the system so that the internal pressure is never less than -0.2 nor greater than $0.3 \mathrm{~atm}$ gage. Analysis indicates that the cylinder will withstand an external buckling pressure of $1.3 \mathrm{~atm}$ gage. This safety factor of more than six gives comfortable assurance that the gas enclosure will operate satisfactorily under reasonable amounts of out-of-roundness due to manufacturing tolerances.

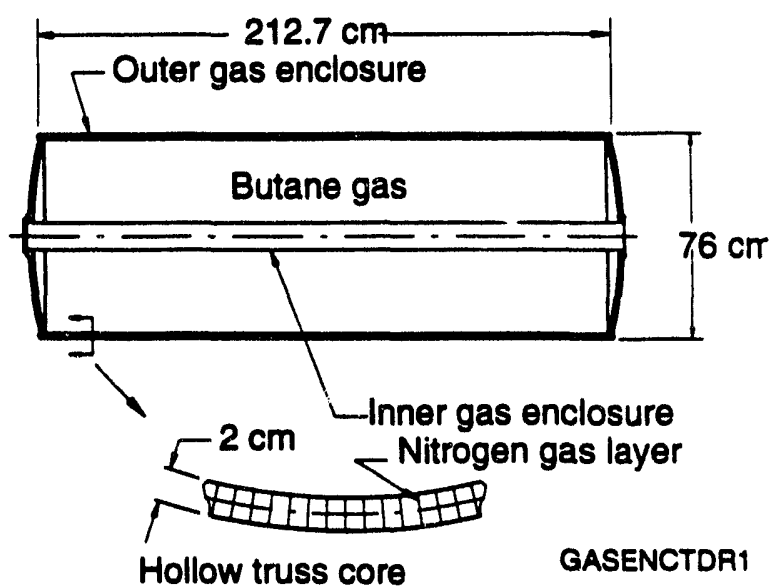

FIG. 6-42. The gas enclosure for the STS. 


\section{Space Frame}

The central and forward silicon subassemblies will be installed into a low mass, highly stable tubular support structure. Figure 6-43 shows the design concept for the space frame. This structure has been optimized to meet the requirements of long radiation length, butane vapor compatibility, radiation compatibility, thermal stability, low creep, and ease of assembly and installation. The present design incorporates aluminum metal matrix composite (MMC) material and an open frame construction. The material has a high elastic modulus with an acceptable radiation length. It has no moisture sensitivity, and can be modified to a near zero coefficient of thermal expansion. The open frame allows easy installation of subassemblies, direct cable routing, and access to installed components. The central subassembly will be supported between the two spiders. The forward region detectors will be directly attached to the frame. A cylindrical support has also been considered. When adequate openings are provided for access to the components and the remaining sections are strengthened to restore the required rigidity, the structure looks very much like the current design of a tubular space frame.

The design is an open frame with octagonal cross sections interconnected by longitudinal and diagonal stringers. The frame is constructed of 0.76-mm-thick 25.83-mm-diameter tubes bonded into composite connectors made of aluminum metal matrix and silicon carbide particulate $(\mathrm{SiCp} / 6061)$. The modular design includes the ability to assemble and disassemble the frame for maintenance access to the center of the detector. Frame modularity was considered by incorporating positioning/reposition-

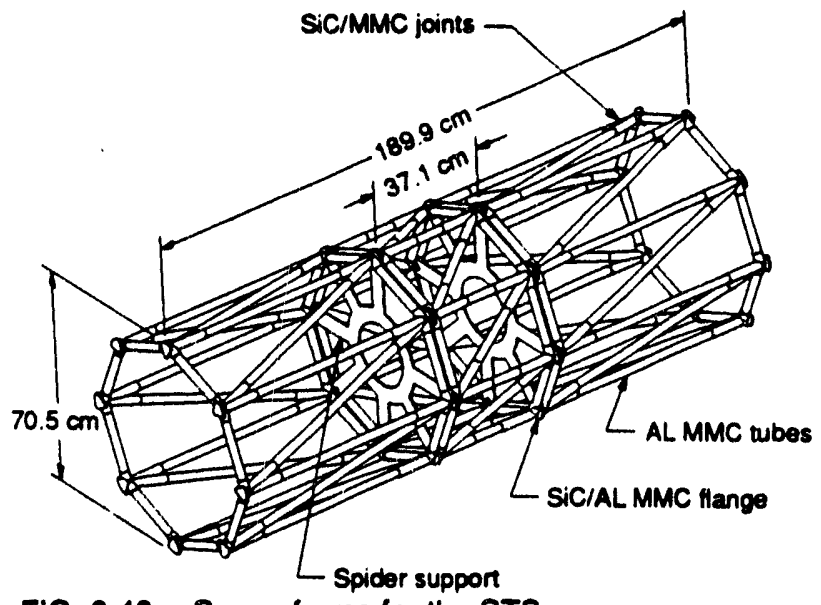

FIG. 6-43. Space frame for the STS. ing pins to accurately relocate the three modular frame sections for precision assembly.

A near-zero CTE frame is required to minimize the thermal distortion due to the cooldown of $20^{\circ} \mathrm{C}$ from assembly (alignment) to operation. This will minimize the relative displacement of the central and forward subassemblies. In addition, the forward region subassemblies will be kinematically mounted to the frame to isolate them from any thermal distortions. The central region support cylinders are kinematically mounted to one of the spiders, and the spiders are clamped between the central and outer sections of the space frame. Power and signal cables, coolant lines, and fiber-optic cables will enter the central region along the spiders.

\section{Central Region}

The silicon ladder assemblies are grouped into superlayers consisting of two detector layers mounted one on the inner circumference and one on the outer circumference of the cooling/support rings. One such central region superlayer is shown in Figure 6-41(a). Two of these shell assemblies are abutted at the center line to form each central region superlayer. In all there are three concentric superlayers composed of $18-\mathrm{cm}$-long ladder assemblies. The ends of the $18-\mathrm{cm}$-long ladder assemblies are mechanically bonded to the inside and outside circumferences of the cooling rings. In addition to providing the mechanical support for the ladders, the hollow cooling ring provides the passage for the wick coolant distribution.

The cooling ring wall thickness and cross-sectional height must be minimized from a radiation length consideration, and must be structurally stiff enough to provide adequate stability to the ladders. A cross section of the superlayer is shown in Figure 6-44. The current ring design is based on a wall thickness of $0.8 \mathrm{~mm}$ and height of $2 \mathrm{~cm}$, with outer radii of 12,15 , and $22 \mathrm{~cm}$.

The cooling ring material of choice is molded $\mathrm{G} / \mathrm{CE}$ for many reasons. The material provides high stiffness at low radiation lengths and is reasonably priced. Fabricating the rings by compression molding with a short chopped fiber is one cost-effective method of producing this complicated, high-precision, multi-surfaced geometry. 
The superlayers will be supported by a G/CE support cylinder. The ladders are bonded to the top and bottom of the cooling ring and overlap at the edges. The angu'ar orientation of the wafers will be minimized. The present design is set at $5^{\circ}$ off the radial. The minimum edge-to-edge overhang is set by overlapping the inactive regions of the wafer, the inactive region due to the stereo strips, and adding five strips to assure detector coverage. The minimum overlap is $2.85 \mathrm{~mm}$ with a tolerance of $\pm 100 \mu \mathrm{m}$, producing a maximum overlap of seven strips and a minimum of three strips. The actual overlap will be established for each layer by keeping the nominal wafer width constant.

A laminated power and signal distribution bus mounted on the support cylinder provides low voltage for the electronics, $150 \mathrm{~V}$ wafer bias, digital signals, thermal instrumentation, and an electrical shield. In addition to the bus, there are fiber-optic (FO) cables and two short coaxial cables. Flexible Kapton cables are used to connect the ladder electronics. The flexible cable to bus connector must be low radiation length and detachable. Figure 6-45 shows the proposed flexible cable concept. Cables will be constructed by laminating flat sheets of conductor material with Kapton. Two conductor materials being considered are aluminum and beryllium. Vacuum processing of the beryllium is required to remove essentially all the BeO from the base material, thus greatly enhancing its ductility.

Each superlayer will be supported off a G/CE cylinder designed with an ultralight truss core design. The baseline design as shown has a thickness of $0.635 \mathrm{~cm}$, with a length of $36 \mathrm{~cm}$.

The laminate thickness chosen is eight layers of $25-\mu \mathrm{m}$ thick plies to minimize the radiation

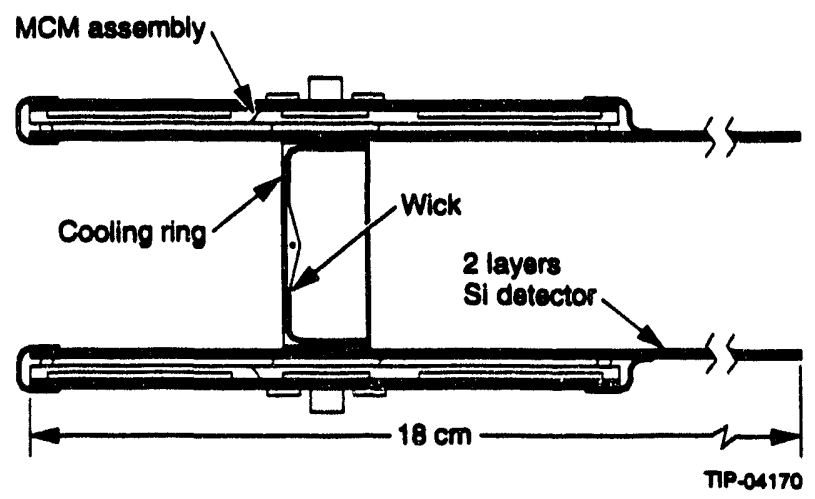

FIG. 6-44. Superlayer cross section. length. The superlayers will be kinematically mounted to the cylinders at six locations. Three kinematic mounts located $120^{\circ}$ apart will be attached to the cooling rings at each end to structurally isolate the superlayer from the support cylinder and from thermomechanical strains induced by the silicon. The kinematic mount transition from the ring to the cylinder may require small discrete openings through the silicon ladder ends.

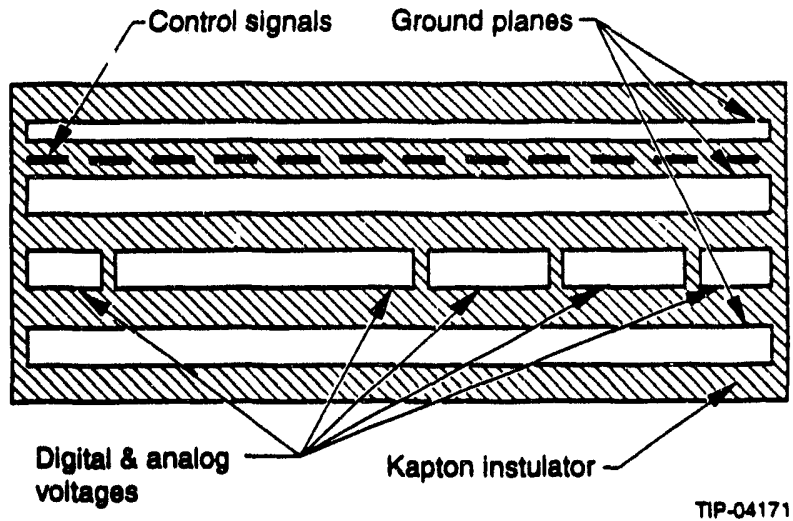

FIG. 6-45. Flexible cable concept.

\section{Forward Region}

Design details of the forward region disk subassemblies are shown in Figure 6-41(b). They are not as well advanced as the central region designs. The design specifications for ladder stability, operating temperature, ladder overlap, signal and power cable routing are basically the same, so the same design philosophy will be incorporated. The ladder will be tapered from a maximum of $33.4 \mathrm{~mm}$ wide at the outer radius, with a ladder length of 14 or $16 \mathrm{~cm}$. Identical electronics packages are mounted on the outer radii of the ladders. Structural support for the ladders will come from inner and outer rings constructed of low-mass G/CE with three interconnected spokes $120^{\circ}$ apart. Kinematic mounts will be installed at the ends of the spokes and attached directly to the space frame.

The forward region assemblies are grouped into superlayers in a manner similar to the central region. A minimum number of different inner and outer radii are preferable to minimize the construction costs. The axial placement is established to ensure that any particle from the interaction point crosses six layers. Ladders will be alternately bonded to the cooling rings front and back, with a nominal five-strip overlap at the edges. The outer 
edges of the ladder assemblies will be bonded over the cooling ring in much the same manner as in the central region ladders. However the inner edges will be attached to small, radially flexible members that will allow for differential thermal expansions due to small mismatches in CTE.

\section{Cooling System}

The STS contains 1942 multichip module (MCM) assemblies, each generating a maximum of $2.97 \mathrm{~W}$ of thermal energy for a total heat load of approximately $6.0 \mathrm{~kW}$. Heat generated in the central region subassembly is extracted through six cooling rings of three different diameters. A total of $1.22 \mathrm{~kW}$ of heat is generated in the central region.

The local heat fluxes at the $\mathrm{MCM} /$ cooling ring interfaces may be as high as $1.3 \mathrm{~W} / \mathrm{cm}^{2}$. An operating temperature of $0^{\circ} \mathrm{C}$ has been selected to minimize the detector leakage current. The mechanical stability requirements call for precise thermal control $\left( \pm 0.1^{\circ} \mathrm{C}\right)$ over the entire assembly with a minimal thermal gradient.

These requirements have led to the development of an evaporative phase change system to isothermally cool the detector assembly. Evaporative systems offer several advantages over other systems. Evaporative cooling systems are characterized by high local heat transfer rates that yield lower thermal gradients and greater isothermalization over large areas. Coolant flow rates are much lower, requiring lower liquid inventories, and thereby lowering the radiation length.

Butane has a normal boiling point at $0^{\circ} \mathrm{C}$, and isobutane has a boiling point of $-10^{\circ} \mathrm{C}$. Both are stable under radiation, and are non-toxic and chemically compatible with silicon. The butane system will al ways be enclosed in a secondary gas enclosure filled with nitrogen gas, as described earlier. This will allow for control over the butane gas mixture in the event of a leak. If butane were to leak out, it would be collected and detected in the surrounding flowing nitrogen gas.

The MCM heat generating sources will be mounted on top of the cooling rings. These cooling rings are lined with a capillary wicking structure that incorporates a low-impedance circumferential flow artery, as shown in Figure 6-44. The coolant is pressure-fed into the artery and distributed through the wick structure by capillary forces. The liquid flow in the supply artery is only $1.4 \mathrm{mg} / \mathrm{s}$. The liquid evaporates due to the electronics heat load. The fluid loop is closed by transporting the resulting vapor to a condenser using the small pressure differential that $r$ xists between the STS containment vessel and the condenser. The condensed liquid is fed back to the detector array through a liquid distribution system.

\section{Silicon Alignment and Detector Monitoring Systems}

Mechanical design, alignment systems and assembly procedures must be closely coordinated to build a precise silicon tracking system. The difference between actual placement tolerances and knowledge of position needs to be carefully separated and considered. The tolerance requirements will determine where the most effort is placed, whether in assembly or in calibration and stability monitoring; however even the loosest assembly tolerances will be too demanding for manual placement.

Six distinct alignment/assembly steps must be further defined and planied: 1) a stable assembly reference system; 2) an assembly procedure, align$n$ snt system and mechanical placement machine to attach silicon ladders to support rings and planer arrays; 3 ) an assembly/alignment procedure to attach shells and forward superlayer assemblies to a space frame; 4) a method to place the assembled STS relative to the outer support structure and the SSC beam line; 5) alignment monitors to follow alignment changes during assembly, transport, installation and use; and 6) a calibration procedure to be used for the installed detector. Tradeoffs between these steps will influence the cost, assembly time and difficulty of building the detector.

In addition to the alignment/assembly, some testing is planned to establish the stability of completed subassemblies. A dependable alignment monitoring system will relieve some burden on the mechanical stability of the assembly, but monitoring systems monitor only a few points on the structure; subassemblies are treated as rigid bodies. TV (electronic) holography is a test procedure with the required resolution and field of view for testing the stability of completed subassemblies. The technique is basically an optical interferometer for non-optical surfaces. A state-of-the-art system can measure 
quantitative and qualitative information about object shape, shape changes induced by mechanical or thermal loading, mechanical resonance frequencies and resonant $\mathrm{Q}$. State-of-the-art TV holography systems are not available commercially. They will be custom built to carry out these important tests.

The alignment monitoring system is critically important for the calibrated detector. A useful alignment system should monitor detector alignment shifts after assembly, during transport and installation, and between calibration runs. The resolution of the monitor should be sufficient to determine when another calibration is needed. If the rigid body assumption about the subassemblies is valid, and if the alignment monitor has sufficient dynamic range, then after an initial calibration run, the monitoring system can be used to correct particle track information and eliminate the need for continuous, time consuming calibrations.

One concept for continuously monitoring one subassembly relative to another, and the position of the silicon dutector to the outer detectors, is to use a rad-hard, compact, non-intrusive fiber-optic motion stnsor. As shells are attached to the space frame, fiber-optic motion sensors are also attached to every rigid body subassembly to monitor the internal relative positions and any changes relative to each other. Additional monitors provide the same information on the tracker reference system to a reference outside the detector. If the motion sensor is reflected off a spherical surface, two degrees of freedom can be monitored with a seven fiber bundle assembly shown in Figure 6-46.

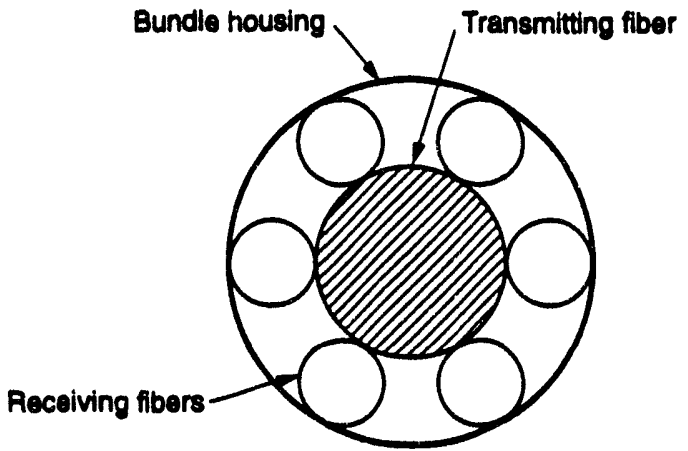

Tip-04172

FIG. 6-46. Fiber bundle assembly.

\section{Silicon Ladder}

In the central region three single-sided wafers are edge-bonded forming an 18-cm-long ladder, shown in Figure 6-47. Two of these ladders are bonded back-to-back with $a \pm 5 \mathrm{mrad}$ stereo angle. In the forward region. the ladder lengths are 14 and $16 \mathrm{~cm}$. The electronics are mounted at the outer radii where the detector strips are read out.

A large, single $\mathrm{MCM}$ is mounted on the top of the ladder at one end. It is critical to maintain a low-profile compact ladder assembly in order to achieve compact packaging of the ladders onto the cooling rings. The edges of the silicon ladder are structurally reinforced with thin G/CE rails to increase the transverse stiffness of the assembly.

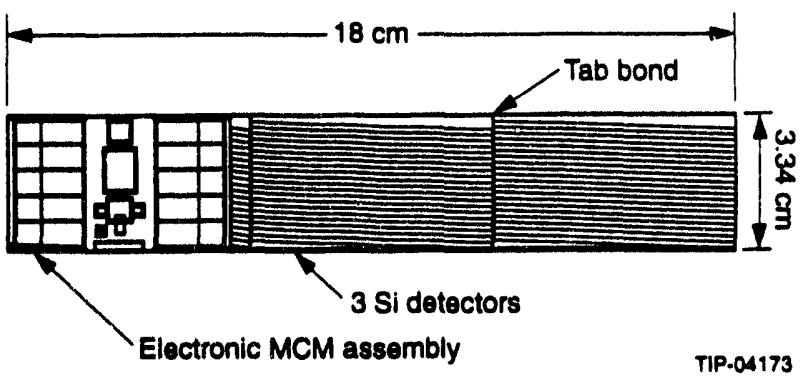

FIG. 6-47. Silicon ladder assembly.

\subsubsection{Electronics}

The silicon tracker electronics must satisfy a number of requirements. The most important of these are low power and high speed, a signal-tonoise of greater than $3: 1$, radiation resistance, low mass, and low cost. The bulk of the electronics are on the silicon ladder assembly with fiber-optic communications to the external data acquisition electronics. The silicon tracker electronics system architecture is shown in Figure 6-48. The heart of the design is the multichip module (MCM) which contains the front-end electronics. The front-end electronics performs the following functions: provides bias and ground connections to the strip detectors; shapes the signals from the strip detectors and discriminates the signal to produce a logic pulse; buffers and compresses the logic bit pattern for readout; transmits the event of interest to the data acquisition system; performs housekeeping functions such as monitoring voltages, currents and temperatures; and allows for a full functional test of the MCM. Analog signal processing is done by the bipolar chip, and data buffering and compression is done by the CMOS-1 chip. Both the bipolar chip and CMOS-1 chips are expected to be able to survive in the radiation fields. A technical description of the electronics is given in Section 7.4. 


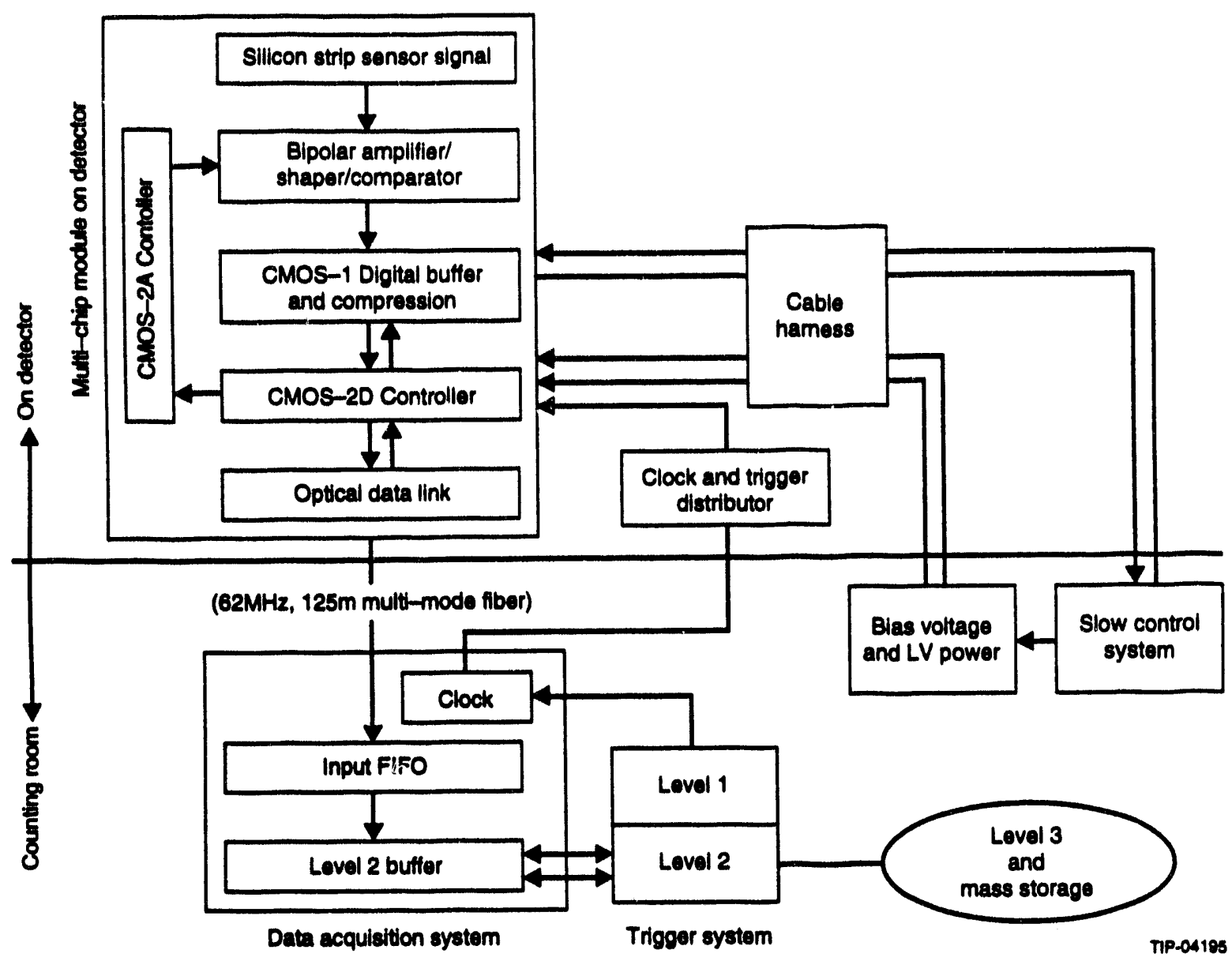

FIG. 6-48. Silicon tracker electronics system architecture.

The multichip module is a departure from the more conventional hybrid circuit designs used in high-energy physics vertex detectors. The MCM offers the advantages of a reduction in assembly labor because of increased automation and an increase in reliability and testability. The MCM does not use wire bonding for interconnects where the failure rate approaches $1 \%$, but instead utilizes Kapton interconnect layers and vias where the defect rate is only 3:5 per million. The MCM will be designed to be completely tested prior to assembly into a complete ladder. It is repairable, so a minimum number of spares will be required.

The design goals for the electronics include single crossing resolution, less than $2 \mathrm{~mW}$ per channel heat load, and radiation resistance to $3 \mathrm{Mrad}$. The current designs meet these goals. The bipolar amplifier/shaper/comparator time-walk is less than 16 ns and the power consumption is approximately $1.7 \mathrm{~mW}$ per channel. The CMOS-1 buffer and compressor chip is expected to operate at
$60 \mathrm{MHz}$. It utilizes a design that minimizes die size (and therefore cost) and power. Currently, the CMOS-1 design features include

- Reset-free, latch-up resistance

- Programmable trigger latency

- Programmable trigger aperture (up to four crossings)

- Phased clock (between local and master)

- Data driven storage efficiency $>99.5 \%$.

The system design allows for complete electronic testing prior to and during the assembly process, and in the fully configured detector.

\subsection{INTERPOLATING PAD CHAMBER OUTER TRACKER}

\subsubsection{Interpolating Pad Chambers}

An interpolating pad chamber (IPC) is a multi-wire proportional chamber with one cathode plane consisting of an array of electrically isolated 
regions, or "pads." These pads sample the induced charge on the cathode plane due to as avalanche at the anode initiated by ionizing radiation in the chamber volume. The centroid of the induced charge distribution, determined from the pad signals, defines the ionizing particles' transit position.

Several techniques have been developed over the years for reading out segmented cathode MWPCs. These include resistive charge division, capacitive charge division, and geometrical charge division. The resistive charge division technique, which employs resistive strips running under each anode wire with periodic readout points, is not favored for large area applications due to the difficulty in maintaining a uniform resistance. The geometrical charge division technique is the most straightforward, using a separate readout channel for each pad as is shown in Figure 6-49. Capacitive charge division is an extension of geometrical charge division, in which the pads are made narrower and one or more pads are skipped between readout pads. In this scheme, the induced charge on the intermediate pads is coupled to the nearest readout pads via the inter-Fad capacitances. Its major advantage over the simple geometrical approach is a reduced capacitive load on the readout amplifier input, which results in lower electronic noise at the expense of position linearity. Optimization studies of the cathode readout are now underway and will be used to evaluate the tradeoffs between the geometric and capacitative readout techniques. The current GEM outer tracker design and the IPC prototypes constructed to date employ the geometric charge division technique. Changing to a capacitative charge division readout scheme would not affect any aspect of the IPC design other than the physical layout of the chamber cathode.

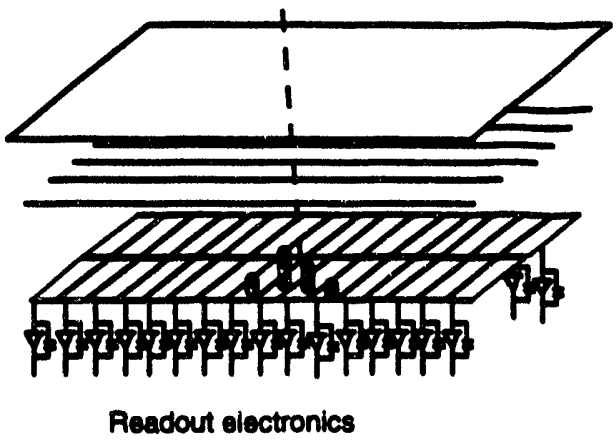

FIG. 6-49. Geometric charge division.

\section{Geometrical Charge Division}

For IPC readout by geometric charge division, the transit position of an ionizing particle can be obtained from the equation

$$
x=\Sigma Q_{k} x_{k} / \Sigma Q_{k} .
$$

where $x_{k}$ are the position of the pads, and $Q_{k}$ are the signals induced on each pad. Typically the induced charge spans 3:5 pads, depending upon the chamber geometry. It is desirable from the standpoint of occupancy and channel count considerations, discussed below, to minimize the number of pads associated with a given particle transit. In practice, the number of pads used to calculate the particle position from the charge centroid is always finite, leading to a non-linearity in the relationship between the true and reconstructed position. For this reason, a fit of the pad signals to the known spatial distribution of the induced charge distribution for the chamber geometry used is generally superior to the centroid method of determining the avalanche position.

The position resolution obtained from the centroid measurement is directly related to the signal-to-noise ratio of the readout chain. A simple error analysis of the equation for the centroid position shows that the position resolution obtained is given by

$$
\sigma_{x} / w=\sqrt{n} \sigma_{\mathrm{Qk}} / \Sigma Q_{k},
$$

where $w$ is the pad width and $n$ is the number pads used in the measurement.

\section{Design Parameters}

The design parameters of the IPC system are set by the resolution, occupancy, and speed requirements imposed by the physics goals of the central tracker. In this section we discuss the optimization of these parameters under the operational constraints and design goals summarized in Table 6-9.

\begin{tabular}{|c|c|c|}
\hline \multirow{2}{*}{$\begin{array}{l}\text { Anode } \\
\text { wires }\end{array}$} & Table 6-9. Parameters of th & C detuctors. \\
\hline & \multicolumn{2}{|l|}{ Design constraints } \\
\hline \multirow{3}{*}{$\begin{array}{l}\text { Readout } \\
\text { cathode }\end{array}$} & Beam crossing rate & $62 \mathrm{MHz}$ \\
\hline & $\begin{array}{l}\text { Charged track density/min bias } \\
\text { ovent }\end{array}$ & $d N / d \eta d \phi=1.2$ \\
\hline & $\begin{array}{l}\text { No. min bias events/crossing } \odot \\
10^{34} \mathrm{~cm}^{-2} \mathrm{~s}^{-1}\end{array}$ & 16 \\
\hline TP-04080 & Tracks/seqc-mm wire $10^{34}$ & $1 \times 10^{4} \mathrm{~s}^{-1} \mathrm{~mm}^{-1}$ \\
\hline
\end{tabular}

Table 6-9. Parameters of the IPC detectors. 
Table 6-9. Parameters of the IPC detectors. (Cont.)

\begin{tabular}{|c|c|}
\hline \multicolumn{2}{|l|}{ Design constraints } \\
\hline $\begin{array}{l}\text { Integrated charge/yr-cm wire } \\
10^{34} \mathrm{~cm}^{-2} \mathrm{~g}^{-1}\end{array}$ & $0.85 \mathrm{C} / \mathrm{cm}-\mathrm{yr}$ \\
\hline Level 1 trigger rate & $100 \mathrm{kHz}$ \\
\hline Level 1 trigger latency & $2 \mu \mathrm{s}$ \\
\hline \multicolumn{2}{|l|}{ Design goals } \\
\hline Occupancy $\odot 10^{34} \mathrm{~cm}^{-2} \mathrm{~g}^{-1}$ & $<10 \%$ \\
\hline $\begin{array}{l}\text { Design goal position resolution } \\
\text { layer in r/p }\end{array}$ & $50 \mu \mathrm{m}$ \\
\hline
\end{tabular}

\section{Chamber Geometry}

The chamber geometry is set by the requirement of a fast electron collection time and reasonable mechanical tolerances. A large anode to cathode distance would result in an unacceptably long electron collection time, while separations less than $2 \mathrm{~mm}$ would result in unacceptably tight mechanical tolerances due to the large chamber capacitance.

The chamber gain is given approximately by

$$
M=k e^{\left(\mathrm{CV}_{0}\right)} \text {, }
$$

so that a large chamber capacitance results in a more rapid increase in gain for a given voltage change, with correspondingly larger sensitivity to mechanical tolerances. It can be shown that

$$
\begin{gathered}
\Delta M / M=(\Delta Q / Q) \ln M, \\
\text { and, } \Delta Q / Q=\left(C / 2 \varepsilon_{0}\right) \Delta a / a, \\
\Delta Q / Q=\left(C / / 2 \varepsilon_{0} s\right) \Delta U,
\end{gathered}
$$

where $Q=C V_{0}$ is the charge per unit wire length, $M$ is the chamber gain, $a$ is the wire radius, $l$ is the anode/cathode spacing, and $s$ is the spacing between wires.

The geometry specified in Table 6-10, corresponding to the present IPC design, has a capacitance of $7.6 \mathrm{pf} / \mathrm{m}$. For this geometry, a 2 -mil variation in the anode/cathode spacing results in a $10 \%$ variation in the gain of the chamber.

Table 6-10. Parameters of an interpolating pad chamber.

\begin{tabular}{ll}
\hline Wire spacing & $2 \mathrm{~mm}$ \\
Anode-cathode spacing & $2 \mathrm{~mm}$ \\
Wire diameter & $20 \mu \mathrm{m}$ \\
Voltage & $3000 \mathrm{~V}$ \\
Gas & $50: 50 \mathrm{CO}_{2}-\mathrm{CF}_{4}$ \\
\hline
\end{tabular}

\section{Occupancy}

The design goal for occupancy in the IPCs is set by the desire for robust operation at a beam luminosity of $10^{34} \mathrm{~cm}^{-2} \mathrm{~s}^{-1}$. The IPC design parameters that affect the occupancy are the number of pads having a significant signal associated with a single track, the pad length, and the length of time during which a subsequent hit on a pad would result in serious degradation in position resolution due to pileup.

The most conservative estimate of occupancy assumes that three pads are needed to reconstruct a position, and multiple tracks with separations less than this are not analyzable. However, studies conducted by the tracker group, described in detail in Section 6.2.2, have shown that the positions of two tracks separated by a small fraction of a pad width can be determined with minimal loss of resolution, and one can expect to do better that this worst case estimate in practice. The real two-track resolution will be measured as part of the GEM central tracker test beam program.

A fast detectnr response is a critical aspect of the central tracker IPC system. A deadtime of $40-50$ nsec ( 3 bunch crossings) is desirable in order to achieve the IPC occupancy specification. The deadtime of this system is given by the time taken for the shaped output of the front-end an:plifier to return to a level such that it can be subtracted from the subsequent pulse without significant loss of resolution. A lower limit to the shaping-time that can be used is given by the characteristic time for collection of the ionization electrons at the wire. Integration times shorter than this will result in a large reduction in the observed signal, large event-to-event differences in the peak signal, and a strong dependence of the peak signal on the shaping-time. An operational requirement of the IPCs, therefore, is that they have an electron collection time on the order of $20 \mathrm{nsec}$. At the same time, the Lorentz angle of the gas at $0.8 \mathrm{kG}$ must be kept under $15^{\circ}$, as the chambers must be canted at this angle with respect to the magnetic field to minimize the position-resolution degradation due to spreading of the induced signal along the cathode. Mechanical constraints make a cant angle larger than $15^{\circ}$ difficult. A gas mixture suitable for central tracker use in the IPCs must, therefore, be relatively fast, with a small Lorentz 
angle. Suitable gas mixtures do exist that satisfy our requirements: for example, $\mathrm{CF}_{4}-\mathrm{CO}_{2}$ mixtures. The GEM central tracker group at Indiana University has been investigating the properties of $\mathrm{CF}_{4}-\mathrm{CO}_{2}$ using a chamber designed to make precision drift velocity and Lorentz angle measurements. Results show that these gas mixtures are relatively fast and exhibit Lorentz angles below $10^{\circ}$ at $8 \mathrm{kG}$ over a wide $\mathrm{CF}_{4}$ concentration range. The results of these gas studies have been used in a simulation of an IPC chamber using the wire chamber simulation program GARFIELD. The critical operational parameters used in this simulation are listed in Table 6-10. The contours of equal electron drift time obtained in the simulation are shown in Figure 6-50. The contours are at 5-ns intervals. It is apparent from this figure that the maximum delay between the passage of the particle and the arrival of the first electron at the wire is less than $20 \mathrm{~ns}$, and that for electrons coming from a given particle track the spread in arrival times is also less than $20 \mathrm{~ns}$. The distribution of arrival times of the first electron at the anode is shown in Figure 6-51 for a number of random trajectories through the chamber. This represents the major contribution to time jitter in the system and demonstrates that it will be possible to assign virtually all hits to the proper crossing.

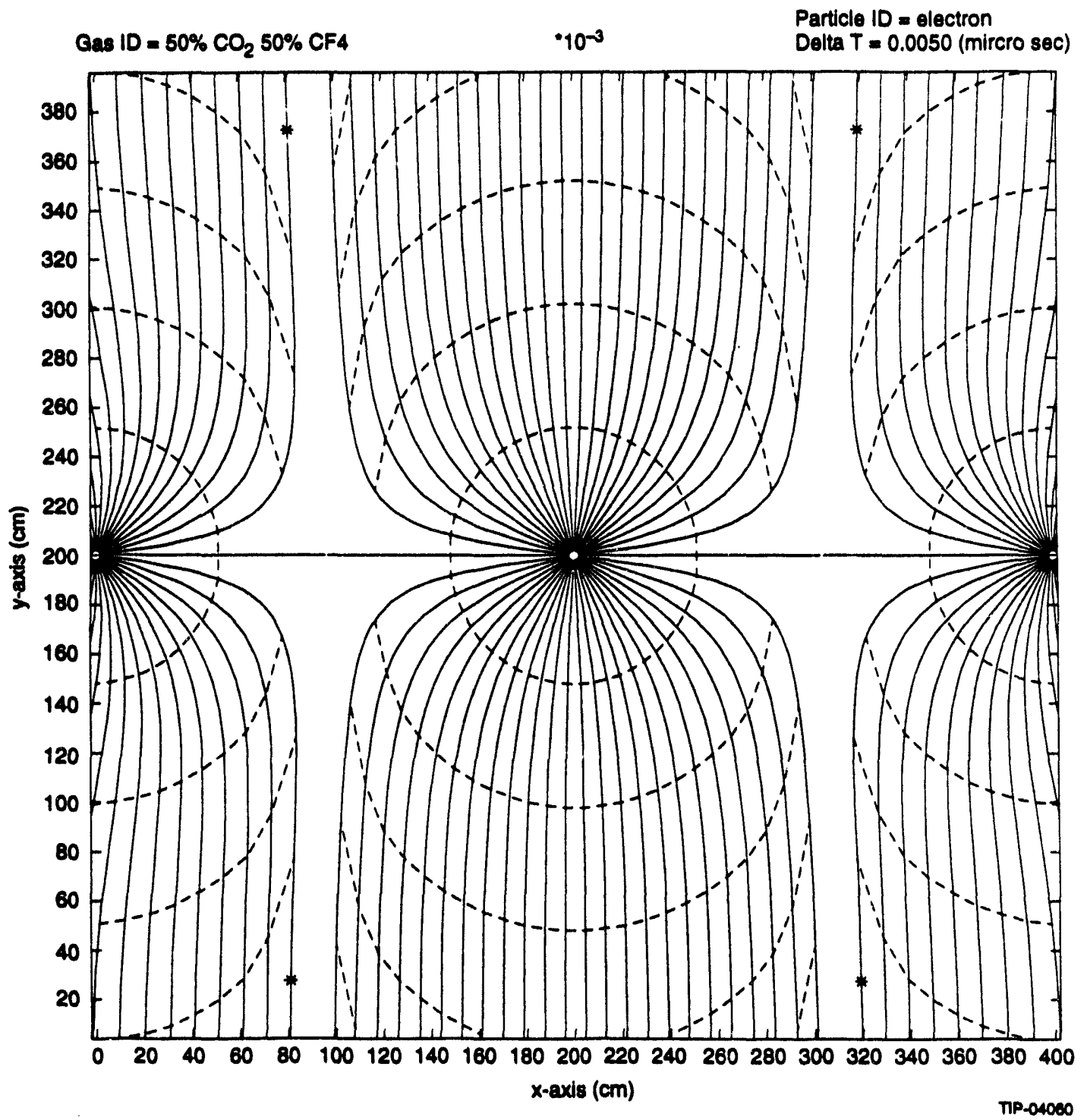

FIG. 6-50. Equal time contours. 


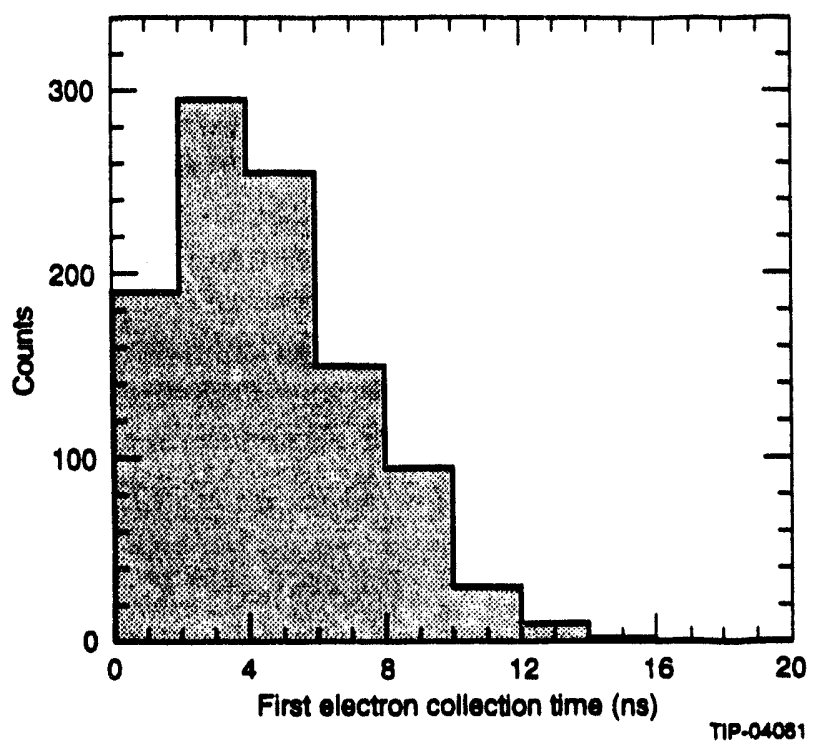

FIG. 6-51. First electron collection time distribution.

\section{Position Resolution}

As stated earlier, the position resolution is determined by the signal-to-noise ratio of the readout chain. The design goal of the tracker calls for $50-\mu \mathrm{m}$ resolution, corresponding to $2 \%$ of the pad width. Referring to the relationship between position resolution and signal-to-noise ratio, this requires a signal resolution of about $1 \%$ of the total measured cathode signal. The gas gain, which determines the signal amplitude, is therefore constrained by signal-to-noise considerations. Figure 6-52 shows the measured position resolution in a prototype IPC chamber versus the gas gain, while Figure 6-53 shows the distribution of event positions at a single source position. These measurements were made on a chamber with the same geometry as the baseline IPC design, with a fast gas mixture, and a front-end amplifier with a peaking time of $20 \mathrm{~ns}$. They demonstrate that the $50-\mu \mathrm{m}$ requirement is achievable under realistic conditions. At low values for the gas gain the resolution is measurement error dominated, and hence varies roughly as the inverse of the gas gain. Under the operating conditions used to generate the data in Figure 6-52, the position resolution of the chamber under study approaches an asymptotic value at a gas gain of $2 \times 10^{4}$. The gas gain above which the chamber resolution is dominated by irreducible effects is determined by the noise in the front-end readout electronics, normally expressed in equivalent electrons. The dominant noise component, due to the input transistor of the preamplifier, can be expressed as:

$$
\sigma_{q} \propto C_{\text {in }} /\left(\mathrm{g} \sqrt{\mathrm{t}_{\text {shaping }}}\right) .
$$

This component of the amplifier noise increases with the inverse square root of the shapingtime and is proportional to the input capacitance. This capacitance includes the mutual capacitance between pads, along with the capacitance between a pad and the readout lines, ground planes, etc. The present design of the cathode calls for readout lines lying on the opposite side of a $0.5-\mathrm{mm}$ thick sheet from the pad plane, with no ground plane between the readout and pad layers. Measured values of the total capacitance for $0.2-\mathrm{cm}$ wide rectangular pads plus readout lines are in the range of 2 to $25 \mathrm{pf}$ for the range of pad lengths used in the IPCs.

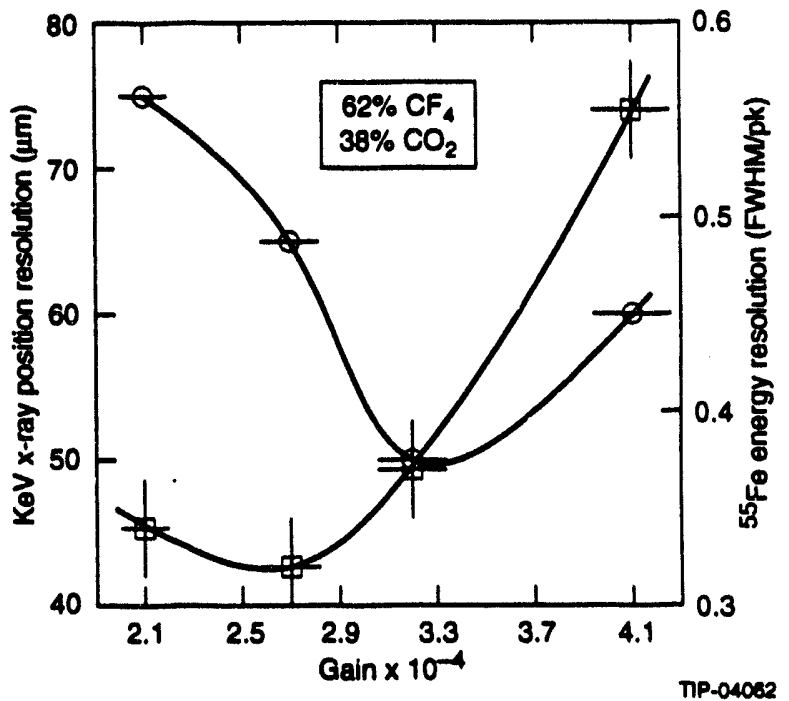

FIG. 6-52. Position resolution vs gain. Results determined by an $x$ ray source are denoted by open circles and results derived from an $55 \mathrm{Fe}$ source by open squares.

Based on the performance of monolithic preamplifiers fabricated as part of the GEM R\&D program and described in cietail in the electronics section of the TDR, it is reasonable to expect a noise figure of 1300 electrons for an input capacitance of $25 \mathrm{pf}$, with a peaking time of $20 \mathrm{~ns}$. Using this vaiue for the expected noise in the readout chain, we require a total cathode signal of roughly $1.3 \times 10^{5}$ electrons to obtain a total signal to single channel noise ratio of 100 . This value can be used to determine the gas gain required to achieve the $50-\mu \mathrm{m}$ position resolution design goal. The number 
of ion pairs produced in a 4-mm gap of 50:50 $\mathrm{CO}_{2}-\mathrm{CF}_{4}$ is 60 . The measured signal on the cathode is smaller than $60 \times M$ as a result of two factors. First, the induced signal is shared between the two cathodes and the adjacent wires, so the induced signal on a given cathode is roughly one half the total. Second, the shaping amplifier filters out all but the initial portion of the induced signal. For the baseline chamber geometry and known values for the ion mobilities of $\mathrm{CO}_{2}$ and $\mathrm{CF}_{4}$, the fraction of the total charge seen at the output of the shaping amplifier is $20 \%$ for a 20 -ns peaking time. Combining these factors, and requiring a cathode signal of $1.3 \times 10^{5}$ electrons, we find that the gain required would be 20000 if electronics noise were the only contribution to the position error. Results of the position resolution measurements in IPC prototypes, shown in Figure 6-52 and Figure 6-53, indicate that irreducible effects such as diffusion also contribute to the position error, requiring that a siightly higher gas gain than this value be used.

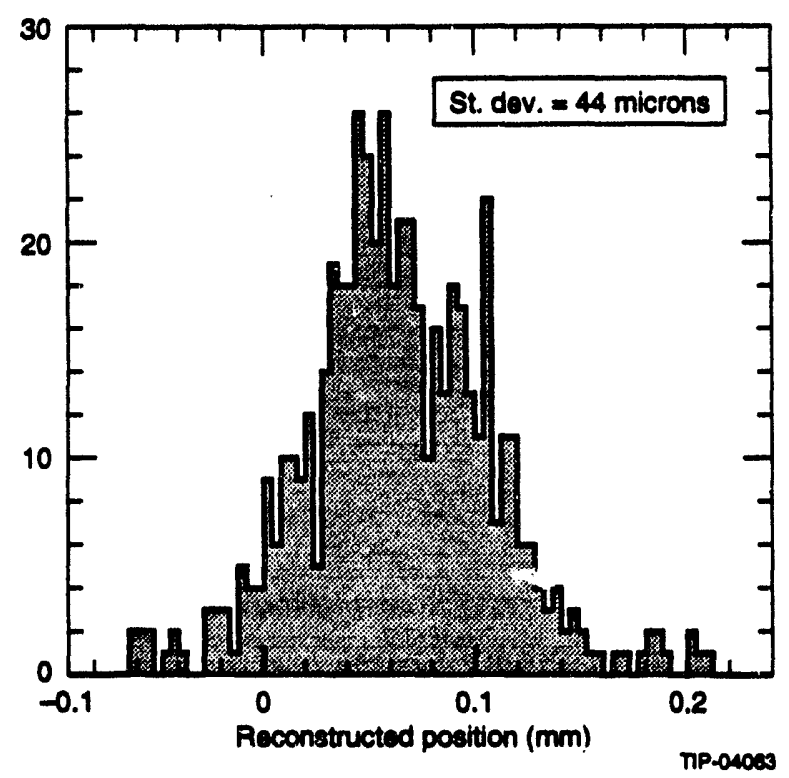

FIG. 6-53. Distribution of position residuals for 2-KeV $x$ rays, demonstrating a resolution of 44 microns.

\section{Rate Effects}

Effects of the high rate SSC environment on IPC performance fall into two categories: chamber aging, and the effects of space charge on chamber gain. Chamber aging reiers to a gradual decrease in the gas gain in the chamber due to a buildup of deposits on the electrodes, resulting in a narrowing operating plateau and eventual chamber breakdown.
This effect is directly proportional to the integrated charge collected per unit length of wire. At a luminosity of $10^{34} \mathrm{~cm}^{-2} \mathrm{~s}^{-1}$ the amount of charge collected/SSC $\mathrm{yr} / \mathrm{cm}$ of wire in the IPCs, assuming a gas gain of 30000 , is $0.85 \mathrm{C} / \mathrm{cm}$ at a radius of 30 $\mathrm{cm}$. For: a given integrated charge, chamber aging depends primarily on gas composition. Measurements have been carried out on chamber aging for $\mathrm{CF}_{4}-\mathrm{CO}_{2}$ mixtures. For a 50:50 mixture the measured decrease in the operating current of a test chamber was found to be

$$
\left(I-I_{0}\right) / I_{0}=0.07 \mathrm{C}^{-1} \mathrm{~cm} / \mathrm{yr} \text {. }
$$

This result confirms the expectation that these gases should exhibit very small aging effects. It is well known that freon can be used to recover a chamber that suffers from aging. The conclusion is that at the gas gain required from signal-to-noise requirements, the chambers should be able to operate for a number of years at $10^{34} \mathrm{~cm}^{-2} \mathrm{~s}^{-1}$ while suffering minimal aging effects.

The buildup of space charge in the IPCs will result in a decrease in gain of the chamber at sufficiently high ionizatior rates. At a luminosity of $10^{34} \mathrm{~cm}^{-2} \mathrm{~s}^{-1}$, the charged track rate in the IPCs is $2 \times 10^{9} \mathrm{~s}^{-1}$ in each IPC, or $1 \times 10^{4} \mathrm{~s}^{-1} \mathrm{~mm}$ wire ${ }^{-1}$ in the innermost layer of the IPCs. Measurements have been conducted on IPC prototypes running $\mathrm{CF}_{4}-\mathrm{CO}_{2}$ mixtures. The chambers were exposed to a high intensity $x$ ray source generating local ionization levels in excess of this amount. The results indicate little if any space charge-induced reduction in gain at these ionization levels. To confirm this, a critical part of our R\&D plan during the coming year is an investigation of space charge effects on chamber operation at high particle fluences using minimum ionizing particles.

\subsubsection{Mechanical Design Introduction}

The following sections describe the interpola:ing pad chamber (IPC) tracker array that has been proposed as a part of the GEM detector. The IPC tracker array consists of four major subsystems: the barrel chambers, the barrel array support structure, the endcap chambers and the endcap array support structure. In addition, there are utilities for both the forward and barrel regions (signals, high voltage for anode wires, low voltage and cooling for "on-board" electronics, and gas for the chambers). 
Interpolating Pad Chamber Design Parameters

Table 6-11 summarizes the design parameters for the IPC tracker array (barrel and endcap). Figure 6-54 is a quadrant view of the tracker. Figure 6-55 is an end view of the barrel section illustrating the proposed barrel superlayer arrangement. The locations of the pad chambers are summarized in Table 6-12.

Table 6-11. GEM IPC array design parameters.

\begin{tabular}{|c|c|}
\hline Parameter & Quantity \\
\hline Lorentz angle & 12.5 degrees \\
\hline $\begin{array}{l}\text { Number of sectors-barrel } \\
\text { superlayer }\end{array}$ & 20 \\
\hline $\begin{array}{l}\text { Number of sectors-endcap } \\
\text { superlayer }\end{array}$ & 10 \\
\hline $\begin{array}{l}\text { Number of superlayers-barrel and } \\
\text { endcap }\end{array}$ & 4 \\
\hline $\begin{array}{l}\text { Number of chambers per } \\
\text { superlayer }\end{array}$ & 2 \\
\hline $\begin{array}{l}\text { Chamber depth (cathode to } \\
\text { cathode) }\end{array}$ & $4 \mathrm{~mm}$ \\
\hline Anode wire diameter & $\underset{\mu \mathrm{m}}{20 \leq \text { diam. } \leq 40}$ \\
\hline Anode wire potential & $3 \mathbf{k V}$ \\
\hline Barrel IPC superlayer length & $200 \mathrm{~cm}$ \\
\hline $\begin{array}{l}\text { Barrel IPC array inner radius } \\
\text { boundary }\end{array}$ & $39 \mathrm{~cm}$ \\
\hline $\begin{array}{l}\text { Barrel IPC array outer radius } \\
\text { boundary }\end{array}$ & $88 \mathrm{~cm}$ \\
\hline $\begin{array}{l}\text { Endcap IPC minimum axial } \\
\text { boundary }\end{array}$ & $112 \mathrm{~cm}$ \\
\hline $\begin{array}{l}\text { Endcap IPC maximum axial } \\
\text { boundary }\end{array}$ & $170 \mathrm{~cm}$ \\
\hline Number of barrel pads & 204240 \\
\hline Number of pads (each endcap) & 93540 \\
\hline $\begin{array}{l}\text { Electronics power dissipation- } \\
\text { barrel }\end{array}$ & $12.5 \mathrm{~kW}$ \\
\hline $\begin{array}{l}\text { Electronics power dissipation- } \\
\text { each endcap }\end{array}$ & $5.7 \mathrm{~kW}$ \\
\hline
\end{tabular}

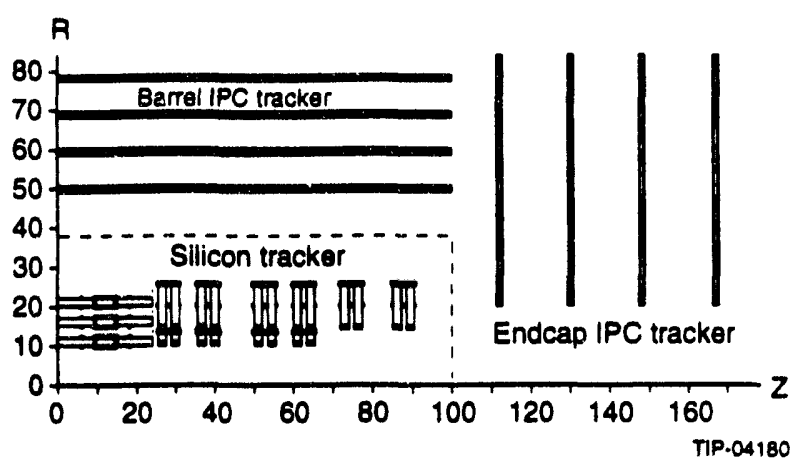

FIG. 6-54. Central tracker quadrant view.

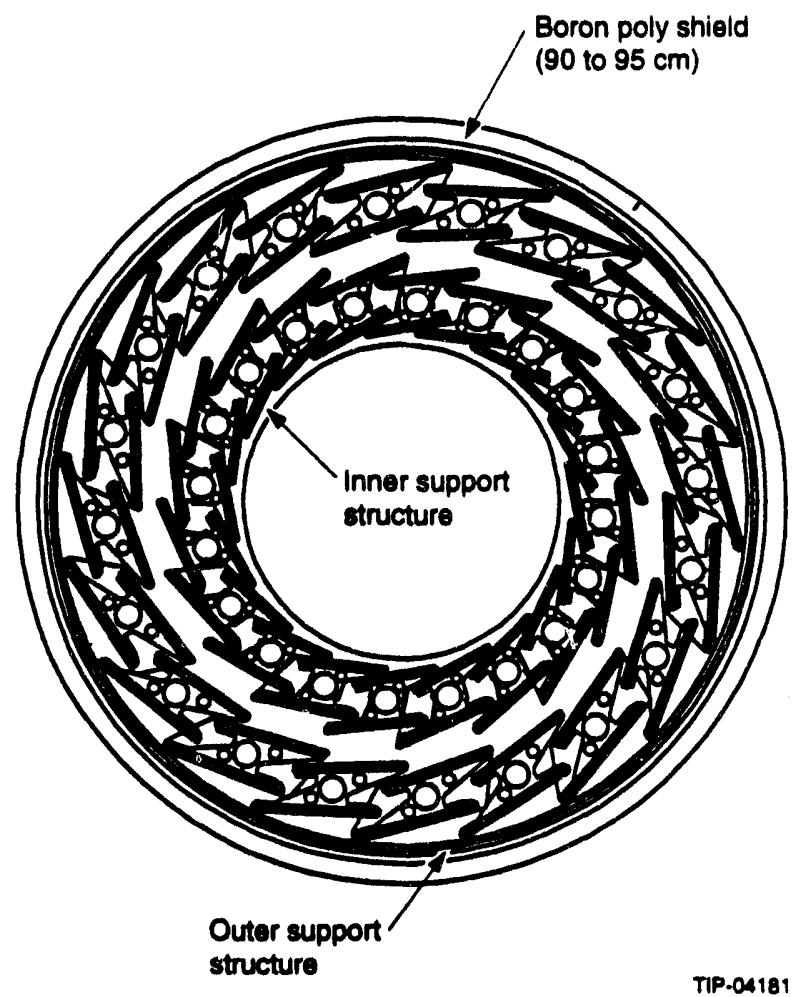

FIG. 6-55. IPC tracker cross-sectional view.

Table 6-12. GEM IPC locations (middle of anode wire plane).

\begin{tabular}{|c|c|c|}
\hline Superlayer Number & $\begin{array}{l}\text { Effective } \\
\text { Width } \\
\text { (cm) }\end{array}$ & $\begin{array}{l}\text { Distance to } \\
\text { Interaction Point } \\
\text { (cm) R for barrel; } \\
\text { z for endcaps }\end{array}$ \\
\hline $\begin{array}{l}\text { Superlayer I } \\
\text { Inner chamber } \\
\text { Outer chamber }\end{array}$ & $\begin{array}{l}16.00 \\
16.00\end{array}$ & $\begin{array}{l}42.85 \\
44.03\end{array}$ \\
\hline $\begin{array}{l}\text { Superlayer II } \\
\text { Inner chamber } \\
\text { Outer chamber }\end{array}$ & $\begin{array}{l}20.00 \\
20.00\end{array}$ & $\begin{array}{l}55.19 \\
56.36\end{array}$ \\
\hline $\begin{array}{l}\text { Superlayer III } \\
\text { Inner chamber } \\
\text { Outer chamber }\end{array}$ & $\begin{array}{l}22.75 \\
22.75\end{array}$ & $\begin{array}{l}66.77 \\
67.94\end{array}$ \\
\hline $\begin{array}{l}\text { Superlayer IV } \\
\text { Inner Chamber } \\
\text { Outer Chamber }\end{array}$ & $\begin{array}{l}26.50 \\
26.50\end{array}$ & $\begin{array}{l}79.10 \\
80.27\end{array}$ \\
\hline
\end{tabular}


Table 6-12. GEM IPC locations (middle of anode wire plane). (Cont.)

\begin{tabular}{lcc}
\hline & $\begin{array}{c}\text { Effective } \\
\text { Width } \\
(\mathrm{cm})\end{array}$ & $\begin{array}{c}\text { Distance to } \\
\text { Interaction Point } \\
(\mathrm{cm}) \\
\text { z for for barrel; }\end{array}$ \\
Superiayer Number & 113.10 \\
\hline $\begin{array}{c}\text { Endcap Superayer } \\
\text { Inner Chamber } \\
\text { Outer Chamber }\end{array}$ & 114.20 \\
$\begin{array}{l}\text { Endcap Superlayer II } \\
\text { Inner chamber }\end{array}$ & 131.20 \\
$\begin{array}{l}\text { Outer chamber } \\
\text { Endcap Superlayer III } \\
\text { Inner chamber }\end{array}$ & 132.30 \\
$\begin{array}{c}\text { Outer chamber } \\
\text { Endcap Superlayer IV }\end{array}$ & 149.30 \\
Inner chamber & 150.40 \\
Outer chamber & 167.40 \\
\hline
\end{tabular}

NOTE: Endcap chambers overlap; add approx. 3 $\mathrm{Cm}$ to $z$ for second array.

\section{IPC Tracker Design Constraints}

The GEM IPC tracker is designed to operate in a high-radiation (1 Mrad/yr), high magnetic field $(0.8 \mathrm{~T})$ environment for an extended period of time with maximum dimensional stability. In addition, the radiation length must be minimized. Table 6-13 shows the proposed stability requirements for the IPC tracker.

Table 6-13. GEM IPC array stability requirements.

\begin{tabular}{ll}
\hline Parameter & Quantity \\
\hline $\begin{array}{l}\text { Between superlayer modules } \\
\text { in } \phi\end{array}$ & $25 \mu \mathrm{m}$ \\
$\begin{array}{l}\text { Between superlayer modules } \\
\text { in } Z\end{array}$ & $100 \mu \mathrm{m}$ \\
$\begin{array}{l}\text { Between endcaps and barrel } \\
\text { in } \phi\end{array}$ & $25 \mu \mathrm{m}$ \\
$\begin{array}{l}\text { Between endcaps and barrel } \\
\text { in Z }\end{array}$ & $100 \mu \mathrm{m}$ \\
$\begin{array}{l}\text { Module transverse flatness } \\
\text {-or- }\end{array}$ & $\begin{array}{c}160 \mu \mathrm{m} \\
20 \mu \mathrm{m} \text { over adjacent } \\
\text { pads }\end{array}$ \\
\hline
\end{tabular}

\section{Description of IPC Tracker Arrays}

The GEM IPC tracker array consists of a total of 80 barrel and 80 endcap superlayer modules. Each module consists of two interpolating pad chambers. The barrel superlayers are rotated 4.5 per superlayer to reduce concentrations of higher radiation length material at specific azimuthal angles. The endcap chambers are rotated $9^{\circ}$ per level for the same reason. The endcap IPC modules are trapezoidal in shape and are arranged as shown in Fig- ure 6-56. There are four levels of superlayers for both the barrel and endcap regions.

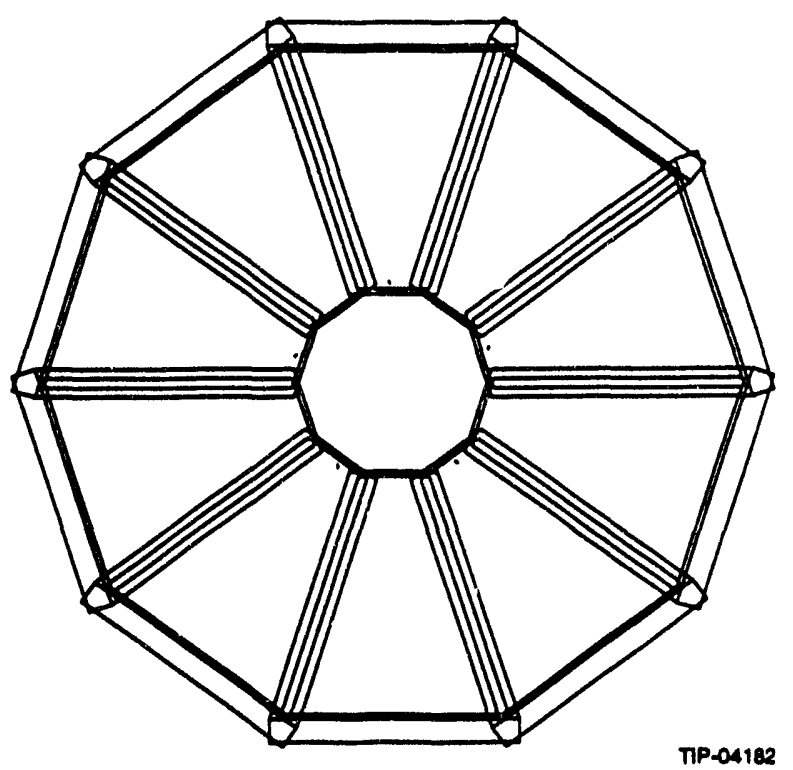

FIG. 6-56. IPC tracker array, frontal view of endcap.

\section{IPC Module Design}

The GEM interpolating pad chamber module is designed to minimize radiation length while maximizing mechanical stability. Composite materials are used throughout to achieve this end.

Figure 6-57 shows a typical barrel module cross-sectional view. The module consists of the following components for each of the two chambers: gas envelope, gas envelope supports, outer cathode plane, anode wires, anode wire supports, the interpolating cathode pad printed circuit board, gas plenums and connectors for utilities. In addition, a honeycomb core between the cathode pad boards adds rigidity.

The "on-board" electronics are arranged on either side of a rectangular cross-sectioned graphitecomposite cooling tube and are designed to be mechanically and thermally isolated from the pad chambers. Flexible Kapton printed circuits provide the connection between the cathode pads and the electronics. The cooling for the electronics is described below.

The anode wires (20 to $40 \mu \mathrm{m} \phi$ gold-plated thingsten) may operate at a pote itial of $3 \mathrm{kV}$. The distance between the anode wires and cathode planes is $2 \mathbf{~ m m}$, so the anode wire supports must be good insulators. The tracker group has explored 
many material combinations, including graphite/ epoxy, Kevlar (aramid)/epoxy, and G10.

The most satisfactory material to date is a quartz fiber/cyanate ester composite. Quartz fiber has a dielectric constant of 3.7 , a coefficient of thermal expansion (CTE) of 3-4 $\mathrm{PPM} /{ }^{\circ} \mathrm{C}$, and negligible moisture absorption. Cyanate ester resin has superior radiation-hardness properties and lower moisture absorption when compared to most epoxy resins. The composite may be readily machined, and there is a precedent for using this material as a printed circuit board (PCB) substrate. The cathode pad PCB, anode wire supports and cathode plane supports will all be constructed from this material.

The cathode pad PCB will utilize the quartz/ cyanate ester discussed previously as a substrate. The pads and traces will be fabricated by depositing copper onto the quartz composite surfaces. Discussions with printed circuit fabricators have revealed that conductor thicknesses of as little as $125 \times 10^{-6}$ in. $\left(318 \times 10^{-6} \mathrm{~cm}\right)$ are possible using this technique. The substrate thickness is limited to a minimum of $0.5 \mathrm{~mm}$ due to capacitance concerns.

The gas envelope has been included in order to contain the chamber gas mixture $\left(\mathrm{CO}_{2}\right.$ and $\left.\mathrm{CF}_{4}\right)$ while preventing distortion of the outer cathode plane due to gas pressure. The cathode plane will be perforated, allowing gas into the cavity between the cathode and the gas envelope. The gas envelope will be constructed from 0.15-mm thick Mylar.

The cathode plane will be a metalized Mylar or Kapton sheet of $0.15-\mathrm{mm}$ thickness. Both the gas envelope and the cathode plane are to be stretched prior to installation. The correct tension is currently being researched.
The modules are designed to be easily removable from the support structure. Therefore, all utilities for each module are accessible from one end. The gas mixture enters one end of the module, flows down the module length through one chamber and doubles back through the other chamber. The gas plenums direct this flow. At this printing, design of the plenums has begun and initial prototypes have been constructed.

Consideration of chamber mechanical stability applies to dynamic as well as to static loading. Preliminary studies indicate that vibrations between 0 and $30 \mathrm{~Hz}$ and between 600 and $1200 \mathrm{~Hz}$ could be introduced into the central tracker. Therefore, the IPC modules must be designed to have resonant frequencies above $45 \mathrm{~Hz}$. From studies performed by both Yale University and Los Alamos National Laboratory, a module has been designed that employs a honeycomb core thickness of $1.3 \mathrm{~cm}$ and uses P120 unidirectional graphite composite for the gas envelope support.

Subsequent to the design study, the IPC design team changed the anode wire support width from 1 to $2 \mathrm{~cm}$ in order to accommodate additional high "nltage circuitry. This change necessitated a new out of the IPC modules to achieve the required cnamber overlap. As a result, the module thickness was reduced from $2.69 \mathrm{~cm}$ overall to $1.82 \mathrm{~cm}$ $(0.63 \mathrm{~cm}$ honeycomb core). The first bending mode frequency is between 31 and $36 \mathrm{~Hz}$, depending on the width of the module. One method to increase the frequency is to couple pairs of modules via lowmass struts and a central support tube. This utilizes not only the modules themselves but also the distance between superlayers. In addition, module installation is easier, since pairs of modules are

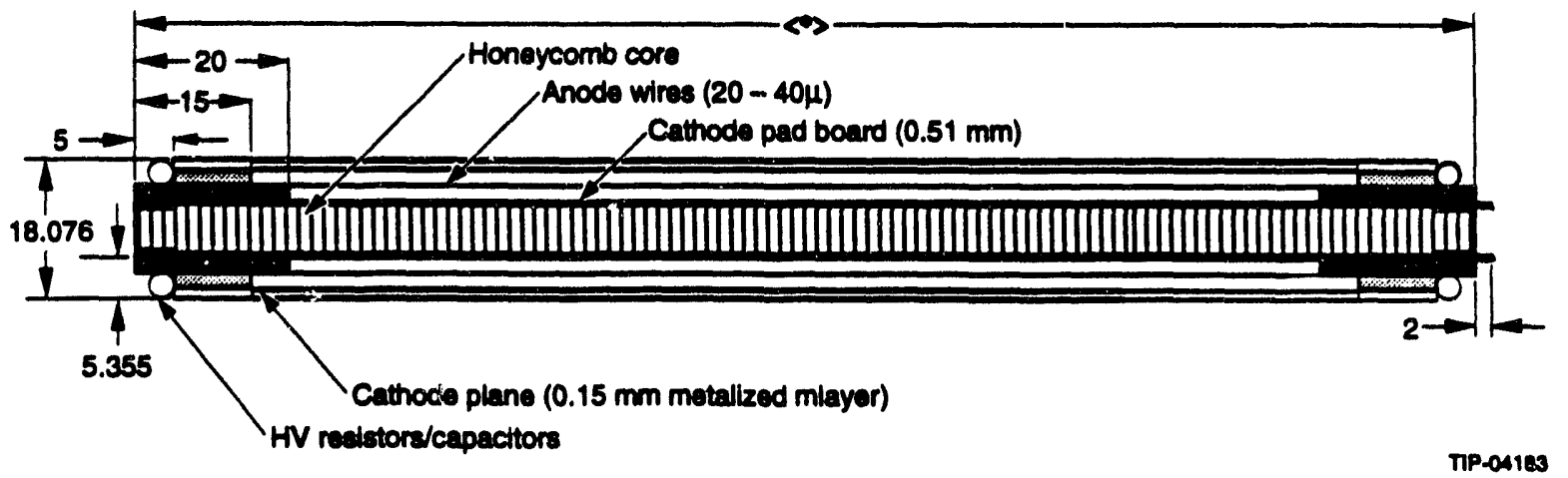

FIG. 6-57. Barrel module croses section. 
installed at one time. However, disadvantages include more complicated module assembly and greater amounts of material at specific locations. Preliminary finite element analyses show a first bending mode of approximately $56 \mathrm{~Hz}$. Figure 6-58 illustrates this concept.

\section{Radiation Length Budget}

One of the design constraints for the GEM IPC tracker array is minimizing radiation length. Table 6-14 shows the percentage of a radiction length from $\mathrm{r}=39 \mathrm{~cm}$ to $\mathrm{r}=88 \mathrm{~cm}$ in the barrel section. Note that the electronics and anode supports are averaged over $360^{\circ}$.

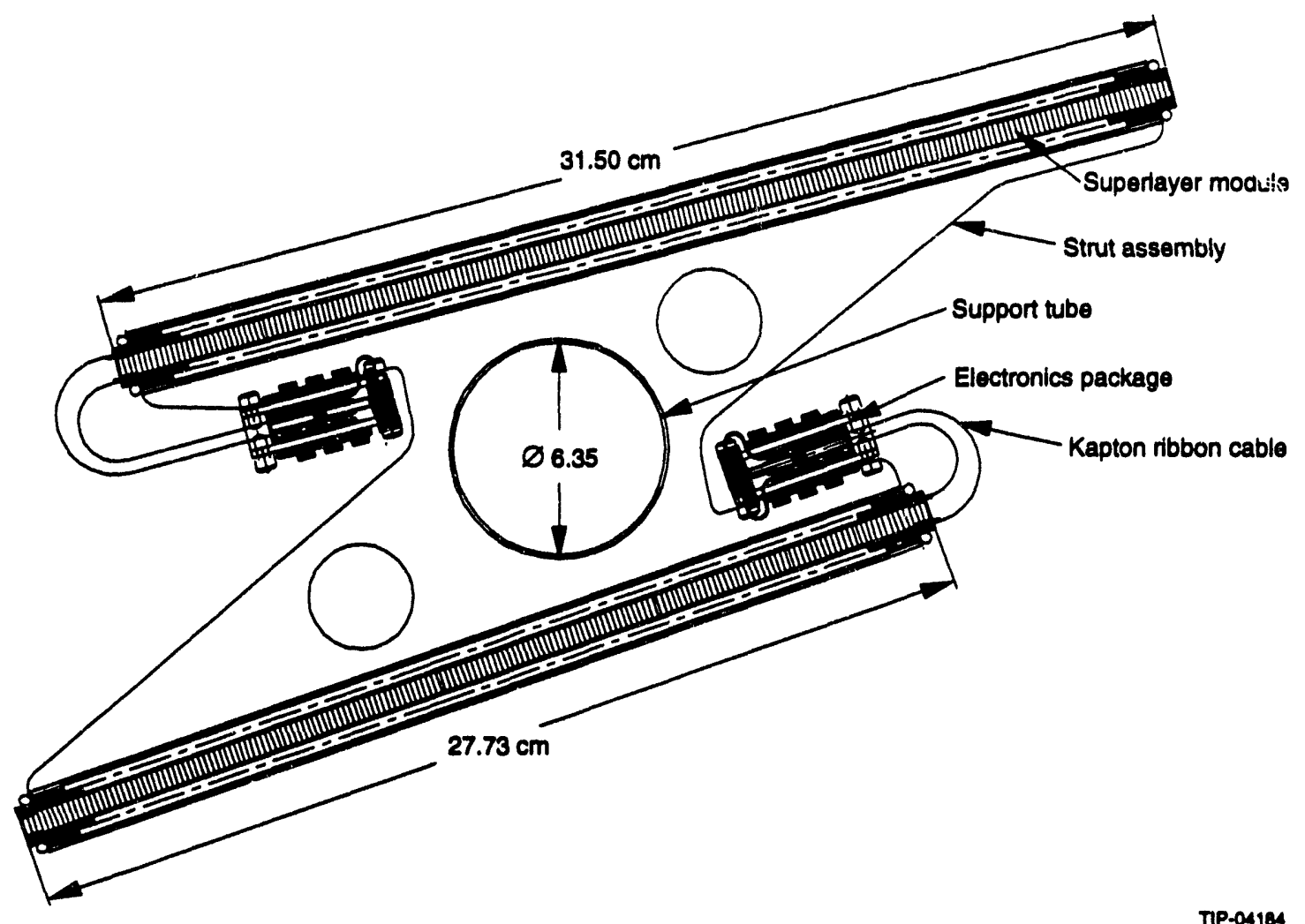

FIG. 6-58. Module doublet.

Table 6-14. IPC radiation length budget.

\begin{tabular}{|c|c|c|c|}
\hline Layer Matorial & Thickness (cm) & Lrad $(\mathrm{cm})$ & $\%$ Lrad \\
\hline $\begin{array}{l}\text { Hardback } \\
\text { Quartz/cyanate-ester (skin) } \\
\text { Copperpads/traces } \\
\text { Graphite/epoxy (honeycomb core) } \\
\text { Coppepads/traces } \\
\text { Quart/cyanato-ester (skin) }\end{array}$ & $\begin{array}{l}0.0508 \\
6.35 \times 10^{-4} \\
0.63(0.025) \\
6.35 \times 10^{-4} \\
0.0508\end{array}$ & $\begin{array}{r}19.40 \\
1.43 \\
25.00 \\
1.43 \\
19.40\end{array}$ & $\begin{array}{l}0.262 \\
0.044 \\
0.100 \\
0.044 \\
0.262\end{array}$ \\
\hline $\begin{array}{l}\text { Upper chamber } \\
\text { Copper cathode } \\
\text { Mylar substrate }\end{array}$ & $\begin{array}{l}3.17 \times 10^{-4} \\
0.00508\end{array}$ & $\begin{array}{r}1.43 \\
28.70\end{array}$ & $\begin{array}{l}0.022 \\
0.0177\end{array}$ \\
\hline $\begin{array}{l}\text { Lower chamber } \\
\text { Copper cathode } \\
\text { Mylar substrate }\end{array}$ & $\begin{array}{l}3.17 \times 10^{-4} \\
0.00508\end{array}$ & $\begin{array}{r}1.43 \\
28.70\end{array}$ & $\begin{array}{l}0.022 \\
0.0177\end{array}$ \\
\hline
\end{tabular}


Table 6-14. IPC radiation length budget. (Cont.)

\begin{tabular}{|c|c|c|c|}
\hline Layer Material & Thickness (cm) & Lrad (cm) & \%Lrad \\
\hline $\begin{array}{l}\text { Gas Envelope Windows } \\
\text { Upper chamber ( } 2 \text { mil mylar) } \\
\text { Lower chamber ( } 2 \text { mil mylar) }\end{array}$ & $\begin{array}{l}0.00508 \\
0.00508\end{array}$ & $\begin{array}{l}28.70 \\
28.70\end{array}$ & $\begin{array}{l}0.0177 \\
0.0177\end{array}$ \\
\hline $\begin{array}{l}\text { Subtotal per superlayer } \\
\% \text { due to hard back (structural) } \\
\% \text { due to conductor material } \\
\% \text { due to insulator material }\end{array}$ & & & $\begin{array}{l}0.8268 \\
75.50 \\
15.90 \\
8.60\end{array}$ \\
\hline Module sides (anode supports, etc.) & & & 5.05 \\
\hline Electronics section & & & 3.51 \\
\hline Module support tube & 0.20 & 25.00 & 0.81 \\
\hline $\begin{array}{l}\text { Average of module side effects } \\
\text { Supertayer I } \\
\text { Superlayer II } \\
\text { Superlayer III } \\
\text { Superlayer IV }\end{array}$ & & & $\begin{array}{l}0.72 \\
0.60 \\
0.52 \\
0.45\end{array}$ \\
\hline $\begin{array}{l}\text { Average of electronics } \\
\text { Superlayer I } \\
\text { Supertayer II } \\
\text { Superlayer III } \\
\text { Superlayer IV }\end{array}$ & & & $\begin{array}{l}1.17 \\
0.94 \\
0.78 \\
0.67\end{array}$ \\
\hline Average of module support tubes & & & 0.54 \\
\hline $\begin{array}{l}\text { Support cylinders } \\
\text { Inner cylinder } \\
\text { Outer cylinder }\end{array}$ & & & $\begin{array}{l}0.61 \\
0.61\end{array}$ \\
\hline $\begin{array}{l}\text { Grand Total } \\
\text {-or at } 14 \text { degrees Lorentz angle }\end{array}$ & & & $\begin{array}{l}10.91 \\
11.18\end{array}$ \\
\hline
\end{tabular}

\section{Outer Support Structure}

The outer support structure (OSS) is the primary structural element that is used to support all of the detector elemer +s. The OSS is a graphite/ cynate ester composite, composed of two annular disks at $Z= \pm 100 \mathrm{~cm}$ and two twenty sided polygons which enclose both the inner and outer diameters of the IPC barrel section, as is shown in Figure 6-59. The outer support structure employs what is commonly referred to as a "skin-and-stringer" technique, which is an extremely efficient structure.

The annular disks serve as supports for the silicon tracker and the IPC assemblies and are connected via kinematic mounts to the barrel calorimeter structure. In addition to enhancing mechanical stability, the honeycomb cylinders permit an enclosed volume. The volume serves two purposes: containment in the event of a module gas window rupture and a controlled (temperature and humidity) atmosphere to improve module stability. An inert gas will be circulated at a low rate of flow through the volume to maintain temperature and relative humidity set points.
The end view shown in Figure 6-60 shows one of the annular disks. The module sectors ( 4 modules per sector, 20 sectors) are installed alternately at either end. As discussed previously, the barrel modules are installed in pairs or "doublets." This allows for sector overlap while leaving enough material on each support disk for structural continuity.

\section{Endcap IPC Support Structure}

The individual endcap IPC modules present a diminished structural stability challenge when compared to the barrel section. The modules are a factor of three shorter $(<65 \mathrm{~cm})$ than their barrel counterparts, and gravity acts within the module plane.

The current endcap support structure concept utilizes the gas enclosure as the structural element. The gas enclosure consists of an outer cylinder, an inner cylinder and two endcap annular disks. Each superlayer of 10 modules is attached to a graphite/ epoxy honeycomb reinforced disk, with each module mounted on alternating sides of the disk to permit 


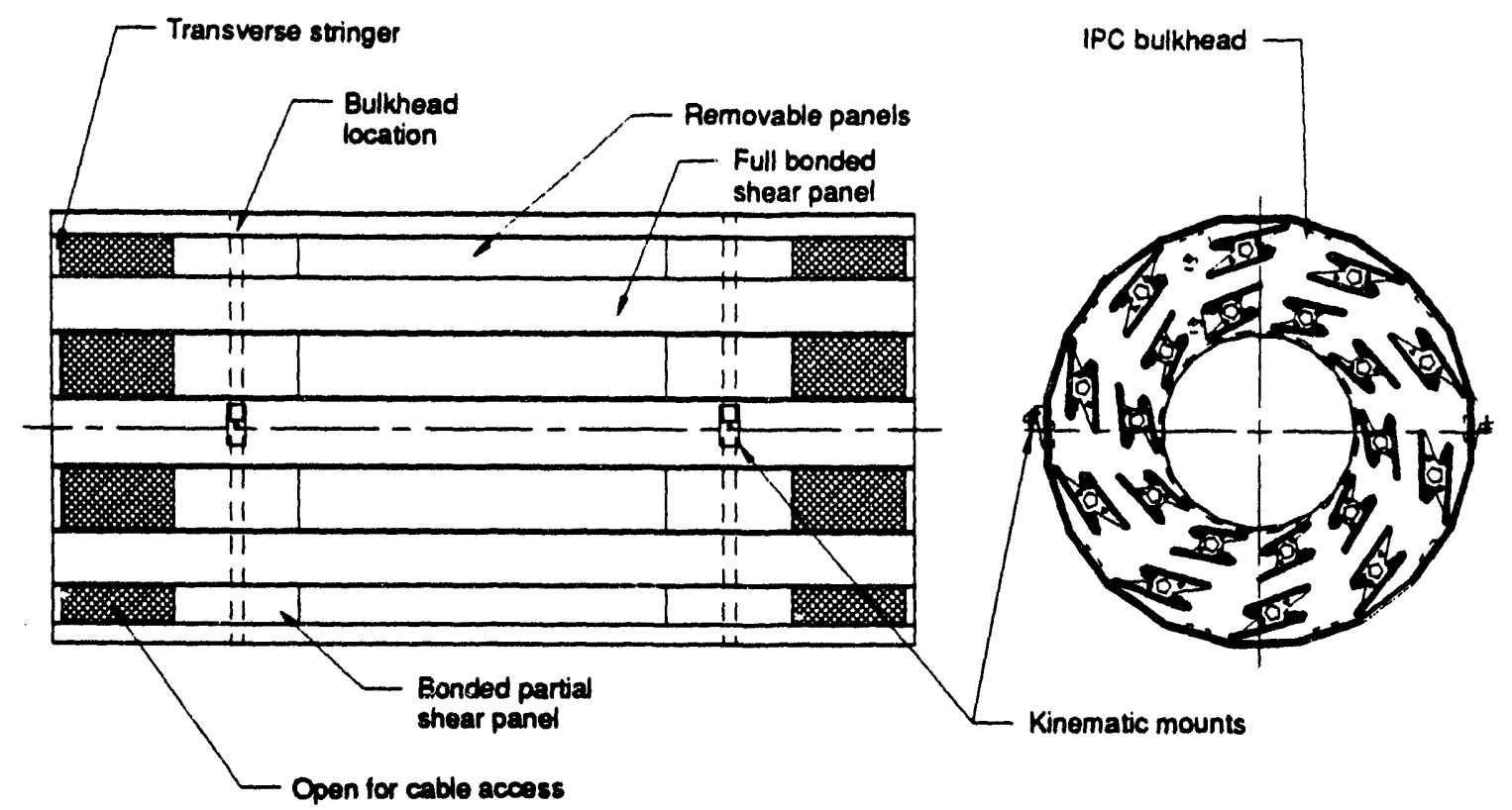

FIG. 6-59. IPC tracker array, barrel assel ibly.

overlap. Each of four disks is suspended kinematically within the support cylinder. The entire subassemblies are then inserted into the outer support structure and fixed in place. Removal of an individual module could be accomplished in one of two ways: in-situ remove the outer endplate, remove disks until the faulty chamber is reached, and remove chamber; or up-hole remove cntire endcap assembly and transport to clean area, remove endplate closest to faulty chamber, remove disks as required, and replace modulc.

Figure 6-60 illustrates the endcap IPC support concept. Structural analyses and some prototype testing will be completed in the near future to validate this design.

\section{IPC Detector Gas System}

The IPC ionization gas system has been designed to be reliable and cost-effective and to meet stringent performance requirements. The requirements include providing remote control and monitoring of a mixture of $\mathrm{CF}_{4}-\mathrm{CO}_{2}$ with $1 / 4$ gas change per minule in the IPCs. The required flow rate is about $80 \mathrm{~V} / \mathrm{min}$ through 4-mm ionization spaces and $1 \mathrm{~mm}$ gas windows (on the top and bottom of each IPC). The flow is contained by $0.15-\mathrm{mm}$ thick mylar films. Less than 5-Torr pressure difference from atmospheric pressure across a mylar window is required to prevent bursting the windows. Since

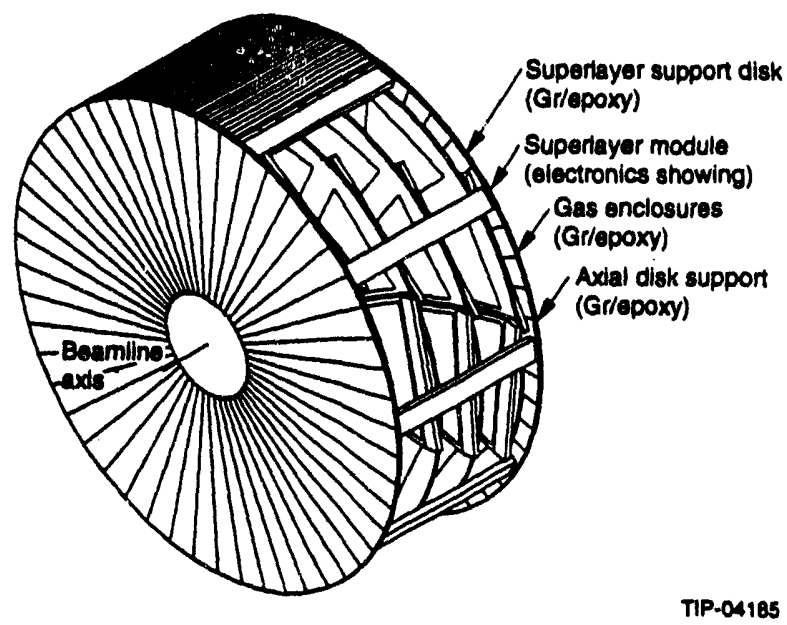

FIG. 6-60. IPC tracker array, endcap assembly.

detector operation is somewhat sensitive to absolute pressure, constant pressure will be regulated in the IPCs. Gas flow will be measured and gas composition will be monitored to $1 \%$ accuracy for the major constituents and to several ppm for trace contaminants. The ionization gas system will include a gas scrubbing subsystem that will filter out water vapor, $\mathrm{O}_{2}$, and total hydrocarbons to low ppm levels. The contamination levels will be continuously measured using gas analyzers. Radiation lengths for the system will be minimized by selection of low-Z materials where possible. The system will account for luminosity induced heating of the ionization gas. 
Because of the relatively large gas flow rate and the high cost of $\mathrm{CF}_{4}$, a recirculating gas system has been selected. The system consists of supply cylinders of high-purity $\mathrm{CF}_{4}$ and $\mathrm{CO}_{2}$ and a mixing pressure tank where the source gases will be mixed by partial pressures prior to charging the system. The driving potential for the flow will be provided by sealed metal bellows pumps, which will impart a $1 \mathrm{~atm}$ pressure rise. The flow will be metered, controlled, scrubbed, and analyzed prior to its distribution to the IPCs. Flow distribution will take place from a manifold located in the annular space between the endcap and barrel calorimeters via $1.59-\mathrm{mm}$ stainless steel capillary tubes. These capillaries are designed to return the pressure to atmospheric over a length of about $2 \mathrm{~m}$, which is sufficient to reach the IPC inlet connections from the manifolds. The large equal impedance of each of the inlet capillaries will equalize the flow to each of the IPCs. The gas flow will be cooled by a liquid-gas heat exchanger to take out adiabatic compression heating because of pumping, as well as ionization heating of the gas in the IPCs. Most of the IPC ionization gas system hardware will be located at surface level in the gas mixing building and, therefore, will be easily maintained.

\section{IPC Detector Electronics Cooling System}

The IPC electronics cooling system has been designed to remove the $25-\mathrm{kW}$ total electronics heat load while fulfilling the additional requirements of dimensional and positional stability, low vibration, low risk of water leakage, and low radiation length. The baseline cooling concept selected is a "leakless" chilled-water cooling system. The system consists of two unconnected loops, one containing high-purity de-ionized water for cooling the electronics, and one containing house-mixed chilled water.

The electronics cooling loop will be a leakless cooling system consisting of redundant vacuum pumps to pull water up into the systern and into a level controlled reservoir to a vacuum pressure of from 400 to 600 mbar (which corresponds to working liquid heights of from $6 \mathrm{~m}$ to $4 \mathrm{~m}$, respectively). Thus, if any leaks occur, they will admit only $\mathrm{CO}_{2}$ into the cooling loop and not release water, as long as the vacuum pumps are working. Redundant water pumps will be used to circulate water in the electronics cooling loop.
The house-water loop will direct hous!: water through a $25-\mathrm{kW}$ plus refrigeration unit that will cool the house-water loop. This loop flow will pass through a heat exchanger coupled to the electronics cooling loop, which will serve to remove the IPC heat load.

Most of the IPC electronics cooling system will be located in the mechanical room that is to be located in the GEM utilities shaft. Total water flow to the IPC tracker will be on the order of $400 \mathrm{Vmin}$, but the flow in the individual IPC cooling passages will be laminar with a temperature rise of about $5^{\circ} \mathrm{C}$ and a pressure drop of about $12 \mathrm{kPa}$.

\section{IPC Tracker Assembly and Installation}

The IPC tracker array is designed to be modular. Superlayer modules are to be assembled and tested mechanically and electrically at collaborating institutions. The support structure will also be fabricated off-site. All components and subassemblies are to arrive at the SSC site with final module-level electrical tests occurring within a specially designed facility. The barrel support structure will be assembled first, with individual barrel modules inserted as soon as possible. Barrel module position surveying could be accomplished at this point.

The assembled endcap modules can be installed in the IPC support structure once the surveying of the barrel has been completed. The primary disadvantage of doing so is the possibility of their becoming misaligued in transit. Because of the axial nature of the endcap module installation, assembly could also occur down-hole. Here the primary disadvantage would be the additional time needed down-hole.

\subsubsection{IPC Readout Electronics}

The readout electronics for the IPC system must satisfy three primary requirements. First, the signal-to-noise ratio must be maintained below $1 \%$ throughout the readout chain. Second, the IPCs must be read out in time to participate in a Level 2 trigger decision. Third, the front-end elicetronics must be rad-hard. Recent simulations of the radiation environment in the GEM central cavity indicate that the charged particle radiation dose is approximately 0.44 $(10 \mathrm{~cm} / \mathrm{r})^{2} \mathrm{Mrad} / \mathrm{SSC}$ yr. To allow several years of operation at $10^{34} \mathrm{~cm}^{-2} \mathrm{~s}^{-1}$, IPC electronics at a radius 
of $25 \mathrm{~cm}$ must survive a radiation dose of $2 \mathrm{Mrad}$ while maintaining the noise specification above. In this section the basic IPC readout approach is described. Further details can be found in Chapter 7 , Electronics.

The basic approach to the readout of the IPCs is shown in Figure 6-61. The shaped output of each pad signal is sampled every $16 \mathrm{~ns}$. Upon receipt of a Level I trigger, four samples are output for each hit pad. The first sample is used to determine the baseline for the signal on that pad and to eliminate pileup. The next three samples provide three independent measurements of the charge centroid. Multiple measurements are desirable due to the time jitter of the signal with respect to the beam clock, caused by electron transit time variations. It is important to note, however, that this time jitter does not affect the temporal relationship between adjacent pad pulses arising from the same avalanche, and does not, therefore, affect the charge centroid measurement obtained using samples acquired at the same time on all pads. Results of a full simulation of the IPC readout, which include the affects of channel-to-channel shaping-time differences, clock slewing, etc., will be presented at the end of this section.

The full IPC readout architecture is shown in Figure 6-62. (Also see Chapter 7, Electronics.) The readout electronics consists of five functional blocks. The first is the preamp/shaper, which in the current design would be packaged eight channels per die. The second is a $16 \times 128$ analog memory, which stores the samples during the Level 1 trigger latency period, and buffers samples during the readout to Level 2. The third element in the readout chain is a

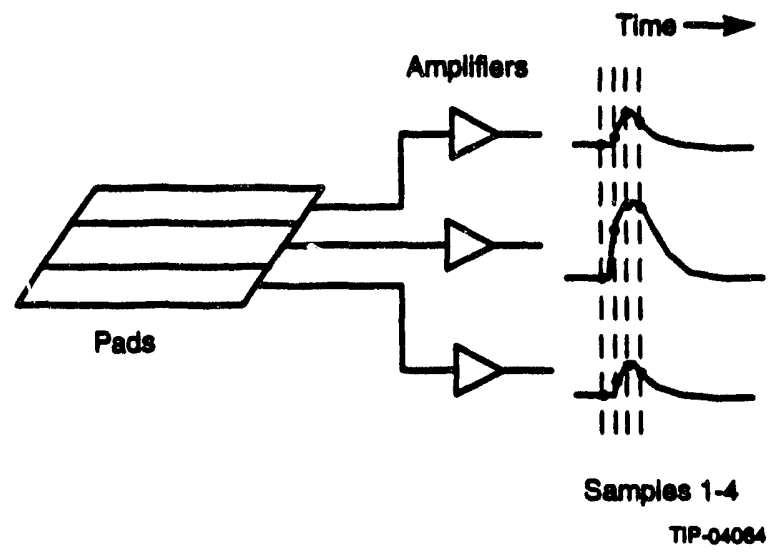

FIG. 6-61. IPC readout approach.
16 to 1 analog multiplexer, which will be included on the same die as the analog memory. The memory/ MUX unit is followed by a $15-\mathrm{MHz}$ FADC, which digitizes the samples to eight bits, with an effective 9-10 bits of dynamic range obtained through the use of a center-tapped reference ladder. The final element in the readout chain is the controller, which services $128 \mathrm{pad}$ channels. It consists of address drivers for the analog memory, a state machine to control the readout sequence, a zero-suppression circuit, and a buffered frame builder that appends a beam crossing tag to the zero-suppressed data. These frames are output to the DAQ through a $50-\mathrm{MHz}$ LED/fiber-optic link.

The current design for the zero-suppression logic uses a comparator, which scans the digitized pad signal samples as they are generated. If a sample exceeds a preset value, then that sample, along with the corresponding sample from the previous and two subsequent pads are kept, as shown in Figure 6-63. This ensures that all samples required to reconstruct a position are available in the output stream. This operation is performed on the sample nearest the pulse peak, which is digitized first in the readout sequence. The location of the pads that were not zero-suppressed on this sample are stored, and the remainder of the 4 samples for these pads are transmitted regardless of sample amplitude.

A full simulation of the IPC readout electronics has been performed, using GARFIELD to generate current wave forms for random trajectories through the chamber. Landau fluctuations and electron diffusion are included in the simulation. These wave forms are then convolved with the transfer function of the preamplifier and shaping amplifier to obtain shaped anode signals. The cathode pad signals are generated by first selecting a random position across a pad. This position is used to determine the correct relationship between the signal on this pad and those on its nearest neighbors. The anode signal is then divided among the three pads according to these relationships, with an additional attenuation factor of two on each pad signal due to charge sharing with the other cathode plane. These cathode signals are then sampled at 16-ns intervals. The samples are subjected to smearing due to amplifier noise, clock slew, and shaping-time differences between the three pads. Clock slew is simulated by adding a random component to the times at which the samples are 


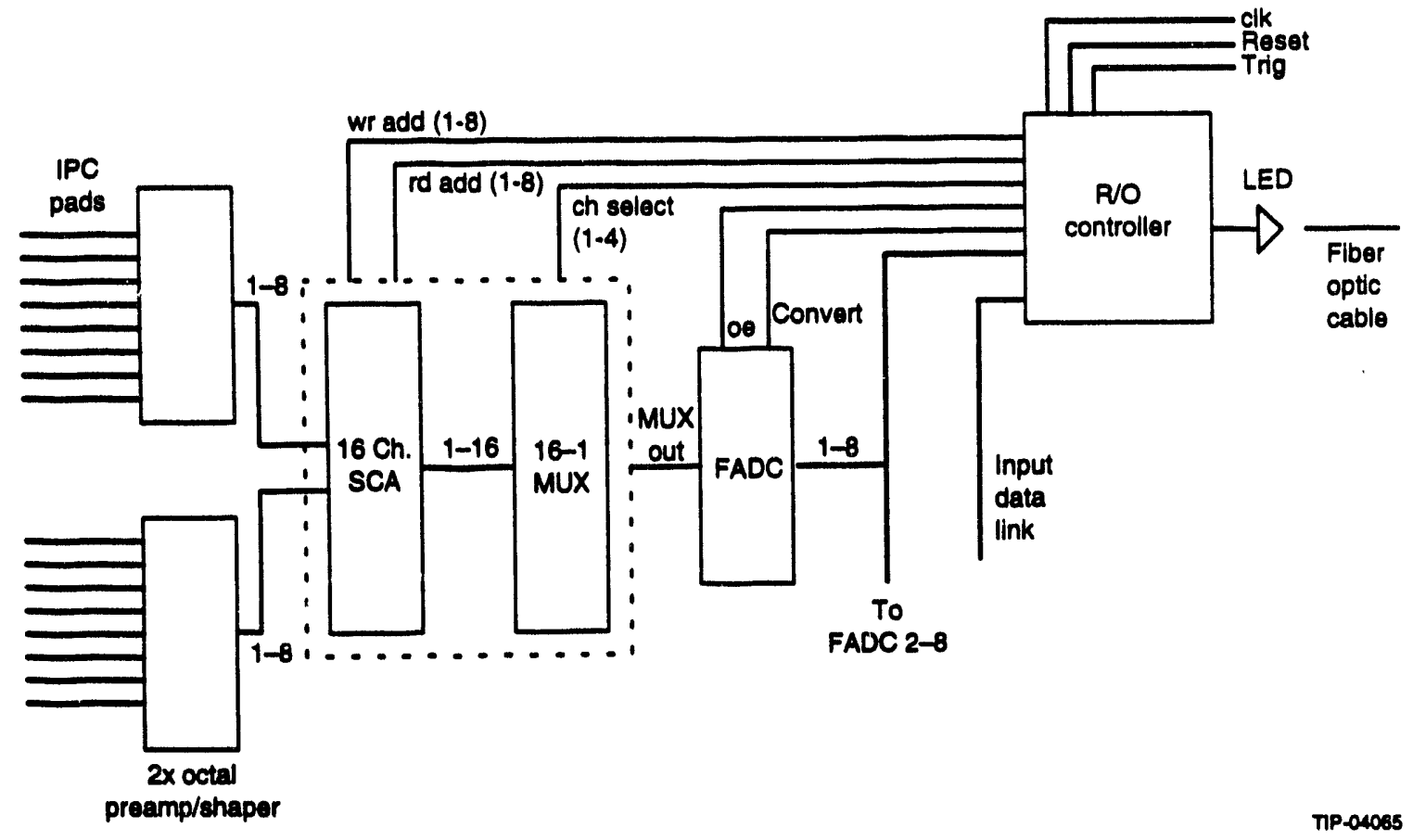

FIG. 6-62. IPC readout architecture.

acquired. This results in an error in the positions determined from the sample sets, which is greatest for samples acquired on the leading or trailing edge of the pulse. shaping-time variations are modeled by varying the amplifier transfer function for each of the three pads on every event, so that each has the same nominal shaping-time, but actual shaping-times differ over a set range. Amplifier noise is simulated by adding a random component of noise to each sample. After these operations are performed on the samples, the digitization is simulated using a set number of effective bits, and the resulting digital values are used to determine the position. This reconstructed position is then compared with the true position to evaluate the effects of the various smearing processes. Some results from this simulation are presented here. In Figure 6-64, the relationship between position resolution and noise is pres-

Digitized pad saniples

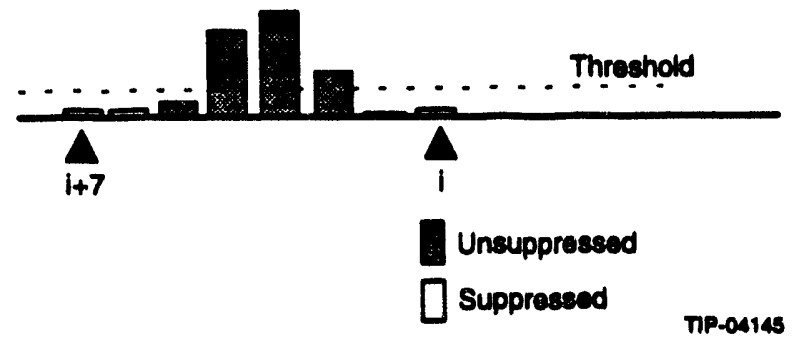

FIG. 6-63. Zero suppression.

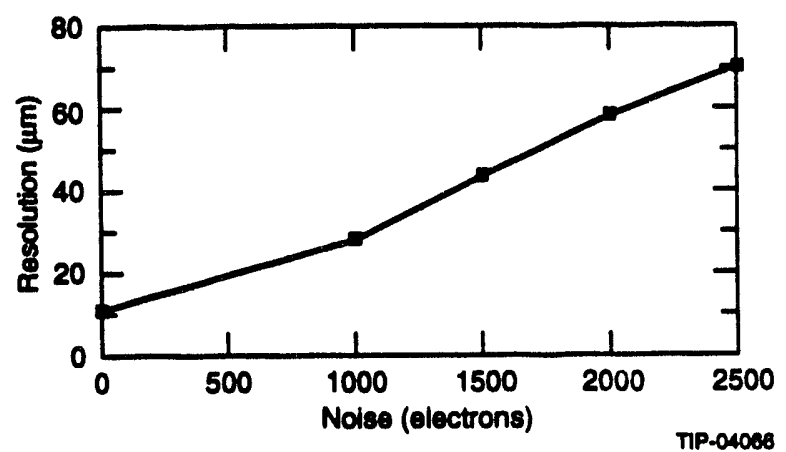

FIG. 6-64. Position resolution vs. noise.

ented. The vertical scale is in microns. Figure 6-65, presents the dependence of the resolution of the position derived from the sample closest to the signal peak on jitter in the sampling time. Figure 6-66, shows the dependence of the position resolution, again derived using the peak sample, on channel to channel shaping-time variations.

\subsection{INTEGRATION INTO CEM}

\subsubsection{Installation and Alignment}

The installation of the central tracker into the barrel calorimeter will be a critical operation requiring careful planning and control. During the calibration and beam testing, the central tracker will have been surveyed to high precision. This alignment must be preserved as much as possible. The central 


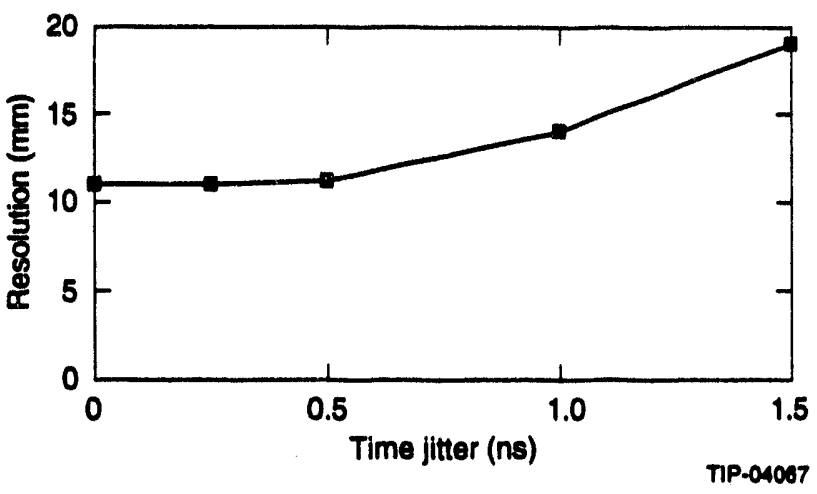

FIG. 6-65. Position resolution vs time jitter.

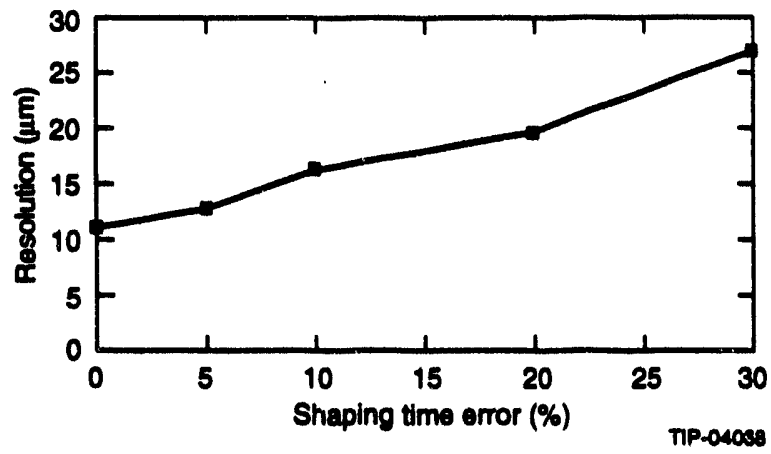

FIG. 6-66. Position resolution vs. shaping-time variations.

tracker is not a robust structure. The requirement of low radiation length has resulted in the design of a delicate device that should be installed in GEM as late as possible.

An installation procedure will first be developed, then rehearsed with a mock structure. This structure will be the same physical size and weight, will contain the same mounting and installation hardware, and will have optical targets simulating the center line and external targets. The entire instalıation sequence will be stepped through, followed by optical alignment of the center line to the reference beam line.

Since the location of the reference beam line with respect to the actual beam line is uncertain to several millimeters, the central tracker will not require precise placement. Placement within $250-500 \mu \mathrm{m}$ of the reference beam line will be adequate. The external targets on the mock structure will then be measured and referenced to similar targets on the end of the barrel calorimeter.

During the installation and removal of the mock structure, instrumentation will measure the vibration levels that the central tracker would experience. This monitoring would begin during handling at the assembly facility and continue throughout the air ride transport, off-loading onto the elevator or crane, down hole lowering, transferring to the hall crane, and installation into the calorimeter. Rough operations that could potentially disturb the tracker precision will be identified and corrected prior to final installation. The effectiveness of the shock and vibration isolation platform will also be evaluated.

During the actual installation, the IPC barrel modules and silicon tracker will be mounted in the support structure and installed as one unit. After the assembly is transferred to its mounts, the ends will be optically mapped with the same procedure used on the mock structure. The external alignment fiducials will be mapped relative to the reference beam line and relative to the barrel calorimeter. Any necessary adjustments will be made at this point. The IPC endcaps will then be installed. They will also contain external alignment fiducials and will be optically mapped. This series of optical alignment measurements will define the locations of the silicon tracker, IPC barrel and endcaps relative to the reference beam line, as well as their positior. relative to the barrel calorimeter.

Some final alignment checks are required after the services and beam pipe have been connected to verify that no external strain has repositioned the tracker. Additional optical monitoring will also take place as the silicnn tracker is cooled down, the calorimeter vacum, s applied, and the IPC cooling and gas systems sme operational. An internal alignment monitc of system will continually monitor the relative positions of each major subassembly. This information will serve as input to the ultimate determination of the alignment of the central tracker using particle tracks, as discussed in Section 6.2.4.

\subsubsection{Services and Connections}

Services to the central tracker include: power cabling, fiber-optic readout, fast and slow control cabling, high voltage lines, coolant lines, gas lines, and safety systems lines. These service lines are supplied to both the silicon and IPC trackers and must be packaged internally in the central tracker assembly and routed out through the interface between the barrel and endcap calorimeter cryostats. Adequate clearances need $w$ be 
designed for cable routing inside the central tracker, through the calorimeter and for locations where cable connectors and piping hookups are placed. The central tracker will be installed with only short service leads on each end and then connected to lines that will have been installed on the ends of the barrel calorimeter. Table 6-15 is a summary of the silicon and IPC tracker cabling.

The first systems to be connected will be the gas and cooling systems. The butane coolant supply and return lines will be hooked up, leak checked, and then sealed in the nitrogen gas enclosures. The nitrogen supply lines and other safety lines will be attached to complete the silicon cooling system. At the same time the IPC cooling and gas system lines will be connected and leak checked. Safety system tests will be performed and verified as acceptable before any external electrical systems are hooked up. Butane flow tests may be done at this time, but no butane will be in the tracker while external hookups are being performed.

Following the gas and cooling systems testing, the electrical power systems will be connected and tested for continuity. Slow controls, fiber-optic cables and other sensors readouts will then complete the service connections. When all safety checks have been completed, the central tracker electronics can be powered and verified.

\subsubsection{Access}

Access to all parts of the GEM detector is discussed in detail in Chapter 10, Detector Operations, Access and Maintenance. The central tracker is the innermost element of this large and highly

Table 6-15. Senvices summary.

\begin{tabular}{|c|c|c|c|c|}
\hline Tracking System & Service & Size $(\mathrm{cm})$ & Quantity & Area $\left(\mathrm{cm}^{2}\right)$ \\
\hline \multirow[t]{11}{*}{ Silicon tracker } & Power & $1 \times 0.43$ & 194 & 280 \\
\hline & Fiber-optic readout & $500 \mu \mathrm{m} \varnothing$ & 3892 & \\
\hline & Fiber-optic timing & $500 \mu \mathrm{m} \varnothing$ & 500 & 61 \\
\hline & Internal alignment & $1 \mathrm{~mm} \varnothing$ & 220 & 9 \\
\hline & Liquid butane & 0.250 in. $\varnothing$ & 16 & \\
\hline & Gas butane & 0.5 in. $\varnothing$ & 16 & \\
\hline & Nitrogen enclosure & $2 \times 8$ & 4 & 62 \\
\hline & Nitrogen supply & 0.750 in. $\varnothing$ & 2 & \\
\hline & Nitrogen retum & 0.750 in. $\varnothing$ & 2 & \\
\hline & Butane vent & 0.750 in. $\varnothing$ & 2 & \\
\hline & Butane purge & 0.750 in. $\varnothing$ & 2 & 32 \\
\hline \multirow[t]{9}{*}{ IPC tracker } & Power & $0.5 \times 3$ & 160 & 800 \\
\hline & Fiber-optic readout & $500 \mu \mathrm{m} \varnothing$ & 4800 & \\
\hline & Fiber-optic timing & $500 \mu \mathrm{m} \varnothing$ & 960 & 50 \\
\hline & Anode hv & $0.3 \varnothing$ & 160 & 30 \\
\hline & Water supply & $2 \varnothing$ & 8 & \\
\hline & Water retum & 20 & 8 & 128 \\
\hline & Gas supply & $2 \varnothing$ & 2 & \\
\hline & Gas retum & 20 & 2 & \\
\hline & Sensors & $2 \times 2.5$ & (many) & 34 \\
\hline Total routing area & & & & 1486 \\
\hline
\end{tabular}


integrated experiment and is therefore the least accessible. Even when the entire detector is open and access to the tracker is optimal, the tight integration of the electronics and mechani:al structure inside the assembly makes repairs in situ very difficult. Any major repairs to the central tracker will require its removal to the surface facility and can therefore be considered only during a lengthy shutdown. It is expected that the silicon tracker will require replacement when the integrated fluence of hadrons exceeds $10^{14}$ charged particles $/ \mathrm{cm}^{2}$ on the first layer of silicon.

\subsubsection{Surface Facilities}

The central tracker will require a dedicated facility on site to complete the initial detector assembly and to conduct maintenance operations throughout its expected $10 \mathrm{yr}$ lifetime. The facility will contain two $10 \mathrm{~m} \times 10 \mathrm{~m}$ class 10000 clean rooms for parallel assembly operations of the silicon tracker and interpolating pad chamber system. A highbay, $2 \mathrm{~m} \times 20 \mathrm{~m}$, with a $4.5-\mathrm{Mg}$ crane covering the entire floor area, will be used to combine the two subsystems and to perform systems tests and calibration. Within the highbay will be an $x$ ray calisration lab lined with personnel shielding blocks, an electronics power supply area with appropriate safety features, and a utility area that will house the silicon and IPC cooling and gas systems. A storage room of about $150 \mathrm{~m}^{2}$ is needed to house components at various stages of assembly. The entire assembly facility will require thermal and humidity control. The total space needed is approximately $750 \mathrm{~m}^{2}$, and the facility should be available in 1997. A more extensive description of the facility can be found in Chapter 9, Facilities, Assembly and Installation.

\subsubsection{Safety and Environmental Impact}

The central tracker presents a concentration of various safety concerns in its compact volume. In addition to the butane used to cool the electronics of the silicon tracker, there are high and low voltage, heat producing electronics, beryllium in the beam pipe, and the proximity of the vacuum, all within centimeters of the enormous power stored in the two proton beams of the SSC.

The central tracker engineering team has provided full details of all tracker systems to the GEM safety coordinator to assist in the development of a complete Conceptual Safety Analysis Review (CSAR) for the entire GEM detector. The results of this review, including the central tracker, are detailed in Chapter 16, Environmental, Safety and Health Considerations.

\subsection{RADIATION ENVIRONMENT}

The lifetime of the central tracker depends on controlling the radiation environment in the central cavity. The radiation environment comes from three sources: charged particles from the interaction point (mostly hadrons), neutron albedo entering the central cavity from the calorimeters, and low-energy debris (gammas, photons, and protons) from the material in the central tracker.

\subsubsection{Radiation Dose Due to Charged Particles}

The major constraint that determines the inner radius of the central tracker is the flux of charged particles from the primary $P-P$ interactions. One also has to take into account secondary interactions and $\gamma$ conversions in the detector, as well as soft particles trapped in the magnetic field (loopers).

The flux of charged particles at a radius $R$ from the beam line $(R=r \sin \theta$, where $r$ is the distance from the interaction point) can be written as

$$
\text { Charged particle flux }=\frac{K L \sigma}{2 \pi R^{2}} \frac{d n}{d \eta} \text { particles } / \mathrm{cm}^{2}
$$

where $L$ is the integrated luminosity, $\sigma$ is the total $p p$ cross section, $d n d \eta \eta$ is the number of primary charged particles per unit of rapidity per interaction and, $K$ is the factor by which the flux is increased due to secondary interactions, $\gamma$ conversions, loop ers, etc., in the material of the tracker.

From Monte Carlo simulations of the GEM central tracker, $K$ is estimated to be around 1.5. For 1 yr of SSC running ( $10^{7}$ seconds) at $\mathcal{L}=$ $10^{33} \mathrm{~cm}^{-2} \mathrm{~s}^{-1}$ and a total $p p$ cross section of $\sigma=$ $10^{-25} \mathrm{~cm}^{2}$, and the expected value of $\frac{d n}{d \eta}-6.5$ charged particles per event, we calculate $\eta_{\text {at }} \mathbf{R}=$ $10 \mathrm{~cm}$ from the beam line a flux of charged particles of

$$
\begin{aligned}
& \text { Charged particle flux }= \\
& 1.5 \times 10^{13} \text { particles } / \mathrm{cm}^{2} / \mathrm{yr} \text {. }
\end{aligned}
$$

We can then write the radiation dose corresponding to a flux of charged particles as 


$$
\begin{gathered}
\text { Dose }(\text { Mrad })= \\
\frac{\text { charged particles } / \mathrm{cm}^{2}}{6.24 \times 10^{13}} \times \frac{d E}{d x},
\end{gathered}
$$

where $\frac{d E}{d x}$ is the energy loss of charged particles in a given material in units of $\mathrm{MeV} / \mathrm{g} / \mathrm{cm}^{2}$.

In silicon, the energy deposited by a minimum ionizing particle is

$$
\frac{d E}{d x}=1.66 \mathrm{MeV} / \mathrm{g} / \mathrm{cm}^{2} ;
$$

thus the radiation dose in silicon can be written as

$$
\begin{gathered}
\begin{array}{c}
\text { Dose }(\text { Mrad })= \\
\text { charged particles } / \mathrm{cm}^{2}
\end{array} \\
\frac{3.8 \times 10^{13}}{} .
\end{gathered}
$$

The charged particle flux calculated above $\left(1.5 \times 10^{13}\right.$ particles $/ \mathrm{cm}^{2} / \mathrm{yr}$ ) for $1 \mathrm{yr}$ of SSC of $L=$ $10^{33}$ at a radius of $R=10 \mathrm{~cm}$ from the beams then corresponds to a radiation dose of

Radiation dose $=0.4 \mathrm{Mrad} / \mathrm{yr}$ at $\mathrm{R}=10 \mathrm{~cm}$.

At a tolerable lifetime dose of $4 \mathrm{Mrad}$ for silicon this corresponds to a 10-yr lifetime for the inner layer of silicon detectors at a radius of $10 \mathrm{~cm}$ and a luminosity of $10^{33} \mathrm{~cm}^{-2} \mathrm{~s}^{-1}$.

In the vicinity of the IPC outer detector, the radiation dose is much smaller.

Radiation dose $=25 \mathrm{Krad} / \mathrm{year}$ at $\mathrm{R}=40 \mathrm{~cm}$.

We are designing the IPC electronics to tolerate a lifetime dose of $2.5 \mathrm{Mrad}$, which corresponds to a lifetime of $10 \mathrm{yr}$ at a luminosity of $10^{34} \mathrm{~cm}^{-2} \mathrm{~s}^{-1}$.

\subsubsection{Neutral Particle Backgrounds}

The central tracker volume will have a significant background from albedo neutrons generated in the surrounding environment. These will produce photons from $(n, \gamma)$ reactions, as well as charged particles ranging from recoiling hydrogen nuclei to a variety of light products from nuclear "eactions. This background will have three serious effects on the operation of the central tracker. First, charged products of .eutron and associated photon interactions will produce radiation damage in any component in the central tracker, not only in the active elements of the silicon and IPC detectors, but also in the electronics and passive support materials. Such damage may compromise the effective performance and lifetime of these devices. The actual dose expected may be accessed through simulated generation and tracking of individual reaction products, or through folding appropriate Kerma factors with the expected neutron energy spectrum. Secondly, the products of neutral-particle interactions may produce unwanted signals in the detectors that could interfere with the pattern recognition capabilities for determining acceptable tracks. Lastly, the neutron fluences have the potential to activate any component, especially those in close proximity to the interaction region. Since the central tracker is the closest set of detectors to the interaction point, access to this region could be seriously affected by unwanted activation. The goal of the simulations to date have been to address these three concerns.

\section{Neutral Particle Fluences and Dose Rates}

Previous work has shown that the central volume is effectively isolated from areas outside the calorimeter, hence changes made in the FCAL, muon chamber shielding, and placement and shielding of the focusing quadrupoles have no effect on the central tracker fluences. The behavior of the neutron fluences is most influenced by the placement and composition of the electromagnetic calorimeter sections, a matter dictated by physics considerations. To shield the central volume from lowenergy neutrons created in the calorimeter, a borated polyethylene shield lines the outer boundary of the central tracker walls. This shield acts to reduce the neutron energy to levels where it is then lost via the capture reaction on $\mathrm{B}^{10}$. In the present configuration an order of magnitude reduction in the neutron fluence in the central volume is achieved. One consequence of using the boron in the polyethylene shield is to suppress the $2.2-\mathrm{MeV}$ photon from the $(n, \gamma)$ capture reaction but introduce the $0.48-\mathrm{MeV}$ photon from the $(n)$ capture reaction on $\mathrm{B}^{10}$. The neutral particle fluences for the central tracker volume are listed in Table 6-16. The results include all low-energy neutrons from the calorineter and shield as well as the high energy neutrons from the primary collider event. The low-energy neutrons from the calorimeter are essentially uniformly distributed in the central tracker volume while the high-energy neutrons have a flat distribution in $\eta$. 
Table 6-16. Neutron fluences $\left(\mathrm{cm}^{-2} \mathrm{SSCY}^{-1}\right)$.

\begin{tabular}{lcc} 
Energy Range & $\begin{array}{c}\text { Yearty Fluence } \\
(r=10 \mathrm{~cm})\end{array}$ & $\begin{array}{c}\text { Yearly Fluence } \\
(r=44 \mathrm{~cm})\end{array}$ \\
\hline$>100 \mathrm{keV}$ & $7 \times 10^{12}$ & $2 \times 10^{12}$ \\
All energies & $8 \times 10^{12}$ & $4 \times 10^{12}$ \\
\hline
\end{tabular}

\section{Backgrounds from Neutral Particles}

Signals in the active regions of the detectors come from primary interactions of the neutral particles and from debris produced by all particles, neutral and charged, in materials surrounding the detectors and from shielding materials. In our physics simulations we have allowed charged-particle interactions to occur, as well as electronic noise, to contribute to the signal rate for typical physics events, but we have not included the effect of neutral particles. This is primarily because the GEANT simulation does not treat low-energy neutron interactions in a clearly physical manner. We expect the LAHET code system to do a much more physical job. Our concerns included the debris coming off the borated polyethylene shield into the IPC chambers, conversions of photons in the silicon and IPC active regions, and neutron interactions in the active regions of the silicon and IPC detectors. This program of study is still in progress, but some results are still available. The rates for two processes studied are shown in Table 6-17. In the first row are tabulated the yearly photon fluence in the first silicon layer and the first IPC layer. In the second row are tabulated the $(n p, d, t, \alpha)$ reactions in the silicon and IPC active regions. Both processes produce unwanted signals in the detectors but at low rates $(<1000 \mathrm{~Hz}$ ). We don't expect these processes to effect the occupancy af the silicon or IPC detectors. The rates translate (conservatively) into less than $10 \%$ increases in occupancies described in Section 6.2.1 and shown in Tables 6-3 and 6-4.

Table 6-17. Photon fluences $\left(\mathrm{cm}^{-2} \mathrm{SSCY}^{-1}\right)$.

\begin{tabular}{lll}
\hline Process & $\begin{array}{c}\text { First Silicon } \\
\text { Layer } \\
(r=10 \mathrm{~cm})\end{array}$ & $\begin{array}{c}\text { First IPC } \\
(\mathrm{L}=44 \mathrm{~cm})\end{array}$ \\
\hline Yearly photon fluence & $3.5 \times 10^{12}$ & $3.2 \times 10^{12}$ \\
$\begin{array}{c}\text { (np, } d, t, \alpha) \text { reactions/ } \\
\text { SSC yr-cm }\end{array}$ & $0.02 \times 10^{12}$ & $0.005 \times 10^{12}$ \\
\hline
\end{tabular}

\section{Activation of Central Tracker Components}

The question of activation of central tracker components will be addressed through the use of the CINDER activation code. This code accepts as input the yield of spallation products from primary nuclear interactions from the LAHET code, along with the fluence of neutrons calculated by MCNP. The decay of the reaction products is tracked in the CINDER code, which produces the nuclear inventory, activity in curies, decay heating in watts, and the decay photon spectra. This may be done at user chosen time intervals. The photons may further be tracked with Monte Carlo methods to various places around the detector, and the equivalent dose in rads calculated. Preliminary calculations have indicated that the dose rates in the central region around the beam pipe are 10-100 mrem $/ \mathrm{h}$ in the forward region. More detailed calculations are underway. The implication is that while the forward region is radioactive, access for minor repairs is clearly possible.

\subsubsection{Lifetime Expectancy of the Central Tracker}

The radiation levels discussed in the preceding sections are summarized in Table 6-18.

We can see from this table that the lifetime expectancy of the silicon inner tracker is at least $10 \mathrm{yr}$ at a $L=10^{33} \mathrm{~cm}^{-2} \mathrm{~s}^{-1}$ and of the IPC outer tracker is $10 \mathrm{yr}$ at $\mathscr{L}=10^{34} \mathrm{~cm}^{-2} \mathrm{~s}^{-1}$.

Table 6-18. Radiation hardness of central tracker.

\begin{tabular}{|c|c|c|c|}
\hline \multicolumn{4}{|c|}{$\begin{array}{l}\text { Charged particles, including secondary interactions, } \\
\gamma \text { conversions, delta rays, loopers, otc. }\end{array}$} \\
\hline & \multicolumn{2}{|c|}{$\begin{array}{c}\text { Yearty Radiation Dose } \\
\left(\mathrm{cm}^{-2} \mathrm{SSCr}^{-1}\right)\end{array}$} & \multirow{2}{*}{$\begin{array}{c}\text { Lifetime } \\
\text { Dose } \\
\text { Tolerance } \\
\text { Tolerance }\end{array}$} \\
\hline & $L=10^{33}$ & $L=10^{34}$ & \\
\hline Silicon & $0.4 \mathrm{Mrad}$ & $4 \mathrm{Mrad}$ & 4 Mrad \\
\hline Pads & $0.025 \mathrm{Mrad}$ & $0.25 \mathrm{Mrad}$ & $2.5 \mathrm{Mrad}$ \\
\hline \multicolumn{4}{|c|}{ Neutron flux with polyethylene shield } \\
\hline & \multicolumn{2}{|c|}{$\begin{array}{l}\text { Yearty Neutron Flux } \\
\left(\mathrm{cm}^{-2}\right)\end{array}$} & $\begin{array}{c}\text { Lifetime } \\
\text { Dose } \\
\text { Tolerance }\end{array}$ \\
\hline & $l=10^{33}$ & $L=10^{34}$ & Tolerance \\
\hline Silicon & $7 \times 10^{12}$ & $7 \times 10^{13}$ & $10^{14} / \mathrm{cm}^{2}$ \\
\hline Pads & $2 \times 10^{12}$ & $2 \times 10^{13}$ & $5 \times 10^{14} / \mathrm{cm}^{2}$ \\
\hline
\end{tabular}




\subsection{R \& D PROGRAM}

\subsubsection{Silicon R\&D}

\section{Silicon Wafer R\&D}

The research and development plan for the GEM microstrip detectors has been designed to bring the performance of the detectors to the level demanded by the GEM tracker by the end of $: 994$. Early prototype production and testing an being planned. The silicon detector $R \& D$ includes detailed simulation studies of the microstrip response to ensure full characterization and understanding of the detector operation.

The key issues being addressed for the microstrip detectors are the basic detector response, multi-strip response, (intra- and inter-charge coupling), strip length dependence, minimization of microstrip capacitance, reduction of microstrip resistance, effect of 0.8-T magnetic field on detector response, effect of radiation on detector performance, and detector/electronics coupling. Many of these effects are being and will be studied with viy fast amplifiers with nanosecond resolution. Studies of the time-structure of induced currents correlated with simulation studies will lead to a full understanding of detector performance and optimization.

\section{Silicon Mechanical Engineering R\&D}

The mechanical engineering R\&D efforts will focus on the continued development of ultrathin, dimensionally stable composites. This effort has two separate issues: 1) the development of the mechanical processing, and 2) the development of the measurement technology required to validate our design. Some of the more fundamental efforts are presently being addressed by the SDC R\&D engineering plan. The GEM effort will seek to augment those areas of SDC that we feel are not progressing fast enough to meet the GEM program schedule or on areas that the GEM collaboration feels are more relevant to our design.

Solving the technical issues associated with the central region will diminish the technical risk in other relevant areas. This is because elements contained in this region are basic building blocks for the entire detector. The central region has been identified as being on the critical path. Because the central elements are similar to those of the forward region, the central region can form the basis for the design of the forward region components.

One additional area of research to be addressed in detail by GEM is the detector cooling and support for the 18-cm ladder design. This area is so key to the overall success of the silicon tracker that additional work within GEM will be undertaken to ensure its success. Additional testing will be done to simplify the cooling ring/wick interface to improve the repeatability of the electronics temperature, which has a direct relationship to the overall stability of the tracker.

The component with the largest payback in terms of tracker stability is the space frame. The space frame carries all the measurement components and is required to remain absolutely stationary. A structural prototype of the tube and joint assembly will be evaluated, and later a full module $(\sim 1 / 3$ of the total) will be built and evaluated.

\section{Silicon Electronic Engineering R\&D}

The R\&D effort for FY93 and FY94 is intended to bring the silicon tracker electronics to a point where construction can begin. The electronics effort in FY93 will concentrate on the bipolar amplifier, the three CMOS chips, the multi-chip module (MCM), and the fiber-optic data link in semi-custom ASICs and FPGA devices to develop an engineering proof-of-principle (POP) test of the system architecture.

The initial design of the bipolar chip has been completed and submitted to AT\&T Mixed-Signal Operations for prototype semi-custom ASIC fabrication. The goals of the R\&D work are to test and analyze the transistors for gain reductions due to radiation, to redesign the circuit to compensate for radiation-induced signal gain and DC operating point shifts, and to attempt to improve the equivalent noise charge from the present level of 1550 electrons RMS. The ASICs will be tested in a test beam and used to support the engineering proof-of-principle setup.

The three CMOS chips perform the primary tasks of data acquisition and storage, digital control and external interface, analog housekeeping, and in-situ test functions. The goals of the R\&D work are to optimize the architecture to compress and store the data, to develop a full logic description of the 
architecture in VHDL (VHSIC Hardware Description Language), and to implement the VHDL description using FPGA technology. For the CMOS-2D and CMOS-2A chips the goals are to define the monitoring, state-of-health, and self-test features.

As the carrier of all the microchips plus the fiber-optic links and signal and power connections, the MCM is one of the most critical components of the front-end electronics. The goals of the R\&D efforts this year are to develop a low-cost but highly reliable MCM that can accommodate automated production techniques. The R\&D efforts will be to study the performance and costs of various available technologies, including flip-chip (bump-bonding), wire bonding and High Density Interconnect (HDI) from Texas Instrument; to lay out a representative circuit to assess the different technologies; and to fabricate a prototype MCM for the proof-of-principle setup.

\subsubsection{IPC R\&D Program}

\section{Chamber Development}

The first stage of IPC development for the GEM central tracker involves addressing a number of performance issues that arise from the unique characteristics of the tracking environment at the SSC. These issues, discussed in Section 6.4.1, include the high beam crossing rate, high track density, and high radiation levels. This initial stage of the IPC development program, in which small IPC prototypes are used to test proof of principle, is essentially complete. Some of the key results of these studies, including the measured position resolution of an IPC prototype using fast gas and electronics, are presented in Section 6.4.1. Further tests on these prototypes using a cosmic ray test stand will be conducted through 1993, with the primary goal of determining the position resolution of the IPCs for minimum ionizing particles in an 8-kG magnetic field. The next stage of the IPC R\&D program involves the fabrication of a full scale prototype chamber, incorporating the results of the initial prototype studies, including optimal cathode layout, wire spacing, and other chamber parameters into the mechanical design. This detector will be instrumented with the same hybrid front-end electronics used for the initial prototype studies. A beam test of this prototype will occur in early to mid 1993. as described in Section 6.7.3. The key issues to be addressed in these tests are the resolution and uniformity of a full-sized prototype. Multi-track resolution will be measured, as well as single-track resolution in the presence of a high-rate background. During this time period the readout electronics development will progress to the point where a prototype readout system can be fabricated. The final stage of the IPC R\&D program involves the integration of this front-end electronics with a full-scale IPC chamber. The culmination of the IPC R\&D program will consist of a series of beam tests using this chamber, to take place in 1994 or 1995.

\section{IPC Mechanical R\&D}

The engineering of the IPC chambers will be conducted at Yale during FY93. The goal of this program is the construction of a full-scale mechanical prototype of a barrel IPC during early FY93. In addition, Yale will carry out a design study of the mechanical support for the IPC system. Included in this program is the evaluation of low-mass, low coefficient of thermal expansion materials suitable for use in the chamber and support structure.

\section{Interpolating Pad Chamber Electronics R\&D}

The FY93 R\&D program of IPC electronics development is presently divided into four areas. These are the front-end amplifier design, development of a rad-hard analog memory, evaluation of a rad-hard FADC, and the design of the overall readout architecture. Details of the progress to date are presented in the Electronics section of the GEM TDR and are summarized below, along with the program of future development work.

A prototype preamplifier and shaping amplifier for use in the IPCs has been developed by the instrumentation group at BNL. This design is presently being fabricated in the Harris rad-hard AVLSI-RA CMOS process. Initial rad-hard prototypes will be available for testing in mid 1993. At this point, BNL will carry out a series of tests to evaluate the performance and radiation tolerance of this device.

The development of the rad-hard analog memory is being carried out by a group at Oak Ridge National Laboratory (ORNL). A prototype 8 channel by 128 deep device has been developed by ORNL in the AVLSI-RA process, and is being 
fabricated in conjunction with the BNL amplifier. Testing of these prototypes will be conducted by ORNL in mid 1993. The development of the amplifier and analog memory will then proceed through a series of three fabrication cycles, during which the designs will be refined. A final preproduction fabrication run will occur in late 1994, prior to the start of full production.

A FADC developed by Harris in the AVLSIRA process is being tested by a group at the University of Michigan, using a FADC test facility being made available to them by Ames Research Laboratory. A full performance evaluation is being conducted at various ionizing and neutral radiation levels. Initial results indicate that this device maintains its specified performance to a dose of at least $2 \mathrm{Mrad}$, and therefore appears suitable for use in the outer tracker.

A readout controller prototype for the IPCs is being developed at Indiana University. The design and layout of the initial prototype controllers, which utilize Xilinx FPGAs, is now complete. The use of FPGAs will allow modifications to the readout to be made in a straightforward way during the initial development stage. This readout controller prototype will be combined with the rad-hard amplifier, analog memory, and FADC prototypes in a prototype readout system to be fabricated during 1993 . The readout system prototype will be used in conjunction with full-scale chamber prototypes in a series of beam tests, as described earlier. In 1994, the readout controller design will be implemented in a LSI Logic rad-hard gate array, and combined with the final versions of all front-end readout devices in a preproduction readout card, which will be available for testing in early 1995 . The milestones identified for the development of the IPC electronics are summarized in the GEM central tracker subsystem schedules at the end of this chapter.

\subsubsection{Test Beam Plans}

\section{Silicon Detectors}

Because silicon detector performance cannot be readily factorized from electronics performance, a beam test cannot be expected to yield meaningful results on the key questions of resolution and rate capability until realistic prototypes of detectors and electronics are available. We expect this to be possible beginning mid-1993. Wherever possible, silicon beam tests will be made in conjunction with IPC tests. The silicon group also will provide a telescope of standard silicon detectors and SVXD electronics for the IPC tests, starting in 1993.

We expect to begin receiving prototype silicon detectors from Hamamatsu, Taiwan, and Moscow during the first half of 1993. In addition, some of the Bipolar ASIC prototype electronics should become available during this time. These could possibly be used in the BNL test beam in summer 1993. However, we anticipate that a full beam test of detectors and electronics will not occur until 1994. At this time we will measure resolution, and signal response and noise, as a function of rate for various detector and electronics configurations. We assume that refinements of design will warrant additional test beam running in 1995. Table 6-19 summarizes the test beam plans for the silicon development work.

Table 6-19. GEM central tracker silicon test beam needs 1993-1995.

\begin{tabular}{|c|c|c|c|}
\hline Time & Device/Test & Beam Parameters & $\begin{array}{c}\text { Requireo } \\
\text { Beam } \\
\text { Time }\end{array}$ \\
\hline Late 1993 & $\begin{array}{l}\text { Possible tests of new detectors in } \\
\text { conjunction with bench tests }\end{array}$ & Low rate secondary beam & 2 werks \\
\hline 1994 & $\begin{array}{l}\text { Full resolution and rate tests of } \\
\text { prototype detectors and front-end } \\
\text { electronics }\end{array}$ & $\begin{array}{l}\text { Charged pions of at least } 5 \mathrm{GeV} / \mathrm{c} \text { to high rate } \\
\left(10^{8} \mathrm{~Hz} \text { over few } \mathrm{cm}^{2}\right) ; \text { fow GeV/c electrons. } \\
\text { Use nearby Si triplets for resolution; Si tele- } \\
\text { scope for extemal reference. }\end{array}$ & 2 months \\
\hline 1995 & $\begin{array}{l}\text { Follow-up of above tests, including } \\
\text { tests of engineering prototype units }\end{array}$ & same as above & 2 months \\
\hline
\end{tabular}




\section{Interpolating Pad Chambers}

The central tracker team is preparing to install a test setup in the BNL B2 beam line in summer 1993. The measurements to be made will include the determination of the intrinsic resolution of prototype interpolating pad chambers, uniformity scans and efficiency measurements in a high counting rate environment. The BNL beam will reach a maximum of $9 \mathrm{GeV}$ with an intensity of $5 \times 10^{6}$ particles per spill and is currently scheduled to begin in June.

The test setup will include several prototype IPCs mounted on a precision transporter, a scintillator trigger system and a silicon telescope to provide an external determination of the resolution of the prototypes. A flexible data acquisition system, similar to the one currently in use in the Texas Test Rig (TTR), is being assembled. The main limiting factor for these tests will be the unavailability of the final electronics and the scarcity of any suitable replacements. Approximately 100 channels will be available.

The BNL beam may not achieve the intensity required to make a realistic assessment of the high-rate capabilities of the IPCs. If this is the case, another test beam run will be planned for 1994 using the final electronics. This should complete our test bearn development work for the IPCs. Table 6-20 summarizes the test beam plans for the interpolating pad chambers.

\subsection{ORGANIZATION \& MANAGEMENT}

\subsubsection{The Central Tracker Group}

The GEM central tracker group consists at this time of 14 institutions and more than 110 scientists. They are as follows:

Academia Sinica, Taiwan, R.O.C.

Brookhaven National Laboratory (BNL)

Indiana University

Los Alamos National Laboratory (LANL)

Moscow State University

Nanjing University

Oak Ridge National Laboratory (ORNL)

Rutgers University

Superconducting Super Collider Laboratory

(SSCL)

University of Albany

University of Michigan

University of Oregon

Yale University

Vanderbilt University

Management of the central tracker group and technical design is performed by the Central Tracker Steering Committee, which consists of the following scientists: C. Baltay, J. E. Brau, D. Lee, S.C. Lee, K. Morgan, J. Musser, T. Thompson. The chairman of the Steering Committee is C. Baltay.

The chief engineer for the central tracker group is $\mathrm{T}$. Thompson. Budgeting support is provided by H. Pretty.

Table 6-20. GEM central tracker IPC test beam needs.

\begin{tabular}{|c|c|c|c|}
\hline Time & Device/Test & Beam Location or Parameters & $\begin{array}{c}\text { Required } \\
\text { Beam Time }\end{array}$ \\
\hline Mid 1992 & $\begin{array}{l}\text { Operating parameters of a small } \\
\text { chamber }\end{array}$ & $\begin{array}{l}\text { low rate secondary beam, a few hundred } \\
\text { MeV es, } \mu s, \pi s\end{array}$ & 1 month \\
\hline Late 1993 & $\begin{array}{l}\text { Resolution of full sized prototype of } \\
\text { barrel pad chamber, scan over the } \\
\text { full length of the device }\end{array}$ & $\begin{array}{l}\text { es, } \mu s, \pi s \text { of a few } \mathrm{GeV} \text {, protons } \geq 5 \mathrm{GeV} \text {, } \\
\text { rate: a few particles/sec. Well collimated } \\
\text { beam width position measurement }\end{array}$ & 3 months \\
\hline Late 1994 & $\begin{array}{l}\text { Rate capabilities of full sized proto- } \\
\text { type, both barrel and endcaps }\end{array}$ & $\begin{array}{l}\text { high-rate secondary beam } 10^{3} \text { par- } \\
\text { ticles/s, which can be spread over } \\
-30 \mathrm{~cm}^{2} \text { in the chamber }\end{array}$ & 3 months \\
\hline 1995 & $\begin{array}{l}\text { Systems tests: gas, ccoling, } \\
\text { mechanics, otc. of final design, } \\
\text { with final electronics }\end{array}$ & same as 1994 & 3 months \\
\hline
\end{tabular}




\subsubsection{Personal and Jnstitutional Responsibilities}

At the present time the effort on the central tracker consists mostly of $R \& D$, detailed engineering design, and computer simulations of the performance of the detector. The personal and institutional responsibilities for this effort have been defined and are shown in Table 6-21.

Table 6-21. Personal and institutional responsibilities.

\begin{tabular}{|c|c|c|}
\hline Area of Effort & $\begin{array}{c}\text { Person } \\
\text { Responsible }\end{array}$ & $\begin{array}{c}\text { Institutions } \\
\text { Involved }\end{array}$ \\
\hline $\begin{array}{l}\text { Silicon } \\
\text { Detector } \\
\text { Overall: } \\
\text { Mechanical: } \\
\text { Electronics: }\end{array}$ & $\begin{array}{l}\text { D. Lee, J. Brau } \\
\text { T. Thompson } \\
\text { S. Hahn }\end{array}$ & $\begin{array}{l}\text { LANL, Moscow } \\
\text { State University, } \\
\text { University of } \\
\text { Oregon, Taiwan }\end{array}$ \\
\hline $\begin{array}{l}\text { Pad chambers } \\
\text { Overall: } \\
\text { Mechanical: } \\
\text { Electronics: }\end{array}$ & $\begin{array}{l}\text { J. Musser, } \\
\text { C. Baltay } \\
\text { W. Emmet } \\
\text { J. Musser, } \\
\text { P. O'Conner }\end{array}$ & $\begin{array}{l}\text { BNL, LANL, } \\
\text { SSCL, Indiana } \\
\text { University, } \\
\text { University of } \\
\text { Michigan, } \\
\text { Rutgers } \\
\text { University, } \\
\text { Taiwan, Yale } \\
\text { University }\end{array}$ \\
\hline $\begin{array}{l}\text { Integration into } \\
\text { GEM }\end{array}$ & $\begin{array}{l}\text { K. Morgan } \\
\text { T. Thompson }\end{array}$ & All \\
\hline Simulations & S. McKeө & All \\
\hline Beam Tests & K. Morgan & All \\
\hline Budgets & H. Pretty & Ail \\
\hline
\end{tabular}

\subsection{SCHIEDULES}

We envision a 3-yr conceptual design and R\&D period, 1992 to 1994 . The bulk of the detailed engineering design will also take place during this period, although some engineering design will stretch out through 1997. Building and testing of various prototypes will also take place from 1992 to 1994.

Construction of the various components of the tracker is anticipated to take place at the various collaborating institutions away from the SSC site over a 4-yr period from 1994 to 1997. The components will be shipped to the SSCL and the assembly of the central tracker will occur in the surface building designed for this purpose at the experimental site between mid 1997 and mid 1998. Calibration, alignment and various test activities will also take place at that time.

We expect that the complete central tracker, including the pad chambers and the silicon detector, will be installed in GEM in mid 1998. This will be followed by a year of finishing up the cabling, cooling, and gas connections and the final commissioning of the detector. The schedule calls for the detector to be ready for data-taki:.g by mid 1999 . This schedule will be refined and updated as appropriate as the central tracker, the GEM detector and the SSC collider progress. 


\section{REFERENCES}

1 Cherniatin and Chikanian, BNL, computer simulation, private communication.

2 J. Thomas, GEM-TN-93-361

3 J.S. Gordon and E. Mathieson, NIM 227 (1984) pp. 267-76.

4 E. Mathieson and J. S. Gordon, NIM 227 (1984) pp. 277-82.

5 J.R. Thompson, J. S. Gordon, and E. Mathieson, NIM A234 (1985) pp. 505-11.

6 E. Mathieson, NIM A294 (1990) p. 121.

7 H. Yamamoto and R. Zhu, GEM-TN-92-126.

8 I. Sheer, GEM-TN-93-370.

9 B. Zhou, GEM-TN-92-100.

10 L. Rosenson, GEM-TN-92-97.

11 GEM Collaboration, GEM-TN-92-231.

12 J. Bagger, private communication.

13 M. Botlo, et al, SLAC-PUB-5795, 1992.

14 M. Strovink, private communication.

15 J. Leslie, A. Seiden, and Y. Unno, SCIPP 92-20 (1992).

16 V. Radeka, Rev. Nucl. Part. Sci. 38, 217 (1988)

17 R. Sonnenblick, et al, SCIPP 91/04 (1991).

$18 \mathrm{~K}$. Yamamoto, Hamamatsu, private communication. 


\section{ELECTRONICS, TRIGGER, AND DATA ACQUISITION}

\subsection{INTRODUCTION AND OVERVIEW}

\subsubsection{General Requirements and Philosophy}

The challenging GEM performance goals and the high SSC collision rate place severe demands on the readout electronics due to the high event rate coupled with the large channel count and data flow. A suitable architecture has been developed for the GEM electronics system, which includes a multilevel triggering system coupled with data storage between levels. The strategy is to provide a system free of deadtime that furnishes as much information as possible to each trigger level. The architecture is designed to work at luminosities of $10^{33} \mathrm{~cm}^{-2} \mathrm{~s}^{-1}$, with provision for running at $10^{34} \mathrm{~cm}^{-2} \mathrm{~s}^{-1}$. The system can be broken down into four subsections:

1. Amplification and shaping of detector signals

2. Signal sampling, storage, processing, and digitization

3. Triggering and event selection

4. Data acquisition and event building.

Each detector subsystem imposes unique requirements on the associated electronics readout system. The inner detectors need to be instrumented with radiation-hard electronics. In order to reduce the cable plant and to minimize signal degradation, much of the electronics must be integrated on the detectors. This is especially true for the inner silicon vertex detector and pad chambers, where the number of channels precludes bringing out all signals at the
60-MHz beam-crossing rate. In addition, GEM's primary design goals of precision calorimetry and muon measurement place exacting demands on the electronics precision and calibration, which, owing to the large number of detector elements, must be satisfied at a low cost per channel. Commercial systems meeting the technical requirements are currently not available, necessitating the development of application-specific integrated circuits (ASICs) for each of the major subsystems. A summary of the subsystem requirements is given in Table 7-1.

\subsubsection{Overall Architecture}

The triggering and data acquisition system, shown in Figure 7-1, follows a three-level approach: Level 1 is synchronous and pipelined; Level 2 is asynchronous using a processor ranch and, possibly, special-purpose hardware processors; and Level 3 is a processor ranch. A unique feature of the GEM DAQ system is the availability of complete sets of full granularity data at Levels 2 and 3. Event rates and latency times are summarized in Table 7-2. (The second column in the table specifies the maximum average input rate that can be sustained at each level.) As indicated in the table, the rate-handling capability of each level is 10 times greater than the design goal for the output rate of the preceding level. This design margin will ensure reliable operation at $\ell=10^{33} \mathrm{~cm}^{-2} \mathrm{~s}^{-1}$ while leaving room for operation at $\mathcal{L}=10^{34} \mathrm{~cm}^{-2} \mathrm{~s}^{-1}$.

Table 7-1. Channel information table.

\begin{tabular}{lccccc}
\hline & $\begin{array}{c}\text { Silicon } \\
\text { Strip }\end{array}$ & $\begin{array}{c}\text { Int. Pad } \\
\text { Chambers }\end{array}$ & $\begin{array}{l}\text { Liquid } \\
\text { Calorimeters }\end{array}$ & $\begin{array}{c}\text { CSC Wire } \\
\text { Readout }\end{array}$ & $\begin{array}{c}\text { CSC } \\
\text { Pad } \\
\text { Readout }\end{array}$ \\
\hline $\begin{array}{l}\text { No. of channels } \\
\text { Power dissipation/channel }\end{array}$ & $2500 \mathrm{~K}$ & $400 \mathrm{~K}$ & $125 \mathrm{~K}$ & $313 \mathrm{~K}$ & $922 \mathrm{~K}$ \\
$\begin{array}{l}\text { (In-detector) } \\
\text { (On-detector) }\end{array}$ & $2 \mathrm{~mW}$ & $60 \mathrm{~mW}$ & $275 \mathrm{~mW}$ & $100 \mathrm{~mW}$ & $100 \mathrm{~mW}$ \\
\hline
\end{tabular}


Table 7-1. Channel information table. (Cont.)

\begin{tabular}{|c|c|c|c|c|c|}
\hline & $\begin{array}{c}\text { Silicon } \\
\text { Strip }\end{array}$ & $\begin{array}{l}\text { Int. Pad } \\
\text { Chambers }\end{array}$ & $\begin{array}{l}\text { Liquid } \\
\text { Calorimeters }\end{array}$ & $\begin{array}{l}\text { CSC Wire } \\
\text { Readout }\end{array}$ & $\begin{array}{c}\text { CSC } \\
\text { Pad } \\
\text { Readout }\end{array}$ \\
\hline Resolution & Digital & $.5 \%$ & $.1 \%$ & $<16$ ns & $.5 \%$ \\
\hline Dynamic range & 1-bit & 10-bit & 18-bit & $4 \mu s$ & 10-bit \\
\hline Occupancy/channel (\%) & 0.16 & 1.8 & 5 & $<1$ & $<1$ \\
\hline Number of samples & 1 & 2.4 & 5 & 1 & 5 \\
\hline Channols/link & 640 & 128 & 496 & $\cdots$ & 864 \\
\hline Link bandwidth (Mbit/s) & 60 & 60 & 400 & $\cdots$ & 60 \\
\hline Radiation (Radiyr $\odot 10^{33}$ ) & $0.5 \mathrm{M}$ & $30 \mathrm{~K}$ & $\begin{array}{l}100 \mathrm{~K} \text { Barrel } \\
5.0 \mathrm{M} \text { Endcap }\end{array}$ & $<1 K$ & $<1 K$ \\
\hline
\end{tabular}

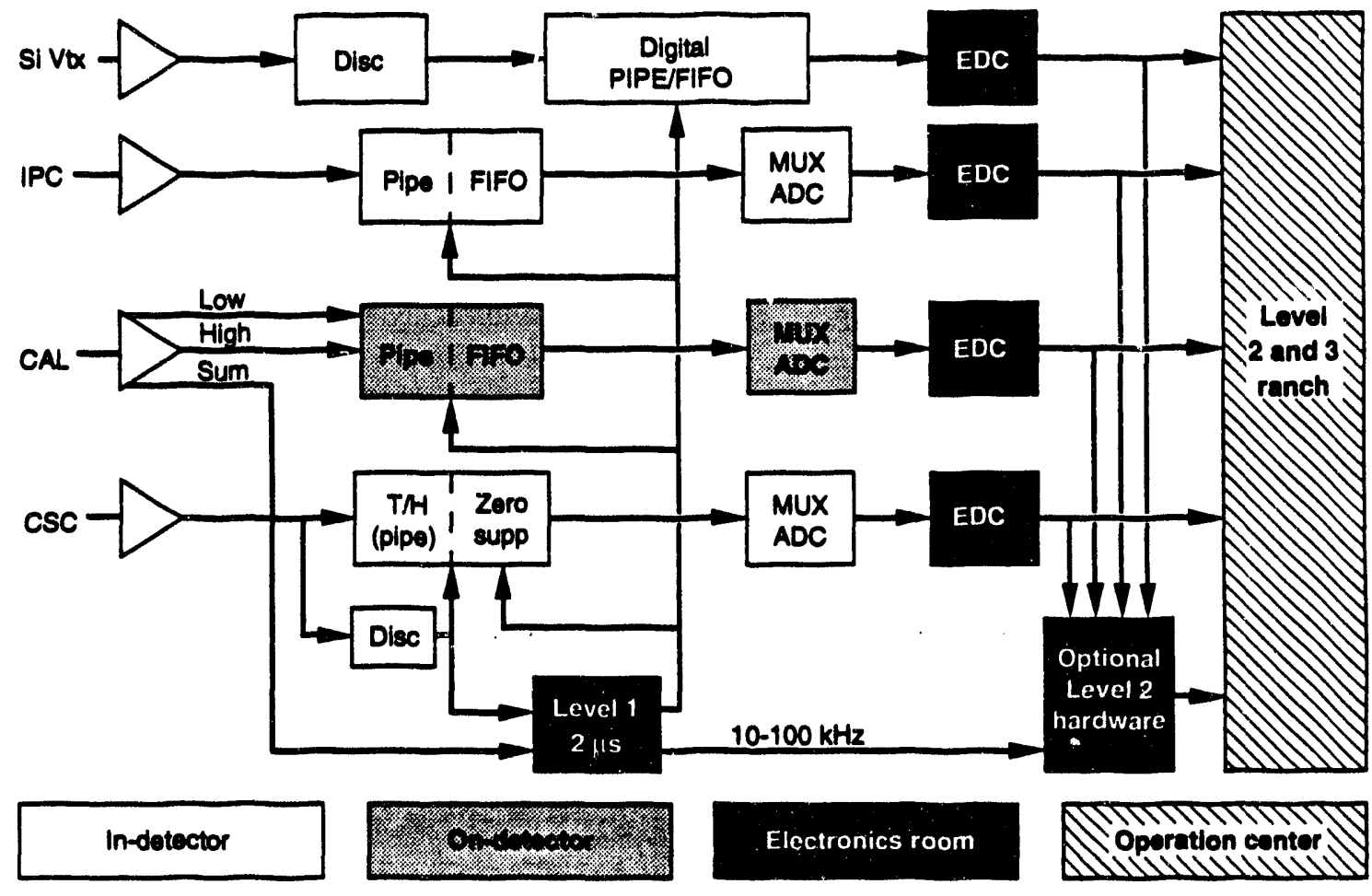

TाP-03936

FIG. 7-1. Block diagram of the GEM triggering and data acquisition system.

Table 7-2. Design goals for the GEM trigger/DAQ system. Output rates are for operation at $L=10^{33} \mathrm{~cm}^{2} \mathrm{~s}-1$.

\begin{tabular}{ccccc}
\hline Level & Rate In & Rate Out & Latency & Comments \\
\hline 1 & $60 \mathrm{MHz}$ & $10 \mathrm{kHz}$ & $2 \mu \mathrm{s}$ & Synchronous, Pipelined \\
2 & $100 \mathrm{kHz}$ & $300 \mathrm{~Hz}$ & $100 \mu s-0.1 \mathrm{~s}$ & Asynchronous \\
3 & $3 \mathrm{kHz}$ & $100 \mathrm{~Hz}$ & - & Processor Ranch \\
\hline
\end{tabular}


As shown in Figure 7-1, each subsystem has an amplification/shaping section followed by an analog or digital pipeline storage unit (possibly a "track and hold" for the muon system). The pipeline provides the synchronous $2-\mu$ s delay needed for the Level 1 trigger without any loss of data. Level 1 tags the bunch-crossing of each trigger, allowing the processing and readout of only those data that correspond to the interaction of interest.

After a Level 1 accept is generated, all relevant data are digitized and stored in local memories until they are transferred into the event data collector units (EDCs). The EDCs hold the data until a Level 2 accept occurs. In most systems, precise timing and pulse height measurements demand that multiple samples be stored and processed. To reduce the data volume transmitted, these samples are processed and reduced locally to yield the desired information.

In the baseline design, the Level 2 trigger algorithms are run on processors that are situated downstream of the event-building network. This architecture is sometimes referred to as a "virtual Level 2" trigger, because rather than employing dedicated special-purpose processors, Level 2 can be implemented on the same processors that comprise the Level 3 ranch. This approach has the advantage of using the fully programmable processor units with substantially complete sets of data, but it requires a higher event-builder bandwidth and/or a more complicated data-flow control network.

The baseline system is capable of transmitting fill event data at Level 1 trigger rates of $20-30 \mathrm{kHz}$ or any selected subset of data up to $25 \%$ of the full amount at the maximum design rate of $100 \mathrm{kHz}$. It can be easily expanded to accept full data events at a Level 1 igger rate of $100 \mathrm{kHz}$, should this become necessary. The event builder utilizes a control feedback path that dynamically selects and transmits only the portions of the event required by Level 2 . This process reduces the bandwidth requirements of the event building switch at the expense of complicating the control network.

\subsubsection{Front-End Subsystem Summaries Silicon Strip Vertex Detector}

The silicon tracker front-end electronics system is "digital" in that all data transferred from the front-end is a digital yes/no signifying a-hit/no-hit on an individual strip. The system is clocked by the $60-\mathrm{MHz}$ beam crossing, and the data from each crossing is stored for $2 \mu$ s until a Level 1 accept signal arrives. The electronics are mounted on a multi chip module, which is in turn mounted to a silicon ladder assembly. The signal from the sensor strips is amplified, shaped, and compared to a threshold in a bipolar VLSI chip. The logic output of the bipolar chip is fed to a CMOS chip, whose function is to time-stamp, buffer, and compress the data. Data with the appropriate time-stamp are transmitted upon a Level 1 trigger to the data acquisition system via a fiber-optic data link. The system has a total of 2.5 million individual strips. The electronics must be radiation-hardened to total doses of $5 \mathrm{Mrad}$.

\section{Interpolating Pad Chambers}

The GEM outer tracker is made up of interpolating pad chambers (IPCs), which require an analog readout accurate to $1 \%$ on each of 400000 input channels. Amplification of the input signal is performed by a charge-sensitive preamplifier and shaping amplifier, with a nominal gain of $10 \mathrm{~V} / \mathrm{pC}$ and an output peaking time of $25 \mathrm{~ns}$. These shajed pulses are sampled at the bunch crossing fr:quency, the samples being stored in a switched-capacitor array with a length determined by the Level 1 trigger latency. Upon receipt of a Level 1 trigger, relevant samples are digitized by an onboard FADC. These digitized samples are then zero-suppressed and packeted for transmission on a fiber-optic link to the data collection system.

\section{Calorimeter}

The electronic readout of the $125 \mathrm{~K}$ channels of the GEM calorimeter must provide high-precision reconstruction of deposited energies over a wide dynamic range and must deliver time resolution sufficient to correctly tag the bunch crossing. The design of the readout seeks to maintain maximum accessibility of the electronics without introducing an unacceptable degrardation of performance; only the preamplifiers and precision calibration circuits will be mounted inside the cryostat. Preamplified signals will be transferred on short cables to "junction boxes" located on the outside of the cryostat. Cable drivers mounted there will drive the signals approximately $40 \mathrm{~m}$ to crates of electronics mounted on the wall of the experimental hall. 
There, after a fast pick-off for the Level 1 trigger, the signals will be split into two gain scales and shaped before being sampled at $60 \mathrm{MHz}$. The samples will be stored in analog form using switched-capacitor technology while awaiting a Level 1 trigger decision. Upon receipt of a Level 1 trigger, up to five samples per signal will be buffered while awaiting digitization. Multiplexed and digitized samples will be processed locally using the methods of optimal filtering to provide the best energy and time resolutions and then sent over fiber-optic links to the Level 2 processing system.

\section{Muon Chambers}

The muon cathode strip chambers are read out using a chamber-mounted, front-end processing chain consisting of a preamplifier, a fast and a slow shaper, a sampling circuit, and a multiplexed ADC. Digital data are collected on a per-module basis and transmitted off detector via optical fiber. The first rank of the Level 1 muon trigger is also implemented by the on-chamber electronics. In addition to serving as input to the next layer of the trigger, these signals are integral to the data-driven readout architecture, which greatly reduces the demand on subsequent stages of the data acquisition chain. The large channel count $\left(>10^{6}\right)$ requires the use of low-cost, fully-custom integrated circuits. Further economies arise from mounting the complete readout chain on the chambers, which minimizes the required cable plant.

\subsubsection{Overview of Trigger System}

The Level 1 trigger decision is based on a reduced set of calorimeter and muon system signals, encoded to a minimum number of bits, which are here called primitives. To simplify the system design and to avoid sampling more bunch crossings than necessary, the trigger signals from the Level 1 subsystems are required to tag the bunch crossing of the event. This imposes special constraints on the design of the calorimeter trigger pickoffs and the design of the muon system. The $2 \mu$ s latency of the Level 1 trigger system is achieved by locating the trigger electronics in the detector hall or the adjacent electronics room. The $2 \mu \mathrm{s}$ trigger delay is comprised of the following components: $250 \mathrm{~ns}$ for the amplification, shaping and discrimination of the detector signals, $450 \mathrm{~ns}$ for triggering logic; and $1.3 \mu \mathrm{s}$ for the cable and fiber delay out and back into the detector.

The calorimeter trigger towers will be formed from electromagnetic (EMC) and hadronic (HCAL) calorimeter regions of size $\Delta \eta \times \Delta \phi=0.16 \times 0.16$ and $0.32 \times 0.32$, respectively. Analog sums of the calorimeter elements comprising each tower are then compared to preset thresholds to form the information needed for the Level 1 logic. The timing for the trigger is set by a specially designed timing discriminator that operates with a low threshold. Further summing of adjacent trigger towers results in total energy and $E_{T}$ sums for broader regions of the calorimeter. An electromagnetic shower candidate is identified by imposing a veto of $5 \mathrm{GeV}$ on the associated $\mathrm{HCAL}_{1}$ tower behind the given EMC tower. An isolation requirement is also used to reduce the jet background to isolated electron or gamma triggers. Specifically no more than one of the nearest neighbor EMC trigger towers in a $5 \times 5$ square may have more than $1 \mathrm{GeV}$. A summary of the calorimetry Level 1 primitives is given in Table 7-3.

Table 7-3. Calorimetry information available for the Level 1 trigger system.

\begin{tabular}{lcccccc}
\hline Level 1 Primitive & Size $(\Delta \eta \times \Delta \phi)$ & $\begin{array}{c}\text { No. of } \\
\text { Trigger } \\
\text { Towers }\end{array}$ & $\begin{array}{c}\text { Hadron } \\
\text { Veto }\end{array}$ & $\begin{array}{c}\text { With } \\
\text { Without } \\
\text { lsolation }\end{array}$ & $\begin{array}{c}\text { No. of } \\
\text { Thresholds }\end{array}$ & Output \\
\hline EM shower & $0.16 \times 0.16$ & 1065 & Yes & Both & 7 & 4-bits \\
Single particle & $0.32 \times 0.32$ & 247 & No & Both & 7 & 4-bits \\
Jet cluster & $0.64 \times 0.64$ & 62 & No & Without & 7 & 4-bits \\
Total ET & Full calor. & 1 & - & Without & - & 12-bits \\
ET & Full calor. & 1 & - & Without & -- & 12-bits \\
\hline
\end{tabular}


The muon Level 1 trigger primitives are generated from hit patterns produced in the cathode strip chamber (CSC) pads for the muon momentum selection. This selection is then combined with a bunch-crossing determination from the anode wire signals. The intrinsic 20 -ns time jitter of the anode wire signals from drift-time variations is reduced by using the arrival time of the first pulse within a superlayer. A Level $1 p_{T}$ cut is imposed on the data by examining the change in $\phi$ of correlated hits in the middle and outer superlayer (also possibly the inner layer) or by making a crude measure of the sagitta using correlated hits in the three superlayers. Trigger thresholds of up to $50 \mathrm{GeV} / \mathrm{c}$ can be imposed in the barrel with a spatial segmentation of $\sim 2 \mathrm{~cm}$, or about two barrel strip widths. These metho is of imposing a $p T$ cut in the Level 1 trigger are schematically presented in Figure 7-2, labeled " $\delta \phi$ " and "sagitta". The crude $p_{T}$ cut imposed at Level 1 is made more accurate in subsequent trigger levels by using the precise position of hits. In Figure 7-2, the curve labeled " $p_{T}$ cut" refers to a Level 2 algorithm that uses the full fitted track to impose a pr cut.

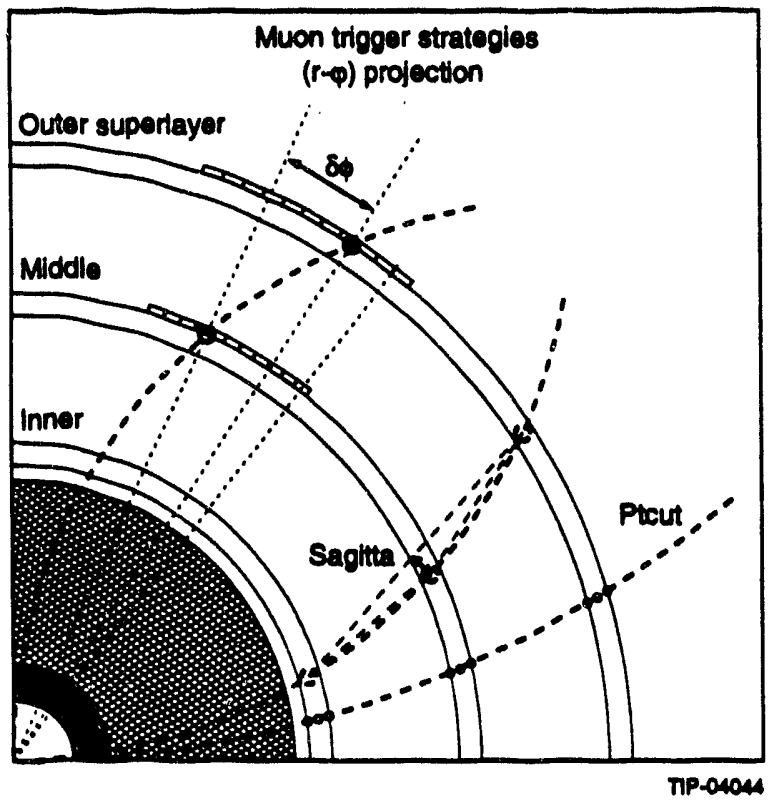

FIG. 7-2. Schematic depiction of the Level 1 and Lovel 2 muon trigger algorithms.

The Level 1 trigger decision is formed for each bunch crossing from the global pattem of trigger primitives. It will be possible to trigger on single high-pr electrons, photons, muons, or jets; on pairs of lower- $p_{T}$ leptons; on $E_{T}$ or $E_{T ;}$ or on any other combination of interest. The description of these global triggers, the efficiency for the various physics processes, and the expected rates are presented in Section 7.2.

Additional trigger rate reduction is accomplished at Level 2 using digitized data from the calorimeter, muon, and central-tracking systems. Information from the Level 1 trigger is used to "seed" further filtering of events. Calorimeter data with high precision and full granularity are used to apply shower-shape and isolation cuts to electron and photon candidates. Electron candidates are further refined by requiring stiff $\left(p_{T}>10 \mathrm{GeV} / \mathrm{c}\right)$, spatially-matched tracks in the central tracker. Finally, as described above, digitized muon information is used to determine precise momenta.

The following sections of this chapter present the readout electronics and data acquisition system in more detail. Section 7.2 describes the Level 1 and 2 trigger system, including algorithms, rates, and efficiencies. Section 7.3 gives a description of the DAQ and event-building architecture. Sections 7.4 through 7.7 describe in detail the front-end electronics associated with each of the four subsystems, including the pre-amplifiers and shapers, the various sampling devices, and the $A D C$, processing, and MUX system. Finally, Sections 7.8, 7.9, and 7.10 deal with integration, organization, and R\&D issues.

\subsection{TRIGGERING}

This section presents design and performance details of the various Level 1 and Level 2 trigger subsystems. In GEM, the Level 3 trigger is considered part of the computing subsystem and is described in Chapter 8.

The Level 1 trigger employs prompt signal pickoffs from the calorimeter and muon systems to reduce the readout rate of the GEM detector from the 60-MHz primary interaction rate down to the target rate of $10 \mathrm{kHz}$. Information from the silicon microstrip and IPC tracker arrays is not used at Level 1.

Level 2 has access to full-granularity data from all detector subsystems. Using these data more precise algorithms that incorporate, for example, the details of the spatial arrangement of the data and the 
combination of subsystems can be employed to reduce the rate.

\subsubsection{Calorimeter Level 1 Trigger}

Events of interest generally contain one or more of the following:

- Isolated photons and leptons

- High-pT hadrons

- High-pT jets

- Large transverse energy

- Large missing transverse energy.

The calorimeter will be able to provide trigger information for each of the above, except for the presence of a muon, which is provided by the muon system. The Level 1 trigger must identify the beam crossing containing the event of interest within the total allowed latency of $2 \mu \mathrm{s}$. Taking into account the transmission delays and the peaking time of the calorimeter signals, there remains approximately $450 \mathrm{~ns}$ for the total latency of the Level 1 logic.

The general strategy is to form various useful "primitives" using the Level 1 logic of the different detectors, which are then combined to form the trigger. In the calorimeter Level 1 logic, trigger sums of various sizes, called EM, SP, and JET, are formed and discriminated at several levels. The number of non-contiguous hits at each level for the different types of sums are evaluated. Nearest-neighbor logic is used to define topological patterns, such as isolated hits. A timing consistency check is performed on the jet sums to establish the absence of significant pileup. In addition, global sums of total transverse energy and missing transverse energy are provided by this logic.

The GEM baseline system for the calorimeter Level 1 trigger uses analog summing for the lowest level sums and digital summing for the sums coarser than the JET sums. Another option using digital processors to manipulate low-level digitized sums is also being investigated. (See Digital Processor Option).

\section{Trigger Sums}

The lowest level of summing will be analog sums, which are shaped into bipolar signals. As the number of channels in the sums becomes large, the noise increases, and at the point where quantization accuracy and random noise are comparable, it is convenient to use flash digitization and continue to form larger sums digitally. Adding together signals from sections of the calorimeter with different shaping times will be done by reshaping the faster signal. In the baseline design, flash digitization occurs at the level of the largest local (JET) sums, so that only the global sums are formed digitally at Level 1.

To minimize the summing circuitry, we employ a summing tree, where higher-level sums are formed from lower-level sums. Thus, for example, the transverse energy $E_{T}$ sum is made from jet sums, which in turn are made from single-particle sums. The nomenclature for the sums refers to the geometrical area: $\mathrm{EM}$ is a $0.16 \times 0.16 \mathrm{sum}, \mathrm{SP}$ is a $0.32 \times$ 0.32 sum, and JET is a $0.64 \times 0.64$ sum. $E_{T}$ and $E_{T}$ are summed over the entire calorimeter.

Table 7.4 lists the sums and the number of each that are needed. The geometrical configuration of these sums is illustrated in Figure 7-3. Below is a brief description of each of the sums:

Electromagnetic Sums (EM). The EM sum has a fiducial area of $\Delta \eta \times \Delta \phi=0.16 \times 0.16$ and is summed over cells at all EM depth sections. It is made up of $6 \times 6$ individual EM towers.

Single Particle EM Sums (SPem). The singleparticle EM sum SPem is the electromagnetic component of the single-particle sum. Its primary use is in the lateral isolation requirement for electromagnetic showers. It is formed as a $2 \times 2$ sum of EM sums and has an area of $0.32 \times 0.32$.

Hadron Isolation Sums $\left(H A D_{1}\right)$. This is a sum of $4 \times 4$ hadronic towers, resulting in an area of $0.32 \times 0.32$. The first hadronic section of the calorimeter is summed separately from the deeper sections, as this signal is used to help define the isolation requirement for the electromagnetic shower. Since a low energy threshold will be used for this purpose, it is advantageous to utilize only the first few interaction lengths of the hadronic section in order to minimize the contributions from thermal noise. 
Table 7-4. Liquid argon trigger sums.

\begin{tabular}{lccc} 
Sum Type & $\Delta \eta \times \Delta \phi$ & $\begin{array}{c}\text { Number } \\
\text { (Barrel) }\end{array}$ & $\begin{array}{c}\text { Number } \\
\text { (Endcaps) }\end{array}$ \\
\hline EM & $0.16 \times 0.16$ & 527 & -438 \\
SPem & $0.32 \times 0.32$ & 135 & -112 \\
HAD, & $0.32 \times 0.32$ & 135 & -112 \\
SP & $0.32 \times 0.32$ & 135 & -112 \\
JET & $0.64 \times 0.64$ & 34 & -28 \\
ET & $6.0 \times 2 \pi$ & 1 & 1 \\
ET & $\sim 10.0 \times 2 \pi$ & 1 & 1
\end{tabular}
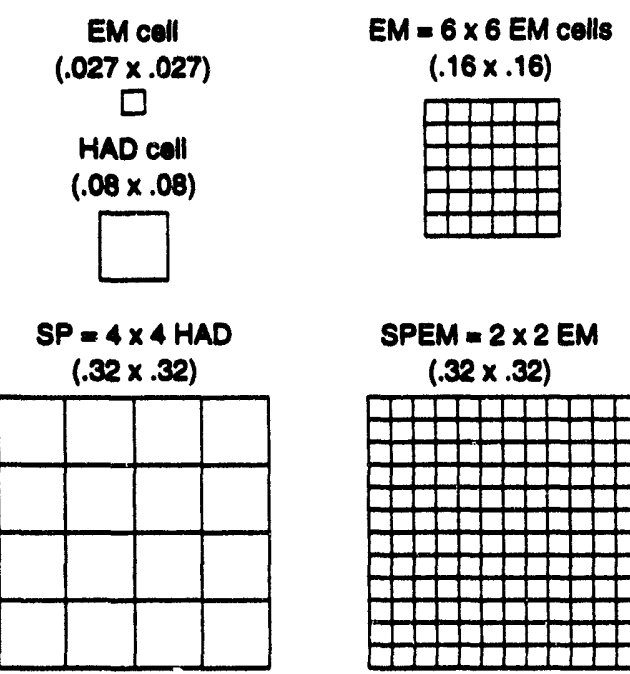

SPEM $=2 \times 2 \mathrm{EM}$

(.32 $\times .32)$
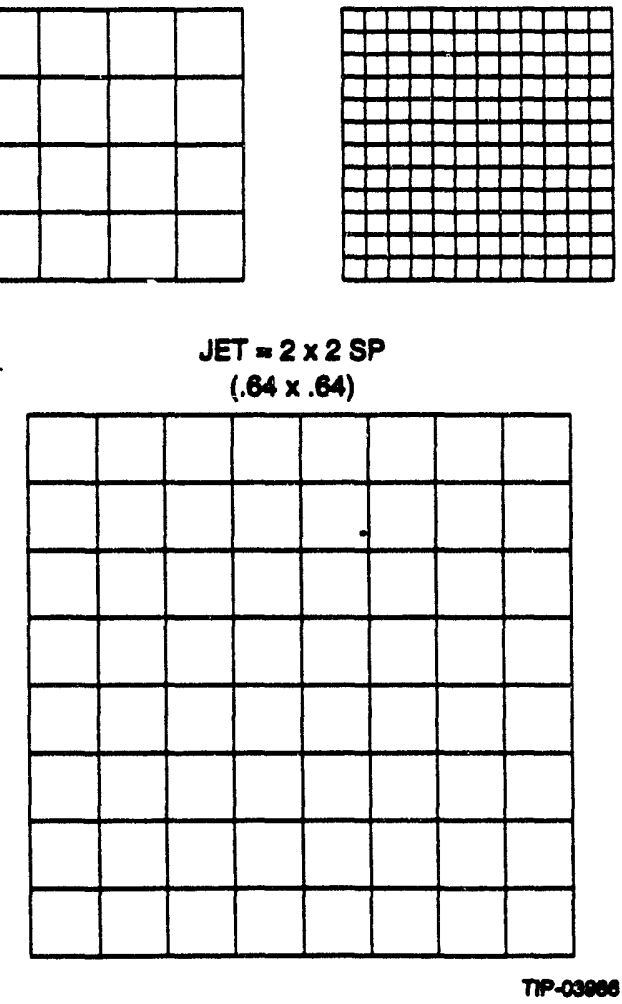

FIG. 7-3. Geometrical configuration of the trigger sums.
Single Particle Hadronic Sums (SPhad). Sums of $4 \times 4$ towers in the second, third, and fourth (where they exist) hadronic sections. This sum is not discriminated but is formed only as an ingredient of the SP sum.

Single Particle Sums (SP). The SP sum is the sum of the electromagnetic and hadronic SP-sized sums, which corresponds to the total transverse energy deposited in a fiducial area of $0.32 \times 0.32$.

Jet Sums (JET). The JET sum is the $2 \times 2$ sum of SP sums, which contain the transverse energy seen in a fiducial area of $0.64 \times 0.64$.

Global Sums ( $E_{T}$ and $\left.E_{T}\right)$. These sums are formed from the JET sums that can be positively identified with a particular crossing through the use of a digital filter that is sensitive to timing of the signal. Identification of the energy deposition with a crossing implies that a threshold of approximately 20-30 GeV mist be applied to the components of the global sums. The $E_{T}$ sum is made only over the barrel and endcap calorimeters, whereas the $E_{T}$ sum may include the forward calorimeter.

The missing transverse energy sums are formed from the JET sums by the use of moments, which are the transverse energy multiplied by weights proportional to the vertical and horizontal directions of the center of the fiducial volume of the JET sum. Each JET sum feeds into one horizontal $\left(E_{T}-X\right)$ and one vertical $\left(E_{T}-Y\right) E_{T}$ sum. All positive and negative moments are summed together, and the two moment sums are added in quadrature, providing the square of $E_{T}$ as a Level 1 primitive.

\section{Association With the Beam Crossing}

Reliable bunch-tagging places a timing requirement of $\sigma_{t}<5$ ns on the trigger signal. Thus a precise timing measurement on the shaped signal is essential.

For the analog sums we require a discriminator circuit whose timing is magnitude-independent. A circuit that accomplishes this was developed and tested in the July 1992 liquid argon/krypton test. ${ }^{1}$ It operates on the principle that one can perform a timing measurement for a signal of constant shape by measuring the point at which a signal is equal to its integral, a condition that is independent of the signal amplitude. In the beam test, an on-line resolution of $\sim 1 \mathrm{~ns}$ was obtained on a trigger sum for signals of at least $10-\mathrm{GeV}$ amplitude. Correction for 
inter-channel timing delays revealed that the system has an inherent resolution of $4.15 \mathrm{GeV}-\mathrm{ns} / \mathrm{E}$ for the EM signals. We are currently designing a discriminator circuit that will contain eight comparators, permitting up to seven amplitude thresholds to be used in the Level 1 trigger.

For the digital sums $\left(E_{T}, E_{T}-\mathrm{X}\right.$, and $\left.E_{T} \mathrm{Y}\right)$ we require a digital filter that can sense both the amplitude and time origin of the signal. For this purpose we plan to build a transversal filter. The principle of operation of this circuit is that the digitized signals are stored, forming the samples $\left(s_{k}\right.$, $\left.s_{k+1}, \ldots\right)$ associated with crossing time $t_{k}$. These samples are multiplied by filter coefficients $\left(a_{1}\right.$, $\left.a_{2}, \ldots\right)$ for the amplitude information and $\left(b_{1}, b_{2}, \ldots\right)$ for the timing information and are summed to produce the weighting functions for amplitude and timing. The properties of these weighting functions are discussed elsewhere. ${ }^{2}$ If the signal is of the correct shape (i.e., no significant pileup is present), it crosses zero at the expected time relative to the origin of the signal, and the associated value of the sum is the signal amplitude. In general the origin of the signal is unknown, but in our case the JET sum is composed of four SP sums, each of which is viewed by a multilevel discriminator. The lowest of these levels can be set to detect energy deposition just above the noise. Thus in principle there are four possible starting times, and the transversal filter must therefore keep track of four sums. Which of the sums is correct is determined by a window set on the value of the timing function. Studies have shown that adequate amplitude and timing resolution can be obtained with the use of five samples, and the arithmetic can be completed in the time permitted by the Level 1 latency.

\section{Reduction of Pileup Effects}

In the Level 1 logic, we add the signals from the EM and hadronic cells in the fiducial area of a jet sum $(0.64 \times 0.64)$ to form a total $E_{T}$. In addition, a check is made to verify that the waveform is consistent with energy being deposited at the crossing in question. Jet sums in which this criterion is not met are not added to the global sums. This "timing filter" has the property of reducing the effects of pileup, but it also deletes a certain fraction of the energy from the sum. We have simulated the errors introduced by both of these effects by comparing the global quantities with their "true" values obtained by using the fine-grained calorimeter data. We have used a threshold of $20 \mathrm{GeV}$ in the time filter, so only jet sums of $20 \mathrm{GeV}$ or greater are added to the global trigger sum. The rate of $20-\mathrm{GeV}$ jets is approximately 1 per 20 crossings at $\mathcal{L}=$ $10^{33} \mathrm{~cm}^{-2} \mathrm{~s}^{-1}$. The dwell time for the positive lobe of a signal with $t_{m}=70 \mathrm{~ns}$ is close to 20 crossings, so the number of overlapping jets at some position in the calorimeter is of order unity. We have calculated the difference between the $E_{T}$ deposited in the calorimeter and that found in the trigger. We find that the width of the distribution of this difference is about $40 \mathrm{GeV}$ due to the imposition of the jet thresholds. The distribution is broadened by an additional $13 \mathrm{GeV}$ when five jet sums are rejected at random, due to pileup. The mean number of jets contributing to the sum for any one event is about two, and it is only the noise of these jets that contributes to $E_{T}$ in this method. The total width of the $\Delta E_{T}$ distribution is therefore about $42 \mathrm{GeV}$, and this value is essentially luminosity independent. The estimated noise obtained by simply adding all of the cells in the calorimeter would be $54 \mathrm{GeV}$ at $10^{33}$ and $128 \mathrm{GeV}$ at $10^{34}$.

As is the case for the total $E_{T}$, we add the signals from the EM and hadronic cells in the JET sums, and to form $E_{T}$, we multiply this quantity by the (signed) values of either the sine or cosine of the azimuthal angle of the center of the fiducial area, in order to form the appropriate moments. The timing filter is also applied to this sum, so there is no contribution to the missing energy if the deposited energy in the JET sum falls below $20 \mathrm{GeV}$. We have also caiculated the difference between $E_{T}$ found in this way from that found by using the information in the individual cells. The width of this distribution is considerably smaller than that for the $E_{T}$ distribution, since the deposited energy is multiplied by coefficients of both sign to form the moments. Hence the losses can be of either sign and have a tendency to cancel. The width of the $E_{T}$ distribution is broadened by $21 \mathrm{GeV}$ due to the imposition of the jet energy threshold, and the loss due to pileup broadens the distribution by an additional $10 \mathrm{GeV}$. Taking into account the smaller effects of geometri- 
cal quantization of the energy of the jet and the noise in the jet sums, we arrive at a total width for the $\Delta E_{T}$ distribution of $25 \mathrm{GeV}$, and like the $E_{T}$ sum it is expected to depend only weakly on luminosity.

\section{EM, SP, and JET Identification at Level 1}

The presence of an electron or photon is signaled by an EM sum which crosses a discriminator threshold. Two isolation requirements can be applied at Level 1:

1. Hadronic isolation. None of the four $H A D_{1}$ sums (covering an area of $\Delta \eta \times \Delta \phi=0.64 \times$ 0.64) nearest the hit EM sum exceeds a low threshold (typically $5 \mathrm{GeV}$ ).

2. Lateral isolation. Lateral isolation can be defined in terms of (a) the EM sums, which are located within the same SP fiducial area as the hit sum; (b) the individual EM sums, which are located within the eight neighboring SP sums in a $3 \times 3$ matrix centered on the hit sum; and (c) the eight neighboring SPem sums. The exact isolation criterion can be defined through the use of combinatoric logic in field prograinmable gate arrays, which can be loaded under software control. This will permit adjustment of the lateral isolation region up to a size of $0.96 \times 0.96$. (Figure 7-4). Typically, isolation thresholds of $1-3 \mathrm{GeV}$ will be set.

The output of the EM discriminators is a priority encoded 3-bit digital word, indicating the highest threshold crossed. The isolation logic on the Level 1 summing board produces a 4-bit code, which could be simply the 3-bit discriminator information with an additional "topological" bit indicating isolation. However, the code, specified in software, could also be more complex, defining up to 15 categories of combined amplitude and isolation criteria.

The Level 1 logic on the SP discriminators is similar to that of the EM discriminators. The isolation information provided for the EM signal can also be used, if desired, to define an isolation requirement for the SP hit. The digital SP discriminator also produces 3 bits of amplitude formation, and the logic produces a 4-bit code, which may be defined independently of the code for the EM signals.

The Level 1 JET logic utilizes analog summing of the individual SP sums, followed by flash digitization, in order to perform digital filtering to reduce the effect of out-of-time hits, as explained above. The JET data that are sent to the counting logic are provided by either table lookup or gate array logic (or both). The information is presented as a 4-bit code, similar to the EM or SP data, but defined independently. It can contain amplitude information, information on the SP hit pattern within the JET, and timing information produced by the transversal filter.

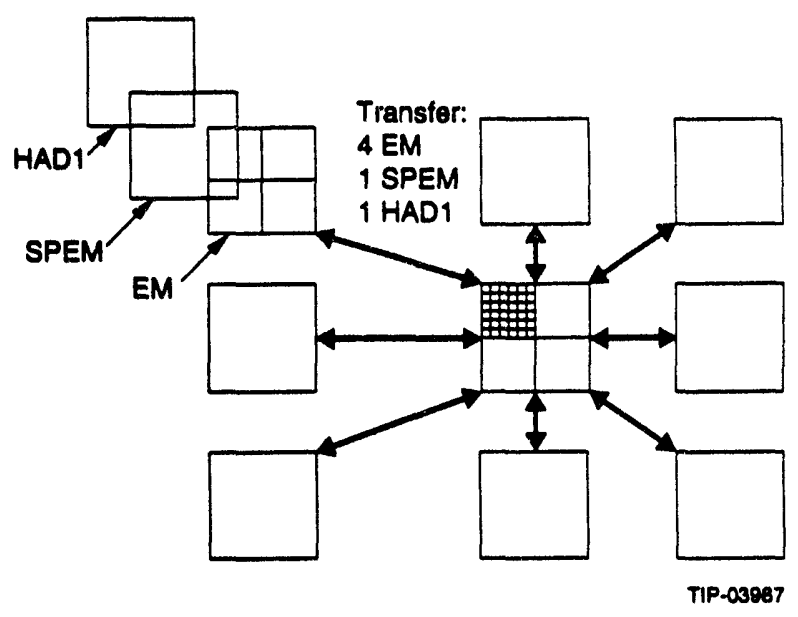

FIG. 7-4. IIlustration of Level 1 isolation requirement. Each large square corresponds to the Level 1 summing board in each crate of front-end electronics. The hit EM sum is the cross-tiatched box in the center. The empty boxes in the center are the neighboring EM sums within the same SP fiducial area. Six discriminator signals (1 level only) are transferred between the central module and each of its eight neighbors: the four individual EM, the SPOm, and $H A D_{1}$, in order to define the isolation requirement. The isolation logic is located on an FPGA mounted in each Level 1 summing module.

\section{Count Logic}

The number of clusters of the various types (EM, SP, JET) above each threshold must be determined for the Level 1 primitives. The counting of clusters will be done through the use of field programmable gate arrays (FPGAs), which can be loaded under software control, so the logic can be easily altered. One way fast cluster counting can be done is by two-dimensional edge counting, which is illustrated in Figure 7-5. Once the pattern of discriminator hits is determined, the number of non-contiguous hits is obtained for each threshold. It is sufficient for counting purposes for each discriminator hit to veto its neighbors along a particular sense in each of four directions (vertical, 
horizontal, and the two diagonals). The number of cells that survive these vetoes is the number of non-contiguous hits. The results of the counting, carried out on a matrix of size $4 \times 4$, are added in a digital summing tree using 4-bit arithmetic. It is possible to form, for each of the discriminator types (EM, SP, and JET) up to eight counting sums using the 4-bit code defined in the local logic producing each of the signals. The definition of these sums is also carried out under software control through the bit pattern loaded into the FPGA.

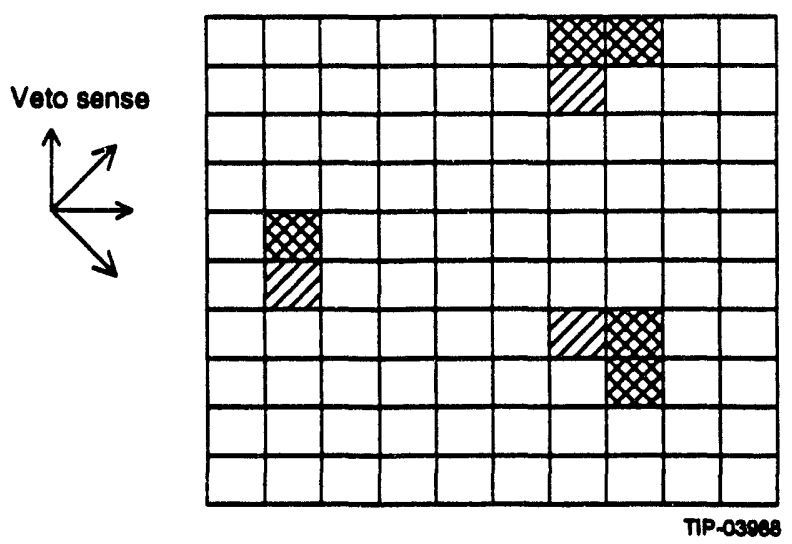

FIG. 7-5. Diagram illustrating the edge counting technique. Each box represents a discriminator, arranged like the physical cells. To count the number of non-contiguous hits, each discriminator vetoes its neighbor in the sense shown. In the drawing, the shaded boxes are counted and the cross-hatched boxes are vetoed. In this case, three hits are counted.

\section{Physical Layout}

The Level 1 calorimeter logic is physically integrated with the calorimeter front-end electronics. The EM and SP sums are formed from signals within a given crate, with the discriminators being placed on the same board as the summing and shaping amplifiers. The arrangement of the frontend electronics crates corresponds closely to the physical calorimeter cells, so the nearest-neighbor topological log can be done with short cable runs to the nearby crates. All of the combinatoric logic will be done in FPGAs, which will allow for reconfiguration for testing etc. under software control. The JET analog sums are also formed from nearby crates, so these cable runs can also be kept short. Because of the desirability of avoiding copper connections between the experimental hall and the electronics counting $\mathrm{rnn}_{7}$, the output of the discriminator-FPGA logic and we digital value for the JET transversal filters will be converted to optical signals for transmission to the global Level 1 logic in the electronics room.

\section{Latency Considerations}

It is expected that the analog signals will arrive at the front end electronics approximately $300 \mathrm{~ns}$ after the event $(t=0)$. Due to the peaking time of the signal and the integration time of the timing discriminator, the logical signals are available at $t=550 \mathrm{~ns}$. The most time-consuming part of the calorimeter Level 1 logic is the formation of the global sums. (Estimates for the EM and SP logic indicate that these signals will be available about 30 ns ahead of the global sums.) The time critical path for the global sums is the formation of the JET sum, which is estimated to be completed at about $t=700 \mathrm{~ns}$. Assuming an optical suber run of $80 \mathrm{~m}$ to the electronics room, and accounting for an additional processing time of $200 \mathrm{~ns}$, we estimate that the global sum signals will be present at the global Level 1 logic at $t=1300 \mathrm{~ns}$. The run back to the front-end electronics will require approximately $600 \mathrm{~ns}$, assuming a $120 \mathrm{~m}$ fiber. Thus if the global Level 1 logic completes its processing in $100 \mathrm{~ns}$, the Level 1 trigger will arrive at $t=2000 \mathrm{~ns}$. Note that only $450 \mathrm{~ns}$ is consumed in the actual logic, with the remainder being taken up by the cable runs ( $1300 \mathrm{~ns}$ ) and the time for shaping and discrimination (250 ns).

\section{Digital Processor Option}

Another option, using digital processors, is also being pursued as a possible solution for the Level 1 calorimeter trigger. The current R\&D is concentrating on a special "3D-Flow" processor. This unit is a data-driven processor that would be used in a two-dimensional array, corresponding to the calorimeter segmentation, for high-speed identification of topological triggers associated with energy deposition in the towers. Feasibility studies demonstrate that with present technology a chip consuming $1 \mathrm{~W}$ and accommodating 4 processors at a $120 \mathrm{MHz}$ clock rate can be built. The 3D-Flow processor is a general-purpose programmable data stream pipeline device allowing fast data movements in six directions with digital signal-processing capability. It is suitable for digital filtering (e.g., transversal filters) and pattern recognition (identifying particles such as electrons, jets, etc.) in trigger applications. 
The main features of the 3D-Flow parallel processing system are its programmability, scalability, high bandwidth communication, and its cylindrical physical layout. These features enable design of a trigger that can satisfy current GEM requirements and allow growth into future physics by accepting new threshold sets, implementing revised and optimized algorithms, and by incorporating hardware advances with little effect on the installed system. Scalability allows incremental upgrading of the 3D-Flow system by adding elements to a previous simpler system with fewer pipeline stages. The high communication bandwidth allows fast data exchange between neighboring elements. The cylindrical geometry also promotes high-speed communication and lor power consumption by providing short length signal paths between neighboring elements. In the 3D-Flow pipelined parallel-processing system, each processor executes the algorithm of pattern recognition entirely. Distribution of the information sent by the calorimeter, and the flow of results to the output are controlled by program sequence instructions rather than from a multiplexer.

\section{Description of Programmable Digital Level 1 Trigger}

The digital Level 1 trigger hardware array would be located in the electronics room (ER). Digitized trigger sum signals ( $4 \mathrm{EM}$ signals, $H A D_{1}$, and $\mathrm{HAD}_{2-4}$ signals from a calorimeter area of $0.32 \times 0.32$ ) arrive from the calorimeter readout boards through optical fibers approximately $850 \mathrm{~ns}$ after the beam crossing at the rate of $60 \mathrm{MHz}$. The signals arriving from the calorimeter are converted from optical to electrical, are processed in the 3D-Flow system and then sent to the global Level 1 trigger. A schematic view of the system is presented in Figure 7-6 where one section of the calorimeter is connected to the first stage of the processor array.

The program execution at stage 1 must not only route the new incoming data from the calorimeter to the next stage in the pipeline staging (stage 2), but must also execute its trigger algorithm. It then sends its results to the stage 2 processor. At this point the stage 1 processor begins to re-execute its algorithm, receiving th electromagnetic and hadronic values from the calorimeter and processing those values. The output results from all processors flow (like the input data) through the different processor stages.
The last processor will output the results from all processors at a rate of $60 \mathrm{MHz}$. More than one operation can be executed in one 3D-Flow instruction cycle. The main functions that can be accomplished by the parallel processing system are:

- digital filtering on the incoming data related to a single channel.

- pattern recognition using information communicated from/to neighboring channels, with no boundary limitation.

- data tagging, counting, adding and moving data between processor cells in order to gather the information of an area of processors to be sent to the next electronic stage.

Several Level 1 trigger algorithms, including those involving digital filtering, have been simulated on the ITT-Data Wave simulator. System layout and program examples with their results are reported in SSCL-576 3 and SSCL-6074. These examples illustrate how to implement the digital filter and local maximum, cluster-finding, and particle identification algorithms. The routing of the data between cells has been checked with a simulator, to verify that there is not a dead-lock in the communication. Examples of programs that gather information from an area of $8 \times 8$ processors (or trigger towers) are also given. In Figure 7-6b), the function of calculating $E_{T}, E_{T}-\mathrm{X}, E_{T}-Y$ and counting EM, SP and JET's is done partly by the 3D-Flow and partly in the boards of ALUs (or FPGAs) shown in the right of the figure.

\section{Overview of 3D-Flow Processor Array (including real-time (roubleshooting and event tagging)}

The 3D-Flow operates on a data-driven principle. Program execution is controlled by the presence of the data at five ports (North, East, West, South, and Top) according to the instructions being executed (See Figure 7-6). A clock running at a frequency of $120 \mathrm{MHz}$ synchronizes the operation of the cells. At each input port of the 3D-Flow processor there is a FIFO that derandomizes the data from the calorimeter to the processor array. North, East, West, and South ports are 12-bit parallel bi-directional on separate lines for input and output, while the Top port is 12-bit parallel input only, and the Bottom port is 12-bit parallel output only. 


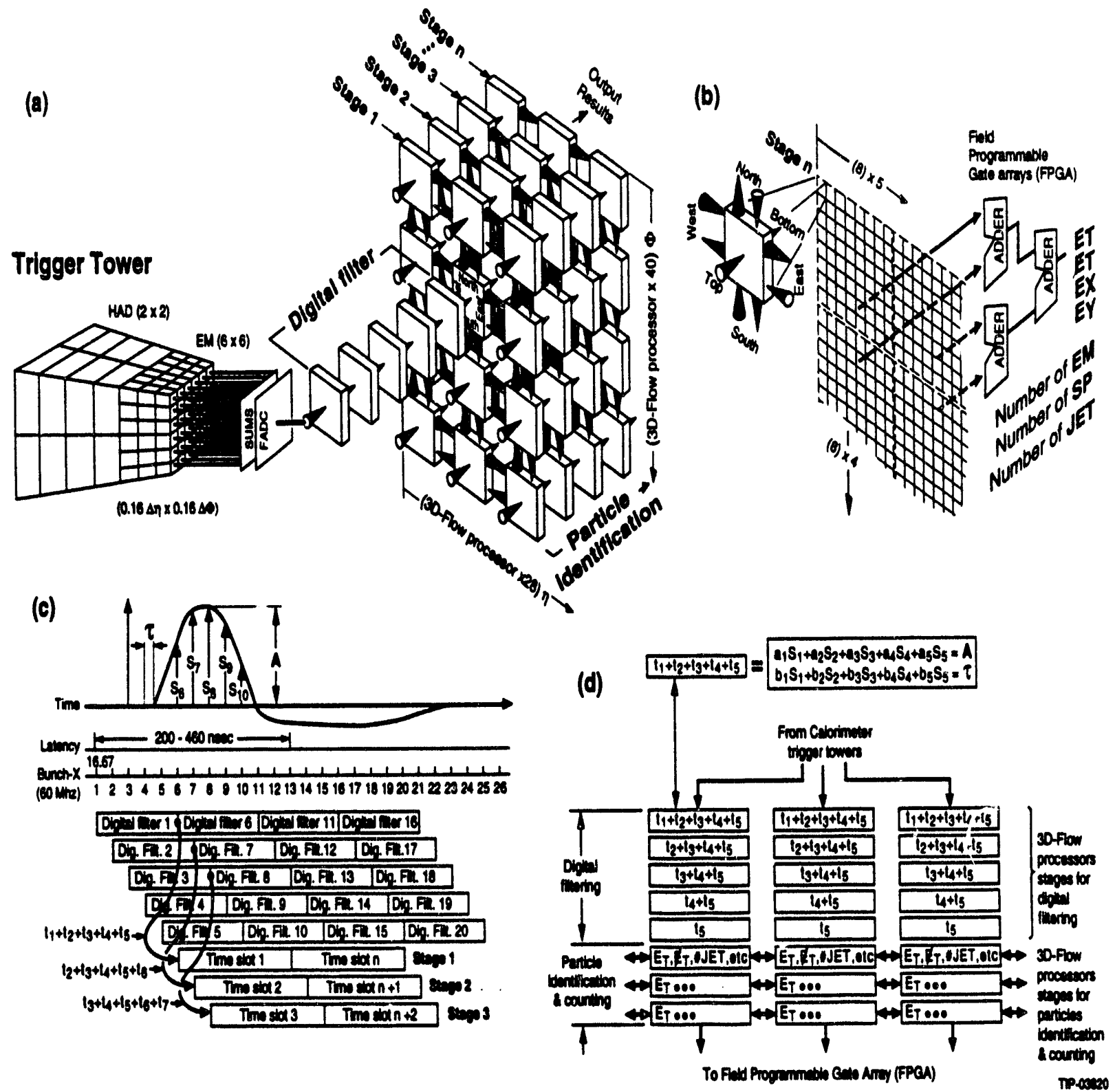

FIG. 7-6. a) Connection between the calorimeter trigger towers and the 3D-Flow array after analog summing and digitization by the FADCs. b) Calculation of the superblock and global information. Each processor is connected to its neighbors as in the physical layout of the elements of the calorimeter. In order to calculate the global sums, the 3D-Flow processor array is segmented into $8 \times 8$ superblocks and the results for a given block are routed to a single processor that becomes the exit point for that block to the gate array system. C) Timing of the pulse samples and the processor calculations. The first five stages of the 3D-Flow processor array are used to apply the digital filter algorithm. Each subsequent array stage has a time slot for algorthm execution of the data from a single event. d) Flow of the data among the 3D-Flow processors. As in c), the processing is broken into two parts for applying the digital filtering and for doing the particle identification and counting. 
The North, East, West, and South ports are used to exchange data between adjacent processors belonging to the same array stiige. Each processor has a one-to-one correspondence to a physical trigger tower (A trigger tower is a $6 \times 6$ individual $\mathrm{EM}$ tower $+\mathrm{Had}_{1}, \mathrm{Had}_{2-4}, \mathrm{co}$ sponding to $\Delta \eta \times \Delta \phi$ area of $0.16 \times 0.16$ ). Top ar. sttom ports are used to route input data and output results between stages under program control.

For diagnostic purposes during real-time operation of the system, each processor is provided with a presettable counter at each of the five input ports. The counters are preloaded (with the number of expected input data) between the data set of different events and before algorithm execution. If at the end of the execution of each algorithrn a:l counters are non zero (e.g., missing connection), a data-flow error is issued at the bottom output port of that particular processor.

An additional internal counter (counting the beam crossing) is provided in the 3D-flow system in order to be able to associate each event with a beam crossing. A precise timing measurement on the shaped signal is also necessary. This can be done with an external circuit, or the timing information can be obtained by the digital filter calculation internal to the 3D-flow processor. (See Figure 7-6c) and d)) (Examples of digital filters with 3D-flow or FEP are reported in SSCL-576). Any additional counters needed in the 3D-flow processor can be implemented by using a data-memory location.

Each 3D-flow cell consists of a multiply accumulate unit (MAC); arithmetic logic unit (ALU); a register file; an interf. ce to the universal asynchronous receiver and transmitter (UART) used to load programs and to debug and monitor during their execution; a data-memory to be used also as look-up table to linearize the compressed signal, to remove pedestals, and to apply calibration constants; and a program storage surrounded by a system of three ring buses. At each clock, a three-ring bus system allows input data from a maximum of two ports and output to a maximum of five ports. During the same cycle, results from the internal units (ALU, etc.) may be sent through the internal ring bus to a maximum of five ports. Figure 7.7 shows a schematic of the electronics for the digital Level 1.

\subsubsection{Muon Level 1 Trigger}

In this section we discuss the concepts and implementation of the muon Level 1 trigger. The muon chambers provide both measurement and trigger information, hence this section should be read in conjunction with the muon front-end electronics section (Section 7.7).

\section{Introduction}

The purpose of the muon Lavel 1 trigger is twofold:

- Identify events having one or more muons above a set of adjustable $p T$ thresholds.

- Identify the beam crossing responsible for the generated trigger.

The calorimeter thickness has been chosen so that the rate of single particle triggers in the muon system is dominated by the direct muons from the interaction point, as well as the decay muons generated primarily in the central tracker cavity (See Section 4.2.2).

The charged particle rate outside of the calorimeter is dominated by muons, leaving the imposition of a geometrical $p_{T}$ cut as the primary means of reducing the Level 1 muon trigger rate. An ideal $p_{T}$ cut would provide full efficiency above and complete rejection below the selected threshold. In practice, neither condition is satisfied, an effect often referred to as trigger resolution. The optimization of the muon Level 1 trigger involves improving this resolution without significantly increasing the hardware complexity or the trigger formation time.

The muon Level 1 trigger hardware is capable of measuring the $p_{T}$ using the two methods shown in Figure 7-8.

- $A \delta \phi$ cut based on just the last two superlayers. This method identifies the difference in location between the second and third superlayer and extrapolates an effective $p_{T}$ for the potential muon, assuming it originated at the interaction point. 
(a)
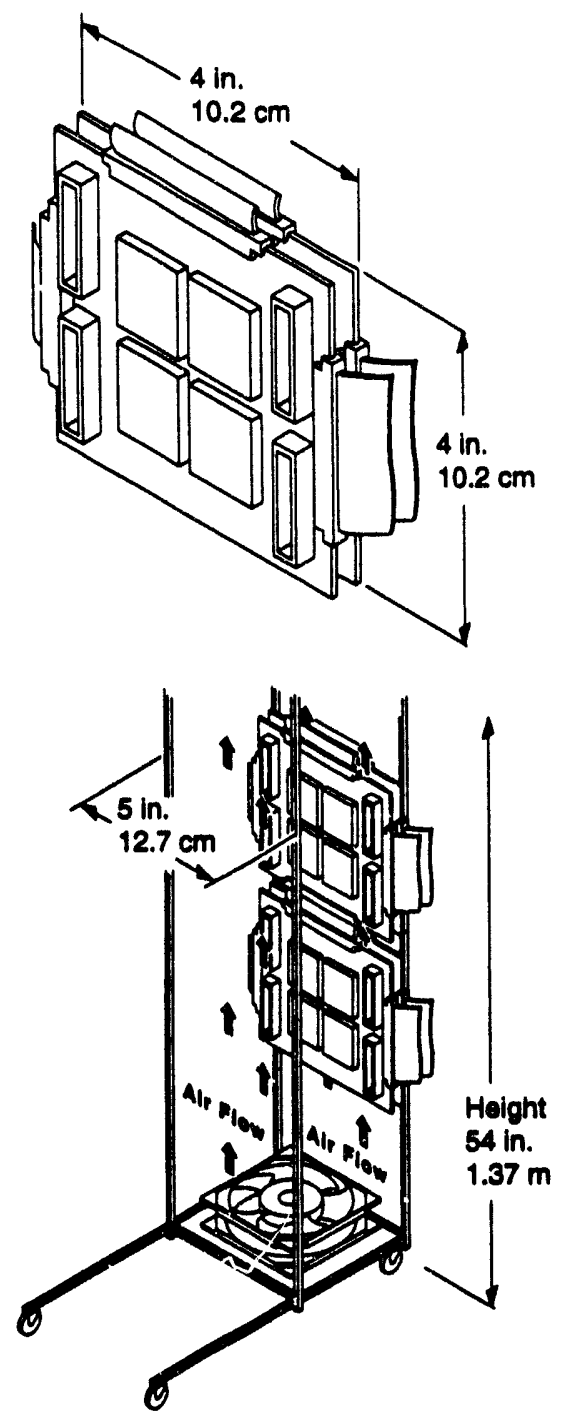

(b)

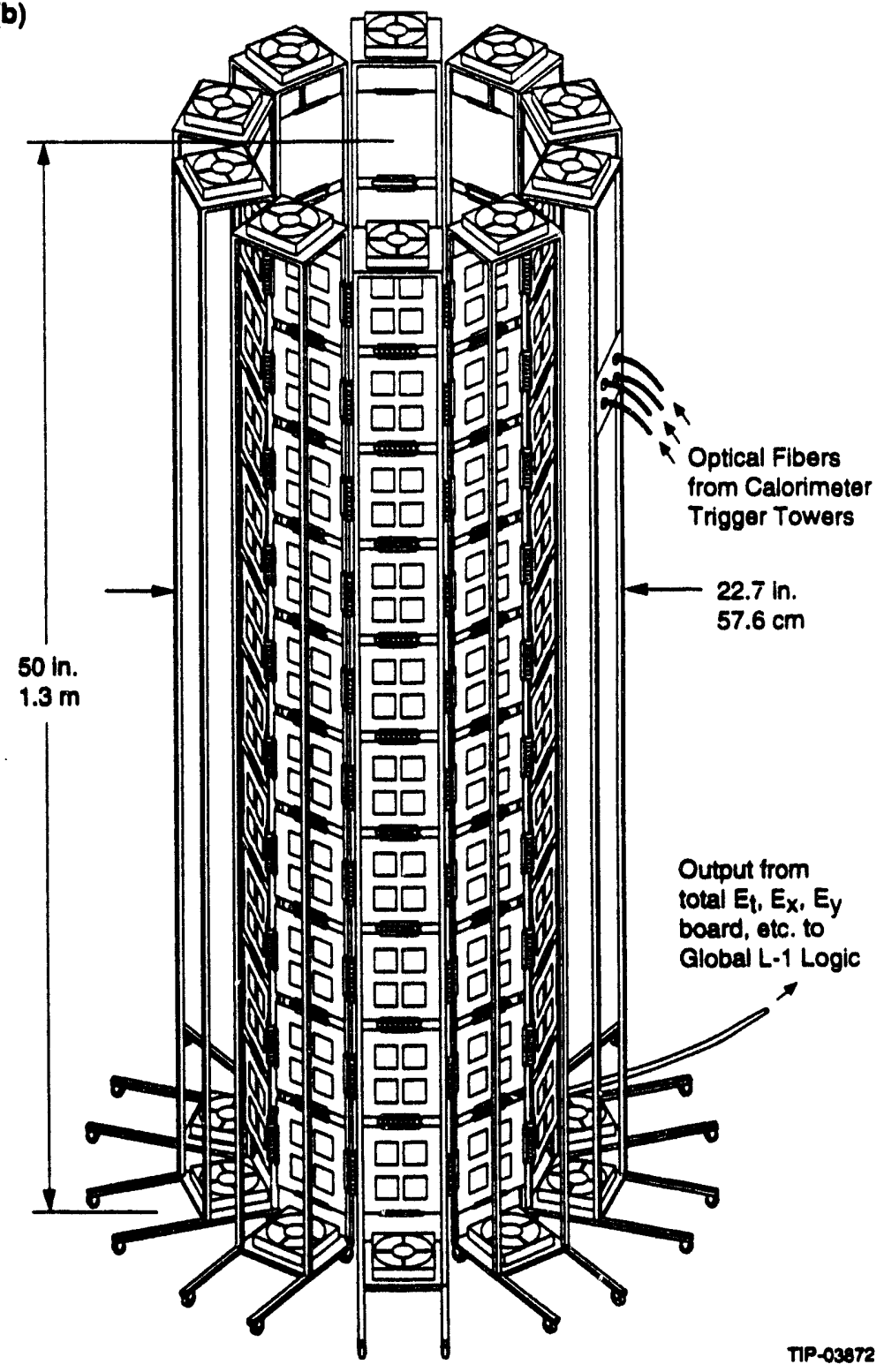

FIG. 7-7. a) Details of the 3D-Flow programmable processing system crate assembly. b) The layout and dimensions of the complete digital Lovel-1 trigger system.

- A sagitta method that uses all three superlayers. This method forms a line between the first and third superlayers and searches for $a$ hit in the second superlayer within a certain sagitta distance, which determines the muon $p_{T}$.

The $\delta \phi$ method uses slightly less hardware and is insensitive to the rate in the first superlayer, which is the one most affected by the calorimeter-generated rate. The sagitta method, however, is insensitive to multiple scattering in the calorimeter, and provides better trigger resolution, resulting in an overall trigger rate that is lower than the $\delta \phi$ method.
Additional protection against accidental triggers is provided by examining the non-bend-plane view, where bona fide muons will follow straightline trajectories. Since any realistic implementation of the non-bend plane trigger will involve the use of finite-size cells, boundary crossing will occur, which must be accommodated in the design. 
The baseline hardware design will support both the $\delta \phi$ and sagitta methods, as well as a non-bend plane trigger. In forming the trigger, the actual hit strip number will be extrapolated from a local track segment identified by each superlayer using " $n$ of 6" logic. With $n \geq 4$, this method will reduce the rate of fake hits from neutrons and photons to a level well below that of the charged particles.

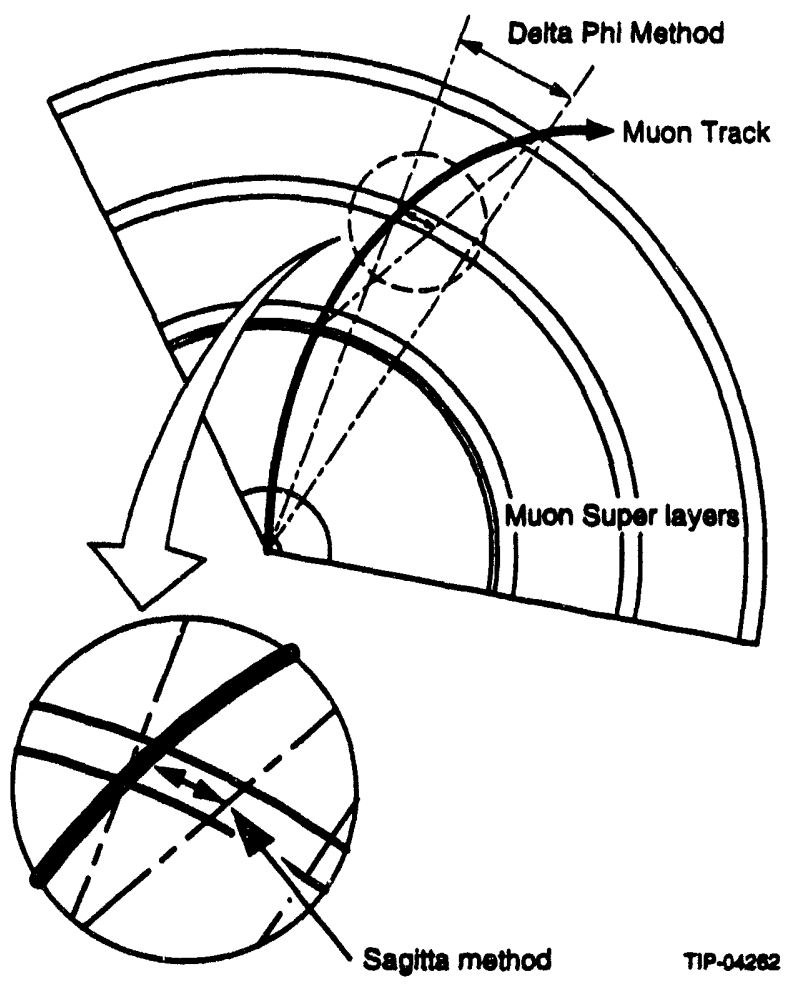

FIG. 7-8. The $\delta \phi$ method and the sagitta method for muon triggers.

\section{Trigger Element Definition}

The geometrical nature of the Level 1 trigger dictates a hodoscope-like structure for the basic muon trigger elements. The GEM muon chambers are designed specifically to accommodate the Level 1 requirements, and hence the hodoscope elements correspond to physical segments of the chambers. This allows the Level 1 to be generated quickly with no remapping of the basic chamber signals.

The trigger system is segmented into supertowers that are projective in both the bend- and non-bend-plane views. The trigger supertowers generally correspond to chamber modules, with the exception of the modules of the inner superlayers, which participate in two supertowers each, as shown in Figure 7-9. The trigger supertowers are subdivided into strip-like elements in both views. In the $\phi$ view, the trigger elements correspond to two strips in the barrel and one strip in the endcap. The finer segmentation in the endcap is driven by the need for better trigger resolution in the face of reduced bending power (for fixed $p_{T}$ ) and intrinsically higher rates. In the non-bend view the trigger elements correspond to anode wire groupings. The size and number of the trigger supertowers and elements is summarized in Table 7-5.

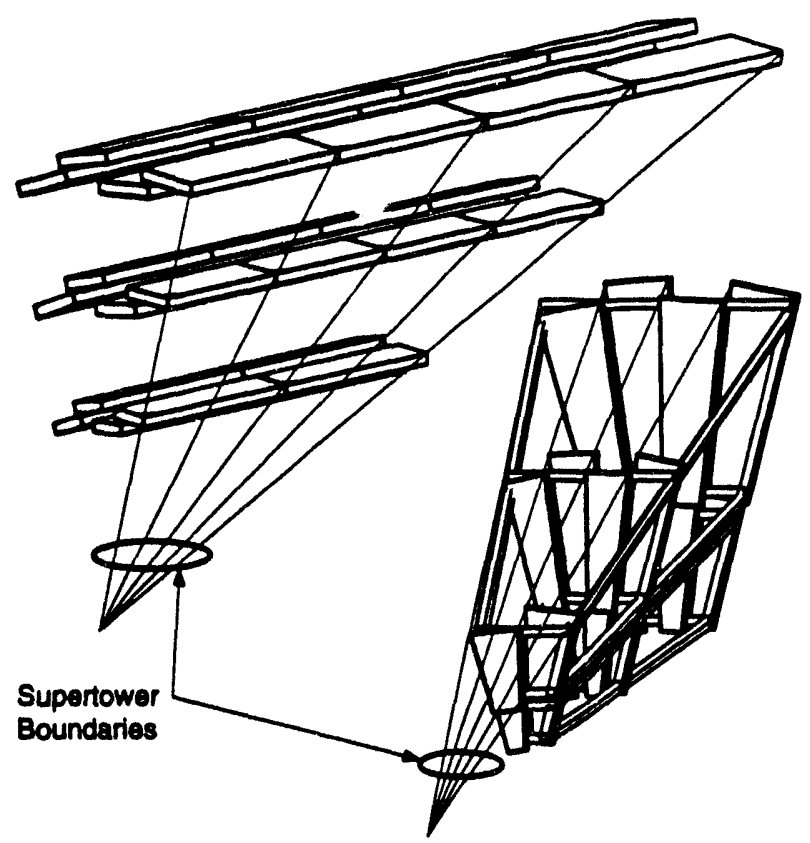

nP-0.4206

FIG. 7-9. Muon trigger supertower segmentation for the barrel and the endcap. The chamber modules in the inner superlayers participate in two supertowers each. 
Table 7-5. Trigger elements count.

\begin{tabular}{|c|c|c|}
\hline & Barrel & $\begin{array}{l}\text { Endcap } \\
\text { (each) }\end{array}$ \\
\hline $\begin{array}{l}\text { Number of trigger } \\
\text { tuwers } \\
\text { (bend plane/non- } \\
\text { bend plane) }\end{array}$ & $48 / 8$ & $48 / 2$ \\
\hline $\begin{array}{l}\text { Number of trigger } \\
\text { transmission cables }\end{array}$ & 1152 & 288 \\
\hline $\begin{array}{l}\text { Total number of effec- } \\
\text { tive trigger elements } \\
\text { per superlayer } \\
\text { (bend plane/non- } \\
\text { bend plane) }\end{array}$ & $2688 / 256$ & $2304 / 64$ \\
\hline $\begin{array}{l}\text { Number of physical } \\
\text { cathodes per trigger } \\
\text { strip in the bend } \\
\text { plane }\end{array}$ & 2 & 1 \\
\hline $\begin{array}{l}\text { Approximate element } \\
\text { size and } \phi \text { coverage } \\
\text { per effective ele- } \\
\text { ment }\end{array}$ & $\begin{array}{l}1.17,1.67 \text {, } \\
2.14 \mathrm{~cm} \\
\text { for } 5 L 1.3 \text { (or } \\
2.3 \mathrm{mrad} \text { ) }\end{array}$ & $\begin{array}{c}0.5,0.8 \text {, } \\
1 . \mathrm{cm} \text { for } \\
\text { SL1-3 } \\
\text { (or } 2.7 \mathrm{mrad} \text { ) }\end{array}$ \\
\hline $\begin{array}{l}\text { Approximate element } \\
\text { size and } \mathbf{z} \text { (or } \eta \text { ) } \\
\text { coverage per effec- } \\
\text { tive element }\end{array}$ & $\begin{array}{l}-0.01 \text { units } \\
\text { of } \eta\end{array}$ & $\begin{array}{c}\sim 0.007 \text { units } \\
\text { of } \eta\end{array}$ \\
\hline $\begin{array}{l}\text { Total Number of avail- } \\
\text { able effective trigger } \\
\text { elements in the sys- } \\
\text { tem (bend pland } \\
\text { non-bend plane) }\end{array}$ & $8604 / 768$ & $6912 / 192$ \\
\hline
\end{tabular}

\section{Muon Level 1 Trigger Implementation}

The implementation of the Level 1 trigger is closely linked to the overall electronics design of the CSC readout chain. The trigger hardware is located in two separate locations: on the chamber and in the electronics room. The placement of the various components represents a tradeoff between the conflicting goals of minimizing cable plant and maintaining easy access for repair. To this end, the more complex, and hence less reliable, circuit elements have been located in the electronics room, while a small amount of encoding circuitry is located inside the detector. Figure 7-10 shows a schematic view of the general trigger hardware location.

There are two distinct methods for forming the necessary geometrical trigger cuts. The first method employs content-addressable-memory (CAM) look-up tables to select combinations of track segments satisfying the required $p_{T}$ thresholds. The second method uses combinational logic (i.e., sim- ple arrays of AND and OR gates) to detect abovethreshold trajectories.

The combinational logic approach combines conceptual simplicity with a ready implementation as a pipelined system. However, this approach will increase the number of lines to be transmitted out of the detector, e.g., from 6 to 56 lines for a single barrel trigger module. With the increased cable plant comes an increased number of off-detector trigger modules (each cable needs to connect to something) and an increased number of interconnects. For these reasons, the CAM approach has been adopted as a baseline. The process of understanding the relative merits of the two schemes is not yet complete, however, and a systematic comparison is ongoing.

\section{On-Chamber Trigger Electronics}

The front-end-specific aspects of the on-chamber electronics are described in detail in Section 7.\%. This subsection deals with the trigger-specific portions of that electronics. The on-chamber trigger electronics is located on the readout printed circuit boards (ROPCBs) for each CSC chamber module. This electronics collects the local charge track (LCT) signals from the cathode and anode front-end printed circuit boards (FEPCBs), combines them, and transmits a reduced set of signals to the off-chamber electronics.

\section{Cathode}

The cathode LCT signals correspond to track segments and are formed by imposing a four-out-ofsix majority logic requirement on the discriminated outputs of the strips from all six gaps lying within a small angular range (corresponding to approximately two strips in the barrel and one in the endcap). The discriminators are driven by the cathode fast shapers, whose outputs are typically $30 \mathrm{~ns}$ wide. There are 56 LCT signals for each barrel CSC module, and between 96 and 256 LCT signals for each endcap module. The majority logic will be implemented either digitally or by using analog current sums coupled to a comparator.

In the baseline design (the "CAM" option) the LCT patterns from each chamber module are encoded into a list of numbers corresponding to the strips that are struck. This requires six bits per address in the barrel and seven or eight bits in the endcap. The address lists are "packetized" and time stamped with a beam crossing identification using 

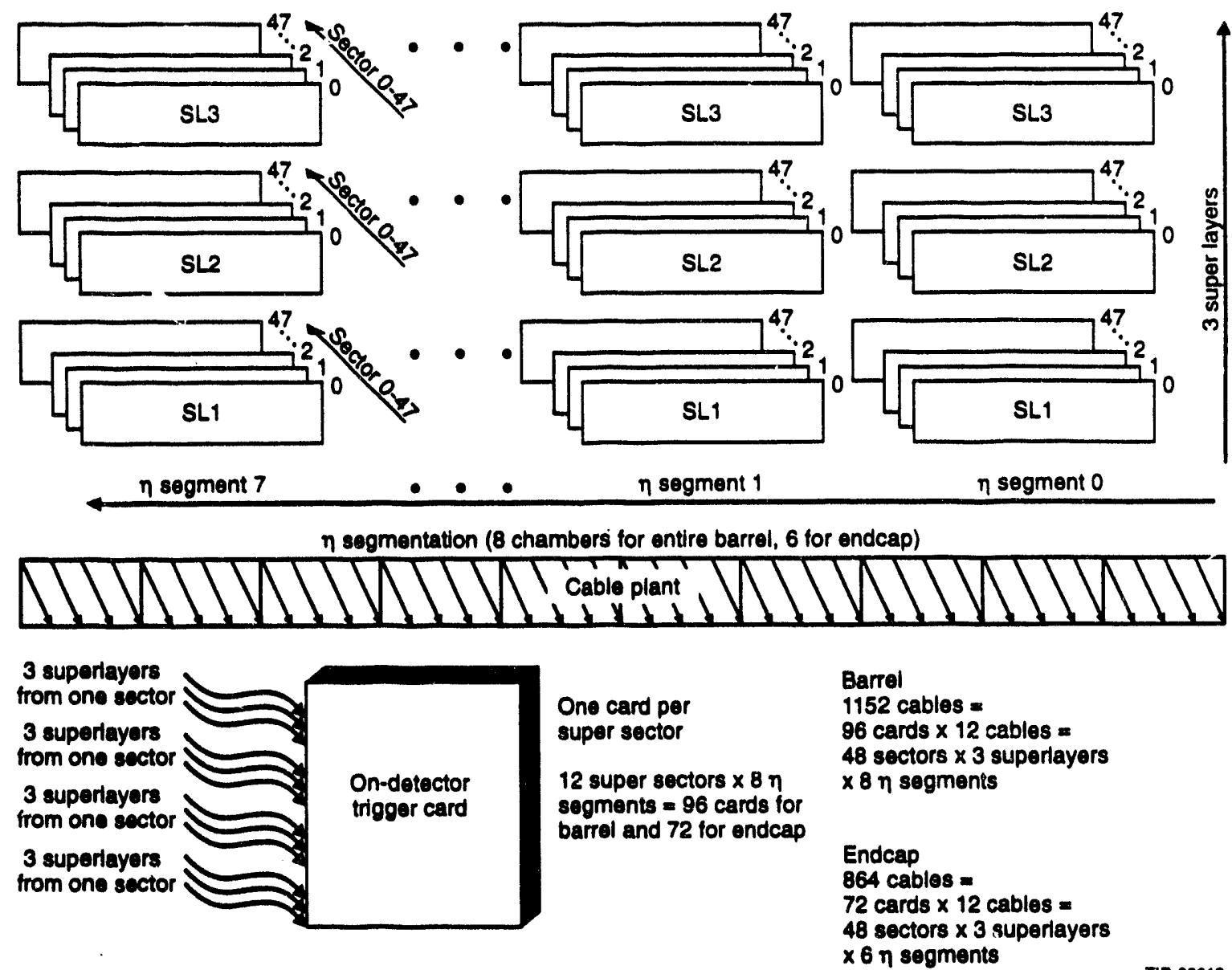

FIG. 7-10. Schematic view of trigger hardware.

timing information from the anode signals and then transmitted to the electronics room via fiber-optic links. Alternatively, the timing information could be carried implicitly by the arrival time of the pulse packet. In either case, there is one such link for each chamber module, with the exception of the modules of the inner superlayers, which will drive two trigger supertowers each. This results in a total of 1152 links for the barrel and 288 links for each endcap. The maximum number of track segments (i.e., asserted LCT bits) is $\mathbf{5 6}$ for the barrel modules and up to 256 in the endcaps.

\section{Anode}

The anode trigger electronics provide the beam crossing time of the hits used to form the bend plane trigger signals (cathode). Additionally, the anode signals provide an address for the non-bend-plane trigger.
The signals from each wire grouping are amplified and shaped, before being sent to a constant fraction discriminator. The discriminator outputs from each of the gaps within a superlayer are ORed together to form a first-arrival-time signal. Beam tests with a four-gap chamber, have shown that this method will correctly associate $96 \%$ of the events with the appropriate bunch crossing. A simple extrapolation to a six-gap system yields a $98 \%$ efficiency.

\section{Off-Chamber Electronics}

The off-chamber electronics receive track segment data (LCT addresses) from the on-chamber electronics and use them to form a pr trigger. The choice of hardware will allow for both sagitta and $\delta \phi$ trigger formation. 
The off-chamber electronics reside in the electronics room. The current design has as a basic element a complex digital board servicing 12 trigger supertowers for a total of $1152 / 12=96$ boards in the barrel and $288 / 12 \times 2=48$ boards for the endcaps. The boards reside in crates, each typically housing 20 boards. The off-chamber electronics also includes testing and supervisory boards to generate relevant triggers (such as single, and multi-muon signals) and to perform various housekeeping tasks associated with the Level 1 trigger. Provisions are made for Level 2 trigger hardware, should such a need arise in future upgrades.

As noted above, the baseline logic design is implemented using content-addressable look-up memories. This look-up memory approach significantly reduces the number of interconnects and the cable plant at the cost of a slightly more complex implementation. In addition, the look-up memory approach drives the signals from the on-detector electronics serially, and is therefore not strictly pipelined. However, the rates are such that a typical module will have fewer than five hits to drive off the chamber.

The implementation of the look-up memory could proceed either through a conventional random-access memory (RAM) or a content-addressable memory (CAM). (The CAM is often referred to as an associative memory.) The RAM approach, although straightforward, has been rejected in favor of the CAM technique since the latter requires considerably less space and power.

The trigger is formed by loading the encoded words (LCT addresses) from the three superlayers onto a bus serving the memory cells, as shown in Figure 7-11. Each three-cell column in the memory corresponds to an acceptable trigger pattern. For the usual situation where each superlayer within a trigger tower asserts just one LCT, the processing proceeds as follows. Three LCT addresses (one from each superlayer) are loaded in parallel onto the data

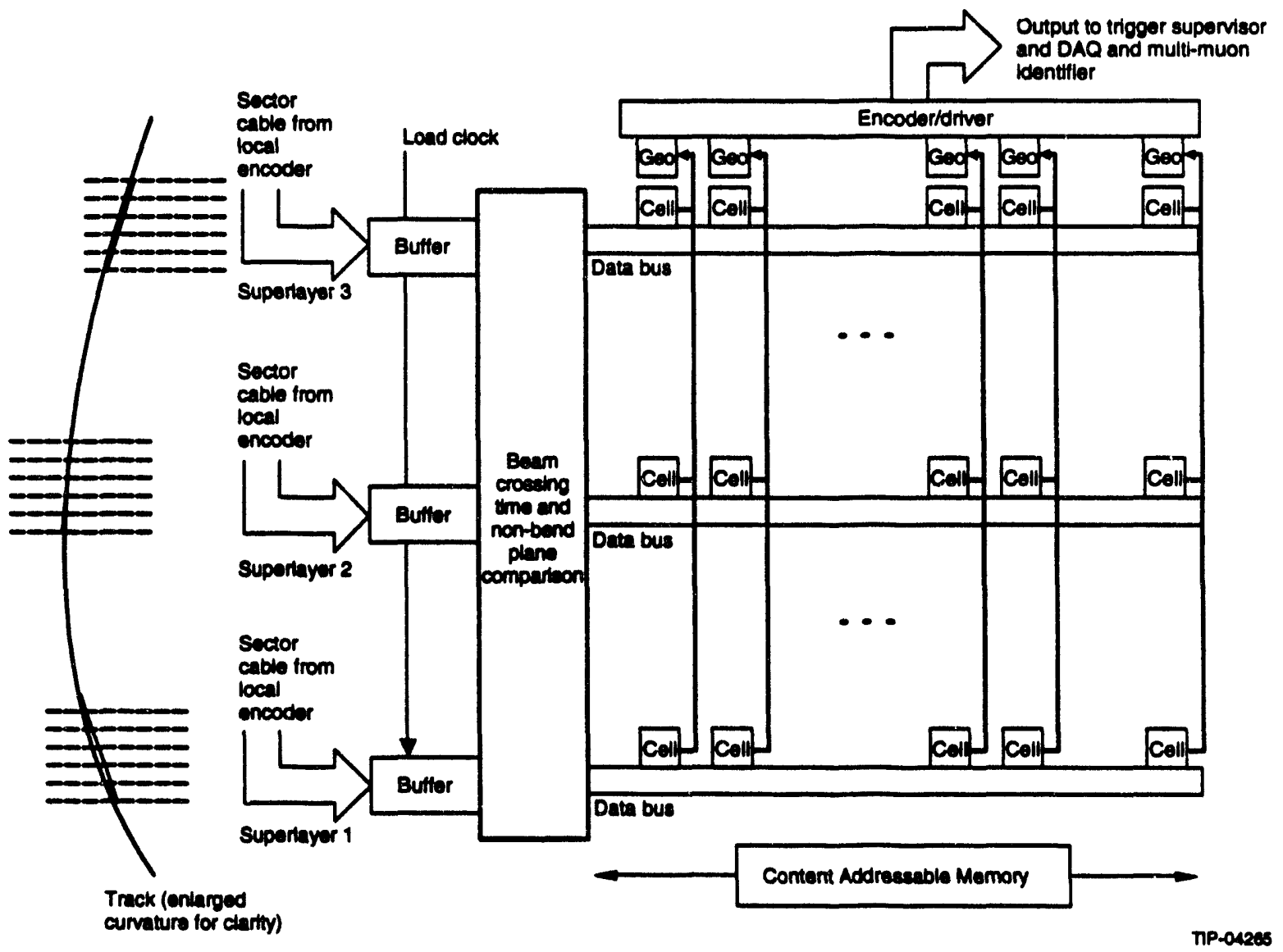

FIG. 7-11. Superlayer trigger formation using associative, or content-addressable memory. 
buses, after which each cell compares its contents to the bus contents and sets a "match" flip-flop if agreement is found. Majority logic (labeled "Geo" in Figure 7-11) detects acceptable column patterns. Selection of either the $\delta \phi$ or the sagitta method can be made by downloading appropriate cell contents and by requiring either two-out-of-two or three-outof-three matches within a column. Finally, the "OR" of the accepted patterns is encoded and transmitted to the trigger supervisor.

If more than one LCT bit is asserted on one or more superlayers, the addresses are loaded in sequence until all input lists are exhausted. The total number of load cycles is equal to the length of the longest input list-i.e., the maximum number of asserted LCTs. Since each detected match is latched, valid combinations will be recognized irrespective of the order in which the addresses are loaded. This is to be contrasted with the conventional RAM approach, where the number of combinations to be checked (and therefore the number of cycles) would be the product of the number of LCTs asserted on each layer.

A fundamental issue in the CAM design is the number of columns, or acceptable patterns, necessary to implement the trigger. A hardware simulation of the CAM scheme has been carried out to address this and other design issues. The simulation allows for determining the number of patterns and estimating a realistic hardware precision. The results of the simulation show that the number of necessary patterns is dominated by the desire to trigger at $10 \mathrm{GeV} / \mathrm{c} p_{T}$. Table 7-6 shows the number of patterns necessary as a function of the minimum $p_{T}$ cut. A preliminary layout of a suitable IC has also been carried out. In a $1.2 \mu \mathrm{m}$ technology, $16 \mathrm{ICs}$ will be required to store the necessary number of patterns.
Table $7-6$. The number of patterns necessary to implement a minimum pr cut.

\begin{tabular}{cc}
\hline PT $(\mathrm{GeV} / \mathrm{c})$ & Number of Patterns \\
\hline 10 & 1618 \\
20 & 1090 \\
30 & 793 \\
40 & 610 \\
50 & 503 \\
60 & 448 \\
\hline
\end{tabular}

\subsubsection{Level 1 Global Trigger}

The task of the global Level 1 trigger is to take the Level 1 information provided by the calorimeter and muon systems and to make the final trigger decision. If an event satisfies one or more of the trigger criteria, a unique pointer is passed to the data acquisition system corresponding to the event of interest.

The calorimeter primitives provided to the global Level 1 logic are:

- The number of clusters of EM, SP, and JET sums above a set of thresholds with possible topological selection. Up to eight conditions per type of sum can be defined, creating a total of 24 four-bit "counting" sums sent to the global Level 1 logic. Thus for example, one could ask for the number of EM sums above four thresholds, with two different isolation criteria. In the JET system one could request the number of sums above two different thresholds, with or without a neighboring hit at another threshold, and with or without the timing requirement.

- The global sums, $E_{T}$ and the square of the $E_{T}$, formed from the JET sums with good timing, are sent as 12-bit digital numbers to the global Level 1 logic. Independent calibration constants for each of the inputs to these sums can be applied.

A summary of the information coming from the calorimetry is shown in Table 7-7. The information consists of the numbers of several classes of objects over each of several thresholds. The muon system simply provides counts of muons exceeding each of several thresholds, a total of seven words of four-bits each. The Level 1 trigger decision will involve arbitrary logic based on this information. 
Table 7-7. Level 1 calorimeter information.

\begin{tabular}{lcc}
\hline Description & $\begin{array}{c}\text { Number of } \\
\text { Words }\end{array}$ & $\begin{array}{c}\text { Bits per } \\
\text { Word }\end{array}$ \\
\hline EM particle counts & 7 & 4 \\
SP particle counts & 7 & 4 \\
Jet particle counts & 7 & 4 \\
Global ET & 1 & 12 \\
Global Ex & 1 & 12 \\
Global Ex & 1 & 12 \\
\hline
\end{tabular}

Figure 7-12 shows a block diagram of the global trigger logic. In general, information from the muon system will arrive before information from the calorimeter. It will therefore be passed through a delay to synchronize it with the calorimeter data. The bulk of the calorimeter data will also be delayed to allow time to form the $E_{T}$ from the $E_{X}$ and $E_{Y}$ primitives. Data will then be passed to the actual logic. However, it will also be stored in a parallel FIFO so that it may be retrieved by the data acquisition system if the event satisfies a trigger.
The trigger logic will be implemented in field programmable gate arrays. The complete information from the calorimeter comprises 136 bits of data and so would be accessible to a single gate array chip. For example, the XILINX 3195 has 176 I/O pins and could easily process pipelined data at $60 \mathrm{MHz}$. It is possible that one such chip is insufficient to perform all the necessary trigger logic. If so, several chips in parallel could be used. Studies are under way to determine how many chips are needed.

Information about which (if any) triggers have been satisfied is passed to the master logic which makes the final trigger decision. It is this logic that also performs any prescaling, if desired. This logic has access to the global crossing clock.

If an event satisfies any trigger, an ACCEPT signal is passed to the data acquisition, along with an encoded trigger word and the crossing number. All of the raw input information will also be passed to the data acquisition, in order to fully reconstruct the trigger decision later.

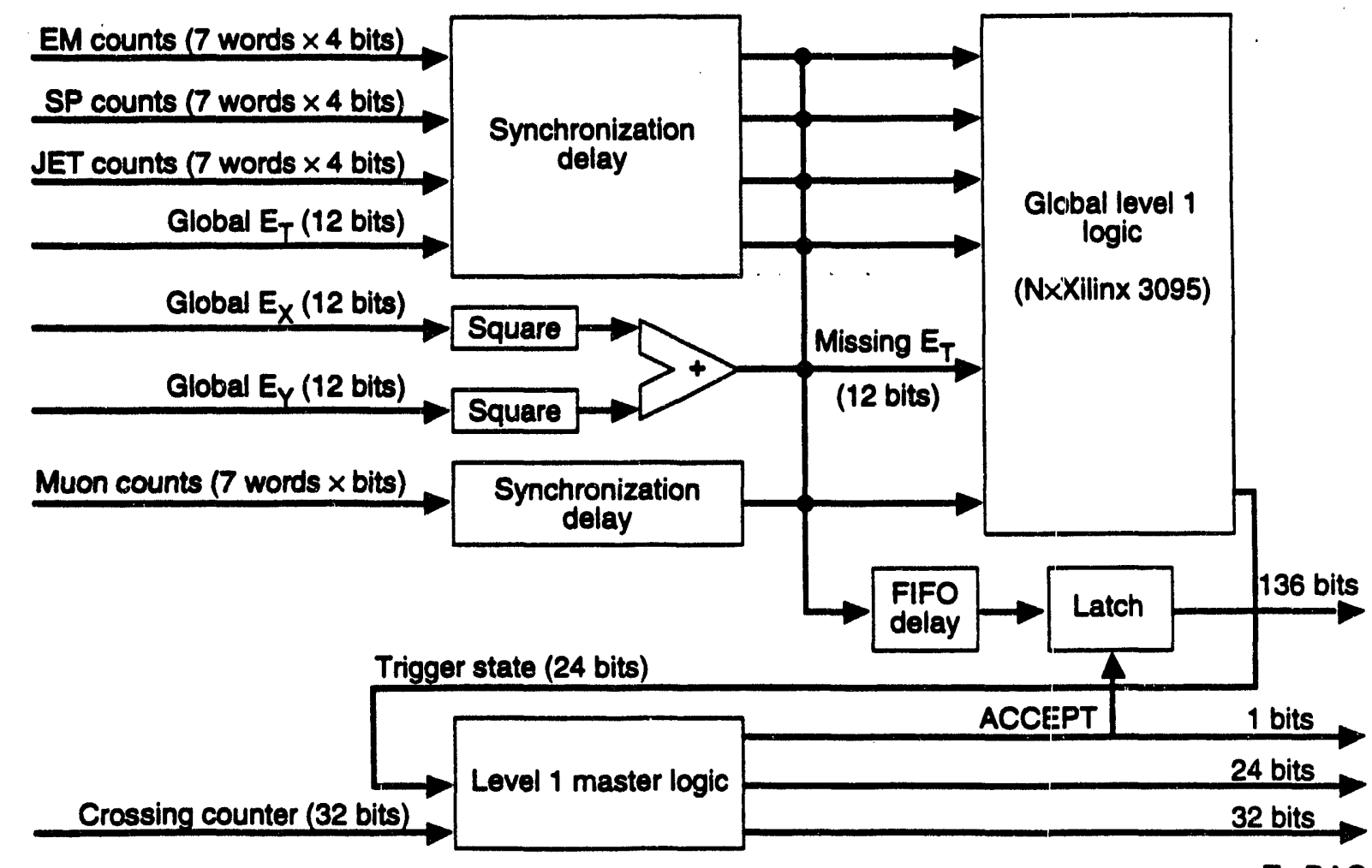

TO DAQ

FIG. 7-12. Level 1 global trigger logic.

TIP-04048 


\section{Physical Implementation}

The global trigger system will consist of the three VME modules. The first, the synchronization module, will perform the necessary delays to synchronize the data for the actual logic. It will also store all the input data so it may be read out if the event satisfies a trigger. The second module is the logic module, which performs the trigger logic. The final module is the master module, which performs any prescaling and associates selected events with the appropriate crossing, and passes that information on to the data acquisition system.

\section{Synchronization Issues}

The most difficult synchronization, namely the synchronization of the data from the hadronic calorimeter with that from the electromagnetic calorimetry will have already been done by the calorimeter Level 1 logic. The calorimeter information is therefore assumed to arrive synchronously at the global logic. As mentioned, the muon information will have to be delayed until the corresponding calorimeter information arrives. As for the logic itself, FPGAs are inherently synchronous in their operation. That is, intemally they are characterized by levels of logic followed by flip-flops. The entire design will therefore run in a synchronous, pipelined fashion, clocked at the beam-crossing rate, with a fixed time between the arrival of the lower level information and the generation of an ACCEPT.

\subsubsection{Rates and Efriciencies of Level 1}

The Level 1 trigger should accept all events from the GEM physics program (see Chapter 2) with sufficient ( $>O(95 \%)$ efficiency while at the same time reducing the total rate into the Level 2 trigger to below $10 \mathrm{kHz}$. This section presents a set of trigger criteria that accept, in principle, all interesting events. The performance of these criteria has been tested for various processes using the GEMFAST simulation codes. It will be shown that both the efficiency for physics and background reduction are close to the required values. A more detailed study of trigger rates and efficiencies can be found in GEM-TN-93-2945 and -332.6

\section{Background Processes}

The following categories of events compose the uninteresting (or non-physics) background. There can also be a physics background (i.e., an event from one interesting physics process is misidentified as an event from another process) but for trigger studies, these are not relevant.

- Minimum bias. At a luminosity of $10^{33} \mathrm{~cm}^{-2} \mathrm{~s}^{-1}$, each bunch crossing will produce, regardless whether or not an interesting physics interaction occurs, an average of 1.6 minimum bias interactions. The trigger should reject those crossings that contain only minimum bias events.

- Interactions between the proton beams and the residual gas in the beam pipe ("beam gas"). A rough estimate ${ }^{5}$ shows that 1 to $5 \%$ of the bunch-crossings will contain a beam gas interaction. Beam-gas interactions will generally deposit energy around the beam pipe and constitute a background to physics processes at high values of rapidity.

- Interactions between the beam halo and off-momentum protons, and the detector and collider elements close to the beam like quadruples, collimators etc. ("beam scraping"). The rate depends on the quality of the beam and is hard to estimate at this stage. This kind of interaction will mainly deposit energy around the beam pipe.

- Cosmic ray muons and muons in the beam halo. (Although cosmic muons should be triggerable for calibration purposes).

The most important source of backgrounds is minimum bias. Therefore, in this study, only backgrounds from this source are considered.

\section{Trigger Primitives}

The following primitives are available for the Level 1 trigger determination:

- Electromagnetic calorimeter $\left(E_{x}\right)$. Isolated electrons and photons. The trigger will determine the number of isolated electrons and 
photons with $E_{T} \geq x \mathrm{GeV}$, where $x$ is a programmable threshold.

- Electromagnetic and hadronic calorimeter.

- Jet candidates or calorimeter towers with a high energy deposit $\left(J_{x}\right)$. The trigger will determine the number of jet-candidates above programmable $E_{T}$-thresholds and their position.

- Global $E_{T} \operatorname{sum}\left(E_{T_{x}}\right)$ and $E_{T}$ measurement $\left(P_{x}\right)$, including the forward calorimeters.

- Muons $\left(M_{x}\right)$. Number of muons with a $p_{T}>x$, where $x$ is a programmable threshold.

Relation between Physics Processes and Trigger Primitives

The signature of every physics process can be written as a Boolean equation in terms of the trigger primitives, giving the trigger conditions for the various processes. A simulation of the global Level 1 trigger can then be obtained by taking the logical "OR" of these trigger conditions. A list of trigger criteria for physics processes discussed in Chapter 2 can be found in GEM-TN-93-294. The trigger criteria, however, for the various processes will often overlap. If we remove the overlapping trigger criteria, the list of trigger criteria for the individual processes can be reduced to the list given in Table 7-8. A logical "OR" of these 12 trigger conditions will accept all physics processes discussed in Chapter 2.

The threshold values mentioned in Table 7-8 are preliminary. The thresholds are a trade-off between efficiency for the process of interest and background reduction. The optimal combination of the thresholds will be determined through further Monte Carlo simulation. One might also consider lowering the thresholds in the barrel-region of the detector with respect to the endcap regions.
Table 7-8. Ovenview of the 12 criteria for the Level 1 trigger. The letters $E, J, M$ and $P$ stand for the $d / \gamma$, jet, muon, and $\boldsymbol{E}_{\mathrm{T}}$ primitives, the subscripts stands for the thresholds in GeV on the given primitive.

\begin{tabular}{|c|c|c|}
\hline No. & Trigger & Comments \\
\hline 1 & $J_{80}$ & Jet with $E_{T}>80 \mathrm{GeV}$. \\
\hline 2 & 4 of $J_{50}$ & $\begin{array}{l}4 \text { jets with } E_{T}>50 \\
\mathrm{GeV} \text {. }\end{array}$ \\
\hline 3 & $E_{50}$ & High $E_{T}$ electron or $\gamma$. \\
\hline 4 & $M_{30}$ & High ET muon. \\
\hline 5 & 2 of $E_{16}$ & 2 electrons or $\gamma s$. \\
\hline 6 & 2 of $M_{10}$ & 2 muons. \\
\hline 7 & $P_{100}$ & $E_{T}$ \\
\hline 8 & $E_{16}$ AND $P_{50}$ & $\begin{array}{l}\text { Electron and } E_{r} \\
\left(W^{ \pm}, \tau \text {-decays }\right) .\end{array}$ \\
\hline 9 & $M_{10}$ AND $P_{50}$ & $\begin{array}{l}\text { Muon and } E_{T} \\
\left(W^{ \pm}, \tau-d_{e c a y s}\right)\end{array}$ \\
\hline 10 & $J_{16}$ AND P 50 & $\begin{array}{l}\text { Hadrons and } E_{Y} \\
\left(W^{ \pm}, \tau \text {-decays }\right) .\end{array}$ \\
\hline 11 & $M_{10}$ AND (3 of $\left.J_{50}\right)$ & Muon and 3 jots. \\
\hline 12 & $E_{16}$ AND $\left(3\right.$ of $\left.J_{50}\right)$ & Electron $\gamma \gamma$ and 3 jets. \\
\hline
\end{tabular}

Trigger Simulation

The trigger simulation is part of the GEMFAST package. ' It can optionally be called after the event has been tracked through the detector. The following components of the trigger are available in the latest version of this trigger simulation:

Calorimeter: For the digital Level 1 calorimeter trigger, all primitives listed in Section 7.2.1 have been implemented in the simulation. A simulation of the analog Level 1 calorimeter trigger is currently being tested.6,8 For these simulations, the timing veto has not been implemented, e.g., it is assumed that no interactions inconsistent with the bunchcrossing structure occur. Figure 7-13 shows the efficiency of the electron-trigger as a function of $|\eta|$ for both the analog and digital options. The figure shows that there is very little difference between the two simulations. It is, therefore, expected that physics efficiency and background reduction will be similar for both options . 


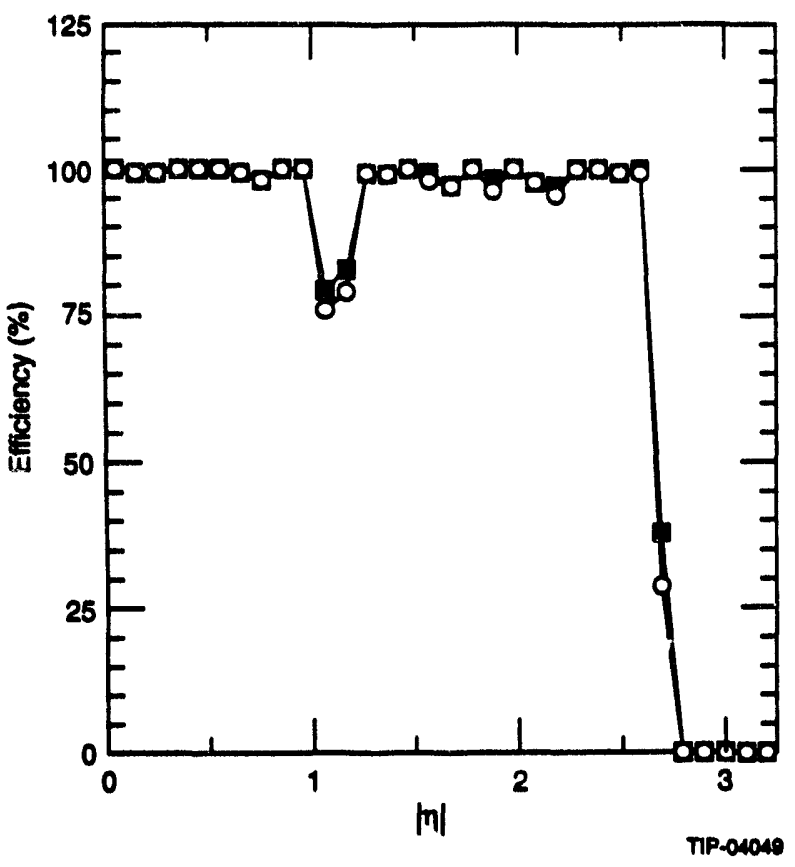

FIG. 7-13. Efficiency of the $E_{8}$ a/p-trigger for electrons with a pr of $50 \mathrm{GeV}$. Circles show the efficiency of the digital calorimoter Level 1 design, squares the efficiency of the analog design. Piloup noise was included. The dip in the curves is caused by the barrel endcap transition.
Forward Calorimeter: $E_{T}$ and $E_{T}$ calculated on a cell by cell basis.

Muon Chambers: The number of tracks above a given threshold for a sagitta method is available. The simulation determines these by calculating the probability that a track will be seen by the trigger for a given threshold and direction. Tracks are then accepted at random according to this probability.

Global Level 1 Trigger: The set of triggers given in Table 7-8 has been implemented in the simulation.

\section{Physics Acceptance}

The physics events used for this study were generated using the standard GEMFAST setup with a signal and minimum bias background generator (see Section 2.2). Gaussian pileup has been assumed with the default GEMFAST mean and RMS values. Table 7-9 gives an overview of the generator setups. The kinematic constraints have been set so that the relevant final-state particles are within the geometrical acceptance of the detector. The trigger efficiency can then be defined as the number of events that are accepted by one of the trigger modes divided by number of generated particles. This efficiency, therefore, does not include the geometrical acceptance of the apparatus.

Table 7-9. Event Generators and their setup used to generate the events used in this study.

\begin{tabular}{|c|c|c|c|c|}
\hline Process & Generator & $\mathrm{m}_{\mathrm{H}}{ }^{0}$ & $m_{t}$ & Kinematic constraints. \\
\hline \multirow{3}{*}{$H^{0} \rightarrow \gamma$} & PYTHIA & 80 & 140 & $\left|\eta_{y}\right|<3$. \\
\hline & & 110 & 140 & - \\
\hline & & 140 & 140 & “ \\
\hline$t w H^{0} \rightarrow$ ryex & PYTHIA & 80 & 140 & $\left|\eta_{\gamma}\right|<3$, both ts to $W+$ quark \\
\hline$H^{0} \rightarrow \tau-\tau-$ & PYTHIA & 140 & 140 & $\left|\eta_{8}\right|<3$ \\
\hline$H^{0} \rightarrow Z^{*} \rightarrow$ eece & PYTHIA & 140 & 140 & $\ell=\theta, \mu$. \\
\hline$H^{0} \rightarrow \infty \theta \mu \mu$ & Isajet & 400 & 140 & $\begin{array}{l}50<p_{T}<20000 \mathrm{GeV} \text { and } \\
|\eta|^{<}<\text {for both } \mathrm{Zs} \text {. }\end{array}$ \\
\hline$H^{0} \rightarrow \bullet \bullet$ jet jet & Isajet & 800 & 140 & $\cdot$ \\
\hline t-pair production & PYTHIA & 140 & 140 & Both $t s$ to $W+$ quark. $\left|\eta_{t}\right|<3$. \\
\hline Susy & Isajet & - & 140 & $\begin{array}{l}500 \mathrm{GeV} \text { gluino production, decaying to a photino and } \\
\text { a jet. I } \text { Vot }^{1}<3 \text {. }\end{array}$ \\
\hline$W^{ \pm} \rightarrow e v$ & PYTHIA & - & - & $\eta_{\text {end }}<2.5, \ell=e, \mu$. \\
\hline
\end{tabular}


Table 7-9. Event Generators and their setup used to generate the events used in this study. (Cont.)

\begin{tabular}{lllll}
\hline Process & Generator & $m_{H}{ }^{0}$ & $m_{t}$ & Kinematic constraints. \\
\hline$Z^{0} \rightarrow \ell \ell$ & PYTHIA & - & - & $m_{\ell}<2.5, \ell=\ell, \mu$. \\
Minbias & PYTHIA & - & - &
\end{tabular}

Table 7-10 lists the efficiency for the different processes. From this table it can be seen that the trigger efficiency for most processes is well above the $90 \%$ level. The efficiency for $\mathrm{H}^{0} \rightarrow \gamma \gamma\left(m_{\mathrm{HO}}=\right.$ $80 \mathrm{GeV}$ ) is somewhat low and can be improved by lowering the thresholds. The $\mathrm{W}^{ \pm}$events are primarily intended for calibration purposes and the low efficiency is acceptable.

Table 7-10. Efficiency for various physics processes of the Level 1 trigger criteria (Table 7-8).

\begin{tabular}{|c|c|}
\hline Process & $\%$ Accepted \\
\hline $\mathrm{H}^{0} \rightarrow r \quad 80 \mathrm{GeV}$ & 78.72 \\
\hline $110 \mathrm{GeV}$ & 89.31 \\
\hline $140 \mathrm{GeV}$ & 94.66 \\
\hline $\mathrm{tt} H^{0} \rightarrow$ ryex & 94.38 \\
\hline$H^{0} \rightarrow \ell^{+} \ell^{-} \ell^{+} \ell^{-}$ & 81.77 \\
\hline$H^{0} \rightarrow \tau^{+} \tau^{-}$ & 34.70 \\
\hline$H^{0} \rightarrow \theta^{+} \theta^{-} \mu^{+} \mu^{-}$ & 99.80 \\
\hline$H^{0} \rightarrow \theta^{+} \theta^{-}$jet jet & 99.85 \\
\hline t-pair production. & 75.25 \\
\hline Susy & 100.00 \\
\hline$W^{ \pm} \rightarrow$ ov & 15.82 \\
\hline$W^{ \pm} \rightarrow \mu v$ & 48.73 \\
\hline$Z^{0} \rightarrow \theta^{+} \theta^{-}$ & 80.35 \\
\hline$Z^{0} \rightarrow \mu^{+} \mu^{-}$ & 86.88 \\
\hline
\end{tabular}

Figure 7-14a shows the number of accepted events by each trigger mode $H \rightarrow \gamma \gamma$. This figure shows clearly that the dominant triggers are \#3 and \#5: the trigger for a single isolated high pr photon and the trigger for a pair of isolated photons. This is exactly what one expects given the choice of trigger criteria. Similar conclusions can be drawn for the other processes. Figure 7-14a also suggests that the efficiency for $\mathrm{H}^{0} \rightarrow \gamma \gamma$ can be improved by lowering the thresholds of triggers \#3 and \#5. Lowering the thresholds increases the efficiericy at the price of increasing the trigger rate. For example, replacing the $E_{50}$ and $E_{16}$ thresholds by $E_{30}$ and $E_{10}$, increases the efficiency to about $93 \%$ but raises the rate to over $10 \mathrm{kHz}$. This tradeoff between trigger efficiency and rate is discussed in more detail in the next section on threshold studies and also in GEM-TN-93-332.
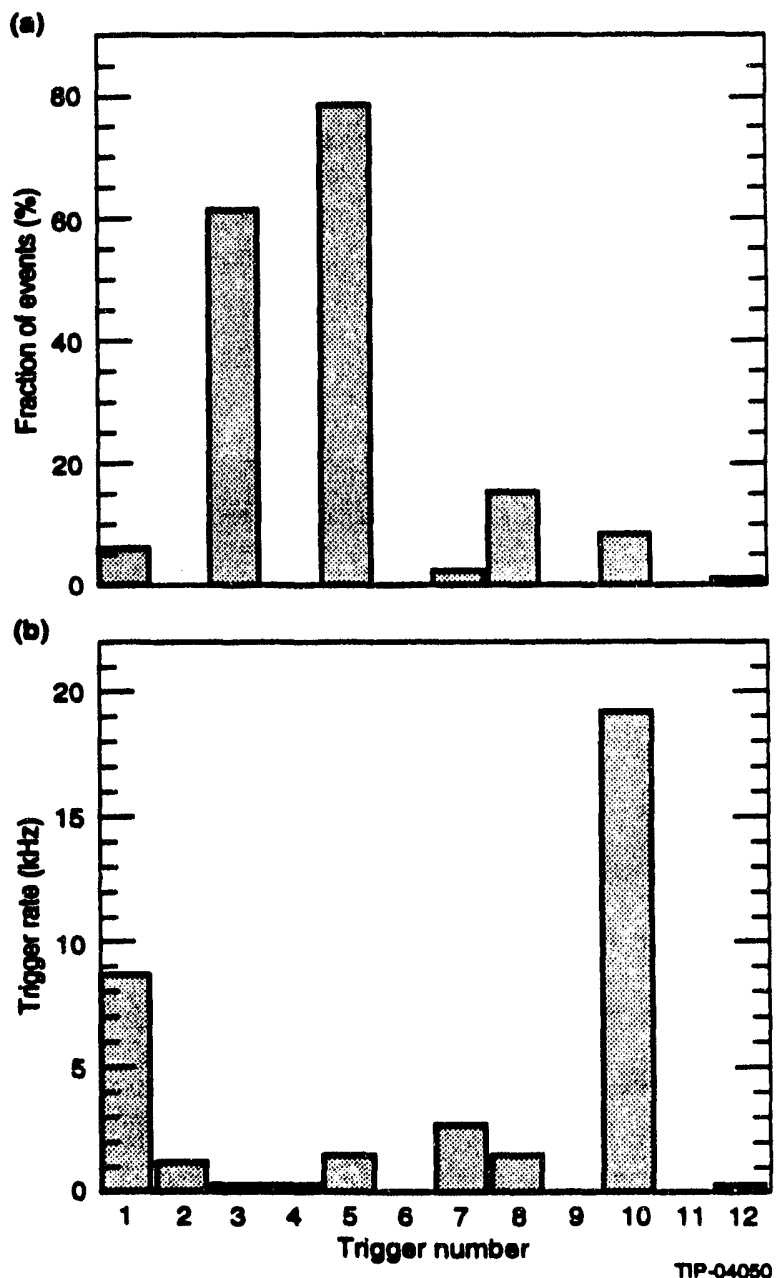

FIG. 7-14. a) Fraction of events accepted by each of the 12 trigger modes from Table $7-8$ for $\mathrm{H}^{0} \rightarrow \gamma$ $\left(m_{H}{ }^{*}=110 \mathrm{GeV}\right)$ and $\left.b\right)$ trigger rates for minimum bias for these triggers. 


\section{Background Reduction}

The background events used for this study were generated by replacing the signal generator process by a (PYTHIA) minimum bias generator process. As a result, every interaction contains one minimum bias event from the "signal" stream and an average of 1.6 interactions from the background stream. The generated events thus correspond to a luminosity of approximately $1.6 \times 10^{33}$.

A total of 200000 events were tracked through GEMFAST. Only 78 of them were accepted by the trigger. This is equivalent to a reduction of the minimum bias interaction rate from $60 \mathrm{MHz}$ to $23.4 \mathrm{kHz}$. Figure $7-14 \mathrm{~b}$ shows the trigger rate by each trigger mode. From this figure it can be seen that most of the surviving minimum bias events are accepted by trigger criteria \#1 and \#10.

- Trigger \#1 is a trigger on high pr jets. This trigger mode cannot be removed without affecting jet-related physics.

- Trigger \#10 is a trigger on a low $p_{T}$ jet and $E_{T}$. This trigger is intended to trigger on a $W^{t}$ decaying to a neutrino and a jet or a top quark decaying to neutrinos and hadrons.

If trigger \#10 is completely removed from the list, the background rate is reduced by another factor two to about $11.7 \mathrm{kHz}$. Removing this trigger affects the physics efficiency for the processes studied here, including $\mathrm{H}^{0} \rightarrow \tau$ by less than $5 \%$. Some of the lost events can probably be recovered by a $\tau$-pair trigger.

\section{Trigger Threshold Dependence for Selected} Processes

The Level 1 trigger algorithms for several specific processes have also been studied in somewhat more detail to understand the tradeoffs between physics efficiency and Level 1 trigger rate. These studies also use the GEMFAST simulation code as described previously. In particular, we have studied triggers for the decay of $\mathrm{H}^{0} \rightarrow \gamma \gamma$ and heavy flavors and, in addition, have calculated trigger rates for "topological" first level triggers involving isolated photons/electrons, muons, jets, and missing transverse energy.

\section{$H^{0} \rightarrow \gamma \gamma$ and Inclusive ex $\gamma$ Triggers}

As shown in the previous section, the combination of the single-and two-photon trigger is the primary channel for triggering on $\mathrm{H}^{0} \rightarrow \gamma \gamma$ decays. Figure 7-15a shows the efficiency for triggering on these decays as a function of trigger threshold. Better efficiency can be gained by reducing threshold but only at the expense of higher trigger rates. For example, a reduction from the standard triggers of $E_{50}(1 \gamma)$ and $E_{16}(2 \gamma)$ to $E_{30}(1 \gamma)$ and $E_{10}(2 \gamma)$ increases the efficiency from $79 \%$ to $93 \%$ but raises the trigger rate from $1 \mathrm{kHz}$ to $13 \mathrm{kHz}$. (See Figure 7-15b.)

\section{Heavy Flavor Triggers}

We have examined the trigger rate for a Level 1 trigger based on the presence of one or more jets which is an important component for triggering on top quarks. Figure 7-16a shows the efficiency for triggering on a top quark pair of mass $140 \mathrm{GeV}$ as a function of jet threshold for a one and four jet trigger. The standard configuration of triggers uses an 80 (50) GeV threshold for the single (four) jet trigger and leads to greater than $75 \%$ triggering efficiency when combined with the missing energy triggers. Figure 7-16b shows the minimum bias background rate for these two triggers, which is within allowable limits.

\section{Missing Transverse Energy Triggers}

Missing energy triggers are important for triggering on exotic physics with non-interacting particles or physics processes involving $W$ s. The standard GEM trigger mixture given in Table 7-8 includes a missing energy component with a threshold of $100 \mathrm{GeV}$ and combinations of $50 \mathrm{GeV}$ missing energy coupled with leptons or jets. Studies have been made of the efficiency and background rate for the stand-alone missing energy trigger for $500 \mathrm{GeV}$ gluino production as a function of triggering threshold (See Figure 7-17).

W-boson production is also an important signature for many possible physics processes. Triggers incorporating missing transverse energy and jets or leptons are key components needed for efficient triggering. In Figure 7-18, we show the trigger efficiency for a $140 \mathrm{GeV}$ top quark and background rates for these combined triggers as a function of the $E_{T}$ threshold.

\section{Inclusive Muon Triggers}

A hit-level Monte Carlo has been developed to study the pattem recognition and trigger issues in the muon system. This Monte Carlo has been used to 
predict the trigger rate in both the barrel and endcap. The program has the correct magnetic field configuration, and treats muons with a full GEANT simulation in the tracker, calorimeter, and muon system. This includes muon multiple scattering and radiation. To reduce computing time hadrons are not

(a)

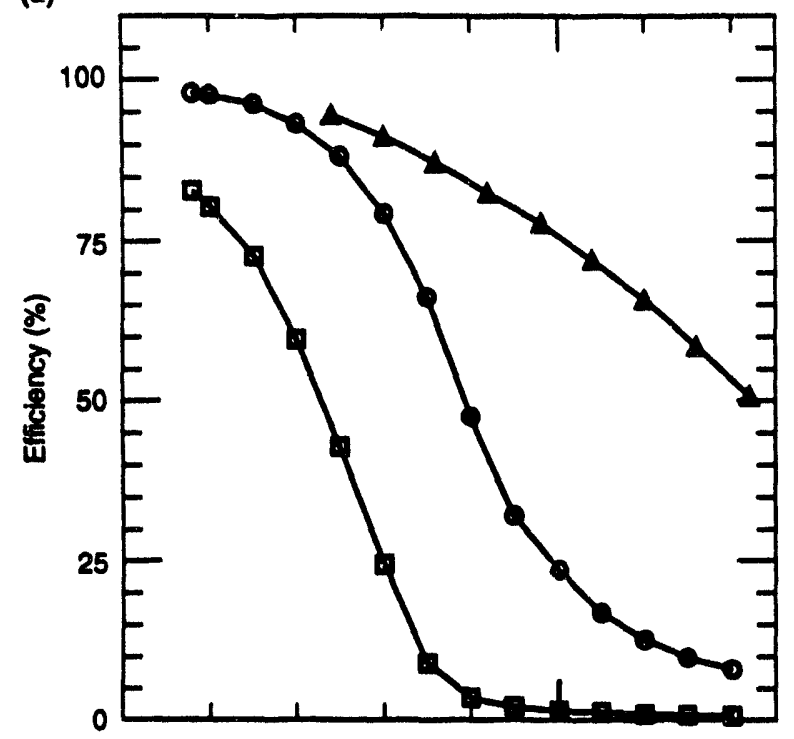

(b)

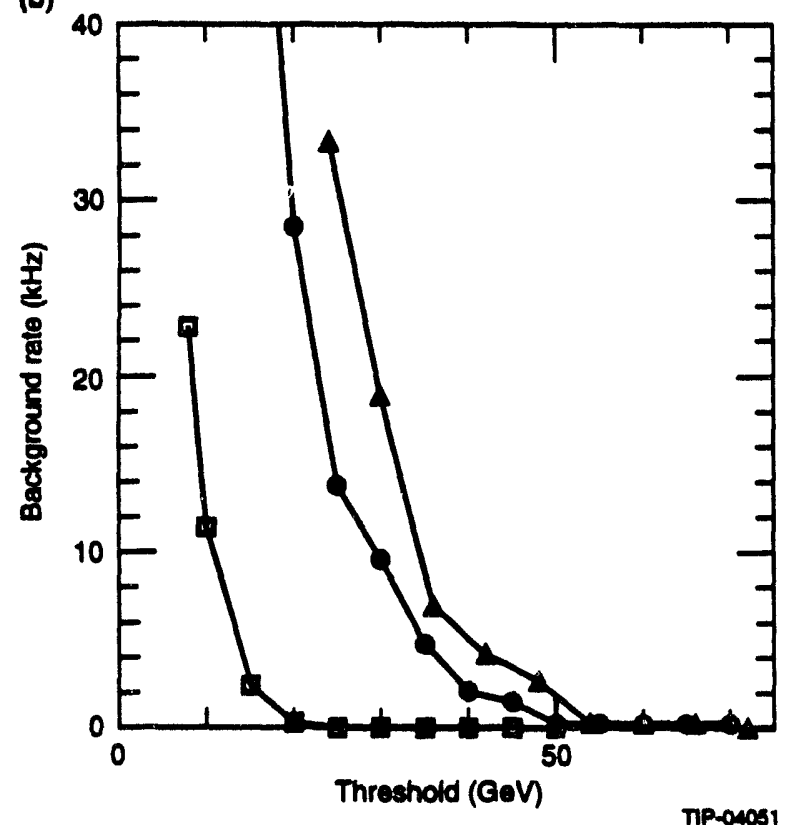

FIG. 7-15. a) Trigger efficiency for $\mathbb{H} \rightarrow y \gamma\left(m_{H}{ }^{0}=\right.$ $80 \mathrm{GeV}$ ) for a single-photon trigger (circles) as a function of threshold, a two-photon trigger (squares), and a single-proton trigger logically ORed with a two-photon trigger at $1 / 3$ of the threshold (triagles). b) The minimum-bias background rates for these triggers. fully traced through the calorimeter. A parameterization of the hadronic punch-through exiting the calorimeter is built from a full GEANT simulation and is used to provide a background hit rate, including exiting particle momentum, angle, and species.
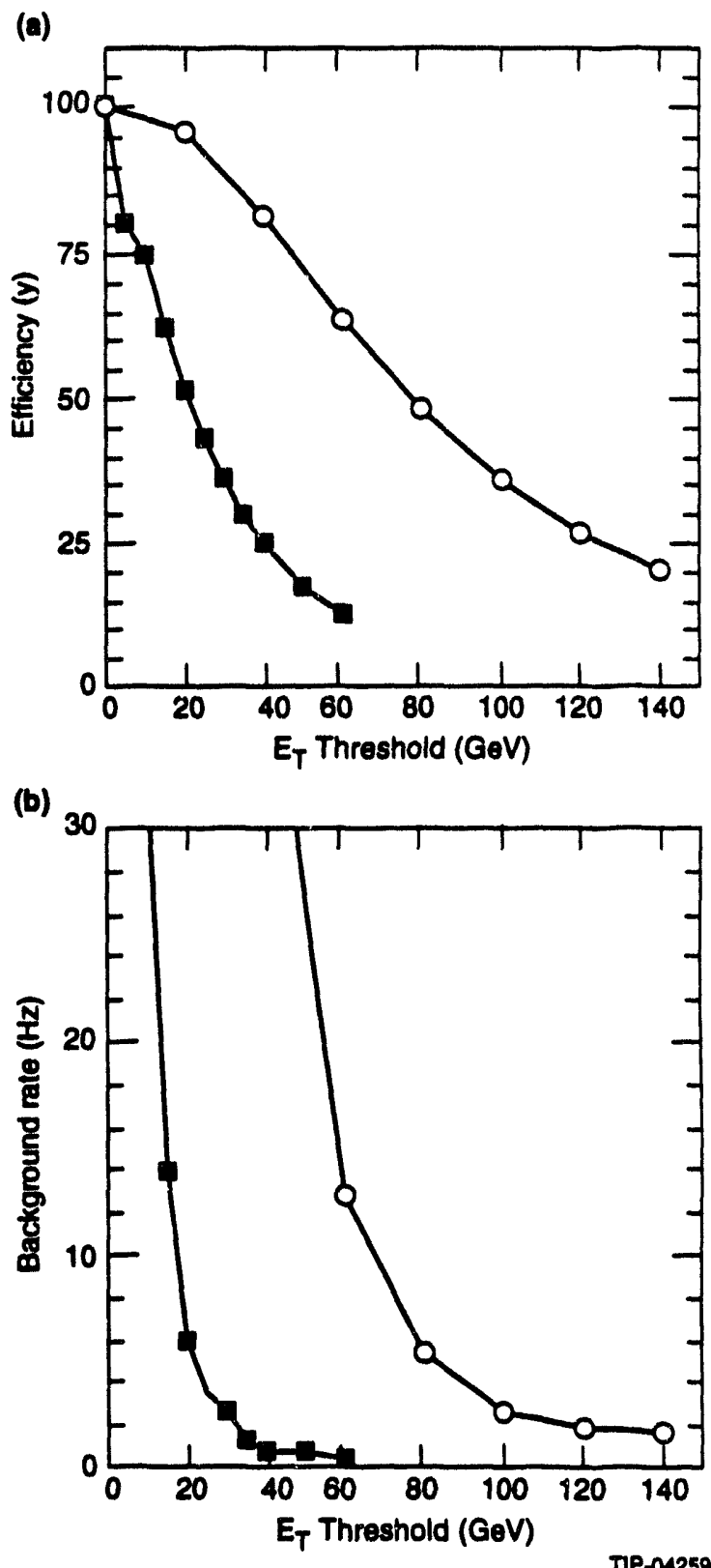

FIG. 7-16 a) Trigger efficiency for top quark production $\left(m_{i}=140 \mathrm{GoV}\right)$ using a single-jet trigger (circles) and a four-jet trigger (squares) as function of the jet $E$ threshold. Both top quarks are forced to decay to $W+b$. b) Minimum bias backgrounds for these triggers. 
The resulting hit banks from the Monte Carlo simulation provide output for analysis by the trigger hardware simulation. This analysis, in turn, provides trigger rates, both for single and multiple muons, spectra for triggered particles, and trigger efficiencies for single muons originating at the interacion point.

The relevant parameters for the study of trigger rate and efficiencies are:

- The number of trigger cells in the $\phi$ (or bend) view. The current design has 8604 such cells in the barrel and 10368 in the endcaps. The barrel segmentation represents a trigger cell corresponding to two measurement cathode strips, while the endcaps have one trigger cell per strip

- The difference in the number of cells for the sagitta measurement. The studies presented here are carried out for a cell difference

(a)

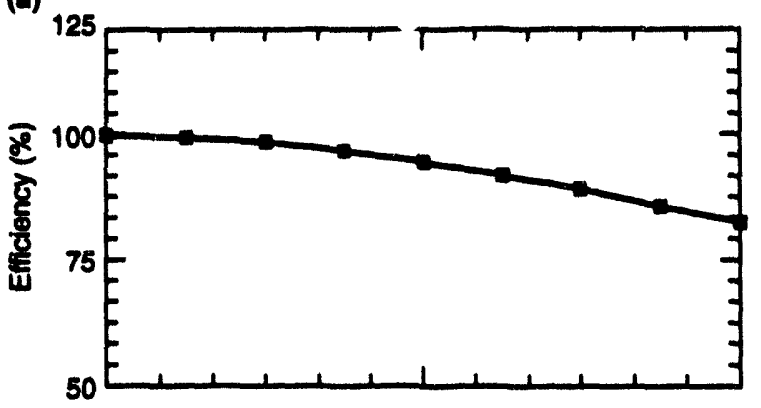

(b)

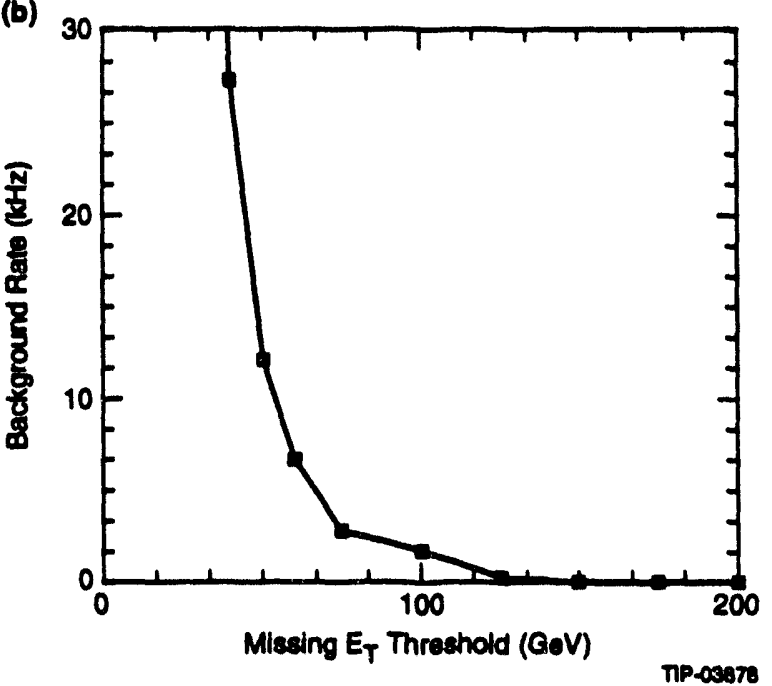

FIG. 7-17. a) Trigger efficiency for $500 \mathrm{GoV}$ gluino production using the standalone missing energy trigger as a function of the $\boldsymbol{F}_{T}$ threshold. b) Minimum bias background rate for this trigger versus $F_{T}$ threshold. between zero and five trigger cells corresponding to $p_{T}$ thresholds of 50 to $10 \mathrm{GeV} / \mathrm{c}$.

- The number of non-bend-plane trigger cells. In principle, the system is capable of supporting up to 256 such cells in the barrel and 16 such cells in the endcaps. However, the resultant trigger ratc $;$ do not warrant a non-bend-plane cell count beyond 8 in the barrel and 2 in the endcaps.

- The difference in the number of cells in the non-bend plane sagitta. As mentioned earlier this difference should be zero, except for the effect of muons crossing the cell boundaries. To reduce the efficiency loss due to this effect

(a)

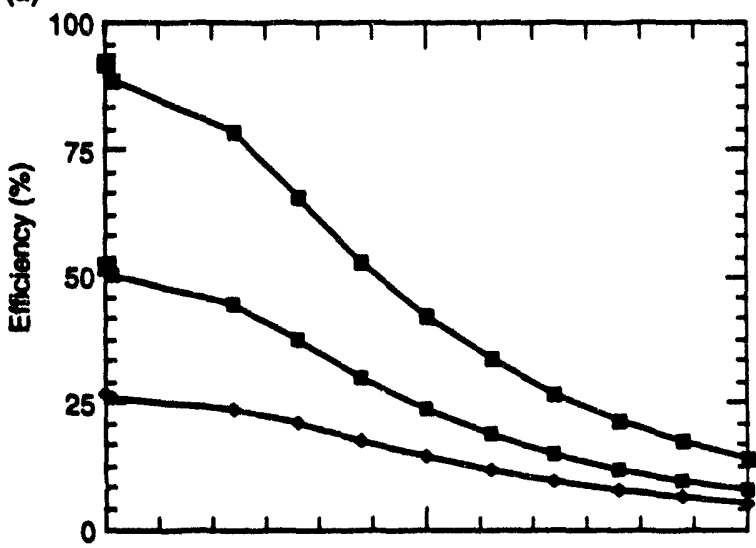

(b)

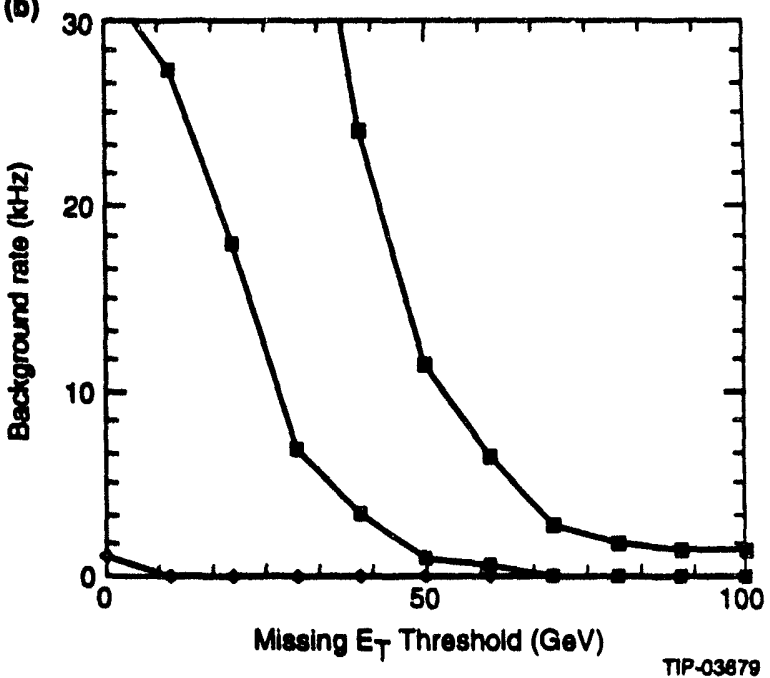

FIG. 7-18. a) Trigger efficiency for tt production $\left(m_{1}=\right.$ $140 \mathrm{GeV}$ ) using a missing energy trigger combined with a $16 \mathrm{GeV}$ threshold for an isolated EM shower, $E_{10}$ (circles), a $10 \mathrm{GoV} p_{T}$ threshold for a muon trigger, $M_{10}$ (squares) or a $25 \mathrm{GeV}$ threshold jet trigger, $J_{25}$ (triangles) as a function of the $E_{T}$ threshold.

b) Minimum bias backy :ound rate for these triggers versus $\boldsymbol{F}_{T}$ threshold. 
we have chosen a cell difference of one in the barrel and zero in the endcap. A larger number will increase the trigger rate without appreciably improving the efficiency.

Figure 7-19 shows the integrated trigger rates as a function of the $p_{T}$ threshold for the sagitta method in both the barrel and endcaps. The rates shown include muon and punch-through rates. Figure 7-20 shows the efficiency as a function of $p$ T. These rates represent the current design parameters..

(a)

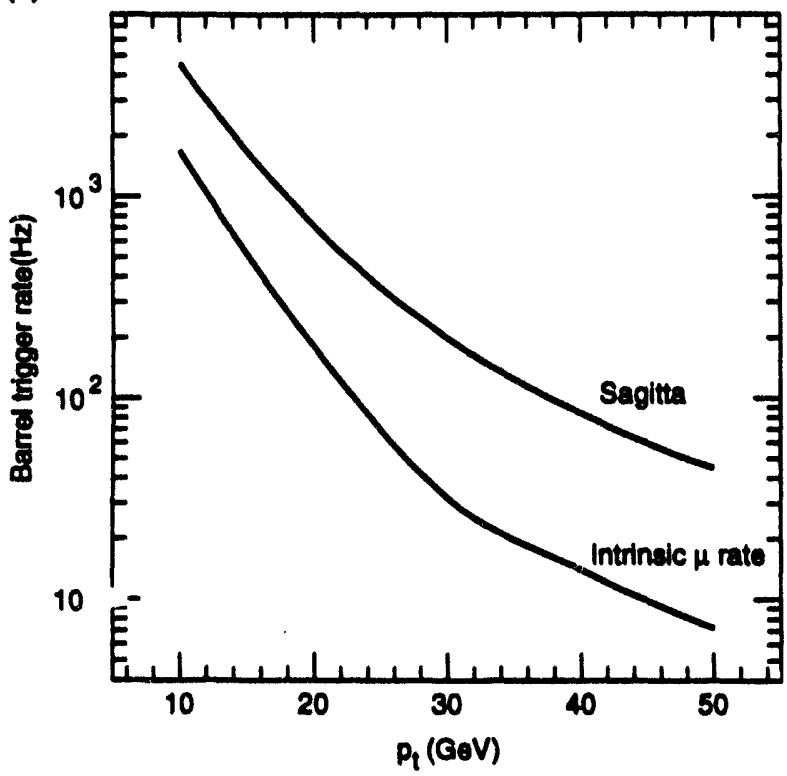

(b)

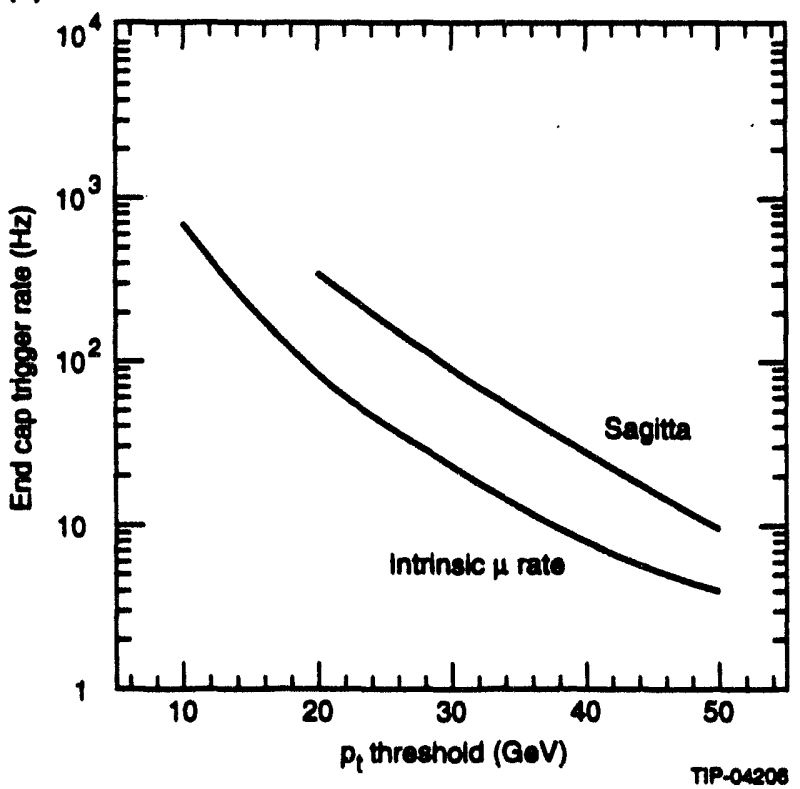

FIG. 7-19. The trigger rate in the a) barrel and b) endcap as a function of $p_{T}$ for different $p_{T}$ thresholds in the sagitta method.
The muon trigger study shows that the hadronic punchthrough increases the trigger rate only marginally above the rate due to hits from real muons. Therefore, the GEMFAST trigger simulation is setup to include only triggers from real muons produced at the primary interaction by particle decays.
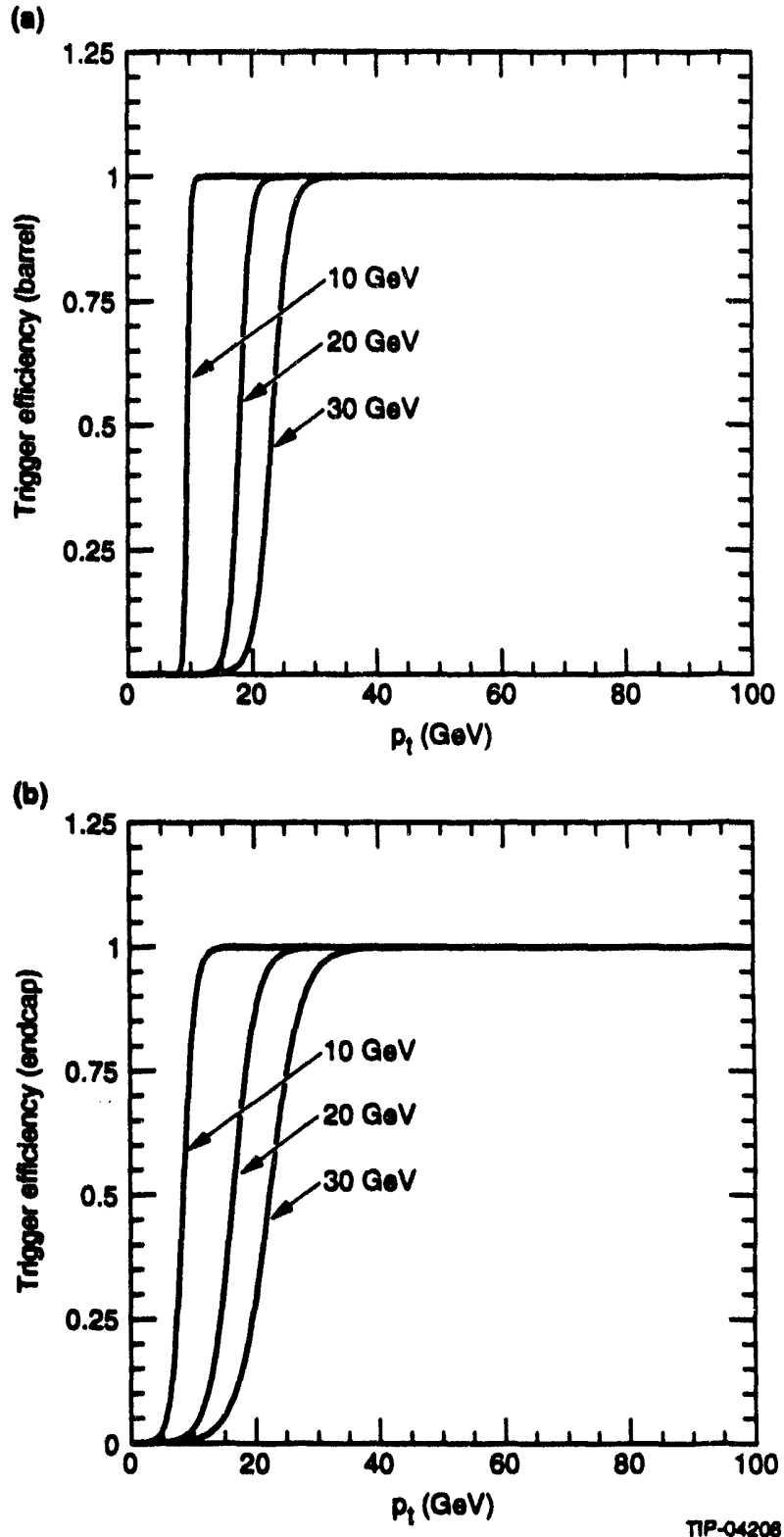

FIG. 7-20. Trigger efficiencies in the a) barrel and b) endcap as a function of $p_{T}$ for different $p_{T}$ thresholds.

\section{Conclusions}

The studies presented here show that the GEM Level 1 trigger can trigger on a wide variety of physics processes with efficiency greater than $90 \%$ 
while at the same time reducing the minimum bias background to the level of $10 \mathrm{kHz}$. These numbers are already close to target values for the Level 1 trigger efficiency and background rate. Further improvements should be possible with more refined algorithms.

\subsubsection{Level 2 Trigger}

\section{Introduction}

The goal of the Level 2 trigger algorithms is to reduce the event rate, by a factor between 30 and 300 , to $\mathrm{O}(300) \mathrm{Hz}$. The Level 2 trigger decision for a particular event is made using a program running on one of the processors in the farm. The program first calls a set of subroutines that extracts trigger primitives from the data of the individual components. The program then calls a global routine where the trigger primitives are combined into a global decision. Finally, the decision is sent to the Level 2 control system. The Level 1 trigger conditions are used as seeds for the Level 2 trigger program. It may determine which Level 2 algorithms will be executed and which portions of the data will be made available to the Level 2 trigger.

An advantage of the "virtual Level 2" architecture is the flexibility afforded by the fact that the algorithms will reside in full-performance processors instead of special purpose hardware. These processors will also have access to the full data set for a given event and can be made to implement almost any trigger strategy. Information on backgrounds and necessary physics signals that becomes available through simulations or from initial running will lead to new algorithms that can be easily implemented with this flexible trigger scheme. We present some examples of Level 2 algorithms in the next sections.

\section{Level 2 Trigger Primitives}

The following trigger primitives are possible inputs for the global Level 2 trigger algorithms:

\section{Central Tracker:}

- $p_{T}$ and position of isolated tracks.

The Level 1 electron-trigger will define regions where an isolated, stiff $\left(p_{T}>10 \mathrm{GeV}\right)$ track could be found. A track-finding algorithm can then be used to confirm the presence of a track in this region, thus establishing either an electron or gamma primitive.

\section{Calorimeter:}

Digital information from individual cells will be used in Level 2 for the purpose of shower shape analysis and better isolation cuts. The regions of the calorimeter from which information will be processed will be identified by the preceding Level 1 trigger stage.

- Electron finding based on better energy estimates and more restrictive isolation cuts.

Single photons can be better distinguished from the background photon pairs through the use of pattern recognition on the fine-grained data. Using the full granularity of the calorimeter, the algorithm determines the ratio of energy deposition in a given set of $3 \times 3$ towers $(0.08 \times 0.08$ in $\Delta \eta \times \Delta \phi)$ to that in the corresponding $5 \times 5(0.13 \times 0.13$ in $\Delta \eta \times \Delta \phi)$ group. For electrons and photons, this ratio should be above a given threshold. A cut on the ratio of energy deposit in a $3 \times 3$ sum to that in a $5 \times 5$ sum yields approximately a factor of three rejection for $20 \mathrm{GeV}$ photons.

The isolation cut can be improved by permitting energy deposit only in the cells containing the electromagnetic shower. A simple difference between the energy found in the highest EM cluster and the total transverse energy in the trigger tower can reject events in which two separated photons have entered the same trigger tower.

Better energy discrimination is achieved by summing only those cells contributing to the shower. Having removed all energy in the trigger tower that is not associated with the EM cluster, many photons that are close to the Level 1 trigger threshold will be rejected.

A tighter hadronic veto can be applied through the examination of energy deposit in each hadronic cell behind the EM shower. Preliminary studies of this criterion in the case of the two photon decay of the Higgs suggest that this criterion can reject an additional factor of two (per photon) in the background, with negligible effect on the signal.

Electromagnetic showers will be better distinguished from hadronic showers through the requirement that the ratio of energy deposit in the first two 
electromagnetic section be consistent with that expected from an electromagnetic shower.

- Jet finding.

A Level 2 jet-finding algorithm that has access to the individual calorimeter cells and can therefore find jets more precisely thin a Level 1 jet-trigger with fixed towers. A jet-finding algorithm has been studied ${ }^{9}$ and it has been shown that such an algorithm can do the jet-finding within the time constraints of the Level 2 trigger and with sufficient efficiency. With a steeply falling energy spectrum, a better discrimination on the energy of the jet should provide a significant rate reduction.

- Calculation of $E_{T}$ and $E_{T}$.

In the Level 2 trigger, individual cells with only pile-up noise can be excluded from the $E_{T}$ and $E_{T}$ calculations. The result will be more precise than the Level 1 calculation (see Section 7.2.1). More precise information from the forward calorimeter can also be included.

- Timing cuts.

Checks on the reconstructed timing can be made to reduce the effects of accidental overlap of events. A detailed study of the effects of overlapping events from different bunch crossings on the trigger has yet to be made. Except for the transversal filter applied to the JET sums, there is no protection against such overlaps at Level 1 . However, since the Level 1 trigger establishes the bunch crossing which contains the event, it will be a simple matter to check for timing consistency for all signals contributing to the trigger at Level 2.

\section{Muon Chambers:}

- $p T$ determination of the muons found by Level 1.

The high-resolution hits in the muon chambers that are associated with Level 1 trigger accepts can be used in a full fit to the trajectory to make a precise PT determination. The hits can be corrected for misalignments and nonlinearities and a more precise field map can be utilized.

\section{Combination Algorithms.}

Trigger algorithms that combine more than one particle or subsystem are available for making Level 2 trigger decisions. Some examples of these type of algorithms are given below:

- Electron separation.

The presence of a track pointing to a electromagnetic cluster found in the calorimeter indicates whether it is a photon or electron. The tracker information can also be used to reject electrons from background interactions.

- Combinations of particles/jets into pairs.

The Level 2 trigger can combine any two particles and calculate properties of the pair such as invariant mass, vertex and relative charge. The Level 1 trigger criteria can then be extended with constraints on two final state particles together.

\section{Conclusions}

The above discussion describes some of the possible improvements that are accessible to the Level 2 trigger. Although many details remain to be worked out, it is clear that the fine-grained data contain information that can be used to reduce the trigger rate at Level 2 . For the two-photon decay of the Higgs, the preliminary studies cited above indicate that a factor-of-six rejection for each photon can be obtained through the shower shape criterion and improved hadronic isolation, reducing the trigger rate by a factor of 36 . The additional cuts on energy outside of the EM cluster and more precise determination of the energy of the cluster should bring the Level 2 rejection to at least a factor of 100 . For the muon system, the Level 2 pr calculations should allow triggering rates only slightly above the intrinsic muon rates for a given $p r$ threshold. The calorimeter data can also be used in establishing isolation criteria for muon candidates. Information from other detectors, such as the silicon tracker and the IPC system, will also be applied at Level 2. allowing the better identification of electrons and photons.

\subsection{DAQ, LEVEL 2 ARCHITECTURE, AND EVENT BUILDING}

\subsubsection{Introduction}

The data acquisition system is responsible for making the data for the relevant physics events 
available to further processing in an efficient way. It also provides the context in which the Level 2 and possibly the Level 3 triggers run.

A design goal specific to the GEM DAQ system is that all front-end chips are read after each Level 1 trigger. Therefore, no data has to be stored on the front-end chips during the Level 2 decision. Only buffers of moderate size will be needed to derandomize the Level 1 trigger rate fluctuations. The architecture supports both hardware and software implementations of the Level 2 trigger, or combinations of both, by allowing large Level 2 latencies.

The Level 2 and 3 algorithms will be executed in the general purpose processors of the on-line farm. Hence, the event data must be transported to the farm at rates up to the maximum specified Level 1 trigger rate of $100 \mathrm{kHz}$. It also means that there are basically only two levels of triggering: a low latency trigger (Level 1) and the combined Level 2 and 3 that forms a high latency trigger. The latency for Level 1 is fixed by the depth of the pipeline buffers on the front-end chips. The latency for Levels 2 and 3 is limited only by the size of off-detector buffers which can be easily expanded.

We propose a readout (Figure 7-21) where for each Level 1 accept, the data from the front-end boards are read out via serial links of moderate speed. The data are stored in a number of event data collector modules (EDCs). These modules contain the large buffers where most event data remains during the Level 2 execution. Data needed by Levels 2 and 3 are transported on high speed fibers to the processor farm via a modular switching network (event builder). They are received by event data distributor modules (EDDs). Data for each event originate in different EDCs. Event data fragments are combined in the EDDs. The EDDs forward the data to the processors in the farm, again via moderate speed links.

Because front-end occupancies, trigger rates and trigger processing times are not fully determined, the initial design for the GEM data acquisition system places a large emphasis on scalirig and flexibility. The system is described in more detail below.

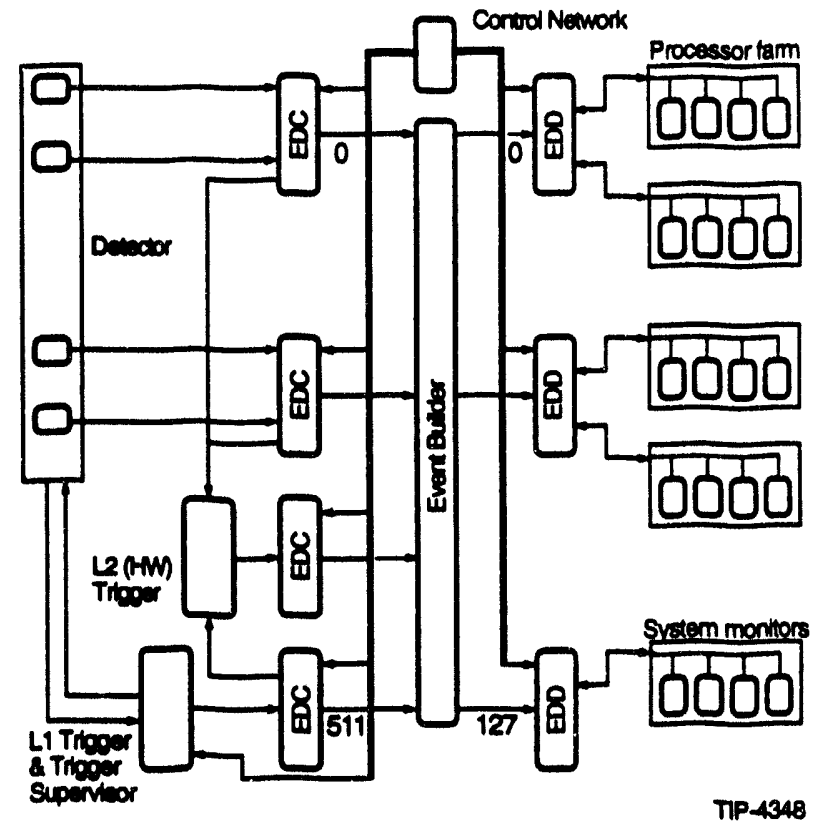

FIG. 7-21. Overview of the GEM DAQ system.

\subsubsection{Data Collection}

Table 7-11 lists the major GEM subsystems with estimates of channel counts, number of frontend boards, number of bytes per events and data volume at a Level 1 trigger rate of $100 \mathrm{kHz}$.

Table 7-11. Numbers of channels and front-end boards for the GEM subsystems, number of bytes per event and data volume at a level 1 trigger rate of $100 \mathrm{kHz}$.

\begin{tabular}{lllll}
\hline Subsystem & Channels & FE Boards & Bytes/event & Data Volume \\
\hline Calorimeter & $125 \mathrm{~K}$ & 3,555 & $130 \mathrm{kB}$ & $13 \mathrm{GBytes} / \mathrm{s}$ \\
IPC & $400 \mathrm{~K}$ & 3,125 & $80 \mathrm{kB}$ & $8 \mathrm{GBytes} / \mathrm{s}$ \\
Silicon & $2,500 \mathrm{~K}$ & 1,950 & $40 \mathrm{kB}$ & $4 \mathrm{GBytes} / \mathrm{s}$ \\
Muon & $1235 \mathrm{~K}$ & 1,270 & $40 \mathrm{kB}$ & $4 \mathrm{GBytes} / \mathrm{s}$ \\
\hline Total & $-4,300 \mathrm{~K}$ & $-10,000$ & $-300 \mathrm{kB}$ & $-30 \mathrm{GBytes} / \mathrm{s}$ \\
\hline
\end{tabular}


The estimate for the initial Level 1 trigger rates is $\sim 10 \mathrm{kHz}$ but the system is to be designed to have the capability to accept up to $100 \mathrm{kHz}$. If about a third of the data is needed for the Level 2 trigger calculations, the baseline system must have the capacity to transfer about $10 \mathrm{GBytes} / \mathrm{s}$. To accommodate increases in trigger rates or data volumes, the design bandwidth must be scalable without replacement of any existing components. Load balancing is essential for economic use of the available bandwidth.

All components in the data acquisition system are interconnected using point-to-point links, which are generally more reliable and allow greater flexibility in system configuration. Standard backplanes are used only for power and auxiliary features, reducing the cost of the crates and software.

\section{Data Format}

A data packet (event fragment) is generated for every Level 1 trigger accept. If there is no data, only the packet header information is sent. Each event fragment contains (minimally) word count and Event ID information. It may also include source ID and status information.

\section{Low Bandwidth Data Links}

Each front-end module transmits NRZ data on a separate $60 \mathrm{Mbits} / \mathrm{s}$ fiber link to an EDC. The receiver is assumed to be DC coupled using the global system clock.

\subsubsection{Event Data Collection Modules}

At the EDC, twenty-four front-end serial data links are multiplexed to form a single $100 \mathrm{MByte} / \mathrm{s}$ data stream. This data is huffered in a large dual-port memory which serves two main functions: time-ordering of packets transmitted to the event builder (to eliminate blocking) and buffering of event fragments pending Level 2 and 3 decisions. The EDCs send data to the event builder switch via $1 \mathrm{Gbits} / \mathrm{s}$ links.

The EDC buffers hold the event data for the period of the Level 2 decision, which is limited to $500 \mathrm{~ms}$. This requires approximately 50 MByte (100 MByte/s $\times 500 \mathrm{~ms}$ ). A buffer size of $64 \mathrm{MByte}$ should provide adequate margin. If all data is transmitted following each Level 1 trigger, smaller buffers of about $4 \mathrm{MByte}$ can be used.
Transfer of data from the receivers in the EDC to the common data buffer is handled by an FPGA buffer controller. This can be programmed for specific subsystem data formats.

\subsubsection{Event Building}

The event builder function is performed by a modular crossbar switch. Data flows through the switch in one direction only. The switch is implemented as three stages of $8 \times 8$ modules. The initial configuration will provide 64 switch modules (operating $8 \times 2$ ) in the first stage and 16 switch modules $(8 \times 8)$ in each of the second and third stages for a total size of $512 \times 128$. This is expandable to $512 \times 512$.

In its simplest configuration, an $\mathrm{N} \times \mathrm{N}$ switch provides the equivalent of $\mathrm{N}^{2}$ separate virtual data links, one from each EDC to each EDD. For a $100 \mathrm{MByte} / \mathrm{s}$ switch, each of these virtual channels operates at a rate of $100 / \mathrm{N} \mathrm{MByte/s.} \mathrm{A} \mathrm{single}$ $8 \times 8$ module implements 64 virtual channels, each operating at $12.5 \mathrm{MByte} / \mathrm{s}$ for a total bandwidth of $800 \mathrm{MByte} / \mathrm{s}$. A $512 \times 512$ switch has 262,144 virtual channels, each operating at $200 \mathrm{kBytes} / \mathrm{s}$, for a total of $50 \mathrm{GBytes} / \mathrm{s}$. The latency is determined by the individual data rate of each virtual channel and the total bandwidth is determined by the number of channels.

If the switch is programmed to operate with fixed packet lengths in a fixed rotation, no external control is necessary. For this simple mode of operation, data packets must be ordered by the EDCs to eliminate blocking. Without the input queuing (time-slot interchange) function performed by the EDCs, a general-purpose switch would require an expanded intermediate stage, arbitration and feedback paths.

Commercial standards, such as Fiber Channel, SONET/ATM, SCI and HIPPI have been examined for the high-speed data links and switching system. There is significant additional cost and overhead in using a general-purpose, bi-directional network but some of this cost is offset by the reduced development expense.

\subsubsection{Event Data Distribution Modules}

The event data distributor receives packages from the switch through a high-speed link. Switch 
packet boundaries need not coincide with event boundaries. The EDD reconfigures the data into event blocks that are then forwarded to the processors. Data is passed to the processors on one of four standard serial output links.

\subsubsection{Level 2 Trigger}

In the baseline design we assume that the Level 2 decisions are taken in the farm. A single processor is allocated to each event and takes care of the complete Level 2 and 3 trigger algorithms. The design is flexible and allows for different trigger strategies.

\section{Alternative Trigger Strategies}

There are a number of different trigger strategies. One can, for example, read out complete events or read data only as needed by the algorithms. For the first strategy all data is sent to a processor before the trigger algorithms run. There is no sharp distinction between Level 2 and Level 3. This method involves a fairly simple control protocol since events can be sent to the farm in the same order they were accepted by Level 1 .

The second strategy is currently the preferred one. The Level 2 algorithm starts by reading the Level 1 trigger output data. Based on the signature of the event, the algorithm retrieves only data needed by subsequent steps of the algorithms. For rejected events, data that are still in the EDCs are overwritten. For accepted events, all data is brought into the processor and the Level 3 algorithm is started. We no longer assume that the data stored in the EDCs is read out in event order. This strategy may reduce the data traffic through the switch by a large factor, so that a smaller switch and fewer EDDs can be used.

The flexibility of the GEM DAQ design allows for more complex schemes as well. For example, it is possible to apply different levels of zero suppression on the data sets used by Level 2 and Level 3. It has been shown that the Level 2 calorimeter algorithms perform well even if a $1 \mathrm{GeV}$ threshold cut is made on the EM tower energies. The reduced data set for Level 2 would be sent to the farm. The much larger full data set would remain in the EDCs, to be read for accepted events only.
Execution Times of Level 2 Algorithms

To estimate the contribution to the latency from the Level 2 algorithms, we have run simple calorimeter trigger algorithms on a workstation. The algorithms perform rudimentary checking on the data, compute $E_{T}$, build a lego plot and use it for jet and electron finding. Execution times depend strongly on the zero suppression level and will take several milliseconds on a 500 MIPS CPU. Execution times for tracking algorithms may be significantly larger. This indicates that the latency contribution from the algorithms themselves will be of the order of milliseconds. Hundreds of 500 MIPS CPUs will be needed to keep up with a Level 1 rate of $100 \mathrm{kHz}$. As typical system latencies will be larger than a few milliseconds, each processor will be working on many events simultaneously. This will require extra processor memory, fast context switching and reentrant code.

\section{Level 2 Upgrade Path}

An upgrade of the Level 2 trigger may have different goals: reduced latency, smaller load on the processors, or reduced traffic through the switch. This can be done in different ways. Special purpose coprocessors can be added to the farm or special purpose engines can be added to the EDDs. To reduce traffic through the switch, hardware must be inserted upstream from it. Special Level 2 processors can be implemented for specific subsystems. For example, one can install a silicon tracker trigger in the silicon data flow path at the EDCs. This trigger would output lists of high $p_{T}$ tracks to be used by Level 2 algorithms in the farm.

\subsubsection{Level 3 Trigger and Mass Storage}

Details on the farm and mass storage implementation are given in Chapter 8. The Level 3 trigger algorithms may be executed in the processors connected to the EDDs. If consistent with the primary role of the farm as event builder and Level $2 / 3$ filter, the farm will be structured so that it can be used for reconstruction and simulation when processing power is available during and between runs. 


\subsubsection{Control Network}

The most challenging aspect of this design is the control. Data flow must be regulated to use buffers and links in the most efficient way. Provisions must be made to download constants and software and to monitor the overall state of the DAQ system. Most of the control is done via a segmented control network. EDCs and EDDs are connected with medium speed data links. Total bandwidth for all control messages (at the maximum $100 \mathrm{kHz}$ event rate) is estimated to be $20 \mathrm{MByte} / \mathrm{s}$. A trigger supervisor CPU is connected to the network to assign events to processors and a system supervisor CPU monitors the readout.

\section{Front-end Control}

A simple bi-directional serial link is used to download and initialize each front-end board. These links operate asynchronously at approximately $20 \mathrm{Mbits} / \mathrm{s}$ using the system clock for $3 \times$ oversampling. For fast control two lines are used; the crossing clock and a crossing synchronous event signal. The event signal is normally interpreted as a Level 1 accept unless otherwise indicated by a preceding control message (such as "start," "stop," "test," etc.) on the serial link.

\section{Event Data Collector Control}

Events are buffered in the event data collectors. Control messages are used to request transmission to the event builder. As only part of the data is used by Level 2, only a fraction of the EDCs are asked to transmit it. EDCs may be addressed individually or as members of predefined groups. For accepted events a control message is sent to request event data in the remaining EDCs. The required control network bandwidth for event data requests is estimated at $10 \mathrm{MByte} / \mathrm{s}(100 \mathrm{kHz}$ event rate $\times 10$ messages/ event $\times 10$ Bytes/message).

\section{Event Data Distributor Control}

The data request messages from a processor to the EDCs pass through an EDD. By inspection of the messages, the EDD knows which EDCs have been requested to send data. The EDD waits until all requested data fragments have arrived, assembles the data and forwards it to the requesting processor.

\section{Event Allocation}

Each processor in the farm provides buffer space for multiple events. As described below, the latency of the data collection network is large. A processor has to ask for new events long before it is done with all previous ones. To get a new event allocated to it, the processor sends an "event number request" message to the trigger supervisor $\mathrm{CPU}$. This CPU sees the Level $l$ accept signals and knows how many events have gone to the EDCs. The trigger supervisor returns an "event number assignment" message to the processor that contains the event number for an event not yet allocated to any other processor. The processor then issues "send data" messages to the E:DCs from which it needs data, specifying the event number and processor ID. While the event data is being transmitted, the processor continues to process one of the previous events in its buffers. The processor may request event data fragments in any order and may process part of the data before issuing additional requests.

\section{Data Flow Control}

Each processor regulates its own data flow by requesting events only when it has a free buffer. This prevents buffer overflow in the processors. Processor efficiency is maintained by allocating multiple processes per processor. Each process has a buffer associated with it and deals with only one event. When no new event dati are available for processing in the EDCs, the prociessor can allocate time to off-line processing.

The EDDs have large buffers, calculated to avoid buffer overflows. If under exceptional circumstances buffers in an EDD start to fill up, messages must be sent to the EDCs to limit data flow to that particular EDD. EDD overflow is unlikely to occur in the selective readout mode, since data is delivered directly to the requesting processors. The event builder and high-speed data links are entirely synchronous, so no overflows are possible in these components. The EDC's avoid overflow by sending "inhibit trigger" control messages to the system supervisor and/or Level 1 trigger.

The front-end modules may also send overflow error messages to the system supervisor or 
L1 trigger. If an overflow occurs, the front-end module should attempt to send a valid packet (with or without partial data) since a missing event or an event with an incorrect word count will cause channel disconnection.

\section{Data Flow Latency}

The time required to access a block of data in an EDC and transmit that data to an EDD is a function of the block size and access method. For example, sending a $1 \mathrm{kByte}$ block at $1 \mathrm{Gbits} / \mathrm{s}$ takes 10 microseconds. However, in an $\mathrm{N}$ channel system, the event builder provides the required EDC-EDD connection only $1 / \mathrm{N}$ of the time. Hence, retrieval of a $1 \mathrm{kByte}$ block of data in a 512 channel system will require several milliseconds. Under full load the derandomizing output buffers in the EDCs normally contain several events and one may have to wait several full cycles of the switch. This can add 20-30 $\mu$ s of latency to each set of event data requests to the EDC. A typical Level 2 algorithm may require four to six sets of data before an event is rejected, leading to overall latencies of about 100-200 $\mu \mathrm{s}$. This means that up to 20,000 events may reside in an $E D C$ at one time. For strategies where all data are shipped to Level 2, an important contribution to the latency comes from the time it takes to transmit complete events over the medium speed links to the processors and leads to latencies of about $30 \mu \mathrm{s}$.

\section{Error Detection and Recovery}

At a data link bit-error-rate of $10^{-15}$, an error can be expected every 1000 seconds. If the error is in the data portion of an event packet, it is not considered critical by the data acquisition system. If the error is in the header (word count or event ID) the input channel is automatically disconnected and a message is sent to the system supervisor without interruption in data flow. The system supervisor may then attempt to reset the front-end module.

\section{Monitoring}

The system supervisor consists of one or more workstations. They download embedded software and configuration data to the data acquisition system. During operation, various components of the data acquisition system transmit summary status information to the system supervisor for monitor purposes. User control of the DAQ is performed via these CPUs.

\subsubsection{Partitioning}

Partitioning is the mechanism by which several users can independently read different parts of the detector. This mechanism is needed during commissioning, testing and calibration. In its most basic implementation one group of processors can read a given set of front-end boards, while another processor group reads a different set. To apply partitioning, one must control the different groups of front-end boards independently. They get independent Level 1 trigger signals and may have independent deadtimes and event number sequences.

We propose to provide partitioning at the EDC level. All front-end boards connected to one EDC will always belong to the same partition. As different partitions share resources such as the control network and switch, overall throughput may suffer in a partitioned system. This will happen, for example, in a simple partitioning scheme, where a group of eight EDCs connected to a single $8 \times 8$ switch module, is read by a group of eight EDDs connected to the same switch module. For this configuration, there is only a single physical link between the two groups, with a maximum bandwidth of one Gbits/s. This can be avoided by distributing the eight EDDs among eight different output switch modules.

\subsubsection{Scalability}

Components of the data acquisition system can be used to implement smaller systems. This way one can provide the data acquic:cion facilities needed for front-end and detector prototype tests. The smallest system with relatively few channels would be a single front-end group. It uses standard front-end boards to acquire the data. As a simple serial link is used to connect the front-end boards, any processor with an interface to that link can be used to handle the data.

For a larger setup, up to 24 front-end modules can be attached to one EDC. For test purposes, the data could be transferred over the medium speed serial VO link of the EDC that is normally used for control only. Again, any processor with an interface to that link can be used to handle the data. For normal data rates, the data is shipped over the high bandwidth link to an EDD.

The largest "small system" would be of detector subsystem size. The data acquisition system 
for such a setup would use several EDCs and would be connected to several EDDs via a single switch module.

\subsubsection{DAQ Software}

The main purpose of software in the DAQ is to provide flexibility, reliability and modularity in a complex environment. In most cases, deterministic behavior is required of hardwa:e devices and software algorithms, running on a wide variety of processor platforms, linked together by different communication mechanisms. A balance between hardware, supported by embedded software, and software running on commercial hardware platforms is essential.

Some modes of operation require "real-time" response in the processor farm. A context switching time of less than $100 \mu \mathrm{sec}$ is desirable. Within the data acquisition system, events must be processed at $10 \mathrm{msec}$ intervals. Embedded processor software is used primarily to transmit and receive control messages and to initialize DMA hardware. These processors may contain real-time operating system kernels, but control will be passed to a standalone DAQ process during data readout. Low level control of data flow and memory management is handled by firmware in the form of FPGA based algorithms.

\subsubsection{Simulation Studies of the DAQ System}

To check that the design of the GEM data acquisition hardware is sufficient to function under expected conditions, the flow of events through the system has been studied. Simulations have been run for the virtual Level 2 trigger archicecture in both the selective readout and full event builder mode. A system with 512 processors, 128 event data distributors (EDDs), and 512 event data collectors (EDCs) was simulated. The cross-bar switch then has 512 inputs and 128 outputs and is assumed to operate as a barrel switch. Under the assumptions outlined below, it was found that this system has more than enough capacity to handle the expected load.

The standard simulation is run with an event rate after Level 1 of $100 \mathrm{kHz}$. The average event size is assumed to be $200 \mathrm{kBytes}$. The flow of data through the switch is sensitive to how the data are distributed among the EDCs. A two-component distribution in which the RMS width is three times the mean has been used; that is, it includes a small number (12) of EDCs with large event fragments, as might be expected in events with jets. These 12 EDCs on average contain $38 \%$ of the event data. The remaining 500 EDCs are distributed uniformly and contain the rest of the data.

Four Level 2 steps are assumed, each with a rejection factor of 4.35 , giving an overall rejection factor of about 350 . In each Level 2 step, data from two of the 12 EDCs containing large amounts of data are transmitted along with data from $5 \%$ of the remaining EDCs. With this model, slightly less than $10 \%$ of the data is transmitted per step and the RMS data per EDC is about six times the mean for the transmitted data. If an event passes Level 2 , the rest of the event data are also transmitted.

So far it has been assumed that the processors can handle one of the four Level 2 steps in $1 \mathrm{~ms}$ and that Level 3 requires an additional $100 \mathrm{~ms}$. At $250 \mathrm{kHz}$, the processors are nearly saturated under these assumptions.

The current model of the GEM data used is based on estimates, and not on a detailed simulation. The results of the simulation will clearly depend on the model of the data. It will be shown, however, that the proposed system performs well above the expected data rate, leaving considerable design margin.

In the selective readout mode, the simulated system was able to cope with a $100 \mathrm{kHz}$ rate from Level 1 without difficulty. In fact, rates of up to $250 \mathrm{kHz}$ were simulated successfully. In order to cope with the high rates, it was necessary to allow the processors to request as many as 100 events at the same time. This large number allows the bandwidth available in the switch to be used effectively. The system also functioned at $100 \mathrm{kHz}$ with double the expected event size.

With the standard $100 \mathrm{kHz}$ rate, the mean latency of events in the EDCs was $0.137 \mathrm{~s}$ and the maximum event latency was $1.3 \mathrm{~s}$. The maximum amount of memory used in any EDC was about 6.5 MBytes, well below the proposed buffer size. The mean number of events requested by a processor was 26.

A simulation in the full event-builder mode was also run. That is, it was assumed that every Level 1 event was transferred in full to the proces- 
sors. This worked up to $50 \mathrm{kHz}$ with the same system parameters. With a somewhat larger system, we could run at $100 \mathrm{kHz}$ without the virtual Level 2 trigger. Nevertheless, the selective readout mode seems to be able to cope with five times the trigger rate attainable in the full event-building mode.

\subsection{SILICON MICROSTRIP DETECTOR FRONT-END}

\subsubsection{Introduction}

This section describes the design of the electronics associated with the silicon strip detectors. The scheduling and research and development efforts associated with the silicon tracker electronics are discussed in the central tracker section of this report (Section 6.7).

There are nearly 2.5 million individual PIN diode strips in the system, each requiring an individual amplifier plus readout chain. The strips are grouped into subunits called "ladders," such that a ladder corresponds to a physically separate mechanical unit. Electrically, each ladder is divided into two "half-ladders," each of which has its own 1280-strip readout system. The electronics for a half-ladder resides physically on a multi-chip module (MCM), which is bonded to the silicon-strip sensors of the half-ladder. With $1942 \mathrm{MCMs}$ in total, the channel count in this system is very high, so that a careful design is needed in order to minimize cost and maximize performance. The general parameters of the baseline system for these electronics are given in Table 7-12.

Tablo 7-12. GEM silicon tracker parameters.

\begin{tabular}{lr}
\hline $\begin{array}{l}\text { Total number of strip } \\
\text { electronic channels }\end{array}$ & $2,486 \mathrm{k}$ \\
Number of VLSI circuits: & 19,420 \\
Bipolar CMOS & 23,300 \\
Number of multi-chip & 1,942 \\
modules & 3,884 \\
Fiber optic data links & $<2 \mathrm{~mW}$ \\
\hline
\end{tabular}

The silicon tracker electronics requires the application of high-speed, low-power, low-noise, low mass, integrated microelectronics in a high radiation environment (equivalent to $10^{14} \mathrm{mip} / \mathrm{cm}^{2}$ total dose). Indeed, the requirements are more severe than have been imposed by any previous system in experimental high energy physics to date. This, coupled with the drive for low cost, make the engineering task at hand a challenging endeavor. Fortunately, the field of radiation hardened electronics has followed on the heels of commercial electronics, and bipolar and CMOS VLSI technologies that satisfy the requirements above exist.

The mechanical and thermal design of the electronics package is also an important consideration. The electronic packaging must represent a minimum number of radiation lengths in the system. Since there is a substantial amount of heat generated by the system, a careful arrangement of heat conduction paths is necessary so that any heat generated by the electronics travels efficiently to the heat rejection system. This requires that the MCM substrate be made of a material with high thermal conductivity. The mechanical design must also offer good containment of any RF signals generated by the MCM's digital electronics to ensure that no extra noise is coupled into the signal amplifier input. This is critical, since the system design requires intermittent transfer of digital data during the silicon detector live operation.

Table 7-13 lists various rate and occupancy conditions to be expected under normal SSC operal. ing conditions. Note that the individual channel occupancy is low, a fact that will be exploited by the digital buffering scheme (see below).

Table 7-13. Nominal SSC operational parameters for the GEM silicon tracker.

\begin{tabular}{ll}
\hline Nominal luminosity & $10^{33} \mathrm{~cm}^{-2} \mathrm{~s}^{-1}$ \\
Bunch crossing frequency & $60 \mathrm{MHz}$ \\
$\begin{array}{c}\text { Total charged particle } \\
\text { radiation dose } \\
(R=10 \mathrm{~cm}, \eta=0)\end{array}$ & $\begin{array}{c}.44 \mathrm{Mrad} / \mathrm{SSCY} \\
\text { (charged particle) }\end{array}$ \\
$\begin{array}{l}\text { Number of primary tracks } \\
\text { in tracker for a } \\
\text { hard scattering event }\end{array}$ & $100-200$ tracks \\
$\begin{array}{l}\text { Inner barrel occupancy at } \\
\ell=10^{33} \mathrm{~cm}^{-2} \mathrm{~s}^{-1}\end{array}$ & $0.16 \%$ \\
\hline
\end{tabular}

A schematic of the physical layout is shown in Figure 7-22. The silicon tracker electronics architecture is functionally divided into the following areas: amplifier, comparator, latch, memory, compression, transmission, clock distribution, and data acquisi- 
tion buffers. The amplifier and comparator reside on a bipolar VLSI chip (BIPOLAR). The latch, memory, and compression functions are implemented on a CMOS chip (CMOS-1). The data transmission is handled by an LED fiber optic link. Control and housekeeping functions are performed by two auxiliary CMOS chips, CMOS-2A and CMOS-2D, for the analog and digital sections respectively. The clock and Level 1 trigger distributor is a separate hybrid circuit, which contains a high speed fiberoptic receiver and a clock fan-out circuit that services up to eight MCMs.

\section{Input Signal}

The response of silicon detectors to minimum ionizing tracks is discussed in Chapter 6. The essential features are listed in Table 7-14.
Table 7-14. Input signal characteristics (one strip).

\begin{tabular}{ll}
\hline Input signal (normal incidence) & $4 \mathrm{fC}\left(24,000 \mathrm{e}^{-}\right)$ \\
Charge coliection time & $25 \mathrm{~ns}$ \\
$\begin{array}{c}\text { Strip-to-strip coupling capacitance } \\
\text { per unit length of strip }\end{array}$ & $1.0 \mathrm{pF} / \mathrm{cm}$ \\
$\begin{array}{l}\text { Lorentz drift angle (holes) } \\
\begin{array}{l}\text { Post radiation leakage current } \\
\left(0^{\circ} \mathrm{C}\right)\end{array}\end{array}$ & $1.5^{\circ}$ \\
$\begin{array}{c}\text { Rate from charged tracks } \\
\left(10^{33} \mathrm{~cm}^{-2} \mathrm{~s}^{-1}\right)\end{array}$ & $-1.0 \mu \mathrm{A}$ \\
\hline
\end{tabular}

\section{Amplifier and Comparator (BIPOLAR)}

The choice of a radiation hardened bipolar design is based upon its superior noise and power

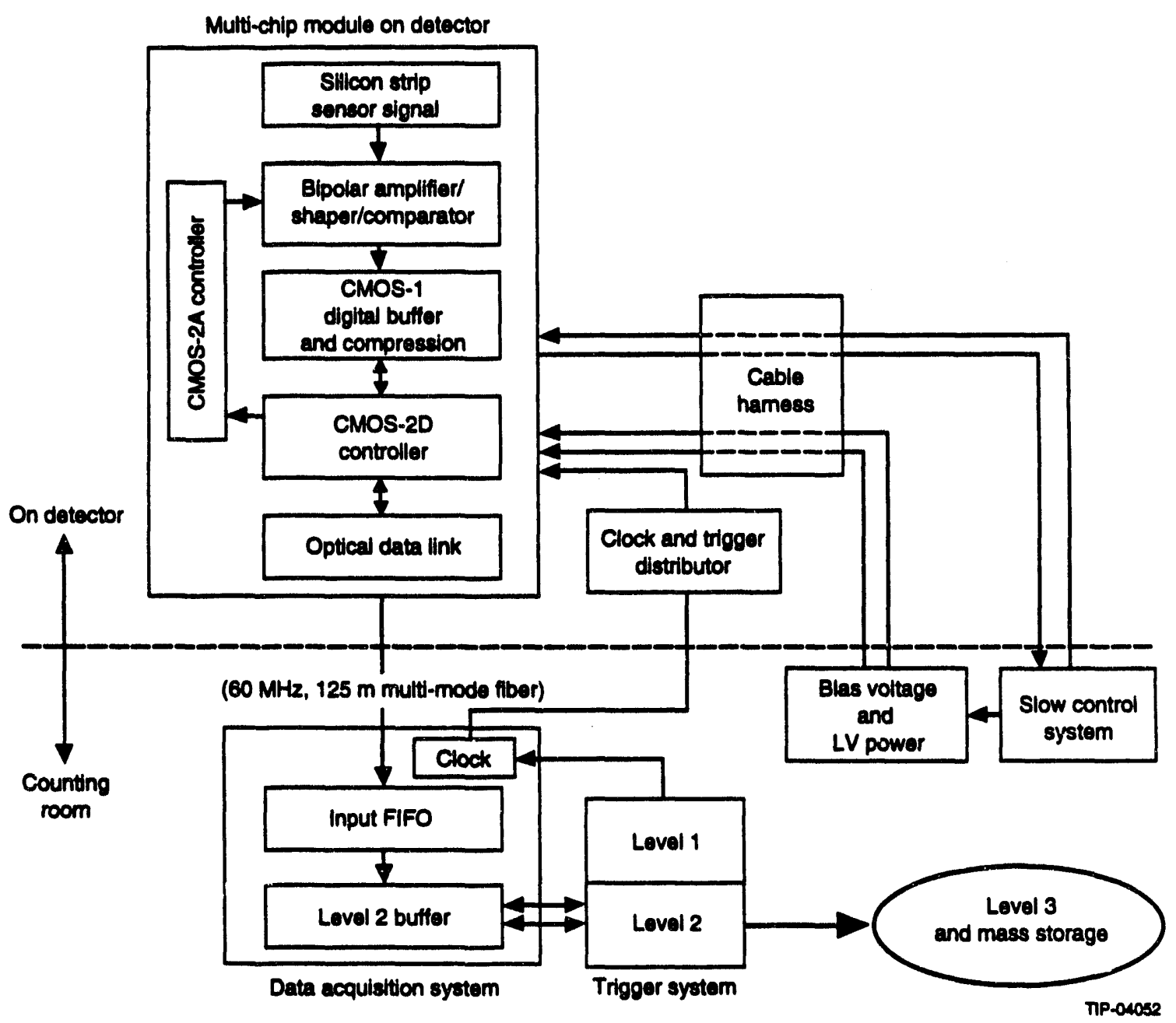

FIG. 7-22. Silicon tracker electronics architectu. 
performance. For amplifier rise times of $20-30 \mathrm{~ns}$, bipolar amplifiers out-perform CMOS amplifiers in noise-power product for capacitances above $6 \mathrm{pF}$. Equivalent noise charges (ENCs) in the range of $1500 e^{-}$can be achieved for lumped input capacitances of $15 \mathrm{pF}$. Taking into account the effects of interstrip capacitance and strip resistance, the equivalent noise increases to approximately $2200 e^{-}$(see Section 7.4 .2 below).

The effect of the comparator threshold on track-finding efficiency has been studied. The study employed a full event simulation via the GEANT code system, and included the effects of comparator' deadtime. It revealed that the threshold should be set $3 \sigma$ above pedestal (where $\sigma$ is the RMS noise) for good track-finding efficiency. Channel-to-channel variations in the gain and pedestal values and other system noise sources require a setting of $3.5 \sigma$ $\left(7800 e^{-}\right)$above pedestal in order to limit the noise-hit rate, and hence the channel occupancy, to a level wat is comparable to the intrinsic occupancy due to charged particles.

\section{The Digital Data Processing Architecture}

The proposed digital architecture consists of a latch, followed by data-driven storage and compression circuitry. The latch accepts asynchronous pulses of variable duration and generates synchronized 16-ns pulses, which are stored for the 2- $\mu \mathrm{s}$ Level 1 trigger latency. Triggered data is then compressed using either a clustering algorithm or an $8 / 16$ algorithm. Finally, it is transmitted off-detector via a fiber-optic link. Assuming a $100 \mathrm{kHz}$ Level 1 trigger rate, and a compression factor of between 5 and 25, a data bandwidth of 5-30 Mbits/s for each 1280-strip module is required.

\subsubsection{Electronics Design}

Detailed descriptions of these subsystems follow. In addition to meeting the formal requirements, the design optimization, which is ongoing, takes into account cost, fault tolerance, assembly complexity, and testability.

\section{The Bipolar Analog Chip Design \\ Design Considerations}

Baseline design requirements such as radiation-hardness levels, noise performance, discriminator time-walk as well as power consumption are well established by various studies. ${ }^{10}$ The circuit design and fabrication alternatives, however, have yet to be optimized. Specific design issues concerning the design of the analog bipolar ASIC include:

- Thermal environment, where the temporal fluctuation is small, but the thermal gradient over the entire tracker volume may not be.

- The rate of radiation damage as a function of relative location of the electronics, and, consequently, uneven gain changes of the transistors throughout the silicon tracker volume, causing non-uniform bias point shifts.

- Testability and controllability of each channel, while considering the enormous number of channels to scan.

- Protection of each input line from most transient surges or spikes.

- Fluctuation of the bias points of the circuits due to process variation.

- Cost of production, which is a strong function of die yield.

A detailed description of the design of this circuit has been given elsewhere. ${ }^{11}$

\section{Design Baselines and Goals}

The input current of the preamplifier is positive (hole-current) since the GEM silicon tracker uses single-sided detectors. Signal charge is AC coupled to the preamp input, so that separate HV blocking capacitors are not necessary. The amplifier input does, however, require protection from large accidental voltage spikes.

The primary design goals are shown in Table 7-15..

Table 7-15. Performance goals for the silicon electronics analog chip.

\begin{tabular}{ll}
\hline Power consumption: & $\begin{array}{l}1 \mathrm{~mW} / \mathrm{channel} \\
( \pm 1.5 \mathrm{~V}, 3.3 \mathrm{~V} \text { supplies })\end{array}$ \\
$\begin{array}{l}\text { Equivalent noise } \\
\text { (lor } 15 \mathrm{pF} \text { lumped input } \\
\text { capacitance) }\end{array}$ & $\begin{array}{l}1200 \mathrm{e}^{-} \text {B.O.L. } \\
1500 \mathrm{e}-\mathrm{E} . \mathrm{O} . \mathrm{L} .\end{array}$ \\
$\begin{array}{l}\text { Double pulse resolution: } \\
\text { Time walk ( } 1 \mathrm{fC} \text { to } 8 \mathrm{fC}):\end{array}$ & $100 \mathrm{~ns}$ \\
\hline
\end{tabular}

\section{Prototype Circuit Design}

Figure 7-23 shows the prototype circuit, which includes the preamplifier, amplifier, shaper with a baseline restorer, and a level discriminator. The 
preamplifier is a conventional cascode arrangement with an emitter-follower buffer. A charge conversion capacitor of $0.2 \mathrm{pF}$ is used in parallel with a $120-\mathrm{k} \Omega$ resistor, yielding a $24-\mathrm{ns}$ charge integration time, which is dictated by the charge waveform from the strip detector. A larger feedback resistor with a smaller conversion capacitor would increase the conversion gain and lower the equivalent input noise. However, the feedback resistor provides the base current of the input transistor so that a decrease in its value would result in larger DC-bias fluctuations (due e.g., to process variations and radiation damage effects) at the preamplifier output. The input circuit node has a protection diode with a $50-\Omega$ current limiting resistor and calibration injection capacitor. The noise contribution of the current limiting resistance is insignificant compared to other noise sources.

The preamp output is fed to an emitter degeneration amplifier with a gain of approximately 3.5 , which was determined from bias-drift considerations for the direct-coupled stages. Larger gains result in too much DC shift for the baseline restoration when the gains of individual transistors change.

The third stage amplifier not only amplifies and shapes the signal, but also maintains the output quiescent point near zero through a baseline restoration amplifier, which feeds back an error signal through a low-pass filter. The pole of the low-pass filter is at a sufficiently low frequency so as to not significantly attenuate the signal, and thus the signal-to-noise ratio. The closed loop gain of the baseline restorer, including the gain of the main differential amplifier, is kept within the gain margin for stable operation.

The DC-restored signal output is discriminated against the reference voltage at the differential inputs of the comparator. The comparator has two differential gain stages followed by three currentgain stages, which use current mirrors.

Simulation Results

Component model libraries from Harris (UHFseries) and AT\&T (CBIC-U, U2, V series) were used to simulate the circuit operation. Both technologies include state-of-the-art multi-GHz complementary transistors. The simulation models incorporate distributed parasitic elements, as required for simulation of high-frequency operation. Figure 7-24a shows the simulated response at the shaper output to typical input waveforms of various amplitudes. The shaper output has a stable DC bias level that is within a few millivolts of ground. It has a bipolar swing due to the differentiating effect of the baseline restorer, which essentially cancels the low-frequency component of the signals at the shaper input.

Figure 7-24b shows the comparator output for the same set of inputs. Note the delayed leading edge of the smaller comparator output pulses, which is a result of time walk. The double-pulse resolution of about $150 \mathrm{~ns}$ is determined by the comparator reset time.

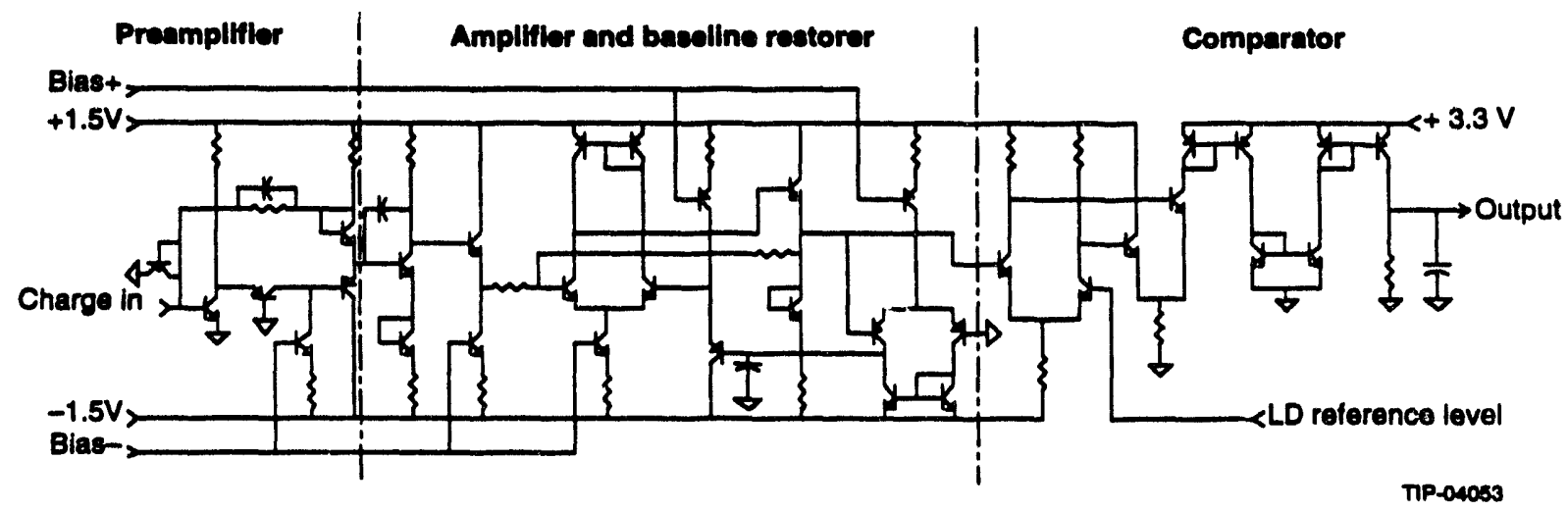

FIG. 7-23. Silicon tracker amplifier and comparator design used for SPICE simulation. 
For 18-cm strips, charge sharing among neighboring channels due to inter-strip capacitances and time dispersion of the propagated signal must be minimized. For a given preamplifier charge-collection time, the charge collected per pulse and the resulting signal-to-noise ratio is limited by the total strip capacitance and resistance. Moreover, with non-ideal strip-line termination, care must be taken to avoid oscillatory operation of the amplifiers resulting from reactive interference between adjacent channels. The best performance will be obtained from detectors with the lowest interstrip capacitance and metalization resistance. For example, when the equivalent strip resistance : reduced from $50 \Omega / \mathrm{cm}$ to $20 \Omega / \mathrm{cm}$, the equivalent noise for far-end charge injection decreases by as much as
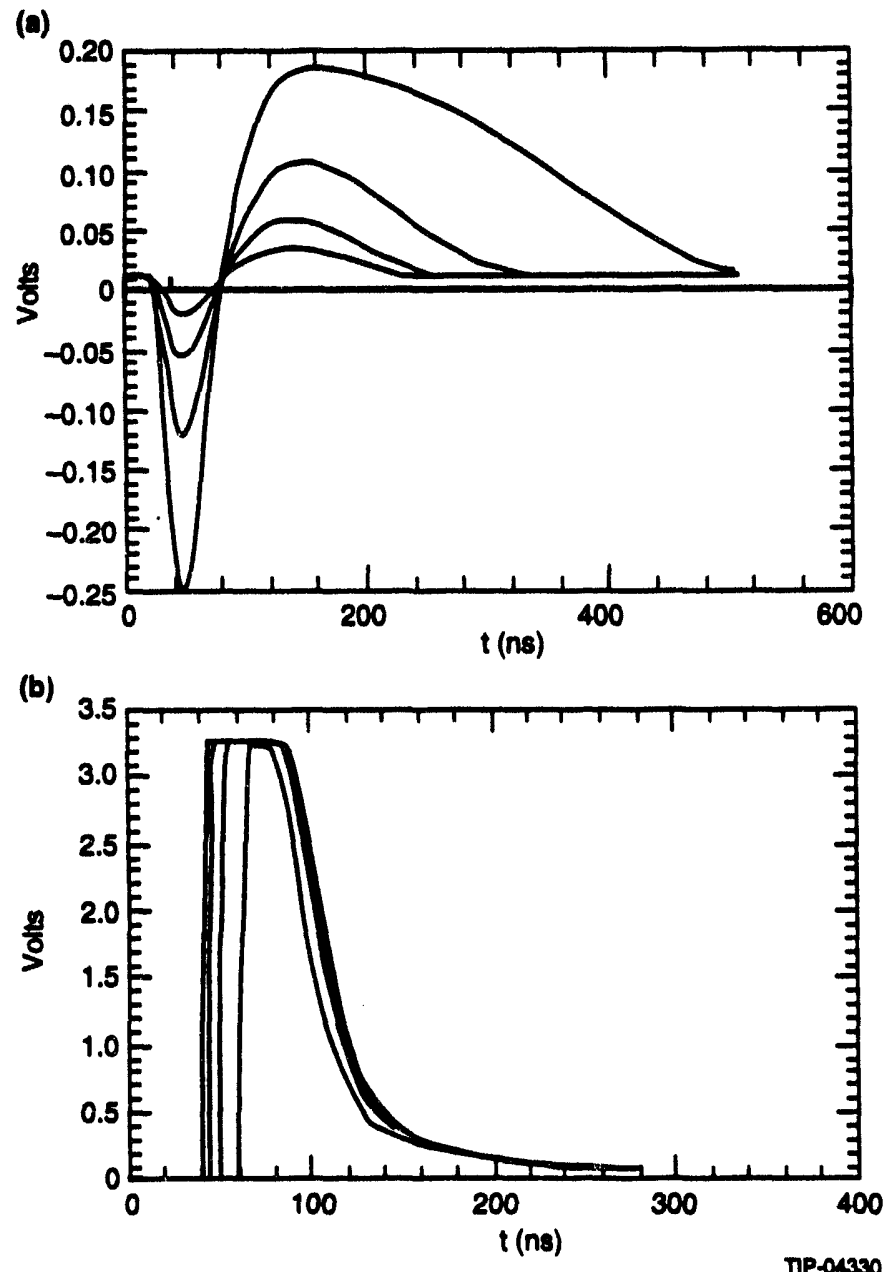

FIG. 7.24. a) Silicon tracker preamplifier/shaper response to 1, 2, 4, and $8 \mathrm{fC}$ input charges.

b) Comparator outputs for 1, 2, 4, and 8 fC input charges.
$10 \%$. Similarly, lowering the inter-strip capacitance from $1.2 \mathrm{pF} / \mathrm{cm}$ to $0.8 \mathrm{pF} / \mathrm{cm}$ lowers the noise by $15 \%$. Reducing both of these parameters is an important goal of the GEM central tracker R\&D program.

The simulation results listed in Table 7-16, were obtained with the AT\&T CBIC-V series transistor libraries. The $18-\mathrm{cm}$ detector is simulated assuming a range of distributed capacitance and resistance values, while the charges were injected at either the far or the near end. To better simulate the strip-detector operation, five strips were arranged in parallel with the middle three channels connected to the amplifiers and the outer two channels terminated with a $100 \Omega / 100 \mathrm{pF}$ combination. In each of the simulations, a Landau detector-charge distribution was used.

Table 7-16. ENC as a function of linear strip resistance and capacitance.

Equivalent noise in electrons for far(near) end charge injection for $18 \mathrm{~cm}$ strips

\begin{tabular}{llll}
\hline \multicolumn{2}{c}{$\begin{array}{c}\text { Noise vs capacitance } \\
(20 \Omega / \mathrm{cm})\end{array}$} & \multicolumn{2}{c}{$\begin{array}{c}\text { Noise vs resistance } \\
(1.0 \mathrm{pF} / \mathrm{cm})\end{array}$} \\
\hline $0.6 \mathrm{pF} / \mathrm{cm}$ & $1948(1916)$ & $10 \Omega / \mathrm{cm}$ & $2191(2180)$ \\
$0.8 \mathrm{pF} / \mathrm{cm}$ & $2122(2092)$ & $20 \Omega / \mathrm{cm}$ & $2276(2233)$ \\
$1.0 \mathrm{pF} / \mathrm{cm}$ & $2276(2233)$ & $30 \Omega / \mathrm{cm}$ & $2356(2272)$ \\
$1.2 \mathrm{pF} / \mathrm{cm}$ & $2432(2371)$ & $40 \Omega / \mathrm{cm}$ & $2431(2303)$ \\
$1.4 \mathrm{pF} / \mathrm{cm}$ & $2664(2579)$ & $50 \Omega / \mathrm{cm}$ & $2501(2327)$ \\
\hline
\end{tabular}

A preliminary analysis of the time walk, including Landau fluctutations, charge sharing and geometrical effects shows that $99 \%$ of all hits fall within a single 16-ns bunch crossing interval. An equivalent noise charge of $2200 \mathrm{e}^{-}$RMS and a threshold of $7000 \mathrm{e}^{-}$was assumed. It is therefore expected that time walk will not seriously affect tracking efficiency.

The simulation results do not yet reflect the worst-case operation of the front-end electronics, which is expected at the end of the lifetime, when the transistor gains are likely to drop to less than one-half of their beginning-of-life values. Nor do they include a possible degradation in noise from increased detector leakage currents, which are expected to be near $1 \mu \mathrm{A}$ per strip. These and other effects, such as interference noise from the logic 
circuits located on the same multi-chip modules and process variations, will be added to future simulations.

\section{Digital Architecture}

A single channel of the digital portion of the silicon strip readout is shown in Figure 7-25. The digital section on each MCM processes the analog hits from a total of 1280 channels. It comprises ten 128-channel CMOS-1 chips, one CMOS-2D chip, one time-division multiplexor (TDM), and two LEDs. The CMOS-1 chips latch, buffer, and compress incoming pulses from the BIPOLAR comparator. The CMOS-2D IC interrogates the ten CMOS-1 chips, encodes the data and drives the TDM. In addition, the CMOS-2D chip operates the slow-control and test systems. The TDM chip multiplexes the $30 \mathrm{Mbits} / \mathrm{s}$ "top" and $30 \mathrm{Mbits} / \mathrm{s}$ "bottom" into a single $60 \mathrm{Mbits} / \mathrm{s}$ channel and provides the necessary drive current to the LED. The TDM chip can also be run in a mode that will provide two independent optical channels ensuring additional reliability and bandwidth-i.e., a $2 \times 30 \mathrm{Mbits} / \mathrm{s}$ "top" plus a $2 \times 30 \mathrm{Mbits} / \mathrm{s}$ "bottom" for a total bandwidth of $120 \mathrm{Mbits} / \mathrm{s}$.

\section{Data Latch}

Variations in the time and amplitude of the charge waveform produce both leading-edge and trailing-edge time jitter (or time-walk) at the output of the analog stage. The function of the latch is to produce a synchronous (relative to the system clock)

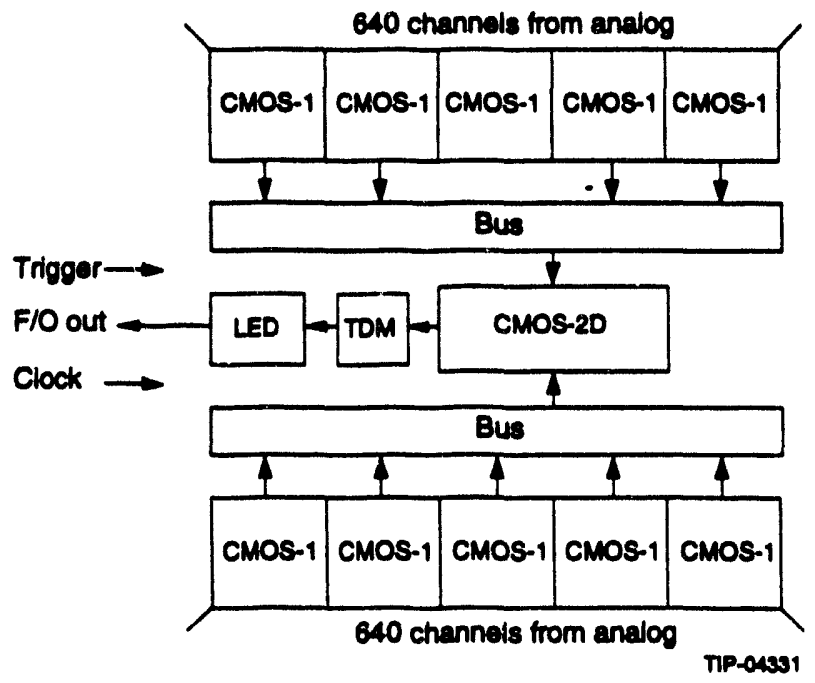

FIG. 7-25. The processing architecture of the digital data storage on the CMOS-1 chip for the silicon tracker. pulse, independent of the leading- and trailing-edge jitter of the analog stage. The latch can be programmed to trigger on rising or falling edges. Edge triggering renders the system insensitive to "shorted" amplifier and strip-detector channels.

\section{Data Storage (Concurrent Pointer Tracking)}

The expected strip occupancy is very low, allowing for the implementation of data-driven storage $e^{12,13,14}$ wherein only hit channels initiate data processing. Since the relative processing activity in an ideal data-driven system is directly proportional to the channel occupancy, a significant reduction in data processing volume is expected. This leads to lower power dissipation and simplifies the design.

The proposed architecture, which is shown schematically in Figure 7-26, makes use of two pointers (READ and WRITE), a register (COUNTER) that is incremented with each bunch crossing, and a bank of memory registers (REG0 through REG7). When an edge is detected and latched, the current value of COUNTER is copied into the register pointed to by WRITE (REG6 in the example shown). WRITE is then incremented to point to the next register (REG7) in anticipation of the next latched edge. READ is incremented on the subsequent clock cycle.

To read the array, the value of COUNTER and the contents of the register pointed to by READ (REG3 in this example) are continuously compared

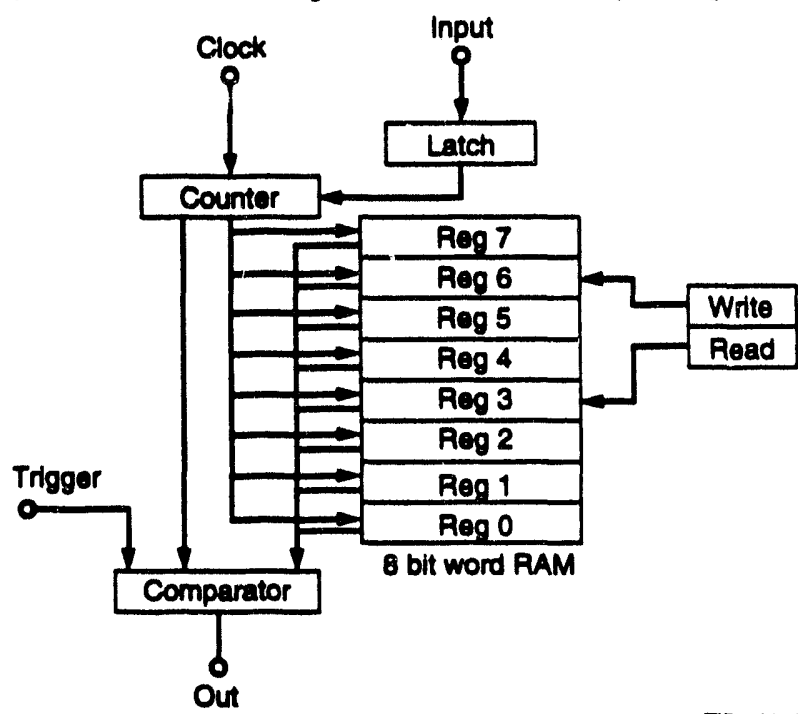

TIP-04333

FIG. 7-26. Sillcon tracker CMOS-1 data driven memory. 
until they are equal. For an $n$-bit counter, this condition will occur $2^{n} \times 16$ ns after REG3 was written-i.e., COUNTER must complete one complete cycle. The time interval required for it to do so is chosen to be equal to the Level 1 trigger latency. Depending on whether or not the Level 1 accept line is asserted when the match occurs, the value of OUT is set to either " 1 " or " 0 ". In either case, READ is incremented to point to the next register (REG4 in this example). A larger trigger aperture can be established by asserting the Level 1 accept line for successive bunch crossings. Operation proceeds in this manner until READ and WRITE point to the same register location, which happens when no hits have occurred within the trigger latency interval. At this point, the circuit lies dormant and all Level 1 accept signals are ignored until a new edge is detected.

A key advantage of this technique, which is called concurrent pointer tracking, is that the READ pointer is guaranteed to point to the address of the only possible event candidate when a Level 1 accept occurs. Moreover, each single-bit circuit (i.e., Figure 7-26) is a self-contain d autonomous cell, minimizing the potential impact of single-point failures. The cells are self-correcting in the event of a single-event-upset (SEU) logic glitch, since COUNTER will normally cycle through all the "garbage" and return to the dormant state before the next trigger is delivered.

\section{Data Compression}

Upon receipt of a Level 1 trigger, the system transfers the 128-bit data vector from the data storage and initiates a data compression cycle. Two compression algorithms, both of which exploit the sparse nature of the silicon microstrip data, are under investigation. These are clustering and $8 / 16$. The clustering algorithm is more efficient, but the $8 / 16$ is easier to implement.

Clustering: Data compression through clustering is accomplished by transmitting both cluster and single-hit information. The algorithm proceeds as follows: the uncompressed data retrieved from storage are edge-detected for either single-hit or cluster edges (here edge refers to a transition from a strip that has not fired to one that has, or vice versa), and the relative position offsets of the edge boundaries are found. The resulting compressed data vector is then composed of the number of edges and the position offsets of each edge (relative to the CMOS-1 boundary in this design).

8/16: 8/16 data compression is accomplished by dividing the 128-bit segments into 16 eight-bit words. The 16 words are independently logic ORed. If an eight-bit word contains a hit, that particular word is sent up. Each 128-bit segment contains a 16-bit segment header indicating which 8-bit words are hit, followed by a series of non-zero eight-bit words.

Cluster-8/16 Compression Efficiency: A Monte Carlo simulation for both the cluster and 8/16 compression efficiency shows that the clustering algorithm is $40-50 \%$ more efficient than the $8 / 16$ algorithm over the occupancy ranges expected at the SSC. However, the 8/16 appears to be easier to build, runs faster, and may be more reliable than clustering. The choice of algorithm will be made as the design progresses.

\section{Data Transmission}

Fiber optic data links are used to transmit clock and trigger signals to the front-end readout and to carry digital data off-detector. Each MCM requires two dedicated data readout links, but shares its clock and trigger links with seven other modules. The clock and trigger are brought onto the MCM through micro coax from a central optical receiver.

\section{Optical Readout}

The design of the optical readout calls for an $850 \mathrm{~nm}$ optical link that employs a rad-hard LED from Honeywell. It uses a large-numerical-aperture fiber, a surface mount LED, and a TDM driver.

A large diameter $(O D=500 \mu \mathrm{m})$ fiber has been selected for reasons of economy and ease of handling. In particular, using such fibers will reduce the cost of assembly, which is typically an important component in the overall cost of optical links. For example, inexpensive connectors can be manufactured by drilling $500-\mu \mathrm{m}$ holes in lucite blocks, which can then be bolted together. Moreover, a large diameter fiber captures more of the diffused LED light and minimizes losses from fiber positional mismatch.

The time-division-multiplexed (TDM) chip not only provides the multiplexing 'etween the two 
CMOS-2D channels but also provides the appropriate buffering. The circuit is fairly standard and an appropriate off-the-shelf part should be available. If the incoming clock (see below) needs buffering, an additional buffer can be provided on the TDM chip.

\section{Clock and Trigger Distribution}

The clock and trigger are carried on the same fiber by phase locking the clock. The clock is fed into a phase-locked-loop (PLL) and distributed to the MCM assemblies through micro coax. The trigger is signaled by the absence of a clock tick, i.e., when a clock pulse is deliberately left out. When the PLL detects and generates a missing pulse, a trigger signal is distributed through a second set of micro coax. The signal-to-noise ratio of the optical link must remain high enough to maintain the integrity of the trigger. Suitable GaAs PLL clock distribution chips are commercially available.

\section{Multi-Chip Module (MCM) Design}

The base assembly unit is composed of two 640-channel silicon strip detectors bonded together back-to-back to form an $18-\mathrm{cm}$ half ladder. A single MCM provides the interface electronics for both the top 640 and bottom 640 channels. A schematic of the MCM is shown in Figure 7.27. The MCM provides an efficient interface between the electronic and mechanical systems. It is supported by the stripdetector wafer through a thermally-conductive spacer, while the strip detector itself is mounted (glued) to the cooling ring. Note that all mechanical contacts in this design straddle the thennal symmetry axes in order to minimize position drifts due to thermal expansion and contractions. Power and the digital slow-control bus are connected to the space frame cabling through an edge connector, while the clock and trigger are brought in through $100-\Omega$ micro coax from the 1:8 phase-locked optic-to-coax distribution modules situated on the cooling ring. Data from the MCM is transmitted through the fiber-optic link that also runs through the space frame.

Each MCM assembly is a self-contained unit. All functions, including the analog and digital signal processing circuits, digital control circuits (CMOS-2), and slow-control are fully integrated, as shown in Figure 7-27.
The MCM technology under consideration utilizes the General Electric/Texas Instruments High-Density-Interconnect (HDI) process. This process imbeds the IC chips in the substrate and builds the interconnect layers above the chips. This technology maximizes heat transfer from the chip to the MCM and from the MCM to the cooling ring and minimizes spurious radiation due to digital switching and power-supply coupling by maintaining quasi-TEM modes in its signal paths. A complete layout has been performed.
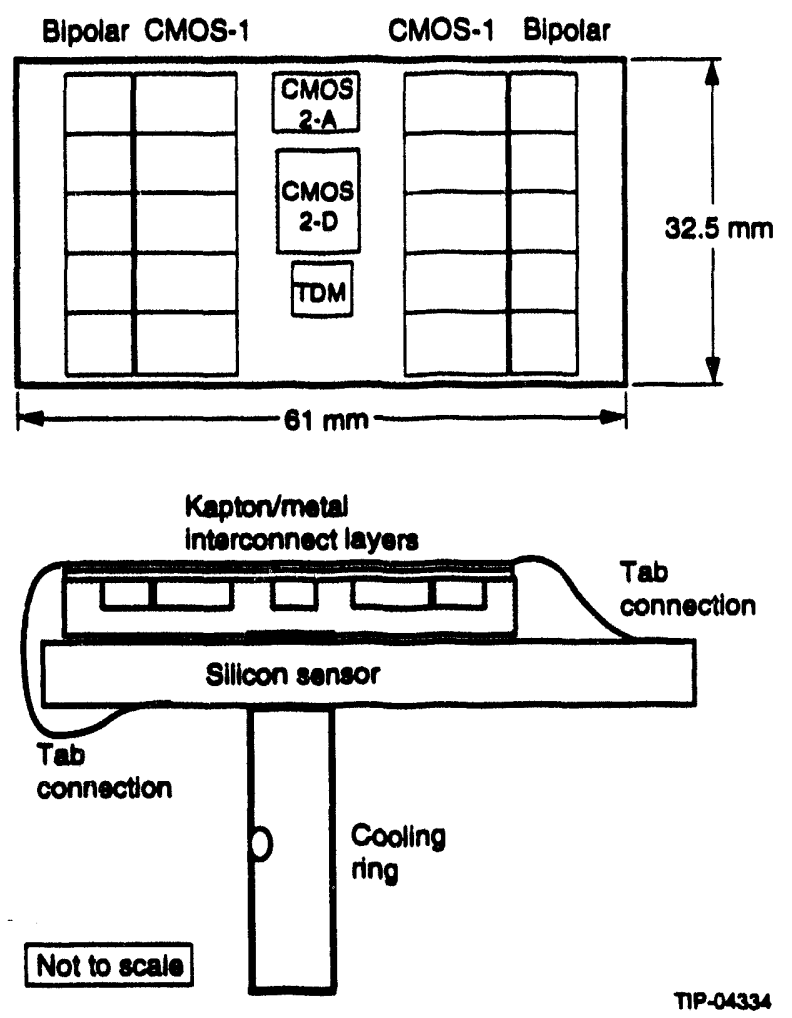

FIG. 7-27. Silicon tracker MCM unit. Arrangement of VLSI Circults on MCM and the mounting of the MCM to the ladder assembly for the silicon tracker.

\subsection{IPC CENTRAL TRACKER}

The readout electronics for the Interpolating Pad Chamber (IPC) system must satisfy four basic requirements. The most important is that the signalto-noise ratio be maintained above 100 throughout the readout chain. It is likely that the position resolution of the IPCs will be determined by the noise inherent in the readout process. The second requirement is that the IPCs be read out in time to participate in a Level 2 trigger decision. A third 
requirement of the front-end electronics is that it be rad-hard. Finally, the power requirements of the electronics must be compatible with the use of a low-mass cooling system.

The signals induced on the IPC cathode pads by an avalanche at the anode are typically $30 \mathrm{fc}$ in size, and develop over a time characteristic of the ion drift time in the chamber. These current pulses are amplified and shaped by a charge integrating preamplifier, with a nominal gain of $1 \mathrm{mV} / \mathrm{fC}$, followed by a shaping amplifier with a voltage gain of 10, and a peaking time of 25-30 ns. The sliuped signals are then sampled at the $60 \mathrm{MHz}$ bunch crossing rate. These samples are stored in analog form in a switched-capacitor array until the receipt of a Level 1 trigger. At that time, the samples of interest are digitized, zero suppressed, and transmitted off the detector through a fiber-optic link. For each pad with valid data, two to four time samples are transmitted. These correspond to a presample, which allows the baseline to be subtracted from subsequent samples, and the samples that span the shaped pulse. A full simulation of this readout approach has been performed, in which an ensemble of signals is generated for random trajectories through the chamber using the wire chamber simulation program GARFIELD. These induced-current signals are amplified and shaped, and then sampled and digitized. The effect of electronics noise on the samples, jitter in the sample time from pad to pad, shaping time differences from pad to pad, and digitization error have been studied. The results of this study, which are presented in the description of the IPC system in Chapter 6, indicate that this readout approach is viable. A full prototype of this readout system is now being developed and will be available for testing this year.

\subsubsection{Radiation Hardness}

Recent studies of the radiation environment in the GEM central tracker volume indicate that the charged particle radiation dose is approximately $0.44(10 \mathrm{~cm} / \mathrm{r})^{2} \mathrm{Mrad} / \mathrm{SSCY}$. To allow several years of operation at $10^{34} \mathrm{~cm}^{-2} \mathrm{~s}^{-1}$, IPC electronics at a radius of $40 \mathrm{~cm}$ must survive a radiation dose of
2 iMrad while maintaining the noise specification above. The front-end electronics being designed for the GEM outer tracker consists exclusively of CMOS ICs at the present time, although a bipolar preamplifer/shaping amplifier is currently under study. Neutrons are generally a less serious problem in rad-hard CMOS than ionizing radiation, since CMOS is not sensitive to bulk damage. Neutron fluences below $10^{15} \mathrm{~cm}^{-2}$, curresponding to an exposure of 500 SSCY, can be handled without serious degradation in performance in the CMOS processes we consider. Ionizing radiation in CMOS affects the gate oxide and the field oxide regions. The primary effects are a shift in the threshold voltage of the transistor and channel mobility degradation, due to a buildup of holes at the $\mathrm{Si}-\mathrm{SO}_{2}$ interface. Because of this threshold shift, $n$-channel devices become easier to tum on, and p-channel devices become harder to turn on. In analog circuitry, threshold shifts result in a change in the biasing of the circuit. In the rad-hardened CMOS processes under investigation, threshold shifts of $0.2 \mathrm{~V}$ are typical for a 1-Mrad dose. At the present time, the development of custom electronics for the IPC system is being carried out in the Harris AVLSI-RA process, which is radiation tolerant to the required level. As part of our R\&D program, we are developing essentially full-scale prototypes of the critical front-end components. These are expected to be fabricated starting in 1993.

\subsubsection{IPC Readout Electronics Architecture}

The baseline IPC readout architecture is illustrated in Figure 7-28. The physical layout of this electronics on a barrel IPC chamber is shown in Figure 7-29. As shown in Figure 7-28, the signals from the cathode pads are input to octal preamp/ shaper ICs. The amplified, shaped signal is input to an analog memory element, which is clocked at the $60-\mathrm{MHz}$ bunch-crossing rate. The analog memory is packaged 16 channels to a chip, and includes a 16-to-1 analog multiplexer at the output. The multiplexer output feeds a single FADC. The READ/WRITE operations of these front-end devices are controlled by a readout chip that services 128 pad channels. 


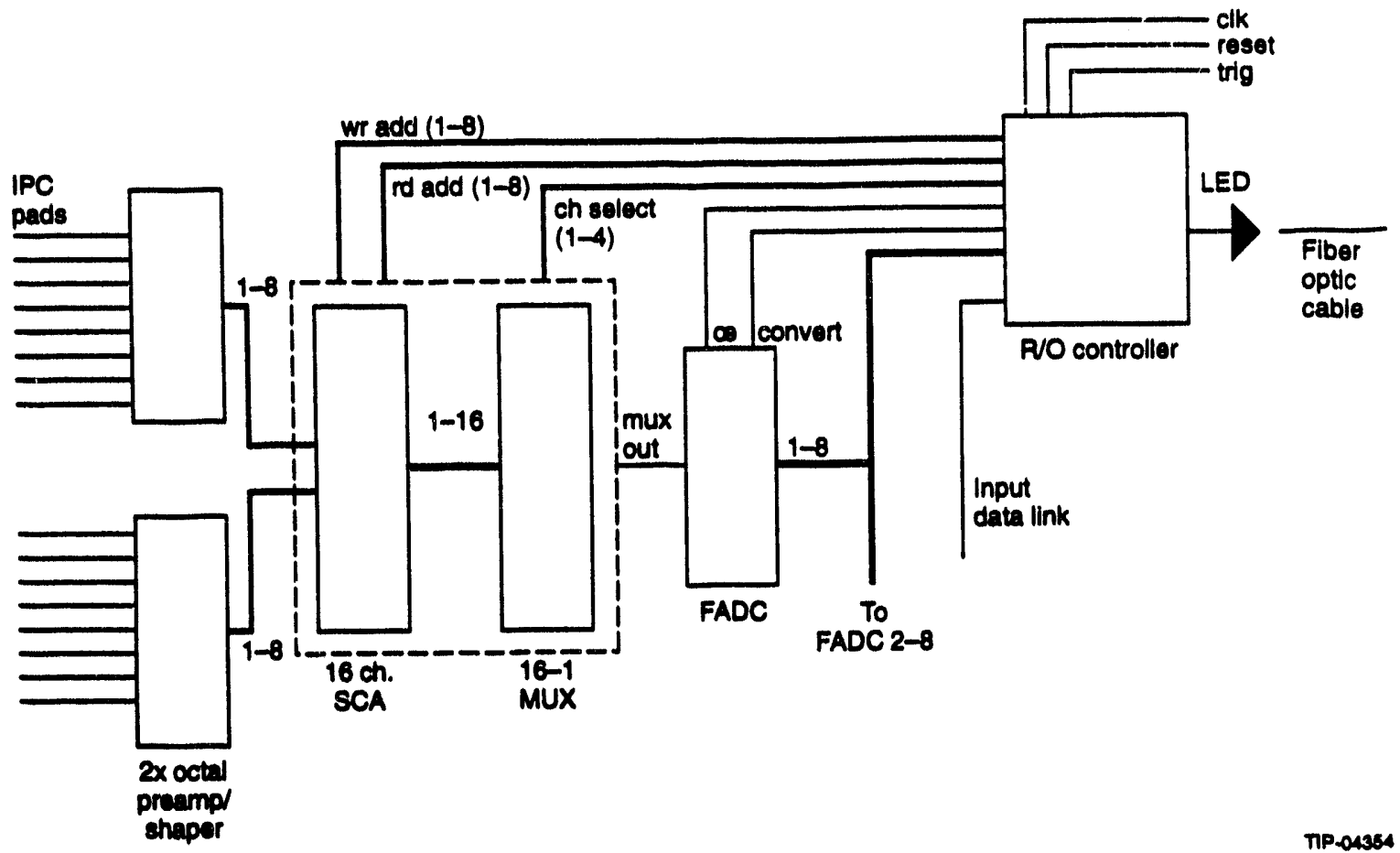

FIG. 7-28. IPC electronice architecture.

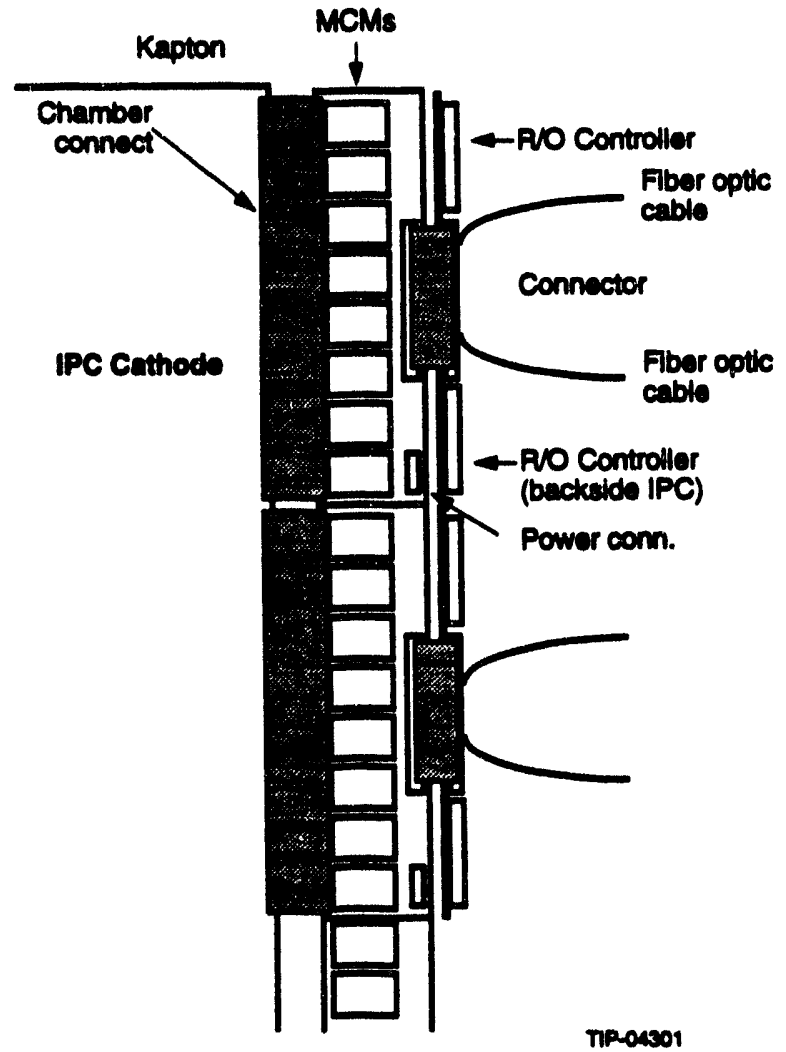

FIG. 7-29. IPC electronics layout.

\subsubsection{IPC Preamp/Shaper}

The IPC prearnp and shaping amplifier circuits are based on a design developed by the instrumentation group at Brookhaven National Laboratory. In the present application, the front-end preamp/shaper IC is packaged on a separate die from the analog memory to minimize coupling of the digital signals into the analog readout. Two technologies are being considered for the amplifier. Analog CMOS is available from several vendors and offers the advantages of fast multiproject service for rapid prototyping from several vendors; the availability of model parameters for BSM, which is the most advanced MOS model for analog design; and a technology that is well integrated into commercial and public-domain CAD packages.

Bipolar processes remain a candidate for this device. Many foundries have recently announced advanced processes optimized for high-frequency analog work, where the devices generally have higher key performance ratios and are inherently more tolerant to gamma radiation. The specifications for the preamplifier and shaper are given in Table 7-17. 
Table 7-17. Amplifier specifications.

\begin{tabular}{ll}
\hline Peaking time & $25 \mathrm{~ns}$ \\
Input capacitance & $10-40 \mathrm{pF}$ \\
RMS noise & $-1000 \mathrm{\sigma}$ \\
Power dissipation & $60 \mathrm{~mW} / \mathrm{ch}$ \\
Linearity & $<1 \mathrm{LSB}$ \\
Gain & $10 \mathrm{~V} / \mathrm{pC}$ \\
Dynamic range & 1,000 \\
Full-scale charge & $150 \mathrm{fC}$ \\
Filter-Shape & Semi-gaussian \\
\hline
\end{tabular}

The baseline preamp design uses an $n$-channel input device in a folded-cascode configuration, as shown in Figure 7-30. The width of the input device must be selected to optimize the signal-to-noise ratio by matching the capacitance of the input device and the signal pad. The first prototypes have an input device with a $10000-\mu \mathrm{m}$ gate width biased at $5 \mathrm{~mA}$ (nominal).

The $\mathrm{CR}-(\mathrm{RC})^{\mathrm{n}}$ shaper response can be obtained with a variety of circuit configurations. We have chosen a low-pass stage with a second-order transfer

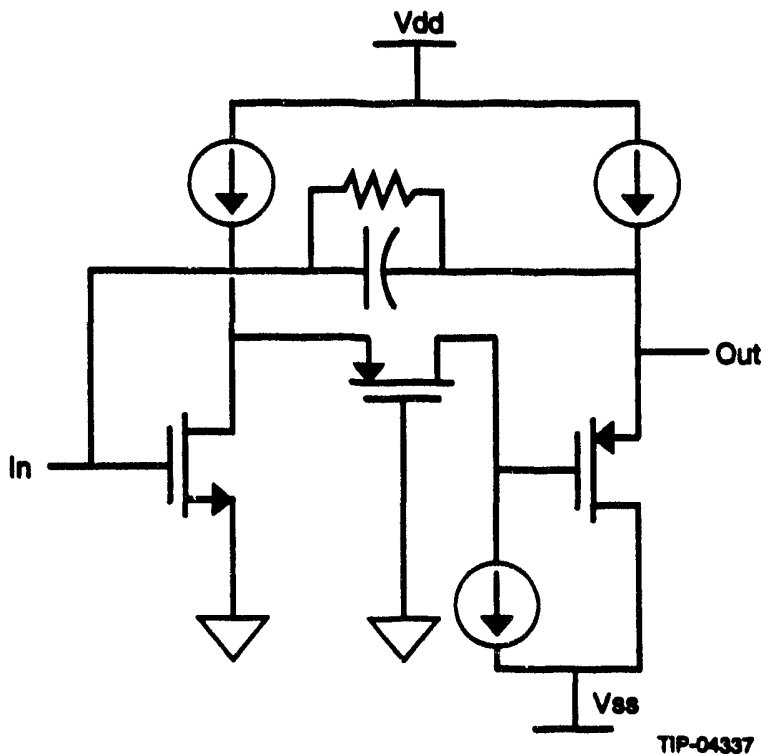

FIG. 7-30. Preamplifier design. function. The natural frequency of the filter in this design can be changed by adjusting the bias current at points in the circuit. On the other hand, the damping factor is determined only by component geometry, i.e., transistor W/L. Power dissipation is reduced by having two poles per op-amp. A passive differentiator and two stages of this type form a shaper whose peaking time can be controlled (for all channels on a chip) by a single bias without distorting the shape of the resulting pulses. This is a simple way to trim out manufacturing variations. Figure 7-31 shows the results of noise measurements made on a prototype preamplifier circuit, fabricated using MOSIS. In this figure we see the relationship between the RMS noise and the input capacitance and shaping time.

Tests conducted on a single-channel version of this chip demonstrate that the performance of the amplifier is well represented by the circuit models used in the design of the chip, and they indicate that the design goals will be met. A fabrication run in the Harris AVLSI-RA process will take place soon.

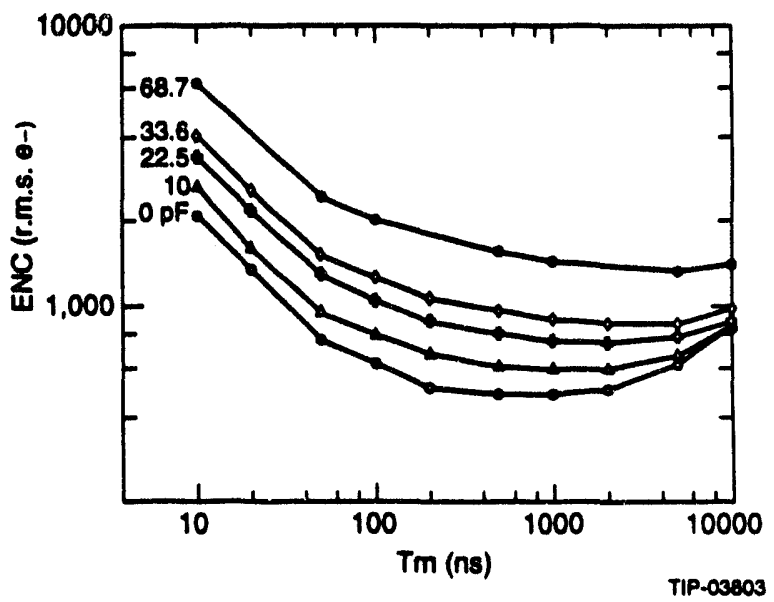

FIG. 7-31. Preamplifier noise measurements.

\subsubsection{IPC Switched-Capacitor Array}

The IPC switched-capacitor array is a 16-channelby-150-cell array. The device has simultaneous read/ write capabilities and will clock at $60 \mathrm{MHz}$. The required length of the array is set by the Level 1 trigger latency pius additional cells required to buffer Level 1 triggers during readout. Some of the critical design parameters are listed in Table 7-18. 
Table 7-18. Analog memory specifications.

\begin{tabular}{ll}
\hline No. channels/chip & 16 \\
Pipeline length & -128 \\
Channel pitch & $200 \mu \mathrm{m}$ \\
Cell length & $30 \mu \mathrm{m}$ \\
Dynamic range & $10 \mathrm{bits}$ \\
Access time & $500 \mathrm{~ns}$ \\
\hline
\end{tabular}

The research and development plan for IPC electronics development in FY 92-93 calls for the fabrication of a prototype of this chip in the Harris AVLSI-RA process. The IPC SCA chip is now under development at Oak Ridge National Laboratory. The basic architecture for the first memory prototype has been finalized. The initial prototype design will be an 8-channel-by-128-cell device. Several readout designs are being tested in this prototype, including both voltage-read and charge-read configurations. These alternatives are shown in Figures 7-32 and 7-33. In the voltage-write, voltage-read (VWVR) design, the output offset voltage (or pedestal) can be controlled by using the residual charge generated by the switched feedback of the readout amplifier. In the simpler voltage-write, charge-read (VWCR) scheme, on the other hand, the output offset is not easily compensated by $p$ - and $n$-device matching. A second reason for considering the VWVR topology is that amplifier compensation providing rapid and stable settling is easier for the VWVR topology than for VWCR. This results from the fact that the VWCR amplifier has a noise gain of approximately 2.5 for readout and 1.0 for reset, implying that fast, stable, settling times must be maintained over a 2.5:1 change in loop transmission. The VWVR topology, on the other hand, requires a noise gain of approximately 1.5 during sampling and 1.0 during reset. Further simulations and tests on the prototype device are needed to make the choice.

Several amplifier designs have been simulated using Hewlett Packard CMOS34 semi-empirical (Level 3) and BSIM (Level 13) models and Harris Semiconductor AVISI-RA nominal pre- and postradiation models. We have settled on three candidate designs, two of which will be used on the first prototype chip. The address control for the initial design will use two 6-bit counters, one for even addresses, one for oidd. This will allow at least $16 \mathrm{~ns}$ of settling time for the logic of either counter and will also allow maxinum packing of the blocks under development. The settling time of this amplifier is critical in determining the readout time for the system. A BSIM sirnulation of this circuit indicates that the settling time to 11 bits is less than $500 \mathrm{~ns}$. Post-irradiation simulations in the Harris process show no significant increase in settling time. A typical BSIM result for this amplifier is shown in Figure 7-34.

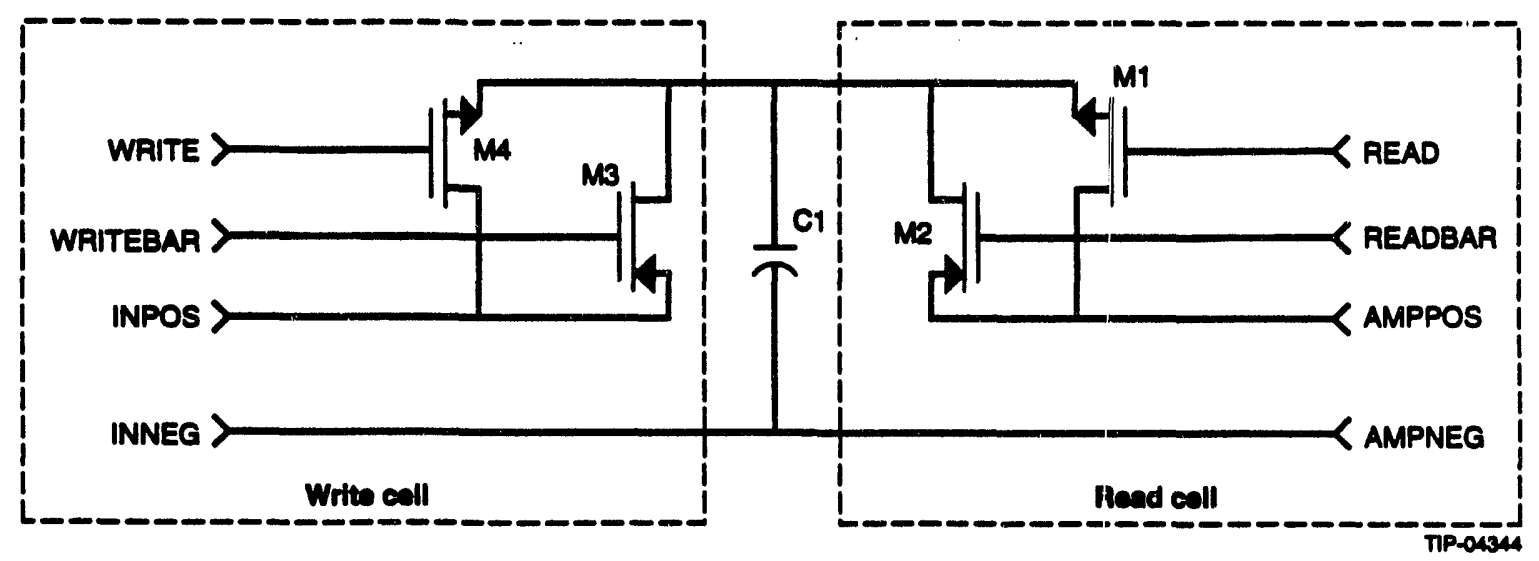

FIG. 7-32. Voltage-write/charge-read SCA cell. 


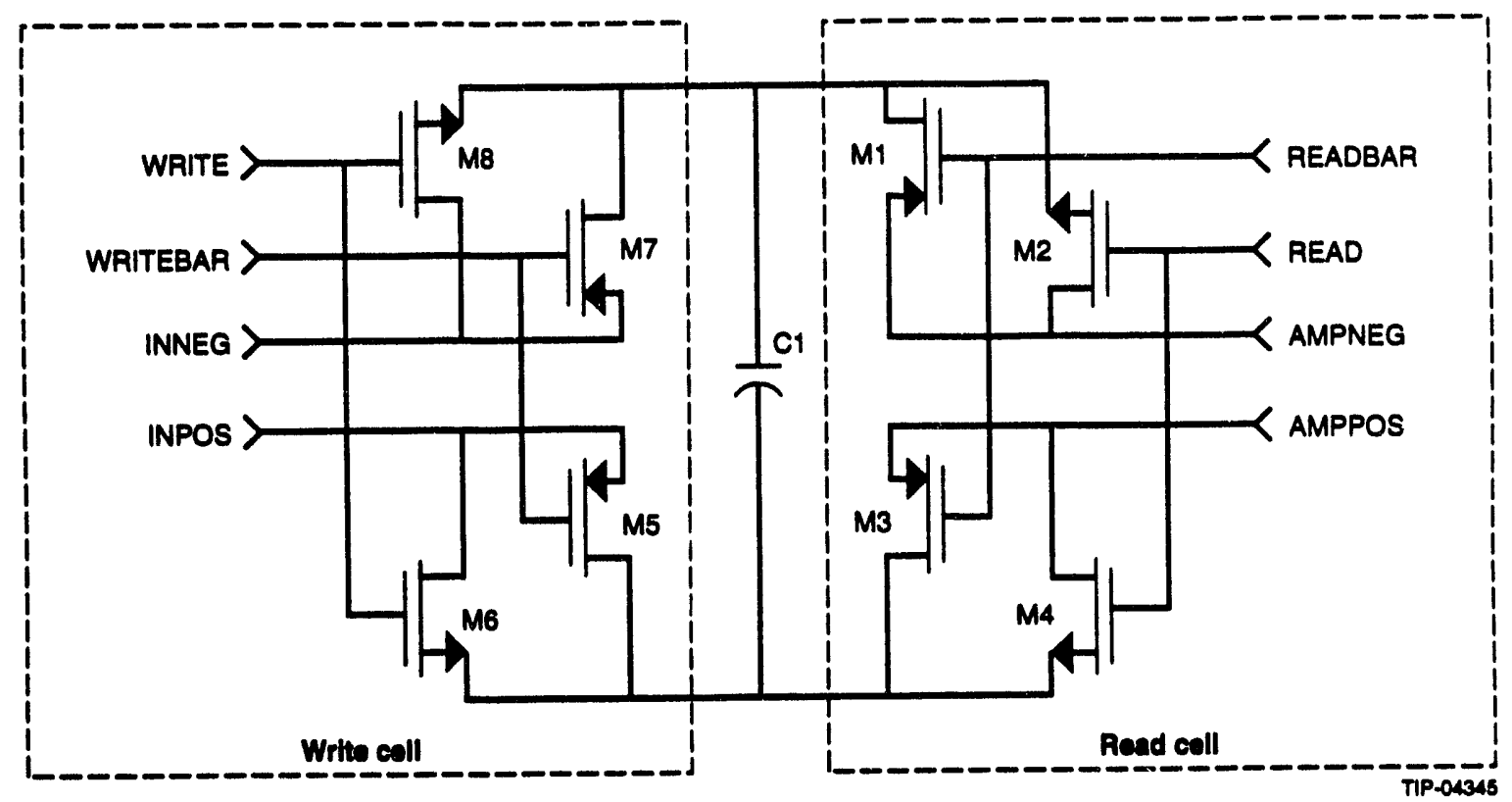

FIG. 7-33. Voltage-write/voltage-read SCA cell.

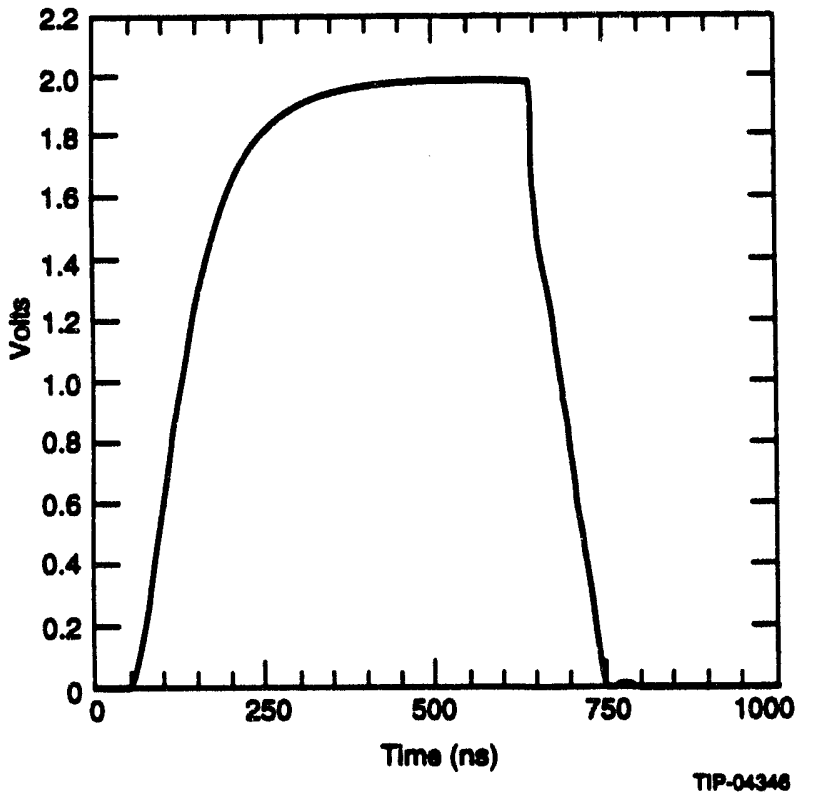

FIG. 7-34. BSIM SCA post-rad model.

\subsubsection{IPC Flash ADC}

Extensive tests have been conducted on the performance of a rad-hard FADC developed by Harris, the HA4009, which would, in principle, be suitable for IPC use. The HA4009 is an 8-bit, 25-MHz FADC developed in the AVLSI-RA process. A complete set of tests of the critical performance parameters of the FADC, including the number of effective bits vs. conversion rate, the generation of speckle code, and the integral and differential non-linearity, have been conducted for radiation doses extending up to $6 \mathrm{Mrad}$. No significant deterioration in the device performance was observed. We are now in the process of repeating these measurements under neutron irradiation. The FADC specifications are given in Table 7-19.

Test data of cathode readout devices indicate the need for more than eight bits of dynamic range. A number of approaches for increasing the effective dynamic range of the ADC are under consideration. For example, the HA4009 is equipped with a center tap on the reference ladder, allowing the device to run in a bilinear response mode, which can be used to extend the dynamic range. An increase in the dynamic range might also be realized through the use of non-linear input amplifiers. Finally, the prospects that rad-hard variants of existing 10-bit parts will be developed are good.

Table 7-19. FADC specifications.

\begin{tabular}{ll}
\hline Resolution & 8 bits \\
Dynamic range & 10 bits \\
Integral linearity & $1 \mathrm{LSB}$ \\
Diff. linearity & $1 \mathrm{LSB}$ \\
Bandwidth & $25 \mathrm{MHz}$ \\
Conversion speed & $25 \mathrm{MHz}$ \\
\hline
\end{tabular}




\subsubsection{IPC Readout Controller}

A block diagram of the readout controller is shown in Figure 7-35. It consists of a state machine to drive the readout sequence, address drivers for the analog pipelines, zero-suppression logic consisting of an 8-bit comparator and a register containing the threshold value used in the zero suppression operation, an output frame buffer, and test and calibration circuits. The readout controllers on an IPC chamber are serviced by a single low-bandwidth serial input link that allows the threshold values to be set and can trigger the test and calibration functions.

The readout controller is also responsible for driving the switched-capacitor array write-address lines, which are common to all arrays serviced by a single readout controller. Upon receipt of a Level 1 trigger, the controller stores the write address on the Level 1 pending stack. Asynchronously, the controller initiates a readout sequence, described in the section on readout timing below. The controller issues the read address appropriate to the time of the Level 1 trigger and the current write address, and latches the capacitor voltage onto the readout amplifier for all channels, allowing that voltage to settle. The controller then scans through the analog mernory chips and the channels within each memory chip, allowing the output multiplexer to settle on each channel. A FADC conversion is then loaded into a pipeline in the controller chip. If the value is above the threshold stored in the controller, a block of four pad locations is retained, including this pad address, the preceding pad, and the subsequent two. This ensures that all signals required to reconstruct an address are retained, independent of the amplitude of the pad signals in the tail of the induced charge distribution. The non-suppressed digital values and pad addresses are appended to a header containing the bunch and controller ID and are sent out the LED output link.

The test and calibration function consists of a circuit capable of injecting a known voltage pulse onto a line that is capacitively coupled to the readout lines into each amplifier, at the same time controlling the read and write address lines on the pipeline, allowing all elements of the pipeline to be read out sequentially. The controller will be programmed to initiate a test sequence upon receipt of a calibration trigger on the input link. All the functions of the controller described above could be provided by a single gate array chip. Rad-hard gate arrays of the required complexity and speed are commercially available. Prototypes of this chip have been developed in a programmable gate array and are now under test.

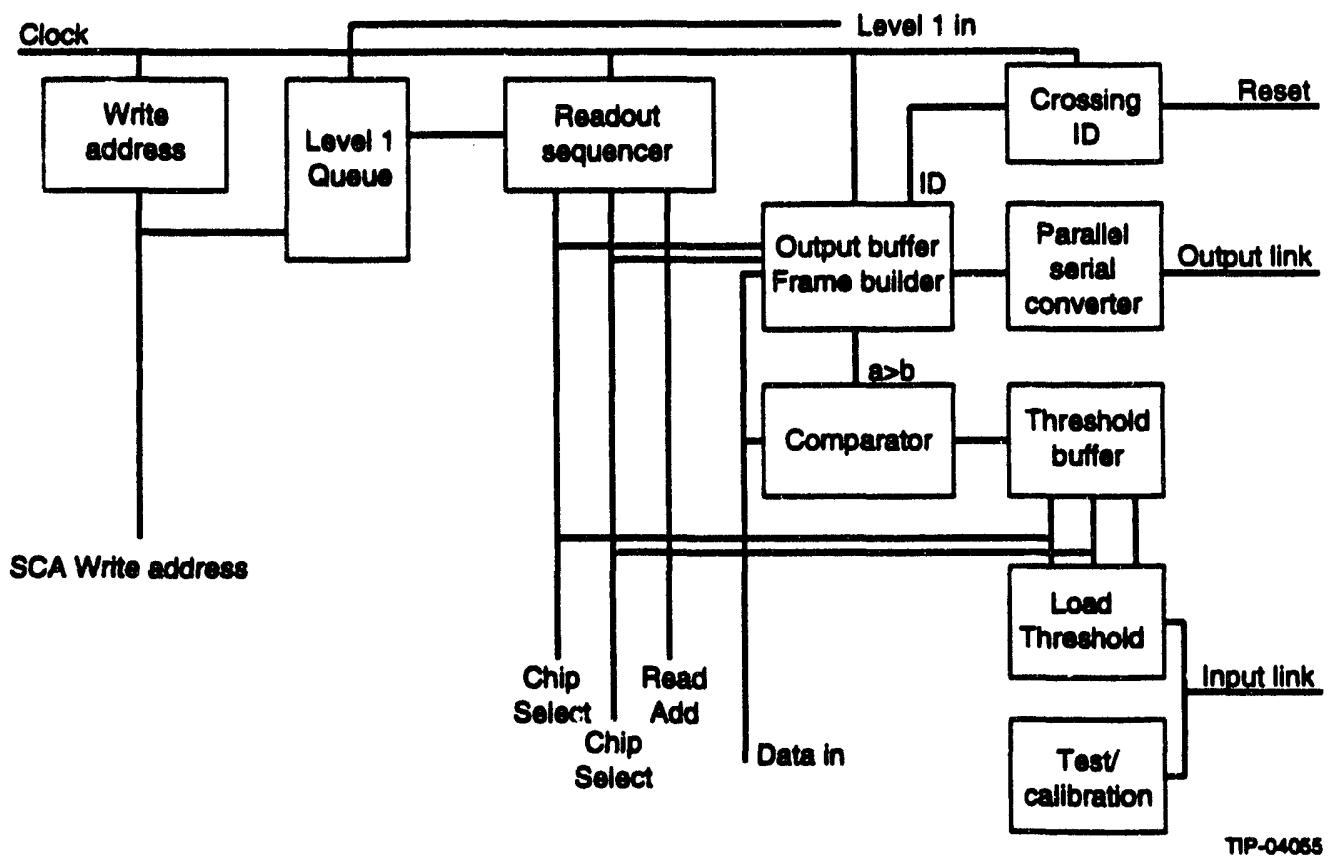

FIG. 7-35. Readout controller. 


\subsubsection{IPC Readout Link}

In the baseline digital readout design, each readout link services 128 pad channels. With no zero suppression, and assuming three time slices are read out for each hit, the number of bits being transmitted per channel per event is $128 \times(8$ bits $) \times(3$ samples), which equals 3 kbits. If we assume a $10-\mu \mathrm{s}$ readout time, the worst-case bit rate required by the link is $0.3 \mathrm{Gbits} / \mathrm{s}$. Handling this bandwidth would require rather expensive technology, and it is therefore desirable to avoid these rates by zero suppression on the detector. As described above, the zero-suppression processing uses variable thesholds that are downloaded using a low-bandwidth link running into the detector. The zero-suppressed bit rate is given by $128 \times\left(\mathrm{f}_{\text {zero }}\right) \times\left(\mathrm{N}_{\mathrm{s}} \times 8\right.$ bits +8 bits $\left._{\text {add }}\right) /$ $(10 \mu \mathrm{s})$. For $\mathrm{f}_{\text {zero }}=0.1$ and $\mathrm{N}_{\mathrm{s}}=3$ we find that a 45-Mbits/s link is required. This is within the capabilities of a simple LED-based system, as described in Section 7.8.

\subsubsection{Readout Timing}

One of the requirements of the IPC readout electronics is the ability to output the data to the Level 2 event data collectors (EDCs) in a time consistent with a $100 \mathrm{kHz}$ Level 1 rate. In this section we will examine in some detail the readout timing for the baseline design.(Table 7-20) The readout of the pipeline is performed at the $60-\mathrm{MHz}$ accelerator clock rate. At present, it is expected that two to four time slices will be read out for each pad. Simulations of the readout electronics indicate that two samples are sufficient for pulse height analysis but that three or more samples are needed to define the timing and pileup associated with a given pulse.

In the event of a Level 1 trigger, the readout supervisor initiates a readout sequence by asserting the chip, time slice, and channel addresses on the address bus connecting the supervisor with the eight pipeline chips it services. The access and settling time of the pipeline is the time-critical operation in the readout, and we wish to pipeline this process to the extent possible. This dictates a readout sequence in which the pipeline bucket is clocked at the highest rate, followed by the channel number, with the time slice address within the pipeline clocked at the slowest rate. Below, we describe the readout cycle, including timing estimates for each step. A $25 \mathrm{MHz}$ FADC is assumed. The access time of the switched- capacitor array is based on BSIM models of the readout amplifier of the array, which predicts a settling time to 11 bits of $500 \mathrm{~ns}$. A total readout time below $11 \mu \mathrm{s}$ is predicted by this timing sequence for the case with three time slices. The readout time varies with the number of samples, $N_{s}$, as follows: $\Delta t=N_{s} \times(500 n s+15 \times 20 n s+16 \times 40 n s+128 \times$ $17 \mathrm{~ns})$. This simple estimate has been verified on the FPGA prototype. Table 7-20 gives the pipeline/ MUX/FADC timing sequence.

Table 7-20. Pipeline/MUX/FADC timing sequence. The sequence involves nested loops as indicated by the level of indentation.

\begin{tabular}{ll}
\hline Operation & $\Delta t$ \\
\hline Assert time slice address ( $\mathrm{N}_{\mathrm{s}}$ times) & 0 \\
Wait for SCA to settle & $500 \mathrm{~ns}$ \\
Assert channel (16 times) & 0 \\
Wait for MUX settle & $20 \mathrm{~ns}$ \\
Convert FADC & $40 \mathrm{~ns}$ \\
Assert chip (8 times) & 0 \\
Load data/add & $17 \mathrm{~ns}$ \\
Increment chip & 0 \\
Increment channel & 0 \\
Increment time slice & 0 \\
\hline
\end{tabular}

\subsection{CALORIMETER}

\subsubsection{Overview and Requirements of the Readout}

The GEM calorimeter comprises approximately $125 \mathrm{k}$ channels. The readout must provide:

- 18 bits of dynamic range,

- a contribution to the constant term in the energy resolution of $<0.2 \%$

- time resolution sufficient to identify the bunch crossing uniquely

- storage for the calorimeter signals during the Level 1 latency of $2 \mu \mathrm{s}$

- deadtimeless operation at a maximum Level 1 rate of $100 \mathrm{kHz}$

- transfer of fully digitized data to Level 2 , with up to five samples per channel per Level 1 trigger 
A schematic of the readout chain is shown in Figure 7-36. Preamplified signals will be transferred on short (several meters) cables to "junction boxes" located on the outside of the cryostat. Cable drivers mounted there will drive the signals approximately $40 \mathrm{~m}$ to crates of electronics mounted on balconies on the wall of the experimental hall. There the signals will be received and split into two paths: one to the data acquisition (DAQ) hardware and the other to the Level 1 trigger-summing electronics.

The Level 1 electronics will perform analog sums to form trigger towers. Primitives will be formed of individual or groups of trigger towers above various thresholds. Sums of total and missing energy will also be formed for use in the Level 1 trigger decision (see Section 7.2.1).

The DAQ signals will be split into two gain scales and shaped before being sampled at $60 \mathrm{MHz}$. The samples will be stored in analog form using switched-capacitor technology while awaiting a Level 1 trigger decision. A digital pipeline approach is also being investigated. Upon receipt of a Level 1 trigger, up to five time samples per signal will be buffered awaiting digitization. Multiplexed and digitized samples will be processed locally and sent over optic fibers to the Level 2 processing system.

The digital pipeline approach offers a much simpler and more flexible architecture than that of the analog memory readout. However, it suffers from the lack of availability of $60-\mathrm{MHz}$ high-precision ADCs, which are affordable in terms of both money and power. As our "baseline," we have adopted the analog readout strategy, which seems the most conservative given today's technology. This approach, namely a dual-range scheme with switched-capacitor analog memories followed by analog multiplexors and $10-\mathrm{MHz}, 12$-bit commercial ADCs, will be discussed in detail here. However, we continue to pursue the digital pipeline approach vigorously.

\subsubsection{Front-End Electronics}

The in-detector electronics are described in Chapter 5. These include the preamplifiers and calibration circuits for the liquid ionization calorimeter and the phototubes for the scintillator calorimeter.

\subsubsection{Calorimeter Readout Electronics}

One $0.32 \times 0.32$ section of the calorimeter (CAL), including both electromagnetic (EMC) and hadronic (HAC) sections, is mapped onto one CAL readout electronics crate. Each crate then contains:

- 14 CAL readout modules (CRM),

- 1 CAL output module (COM),

- 1 Level 1 summing module (L1SUM),

- 1 crate controller for monitoring power levels, currents, temperatures, etc.

With this mapping, there is a total of $496 \mathrm{CAL}$ channels per crate. The total system, with approximately $125 \mathrm{k}$ channels, then contains about 252 crates, or 84 racks.

Assigning a region in $\eta, \phi$ to each crate implies that, insofar as the physical structure of the calorimeter itself respects the division into these regions, each crate looks identical. Thus, each COM is

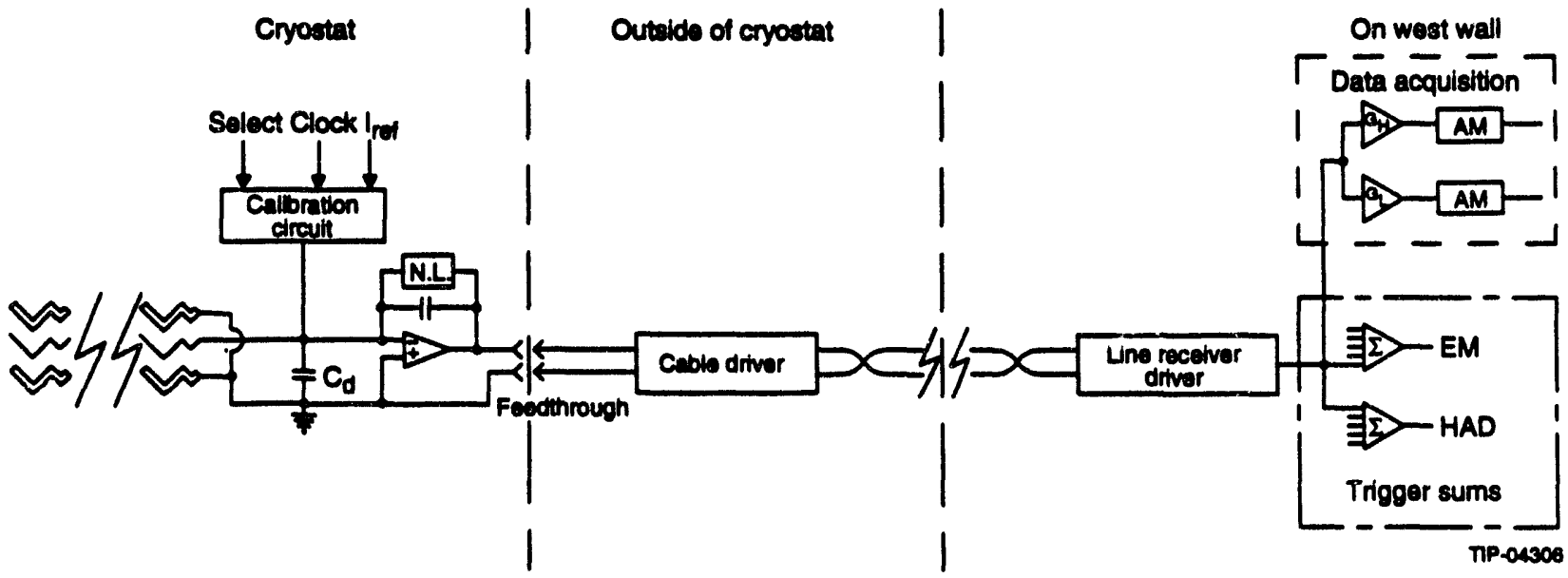

FIG. 7-36. Schematic depicting the calorimeter readout chain. 
identical, as is each LISUM. Furthermore, dividing the readout into EMC and HAC boards as descrihed below also allows all CRMs in the system to be identical.

The CRMs and COMs are described in more detail in the following sections; the LISUM is described in Section 7.2.1.

\section{Analog Pipeline Readout Architecture}

\section{Calorimeter Readout Module}

One CRM services 36 channels of CAL data. The board receives the signals, shapes and samples them, digitizes and processes the samples for triggered events, performs zero suppression (if desired), and formats and buffers the data for output to Level 2. The board also performs the first level of analog summing for Level 1, adding together the upper 18 channels and the lower 18 into two separate sums. These sums are then transferred to the Level 1 summing module residing in the same crate.

One CRM will service either EMC channels or HAC channels, but not a mixture of both. For the EMC boards, all 36 channels will be used, and the 36 channels mapped onto a given board will be those belonging to the same $6 \times 6$ trigger tower for a given longitudinal depth segment. For HAC boards only 32 channels will be active, with 16 channels mapped to the top 18 channels and 16 into the lower 18. A given set of 16 will correspond to the $4 \times 4$ channels forming a HAC trigger tower for a given depth segment.

To service a complete $0.32 \times 0.32$ section, or "supertower," one crate will require 12 EMC CRMs and 2 HAC CRMs. From these will emerge 28 ana$\log$ sums for the Level 1 summing board. Although it is possible to combine the two sums per board into a single sum in the case of the EMC boards if desired, this cannot be done for the HAC boards because one wishes to maintain a separate sum for the first depth segment of the HAC for use in the hadronic veto for electromagnetic triggers.

A block diagram of the CRM is shown in Figure 7-37. The 36 differential CAL signals are received and then, after a fast pick-off for the intermediate Level 1 trigger sums, are put to four different bipolar shaping amplifier hybrids, one for each of the nine channels. The shaper hybrids split the signals into high (HI) and low (LO) gain scales, and apply the bipolar shaping function to each scale. Two gains are needed in order to cover the full dynamic range, with the HI signal amplified by a factor of 16 relative to the $L O$. The shaped signals are sampled at $60 \mathrm{MHz}$ using eight different analog memory chips (AM), with four of the AM chips sampling HI signals (nine channels each) while the other four sample the LO scales. The HI and LO scales are routed to separate AMs in order to reduce cross-talk problems.

Analog multiplexers (MUX) transfer samples from triggered events for digitization by 12-bit, $10-\mathrm{MHz}$ commercial ADCs. One ADC, operated at nine $\mathrm{MHz}$, is sufficient to service nine calorimeter channels.

The operation of the AMs, MUX, and ADC are controlled by a single on-board pipeline control (PC) module. This module is responsible for generating the READ addresses and WRITE addresses for the $A M$, the channel select signal for the MUX, and the convert signal for the ADC. The PC, realized in gate array technology, also provides the interface to the Level 1 trigger.

Digitized samples are buffered in FIFOs, and then processed by DSPs (one for every nine channels). The DSP reads the samples, chooses the gain scale, applies the necessary calibration constants, calculates the charge and time for each channel, linearizes the resultant charge, suppresses channels for which the charge is below some threshold, and formats the data before writing it to the output FIFOs which buffer the data for the Level 2 system. Assuming a reduction of the data by a factor of 10 due to zero suppression, the data rate from a single board will be less than $4 \mathrm{MBytes} / \mathrm{s}$ at a Level 1 trigger rate of $100 \mathrm{kHz}$. The data will be sent optically to the Level 2 system, with one link servicing a crate of readout boards.

An additional DSP serves as the board controller. Its responsibilities include interfacing with the Level 2 system, controlling a precision DAC and charge injection system used for calibration, downloading the program and constants needed by the channel-processing DSPs, generating and controlling test and calibration sequences, and performing other diagnostic and monitoring tasks. 


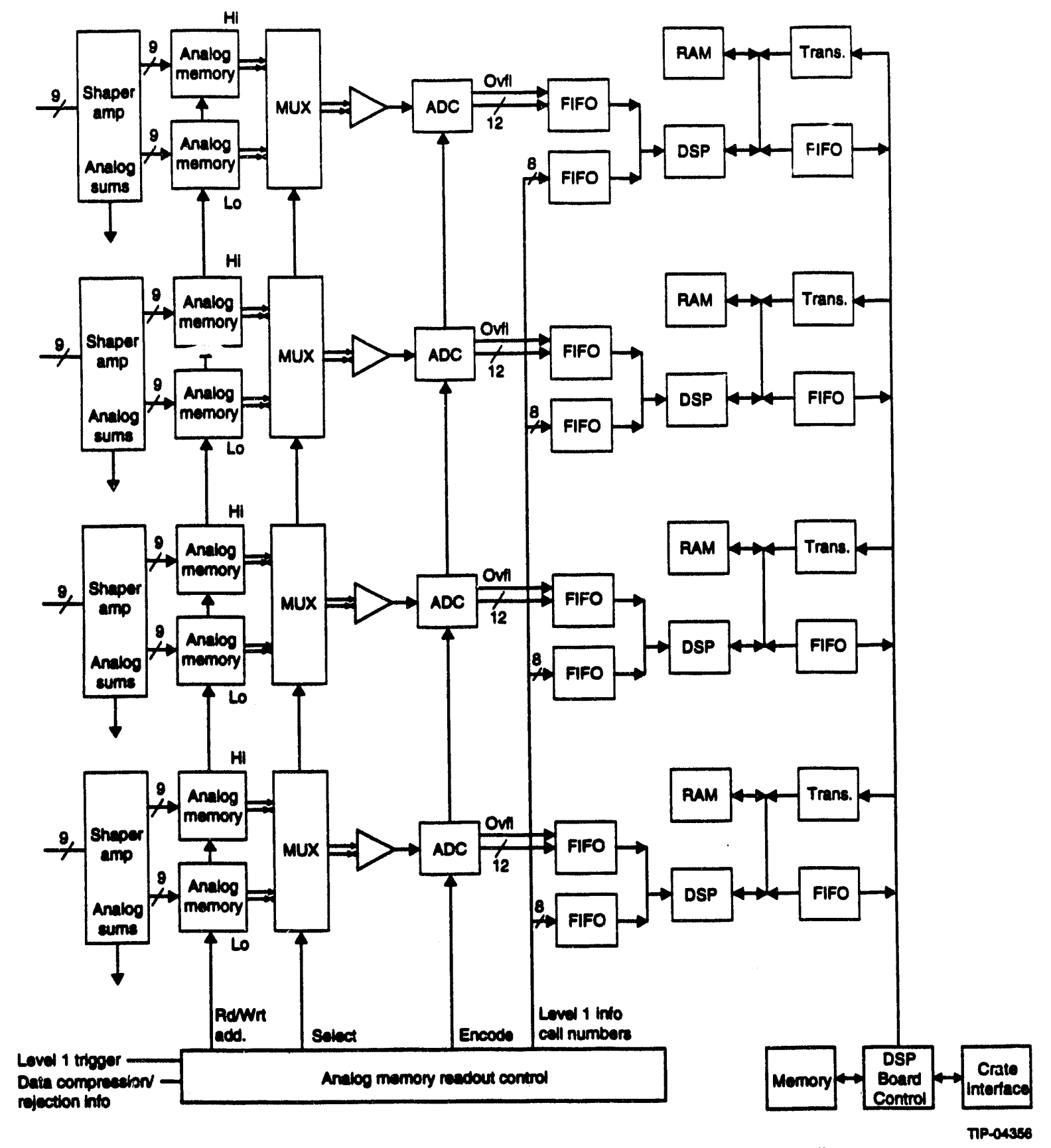

FIG. 7-37. Schematic drawing of a calorimeter readout module (CRM) using analog pipelines.

Each of the components of the calorimeter readout module will be described in more detail below:

\section{Bipolar Shaping Amplifier Hybrid}

As mentioned above, the functions of the bipolar shaping amplifiers include receiving the differential calorimeter signals, splitting the signals into high- and low-gain paths, applying the bipolar shaping function to each path, and providing an unshaped fast output for use in forming the analog
Level 1 trigger sums. A hybrid circuit will be used, with one amplifier circuit measuring approximately $5 \times 5 \mathrm{~mm}^{2}$ and consuming 200-300 mW.

The optimal shaping time is determined from considerations of the electronic noise and pile-up noise. ${ }^{2,15}$ The present design calls for a peaking time (for an impulse response) of $40 \mathrm{~ns}$ for the EMC and $70 \mathrm{~ns}$ for the front portion of the HAC. The other depth segments of the HAC will have peaking times up to about $200 \mathrm{~ns}$. 
Since the $S / N$ ratio will be optimized at a given luminosity during the energy reconstruction by using suitably weighted multiple samples, the role of the shaper is just that of an anti-aliasing filter band-limiting the preamplifier output.

The Level 1 trigger involves the formation of analog sums, requiring that the channel-to-channel gains be identical within a suitable tolerance. This can probably be achieved simply by matching the gains of the preamplifiers (at least in groups belonging to the same trigger tower), thereby avoiding the need for computer control of the gain. This solution would allow a fully monolithic version of the shaper, using bipolar technology, which would minimize the power dissipation for this fast, linear stage.

Since different shaping times will be necessary in the EMC and HAD sections, a number of different versions of the shaper circuit will be needed. Alternatively, one could employ a monolithic circuit, containing the active electronics, coupled to external resistors and capacitors. This solution would allow inclusion of a gain-control stage using varicap diodes, and may prove the most economical if further trigger studies show the need for computercontrolled gain. This would also solve the potential problem of tolerances of passive components in monolithic processes, even though the multisampling technique used for the charge reconstruction is relatively insensitive to the precise values of shaping times.

\section{Analog Memory}

Experience with the ZEUS experiment ${ }^{16}$ has verified the viability of switched-capacitor (SC) technology for analog storage. Deadtimeless operation can be accomplished through an analog memory (AM) design, similar to that developed by the SSC Subsystem R\&D Front-end Electronics Collaboration, ${ }^{17}$ which incorporates the ability to perform simultaneous READ and WRITE operations. The layout for such an AM is shown schematically in Figure 7-38. Each cell comprises a sampling capacitor and four switches. Two of the switches (W1 and W2) connect the input voltage across the capacitor during a WRITE operation, while the other two (R1 and R2) connect the capacitor across the output op-amp for a READ operation. The READ and WRIT switches are controlled independently by separate buses (not shown in the figure). Thus, while one sample is being read out, it is possible to continue to sample the input voltage and write the samples to other cells in the same pipeline.

In this scheme, buffering of the Jata for Level 2 is achieved by transferring the addresses of the relevant samples to a separate list of cells awaiting digitization. The chip is designed for random access to any cell, with the READ and WRITE addresses generated by a separate chip and subsequently decoded on the SC chip. The control logic will be described in the following section.

A significant worry concerning SC analog memories lies in the possibility of capacitor-specific calibration constants (offsets, gains) needed in order to preserve the precision of the analog measurement. The need to add up large numbers of channels of the calorimeter to form jet and global sums implies that very small effects in individual channels can lead to significant systematic errors if channel-by-channel correlations are present. Special care has been taken in the design of the SC sampling cell to avoid these effects, through a careful design of the cell switch architecture (for details, see reference 18).

The effects of noise injected into the substrate due to the digital control logic on the same chip (see the following section) will be reduced by thoroughly guarding the analog structures from the digital logic. If necessary, a further reduction can be achieved by driving the signal from each calorimeter channel differentially into two adjacent switched-capacitor pipelines, with a corresponding differential readout structure.

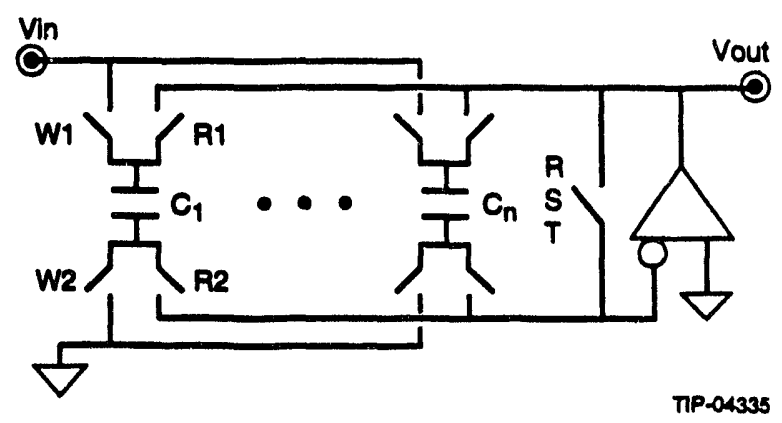

FIG. 7-38. Schematic drawing of a switched-capacitor analog memory with simultaneous READ and WAITE operations. 


\section{Pipeline Control}

As mentioned above, the virtual buffering scheme requires that the SC chip be provided with addresses for controlling which cells are written or read at any time. Space considerations imply that the control functions will need to be performed off-chip, with the addresses and other control signals transmitted to the SC chips. The control architecture (for details, see reference 18 ) has been optimized according to the following considerations.

In order to preserve the measurement precision, it is necessary to minimize the noise injected into the analog environment by the control system. The design, which attempts to reduce both the number and the rate of change of external control lines that must be received, level-shifted, and driven on the SC chips, incorporates the following features:

- Since READ operations occur at frequencies less than $1 \mathrm{MHz}$, there is sufficient time to transmit the READ Address (RA) bit-serially to the SC chip. This method requires a single data line with an accompanying shift clock instead of eight address lines.

- The WRITE Address (WA) space is divided logically into pairs of cells ("pages") and the least significant bit (LSB) of the WA is generated using a counter on the SC chip. In this way, write addresses (i.e., page numbers) need to be transmitted at a rate of only $30 \mathrm{MHz}$ instead of $60 \mathrm{MHz}$.

- A WRITE page increment is transmitted instead of the absolute page number, reducing the required number of lines from eight to five. Only five bits are needed for the page increment, since limiting to five the number of events in the buffer implies a maximum of 25 samples reserved. Including the inefficiency introduced by the page structure, which always leads to reserving the cells two at a time, still does not lead to the need to ever jump more than the 64 physical cells allowed with a 5-bit page increment.

- The sequence of WRITE pages is generated in a manner that minimizes the likelihood of changes in the levels of the page increment bits, by always choosing the next WRITE page as the first available page that is encountered by incrementing past the current page.
The pattern of samples to be digitized for a given trigger may depend on the section of the calorimeter in question (EMC or HAD) due to their different shaping times. Also, the procedure of optimal filtering implies that one may wish to digitize different samples following a major upgrade in the luminosity of the machine. In order to maintain this flexibility, the system is designed such that the pattern of samples to be digitized is loaded from an external register and is, therefore, programmable.

\section{Digital Pipeline Readout Architecture}

As mentioned previously, we are also pursuing a readout scheme wherein the calorimeter data are sampled and digitized at $60 \mathrm{MHz}$, with the resultant samples stored in digital memory awaiting a Level 1 trigger.

The digital pipeline version of the CRM is shown schematically in Figure 7-39. The bipolar shaping amplifier hybrids receive the differential CAL signals and, after providing an unshaped fast pick-off for the first level of analog summing for the Level 1 trigger, apply the bipolar shaping function to the CAL signals. The shaper hybrids are followed by a custom bipolar IC, whose function is to split the signal into different gain scales, provide a sampleand-hold on each gain scale, choose the correct gain scale to use, transmit the corresponding voltage level to be digitized by the $60 \mathrm{MHz}$ ADC, and encode the gain scale chosen as the exponent of the resulting digitized "floating point" value. The number of gain scales needed to cover the dynamic range could be as low as two, if 12-bit, 60-MHz ADCs become commercially available within the next few years. On the assumption that at least 10-bit, $60-\mathrm{MHz}$ ADCs will be available, the number of scales will not exceed four.

Following the ADC, the floating point result is stored in a digital pipeline awaiting a Level 1 accept. For triggered events, the relevant samples are processed by a DSP and formatted for transmission to Level 2.

The digital pipeline approach has the advantage of avoiding the use of analog memories; achieving the desired precision with the latter may well necessitate the use of pipeline-cell dependent calibration constants, with a large increase in system 


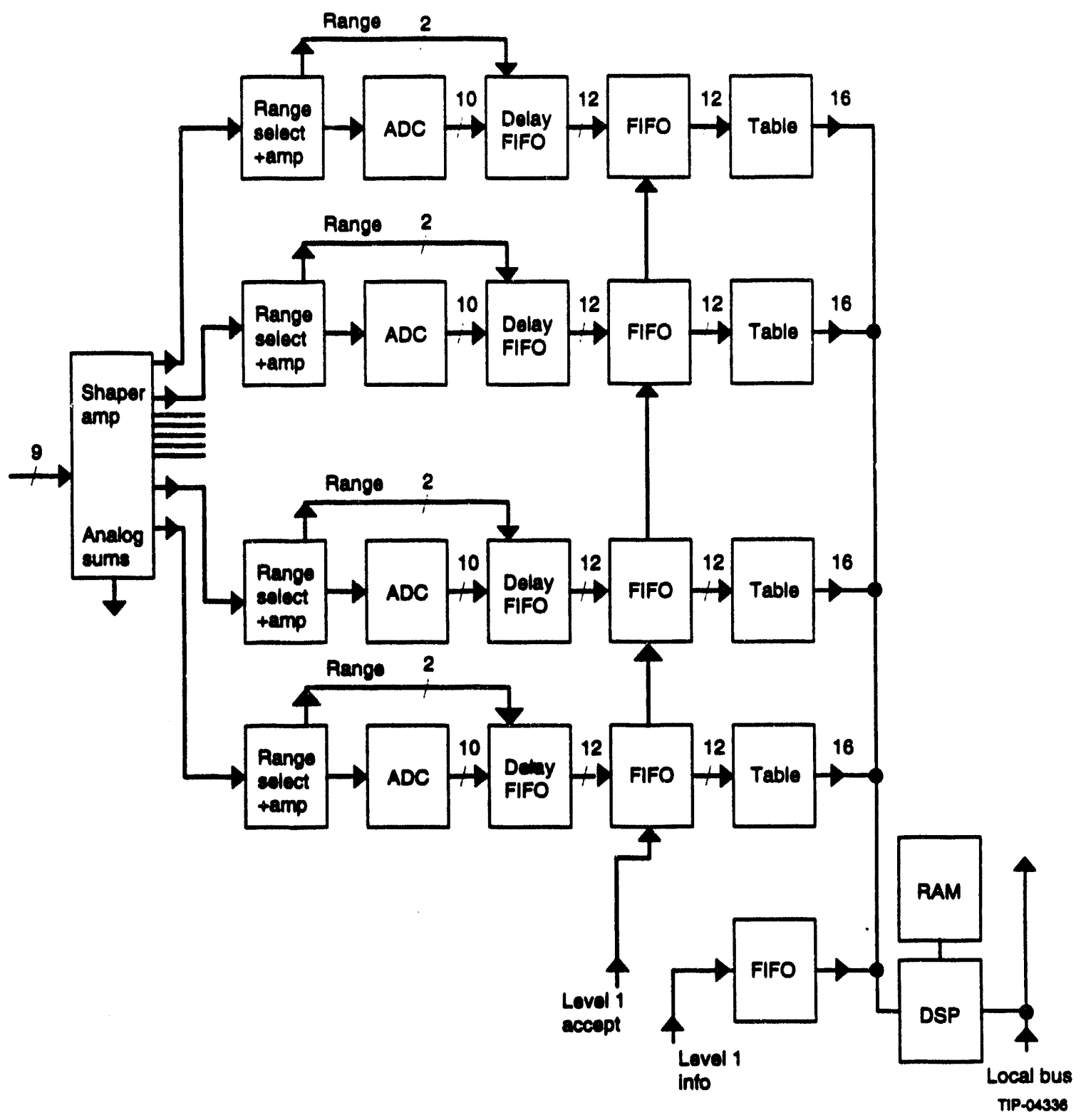

FIG. 7-39. Schematic drawing of a calorimeter readout module (CRM) using digital pipelines.

complexity as well as processing requirements. The disadvantages of the digital approach are the affordability of high precision (at least 10-bit) $60 \mathrm{MHz}$ ADCs in terms of both cost and power. The necessity for at least one $A D C$ per channel also serves to limit the channel density that can be achieved. In addition, the digital approach will give samples from different scales for a given trigger, complicating the achievement of the desired precision; whereas in the analog approach all samples use the same scale.

\subsubsection{Cables and Packaging Signal Cables}

The signal cable path is from the motherboard on the detector, which contains the preamplifiers, through the feedthroughs to the junction box. The junction box may have active elements to provide additional gain or a completely passive interconnecting network. From the junction box the signals 
are sent differentially to crates housing the data acquisition boards.

The signal cables between the preamplifiers and the junction box are $50 \Omega$ stripline transmission cables. These are three-layer flexible printed circuit boards. The outer layers are shield or ground layers and the inner layer carries the signal traces. Stripline cables with kapton as a dielectric material represent a high-quality, low-loss transmission line. Kapton is extremely resistant to radiation damage and contains none of the additives that can poison argon or krypton.

\section{Calibration Cables}

Several connections are required for the calibration scheme, including bias lines, lines for the current source, channel select lines, and the clock line. Only the clock line need be a high quality transmission line, since the bias and current lines carry quiescent sources while the channel select toggles at about $1 \mathrm{kHz}$. Since all of these control and calibration lines service many channels, the total number of calibration lines required per module is small and may be handled with only a few stripline cables of the type used for the signal cables.

\section{HV and Power Cables}

The high-voltage cables, like the signal cables, run continuously from the preamplifier motherboard to the junction box. The cables have a solid copper inner conductor and use extruded Tefzel or PEEK as insulator material; they are not jacketed. The retum path for the high voltage power supplies is via the DC ground return. The insulator material must be capable of sustaining a 50\% overvoltage above the normal operating voltage. It $i$ expected to be possible to use the same type oi cable for power distribution and high voltage. The diameter of the inner conductor should be such that a voltage drop of no more than $50 \mathrm{mV}$ occurs under normal operating conditions. This may require mrre than one cable in parallel, especially on the ground return. Since prudent design would require a redundant feed for each power and high-voltage connection, and the conductivity of copper increases by a factor of five at cryogunic temperatures, staying below the maximum voltage drop requirement is easily achieved.

\subsubsection{Electronics Placement}

It is envisioned that access to the inner regions of the detector might be available at most once per year. The placement of the electronics has been organized with an emphasis on providing as easy access as possible without reducing the performance in an unacceptable manner.

While maintenance issues ha\%e led to the desire to place as little of the electronics as possible inside the detector, noise considerations necessitate that the $L K r / L A r$ preamps and calibration circuits be located inside the cryostat, mounted directly on the calorimeter. From the preamp outputs, the signals will be transmitted single-ended on cables that exit the cryostat and terminate in the junction boxes. In order to minimize the noise pickup as well as the drive requirements of the preamps, the junction boxes are mounted directly on the outside of the cryostat. From the junction boxes, the signals will be driven differentially to racks of electronics that are mounted on the wall of the experimental hall. Thus, only the preamps, calibration circuits, and cable drivers are located inside the detector, where access is very limited. Reliability and redundancy issues must receive special attention in the design of these components.

The Level 1 trigger sums will be formed on separate printed circuit boards housed in the same racks as the data-acquisition boards. The digital information will be sent optically to the Level 1 trigger processing hardware, located in the electronics room (ER). Similarly, the Level 2 processing system, which will receive the full-granularity digital calorimeter information, will be located in the ER, or perhaps in the operations center on the surface. The ER will be sufficiently shielded from both the beam and the GEM magnetic field so that access is expected to be possible even during data taking. 


\subsection{MUON SYSTEM}

\subsubsection{Overview}

\section{General System Requirements}

The CSC readout electronics must:

- Measure the charge on each strip with sufficient accuracy to render the electronic contribution to the position resolution negligible.

- Measure single-hit times with a resolution that is comparable to one bunch crossing time-i.e., 16.7 ns.

- Provide a reduced set of accurately timed signals as input to the Level 1 muon trigger.

- Identify and reject random backgrounds induced by neutrons and other debris not associated with the muon track.

- Conform to the timing and readout-rate requirements of Table 7-1.

- Be suitable for chamber mounting.

- Be inexpensive on a per-channel basis.

The first four of the above criteria derive from the philosophy that the readout electronics should not significantly degrade the performance of the CSCs beyond the inherent limitations of the chamber technology.

The last two criteria, which are related, are a consequence of the large channel count $\left(\sim 10^{6}\right)$ of the GEM muon system. In particular, they mean that the electronics must make extensive use of custom integrated circuits and that the circuitry must be compact and use little power. Fortunately, the large physical volume of the muon system and the relatively low charged-particle fluxes present outside the calorimeter help to relax some of the design constraints that apply to other front-end systems.

The above criteria can be translated into a set of engineering specifications, which are listed in Table 7-21.
Table 7-21. Electrical Specifications for the CSC readout.

\begin{tabular}{|l|l|c|}
\hline \multicolumn{1}{|c|}{ Parameter } & \multicolumn{1}{|c|}{ Value } & Notes \\
\hline Equivalent input noise & $<2000 \sigma \mathrm{rms}$ & 1 \\
\hline Peaking time & $300 \mathrm{~ns}$ & 2 \\
\hline Overall system gain & $0.4 \mathrm{fC} /$ Count & 3 \\
\hline Dynamic range & $10 \mathrm{bits}$ & 4 \\
\hline Accuracy & $<1 \%$ & \\
\hline Cross talk & $<.50 \mathrm{db}$ & 5 \\
\hline Readout rate & $100 \mathrm{kHz}$ & 6 \\
\hline Readout latency & $<100 \mu \mathrm{s}$ & 7 \\
\hline Temperature range & $20 \pm 5^{\circ} \mathrm{C}$ & 8 \\
\hline Power per channel & $<100 \mathrm{~mW}$ & 9 \\
\hline $\begin{array}{l}\text { Radiation hardness: } \\
\text { ionizing }\end{array}$ & $<1 \mathrm{kRad}$ & \\
\hline $\begin{array}{l}\text { Radiation hardness: } \\
\text { neutrons }\end{array}$ & $10^{14} \mathrm{~cm}^{-2}$ & \\
\hline
\end{tabular}

Notes:

1. A level of $2000 e^{-}(0.32 \mathrm{fC})$ equivalent input noise produces an ADC "pedestal width" of 0.8 counts. Noise level applies to $100 \mathrm{pF}$ input capacitance and $300 \mathrm{~ns}$ shaping time.

2. Peaking time for "semi-gaussian" shaping. Transfer response of system is given by

$$
H(s)=\frac{\pi}{(1+\pi)^{n+1}}
$$

where $n \tau=$ peaking time and $n$ is the number of integrations. The most likely choice is $n=4$, since $n=2$ leads to a long uncancelled tail.

3. Ratio of input charge to ADC least count.

4. Ten-bit range will keep a larger fraction of the Landau tail on scale and will allow operation over a wider range of chamber gas-gains.

5. This is maximum average rate of Level 1 accepts.

6. Maximum time between Level 1 accept and availability of data at readout output port. In data driven architectures, this drives the ADC conversion speed.

7. Ambient temperature range over which all other system specifications must be met.

8. Total power for on-chamber electronics divided by total number of channels. Includes on-chamber readout and trigger sections.

9. The muon electronics are situated outside of the calorimeter and therefore are expected to experience only low radiation levels. This remains to be verified. 


\section{Physical Layout}

The layout of the CSC readout for a single module is shown schematically in Figure 7-40. The in-detector electronics consist of 96-channel frontend readout boards (FEPCBs), which are mounted directly on the chambers, and "motherboards," or read out PCBs (ROPCBs), which provide an interface to the off-detector electronics. Tables 7-22 to 7-25 summarize the cathode and anode element totals for the barrel and endcaps.

The input signals for a given front-end board are collected from all six layers by a set of shielded multi-conductor cables running between the cathode planes and the FEPCBs. Collection of signals in this manner decreases the effective channel pitch by a factor of six, which allows for a denser and consequently more economical design. The gathering of signals onto a single front-end board also simplifies the implementation of the logic used to identify charged-track segments. The outputs of this logic constitute the first rank of the Level 1 trigger logic and are a key element of the data-driven

Table 7-22. Barrel cathode readout physical layout and channel count

\begin{tabular}{lrrr}
\hline \multicolumn{1}{c}{ Component } & SL1 & SL2 & SL3 \\
\hline Segments & 48 & 48 & 48 \\
Segments & 4 & 8 & 8 \\
6 gap modules & 192 & 384 & 384 \\
Chan/FEPCB & 96 & 96 & 96 \\
FEPCB/module & 7 & 7 & 7 \\
Chan/module & 672 & 672 & 672 \\
Total FEPCB & 1344 & 2688 & 2688 \\
Total channels & 129,024 & 258,048 & 258,048 \\
TOTAL & & 645,120 & \\
\hline
\end{tabular}

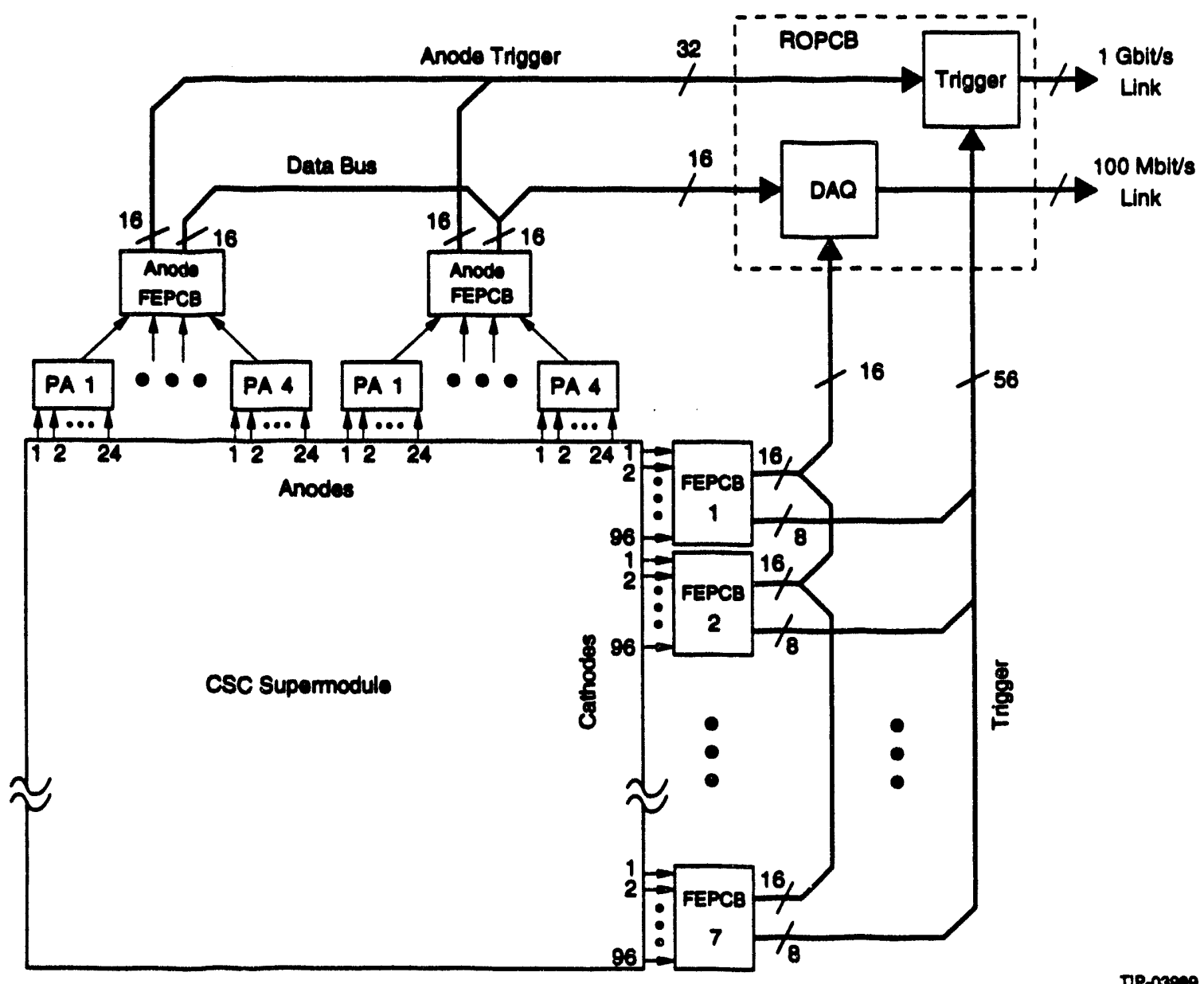

FIG. 7-40. Schematic representation of the layout of the readout, trigger, and DAQ system for the CSCs. 
readout architecture. Finally, it makes it possible to position the FEPCBs in such a way that they provide minimal mechanical interference with other cham- bers or with the line-of-sight paths required by the alignment system.

Table 7-23. Endcap cathode readout channel count. Channel counts are for one endcap.

\begin{tabular}{|c|c|c|c|c|c|c|}
\hline \multirow[t]{2}{*}{ Component } & \multicolumn{2}{|c|}{ SL1 } & \multicolumn{2}{|c|}{ SL2 } & \multicolumn{2}{|c|}{ SL3 } \\
\hline & Med & Small & Med & Small & Med & Small \\
\hline Segments & 24 & 24 & 48 & 48 & 48 & 48 \\
\hline Number of segments & 1 & 1 & 1 & 1 & 1 & 1 \\
\hline 6 gap modules (8 gaps in SL 1) & 24 & 24 & 48 & 48 & 48 & 48 \\
\hline Chan/FEPCB & 96 & 96 & 96 & 96 & 96 & 96 \\
\hline FEPCB/module & 12 & 8 & 6 & 4 & 6 & 4 \\
\hline Chan/module & 1152 & 768 & 576 & 384 & 576 & 384 \\
\hline Total FEPCB & 288 & 192 & 288 & 192 & 288 & 192 \\
\hline Total Chan. & 27,648 & 18,432 & 27,648 & 18,432 & 27,648 & 18,432 \\
\hline \multicolumn{5}{|l|}{ TOTAL } & \multicolumn{2}{|c|}{138,420} \\
\hline Component & & SL1 & & SL2 & & SL3 \\
\hline Segments & & 48 & & 48 & & 48 \\
\hline Segments & & 4 & & 8 & & 8 \\
\hline 6 gap modules & & 192 & & 384 & & 384 \\
\hline Chan/FEPCB & & 96 & & 96 & & 96 \\
\hline FEPCB/module & & 4 & & 2 & & 2 \\
\hline Total FEPCB & & 768 & & 768 & & 768 \\
\hline Total channels & & 73,728 & & 73,728 & & 73,728 \\
\hline TOTAL & & & & - & 221,18 & \\
\hline
\end{tabular}


Table 7-25. Endcap anode readout channel count. Channel counts are for one endcap.

\begin{tabular}{lrrrrrr}
\hline \multicolumn{1}{c}{ Component } & SL1 & & SL2 & & SL3 \\
\hline & Med & Small & Med & Small & Med & Small \\
\hline Segments & 24 & 24 & 48 & 48 & 48 & 48 \\
Number of segments & 1 & 1 & 1 & 1 & 1 & 1 \\
6 gap modules (8 gaps in SL 1) & 24 & 24 & 48 & 48 & 48 & 48 \\
ChanfFEPCB2 & 96 & 96 & 96 & 96 & 96 & 96 \\
FEPCB2/module & 2 & 2 & 2. & 2 & 2 & 96 \\
Total FEPCB2 & 48 & 48 & 96 & 96 & 96 \\
Total Channels & 4508 & 4608 & 9216 & 9216 & 9216 & 9216 \\
TOTAL & & & & 46,080
\end{tabular}

Figure 7-41 shows the physical layout of the front-end printed circuit board. The figure is approximately to scale, although a detailed layout remains to be done. The general flow of signals is from top to bottom. The first row consists of preamplifiershaper (PASA) ICs, which accept low-level input signals from the chambers, amplify them and then pass on fast- and/or slow-shaped versions of the signals to the next row of ICs labeled ASIC \#2. This row of ICs incorporates timing discriminators and digital logic designed to identify signatures corresponding to stiff charged tracks traversing the six planes of the module. Upon receipt of a Level 1 accept that is coincident with a local charged track (LCT) signal, the sampled signals are digitized by a single ADC, which receives its inputs through a network of analog multiplexers. Finally, the digital data are buffered in a digital FIFO memory while awaiting readout.

The final layout must take into account the need to isolate preamplifier inputs from digital crosstalk. One way of doing so is by mounting the digital ICs on the reverse side of the board with buried ground and power planes. The need to maintain signal ground integrity with the anode front ends is also of prime importance. Additional details on the various functional blocks of the FEPCB can be found in Section 7.7.2.

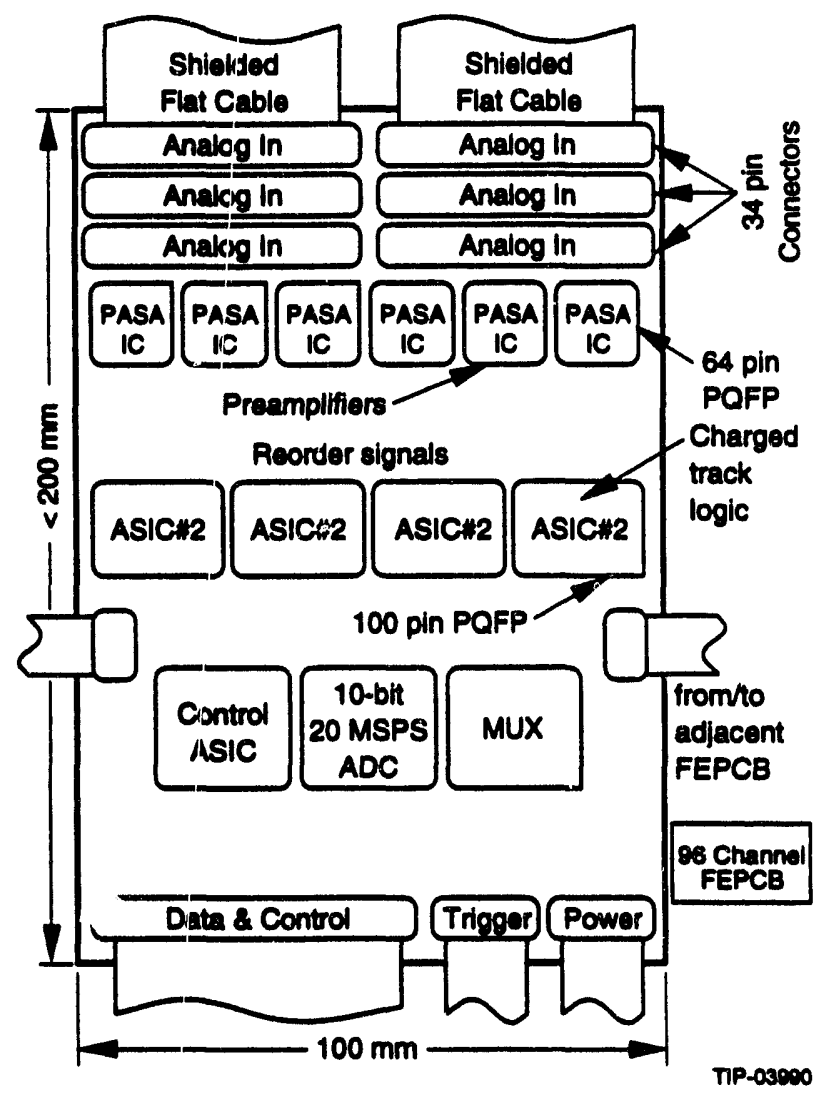

FIG. 7-41. Physical layout of the CSC front-end printed circuit board (FEPCB). Each 96-channel board services 16 cathode strips on each of the six layers that comprise a supertayer. 
The ROPCBs provide services common to all of the channels within a chamber module or set of chamber modules. In particular, they provide the first level of circuitry for data collection, for retransmission of Level 1 trigger signals, and possibly for regulation and/or monitoring of the low-voltage power. In addition, the ROPCBs serve as a point of contact for the downloading of configuration, calibration, and control signals (e.g., the clock and the Level 1 accept).

\section{Electronic Architecture, Dataflow, \& Timing}

The cathode readout electronics must perform the following functions:

- Amplify and shape the strip signals.

- Provide prompt information to the Level 1 trigger.

- Store the precision analog information during the Level 1 trigger formation time.

- Transmit sparsified digital data to the DAQ upon receipt of a Level 1 accept.
Figure 7-42 shows the various functional blocks. Thin lines represent single-signal analog or digital lines, while thicker lines represent multi-signal digital paths. The number of lines of a given variety is indicated by the number located next to the "q" symbol.

Input signals are amplified using a charge-sensitive preamplifier that drives a slow $\left(t_{\mathrm{p}}^{\text {slow }} \sim 300 \mathrm{~ns}\right)$ shaping amplifier optimized for low noise and a fast $\left(t_{\mathrm{p}}^{\text {fast }}-30 \mathrm{~ns}\right)$ shaping amplifier optimized for timing. Calibration is achieved using a set of precisely matched capacitors that couple a test pulse to each channel's input. An arbitrary pattern of channels can be programmed with a set of on-chip switches and a control shift register. In addition to calibration, these capacitors can be used to exercise the readout and trigger logic.

Signals from the fast shapers are routed to discriminators, which produce logic pulses that serve as inputs to the local-charge-track (LCT) logic.

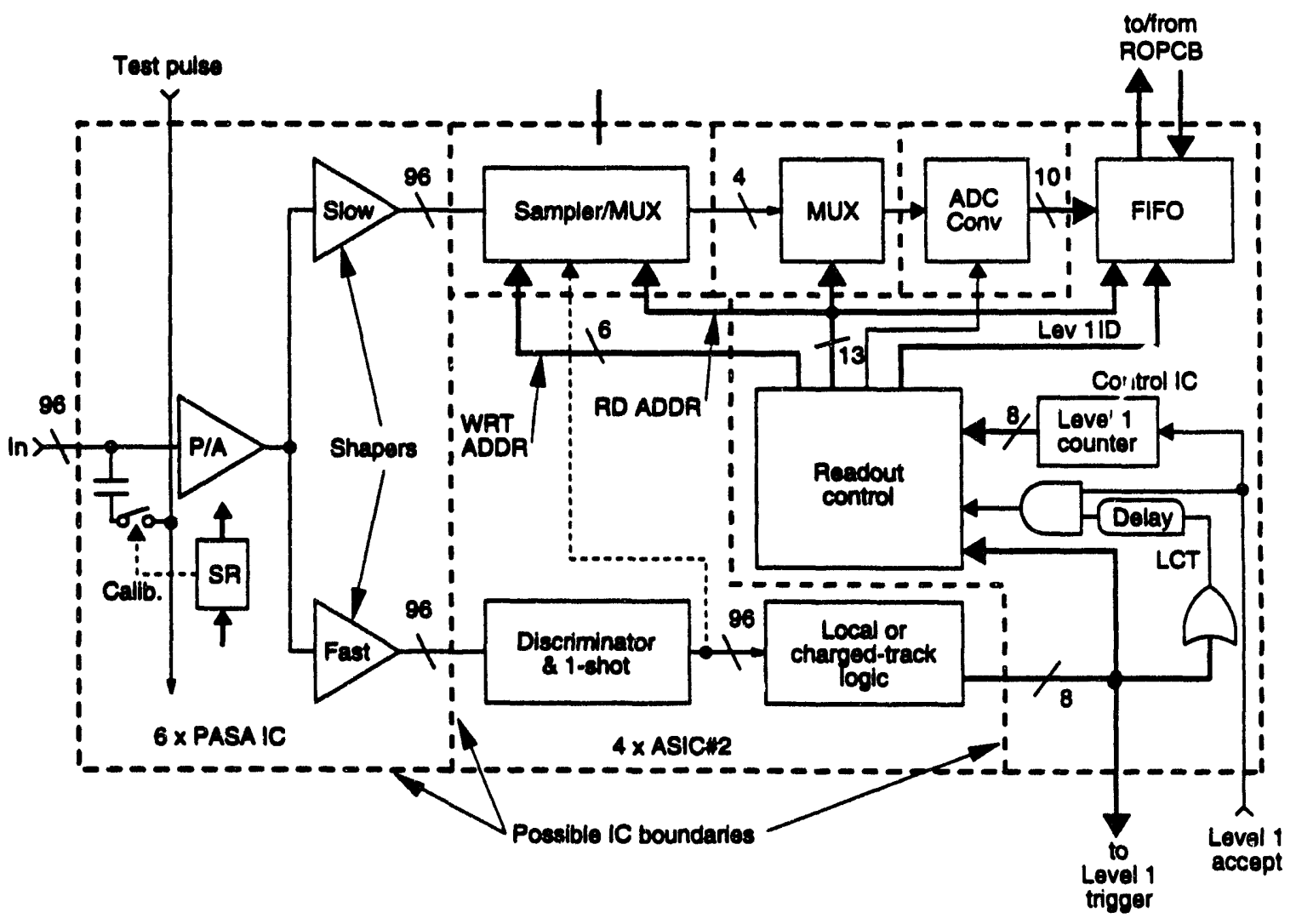

FIG. 7-42. Block diagram of the CSC front-end printed circuit board (FEPCB). 
The LCT logic combines signals from narrow (few-strip-wide) $\phi$-slices in each superlayer and identifies combinations corresponding to stiff tracks traversing the superlayer. By requiring that four or more of the six layers fire, signals induced by neutrons, which typically fire only one or two layers, are highly suppressed. The outputs from the LCT logic are used on the FEPCB to "steer" the readout and are also transmitted off-board to the next layer of the muon trigger logic.

The precision analog information from the slow shaper of the PASA IC will be sampled by a CMOS switch and held during the Level 1 latency on one or more integrated MOS capacitors. Two schemes for achieving this storage are currently under study. The first is a derivative of the AMPLEX approach, ${ }_{19}^{19}$ and employs data-driven single-switch track-and-hold (T/H) sampling circuits. Specifically, the LCT trigger information is used to restrict sampling to only those strips that are in the vicinity of charged tracks. The number of storage capacitors per channel depends on the desired deadtime. The probability of two spatially overlapping LCT hits occurring within the Level 1 latency time is, under worst-case conditions, about 10\%-20\%. Deadtime losses can be eliminated by using a second storage capacitor on each channel and steering the sampling switches appropriately.

The second scheme uses clock-driven sampling as in a pipelined analog memory. The precision analog information is sampled repetitively on a switched capacitor array (SCA), dimensioned large enough to hold an entire waveform record corresponding to the Level 1 latency interval. When the Level 1 trigger decision arrives, it is put into coincidence with the delayed LCT signal. If the result is TRUE, then the appropriate group of samples in the SCA is logically transferred into a derandomizing buffer to await digitization. Amplitude and leading-edge time can be recovered by sampling at approximately $100 \mathrm{~ns}$ intervals. At that sample rate about 20 storage capacitors are needed to provide the Level 1 latency and an additional 10 to 20 storage locations are required for the virtual derandomizing buffer.

The data-driven $T / H$ system uses about 10 times fewer storage capacitors and therefore can combine amplification and sampling on the same chip. Any gain variations due to capacitor and switch non- uniformity will be removed in the calibration procedure. Analog signals can be well isolated from digital crosstalk. By digitizing only a single sample for each hit channel, the ADC bandwidth will be about five times less than in the SCA scheme.

In addition to deadtimeless operation, with more samples per hit, the SCA approach provides greater flexibility for off-line analysis. The benefits are expected to be greatest in reducing the effect of background pileup on resolution, although the extra samples should also provide a modest increase in the precision of the amplitude data.

Either technique must provide a way to know if the desired hit is spoiled by pileup from a recent background pulse. In the SCA, enough samples are digitized to examine the waveform record for some interval of time preceding the pulse peak of interest. In the data-driven sampling scheme, one must rely on the hit record provided by the digitized fast cathode signals. These can be either be stored in a digital pipeline, or their time differences can be stored. The details of the pileup detection schemes depend on the rate and spectrum of background events. Clearly, the SCA method provides more insurance against the effects of different background scenarios.

In both schemes, the LCT $\cdot \mathrm{L} 1$ coincidence initiates a readout sequence. The readout controller maintains a list of hit channels and capacitor read addresses. During readout, the multiplexer tree sequentially routes the information stored on these storage capacitors to the ADC.

In both schemes, an LCT $\cdot \mathrm{L} 1$ coincidence initiates a readout sequence. The readout controller maintains a list of hit channels and SCA read addresses. During readout, the multiplexer tree sequentially routes the information stored on these storage capacitors to the ADC.

As shown in Section 7.7.3, the expected coincidence rate between $\mathrm{L} 1$ and LCT is less than $1 \mathrm{kHz}$ on any given board. Nonetheless, a readout time of $\leq 100 \mu \mathrm{s}$ per board has been chosen so as to keep the readout latency to a reasonable value-i.e., one that is compatible with a hardware Level 2 trigger. Channels from portions of the detector whose LCT bits are active and their neighbors are 
read. Since a track typically traverses one or two twelve-strip LCT towers, a total of $4 \times 12=48$ strips must be read per LCT $\cdot \mathrm{L} 1$ coincidence. Note that the LCT-gated signal eliminates the need for channelby-channel zero suppression.

In the SCA case, there are five samples per channel, which leaves $100 \mu \mathrm{s}+(48 \times 5)=416 \mathrm{~ns}$ for each conversion. This conversion rate is well within the rate capabilities of commercial 10-bit ADCs. Since the T/H version uses just one analog sample, the $A D C$ and multiplexer throughput requirements are approximately a factor of five less.

The write addresses for the SCA (or the T/H) and the sequencing of the readout are controlled by a digital controller chip. This IC also serves to buffer readout commands and to provide a derandomizing FIFO buffer for the data from the ADC. Finally, it appends address information and an event ID to each block of $A D C$ data before it is transmitted to the readout motherboard. Since all channels on a given board operate in "lock step," only one such IC is needed for each 96-channel FEPCB.

\section{Second Coordinate Readout}

In the CSCs, the anode wires run in an azimuthal sense in both the barrel and the endcap chambers. Readout of the anode wires has two primary objectives: tagging the beam crossing and providing a measurement of the non-bend coordinate ( $z$ in the barrel, $r$ in the endcaps).

The anode wires are operated at high voltage with respect to the grounded cathodes. Groups of wires are ganged to a single readout channel. In the barrel the ganging is done in groups of $\mathbf{4 0}$ (this number, which is typical, corresponds to about $10 \mathrm{~cm}$ pitch for anode groupings). The wires in the middle and outer endcap superlayers are also ganged in groups of 40 , while those in the innermost superlayer are ganged in groups of $20(5 \mathrm{~cm})$. This choice of ganging represents a trade-off between polar angle precision, wire capacitance, channel occupancy, and cost. Further study is ongoing.

Figure $7-43$ shows the CSC 2 nd coordinate readout system in block diagram form. Specifics of the signal handling are shown in Figure 7-44 and described below.

Four gangs of signals are brought out on each plane of the CSC chambers via wire-board tongues.
These tongues have flexible cable connectors that mate to the signal-handling boards (PCB1). Each PCB1 contains three 8-channel integrated preamplifier/driver ICs, which handle sets of four signals on each of six planes. These 24 channels of preamplified signals are then transmitted from PCB 1 to an anode FEPCB via ribbon cable.

Each anode FEPCB receives the output of four PCB1s for a total modularity of 96 channels. At the point where the ribbon cable header mates to the FEPCB there is a 24-channel multiple-function IC that processes the incoming signals. The first stage splits the signal and sends it into fast and slow shaping amplifiers. From that point the processing proceeds in a fashion that is closely analogous to the cathode readout, but with an emphasis on timing rather than pulse-height resolution.

As noted, tagging of the bunch crossing is of prime importance. Provided the neutron/photon accidentals rate is not too high, it will be sufficient to form an OR of all six layers in a tower and then gate that with an LCT-like charged-track signal. For rates above $100 \mathrm{~Hz} / \mathrm{cm}^{2}$, which correspond to the high end of the expected range, it may be necessary to base the timing on the arrival time of the second hit.

\section{Readout Motherboards (ROPCB)}

The motherboards provide a central interface between the FEPCBs and the outside world. The various functions that must be performed and the cables over which the interactions with the individual boards occur naturally divide into distinct groups:

- Distribution of low voltage power to the FEPCBs.

- Collection and retransmission of fast signals from the cathode and anode front-end boards to the Level 1 decision hardware.

- Collection and retransmission via optical link of readout data from the FEPCBs to the Level 2 and DAQ.

- Downloading of information to the FEPCBs.

These functions will be implemented on a single printed circuit board unless further analysis shows a multiple-board approach to be advantageous. Present plans call for the motherboards to be constructed with gate arrays and available LSI devices. Prototyping will be done with FPGAs. 
For $6 / 6 / 6$ and $8 / 6 / 6$ Goometries

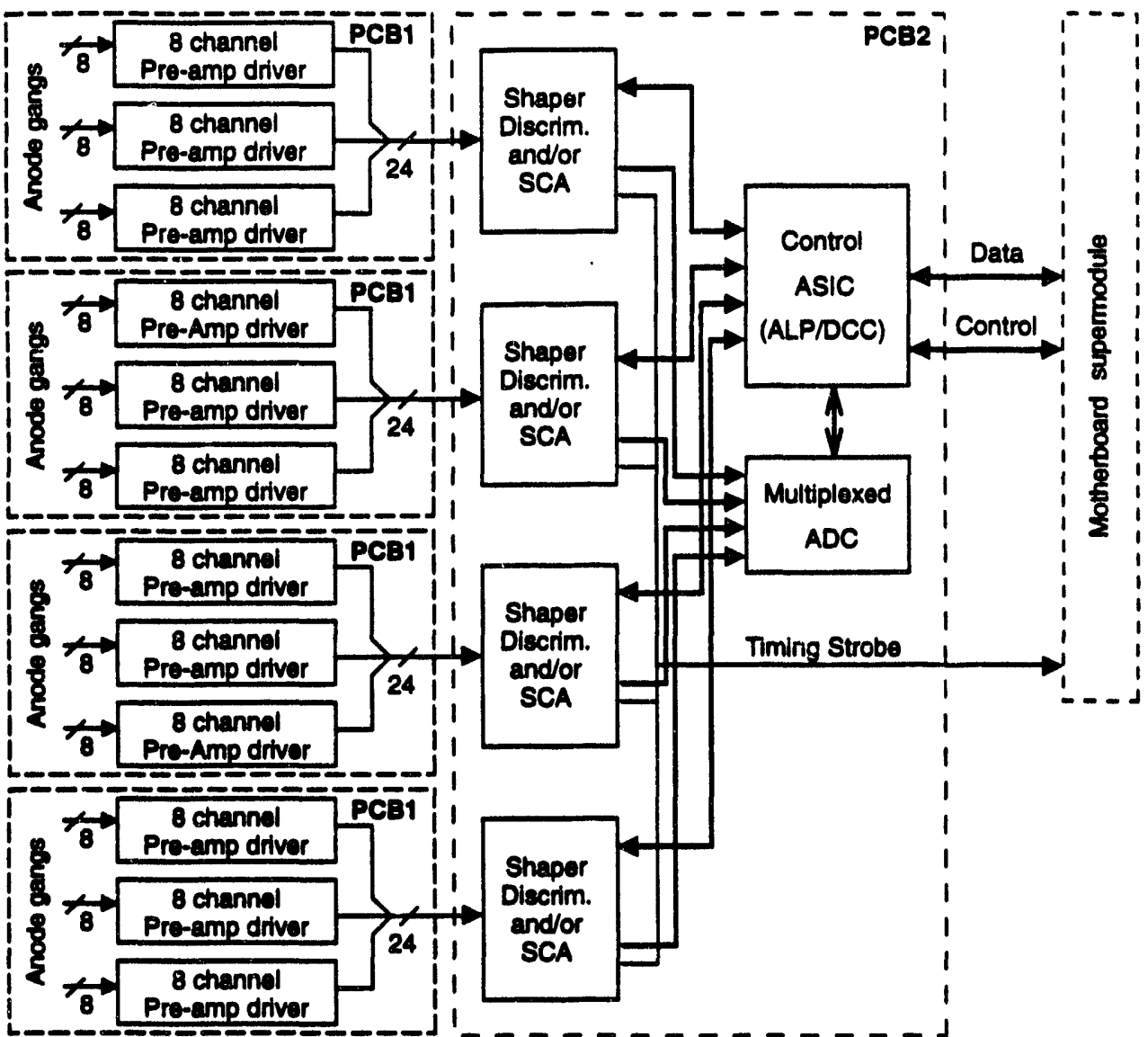

TIP-04311

FIG. 7-43. Block diagram of anode signal processing.

\section{DAQ Function}

When a Level 1 trigger has been generated, it will be distributed to each of the ROPCBs, where it will be channeled to the FEPCBs via dedicated lines linking the motherboards to the FEPCBs. Within a few microseconds, the first data to be read out will start becoming available in FIFO memories on the FEPCBs. At that point, the ROPCB will begin the process of transferring the data to its own FIFO memory, for retransmission to the DAQ via the fiber optic link.

As noted in Table 7-21, the design readout latency is to be less than $100 \mu \mathrm{s}$. This means that under worst case conditions, the last byte of data from the FEPCBs must be present on the motherboard for transfer out via the fiber optic link in that time frame.
Each ROPCB motherboard typically services seven cathode and two anode 96-channel FEPCBs. Since most events will produce both cathode and anode data, separate cathode and anode buses will be used. In the SCA approach, which produces the most data, FEPCBs will digitize five SCA samples per channel with a 10-bit ADC for each LCT.L1 coincidence. A typical charged track will result in the readout of $36-48$ strips, yielding about 430 bytes per LCT.L1 coincidence. The data transfer rate between the FEPCBs and the ROPCBs is $20 \mathrm{MBytes} / \mathrm{s}$ (a 16 bit data bus running at $10 \mathrm{MHz}$ ), resulting in a transfer time of $23 \mu \mathrm{s}$. The fiber optic link now envisaged is of the $60-100 \mathrm{Mbi} / \mathrm{s}$ variety, which is also sufficient for $100 \mu$ s transfer.

Figure 7-45 is a block diagram for the control circuitry on the ROPCB. A pair of 24-channel buses 


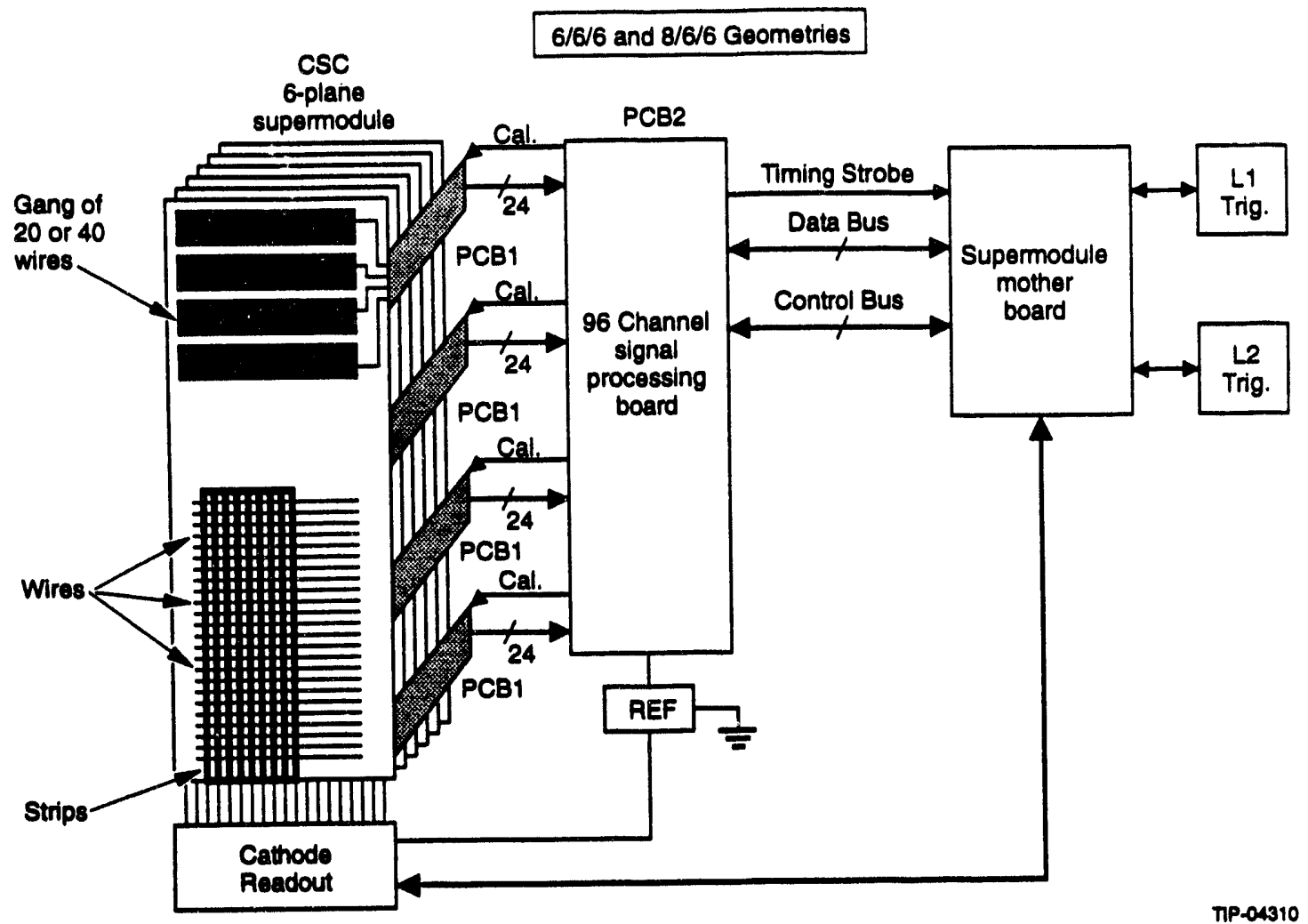

FIG. 7-44. Interface of the anode electronics with the CSC readout path.

(one each for anode and cathode) connecting the FEPCBs in a chain to the motherboard. In addition to a 16-bit data block (DATA), there is a 3-bit address block (ADDR), and single-bit blocks for the $10 \mathrm{MHz}$ clock (CLK), a data-in-FIFO status bit (DIF), a data acknowledge bit to confirm the presence of data on the data lines (DACK), a write-enable bit to signal the FEPCBs that the addressed board should read the information on the DATA lines (WREN), and finally the Level 1 trigger being distributed to the FEPCBs.

Each FEPCB will have a unique address (probably implemented with dip switches) and an address decoder that will cause it to respond on the DIF line when it is addressed by the motherboard. The FEPCB's controller will then begin transmitting the data from its FIFO memory on successive clock pulses, confirming each transmission with a DACK pulse. When the FIFO memory has been emptied, the DIF line will fall and the motherboard will move on to the next FEPCB.

The FIFO memories will be connected to the DATA lines via a transceiver, which can either transfer data from the FIFO memory outputs to the DATA lines or from the DATA lines to a READ register. When the motherboard wishes to transmit information (such as commands for calibration and reset purposes) it will load the DATA lines appropriately and assert the address of the recipient FEPCB on the ADDR lines along with the WREN line.

Level 1 triggers are encoded and passed on to the global Level 1 trigger asynchronously to other activities. The ROPCB's FIFO memory will have a capacity of at least $4 \mathrm{KBytes}$ to ensure substantial buffering capacity. 


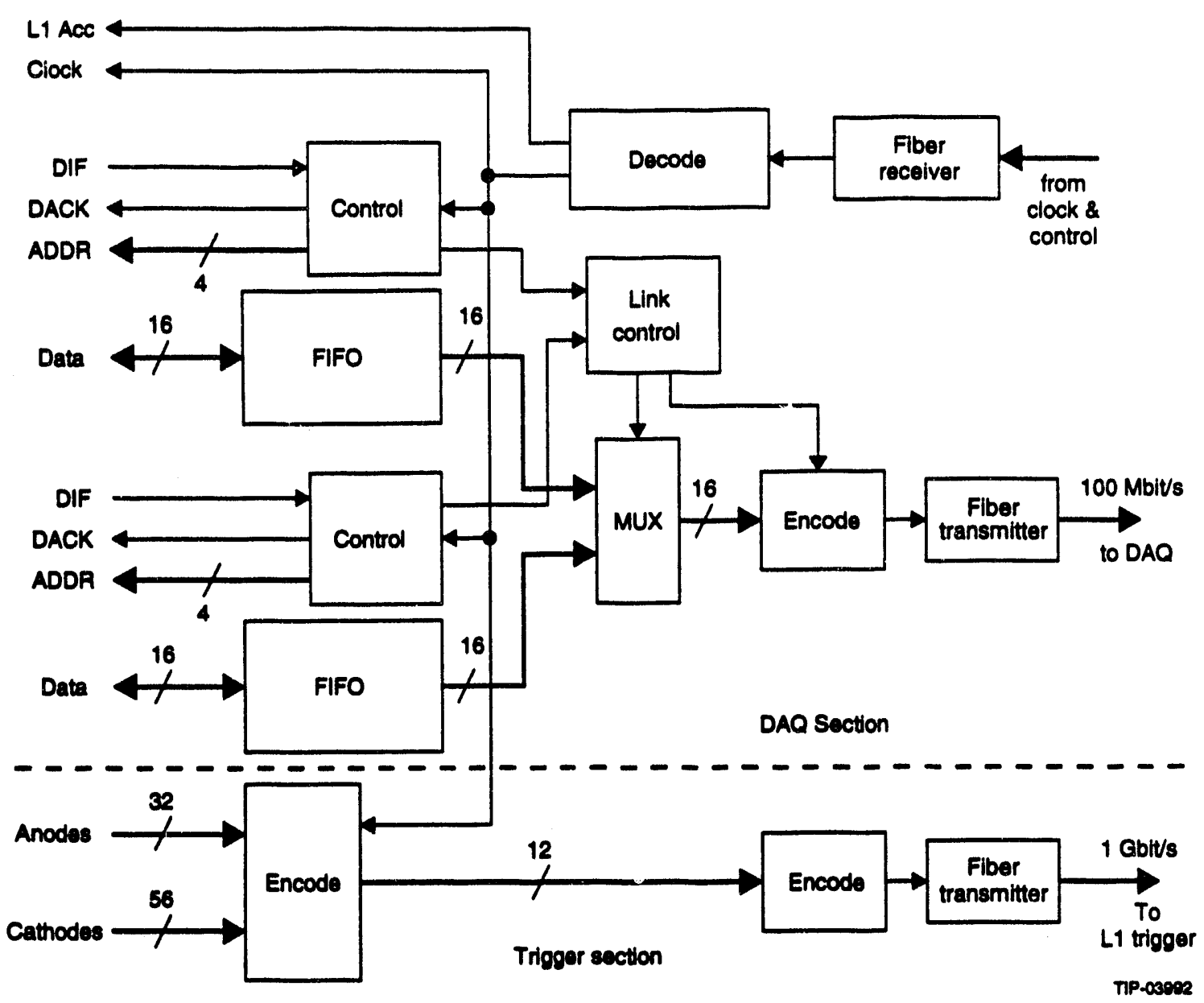

FIG. 7-45. Block dilugram of the control circuit for ROPCB and the FEPCBs. The elements of the trigger to be housed on the ROPCB are also shown.

\section{Trigger Function}

The ROPCB also serves to encode the trigger data from the FEPCBs for transmission to the off-detector Level 1 trigger. Each cathode FEPCB produces eight trigger bits, corresponding to the eight LCT towers that are instrumented by each card. As a rule, each charged track that traverses a chamber will result in a single cluster of hits in the 56-bit word that represents the supermodule. The trigger section of the ROPCB encodes this 56-bit word into a list of clusters that are sent sequentially on a $1 \mathrm{Gbit} / \mathrm{s}$ fiber-optic trigger link. The handling of multiple hits is described in the muon trigger section (Section 7.2.2) of this chapter.

\section{HVPS and Distribution}

The worst-case current draw per CSC supermodule is given by

$$
I=N \times G_{\text {gas }} \times \Phi \times A \times N_{\text {plane }} \times e=10 \mu \mathrm{A}
$$

where $N=100$ is the number of electron-ion pairs per detected track, $G_{g a s}=2 \times 10^{4}$ is the chamber gas gain, $\Phi=100 \mathrm{~Hz} / \mathrm{cm}^{2}$ is the worst-case flux of detected particles (mostly neutrons and photons) $A=42.000 \mathrm{~cm}^{2}$ is the chamber area, $N_{\text {plane }}=6$ is the number of planes, and $e$ is the electron charge. This current can readily be supplied by the typical commercial high-voltage power supply (HVPS) unit designed for similar applications. A dedicated channel of high voltage is provided for each of the 1440 CSC supermodules that comprise the muon system. Current and voltage monitoring is done for each such channel.

The -40 HVPS crates are located in the electronics room and a single HV cable is run to each supermodule. Within each supermodule there is a printed circuit board (HVPCB) that distributes the incoming HV line to each of the six planes. In addition, the HVPCB monitors the voltage and 
current for each plane, and provides for individual disconnect in the event of a single plane failure, allowing the remaining five planes in the supermodule to continue functioning. An individualchannel disconnect mechanism capable of operation in a $0.8 \mathrm{~T}$ magnetic field is an R\&D topic. If a suitable circuit cannot be developed, the HVPCB can be situated outside of the magnet and connected to the supermodules via six individual cables.

\section{LVPS and Distribution}

In total, the muon electronics will dissipate approximately $100 \mathrm{~kW}$ of power. Much of this power is consumed in the large front-end MOSFETs, which are operated with large drain currents to minimize noise. The design of the LVPS system represents a tradeoff between power loss in the delivery system and the mass of conductor used to deliver current to the front-end electronics. A straightforward analysis shows that for a given current supplied to the front-end electronics and a specified maximum tolerable power dissipation in the power delivery system, the mass of the delivery system goes like the square of the length of the feed lines. In view of these considerations we have $c$, ted for a distributed delivery system. This design employs 300-VDC-to-5-VDC DC-DC converters mounted just outside the solenoidal magnet. Using such a system to deliver $20 \mathrm{kA}$ of current and allowing no more than $10 \mathrm{~kW}$ of power dissipation in the delivery system results in a total cable mass of approximately six tons.

\section{Cooling}

To reduce thermal gradients within the muon system it is important to minimize the amount of heat expelled into its volume. To remove the heat, each FEPCB is laminated to an aluminum coldplate that is mechanically attached to a second water-cooled coldplate, which is shared by all of the boards of the module.

\subsubsection{Design Details}

This section presents the various functional blocks of the CSC readout in detail. Table 7-26 gives a list of the various printed circuit boards and their associated components with details for each component given in the sections that follow.

\section{Cathode-to-FEPCB Connection}

The signals will be carried from the cathode boards to the FEPCBs on shielded flat cable. Each 96-channel FEPCB will be connected using a set of six mass-terminated 16-pair cables.

\section{Low-Noise Preamplifier-Shaper}

\section{Circuit Details}

As noted above, the front end IC noise requirement is $\mathrm{ENC}<2000 e^{-} \mathrm{RMS}$ for a detector capacitance of $100 \mathrm{pF}$ and a shaping time of $300 \mathrm{~ns}$.

The equivalent noise charge (ENC) of the preamplifier is given by

$$
E N C^{2}=\frac{a_{1} e_{n}^{2} C_{T}^{2}}{t_{m}}+a_{2}^{2} i_{n}^{2} t_{m}
$$

TABLE 7-26. The types and functions of the muon system PC boards.

\begin{tabular}{|c|c|c|}
\hline Board & Location/size & IC housed in board \\
\hline $\begin{array}{l}\text { Cathode front end PCB } \\
\text { (FEPCB) }\end{array}$ & $\begin{array}{l}\text { Edge of Cathode plane } \\
\text { (<100 } \mathrm{mm} \text { by < } 200 \mathrm{~mm} \text { ) }\end{array}$ & $\begin{array}{l}4 \text { PASA + } 4 \text { ASIC\#2 + ADC + MUX + } \\
\text { ALPIDAQ }\end{array}$ \\
\hline $\begin{array}{l}\text { Anode front end PCB } \\
\text { (PCB1) }\end{array}$ & $\begin{array}{l}\text { Edge of anode frame } \\
(<100 \mathrm{~mm} \text { by < } 200 \mathrm{~mm})\end{array}$ & 3 APREAMP \\
\hline $\begin{array}{l}\text { Anode tront end PCB } \\
\text { (PCB2) }\end{array}$ & $\begin{array}{l}\text { Edge of anode frame } \\
(<200 \mathrm{~mm} \text { by }<300 \mathrm{~mm})\end{array}$ & 4 AIFIC+ ALP/DCC+ AADC \\
\hline $\begin{array}{l}\text { Module Mother board } \\
\text { (ROPCB) }\end{array}$ & $\begin{array}{l}\text { Top of a } 6 \text { gap chamber module } \\
\text { ( }<300 \mathrm{~mm} \text { by <300 mm) }\end{array}$ & $\begin{array}{l}\text { LCENC + cables to trigger and read- } \\
\text { out + Low Voltage Power Compo- } \\
\text { nents }\end{array}$ \\
\hline Trigger Board & $\begin{array}{l}\text { Electronics room } \\
(<300 \times 300 \mathrm{~mm})\end{array}$ & $\begin{array}{l}\text { CAM lookup memory + trigger re- } \\
\text { ceivers + trigger glue logic }\end{array}$ \\
\hline
\end{tabular}


- where $a_{1}$ and $a_{2}$ are shaping-time form factors of order unity, $e_{n}$ and $i_{n}$ are the equivalent input voltage and current noise spectral densities, $C_{T}$ is the total input capacitance, and $t_{m}$ is the shaping time.

For a MOS transistor

$$
e_{n}^{2}=4 k T\left(\frac{2}{3 g_{m}}+R_{g}\right)+\frac{a_{3} t_{m} K_{f}}{C_{o x}^{2} W L}
$$

where

$$
g_{m}^{2}=\frac{2 W K_{p} I_{d}}{L}
$$

- where $I_{d}$, is the drain current, $R_{g}$ is the gate resistance, $K_{p}$ is the transistor mobility-capacitance product and $K_{f}$ is the $1 / f$-noise coefficient.

Given the required ENC, form factors, detector capacitance, and technology parameters $R_{g}, K_{p}$, and $K_{f}$, we choose $I_{d}$ and $W$ to meet the performance goals. This leads to a choice of minimum $L$, maximum permittad $I_{d}$, and an optimization of $W$. Optimization studies for the Orbit $2 \mu \mathrm{m}$ MOS process reveal that the noise minimum occurs for a very large gate periphery, but is rather shallow. The low ENC requirement leads to a large drain current, comparable to what is typically used in JFET preamplifiers.

The shaper employs a passive differentiator followed by two second-order active low-pass sections to achieve a $C R-(R C)^{4}$ response. The chosen configuration gives a peaking time that is tunable without altering the shape of the response by adjusting a bias current. A shaper with a target peaking time of $70 \mathrm{~ns}$ has been designed using this principle and fabricated. Its step response is tunable over a range of about a factor of two. The peaking time varies according to the inverse square-root of the bias current, as predicted.

\section{Results Obtained to Date}

Several test chips have been fabricated to verify the design principles of the analog circuit blocks (preamp and shaper):

- 10000/2 $\mu \mathrm{m}$ p- and n-channel MOSFETs for $1 / f$ noise measurements

- Preamp with $10000 / 2 \mu \mathrm{m}$ p-channel input device, $C_{F}=2 \mathrm{pF}$
- Preamp with $10000 / 2 \mu \mathrm{m} n$-channel input device, $C_{F}=1 \mathrm{pF}$

- Fourth-order semi-gaussian shaper with $t_{p}=$ $700 \mathrm{~ns}$

- Fourth-order semi-gaussian shaper with $t_{p}=$ $25 \mathrm{~ns}$

- The $p$-channel preamplifier combined with the 700 ns shaper and a simple track-and-hold circuit with a 6-pF hold capacitor

- The $n$-channel preamplifier driving the 25-ns shaper.

Equivalent input noise charge (ENC) as a function of shaper peaking time is shown in Figure 7-31 for the $n$-channel preamp with an external shaper. The curves exhibit the characteristic $t_{p}-1 / 2$ dependence for peaking times below about $200 \mathrm{~ns}$, followed by a $t_{p}$-independent region, where the noise is dominated by $1 / f$ noise from the input device. Finally, at very large $t_{p}$ the parallel noise leads to a $\sqrt{t_{p}}$ behavior. The noise slope $d(E N C) / d t_{p}$ ranges from about $25 e^{-/ p F}$ at $50 \mathrm{~ns} t_{p}$ to $16 e^{-/ p F}$ at $500 \mathrm{~ns}$ and $10 e^{-/} \mathrm{pF}$ at $5 \mu \mathrm{s}$.

Waveforms from the $p$-channel preamplifier and the slow-shaper output are shown in Figure 7-46 for nominal bias conditions. The preamplifier feedback resistor has about $800 \mathrm{k} \Omega$ equivalent resistance, leading to a 1.6- $\mu \mathrm{s}$ falltime. This causes the overshoot in the shaper output seen in the lower portion of the figure, and contributes to the parallel noise of the circuit. In subsequent designs, additional circuitry will be added to control the preamp $R_{f}$ to accommodate larger process variations.

The noise measure at the output of the 700-ns shaper is about $1800 e^{-}$RMS at zero input capacitance, and increases with a noise slope of approximately $12 e^{-/ p F}$. This includes contributions from the parasitic capacitance of the IC package, the PC board, the ESD protection network on chip, the low value of the preamp $R_{f}$, and second-stage noise due to the shaper input transistors and differentiator resistors. All of these sources can be reducej by circuit and packaging modifications now in progress.

Finally, Figure $7-47$ is a graph of the input versus output of the p-channel amplifier combined with the 700-ns shaper measured at the shaper 
output. Reasonable linearity is achieved up to about $150 \mathrm{fC}$ equivalent input charge.

(a)

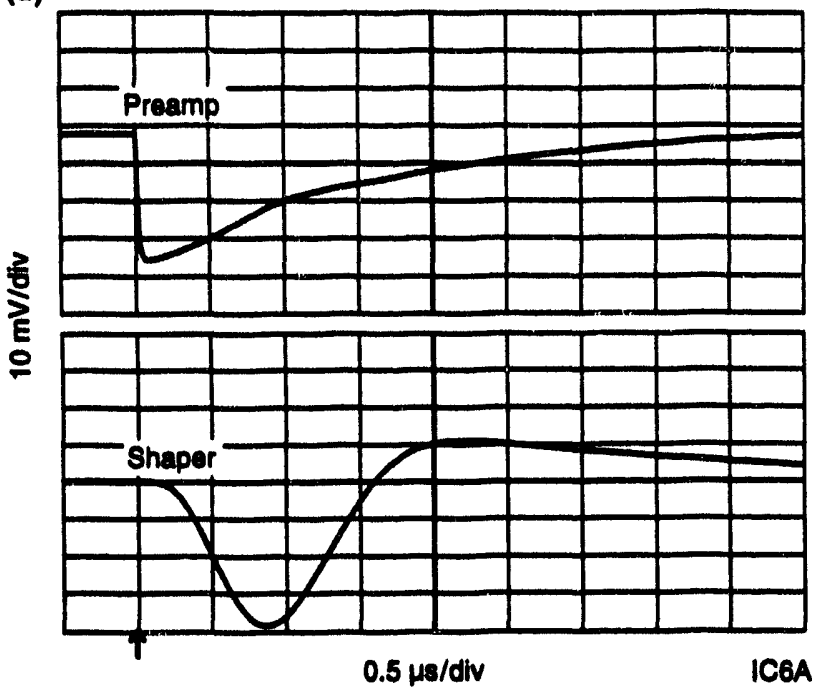

(b)

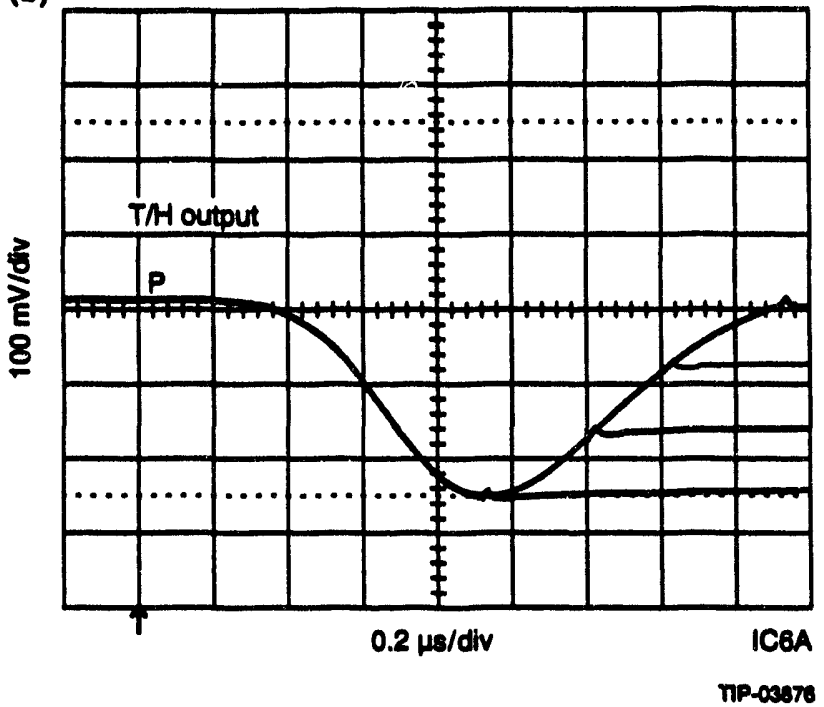

FIG. 7-46. (a) Output wavelorms from test chip showing response of preamp and 700-ns semi-gaussian shaper to a $40-1 \mathrm{C}$ chargo impulse injected through a 2-pF capacitor. (b) Track and hold response for the preamp/700-ns shaper test chip. Various delay settings of $T / H$ relative to shaper peaking time are shown.

\section{ASIC \#2}

The functionality of ASIC \#2 depends on whether the $\mathrm{T} / \mathrm{H}$ or the SCA option is selected. Timing discriminators and the trigger logic must be implemented in either case, but in the T/H case, the SCA array is replaced by a digital timer circuit.

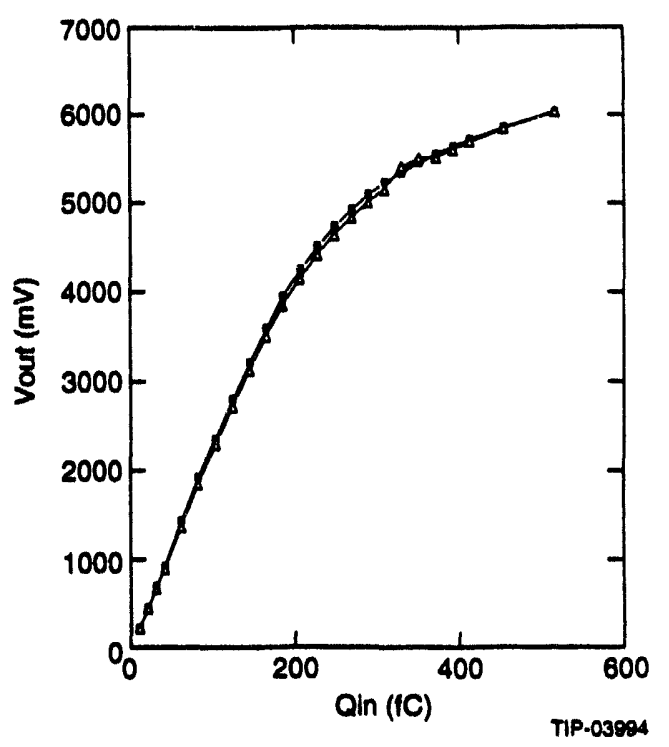

FIG. 7-47. Output voltage versus input charge for the combined preamplifier/shaper.

\section{Timing Discriminator}

A block diagram of the timing discriminator is shown in Figure 7-48 The function of the circuit is to provide an output logic pulse with leading-edge timing that is independent of pulse amplitude. The circuit meets this requirement by comparing the fast shaper output signal with its "integral," which is derived using a series $R C$ network. The basic circuit. which was originally developed in discrete form by Turko and Smith, ${ }^{20}$ does not require a delayed version of the signal as needed by the conventional constant-fraction discriminator. ${ }^{21}$ This feature is essential and makes it possible to implement a compact, multi-channel, integrated version of the circuit in an inexpensive CMOS process.

The upper comparator operates as a conventional discriminator, providing an adjustable threshold. Pulses are standardized to a uniform width by using an edge-triggered one-shot subcircuit.

The performance of the discriminator is largely determined by the performance of the input comparator. The design that is currently being developed employs a low-gain differential-pair input to ensure high-speed operation. Simulation of that circuit using typical shaper response functions indicates that a 30:1 variation in the input pulse amplitude results in a variation of less than $6 \mathrm{~ns}$ in the output signal's leading-edge time. 


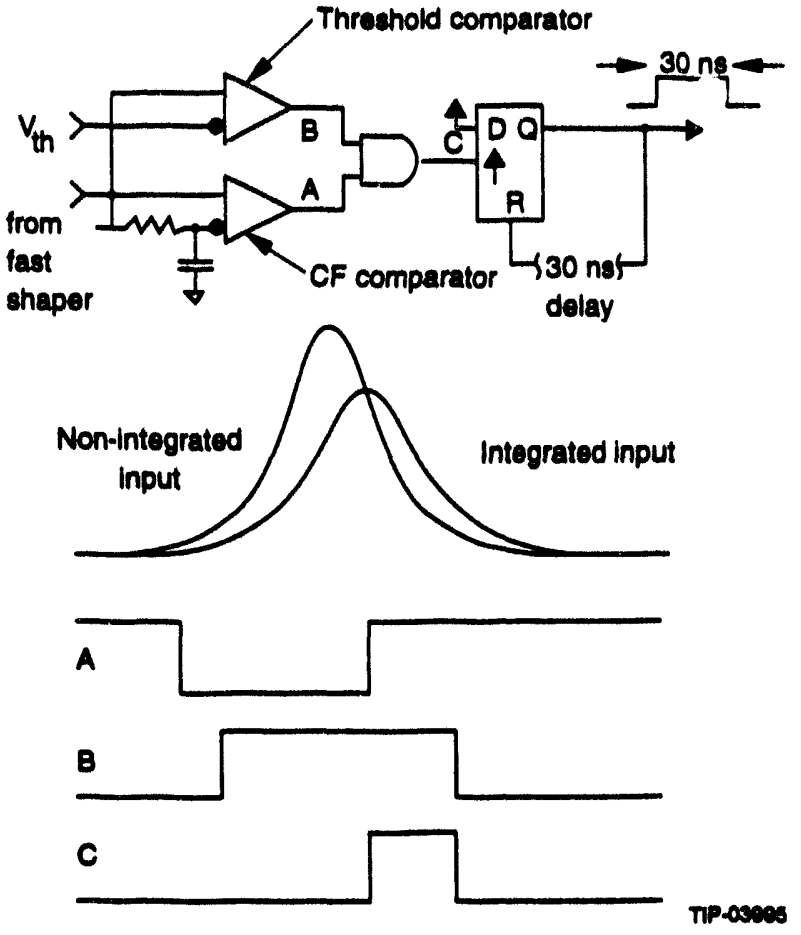

FIG. 7-48. Block diagram of the timing discriminator circuit and typical waveforms.

\section{Trigger Jogic}

the local charged-track (LCT) logic identifies logical patterns consistent with straight tracks traversing a superlayer. Individual strips are first OR'ed in pairs to reduce the amount of circuitry required, resulting in a trigger element size between 12 and $22 \mathrm{~mm}$, depending on the superlayer. A typical LCT "road" is shown in Figure 7-49. Hits induced by neutrons and other non-track debris are suppressed by requiring that at least four out of the six trigger elements that lie along a given trigger road fire. The required count logic will be implemented using either digital population counter blocks or high-speed current-summing junctions followed by analog comparators. The former approach offers the intrinsic robustness of a digital design, while the latter may prove to be more economical and better suited to asynchronous (i.e., pulsed) operation. Design studies and prototype work are ongoing. Each ASIC2 IC will produce a pair of outputs corresponding to the two "LCT towers" serviced by a chip. Eight such signals from each FEPCB will be passed on to the global Level 1 muon trigger and will also be used locally to "steer" the ensuing readout sequence.
It is evident from Figure $7-49$ that there is a non-negligible probability that a muon track will cross an IC boundary when traversing a superlayer. To accommodate such occurrences without loss of efficiency requires that each logic IC transmit six signals to its nearest neighbor. Since the ICs are in close physical proximity, this is not a fundamental problem. A similar situation obtains at FEPCB boundaries. The costs and benefits of transmitting the signals across board boundaries are under study.

\section{Switched Capacitor Array}

The slow shaper outputs from the preamplifier must be sampled and stored pending arrival of the LCT. $L 1$ coincidence. This will be done using a switched capacitor array (SCA). The comparatively long shaping times mean that the SCA need sample at only $10 \mathrm{MHz}$. A voltage write and voltage read configuration is sufficient to provide the required 10 bits of effective dynamic range. The $2-\mu$ s Level 1 latency requires a total of 20 storage cells, with an additional 10-20 cells being required to implement a virtual FIFO buffer to derandomize the load on the ADC system. Thus a total of $30-40$ storage cells, arranged in a conventional array of 2-pF floating capacitors, are required for each channel. We note that the design requirements of the SCA for this system are considerably less stringent than what has already been achieved ${ }^{17,22}$ in the SSC Subsystem R\&D program.

\section{Readout Amplifier and Multiplexer}

The major subcircuit of the SCA memory is the readout operational amplifier. The amplifier sche-

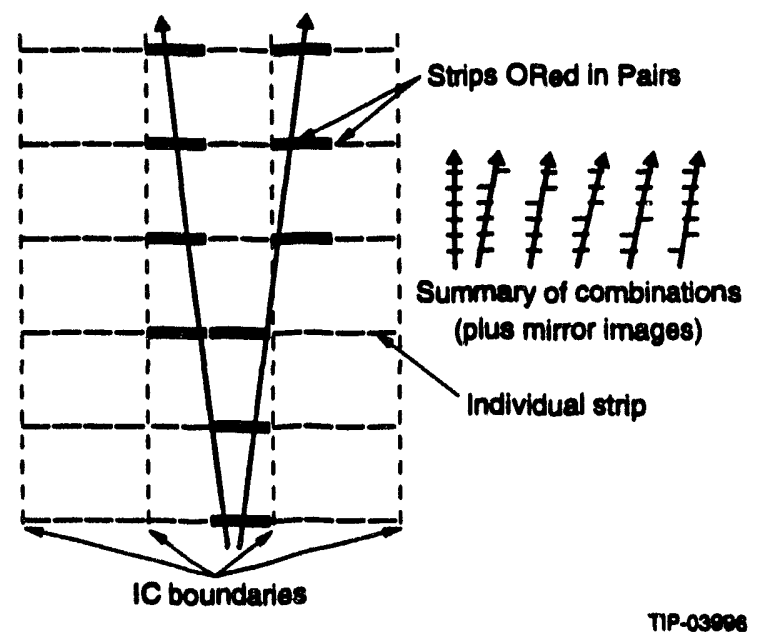

FIG. 7-49. Typical combination of cathode strips corresponding to a valid LCT combination. 
matic is shown in Figure 7-50. The amplifier, which is self biasing, accepts a wide input range by using both $p$-MOS and $n$-MOS differential pairs and a cascoded output stage. The output drive requirements have been minimized by the use of a common unity gain buffer after the output multiplexer. The multiplexer provides a single analog output from the chip.

\section{Timer Circuit}

In the $\mathrm{T} / \mathrm{H}$ option the timing of each cathode strip signal with respect to LCT is determined using a digital counter. The counter for a given strip starts upon receipt of a pulse from that strip and stops with the receipt of LCT. If LCT does not occur, the counter counts to a preset value, at which point it resets itself and awaits its next input. An LCT.L1 coincidence causes the contents of the counter to be transferred to an output register, where they are held pending readout. The clock rate of the counter will depend on the required time resolution, and will most likely be in the 10-30 $\mathrm{MHz}$ range.

\section{Control IC}

The control IC orchestrates the operation of the FEPCB. In particular it controls the read and write addresses of the SCAs ${ }^{17}$ (or $\mathrm{T} / \mathrm{Hs}$ ) and sequences the operation of the ADC and its input multiplexer. Since a LCT.L1 signal can occur while the ADC is reading out an earlier event, the control IC must be able to "queue" readout commands. The data driven nature of the readout means that different FEPCBs will in general be reading out different events, making it necessary to assign a "serial number" to each event on a card-by-card basis. This is conveniently done by counting Level 1 accepts, since this signal is common to all cards in the system.

ADC

Each FEPCB will employ a single high-speed $A D C$ driven by an analog multiplexer tree. As noted previously, the ADC must operate in the 5-10 MSPS range with 10-bits of dynamic range ( 8 bits of accuracy). Suitable ADCs based on CMOS technology will soon be commercially available.

\section{Multiplexer Tree}

The ADC throughput requirements can only be met if the settling time of the analog multiplexer is sufficiently short. Typical settling times for the first rank of multiplexers (i.e., the read amplifiers) are in the few hundred ns range. However, commercial multiplexers that offer considerably shorter settling times exist. By sequencing the readout such that SCA ICs are polled in a round-robbin fashion, the system fully exploits the fast-settling properties of the commercial multiplexer while maintaining the settling time requirements on the SCA output amplifiers (see Figure 7-51).

\subsubsection{Rate-Handling Capacity}

The general topic of rates in the muon system is discussed in Chap:or 4. In this section, the rate-handling limitations of the readout electronics

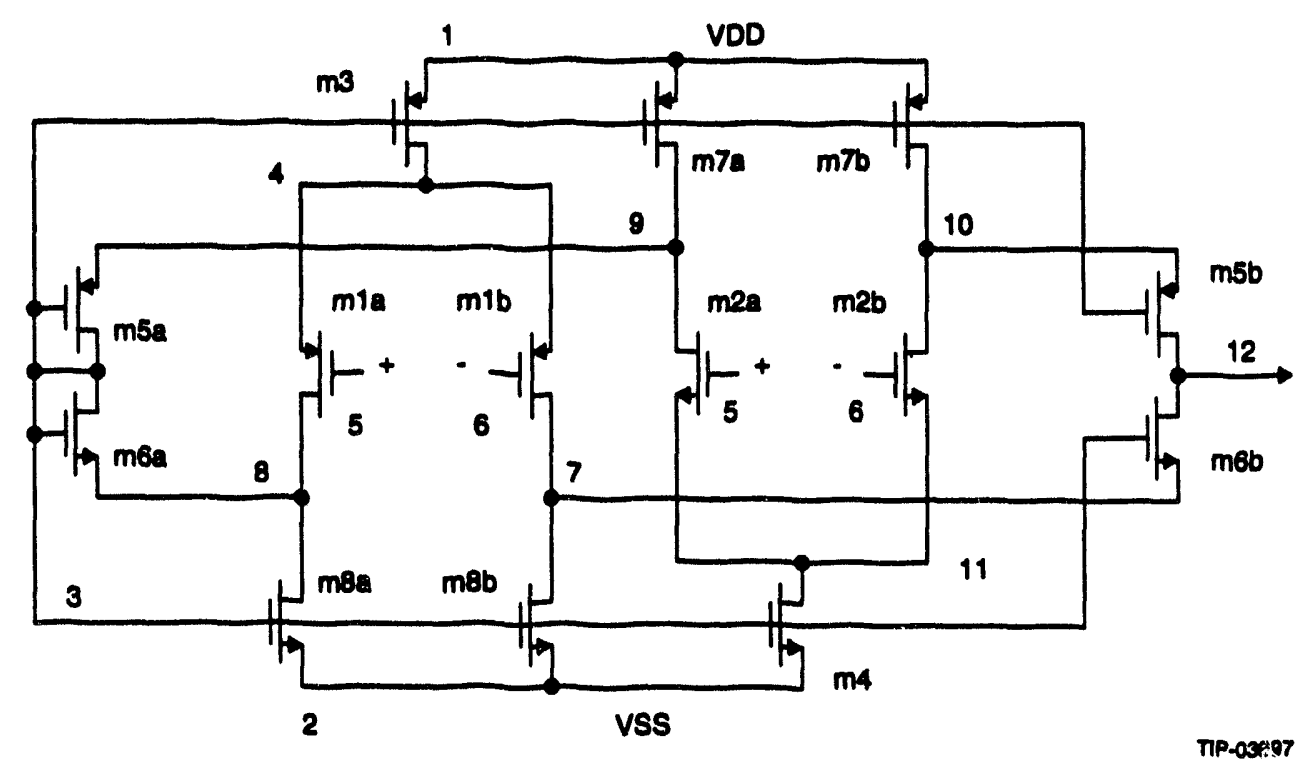

FIG. 7-50. SCA readout amplifier schematic. 


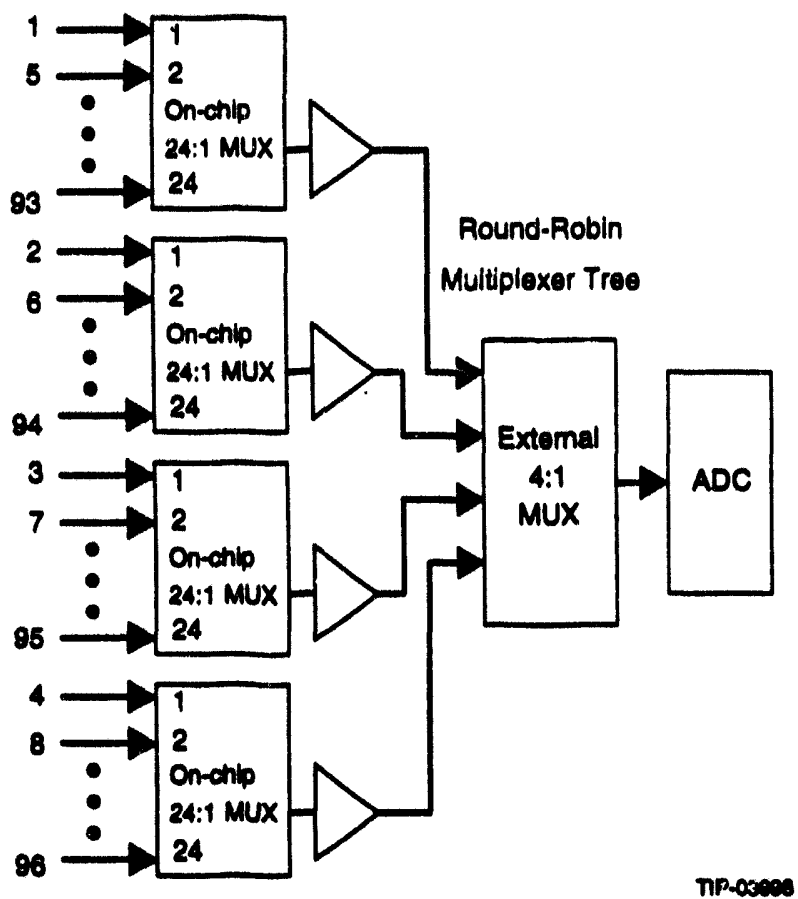

FIG. 7-51. Block diagram of the muttiplexer tree. The numbers on the extreme right indicate the scan order. Not shown is the address control logic. The select address value for a given on-chip multiplexer is incremented immediately after th has been read.

are discussed. It is shown that the electronics will continue to function at the highest anticipated rates.

The rate-handling capability of the CSC readout is limited by pulse pileup in the cathode front ends. Pileup events, which arise mainly from accidental neutron and photon hits, induce spoilage with varying degrees of severity. In the least severe cases, bonafide hits can be fully recovered through subtraction of the tails of accidental hits, while in the most severe cases, bonafide hits are spoiled in a way that cannot be detected, resulting in a possible position mismeasurement. An intermediate class of pileup events occurs when good hits are spoiled by accidental hits that can be detected but not subtracted away. Such hits must be discarded in the off-line analysis and therefore represent a loss of efficiency.

The situation is represented schematically in Figure 7-52. The vertical and horizontal gridlines indicate boundaries between strips and SCA samples. The dark circles represent the amount of charge deposited by a hit in the various "space-time" buckets. The solid ellipse denotes a boundary outside of which accidental hits can be subtracted with sufficient accuracy to avoid compromising the reconstructed position. A much smaller region, approximately one gridblock in size, corresponds to the area in which it is not possible to detect the presence of an accidental hit.

The total probability for event spoilage is simply the probability that an accidental hit falls into the elliptical area. Assuming a 100-ns sampling time, a -300 ns peaking time, and $350-\mathrm{cm}$-long strips of $1 \mathrm{~cm}$ width, this area is approximately 20 grid blocks-i.e.,

$$
\begin{aligned}
A_{\text {spoilage } \cong N_{\text {grid }} a_{\text {grid }}} & =20 \times 350 \mathrm{~cm}^{2} \times 10^{-7} \mathrm{~s} \\
& =7 \times 10^{-4} \mathrm{~cm}^{2} \mathrm{~s}
\end{aligned}
$$

The spoilage probability is given by

$$
p_{\text {spoilage }}=\Phi A_{\text {spoilage }}
$$

where $\Phi$ is the flux of detected particles in $\mathrm{Hz} / \mathrm{cm}^{2}$. Assuming that one can tolerate a $20 \%$ spoilage probability (for a six-layer superlayer, this gives 98\% detection probability for $\geq 3$ hits) the maximum flux of detected particles is then $\Phi \sim 300 \mathrm{~Hz} / \mathrm{cm}^{2}$, which is approximately 30 times larger than what is expected at standard luminosity.

A given 96-channel FEPCB executes a readout sequence whenever there is a coincidence between the Level 1 accept signal and the logical OR of the eight LCT signals on that board. The area coverage

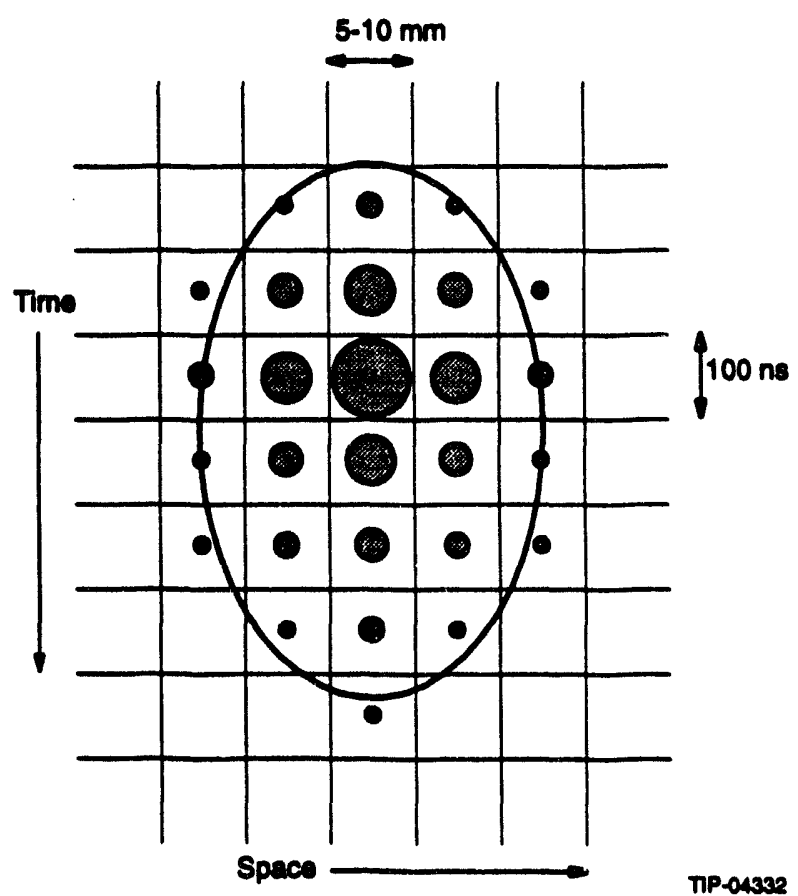

FIG. 7-52. Schematic representation of how hits appear in the muon readout. 
of a 96-channel cathode FEPCB in the 3rd barrel superlayer is approximately

$$
\begin{aligned}
A_{\text {FEPCB }} \approx \mathrm{N}_{\text {strip }} \ell \mathrm{W} \cong & =16 \times 350 \mathrm{~cm} \times 1 \mathrm{~cm} \\
& =5600 \mathrm{~cm}^{2}
\end{aligned}
$$

Thus with charged particle fluxes of $10 \mathrm{~Hz} / \mathrm{cm}^{2}$, which is higher than what is expected in the barrel, the LCT rate is in the $50-60 \mathrm{kHz}$ range. Assurning that the L1 $\mathrm{LCT}$ coincidence is formed with a 33 ns (two bunch crossing) resolving time and that there is no correlation between LCT hits and the Level 1 signal, yields an L1 LCT coincidence rate of $R_{L 1} \cdot L C T=200 \mathrm{~Hz}$. Given the specified $100-\mu \mathrm{s}$ digitization time, the FEPCBs will be actively digitizing with a duty factor of $5 \%$ or less. With simultaneous read-write operation and double buffering of the input samples, the fraction of lost data will be of order $0.1 \%$. Moreover, the system will operate with acceptable, if non-negligible, losses even if simultaneous read-write operation should prove problematic.

\subsection{INTEGRATION ISSUES}

\subsubsection{Physical Layout}

The GEM subsystem electronics will be distributed over a large area covering the in-detector area, the on-detectur area, the electronics room, and the operation center on the surface. Figure 7-53 shows the general layout. The in-detector electronics are relatively difficult to access and must be specially packaged. The label "on-detector" is used for historical reasons, and mainly refers to rackmounted calorimeter electronics located on the walls of detector hall. There will, however, be a small amount of electronics (muon LVPSs) mounted on the solenoid. The typical cable run from the calorimeter to the wall-mounted racks is approximately $40 \mathrm{~m}$. The electronics room, which is located at the bottom of the cable access shaft, is the closest area where electronics with "beam-on" accessibility can be located. It will be used to house the Level 1 trigger, assorted power supplies (both high- and low-voltage) and much of the DAQ. Finally, the control room, which is at the surface will be used to house the Level 3 processors and non-time-critical elements of the DAQ.

\subsubsection{Fiber-Optic Iinks}

Ground loops and electromagnetic interference (EMI) are major concerns in the GEM electronics system. The strategy for reducing these problems is to use Faraday-shielded power isolation transformers and fiber-optic cables extensively. Isolation transformers will be used between floors in the electronics room and between subsystems in the experimental hall. If they are needed, isolation transformers will be installed in each rack for further isolation. Isolation transformers reduce primary circuit noise that will capacitively couple into the secondary power distribution system. Fiber-optic cables provide immunity from EMI. They offer low loss, high bandwidth, low mass and small size. By using both isolation transformers and fiber-optic cables, conducted electrical noise coupling will be kept local. In addition, the operation center, the electronics room, and the experimental hall will be electrically isolated, thus reducing ground loops between these areas.

Figure 7-54 and Table 7-27 show the schematic layout and specifications for the various links in the GEM electronics system. The specifications of the silicon strip and the IPC require the optic links to be operated in $5 \mathrm{Mrad}$ and $2 \mathrm{Mrad}$, respectively. Their radiation rates are $0.4 \mathrm{Mrad}$ and $0.025 \mathrm{Mrad}$ per SSCY. The silicon strip group is designing a rad-hard link to meet this requirement. The IPC will use the same link, which will be based on a rad-hard surface-mount 850-nm wavelength LED from Honeywell. There may be a lens on the LED to focus the light into the fiber. If a lens is not workable, the LED will be coupled directly into a 200-nm fiber cable. The PIN photodiode does not have to bc a rad-hard component. To reduce costs, instead of using the rad-hard fiber cable all the way from the MCM to the electronics room, a rad-hard fiber cable of about $10 \mathrm{~m}$ will be welded with a $70-\mathrm{m}$ non-rad-hard fiber cable. The rad-hard fiber cable may be the hard-clad silica family of fiber products from the Ensign-Bickford Optics company. The hard-clad silica optical fibers have been tested for space and medical applications, where the total dose of ionizing radiation is relatively low when compared to that of GEM. There is currently no test 


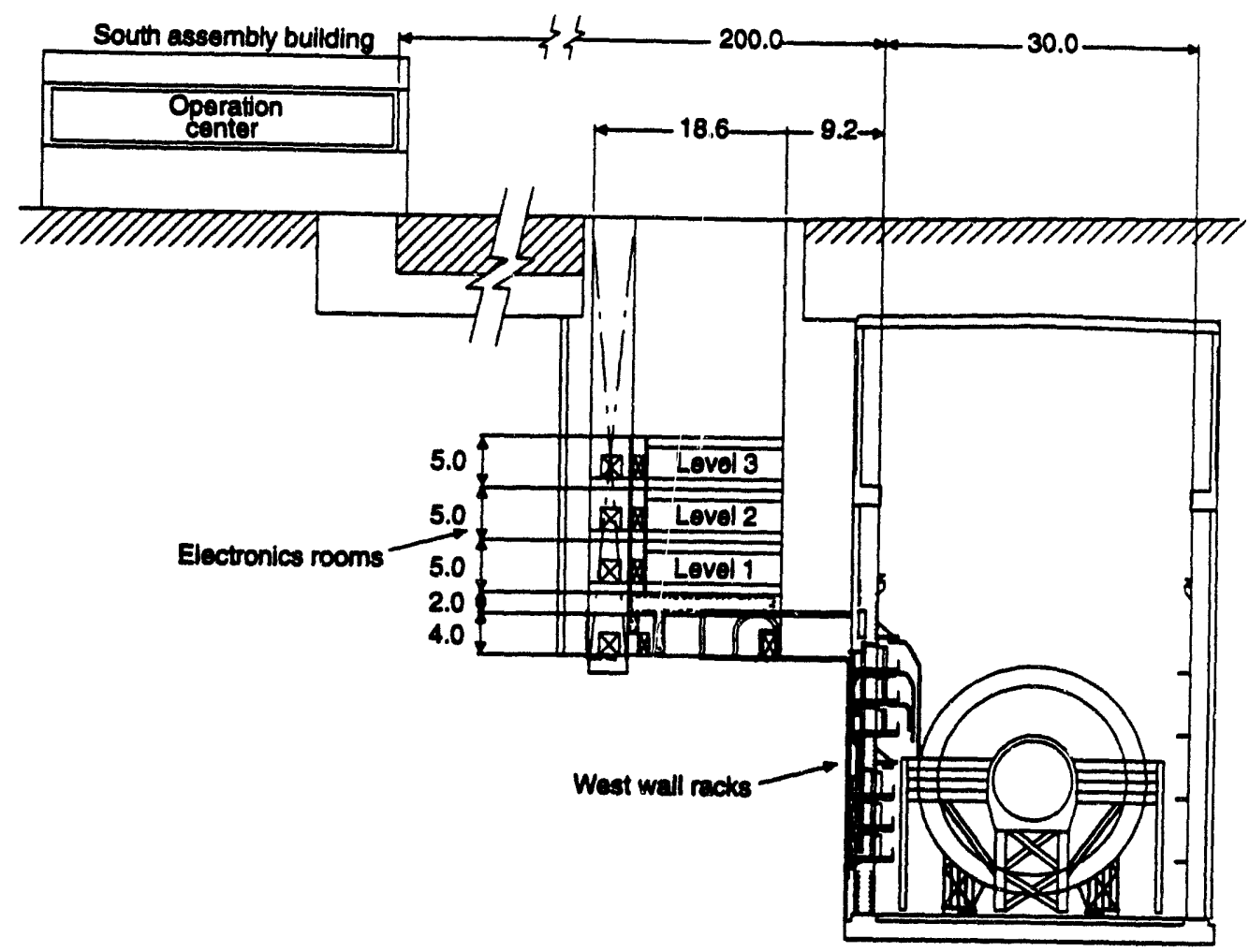

FIG. 7-53. View of GEM detector facility showing location of various dectronic subsystems - i.e., the in-detector area, the on-detector (west wall) area, the electronics roorn (ER), and the operations center.

data for neutron radiation, which may cause displacement damage. More tests will be done on the effects of high total dose radiation, $\mathrm{pr}$ microbending, and the rate of annealing.

The rad-hard link developed by the silicu $\mathrm{b}_{k} \ldots$ 4) group may be utilized by the calorimeter and muon subsystems for their $60 \mathrm{Mbit} / \mathrm{s}$ data links. Many low-cost slow links are available commercially such as the AT\&T model ODL-70 operating at a bandwidth of DC-50 Mbit/s or 10-70 Mbilis with long-life LED, PIN photodiode, and 870-nm multimode fiber. The FDDI optical links operating up to $100 \mathrm{Mbit} / \mathrm{s}$ with LED, PN photodiode and $1300 \mathrm{~nm}$ multimode fiber will be suitable for the GEM data links. Other links for GEM that require a bandwidth of more than $100 \mathrm{Mbit} / \mathrm{s}$ will use the short-distance fast link. The Finisar Corporation offers a low/-cost gigabit link operating from $100 \mathrm{Mbit} / \mathrm{s}$ to $1.5 \mathrm{Gbit} / \mathrm{s}$ at a distance of less than $500 \mathrm{~m}$ of multimode fiber. This optical link consists of a short-wavelength CD-type laser diode transmitter, PIN photodiode receiver and a link controller. The link controller monitors the status and the optical performance of the link. A chip set for multiplexing and demultiplexing is required to interface with the optical transmitter and receiver. It provides a transparent link that accepts parallel data into the transmitter and delivers parallel data from the receiver. The chip set performs all the complexity of encoding, multiplexing, parallel-serial converjion, clock extraction, serial-parallel conversion, demultiplexing, decoding, frame synchronization, and DC balance of the transmission The chip set is a low-cost item and is available from many sources, including Hewlett Packard, TriQuint, AMCC, NCR, and Vitesse.

The US government is contemplating the introduction of a national fiber link highway, while semiconductor vendors and the telecommunication industry are promoting some standards such as the fiber-channel standard and the extended Ethernet LAN. As a result, very-low-cost fiber optic links for $100 \mathrm{Mbit} / \mathrm{s}$ and gigabit bandwidths will be available in the near future. 


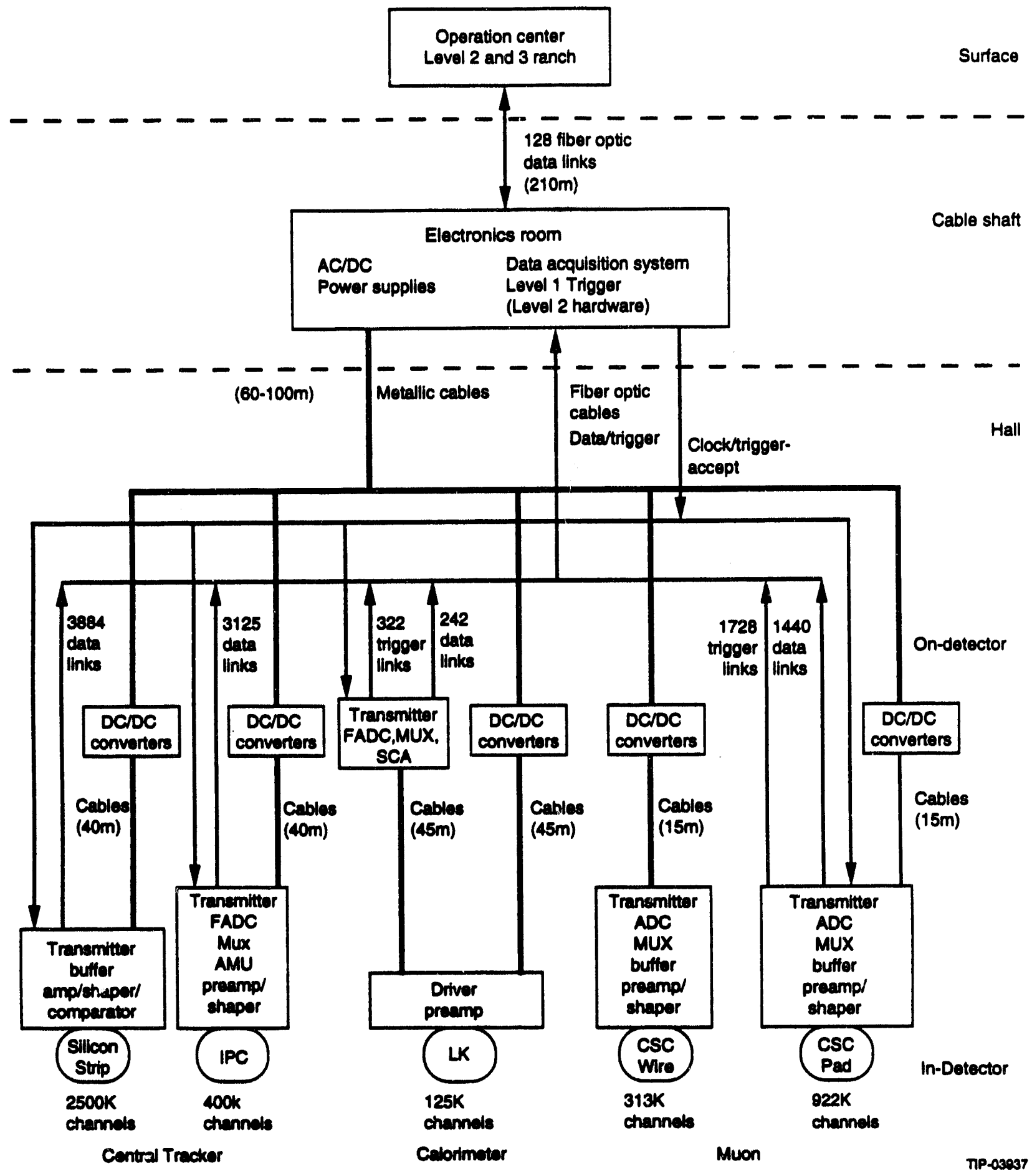

FIG. 7-54. Fiber and cable connections for the GEM electronics system. 
Table 7-27. Optical links and requirements.

\begin{tabular}{lrrrr} 
& Si Strip & IPC & CAL & CSC \\
\hline $\begin{array}{l}\text { No. of channels } \\
\text { (K) }\end{array}$ & 2486 & 400 & 125 & 1235 \\
Channels/Link & 640 & 128 & 496 & 864 \\
No. of data links & 3884 & 3125 & 242 & 1440 \\
$\begin{array}{l}\text { Data link (Mbit/s) } \\
\text { No. of trigger links }\end{array}$ & 60 & 60 & 400 & 60 \\
$\begin{array}{c}\text { T-link bandwidth } \\
\text { (Mbit/8) }\end{array}$ & & & 322 & 1728 \\
\hline
\end{tabular}

\subsubsection{Electronics Rack Design}

The standard electronics rack design, shown in Figure 7-55, is $2.1 \mathrm{~m} \times 0.54 \mathrm{~m} \times 0.95 \mathrm{~m}$ in its outer dimensions, with standard 19-in mounting areas front and rear. The front panel mounting space provides for three large electronics crates housing 400-mm Eurocard format modules, such as VME, VXI, or Fastbus. The front panel also provides a status display panel and convenience outlets for local troubleshooting. In some racks a crate will be dedicated to Global Control system functions.

The power and cooling systems are designed for $9 U \times 400 \mathrm{~mm}$ VME modules. The spaces between crates and the spaces above and below the crates contain fan trays and air-to-water heat exchangers. A closed air-loop transfers heat from modules and power supplies to a mixed chilled water loop via the heat exchangers, thereby reducing the loading on the room HVAC system.

The rear mounting area of the rack houses AC-to-DC power converters, which provide conditioned DC voltages to the crates. Specific load voltages will be chosen to suit the particular equipment requirements. Space constraints combined with high power requirements suggest the use of switching power converters wherever possible. Racks accept three-phase 208/120 wye power through an EMI filter, a circuit breaker, and a disconnect switch. An isolation transformer may
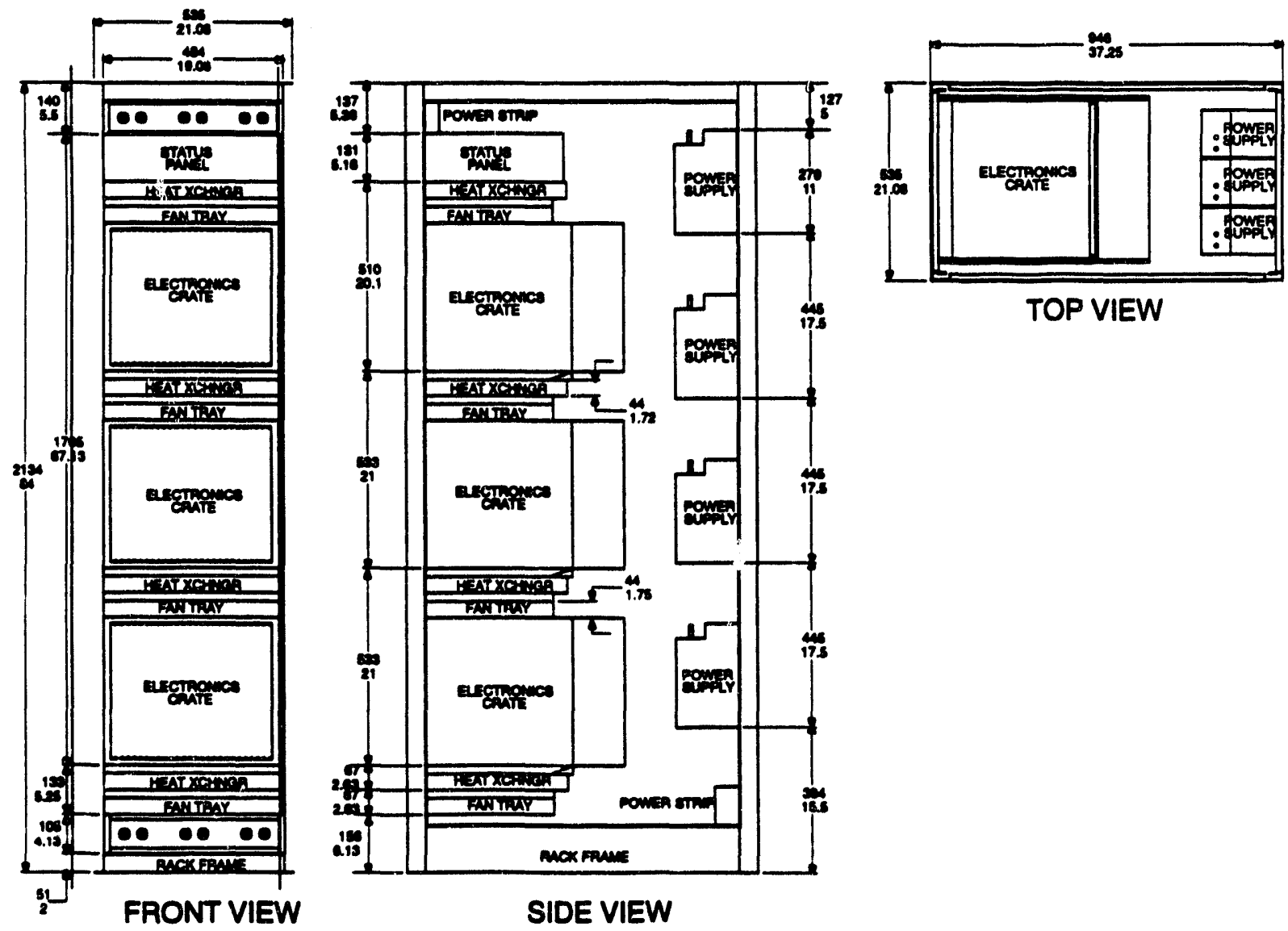

FIG. 7-55. Standard electronics rack design. 
also be used. Power converters will be specified to accept three-phase power. Three-phase power will be supplied in the detector hall from transformers in the basement (Level 0) of the cable electronics shaft, and it will be supplied to electronics room racks from transformers in the electronics rooms. The use of isolation transformers with interwinding shields in these locations reduces the potential for interference between subsystems. Conductors, circuit breakers, and related branch circuit hardware will be sized to suit load operation requirements, meet applicable standards of good safety practice, and provide long-term reliability.

\subsubsection{Electronics Rack Placement and Services}

In the experimental hall, racks will be located on the west wall on extended gangways between the entrances to tunnels 5 and 6 . Gangways on Levels 2 , 3,4 , and 5 will be utilized for racks. In addition, in this area gangway levels carrying racks will be added between Levels 2 and 3 and between Levels 4 and 5, called Levels $2 a$ and $4 a$, respectively. These locations are shown in Figure 7-56. Gangways will extend back to the rear faces of the support columns in rack-mounting locations to a depth of $1.83 \mathrm{~m}$ behind the front face of the support columns. On Levels 2, 2a, and 3 gangways extend $1.75 \mathrm{~m}$, the hall, and racks are mounted to partially exten $\_$into this space. On the other rack-mounting levels, racks are fully mounted on gangways. Gangways on Levels $4,4 \mathrm{a}$, and 5 extend $2.1 \mathrm{~m}$ into the hall space. Up to 252 racks may be placed using this arrangement.

With the exception of the column at $z=0$, the spaces in front of support columns may each contain two shallow racks providing DC power to front-end stages inside the detector. The front of the column at $z=0$ is reserved for alignment use. An area $0.7 \mathrm{~m}$ in width is also reserved on each level and in front of each column for alignment sighting use.

Access to racks is available in front of the racks on the gangway and in back of the racks on the platforms between columns. Personnel access to the racks and egress to safe haven is available by clear areas on gangways in front of the racks. Cabling from the detector will run in a system of cable trays integrated with the gangways. routed in front of the racks. Calorimeter cables will exit the central detector support (CDS) and run to racks on Levels 4 , $4 a$, and 5 in non-magnetic riser trays located in front of the gangways. Tracker cables will be routed from the CDS to racks on the lower levels. Muon system cables, which can be disconnected to allow magnet retraction, will run from the magnets to racks also located on the lower levels and directly to the electronics rooms. The muon system cables may be reconnected with the magnet open to allow testing of the muon chambers while other service activities are proceeding. Inter-rack cable routing is available in technical chases located between the support columns. Power and water services are routed to racks underneath the platform decks. Rack outputs to the electronics rooms are through fiber trays mounted behind the support columns.

The cable electronics shaft provides power to the experimental hall and contains the Level 1 trigger, high-voltage power supplies, low-voltage power supplies, and the data acquisition system. Tunnels 5 and 6 convey power, signal, and control connections between the experimental hall and the cable electronics shaft. On the bottom level of the cable electronics shaft, an electrical service room contains a bank of power isolation transformers and panel boards for transmitting $A C$ power to the racks on the west wall of the experimental hall. Additional panel boards may be located at the gangways. Racks are powered from subsystem power isolation transformers, eliminating ground loops as a potential noise-coupling path. The power systems serving the experimental hall are isolated from those serving the electronics rooms by the use of power isolation transformers. They are commonly fed from the service entrance transformer at the surface.

The electronics rooms are located on Levels 1 through 3 of the cable electronics shaft. Including utility siting and accesses, each electronics room has a clear space $12.7 \times 8 \mathrm{~m}^{2}$ for rack installation. Each electronics room provides space, power, and cooling for up to 41 electronics racks, shown in Figure 7-57. A raised floor allows conditioned air, cooling water, and $\mathrm{AC}$ power to be distributed to racks without obstructing human access or signal-cable routing. A single large cooling unit in each room (Liebert or similar) maintains the climate at $72^{\circ} \mathrm{F}$ and $45 \%$ relative humidity. Cable trays above the racks will deliver signal cables and fiber from the experimental 


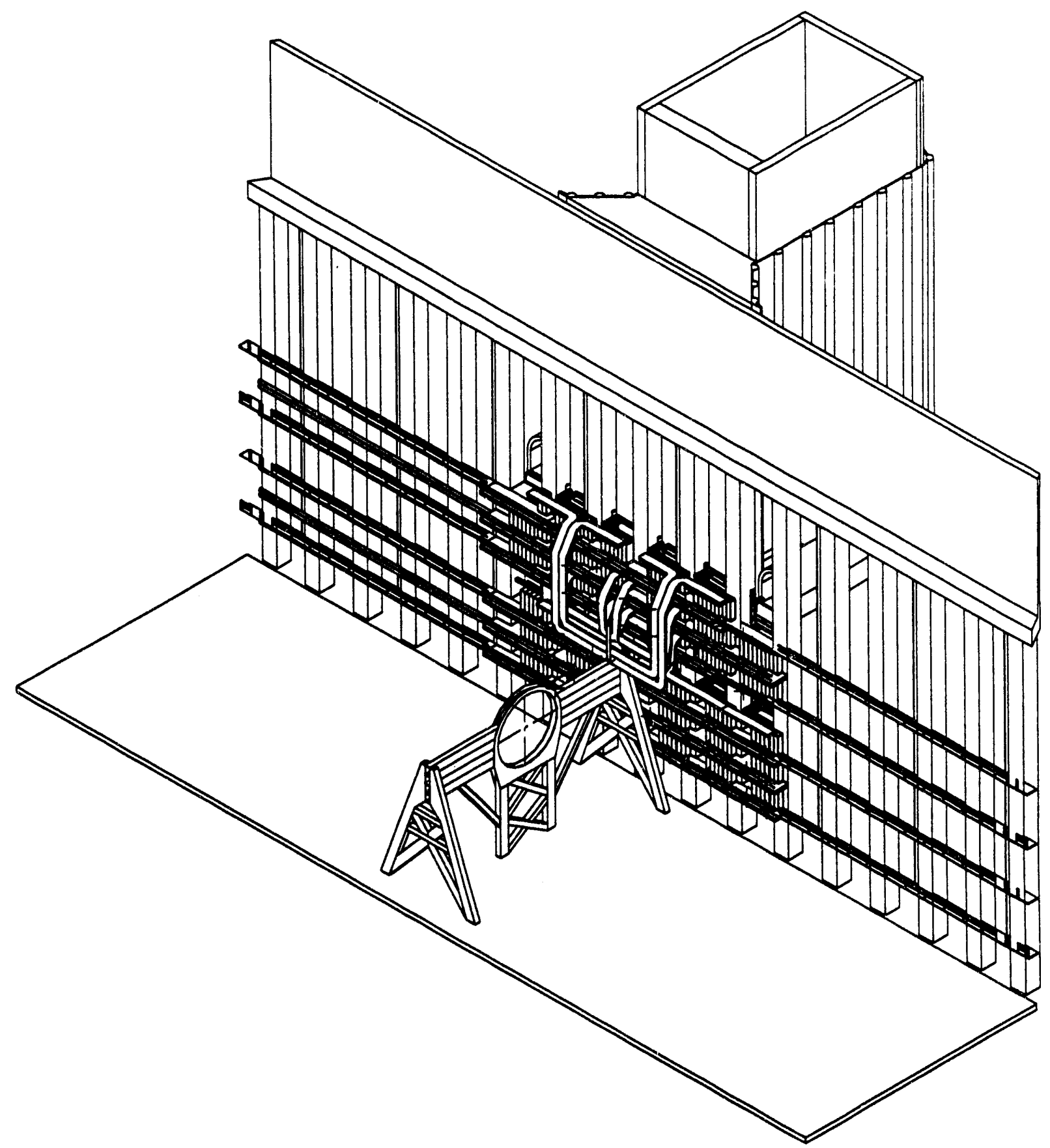

FIG. 7-56. Electronics rack placement on West wall of experimental hall.

hall, and carry signal and control fibers to the operations center.

Each electronics room is electrically isclated from the others by use of power isolation transformers and separate ground planes on each floor. All racks are referenced to these ground planes, which are in turn individually grounded to the safety ground system. Power transformers incorporate at least one interwinding shield and may be double shielded. Each power isolation transformer is individually fed from the service entrance transformer at the surface. The power transformers, panelboards, and cooling units are located in the electrical equipment area of each room. Level 3 will initially provide a local workshop and staging area, but will 


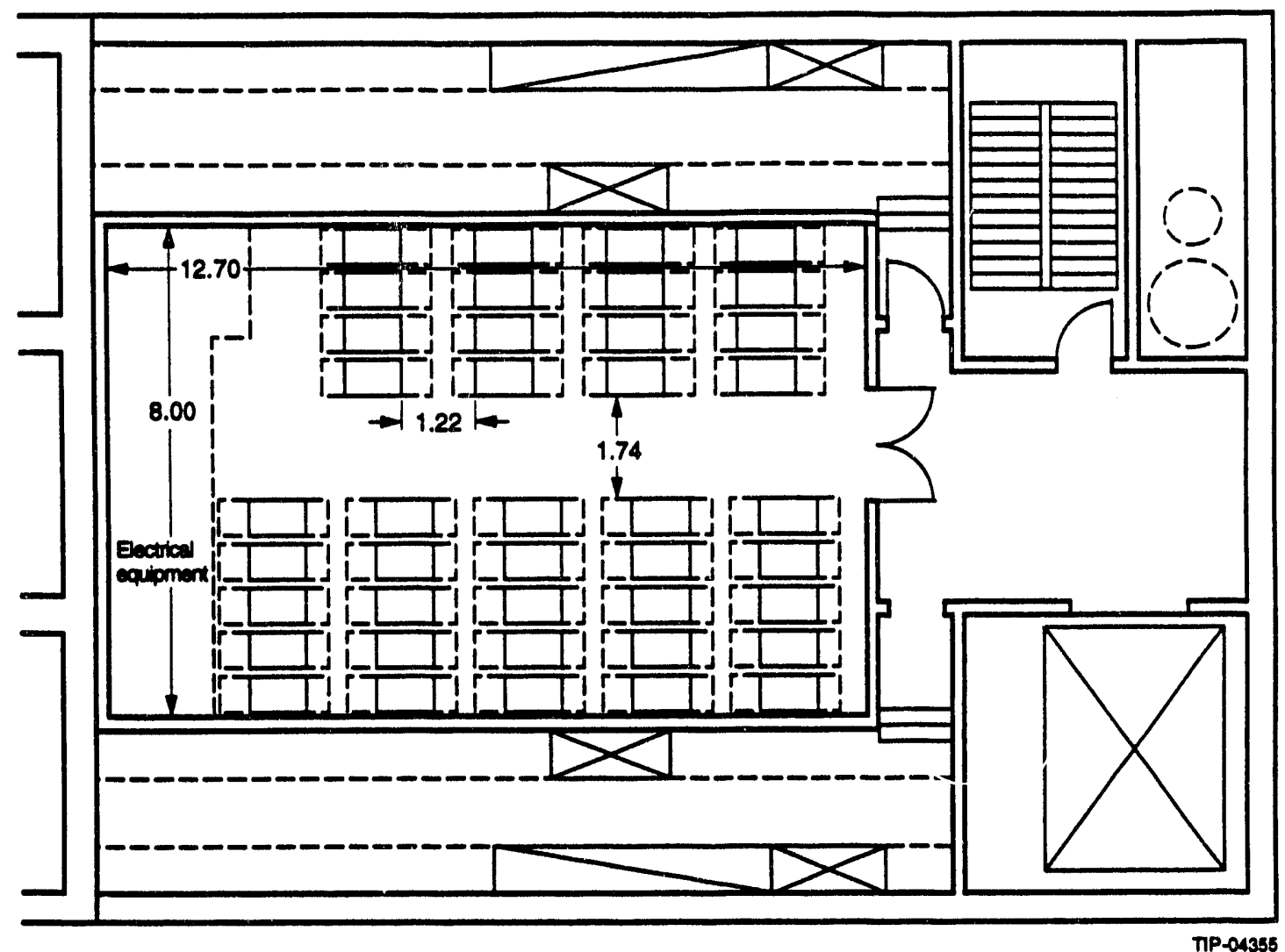

FIG. 7-57. Electronics room plan view - rack placement.

be available to house electronics from future detector upgrades.

\subsubsection{Magnetic Field Mitigation}

The GEM design uses an open solenoid magnet for charged particle measurement, resulting in a large fringe field. A two-dimensional plot, resulting from a finite element calculation, of the flux density at locations outside of the magnet is shown in Figure 3-4 contour map of constant flux density. Based on this calculation, the GEM electronics are designed to function in the following magnetic environments:

- cable electronics shaft - $50 \mathrm{G}$

- on-detector electronics (west wall) - $1400 \mathrm{G}$

- in-detector electronics $-8000 \mathrm{G}$

In the cable electronics shaft, the $50 \mathrm{G}$ level is low enough for any electronic equipment to function acceptably, with the exception of high-resolution cathode ray tubes. The limited number of such devices can be equipped with supplemental magnetic shielding as needed.
On-detector equipment located on the west wall of the underground hall consists of rackmounted electronic modules and low-noise power supplies. Unlike power supplies, small-signal and digital electronics are essentially unaffected by magnetic fields, provided ferrites are avoided. Common power supplies can be made usable by adding soft-iron transformer shielding, replacing the included transformers with larger ones, and proper orientation. Soft-iron shielding using spherical geometry has been investigated. As Figure 7-58 shows, a soft-iron shield with a thickness to diameter $(t / D)$ ratio of 0.25 placed in an external flux density of $4 \mathrm{kG}$ results in a cavity flux density of around $70 \mathrm{G}$, while in an ambient 1.5-kG field, a $2 \mathrm{G}$ internal field results. The sharp rise in cavity flux density reflects the onset of shield-wall saturation. Results of the same order of magnitude can be expected from rectangular shields if comers are avoided.

Linear power supplies can be made to operate in high magnetic fields when equipped with oversized transformers, especially if they are judiciously 
oriented. This approach is more economical in terms of iron-usage, but necessitates custom, or at least semi-custom, transformer and LVPS designs. Commercial $60-\mathrm{Hz}$ transformers were tested in the fringe field of a decommissioned cyclotron magnet. The primary current increases with external flux density, shown in Figure 7-59. At 115-V drive, the RMS primary current increases by a factor of four if the extemal field increases from $50 \mathrm{G}$ to $2000 \mathrm{G}$. An increase in primary current results whether or not the transfomer is loaded and comes about due to core saturation. There is also a noticeable distortion of the current waveform. At 30-V drive, the same increase in external field causes a much smaller rise. (The relevant parameter is the increase in current over its zero-field value.) Reducing the drive to $30 \mathrm{~V}$ is equivalent to increasing the core area by a factor of four.

Zero current switching (ZCS) power supplies resist external magnetic fields better than conventional types, due to the very high internal frequencies employed and the resulting low internal magnetic flux densities. Discussions with vendors indicate that ZCS supplies will operate unshielded in fields of order $1 \mathrm{kG}$. They are also very compact, making magnetic shielding an attractive option.

Inside the detector the magnetic flux density is $0.8 \mathrm{~T}$. Considerations of space and radiation length appear to preclude placing power supplies in that region. Power supplies operating front-end circuits are on the west wall and are protected from fringe

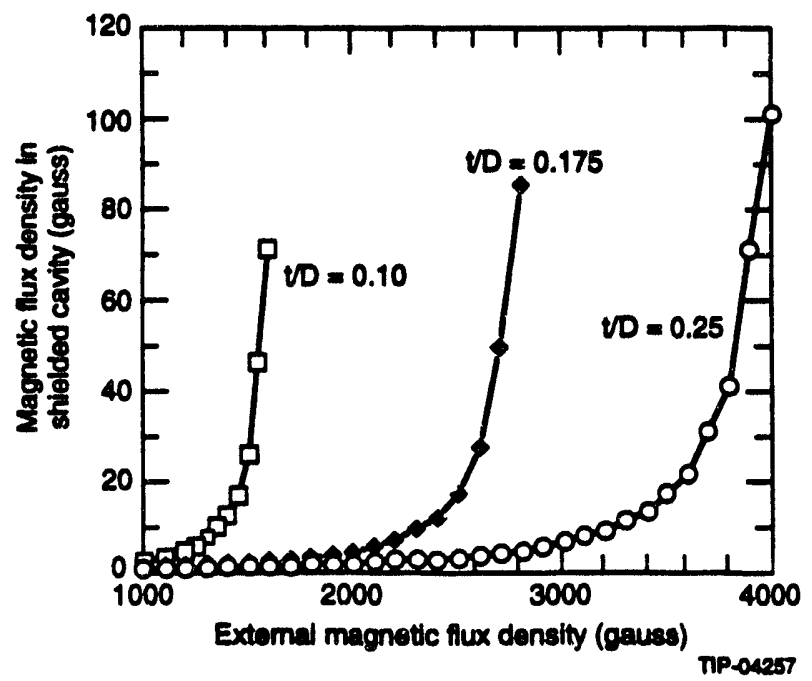

FIG. 7-58. Effect of spherical magnetic shield.

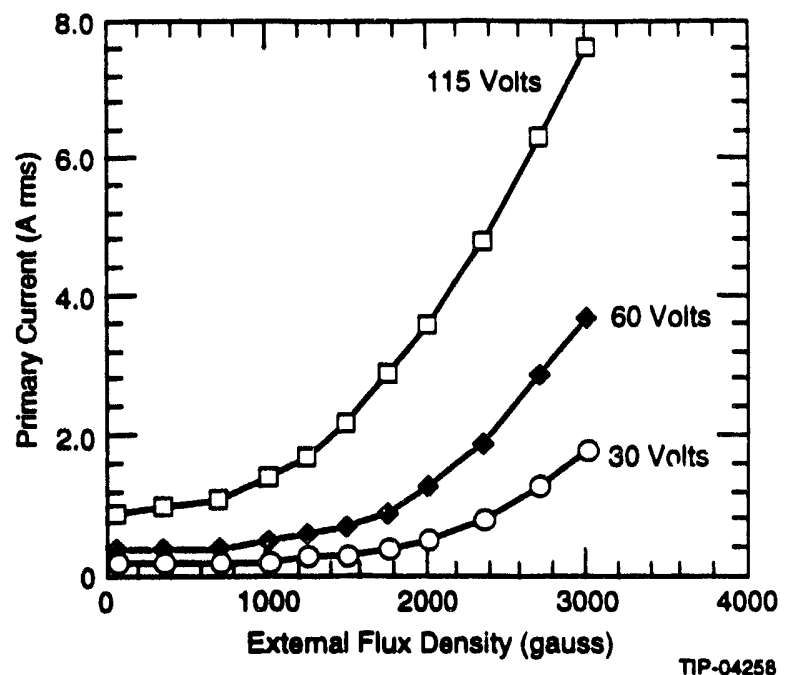

FIG. 7-59. Effect of magnetic field on transiormer primary current.

field effects using soft-iron shielding, orientation, and/or oversized transformers.

\subsection{ELECTRONICS GROUP ORGANIZATION}

The Electronics and DAQ group is organized in subgroups corresponding to the various subsystems. A Steering Committee has been established to oversee the design, R\&D, and production phases of the project. This group is composed of the physicistin-charge of each subsystem along with the corresponding engineer. The current list of members is given below:

Group Leaders:

D. Marlow, M. Shaevitz

Silicon Tracker:

G. Mills, S. Hahn

Central Tracker:

J. Musser, C. Britton, P. O'Conner

Calorimeter:

J. Parsons, S. Rescia, W. Sippach

Muons:

D. Marlow, V. Polychronakos, P. O'Conner, R. Wixted

Trigger:

M. Atiya, W. Cleland 
DAQ / Level 2:

J. Branson, M. Bowden

Integration:

K. Freeman, N. Lau

\subsection{R\&D PROGRAM}

\subsubsection{Trigger System and Simulations}

The research and develoment program for the trigger involves work in four areas (1) continuation of the simulation program to refine the trigger needs for physics processes not yet studied and to understand in better detail the backgrounds accepted by the Level 1 trigger; (2) design and prototyping of circuits; (3) tests of certain elements of the trigger logic in currently running experiments or in beam tests; and (4) continuation of the design of the final system. Each of these areas are briefly discussed.

The calorimetry simulation program will have three aspects: (1) development of a more detailed understanding of the effects of shower fluctuations on the Level 1 trigger and how events passing the Level 1 trigger as a result of such fluctuations can be rejected at Level 2 . This will be done by generating full showers for a small portion of the calorimeter in the region of a high-energy photon or electron. (2) the trigger requirements for the endcap calorimeters need additional study, which will begin with a definition of the geometry of the trigger towers and then proceed to an optimization. This will clearly need to be coordinated with the members of the calorimeter group working on the design of this part of the calorimeter. (3) A study of triggers for processes other than the ones presented here needs to be started (for example, triggers with tau particles in the final state need to be developed), and optimization of the triggers currently considered needs to be continued. This simulation program is underway, and will likely continue throughout the life of the experiment.

Electronics design projects for the calorimeter trigger include a development of a multilevel discriminator of the type tested in the liquid $\mathrm{Ar} / \mathrm{Kr}$ test run at BNL in summer, 1992; a 60-MHz multiple transversal filter with the ability to increment a number of sums simultaneously, as is needed for the JET sum logic; and continuation of development of the FPGA circuitry, which will be tested in a relativistic heavy ion experiment at BNL in 1993-94. As the design of the sys em progresses, we are planning to begin a simulation of the system, using Viewlogic, with part of the units represented as schematic circuits and other parts represented as VHDL code. This will be a continuing activity, probably starting in 1994.

We plan to test an FPGA-based CAMAC module we have built as part of the trigger for E877 at the Brookhaven Alternating Gradient Synchrotron. It is expected that the experience obtained in using this type of logic in a real-time environment will be invaluable for GEM. In addition, we plan to participate in the GEM test beam program at Fermilab in MWEST beam, testing prototype units of the L1SUM logic and the transversal filter circuit. We anticipate that this will occur toward the end of 1995, but this depends heavily on the Fermilab schedule.

\subsubsection{DAQ System Studies and Development}

The data acquisition system is designed to make use of commercially available components to the greatest extent possible. This minimizes the need for a significant research and development program i i this area. However, certain cost and performance aspects of the design are based on anticipated, rather than currently existing, commercial products and standards. These components will be evaluated as they become available. Research and development work on the data acquisition system is therefore concentrated in two areas: (1) extensive simulation of the overall architecture, and (2) the detailed evaluation of new components and standards, that would simplify the design or improve the performance of individual subsystems.

These activities will be ongoing during the system design phase and are not broken out as separate research and development projects. The system-level simulation work is in progress and should be completed by the end of 1993. Evaluation of components and standards will rely mainly on information from the supporting vendors but may also involve limited testing. These evaluations will continue through the end of the prototype design phase in 1995. 


\subsubsection{Silicon Tracker Electronics R\&D}

The silicon tracker electronics $R \& D$ program focuses on the front-end electronics, as they are the most important for detector installation. The effort is concentrated upon the bipolar analog chip, the CMOS data driven buffer and controller chips, the LEL optical data link, the multi-chip module (MCM), and the power and slow control cabling. Under the assumption of a 1998 installation of the silicon tracker, the goal of the R\&D effort is to produce a working production prototype by late 1994 or early 1995.

The bipolar design has been implemented in the AT\&T CBIC-V series tile array format, and is presently undergoing evaluation. It is expected that a full custom design will be submitted in late 1993 or early 1994.

The CMOS-1 data driven buffer architecture has been developed and a VHDL implementation is underway. Prototype versions of this are expected in mid-1994. The CMOS-2 controller chip development closely follows the CMOS-1 development.

A preliminary layout of the MCM in the TI/GE HDI (High Density Interconnect) process has been made. This process is well suited to the fine pitch of the inter-chip connections. It is necessary to reduce the radiation length of the substrate material as much as possible. This will be investigated in order to find the optimum substrate material and minimum thickness allowable by mechanical and assembly constraints.

The cabling must also present as little material as possible. Beryllium and/or aluminum conductor kapton laminates are currently under investigation $\approx$ possible solutions.

\subsubsection{IPC Tracker Electronics R\&D}

The GEM IPC electronics research, development, and construction schedule, shown in Figure 7-60, shows the major research, development, prototyping, fabrication, and assembly milestones of this effort. The early critical-path items are the development of the rad-hard preamp and SCA custom ICs.

\subsubsection{Calorimeter Electronics R\&D}

The research and development of the electronics for the calorimeter is focused on retaining the exceptionally high resolution and large energy range of which the calorimeter is capable while allowing for very high luminosity. These requirements have

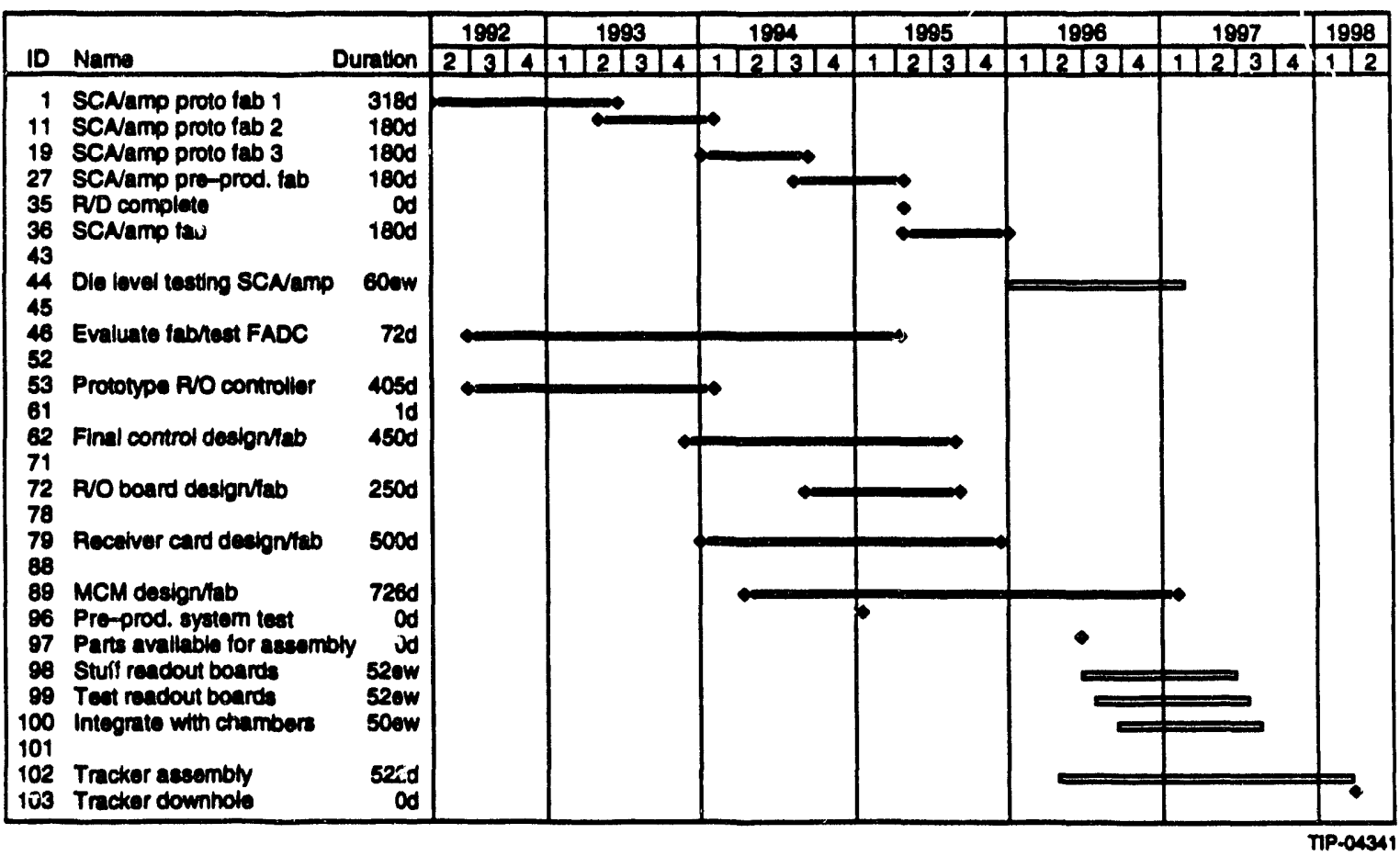

FIG. 7-60. IPC electronics research and development timeline. 
resulted in a vigorous research and development effort to develop analog circuits with extremely high dynamic range and precision. The expected Level 1 trigger rates necessitate considerable digital logic to efficiently reduce the data size to a manageable level before sending data to the data acquisition system. Research efforts into commercially available methods of achieving this data manipulation using DSPs, FPGAs and gates arrays are underway.

The custom design of a preamp, calibration circuit, cable driver, shaper, and switched-capacitor analog memory are needed to meet the strict analog design requirements. The large number of calorimeter channels allows the production cost of each of these elements to be near the cost of similar, although insufficient, commercial components. The preamp and calibration circuit must be designed to operate in an extreme radiation environment. While radiation is still a concem for the shaper and analog memory, they are located about $50 \mathrm{~m}$ away from the cryostat, and the design of these devices can be implemented in non-radiation-hardened processes.

The large dynamic range of the calorimeter requires a small noise contribution from all components along the signal path. This will allow a good precision measurement for small signal levels, as dictated by the necessarily small signal-level to energy ratio. The fine granularity of the calorimeter leads to the summation of large numbers of channels in order to extract physics. This imposes serious constraints on the amount of systematic noise that can be tolerated in the readout system.

We are currently testing prototype versions of the preamp, calibration circuit, shaper and analog memory. The noise requirements of the analog memory require many interations before a satisfactory design will be reached. The switched-capacitor array is currently being prototyped in an inexpensive multi-user process. Good results have already been obtained concerning DC and AC tests with a prototype chip that is about one quarter the final depth and has one half the number of channels. Full calibration of these chips is now commencing. The next generation chip will allow us to accurately estimate the noise characteristics of the final chip. As in analog memory will be stretching the carabilities of currently available technologies, we are also pursuing a digital storage approach using fast digitization followed by a digital buffer. This approach is more straightforward but suffers significantly in both cost and power.

The preamp will be located inside of the cryostat and is therefore, a high-priority item due to its early installation. We have fully tested the prototype preamp in a severe radiation environment with excellent results. Prototype design is also complete for the calibration and cable drivers. The shaper design has been prototyped and is currently being used in testing of the analog memory.

Prototype versions of all components as well as full readout boards and calibration circuitry will be finished for use in the Fermilab fixed-target run of 1995 , where they will be thoroughly tested.

\subsubsection{Muon System Electronics R\&D}

Initial versions of most analog and mixed signal circuit blocks of the various integrated circuits have been designed and submitted for fabricat .Jn (and in some cases tested). It is expected that much of the initial effort will concentrate on further testing and optimizing these blocks. Although most elements of the CSC readout electronics are within the current state of the art, a number of questions remain to be settled through $R \& D$. These include:

- Noise reduction in the preamplifier shaper IC. It should be possible to reduce the noise by employing improved processes and/or through improved circuit design.

- Systematic study of the relative merits of the T/H approach to sampling versus the SCA approach.

- Timing discriminator development. The existing timing measurement will be extended to include circuits suitable for fabrication in monolithics. The impact of ganging several wires together to reduce anode channel count will also be studied further.

- Packaging options. Detailed tradeoff studies are needed to ascertain the optimal level of integration (with respect to both channel count and function) for the IC's. In particular, limits imposed by power dissipation and fabrication yield must be traded against the reduced package count that results from higher levels of integration. At the board level, the economics 
and benefits of alternate packaging options such as multi-chip modules and chip-on-board will be studied. Techniques that provide higher density at the same, or possibly even slightly higher, costs may prove advantageous if they simplify the general chamber layout.

- System level tests. Realistic tests with onchamber electronics are of obvious importance. To allow such tests to proceed at the earliest possible date, an evolutionary approach will be adopted. Specifically, a series of tests, starting with existing components (e.g., commercial hybrids and existing $\mathrm{T} / \mathrm{H}$ circuits) and progressing in steps to the fully integrated production electronics, will be conducted. This strategy will allow us to take advantage of lessons learned through practical experience before finalizing the design. 


\section{REFERENCES}

1. O. Benary, et al.,"Precision Timing with Liquid Ionization Calorimeters," BNL-48159, submitted to Nuclear Instruments and Methods, November 1992.

2. W. E. Cleland and E. G. Stern, "Signal Processing for Liquid Ionization Calorimeters" III International Conference on Calorimetry, Corpus Christi, Sep 29-Oct 2, 1992.

3. D. Crosetto and L. Love, "Fully Pipelined and Programmable Level-1 Trigger," SSCL-576, July 1392.

4. D. Crosetto. "General Programmable Level-1 Trigger with 3D-Flow Assembly System for Calorimeter of Different Sizes and Event Rates," SSCL-607, Dec., 1992.

5. K. McFarlane, H.Uijterwaal, GEM-TN-93-294.

6. K. McFarlane, H.Uijterwaal, GEM-TN-93-332.

7. H. Uijterwaal; Libtrf, trigger simulation for Gemfast, v1.03.; T. Skwarnicki; Gemfast, fast simulation of the GEM detector, version vII2.02.

8. B. Cleland; Results presented at the February trigger and DAQ meeting at SSCL.

9. H. Uijterwaal and J. Dorenbosch, in GEM TN-93-259.

10. "The GEM Central Tracker: Design Issues" GEM TN-92-54; "The GEM Central Tracker GEM TN-92-63"; "SDC Technical Design Report SSCL-SR-1215."

11. S. Hahn et al., IEEE 1992 Symposium on Instrumentation in Nuclear Physics, Bipolar Amplifier and Comparator Circuit for the SSC, to be published.

12. V. Cook, P. M. Mockett, "A Precision Vertex Chamber For EMPACT," Symposium on Detector R\&D for the SSC, Fort Worth, Texas, Oct 15-18, pp. 136-138.

13. O. Barkan, E. L. Atlas, W. L. Marking, S. Worley, G. Y. Yacoub, G. Kramer, J. F. Arens, J. G. Jernigan, S. L. Shapiro, D. Nygren, H. Spieler, M. Wright, "Development of a Customized SSC Pixel Detector Readout for Vertex Tracking," Symposium on Detector R\&D for the SSC, Fort Worth, Texas, Oct. 15-18, 1990, pp. 142-144.

14. R\&D Report on Double-Sided Silicon Detectors and Associated Electronics, Sept. 1990, 62-78.

15. W. E. Cleland and E. G. Stern, "Signal Processing for Liquid Ionization Calorimeters," to be submitted to Nucl. Instr. and Meth.

16. A. Caldwell, et al., Nucl. Instr. and Meth. A321 (1992) 356.

17. S. Kleinfelder, M. Levi, O. Milgrome, Nucl. Phys. B (Proc. Suppl.) 23A (1991) 382.

18. A. Gara, J. A. Parsons, W. Sippach. "Keadout Electronics for the GEM Calorimeter," Proc. of the International Conference on Electronics for Future Colliders (1992).

19. "AMPLEX, a Low Noise, Low-Power Analog CMOS Signal Processor for Multi-Element Silicon Particle Detectors," E. Beauville, et al., Nucl. Instr. and Meth. A288 (1990).

20. "A Precision Timing Discriminator for High Density Detector Systems," Turko, B. T. and Smith, D. C., IEEE Trans. Nucl. Sci., NS39, (1992).

21. "Design of the Constant Fraction of Pulse Height Trigger for Optimum Time Resolution," Gedcke, D. A. and McDonald, W. J., Nucl. Inst. and Meth., 58, (1968). 253.

22. "Front End Electronics Development for SSC Detectors," C. Blocker, et al., SSC Subsystem R\&D Progress Report and Renewal Request, (1991). 


\section{COMPUTING, CONTROLS, NETWORKING}

\subsection{OVERVIEW}

The responsibility of the GEM Computing Subsystem is to ensure that computing systems are available to fulfill the overall goals of GEM. The scope of this chapter includes event filters, control systems, monitoring systems, reconstruction, simulation, calibration, database and analysis systems, mass storage, and communication systems for machines and people. The boundary between data acquisition and event filters depends on the specific implementation chosen for event building. The off-line systems will be built in cooperation with the SSCL Physics Research Computing Department (PRCD).

The high data rate (beginning with a bunch crossing rate of $60 \mathrm{MHz}$ ) can only be accommodated in permanent storage by using extensive real-time or on-line filters, so that the risk of a bias in filter algorithms must be balanced against the cost of storage systems. The high duty cycle that is planned ( $80 \%$ of the year) ${ }^{1}$ means that all computing tasks must be supported essentially on-line and full-time. The variability of data rates and data volumes according to changes in triggers and luminosities means that systems should be flexible. The very large volume of stored data and the many activities that will operate on that data prescribe that all storage management be automatic and transparent. Finally, the systems must be structured in such a way as to make validation easy. In this context, validation is the process of testing and modifying the systems until the results are reliable.

There appear to be no technical obstacles to achieving the desired goals. However, careful planning and timely effort will be needed on hardware and software to exploit the full potential of GEM in the most cost-effective way. Two areas are of particular concern: first, the technology to store and access very large amounts of data at high rates, especially for analysis; and second, the ratios of $V O$ capability and memory bandwidth to CPU power for volume-produced systems, that may affect imple- mentation of Level $2 / 3$ systems and analysis systems. These two concerns may result in a shift of approach, as re-computing becomes less expensive than storage. The data storage hardware and the scale of its capabilities within a reasonable cost will determine filtering and analysis strategies, and the approach to data storage (file-based or database) will have fundamental effects on most GEM software.

The rapid pace of change in computing technology (both in hardware and software) means that a very open and flexible approach must be followed if we are to take advantage of rew developments. The distributed nature of highenergy physics collaborations, along with the fact that much development will be done by physicists rather than by computing professionals, means that system development methodologies used in the computing industry must be greatly modified for use in GEM. Finally, the large investment needed to build the computing systems requires that careful analysis, as well as extensive prototyping, be done to guarantee reliable performance within cost and schedule.

To optimize the choices made for GEM-for example, in the balance between on-line and off-line processing or between storage and computation-it is important to analyze the entire system and the overall requirements. Analysis of the GEM computing system is under way; it depends on constri stion of detailed data models and data flow models, which in turn depend on simulations (both physics simulations to establish data volumes and simulations of computing systems) that are themselves in progress. The feasibility of some hardware implementations have already been demonstrated through simulations.

The GEM approach to computing can be defined as one of unification: where possible, to use a single logical processing and database system, and to create software tools and re-usable modules that form a single framework. The data and processing power will be located centrally at the SSCL, with 
access for analysis over networks. The hardware implementation will consist of "ranches" of off-theshelf processors linked by high-speed networks and data links, with a single mass storage system and no mass storage at the detector. As well as leading to economies, this allows us a great deal of flexibility in allocating resources, and gives redundancy and the ability to upgrade easily as technology and needs change. Reconstruction will be viewed as an on-line task, to be done as the data comes from the detector, and before storage of the data. The processes which transform the data will all use the same databases for apparatus parameters such as calibrations. Software will be modular, communicating by passing defined data structures between steps, and usable in every appropriate context.

The remainde- of this chapter is divided into sections that address the following issues:

- Requirements imposed by the physics goals

- Functional requirements on the system

- A technology-independent system model

- A hardware implementation model

- A software implementation model

- Approach to system development

- Development projects

- Management

- Schedule.

\subsection{PHYSICS REQUIREMENTS}

This section describes the overall datahandling and processing requirements imposed by the physics goals of GEM. The GEM physics program has been outlined in Chapter 2, where the trigger primitives used for the Level 1 trigger are listed. As described in Chapter 7, the Level 1 maximum trigger rate is $100 \mathrm{kHz}$, and this, together with the anticipated event size of $0.3 \mathrm{MB}$, sets the scale of the computing facilities needed.

In finding a balance between recording every event and having an overly selective Level 1 trigger, the optimal course is to choose a set of triggers (and filters) that covers a sufficiently wide set of possible new processes and also gives an unbiased sample of standard model (SM) processes, at a rate that can be stored and reprocessed at reasonable cost. Hardware costs are hard to predict, but a reasonable estimate for 1999 is that a storage system capable of storing $100 \mathrm{MB} / \mathrm{s}$ for one year of SSC operation and of reading it back for further processing will cost about $\$ 10 \mathrm{M}$, with a similar cost for the computational power to reconstruct the raw data five times per year. Thus, although there is little incentive to reduce the rates below the $100 \mathrm{MB} / \mathrm{s}$ level, it is clear that a substantial increase above this level would be problematic. As noted above, detailed optimization has yet to be done.

New processes, triggered on complex signatures, are typically at the picobarn level, while SM processes (such as inclusive jet cross sections) must be prescaled at low $p_{T}$, where the rates are high, but will fall to the nanobarn level at $p_{T}<200 \mathrm{GeV}$. To establish low-pT normalizations, statistics of $1 \% / \mathrm{GeV}$ or better will be adequate, so a few nb for each SM process is a reasonable goal (1 nb corresponds to $10^{7}$ events in $10^{7}$ seconds at full luminosity). Table 8-1 lists some processes that might make up the recorded events (at full luminosity). The cross sections are for the physics processes within the acceptance of the GEM detector; the data samples needed to study the processes will be larger, but it is assumed that the chain of filters will restrict sample size so that the ability to record and process data equivalent to a cross section of $100 \mathrm{nb}$ will more than satisfy GEM requirements. At design luminosity, this means $100 \mathrm{~Hz}$ of events is to be recorded and made available for further physics analysis. At lower luminosity, the same event rate can be used to study $W$ and $Z$ production, high- $p_{T}$ jets, jet fragmentation functions, heavy quark production and possibly detect gluinos. At a luminosity of $10^{34} \mathrm{~cm}^{-2} \mathrm{~s}^{-1}$, thresholds would be raised to keep the rate about the same. 
Table 8-1. Examples of physics processes (or possible processes) to be recorded in the event store. The cross sections are for the GEM acceptance and typical cuts. The corresponding data samples will be larger.

\begin{tabular}{lc}
\hline Process & $\begin{array}{c}\text { Cross } \\
\text { section }\end{array}$ \\
\hline Single $Z \rightarrow \ell^{+} \ell^{-}$ & $2 \mathrm{nb}$ \\
Single $W \rightarrow \ell v(10 \mathrm{nb})$, prescaled & $1 \mathrm{nb}$ \\
if with leptonic decays & $10 \mathrm{nb}$ \\
$E_{T}$ & $1 \mathrm{nb}$ \\
$\begin{array}{l}\text { Inclusive jets, prescaled below } \\
\text { PT }=200 \mathrm{GeV}\end{array}$ & $1 \mathrm{nb}$ \\
$\begin{array}{l}\text { SUSY processes } \\
\text { New processes }\end{array}$ & $8 \mathrm{nb}$ \\
\hline
\end{tabular}

A model, including time evolution, for luminosities, detector data rates, event filter performance, rate to permanent storage, and equivalent cross section of stored data is shown in Table 8-2. (Cosmic ray tests are not addressed in the table.) The luminosities and operating time correspond to the "SSCL Laboratory Operations Program Plan."' The 1999 run is an engineering/commissioning run during SSC commissioning; if useful collision time is not available in 1999, this would refer to the first month of the year 2000. As luminosity is increased, the triggers would be progressively tightened (in the case of Level 1, to keep at or below the maximum rate), and some triggers (e.g., single Ws) would be prescaled, possibly at Level 1 . High cross section physics studies appropriate to lower luminosities will be done. Thresholds would also be raised as more is learned about the detector, so that events would decrease in size. The result is that the rate to permanent storage is approximately constant, as is the computational power needed to reconstruct the events. This model is very approximate and is clearly subject to large variations; for example, a rapid increase in the Level $2 / 3$ filter factor would reduce off-line requirements in the initial years.

In this table, the peak luminosity quoted is the luminosity (averaged over the lifetime of a store) reached at the end of the year. This is assumed to rise exponentially from the value of the previous period. The live time is the time for which collisions are available multiplied by the detector efficiency. The peak rates in the table are based on the end-of-year luminosity, while the amount of data stored is based on the integrated luminosity over the year. The Level 1 trigger level is the effective cross section for collisions that satisfy Level 1, and the Level 1 trigger rate is the maximum expected rate at the peak luminosity; efforts will be made to reduce this rate. As understanding of the data grows, the DAQ event size is assumed to shrink from an initial mean value of $1 \mathrm{MB}$ to the eventual mean value of $0.3 \mathrm{MB}$ noted in Table 7-11. There will be special runs in which a readout is done without zero suppression, using a prescaled trigger at about $1-10 \mathrm{~Hz}$, and giving about $10 \mathrm{MB}$ per event for the same final data rate.

The data rate out of the DAQ system is the rate to be handled by succeeding stages. The event filter factor is the overall reduction factor required to go from the maximum Level 1 rate to the design rate for events available to physics analysis, possibly in conjunction with a rejection factor from the first full reconstruction (RECO) that we expect to do on-line (before storage). The event rate stored is the rate of events into permanent storage, and the equivalent cross section is the cross section to which this is equivalent at the peak luminosity.

As each event is processed, data is added to it by the filters and reconstruction (Table 8-3); the initial data per event is the data written during data-taking. In 1999 (the engineering run) no data is added; the events will be reprocessed outside the data-taking time. To keep track of filter performance, a prescaled fraction of all events (whether or not rejected by a filter) will result in a record being written to permanent storage containing at least the header and the filter information. This might generate a rate of a few $\mathrm{MB} / \mathrm{s}$ for a $100-\mathrm{kHz}$ Level 1 trigger rate. There will be other beam-associated data streams from the detector: scaler data (i.e., counts of rates in specific channels), summary data from Level 2 and Level 3 filters, and luminosity data. The format and quantity of these have yet to be determined, but they are expected to require much less than $1 \mathrm{MB} / \mathrm{s}$. 
Table 8-2. Overall model for expected rates; system capabilities will exceed these rates. Rates are given for the end of the operating year, while integrated quantities (integrated luminosity, total storage) are calculated assuming an exponential increase in luminosity each year until a constant value is reached in 2003 . For explanations, see text.

\begin{tabular}{|c|c|c|c|c|c|}
\hline Parameter/Year & 1999 & 2000 & 2001 & 2002 & 2003 \\
\hline Peak luminosity $\left(\mathrm{cm}^{-2} \mathrm{~s}^{-1}\right)$ & $10^{30}$ & $10^{31}$ & $10^{32}$ & $10^{33}$ & $10^{33}$ \\
\hline Total live time (s) & $10^{6}$ & $10^{\prime}$ & $10^{7}$ & $10^{7}$ & $10^{7}$ \\
\hline Integrated luminosity $\left(\mathrm{pb}^{-1}\right)$ & 1 & 40 & 400 & 4,000 & 10,000 \\
\hline Lovel 1 trigger level & $100 \mu b$ & $100 \mu b$ & $100 \mu b$ & $100 \mu b$ & $100 \mu b$ \\
\hline Level 1 trigger rate (maximum) & $100 \mathrm{~Hz}$ & $1 \mathrm{kHz}$ & $10 \mathrm{kHz}$ & $100 \mathrm{kHz}$ & $100 \mathrm{kHz}$ \\
\hline DAQ event size & $1 \mathrm{MB}$ & $0.6 \mathrm{MB}$ & $0.3 \mathrm{MB}$ & $0.3 \mathrm{MB}$ & $0.3 \mathrm{MB}$ \\
\hline Peak data rate out of DAQ & $100 \mathrm{MB} / \mathrm{s}$ & $600 \mathrm{MB} / \mathrm{s}$ & $3 \mathrm{~GB} / \mathrm{s}$ & $30 \mathrm{~GB} / \mathrm{s}$ & $30 \mathrm{~GB} / \mathrm{s}$ \\
\hline Event filter (Level 2/3) factor & 1 & 10 & 100 & 500 & 500 \\
\hline $\begin{array}{l}\text { Level } 2 \text { computational load } \\
\text { (SSCUP)(a) }\end{array}$ & $10^{3}$ & $10^{3}$ & $10^{4}$ & $10^{5}$ & $10^{5}$ \\
\hline Lovel 3 computational load (SSCUP) & 0 & $10^{4}$ & $3 \times 10^{4}$ & $1.3 \times 10^{5}$ & $1.3 \times 10^{5}$ \\
\hline RECO rejection factor & 1 & 1 & 1 & 2 & 2 \\
\hline RECO computational load (SSCUP) & $2 \times 10^{4}$ & $2 \times 10^{5}$ & $3 \times 10^{5}$ & $4 \times 10^{5}$ & $4 \times 10^{5}$ \\
\hline Event rate stored & $100 \mathrm{~Hz}$ & $100 \mathrm{~Hz}$ & $100 \mathrm{~Hz}$ & $100 \mathrm{~Hz}$ & $100 \mathrm{~Hz}$ \\
\hline Equivaient cross section & $100 \mu b$ & $10 \mu b$ & $1 \mu b$ & $100 \mathrm{nb}$ & $100 \mathrm{nb}$ \\
\hline Initial data per event & $1 \mathrm{MB}$ & $0.9 \mathrm{MB}$ & $0.6 \mathrm{MB}$ & $0.6 \mathrm{MB}$ & $0.6 \mathrm{MB}$ \\
\hline Rate of initial data to storage & $100 \mathrm{MB} / \mathrm{s}$ & $90 \mathrm{MB} / \mathrm{s}$ & $60 \mathrm{MB} / \mathrm{s}$ & $60 \mathrm{MB} / \mathrm{s}$ & $60 \mathrm{MB} / \mathrm{s}$ \\
\hline Additional event data & $1.6 \mathrm{MB}$ & $1.6 \mathrm{MB}$ & $1.6 \mathrm{MB}$ & $1.6 \mathrm{MB}$ & $1.6 \mathrm{MB}$ \\
\hline Peak event data rate & $110 \mathrm{MB} / \mathrm{s}$ & $150 \mathrm{MB} / \mathrm{s}$ & $130 \mathrm{MB} / \mathrm{s}$ & $130 \mathrm{MB} / \mathrm{s}$ & $130 \mathrm{MB} / \mathrm{s}$ \\
\hline Annual event storage & $0.26 \mathrm{~PB}$ & 1 PB & $0.9 \mathrm{~PB}$ & $0.9 \mathrm{~PB}$ & 2.2 PB \\
\hline $\begin{array}{l}\text { Simulation computational load } \\
\text { (SSCUP) }\end{array}$ & $1.5 \times 10^{5}$ & $3 \times 10^{5}$ & $6 \times 10^{5}$ & $6 \times 10^{5}$ & $6 \times 10^{5}$ \\
\hline Annual simulation storage & $0.14 \mathrm{~PB}$ & $0.25 \mathrm{~PB}$ & $0.4 \mathrm{~PB}$ & $0.4 \mathrm{~PB}$ & $0.4 \mathrm{~PB}$ \\
\hline Annual test beam data & $0.01 \mathrm{~PB}$ & $0.01 \mathrm{~PB}$ & $0.01 \mathrm{~PB}$ & $0.01 \mathrm{~PB}$ & $0.01 \mathrm{~PB}$ \\
\hline Total annual storage & $0.5 \mathrm{~PB}$ & 1.3 PB & 1.3 PB & 1.3 PB & 2.6 PB \\
\hline Peak rate to storage & $125 \mathrm{MB} / \mathrm{s}$ & $170 \mathrm{MB} / \mathrm{s}$ & $155 \mathrm{MB} / \mathrm{s}$ & $155 \mathrm{MB} / \mathrm{s}$ & $155 \mathrm{MB} / \mathrm{s}$ \\
\hline
\end{tabular}

(a) The SSCUP is a unit of processing power, approximately equal to 0.9 VUP. 
Table 8-3. Profile of a GEM physics data event.

\begin{tabular}{|c|c|c|c|}
\hline Item & $\begin{array}{l}\text { Level } \\
\text { Created }\end{array}$ & Data type & Size \\
\hline 1. & Level 1 & $\begin{array}{l}\text { Event no., run no., } \\
\text { time, Level } 1 \text { trigger } \\
\text { data, version number }\end{array}$ & 50 bytes \\
\hline 2. & $\begin{array}{l}\text { Event } \\
\text { filter }\end{array}$ & $\begin{array}{l}\text { Event filter data for } \\
\text { rejected event, } \\
\text { version number }\end{array}$ & 50 bytes \\
\hline 3. & $\begin{array}{c}\text { Event } \\
\text { fitter }\end{array}$ & $\begin{array}{l}\text { Filter results for } \\
\text { accepted event, } \\
\text { version number }\end{array}$ & $20 \mathrm{kB}$ \\
\hline 4. & DAQ & $\begin{array}{l}\text { Tracker, calorimeter, } \\
\text { muon data, version }\end{array}$ & $300 \mathrm{kB}$ \\
\hline 5. & $\begin{array}{l}\text { Recon- } \\
\text { struction }\end{array}$ & $\begin{array}{l}\text { Calorimeter clusters, } \\
\text { track segments, fitted } \\
\text { tracks, photons, } \\
\text { muons, jets, missing } \\
E_{\text {, etc., version }}\end{array}$ & $300 \mathrm{kB}$ \\
\hline 6. & $\begin{array}{l}\text { Event } \\
\text { summary } \\
\text { creation }\end{array}$ & $\begin{array}{l}\text { Identified particles, } \\
\text { vertices, error ma- } \\
\text { trices, fit parameters, } \\
\text { event hypotheses.... } \\
\text { version }\end{array}$ & $50 \mathrm{kB}$ \\
\hline 7. & $\begin{array}{l}\text { Mini- } \\
\text { summary } \\
\text { creation }\end{array}$ & $\begin{array}{l}\text { Classified ovents with } \\
\text { identified particles, } \\
\text { version }\end{array}$ & $20 \mathrm{kB}$ \\
\hline 8. & $\begin{array}{l}\text { SUB. } \\
\text { TOTAL }\end{array}$ & $\begin{array}{l}\text { Initial event size } \\
\text { (items 1-5) }\end{array}$ & $0.62 \mathrm{MB}$ \\
\hline 9. & TOTAL & $\begin{array}{l}\text { Ratio of total event } \\
\text { data to number of } \\
\text { events, including } \\
\text { duplications, event } \\
\text { summaries, etc. }\end{array}$ & $\begin{array}{l}2.2 \mathrm{MB} \\
\text { event }\end{array}$ \\
\hline
\end{tabular}

The additional event data is the data that will be added over the course of the year, e.g., from repeats of RECO with new calibrations and Data Summary File (DSF) creation; the value is the total event-related data in the store divided by the number of events. The peak event data rate is calculated assuming that a repeat RECO and DSF creation should be done in three months $\left(10^{7} \mathrm{~s}\right)$, and is run whether the detector is taking data or not.

Computational demands are listed for Level 2 , Level 3, and RECO. In 1999, the Level 2/3 load is for event building only, with no filtering. It is assumed that the Level 2 filter takes 1 SSCUP-second ${ }^{2}$ per input event, Level 3 takes 30, and RECO takes 1,000 in the year 2000 (and 2,000 in the year 2002), and that Level 2 and Level 3 each obtain a rejection factor equal to the square root of the required combined factor. The computation times are estimated from studies with gemfast (see Section 2.2), hit-level Monte Carlos, and D0 and CDF experience. Simulation computational load and storage requirements are detailed in Section 8.4.5, and test beam storage requirements are estimated to be about $10 \mathrm{~TB} / \mathrm{yr}$.

The scenario embodied in Table 8-2 is that the Level 1 trigger level remains the same while the demand on Levels 2 and 3 increase with luminosity. Level $2 / 3$ filter factors are applied progressively as they are proven to be optimal and safe using real data. This results in a RECO and storage load that varies only a factor of two, while the luminosity changes by a factor of a thousand. An alternate scenario is to use higher filter factors earlier, resulting in a staging of off-line requirements. In 1999, RECO is spread over three months, as is the creation of the additional event data.

\subsection{FUNCTIONAL REQUIREMENTS}

Functional requirements are those placed on the computing systems by their users-the operators and the physicists-independent of the system model and distinct from the requirements imposed by physics and data volumes.

Control should be easily exercised over stan. ing and stopping runs, trigger setup, and management of data flows through data acquisition and production. The control functions must not be bound to particular hardware, and must be available in locations remote from the detector and the interaction region.

Monitoring processes must inform detector shift personnel (and data production personnel) of any problems, especially those that need intervention, while also updating a record of the state of the apparatus. The data must be presented in a hierarchical fashion, with the most important issues first. The display update frequency must be on the order of once per second for the shift and production personnel. Access to specific monitored items must occur within 10 seconds. Displays must also be available to other users, but may be at a lower rate. All information available to the monitoring system must be recorded to be available to analysis and calibration processes. Parameters that are satisfactory can be recorded at a low rate, while all 
alarms, errors, and changes must be recorded with a time stamp. The monitoring system should include full access to reconstruction data, event displays, and analysis tools. The monitor system must also capture operator actions, queries, and comments, in electronic form to obviate the need for paper logbooks.

Data production is the process of making data available for analysis, possibly after reduction, and includes event filters, and full reconstruction. This includes event data and "apparatus state" data. Events coming from the detector should be completely processed through the production systems (for the first time) almost in real time. Full data is then immediately available for analysis. An elaboration of this requirement is that there might be a delay of as long as $30 \mathrm{~min}$. in beginning full reconstruction after the start of data-taking from each store, to give time to establish the short-term calibrations for the particular store. During this time the "raw" event data will be stored in temporary files that are later deleted; a copy of this raw data will be stored with the reconstructed data. The system should be designed to catch up witbin a few hours. For diagnostic purposes for the snift personnel, it may be necessary to process a subset in a shorter time.

The "apparatus state" data is required to be an adequately complete description of the state of the accelerator, the detector, and the production systems from $D A Q$ to reconstruction. It must also be possible to add new variables to the description. Recording the state of the apparatus in full detail is an ambitious goal; especially since we require that all information available to the monitoring system be also available to later analysis.

All data must be accessible by any user or task at any time from all collaborating institutions; the response time must be appropriate to the task at hand. This includes monitor data logged by the control system, collider data, event data, and versions of software in use at the time the data was generated. A running analysis task $\mathrm{m}$ st be able to access such data pertinent to the time or collection of each event, and a physicist analyzing a particular event must be able to recall a detailed description of the state of the letector at the time the event was coll cted.
Simulation of the apparatus will produce results which are an accurate representation of the physics and detector performance. The simulated and real data will be compared in detail, as one of the principal steps in the analysis. Simulated data also will be lised to test the production systems by generating data that will fed through the systems in the same way as the real data.

All subsystems should be so designed as to make validation, verification, and testing easy. This requires that all software (that transforms event data) of all production systems be available in the off-line/analysis environment, and also that it be possible to inject simulated data at any point in the production chain.

The analysis environment should allow easy use of the software used in the other systems, including data reduction, simulation, event display, and monitor information display. It should be easy to make new analysis processes or to introduce new algorithms.

There should be a full suite of tools available for viewing and manipulating the data, using commercial data-analysis tools as well as HEP tools. These tools should be as available to the controls tasks as to the analysis. A query language needs to be developed to allow the kinds of questions that are asked while exploring the data to be expressed in a compact and high-level form. Commercial tools such as Mathematica and spreadsheets should be available. As part of the GEM development effort, requirements will be developed for a suite of tools.

Consistency of the user interface is a requirement. Physicists and others will work across the GEM computing systems, from operations room to analysis station. The user interface must be consistent across these different environments. To reduce the possibility of confusion and error, and to make it easier to train operators, all user interfaces will be planned with a consistent style, from detector and DAQ control to analysis.

The responsiveness of the systems must be matched to the task at hand. Response time will include the time to access data, the time to launch an appropriate process, and the time to complete the process; the balance between these must be opti- 
mized. Detailed requirements must be developed for each activity. For the controls systems, time to respond must not reduce detector uptime by more than $1 \%$. For production, the time to reprocess the complete data set in response to new calibrations or new requirements should be reasonable. For analysis, the time for a physicist to evaluate the results of an activity should be considered.

Automation should be applied to every technically feasible activity, including moving the data from the DAQ to the final file system, production processing, creation of calibration data, and adjustment of out-oif-range detector parameters. This will avoid bookkeeping problems created by manual activities such as transferring tapes from one file system to another.

Flexibility is an important requirement: it must be possible to allocate computing power and other resources to the highest-priority tasks at hand and to reconfigure subsystems to take advantage of developing te:hnology without impact on other systems. The har'ware costs of the needed systems will be high, $\rightarrow$ that the software and management effort needed to ensure maximum flexibility will be repaid.

Documentation must be available (on-line) for all systems, covering users' guides, operations manuals, repair manuals, system descriptions, etc.

\subsection{SYSTEM MODEL}

The system model for the GEM computing system is illustrated in $F_{0}$ ure 8-1, in which the primary elements are processes, data stores, and entities external to the computing system (sources or sinks of data or sources of commands at the boundary of the system). In what follows, each statement of a parameter of the model gives rise implicitly to a matching requirement. The model is intended to have little reference to any particular physical implementation, as this is very likely to change as technology evolves. A brief description of each element, including the functional requirements, follows. The collaboration will influence, and interact with, all elements of this diagram; some specific roles for collaboration members are shown.

The major flows of data from front end to analysis are modeled, so that a comprehensive view is given. To keep the diagram relatively simple, some subsidiary flows and processes have been omitted. This diagram shows partitioning into three subsystems: the global control system (GCS), the on-line system, and the off-line systems. Besides being connected by data flows and commands, these subsystems are connected by the shared essential stores of persistent data. A notable feature of Figure 8-1 is that there is no separate event store for the on-line system. This is intentional: in GEM, event data will be sent directly to the final storage system.

\subsubsection{External Entities}

The detector (or more precisely, the electronics) produces a flow of digital event data, strobed by Level 1 at rates up to $100 \mathrm{kHz}$. Data is downloaded to it to set it up, and its state will be controlled from the global control system.

The operator represents the operating crew, which includes the shift physicists, other operating staff members, and physicists with detector responsibilities who access the control system from off-site. The operator initiates transitions between detector states, is responsible for data quality through operator commands, and can call on display of detector status and other data in many forms. Data quality assurance requires that results of physics analysis be available to the operator. The special operator controls special runs as neesded (for example subsystem test runs or runs with a special trigger), independently of the main data flow, to carry out specific studies. Special run commands go to the control system and special readout to off-line systems.

The interface to the accelerator control system will enable the passing of accelerator status information to the control and monitor system, as well as transmission of detector information to the accelerator control system. Not shown is the passage of timing signals from the accelerator to the electronics. Some messages may be time-critical and require prompt action at the detector, e.g., an impending beam dump; a fail-safe mechanism might be incorporated for accelerator transients that would damage the detector. Personnel access information and emergency information from PASS (Personnel Access Security System) and EMS (Emergency Management Systems) are included here. 


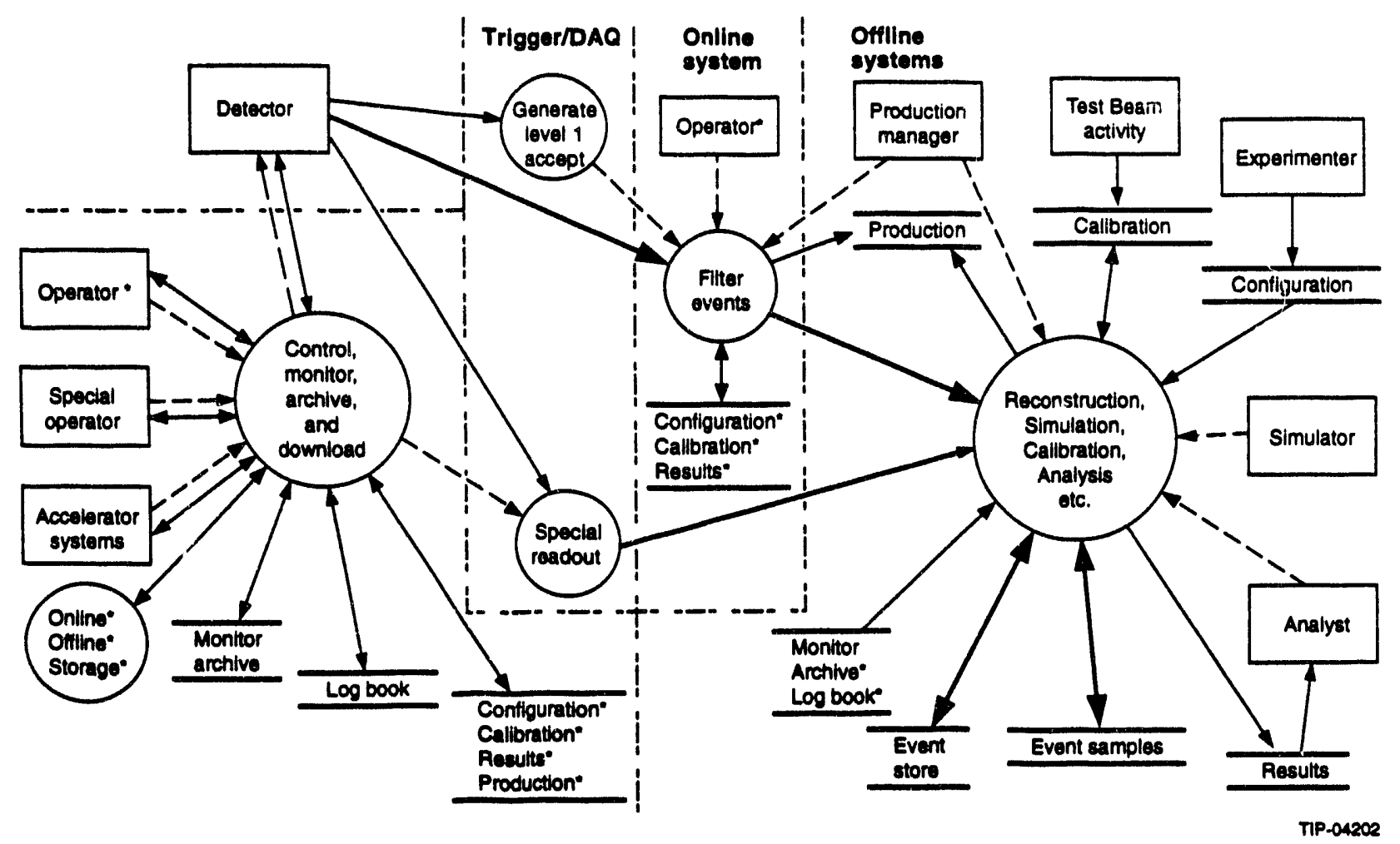

FIG. 8-1. System model for GEM computing. Rectangles represent extemal entities, circles represent processes, double bars represent stores, solid arrows represent data flows, and dashed arrows represent commands. The primary data flows are indicated by a thick arrow. A label with an asterisk (") indicates that the entity appears in more than one place in the diagram.

The production managers manage the on-line and off-line production activities, such as high-level event filters and reconstruction. Tools must be provided to support this function and to produce the necessary records. The experimenter creates the configuration database, from detailed understanding of the hardware and software configuration of the detector. Test beam activity will generate calibration data that will be used to build the calibration database. The simulator represents the members of the collaboration engaged in the simulation effort, which is described below in more detail, and the simulation production manager. The analyst analyzes the data for physical significance, makes comparisons to simulated data by initiating analysis processes-including additional passes through the event data-and stores results (histograms, "NTUPLEs," fitted events). Analysts will be off-site as well as on-site.

\subsubsection{Data Stores}

The data stores that are shown are only those that are persistent for reasons not associated with an actual implementation. A real system will require a variety of additional buffers. The categories of database described here will evolve as the system is further analyzed. An essential element of the database philosophy is that there will be a master version of the data used in any database, and a mechanism to ensure coherence, of any data that is used, with the master version. For every store, processes must be created to allow access to the data.

\section{Event Store/Database}

At some point in the process of data reduction, it is appropriate to store events for an extended period of time, to allow for reprocessing. This is a compromise between the cost of storage and the risk is that too severe a filtering process will introduce biases that reduce the value of the data, or that a comple. filtering algorithm might contain an error.

The composition assumed for a single event is shown in Table 8-3. An important feature of the event data is that it includes version numbers for all software that has modified the event. An event can 
cunsist of items 1 and 2 only (for an event rejected at Level 2). In an ideal storage system, items 1, 2, 3 and 4 (the "raw" data) would be present only once; however, we assume that these items are present three times: once for the original data and twice for succeeding reconstruction passes. Item 5 will also be triplicated, while items 6 and 7 will need storage of about 1.5 to 2 times the amount needed for a single DSF event to allow for duplication of the same event in different DSFs (Table 8-4). The estimate of event sizes comes from Section 7.3.2, D0, H1, and other experiments.

The event store contains the "raw" data for each event, reconstructed data, and summaries of the events, which should be accessible for reprocessing for reconstruction as needed, calibration studies and making of data summary files (DSFs). As noted above, all triggers will result in a record being written to storage. Simulated full events will also be part of this store.
One point to note is that the cust of recomputing reconstruction data may be jess than the cost of storing it. The technology and cost trends assumed for GEM computing indicate that the costs of recomputing from the raw data is equal to the cost of storage in the year 2000 if recomputing is done five times a year.

The input requirement for the event storage system is assumed to be constant at about $150 \mathrm{MB} / \mathrm{s}$ (Table 8-2). At full luminosity the event-storage system must be capable of storing $1 \mathrm{MB} / \mathrm{s}$ from monitoring, $60 \mathrm{MB} / \mathrm{s}$ from the event filters, $50 \mathrm{MB} / \mathrm{s}$ from the reconstruction and fitting processes, $20 \mathrm{MB} / \mathrm{s}$ from simulation, and $33 \mathrm{MB} / \mathrm{s}$ frori $\mathrm{DSF}$ creation and analysis, for a total of $164 \mathrm{MB} / \mathrm{s}$. For read access, the requirement is $60 \mathrm{MB} / \mathrm{s}$ for the reconstruction process, up to $10 \mathrm{MB} / \mathrm{s}$ for calibration, an average of $200 \mathrm{MB} / \mathrm{s}$ for DSF creation, and $1 \mathrm{~GB} / \mathrm{s}$ for analysis, for a total of about $1.4 \mathrm{~GB} / \mathrm{s}$. The specific rates will depend on whether event data is stored after filtering or after reconstruction, whether DSFs are created by the reconstruction pass, and the level of simulation detail required.

Table 8-4. Anticipated 1-yr event samples, including simulation output.

\begin{tabular}{|c|c|c|c|c|c|}
\hline Category & $\begin{array}{l}\text { Number of } \\
\text { files }\end{array}$ & Record size & $\begin{array}{l}\text { Number of } \\
\text { records }\end{array}$ & File size & $\begin{array}{l}\text { Equivalent cross } \\
\text { section }\end{array}$ \\
\hline Calibration ( $\theta . g ., Z \rightarrow \theta \theta, \mu \mu)$ & 20 & $10 \mathrm{kB}$ & $10^{7}$ & $100 \mathrm{~GB}$ & $1 \mathrm{nb}$ \\
\hline $\begin{array}{l}\text { Calibration ( } \theta . g . \text { single par- } \\
\text { ticles from } W \text { decays) }\end{array}$ & 10 & $2 \mathrm{kB}$ & $10^{8}$ & $200 \mathrm{~GB}$ & $10 \mathrm{nb}$ \\
\hline $\begin{array}{l}\text { Physics process (e.g., th } \\
\text { SUSY) }\end{array}$ & 20 & $50 \mathrm{kB}$ & $10^{8}$ & 5 TB & $10 \mathrm{nb}$ \\
\hline Physics process (full events) & 20 & $600 \mathrm{kB}$ & $10^{7}$ & 6 TB & $1 \mathrm{nb}$ \\
\hline Physics process (0.9. $\left.H^{0} \rightarrow \gamma \gamma\right)$ & 20 & $50 \mathrm{kB}$ & $10^{7}$ & $500 \mathrm{~GB}$ & $1 \mathrm{nb}$ \\
\hline Physics process (mini-DSF) & 100 & $20 \mathrm{kB}$ & $10^{7}$ & $200 \mathrm{~GB}$ & $1 \mathrm{nb}$ \\
\hline $\begin{array}{l}\text { Physics process (e.g., rare } \\
\text { events) }\end{array}$ & 100 & $600 \mathrm{kB}$ & $10^{6}$ & $600 \mathrm{~GB}$ & $<100 \mathrm{pb}$ \\
\hline $\begin{array}{l}\text { Physics process (e.g., new par- } \\
\text { ticle search) }\end{array}$ & 200 & $50 \mathrm{kB}$ & $10^{6}$ & $50 \mathrm{~GB}$ & $<100 \mathrm{pb}$ \\
\hline Physics (mini-DSF) & 200 & $20 \mathrm{kB}$ & $10^{6}$ & $20 \mathrm{~GB}$ & $<100 \mathrm{pb}$ \\
\hline Physics (micro-DSF) & 200 & $2 \mathrm{kB}$ & $10^{7}$ & $2 \mathrm{~GB}$ & $1 \mathrm{nb}$ \\
\hline
\end{tabular}




\section{Event Samples}

Event samples fall into several classes. In an ideal storage system, it would be possible to store only a list of pointers to interesting events or to use a database technique to access events any associated items of data, so that the samples might not be contained in separate files. The customary approach is to create data summary files. Event samples will contain events in full or compressed form and will be rapidly accessible; events will be classified according to some characteristic, e.g., the Level 1 trigger or the physics process being studied. At a later stage of analysis, there may also be "miniDSFs," for which the data will be compressed or filtered by an additional factor of 2-5. There will be matching simulation output for each file derived from detector data.

Data summary file sizes are estimated in Table 8-4 for a single year's data. There is an indication of the category of a study. The number of files is the number of distinct DSFs in the category which are concurrently stored. Since we have a large collaboration and many physics processes to study, these numbers can be expected to be large. The total storage required for this is $330 \mathrm{~TB}$ about equal to the raw data. Of this, some (small) fraction should be in rapid-access storage-that is, accessible in a few seconds, to meet the criterion of responsiveness. The list in the table serves only to demonstrate a problem: to access a DSF of $10^{7}(50 \mathrm{kB})$ events would take 5 hours at the assumed bandwidth of a single tape reader. Using multiple readers means fragmenting files across cartridges and dedicating more resources to a single user. The use of "micro-DSF" files of extremely compressed events for most analyses may be necessary. For the study noted above, it would reduce the read time to $12 \mathrm{~min}$.

\section{Configuration Database}

The purpose of the configuration database is to maintain full documentation of all static parameters of the detector, including a history of changes. This will provide the database from which the detector is brought into a running condition, as well as the database for simulations and monitoring tasks. Configuration data will be used by many processes. Among the subdivisions of this database, the following items will be included:
Level 1 (and hardware Level 2) codes and data that are downloaded to the front end will be stored here. No code will be downloaded from any other place, to preserve the integrity of the record. This database will grow with time. An initial size of $1 \mathrm{~GB}$, with an average growth rate of $20 \mathrm{MB} /$ day, might be anticipated. This store must be accessible within fractions of a second, and must be capable of data rates exceeding $20 \mathrm{MB} / \mathrm{s}$.

There will be a physical description database that defines baseline locations and relationships among parts of the apparatus; it will include survey information. This database will initially be of the order of $1 \mathrm{~GB}$, and may be updated at the rate of $10 \mathrm{MB} /$ day on average. Since many tasks will access this database, it must be accessible in very short times and may need to be copied in several places.

The hardware database will define the hardware in the front-end, data-acquisition, and monitoring systems. It will include access methods and data formats and it will be several times the size of the physical description database, with "real-time" access requirements.

Filter (Level 2 and 3) and RECO and off-line codes, including all codes used for the production of results will be version-controlled and will be kept in a database.

\section{Calibration Database}

This database will contain the results of a variety of calibration techniques: electronically measured, calculated from special runs, and calculated from a full reconstruction. It will include calibration data to be downloaded to the front-end processors, such as to the calorimeter electronics. Calibration data will be used in the event filters and the reconstruction passes, as well as in simulation and analysis. It will also be used to create data in the configuration database to be downloaded to the detector.

The size of this database is likely to be dominated by the calibration constants to be downloaded to the front-end. A preliminary estimate gives a total of $330 \mathrm{MB}$ for the calorimeter data and an equal amount for the remainder of the apparatus. ${ }^{3}$ Assuming that this database is completely updated on a daily basis gives at least a $600-\mathrm{MB} /$ day increase 
in size, for a total of about $200 \mathrm{~GB} /$ year, which must be accessible by filter and reconstruction tasks. Further, the download must proceed at a rate of $2 \mathrm{MB} / \mathrm{s}$ or greater. Other calibration constants may amount to about $30 \mathrm{MB} /$ day. Of course, if reconstruction proceeds very close in time to data-taking, only the most recent constants need be readable with short access times.

\section{Monitor Archive}

This database records the state of the apparatus as seen by the control and monitor systems. In addition to such quantities as high voltages, state transitions and alarms will be recorded here. An overall data rate of less than $100 \mathrm{kB} / \mathrm{s}$ is anticipated for a total of $3 \mathrm{~TB} /$ year. A regularly updated image of the status will be distributed to the control and on-line systems, and a record of changes, updates, and alarms will be recorded permanently. All information will be time-stamped to within $10 \mathrm{~ms}$, and at least the previous week's worth of data should be accessible in seconds in a variety of ways: by subject or by time. "Aged" data can have relaxed access requirements. Monitor data will be used in the reconstruction and analysis stages, both to make any needed corrections to calibrations and to stamp event data with an indication of quality.

\section{Electronic Log Book}

The electronic log book will be an interactive, multimedia database that will capture the activities and comments of the shift personnel in a form that is accessible electronically. There will be no paper log book. Input devices will include OCR devices, scanners, TV cameras, microphones, graphics programs, etc. All information will be time stamped. In conjunction with the monitor database, the electronic log book will provide a complete and recoverable picture of the detector at the time any event is collected. An overall data rate of $1 \mathrm{MB} / \mathrm{s}$ should be anticipated. The data should be accessible in seconds in the same way as the monitor data.

\section{Production Database}

The production database will log the production activities of the computing systems themselves: on-line computing summaries, off-line jobs completed, dataset creation, RECO status, and simulation runs.
Results

The results database will contain diagnostic results and physics results, which result from standard analyses of production data and other official analyses. These will consist of standard histograms, other plots and graphs, and documents describing results. Results of standard analyses will be stored here. The size is hard to estimate but is presumably small compared with other databases.

\section{Processes}

The processes of Figure 8-1 are grouped according to function and response time into three systems: the global control system, the on-line system, and the off-line system. These systems interact through data flows, commands, and shared data stores.

\subsubsection{Global Control System}

The Global Control System (GCS) is expanded in Figure 8-2. A group of processes, important to the GCS but included in the on-line and off-line systems, is the group of diagnostic analysis processes that produce diagnostic results accessible to the operator. A primary requirement of the GCS is that data quality be assured through extensive monitoring, rapid reporting of problems, and record. ing of any deviations.

The Control Processes running in the control system change the detector state on operator request. adjust out-of-range parameters where appropriate. pass on requests for display of data, initiate down. load of data to the front ends, initiate changes in trigger, and request changes in running conditions for the on-line system and for the off-line compute server and storage system.

Monitor processes manage the reading of monitor data from the detector; ensure processing and logging of alarms; monitor the status of other GEM systems including DAQ, on-line, off-line and storage; and monitor the status of the accelerator.

Display processes set up displays for the operator(s) and members of the collaboration, and send data to the accelerator system (including data to be processed as well as displayed). These display processes will access all databases, including the results database. 


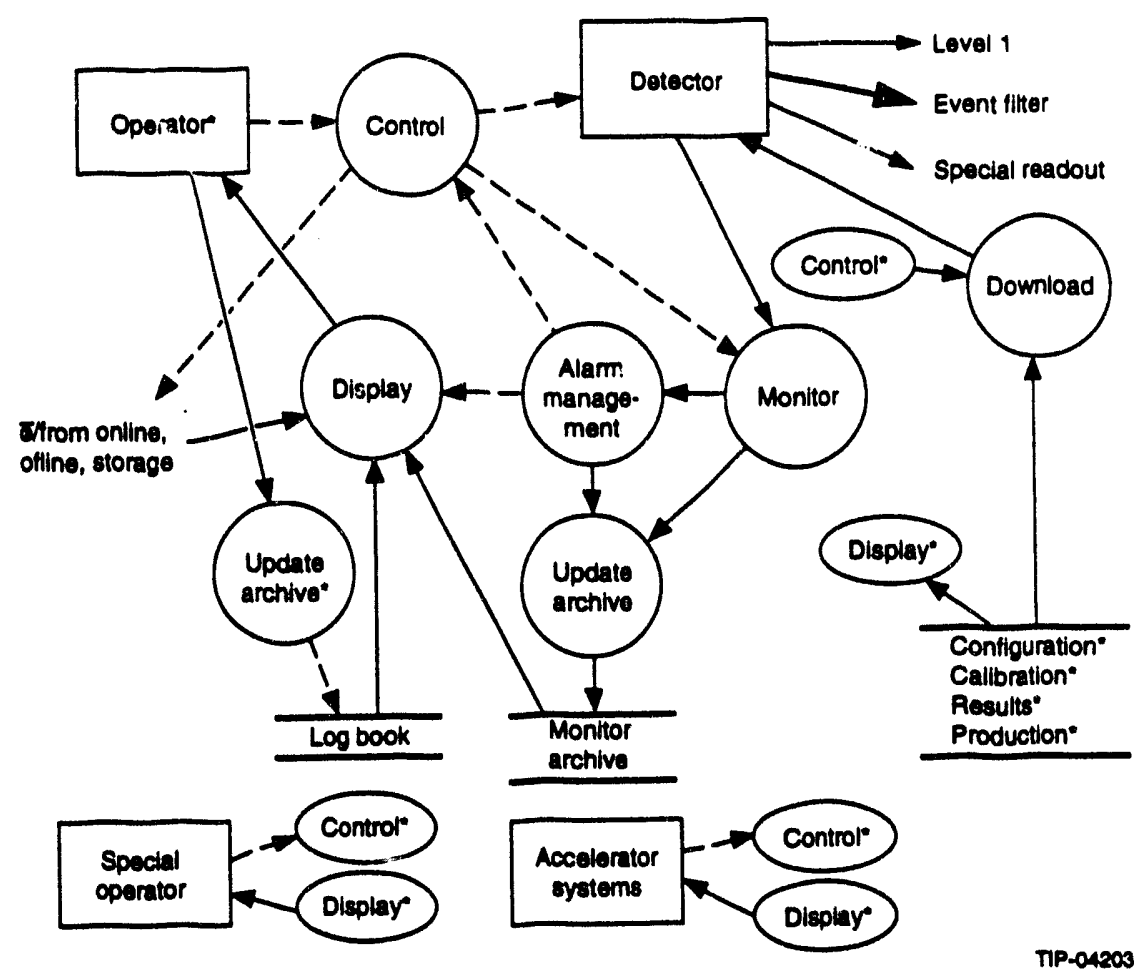

FIG. 8-2. Model of the GEM GCS, showing major process groups. Conventions are the same as for Figure 8-1, with the addition of labels for data and command flows to and from processes outside this diagram.

The archiving processes make a permanent record of monitor data, including recording of alarms. These processes will include the processes that support the "electronic log book."

The download process is initiated through the GCS; it must be able to read as well as write all locations where there are programmable constants, so that verification is possible. A requirement is that a full download of all data, and verification of the download, to the detector should take no more than $10 \mathrm{~min}$. A first estimate of the total data to be downloaded to the $d r$ ector is about $600 \mathrm{MB}$. This implies a total bandwidth of $2 \mathrm{MB} / \mathrm{s}$, within the range of FDDI, but not of Ethernet.

\section{Requirements}

In general, the top-level operation of the GEM detector systems will not be bound to particular hardware. It will be possible, consistent with safety requirements, to carry out all operations remotely from the detector site; specifically, control will be possible from at least two places at the SSCL: the GEM IR Detector Operations room, and the GEM Main Campus Operations Room. Access to GCS will be controlled, as will access to specific subsys- tems. For $90 \%$ of the time, only two people should be required to operate the detector at the top-level. There will te other operating personnel, for the magnet pover supply and the cryogenics systems, who may ' lave to be at the IR. A subset of the GCS will be built and used for test beam work at FNAL and SSCL, to support this work and to develop the GCS itself.

\section{Quality Assurance}

The goal is to update the monitoring record frequently enough that no more than $1 \%$ of data is lost because of uncertainty in the condition of the apparatus. For example, if failures occur once per hour, the updates must be at least once per minute. The GCS must have no single-point failure mode.

\section{Modes of Operation}

The GCS will be designed to support a variety of modes of operation of the detector, as described in the following paragraphs.

During Construction and Installation of the detector, the GCS should support all test operations on subsystems to the greatest extent feasible, to minimize development and use of alternate systems. 
The construction state will be characterized by many special requirements, in which subsystems may be "locally" controlled; there may be temporary lockouts or manual operation for some functions and a system of tagging of special conditions. The GCS should support recording and reporting of such special conditions. The maintenance phase (between collider runs) will have similar requirements.

During commissioning of the detector and electronics, the GCS will be brought into full operation. It is expected that up to 30 individuals will require access to monitor and control functions, mostly to subsystems. Commissioning will be characterized by special conditions, as during construction, and in addition, by many separate and overlapping test runs on subsystems.

Special runs must be supported by the GCS (and the DAQ) for overlapping test runs, overlapping setup phases, and overlapping monitor functions on different subsystems. Subsystem and other special runs will take place during construction (installation), commissioning, and data-taking, and possibly during standby.

During normal data-taking, the GCS will provide enough support so that top-level control and monitoring can be done by two people. The highest priority will be to maximize throughput of event data from the detector to further processing, while permitting other activities to take place in a noninterfering mode. The transition from the Standby mode to the Data-taking mode (a "warm start") should take no longer than $1 \mathrm{~min}$. During data-taking, no more than $1 \%$ downtime should be attributable to the GCS itself.

The standby mode is characterized by the magnet being on, the calorimeters cold, high voltages on, and all systems ready to take data. The detector hall is closed. Subsystem runs may take place during this phase. The transition from Shutdown to Standby (a "cold start") should take no more than 30 minutes, for startup or setup operations attributable to the GCS itself.

The shutdown mode is characterized by a stable situation in which no operation is expected. In this mode, no top-level operators will be needed.

\section{Detector Setup}

The GCS will support detector setup, by downloading code or constants to any front-end processors, by enabling trigger selection, by controlling the setup of on-line computing facilities, and by establishing that "off-line" facilities such as the processing ranch and storage systems are available, or by initiating appropriate action to make them available.

Detector setup (configuration) requires databases and processes to transfer data to the appropriate destination, verify the transfer and record the fact of the transfer, and the version of the data used, in the monitor database. The configuration database will be used to guide this process. The databases will include front-end constants, Level 1 setup, Level 2 and Level 3 codes and processes and calibration constants. The processes will run on nodes of the GCS, and access databases on local disk arrays. In the case of Level 2 and Level 3, the setup may be handled through messages to processes running in those systems.

The download sequence will include a readback and verification step; all programmable elements will support this read-back. Data paths will be adequate to complete this task in less than $10 \mathrm{~min}$ utes. Changes, including partial changes, may occur between data-taking runs. All such changes will be recorded in the configuration database. Changes that affect a data-taking run will be locked out during the run.

\section{Control}

The GCS will support control of all changes of detector state, and will have the capability to modify data rates, through dead time, trigger changes, etc. Some changes in detector state, e.8., run start and stop, should take place within $10 \mathrm{~s}$ of being requested and should be time-stamped to within $10 \mathrm{~ms}$.

\section{Monitoring of Detector}

The monitor requirements will be spelled out in detail in GEM TN-93-398. ${ }^{4}$ The basic requirement is that the system itself should not contribute more than $1 \%$ to downtime, where downtime is defined to include out-of-calibration time. Thus, if a 
parameter drifts outside its allowable range on average once an hour, the system must measure all such parameters every $30 \mathrm{~s}$ or better. The total data rate to be sustained for all monitored parameters is expected to be of the order of 1-2 MB/s. A complete 48-hr history is to be maintained with access times measured in seconds.

\section{Monitoring of Processed Data}

The GCS must monitor data emerging from each processing stage: Level 1 and 2, the event builder, Level 3, and reconstruction. Samples of appropriate events or other kinds of data will be collected for recording, analysis, and display.

\section{Log Book}

The "electronic logbook" must allow for easy input from speech, handwritten comments, windows on workstations, TV images, monitor data streams, event analysis, event displays, scanned documents, and so forth. Keyword access and access by date and time must be supported. The data rate is expected to be of the order of $1 \mathrm{MB} / \mathrm{s}$. A complete 7-day history is to be maintained with access times of the order of seconds. A hypertext multimedia format is desirable.

\section{Display}

The control and monitor system must have a graphical user interface that permits hierarchical control and display of monitor data, including analyzed events. Display capabilities must include full-frame-rate video and sound, Xwindows, Display Postscript, bit-mapped images and color. The system must be able to support up to 50 concurrent displays without deterioration of response time.

\section{Interfaces}

The GCS must interface to the Accelerator Control and Monitor system so that information can be passed in both directions. This should include a bunch-crossing timing signal from the collider. The bandwidth of the interface must be of the order of $100 \mathrm{MB} / \mathrm{s}$ and include video capability. There will be interfaces to the IR utilities (e.g., HVAC, electrical power) and to the EMS.

\section{Slow Control and Monitoring System}

The Slow Control and Monitoring System (SCMS) is a subsystem of the GCS to be implemented using the EPICS (Experimental Physics and
Industrial Control System) system ${ }^{10}$ as adopted by accelerator systems. The goals of the SCMS are safety, data quality assurance, and ease of operation. To those ends, a common framework of software and hardware will be provided. This includes the concept that control functions will have several levels, some of which may be local to the subsystem. The location of control functions would depend on considerations of safety and response time. Three levels of control are envisaged:

1. Open or general (accessible to all shift personnel)

2. Access-controlled or special (confined to subsystem personnel and systems)

3. Safety-related (may be automatic).

The first two levels will reside in tasks running on a networked computer system, the third is likely to be implernented in standard industrial hardware or in an adaptation of standard hardware. Subsystems will work within the GCS/SCMS framework to meet these goals and perform the functions required.

The SCMS will define four levels for information:

- Level A: Information only

- Level B: Warning of out of-range-condition requiring adjustment or maintenance in due time

- Level C: Alarm requiring immediate intervention to retum detector to valid state

- Level D: Alarm requiring immediate response by emergency (EMS) personnel, such as a fire or rescue.

Corresponding responses will be carried out by the appropriate agents, which may be automatic hardware systems, software with the appropriate authorization level, general shift personnel, specialists in the subsystem concerned, or emergency crews (EMS).

An example of Level $\mathbf{A}$ information is the current of the solenoid, within range. Level B information that the current to a muon chamber section is out-of-range might result in an adjustment to the HV, perhaps carried out by a task running in the (accessed-controlled) muon control suite of tasks. If a crate goes over allowed temperature, its power supply may shut off (an automatic action), 
while notifying shift and other personnel via a temperature or voltage monitor (a Level $\mathrm{C}$ warning) so that the cause is found and the crate replaced (a specialist action). If smoke is detected in a muon electronics rack (a Level D alarm), an automatic system might notify EMS personnel and the shift personnel, and release a smothering agent in the rack.

Examples of monitoring activities that will be carried out within the SCMS include: monitoring of the magnet and cryogenics, alignment of the muon system, gas flow rates, calorimeter vacuum and temperature, silicon tracker temperatures and pressures, electronics rack conditions and power supply currents and voltages. Other functions include the monitoring of temperatures and humidities and other aspects of the environment. Preliminary estimates of the quantities to be monitored and controlled have been made for some subsystems; about 20000 analog quantities, several thousand binary quantities, and several hundred control points have been identified.

\subsubsection{On-line Computing System}

Analog and digital data are used to produce a Level 1 Accept signal, which is built up from a set of subtriggers and which reduces the rate to below $100 \mathrm{kHz}$, as discussed in Chapter 7. This signal initiates transfer of data to the event filters, which are driven by the Level 1 pattern; the event filter process can occur in several stages, dependent on the Level 1 trigger and the results of previous filters. The maximum input data rate envisaged is $30 \mathrm{~GB} / \mathrm{s}$ (Chapter 7), and the purpose of the event filters is to select interesting events for further study. The event filters send their output to the off-line system for further processing and storage. Diagnostic processes within the on-line system will produce diagnostic results.

The Level 2 system must be able to be configured via the GCS, and must conform to GEM guidelines for hardware and software. Reconfiguration should take less than $1 \mathrm{~min}$. To the extent that Level 2 uses general-purpose CPUs that are a major investment, it should be so designed that standard computer management tools can access it for other tasks, such as simulations. Because any error made in the trigger/filter sequence is not recoverable, attention must be paid not only to the hardware and software but also to access control and management, so that the on-line system is protected from inadvertent changes.

\subsubsection{Off-line Computing System}

The off-line computing system is expanded in Figure 8-3, to show the major categories of process.

Calibration creation processes will use a variety of data: detailed electrical measurements and processing of test beam, pulser, and physics data to create the calibration database. Calibrations that have to be updated daily using physics data should be capable of being updated in less than $30 \mathrm{~min}$. at full luminosity. Configuration data creation processes are needed to create and update the configuration database from detector design and implementation descriptions, from code management systems and from calibration results.

Reconstruction and fitting will be driven by the trigger and filter patterns; physical entities (electrons, jets) are built up from tracks and calorimeter clusters into hypothetical events. Detailed simulations of the reconstruction process have yet to be made, so we use estimates based on experience. One assumption is that reconstruction time scales with event size, another is that it is more or less independent of event size. CDF's experience translates to about 1,000 SSCUP-s per $0.5 \mathrm{MB}$ of data, based on events averaging $115 \mathrm{kB}$, while DO's reconstruction requires 700 SSCUP-s for a $0.5-\mathrm{MB}$ event. We have assumed 1000 SSCUP-s per event in the year 2000 growing to 2000 at full luminosity. It is planned that reconstruction occur essentially on-line, with only the delay necessary to recalculate the calibrations for each collider store. This is required to be less than $30 \mathrm{~min}$. During this time, raw data will go into the temporary files, to be deleted after calibrations are established and the data processed and recorded elsewhere.

The creation of event samples (DSFs) will be carried out in several ways. The primary path will be as direct output from the reconstruction process (RECO). This process will create many streams of events, by event character (e.g., isolated photons, two-lepton), going into appropriate DSF files, which can then be analyzed. It will also be necessary to allow for the creation of DSFs by scanning the recorded output of the reconstruction pass. This 


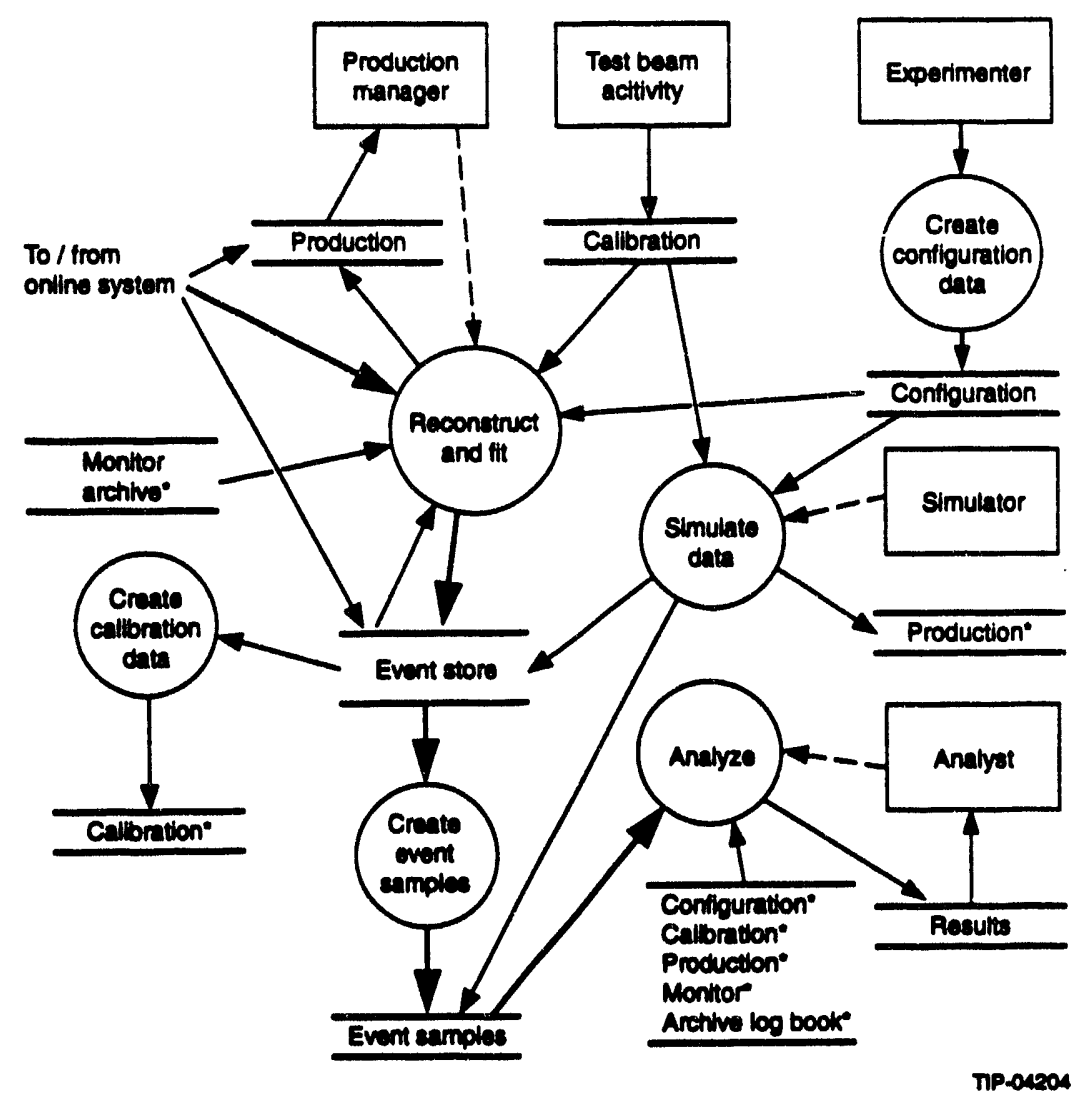

FIG. 8-3. The GEM off-line system, showing major process groups. Conventions are the same as those for Figure 8-2.

increases the VO load on the file system. In any case, the creation of DSFs is assumed to require a small fraction of the overall CPU capacity, say about $10^{4}$ SSCUPs.

The analysis activity will access all databases, but primarily the reconstructed events and the DSFs. There are two kinds of analysis: diagnostic analysis to produce results to be fed back to operators and production personnel, and physics analysis. Much of the physics analysis will be off-site, so that a major requirement is for wideband network capacity. If we assume that 50 physicists are active at any one time, accessing a micro-DSF of $20 \mathrm{~GB}$ ( $10^{7}$ events) once per hour, that implies a data rate from storage of $280 \mathrm{MB} / \mathrm{s}$. If the average computation time is 1 SSCUP-s/event (as it might be for a simple algorithm), then we require 150000 SSCUP. The required data rate and computing capacity for an individual workstation per analyst may be satisfied by the year 2002, but it is clear there is a data access problem for larger files, longer compute time, or more active users.
GEM emphasizes calorimetry for energy measurement, particle identification, and photon pointing. The experiment also uses a wide variety of calorimeter technologies to satisfy the requirements of precision, coverage, radiation hardness, and cost. This results in a requirement for a full, detailed, and global simulation of large numbers of events with a variety of tools. By "full" we mean that all secondary particles are tracked and that all energy deposition processes are simulated down to some energy cutoff; by "detailed" we sean that each subdetector element is described in the geometrical model (each accordion plate, every cable); by "global" we mean the entire detector and its environs. Experience with other detectors $(\mathrm{L} 3, \mathrm{DO})$ ) has indicated that calorimeter-based detectors need full simulations at very detailed level (e.g., following EM showers down to the 10-keV level), and that parameterized studies, while they have their place, cannot tell the whole story. A typical computation time for a full simulation is 300 SSCUP-s/GeV deposited, while a parameterized simulation takes of order 
40 SSCUP-s/event at a luminosity of $10^{33} \mathrm{~cm}^{-2} \mathrm{~s}^{-1}$. It is often said that the cost of a full and detailed simulation with full statistics is too high. In GEM we believe that full, detailed, and global simulations are necessary and, with modern technology, affordable.

The types of simulations that are needed include simulation of EM showers from photons, electrons, and narrow multi-gamma jets, including pointing, photon conversion in the tracker, muon tracking through the calorimeter and through the non-uniform magnetic field, and neutron and photon fluxes produced in the GEM detector and the beam elements. Studies that will be done include evaluation of detector design choices, estimation of calorimetry weighting factors, overall detector performance, fluctuations (e.g., in jet rejection), $k_{r}$ neutron and photon background rates, pileup at $10^{33}$ and $10^{34} \mathrm{~cm}^{-2} \mathrm{~s}^{-1}$, occupancies, pattern recognition, and trigger efficiencies.
The expected scale of our simulation effort is shown in Table 8-5, integrated over the time up to and including the first data run. The number of runs and number of events per run are very rough estimates based on the purposes of each type. For example, to search for the process $H \rightarrow y y$ in the intermediate mass region requires us to study jet rejection at the $10^{-4}$ level, and an overall rejection of QCD processes to the $10^{-8}$ level. We should have a well-understood model of the expected backgrounds, with adequate statistics. For $E_{T}$ processes, we need to study the tails of distributions in the forward calorimeter, where energies are very high. To understand the effects of non-homogeneous calorimetry, we need to have many studies using GEANT, EGS, CALOR, and LAHET to optimize weighting schemes. To study the trigger and filters that together reduce the rate by a factor of $10^{5}$ to $10^{6}$, we need to simulate at least that order of magnitude of events. Matching to test beam results will be a significant part of the simulation program.

Table 8-5. Expected scale of GEM simulation effort.

\begin{tabular}{|c|c|c|c|c|c|}
\hline Simulation Type & Typical Purposes & Runs & $\begin{array}{l}\text { Events/ } \\
\text { nun }\end{array}$ & $\begin{array}{l}\text { Compute time/ } \\
\text { event } \\
\text { (SSCUP-s) }\end{array}$ & $\begin{array}{c}\text { Total Require- } \\
\text { ment } \\
\text { (SSCUP-s) }\end{array}$ \\
\hline Subdetector (GEANT) & Test beam comparison & 1000 & $10^{5}$ & $10^{5}$ & $10^{13}$ \\
\hline Subdetector (GEANT) & Design studies & 1000 & 104 & $10^{5}$ & $10^{12}$ \\
\hline Subdetector (full GEANT) & $\begin{array}{l}\text { Design studies, } \\
\text { multi-gamma rejection, } \\
\text { pointing }\end{array}$ & 100 & $10^{6}$ & $10^{5}$ & $10^{13}$ \\
\hline Subdetector (EGS) & Comparison to GEANT & 100 & $10^{4}$ & $2 \times 10^{5}$ & $2 \times 10^{12}$ \\
\hline Global (full GEANT) & $\begin{array}{l}\text { Pattem recognition, } \\
\text { corrolation between } \\
\text { subdetectors }\end{array}$ & 100 & $10^{6}$ & $2 \times 10^{5}$ & $2 \times 10^{13}$ \\
\hline Global (CALOR, LAHET) & $\begin{array}{l}\text { Noutron flux, comparison } \\
\text { to GEANT }\end{array}$ & 500 & $10^{4}$ & $10^{6}$ & $5 \times 10^{12}$ \\
\hline $\begin{array}{l}\text { Global parameterized } \\
\text { GEANT }\end{array}$ & $\begin{array}{l}\text { Jet rejection, energy } \\
\text { deposition fluctuations, } \\
\text { track recognition and } \\
\text { fitting }\end{array}$ & 1000 & $10^{7}$ & $10^{3}$ & $10^{13}$ \\
\hline $\begin{array}{l}\text { Global parameterized } \\
\text { non-GEANT }\end{array}$ & $\begin{array}{l}\text { Physics studies, jet } \\
\text { rejection }\end{array}$ & 1000 & $10^{7-8}$ & 50 & $10^{12}$ \\
\hline Generator studies & Physics studies & 1000 & $10^{7}$ & 30 & $3 \times 10^{11}$ \\
\hline $\begin{array}{l}\text { Paramoterized high- } \\
\text { luminosity studies }\end{array}$ & Piloup at $10^{34}$ & 100 & $10^{6}$ & $10^{3}$ & $10^{11}$ \\
\hline
\end{tabular}


The total requirement through 1999 is approximately $6 \times 10^{13}$ SSCUP-s, which can be produced by a facility of $10^{6}$ SSCUP in two years. While this may seem an ambitious and demanding program, it is not out of the question. Careful planning may reduce the requirements in the later stages, and some studies could be done over a period of several years as the cost of computing drops. A reasonable 8-yr schedule would be to do $0.5 \%$ in 1994 , double each year until the year 2001 , and then level off at $32 \%$ per year. As for storage, we assume that most of the few $\times 10^{10}$ events are not written out, but that $10^{10}$ will be, in compressed $(40 \mathrm{kB})$ format. An additional $10^{8}$ will be written in a full-data format (10 MB), and some smaller number in a very expanded format (100 MB) for high-luminosity studies, for a total of 1.5 PB.

\subsection{SYSTEM DEVELOPMENT}

An important aspect of GEM system development will be the use of test beam activity to exercise the development approach and to evaluate prototype systems. Systems will be specified and developed using well-defined methodologies, which should provide:

- Structured techniques for developing a complete system

- Definition of major and intermediate deliverables

- Description of all data entering and leaving the system

- Description of all processes (including manua) that transform the data

- Definition of all required hardware (CPUs, memory, networks, mass storage)

- Standard schedule and quality-control points

- All managerial planning and implementation activities

- GEM collaboration and project management approval points.

There are many methodologies that satisfy some requirements, ${ }^{5}$ particularly for software, but in JEM we need to develop a total approach to both hardware and software.

\subsubsection{System Development Stages}

GEM will divide the system development process into four major stages: project planning, design, implementation, and maintenance and enhancement.

\section{Project Planning}

In this phase, an initial project plan is developed. This includes a project charter that defines the goals of the project and its scope, and a project initiation plan that codifies the requirements, describes the specific methodology to be used, gives a preliminary analysis and a cost and schedule estimate, and contains an R\&D plan, a management plan, and a review plan.

\section{Project Design}

The project design phase will produce an analysis of the current approach, a new essential model, process specifications and data specifications (or object specifications), a configuration management plan, a testing plan, an implementation plen, and, as appropriate, hardware specifications, a draft operations manual, and a draft procedures manual. The implementation plan will include a schedule of walk-throughs and reviews. During this phase, R\&D will be carried out to supplement the top-down analysis approach with a bottom-up approach, prototyping elements needed in the final system. The major deliverables will be a revised project plan, revised requirements document, specifications documents, draft manuals, reports on the $R \& D$, and an implementation plan that includes revised cost and schedule estimates and a procurement plan.

\section{Implementation}

In this phase-which may be carried out twice, once for a major prototype and again for the final system-a full system is implemented that satisfies the requirements. This includes hardware and software procurement, software development, installation, testing, and a final qualification review. Major deliverables include installation specifications, a final requirements document, data conversion specifications, data and process specifications, libraries (if object-oriented, class libraries), training specifi- 
cations, test specifications, final operations and maintenance manuals, a final review report, an enhancement or upgrade plan, and a working system.

\section{Maintenance/Enhancement}

In this phase, there are two activities: clarification of requirements and rectification of deviations from requirements, and enhancement-i.e., adding new functionality to the system. Enhancements will go through a process of planning, control, and review for production systems.

\subsubsection{Software}

Software for GEM will be developed using software engineering methods, but the methodology will be adjusted to the particular subproject. The intention is to use a coherent strategy across all activities to provide common solutions, to ease development, to reduce costs and to enhance quality and reliability. The chosen methodology must support real-time and concurrent systems and be supported by CASE tools. For systems that are crucial to the success of the program, a full methodology will be applied. There are a variety of development methodologies and life-cycle options that might be adapted to the needs of GEM; ${ }^{6}$ among these, the spiral approach of Boehm 7 seems particularly suitable. An appropriate choice must be made and adopted by the coliaboration.

By applying accepted software engineering practices, we hope to obtain a thorough analysis of requirements, use of a mature methodology, a sound life-cycle model, proper documentation, use of effective CASE tools, ability to keep track of evolving requirements, assured quality, and userfriendliness.

The guiding GEM philosophy is that processes will be modular and will exchange well-defined data structures. This approach allows for the substitution of new algorithms for old, and for easy testing. The software produced should be designed to have functionality, performance, economy, ability to adapt to system evolution, and well-defined external interfaces.

The first approach to software quality and reliability is through modularity of processes and strict definiti. of data objects. The goals for process modularity are:
- Linguistic modularity: the module uses only one language (though it may link to libraries built in other languages)

- Few interfaces: each module communicates with as few others as possible

- Small interfaces: modules should exchange as little information as possible

- Explicit interfaces: when two modules communicate, it should be obvious from the text of both

- Information hiding: all information about the module should be private to the module unless it is specifically declared public, i.e., only the information accessible through the interfaces should be visible or alterable externally

- Open/Closed distmition: a moduie should be either open or closed; that is, subject to modification in its data structures, functions, or interfaces (open), or in finished form that can be compiled and stored in a library for others to use.

Our goals for data objects include:

- Language independence: the ability to manipulate the object with any appropriate language

- Machine independence: the ability to access the object on any appropriate machine

- Self-description: the object is expressed in such a way that its internal structure (integer, floating point, character, and higher-level structure) can be determined from itself.

\subsubsection{Hardware}

Where possible, commercial off-the-shelf components will be used to reduce costs and to provide easy upgrades and scalability. To the greatest extent practical, the computing hardware systems will be unified through the use of data links, networks, and overall management, so that a single computing environment will be available and computing power can be allocated to the highest priority tasks, whether high-level filters, reconstruction, or simulations. This implies a system that crosses the customary boundary between on-line and off-line computing.

Computing systems also will be unified by configuring processors with compatible operating systems and compatible communications, and by using a single mass storage system to obtain 
maximum use of robots and drives and to obtain redundancy and flexibility. The storage system will appear to the user as a single logical file (and/or database) system. Data from the detector will be put directly into the file system. This approach will enhance our ability to keep track of events and their production status, and to associate them reliably with the monitor data that describes the environment in which they were acquired.

\subsubsection{Testing and Validation}

A crucial requirement is that GEM be able to take valid data in its first data run. The reduction by trigger processing, whether Level 1 or higher, is so great that the opportun $y$ for bias is very large. While careful attention to system design, and particularly to good software practices, will be part of the GEM approach, complete confidence can be achieved only by detailed simulation and testing. The main test of computing systems in HEP is that the results of the systems be consonant with expectations, on the basis of physics and the design of the apparanus. The expectations can be codified to some extent, but not in a form that easily le- $/ s$ to the type of requirement normally expected by designers of computing systems. We will use a multistep approach to the problem, in which we develop a set of expectations and use these to test prototypes and parts of the final system and then bring them together in a final synthesis.

The full simulation of the detector, to be developed in 1993-1994, will be used to produce large files of simulated "raw" events of many kinds for a variety of tests, from tests of algorithms to tests of hardware and architectures. Along with these files, databases will be filled with simulated calibrations. Computing systems will also be simulated with discrete simulations as part of the design process, over the period 1994-1996. Prototype systems, particularly for on-line and storage systems, will be built and tested over the period 1994-1997, with the input files and databases being used in the same way that the real data would be. A possibility is to build a hardware-event generator that can feed simulai 1 events into the front of the event filter chain at rates expected from the detector. The analysis process will be simulated, with workshops analyzing data prepared by a special team or teams.
The GEM computing subsystem, along with the SSCL Physics Research Computing Department, should support test beam work with systems that are on an evolutionary path to the final system. The Fermilab test beam activity, to begin in 1995, will be the first such work, while the SSCL test beam activity is expected to begin in 1996. Cosmic ray tests of the complete GEM detector in 1998 and 1999 will provide a crucial exercise of the system. An engineering run (possibly in 1999) will be a major test; and will provide data on which to test systems, as well as many changes in requirements and data structures. The first data run will require very fast turnaround in the early stages to iron out any remaining problems. Full reconstruction within hours or minutes of taking data is the goal.

\subsection{HARDWARE IMPLEMENTATION MODEL}

\subsubsection{Overview}

This model describes an implementation of the GEM computing system developed for use in costing and scheduling the major components, which provides for support of the stores and processes shown in Figures 8-1 to 8-3. It is based on current or predicted technologies; a real implementation will almost certainly differ. The description is divided into five sections: the Global Control System, the on-line computing system, the off-line system, storage systems and the communications system (which includes off-site). The boundaries between these systems are implementation-dependent, and there are several options that could be exercised. In addition, staging is natural for the hardware implementation and is addressed in this section. Software implementation is considered in the succeeding section.

Figure 8-4 shows a model of the hardware of the GEM computing system as planned for 1998-2002. The system is intended to meet the goals outlined in Table 8-2 and elsewhere, and is described in the following paragraphs.

The Global Control System has top-level groups of workstations at the IR and at the main campus and uses the EPICS system for slow control and monitoring. 


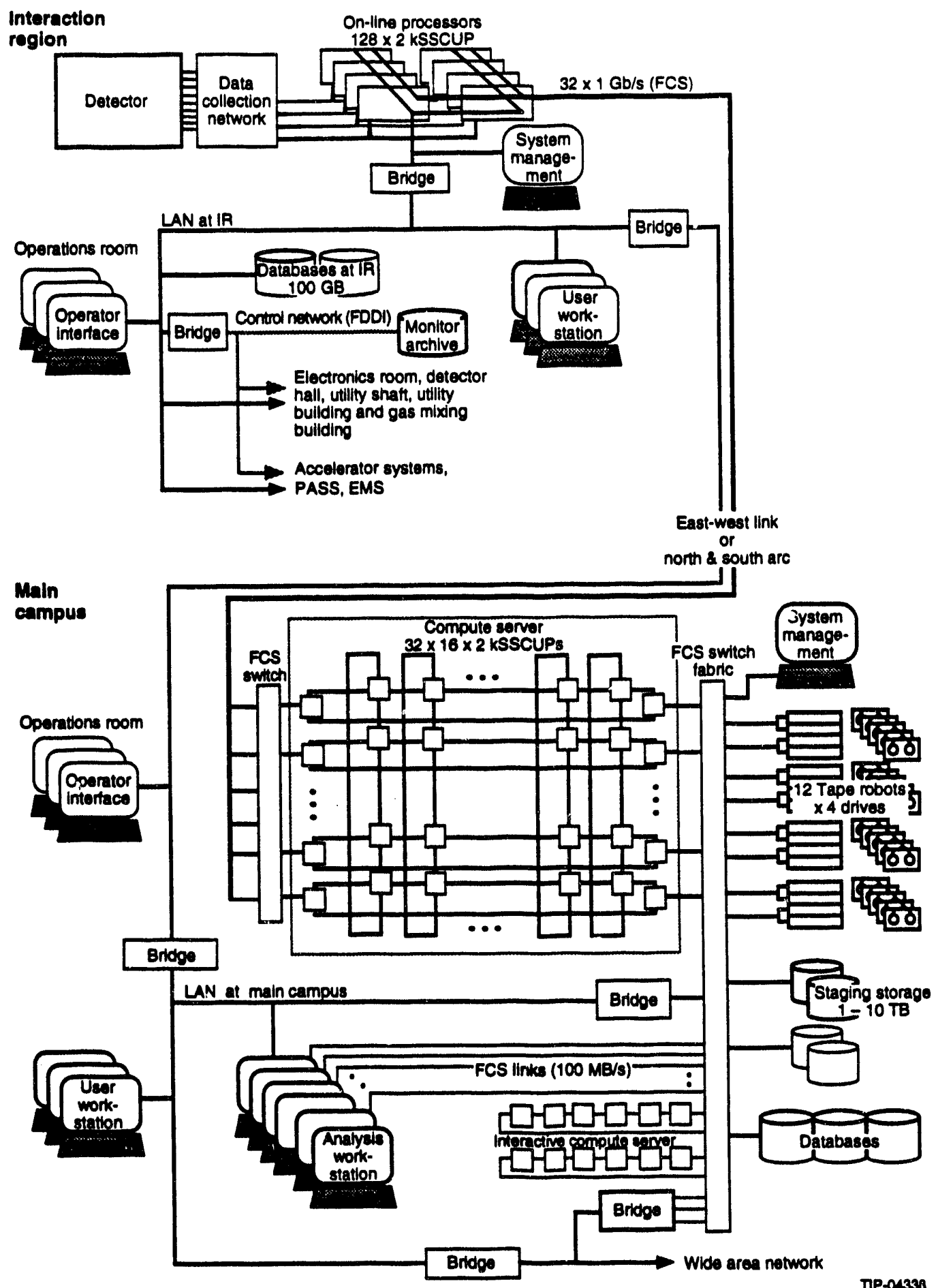

FIG. 8-4. Schematic of implementation of GEM computing system. 
The on-line farm will consist, in 1998, of 64 processor boxes, with a total prower of 70000 SSCUP, in the South Assembly Building (SAB). By 2002, it will evolve to 128 units with total power of 250000 SSCUP.

The database system is distribut he master version resides on a disk (or semiconductor) array of about 1 TB at the main campus, backed by the mass storage system, with a system of about $100 \mathrm{~GB}$ containing copies of the most recent versions at the SAB. The coherence of this copy with the master will be maintained by the database system.

The off-line system consists of 512 processor boxes located in the main campus, tightly coupled to the storage system, with a total power of above 1 MSSCUP. The allocation of these to high-level filters, reconstruction, simulation and analysis will vary. The processes are shown connected in a regular mesh topology 8 to provide high performance, flexibility and ability to handle failures. There may be some distinction between processors intended for simulation and reconstruction and other processors as their $1 / O$ requirements are lower.

The storage system is hierarchical, based on a robotic tape system ${ }^{9}$ with 12 robots with 4 helicalscan tape drives each (48 drives with a total bandwidth of $1.4 \mathrm{~GB} / \mathrm{s}$ ), and a staging disk (or semiconductor) array of greater than $1 \mathrm{~TB}$ at the main campus.

The analysis system consists of an array of processors tightly coupled to the storage system, and may be part of the off-line system, with a power of about 150000 SSCUP. To support user interfaces, there will be distributed workstations, some at the IR, some at the Main Campus, and wife-band network $\mathrm{C}$ snnections outside the SSCL.

These systems are connected by a network with a capacity of $1-4 \mathrm{~GB} / \mathrm{s}$ between systems. The configuration shown for connecting analysis and storage systems follows a recommendation by R. Mount. ${ }^{10}$

All systems have appropriate uninterruptable power supplies (UPS).

There are a number of options for implementation of the DAQ system, event filters and off-line; some are listed here. The terminology used here is:
- Level 2: an event filter uses simple algorithms on a mostly local subset of the data and hence can be implemented in hardware or has a short execution time on a general-purpose computer.

- Level 3: an event filter that applies calibrations and algorithms to refine energy, momentum, and position measurements, reconstructs tracks and other high-level objects, correlates data from all detector subsystems, and can select events based on quantitit; such as invariant mass. These algorithms run on general-purpose machines and take many times longer than the Level 2 algorithms.

- RECO: a full reconstruction of the event, including correlated fitting of tracks, vertexfinding, and shower shape analysis. RECO may also divide the events into physics streams.

Levels 2 and 3 are generally run in the on-line system and RECO in off-line. There are clearly no definite boundaries between these levels, and different strategies can be followed for different triggers to optimize system throughput.

\subsubsection{Technology Assumptions}

The technology trend information in this section is an independent estimate, based on information from a variety of sources." Acquired software costs are not included. We assume that large computing capacity will be obtained in a cost-effective way by building ranches of volumeproduction workstation boxes connected by standard networks. Storage will be done in a hierarchical way with a mix of semiconductor memories, disk arrays, and tape (magnetic or optical) robots. Technology is evolving very rapidly for CPU and memory, and fairly rapidly for disk, tape, and networks. (Network improvement proceeds in large steps at relatively long intervals.) Thus any projection more than two years in the future is likely to be off the mark; however it is as much an error to underestimate what the future will bring as to overestimate it. Our projections are embodied in Table 8-6; these projections assume no breakthroughs, but simply continued evolution of current technologies, for example, towards smaller feature size in VLSI components and larger chip size, and the continued effects of volume production and competition. Note that it is assumed that our designs 
Table 8-6. Technology and cost projoctions.

\begin{tabular}{|c|c|c|c|c|c|c|}
\hline Parameter & 1993 & 1997 & 1998 & 1999 & 2000 & 2003 \\
\hline Single CPU power (kSSCUP) (midrange) & 0.10 & 0.38 & 0.54 & 0.75 & 1.05 & 2.89 \\
\hline Number of CPUs per box & 1 & 2 & 2 & 4 & 4 & 8 \\
\hline CPU cost per box (K\$) & 6 & 7 & 6 & 11 & 9 & 13 \\
\hline Memory size (GB) & 0.06 & 0.3 & 0.5 & 1 & 1 & 3 \\
\hline Memory Cost (K\$/GB) & 100 & 35 & 27 & 21 & 16 & 7 \\
\hline Disk Cost (k\$/GB) & 2 & 1 & 0.8 & 0.6 & 0.5 & 0.3 \\
\hline Mass (slow) memory cost (kS/GB) & 25 & 7 & 5 & 3.5 & 2.4 & 0.9 \\
\hline Mass storage media cost (M\$/PB) & 2 & 0.41 & 0.38 & 0.34 & 0.31 & 0.23 \\
\hline $\begin{array}{l}\text { Mass storage bandwidth cost (M\$/GB/s) } \\
\text { (including robots) }\end{array}$ & 20 & 10 & 8 & 7 & 6 & 3 \\
\hline Number of boxes for 1 MSSCUP & 10,000 & 1,302 & 930 & 332 & 2.37 & 43 \\
\hline CostMSSCUP (MS) & 130 & 24 & 19 & 11 & 6 & 1.5 \\
\hline
\end{tabular}

are flexible enough, and that procurement, installation, and testing can be done quickly enough, such that we can use 1998 technology and cost in 1998.

Based on these projections, the hardware technology that is assumed in costing and optimizing the systems is:

1. Processor boxes with 2000-SSCUP rating and $100 \mathrm{MB} / \mathrm{s}$ VO capability, running UNIX (volume produced as parts for workstations).

2. Expandable mass-storage systems based on helical-scan tape units with $30-\mathrm{MB} / \mathrm{s}$ VO capability, robotics to handle petabytes of data, and a UNIX-compatible interface.

3. Standard data link/network components with $100-\mathrm{MB} / \mathrm{s}$ bandwidth and light-weight protocols to use them.

4. Disk (or semiconductor) arrays supporting up to $10 \mathrm{~TB}$ of data.

\subsubsection{Standards and Integration}

As noted above, there will be an effort to integrate all GEM computing activities. By this we mean that all databases are to be accessible from all parts of the system, that the user environment is the same in all parts, and that software modules are usable in all parts. With the emergence of UNIX (and UNIX-like real time operating systems) and com- munications standards, achieving this goal is largely a matter of detail. To the extent that we use off-the-shelf systems, integration is achieved by selecting compatible operating systems, networks and communications. Hardware standards will be adopted as needed. The following standards related to hardware will be used within GEM computing (these are standards that would be used for a current implementation; we expect standards to evolve):

- The operating system will be a variant of UNIX, with compliance with POSIX 1003.1 (and POSIX 1003.2), and for real-time systems, POSDX 1003.4 D9 and 1003.4a D4 or the final forms of these draft standards

- The OS will give full support to NFS, and in the future to DFS

- Standard network software and hardware standards will be followed.

\subsubsection{Global Control System}

The GEM Global Control System (GCS) will provide top-level functionality for control, monitoring, setup (configuration), and management of all GEM systems, including the cryogenics and the magnet, recording of the state of the system at regular and frequent intervals, and maintenance of a history of changes. The GCS will provide a frame 
work for appropriate control of all systems, including trigger, DAQ, on-line systems and the slow control and monitoring system (SCMS). There will be interfaces to the accelerator systems, providing information flow in both directions. Monitoring will include ability to access a full analysis capability as well as results from the trigger, DAQ, and on-line systems. The GCS will span all activities of GEM that are involved in running the detector. In the environment planned for SSC detectors (including a 10-month running schedule) this implies that the GCS needs to have access to the state of processing farms and the storage system, along with the capability to put them in the desired state.

The GCS will consist of networked workstations, compute servers, and databases. About 50 workstations, 15 compute servers, and a disk array of about $100 \mathrm{~GB}$ will be needed, along with appropriate network capacity. GCS workstations at the IR will be located in the SAB, the electronics shaft, the hall, and the utility building; to mitigate the effect of magnetic field, some of these will use flat panel displays and be diskless. There will also be full GCS capability at the main campus. Figure 8-5 shows a schematic of the GCS implementation, which will be based on the EPICS system as far as practicable.

\section{Overview}

The GCS will be implemented according to applicable standards, including POSIX, Motif, VME, VXI, Underwriters Laboratory and Factory Mutual, in accordance with SSCL policies and standards.

Where possible, GCS functions will be implemented in the SCMS framework, in which the standards followed will be those adopted for the accelerator controls, currently the EPICS framework. ${ }^{12}$ This provides:

- Interface to instrumentation for data acquisition, supervisory control, and steady-state control

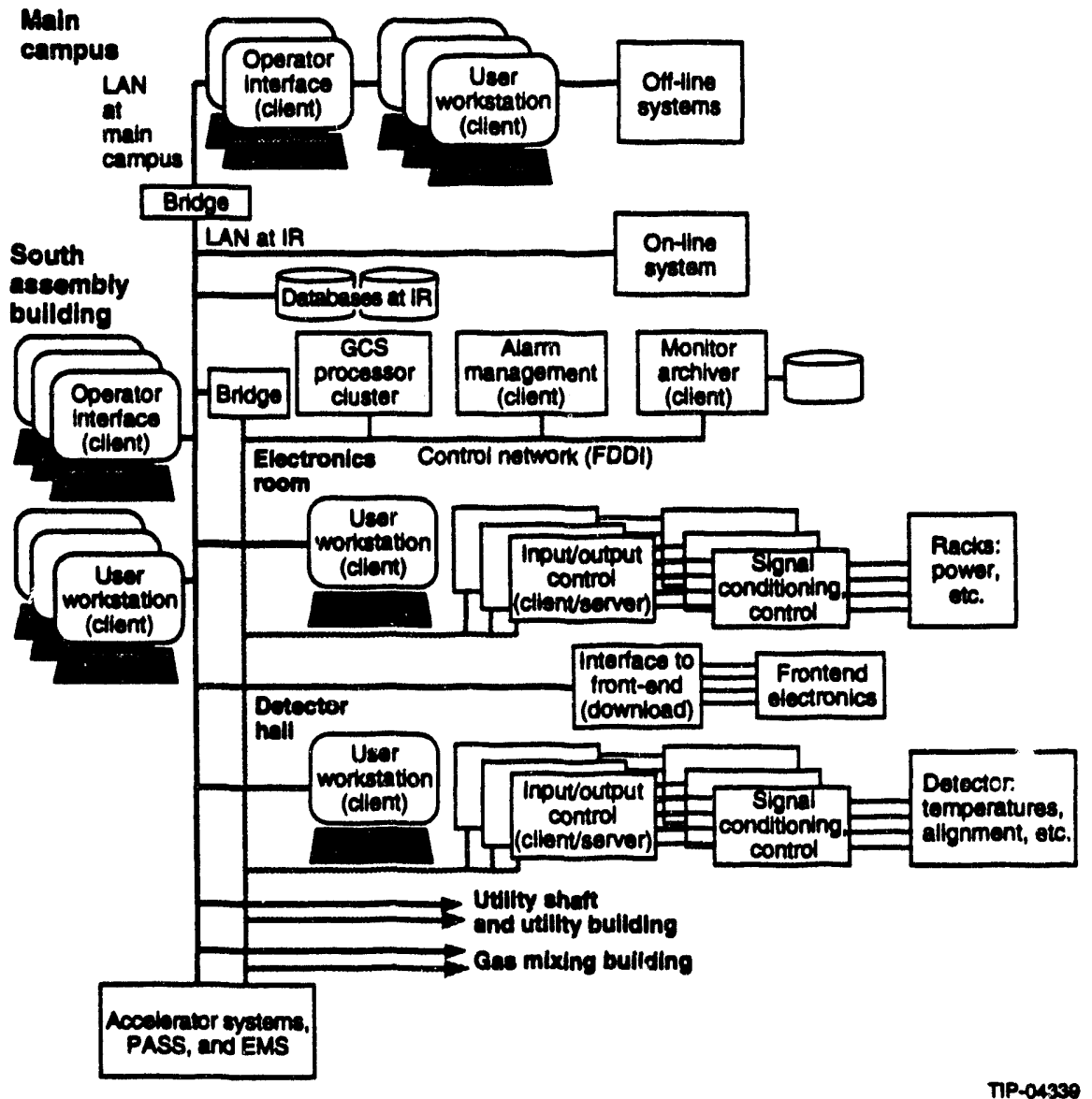

FIG. 8-5. A schematic of an EPICS-based GCS. 
- Operator interface to all control system parameters throu $h$ interacti re display editor

- Data logging

- Sequential control through a state definition language

- Routines for interfacing the control system data to data analysis, adaptive control algorithms, and any other functions not provided in the control system.

The EPICS system is currently implemented using UNIX and VXWorks, and it supports VME, VXI, Allen-Bradley Serial Bus, Bit-bus, and CAMAC. It provides "Channel Access" functions, an Input/Output Controller, an Operator Interface, an Archiver, an Alarm Manager, and a Sequencer. The EPICS system will be used for all GEM projects where there is a need for control and monitoring.

Prototype work is needed to develop a system that would integrate EPICS with other elements of the GEM computing system, but there seems to be no obstacle.

\section{Detector Operations Rooms}

Each detector operations room will be able to accommodate up to 15 people; access will be limited. A floor space of $100 \mathrm{~m}^{2}$ is a minimum; this space is based on the DO control room. In addition, there will be two other rooms located close by: a workspace to accommodate up to 24 people working with GCS and the subsystems, and a video conference room with space for 30 people. The Main Campus Operations room may have an observation capability. The Operations Room at the $\mathbb{I R}$ will be located in the South Assembly Building (SAB), at the west end, where the field from the GEM magnet is expected to be at or below the 1-G level.

\section{CCTV system}

As part of the GCS, a Closed Circuit Television (CCTV) system will be installed in all areas at the IR. There will be frequent $(1 \mathrm{~Hz})$ time-stamped recording of the images and the ability to "grab" frames to display them or insert them in the log book.

\subsubsection{On-line System}

\section{Overview}

The on-line processing system is placed between the DAQ and the off-line systems. Thus the precise requirements depend on the options exer- cised. At a minimum, the on-line system must transport the data to the off-line and storage systems. A variety of options includes:

- Option 1. Data collection network with combined Level $2 / 3$ processor ranch. This is the preferred option for GEM described in Chapter 7 . The Level 3 prosessing is carried out in the Level $2 / 3$ ranch, and events are sent directly to off-line and storage at a rate of about $100 \mathrm{MB} / \mathrm{s}$.

- Option 2. DAQ/Level 2 and separate Level 3 ranch. In this case the data rate from the Level 2 to Level 3 could be up to $4 \mathrm{~GB} / \mathrm{s}$; with care the link from Level 3 to off-line and storage could be made in such a way that some of the flexibility of Option 3 is obtained.

- Option 3. Separate DAQ/Level 2 and combined Level 3/off-line ranch. The option of combining the Level 3 with the off-line has been simulated ${ }^{13}$ and found to behave in a predictable fashion against fluctuations in data rate. Also, an optimum configuration of robots and high-speed drives was found. This pernits the full performance of the off-line and storage systems to be available to accommodate fluctuations in detector-generated loads, while also allowing the Level 3 capacity to be easily available for other work when the detector load is low.

For the baseline (Option 1), the total CPU power required is $2.3 \times 10^{5}$ SSCUP. The input bandwidth must be of the order of $15 \mathrm{~GB} / \mathrm{s}$ to Level 2 and much smaller for Level 3. There must be adequate management mechanisms to start the system in a few minutes and to reset it in case of an error. To most easily interface with other systems, a standard network port will be available for each processor. Output rates up to $4 \mathrm{~GB} / \mathrm{s}$ must be handled to allow for passing processing to the off-line. A farm of 128 off-the-shelf processor boxes with two $100 \mathrm{MB} / \mathrm{s}$-interfaces will satisfy the requirements for the year 2002; in 1998 we will use a smaller number of units (64) and add other units as costs drop and requirements grow. This farm will be located in the South Assembly Building.

The Level 2 system must be able to be configured via the GCS, and will conform to GEM guidelines for hardware and software. To the extent 
that Level 2 uses general-purpose CPUs that are a major investment, it will be so designed that standard computer management tools can access it for other tasks, such as simulations.

Because any error made in the trigger/filter sequence is not recoverable, attention must be paid not only to the hardware a $A$ software but also to access control and management, so that the on-line system is protected from inadvertent changes.

\section{Event Filters (Level 3)}

Chapter 7 describes the Level 1 trigger and suggests possible algorithms for a Level 2 event filter. However, the combination of Level 1 and Level 2 may not reduce the overall rate to the desired $100 \mathrm{~Hz}$ or so. So additional filter stages are necessary, depending on the particular trigger combination. These additional filter stages (collectively referred to as Level 3) will have complete events available to them, may apply prescale factors, and will have varying degrees of sophistication up to full reconstruction. They will be steered by the results of previous stages; one characteristic of Level 3 is the matching of physics processes to triggers. Examples of what can be done at Level 3 include refinement of energy measurement in the calorimeter, calculation of $E_{T}$ using the forward calorimeter, full showershape analysis, track finding in the central tracker, veriex finding, possible $\tau$ identification, muon momentum fitting, sharpening of thresholds as a result of the improvements, and cuts based on correlations between systems (e.g., isolation cuts based on the calorimeter and tracker, the muon system and the calorimeter, or matching of muon and central tracker).

In GEM, events are processed to a considerable extent in the front end (zero suppression and signal processing in the calorimeter, triggering on track segments in the muon system) thus reducing demands on pattern recognition. The fact that many events will be processed according to multiple scenarios increases the processing time.

We consider two processes that produce high trigger rates and for which we have estimated processing times: $H \rightarrow \gamma \gamma$ and $E_{T}$ signatures for SUSY.
$H \rightarrow \gamma \gamma$

For this process, Level 3 will use cuts as described in Section 2.3.3, to reject the $\gamma$-jet background to approximately the level of the irreducible background ( $200 \mathrm{pb})$. These include the full shower-shape algorithm based on the $\theta$-measuring calorimeter strips and the full longitudinal and transverse segmentation of the calorimeter, energydependent isolation cuts, a full charged-particle isolation cut based on the central tracker, and other cuts discussed in Chapter 2 . The $\gamma \gamma$-invariant mass will be reconstructed and limited to the range $>70 \mathrm{GeV} / \mathrm{c}^{2}$.

\section{$E_{T}$ signature for SUSY}

Events from the $E_{T}$ trigger (number 7 in Table 7-8) will be cut on transverse sphericity $\left(S_{T}>\right.$ 0.2 ), and at least two jets with $p_{T}>75 \mathrm{GeV} / \mathrm{c}$ with $|\eta|<3$ will be required (see Section 2.7.1). The high-p $p_{T}$ jet trigger $\left(J_{80}\right)$ and the multi-jet trigger $\left(4 J_{30}\right)$ may also contain SUSY events; these will be processed in the same way. Events with $p_{T}<150 \mathrm{GeV} / \mathrm{c}$ will be prescaled. These cuts should reduce the cross section for background events to about 1-10 nb, and the signal for a gluino mass of about $300 \mathrm{GeV} / \mathrm{c}^{2}$ to $1 \mathrm{nb}$. The total detected cross section for all SUSY processes will be several nanobarns.

Filtering events from the gemfast simulation, that satisfy the Level 1 trigger intended for the $H^{0} \rightarrow y \gamma$ study, takes 1 SSCUP-s to reject QCD background by a factor of 3-10 (say at Level 2), and 3 SSCUP-s to reduce QCD background to close to the irreducible $\gamma y$-background level (at Level 3). ${ }^{31}$ Although these times seem very short-compared with CDF's experience of around 20-30 SSCUP-s per event for the filter-it should be noted that this filter sequence is based entirely on the calorimeter array, where pattern recognition, at the level used in Level 2, is straightforward. For the muon system, about 30 SSCUP-s are required to reconstruct and fit a two-muon event from the SIGEM full simulation. Based on these numbers, estimates of 30-100 SSCUP-s ${ }^{14}$ per event seem reasonable, and agree with $\mathrm{CDF}$ and $\mathrm{D} 0$ experience. With a factor of 30 reduction by Level 2 , this implies a requirement of 100 000-300 000 SSCUP for Level 3. 
Databases

The databases that must be available to the GCS and the on-line system are the configuration database, the calibration database, the processing database, and the monitor database. The access requirements for the downloading process of configuration data to the front-end, for setting up the on-line system, and for updating the monitor database will be satisfied by a disk array of about $100 \mathrm{~GB}$ at the IR to support local copies of current data, which are to be maintained coherent with the master databases.

\section{Data Links}

The data link to the on-line system from the electronics room consists of 128 fibers with $1 \mathrm{~Gb} / \mathrm{s}$-bandwidth supporting a total data rate of about $10 \mathrm{~GB} / \mathrm{s}$; this link will use special protocols (Chapter 7). From the on-line system to the off-line and storage system, a standard data link with a bandwidth of $4 \mathrm{~GB} / \mathrm{s}$ will be implemented. Connecting the systems at the IR, there will be a LAN, possibly implemented in FDDI and Ethernet, with $>10-\mathrm{MB} / \mathrm{s}$ capability.

\subsubsection{Off-line System}

The off-line compute server will be capable of supporting event filters (Level 3), an initial reconstruction pass (RECO), up to two simultaneously executing subsequent reconstruction passes, detailed fitting, creation of event samples, and compute-intensive analysis tasks. The compute server will consist of a ranch of about 500 processors, with a total capacity, in the year 2001, of the order of $10^{6}$ SSCUP. The compute capacity would be allocated as follows: 0-200000 SSCUP to Level 3; 200000 to RECO; 200000 to a second reconstruction pass; $200000-400000$ to simulations and 200000 for sample creation and analysis support. The total network capacity required is of the order of $10 \mathrm{~GB} / \mathrm{s}$, with up to $4 \mathrm{~GB} / \mathrm{s}$ from the $\mathrm{GEM}$ on-line system, and 2-4 GB/s to the storage system. While these bandwidths are reasonable, the emerging standards (such as Fibre Channel) generally limit the length of (inexpensive) connections to less than $10 \mathrm{~km}$. For these and other reasons, it would be most convenient for the detectors if the off-line systems were sited at the $\mathbb{I}$.

\section{Analysis Systems}

Analysis will be carried out on-site and offsite. The intention is to centralize data storage . nd computational capability at the SSCL, and $t$ establish adequate networking capability to all GEM institutions to support this model. A capacity of $1 \mathrm{Mbit} / \mathrm{s}$ per active user may be adequate for many applications; $10 \mathrm{MB} / \mathrm{s}$ is desirable. For work at SSCL, a group of 50-100 workstations with a 1-2 GB/s-link to the storage system and computer server would appear to be adequate. The compute server would support compute-intensive analysis tasks for on-site and off-site users. Off-site access can be of several kinds: transfer of very small numbers of full events for local reconstruction and/or viewing, transfer of very compressed files for rare processes, and viewing of analysis results (histograms, etc.). The use of DFS (the Distributed File System under development by the OSF) over the WAN will impose a load which is not yet estimated.

\subsubsection{Storage Systems}

The GEM event storage system will be based, where possible, on industry-standard approaches (e.g., the emerging IEEE Mass Storage model), and will likely be implemented in hardware as a hierarchical system: semiconductor memories, disk arrays, and tape robots. The requirement for input bandwidth (writing) is detailed in Section 8.2. The requirement for output (reading) is set by the response time desired for access to data. We have chosen to specify a bandwidth of at least $1 \mathrm{~GB} / \mathrm{s}$ for reading. Using this bandwidth, a scan of a 1-PB data store would take about two weeks.

The format of the store can follow one of two models: the file model in which data is kept in sequential files and data summary files are created to speed access for specific purposes, and the database model in which events are regarded as records in a database to be accessed through a query language. The database approach has the advantage that information need not be duplicated, but the disadvantage that existing technology does not support the volume and variety of data we expect. 
A system using 12-18 robots, each with 3-4 D2 helical-scan tape recorders (capable of $30 \mathrm{MB} / \mathrm{s}$ transfer rate and 50-s seek time) and a staging area of $160 \mathrm{~GB}$ has been modeled for the GEM storage system and would appear to satisfy many requirements ${ }^{11}$ when using the file model. The maximum configuration ( 72 drives) would have an V/O bandwidth of $2 \mathrm{~GB} / \mathrm{s}$. To make event samples rapidly available, a rapid-access staging area of 1 TB or greater may be needed (implemented in disk or semiconductor memory); the cost of this may be a major item in the overall cost.

If duplication of raw data is required for backup purposes (with copies stored separately) or if copying of data summary files to tape is required for distribution to other institutions, more drives will be needed.

The databases (configuration, calibration, monitoring, log book, processing) must be distributed so that all systems can access them with tolerable delays. This will require disk arrays at the IR and at the off-line site. Because of the volume of data, it must be possible to migrate infrequently accessed data to the mass storage system.

\subsubsection{Data Links, Networks, Communications}

Data-transport requirements can be separated into three categories: data links for point-to-point bulk transport of data, networks (or fabrics) for multi-point communications, and other forms of links for human communications. Emerging standards (e.8., B-ISDN, SONET, and ATM) combine some of these, but it is still economical to use different approaches for the different functions.

\section{Data Links}

Data links will transport data from Level 1 to Level 2, from Level 2 to Level 3, and from Level 3 to the off-line. To keep options open and allow the use of lower-cost links, there will be duct capacity from the electronic shaft to the SAB to contain up to 4000 fibers. GEM has requested that two 4 -in. ducts be brought to the SAB from the hub at IR5. This will provide access to both north and south arcs for safety systems and accelerator signals, and access around the ring to the west campus. GEM's share of the ducts in the collider tunnel is one (1.25 in.) inner duct, in each of the north and south arcs, which can support 2.4-4.8 GB/s data transfer capability. A surface east-west link substituting for this capacity is a possibility.

\section{On-site Networks}

A local area network at the IR is required between the Gas Mixing Building, the Utility Building, the Utility Shaft, the Detector Hall, the Electronics Shaft, the $S A B$, and the NAB, to support general needs, as well as specific needs of the GCS. The requested duct capacity is adequate to support these needs.

\section{Off-site Networks}

The GEM model of centralizing data and computing is very dependent on networking capability, including networks to non-U.S. institutions. The SSCL must work with other institutions to make the necessary bandwidth available. With approximately 100 institutions and as many as 100 active users, we envision using a total WAN bandwidth of order $1 \mathrm{~GB} / \mathrm{s}$, with $10-20 \mathrm{MB} / \mathrm{s}$ capability to each institution. The major network loads will come from display of analysis results, software development and distribution, moving small numbers of events for detailed study to a local facility, and transferring highly-compressed event samples for local processing and display. Some of these functions may be supported by DSF (Distributed File System), which will impose some requirements on network bandwidth.

\section{Communications}

As noted above, the dispersed nature of the SSC site makes a carefully planned human communications system a necessity. This will be implemented by video-conference capability between the detector hall, the electronics shaft, the SAB, and the main campus. Some of this will be supported by standard video-conference technology; use of workstation-based multi-media capability is also planned. A CCTV system to be implemented as part of the GCS will provide additional communications capability. 


\subsubsection{Evolution and Optimization}

Table 8-2 shows how the demands on the computing systems will evolve if the turn-on of the collider and detector follow the proposed scenario. The demand on Level 2/3 computing power will grow, RECO requirements will grow slowly, and input rate to storage systems will remain roughly constant, while output requirements for analysis will likely grow. The software needed is the same, independent of the scale of the hardware. Thus, the strategy for hardware must be to compromise between buying systems early enough to have them fully integrated and debugged and a just-in-time strategy that minimizes the cost. A specific approach should be defined about 3 yrs. before turn-on. In the meantime, the approach is to use an approximately fixed cost for the systems needed in the year 2002 based on the extrapolations in Table 8-6.

\subsection{SOFTWARE IMPLEMENTATION MODEL}

This section describes an approach to building the necessary software, with an indication of direction of implementation. The software considered is the set of common tools and public software, rather than the analysis software that will be written by individuals. An important part of the public software will be a general framework for individual efforts. A decision with far-reaching impact on most GEM software is whether the approach to data storage is based on files or on a database approach. The current approach is to use a database approach for the configuration, calibration, monitor, $\log$ book, and production databases, while using a file approach for the event data and the event samples, though these files will be indexed in a database.

GEM will use a data-oriented and modular approach to software implementation, as is done for gemgen (see section 8.8.1). The data structures used in production systems will be defined and documented in computer-accessible format (for example, as a data dictionary). Modular processes will then have read access to their input data and will be able to write only the required output data structure. Processes will be modular on the level of well-defined single tasks. The software structure will be designed to make effective use of both looselycoupled systems and the multiple-CPU systems that are envisaged to be available in the future. Thus there are two areas where work is needed to ensure performance and reliability: communication of data between processes and control of interacting tasks across multiple processors. Also, data shared across the computing system will be placed in the databases described above (configuration, calibration, monitor, logbook, production) and hence the implementation of an appropriate database approach is essential. Support of this approach defines a framework for GEM software, with an appropriate set of tasks.

There are performance concerns resulting from this approach: copying data between processes consumes CPU and memory resources, and modularity at the function or subroutine level also results in overhead. However, in most cases, this will be a small factor and hardware progress will reduce these concerns. In high performance systems, like Level 2 , these concerns may be important.

Software implementation will be accomplished through a system of project teams. Software tasks are divided among areas that recognize various tasks specific to computing and computing tasks as well as to the detector-related interests of the physicists. The areas are Framework for coordination and common tools; Detector subsystems to reflect interests and expertise; Global Physics for overall tasks, Global Controls for the GCS system, On-line computing for the on-line system. A distinct set of tasks, Computing systems tasks, is concerned with physical impllementation.

\subsubsection{Standards and Integration}

There is almost no function needed for off-line computing that is not also needed for on-line computing. This includes full analysis, access to the monitor, processing, calibration and configuration databases, event displays, control of processing ranches, etc. To minimize software development costs and allow the integration of all systems, funstions should be implemented following appropriate standards. For software, the standards that would be used for a current implementation are:

1. The operating system (OS) will be a variant of UNIX, with compliance with POSIX 1003.1 (and POSLX 1003.2), and for real-time systems, POS.LX 1003.4 D9 and 1003.4a D4, or the final forms of these draft standards.

2. The GUI will use $X^{T M}$ and Motif ${ }^{T M}$. 
3. The OS will give full support to NFS, and in the future to DFS.

4. Languages used will be confined to $F 77$ (with some extensions), $\mathrm{C}$, and $\mathrm{C}++$.

5. Standard network protocols will be supported (e.g., TCP/IP).

6. The code management system will use CVS ${ }^{15}$ and follow a practice of explicit releases.

7. GEM will use a common Data Model, Data Description Language and Data Dictioncry (yet to be defined).

8. GEM coding standards and practices will be developed and followed. ${ }^{16}$

On the language issue, FORTRAN 90 is omitted since it is felt that $\mathrm{C}$ and $\mathrm{C}++$ will provide the functionality beyond F77 that is needed to support GEM computing, and will have wide industry support. The implementation will provide a framework in which processes written in FORTRAN can access needed data structures.

\subsubsection{Framework}

The GEM software framework will provide support for all GEM computing tasks. GEM has developed a preliminary implementation of some parts of a software framework using UNIX and Free Software Foundation tools. This is expressed in a software base of utilities, the gemfast program, the gemgen generator interface package, code management and distribution by CVS, and a self-documentation system. The software base is maintained on five UNIX platforms. This approach will be developed and extended. The Framework tasks include: coordinating the development of requirements, making a top-level analysis of the systems and possible implementations, making a model of the data, supporting strategies for validation of production processes, and providing general tools and applications. outline:

Within the Framework the subtasks are, in

- Requirements development

- System Analysis and Design

- Data modeling

- Database Requirements
- Quality Assurance strategies

- Software Framework

- User interface

- Operating System interface layer

- Utilities

- Analysis tools

- Database access tools

- Documentation tools

- Parallel Processing and Interprocess Communication

- Software Engineering

- CASE tools

- Languages

- Integrated Development Environment (IDE)

- Methodologies

- Database Implementation

- Implementation of production systems

- Validation, verification and testing.

\subsubsection{Detector Subsystems}

The detector subsystem tasks are to provide simulations that fit into the general framework; trigger, event filter, and pattem recognition strategies for the subdetector; a model of the data from the detector; and requirements for the framework, the databases, and the computing capacity. Detector subsystems are the central tracker, the calorimeter, the muon and magnet system, electronics/trigger/ $D A Q$, and shielding and beam pipe. The groups working in these areas will evolve from the existing subsystem software groups and will exploit their knowledge of the detector hardware. The detector subsystem groups will also populate and maintain the configuration and calibration data bases.

\subsubsection{Global Physics Tasks and Validation}

The Global Physics tasks are to ensure that the parts come together to provide a whole that produces valid results. The group concerned with these issues will evolve from the existing Physics/Simulation group, with increased participation from the subsystem groups. The main task areas are:

- Data description

- Interaction generators 
- Event filters and triggers

- Global simulation

- Global reconstruction and fitting

- Particle identification

- Creation of data samples (DSF)

- Physics analysis

- Overall validation.

\subsubsection{Global Control System}

The GCS software will be implemented within two frameworks: one defined by the overall GEM framework, and the other defined by the EPICS system. This will be a major effort as we must provide:

- Support for downloading of frontend and trigger electronics

- Interfaces to the DAQ system, and to on-line and off-line systems

- Processes to support control and monitor functions, including adaptive and/or expert system feedback.

- Processes to display data from a variety of sources, including off-line analysis

- Support of the electronic logbook

- Interfaces to Accelerator and Safety systems

- Control of access to GCS functions and remote operation.

The GCS will be responsible for the monitor archive and the electronic log book.

\subsubsection{On-line Computing System}

The software for the on-line system (ONS) will be partly the responsibility of the DAQ group, for example, for event building. Event filter algorithms will be a Global Physics task, while transport to the off-line system and mass storage will be a Computing subsystem task. The GEM framework will be used to implement the appropriate functions. A requirement placed on on-line software is that event formatting and selection software shall be capable of being run in the off-line environment for validation and testing. The ONS will be responsible for updating the on-line part of the production database.

\subsubsection{Off-line System}

Software for the off-line system will be implemented by a combination of the Framework group, the Detector subsystem groups and the Global Physics groups.

\subsubsection{Interface Systems}

Software for interfaces between GEM systems will be implemented by the subsystem group that has the most stringent requirements. These interfaces will include data links from on-line to off-line systems, interfaces with hardware test systems, and the interface to off-site computing.

\subsubsection{Scale of Software Development}

A detailed division of software responsibilities and an indication of approach is given later; first we discuss some general requirements. An approximate estimate (based on $\mathrm{H1}, \mathrm{DO}$, and L3) of the software in terms of "lines of code" is given in Table 8-7 according to categories used in those experiments; the total is less than $10^{6}$. However, due to the use of rapid prototyping, the evolution of user requirements, and changes in data structures, the total number of lines of code produced may be twice this. Table 8-8 shows the number of lines of code specified as a function of time, along with the manpower needed from various bodies.

Table 8-7. Estimated lines of code in the final GEM system.

\begin{tabular}{lc}
\hline Category & Lines of Code \\
\hline GCS sottware & $100 \mathrm{~K}$ \\
On-line software & $50 \mathrm{~K}$ \\
Generators (GEM-specific code) & $20 \mathrm{~K}$ \\
Simulation (GEM-specific code) & $150 \mathrm{~K}$ \\
Reconstruction & $200 \mathrm{~K}$ \\
Physics ana!ysis & $50 \mathrm{~K}$ \\
Event display and graphics & $40 \mathrm{k}$ \\
Database & $50 \mathrm{k}$ \\
Framework & $50 \mathrm{k}$ \\
Utilities & $100 \mathrm{k}$ \\
Special OS interfaces and drivers & $50 \mathrm{k}$ \\
Event filters & $50 \mathrm{~K}$ \\
\hline
\end{tabular}


Based on industry standard methods of estimating manpower, ${ }^{17}$ the manpower required is of the order of 500 FTE-yr. Some of this will come from PRCD (some will be joint with SDC), and the remainder from the SSCL DAQ group, the GEM project, the collaboration, and, possibly, industry. The effort from PRCD should peak at about 40 FTEs over the 1996-1998 period, while the remainder should rise from the current level to about 70 FTEs in 1997, maintaining that level until the year 2000. Table 8-8 gives a very approximate manpower scenario that is consistent with PRCD and collaboration plans.

\subsubsection{Quality Assurance}

Quality is primarily assured in the design and implementation of systems, rather than through testing, important as testing is. The level of quality assurance for GEM systems will depend on the risks (technical, cost and schedule) associated with each project. Higher cost and risk projects will be subject to more detailed and more frequent checks. Quality assurance activities will be built into the analysis, design and implementation phases. Though quality assurance activities do use project resources, the cost is less than that of correcting errors at the operation phase.

Techniques to assure quality will include documentation of requirements and analyses, documented designs and specifications, reviews, walkthroughs, validation, verification, and testing. Hardware and software systems will be exercised with sample data and the performance and results compared with expectations and requirements.
Quality assurance activities will be planned and resources will be allocated to ensure that they are carried out.

\subsection{DEVELOPMENT PROJECTS}

The main goal of these development projects is to make steady progress towards a final system through a process of prototyping and using elements expected to be part of the final system. Involvement of GEM with SDC and PRCD projects will be essential. The GEM computing group has several current projects under way and will add more as the group increases in strength. One example of this "prototyping and use" approach is the use of the code management system CVS for support of the fast simulation package incorporating gemfast and gemgen, ${ }^{18}$ as described in Section 8.8.1. This section briefly describes existing and proposed projects that need support. Some of these projects will overlap with existing PRCD and SDC projects ${ }^{19}$ and the PASS project. ${ }^{20}$

The current status and future plans for detector simulations are described in the following section. Succeeding sections give brief descriptions or titles of other development projects for which support is requested.

\subsubsection{Detector Simulations}

Current simulations fall into two groups: subdetector simulations and global simulations. The subdetector simulations were mainly developed during the process of detector R\&D by the various subdetector groups, and are described in their respective chapters. There are three global simulations in current use: gemfast, SIGEM, and UAZMIX.

Table 8-8. Estimated manpower profile for software development, in FTE by year.

\begin{tabular}{lrrrrrrrr}
\hline Source & 1993 & 1994 & 1995 & 1996 & 1997 & 1998 & 1999 & 2000 \\
\hline PRCD & 4 & 10 & 20 & 40 & 40 & 40 & 30 & 20 \\
GEM Project & 1 & 4 & 8 & 12 & 16 & 16 & 16 & 12 \\
DAQ & 1 & 3 & 6 & 6 & 6 & 6 & 6 & 6 \\
SSCL Physics & 2 & 4 & 8 & 8 & 10 & 10 & 10 & 12 \\
Collaboration & 25 & 30 & 35 & 40 & 50 & 50 & 55 & 60 \\
\hline
\end{tabular}


gemfast is described in detail in Chapter 2; it is a non-GEANT system of user-callable subroutines that parameterize the detector response and that have short execution times. The parameterizations are based on subdetector simulations and beam test data. Energy deposition is done with a modified version of GFLASH, and there is support for pileup in the calorimeter. The structure of gemfast and the generator interface gemgen ${ }^{18}$ is modular; together they form a set of independent tasks that communicate via UNIX operating system services, as shown in Figure 8-6. This structure is one we intend to follow for GEM software to gain the many advantages of separation of tasks, including the possibility of parallelism. Both gemfast and gemgen were initially developed at the SSCL and will be supportad at the SSCL until a replacement is developed as described . Llow. These programs are maintained using CVS.

UAZMIX is a GEANT-based mixture-level program developed at the University of Arizona. It describes the detector in terms of fairly simple volumes and mixtures rather than full detector detail. ${ }^{21}$ The relation between energy deposited and the measured energy is adjusted to agree with beam tests and detailed simulations.

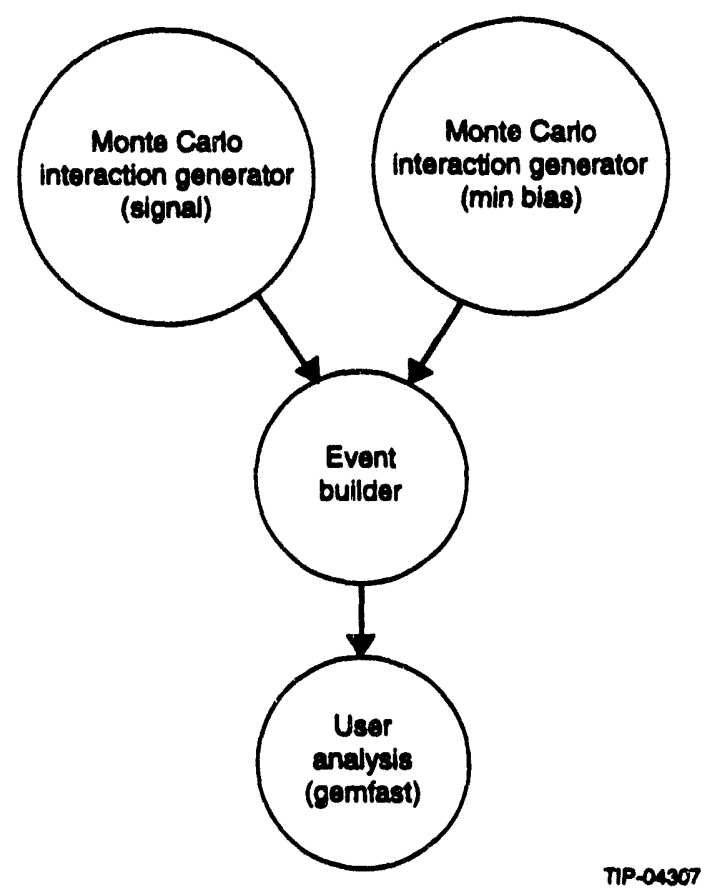

FIG. 8-6. The structure of gemgen and gemfast as separate tasks, communicating through operating system services.
SIGEM is a full and detailed global GEANT simulation that takes advantage of the CERN framework and is maintained with PATCHY. Data structures are passed between the GEANT stages (materials and rotations definition, geometry definition, tracking, hits, etc.) via ZEBRA banks. These banks can be written out to be used by separate tasks if so desired-for example, files of hit banks have been used to study the performance of different reconstruction algorithms in the muon system. SIGEM also has an option for parameterized energy deposition, using algorithms developed for $\mathrm{H} 1$ at DESY and ported for GEM, as a new version of GFLASH, by one of the developers. ${ }^{22}$ SIGEM will be supported at the SSCL until it is replaced.

We aim to develop a global simulation package that draws on and incorporates as much as possible of the simulation code written so far but places this in a unified, coherent structure. The goal of the package is to provide simulated data to address, with as much precision as needed, issues such as:

- Detailed calorimeter studies of jet rejection. reconstruction, weighting, cracks, and the effects of pileup

- Pattern recognition, occupancy in the tracker. and matching the tracker with the muon system

- Studies of event filter algorithms and triggering

- Backgrounds from pileup, neutrons, and gammas

- Generation of test data for development of reconstruction code, validation, and visualiza. tion tools

- Studies of physics performance.

These topics do not all require simulation of each component of the detector in full detail; the global simulation should support three levels of detail. A parameterized simulation derived from gemfast (see Section 2.2) will provide the fastest results. An intermediate level of detail is provided by a "mixture-level" GEANT simulation such as the existing UAZMIX program. Finally, the greatest accuracy (and slowest execution speed) is derived from a fully detailed model incorporating every geometrical element, as in SIGEM. The latter level of detail should also support parameterized (GFLASH) showers. We aim to incorporate as much code as possible from gemfast, UAZMIX, and 
SIGEM. Support for maintenance, portability, and distribution of simulation programs should come from PRCD.

\subsubsection{Global Physics Tasks}

Besides the effort on detector simulation described above, there will be projects in the areas listed in Section 8.7.4. One essential project is the development of detailed descriptions of the data used or produced in each stage of the GEM computing system. Most of the tasks in this category will be carried out by members of the collaboration outside the SSCL.

\subsubsection{Software Framework}

This is the project to develop the GEM framework, as described in section 8.7.2, and for which the bulk of the effort will be provided by PRCD. Prototylye frameworks will be developed and used to suppritt test beam work, especially for analysis. A part of the effort will be to work with PRCD to develop requirements for the framework and its components. Specific elements of the framework which will be addressed are:

- Data modeling/management/structures

- Interprocess communication and control (IPC)

- Remote procedure calling (RPC)

- Parallel processing support

- Mixed langusge programming.

We will also establish a $\mathrm{C}++$ pilot project in cooperation with PRCD, where the particular issues are the development of appropriate class libraries, developing methods of supporting object persistence and methods for object transport between processes.

\subsubsection{Database}

Appropriate databases are now crucial to HEP and our system model defines configuration, calluration, monitor and production databases as well as the electronic log book. Support from PRCD is requested for:

- Evaluation and prototyping of database approaches for these databases

- Support for a geometry database (an example of a configuration database) in the short term for simulation support

- Database for test beam support
- R\&D on approaches for the event data, both file-based and database

- R\&D on multimedia approaches, including an electronic logbook.

\subsubsection{Computing Systems}

Areas of computing systems that are particularly important to GEM and for which some support is needed from PRCD are listed below. Of particular importance is development of hardware and software for support of test beam work at FNAL and elsewhere with a fully integrated system that demonstrates the overall features of a final system. Some of these projects might be suitable for a joint SSCLvendor collaboration or for external funding, as being explored by PRCD.

- High-capacity networking

- High speed VO for standard workstations

- Test generator for validation for hardware systems

- On-line/off-line integration

- Mass storage systems.

\subsection{SCHEDULE}

The schedule for system development must provide:

- Test beam support in controls, on-line, off-line, and storage for FNAL work beginning in 1995

- Controls and monitor support for detector construction in 1996

- Simulation support from the present to 2000 and beyond

- Support of cosmic ray tests in 1998

- Support of a full-detector engineering run in 1999

- Full capability in the year 2000 .

Some items on the critical paths are development of requirements, development of a data model, selection of data management techniques and database approach, and development of a basic framework. Work also needs to be done on mass-storage models and system-management methods to handle on-line/off-line integration. 


\subsection{MANAGEMENT}

\subsubsection{Overview}

The management structure follows the GEM model and consist of a subsystem leader, a steering committee, a project manager and a chief engineer; the responsibility chain is defined in Chapter 14. In the case of the computing subsystem, there are groups and group leaders; within groups there are projects and project leaders. The computing subsystem leader (CSL) is responsible for the success of the computing systems.

The groups will consist of personnel drawn from a variety of areas: the collaboration, the GEM project, the PR computing department, and elsewhere. Line management for projects within the GEM project goes through the computing subsystem project manager (CSPM), while management for projects outside the GEM project goes through the subsystem leader. Groups and their projects under the CSPM report to the CSL through the CSPM, while other groups and projects report directly to the CSL. Review boards will be appointed to review progress on projects.

The computing subsystem steering committee (CSSC) is chaired by the subsystem leader; it advises the CSL and reports to the collaboration and the spokesmen. It is composed of representatives from groups and projects, DAQ, the simulation groups, and the collaboration. The CSSC requests reports from groups and projects through the CSPM.

The GEM computing subsystem project manager provides line management for projects within the GEM project. The CSPM reports to the GEM PM on issues such as cost and schedule of these projects and to the CSL on technical issues. The CSPM is supported by the chief engineer and group leaders for the global controls (GCS) and on-line systems (ONS) groups.

The computing subsystem chief engineer (CSCE) is responsible for execution of systems within WBS 522; execution includes planning, designing, acquiring, assembling, installing, and testing. The systems within the GEM project are the global control system, communications at the $I R$, the on-line computing system, and any local database system (for detector configuration, calibration, and monitor). The CSCE is supported by the GCS and ONS groups, each with a group leader.

The GCS group is responsible for controls and monitoring, the monitor database, the electronic log book, and the networking that supports these functions. The ONS group is responsible for the on-line computing systems, including data links at the IR and the local configuration, calibration, and processing databases.

In addition to the above groups, there will be groups, each with a group leader who reports to the CSL, for the following task areas: frameworks, global physics tasks, and detector subsystems. The group leaders' responsibilities will range from developing requirements for other groups to implementing actual systems. However, these responsibilities will not overlap with those of the groups under the CSPM.

Projects will be established by groups, or by cooperation between groups, with project leaders who will report to the appropriate group leaders.

\subsubsection{Review Process}

The CSSC and the CSL will be jointly responsible for periodic reviews of all projects affecting GEM computing; internal and external review boards will be established. An internal review process will be established in which projects (and proposals for projects) will be reviewed on a regular basis and at several levels. Each project will set milestones and will schedule informal walkthroughs as well as a formal repo $.1 \mathrm{~g}$ schedule. Suitable non-GEM reviewers will be asked to participate in external reviews.

\subsubsection{Physics Research Computing Department Role}

The role of PRCD is to be a partner of GEM (and SDC) in the specification, design and implementation of off-line computing systems, including the interface to the on-line systems. PRCD will carry out R\&D tasks directed towards this goal, as well as implementing prototype and development systems. This includes the development of a common framework. Joint development of a software methodology and software engineering methods will be an important role for PRCD. GEM expects to depend heavily on PRCD expertise in a number of areas and 
to encourage PRCD scientists to join the GEM collaboration.

\subsubsection{Documentation}

There should be a central, electronically accessible document system at SSCL. This is of general utility, but will be supported on computing systems. The overall requirement is to make all GEM documentation, from operations manuals to publica- tions (and including meeting reports), part of this database. The resulting detailed requirements have yet to be defined, so no implementation can be discussed. As an access method, World-Wide Web Inight be considered. ${ }^{23}$

\subsection{GLOSSARY}

The acronyms and technical terms used in this chapter are defined in this section.

\begin{tabular}{|c|c|}
\hline $\begin{array}{l}\text { ANSI } \\
\text { ATM }\end{array}$ & $\begin{array}{l}\text { The American National Standards Institute, Inc. } \\
\text { Asynchroilous Transmission Mode, an emerging standard for multipurpose communications } \\
\text { (voice, video, and data) }\end{array}$ \\
\hline CASE & Computer Aided Sottware Engineering \\
\hline CDF & Collider Detector Facility at Fermilab \\
\hline CPU & Central Processing Unit of a computer \\
\hline CVS & Concurrent Versions System of revision control and release control (by Brian Berliner) \\
\hline DAQ & Data Acquisition \\
\hline DFS & Distributed File System (a goal of the Distributed Computing Environment project) \\
\hline DOD & U.S. Department of Defense \\
\hline DSF & Data Summary File \\
\hline EMS & Emergency Management Services \\
\hline EPICS & The LANL Experimental Physics and Industrial Control System \\
\hline Ethemet & A network hardware protocol (IEEE 802.3) \\
\hline FDDI & $\begin{array}{l}\text { Fiber Distributed Data Interiace, ANSI X3.139/148/166 and ISO 9314), a fiber-based net- } \\
\text { work standard }\end{array}$ \\
\hline Fibre Channel & $\begin{array}{l}\text { The proposed fiber-based network/data link standard (FCS) being developed by the X3T9.3 } \\
\text { ANSI committee }\end{array}$ \\
\hline GCS & Global Control System for GEM \\
\hline GEANT & The CERN detector simulation package \\
\hline gemfast & The GEM fast parameterized simulation package \\
\hline gemgen & \\
\hline $\begin{array}{l}\text { GFLASH } \\
\text { GUI }\end{array}$ & $\begin{array}{l}\text { A package for fast simulation of energy deposition developed by } \mathrm{H} 1 \text { at DESY } \\
\text { Graphic User Interface }\end{array}$ \\
\hline HEP & High Energy Physics \\
\hline HiPPI & $\begin{array}{l}\text { High Performance Peripheral Interface, an interconnection standard capable of } 100 \mathrm{MB} / \mathrm{s} \\
\text { rates, typically limited to a few meters. }\end{array}$ \\
\hline HV & High Voltage \\
\hline HVAC & Heating, Ventilation and Air Conditioning \\
\hline IEEE & Institute of Electrical and Electronics Engineers \\
\hline IR & Interaction region of the SSC; GEM is planned to be at IR5 \\
\hline ISDN & $\begin{array}{l}\text { Integrated Services Digital Network, a multipurpose network standard (B-ISDN is broad } \\
\text { band ISDN) }\end{array}$ \\
\hline kB & kilobyte \\
\hline kSSCUP & One thousand SSCUP8 \\
\hline LAN & Locai-Area Network \\
\hline Mb & megabit \\
\hline MB & megabyte \\
\hline MIPS & $\begin{array}{l}\text { Million Instructions Per Second. Performance on the industry-standard integer performance } \\
\text { benchmark. A DEC VAX } 11 / 780 \text { has a performance on this benchmark of } 1 \text { MIPS. The } \\
\text { ratio between MIPS and other measures of performance depends on CPU type, operating } \\
\text { system, and compiler. }\end{array}$ \\
\hline $\begin{array}{l}\text { Motif } \\
\text { MSSCUP }\end{array}$ & $\begin{array}{l}\text { A GUI system developed by the OSF, based on the Xwindow mecharisms } \\
\text { One million SSCUPs }\end{array}$ \\
\hline NFS & Network File System (developed initially by Sun Microsystems) \\
\hline NAB & North Assembly Building at the GEM IR \\
\hline
\end{tabular}




\begin{tabular}{|c|c|}
\hline NTUPLES & $\begin{array}{l}\text { File of events in a tabular format, with each e rent represented by a number of parameters } \\
\text { (fixed for the file) }\end{array}$ \\
\hline OCR & Optical Character Recognition \\
\hline OFS & The GEM Off-Line Computing System \\
\hline ONS & The GEM On-Line Computing System \\
\hline OSF & Open Software Foundation \\
\hline RECO & the typical first full-reconstruction pass through raw event data \\
\hline PDSF & $\begin{array}{l}\text { Physics and Detector Simulation Facility, a multiuser computing facility in the Physics Re- } \\
\text { search Division at SSCL }\end{array}$ \\
\hline PB & Petabyte ( $10^{15}$ bytes) \\
\hline POSIX & $\begin{array}{l}\text { A set of standards and draft standards being developed by IEEE for a Portable Operating } \\
\text { System Interface for Computer Environments }\end{array}$ \\
\hline PRCD & SSCL Physics Research Division Computing Department \\
\hline RISC & $\begin{array}{l}\text { Reduced Instruction Set Computer, a term for computers (with VLSI chip sets) in which the } \\
\text { instruction set is optimized against some set of sotware to enable the CPU to complete } \\
\text { most instructions in one cycle. Contrasted with CISC, Complex Instruction Set Computer, } \\
\text { in which most instructions may take more than one clock cycle. }\end{array}$ \\
\hline SAB & South Assembly Building at IR5. \\
\hline SDC & Solenoidal Detector Collaboration \\
\hline SIGEM & GEM full simulation program \\
\hline SM & Standard Model \\
\hline SONET & Synchronous Optical Network, a standard for wideband "trunk" lines in networks \\
\hline SSC & Superconducting Super Collider machine \\
\hline SSCL & SSC Laboratory \\
\hline SSCUP & $\begin{array}{l}\text { A unit of computing power based on a benchmark developed at the SSCL for evaluating } \\
\text { computing systems for simulation performance with } 32-\text { bit precision FORTRAN programs; } \\
1 \text { SSCUP is approximately O.9 VUP. The ratio between SSCUPS and other measurements } \\
\text { of performance depends on CPU, OS, compiler, and type of benchmark. For example, for } \\
\text { RISC machines, the ratio of SSCUPS to MIPS is about } 0.7 \text {, while for VAX architecture it is } \\
1.0 \text {. }\end{array}$ \\
\hline TCP/IP & Intemet Transmission Control protocol, layered on the Intemet protocol \\
\hline UNIX & Used generically for operating systems derived from AT\&T UNIX \\
\hline VME & A hardware standard for DAQ and Computing \\
\hline VAX & Digital Equipment Corporation's CISC architecture \\
\hline VUP. & Computing power of a DEC VAX-11/780 with Floating Point Accelerator \\
\hline VXI & A hardware standard for DAQ \\
\hline WAN & Wide-Area Network \\
\hline WBS & Work Breakdown Structure \\
\hline Xwindows & $\begin{array}{l}\text { A system for programming pixel-oriented terminals developed at MIT and carried forward by } \\
\text { the XOpen Consortium }\end{array}$ \\
\hline
\end{tabular}




\section{REFERENCES}

1. "SSCL Laboratory Operations Program Plan FY2000 to FY2005," prepared by Tom Elioff, SSCL-SR-1216, Nov. 1992.

2. A. Vaniachine, private communication.

3. D. Marlow, private communication.

4. K. McFarlane and X-Q Yu, "Requirements for the GEM Global Control System," GEM TN-93-398.

5. Brian Dickinson, "Developing Quality Systems," (McGraw-Hill 1989), Bertrand Meyer, "Object-Oriented Software Construction," (Prentice-Hall 1988), Edward Yourdon, "Modern Structured Analysis," (Yourdon Press, 1989).

6. Jag Sodhi, "Software Engineering Methods, Management and CASE Tools," McGraw-Hill (1991). Appendix C lists IEEE and DOD standards.

7. B. Boehm, "A Spiral Model of Software Development and Enhancement," IEEE Computer, Vol. 21, No. 5, 1988.

8. An example is EMASS $\otimes$, the robotic mass storage system developed by E-Systems, Inc., Garland, Texas.

9. R. Krishnan and N.F. Maxemchuk, "Life Beyond Linear Topologies," IEEE Network, March 1993, p.48.

10. R. Mount, "Off-line Computing for Experimental High-Energy Physics," Rep. Prog. Phys. 55 (1992), p.1411.

11. Current prices, semiconductor technology trends, published vendor projections, non-disclosure meetings, and Al Cutela, "Technology projection modeling of future computer systems," Prentice Hall (1990).

12. EPICS Users Guide, 1992, Controls and Automation Group-AT-8, Los Alamos National Laboratory.
13. L. Cormell and K. McFarlane, "GEM detector computing study, of Combined On-line (Level 3) and Off-line Facility," GEM TN-92-286. This is a report of a discrete simulation of a combined Level 3 and off-line computing facility, carried out by E-Systems, Inc.

14. The SSCUP (SSC Unit of processing power) is measured with a FORTRAN benchmark suite (based on ISAJET and the LUND Monte Carlos), K. McFarlane, SSCL-375 (1991).

15. B. Berliner, "CVS II: Parallelizing Software Development," 1992.

16. Current guidance can be found in I. Sheer and K. McFarlane, "GEM Software User's Guide," GEM TN-93-296. This document is kept up-to-date and is accessible on PDSF.

17. B. Boehm, Software Engineering Economics, Prentice-Hall, 1981.

18. I. Sheer, "Gemgen, a Generic Monte Carlo Generator Interface Package," GEM TN-93-379.

19. See, e.g., "SDC Offline Software Concepts." Version 1.0, Dec. 1992 (Soren Fredericksen. private communication). SDC-92-400.

20. Petabyte Access Storage Solution, see C.T. Das et al., "Database Computing in HEP-Progres, Report," Proc. of the Intl. Conf. on Computing in High Energy Physics (CHEP 1992), Annec!. France, Sept. 1992, CERN 92-07, p. 557.

21. M. Shupe, "First Studies of GEM Baseline II Calorimetry from Arizona Mixture Level Simulation," GEM TN-92-236.

22. S. Peters, Yu. Fisyak and K. McFarlane. "Technical Note on Fast Calorimeter Simulatıon for GEM," GEM TN-93-292.

23. T.J. Berners-Lee and R. Cailliau, "The World-Wide Web," Proc. of the Intl. Conf. on Computing in High Energy Physics (CHEP 1992), Annecy, France, Sept. 1992, CERN 92-07. 


\section{FACILITIES, ASSEMBLY, AND INSTALLATION}

\subsection{INTRODUCTION}

This chapter addresses the plan for on-site assembly of the detector subsystems and their installation into the underground experimental hall. It includes consideration of facilities required to assemble and install the detector, and it presents a schedule for integration of facilities and technical components in a smoothly flowing and continuous process of assembly and installation.

The GEM detector is approximately $38 \mathrm{~m}$ in length and $23 \mathrm{~m}$ in diameter, including support structures. It consists of five major functional subsystems, which, because of their size and complexity, is further subdivided into separately constructed components. Although fabrication of much of the detailed parts will occur off-site at vendor facilities, most of the subsystem assembly must be accomplished on-site because of the very large size of the subsystems. The compressed schedule needed to meet the collider's operational milestones requires that assembly of several subsystems be carried out simultaneously. This, in turn, necessitates several large, environmentally controlled work areas, at least one of which requires a $27-\mathrm{m}$ high gantry crane to accommodate the size of the magnet subsystem and muon barrel monoliths.

The underground experimental hall that houses the detector must be large enough to accommodate the size of the detector, while leaving sufficient temporary storage and working space around it to permit multiple, parallel installation activities. This dictated a hall that is $100 \mathrm{~m}$ long, $30 \mathrm{~m}$ wide, and approximately $47 \mathrm{~m}$ from the floor to the bottom of the ceiling truss beams. Lowering of the large, completed subsystems into the hall requires access shafts of approximately $18 \mathrm{~m}$ by $27 \mathrm{~m}$ in cross-sectional area. Two access shafts are provided to permit simultaneous installation of major components down both shafts, if required, to maintain the overall installation schedule.

The GEM detector will be located at interaction region 5 (IR5), on the east side of the main collider ring in Ellis County, near the town of Palmer. The detector requires a large underground experimental hall with associated surface buildings for manufacturing, assembly, operations, offices, and utilities. An aerial photograph of the ongoing site-clearing and grading activity is shown in Figure 9-1.

The GEM top-level schedule is presented in Chapter 16. The assembly and installation sequence is determined by several factors:

1. Adherence to top-level milestones

2. Scheduled availability of components

3. Funding profile throughout the program lifetime

4. Flexibility to accommodate delays in subsystem availability

5. Optimized use of surface assembly areas

6. Efficient use of manpower throughout subsystem development, assembly, and installation.

The surface layout at IR5 consists of two large surface buildings for detector assembly, a utilities building and several head houses. Figure 9-2 shows the overall IR5 site plan. The underground hall is fed by two large installation shafts, an electronics shaft and a utilities shaft. The two installation shafts, each connected to a heavy-load path on the surface, have been provided to permit, if necessar $;$, simultaneous parallel installation operations on separate subsystem components. However, the present installation schedule is based on an essentially serial installation sequence. This schedule is driven by the availability of components, which is in tum driven by efficient usage of surface assembly space, and the need to maintain a relatively flat funding profile for the detector. The funding profile prohibits rapid buildup of subsystems early in the schedule. The symmetrical nature of the detector lends itself to completion of subsystem components for one side of the detector, followed by mirror-image operations for components on the opposite side. 


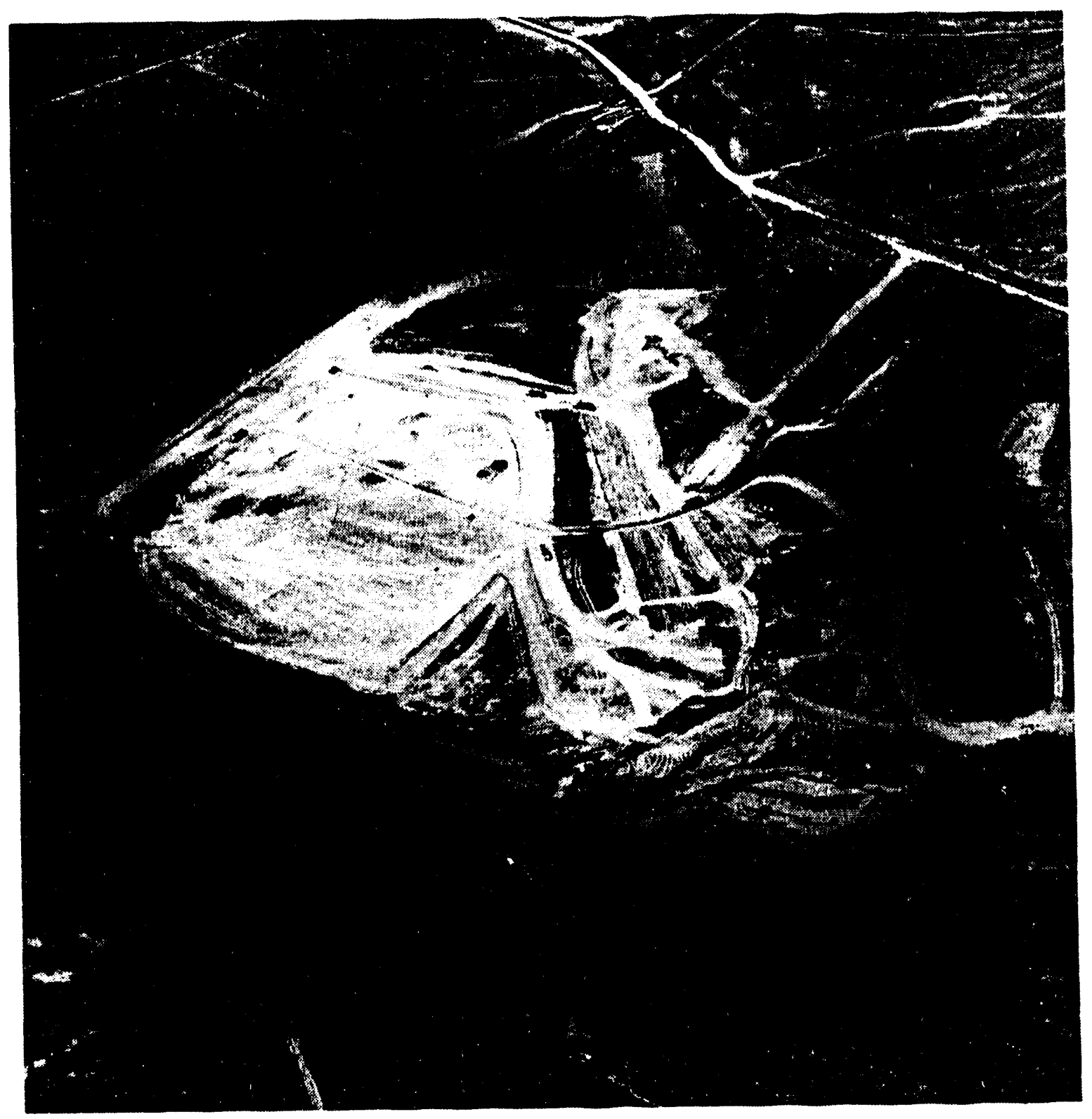

FIG. 9-1. Aerial photograph of the ongoing site-clearing and grading activity at the IR5 site.

The GEM detector assembly and installation schedule must conform to the collider start-up because the collider cannot begin operations until the detector is in position, with the beam pipe connected. The critical path in the GEM detector schedule is largely determined by the logic of the detector component installation sequence. The major contract-for the magnet, the third item on the installation critical path-is planned for the first half of 1993. This will allow three years to manufacture and install one of the largest magnets of its kind in the world. It will contain 24 coil sections, each almost $19 \mathrm{~m}$ in inner diameter and $1.2 \mathrm{~m}$ high. The estimated time to wind, insulate, and test each section is one month. The magnet surface assembly process is expected to take at least six months and the subsequent tests another three months. The overall installation period of the GEM detector into the underground hall is estimated at three years.

\subsection{FACILITIES}

Although many of the detector components will be fabricated elsewhere, it is expected that all of the final assembly and testing procedures will be performed on-site. Assembly of the detector compo- 


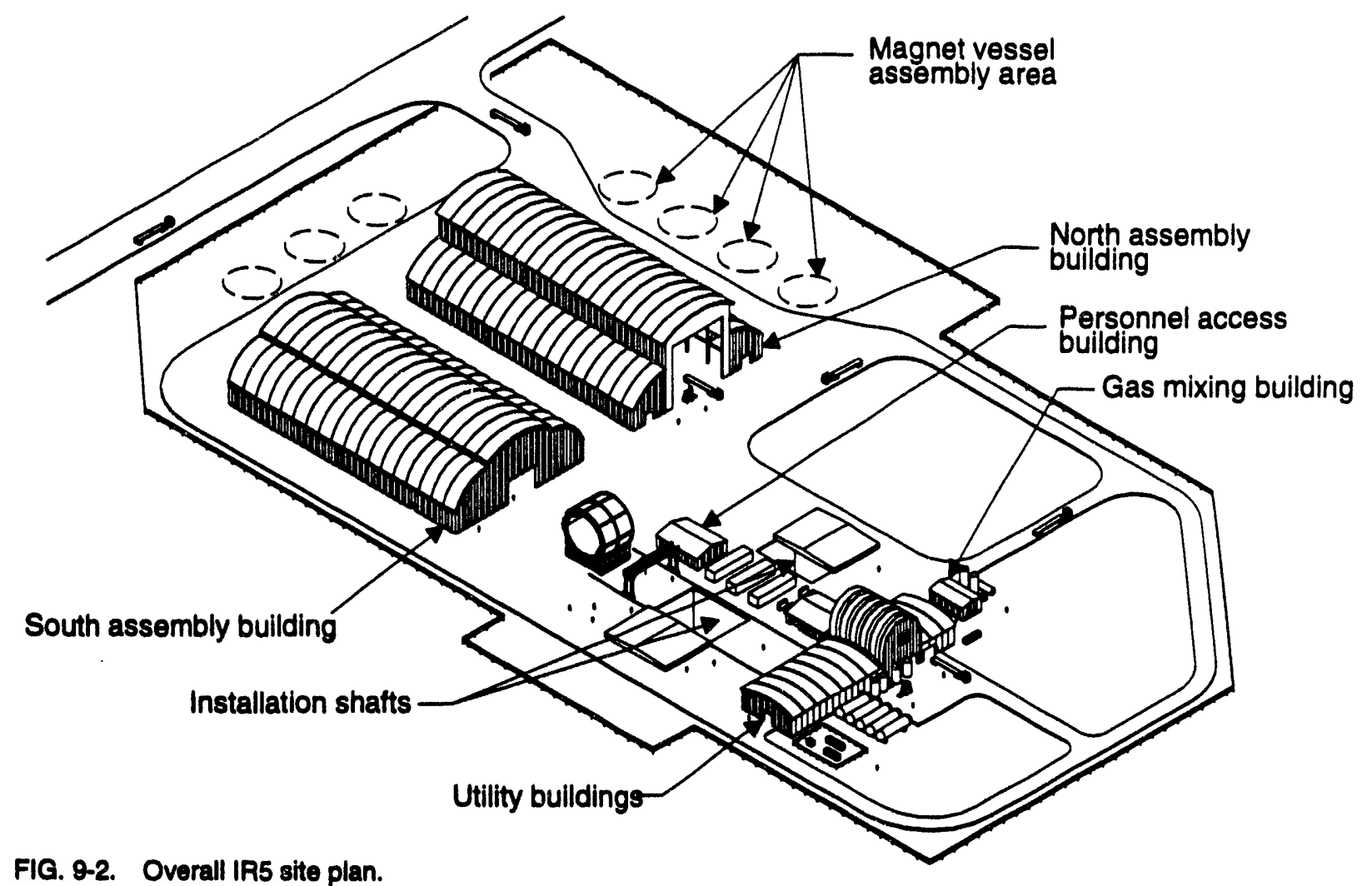

FIG. 9-2. Overall IR5 site plan.

nents will be carried out in the south assembly building, the north assembly building, and the hard-stand fabrication area. The rest of the facilities will provide the necessary support, including utilities and services, for subsystem testing and for detector operations once experiments have begun.

\subsubsection{Surface Facilities}

The surface facilities will be used to build the GEM detector and to support it once it is operational. After the detector has been commissioned and experiments have begun, the surface facilities will house electrical, cryogenic, and data analysis support systems, as well as maintenance and upgrade facilities, offices, and miscellaneous support services.

\section{South Assembly Building}

The south assembly building (SAB), with a high bay and two low bays, will be a multi-use building. A proposed elevation view is shown in Figure 9-3 and 9-4 depicts the assembly floor layout. Initially, as of the anticipated April 1994 Joint Occupancy Date (JOD), the high bay of the building will provide space to wind the coils for the GEM magnet. After the beneficial occupancy date (BOD) the high bay will also be used for the assembly of muon prototype sector structures. The low bays will be used for the supply storage and shops associated with that operation. Included in the low bays is space for a general shop, miscellaneous storage, and a two-level area to be shared between assembly process offices and initial detector operations activities. The remainder of the building will be used as temporary storage.

Once the coil-winding operations have been completed, the areas previously used for that activity will be reapportioned to provide more space for receiving, storage, inspection, assembly, and testing of muon system components. Figure 9-5 depicts a later stage of assembly operations when the SAB will be used for assembly of muon system components.

A major design consideration for the $\mathrm{SAB}$ has been to ensure that all of the intended operations have the space and equipment necessary for succesful completion. The crane requirements are extremely 


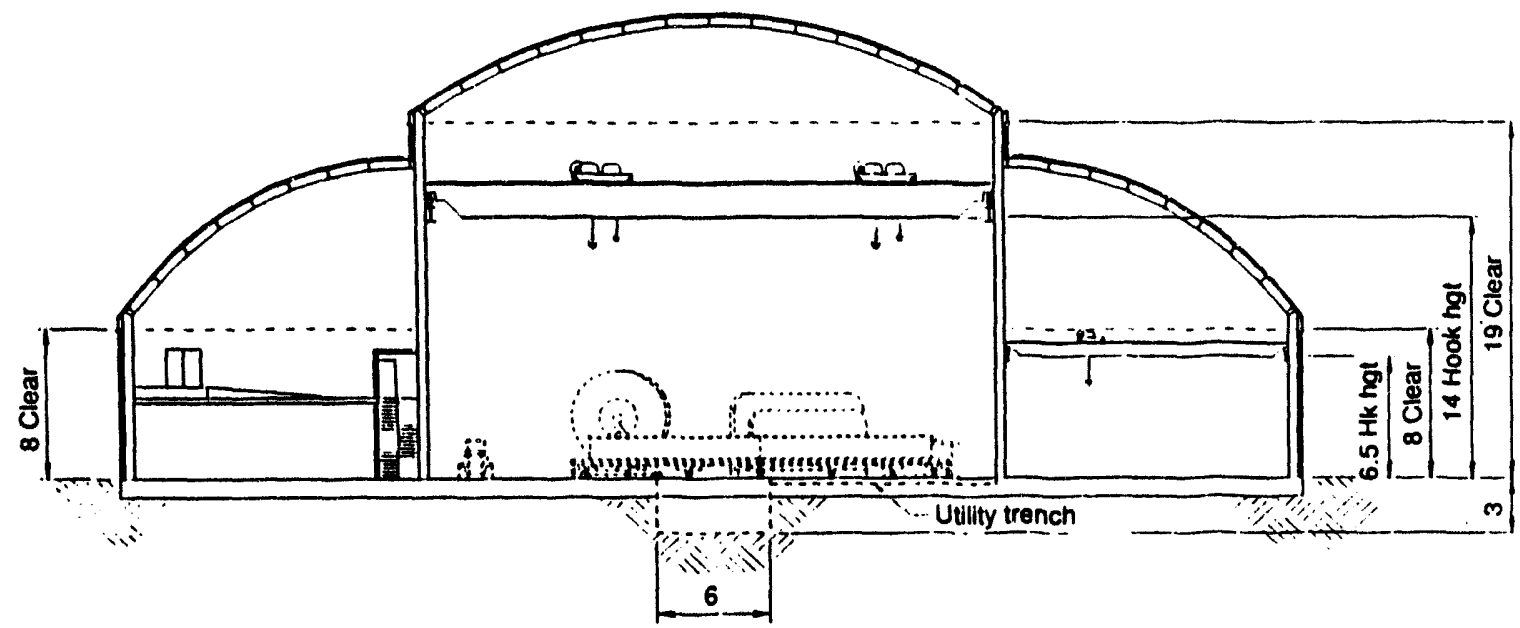

FIG. 9-3. Proposed elevation view of the south assembly building (SAB).

important. The building will be fitted with two identical bridge cranes in the high bay area. Both will use the same runway. Each crane will have a 32-Mg main hook capacity and a $9-\mathrm{Mg}$ auxiliary hook capacity. The required lift of all of the hooks on the cranes will be $14 \mathrm{~m}$. The northern low bay area will be equipped with a bridge crane with a 4.5-Mg hook capacity and a hook height of $6.5 \mathrm{~m}$. The building will be provided with all of the utilities and services necessary to perform the required operations.

\section{North Assembly Building}

The north assembly building (NAB) will also be a multi-use facility. An elevation view is shown in Figure 9-6, with the assembly floor layout illustrated in Figure 9-7. Initially, the building will provide space for assembly of the two magnet halves and an area for receiving, storage, inspection, assembly, and testing of calorimeter parts. There will also be space reserved for tracker and beamline assembly. The remainder of the building will be used for temporary storage. After the magnet halves are completed, the areas previously used for those activities will be reapportioned to provide additional space for muon barrel and endcap assembly. Figure 9.8 illustrates a later stage of operations in the NAB, when muon monoliths are being assembled.

As in the SAB, crane requirements are an important consideration. The high bay industrial area of the building will be served by two identical bridge cranes on the same runway. Each crane will have a $41-\mathrm{Mg}$ main hook capacity and a $9-\mathrm{Mg}$ auxiliary hook capacity. The cranes will provide a hook height of $26 \mathrm{~m}$ above floor level. The low bay area reserved for tracker/beamlire assembly, EM calorimeter assembly, and the assc ciated access area will be serviced by a bridge cran with a $18-\mathrm{Mg}$ hook capacity and an 8-m hook height.

\section{Hard-Stand Fabrication Areas}

The hard-stand fabrication areas, located west and north of the NAB, will be used for the assembly of the magnet's inner and outer vacuum vessels, the central detector support structure, and the two forward field shapers. The assembly procedures performed in this area require only conventional mobile construction cranes.

\section{Utility Building}

The utility building will house equipment for power, cooling water, cryogenics, compressed air, and vacuum for the detector. It will also house some utility systems for the underground hall and other surface facilities, such as boilers and chillers for the HVAC system. Associated storage tanks, transformers, and air handlers to support the detector and underground hall utility systems will be located on outside pad areas adjacent to the building.

\section{Gas-Mixing Building}

Compressed gases will be delivered to the detector subsystems in specific mixtures and quantities; this will require extensive mixing systems that 


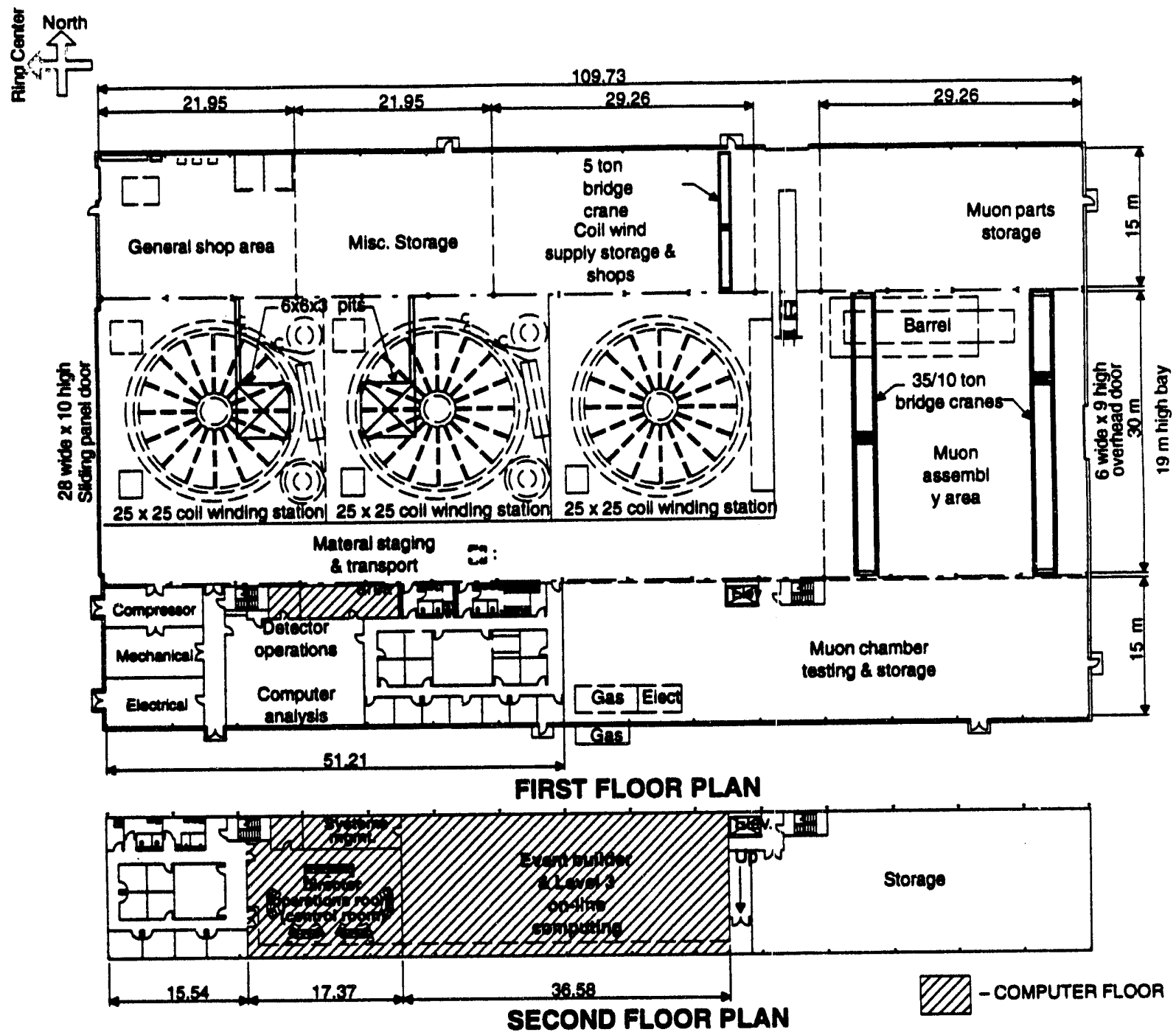

FIG. 9-4. South assombly building floor plan.

are continuously monitored to ensure effectiveness. The gas-mixing building will house those systems along with adequate supplies of gases for uninterrupted operation.

\section{Personnel Access Building}

This building will cover the cable electronics shaft and house the equipment for the elevator and the stairway. Space in the building will be used as a junction point for cable ways running from the underground hall and electronics rooms to the detector operations center (via underground duct banks).

\section{Equipment Access Building}

The equipment access building will cover the utility shafts; provide the structure to support the lifting equipment; house the equipment for the elevator, hoist-way, and stairway; and provide the terminus for cooling, gas, power, and ventilation services for the underground hall. The shaft will be used for personnel access and for lowering electronics and test equipment into the experimental hall. After comrnissioning, this shaft will be the only means for lowering the equipment to the detector without opening the large installation shafts. 


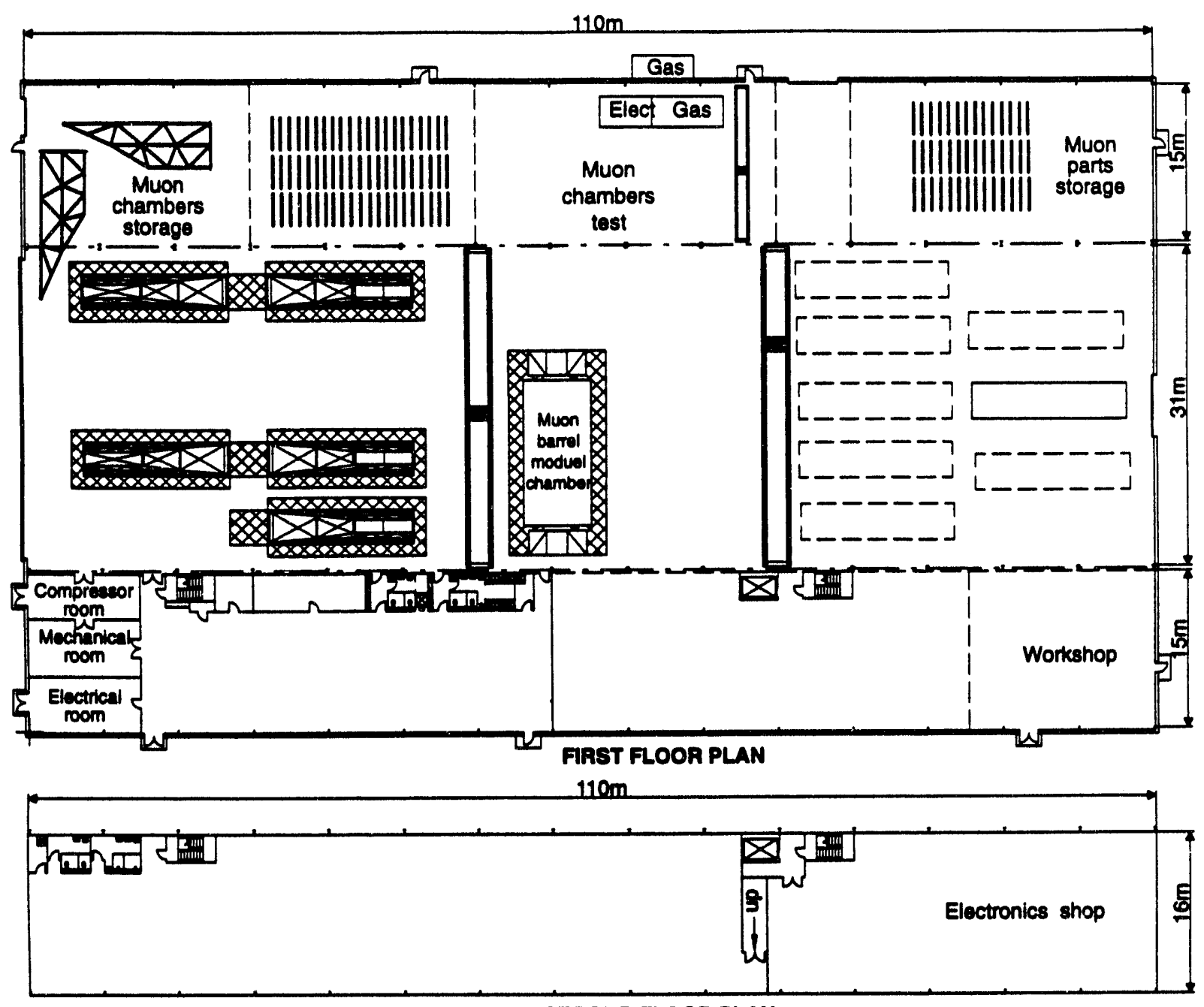

SECOND FLOOR PLAN

FIG. 9-5. Assembly of muon system components in the SAB.

The truck access area of the building and the hoist-way will be served by a pendant-controlled electric bridge crane with a $0.5-\mathrm{Mg}$ hook capacity. The crane will be capable of transporting a load from a truek adjacent to the hoist-way to the bottom of the hoist-way at Level 1 of the experimental hall. It will have a hook height of $4 \mathrm{~m}$ above ground level.

\section{Installation Shaft Headhouse}

During the 3 years of installation operations there will be more than 1000 lowering operations for detector components and auxiliary equipment. A headhouse structure is proposed at the south installation shaft. This will provide covering of the open shaft to permit daily operations regardless of weather conditions. The concept and requirements are still being defined, but the size of the structure should accommodate off-loading a small truck with an independent $1.0-\mathrm{Mg}$ mobile boom crane, in an environment protected from inclement weather.

\subsubsection{Underground Facilities}

The dominant factors driving the general configuration and size of the underground facilities are the configuration, installation, maintenance, and shielding requirements of the GEM detector and the local accelerator technical systems. In addition to the experimental hall, the underground facilities will include installation shafts leading from the surface to the hall, the cable electronics shaft, the utility shaft, and the necessary connecting tunnels.

The detector cable and fiber readout services will exit the underground hall via the electronic access shaft situated on the west side of the hall. This shaft will also contain several levels of magnetically 


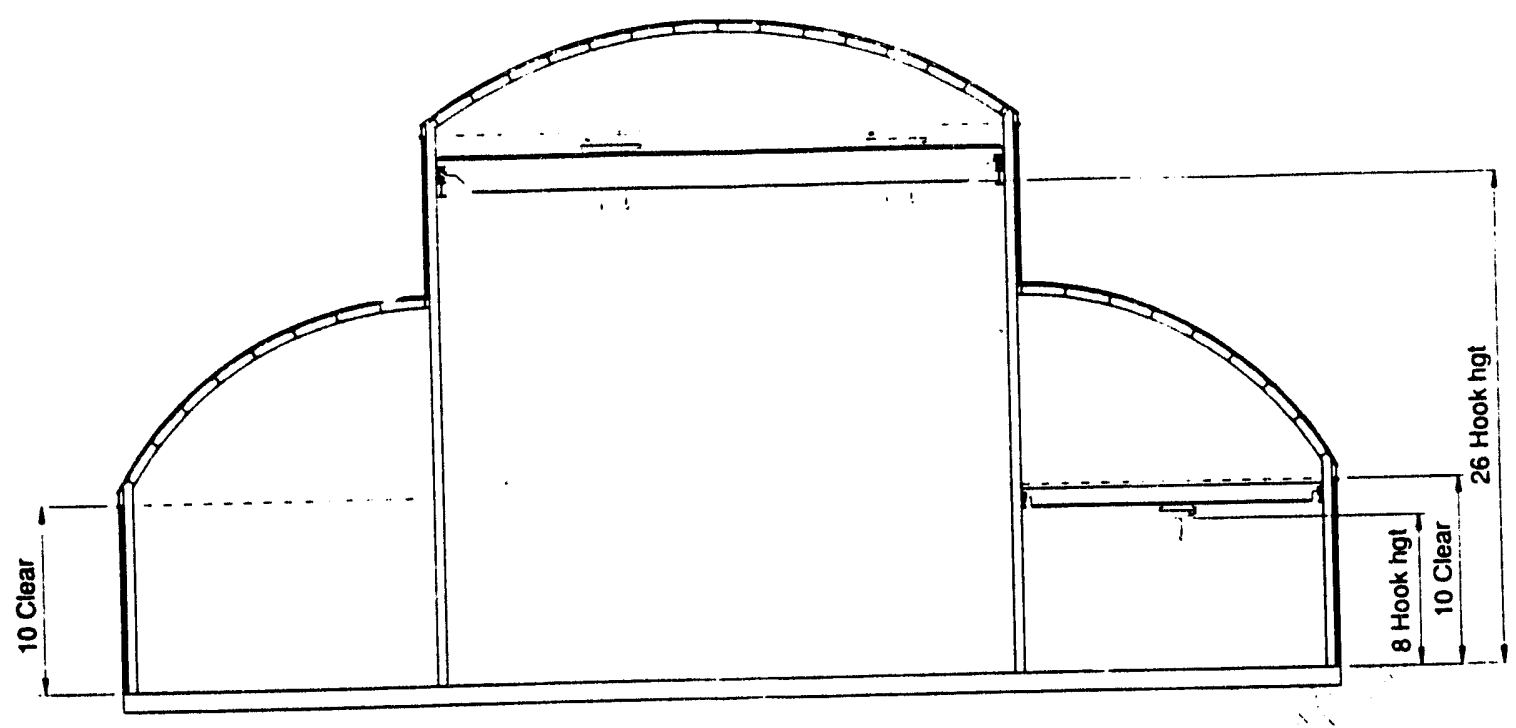

FIG. 9-6. Elevation view of north assembly building.

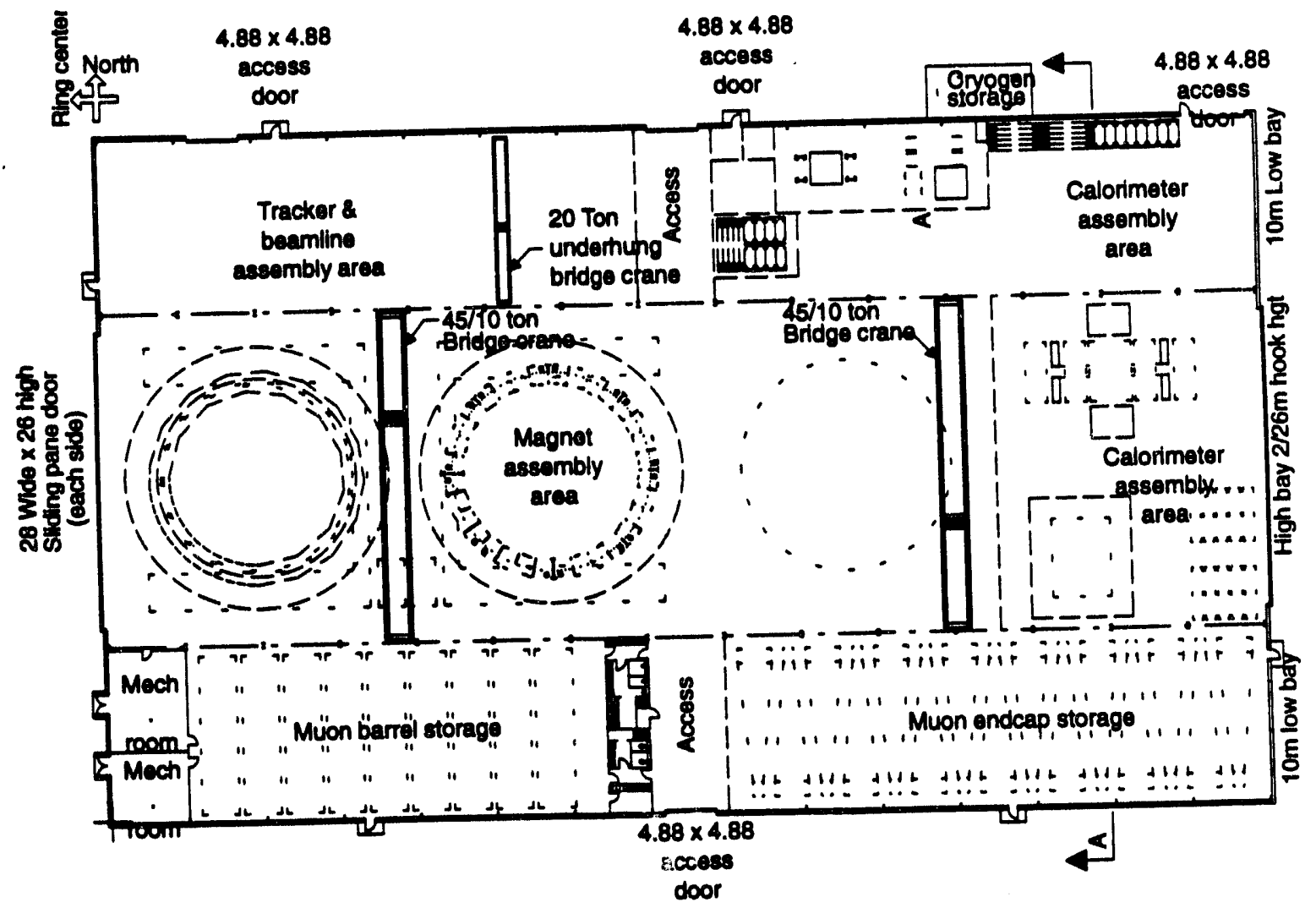

FIG. 9-7. North assembly building assembly floor layout.

shielded rooms equipped with racks of electronics equipment. The utility shaft on the opposite side of the hall will permit the routing of the technical and conventional utilities between the surface facilities and the hall and detector. 


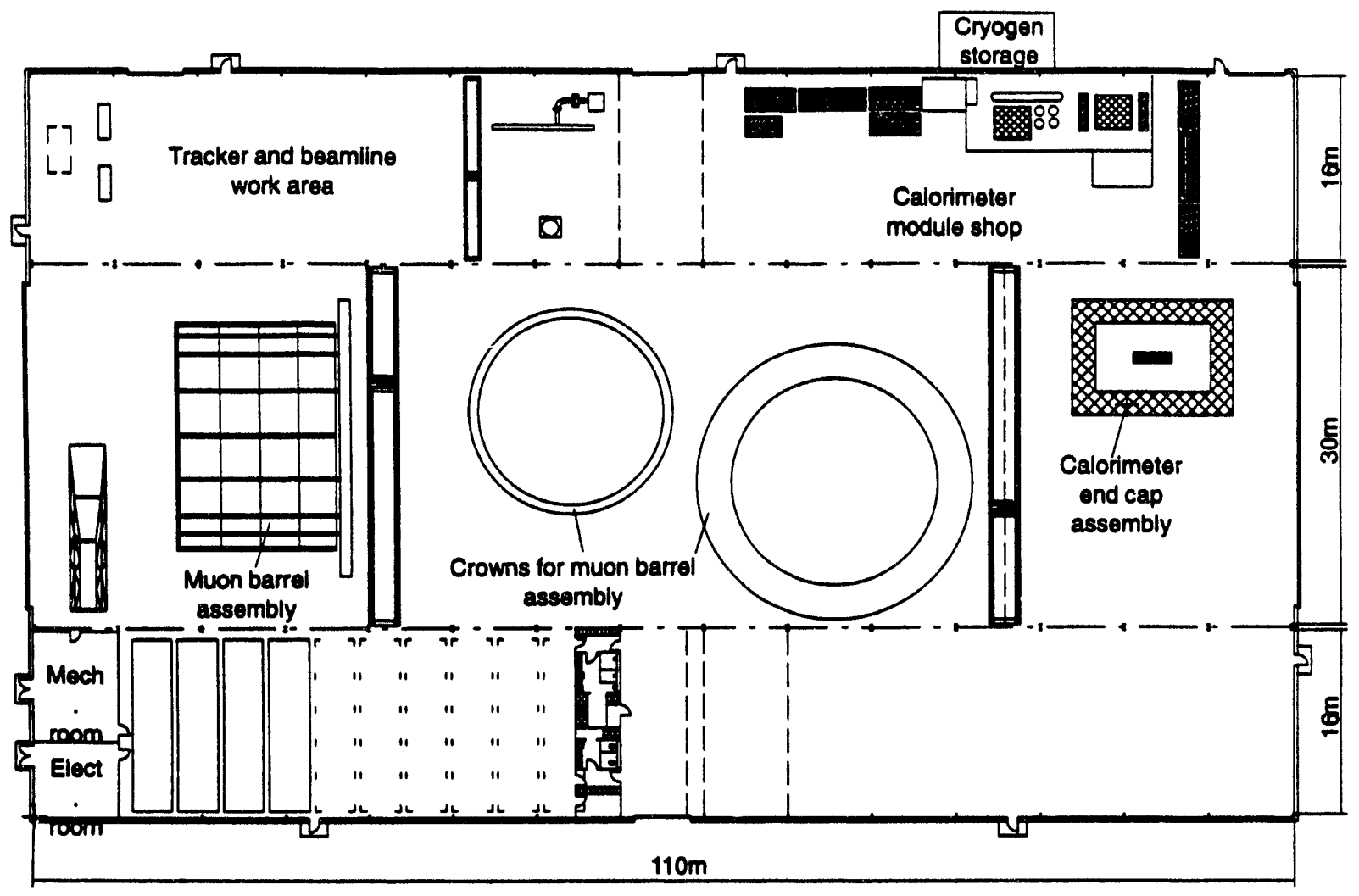

FIG. 9-8. Muon monoliths being assembled in the north assembly building.

\section{Experimental Hall}

The experimental hall will house the GEM detector. Figure 9-9 shows the general configuration of the hall which is situated to allow positioning of the long axis of the detector parallel to the beam line and centered around the interaction point. The length and width of the hall are driven by the detector installation and maintenance requirements.

Each wall of the experimental hall will have gangways for access to the detector. They will be connected to the various elevator and stairway systems that provide access to the electronics shaft, utility shaft, and survey monuments. The survey monuments will be used to align the various detector components into their final positions once the components have been lowered into the hall.

Since most of the detector subsystems consist of massive components, special fixtures will be required to facilitate movement of these components from their initial position at the bottom of the installation shaft to their final position in the underground hall. The present concept uses a combination of large rollers or pads and a hydraulic transport system. The floor of the hall will be equipped with heavy-duty rails to accommodate these fixtures, and it will provide both guidance for the pads and traction for the hydraulic transport system.

The hall will be equipped with bridge cranes that will be used during installation and maintenance. The size and number of overhead bridge cranes are dictated by the installation schedule and by maintenance requirements during major detector shutdown periods. The smaller detector components will be light enough that two $75-\mathrm{Mg} / 20-\mathrm{Mg}$ bridge cranes, operating on a common overhead runway, will be sufficient to position the components in their desired locations. The cranes will be capable of being operated simultaneously, but independently, from separate, portable control stations. They will also be capable of tandem cperation from a single control. The surface will also be equipped with the necessary heavy lift cranes that will lower the detector components down the proper installation shaft. 


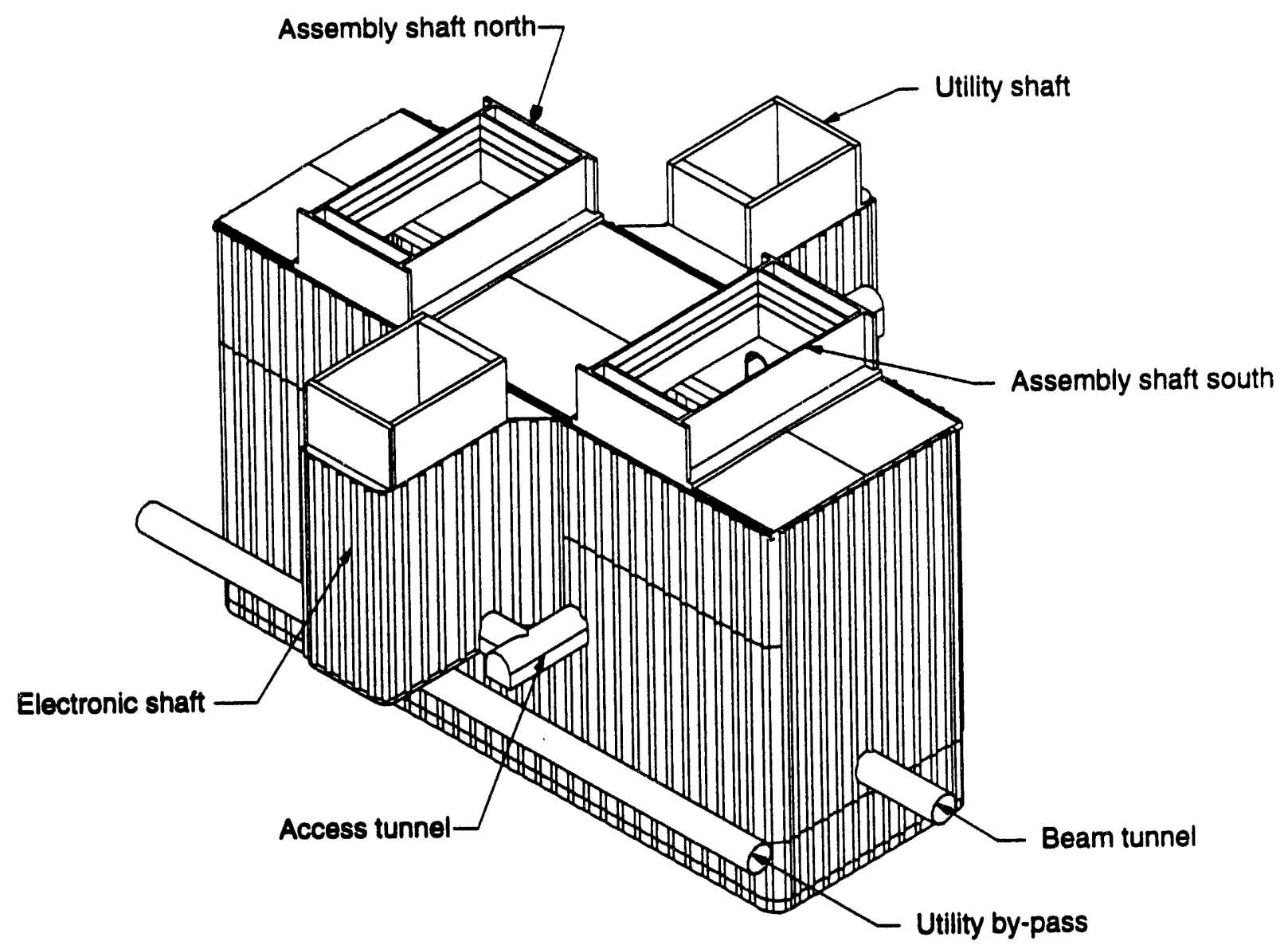

FIG. 9-9. General configuration of the hall.

The JOD of the underground hall is estimated to be January 1996. During this period the initial phases of the installation processes begin while the remaining hall fixtures are installed. However, the hall will be equipped with the necessary rails, gangways, etc., required for the various installation procedures, before the first large component is lowered into the hall.

\section{Installation Shafts}

The two installation shafts will be used to lower the completed detector subsystems from the surface into the experimental hall. The shafts must accommodate the size of the large pre-assembled detector subsystems. The shafts will be located to minimize the amount of movement required of the large components once they are lowered into the hall. After installation is completed, these shafts will be plugged with removable shieldih。 blocks to reduce surface radiation to tolerable levels.
Cable Electronics Shaft and Connecting Tunnels

The cable electronics shaft will house several levels of electronics rooms. The majority of the data acquisition and triggering equipment racks as well as several power transformers will be located in these rooms. The size of the shaft has been determined by the need to accommodate an access elevator, a stairway, the electronics rooms, and cable runs. Provisions have been made to minimize the amount of radiation that is allowed to reach the surface, and to shield the electronics rooms from the effects of the magnetic fringe field.

The connecting tunnels will run from Level 0 of the electronics shaft to Level 5 of the experimental hall. Their locations and routing will be determined by the need to minimize the length of cable runs from the hall to the detector operations center, to minimize 
transmitted radiation, and to provide a minimum egress distance to reach a safe area.

\section{Utility Shaft and Connecting Tunnels}

The utility shaft will provide access to the experimental hall for all of the cooling, electrical, gas, and air supply/exhaust lines and ducts from the surface. In addition, the utility shaft will house a personnel elevator, a cargo elevator, and an equipment hoist-way. These will allow personnel and equipment to be lowered to either Level 0 or Level 5 of the experimental hall.

Connecting tunnels will run between the shaft and the experimental hall at Levels 0 and 5 . Their size has been determined by the quantity and size of the cables, ducts, stairways, elevators, and hoistways that are needed to provide services and access from the surface to the underground hall. Their location and routing will be determined by the need to minimize the ? ngth of utility lines run between the utility building and the hall, to minimize transmitted radiation, and to provide a minimum egress distance to reach a safe area.

\subsubsection{GEFUR}

The GEM experimental facilities user requirements (GEFUR) document (GCT-000001) provides the design and safety system requirements, and the basis thereof, for the conventional construction facilities and systems to be provided at the IR5 site. The GEFUR document contains the requirements that are incorporated into the facility design and construction specifications. It discusses all of the information given here, and should be referred to if more detailed information is desired.

\subsection{ASSEMBLY}

\subsubsection{Assembly Facilities and Tools}

The assembly facilities and tool requirements will be determined in advance by devising a work plan for the overall assembly process and for each subsystem. These plans will be part of the safety approval process before any work begins at the site. Typically, the plans will cover the use of scaffolds, elevators, cranes, lifting fixtures, and local transport devices. Issues such as personnel accommodation, clothing and safety equipment (e.g., hard hats, safety harnesses, gloves, and hard-tipped boots) will also be addressed. Local rest and refreshment areas are foreseen, with adequate changing and showering facilities. Some suitably equipped offices and conference rooms will be available on-site for monitoring the assembly work.

The SAB will contain a small workshop to support the assembly process. One wing will be dedicated as the detector operations center.

\subsubsection{Assembly Strategy and Resources}

The assembly strategy is directly related to the installation sequence in the underground hall and to the need for placing the large, heavy components at an accessible point ready for transport to the shaft head. The heavy structural components such as forward field shapers and the central detector support are considered as suitable for outside work. Two large pad areas have been allocated for this purpose. When welding or other work requiring protection is to be carried out on these devices, local temporary cover will be provided.

The initial assembly process is dedicated to the GEM magnet, which lies on the critical path in the assembly and installation schedule. The types of operations involved in magnet assembly require the sequential use of both assembly buildings-first coil winding in the SAB, and then magnet assembly in the NAB. The CDS, which will be the first component to be installed in the underground hall, will be assembled at one of the hard-stand areas located near the NAB. The calorimeter and muon subsystems will be developed concurrently with the magnet assembly, with muon chamber prototyping studies in the SAB and with assembly of the electromagnetic calorimeter barrel in the NAB. The calorimeter barrel will be the first of these two components to be completed and installed, leaving space in the NAB to increase the capacity for the assembly of the large muon monolithic structures. However, the calorimeter endcaps must be stored in the NAB until their installation, following that of the tracker. The assembly sequence on the surface will be completed when the tracker and the beam vacuum system are assembled and tested in the NAB.

Each subsystem will have a dedicated team of physicists, engineers, technicians, and riggers with appropriate on-site facilities to work efficiently and safely. The number of staff employed will vary according to the subsystem size and assembly stage. 


\subsubsection{Assembly Schedule and Contingency Plan}

The assembly schedule is based largely on a serial assembly flow. Some overlapping parallel operations are possible, thus speeding up the overall assembly process.

The assembly plan anticipates various installation options in the event of delayed availability of a subsystem component. With the exception of the coil winding process of the magnet coil sections in the SAB, the schedule is based on single-shift, five-day operation. Multiple shifts or extended work hours and weekend work will provide assembly time contingency if needed at critical periods.

\subsubsection{Assembly Descriptions}

\section{Central Detector Support}

The central detector support (CDS) will be constructed of stainless steel box beams forming a pedestal, with exterior stabilizers, to carry the load of and provide the structural support for the calorimeter and central tracker subsystems. The CDS is expected to be fabricated initially off-site. The selected contractor will decide whether to preassemble it at the contractor facility for fit check and then partially disassemble and ship it to the IR5 site in large pieces, or to fabricate it in several large pieces and ship it to the site for initial assembly.

The CDS will arrive at the site pre-drilled for simple bolted or welded assembly. The support feet will be similarly assembled from individual plates and beams, possibly welded together to provide the necessary strength. All assembly operations will be performed outdoors on the hard-stand area west of the SAB and NAB.

The box beam sections will be assembled onto the support legs one section at a time. An ordinary construction crane will be required to support the pieces as they are positioned for joining. The entire CDS will be fully assembled, then transported to the vicinity of the north installation shaft, ready for lowering down the shaft into the underground hall. One assembly option also being considered is to mate he scintillating barrel calorimeter with the CDS on the surface and to lower the two components together for installation in the underground hall.

\section{Magnet}

The magnet, by virtue of its size, must be assembled mostly on-site at IR5. There are six main stages in the assembly of each coil half unit: coil segment winding (including preparation), coldmass assembly, vacuum vessel assembly, thermal shield assembly, magnet half assembly, and coldtesting. The coil segment winding will take place in the $\mathrm{SAB}$; the rest of the magnet assembly procedures will be performed either in the NAB or in the hard-stand area north of the NAB.

Each coil section will consist of a large coil form, or bobbin, with a compression flange on each axial end. The conductor will be wound on the inside of the bobbin and will be protected with turn insulation and ground wrap. The conductor will consist of an aluminum sheath, conduit, and cable.

Quadrants of flanges and shells for the 20-m diameter, $1.2-\mathrm{m}$ tall coil form segments will be fabricated off-site and shipped to IR5. In the SAB, these parts will be uncrated, inspected, cleaned, and moved to the coil form preparation area using the lifting strongback and the building crane. The lower flange quadrants will first be mounted to the assembly fixture. The quadrants of the shell will then be added, aligned, joined together, and welded to the flanges. The upper flange will be assembled separately and set aside until it is needed after the coil segment has been wound. The coil form will be thoroughly cleaned to remove all weld debris and contamination so that ground plane insulation can be applied and tested.

When completed, the coil form will be transferred to one of the winding stations, using the building crane and the segment lifting spider. (Refer so Figure 9-4 for location of the winding stations.) The form will be mounted and aligned to the turntable. The coil modules will be wound vertically at each of the coil winding stations. A spool elevation structure and drive will be located at each winding station for the mounting and for control of the conductor supply spool. The conductor will be received at IR5 completely assembled and spooled in lengths sufficient to wind one coil segment from each spool. 
From the supply spool, the conductor will be fed through a series of support rolls, bending rolls, and drivers that will unwind the conductor from the supply spool, and form it onto the inside of the bobbin. Nineteen turns of the conductor are required to complete each coil section. As each turn is added, it will be compressec against preceding turns and the lower compression flange. The motion of the turntable, the payout spool, the roll-formers, the traction drive, the taping station, and the clamps on the fixtures will be synchronized by a programmable controller. After the conductor is installed, the top compression flange will be attached to the completed coil section. The bobbin segment joints will be retensioned as necessary. When winding is complete, the inner thermal radiation barrier will be applied.

Before the coil sections are transferred to the magnet assembly area in the NAB, the completed assembly will be tested for electrical continuity, insulation integrity, and gas leaks of the cryogenic tubing. If any problems are found, the coil will be reworked before being transferred. The completed coil will be removed from the winding station using the lifting spider and the mobile lifter.

The cold-mass assembly process consists of stacking the coil sections into a vertical stack and connecting the sections into a continuous half-magnet module. Stacking the coil segments vertically provides the necessary access to the inside and outside of the coil. As each segment is received, it will be lowered onto temporary support blocks to allow the necessary space for connection of the conductor joint. When the joint is completed and tested, the temporary support blocks will be removed, the segment will be lowered flush to the next-lower segment, and bolts will be installed through the flange to connect the two. As assembly of the coil segments proceeds, the helium plumbing and diagnostics will also be installed. By repeating this process, 12 coil sections will be joined together into a magnet half coil.

After the 12th segment of a magnet half has been bolted to the stack, the larger helium manifolds will be attached. The coil assembly will then be tested for electrical continuity and for leaks in cryogenic tubing. After successful testing, the cold mass will be ready for mating with the vacuum vessel and thermal shields.

At the outdoor hard-stand area, two inner vacuum vessels and two outer vacuum vessels will be completed while work is being done on the magnet coils. The vessels will be assembled from factory prefabricated parts, consisting of the largest components that can be shipped to IR5. The preassembled parts will all be fitted with alignment tabs, allowing them to be put in place and aligned quickly using conventional cranes and rigging.

The inner-vessel assembly sequence will begin with a vessel end ring at the bottom, and will then be built upward as shell sections are welded in place. The outer-vessel sequence will begin with the shells and stiffeners, and will end when the other end ring is welded to the top of the cylinder. Upon completion of the two sections, the two vessels will be temporarily welded together and will undergo vacuum leak checks.

The thermal shields (and super insulation) will also be fabricated simultaneously with the coilwinding and cold-mass assembly. The thermal shield panels will be completely prefabricated off-site and shipped to IR5. Prior to assembly with the vessels and cold masses, the shields will be hydraulically tested, cleaned, and fitted with blankets of super insulation.

Following successful vacuum testing, the inner and outer vacuum vessels will be separated. The inner vacuum vessel will be moved inside the NAB so that the thermal shields can be attached. The outer vacuum vessel will remain outside on the hardstand, where a temporary roof will be prepared to cover it.

The thermal shields will be assembled in the outer vessel and attached to the inside by welding the attachment points directly to the vessel. The G-10 standoffs will then be attached. As the shields are put in place, the liquid nitrogen connections from panel to panel will be welded and cold-tested. When all shields are in place, final hydraulic tests will be completed. Attachment of the thermal shields to the inner vessel will proceed in a similar manner, except that the shields will be attached to the outside of the cylinder. Since the thermal shields will be located on 
the externai face of the inner vacuum vessel, this particular operation will be performed within the NAB. The outer vessel can be kept outside since the shields will be located on its internal face, and, with appropriate covers, will be protected from the weather.

When the inner vacuum vessel and the thermal shields have been mated, and the cold mass has been completed, both will be moved to an outdoor lifting station to mate the inner vessel and the coil assembly. Once mated, the assembly will be moved back inside the NAB where the radial and axial cold mass supports will be attached between the end ring of the inner vessel and the cold mass. The radial rods will be tensioned using hydraulic cylinders to attain the desired pre-load. The axial rods will all be adjusted simultaneously with hydraulics to ensure that the coil load is evenly distributed.

The vacuum vessel/cold mass assembly will then be moved back outdoors to the lifting station, where it will be mated with the outer vacuum vessel. Upon completion, the assembly will be moved back indoors, where all of the final connections will be made. The vacuum vessel will then be welded, and the radial cold-mass supports on the lower side will be completed. All remaining hydraulic and electrical connections will then be completed. The electrical and cryogenic systems will again be tested. In addition, a final vacuum test will be performed to verify the integrity of the vacuum chamber.

When all of the necessary tests and connections have been made, the magnet halves can be moved outside to the large jacking frame and rotated so that the axis is horizontal. Each assembly will be moved to an area adjacent to the utility building, where it will be connected to cryogenic lines from the refrigerator system. A final checkout test will be performed after the magnet has been cooled to liquid nitrogen temperature to verify the thermal, hydraulic, and electrical performance of the coil half. Following successful testing, the magnet will be installed in the underground hall.

\section{Muon System}

As in the case of the magnet, the large size of the muon subsystem requires that most of the assembly take place on-site. Most, if not all, of the components will be prefabricated elsewhere, with only the final assembly and test of the muon modules and the muon monoliths performed at the IR5 site. Muon support structures will be completely fabricated off-site; they will be inspected and evaluated prior to partial disassembly and shipping to the SSCL. Disassembly will be kept to a minimum, dictated by efficient shipping concerns. Upon arrival at the SSCL, the structures will be unpacked, inspected, stored, and reassembled as needed in the SAB. Muon-module preparation, as well as chamber testing, will take place in the SAB. Final assembly of the barrel and endcap monolith structures will take place in the NAB. Twenty-four barrel modules (twelve for each magnet half)-each containing four sectors of CSCs for a total of 960 barrel chambersand two endcap, three-wheel monoliths-containing the 480 endcap CSCs-must be assembled.

Initially the high bay on the east end of the $S A B$ will be used for muon prototypes. The remainder of the building will be used for production of muon modules after the completion of the GEM magnet coil-winding process. The high bay will be equipped with the necessary module assembly stations, rotation areas, testing and alignment facilities, and storage areas. This bay will share use of the two bridge cranes with the other bays in the building. The cranes will assist with the flow of materials and support structures between the assembly stands.

The support structures for the muon modules will consist of a series of aluminum trusses on which all of the muon chambers, alignment, water cooling, gas, and electronics systems will be supported. During assembly, the largest dimension of the module will be kept closest to the floor. This will create the most stable mount for the assembly, utilizing the same attachment points that are used to attach the modules to their respective support rings. Following the evaluation of the structure assembly, the assembly procedure will continue with the insertion of the three superlayers of muon chambers and the chamber-to-support structure interface hardware (described in Chapter 4).

The chambers will then be tested and aligned. As much of the electronic cabling and water-cooling plumbing as possible will be connected to each module while in the SAB. All the connections within each module will be run to predetermined points so that when all of the modules are joined together, the number of final connections to be made is mini- 
mized. Ideally, a manifold-type connection can be used to join all of the electronics and cooling systems.

When completed, the barrel modules will be transported to the NAB for assembly into the monolith structure and stored until they are needed. The barrel modules will be delivered in a special support frame so they can be rotated into their proper orientation for installation into the support structure. The installation procedure is described in detail in Section 9.4.4.

Each endcap assembly will comprise five major subassemblies: three wheel assemblies and two conical connective structures. These five subassemblies will be constructed independently, merged to form an assembly, and finally aligned and checked out as a complete endcap assembly. The five major subassemblies, and the assembly of the endcap monolith are described in Section 4.4. The installation procedure of the endcap is described below.

\section{Calorimeter}

The baseline calorimeter is compor $\mathrm{d}$ of two calorimetry subsystems: a scintillating barrel calorimeter and a noble liquid calorimeter. Both calorimetry systems will be assembled from prefabricated parts in the NAB. The liquid calorimeter will be assembled in three sections: a barrel and two endcaps.

The shell for the scintillating barrel calorimeter will arrive at the IR5 site prefabricated and with rounding rings already attached. The scintillator modules will be attached to the inner face of the support shell. When the scintillating barrel is mated with the CDS, the rounding rings will be removed, and all of the piping, shielding, wiring ways, etc., will be connected.

Assembly of the liquid barrel calorimeter will take advantage of the modular characteristics of the components delivered to the IR5 facilities. Initially, a vertical stabilizer cylinder will be attached to a lower assembly plate to provide the form to which the EM modules will be attached. Both the plate and the cylinder will be built in halves to facilitate insertion of the modules. After $20 \mathrm{EM}$ modules have been inserted into the assembly form, the two halves will be rotated into a closed position. Seventeen tension straps will be applied to the inner radius of the EM cylinder. These straps will keep the EM modules stable while the stabilizer cylinder is removed. External tensioning straps will then be applied to the outer radius.

Once the EM cylinder has been completed, it will be mated with the hadronic spool. The entire assembly will be placed on a handling fixture and rotated so that the axis is horizontal. Supports will be placed at both ends of the assembly. The inner and outer hadron modules will then be applied to the spool. Structural stays will be attached between the two layers.

After all of the barrel modules have been assembled, the outer pressure vessel will be installed and the cold feed-throughs and support stanchions will be connected. Once the load is transferred to a separate support cradle, the outer vacuum vessel can be attached. Finally, the in ter pressure vessel and inner vacuum vessel will be installed so that all of the warm feed-throughs and final plumbing can be installed.

Assembly of the other liquid calorimeter sections will also make use of prefabricated modular components. The procedure used to assemble them will be similar to that used to assemble the liquid barrel. The endcap modules will be attached to structural spools that have been placed on horizontal mandrels. After the modules have been connected, the pressure vessel will be closed and the cold feed-throughs added. The outer and inner vacuum vessel shells can then be finished and the warm feed-throughs and final plumbing installed.

\section{Tracker}

The area reserved for tracker assembly will be located in the NAB. This space will be used to complete the initial tracker assembly and to perform detector maintenance operations throughout the expected 10-year life of the detector. Mandrels and fixtures for rotating, maneuvering, and aligning the interpolating pad chambers (IPC) will be necessary. Materials storage areas are also required.

The preassembly and testing of the silicon ladder assemblies, including the electronics, will be completed prior to shipment to the IR5 facility. Once on-site, the silicon ladder assemblies must be protected during their mounting onto the cooling rings, requiring a Class- 10000 clean room environ- 
ment. High-precision optical equipment will be used to position the ladder assemblies onto the cooling rings with a tolerance of less than $1 \mu \mathrm{m}$. This will require a stable floor with vibration isolation from the rest of the building. The isolated floor area will be about $2 \mathrm{~m} \times 4 \mathrm{~m}$. The clean room will be $10 \mathrm{~m} \times$ $10 \mathrm{~m}$, which is large enough to assemble the $2-\mathrm{m}$ space frame assembly into the $2-\mathrm{m}$ gas enclosure, contain the assembly and optical inspection stations, and provide adequate storage area for critical components. In order to maintain mechanical stability to ensure precise measurements, tight controls over temperature and humidity will be required.

It is anticipated that the IPCs will be fabricated elsewhere. The completed IPCs will be shipped to the IR5 facility, unpacked, and inspected visually. They will be tested with a source under operating conditions (gas and HV) in the $x$ ray calibration lab to ensure that they function correctly. Any chambers with broken wires or other damage will be opened, repaired in the Class- 10000 clean room, and retested.

As each chamber completes its check-out, it will be installed in a positioning frame designed to maintain $25-\mu \mathrm{m}$ stability. This operation will take place on a reference table for the barrel section and again for each endcap of the tracker. Following the assembly, gas manifolds will be installed and leak-tested.

The fully assembled chamber sectors may be scanned with the $x$ ray mapping system in a shielded room at this time, or after the final assembly with the silicon inner tracker. If the IPCs are to be $x$ ray scanned, the IPC assembly will be moved, using the overhead crane, into the $x$ ray calibration lab. This lab requires a vibration-isolated floor of $2 \mathrm{~m} \times 4 \mathrm{~m}$. Normal gas, HV, and cooling conditions will be established for the mapping. The area must be temperature-and humidity-controlled and shielded against leakage of the $x$ rays.

Since the combined length of the IPCs and the silicon inner tracker assemblies exceed $5 \mathrm{~m}$, special fixtures will be required. Once the silicon inner tracker is in place inside the IPC system, the two assemblies must be aligned with each other to less than $25 \mu \mathrm{m}$. A final full-system test will be performed to simulate the central tracker operating conditions. The test requires fully powering both the
IPC and silicon tracker electronics systems. The butane cooling system for the silicon and the water cooling system for the IPC will be connected and operated to test the heat rejection system. X-ray calibration tests will be conducted to verify thermal stability and to perform final silicon microstrip mapping.

\section{Forward Field Shapers}

Each forward field shaper will consist of a cone section and a support structure that will be assembled in the large hard-stand area. The cone will be fabricated off-site in approximately $5-\mathrm{Mg}$ annular pieces, with features designed to interlock with the other parts of the cone. Starting from the large end, these pieces will simply be stacked up, with axis vertical, using dowels to ensure that holes are properly aligned. When the final piece is in place, tie rods will be inserted through the entire stack from the top. The rods will be hydraulically tensioned at the bottom, and the final connection made. When final inspections and measurements are complete, the cone section can be attached to the support structure.

The FFS support structure will be prefabricated and perhaps even preassembled off-site. It will be shipped to IR5 in large 5-7 Mg parts, which will be keyed. They will be bolted and welded together. The support structure will be fabricated wholly on-site at the hard-stand fabrication area. Assembly is expected to proceed rapidly because of the simple construction. When assembly is completed, the structure will be moved to a jacking frame, where the cone will be rotated to its final orientation and placed into the support structure. Final connection will be accomplished with bolts. The FFS assembly will then be ready for installation.

\section{Beam Pipe}

The beam vacuum system will consist of seven discrete sections plus various pumps, valves, and bellows assemblies-divided to allow for installation in the underground hall in different phases as the various detector components are installed. A central section, made of beryllium, will pass through the center of the tracker subsystem. Stainless steel or aluminum sections, sized to pass through the barrel and two endcaps of the calorimeter subsystem, will connect to the central section via a bellows and NEG pump. Beam position monitoring devices will 
connect to the outer ends of these sections. Similarly, stainless steel or aluminum sections will be sized to pass through the FFS. Ion pumps will be attached to each end of these sections, with the pumps at the inner ends connected to the beam position monitors. Finally, additional sections will complete the beamline from the ion pumps at the outer ends of the FFS to the collider quadrupole/collimator system.

The various pipe sections, pumps, valves, etc., will be procured and shipped to the NAB tracker and beamline assembly area. Here each section will be inspected, vacuum tested, and stored until needed. The sections will be cut to size and flanges attached at installation time.

\section{Electronics}

Since the majority of the electronics systems used in the detector are subsystem-specific, they will be assembled along with each subsystem. However, separate preparations must be made so that all of the subsystem electronics can be linked to the electronics room, and then to the operation-center racks. The data acquisition system, the Level 1 trigger system, the calorimeter on-detector electronics, and the lowand high-voltage power supplies for front-end electronics will be prepared in the SAB electronics shop. The racks will be equipped with the necessary wiring, sensors, cooling systems, etc., to support and service the electronics crates.

Cabling will be required to interface power and readouts to each of the detector subsystems. Both the fiber optic cabling and the conventional cabling will arrive at the IR5 site in rolls. Cabling will be dispensed from these rolls as needed, cut to the appropriate length, and terminated to suit the installation.

Two 12.5-kV 480/277 transformers will be installed at the head house of the electronics cable shaft. One of these will supply technical power for each floor of the electronics room; the other will serve electronics in the experimental hall.

\section{Services}

The assembly buildings are designed to incorporate all required service. For example, each building is equipped with electrical outlets capable of supporting welding equipment. In addition, special clean room areas will be required for both calorimetry assembly and tracker assembly. For more specific information, refer to the GEFUR document.

\subsection{INSTALLATION}

\subsubsection{Installation Facilities and Tools}

The installation of the large components in the underground hall will require heavy transport and lowering tackle. Specialized support for this task is being sought where all such movements will be provided under contract by experts in the field.

\section{Access Equipment}

Throughout the installation process, special equipment and fixtures will be required to provide access. Where access is required for continued maintenance and adjustments, this equipment will be permanent. If access is required only during installation, the access equipment may be temporary.

All of the magnet connection points will be located at the top of the detector. For access to the connection points, permanent scaffolding will be constructed. An access walkway will be installed on the top of each outer vacuum vessel, extending the length of each magnet half. Access to these walkways will be achieved directly from the catwalks located on the walls of the detector hall. Bridges will be extended from the catwalks to platforms located at the outer edge of the central detector support. Stairs will connect these platforms to the magnet access walkways. Care must be taken to ensure that the magnet halves can be separated without having to completely disassemble the access scaffolding.

To facilitate the installation of heavy calorimeter components in the detector, a system of support rails will be installed in the underground hall before the first calorimeter component is lowered. The rails will connect to the central detector support structure, and will be set at the height necessary to deliver the calorimeter sections into their position at the center of the detector. The rails will be able to support the weight of the calorimeter sections, and to counterbalance asymmetrical loading of the central detector support structure throughout the calorimeter installation process, until the section can be permanently anchored to the central detector support structure. Clearance considerations require the rails to extend several meters on either side of the support structure, 
to leave room for the endcap sections to be temporarily stored while connections are being made with the barrel section. In addition to the main support rails, a rail extension section will be installed from one end of the main support rails to a position underneath the installation shaft. This will permit the sections to be delivered directly from the installation shaft to their installation height without the use of the hall bridge cranes. The rail extension will be movable and capable of being erected in similar fashion at the opposite end of the hall, so that calorimeter sections may be lowered from the other installation shaft.

Scaffolding will be constructed to provide access to the connections required for the installation of the calorimeter system. Temporary scaffolding will be used to assist in the calorimeter installation; permanent scaffolding will be installed to provide the necessary long-term access to all of the electronic data-acquisition cabling and equipment.

For access to the beam line as it enters the detector, access scaffolding will be constructed on the external face of each FFS. In addition, access will be provided to the inside of the detector to allow for muon maintenance. A platform located at the beam line and walkways/ladders will be extended to platforms located at convenient entry points into the detector. The field shaper scaffolding must be independent from the rest of the detector so that the field shapers can be separated for tracker insertion and later detector maintenance.

Since the beam vacuum system will be installed in several sections, there are several connection points to which access must be provided. Access to the connection points inside the calorimeter will be provided by the temporary scaffolding that will be present to access the connection points required for tracker and calorimeter installation. Access to the connection points between the field shapers and the calorimeter will be provided by temporary scaffolding connected to the FFS. Access to the external connection points at either end of the detector will be provided by the platforms installed onto the face of the field shapers.

All of the permanent scaffolding that is attached to each section of the detector must remain independent from the other sections. No matter where in the hall a component is located, access should be possible from either the floor or the wall catwalks. Magnet components will be moved to different locations within the hall throughout the installation and maintenance procedures.

\subsubsection{Installation Strategy and Resources}

The GEM installation schedule is based on first lowering the CDS into the underground hall and moving it to its permanent location. This will be followed by parallel installation using the two assembly shafts for the two halves of the magnet, the muon barrel structures, and the calorimeter. Each of the two muon endcaps will be temporarily attached to a FFS on the surface, and the two will be lowered down the shaft as a single unit. The FFS will serve as a transporter to translate the muon endcap to the muon barrel structure, where it will then be transferred to, and attached to, the muon barrel.

Twelve major detector components, plus the vacuum system, will be installed. For nearly all of these a large, special-handling facility will be required to lower the components to the hall floor. It may prove more economical to rent only one such facility, which implies that the deployment of the handling facility must be optimized to minimize the installation time lost while transferring the lifting and handling equipment from one shaft to the other.

A work plan for the detector installation in the underground hall will incorporate the equipment necessary for safe working conditions including scaffolds, elevators, platforms, safety harnesses, protective clothing, and hard hats. Personnel will not work under the exposed shafts during lowering of equipment, nor will they be permitted to work at a height without suitable safety equipment.

\subsubsection{Installation Schedule and Contingency Plan}

The detector top-level installation schedule was developed by referencing the important detector and collider milestones. Details were then developed at a lower level for each subsystem. In comparing the details with the top-level milestones it was apparent that small adjustments were necessary. For certain activities a double shift will be necessary to maintain the critical commissioning milestones of the collider. Should a problem arise during the assembly or installation phase of the detector that has an impact on the schedule, different 
contingency strategies may be applied. One method would be to simply increase the number of daily shifts or to work weekends. Another would be to carry out more tasks in parallel, using the two installation shafts. The availability of both of these options results in a healthy contingency for the GEM installation schedule.

\subsubsection{Installation Descriptions}

Installation of the GEM detector will begin as soon as beneficial occupancy of the underground hall is available. At this time, the overhead cranes will be in place and operational, the floor rails for supporting and guiding the subsystem installation rollers and transporters will all be in place and surveyed, and all lighting, ventilation, and other environmental control systems will be functional. Figure 9-10 depicts the underground experimental hall as it will appear when detector installation begins.

\section{Central Detector Support}

The central detector support (CDS) will be the first of the detector components installed into the underground hall (Figure 9-11). CDS installation will begin when access is allowed into the underground hall. However, in preparation for installation of the CDS, tracks in the floor of the hall and moving equipment must already be in place. Final preparations also include a survey of the hall, to verify and document the location of both the hall alignment fiducials and the tracks.

The heavy-lift jacking frame, which will lower all of the large objects down the shaft, will be erected in place over the south installation shaft. The jacking frame has the ability to move while under load, so it will lift objects from an adjacent staging area, translate over the shaft, and lower the objects to the floor of the underground hall. While one shaft is being used the second will remain covered.

Lifting strongbacks will be used to connect the four jacks to the two lift points on the CDS. The CDS will be lowered into the hall and moved by the transporters on the track to its position in the center of the hall. At this point, it will be surveyed and aligned within the detector hall. Final shim stacks will be prepared, and the CDS will be permanently mounted in place.

\section{Scintillating Barrel Calorimeter}

The scintillating barrel calorimeter is the next subsystem to be installed. Special support rails are required to position the calorimeter at the correct height, and to translate it from its initial position at the bottom of the installation shaft to its mounting location (Figure 9.12). The scintillating barrel calorimeter will be lowered down the south installation shaft, placed directly onto the support rail extension, and moved along the rail into position within the CDS.

Following correct positioning and alignment, cabling and services will be routed from the calorimeter support tube, along the CDS structure, then via cable trays and pipe supports to the electronics racks located along the walls of the experimental hall.

\section{Magnet}

Although each of the two magnet halves could be lowered simultaneously by using the two installation shafts, one magnet half will be available before the second is completed. The first magnet half will be lowered down the south installation shaft and transported along the floor rails to its operating location. No lifting equipment (other than the large jacking frame) is required, since the jacks can connect directly to the lift points on the vacuum vessel. Measurements will be taken at each of the survey monuments to make sure that the magnet half is properly aligned to the beam line. Upon confirmation of proper alignment, the magnet half will be shimmed to its correct height and bolted into place. The transport equipment will then be removed.

The surface crane will then be moved to the north shaft so that the second magnet half can be installed (Figure 9-13). Procedures to install the north coil half will be identical to those for the south half. Following installation of the second magnet half, the surface crane will be removed and covers placed over both shafts so that magnet testing can proceed without interference from surface weather.

When both magnet halves have been lowered into the hall and bolted into their final positions, the cryogenic plumbing, the electric power buses, and various other services will be connected to the magnet. Vacuum lines will be cut to length and 


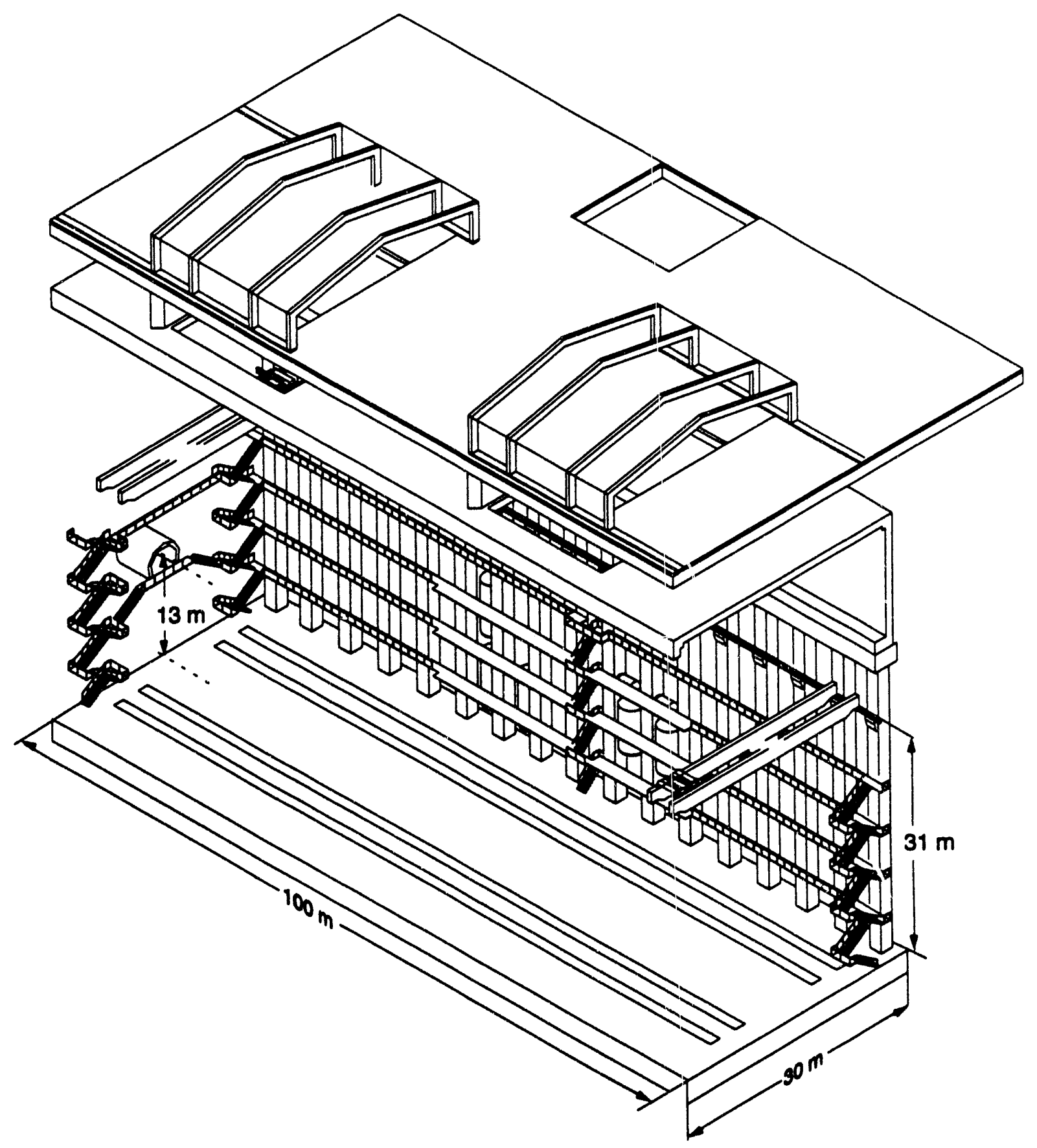

FIG. 9-10. Isometric drawing of the underground experimental hall during cletector installation.

connected to the high-vacuum pumps on the magnet vessels so that pump-down can be initiated as soon as vacuum integrity is accomplished. All of the control and diagnostic cables will then be terminated and connected to the each of the magnet halves. The cryogenic transfer lines, which will be prefabricated, will be cut to length, welded into place, and leak-tested. As soon as the helium and nitrogen connections are finished, and leak-checking is complete, cleanup of the helium and nitrogen system will begin. Letak checks and electronic tests will again be performed before the magnet is cooled down for further testing. All of the service and utility lines will run from the utility shaft to the detector hall through the tunnels located on Level 5 of the detector hall. 


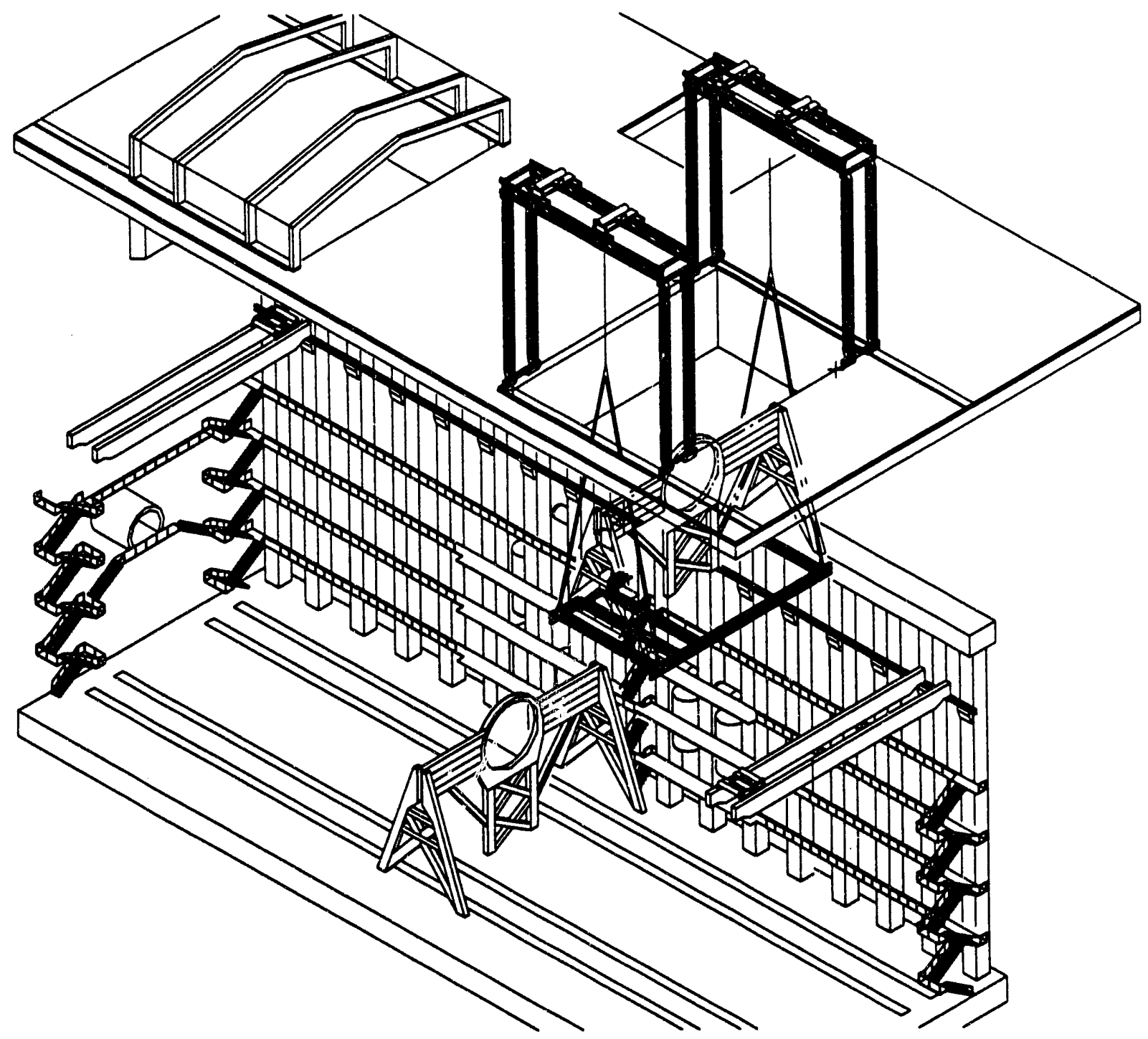

FIG. 9-11. Contral detector support.

The power lines will connect at the top of the detector, at either end of the magnet, where the conductor coils begin and end. In order to join the two magnet halves into one continuous coil, a bolted electrical connection joint will be installed between the two halves at the top of the detector on either side of the CDS structure. The cryogenic feed pipes will form two separate, parallel loops as contrasted with the single, series loop used to power the coils of the magnet. Separation of the loops will enable the magnet to be kept cool when the magnet halves are separated for access to the interior of the detector.
Upon verification of the status of the safety and coil-protection systems, and assurance that the liquid helium dewars are at capacity, the magnet will be prepared for operation. The vacuum vessels will be evacuated and the magnet will be cooled to operating temperatures by circulating cold gas through each system. Magnet cooldown will commence while final connection of the warm buses is completed. The large removable sections of the bus will be lifted into place with the hall crane, and bolted into position so that final electrical checks can be completed. 


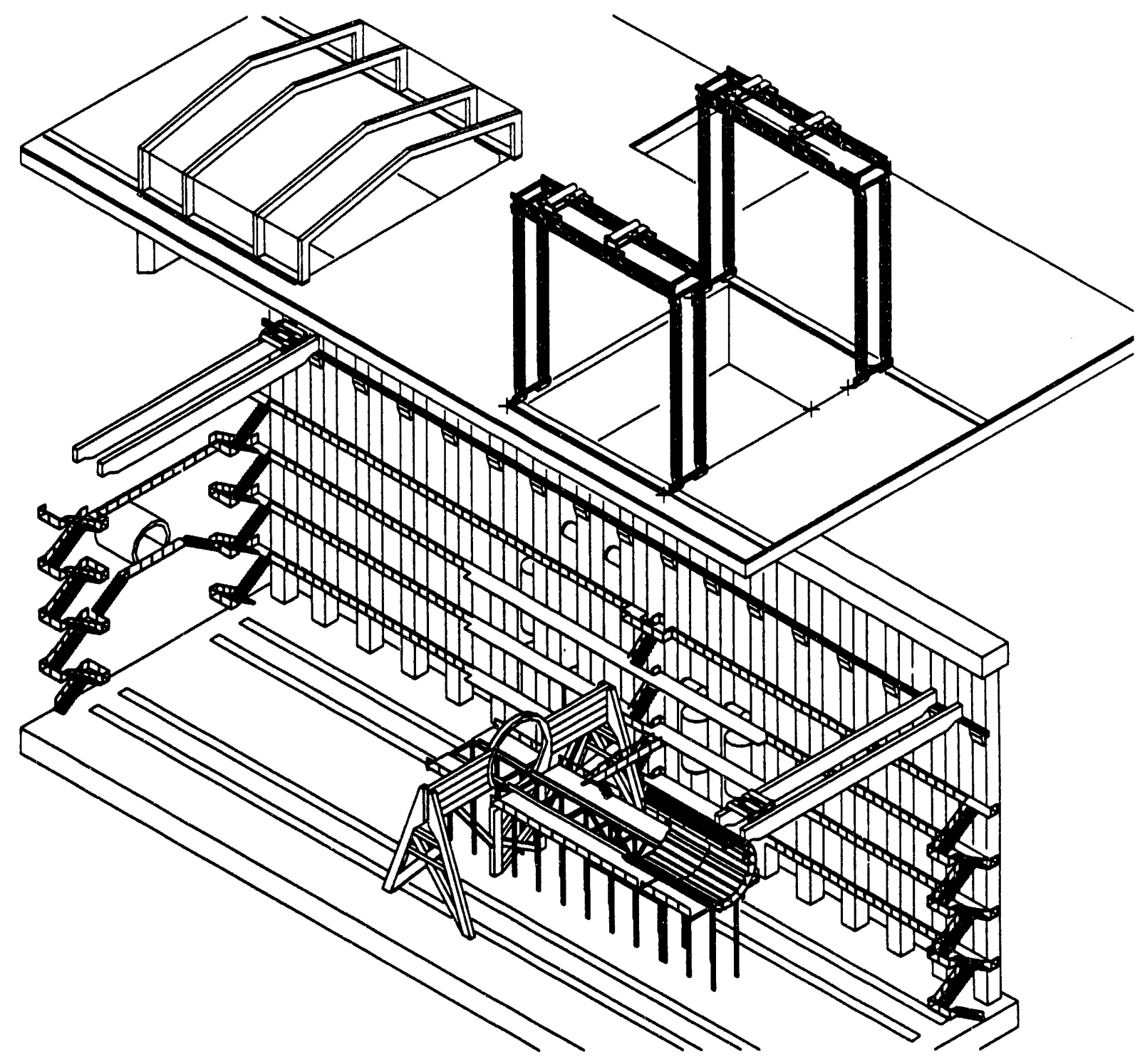

FIG. 9-12. Scintillation barrel calorimeter installation.

After the magnet has been cooled to $4.5 \mathrm{~K}$ and stabilized, performance testing will begin. The hall will be cleared of personnel and ferromagnetic objects, and the cranes and any other movable fixtures will be locked into position. The power supplies will be unlocked and the magnet charged so that the magnetic field can be tested and measured.

Following acceptance of the magnet, is will be discharged and prepared for movement to the parked position, allowing for installation of the other detector subsystems. The magnet halves will be moved using the same transporters and tracks that were used for installation. The magnet installation and test procedures are expected to consume approximately six months. Since the remaining installation procedures are expected to last approximately two years, the magnet will be fully discharged and maintained at a nominal temperature and vacuum in order to protect it while minimizing power consumption.

\section{Noble Liquid Barrel Calorimeter}

It is expected that the hadronic and electromagnetic calorimetry systems will have been assembled on the surface into three calorimeter sections: a barrel section and two endcap sections. After the 


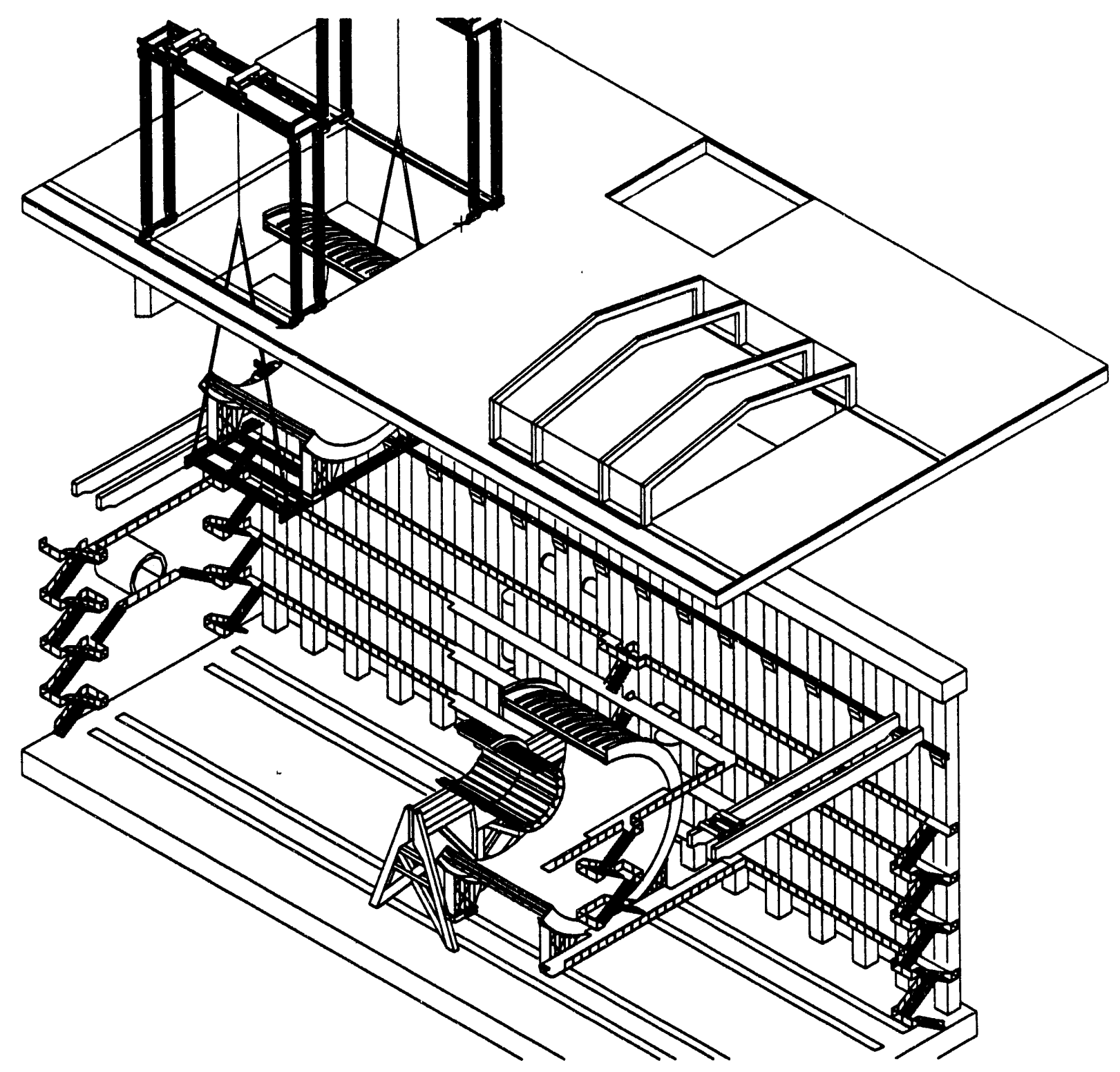

FIG. 9-13. North coil installation.

magnet has been installed, tested, and reopened, the installation of the barrel calorimetry system can begin (Figure 9-14). The surface crane will be repositioned over the south installation shaft. The calorimeter support rails will be required during this part of the installation process.

The barrel section will be lowered down the south shaft, moved along the rail into its final position within the CDS, and fastened into place. All of the electronic and cryogenic connections will then be made to the calorimeter. The electronic data-acquisition connections will be uniformly distributed around the outside of the calorimeter at each end of the barrel. Lines will be run from these points along the outer wall of the calorimeter to the CDS, where they will be routed and connected to the appropriate electronic crates. Cooling loops will be run from the cooling liquid dewars located on gangways along the east wall of the underground hall and fed throughout the calorimeter. In addition, a krypton wet line and a krypton drain/fill line will be run from the head control dewars located along the wall and fed into the barrel. All of the cryogenic piping will feed from the wall at Level 3 into the top of the calorimeter at the CDS structure. From there, it will extend to the appropriate connection points. 


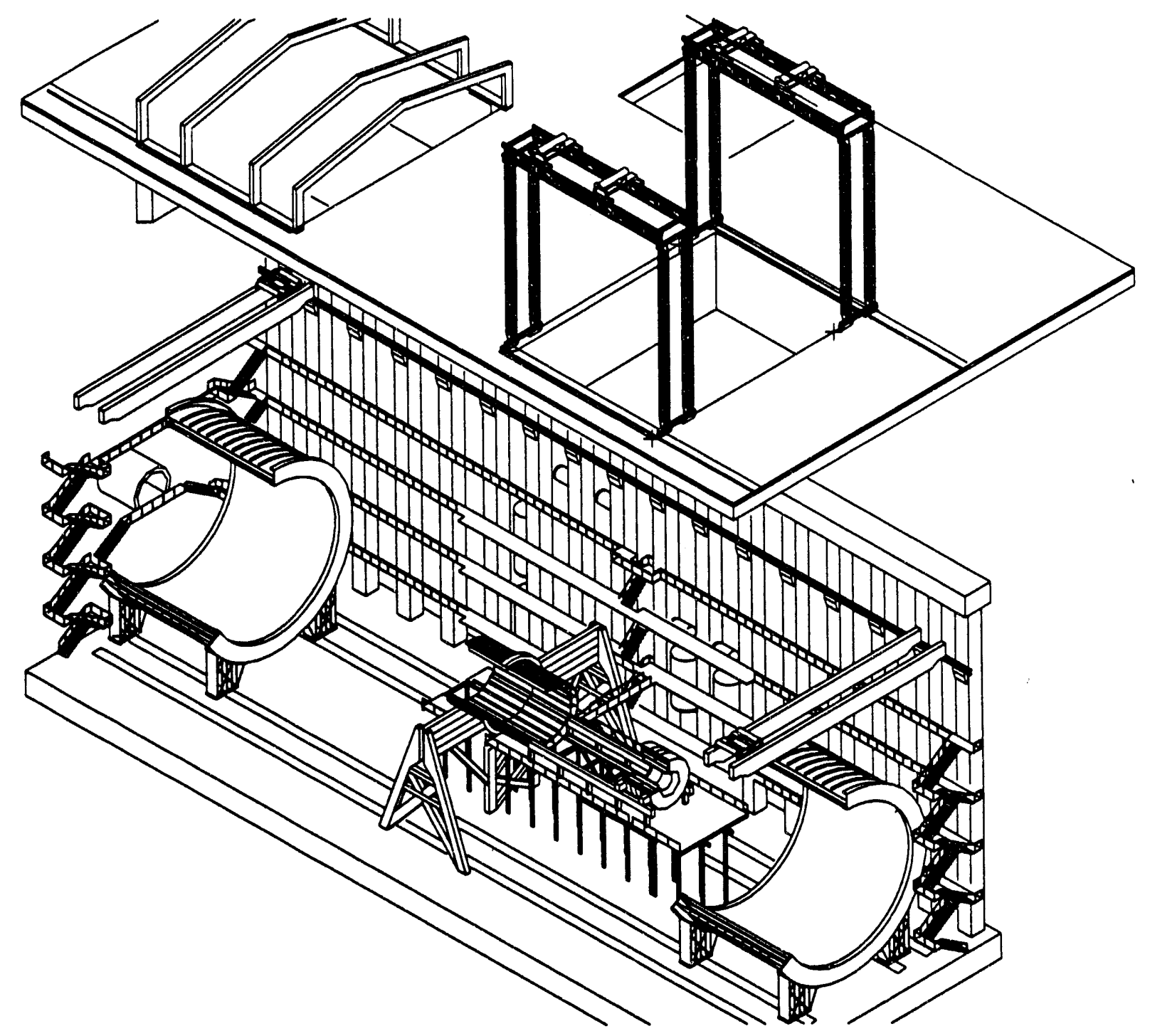

FIG. 9-14. Installation of the barrel calorimetry system.

Once the necessary connections have been completed, the cryogenic lines will be checked for leaks and the electrical connections will be tested. Upon verification of the status of the various safety systems and assurance that all of the cryogenic dewars are at capacity, the caiorimeter will be cold-tested and checked for operational soundness. Once all of the tests have been carried out, the calorimeter support rails will be removed and the two magnet halves will be brought closer together so that installation of the muon subsystem can begin. When the magnet halves are closed, a 2-m gap must be left between the CDS and each magnet half to allow access to the interior of the detector for muon barrel installation.

\section{Muon Barrel Subsystem}

A fully assembled, tested, and aligned set of 12 barrel modules forming a complete monolith, previously stored in the $\mathrm{NAB}$, will be installed into each magnet half. In order to accomplish this, it will be necessary ount the monolith onto a transporter that serves as a base for both the short horizontal trip from the assembly building to the access shaft, and the vertical descent of the muon system half to the floor of the underground hall.

As described in Section 4.4, the two end-support rings will be built atop structures that will form the end pieces of the muon system transporter. Upon completion of the barrel assembly, the remaining 
center portion of this transporter will be installed. The barrel region muon system on this transporter will be moved to the appropriate access shaft. A crane will be used to lift this entire assembly over the access shaft and to lower it to the floor of the underground hall. Here, the muon system will be transferred from the transporter to the installation rails that will be used to translate the muon system through the magnet cryostat.

The first muon barrel monolith structure will be lowered down the south shaft (Figure 9-15). The muon monolith structures have been designed so they may be assembled and installed independently from the other detector subsystems. This will require two sets of installation rails, one within the magnet halves and one on the floor of the experimental hall. The first rail system will start near the end of the magnet. It will consist of two short bridges used to support rails aligned with and connected to the rails attached to the inner surface of the magnet cryostat, on . thickened portion of the cryostat vacuum wall. These will be simple flat rails used in conjunction with either linear roller bearings or pads, to minimize frictional forces during insertion of the assembly and to provide restraint of the lateral motion. The other transport rail system will be used to support and provide a low friction bearing interface for the FFS end of the barrel region assembly. This rail system will utilize the same tracks as the magnet leg transporters. Here, again, either linear roller bearings or grease pads may be used. Selection will most likely be dictated by the final selection for the magnet transporter bearing elements. Lateral tracking restraint hardware for this system will duplicate that required for the magnet transporter.

As an alternative, the floor rails might not be necessary if installation rails are incorporated into the transport fixture that is used to deliver the muon

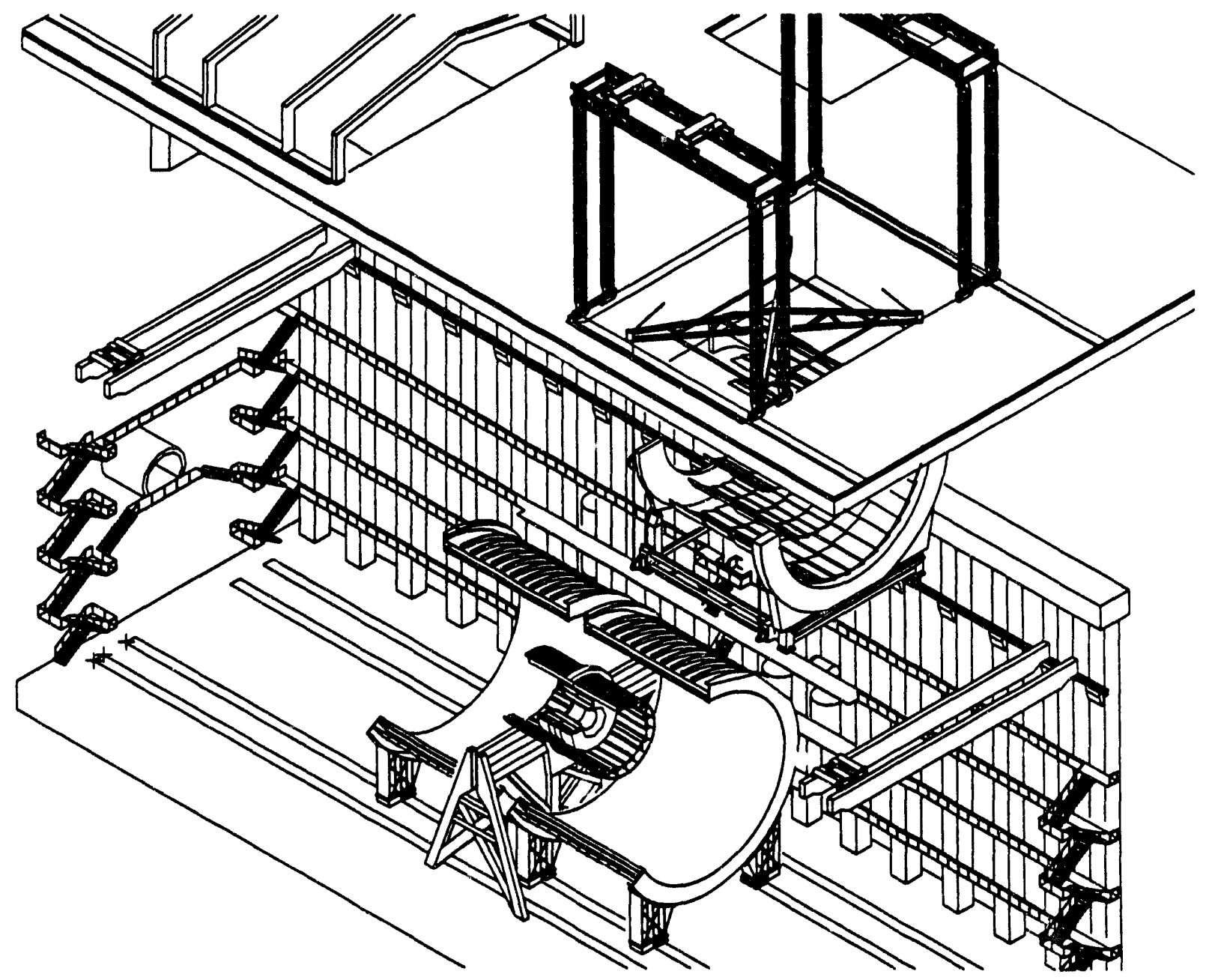

FIG. 2-15. South barrel muon installation. 
barrel section to the underground hall. The rails along the length of the inner face of the magnet halves will act as guides for the muon barrel sections as they are maneuvered into position within the magnet. The internal end of the muon barrel will be supported within the magnet, while the external end will be supported directly on the floor.

The installation rails will deliver the monolith directly into the magnet at the proper height. Once enough of the barrel structure has been transferred into the magnet, the temporary support rails/installation fixtures will be removed. This will allow the barrel sections to be maneuvered into their final positions within the magnet. When the muon system has been positioned, temporary supports will be attached at the internal end of barrel section. This will remove the weight of the system from the rail guidance system, and will allow for height adjustments and for muon system alignment.

\section{Barrel Muon Structure to Magnet Attachment}

The barrel muon monolith is attached to the magnet by fastening the two end-support rings-the CDS and FFS support rings-to two major load-bearing points on each end of the magnet. The FFS support ring has its two attachment points low on the magnet legs, and the CDS support ring attachment points align with the horizontal platform welded to the large "C-Ring" that forms the end of the magnet cryos tat. The quietest vibration environment on the magnet is located at the magnet legs. Thus disturbances to the muon system are minimized with the selection of these magnet-leg mount sites. Attachment of the muon system to the magnet at these points will also minimize motior that could cause alignment problems in the muon system.

With the muon system properly located inside the cryostat, the load of the muon system will be transferred from the transporter hardware to hard mounts on the magnet legs and to laterally compliant mounts on the CDS end of the magnet. The global position of the entire assembly will be evaluated, and final attitude corrections will be determined. The final adjustments in position and orientation will be made with shims, wedges, and linear translations, followed by a final check to confirm compliance with specifications.
Interface hardware between the FFS ring and the magnet is relatively simple. Some additional structural elements are added to the FFS support ring to distribute the load of the FFS end of the muon system to the two mounting points on the magnet legs. Also, there is adequate space for adjustment hardware and, possibly, for total system damping elements. A similar reinforcing and distribution structure will be added to the CDS support-ring end to permit a two-point mount on the CDS. Additional temporary hardware will also be needed to provide space for the transport hardware and for adjusting the position of the muon system at the CDS end. The CDS end of the magnet is chosen as the z-restraint position of the muon system. This choice has been made to minimize the potential disturbances to the muon system when the magnetic field is activated, and the magnet is compressed along the $\mathrm{z}$-axis due to the magnetic forces of the coil and the FFS.

Following installation of the south muon barrel monolith, the heavy-lift crane system and installation rails (if required) will be moved to the north installation shaft. The north muon barrel monolith will then be lowered and installed in a manner similar to that for the south barrel. The installation of the endcap structure is described later.

The muon system will be serviced by a variety of signal cables, power lines, temperature sensors, gas piping, etc. All of the connections to the individual chambers will already have been made during the assembly process. The only connections that will remain will be the manifold-type connections that will connect the barrel section to the hall utilities. All of the gas utility feeds will run from the utility shaft and connect with the detector at both ends of the hall. The fiber optic cables and power lines will be run from the cable electronics shaft. The connections will be made at the joint between the barrel and endcap muon sections. All connections will be checked and tested to ensure proper operation of the barrel muon system.

\section{Noble Liquid Calorimeter Endcaps}

After the barrel muon systern has been installed and its check-out and testing has been completed, the magnet halves will be separated so that the calorimeter endcaps and tracker can be 
installed (Figure 9-16). The north endcap and passive devices will be lowered first, down the north installation shaft, placed directly onto the support rail extension, moved along the rail, and placed temporarily on the north main support rail. They will remain at this location until the central tracker is installed in order to allow access to both ends of the tracker. At this time the north passive endcap devices, which contain the forward calorimeter, will be lowered and also parked on the support rails.

Following delivery of the north calorimeter endcap to the underground hall, the heavy lift crane and calorimeter support rail extension will be relocated to the south installation shaft, where the central tracker will be lowered and installed, followed by the south calorimeter endcap. Calorimeter endcap installation will be completed by locking the endcap sections in place on the rails in the scintillating barrel section. This will remove the weight of the endcap from the support rail but will transfer it to the scintillating barrel calorimeter, which will, in turn, be supported by the CDS. This asymmetric loading of the CDS is compensated by temporary supports at the north end of the calorimeter support tube. After all the calorimeter sections have been lowered into the hall, the calorimeter support rails can be removed. Passive endcap devices are attached next.

The electronic data-acquisition connections will be uniformly distributed around the outside of each end of the two endcap sections. Lines will be run from these points along the outer wall of the calorimeter to the central detector support, where they will extend to the appropriate electronic crates. Liquid nitrogen cooling loops will be run from the liquid nitrogen dewars and fed throughout the calorimeter. In addition, an argon wet line and an argon drain/fill line will be run from the head control dewars located along the wall and fed into the two endcaps. All of the cryogenic piping will feed from the wall at Level 3 into the top of the calorimeter at

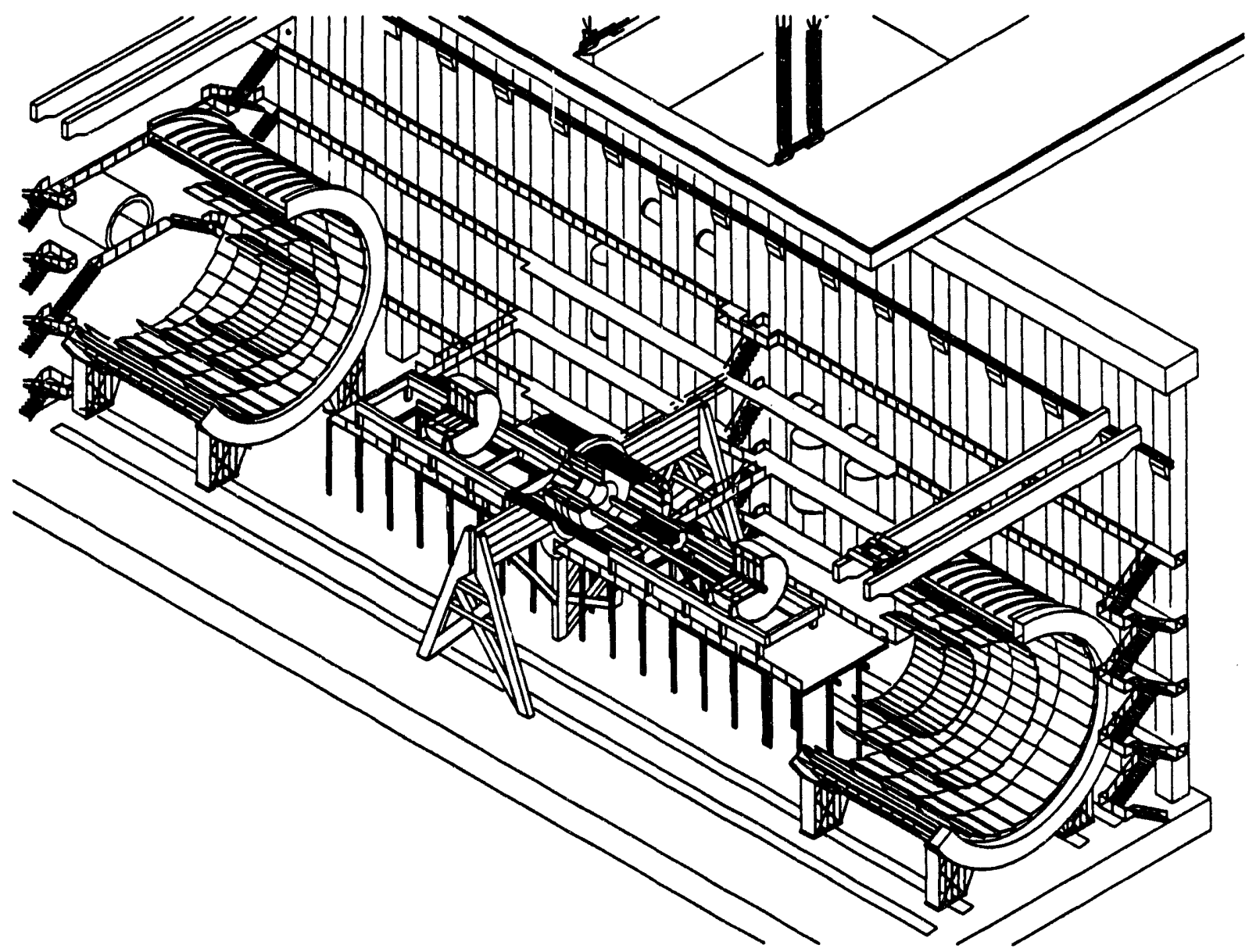

FIG. 9-16. Tracker and noble liquid endcap calorimeter installation. 
the CDS structure. From there, it will extend to the appropriate connection points.

Should schedule constraints require it, both calorimeter endcap systems could be installed simultaneously using both installation shafts. This would require duplicate sets of calorimeter installation support rails, and either two heavy-lift crane systems on the surface or special features on the crane system that permit rapid movement from one installation shaft to the other. All of these would result in increased costs for the detector. However, neither endcap installation can be completed until the tracker installation is complete.

\section{Tracker}

Prior to the lowering of the south calorimeter endcap, secondary support rails will be attached to the south calorimeter support rails. These rails will be able to support the weight of the central tracker as it is transferred into the liquid barrel calorimeter. The inside walls of the integrated barrel calorimeter will have been prepared with installation rails set to accept the tracker, which will be lowered down the south installation shaft and transferred to the hall crane. The hall crane will then deliver the tracker to the secondary support rails, which will be set to deliver the tracker into the calorimeter barrel section at its final height.

Once the tracker has been successfully transferred into the barrel calorimeter, the electronic and cooling connections can be made. These connections will require access at both ends of the tracker. The feed lines will run along the outer edge of the barrel calorimeter and then extend radially out to the edge of the detector. The cooling-liquid pipes and electronic cables will follow the same routes as those for the calorimeter system. The electronics racks required to process the data received from the tracker will be located on the same platforms as those of the calorimeter system.

All connections will be checked to ensure proper operation of the tracker and support services before installation of the south calorimeter endcap is completed and that of the muon endcaps and forward field shapers begins.

\section{Muon Endcaps and Forward Field Shapers}

Each muon endcap monolith will be temporarily mounted on its respective FFS on the surface.
The assembly process of merging the FFS and endcap monolith will be done in the NAB. The FFS will then serve as a transport fixture for the muon endcap. Following its final check-out, the endcap monolith is translated to the FFS, where it is attached to two mounting sites near the ends of the conical surface of the FFS. The entire FFS/monolith assembly will be then be moved to the respective access shaft. Here the assembly will be lifted, translated over the access shaft, and lowered to the floor of the underground hall. Once set onto the floor-mounted transport rails, the FFS will be used as a linear transport fixture to bring the assembly into the magnet.

The first FFS/muon endcap assembly will be lowered down the south installation shaft and placed on transporters prepositioned on the appropriate set of tracks (Figure 9-17). The FFS will be capable of delivering the monoliths at their proper height and orientation at the base of the installation shaft. It will then be pulled to the proper position in the hall so that the muon endcaps can be joined with the barrel sections.

In order for the endcap to be installed into the conical cavity at the FFS end of the barrel, it is necessary to have hardware interfaces on the inside diameter of the endcap structure to permit the attachment of the endcap to the conical element of FFS. This will allow for the transfer of the weight of the endcap from the FFS to the barrel region structure. Currently, two attachment sites to the FFS cone are planned, one near the smallest end of the FFS cone and the second near the largest diameter end of the cone.

The second endcap interface is a mounting system that permits the endcap structure to be tied to the barrel region structures at four points. Two of these load-carrying attachment points will be between the inner and middle superlayers of both regions, and two more will be located on the FFS support ring. These four attachment points will permit the near-kinematic mounting of the endcap to the barrel, and will allow for proper linear and angular orientation of the endcap assembly with respect to the interaction point (IP).

Final adjustments will be made to align the entire endcap region assembly as well as any individual measurement towers that may require 


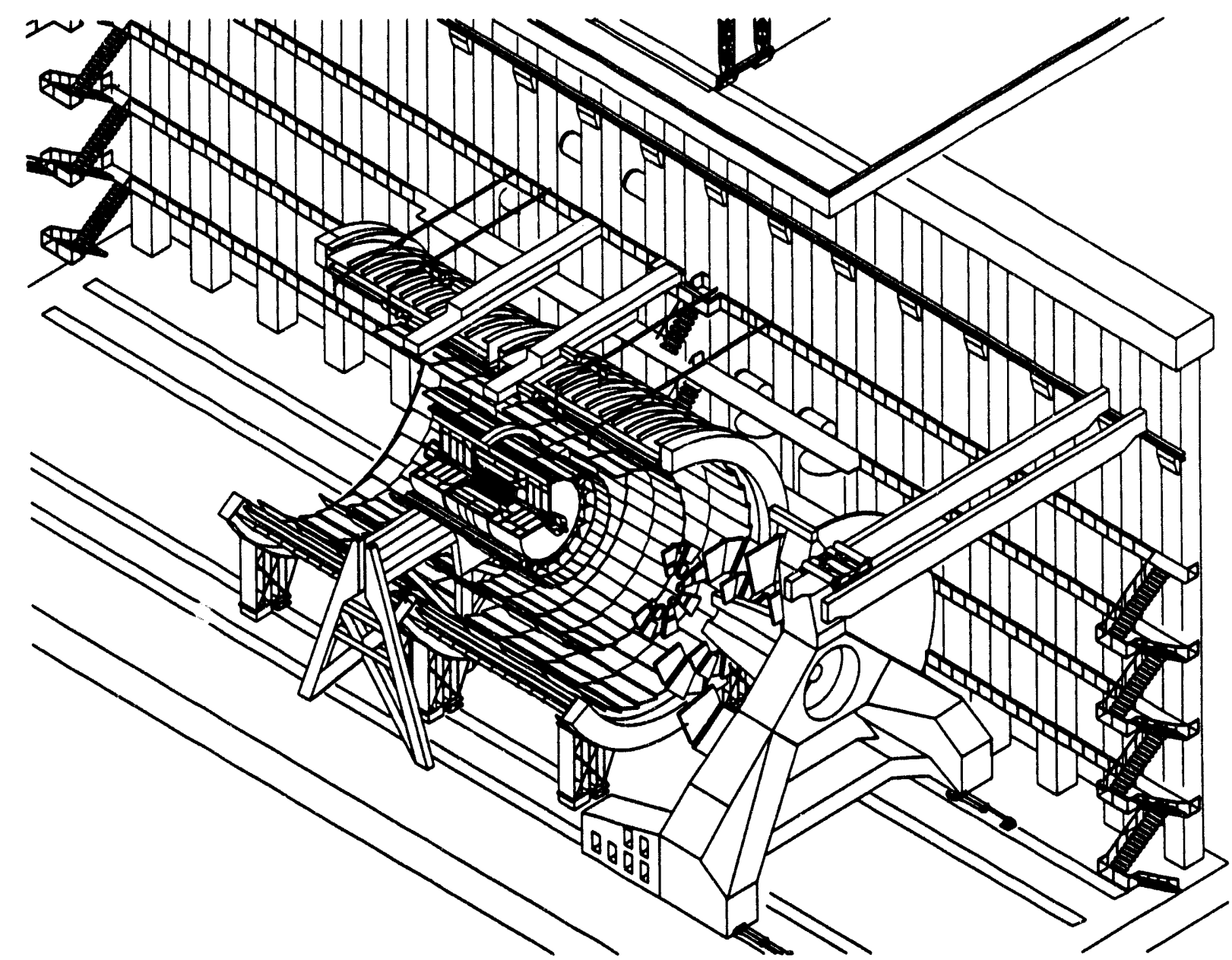

FIG. 9-17. South endcap muon installation.

adjustment. In addition, the barrel region global alignment will be reevaluated and corrected if necessary. The end rings used to mount the muon modules will be used to join the barrel and endcap monoliths once relative alignment has been verified. At this point, the load will be transferred from the FFS to the barrel region muon support structure. Four load-carrying pads are the major attachments between the two structures. Additional attachment points are made between the barrel and endcap support structures to improve the overall stiffiness of both structures. This will improve the frequency response of each of the muon support structures, and will also improve the uniformity of the deformations that occur in response to vibrations and thermal disturbances. The then-independent FFS structure will be secured to the magnet.

After the muon endcap has been transferred to the muon barrel structure, electrical and signal cabling will be installed, and liquid-and gas-plumbing connections completed. These services will run from the ends of the endcap out along the FFS to the outer shell of the magnet vacuum vessel. From there the electrical and electronic cables will extend to the electronics racks located along the west side of the hall. Cryogenic and cooling lines will extend to service dewars located along the east side of the hall.

Following installation of the south muon endcap/FFS, the surface heavy lift crane will be transferred to the north installation shaft in preparation for installation of the north muon endcap/FFS pair. Installation of the north units will proceed exactly as for the south units.

At this point the detector system will remain partially open for installation of the beam vacuum system assemblies. As with the calorimeter endcaps, installation of the FFS, with attached muon endcap, 
can proceed serially with installation at one shaft, followed by installation at the opposite shaft, or installation can proceed in parallel at both shafts. Similar tradeoffs of equipment-versus-costs apply.

\section{Beam Vacuum System}

Once the tracker has been lowered and installed into the barrel calorimeter, the beam vacuum system installation will begin. After the beryllium pipe section has been lowered and installed into the tracker, the beam vacuum system will be continued in both z-directions simultaneously. Vacuum system installation will begin with the connection of NEG pumps to each exposed end of the beryllium chamber. The NEG pumps will be supported by a set of mounting fixtures attached to the barrel calorimeter.

While the NEG pumps are being installed, adjustable supports will be added to the secondary support rails used for tracker insertion. A similar secondary-support system will have to be installed onto the north calorimeter support rails as well. These adjustable supports will be set to deliver the beam pipe at its final height, ready for insertion into the calorimeter endcaps. Temporary support rollers will be placed into the beam pipe opening in each calorimeter endcap to facilitate the transfer of the beam pipe through the endcaps.

Once the NEG pump connections have been completed and the additional supports installed, the inner beam pipe sections will be lowered into the hall, transferred to the hall cranes, and placed onto the adjustable supports. Afte the height of the pipes has been aligned with the opinung in the calorimeter endcaps, the pipe will be passed through the opening and temporarily fixed into position within the calorimeter endcaps.

After the tracker has been installed, the secondary support rails will be removed and the two calorimeter endcaps will be moved closer to the barrel section, leaving a 1-m clearance for the final connection of the inner beam pipe sections. The beam pipe sections will then be joined with the NEG pumps. After these connections have been made, the support rollers can be removed from the calorimeter endcaps so that the endcaps can be closed against the barrel and reconnected.
Once the calorimeter endcaps have been rejoined with the barrel, the inside pump stations will be installed at the exposed ends of the inner beam pipe sections. The pump stations will be supported by fixtures connected to the forward calorimeters.

After all of the internal connections have been made to the detector, the calorimeter support rails will be removed and the magnet halves will be closed. Once the field shapers are in their operational location, temporary support fixtures will be erected next to them. These fixtures will be able to support the outer beam pipe sections as they are passed through the openings in the forward field shapers for connection to the inside pump stations. The procedure used to install the outer beam pipe sections will be similar to that used to install the inner beam pipe sections. The outer beam pipe sections will be supported temporarily in the field shaper by supports located at the internal and external ends of the field shaper cone sections. Refer to Chapter 11 for a more complete discussion of the beam pipe design.

Following completion of beam vacuum system installation, the detector, including magnet halves with their attached muon systems and the FFS, will be closed up. The structures will then be surveyed, aligned, shimmed, and placed on solid mounts in the installed location. Global alignment of the muon subsystem can be started at this point. Figure 9-18 depicts a cutaway view of the completed detector.

\section{Electronics}

There are three floors within the electronics shaft, referred to as Levels 1 through 3. Level 0 is the lowest floor, connecting to the two cable tunnels leading to Level 5 of the underground hall. The data acquisition system and the Level 1 trigger system will be located in Levels 1 and 2 . Level 3 will be used as work space for assembly of electronics racks and as reserve for future growth. The electronics rooms, with the exception of Level 0 , will be shielded with steel to attain a magnetic-field level of less than 50 G. Each floor, except Level 0 , will have a hanging ceiling and a raised floor.

The electronics systems will be installed simultaneously with the detector. Cooling ducts for the electronics room will be routed down the electronics shaft and installed under the raised floor. 


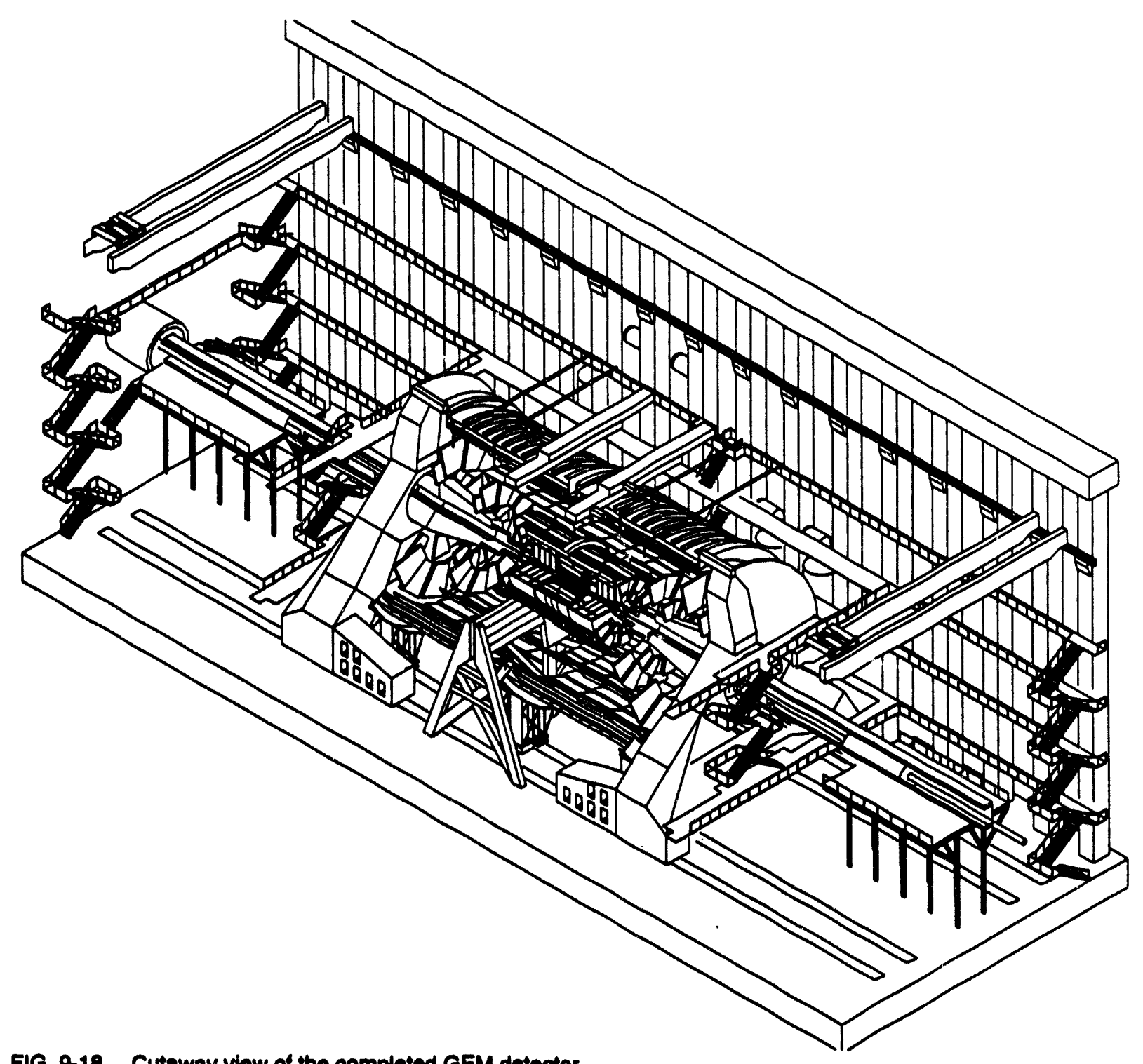

FIG. Q18. Cutaway view of the completed GEM detector.

Cooling ducts for the experimental-hall electronics will be routed down the utility shaft. Cable trays will be installed under the raised floor, along both sides of the cable shaft, and inside the two cable tunnels.

Five 500-kVA transformers for electronics in the experimental hall will be installed in Level 0 . Power cables between the step-down transformer on the surface and these transformers will be installed and connected. Power cables will also be routed between the step-down transformer on the surface and the distribution transformers located on each floor of the electronics room.

Up to 50 electronics racks may be installed on each of Levels 1 and 2 of the cable electronics shaft for the data acquisition system and the Level 1 trigger system. Up to 200 racks will be installed directly in the experiment hall to house the on-detector electronics and their associated low- and highvoltage power supplies.

All of the electronics racks that are housed within the electronics shaft will be lowered by elevator to their proper levels. There they will be installed into the electronics rooms and connected to power. As the electronics crates from each of the subsystems become available, they will be installed into the racks. Any cabling that was run from the hall through one of the access tunnels will be routed to the appropriate rack. Cables will then be routed from 
the electronics room to the operations center as necessary.

The racks that house on-detector electronics and those containing power supplies for front-end electronics will be lowered down one of the installation shafts, mounted, and anchored on platforms along the west wall of the experimental hall. As crates and modules become available, they will be installed and connected to power. All fiber cabling from these racks will be routed through the electronics shaft access tunnels and up to the electronics room. As the installation of the subsystem is completed, power and signal cables will be connected.

More than 8000 fiber optic cables will be run from various points throughout the detector to the electronics racks located along the hall wall and within the electronics shafts. The fibers will carry the data and trigger signals from the detector while it is in operation. As the connections are made, they will undergo a series of tests to ensure proper termination has been achieved. Since the data will be processed through the first two triggering levels in the electronics racks, only 100 fibers will be required to carry the processed data to the operations center for further examination and interpretation.

\section{Services and Utilities}

While the large magnet components are being installed in the hall, cryogenic transfer lines, cryogenic valve boxes, cryogenic dewars, warm bus bars, vacuum piping, and cabling will be installed in the utility shaft. Each of these will be prefabricated off-site in the largest practical unit sizes. The cryogenic valve boxes and dewars will be put in place first; then the transfer lines will be lowered into position, connected to the pipe supports, and welded together. Vacuum lines will be similarly welded together after being mounted to supports. The warm bus bars will then be assembled in place by bolting them to their insulated supports and to each other, before being enclosed in the cooling duct.

Control and diagnostic cabling will be pulled into place by conventional means. All services to the underground hall will be completed and tested prior to connection to the magnet. In order to test the utility systems, U-tubes will be used to complete the cryogenic lines, so that cold fluids can be circulated. The vacuum line will be blanked off and evacuated to facilitate leak testing. The warm bus bars will be shorted at the base of the utility shaft to permit electrical testing.

There will be two liquid-nitrogen storage dewars, two liquid-argon dewars, and one liquidkrypton dewar located along the east wall of the detector hall. It may be necessary for these tanks to be installed during the construction of the underground hall. They will be connected via the utility shaft to larger bulk storage tanks on the surface. In order to maintain the convection loops, smaller krypton and argon head control dewars will be located at Level 3 of the hall wall. The remainder of the services and utilities will be run through the utility shaft and will be connected, where necessary, directly to the detector subsysterns. 


\subsection{INTRODUCTION}

\subsubsection{Overview}

The physics goals for the GEM detector often must be balanced with engineering considerations. Detector alignment requirements, for example, place stiffness demands on the muon support structure that can be met only at the expense of ease of access to the muon chambers and their associated electronics. The design of the GEM detector has been, and will continue to be, a constant trade-off of better physics performance against the requirements for access to the detector subsystems for inspection, maintenance and alignment. This requires an understanding of the system availability budgets imposed by SSCL top-level operational requirements and their relationship to the time available for various levels of detector maintenance.

For purposes of availability/maintainability analyses, system usage time is generally allocated between scheduled up-time and scheduled downtime. Scheduled down-time, as the name implies, is that time when the system is planned to be removed from service for scheduled maintenance activities. Scheduled up-time, however, must allocate time for unscheduled down-time caused by critical failures as well as time for normal operation. In the SSC collider the contra-rotating beams converge at each IR into a single beampipe that passes through the detector. The operational availability of the detector is therefore influenced by both the proton-proton accelerator system and the experimental detector systems. The SSCL top-level requirements documents specify a scheduled operational availability of 80 percent for the accelerator system. Experimental systems (including all detectors) are required to be capable of sensing and recording experimental data 80 percent of the time that the collider is at operational energy and luminosity.

The availability requirements of the collider provide for approximately 3750 hours per year of stable beam operation. An additional 4 hours per day for filling and tuning the beams increases the total collider operating requirement to 4500 hours per year. The collider also needs a shut-down period of three months per year for long-term maintenance operations and an average of one day per week for short-term maintenance actions. Taking the failure of a superconducting string magnet in the accelerator system as an example of an unscheduled failure. approximately 10 days are needed for its replacement. This estimate includes the time required to diagnose the failure, dump the beam, locate the faulty magnet, valve off and relieve vacuum and cryogenic lines, replace and align the magnet, pump down and cool down the new magnet, perform tests, and restart and restabilize the accelerator beams. Current reliability calculations for the string magnets predict up to six such failures in a typical accelerator year.

Operational safety precautions dictate that no personnel be allowed in the underground hall during beam runs. Access is strictly controlled while the magnet is at full energy. Under these conditions maintenance is limited to repair/replacement of components in the surface facilities (operations center, utility building, gas mixing building) and in the cable/electronics shaft. Repairs requiring access to the underground hall, following scheduled and unscheduled beam shutdown, require a waiting time of 8 hours to de-energize the magnet, purge the air in the hall, and allow for one half-life decay of low-level radiation effects.

These top-level requirements place overall limits on the amount of time available for performing maintenance operations on the detector, requiring careful attention during the design phase to considerations of access for maintenance. The design of the GEM detector was driven by its physics goals, including that of hermeticity (which requires an almost totally enciosed detector), but the engineering implementation must provide for access to the interior of the detector for maintenance. The unique size of the GEM detector directly influenced underground hall design and layout in order to permit removal and temporary storage of very large 
components for maintenance access. Relocation of the collider quadrupole magnets and collimators from their initial positions at $\pm 20 \mathrm{~m}$ from the $\mathbb{P}$ to their current locations at $\pm 35 \mathrm{~m}$ also resulted, in part, from the requirement to open the magnet for access to the interior components.

Since the beam passes through all detectors, the beam path through each detector area must be fully operational (i.e., unimpeded and at the proper vacuum level) before the accelerator system can inject beams into the collider. Detector faults during a beam run do not always warrant beam shutdown. These failures may be corrected later (as time permits) during scheduled or unscheduled collider down-times.

The following maintenance modes have been defined, depending on the anticipated length of the beam shutdown. The number of days per year shown for each mode is an average, based on the anticipated number of occurrences for each mode and the down time for each occurrence. The maintenance actions possible under the different modes are addressed in succeeding paragraphs.

- Normal operations: No underground access-188 days per year ( 3750 hours of beam operation plus 1050 hours for beam filling and tuning)

- Short-term access: 16 to 24 hours - 52 days per year ( 1 day per week scheduled maintenance)

- Intermediate-term access: 8 to 10 days -40 days per year (magnet failure rate of 4 per year optimistic at 10 days per failure)

- Long-term access: 90 days (or longer) - Once per year (derived from mission profile)

The availability allocations for the collider system are still somewhat fluid and changes to the collider maintenance philosophy will affect the GEM detector. The subject of detector maintenance and access remains under intense design scrutiny and will continue to be a design driver throughout the development of the GEM detector.

\subsubsection{Analysis and Predictions}

Availability, the probability of being in an operable state, considers both the failure rate and the down-time required to restore the system to an operable condition. To ensure that the GEM detector will be operable during stable beam operations, both factors must be considered. A maintainability analysis is useful in understanding the mechanisms and time requirements for long-term access. When prepared in a bottom-up fashion from individual maintenance steps that are known to be required, and then combined with engineering estimates of the time required for each step, a means of identifying system level scheduled down-time is possible. The analysis must be tailored to known constraints of detector design and to the capacities and dimensions of the underground hall. Among the benefits of this analysis is the identification of the critical path in the maintenance process. Maintainability of the detector and its supporting systems must be well thought out before commencing detailled design. Since access time is a key resource when planning for and allocating time to specific maintenance tasks, early consideration of accessibility in the design can significantly improve overall system availability.

A preliminary analysis has been performed, based on the following ground rules and assumptions:

- The $\mathbb{R}$ quadrupoles are positioned $\pm 35 \mathrm{~m}$ from the IP, allowing partial retraction of both FFSs and magnet coils without disconnecting the muon system frorn the magnet vessels or dismantling the quads or collimators.

- Approximately $4 \mathrm{~m}$ of neutron shielding is present in front of the collimator. Shielding will be removed in sections (non-critical-path item) prior to retraction of the magnet vessels. (This can be performed in parallel with other operations.)

- After roll-back of magnet halves to $\pm 31 \mathrm{~m}$ and " splacement of the endcap calorimeters, all subsystems are accessible without additional maneuvering of large structures. (Some work platforms, scaffolding, and gangways may have to be installed afterward to facilitate access.)

- Hydraulic controls and cylinders are used for roll-back of FFSs and magnet vessels. (Rate is $2 \mathrm{~m}$ per hour.)

- The apportioned subsystem restoration time provides for at least a $50 \%$ contingency due to the preliminary nature of the estimate.

Preliminary results are summarized in Table 10-1. 
Table 10-1. Maintainability analysis.

\begin{tabular}{|c|c|c|c|c|}
\hline Sequence & Operation & $\begin{array}{l}\text { Duration } \\
\text { hours }\end{array}$ & $\begin{array}{l}\text { Parallel } \\
\text { operation }\end{array}$ & $\begin{array}{l}\text { Critical } \\
\text { Path }\end{array}$ \\
\hline 1 & Secure from operations & 2 & & $x$ \\
\hline 2 & Begin HVAC air ODH and activation purge of hall & 2 & & \\
\hline 3 & Ramp-down current to magnet coils & 40 & & $x$ \\
\hline 4 & Remove shielding and bring hall to open access & 16 & & \\
\hline 5 & Health physicists inspection and permit to occupy hall & 16 & & \\
\hline 6 & Disconnect magnet cables and cyogenic services & 30 & $\mathbf{x}$ & \\
\hline 7 & Survey FFS and magnet vessels & 1 & & $x$ \\
\hline 8 & Prepare for and initiate FFS 6 meter roll-back & 41 & & $\mathbf{x}$ \\
\hline 9 & Access inner detector and disconnect inner beampipe & 14 & $\mathbf{x}$ & $x$ \\
\hline 10 & Complete roll-back operation for FFS and magnet half & 35 & $x$ & $\mathbf{x}$ \\
\hline 11 & Reconnect magnet vessel to cryogenics and cool down & 156 & $x$ & \\
\hline 12 & Install calorimeter auxiliary scaffolding and support rails & 24 & & \\
\hline 13 & Refurbish or repair failed magnet components & 282 & $\mathbf{x}$ & \\
\hline 14 & Rofurbish or repair muon system components & 282 & $x$ & \\
\hline 15 & Remove and isolate radioactive calorimeter section(s) & 16 & & $x$ \\
\hline 16 & Disconnect and withdraw passive absorber endcap section & 12 & & $\mathbf{x}$ \\
\hline 17 & $\begin{array}{l}\text { Disconnect services and remove fonward EM and hadronic } \\
\text { calorimeter endcap }\end{array}$ & 24 & $x$ & $x$ \\
\hline 18 & $\begin{array}{l}\text { Arrange removed items for better access to inner detector } \\
\text { components }\end{array}$ & 6 & $\mathbf{x}$ & \\
\hline 19 & Refurbish or repair calorimeter systems & 387 & $x$ & \\
\hline 20 & Reinstall calorimeter support rails to aid removing tracker & 6 & $x$ & \\
\hline 21 & $\begin{array}{l}\text { Detach beampipe from internal NEG and clear path to } \\
\text { tracker }\end{array}$ & 12 & $x$ & $x$ \\
\hline 22 & Remove beampipe to temporary storage & 12 & $\mathbf{x}$ & \\
\hline 23 & $\begin{array}{l}\text { Remove service connections from tracker end shell enclo- } \\
\text { sure }\end{array}$ & 40 & $\mathbf{x}$ & $\mathbf{x}$ \\
\hline 24 & Remove tracker and deliver to surface facility & 6 & & $x$ \\
\hline 25 & $\begin{array}{l}\text { Continue restoration or maintenance of tracker and other } \\
\text { detector systems }\end{array}$ & 395 & & $x$ \\
\hline 26 & $\begin{array}{l}\text { Retum central tracker from surface facillty and install in } \\
\text { beamline }\end{array}$ & 42 & & $x$ \\
\hline 27 & Hookup tracker services and verify operation & 80 & & $\mathbf{x}$ \\
\hline 28 & Reconnect beampipe to NEG station & 16 & & $x$ \\
\hline 29 & Seal off, pump down and perform leak detection & 12 & $x$ & $x$ \\
\hline 30 & Install beampipe through endcap calorimeter section & 12 & $\mathbf{x}$ & \\
\hline 31 & Setup, align and survey to install EC calorimoters & 16 & $x$ & $x$ \\
\hline
\end{tabular}


Table 10-1. Maintainability analysis. (Cont.)

\begin{tabular}{|c|c|c|c|c|}
\hline Sequence & Operation & $\begin{array}{l}\text { Duration } \\
\text { hours }\end{array}$ & $\begin{array}{l}\text { Parallel } \\
\text { operation }\end{array}$ & $\begin{array}{c}\text { Critical } \\
\text { Path }\end{array}$ \\
\hline 32 & Reinstall forward and fine hadronic EC calorimeters & 28 & & $x$ \\
\hline 33 & $\begin{array}{l}\text { Reinstall service connections, pump down, leak check and } \\
\text { check alignment }\end{array}$ & 48 & $x$ & $x$ \\
\hline 34 & $\begin{array}{l}\text { Remove forward calorimeter from radioactive storage and } \\
\text { install in EC region }\end{array}$ & 28 & $x$ & $x$ \\
\hline 35 & Replace passive absorber endcap & 12 & $x$ & $x$ \\
\hline 36 & $\begin{array}{l}\text { Assemble equipment and prepare for magnet / FFS close. } \\
\text { up }\end{array}$ & 33 & $x$ & \\
\hline 37 & $\begin{array}{l}\text { Lift and transport magnet vessel and FFS back to } 6 \text { meter } \\
\text { separation }\end{array}$ & 62 & $x$ & \\
\hline 38 & $\begin{array}{l}\text { Reassemble inner beampipe, pump down, leak check and } \\
\text { align }\end{array}$ & 26 & $x$ & $x$ \\
\hline 39 & $\begin{array}{l}\text { Restore intemal pumping station and attach FFS beam- } \\
\text { pipe }\end{array}$ & 28 & $x$ & \\
\hline 40 & Restore external beamline and vacuum pumping stations & 112 & $x$ & $x$ \\
\hline 41 & $\begin{array}{l}\text { Close-up magnet remaining } 6 \text { meters, and check align- } \\
\text { ment }\end{array}$ & 64 & $x$ & $x$ \\
\hline 42 & Reconnect cryogenics supply and cool down to $4.5 \mathrm{~K}$ & 120 & & $x$ \\
\hline 43 & Perform final inspection and realignment of beampipe & 68 & $\mathbf{x}$ & \\
\hline 44 & Perform prestartup checkout of detector & 8 & & $x$ \\
\hline 45 & Install shielding and bring hall to closed access & 4 & & $x$ \\
\hline 46 & Secure for operation & 2 & & $x$ \\
\hline & \multicolumn{2}{|c|}{$\begin{array}{l}\text { With subsystem } \\
\text { restoration }\end{array}$} & \multicolumn{2}{|c|}{$\begin{array}{l}\text { Without subsystem re- } \\
\text { storatio }\end{array}$} \\
\hline & Total continuous maintenance time & 1366 hours & \multicolumn{2}{|l|}{971 hours } \\
\hline & Total continuous maintenance time & 57 days & \multicolumn{2}{|l|}{40 days } \\
\hline & Calendar time & 97 days & \multicolumn{2}{|l|}{70 days } \\
\hline
\end{tabular}

Notes: 1. Collimators and IR quadrupoles were not considered part of the maintenance envelope and therefore were not considered in the analysis.

2. Continuous maintenance time assumes continuous $\mathbf{2 4}$ hours per day with no allowance for unproductive or scheduled off-time (e.g., holidays, power outages, eic.)

3. Overall calendar time is based on two shifts ( 16 hours per day), 6 days per week. Operations $1-5$ and $42-46$ are based on continuous maintenance time.

4. Central tracker restoration requires 25 consecutive work days at a rate of 16 hours per day.

5. Subsystem restoration time includes a contingency of $50 \%$.

As part of the ongoing design effort, a detailed estimation of the contribution to detector system down-time is planned for all hardware required to operate the detector. Numerical estimates of downtime will be allocated based on a number of factors: 
- predicted availability

- predicted reliability

- access time required

- restoration rate (or mean time to repair)

- redundant components

- complexity of equipment, and

- whether the detector can still function under degraded operating modes.

The objective of the analysis is to apportion down-time among the detector subsystems and their components. The results are intended to help focus the design effort into areas which would minimize down-time.

Further study is in progress to understand the impact of limited access on the performance of GEM. The location of critical elements, the redundancy of systems for greater reliability, and the reliability analyses of individual components all must be validated. The use of movable carriers for the service cables may allow the services to remain connected to the detector subsystems, thus eliminating the time normally needed to disconnect the detector components. Temporary platforms, scaffolding, and hydraulic elevators may then be transported to the maintenance area over the top of the magnet using the hall crane.

For longer term shutdowns, a flexible maintenance scheme could be adopted that leaves the detector open only long enough to restore operation of the overall collider system. Referring to Table 10-1, omitting tracker restoration would save 17 days of down-time for the collider system, but would result in severely degraded operation of the GEM detector.

\subsection{DETECTOR OPERATION}

\subsubsection{Operational Goals}

According to the SSC Level 2 specification, the current operational scenario is based on scheduled maintenance of 940 hours per year at one day per week, and a maximum of 2190 hours per year for long-term shutdowns. This leaves a total of 5,630 hours of run time (including unscheduled down-time) per year over 9 months of scheduled experimental physics time. The goal for sustained operation of the detector is to be available for data-taking $80 \%$ of the time the accelerator is at design energy and luminosity. To meet this goal, two performances objectives have been adopted. First, the detector must be at least as available as the accelerator system. Second, to minimize the possibility of one system being up while the oilier is down, the operational profiles of the two systems must be compatible.

The SSC accelerator system includes more than 12,000 magnets in its 54-mile main ring, in addition to the linear accelerator and three booster rings. Due to the high availability demand on collider subsystems, studies ${ }^{11}$ have shown that the best approach to preserving collider availability is to decrease the amount of time used for long term shutdowns, thereby making more time available for repair of accelerator subsystems. If the recommendations of these studies are adopted, the end effect will be a reduction in the amount of scheduled accelerator down time available for repairing the experimental systems. Considerable engineering effort is therefore focused on ways to reduce the total amount of long-term down time. Engineering designs are being incorporated that will improve the efficiency of installation, restoration, and retest of subsystems. However, maintenance predictions at this stage in the GEM engineering design effort indicate that, regardless of accelerator availability requirements, a shutdown of at least three months will be required for major maintenance or subsystem restoration.

\subsubsection{Detector Start-up}

Following completion of the detector installation phase, a three- to six-month period of joint accelerator and detector commissioning activity is envisioned. During this period a number of operational problems may be expected that will require bringing up either the collider or the detector, or both, and then shutting everything back down to correct or adjust an operating parameter. It is impossible to predict when and how often each system shutdown will be required, who will request the shutdown, or how long it will be expected to last. Very careful coordination will be required between the two large detectors and the collider during this period.

Only after successful completion of the commissioning phase will systematic data-taking operation be possible. Each time the GEM detector is 
brought up to its operating condition, whether during commissioning or for an operational beam run, a carefully programmed and executed system of checks will be necessary to ensure that all safety and operational safeguards have been observed and that the detector can be energized without risk of damage. An orderly sequence of actions must be completed to verify that the detector is ready for physics data collection, the underground hall is safe and secure, and experimental and service subsystems are operational. Table 10-2 lists the top-level steps involved in preparing the detector for operation.

Table 10-2. Detector power-up checklist.

Detector closed and surveyed.

Underground hall searched, cleared and locked.

Installation shafts shielding plugs in position.

Hail environment conditioned and stable.

Detector environment conditioned and stable.

Cranes positioned and locked.

Safety systems armed and checked.

Utilities functioning.

Electronics room functioning.

Operations room ready.

Magnet cooled down and powered up.

System checks performed.

Beam pipe pressure checked

Detector subsystems power checked

Detector electronics power checked

On-line diagnostics and calibrations performed

Ready to commence run.

The GEM operations center in the SAB will be responsible for visual inspection and confirmation that each step in the checklist has been completed. After satisfactory completion of the detector checklist, the accelerator operations center will be notified that the hall has been secured and the detector is ready for beam. (Note that all other citectors within the beam must have their beamlines intact and their halls cleared of personnel before beam can be injected into the collider.)

In parallel with the global detector checks, each individual subsystem will also commence a pre-operational checkout of critical operational parameters, services, and calibrations.

\subsection{MAINTENANCE AND ACCESS CONCEPT}

The accelerator beam will be cycled on and off approximately once every 24 hours. GEM's operational cycle will probably be much longer, encompassing many accelerator cycles. In order to make best use of down-time, which will be a mixture of scheduled and random events, a clear understanding of what equipment should be accessible in what period of time is required. This section will attempt to highlight the processes necessary in acquiring this knowledge.

GEM's down-time can be grouped into four major categories:

- unscheduled down-time caused by incipient or random failures

- short-term scheduled maintenance of a preventive nature to mitigate imminent failures or replenish resources

- intermediate-term, usually unscheduled, downtime and

- long-term scheduled maintenance for major repairs or to incorporate new hardware modifications.

Long-term shutdowns and routine maintenance actions are generally fixed in length and can be predictably inserted into the collider operating schedule. These events are not counted against operational availability. Unscheduled shutdowns are unpredictable and are a reflection of the inherent reliability of detector subsystems. Unscheduled down-time is counted against the detector availability. In order to balance the four types of down-time within a scheduled operational year, a systematic approach of establishing hardware rates of failure and restoration is required.

The GEM detector maintenance concept provides for seven major levels of access governed by:

- access restriction (whether beam is on or off)

- location within detector system, and

- extent of disassembly required to reach detector subsystems. 
Table 10-3 lists, for each GEM subsystem, hardware which can be repaired at a specified access level. Access levels are listed in order of increasing difficulty, e.g., equipment located on the surface will be continually accessible, but equipment located in the detector hall will be accessible only when the beam and field are off, with other restrictions possible. Much hardware, e.g., cabling, is listed at almost every access level; the access level required to repair such hardware depends on the location of the failure. Generally, hardware is listed only at the least difficult access level unless the next level of access exposes additional surfaces.

Table 10-3. Access repair levels.

\begin{tabular}{|c|c|c|c|c|c|}
\hline \multirow[t]{2}{*}{ Access Levol } & \multicolumn{5}{|c|}{$\begin{array}{l}\text { Hardware Description } \\
\text { At the specified access level, the listed types of hardware can be accessed for repair. }\end{array}$} \\
\hline & Magnet & Muons & Calorimeters & Tracker & Beam pipe \\
\hline \multirow{8}{*}{$\begin{array}{l}\text { Surface } \\
\text { Unrestricted } \\
\text { Access }\end{array}$} & Ops center & Ops center & Ops center & Ops center & Ops center \\
\hline & Power supply & Gas storage & Pipes & Gas storage & UHV controls \\
\hline & Bus & Gas Mixer & Slow control & Gas Mixer & Cables \\
\hline & Slow control & Slow control & & Slow control & \\
\hline & Process cooling & Process cooling & Process cooling & $\begin{array}{l}\text { Process cool- } \\
\text { ing }\end{array}$ & \\
\hline & Refrigerator & Pipes & & Pipes & \\
\hline & Helium storage & & Cryogen storage & $\begin{array}{l}\text { N2 \& butane } \\
\text { vents }\end{array}$ & \\
\hline & Pipes & & & HV controls & \\
\hline \multirow{2}{*}{$\begin{array}{l}\text { Electronies } \\
\text { Rooms }\end{array}$} & Bus & Electronics & Electronics & Electronics & N2 pipes \\
\hline & Slow control & Cables & Cables & Cables & Vac interlocks \\
\hline $\begin{array}{l}\text { Unrestricted } \\
\text { Access }\end{array}$ & Pipes & Slow control & Slow control & Slow control & Vac terminals \\
\hline \multirow[t]{2}{*}{$\begin{array}{l}\text { Cable Shaft \& } \\
\text { Utilitios }\end{array}$} & Process cooling & Process cooling & Process cooling & $\begin{array}{l}\text { Process cool- } \\
\text { ing }\end{array}$ & (State of vac sys.) \\
\hline & Cables & Pipes & Pipes & Gas storage & Cables \\
\hline \multirow{3}{*}{$\begin{array}{l}\text { Restricted } \\
\text { eccess }\end{array}$} & Vac pumps & & Valves & HV supplies & \\
\hline & & & Rough pump & Pipes & \\
\hline & & & ODH monitor & & \\
\hline \multirow{7}{*}{$\begin{array}{l}\text { Detector Hall } \\
\text { Beam Off } \\
\text { for Access } \\
\text { Field reduced and } \\
\text { controlled }\end{array}$} & Bus & Electronics & Electronics & Electronics & Sector Valves \\
\hline & Leads & Power supply & Power supply & Power supply & \\
\hline & Cables & Cables & Cables & Cables & Cables \\
\hline & $\begin{array}{l}\text { Extermal vac } \\
\text { vessel }\end{array}$ & Process cooling & Process ceoling & $\begin{array}{l}\text { Process cool- } \\
\text { ing }\end{array}$ & $\begin{array}{l}\text { Extemal lon pump } \\
\text { station }\end{array}$ \\
\hline & $\begin{array}{l}\text { Head control De- } \\
\text { wars }\end{array}$ & Slow control & Cryogen storage & Slow control & Sublimation pump \\
\hline & Slow control & Pipes & Pipes & HV & \\
\hline & VCL dewars & & Valves & Gas storage & \\
\hline
\end{tabular}


Table 10-3. Access repair levels. (Cont.)

\begin{tabular}{|c|c|c|c|c|c|}
\hline & Pipes & & Slow control & Pipes & \\
\hline & Vac pumps & & ODH monitor & & \\
\hline \multirow{7}{*}{$\begin{array}{l}\text { In-Detector } \\
\text { (Detector } \\
\text { closed) } \\
\text { Beam \& Field Off, } \\
\text { Access Between } \\
\text { Coils \& FFS }\end{array}$} & & Electronics & Electronics & & Nitrogen pipes \\
\hline & & Cables & ODH Monitor & & Valves \\
\hline & & Process cooling & $\begin{array}{l}\text { Vac Vessel } \\
\text { Closed(Endcap } \\
\text { Head) }\end{array}$ & & $\begin{array}{l}\text { Internal lon pump } \\
\text { station }\end{array}$ \\
\hline & & Slow control & & \multirow{4}{*}{$\begin{array}{l}\text { Minimal } \\
\text { accessible } \\
\text { Hardware }\end{array}$} & \\
\hline & & Small gas leak & & & \\
\hline & & Alignment & & & \\
\hline & & Pipes & & & \\
\hline \multirow{7}{*}{$\begin{array}{l}\text { In-Detector, } \\
\text { Magnot colls } \\
\text { Retracted } \\
\text { Beam \& field off, } \\
\text { coit: } 9 \text { ind cals } \\
\text { moved for access }\end{array}$} & & Electronics & Electronics & & Pipe thru FFS \\
\hline & & Cables & Cables & & \\
\hline & & Process cooling & $\begin{array}{l}\text { Vac vessel minor } \\
\text { repair }\end{array}$ & $\underset{c}{\text { Minimum ac- }}$ & \\
\hline & & Small gas leak & Hi vac pump & Hardware & \\
\hline & & Alignment & Feedithrus & & \\
\hline & & Pipes & & & \\
\hline & & Slow control & & & \\
\hline \multirow{10}{*}{$\begin{array}{l}\text { In-Detector, } \\
\text { End cals } \\
\text { retracted } \\
\text { Beam \& field off, } \\
\text { Coils \& end cals } \\
\text { moved for access }\end{array}$} & & \multirow{10}{*}{$\begin{array}{l}\text { Same as coils } \\
\text { Retracted }\end{array}$} & \multirow{10}{*}{$\begin{array}{l}\text { Same as re- } \\
\text { tracted } \\
\text { With more vac } \\
\text { Vessel surface } \\
\text { Area Exposed }\end{array}$} & IPC detectors & NEG \\
\hline & & & & Electronics & Cables \\
\hline & & & & Cables & $\begin{array}{l}\text { Pipe thru } \\
\text { calorimeters }\end{array}$ \\
\hline & & & & Process & $\begin{array}{l}\text { Bakeout } \\
\text { Equipment }\end{array}$ \\
\hline & & & & Pipes & Nitrogen pipes \\
\hline & & & & Slow control & \\
\hline & & & & HV & \\
\hline & & & & Gas leaks & \\
\hline & & & & Gas enclosure & \\
\hline & & & & Feedthnus & \\
\hline
\end{tabular}


Table 10-3. Access repair levels. (Cont.)

\begin{tabular}{|c|c|c|c|c|c|}
\hline \multirow{7}{*}{$\begin{array}{l}\text { Subsystem } \\
\text { Removal } \\
\text { Beam \& field off, } \\
\text { Coils \& endcaps } \\
\text { moved } \\
\text { Subsystem } \\
\text { diassembled } \\
\text { for access }\end{array}$} & $\begin{array}{l}\text { Major vessel re- } \\
\text { pair }\end{array}$ & Structural repair & $\begin{array}{l}\text { Pressure vessel } \\
\text { repair }\end{array}$ & $\begin{array}{l}\text { Internal compo- } \\
\text { nents }\end{array}$ & Pipe thru tracker \\
\hline & \multirow[t]{2}{*}{ Cool windings } & Broken wires & Module repair & Electronics & Supports \\
\hline & & Major gas leak & Pre-amps & Cables & \\
\hline & $\begin{array}{l}\text { Internal compo- } \\
\text { nents }\end{array}$ & & $\begin{array}{l}\text { Vac vessel major } \\
\text { repair }\end{array}$ & $\begin{array}{l}\text { Process cool- } \\
\text { ing }\end{array}$ & \\
\hline & & & Internal cables & Slow control & \\
\hline & & & N2 HX repair & HV & \\
\hline & & & Feedthrus & Pipes & \\
\hline
\end{tabular}

Because the detector design incorporates redundant components, single or even multiple failures can be sustained without shutdown and loss of productive operating time. The major GEM subsysiems are designed to maximize the number of parallel readouts so that the operation of the detector can degrade gracefully over time. This concept of graceful degradation results in fewer unscheduled shutdowns and permits extended operation until the next scheduled detector maintenance period or until an unscheduled window of opportunity. Reliability analysis will be performed to ensure that singlepoint failures that could jeopardize the benefit of redundant designs are identified and eliminated, or their effects mitigated.

Surface buildings and the cable/electronics shaft will offer unrestricted access to equipment that can be safely reached and handled. Equipment within the underground hall, utility shaft, and on the detector periphery will be accessible only when the beam is off and the magnet field is down. There is a possibility that shorter periods of access can be achieved with magnet vessels closed and the beamline intact. All other in-detector access is reserved for the long-term annual shutdown period.

\subsubsection{Normal Operations (No Underground Hall Access Permitted)}

Normal operations are underway when the underground hall is closed to access, beam is on and data is being taken. Since the detector magnet on-off cycle time is much longer than that for the accelerator system and is lifetime limited to an average of 10 ramp-up and 10 ramp-down cycles per year, operation of GEM cannot just mirror the demand of the accelerator. During scheduled operations, the GEM magnet will be kept cold and energized. Before data taking can proceed, the detector must undergo a routine checkout procedure to verify status of experiments, calibration of electronics and beam diagnosis.

System abnormalities will be initially detected and diagnosed from inside the operations center, the utility building or the underground electronics room. Problems that are immediately repairable through software controls, resetting relays or remotely controlling valves may be corrected or isolated concurrently with beam operations without the need to enter the hall.

\subsubsection{Short-Term (Restricted Access)}

The most frequently available window for maintenance actions will be the one day per week scheduled for routine collider maintenance. The current GEM access philosophy assumes that these short accesses will only allow maintenance of components outside the magnet.

Before entry into the hall is permitted the beam must be off, the magnet de-energized to a safe level, and radiation safety procedures observed. Approximately 8 hours delay may be required before personnel may enter the hall and an additional 8 hours are required to clear the hall and reenergize the magnet after maintenance activities are completed. This leaves only one 8-hour shift to perform useful work. No work inside the detector will be possible in this limited time. However, minor repairs and maintenance can be performed, including:

- electronic racks mounted along the west wall, 
- electronics junction and power conversion boxes mounted around the periphery of the magnet vessels,

- liquid cryogenic dewars mounted along the east wall,

- vacuum insulated lines and valves,

- power and digital electronics cables, and

- detector support structures.

\subsubsection{Intermediate-Term (Partial Open Access)}

During shutdowns of 48 hours to approximately 250 hours, some inner detector access will be possible by backing the FFS away from the magnet coil by $6 \mathrm{~m}$. With the magnet closed, the beampipe is supported at both ends by the in-detector ion pumping station and the floor support framework external to the FFS. This will permit the FFS to travel freely to the point where a $1-\mathrm{m}$-wide path is available between the cone of the FFS and the barrel muon chambers. Activation analyses are currently underway to determine the potential safety hazards to maintenance personnel working near the beampipe and internal beamline ion pumping stations and to the endcap muon system front-end electronics. The results of these analyses are expected either to confirm that no specific precautions are necessary or to define and determine the type and level of protective measures necessary for access. Assuming unrestricted access is feasible, a temporary access bridge will then be extended from the FFS cone and the muon endcap chambers to the region of the interior vacuum pumping stations. The access bridge will accommodate lightweight, portable electronic test equipment, mechanical tools, and other support equipment required to perform calibrations, surveys and other small repairs (see Figure 10-1).

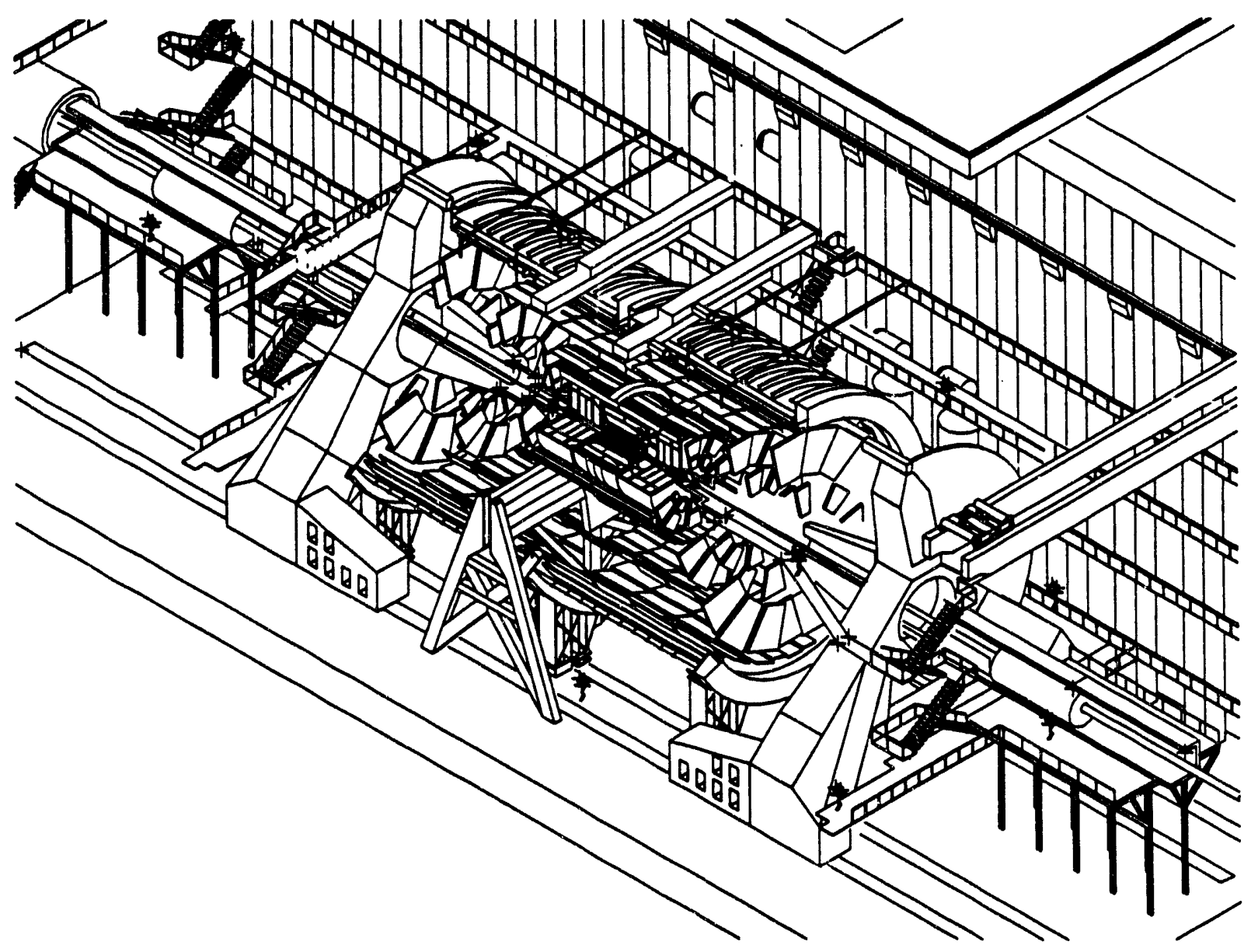

FIG. 10-1. Isometric view: 6-m withdrawal for access to ion pumping station. 


\subsubsection{Long-Term (Full Open Access)}

Access to components in the interior of the detector requires opening of the magnet halves to some extent. If access to the barrel muon system and its front-end electronics is required, one or both magnet halves may be displaced from the CDS by approximately $2 \mathrm{~m}$ along the beamline. Investigations are currently underway to determine if this 2-m displacement can be accomplished without disconnecting the cryogenic cooling and vacuum lines and the monitoring and control electronics cables from the magnet. This would save not only the time needed to disconnect and reconnect these lines, but may result in a shorter time to restore the magnet to its operating state after the magnet is moved back into position. The magnet power busses are rigid structures that must be physically disconnected before the magnet is moved (see Figure 10-2).

In order to assure a free flow of air through the detector during access, the two forward field shapers must be withdrawn approximately $6 \mathrm{~m}$. This requires first that a $6 \mathrm{~m}$ section of the forward neutron shield be dismantled and temporarily stowed at one side of the hall.

For access to and removal of calorimeter components or the inner tracker, complete retraction of both magnet halves and displacement of the calorimeter endcaps is required. This is accomplished in various stages. First, the complete forward neutron shield must be dismantled and removed from both ends of the detector. Also, the collimator shielding blocks must be removed and stored. Then the complete beamline and vacuum system from the tracker to the collimator must be removed and stored. Finally, the FFS and magnet half at each end of the CDS is displaced by $12 \mathrm{~m}$.

With the magnet halves open, the end calorimeters can be removed to provide access to the inner tracker. Removal of the endcap calorimeters requires erection of the same support rails and structures used initially to install these components. Additionally, a lateral support rail may be required at one end of the detector to translate the endcap

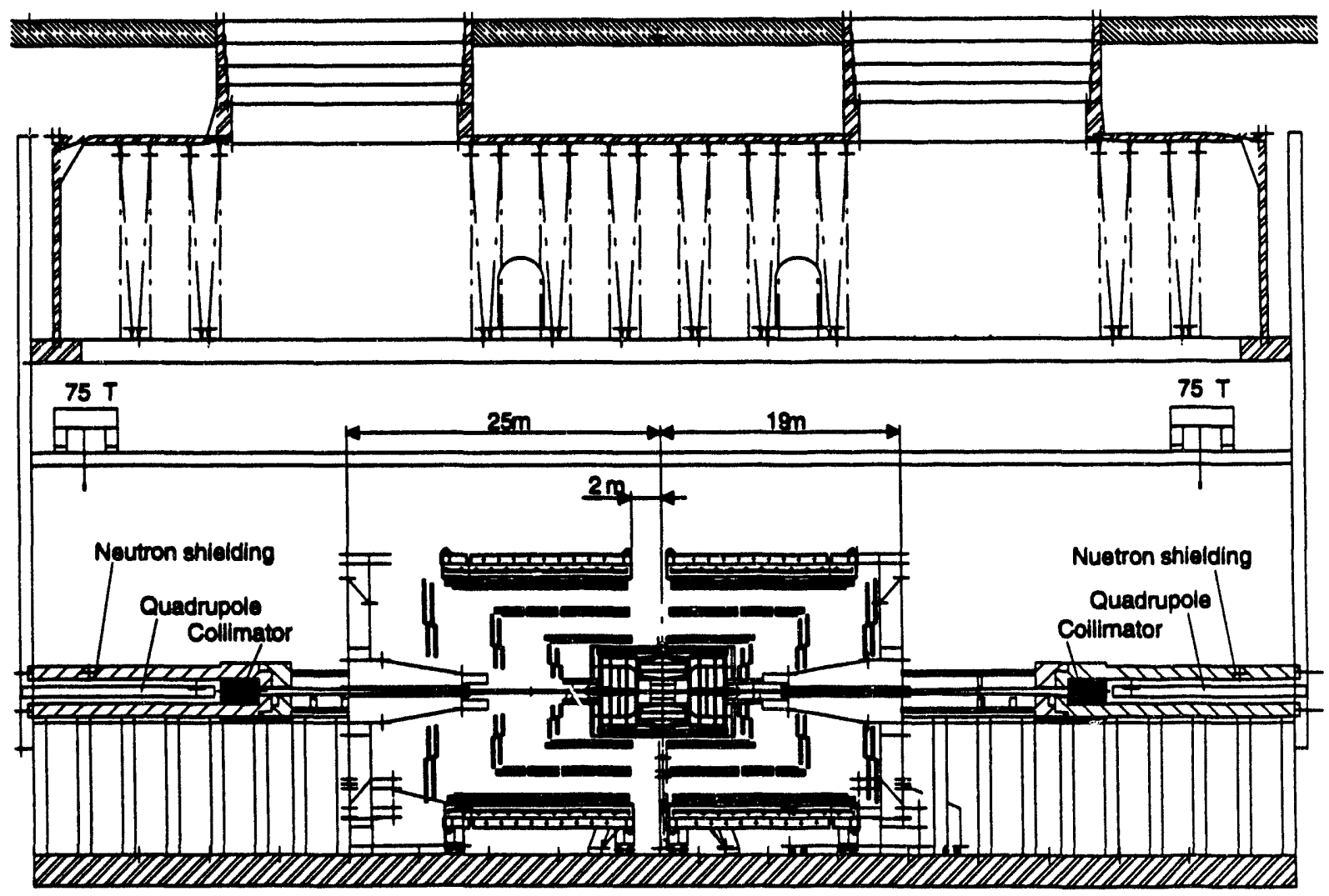

FIG. 10-2. Dimensioned elevation view: 2-m inner access opening. 
calorimeter off the beam center line in order to remove the tracker (see Figure 10-3).

Opening and closing the magnet halves is estimated to require approximately two months. The time for actual detector component service and maintenance is added to this number. Time considerations dictate that this level of maintenance only be attempted during the once-per-year down time of 3 months ( 2160 hours). Where feasible, to save time, operations on the north and south ends of the detector will be performed simultaneously. Also, we are exploring the possibility of providing temporary connections of cryogens and vacuum pumping to the magnet halves in their parked locations in order to minimize, or, if possible, eliminate the time needed for complete pump down and cool down of the magnet coils before reenergizing.

\subsection{SUBSYSTEM MAINTENANCE ACCESS REQUIREMENTS}

\subsubsection{Detector}

Conceptual designs of access requirements around and inside the detector for assembly, installation, and maintenance are currently being evaluated. It is clear that the surface assembly of the magnet, muon system, and calorimeter sections will require large scaffolds and special devices, including personnel elevators and platforms. Most of this equipment would be used during both the installation and maintenance periods.

In general, the installation process in the underground hall will require basically the same type of access equipment used during the assembly process. The CDS will be the first object installed,

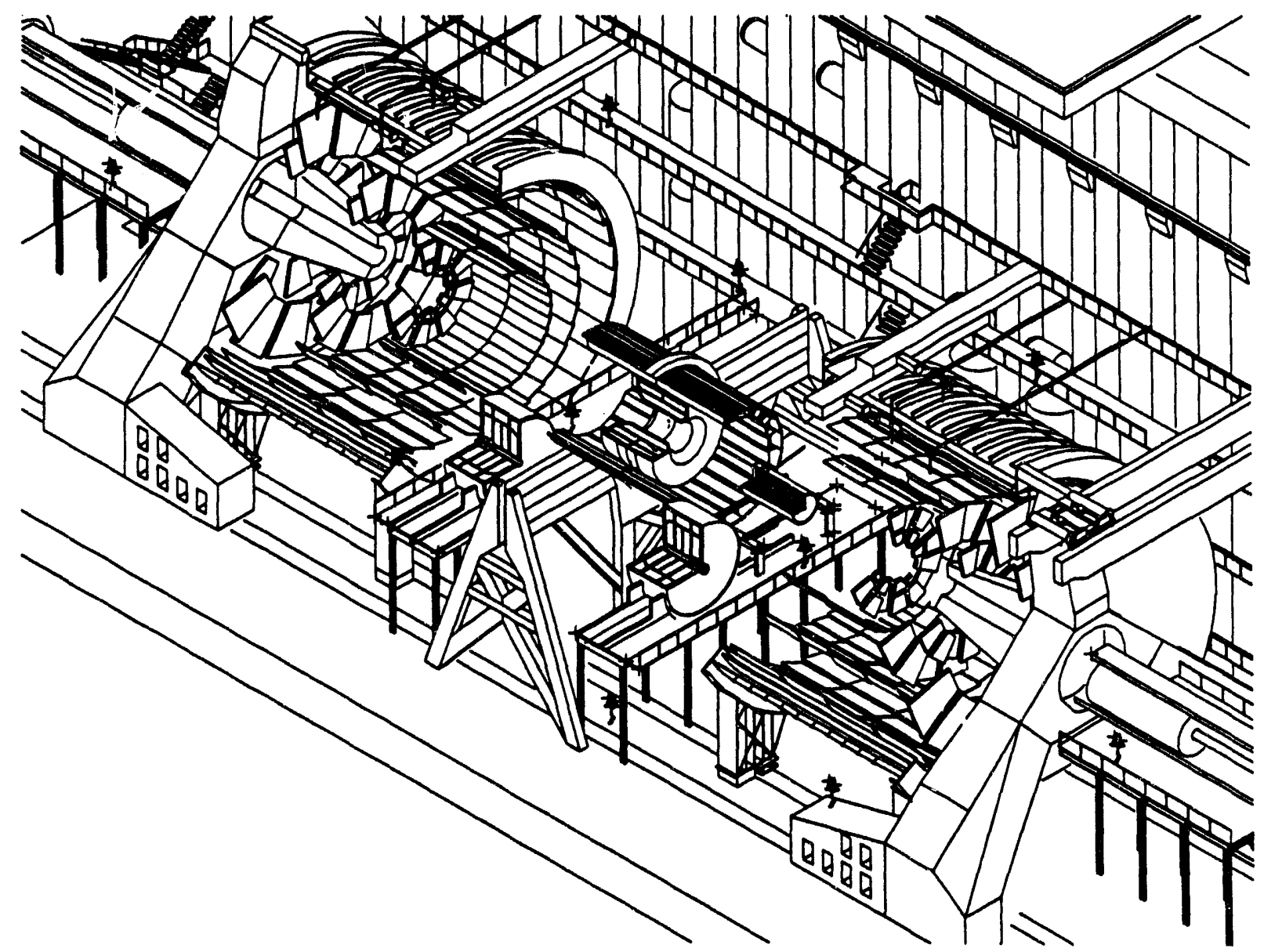

FIG. 10-3. Isometric view: Full retraction of FFS and magnet vessels. 
and it is expected that scaffolding and personnel elevators will be required for the completion of the services carried by that structure. For this purpose it is probable that each side of the CDS upper extent will be covered with temporary scaffolding and linked to the walkways along the sides of the hall. The installation of the scintillating calorimeter barrel and liquid calorimeter will require special heavy duty platforms, placed directly under one of the shafts and extending to the CDS. These platforms will have walkways and stairs, and will be designed to carry further access equipment for the connection of services. The magnet will be delivered complete with access facilities to the top of the coil units. These, too, will be linked with the hall walkways. After the insertion of the muon structures inside the magnet, each system will require scaffolding at each end to attach and align the structures and to connect services. The beampipe will be $13 \mathrm{~m}$ from the floor, and is expected to have support structures equipped with temporary access gangways and stairways.

Maintenance will require basically the same access equipment, which will be adapted from the assembly and installation equipment, but also designed for quick assembly, installation, and removal. The muon system is being designed to permit personnel access to the interior of the muon chambers from either the CDS end or the FFS end.

In-detector routing of power, electronic, vacuum, and liquid service lines for the central tracker and calorimeter subsystems will be designed for maximum accessibility where practical. Lines will either be routed through feedthroughs along the top of the CDS, or will be attached to the outside skin of the CDS. All large-diameter liquid and vacuum lines will be routed above the CDS for maximum accessibility for repair when the detector coils may be displaced from the CDS. Electrical lines routed through feedthroughs may be accessible through sealed hatches or doors built into the structure. Appropriate cable restraints and strain relief measures will alleviate cracking and insulation cold flow during installation and maintenance.

\subsubsection{Electronics}

Access to electronics equipment will be provided at the cable electronics shaft and electronics rooms, the underground hall, the detector peripheral service centers, and the front-end electronics junction box/circuit card interfaces in the detector. The electronics systems inside the operations center will be accessible at all times.

Between the operations center and the underground hall, equipment rooms within the cable shaft will contain floor-mounted racks, fiber optic conduits, and power bus cabling. Power supplies, slow or remote controls, bus, and LAN connectors are typically housed in crates mounted in racks. Access will be provided to the front and rear of all racks. Enough space will be provided in front of and behind each rack to ensure adequate clearance for removal or maintenance of the largest component. Cable support and strain relief will make use of lanyards, cable retractors and other suitable means. Appropriate access panels and enclosures will be provided for maintenance or repair of chilled low-conductivity cooling water (LCW) and HVAC services.

Transformers, power filters and regulators will be mounted on the floor or in racks. Because of the weight of these devices, additional space will be provided for servicing and installation.

Within the underground hall, electronics hardware will be installed along the sides of the hall and on the detector. DC power supplies for all detector subsystems will be located on the sides of the underground hall. Electronies crates and modules at these locations will be rack mounted. Fiber optic electronics cabling from detector to operations center will be routed in conduits and cable trays through these areas. LCW cooling services located along the hall gangway will require periodic servicing. Access to these locations is provided by permanent raised gangways. Maintenance capabilities will be provided similar to those in the underground electronics room. Activities in the detector hall will be limited by access restrictions.

Personnel access safety system (PASS) interlocks, controls, and oxygen deficiency hazard $(O D H)$ monitors will be distributed throughout the underground hall and within the detector envelope. Engineering analysis must still be performed to determine locations for the PASS electronics and slow controls that will not be susceptible to degradation due to the environment it is designed tu monitor. ODH monitors, sensors, lights, and sirens may be mounted in redundant remote locations within the 
hall to provide complete and fail-safe coverage. Access by gangways, ladders, or walkways will be provided where required.

\subsubsection{Magnet}

Magnet access philosophy hinges on an overall design concept that reduces the need for scheduled maintenance by maximizing reliability. Once sealed inside the vacuum vessel, all major magnet assemblies (coil, bobbin, conductor, coolant lines, and structural supports) will be maintenance free for the life of the detector. Periodic servicing of critical service interface connections will be possible through access panels and hatches at the periphery of the vacuum vessel. Removable outer LN2 shield panels may expose superconducting splices, cryogenic valves, insulating breaks, and electrical leads for maintenance.

In order to facilitate access whether magnet coils are closed, retracted, or in any intermediate position, service catwalks and work platforms will be built into rails imbedded in the walls of the hall. When the magnet vessels are retracted, the work platforms will be withdrawn independently to the parking position. In order to provide the option of keeping the magnet cold during long-term shutdown, auxiliary cryogenic connections will be provided at the parking position.

To centralize maintenance activity and to increase floor space available for retraction of the coils, cryogenic supply and return lines will exit from the vacuum vessel at the ends adjoining the CDS and the FFS. Superinsulated vacuum-jacketed cryogenic supply and return lines will extend from the floor of the underground hall to manifolds supplying LHe to the individual coil splices and insulating jackets. Flexible couplings and hoses will permit mating of the hoses with the magnet vessels for cool down while the detector is undergoing its final assembly, alignment, and diagnostic tests.

Access to cryogenic equipment in the hall will be required for servicing and repairs of liquid dewars and pressurized tanks. Railed gangways and built-in ladders will be required to reach the six liquid vessels mounted in niches against the wall. Additional space will be allotted between vessel walls and catch basins to permit access to lines, valves, and other tank penetrations.

\subsubsection{Muon System}

During surface assembly of the four monolithic barrel and endcap structures, the individual muon chambers, arranged in 3 radially staggered superlayers, will be rigidly mounted and will be initially aligned. Since surface assembly affords more freedom of movement for direct contact with each chamber, access equipment used for assembly will probably not be suitable for installation of the monolith structures in the hall or for maintenance. Special temporary catwalks, ladders and scaffolds will be required to thread through the muon support structure for maintenance access. Work areas will provide maintenance personnel with a safe, railed, nonskid surface.

Front-end electronic circuit card modules will be rigidly mounted along the periphery of each cell. Barrel muon chambers closest to the IP will not be directly accessible because they are completely overlapped by the next adjoining layer. Electronics access for these modules will be made at the next adjoining cell. Access capability for slow control electronics hardware and LCW lines will be similar to that for digital electronics modules.

To improve maintenance efficiency and provide modularized access to muon system interfaces, one option being considered is to build niches into one of the magnet vessel support legs, to contain all the junction boxes for cables, LCW cooling, gas pipes, and valves. These would be at the north and south ends of the vacuum vessel, near where service cables and plumbing exit from the detector. The junction boxes would permit disconnection of all applicable services when the coils are retracted and moved to the long-term maintenance parking position.

Cathode strip chambers are inherently robust devices and are expected to experience a very low failure rate. Failure of one or two percent of the total muon chambers can be compensated in the analysis process and will not seriously degrade overall muon system performance. A design goal will be to remove and replace or repair during the three-month annual shutdown those few failures that may occur during the nine months per year of detector run-time. Personnel access, module attachment methods, and service disconnects must be designed in a manner 
that will facilitate replacement or in-situ repair of individual chambers or modules.

\subsubsection{Calorimeter System}

With magnet coils retracted, maintenance personnel equipped with portable tools, electronic instruments, and light weight gangways or ladders will have limited access to endcap calorimeter hardware only. Gangways and work platforms will be erected temporarily at strategic locations in the endcap head regions, barrel scintillating calorimeter outer wall adjoining barrel muon superlayer \#1, and central support structure. Elevated work platforms and gangways will be installed near electronics racks mounted behind the endcap passive absorber near the top.

Access to the barrel calorimeter requires removal of the endcaps. Disconnection of endcap digital electronic cables, DC power cables, cooling water, vacuum, and noble gas lines must te made from this platform prior to retraction of the endcaps. With magnet coils fully retracted and calorimeter endcaps withdrawn, more pressure vessel surface area is exposed. Temporary support structures used to withdraw endcap vessels will be provided with built-in gangways and work platforms around the periphery.

\subsubsection{Central Tracker System}

The central tracker is located closest to the IP and is expected to encounter a very harsh radiation environment. Although considerable effort has been focused on low-maintenance radiation-hardened design, if collider operation reaches a luminosity level of $10^{34} \mathrm{~cm}^{-2} \mathrm{~s}^{-1}$, the tracker will eventually require removal and major refurbishment or replacement. Preliminary analysis (see Table 10-1) indicates that 971 hours ( 40 days) of continuous maintenance time is required for in-detector access, not including downtime for removal and repair of the central tracker. Refurbishment of the tracker in a surface facility is estimated to require another 395 hours, making a total of 1366 hours (57 days) for annual shutdown. Analysis of similar long-term disassembly procedures of the $\mathrm{DO}$ and $\mathrm{L} 3$ detectors tends to confirm these predictions.

Successful installation and alignment of the central tracker (CT) is a critical operation and will require a well-thought-out maintenance procedure. With the endcap calorimeter vessels withdrawn and the beampipe and NEG removed, only the outside enclosure and service connections are immediately accessible. Additional raised work platforms must be built up from the main support structure used to withdraw the endcaps. The support structure will be constructed in two levels. The first level will consist of a raised floor approximately $1 \mathrm{~m}$ above the main calorimeter support structure and protruding partially into the endcap support enclosure. The front of this platform will have two parallel steel rails which will accept an air-ride, wheeled transfer cart. A 4-degree-of-freedom pneumatic platform with a matching set of rails will be provided inside the calorimeter support enclosure to bring the CT into final alignment. The pneumatic platform will have sufficient vertical motion for maintenance personnel to reach the upper service niches between barrel calorimeter and tracker end plate to unhook service connections and to load the CT onto the transfer cart.

After the initial alignment survey is complete the services to the CT will be hooked up. The mechanical and electrical systems connections will be made in the outer comers of the CT within the beveled section of the barrel calorimeter. Access to cable connections arranged around the periphery of the comers will be limited. Due to the physically close fit between endcap calorimeter vessel and the CT (approximately $2 \mathrm{~cm}$ ), some $1500 \mathrm{~cm}^{2}$ of interface cabling, gas and liquid service lines must be arranged within the available space.

\subsubsection{Beamline}

As future detector upgrades are implemented, or as the luminosity level of the collider is increased, it may be necessary to change the beampipe material or dimensions. The beamline vacuum pumping equipment is also expected to encounter a harsh radiation environment. From time to time it will become necessary to replace vacuum pumping and monitoring components. Also, complete removal and reinstallation of the beampipe and vacuum system is required each time the magnet is retracted the full 12-meter distance.

Approximately $14 \mathrm{~m}$ of beamline will extend from the FFS outer-facing surface to the collider tunnel entrance. Surrounding the quads and collima- 
tors will be a roughly box-shaped composite neutron shield extending some $2.75 \mathrm{~m}$ around and $4 \mathrm{~m}$ in front of the collimator. Access will be provided at both ends of the external beamline (assuming approximately $4 \mathrm{~m}$ of shielding in front of the collimator has been removed) and along the entire length of pipe.

Approximately $10 \mathrm{~m}$ of beampipe will remain inside the FFS from the internal ion pumping station just ahead of the forward calorimeter to an external support platform outside the FFS front surface. This pipe section will remain permanently fixed in place during normal operation and during displacement of the magnets. Except for initial assembly, the section requires no scheduled maintenance. To permit initial access inside the magnet vessels for disconnect of the beampipe, the pipe and inner pumping station will remain fixed while the FFS moves back approximately $6 \mathrm{~m}$. The FFS beampipe supports will be designed to allow the FFS to slide freely past the beampipe during retraction.

After the FFS has been withdrawn, a short length of beampipe in front of the forward calorimeter, containing beam position monitor, sector valve and bellows, will be withdrawn. Extensible work platforms will be built up from the calorimeter support stand to facilitate access.

Following removal of the calorimeter endcaps, the last sections of pipe including the NEG are disconnected using the same work platform used to access the tracker. Following removal of the NEG, the final section of pipe will be disconnected from its support mounts and removed from inside the tracker.

Reinstallation of the beampipe and vacuum system will follow the reverse of the procedure outlined above. The same access equipment used for removal will be used during the corresponding step in reinstallation. 


\section{REFERENCES}

1. Design Reference Mission and Operational Timelines for Reliability/Maintainability/ Availability Allocations for the Collider Subsystems, AQA-1010055, K. Dixon, B. Blackford. 


\section{DETECTOR/BEAMLINE INTERFACE}

\subsection{OVERVIEW}

The GEM beamline is designed to satisfy three major requirements:

- a vacuum of $10^{-8}$ torr or better, which is required to achieve an acceptably low flux of secondary particles from beam gas interactions

- an acceptably low flux of neutrons in the central tracker caused by interactions of primary particles in the beam pipe walls

- a pipe composed of discrete sections, with sector valves and connecting bellows. This allows the pipe to be installed in step with the overall installation of the experiment.

The first constraint on the design of the beamline is the required pressure in the vacuum system. Beam gas interactions, distributed throughout the interaction region, are more difficult to shield than pp sources of background. Preliminary calculations (see Sec. 2.2.3) show that at $10^{-8}$ torr the beam gas background is tolerable. In the innermost layers of the central tracker the particle flux due to beam gas interactions at a pressure of $10^{-8}$ torr is $6 \%$ of that due to primary interactions (See Chapter 12). This is a more stringent requirement than that of the collider itself. The beam can tolerate a local vacuum as poor as $10^{-7}$ torr. However, if the vacuum were degraded to $10^{-7}$ torr in the interaction region, then the particle flux from beam gas interactions would become unacceptably large.

The second major input to the beamline design is the need to minimize secondary interactions in the beam pipe itself. In the central region, this is achieved by the use of a thin walled beryllium section of pipe. In the forward regions the path length of particles traversing the beam pipe is much longer, and a potentially large flux of secondaries could be created in the walls of the pipe (see Chapter 12). We have avoided this problem by use of a conical beam pipe section that lies outside of a cone of 0.5 degrees from the interaction point, and that is entirely in the shadow of the forward calorimeter.

The third consideration is a more practical one. The assembly sequence of the GEM detector is such that the beam pipe must be installed in discrete sections, in step with the overall installation of the experiment. This constraint results in a highly integrated detector/beamline system.

\subsection{BEAMLINE DESCRIPTION}

\subsubsection{Length, Diameter and Shape}

The total length of the beam pipe between the collimators of the final focusing quadrupoles is $62 \mathrm{~m}$ ( $\pm 31 \mathrm{~m}$ from the IP). The beam pipe and its associated hardware from the IP to $+31 \mathrm{~m}$ is shown in Figures 11-1 and 11-2. It consists of a central section of beryllium pipe running through the central tracker coupled to successive lengths of stainless steel or aluminum alloy pipe which vary in diameter. The delicate beryllium section is isolated from stress and vibrations via double bellows on each end. The subsequent section in the endcap calorimeter is enlarged to facilitate pumping. The length of the conical section which connects the two is chosen to be larger than the bunch length to avoid perturbing the beam. The design of the interior of the NEG pump eliminates the necessity for a similar conical transition piece in front of the forward calorimeter. In addition to the pipe itself, there are associated pumps, bellows, valves and beam position monitors. The current length, diameter and location of each sector are listed in Table 11-1. 


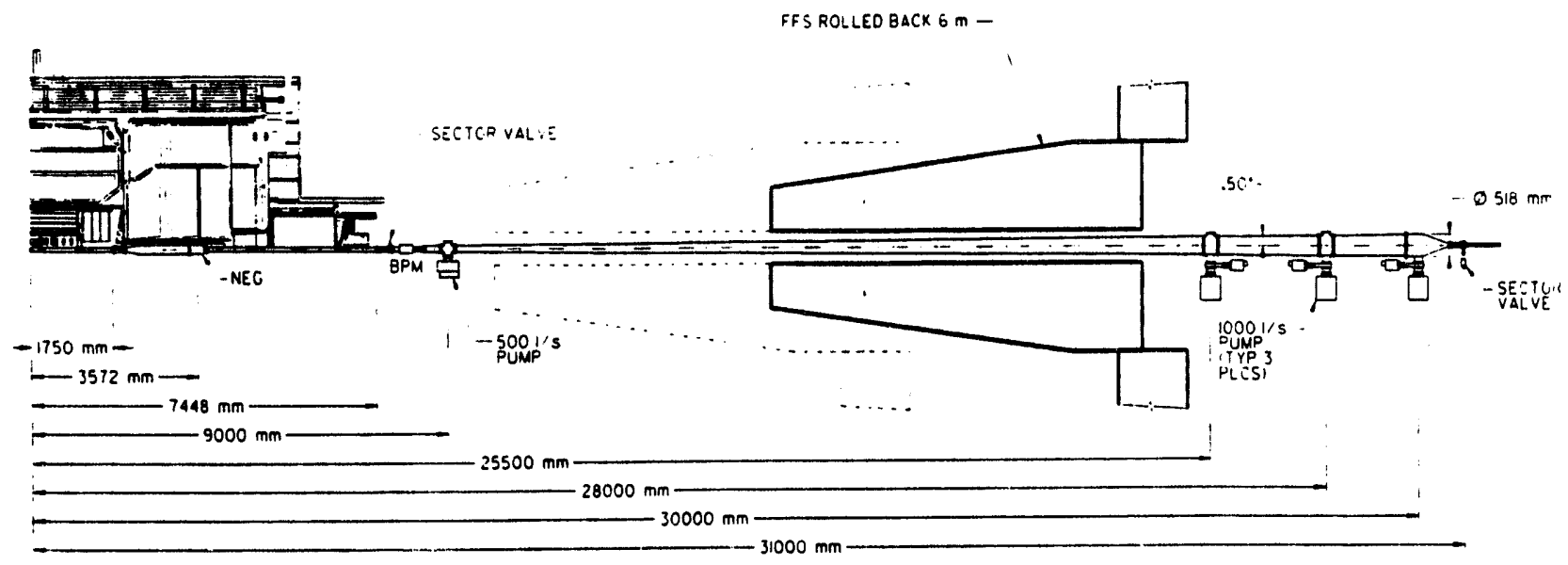

FIG. 11-1. Beam pipe in the entire interaction region.

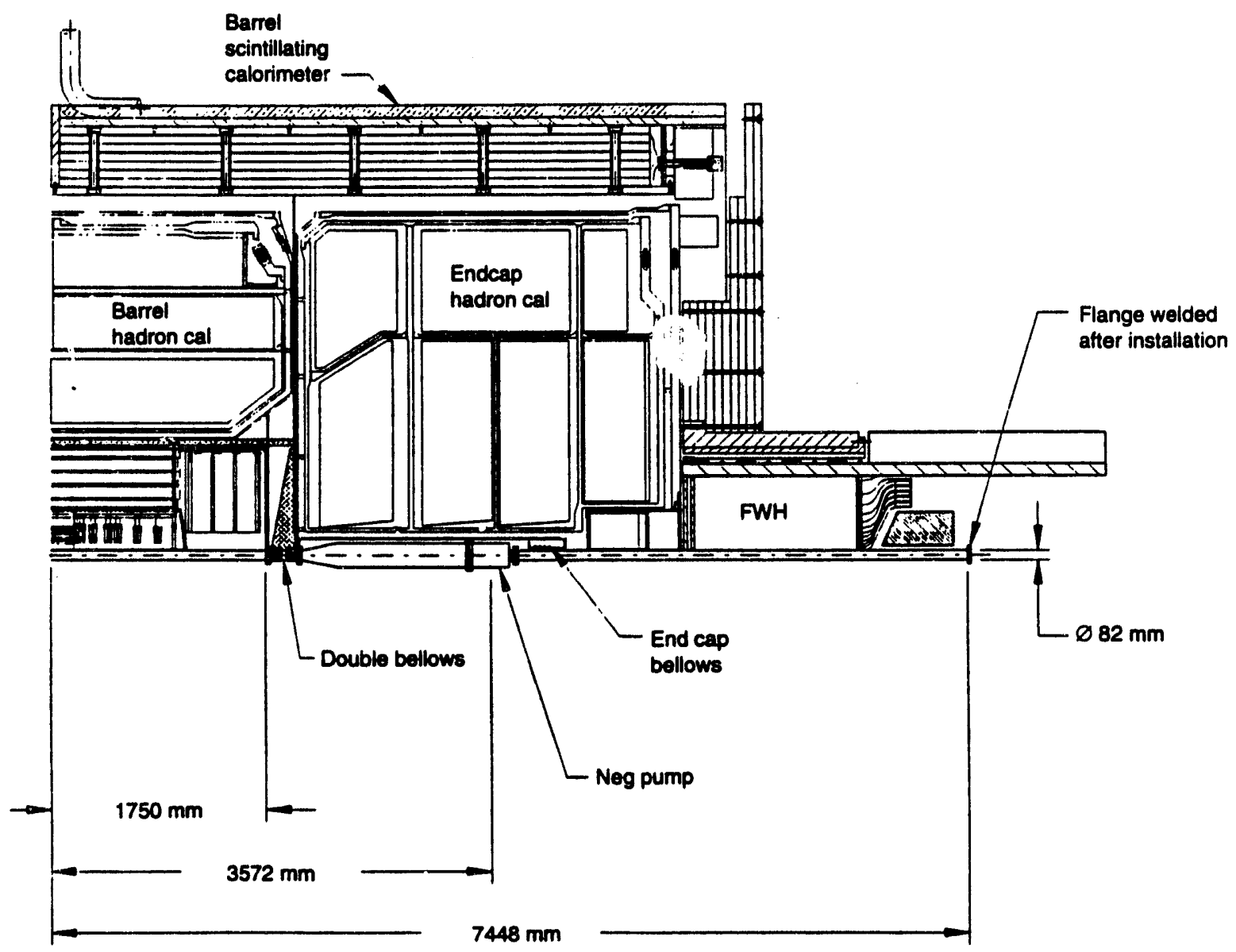

FIG. 11-2. Beam pipe installed in the central tracker region of GEM. 
Table 11-1. Characteristics of each sector.

\begin{tabular}{|c|c|c|c|c|}
\hline Pipe Section & No. & $\begin{array}{l}\text { Location } \\
(\mathrm{mm})\end{array}$ & $\begin{array}{r}\text { Length } \\
(\mathrm{mm})\end{array}$ & $\begin{array}{l}\text { Dia. } \\
(\mathrm{mm})\end{array}$ \\
\hline $\begin{array}{l}\text { Central } \\
\text { tracker with } \\
\text { bellows }\end{array}$ & 1 & $\begin{array}{l}\text { IP to } \pm \\
2000\end{array}$ & 4000 & 80 \\
\hline $\begin{array}{l}\text { Endcap } \\
\text { calorimeter } \\
\text { with NEG }\end{array}$ & 2 & $\begin{array}{c} \pm 2000 \text { to } \\
\pm 3500\end{array}$ & 1500 & 200 \\
\hline $\begin{array}{l}\text { Forward } \\
\text { calorimeter } \\
\text { with BPM } \\
\text { assembly }\end{array}$ & 2 & $\begin{array}{c} \pm 3500 \text { to } \\
\pm 8512\end{array}$ & 5012 & 80 \\
\hline $\begin{array}{l}\text { Forward field } \\
\text { shaper } \\
\text { conical with } \\
\text { ion pumps }\end{array}$ & 2 & $\begin{array}{c}8512 \text { to } \pm \\
31000\end{array}$ & 22488 & $\begin{array}{c}134 \text { to } \\
518\end{array}$ \\
\hline
\end{tabular}

\subsubsection{Material and Thickness}

The beam pipe passing through the central tracker is beryllium, less than $1.5 \mathrm{~mm}$ thick. The low density, low out-gassing rate and high rigidity of beryllium makes it an ideal material for this section. Stainless steel and aluminum alloy have been considered for the subsequent sections of pipe. Stainless steel is preferred for its ease of fabrication and vacuum conditioning. Aluminum is more difficult to fabricate and condition, and its use could result in a higher average pressure; however, its residual activation characteristics are better than those of stainless steel. We chose stainless steel because the beam pipe in the calorimeters is so close to the cryostat walls that there will be a negligible difference in activation.

The nominal thickness of the stainless steel pipe will not be greater than $1 \mathrm{~mm}$ for $80 \mathrm{~mm}$ diameter sections, and not greater than $2 \mathrm{~mm}$ for $200 \mathrm{~mm}$ diameter sections. The thickness of the conical section can be gradually increased with increasing pipe diameter, from $1.2 \mathrm{~mm}$ to $4.5 \mathrm{~mm}$ thick.

\subsection{PRESSURE REQUIREMENTS IN THE BEAM PIPE VACUUM SYSTEM}

To achieve a reliable operating pressure of $10^{-8}$ torr or less under realistic conditions requires a conservative approach to the design of the vacuum system. For example, it is desirable to perform a final in situ bake-out of the entire beam pipe, although at present this appears to be difficult in certain areas of the experiment and impossible in others. To understand the vacuum profile under these conditions, a more detailed set of vacuum calculations assuming less than perfect conditions has been done, and is discussed in detail in technical note GEM TN-93-290. This information is summarized in Figures 11-3 and 11-4. The pumping speed requirements and pumping locations have been determined using these calculations.

\subsubsection{Ion Pumps and Non-Evaporable Getter (NEG) Pumps}

To reduce the pressure in the beam pipe to the required high vacuum range requires a hierarchy of pumps. Ion pumps with pumping speeds of $1000 \mathrm{~V} / \mathrm{s}$ can efficiently evacuate the large diameter conical section of pipe; however their increased capacity does not significantly improve the vacuum at the smaller end of the conical section. There we have chosen $500 \mathrm{~V}$ s ion pumps to minimize material. The locations of these pumps allow the forward field shaper to be withdrawn for access to the inner parts of the detector, without breaking the vacuum of the beamline, as is shown in Figure 11-1. The ion pumps must be able to operate in the GEM magnetic field. These pumps will be specially designed to operate without the use of standard permanent magnets. Pumping will be achieved by using the magnetic field of the GEM solenoid when it is on. The pumps will be fitted with a supplementary coil to supply a low magnetic field $(1.5 \mathrm{kG})$ for pumping when the GEM magnet is off. To maximize the pumping speed, the connections between the ion pumps and the beam pipe must be the same diameter as the ion pump inlets, and as short as possible.

In the central region the problems are more acute. The long distance and low conductance of the $80 \mathrm{~mm}$ beam pipe sections in the central tracker and endcap calorimeter sections require some auxiliary pumping. We plan to use heat activated non-evaporable getter (NEG) pumps with pumping speeds of $300 \mathrm{Vs}$ in front of the endcap calorimeters in order to achieve the best possible vacuum without the inclusion of more massive ion pumps. A prototype NEG pump is currently under construction. It uses conduction heat activation rather than a direct electrical connection through the vacuum wall. If successful, this technique will result in more reliable operation in the inaccessible central tracker region. 


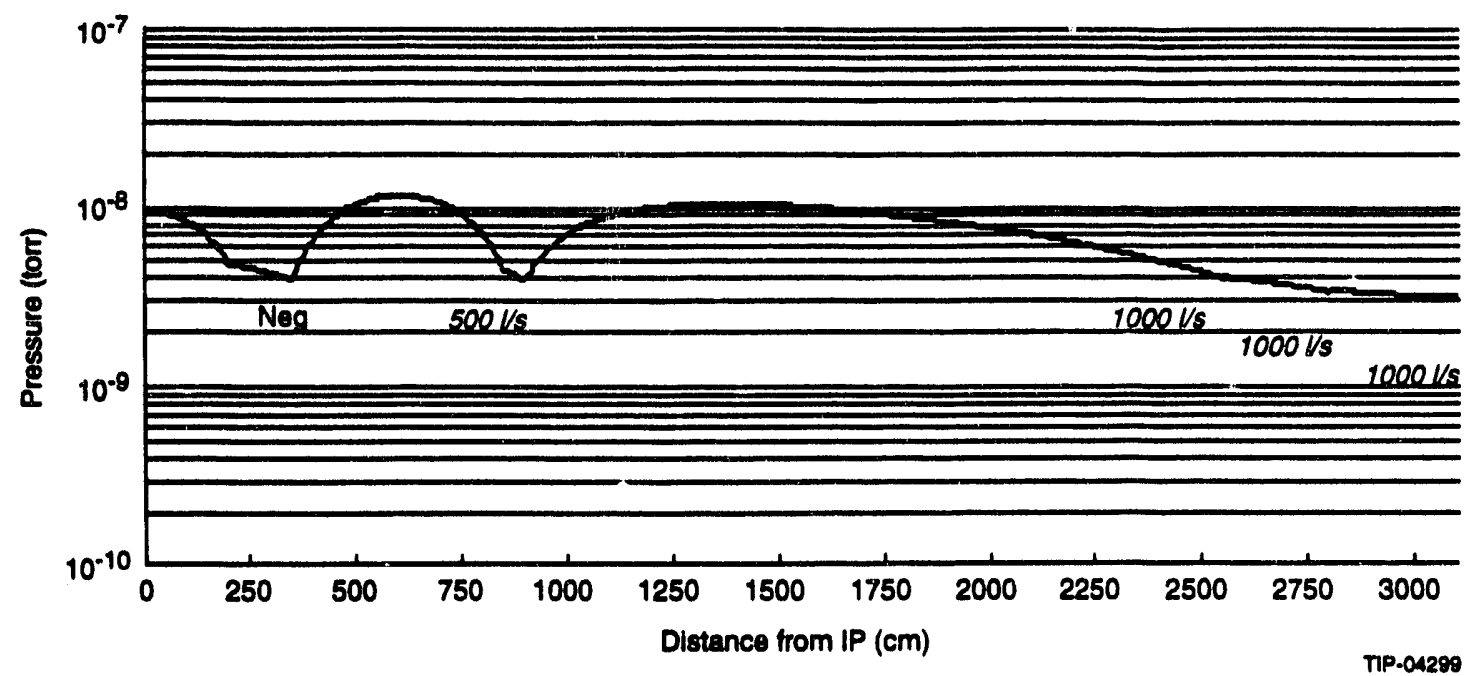

FIG. 11-3. Pressure distribution in the entire interaction region.

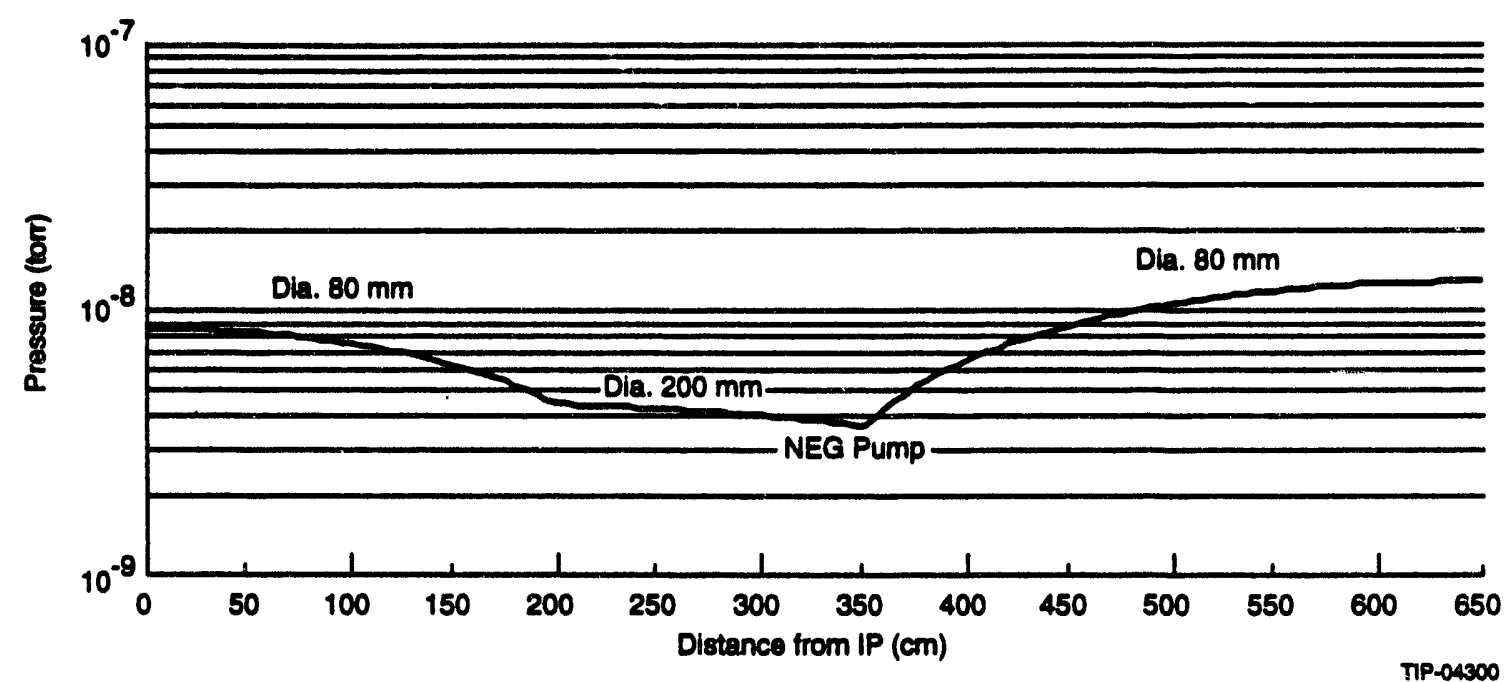

FIG. 11-4 Pressure distribution in the central tracker region.

\subsubsection{Calculated Results}

The calculated pressure profile from the interaction point to $31 \mathrm{~m}$ is shown in Figure 11-4. The result corresponds to a configuration with one NEG pump of $300 \mathrm{Vs}$, and four $500 \mathrm{Vs}$ or $1000 \mathrm{~V} / \mathrm{s}$ ion pumps with incorporated NEG or sublimation elements. The average pressure is $7.06 \times 10^{-9}$ torr. The results from calculation for the interaction region, summarized in Figure 11-3, demonstrates that the average pressure in this region is critically dependent on the diameter of the pipe section within the endcap calorimeter. If an $80 \mathrm{~mm}$ diameter pipe is used in this section, the average calculated pressure changes from $1.02 \times 10^{-8}$ to $1.28 \times$ $10^{-8}$ torr, and the vacuum specification cannot be met no matter where the NEG is placed. However, if a $200 \mathrm{~mm}$ diameter pipe is used in the endcap section, the average calculated pressure drops to $7.66 \times 10^{-9}$ torr.

\section{Out-gassing Rate}

Standard calculations for the pressure distribution in beam pipes assume perfect conditions: pipes of uniform material, perfectly cleaned and properly baked-out, kept in a clean atmosphere, with a minimum of handling through the processing. As mentioned above, a final bake-out of the GEM beam pipe is difficult for most of the pipe sections, and is impossible for the central tracker area. Our assembly scenario requires the pipe to be assembled in 
sections, and there is the possibility that the vacuum might have to be broken for access to some component. Therefore, less perfect conditions must be assumed in our calculations. The out-gassing rates in these calculations, in contrast to perfect conditions, are found in Table 11-2. We have also taken synchrotron radiation-induced out-gassing into account. The worst-case estimate NO TAG of this effect assumes that $25 \%$ of the photons enter the warm beam pipe region and produce an out-gassing rate of $2.0 \times 10^{-10}$ torr- $1 / \mathrm{s} / \mathrm{cm}$ of beam pipe.

Table 11-2. Out-gassing rates (torr-1/s/cm).

\begin{tabular}{lcc}
\hline Material & In this Calculation & $\begin{array}{c}\text { Perfect Condi- } \\
\text { tions }\end{array}$ \\
\hline $\begin{array}{c}\text { Benyllium } \\
\begin{array}{c}\text { Stainless } \\
\text { steel }\end{array}\end{array}$ & $5.0 \times 10^{-11}$ & $7.0 \times 10^{-14}$ \\
\hline
\end{tabular}

\subsection{INTERFACES}

\subsubsection{Detector Beamline/Machine Interface}

The final focusing quadrupoles are located at $\pm 35 \mathrm{~m}$ from the interaction point, with protection collimators located in front of the quads. It is proposed that the warm flange of this collimator be the connecting interface between the GEM detector vacuum assembly and the collider vacuum assembly.

\subsubsection{Beamline/Detector Interface Assembly}

The beam pipe will be assembled in sections as the assembly of the GEM Detector progresses. This assembly procedure is described in detail in Chapter 9. Further documentation is provided in a GEM Technical Note, GEM TN-93-289.

\section{Beam Position Monitors (BPM) and Pumping Stations}

Provisions are being made for the installation of two beam position monitors (BPMs), one on each side of the interaction point, located immediately after the forward calorimeter. Each BPM assembly would consist of a gate valve (about 4" ID), a BPM and two bellows assemblies. A decision on the final location of the BPMs will be made after the details of other detector parameters are frozen. The instrumentation will be positioned in such a way that it lies in the shadow of the forward calorimeter.

There are four pumping stations on each side of the interaction point. Each pumping station consists of a $500 \mathrm{~V} / \mathrm{s}$ or $1000 \mathrm{l} / \mathrm{s}$ ion pump with incorporated NEG or sublimation element (see Section 11.3.2), a 6" diameter gate valve, and a cross with a port for an ion gauge or a mass spectrometer probe, and a port for the roughing line. The positions of the pumping stations are shown in Figure 11-1.

Access must be maintained to all ion pumping stations. To minimize material in the radioactive environment near the beamline, there are no permanently mounted roughing pumps included in the design. Any routine maintenance or repairs will require access for personnel and equipment to the ion pumping stations.

\section{Beam Pipe Support and Alignment}

The support of the beam pipe and associated hardware must meet two requirements:

- it must support the weight of the pipe and vacuum related hardware, and

- it must be adjustable to accommodate deflections. In most of the sections, the beam pipe must be able to slide horizontally along the beam direction during the installation procedure.

A wire or band support will be used between the central tracker pipe section and the barrel calorimeter. It must be vertically adjustable and should allow a small amount of movement along the pipe direction due to thermal contraction.

The beam pipe in the endcap calorimeter section needs a guiding-slide support mechanism to meet the installation requirements. The section of conical pipe through the FFS will be supported on the inner wall of the FFS. In front of the collimator, there will be a $4 \mathrm{~m}$ outer diameter concrete shield around the beam pipe, (see Figure 12-1a). The support for the pipe and pumps should sit within the shielding. 


\section{REFERENCES}

1. A.W. Maschke, March 23, 1992 (private communication). 


\section{RADIATION ENVIRONMENT AND SHIELDING}

\subsection{INTRODUCTION}

As an integral part of the detector design we have performed simulation studies of the neutron, photon, and charged particle fluxes in GEM, and of the resultant doses and radioactivation of all major subsystems and neighboring accelerator components. As a result of these studies, we have developed a shielding and collimator configuration, and an overall layout of the experiment that will ensure that the detector will operate with its design performance for at least 10 years at the standard luminosity of $10^{33} \mathrm{~cm}^{-2} \mathrm{~s}^{-1}$ (see Chapters 4-7). We are confident that modest design changes, resulting from continuing studies, will enable the detector (with the exception of the inner silicon tracker) to function according to its specifications for several years at high luminosity $\left(10^{34} \mathrm{~cm}^{-2} \mathrm{~s}^{-1}\right)$.

The main concerns of our studies have been:

- The charged particle, neutron, and photon fluxes, and the hit rates generated in the inner tracker and the muon spectrometer: We have developed appropriate shielding configurations to reduce the neutron, photon, and charged particle fluxes in the detector. Sections 12.3-12.5 discuss the major sources of background and candidate configurations for shielding the muon system and the central detector cavity. As detailed in Chapters 4-8, we applied the fluences ${ }^{\dagger}$ and energy spectra to the various detector systems and electronics to estimate the hit rates and effects on trigger rates and pattern recognition. The singles rates, trigger rates, and data flow were kept within the design limits of the data acquisition system (Chapter 7). The occupancies in the inner tracker and in the muon spectrometer were below the limit where the pattern recognition efficiency, particle identification efficiency, or track reconstruction would be substantially degraded. For the muon system (see Chapter 4), neutron and photon fluxes less than
$10^{11} / \mathrm{cm}^{2} / \mathrm{SSCY}$ led to low trigger rates and robust pattern recognition efficiency.

- The radiation doses delivered, especially to the inner tracker layers, the endcap and forward calorimeter components, and the beam line components within and near the experiment: For the fast charged particle component, systems that are most likely to suffer radiation damage are located in the central cavity, the endcap calorimeter, and the forward calorimeter. We have computed the total dose rates for detector components in these regions. The integrated fluences and doses over the life of the experiment are at levels that allow for long-term component survival. Examples of the survival limits that have dictated design choices in GEM are: $5 \mathrm{Mrad}$ of ionizing dose or $10^{14} \mathrm{n} / \mathrm{cm}^{2}$, for silicon detectors; $10 \mathrm{Mrad}$ for standard cables; 200 Mrait for superinsulation; and 50 watts of thermal energy dissipated in the nearest superconducting low beta quadrupole magnet.

- Radioactivation of the inner and forward parts of the detector and the beam line, and levels of radioisotopes generated in the experimental hall: Given the close association between the calculation of component activation and the generation of particle fluences, we have included a summary of the activation calculation procedures in Section 12.7. The shielding configuration and choice of materials in GEM have been designed to allow access for detector repair and maintenance operations. Health and safety issues are discussed further in Section 15.13 .

While our studies so far have been geared primarily towards reducing the background of secondary neutrons and photons, we have also considered safety, cost, and access for maintenance of the detector. 
It is difficult to estimate the fluences and dose rates that will occur during accelerator commissioning or under unusual accelerator conditions. Therefore, all fluences and doses presented in this chapter are normalized to a standard year $\left(10^{7} \mathrm{~s}\right)$ of running at a standard luminosity of $10^{33} \mathrm{~cm}^{-2} \mathrm{~s}^{-1}$. This corresponds to $10^{15}$ interactions produced at the interaction point, and $1.3 \times 10^{14}$ protons circulating in each ritig of the collider.

Figure 12-1 a and 12-1b are schematics of the detector and shielding configuration used for the results presented here. For this study we have concentrated on shielding the greatest sources of $50 \mathrm{~m}$

a)

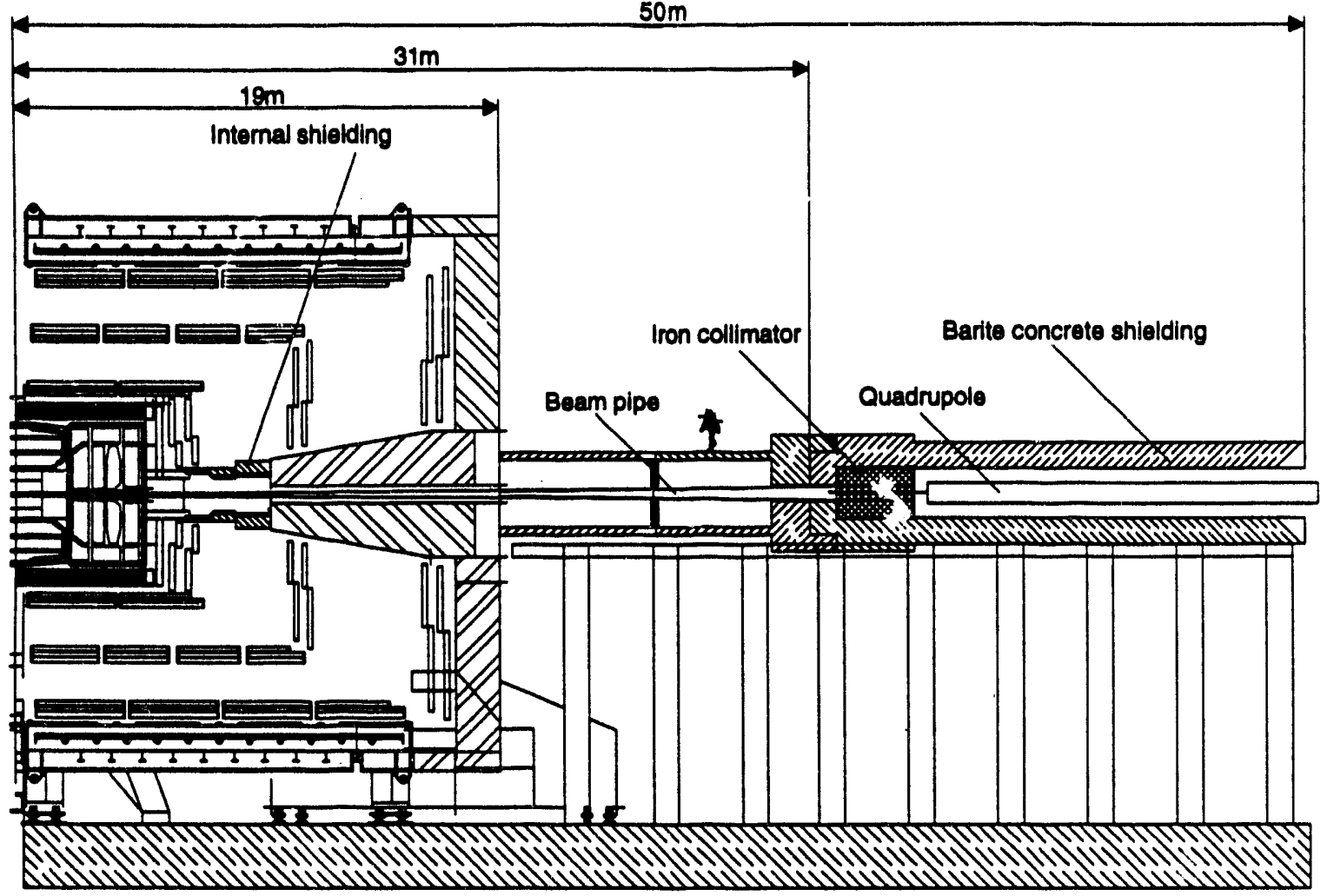

())

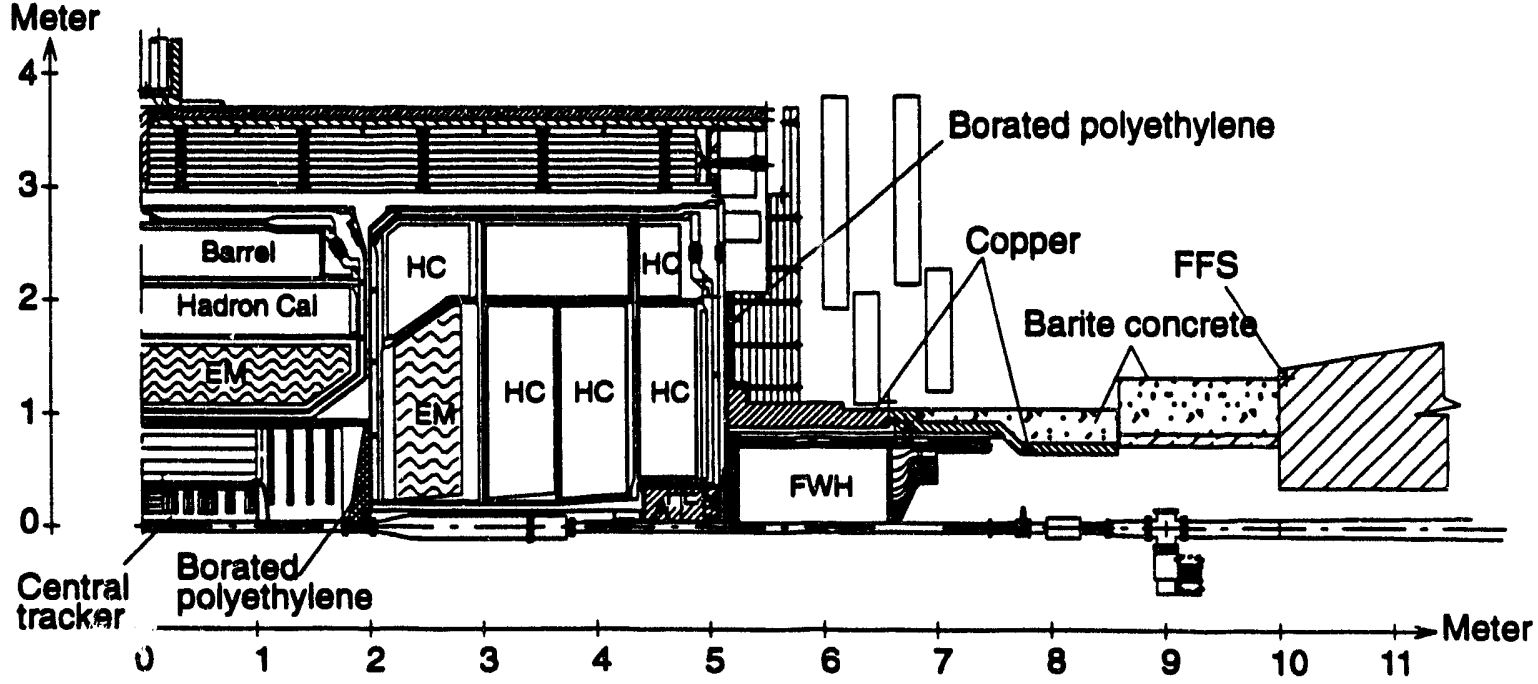

FIG. 12-1. Detector and shielding configuration used in this study to calculate particle fluences and dose rates. Same calculations were also performed without the indicated shielding. a) Elevation view of half of the detector and shielding around the collimator and the quadrupole. b) Details of the shielding near the GEM calorimeter. 
radiation in the detector: the collimator, the forward calorimeter, and the central tracker region. In the following sections we present discussion of shielding configurations and strategy for reducing particle fluxes to tolerable levels.

\subsection{COMPUTATIONAL METHODS}

We have performed this study in three stages. First, we identified the must important radiation sources by considering the deposited energy and the thickness of material between the source and the nearest sensitive detector. Second, we performed calculations to optimize the geometry and the shielding around the main sources. Finally, we performed detailed calculations of the particle fluxes, dose rates, and residual activity. To make the calculations, we used the following simulation tools, including several code systems developed at GEM institutions.

- DTUJET: This is a Monte Carlo program for hadronic multi-particle production in $40 \mathrm{TeV}$ pp collisions. It is based on the two component dual parton model which treats the single diffractive component, soft (low $p_{T}$ ), and hard (minijet and large $p_{T}$ ) frocesses in a unified and consistent way. ${ }^{1}$ We use this model in all simulations to give us the distribution of particles from $40 \mathrm{TeV} p p$ interactions.

- MARS12: This is a Monte Carlo program for three-dimensional simulations of hadronic and electromagnetic cascades and muons. The program emphasizes inclusive particle production and statistical weighting, and therefore allows fast simulations. Versions of the code that interface with magnetic fields and accelerator transport exist, making this system particularly well suited for accelerator shielding studies. ${ }^{2}$

- LAHET-MCNP: This Monte Carlo code package, developed at Los Alamos National Laboratory, ${ }^{3}$ provides transport of hadrons through a combination of the FLUKA code and an extended version of the HETC code to less than $1 \mathrm{MeV}$ for charged hadrons and $20 \mathrm{MeV}$ for neutrons. MCNP transports neutrons down to thermal energies; electromagnetic processes ire handled by ITS or EGS. This code system is widely used for shielding designs of accelerators and nuclear reactors. We have not imple- mented the magnetic field options in LAHET for the studies described here.

- CALOR: A hadronic shower simulation package developed at Oak Ridge National Laboratory, ${ }^{4}$ CALOR combines HETC, which uses the high energy fragmentation model from FLUKA and an intranuclear-cascade evaporation model at low energies; SPECT, which analyzes information from HETC; MORSE, which transports neutrons with kinetic energy below $20 \mathrm{MeV}$; and EGS, which transports electrons and photons.

- GEANT: This code package is widely used in high energy physics. ${ }^{5}$ The main advantages of GEANT are the ease of geometry specification and accurate tracking through magnetic fields. For this study we used our implementation of GEANT containing a complete description of the GEM detector; this code package is called SIGEM. 6 Our version of GEANT uses CALOR for low energy hadron transport. ${ }^{7}$

- CINDER: This code, developed at Los Alamos National Laboratory, ${ }^{8}$ computes residual radioactivity. It uses the spallation products of nuclei produced in the LAHET Monte Carlo code and the low energy neutron spectra produced by MCNP to predict nuclide density, activity, decay heating from electrons and photons, and photon spectra. These data are further processed by LAHET to provide dose rates at specified locations and times.

These tools we used for different tasks, and consistency checls made between the different simulations and $\mathrm{e}$ hand calculations using scaling models. The scaling laws from the literature $9,10,11,12$ were used for many of these checks.

The radiation environment in the GEM detector differs in many ways from the environment described in an earlier SSC Laboratory Central Design Group report, SSC-SR-1033.9 This report concentrated on the radiation environment in the central detector cavity; these considerations are still valid, and we use the results extensively in our report. However, the radiation environment in the muon system outside the calorimeter and, in particular, the albedo from the collimators and the hall walls was not considered there. We devote much of our discussion to reducing neutron and photon fluences 
in the muon system because this is the more difficult problem.

\subsection{ENERGY DEPOSITION}

There are three major sources of radiation: local beam loss in the collider tunnels; interactions of the $20 \mathrm{TeV}$ proton beams with the residual gas in the beam pipe; and particle production at the interaction point. The contributions of each of these sources to the total neutron fluence and the deposited energy are presented in the following sections. The total fluence is dominated by the particle production at the interaction point.

\subsubsection{Contribution by Protons Lost from the Beam}

Beam loss in the walls of the beam pipe occurs continuously around the accelerator. The beam size is large in the final focus quadrupoles near the interaction points (IP), so the beam loss is particularly severe in these regions. Using the MARS12 code system ${ }^{12}$ we have estimated neutron fluxes due to this beam loss by making simulations of proton orbits around the accelerator and of the hadronic cascades due to the lost protons. The maximum loss of about $5 \times 10^{4}$ protons $/ \mathrm{m} / \mathrm{s}$ occurs at the points of maximum beta about $70 \mathrm{~m}$ from the interaction point. Figure 12-2 shows the neutron fluence for neutron energy between $0.5 \mathrm{eV}$ and $14 \mathrm{MeV}$ caused by beam loss as a function of the distance from the interaction point, and at a radius of $200 \mathrm{~cm}$ from the beamline. The calculation includes the tunnel and hall walls but does not include the GEM detector. Hadrons with kinetic energy greater than $14 \mathrm{MeV}$ contribute less than $10 \%$ of the total particle fluence. There is a large increase of the neutron fluence in the tunnel that starts $55 \mathrm{~m}$ from the interaction point; however, near the detector the fluences are less than $10^{9} \mathrm{n} / \mathrm{cm}^{2} / \mathrm{SSCY}$, which is negligible compared to the contribution from $p p$ interactions shown below.

\subsubsection{Contribution from Residual Gas in the Beam Pipe}

The particle fluence due to interactions of $20 \mathrm{TeV}$ beam. protons with atoms of residual gas (beam gas interactions) in the evacuated beam pipe was computed using the MARS12 code system. The calculation assumed a pressure of $10^{-8}$ torr of nitrogen in the warm regions of the beam pipe (which excends up in the low beta guadrupoles located $35 \mathrm{~m}$ from the interaction

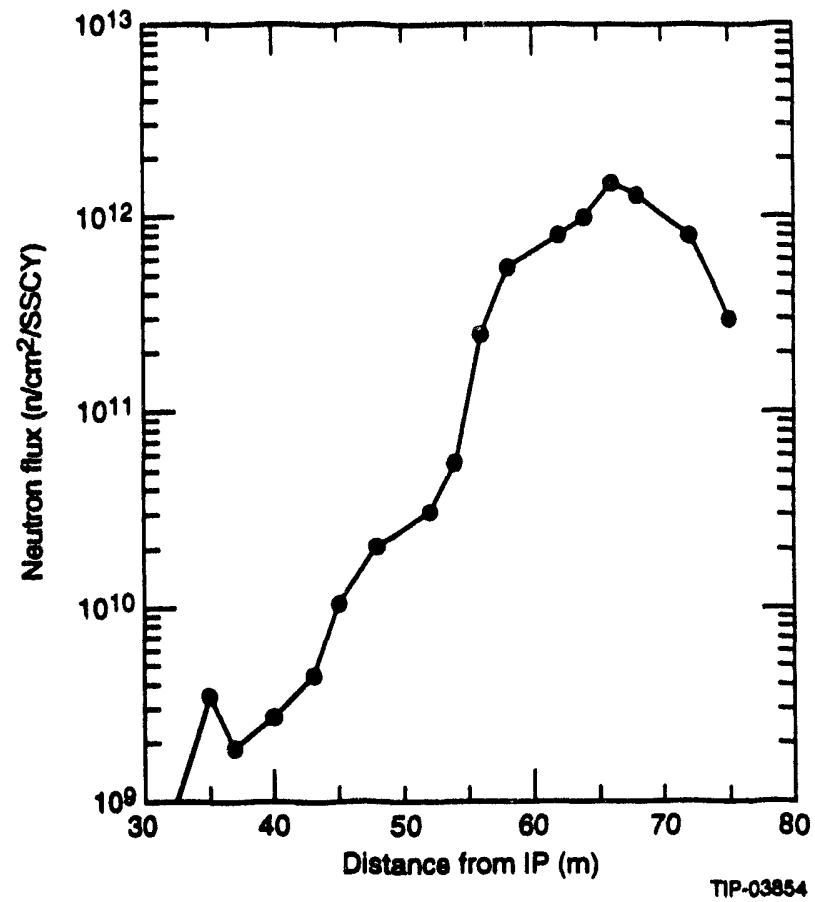

FIG. 12-2. Neutron fluence due to local beam loss in the low beta quadrupoles as a function of distance from the interaction point for a radius of $200 \mathrm{~cm}$ from the beamline (from a MARS12 calculation). The curve is for neutrons with kinetic energy between $0.5 \mathrm{eV}$ and $14 \mathrm{MeV}$. Hadrons with kinetic energy greater than $14 \mathrm{MeV}$ contribute less than $10 \%$ of the total particie fluence.

point). A density of $4.0 \times 10^{8} \mathrm{~N}_{2}$ molecules $/ \mathrm{cm}^{3}$ was assumed for the cold regions of the beam pipe inside the magnets. The cross section of $20 \mathrm{TeV}$ protons ( $50 \mathrm{mb} /$ nucleon) is such that this density corresponds to a loss of $1.7 \times 10^{11}$ protons $/ \mathrm{m} / \mathrm{SSCY}$ from the two rings in the 100-m-long experimental hall. We used the value of $2 \times 10^{11}$ protons/m/SSCY, which includes a small contribution from protons whose orbits are disturbed by the other interaction regions. ${ }^{13}$

The results are shown in Figure 12-3, which gives the particle flux due to beam gas interactions in the shielded GEM detector for all hadrons with energy greater than $0.5 \mathrm{eV}$. There is a large increase in the forward calorimeter region due to the showering of secondary particles procuced in the beam gas interactions. Table 12-1 compares the contribution from beam gas interactions at selected locations in the detector to the fluences due to $p p$ collisions for both the shielded and the unshielded detector configuration. We divide the particle flux in Table 12-1 into high energy ( $E>14 \mathrm{MeV}$ ) neutrons and charged hadrons and low energy $(0.5 \mathrm{eV}<E<14 \mathrm{MeV})$ 
neutrons. The relative contribution from beam gas interactions in the muon system is small, and the shielding does not affect it significantly. In the central detector cavity beam gas interactions contribute $6 \%$ of the high energy particle flux at the inner silicon layers. The neutron contribution from beam gas interactions is small, with or without shielding. Our conclusion is that the attainable vacuum in the detector region could be important. Were it to become ten times worse than we have assumed, the particle flux from beam gas interactions in the central cavity would become significant. This would cause an unacceptable degradation in the lifetime and performance of the silicon tracker.

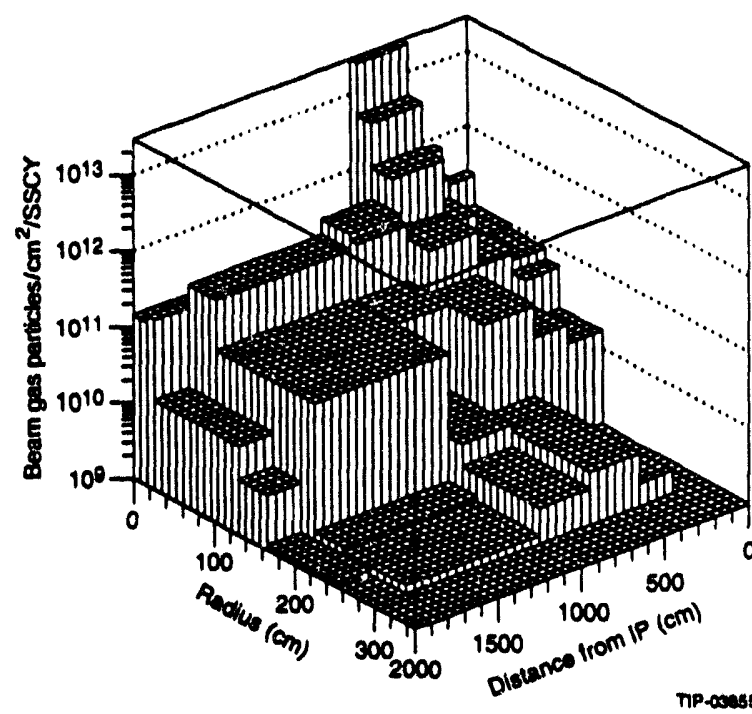

FIG. 12-3. Particle flux due to $2 \times 10^{11}$ beam gas interactions $/ \mathrm{m} / \mathrm{SSCY}$ calculated using the MARS12 code system for all hadrons with energy greater than $0.5 \mathrm{eV}$. We have chosen bins of variable size in order to illustrate the main features of the distribution.

Table 12-1. Ratio in percent of the particle flux caused by beam gas interactions to the flux from pp collisions, at selected locations in the detector. This calculation was performed with MARS12 for the comparison of the two contributions.

\begin{tabular}{|c|c|c|c|c|c|c|}
\hline \multirow[t]{3}{*}{ Location } & \multirow[t]{3}{*}{$\begin{array}{l}\text { Radius } \\
\text { meter }\end{array}$} & \multirow[t]{3}{*}{$z$ from IP } & \multicolumn{4}{|c|}{$\begin{array}{l}\text { Beam gas/pp collisions \% } \\
\text { hadrons with } E>14 \mathrm{MoV} \text { and } \\
\text { neutrons with } 0.5 \text { oV }<E<14 \mathrm{MoV}\end{array}$} \\
\hline & & & \multicolumn{2}{|c|}{ Unshiolded Detector } & \multicolumn{2}{|c|}{ Shielded Detector } \\
\hline & & & $E>14 \mathrm{MoV}$ & $E<14 \mathrm{MeV}$ & $E>14 \mathrm{MeV}$ & $E<14 \mathrm{MeV}$ \\
\hline Central & 0.06 & 0.0 & 5.8 & 1.1 & 3.8 & 2.4 \\
\hline Tracker & 0.30 & 0.0 & 2.3 & 2.2 & 2.3 & 3.0 \\
\hline $\begin{array}{l}\text { Forward } \\
\text { Calorimeter }\end{array}$ & 0.05 & 5.5 & 0.9 & 1.0 & 0.6 & 0.9 \\
\hline Muon & 1.40 & 8.5 & 2.0 & 4.8 & 0.8 & 3.6 \\
\hline System & 2.50 & 16.0 & 1.6 & 3.6 & 8.2 & 2.2 \\
\hline
\end{tabular}

\subsubsection{Contribution from Collisions at the Interaction Point}

We used the event generator DTUJET to estimate the energy deposited in the detector and in the nearby beamline elements. Figures $12-4$ and 12-5 show the DTUJET pseudorapidity and transverse momentum distributions, respectively, of charged particles produced by this model. Figure $12-6$ shows the fraction of the total energy from the interaction point emitted as a function of pseudorapidity. Table 12-2 summarizes the fraction of total energy deposited in the various components of the GEM detector.
The pseudorapidity plateau of 7.5 charged particles per unit pseudorapidity and mean transverse momentum of $0.6 \mathrm{GeV}$ is in agreement with extrapolation of data from lower energies. There is no data in the far forward regions $(|\eta|>6.0)$; therefore, we assign an error of a factor of 2 to the distribution of total radiated energy. ${ }^{14}$

An iron collimator covering $5.8<|\eta|<8.6$ is placed in front of the quadrupoles to intercept energy that would otherwise go into the cryogenic mass of the low beta quadrupoles. The amount of energy lost 
from the collider at the interaction point $\left(4.0 \times 10^{9} \mathrm{TeV} / \mathrm{s}\right)$ is far greater than the beam loss in the accelerator magnets near the interaction point (approximately $4.0 \times 10^{7} \mathrm{TeV} / \mathrm{s}$ ) or the beam loss in the residual gas of the vacuum pipe $(4.0 \times$

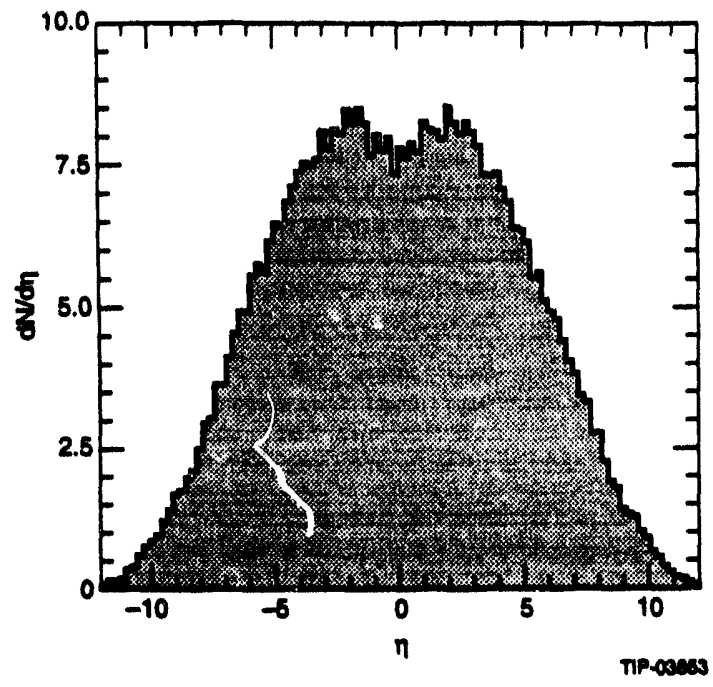

FIG. 12-4. dN/dy distribution for charged particles produced by the Monte Carlo code DTUJET for $40 \mathrm{ToV}$ pp collisions. The plateau is approximately 7.5 charged particles per unit peeudorapidity. The dip at $\eta=0$ is due to a kinematic effect, and corresponds to a flat distribution in rapidity.

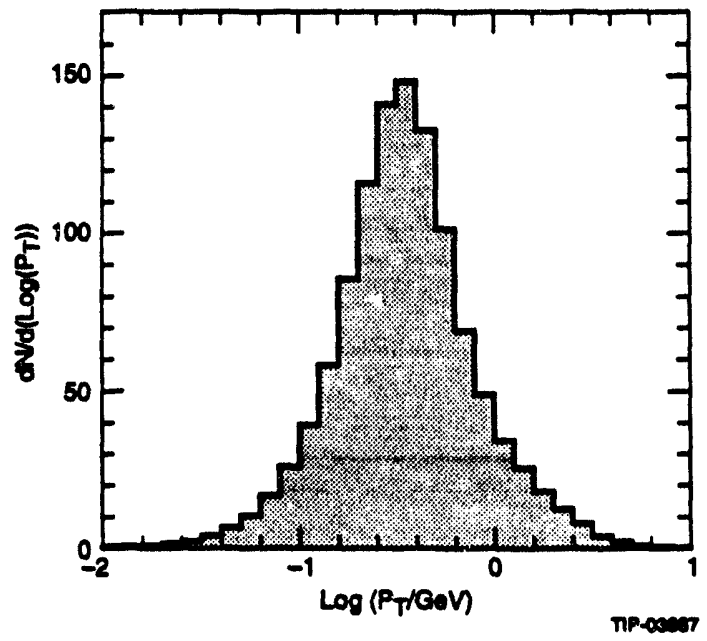

FIG. 12.5. $\quad d N / d p$, distribution for charged particles produced by the Monte Carlo code DTUJET for $40 \mathrm{TeV}$ pp collision. The mean of the distritution is approximately $0.6 \mathrm{GeV}$. $\left.10^{7} \mathrm{TeV} / \mathrm{s}\right)$. The energy intercepted by the forward calorimeters $\left(1.8 \times 10^{8} \mathrm{TeV} / \mathrm{s}\right)$ is much less than the energy into the collimators $\left(1.1 \times 10^{9} \mathrm{TeV} / \mathrm{s}\right)$. Nevertheless, due to the proximity of some muon chambers to the forward calorimeter, both the collimator and the forward calorimeter regions will be the dominant sources of background neutron and photon fluences in the muon system of the detector. The entrance to the central cavity presents a very small solid angle to the forward calorimeter and the collimator, we therefore expect the fluxes in the central cavity

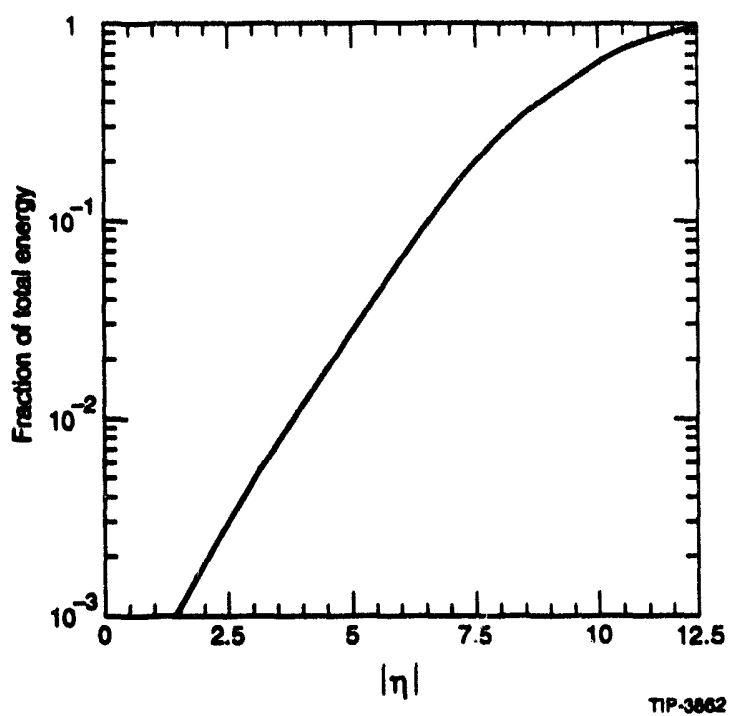

FIG. 12-6. Fraction of the total energy from the interaction point emitted as a function of peeudorapidity.

Table 12-2. Fraction of the total energy emitted from the interaction point intercepted by various detector components.

\begin{tabular}{lcc}
\hline \multicolumn{1}{c}{ Detector } & $\eta$ coverage & $\begin{array}{c}\text { \% energy } \\
\text { deposited }\end{array}$ \\
\hline Barrel calorimeter & $0<|\eta|<1.3$ & 0.1 \\
Endcap calorimeter & $1.3<|\eta|<3.0$ & 0.4 \\
Fonward calorimeter & $3.0<|\eta|<5.8$ & 4.4 \\
Collimator & $5.8<|\eta|<8.6$ & 27.0 \\
\hline
\end{tabular}


to result largely from the albedo of electromagnetic and hadronic showers in the barrel and endcap calorimeters.

Figure 12-7 shows neutron and photon fluxes for neutrons of all energies for an unshielded detector (calculated using the GEANT-CALOR package). The flux in the central tracker is seen to be uniform at a level of $5 \times 10^{13} \mathrm{n} / \mathrm{cm}^{2} / S S C Y$; the flux in the muon system varies between $10^{11}$ and $10^{12} \mathrm{n} / \mathrm{cm}^{2} / \mathrm{SSCY}$. Figure $12-8$ shows the two main components of the neutron flux: the collimators and the calorimeter. Sections 12.4.1 to 12.4.4 of this chapter discuss shielding that can be built around the collimators and the forward calorimeter. Shielding concerns for the central tracker are addressed separately in Section 12.5.

\subsection{SHIELDING CONSIDERATIONS}

\subsubsection{Location and Aperture of the Collimators}

We determined the location and the inner diameter of the collimator, taking into consideration the amount of energy deposited in the low beta quadrupoles and the neutron fluences in the detector. For fixed aperture sizes, moving the collimator away from the interaction point reduces the solid angle for particles impinging on the cold magnets. Nevertheless, the energy going into the magnets is a weak function of the collimator distance to the interaction point, because the distribution of energy from the interaction point is extremely forward-directed (Figure 12-6). For example, with a collimator inner diameter of $25 \mathrm{~mm}$, moving the collimator from $20 \mathrm{~m}$ to $35 \mathrm{~m}$ reduces the energy going into the quadrupoles by only $20 \%$. The theoretical uncertainty in the forward production is much larger than this. The neutron flux in the detector from hadronic showers in the collimators is also a weak function of the collimator location. This is because a significant fraction of the radiation from the collimator undergoes secondary reactions in the concrete walls of the collision hall and the forward regions of the detector (see Figure 12-8), reducing the effect of the collimator as a localized source. Therefore, the location of the collimator and first quadrupole is largely determined by considerations of luminosity, space needed for shielding the collimators, and detector access. We have determined that placing the first low beta quadrupole so that it starts $35 \mathrm{~m}$ from the interaction point is a reasonable choice. A location closer to the interaction point might lead to a modest increase in luminosity, but the increase would most likely be offset by the increased amount of time needed during accesses for removing shielding (see Chapter 10).

A fraction of the energy going into the low beta quadrupoles appears as heat deposited in the cold mass of the magnets. Table $12-3$ shows the amount of heat deposited in the cold mass of the first quadrupole and the expected lifetime of the coil insulation based on a $1000 \mathrm{Mrad}$ limit. These quantities are tabulated as functions of the inner diameter of a 3-m-long iron collimator starting $32 \mathrm{~m}$ from the interaction point. Using the MARS12 program, these numbers were calculated by simulating the showers induced by particles coming from the interaction point and striking the collimatorquadrupole system. The magnetic field in the quadrupoles was included in the simulation. The field approximately doubles the amount of heat deposited in the first quadrupole by bending forward-going particles that would otherwise have deposited their energy farther downstream. There is a large uncertainty on the heat load due to the uncertainties in the DTUJET model. The acceptable heat load for the first quadrupole (determined by the SSC magnet group) is approximately $\mathbf{5 0}$ watts. We propose a collimator with a $25 \mathrm{~mm}$ diameter aperture. Finally, the present design of the low beta quadrupoles with maximum acceptable heat load of 50 watts is expected to be upgraded along with several subsystems of the SSC accelerator. This will allow GEM to operate at a luminosity of $10^{34} \mathrm{~cm}^{-2} \mathrm{~s}^{-1}$.

Table 12-3. Power dissipated in the first low beta quadrupole and the expected lifetime of the coil insulation at standard luminosity $\left(10^{33} \mathrm{~cm}^{-2} \mathrm{~s}^{-1}\right)$ as functions of the collimator aperture.

\begin{tabular}{llll}
\hline Aperture (mm) & 25 & 42 & 300 \\
Heat in QL1 (watts) & 21 & 40 & 85 \\
Lifetime (year) & 7 & 5 & 1.5 \\
\hline
\end{tabular}

\subsubsection{Shielding for the Collimators}

The spectrum of particles striking the iron collimator peaks at about $100 \mathrm{GeV}$, but particles with energies of several TeV are also present. We expect the 3-m thick iron (16.7 interaction lengths) collimator to absorb most of the high energy radiation 

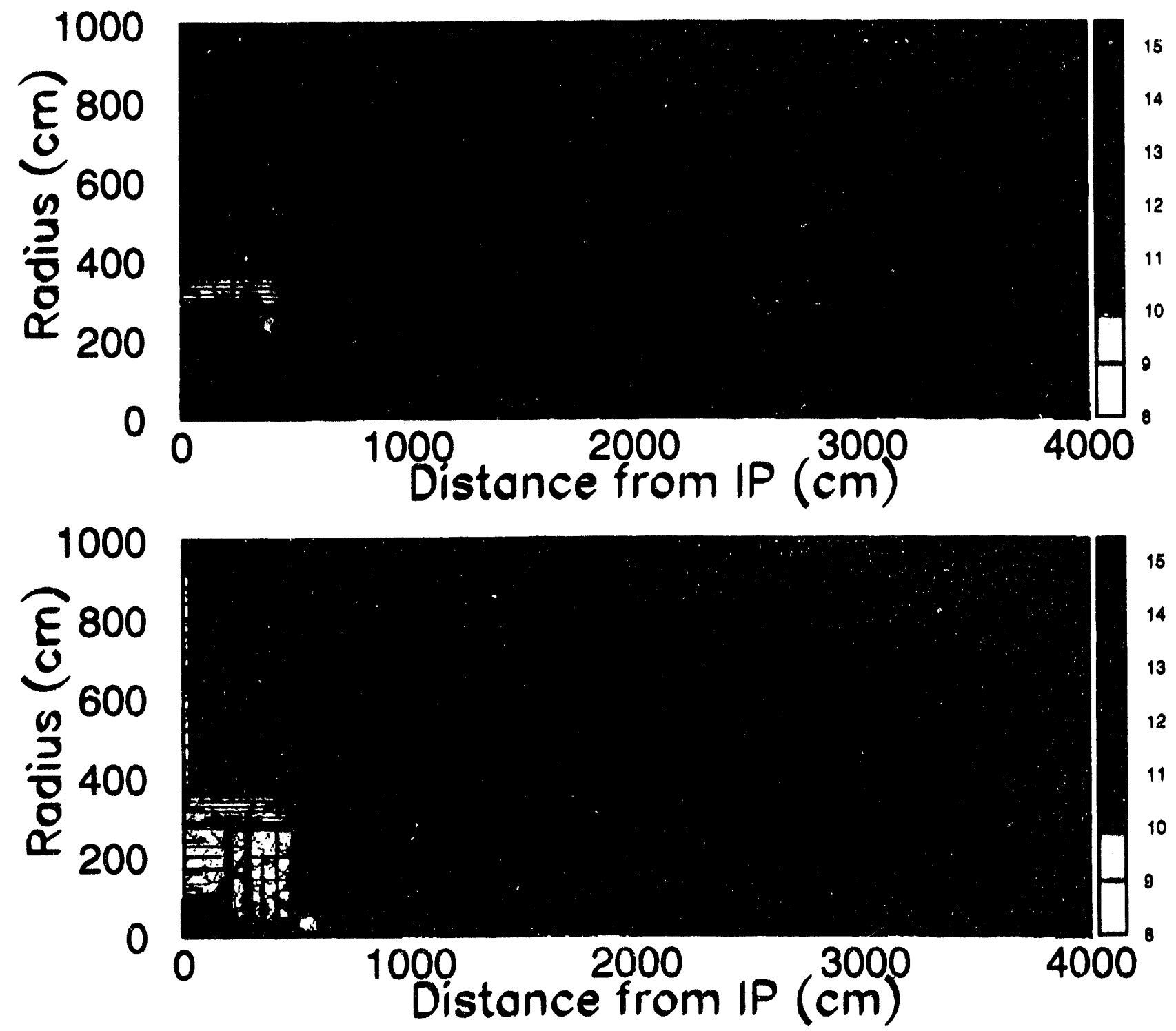

FIG. 12-7. Neutron ( $n$ ) and photon (y) flux, in units of $10^{n} / \mathrm{cm}^{2} / S S C Y$, where the color (or shade) indicates the power $n$, in the unshielded detector (calculated using the GEANT-CALOR package). Neutrons of all energies are included in the plot; photons have an energy cutoff $E>10 \mathrm{koV}$. 


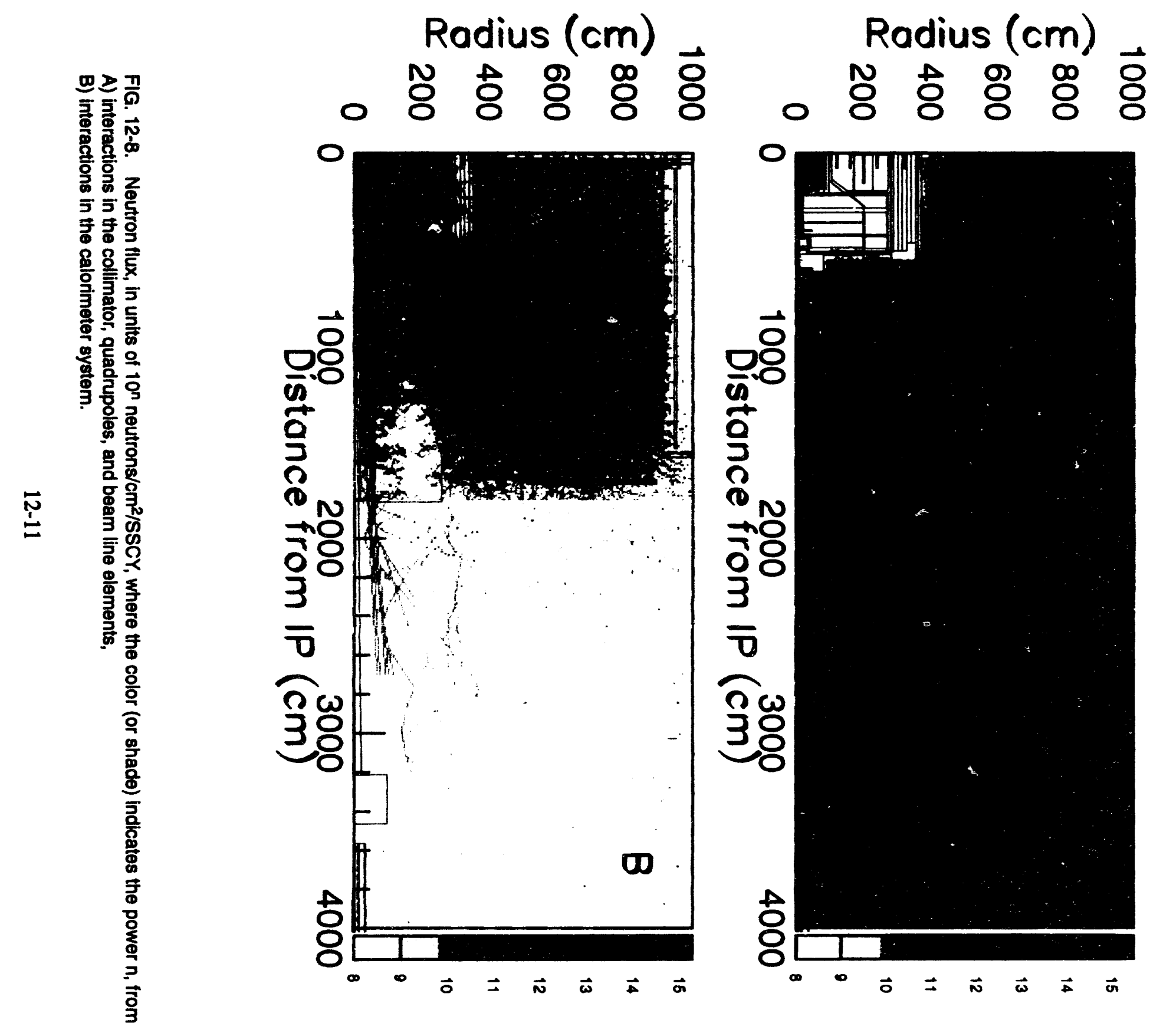


resulting from the hadronic cascade without significant leakage into the cold mass of the low beta quadrupole magnet. At the end of the hadronic cascade in dense material, only neutrons with kinetic energies of 1 to $20 \mathrm{MeV}$ from nuclear evaporation remain. Multiple neutron production and inelastic $\left(n, n^{\prime}\right)$ reactions are important in this energy range. Although these processes generate extra neutrons, they are effective in degrading the neutron energies. As the neutron energy is degraded below a hundred $\mathrm{keV}$, the most effective neutron removal process is the $(n, \gamma)$ reaction, where a neutron is captured with the emission of one or more photons with energy below a few $\mathrm{MeV}$. Unfortunately, $(n, \gamma)$ cross sections are not high enough in most heavy metals to rapidly remove neutrons below a huridred $\mathrm{keV} .15,16$ This is especially true for iron and lead. Therefore, neutrons in the 0.1 to $1.0 \mathrm{MeV}$ range are expected to leak out of the collimator into the hall. We are forced to use light materials such as borated polyethylene or borated concrete with high hydrogen content to rapidly thermalize these neutrons and absorb them on boron or other nuclei that have very high absorption cross sections at thermal energies. We expect that the best shields will be composites made of heavy metals to contain the high energy cascades in a small volume, and light materials such as concrete or polyethylene to moderate and absorb the low energy neutron leakage.

We have investigated several different geoinetrical arrangements of concrete around the collimator and have found the neutron flux attenuation largely insensitive to the geometry when at least $5 \mathrm{~m}$ of concrete is present to trap the albedo from the collimator, which is located at $32 \mathrm{~m}$ from the interaction point. Table 12-4 shows the flux of neutrons in the $0.5 \mathrm{eV}$ to $14 \mathrm{MeV}$ range in various parts of the detector for two different configurations of shielding around the collimator:

A) The column for configuration (A) contains fluxes for an iron collimator with an outer radius of $1.5 \mathrm{~m}$ surrounded by $0.5 \mathrm{~m}$ of concrete. There was no additional shielding around the low beta quadrupoles and no shielding from the collimator albedo.

B) The column for configuration (B) contains fluxes for an iron collimator with an outer radius of $0.75 \mathrm{~m}$ surrounded by $1.25 \mathrm{~m}$ of concrete. In addition, the low beta quadrupoles from the back of the collimator to the tunnel entrance were surrounded by $1.0 \mathrm{~m}$ of concrete, and a 5.0-m-long concrete block with an inner diameter of $0.5 \mathrm{~m}$ and an outer diameter of $2.0 \mathrm{~m}$ was placed in front of the collimator to trap the albedo.

The extra shielding in configuration (B) obtains a one order of magnitude reduction of the flux in the muon chambers, and a two orders of magnitude reduction between the detector and the colliniator. There is also a large reduction around the collimator at $33 \mathrm{~m}$ due to the replacement of some of the iron with concrete.

The largest reduction of neutron fluxes in the detector volume results from the addition of the albedo trap. We also find that the use of special concretes such as barite ${ }^{17}$ with high barium and boron contents leads to shielding designs with low volumes. We have chosen the configuration of shielding shown in Figure 12-1, which gives an estimated two orders of magnitude reduction in neutron fluxes on the boundary of this shield. In addition, we have chosen to extend the shield up to the iron field shaper to eliminate gaps and trap all of the albedo. This choice should help us shield against beam gas interactions in the beam pipe as well. 
Table 12-4. Neutron fluxes at specified locations in the $0.5 \mathrm{eV}$ to $14 \mathrm{MeV}$ range for two different configurations of shielding around the collimator (see text for the exact geometry.'). The second configuration has a 5-m-long albedo trap in front of the collimator, additional concrete around the collimator, and $1 \mathrm{~m}$ of concrete around the low beta quadrupole magnets. The hydrogen and boron content in the concrete removes thermal neutrons effectively.

\begin{tabular}{lcccc} 
Location & $\begin{array}{c}\text { Radius } \\
\text { (meter) }\end{array}$ & $\begin{array}{c}\text { Distance from IP } \\
\text { (meter) }\end{array}$ & $\begin{array}{c}\text { n flux config. A } \\
\text { (n/cm } / \mathrm{SSCY} \text { ) }\end{array}$ & $\begin{array}{c}\mathrm{n} \text { flux config. B } \\
\text { (n/cm } / \mathrm{SSC} \text { ) }\end{array}$ \\
\hline Endcap muon & 2.5 & 14 & $1.2 \times 10^{12}$ & $1.7 \times 10^{11}$ \\
Free space & 2.0 & 23 & $3.8 \times 10^{12}$ & $1.4 \times 10^{11}$ \\
Albedo trap & 2.0 & 33 & $7.1 \times 10^{12}$ & $5.4 \times 10^{10}$ \\
Collimator & 2.0 & $33 \times 10^{12}$ & $4.4 \times 10^{10}$ \\
\hline
\end{tabular}

\subsubsection{The Forward Calorimeter}

We have applied the same general considerations to the forward calorimeter shielding as were applied to the collimator shielding. The location of this shielding in the middle of the sensitive muon system, and the function of the forward calorimeter as an active detector, present special constraints on this shielding design. In particular, we identify two separate escape paths for neutrons generated in the forward calorimeter: 1) Neutrons injected into the cryostat gap between the electromagnetic and hadronic sections of the forward calorimeter can travel through the gap to a larger radius and escape into the muon system (see Figure 12-1 at $z=4.95 \mathrm{~m}$ ). 2) High energy particles and neutrons generated at the inner edge of the hadronic calorimeter can further interact in the iron field shaper and escape into the muon system through the gap between the forward calorimeter and the field shaper (see Figure 12.1 between $z=6.43 \mathrm{~m}$ and $z=10.0 \mathrm{~m}$ ). There are two additional considerations for providing protection to the muon chambers at $z=6.38 \mathrm{~m}$. First, we must place sufficient high density material at the location of the forward hadronic calorimeter transverse to the beam axis. Second, we cannot place a neutron absorbing material such as borated polyethylene in proximity to the muon chambers (because of the photons emitted as a result of neutron absorption on boron).

In order to quantify the number of neutrons injected into the muon system by particle interactions, we have performed Monte Carlo simulations that include the endcap calorimeter, the forward calorimeter, and the forward field shaper. The particles from the interaction point were divided into three groups: 1) $2.5<|\eta|<4.4$, particles striking the lower half of the endcap and the upper half of the forward electromagnetic section; 2) $4.4<|\eta|<5.6$, particles striking the lower half of the forward electromagnetic section; 3) $5.6<|\eta|<5.8$, particles striking the inner edge of the hadronic calorimeter. Table 12-5 shows the number of neutrons per second escaping through the two paths due to these three particle sources. The flux escaping through the cryostat gap is approximately $25 \%$ of the total; this can be eliminated by filling the gap with heavy material. The largest source is the inner edge of the hadronic calorimeter. It can be eliminated by either placing heavy shielding between the forward calorimeter and the field shaper, or by shaping the inner edge to be projective to the interaction point. Table $12-5$ shows that $60 \%$ of the flux can be eliminated by making the inner edge of the forward hadronic calorimeter projective.

Elimination of the cryostat gap and projective shaping of the forward hadronic section have a significant impact on the calorimeter design. These changes are being considered for the future (see Chapter 5). The neutron flux through the cryostat gap is reduced by approximately $30 \%$ by lining the inner edge of the passive absorber with 5-cm-thick borated polyethylene. The passive absorber is made of copper and presents approximately 3.5 interaction lengths of transverse material at the location of the forward hadronic section. We have added $10 \mathrm{~cm}$ of copper and barite concrete with an average thickness of $40 \mathrm{~cm}$ between the forward hadronic section and the field shaper (see Figure 12-1b). This shield reduces the neutron fluxes injected into the muon system by one order of magnitude everywhere except the muon chambers adjacent to the forward hadronic calorimeter. 
Table 12-5. Categorization of neutrons of all energies injected into the endcap muon chambers per second. The source particles from the interaction point are categorized in three $\eta$ regions as indicated in the rows of the table. The neutrons injected in the muon system are categorized in two groups as indicated in the third and fourth columns of the takle.

\begin{tabular}{lccc}
\hline Source of Secondaries & $\eta$ coverage & Cryostat Gap (Hz) & FCAL-FFS Gap (Hz) \\
\hline Top of FCAL & $2.5<|\eta|<4.4$ & $1.4 \times 10^{9}$ & $9.2 \times 10^{7}$ \\
Bottom of FCAL & $4.4<|\eta|<5.6$ & $3.1 \times 10^{9}$ & $2.2 \times 10^{9}$ \\
Inner Edge of Had. FCAL & $5.6<|\eta|<5.8$ & $5.7 \times 10^{8}$ & $8.9 \times 10^{9}$ \\
\hline
\end{tabular}

\subsubsection{Beam Pipe Considerations}

The beam pipe is the first material structure traversed by particles from the interaction point. We have attempted to reduce the density and the thickness of the beam pipe everywhere in the interaction region in order to reduce the number of secondary interactions. Chapter 6 and Chapter 11 discuss the optimization of the beam pipe in the central tracker; it is nominally made of $1.5-\mathrm{mm}$ thick beryllium with an inner diameter of $80 \mathrm{~mm}$. The section of the beam pipe through the endcap region is larger in diameter (inner diameter $200 \mathrm{~mm}$ ) and is composed of $2-\mathrm{mm}$ thick stainless steel. The larger diameter keeps the beam pipe wall away from the low angles where the flux and energy of particles is large. The beam pipe is narrow ( $80 \mathrm{~mm}$ diameter) through the forward calorimeter and then is joined to a flared beam pipe. We have designed gate valves and other beam line instrumentation at these joints (see Chapter 11) carefully so that there are no edges to intercept particles from the interaction point.

The beam pipe is flared between the back end of the forward calorimeter and the collimator, so all the beam pipe material is in the shadow of the forward calorimeter. Thus, particles from the interaction point passing through the hole in the forward calorimeter can not interact until they enter the iron collimator in front of the low beta quads. Secondary products from the showers initiated at the inner edge of the forward calorimeter are the only significant source of interactions in this section of the beam pipe. Figure 12-9 shows the average number of hadronic interactions in the beam pipe as a function of distance from the interaction point for a cylindrical beam pipe with a radius of $4 \mathrm{~cm}$ and for the beam

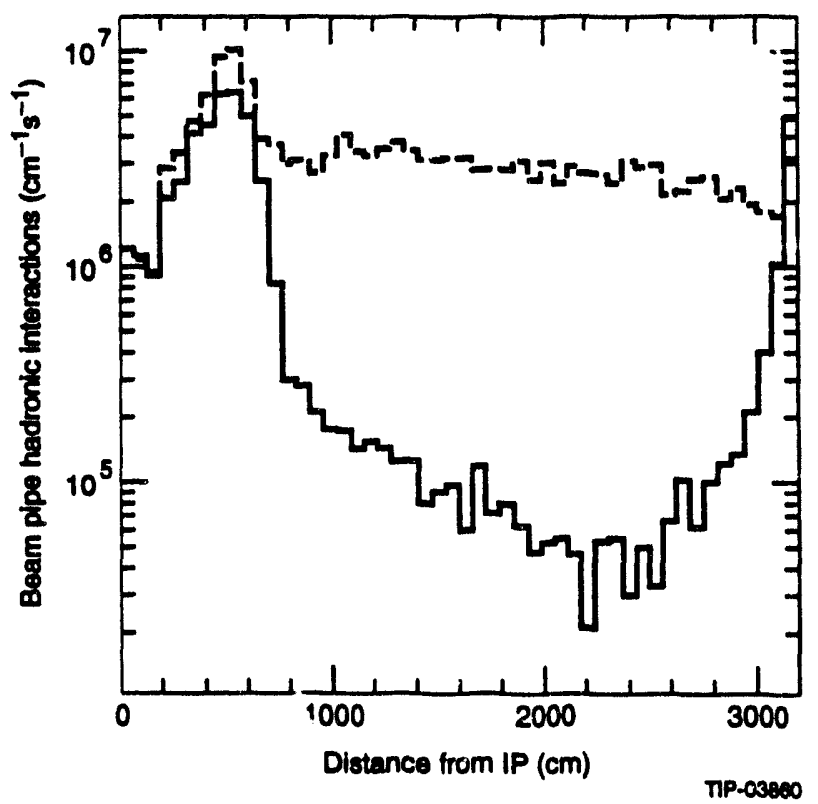

FIG. 12-9. The average number of hadronic interactions in the beam pipe as a function of distance from the interaction point for two cases: as described in the text (solid); and with a constant diameter of $80 \mathrm{~mm}$ (dashod). 
pipe described in this section. The flaring of the beam pipe will reduce the number of hadronic interactions by more than a factor of 10 , with a corresponding decrease in the neutron flux, total dose rates, and activation in the region between the forward calorimeter and the collimator.

\subsubsection{Summary of Particle Fluxes}

In this section we summarize the particle fluxes in the muon system, with and without the additional shielding described above. Fluxes in the central detector cavity are treated separately in the next section.

Charged particle fluxes are negligible outside the central detector cavity in most of the muon system. Preliminary calculations indicate that the muon flux from beam losses in the quadrupoles is small. We will quantify this flux more accurately to examine the possible use of these muons for calibration and alignment.

The neutron fluxes for the unshielded detector are shown in Figures 12-7 and 12-8. The main source is the collimator, as is apparent in the figures. The forward calorimeter contributes significantly to the flux at the innermost endcap muon chambers. Figure 12-10 shows the neutron and photon fluxes for the shielded detector. Neutrons of all energies and photons above $10 \mathrm{keV}$ are included in these figures, whic' $a$ were produced with the same simulation package (GEANT-CALOR). Neutron fluxes in most of the muon system are in the range of $1-5 \times 10^{10} \mathrm{n} / \mathrm{cm}^{2} / \mathrm{SSCY}$, with the exception of the muon chambers closest to the forward calorimeter where the flux is about twice as large. We expect a photon to neutron ratio of approximately $30 \%$ due to the continuous removal of neutrons by $(n, \gamma)$ reactions. Figure 12-11 shows the spectra of neutrons and photons expected in the barrel muon system of the shielded detector. The spectrum of neutrons depends on the details of the shielding and the location in the detector. The thermal part of the neutron spectrum poses special problems since it is highly dependent on the water content of the air and concrete in the hall. It also depends on the presence of rare isotopes with high thermal neutron absorption cross-sections. In particular, boron in the shielding concrete should totally remove thermal neutrons in its vicinity. We have computed that less than $10 \%$ of the neutrons are thermal in the muon system of the shielded detector. Since the calculation of muon chamber hit rates, which is described in Chapter 4, is not strongly dependent on the fraction of thermal neutrons, a thorough understanding of the thermal neutron flux is not needed.

The uncertainties in this calculation result from three sources: 1) the particle production model, 2) knowledge of neutron cross sections in the materials of the detector, 3 ) inexact modeling of the detector geometry for computational convenience. We assign an error of a factor of 2 for the first uncertainty. The other two uncertainties are estimated by using different Monte Carlo codes. We have modeled the same fluxes using MARS12 and the Los Alamos LAHET-MCNP code, which use different cross section libraries and tracking algorithms, and have found agreement within a factor of 2 in these codes. Therefore, we are confident that our estimates are accurate within an overall factor of 3. The contribution to the error from Monte Carlo statistics is small on this scale.

Our results on neutron and photon fluxes are used in Chapter 4 to estimate hit rates in the muon chambers. The calculations are quite conservative. At various locations in the muon system we compare the GEANT-CALOR and LAHET-MCNP results and al ways use the higher estimate for fluxes and the more pessimistic spectra. As shown in Chapter 4, these conservative estimates of rates in the muon system are low even at the higher luminosity of $10^{34} \mathrm{~cm}^{-2} \mathrm{~s}^{-1}$.

\subsection{CENTRAL TRACKER}

The albedo neutron and photon fluxes in the central cavity are affected by the volume of the cavity, the composition of the electromagnetic calorimeter, and by the shielding material placed in front of the calorimeter. We have shown that changes made in the forward calorimeter, and in the shielding of the collimators and quadrupoles, have a relatively small effect on the radiation environment in the central tracker. ${ }^{18}$ As shown in Reference 9, the neutron flux scales as $1 / \mathbf{R}^{2}$, where $R$ is the characteristic dimension of the central cavity. Furthermore, the flux is proportional to $(1+A)$, where $A$ is the mean number of reflections that a neutron experiences before being absorbed. Bor ted polyethylene placed on the walls of the central detector cavity should reduce the neutron flux by fast absorption. 


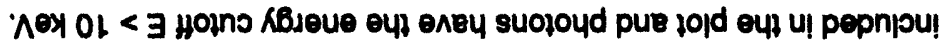

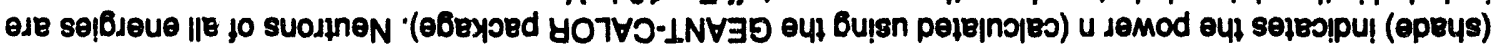

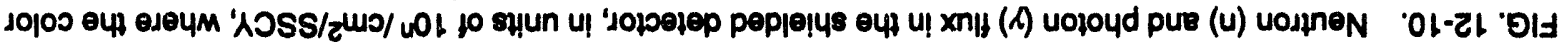

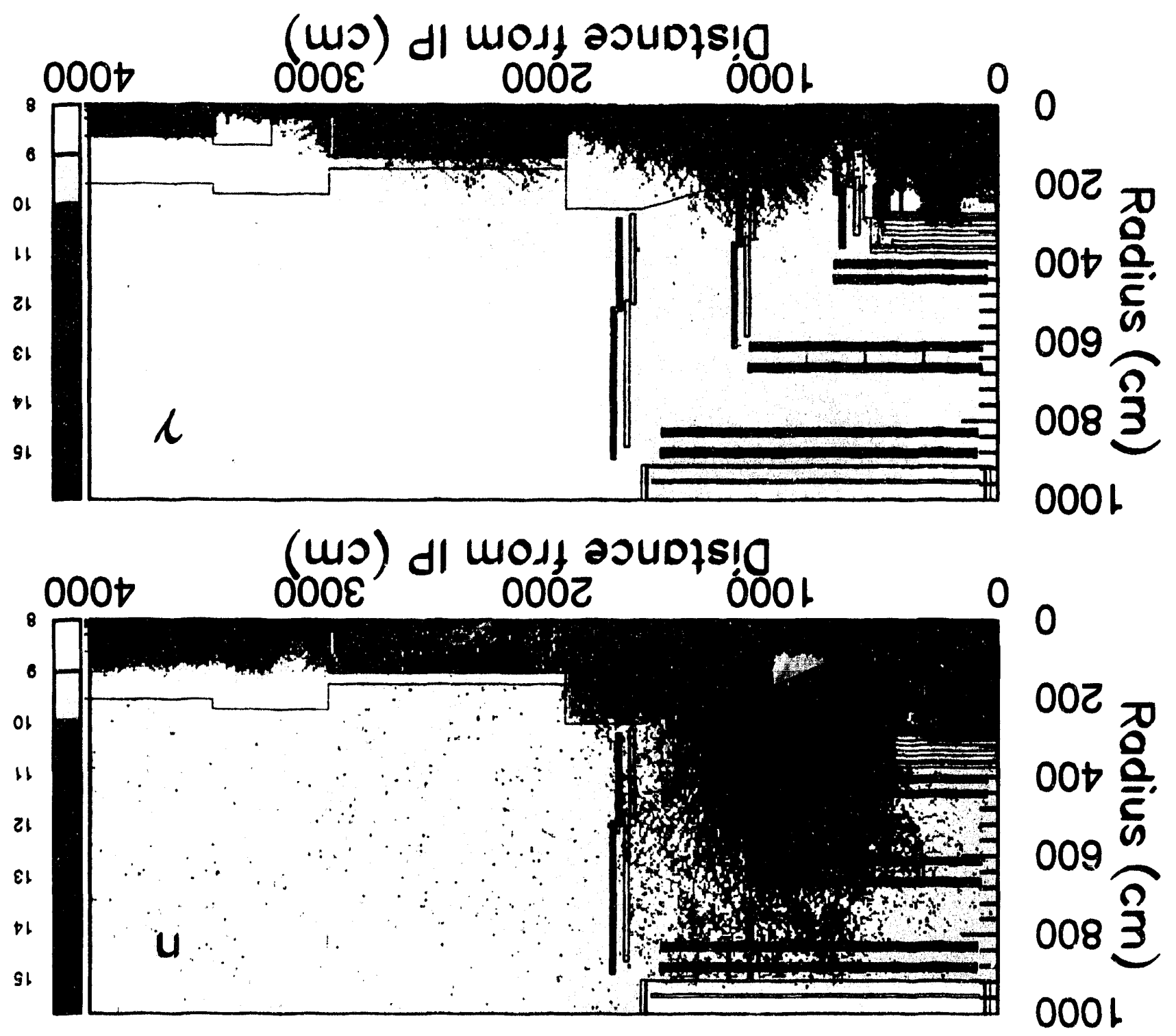



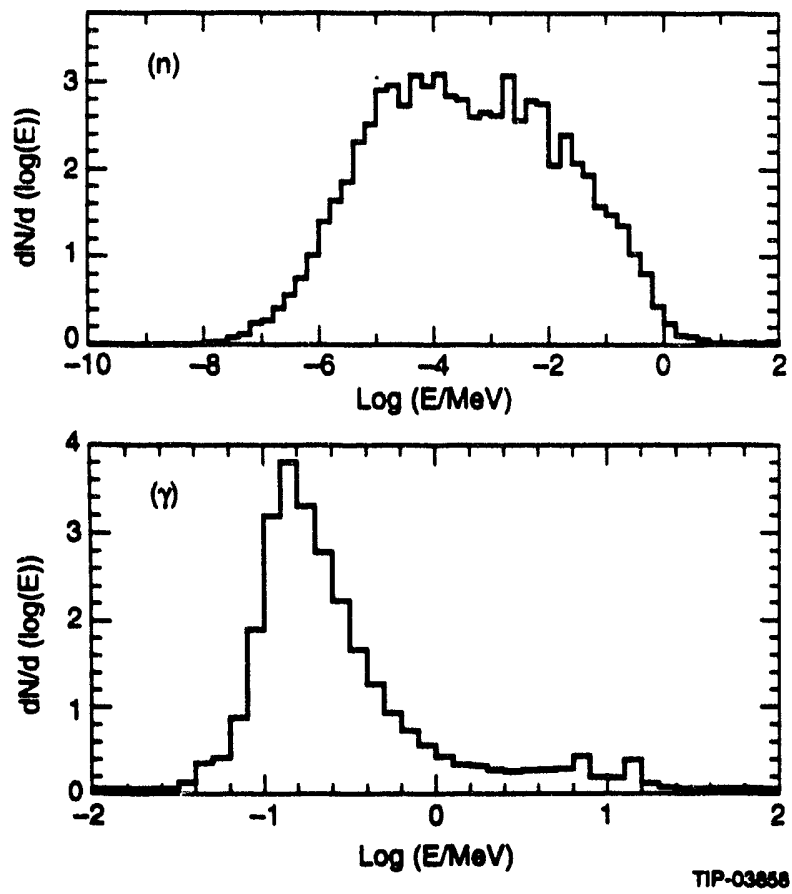

FIG. 12-11. The spectra of neutrons $(n)$ and photons $(y)$ in the barrel muon system of the shielded detector calculated using the GEANT-CALOR package. The normalization is arbitrary in these plots.

However, the reaction $\mathrm{B}^{10}(\mathrm{n}, \alpha) \mathrm{Li}^{7}$ and subsequent decay of $\mathrm{Li}^{7}$ through photon emission $(0.477 \mathrm{MeV}$ ) can increase the photon flux in the central detector cavity. Therefore, we have performed calculations of neutron and photon fluxes for different amounts of borated polyethylene to understand the relative sizes of these effects.

The calculations were performed using the LAHET-MCNP code system with no magnetic field. Omission of the GEM magnetic field from the simulation introduces a negligible error to our calculations. ${ }^{9}$ Figures $12-12$ and 12-13 show the neutron and photon fluxes along surfaces bordering various central tracker components. Neutrons above $100 \mathrm{keV}$ kinetic energy are far more damaging to silicon than lower energy neutrons, due to the sudden rise in the displacement cross section. ${ }^{9,19}$ Therefore, we display neutron fluxes above and below this threshold. The different curves correspond to different configurations of the bocated polyethylene. The plots show that the neutron flux is relatively constant throughout the central tracker volume. It increases by a factor of approximately 1.5 in the forward regions near the endcap calorimeter due to the increased flux and energy of the primary particles striking this region. Furthermore, the borated polyethylene reduces the flux by a factor of 2 to 5 , depending on location. It reduces the flux both by decreasing the rate at which neutrons are injected in the central detector cavity, and by reducing the number of reflections. Increasing the borated polyethylene thickness does not always produce a proportionate reduction in the fluence. ${ }^{18}$ We believe that this is so because the polyethylene has a smaller effect on the high energy component of the albedo, and also because the polyethylene itself starts contributing to the albedo. The shielding configuration that we have chosen is shown in Figure 12-1b. We have designed the polyethylene in front of the endcap calorimeter to be thinner at larger angles so that it has as little effect on the overall electromagnetic resolution as possible. The average neutron flux in the central cavity is calculated to be $4 \times 10^{12} \mathrm{n} / \mathrm{cm}^{2} / \mathrm{SSCY}$; approximately half of these neutrons will be above $100 \mathrm{keV}$. The energy spectra for neutrons and photons are shown in Figure 12-14.

Now we consider the photon flux for the central detector cavity in more detail. Figure 12-13 shows the photon fluxes along the same surfaces for the different configurations of the polyethylene. There are two sources of photons in the central tracker: 1) albedo photons from electromagnetic showers and 2) photons from low energy neutron capture reactions in the material of the central detector cavity. The Monte Carlo calculation described in the previous paragraph simulates only photons from $(n, \gamma)$ reactions. The flux of electromagnetic albedo photons is approximately the same as the neutron flux ${ }^{9}$ without the borated polyethylene shield. The borated polyethylene shield reduces the neutron flux by an order of magnitude, but the additional photons resulting from neutron capture on boron increase the overall photon flux by a factor of two when added to the electromagnetic albedo. The average total photon flux in the center cavity with the borated polyethylene shield will be about $6 \times 10^{12}$ photons $/ \mathrm{cm}^{2} /$ SSCY.

The fluxes in the central tracker have the same uncertainties as the fluxes in the muon system discussed in the previous section. We have found that the GEANT-CALOR calculation gives neutron fluxes that are approximately a factor of 3 lower than the LAHET-MCNP calculation. The neutron spectrum in the central cavity from GEANT-CALOR is 
(a)

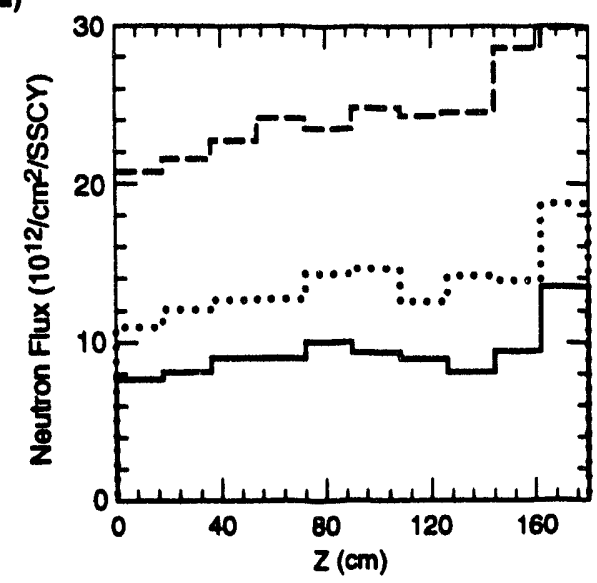

(c)

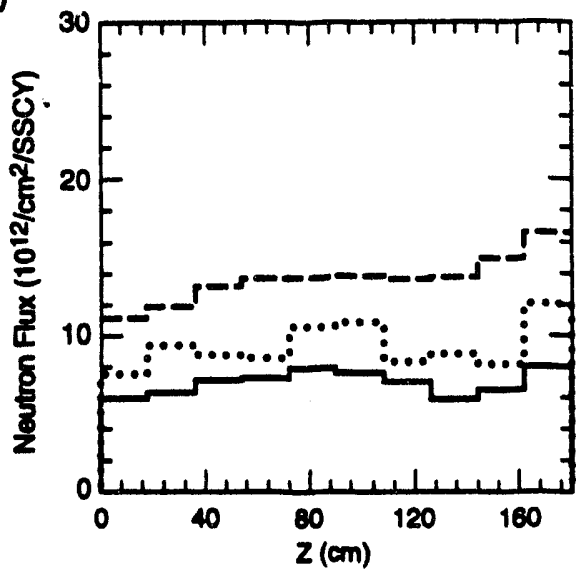

(b)

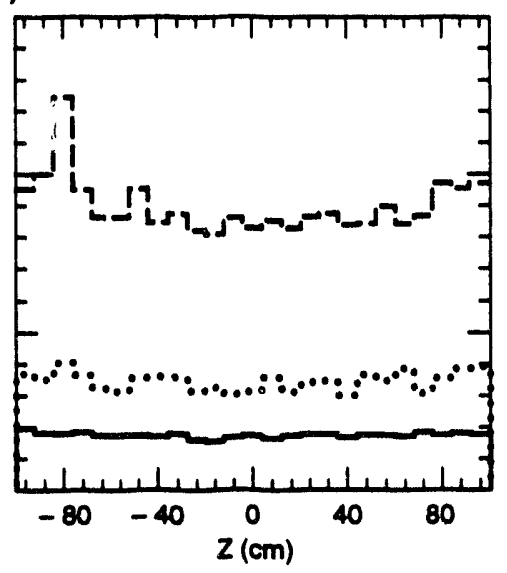

(d)

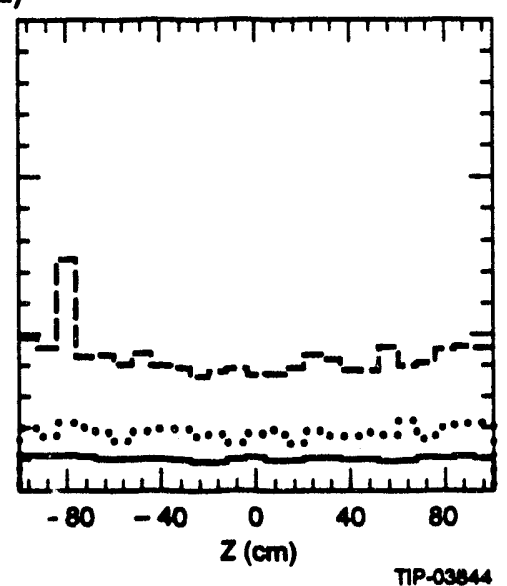

FIG. 12-12. Flux of neutrons in units of $10^{12}$ neutrons $/ \mathrm{cm}^{2} / S S C Y$. a) Flux at a radius of $10 \mathrm{~cm}$ as a function of distance from the IP for all energies. b) Flux at a radius of $/ 4 \mathrm{~cm}$ as a function of distance from the IP for all energies. c) and d) are at the same radius as a) and b) for neutrons with E $>100 \mathrm{keV}$, rispectively. The solid lines represent the borated polyethylene shielding shown in Figure 12-1b. The dotted lines indicate $5 \mathrm{~cm}$-thick borated polyethylene around the barrel and in front of the endcap. The dashed lines represent no borated polyethylene shielding.

also somewhat softer. We have chosen to use the higher flux numbers from LAHET-MCNP in the interest of being conservative. We are investigating the source $\cdot$ this discrepancy in more detail.

\subsection{TOTAL RADIATION DOSE}

The detector components that will receive the highest radiation doses are located in the central detector cavity. These are: the silicon tracker and associated electronics, the IPC tracker and associated electronics, insulation of the cryostats for the electromagnetic calorimetry, the NEG vacuum pump, cables in the central cavity, and the beam pipe. From past experience at colliders, it is known that these systems can experience large instantaneous dose rates during unstable or unusual condi- tions in the accelerator. However, the parameters of the SSC are such that the majority of the integrated doses at interaction regions will result during normal operations. Therefore we have calculated the dose rates in different parts of the detector for standard SSC luminosity ( $10^{15}$ interactions/SSCY).

The radiation dose for each system can be divided into four categories: 1) dose due to ionization energy loss of charged particles from the interaction point, 2) dose due to electromagnetic showers initiated by electrons and photons from the interaction point, 3) dose due to hadronic showers initiated by hadrons from the interaction point, and 4) dose due to low energy neutrons resulting from of the hadronic cascades. In general, the first category, 
(a)

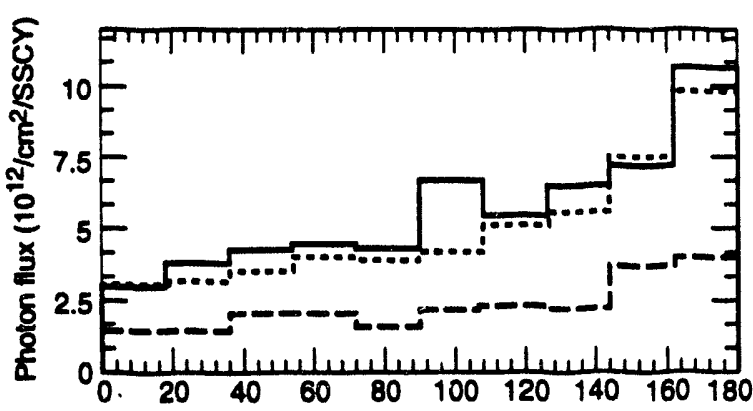

(b)

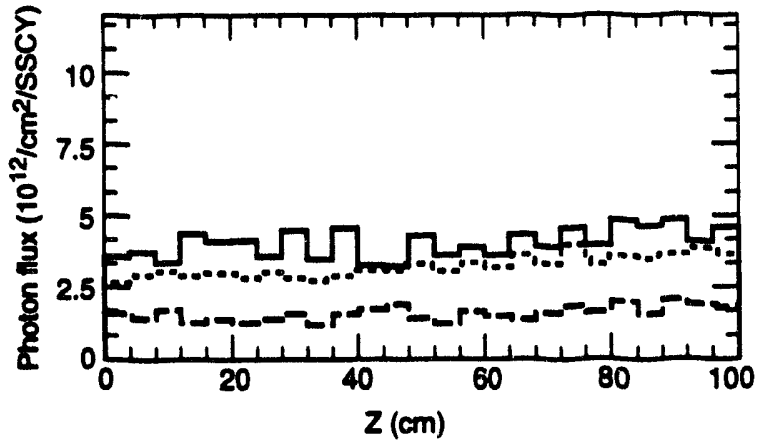

FIG. 12-13. Flux of photons from $(n, y)$ reactions in units of $10^{12}$ photons $/ \mathrm{cm}^{2} / \mathrm{SSCY}$ for two surfaces and for photons above $100 \mathrm{keV}$. a) Flux at a radius of $10 \mathrm{~cm}$ as a function of distance from the IP. b) Flux at a radius of $44 \mathrm{~cm}$ as a function of distance from the IP. The solid lines represent the borated polyethylene shielding shown in Fig. 12-1b. The dotted lines are for $5 \mathrm{~cm}$ thick borated polyethylene around the barrel and in front of the endcap. The dashed lines are for no borated polyethylene shielding.

the $\mathrm{dE} / \mathrm{dx}$ loss, is dominant for thin objects such as the silicon detectors in the central tracker. Electromagnetic and hadronic showers contribute the majority of the dose to thick calorimeters. The maximum dose in calorimeters is dominated by electromagnetic showers simply because radiation lengths are much shorter than nuclear interaction lengths. We consider the dose due to low energy neutrons separately because the mechanism for energy deposition and damage-interactions on nuclei-is fundamentally different from ionization energy loss, which is the main mechanism in the first three cases.

\subsubsection{Charged Particle Dose}

We have calculated the $\mathrm{dE} / \mathrm{dx}$ dose rate to the silicon tracker due to charged particles from the interaction point by using the $\mathrm{dN} / \mathrm{d} \eta$ distribution. We have also taken into account secondary interactions,
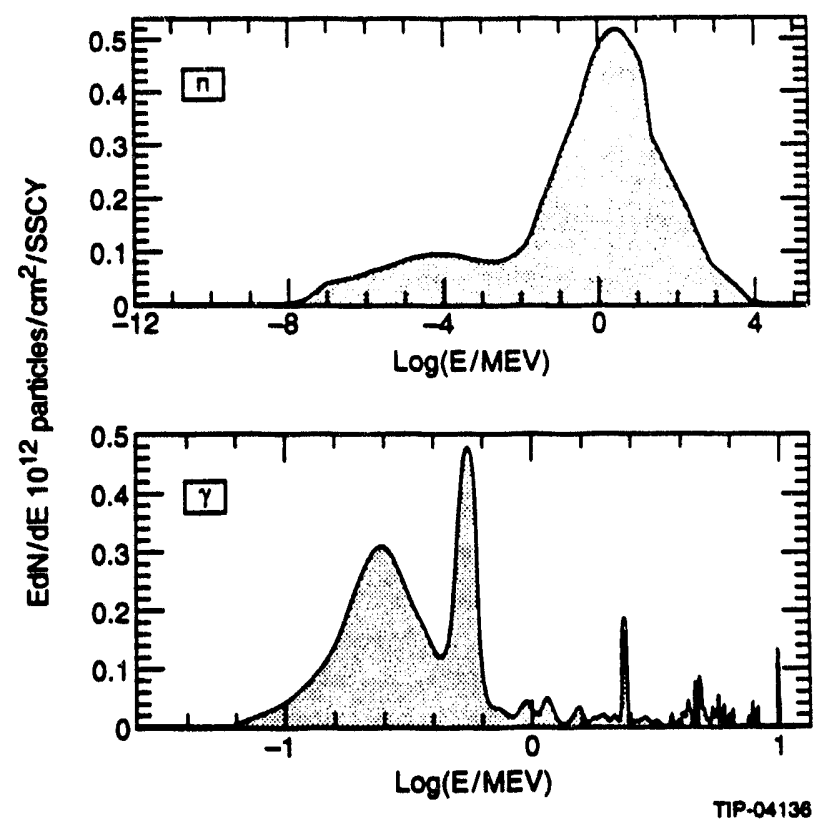

FIG. 12-14. Neutron and photon energy spectra in the central tracker volume. These spectra were obtained at the innermost silicon layer at a radius of $10 \mathrm{~cm}$. The neutron spectrum incl ' Ides high energy neutrons produced in the pp collisions. The photon spectrum only includes photons from $(n, y)$ reactions. The peaks at $500 \mathrm{keV}$ and $2.2 \mathrm{MeV}$ are from captures on boron and hydrogen, respectively. The width of the peaks is mainly due to binning.

gamma conversions, and low energy charged particles, which spiral tightly in the magnetic field. We follow the calculation in Reference 9 with a few modifications relevant to the GEM tracker. The flux of charged particles at a radius $R$ from the beam line can be written as:

$$
\text { Charged Particle Flux }=\frac{K L \sigma}{2 \pi \mathrm{R}^{2}} \frac{\mathrm{dN}}{\mathrm{d} \eta} \text { particles } / \mathrm{cm}^{2}
$$

where $L$ is the integrated luminosity, $\sigma$ is the total $p p$ cross section, $d N / d \eta$ is the number of primary charged particles per unit of pseudorapidity per interaction, and $K$ is the factor by which the flux is increased due to secondary interactions, loopers, and $\gamma$ conversions in the material of the tracker. According to Monte Carlo simulations of the GEM central tracker, $K$ is estimated to be approximately 1.5. For one standard year of SSC running at $L=10^{33} \mathrm{~cm}^{-2} \mathrm{~s}^{-1}$ and a total $p p$ cross-section of $\sigma=10^{-25} \mathrm{~cm}^{2}$, with the expected value of $\mathrm{dN} / \mathrm{d} \eta \sim 7.5$ charged particles/unit rapidity/ event, we calculate at $R=10 \mathrm{~cm}$ from the beam line a flux of charged particles of $1.8 \times 10^{13}$ particles/ 
$\mathrm{cm}^{2} / \mathrm{SSCY}$. We can then write the radiation dose corresponding to a flux of charged particles as:

$$
\operatorname{Dose}(\mathrm{Mrad})=\frac{\text { charged particles/cm }}{2} \times \frac{\mathrm{dE}}{\mathrm{dx}}
$$

where $\mathrm{dE} / \mathrm{dx}$ is the energy loss of charged particles in a given material in units of $\mathrm{MeV} /\left(\mathrm{gm} / \mathrm{cm}^{2}\right)$. We use $\mathrm{dE} / \mathrm{dx}=1.66 \mathrm{MeV} /\left(\mathrm{gm} / \mathrm{cm}^{2}\right)$ for silicon as a representative value for all momenta. The radiation dose for the silicon detector $10 \mathrm{~cm}$ away from the interaction point is $0.5 \mathrm{Mrad} / \mathrm{SSCY}$. At a tolerable lifetime dose of $5 \mathrm{Mrad}$ for silicon, this corresponds to a 10 year lifetime for the inner layer of silicon detectors at a radius of $10 \mathrm{~cm}$ and a luminosity of $10^{33} \mathrm{~cm}^{-2} \mathrm{~s}^{-1}$. In the vicinity of the IPC outer detector, at $40 \mathrm{~cm}$, the radiation dose is $30 \mathrm{krad} /$ SSCY. We are designing the IPC electronics to tolerate a lifetime dose of $3 \mathrm{Mrad}$, which corresponds to a lifetime of 10 years at a luminosity of $10^{34} \mathrm{~cm}^{-2} \mathrm{~s}^{-1}$. The dose rate in the tracker is plotted in Figure 12-15. Chapter 6 discusses the consequences of these dose rates for the silicon in more detail.

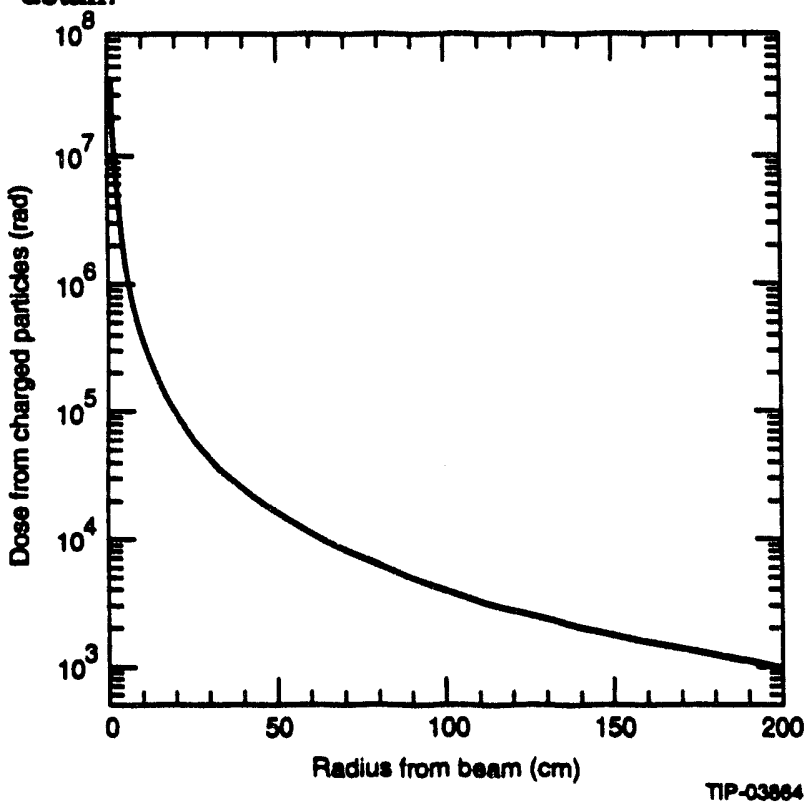

FIG. 12-15. Dose to a silicon device as a function of radius in the central cavity due to ionization energy loss of charged particles from the interaction point for one year of SSC operation at standard luminosity.

\subsubsection{Dose Due to Electromagnetic and Hadronic Showers}

We have used the parameterization of electromagnetic and hadronic showers from References 20 and 21 to compute the total dose in the calorimeter. The maximum dose occurs in the forward calorimeter. Figure $12-16$ is a plot of the total dose in the forward calorimeter, including both electromagnetic and hadronic shower contributions for one year of operation at standard luminosity. This dose is plotted as a function of radius from the beam line and distance from the interaction point along the beam line. The maximum dose of $400 \mathrm{Mrad}$ at the inner corner of the forward hadronic calorimeter is dominated by electromagnetic showers initiated in the electromagnetic section. The hadronic dose is distributed over a much larger volume than the electromagnetic dose as expected from the ratio of interaction length to radiation length. The maximum hadronic dose of $10 \mathrm{Mrad}$ is deposited in the forward hadronic calorimeter. These numbers agree well with considerations in Reference 9.

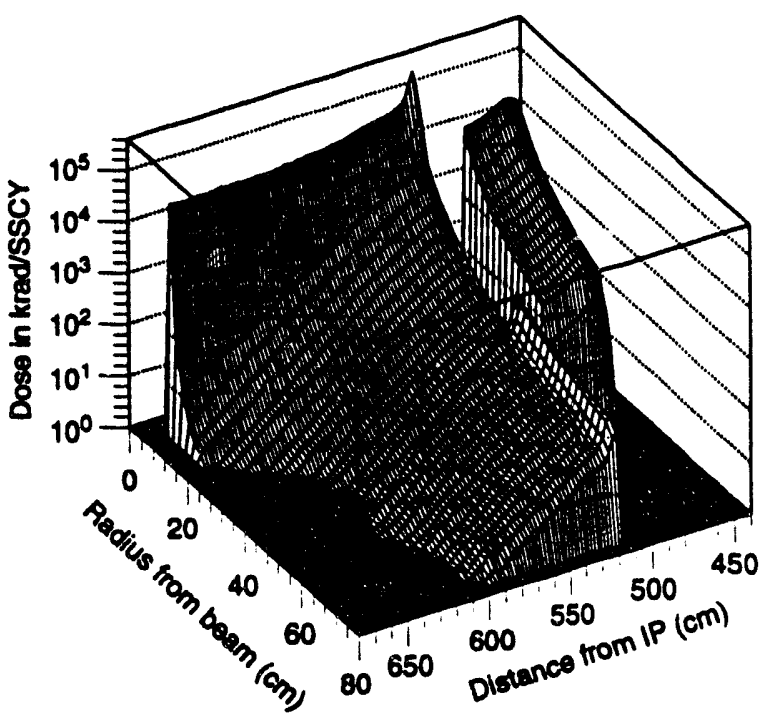

FIG. 12-16. Total dose due to electromagnetic and hadronic showers in the forward calorimeter for one year of SSC operation at standard luminosity.

\subsubsection{Dose Due to Neutrons}

The damage from low energy neutrons can be divided in two parts: 1) damage due to ionization dose from emitted charged particles, recoiling nuclei, internal conversion, and radioactive decay; and 2) damage due to the displacement of atoms from their regular positions in a crystal lattice.

The ionization dose from neutron interactions can be estimated by folding the energy spectra of Figure 12-14 with tabulated heating factors (KERMA factors are another way of tabulating this 
information. $)^{16,22}$ In particular, for the silicon in the central cavity we estimate a dose rate of approximately $1 \mathrm{krad} / \mathrm{SSCY}$, which is negligible compared to the ionization dose due to charged particles from the interaction point. Organic insulation on cables has a large heating factor because of the presence of hydrogen. We estimate a maximum dose rate of $0.1 \mathrm{Mrad} / S S C Y$ from neutrons behind the forward calorimeter for polyethylene insulation, corresponding to a lifetime of 100 years.

Damage to silicon due to displacement of atoms can be judged from existing data. ${ }^{19}$ If neutrons transfer a large amount of energy in a primary collision, the recoil $\mathrm{Si}$ itself continues to cause cisplacements. The result is a cluster of defects or a disordered region in the silicon crystal. To cause significant recoil damage, a neutron has to have an energy in excess of about $100 \mathrm{keV}$. It has bien established that silicon detector performance degrades after exposure to more than $10^{14}$ neutrons/ $\mathrm{cm}^{2}$. Using this as the lifetime exposure, we obtain a lifetime greater than 10 years (from neutrons only) at standard luminosity for the inner most silicon layer in the central cavity.

We summarize the results of the study of total dose in Table 12-6. For design specifications of various detector components, we show the maximum doses from the different contributions for each detector system. For neutrons we list the maximum flux in the systems.

\subsection{ACTIVATION}

Residual activity levels in the detector must be considered both for personnel safety during access, and for computing the accidental hit rates in the sensitive detectors. In the following, we identify items in the detector that will be sufficiently activated to require special handling. We also show that the hit rates due to residual activity are negligibly low while data is being taken.

Radioactive nuclei in the detector are produced by several processes: spallation, nuclear breakup in high energy hadronic interactions and neutron scattering, and absorption of thermal neutrons. We expect the first two processes to dominate inside the calorimeters, because neutrons produced in hadronic showers in our calorimeters will be moderately energetic with small cross sections for absorption. Activation due to thermal neutron absorption will dominate in the muon system. We also note that the dose rates in the vicinity of thick extended objects are expected to be mainly from gamma radiation, while dose rates near thin objects such as the beam pipe and the muon chambers may have a significant contribution from beta emitting isotopes.

\subsubsection{CINDER Calculation of Activation}

We have performed a calculation of the activation of components in the GEM detector using the LAHET/MCNP code system in combination with the CINDER (Version C93A2) code. The LAHET code is used to predict the spectrum of residual nuclei from primary pp interactions. In addition, neutron fluences under $20 \mathrm{MeV}$ computed by MCNP are used by CINDER to recreate the residual nuclei spectrum from low energy neutron interactions. The subsequent decay of these products is followed with the CINDER code. MCNP further transports the photons through the detector materials. The output of the entire program consists of a

Table 12-6. Maximum dose and neutron flux expected in detector systems for one year of SSC operation at standard luminosity. The maximum noutron flux is as much as four times higher than the average.

\begin{tabular}{|c|c|c|c|c|}
\hline System & $\begin{array}{l}\text { dE/dx dose } \\
\text { (Mrad) }\end{array}$ & $\begin{array}{c}\text { EM and Had dose } \\
\text { (Mrad) }\end{array}$ & $\begin{array}{c}\text { n fluence } \\
\text { ( } \mathrm{cm}^{2} / \mathrm{SSCY} \text { ) }\end{array}$ & $\begin{array}{c}n \text { fluence for } \\
\text { E100 keV } \\
\text { (n/cm } 2 / \mathrm{SSCY} \text { ) }\end{array}$ \\
\hline Central tracker Si & 0.5 & - & $8 \times 10^{12}$ & $6 \times 10^{12}$ \\
\hline Central tracker IPC & 0.030 & - & $4 \times 10^{12}$ & $2 \times 10^{12}$ \\
\hline Barrel cajorimeter & - & 0.1 & $5 \times 10^{12}$ & - \\
\hline Endcap calorimeter & - & 5.0 & $5 \times 10^{14}$ & - \\
\hline Fonward EM calorimeter & - & 400 & $10^{1 k}$ & - \\
\hline Fonward hadronic & - & 400 & $10^{15}$ & $\rightarrow$ \\
\hline Muon chambers & - & - & $6 \times 10^{10}$ & - \\
\hline
\end{tabular}


nuclide inventory, activity density, photon spectrum, and equivalent dose rates in $\mathrm{rem} / \mathrm{h}$ at any given location in the detector as a function of time after irradiation. The full calculation, which is very extensive, is being made available as a GEM technical note. Table 12-7 shows the maximum expected dose rates (preliminary results), on contact, with no cooling time, in different detector systems after one year of SSC operation at standard luminosity. The calculation follows the decrease of dose rates for six months after the beam is turned off. Figure 12-17 shows the typical time dependence of the activity in the detector. Activity drops by a factor of 2 within a day after beam turn off at $10^{7} \mathrm{~s}$.

The above calculation was checked with the simple model of activation by Thomas and Stevenson, ${ }^{23}$ which converts the density of inelastic hadron interactions (stars) into equivalent dose rates. The dose rate in rem/h at saturation near a semi-infinite thick slab is given by $w \times\left(\right.$ stars $\left./ \mathrm{cm}^{3} / \mathrm{s}\right)$, where $w$ is a material dependent factor. For iron and most heavy metals $w=1.0 \times 10^{-6} \mathrm{~cm}^{3} \mathrm{~s} \mathrm{rem} / \mathrm{h}$. The last column in Table 12-7 shows the results of this calculation for some locations inside the calorimeter. Considering all the errors associated with these calculations the parameterization compares favorably with the CDNDER calculation. Both sets of numbers have been scaled to be at the same location and time.

\subsubsection{Activation Due to Low Energy Neutrons}

In this section, we consider the residual dose rates caused by thermal neutrons. The calculation using star density does not account for this contribu- tion. The CINDER calculation does. The cross sections for activation by neutron absorption are largest for thermal neutrons; therefore, we pessimistically assume a thermal neutron flux of $10^{4} \mathrm{n} / \mathrm{cm}^{2} / \mathrm{s}$ every where outside the calorimeter, and $10^{5} \mathrm{n} / \mathrm{cm}^{2} / \mathrm{s}$ inside the calorimeter. In Table 12-8, we have reproduced a list of isotopes ${ }^{11}$ with large cross sections for activation via thermal neutron absorption commonly present in high energy experiments. We have added some isotopes of krypton to this list, since we plan to use it in the barrel calorimeter. Using these cross sections and the thickness of

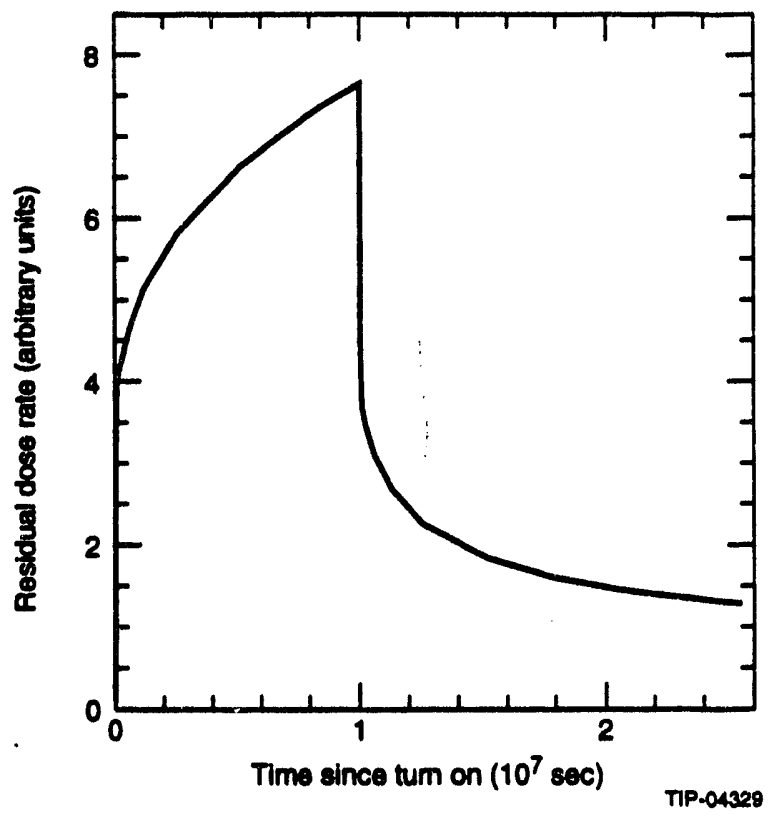

FIG. 12-17. Buildup and decay of residual dose rates in the GEM detector. The curve is normalized arbitrarily. We assume an irradiation time of $10^{7} \mathrm{~s}$.

Table 12-7. Maximum dose rates on contact for different regions of the detector from residual activity. The last column shows the results of a simple calculation based on the density of hadronic stars. The numbers are for one SSCY at standard luminosity. Activation in the muon system is mostly thermal neutron induced.

\begin{tabular}{lcccc}
\hline System & $\begin{array}{c}\mathbf{r} \\
(\mathrm{m})\end{array}$ & $\begin{array}{c}2 \\
(\mathrm{~m})\end{array}$ & $\begin{array}{c}\text { CINDER } \\
(\text { rom/h) }\end{array}$ & $\begin{array}{c}\text { STAR DENSITY } \\
(\mathrm{rem} / \mathrm{h})\end{array}$ \\
\hline Barrel & 1.0 & 0.0 & - & 0.01 \\
Endcap & 0.4 & 2.2 & - & 0.02 \\
EM forward & 0.06 & 4.5 & - & 1.1 \\
Hadronic fonward & 0.10 & 6.5 & 0.5 & 2.0 \\
Field shaper & 0.60 & 10.0 & - & 0.02 \\
Pump & 0.10 & 9.0 & - & 0.04 \\
Collimator & 0.0 & 32.1 & 50.0 & 70.0 \\
\hline
\end{tabular}


copper in the muon chambers $(0.0034 \mathrm{~cm} /$ layer $)$, we estimate the occupancy in the largest muon chamber strips to be about $0.03 \%$, which is negligibly small compared to occupancy levels expected from other sources (see Chapter 4). Similarly, we have estimated the pileup rate in the liquid krypton calorimeter due to krypton activation. We have found that the pileup noise level is less than $0.13 \mathrm{MeV} /$ tower, which is negligible compared to the thermal noise level/tower of $20 \mathrm{MeV}$.

Table 12-8. List of isotopes with particularly high cross sections for activation via thermal neutron absorption. Most of the active isotopes have several decay modes, including photons from short lived metastable states.

\begin{tabular}{|c|c|c|c|c|}
\hline $\begin{array}{l}\text { Parent } \\
\text { lootope }\end{array}$ & $\begin{array}{l}\text { Natural } \\
\text { (\%) }\end{array}$ & $\begin{array}{l}\text { o } \\
\text { (Bams) }\end{array}$ & $\begin{array}{l}\text { Active } \\
\text { Isotope }\end{array}$ & Half Life \\
\hline $23_{\mathrm{Na}}$ & 100 & 0.53 & ${ }^{24} \mathrm{Na}$ & $15 h$ \\
\hline${ }^{40} \mathrm{Ar}$ & 99.6 & 0.61 & ${ }^{41} \mathrm{Ar}$ & $1.8 \mathrm{~h}$ \\
\hline${ }^{44} \mathrm{Ca}$ & 2.0 & 0.70 & ${ }^{45} \mathrm{Ca}$ & $165 \mathrm{~h}$ \\
\hline${ }^{50} \mathrm{Cr}$ & 4.3 & 17 & ${ }^{51} \mathrm{Cr}$ & $28 d$ \\
\hline${ }^{55} \mathrm{Mn}$ & 100 & 13 & ${ }^{56} 6_{\mathrm{Mn}}$ & $2.58 h$ \\
\hline${ }^{59} \mathrm{Co}$ & 100 & 37 & ${ }^{60} \mathrm{Co}$ & $5.3 y$ \\
\hline${ }^{63} \mathrm{Cu}$ & 69 & 4.5 & ${ }^{64} \mathrm{Cu}$ & $13 h$ \\
\hline $64 \mathrm{Zn}$ & 49 & 0.46 & ${ }^{65} \mathrm{Zn}_{\mathrm{n}}$ & $245 d$ \\
\hline $78_{\mathrm{Kr}}$ & 0.35 & 4.7 & ${ }^{79} 9_{\mathrm{Kr}}$ & $35 \mathrm{~h}$ \\
\hline $80 \mathrm{Kr}$ & 2.27 & 4.6 & ${ }^{81} \mathrm{Kr}$ & 138 \\
\hline $82 \mathrm{Kr}$ & 11.6 & 20 & $83_{\mathrm{Kr}}$ & $1.9 \mathrm{~h}$ \\
\hline${ }^{84} \mathrm{Kr}$ & 57 & 0.10 & ${ }^{85} \mathrm{Kr}$ & $10 y$ \\
\hline${ }^{121} \mathrm{Sb}$ & 57 & 6.1 & ${ }^{122} \mathrm{Sb}$ & $2.8 \mathrm{~d}$ \\
\hline${ }^{123} \mathrm{Sb}$ & 43 & 3.3 & ${ }^{124} \mathrm{Sb}$ & $60 d$ \\
\hline${ }^{133} \mathrm{C}_{8}$ & 100 & 31 & ${ }^{134} \mathrm{C}_{3}$ & $2.1 \mathrm{y}$ \\
\hline${ }^{151} \mathrm{Eu}$ & 48 & 8700 & ${ }^{152} \mathrm{Eu}$ & $12 y$ \\
\hline${ }^{153} \mathrm{Eu}$ & 52 & 320 & ${ }^{154} \mathrm{Eu}$ & $8 y$ \\
\hline $186_{W}$ & 28 & 40 & $187 w$ & $1 d$ \\
\hline
\end{tabular}

\subsection{SUMMARY}

We have designed a shielding configuration for the GEM detector that reduces the neutron fluxes in most of the muon system to the range of 1-5 $x$ $10^{10} \mathrm{n} / \mathrm{cm}^{2} / \mathrm{SSCY}$ at the standard luminosity of $10^{33} \mathrm{~cm}^{-2} \mathrm{~s}^{-1}$. The photon flux above $0.1 \mathrm{MeV}$ is approximately $30 \%$ of the neutron flux. The muon chambers surrounding the forward hadronic calorimeter are expected to receive neutron fluxes that are approximately twice as iarge. We are confident that with further optimization of the forward calorimeter geometry, and with additional concrete to shield against the collimator albedo, we will reduce the neutron fluences even more. Because the GEM forward calorimeter is compact, and fits in the endcap calorimeter without leaving large gaps, it provides a natural, effective shield for the muon system. Our current results have been used to determine that the occupancy levels in the muon system are tolerable even at the high luminosity of $10^{34} \mathrm{~cm}^{-2} \mathrm{~s}^{-1}$. Further discussion of muon chamber operation is presented in Chapter 4.

We have also designed the shielding surrounding the central tracker, and estimated the neutron flux with this shielding to be about $4 \times 10^{12} \mathrm{n} / \mathrm{cm}^{2} / \mathrm{s}$ at standard luminosity. The photon flux is approximately twice as large above $0.1 \mathrm{MeV}$. This flux of neutrons and photons presents minimal rate difficulties for the silicon and IPC detectors in the central tracker. We expect the silicon tracker to perform without serious degradation for 10 years at standard luminosity $10^{33} \mathrm{~cm}^{-2} \mathrm{~s}^{-1}$; the limitation is caused mainly by the radiation dose in silicon due to charged particles. Further discussion of the central tracker operation can be found in Chapter 6.

We have quantified the total radiation doses to various systems in the detector. We have identified the forward calorimeter as the location of the maximum dose of about $400 \mathrm{Mrad}$ over one year of SSC operation at standard luminosity. We are designing this region with careful attention to the choice of materials with the expectation that the detector will eventually operate at 10 times higher luminosity.

We have quantified the residual activity and corresponding dose rates at various locations in the detector. Chapter 15 (Environmental Safety and Health) addresses the constraints imposed by these numbers. In particular, we have identified forward regions, such as the forward calorimeter and the vacuum pump stations, that require special handling for radiation safety after one year of SSC operation.

Finally, we have presented the results of a continuing study. We intend to optimize the geometry and shielding at the forward calorimeter to 
further reduce neutron and photon fluxes at the nearest muon chambers. We intend to optimize the shielding around the collimators to further reduce the weight and size. We are studying the mu on flux into the detector from beam losses in the low beta quadrupoles. Preliminary results indicate that this flux will not present rate difficulties. 


\section{REFERENCES}

1. J. Ranft, et al., in "Multiparticle Dynamics," Seewinkel, Austria, 1986. J. Ranft, Presentation in the Simulating Accelerator Radiation Environments Workshop, Santa Fe, New Mexico, Jan. 1993, to be published. See also J. Ranft, et al., UL-92-7 and UL YEP-93-01, Leipzig, Germany. We have used the February 1 , 1993 version of DTUJET.

2. N. Mokhov, "MARS12 code system," Presentation in the Simulating Accelerator Radiation Environments Workshop, Santa Fe, New Mexico, Jan. 1993, to be published. Also see N. Mokhov, MARS10 Code Manual, FERMILAB-FN-509, 1989.

3. "User Guide to LCS: The LAHET Code System," Richard E. Prael and Henry Lichtenstein, Los Alamos National Laboratory, LA-UR-89-3014, Sep. 1989. "MCNP: A General Monte Carlo Code for Neutron and Photon Transport," Judith F. Briesmeister, LA-7396-M Rev. 2, Sep. 1986. "Radiation Calculations using LAHET/MCNP/CINDER90," Proceedings of the III International Conference on Calorimetry in High Energy Physics, Corpus Christi, LA-UR-89-3014, Oct. 1992.

4. "CALOR: A Monte Carlo Program Package for the Design and Analysis of Calorimeter Systems," T. .A. Gabriel, J. D. Amburgey, B. L. Bishop, ORNL/TM-5619, Ajr. 1977. "User's Guide for the FLUNEV Code," J. M.. Zazula, Desy-internal-rep · D3-90-66, Jan. 1990. "FLUKA and KASPRO Hadronic Cascade Codes," J. Ranft, Erice 1978, Proceedings, Computer Techniques in Radiation Transport and Dosimetry.

5. "Hadronic Cascade Simulation in GEANT," K. Lassila, CERN-CN-91-13, Dec. 1991. "GEANT: Simulation Program for Particle Physics Experiments. Users Guide and Reference Manual," R. Brun, R. Hagelberg, M. Hansroul, J. C. Lassalle, CERN-DD/78/2 Rev. July 1978.

6. Y.. Fisyak, K. McFarlane, L. Roberts, GEM-TN-92-162, 1992.

7. C. Zeitnitz, T. A. Gabriel, "The GEANT-CALOR Interface," III Conference for Calorimetry in High Energy Physics, Proceedings (to be published), Corpus Christi 1992. C. Zeitnitz, T. A. Gabriel, "The GEANT-CALOR Interface," International Conference on Monte Carlo Simulation in High Energy and Nuclear Physics, Proceedings (to be published), Tallahassee, 1993. See also J. O. "nson, T. A. Gabriel, "Development and
Evaluation of a Monte Carlo Code System for Analysis of Ionization Chamber Responses," ORNL/TM-10196, July 1987.

8. Description of CINDER90: Los Alamos Code for Estimation of Radio Activation," R. Wilson, Presentation in the Simulating Acc lerator Radiation Environments Workshop, Santa Fe, New Mexico, Jan. 1993, to be published.

9. D. E. Groom, "Radiation Levels in the SSC Interaction Regions," SSC-SR-1033, June, 10, 1988. pp 3-14.

10. Radiological Safety Aspects of the Operation of Proton Accelerators," R. H. Thomas and G. R. Stevenson, IAEA technical reports series no. 283, 1988.

11. A Guide to Radiation and Radioactivity Levels Near High Energy Particle Accelerators," A. H. Sullivan, Nuclear Technology Publishing, 1992. See also "Induced Radioactivity," Marcel Barbier, North Holland, 1969.

12. I. Baishev, A. Drozhdin, and N. Mokhov, SSC-306, 1991.

13. N. Mokhov, Collider ARC PDRR, May 26-27, 1992.

14. J. Ranft, private communication.

15. M. Diwan. "Design of a Compact Beam Dump for the BNL-AGS. Neutral Beam," SSCL-Preprint-192, Presentation in the Simulating Accelerator Radiation Environment Workshop, Santa Fe, New Mexico, Jan. 1993, to be published.

16. D. I. Garber, R. R. Kinsey, Neutron Cross Sections," BNL-32\%, Brookhaven National Laboratory, January 1976. See also "A Nuclear Cross Section Data Handbook," Harl'o M. Fisher, LA-11711-M-Manual, Los Alamos National Laboratory, Dec. 1989.

17. Concrete Radiation Shielding: Nuclear Physics, Concrete Properties, Design and Construction," Wiley, New York, 1989. The concrete that we propose to use has a density of $3.2 \mathrm{gm} / \mathrm{cm}^{3}$; it contains, by weight, $40 \%$ barium, $35 \%$ oxygen, $9.6 \%$ sulfur, $8.5 \%$ calcium, $1.8 \%$ silicon, $1 \%$ iron, $1 \%$ boron, $0.85 \%$ hydrogen, and the rest are trace minerals.

18. David M. Lee, R. E. Prael, Laurie Waters, GE-72-TN-92-91, Apr. 1992.

19. "Radiation Effects at the SSC," Edited by M. G. D. Gilchriese, SSCL-SR-1035, Jun. 1988. "Radiation Damage Testing at the SSC: Task Force Report," W. Chinowsky, R. Thun, SSC-SR-1054, Jun. 1990.

20. F. Barreiro, et al., "Measurements of Longitudinal and Transverse Profiles for Hadron Showers in the Range $10-100 \mathrm{GeV}$ and 
Comparisons with Monte Carlo Simulations," NIM A292(1990) 259-278.

21. M. Della Negra, "Patteru Recognition in Calorimeters," Physica Scripta, Vol. 23, 469-479, 1981.

22. "Trends in Radiation Dosimetry," Caswell and Coyne, The International Joumal of Applied Radiation and Isotopes, Vol 33, No. 11, Nov. 1992.

23. G. R. Stevenson, CERN-TIS-RP-90-10-CF, August 1990. See also G. R. Stevenson, CERN-TIS-RP-90-18-CF, October 1990. 


\section{BEAM TESTING AND CALIBRATION}

\subsection{INTRODUCTION}

Particle beams will be used to test and calibrate all the GEM systems, including not only the various types of hardware modules but also electronics, DAQ, off-line computing, global control systems, and safety. The test running can be divided loosely into two phases: before and after MEB operations begin. The MEB test beams will be the first beams used by experimenters at the SSCL, and will provide a convenient test bed for almost all GEM-related technical issues, including safety.

The pre-MEB test beam phase largely involves prototype testing and debugging. The post-MEB phase will emphasize calibration and alignment determinations for systems that will subsequently be installed in the detector. There will be some overlap, both in schedule and function.

Test beams are now or will be available to GEM at Fermilab, Brookhaven, and LAMPF. CERN beams may also be available through collaboration with CERN groups. Beams in Russia have already been used to test muon chamber prototypes. The Fermilab beams cover a range of energies up to $800 \mathrm{GeV}$, and a wide range of particle types and fluxes. However, they will not be available until 1995 as currently scheduled. Brookhaven test beams are available in 1993 at $9 \mathrm{GeV}$, an energy which is adequate for many purposes.

The tracker group already has a silicon system test program underway at LAMPF. GEM collaborators currently participate in RD1, RD3, and RD5 at CERN. In addition, cosmic ray tests of muon chamber prototypes are underway at the Texas Test Rig at the SSCL Stoneridge site in Dallas. These tests, described in Chapter 4, have been instrumental in the muon technology development and selection process. World record spatial resolutions have been reported from these tests.

Beam testing and calibration will continue even after all of the detector components have been installed and running has begun. There will be detector upgrades to be developed and tested, and it is likely that detailed calibration of some components, such as calorimeter response in the neighborhood of cracks, will require very long running periods. Therefore, we are planning for long-term use of the SSCL calibration hall.

This chapter describes beam testing and calibration beginning in 1993. Results from work already performed are discussed in the appropriate subsystems chapters.

\subsection{GOALS OF BEAM TESTING AND CALIBRATION}

\subsubsection{Calorimeter Systems}

GEM calorimetry consists of liquid krypton (LKr) and liquid argon (LAr) modules backed up by scintillating tiles. Tests of accordion electromagnetic calorimeter modules are presently being conducted in collaboration with RD3 at CERN. The high energy beams available at Fermilab will be essential for understanding the constant term in the calorimeter resolution. The performance of the carorimeter in the neighborhood of cracks, including the complicated barrel-to-endcap transition region, will also be measured. Before the Fermilab beams are available some testing will be performed at Brookhaven.

Calorimeter simulation involves a large number of unknown effects, which must be assumed if they are not measured. For example, simulation of the jet energy resolution, however detailed, must be checked. Since a jet test beam is not feasible, the jet resolution must be approximated from single-track resolutions summed with appropriate weightings. Single-track resolutions at various energies can be measured in test beams, providing this critical calibration point for GEM simulations.

Calorimeter work in test beams involves full-scale systems tests, including mechanical, electrical, and cryogenic systems. It includes calori- 
meter readout and DAQ electronics, and on-line and off-line software. An important goal for the GEM calorimeter is measuring the erergy lost by high energy muons. For muons above about $100 \mathrm{GeV}$, radiative energy loss constitutes an increasingly important part of the muon's energy deposition. Above about $200 \mathrm{GeV}$ (deper $g$ on the material traversed) this component duininates. Radiative losses are subject to large fluctuations, and therefore a significant amount of energy may sometimes be deposited in the calorimeter. Tests with muon beams will determine the capability of the calorimeter to measure such effects.

A more detailed description of the goals of the calorimetry test program can be found in Chapter 5 .

\subsubsection{Muon System}

The muon system consists of cathode strip chambers arranged as described in Chapter 4 . Tests with the Texas Test Rig have verified a resolution of better than $75 \mu \mathrm{m}$ per plane for cosmic ray tracks with momenta greater than $1.4 \mathrm{GeV}$, and angles of incidence no more than 6 degrees from normal, exceeding the nominal GEM requirements. The chambers tested were designed for the barrel region, but were not full-sized.

The GEM muon group will experiment with different gases and voltages to determine the most satisfactory combination from the standpoints of resolution, safety, dead time, trigger speed, and cost. Furthermore, chambers designed for the endcap regions will have a different design, since they measure curvature in $\phi$, and therefore employ radial strips. These chambers will also be tested and an optimized design and optimal running conditions determined.

Relative alignment of the muon chambers must be known to $25 \mu \mathrm{m}$ in the transverse direction, a tremendous technical challenge that will require beam testing. The plan for achieving this tolerance is described in Chapter 4. The final design will require beam testing under simulated stress conditions.

Some testing can be done in the continuously operational Texas Test Rig. However, the muon systems require additional tests using accelerator beams. For example:
- The GEM muon chambers will operate in a magnetic field, where Lorentz angle effects may have substantial impact. Therefore, it will be necessary to test the muon chambers in various orientations in a $0.8-\mathrm{T}$ magnetic field.

- Muon radiation is the dominant source of energy loss above about $100 \mathrm{GeV} / \mathrm{c}$ momentum. Pattern recognition problems resulting from this radiation will have to be studied, preferably in a 0.8-T magnetic field.

- Hadron punch-through is also a problem for pattern recognition. This will be studied using a block of material simulating the calorimeter placed in front of representative muon chambers. The track curvature induced by the field has a significant effect on punch-through probability and on pattern recognition following punch-through. Therefore, it is necessary to do some of this testing in a 0.8-T magnetic field using a hadron beam of the highest available energy.

- Tests of full-scale prototypes and actual production modules, in the barrel and endcap configurations, will be needed. In the barrel configuration, for example, there will be three superlayers with 5-m separation between the first and last layers. In the endcap, the length will be greater. There will be tests of performance and alignment in high energy (to minimize multiple scattering) muon and hadron beams.

A full description of the muon system R\&D program is contained in Chapter 4.

\subsubsection{Central Tracker}

The GEM central tracker consists of silicon microstrip inner chambers and interpolating pad chambers (IPCs) at larger radius (Chapter 6). The program aims to develop and test full scale working prototypes by the end of 1994 . The program will proceed in parallel for the silicon strips and the IPCs through the early stages, culminating in an exposure of the completed tracker to an SSCL beam to calibrate its alignment just prior to installation.

Tests of the silicon microstrip tracker will be conducted with prototypes of the electronics to determine overall system performance. Full beam tests of detectors and electronics will begin in 1994; 
prior to that time, it will be possible to test early prototypes at Brookhaven in 1993.

The IPC tracker requires a more extensive program of R\&D. These tests will include determination of the intrinsic resolution of prototype IPCs, uniformity scans, and efficiency measurements in a high counting rate environment. These studies require particles with high enough energy that multiple scattering is small compared with the chamber resolution. Resolution studies will require low particle rates; efficiency studies will require up to $10^{9} \mathrm{~Hz}$ or higher (if available). One of the principal goals is to simulate the effects of the expected charged particle rates at luminosities of $10^{34} \mathrm{~cm}^{-2} \mathrm{~s}^{-1}$. IPC prototypes will be tested in 1993 , and possibly 1994, at BNL; full scale systems tests with final electronics will occur by 1995 at Fermilab.

The final test before installation will be of the completed tracker at the SSCL calibration hall. This will be a test to exercise the full array of electronics, cooling, gas supply, and mechanical support structures under data-taking conditions prior to installation into the detector, where it will be difficult to access once data-taking has begun. A high-resolution silicon telescope will probably be mounted in front of and behind the tracker. These tests will allow a precision determination of the locations of the tracker elements relative to each other and to external fiducials. This cannot be done using $x$ rays because of inadequate penetration power, and it cannot be done optically becalse the design of the tracker precludes clear optical paths for this purpose. Cosmic rays do not have adequate rates at energies high enough that multiple scattering may be neglected. Cosmic rays are also predominantly vertical, which precludes calibration of the alignment of the forward-backward disks. The tracker will be mounted in a cradle on a transporter that will move and rotate the entire tracker in order to scan the beam across every element.

A more detailed discussion of the full central tracker R\&D program is contained in Chapter 6.

\subsection{ACCELERATOR FACHLITIES}

\subsubsection{Brookhaven Test Beams}

BNL will make its beam line B2 available for detector testing. This beam is capable of delivering
$9 \mathrm{GeV}$ hadrons at rates up to $8 \times 10^{6}$ per spill. The central tracker group will expcse prototype IPC's to this beam to determine intrinsic resolution, uniformity, and efficiency at high counting rates. These tests will continue in 1994.

The forward calorimetry group will expose a prototype forward calorimeter system to tagged electrons and pions in this beam in 1993. Some muon testing will also be done with this calorimeter. The purposes will include tuning the charge calibration system and measuring the energy resolution as a function of the high voltage and other system parameters. The position resolution will also be measured. Though $9 \mathrm{GeV}$ is far from the momentum region of most interest to the forward calorimeter, these calibration points and systems tests will nevertheless provide an extremely useful shakedown of the entire system.

\subsubsection{Fermilab Test and Calibration Beams}

The highest energy beams in the world are those available at Fermilab, making this laboratory the most desirable location at which to test and calibrate SSC detector components (prior to the operation of SSCL's own pre-accelerator complex). GEM proposes to mount a comprehensive test beam program in the Meson West (MWEST) area at Fermilab. This is an ideal location from GEM's perspective. The experimental hall is adequately large $(60 \mathrm{~m} \times 20 \mathrm{~m})$ and spanned by a 25 ton crane with a hook height of approximately $9 \mathrm{~m}$. There is also a spacious external control room and working area adjacent to the MWEST experimental hall, as well as several smaller internal counting houses (see Figure 13-1). The area has extensive cryogenic facilities, full ODH (oxygen deficiency hazard) protection, including an inter-connected trench and deep pit for containing a massive cryogenic spill, and an existing rail system capable of supporting a calorimeter test cryostat and associated transporter. There are also a large number of existing tracking chambers (including silicon strip detectors, multiwire chambers, and straw tube drift chambers) plus associated readout electronics that can be reconfigured for test beam use, as well as a large aperture $\left(48 \times 36\right.$ in $\left.^{2}\right)$ analysis magnet for testing components of the GEM central tracker and muon system in a 0.8-T magnetic field. The existing MWEST 
beam is capable of transporting particles of either polarity, up to and including $800-\mathrm{GeV}$ primary protons.

A schematic layout of the MWEST hall as configured for GEM is shown in Figure 13-1. As indicated in the figure, each of the detector subsystems - the central tracker, calorimeter, and muon tracker - will be provided with its own dedicated area. The types of tests to be performed on each detector subsystem have already been described. In addition, other GEM systems - data acquisition, controls, on-line and off-line computing - will be developed in an evolutionary manner through the MWEST testing program. Negotiations between FNAL, SSCL, and GEM are in progress to specify in detail the full scope of the activities that will take place in MWEST, which is expected to remain assigned to GEM for the indefinite future.

\subsubsection{SSCL Test and Calibration Beams}

\section{Overview}

SSCL beams will be provided by the MEB, which has a maximum energy of $200 \mathrm{GeV}$. The primary protons will strike a target and secondary beams will be extracted at an angle of $5 \mathrm{mr}$. For safety reasons, it will not be possible to transport the primary beam to the calibration hall. The maximum secondary energy allowed by the bearn transport system will be $170 \mathrm{GeV}$. The beamline is shown in Figure 13-2.

The current design provides three secondary beams (Figure 13-3) to the calibration hall. Allowance is being made for the eventual implementation of an additional three beams from the MEB, plus three beams from the HEB, with maximum energy $2 \mathrm{TeV}$. However, the funding and the schedule for this upgrade are as yet uncertain. In addition, the HEB will not turn on until about a year before the collider. Therefore, we will not consider it further here, although GEM considers beams from the HEB to be important for its long-range calibration and R\&D program.

As may be seen in Figure 13-3, the westernmost beam will be dedicated to GEM, the middle beam to as-yet-undetermined smaller experiments, and the eastem-most beam to SDC. The beam pits

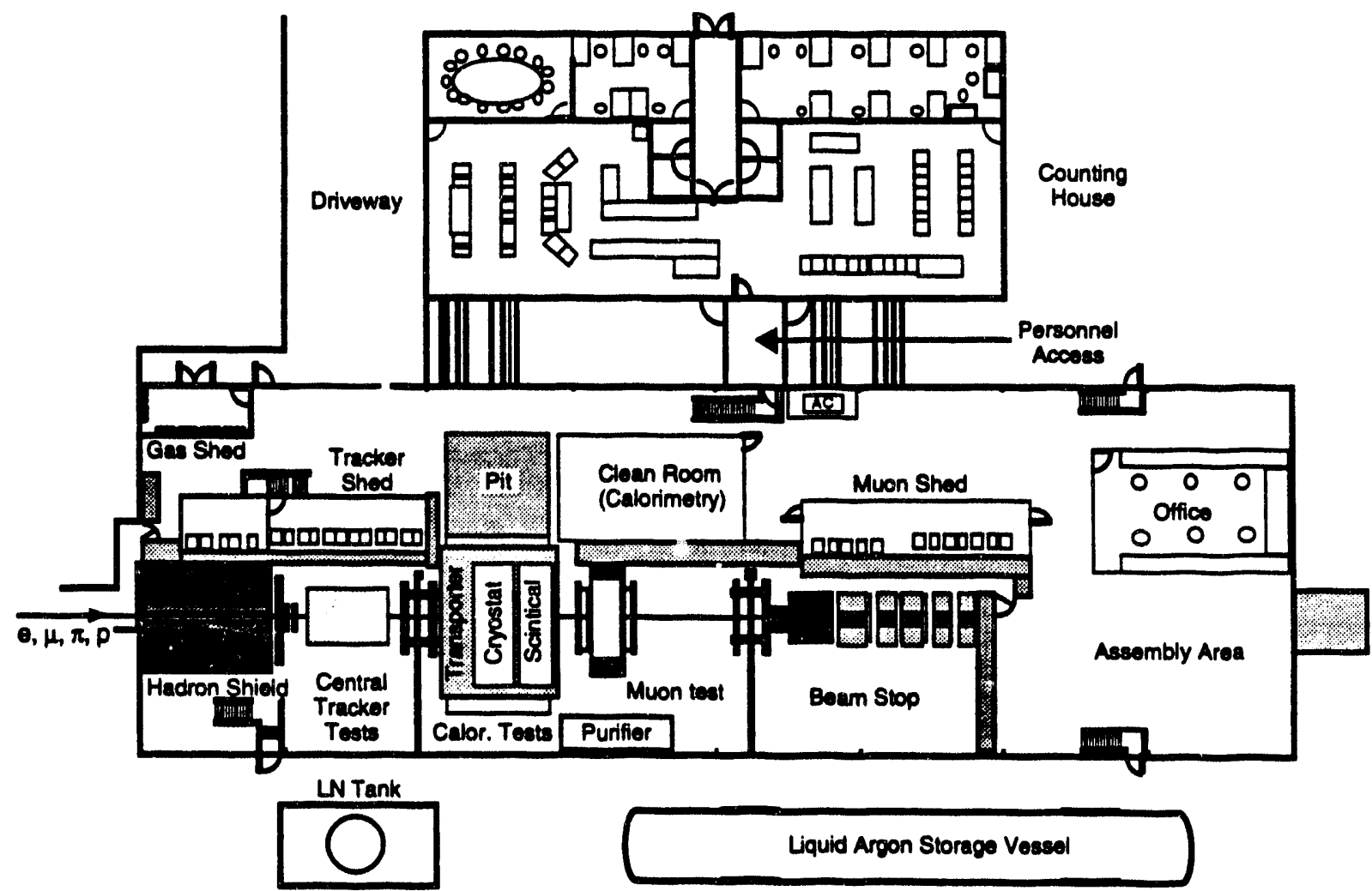

TIP-03840

FIG. 13-1. Schematic of the Fermilab MWEST area as configured for the GEM test beam program (not to scale). 


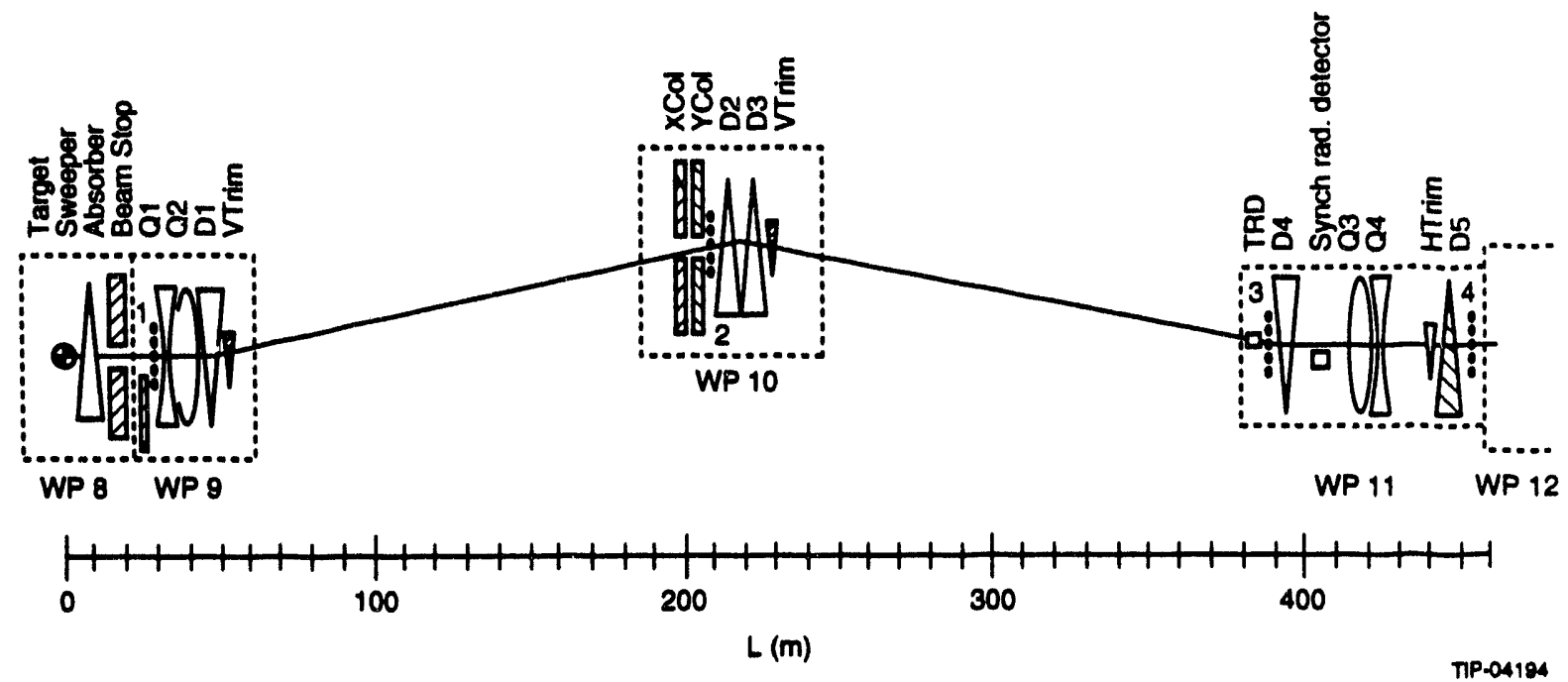

FIG. 13-2. SSCL test beam line optics showing the locations of the particle-tagging TRD and synchrotron radiation detectors. Working Point" 8 is the target hall where the extracted MEB proton beam creates the secondary beam to be transported to the calibration hall (WP12).

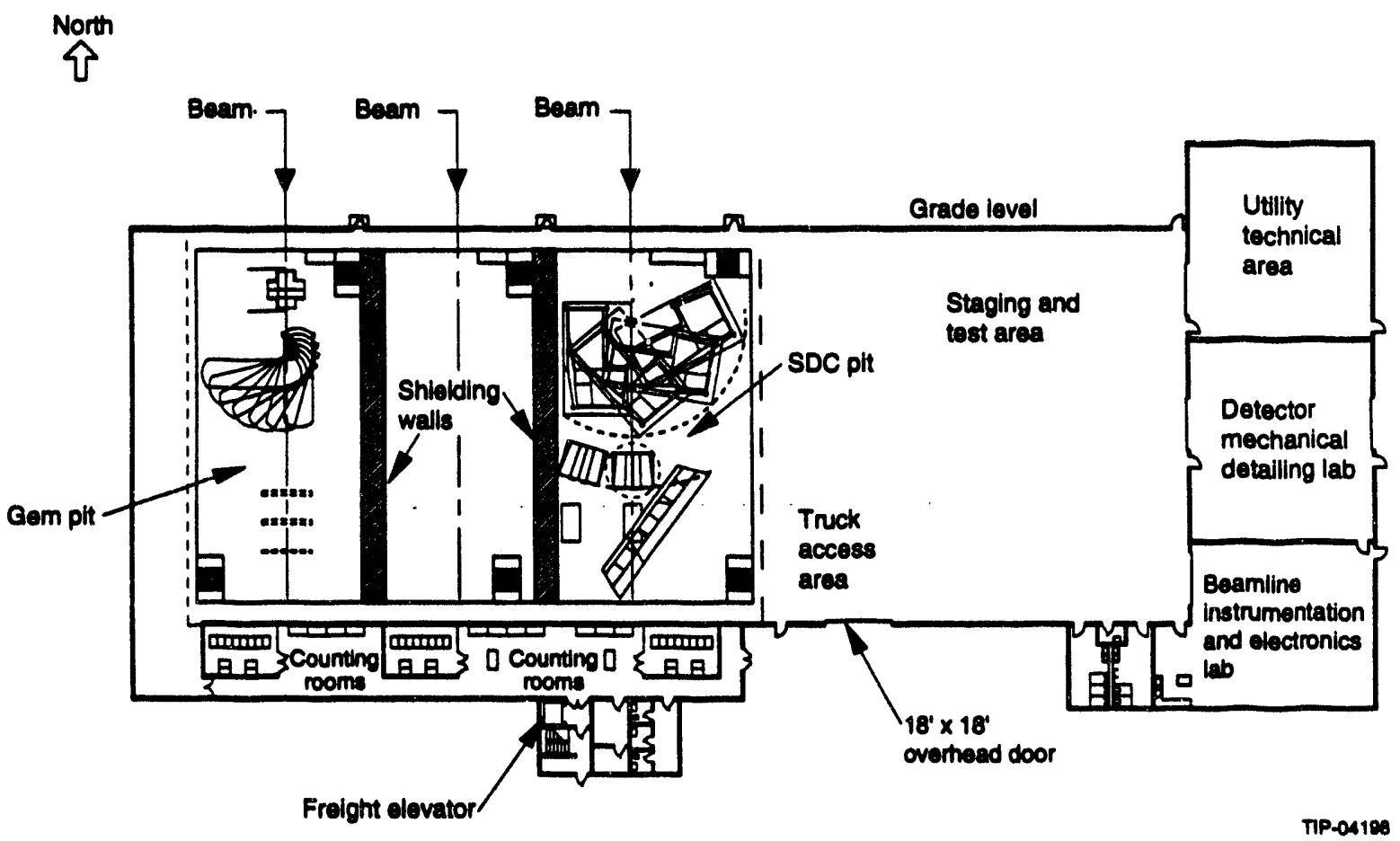

FIG. 13-3. SSCL calibration hall plan view, showing the three experimental pits and the grade-level assembly aread counting houses. The GEM pit is to the west, the SDC pit to the east, and the center pit is reserved for smaller experiments.

will be separated by 6-foot thick-shielding walls 32 feet in height. This will permit operation of any beamline while the others are occupied by workers. The entire pit area is surrounded by shielding walls such that all operations outside the pit may be conducted by people who are not radiation workers, and the general public outside the building will not be exposed to radiation.

A staging area at grade level will be shared by all experiments using beams. This area contains a truck access with a single $18^{\prime} \times 18^{\prime}$ roll-up door. 
During operation, the truck access area will be shielded from direct view of the beam spots by a concrete wall. To avoid impeding initial installation of equipment, this shielding wall, as well as the inter-pit shielding, will not be installed until shortly before first beam. All of these walls will be constructed of movable shielding blocks.

The stagin technical area, a beamline instrumentation lab, and a detector mechanical detailing area. These areas will be used for operations that are not conveniently done in the open assembly area.

The beam pits and assembly area will be covered by a single crane with a 50-ton primary hook and a 10-ton secondary hook. Access to the pits is also provided by a freight elevator capable of carrying moderate-sized loads. There are personnel exits at each end of each pit via staircases. Scheduling of crane time will require close cooperation among the users. GEM plans to begin installation of equipment in the calibration hall at the earliest possible date, and to schedule this installation so as to minimize interference between subsystems and with running time. The beneficial occupancy date for the calibration hall is presently January, 1995.

Beams of hadrons, electrons, and muons will be available at energies ranging from $2 \mathrm{GeV}$ to $170 \mathrm{GeV}$. An electron tagging system, described below, will be provided. The beam layout is shown in Figure 13-2. The maximum momentum bite will be $\Delta p / p=6 \%$. Space is available in the beamline for a tertiary beam, if that proves necessary for lowenergy pions, which otherwise may have large electron contamination. For medium energy pions, the electrons may be differentially removed through the use of a lead degrader, which will cause electrons to lose enough energy that they cannot be transported efficiently. This reduces (by as much as an order of magnitude) the pion flux due to multiple scattering out of the beam, but the loss can be compensated by increased primary current except at low energies where few pions are available even at maximum primary current. At high energies, the electron contamination is not significant and the tagging system will suffice to reduce the contamination to an acceptable level.

Calculations indicate that decay muons are not a serious problem, even at $2 \mathrm{GeV}$, although $98 \%$ of the pions will have decayed over the $450 \mathrm{~m}$ of beamline at that energy. Most such decay muons come from the first portion of the decay path, and do not have adequate energy to be transported (or to penetrate substantial amounts of steel or earth to reach the calibration hall via alternate routes). Furthermore, they have a significant decay angle at $2 \mathrm{GeV}$, and this also contributes to the loss of muons. The main effect of pion decay, therefore, is to lose pion flux. The flux required by GEM at $2 \mathrm{GeV}$ is not more than $100 \mathrm{~Hz}$. The pion and electron fluxes obtainable from the primary target at $2 \mathrm{GeV}$ are difficult to calculate; thus, whether or not a iertiary beam will be required is not known at the present time.

The MEB operates on an eight second cycle time at full energy, shorter at lower primary energy. The beam will have the same 16 ns pulse structure as the SSC main rings. The maximum spill duration will be one second. The beam will be shared among the three lines in a switched mode, in which the entire secondary beam will be directed sequentially down each line in turn for one full spill. Therefore, the worst case scenario for operation will be one pulse every 24 seconds to the GEM area. An alternate mode of running, the split mode (in which each line receives a portion of the beam each pulse), is precluded by cost considerations at the present time. However, it will be possible to convert to this mode at a later time.

In switched mode, the GEM beamline will be able to take three times as much flux for $1 / 3$ the spills, compared with split mode, if both other lines are running. Thus, if the flux to the module under test is limited only by the flux the machine can deliver (as at the highest and lowest energies), this mode takes no more shifts to reach a certain integrated flux than split mode. However, if the flux that can be accepted is limited only by the capacity of the module, then switched mode could take up to three times as many shifts. This is a source of concem to GEM, and we are requesting that more shifts per week be devoted to test beam running in order to compensate for this inefficiency to the greatest extent possible. It is anticipated that in the early running the principal limitation to running time will be due to competition from MEB commissioning, which will overlap with test beams running. In later 
running, cooling pond capacity will limit running to one or two shifts per day, especially on hot days.

The requirements for the SSCL be^ms are listed in Table 13-1. Particle momentum is tagged by a spectrometer (Figure 13-2), with a resolution that exceeds the calorimeter resolution (Figure 13-4) by a factor of two, permitting deconvolution of the true calorimeter resolution. It will be possible to distinguish the liquid krypton resolution $(5.5 \% / \sqrt{\mathrm{E}}$ stochastic term) from the liquid argon resolution ( $7 \% / \sqrt{\mathrm{E}}$ stochastic term) in the same modules. Electron identification is tagged by a transition radiation detector for energies below $70 \mathrm{GeV}$, and by a synchrotron radiation detector for energies above $50 \mathrm{GeV}$.

Table 13-1. GEM requirements for the SSCL test beams.

\begin{tabular}{ccc}
\hline & Electron Beam & Hadron Beam \\
\hline $\begin{array}{c}\text { Momentum Range } \\
(\mathrm{GoV})\end{array}$ & $2-170$ & $2-170$ \\
$\begin{array}{c}\Delta \mathrm{p} / \mathrm{p} \text { (Resolution) } \\
\text { purity (tagged) }\end{array}$ & $2.5 \% / \oplus 0.2 \%$ & - \\
$\begin{array}{c}\text { intensity (Hz) } \\
\text { (instantaneous) }\end{array}$ & $10<10^{-3} \quad\left\{\begin{array}{r}0 / \pi<10^{-4} \\
\mu \pi<0.5\end{array}\right.$ \\
\hline
\end{tabular}

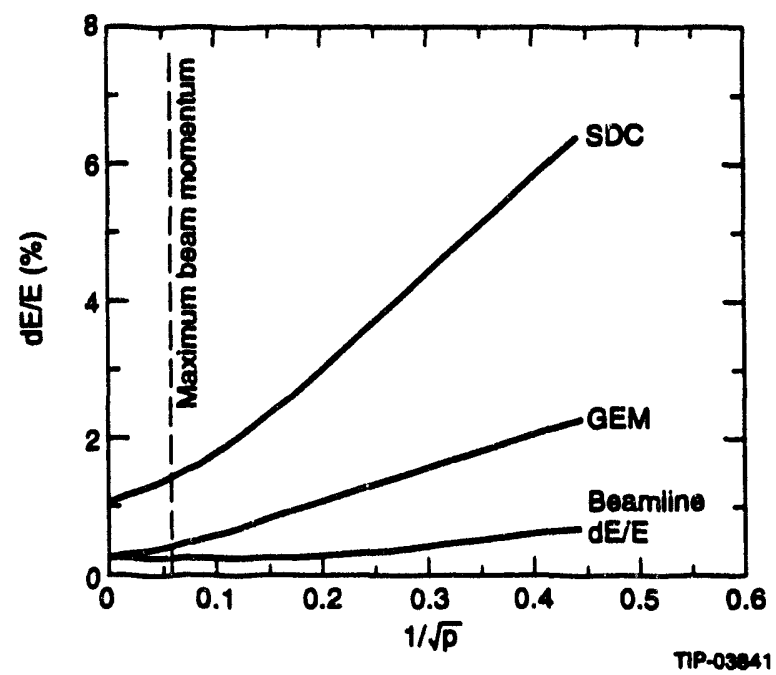

FIG. 13-4. Secondary beamline energy resolution at the calibration hall.

\section{GEM Calibration Pit Layout}

An artist's conception of the layout of the GEM pit is shown in Figure 13-5.

The tracker will be placed at the front of the pit. The tracker is shown here in two orientations, mounted on its transporter. The transporter will be capable of three motions: vertical, transverse (across the beam), and rotational (about a vertical axis). It will be capable of removing the tracker from the beam entirely so that downstream items may be irradiated without interference.

The calorimeter cryostat mounted on its transporter occupies the middle position in the hall. In order to understand the limits of the space requirements, the concept shown here displays the largest cryostat anticipated at the present time. In actual operation, scintillating calorimeter modules may be mounted behind the cryostat for a portion of the running. The calorimeter transporter will be capable of the same motions as the tracker transporter, plus limited axial rotation. The rotation plus translation will enable projective calorimeter components to receive beam from a simulated interaction point. The transporter will also be capable of moving the entire assembly out of the beamline to permit unhindered access of the muon chambers to the beam. A second cryostat, $4 \mathrm{~m}$ in diameter and $2 \mathrm{~m}$ in length, may be used to calibrate endcap modules.

The muon chambers will be mounted in the last position. A barrel tower assembly, as shown in Figure 13-6, will require five meters along the beam line. A magnet (not shown on the sketch) will be mounted near the front of the muon chamber area. There is additional space that can be used as needed.

An clevation view of the calibration hall is shown in Figure 13-6. This shows the beamline emerging from the beam tunnel at an elevation of 16 feet above the floor. This height allows adequate space for equipment, such as transporters, which will be installed either at turn-on or in the future. It also allows a 4-m diameter calorimeter cryostat to be scanned vertically across its full height, which would make possible a full-scale $(2 \pi)$ test of the final endcap electromagnetic calorimeter. Such test data would provide a valuable transition between the FNAL endcap prototype results and performance studies of the actual GEM endcap calorimeter. The 16 foot beam height also protects personnel in the 


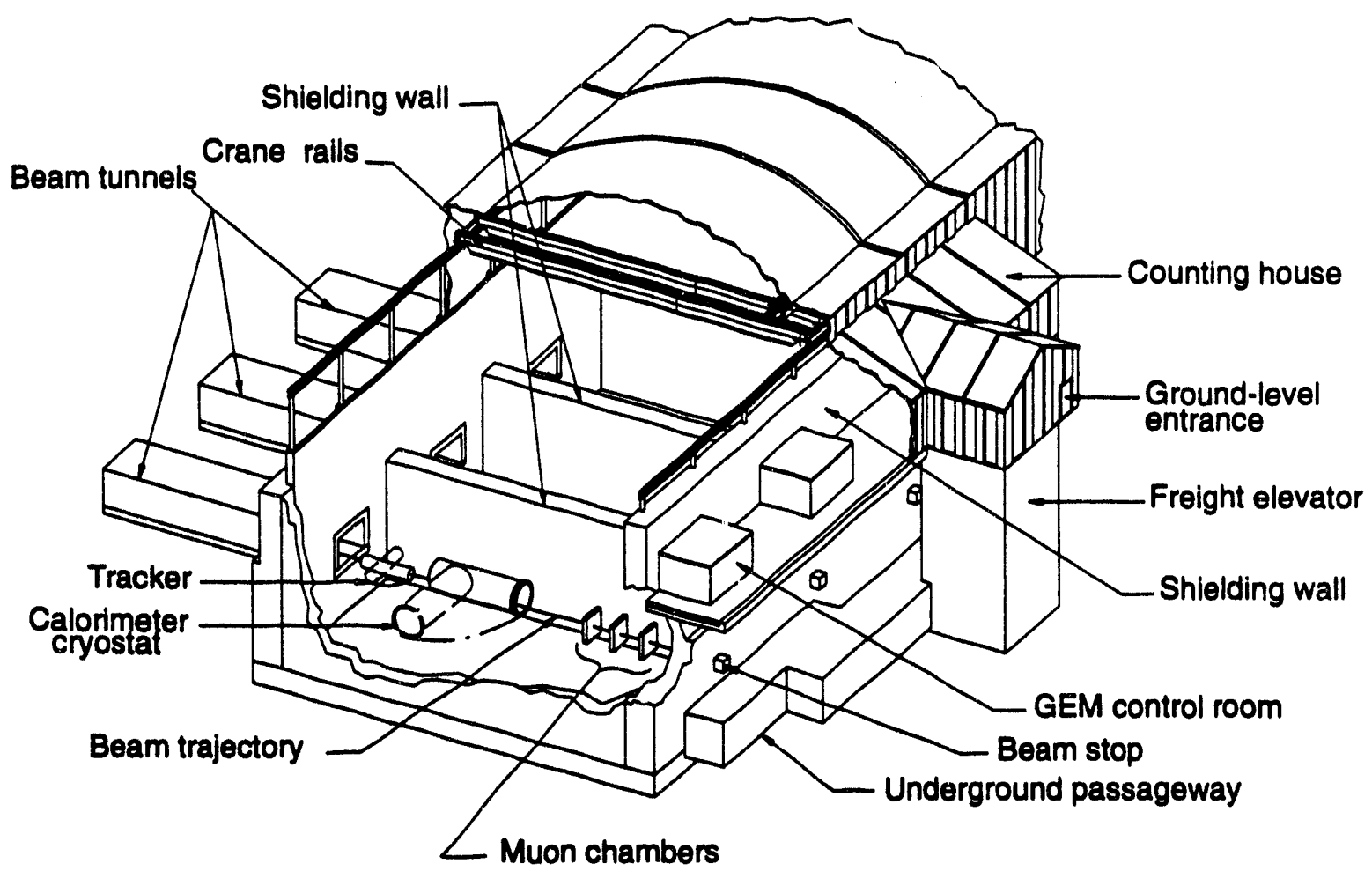

FIG. 13-5. Projective view of the calibration hall, showing the tracker, calorimeter, and muon chambers in test position in the beamline. Stands and transporters are not shown.

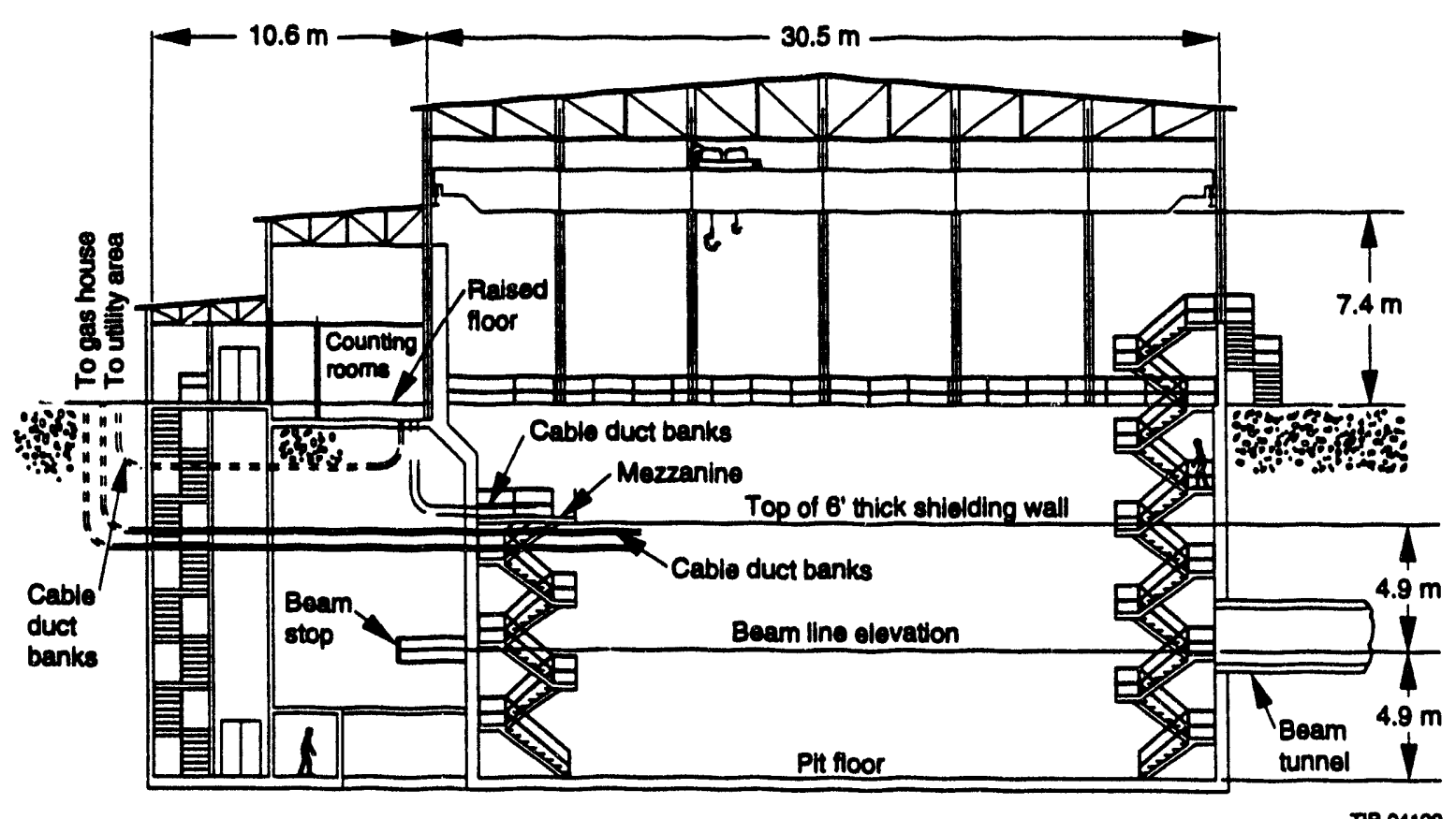

FIG. 13-6. Elevation view of the SSCL calibration hall, showing pit depth, beam height above this floor, and cable and gas conduits into the hall. 
floor-level access tunnels behind the pits, as shown in Figure 13-6. For equipment not requiring this height, a temporary floor constructed of concrete blocks can be installed to any required level. $A$ beam stop (stainless steel) is installed in the wall at the end of the beamline to remove any risk of ground-water irradiation.

Figure 13-6 also shows the counting rooms behind a two-foot-thick wall of concrete shielding. Cable conduits are shown as well. These connect the counting rooms with the utility area and the electronics mezzanine, which is located in the pit at the height of the top of the shielding wall. From there, cables pass into the hall itself, either aiong the west wall (in the GEM pit) or along the floor, as needed. There are also cable and gas conduits from the utility area into the pits. Fiber optics links (not shown) connect the counting rooms with the lab-wide main ring computer and communications networks. These will allow computing for the test beams to be performed either on lab general computing facilities or on the GEM computing system. This is an important feature of test beam running-the opportunity to test and debug prototype computing systems, including data analysis, data storage and retrieval, eic.

No offices are shown in Figure 13-3. Offices and some computer facilities will be provided in trailers in a near-by area. When a permanent campus is built, it might take the place of some of the trailer space, depending on its proximity to the site.

For the tracker calibration, the complete tracker power supply, gas system, cooling system, and data acquisition system will be transported from the tracker assembly area to the calibration hall along with the tracker. These systems will be mounted on skids which will be positioned just outside the building. All the cabling and other lines will cone over the shielding wall, which is external to the pit but contained within the hall. Following calibration, the entire system will be installed into the detector.

\subsection{RUN PLAN}

A summary of the run plan, as currently envisioned, is sketched in Figure 13-7. Exact dates are dependent upon scheduling developments at the labs cited. The beam time durations shown do not represent actual time spent taking data in the beam. They also include time to interleave with other subsystems or user:s (as in the case of BNL, where the beam is shared with non-GEM users).

The actual amount of beam needed by each subsystem is currently being decided. The calorimeter will dominate the beam time needs at both Fermilab and the SSCL. The amount of exposure needed will depend on the number of cells and cracks to be irradiated, the number of events at each one, and the capability of the transporter to reposition the modules rapidly. It will also depend on the capability to change beam energy (and, although to a much lesser extent, particle type) rapidly. These requirements and capabilities will be determined over the next fiew months. Experience with other experiments, however, has shown that the running time sketched in Figure 13-7 is adequate to produce very useful results.

Fermilab fixed-target running beyond 1996 is currently unscheduled, though we do anticipate beam availability in that time frame. When beam becomes available, GEM will take advantage of it. When the main injector becomes operational, beam to the test areas should be available on a regular basis, even during collider running. The maximum energy of this beam will be only $120 \mathrm{GeV}$. However, Fermilab will not stop its fixed target program, and therefore $\geq 800 \mathrm{GeV}$ beams will still be available at times. Nevertheless, if the SSCL beams are available at that time, it may be advantageous to move some operations there.

\subsection{SUMMARY}

Test beams have already become useful for the GEM central tracker and calorimeter groups. In addition, cosmic ray tests of muon chamber technologies have played a role in the muon group technology decisions, and beams in Russia have been used to help develop prototypes.

Test beam work at Fermilab will be performed to do energy scans of calorimeter modules, up to $800 \mathrm{GeV}$, and to accomplish many other tests. In addition, the central tracker group will perform high-rate tests of their IPCs, and the muon group will 
do a number of tests, including tests in a $0.8 \mathrm{~T}$ magnetic field.

Test beams running at the SSCL will provide the first opportunity for the Laboratory itself to conduct operations with a beam. This will serve as a test bed for all laboratory and detector issues except for underground safety. All other safety issues relevant to GEM operation, including cryogenics, $\mathrm{ODH}$, and so on, will be dealt with operationally for the first time. In addition, this will serve to solidify the lab's position as an operational lab and attract physicists to work here, both as visitors and as employees. Depending on the actual schedule, this may help form the seed for the big GEM assembly effort at IR5.

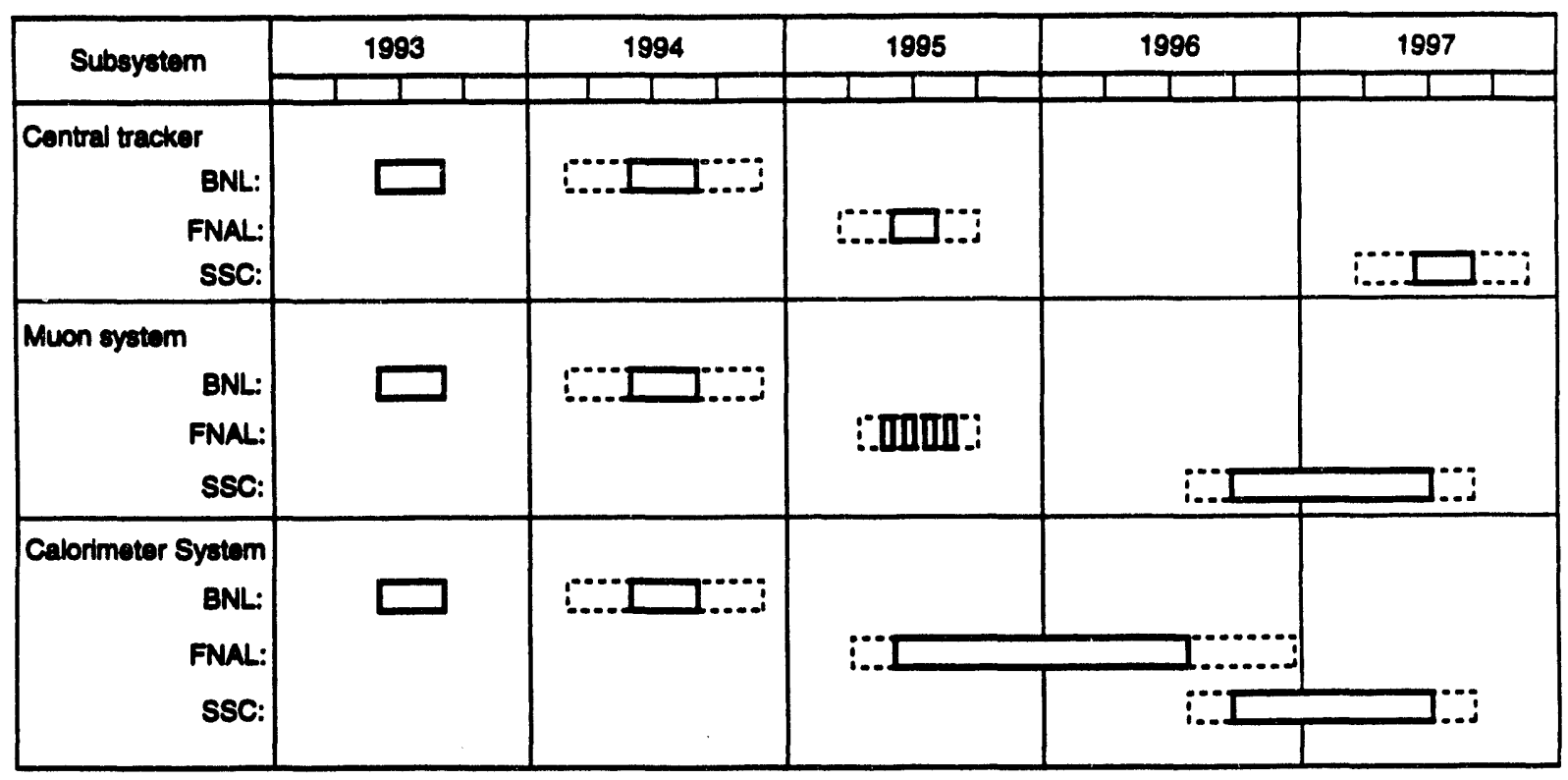

nP-03eas

FIG. 13-7. Summary run plan for GEM test beams activities. 


\section{GEM ORGANIZATION PLAN}

The GEM collaboration is made up of individual members (collaborators) and collaborating institutions. The Organization includes a Collaboration Council, a Spokesman or Spokesmen, an Executive Committee, an International Committee, a Project Manager with a management office, and Subsystem Organizations. The interrelationship of these components is indicated in the GEM Organization Chart (Figure 14-1).

This Organization Plan is designed primarily for the period after the approval of the GEM Technical Design Report. In the past there has been an evolution of several interim organizations. The first one, with two Cospokesmen and a Steering Committee, prepared the GEM EOI (Expression of Interest). The second, adding the Collaboration Council, prepared the GEM LOI (Letter of Intent). The third interim organization, which is in effect at this time, is preparing the GEM Technical Design Report. It consists of two Spokesmen, the Collaboration Council, the Executive Committee, an Acting Project Manager, and an initial set of Subsystem Groups. The intention is that the present interim organization will evolve smoothly into the final

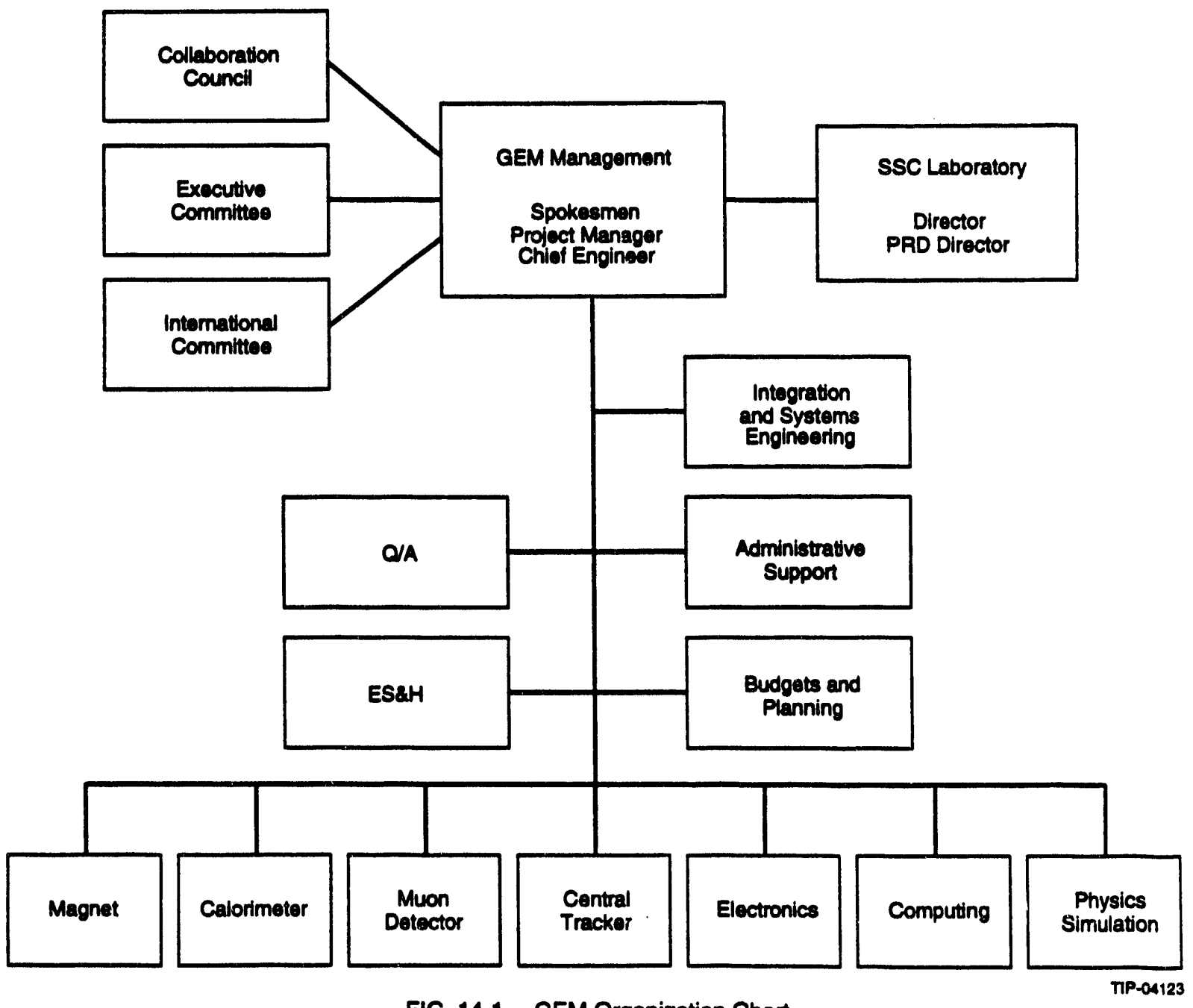

FIG. 14-1. GEM Organization Chart. 
GEM Organization described in this Plan. This Plan is expected to undergo further changes at the end of the construction phase of the detector, when GEM turns into an operating experiment.

The GEM Project Management Plan that will be developed by GEM, the SSC Laboratory, and DOE will go into more detail on the formal management of the Project.

\subsection{MEMBERSHIP}

Scientists with an interest in the research program of the SSC (Ph.D. physicists, graduate students, and engineers) of any nationality are eligible to be members of the GEM Collaboration provided that they are contributing a significant fraction of their research effort (averaged over a period of several years) to the GEM program.

Collaborating institutions can be universities, national laboratories, or other institutions that have one or more members in the GEM collaboration. GEM collaborators may be involved in other experiments or activities, but are expected not to be members of approved competing SSC or LHC experiments.

\subsection{THE COLLABORATION COUNCI}

The GEM Collaboration Council will consist of one representative from each collaborating institution. The Collaboration Council will deal with general issues that concern the collaboration. It will serve as a forum for informing the collaboration and will provide input and guidance for all major scientific technical and fiscal matters. In particular the collaboration, through the Collaboration Council, will approve the GEM Organization Plan, Publication Policy, Management Plan, and other major concerns of the collaboration. It will also approve the selection of the Spokesmen, the Project Manager, the Executive and International Committees, and the Subsystem leadership. The Collaboration Council will have open meetings typically once every one or two months. The Collaboration Council, through its chairman, in consultation with the Spokesmen, will organize collaboration meetings typically twice a year. a) The Collaboration Council Representatives will be chosen by each institution. The term of office of the Representatives will be at the pleasure of each institution.

b) The Collaboration Council will elect its own chairman. The chairman will guide the affairs of the Collaboration Council. In particular, he or she will organize and run the meetings of the Collaboration Council and the general Collaboration meetings; will be responsible for keeping an up to date list of the membership of the Collaboration and the Collaboration Council Representatives; and will administer the voting of the Collaboration Council on relevant issues.

i) The term of office of the Collaboration Council chairman will be two years, not renewable.

ii) Nominations for the chairman will be from the floor at a Collaboration Council meeting or by letter to the outgoing chairman.

iii) The outgoing chairman will check that the nominees are willing to run and will distribute the ballot for vote by the Collaboration Council Representatives by mail or E-mail with at least two weeks notice.

iv) If no candidate receives a majority of the votes, the top two candidates will stand for a run-off election.

\subsection{THE GEM SPOKESMEN}

The Spokesmen will be responsible for the scientific, technical, fiscal, and managerial concerns of the Collaboration. It is their responsibility to have a well designed and functioning detector ready at the turn-on of the SSC. The Spokesmen, in consultation with the Executive Committee and the Project Manager, will direct the technical and engineering design, physics integration, and technical implementation of the detector.

a) There will be one Spokesman or two Cospokesmen as may be deemed appropriate by the Nominating Committee (see Paragraph c-i). 
b) The Spokesmen have a fixed 3-year term of office, with no prejudice or limit on the number of terms served.

c) The Spokesmen will be selected, soon after the GEM Technical Design Report is approved, by the following process:

i) There will be a Nominating Committee consisting of nine members of the GEM Collaboration. Of the nine members, four will be chosen by the Collaboration Council, two by the SSC Laboratory, and three will be selected by the Executive Committee to provide balance. The Nominating Committee will select its own chairman.

ii) The Nominating Committee will consult extensively with members of the collaboration, the present interim Spokesmen, the SSC Laboratory, the subsystem leaders, etc., and will nominate a single slate.

iii) The slate thus nominated will be put up for approval or rejection by the Collaboration Council. The chairman of the Collaboration Council will solicit one vote from each Collaboration Council Representative by mail or E-mail, allowing at least two weeks between the presentation of the slate and the balloting. A majority vote will be required for approval.

d) This selection process will be repeated every three years. A new Nominating Committee will be constituted each time as specified in Section 3-c-i.

e) A petition signed by two thirds of the Executive Committee or a two thirds vote of the Collaboration Council can trigger this selection process at any time under unusual circumstances.

\subsection{THE EXECUTIVE COMMITTEE}

The Executive Committee will advise the Spokesmen and the Project Manager on scientific, technical, fiscal and managerial decisions relevant to the design, construction and operation of the GEM Detector. The Executive Committee will serve as the Configuration Change Control Board.

a) The Executive Committee will consist of between 12 and 18 members of the GEM Collaboration. The members of the Executive
Committee will be nominated by the Spokesmen after wide consultation with the collaboration. The nominated slate will be approved or rejected by the Collaboration Council (using the procedure of Section 3-c-iii).

b) The term of office of Executive Committee members will be the same as that of the Spokesmen.

c) The Project Manager, the GEM Chief Engineer, the chairman of the Collaboration Council, and the leaders of the various GEM Subsystems will be members of the Executive Committee.

d) The Spokesmen will serve as chairmen of the Executive Committee.

e) The Executive Committee will meet at least once every 1-2 months.

f) The membership of the Executive Committee can be reevaluated by the Spokesmen as appropriate, with the approval of the Collaboration Council.

\subsection{THE GEM PROJECT MANAGER}

The responsibility of the Project Manager is to provide technical, administrative, management, and budget coordination to insure the efficient and timely construction of the GEM detector. The Project Manager is responsible to both the GEM Spokesmen and Executive Committee and the SSC Laboratory management. The Project Manager is also the head of the SSC GEM Project Department.

a) The Search Committee for the Project Manager will consist of

i) The Chairman of the Collaboration Council

ii) Two SSC Laboratory representatives chosen by the SSC Laboratory management

iii) Four or five other members, including the chairman of the search committee, selected by the GEM Spokesmen.

b) The Search Committee will conduct a search for a candidate for Project Manager, with extensive consultation with members of the Collaboration, the SSC Laboratory, the DOE, and the GEM Subsystem Leaders. It is possible that one of the Spokesmen will be proposed as 
a candidate for Project Manager. The single candidate selected by the Search Committee will be approved or rejected by the Collaboration Council (same procedure as defined in Section 3-c-iii).

c) It is anticipated that the Project Manager will serve with indefinite term to the completion of the construction of the GEM detector. However, the Spokesmen or the SSC Lab can initiate the formation of a new Search Committee at any time if necessary.

\subsection{THE GEM CHIEF ENGINEER}

The GEM Chief Engineer is responsitle to work with the GEM Spokesmen and the Executive Committee, and the Project Manager, to develop the engineering design for the detector, the detector experimental hall, and the detector support infrastructure, and to carry out that design in an efficient and timely fashion.

a) The Chief Engineer will be nominated by the Spokesmen and the Project Manager after extensive consultation with the GEM Executive Committee and the SSC Laboratory management. The nominee will be approved or rejected by the Collaboration Council (using the procedure of Section 3-c-iii).

b) It is anticipated that the Chief Engineer will serve an indefinite term to the completion of the GEM detector. However, the Spokesmen can initiate a new nomination process if necessary.

\subsection{THE INTERNATIONAL COMMITTEE}

The International Committee will advise the Spokesmen on fiscal matters, especially in the area of international contributions, coordination of work responsibilities between various countries, international agreements, and other related issues.

a) The International Committee will consist of one or two members from each collaborating country.

b) The members of this committee will be selected by the Collaboration Council Representatives from each individual country after consultation with the GEM Spokesmen. c) The term of office of International Committee members is up to each individual country. The Spokesmen can suggest changes or additions as appropriate, with the approval of the Collaboration Council Representatives of the relevant country.

d) Initially the International Committee will be chaired by one of the GEM Spokesmen. Later the Committee may wish to have a rotating chairman to be elected by the Committee; in this case, the chairman of the International Committee will be a member of the Executive Committee.

\subsection{THE GEM SUBSYSTEMS}

The GEM detector, as far as design and construction is concerned, can be divided by the Spokesmen into a number of subsystems such as Magnet, Calorimeter, Muon Detector, Central Tracker, Electronics, Computing, and Physics Simulations. Each subsystem organization will be coordinated by a Subsystem Leader, assisted by a Steering Committee, a Subsystem Project Manager and a Subsystem Chief Engineer. Not all subsystems need to follow this structure; e.g., Physics Simulations might not need a Chief Engineer. The Subsystem Leader will serve as chairman of the Steering Committee.

a) The Subsystem Leaders will be nominated by the GEM Spokesmen, in consultation with the Collaboration, especially with members of each of the relevant subsystems. The nominated slate will be approved or rejected by the Collaboration Council (same procedure as in Section 3-c-iii).

b) The term of office of the Subsystem Leaders is at the pleasure of the Spokesmen. The Spokesmen can recommend changes in Subsystem leadership under unusual circumstances, with the approval of the Collaboration Council.

c) The Subsystem Steering Committee and the Subsystem Project Manager will be selected by the Subsystem Leader in consultation with the GEM Spokesmen, the GEM Project Manager, and the GEM Chief Engineer. The Subsystem Chief Engineer will be selected by the Subsystem Leader and the GEM Chief Engineer in consultation with the GEM 
Spokesmen and the GEM Project Manager. It is possible that the Subsystem Leader and the Subsystem Project Manager, or the Subsystem Project Manager and the Subsystem Chief Engineer, could be the same person.

d) The Subsystem Leaders are primarily responsible to the GEM Spokesmen. The Subsystem Project Manager and the Subsystem Chief Engineer are responsible to their Subsystem Leader, and will be responsive to the GEM Project Manager and GEM Chief Engineer.

\subsection{ADMISSION OF NEW COLLABORATORS INTO GEM}

a) Up to the time of the approval of the GEM Technical Design Report, GEM will have an open membership policy.

i) Any qualified institution can join the Collaboration by notifying the Chairman of the Collaboration council or one of the Spokesmen of their intention in writing.

ii) New members can join an institution that is already a GEM Collaborator at the discretion of that institution. It is the responsibility of the Collaboration Council Representative from each institution to notify the chairman of the Collaboration Council of any changes in membership.

b) After the GEM Technical Design Report is approved the following procedures should be used for the admission of new collaborators to GEM:

i) A new institution that desires to join the collaboration should enter into negotiations with the GEM Spokesmen and possibly one of the Subsystem Leaders, if appropriate, to discuss the contributions and role of the new institution. If these negotiations reach a satisfactory conclusion, the new institution should write a letter to the chairman of the Collaboration Council or to one of the Spokesmen stating their desire to join GEM, listing the individual members of the group, and explaining their anticipated role in GEM. The chairman of the Collaboration Council will present their case at the next Collaboration Council meeting. A favorable vote from a majority of the Collaboration Council Representatives will be required for admission (using the procedure of Section 3-c-iii).

ii) Individuals who wish to join GEM at an institution that is already a GEM Collaborator can do so at the discretion of that institution. It is the responsibility of each Collaboration Council Representative to keep the chairman of the Collaboration Council informed of changes in membership at his or her institution.

c) Withdrawal from the Collaboration. The membership of an individual collaborator will terminate when the Collaboration Council Representative from his or her institution informs the chairman of the Collaboration Council that the individual is no longer associated with GEM. An institution can withdraw from the collaboration (provided it has satisfied whatever construction responsibilities to which it has agreed) by informing the chairman of the Collaboration Council of its intention in writing.

\subsection{ADOPTION OF THIS ORGANIZATION PLAN}

This Organization Plan will go into effect when approved by a majority vote of the Collaboration Council (using the procedure of Section 3-c-iii).

\subsection{AMENDMENTS TO THIS ORGANIZATION , LAN}

A petition for a change or amendment to this Organization Plan after it has been adopted can be initiated either by the Spokesmen or by a letter to the chairman of the Collaboration Council signed by at least 50 members of the collaboration. Follov'ing such a petition the Spokesmen, in consultation with the chairman of the Collaboration Council, will set up a committee to study the desirability of such a change and to draft a specific proposal. The change or amendment will go into effect when approved by a majority vote of the Collaboration Council (using the procedure of Section 3-c-iii). 


\section{$\longrightarrow 15-$ ENVIRONMENT, SAFETY, AND HEALTH (ES\&H)}

\subsection{INTRODUCTION}

This chapter provides a description of identified and hypothesized hazards, their relative significance, and proposed mitigating actions. This chapter is intended to provide a safety overview of the TDR design for the JEM detector. As such it does not necessarily reflect the future final design nor does it entirely describe the extent of risks for hazards identified. This is being accomplished within the Safety Analysis Report (SAR). ${ }^{l}$ The SAR will be updated to accurately reflect the design evolution and safety changes as they occur. The reader is encouraged to refer to the technical drawings and tables provided in th. detector overview and subsystem chapters in the TDR.

Safety specifications provided by DOE to the SSCL include requirements for conducting a safety program. ${ }^{23}$ The Physics Research Division has established a system safety program to support design objectives. Its primary focus is to assure the safety of personnel and public, and to ensure that safety is designed into the GEM detector, its subsystems, equipment, facilities, operation, and interfaces. This chapter outlines the steps taken to ensure that hazards have been identified, summarizes the steps taken to address them, and concludes that the detector will be safe to operate as designed. The current ES\&H design contribution to the GEM detector is presented here to specify requirements, to demonstrate safety compliance, to indicate status, and to provide a basis for future resolution of issues. Preliminary safety analysis and design work has determined the safety systems parameters for the detector and its subsystems. These parameters describe how the safety systems will integrate into the detector and subsystems and the type of protection afforded. Major GEM ES\&H milestones (including important documentation) are being subjected to an ongoing program of independent expert review, e.g., GEM Safety Review, Physics Research Division (PRD) ES\&H Subcommittee, PAC Review, and DOE Review. The responsible line organization (GEM Project Manager) is being formally notified of the results from these independent reviews.

GEM management has implemented the system safety program using MIL-STD-882, System Safety Prngram, 4 as a guide. This approach utilizes hazard analysis methodology to identify, to evaluate, to track, and to resolve safety issues. Hazard analyses have been performed for the current baseline and for proposed changes and alternatives. As design progresses, safety analyses are being performed on the GEM system, its subsystems, support systems, system software, installation, and test activities, and on aspects of construction, facilities, and support services as they relate to operational, personnel, and environmental safety.

Preliminary or current hazard analysis results are documented by the Safety Analysis Report. The SAR will identify all safety hazards, tasks for resolution, and mitigations proposed. When the GEM design is completed, the SAR will then be completed, based upon documented closure of items, and submitted through management for approval approximately six months prior to commissioning.

Since many safety considerations are common to GEM, SDC, the accelerator, and the conventional construction, consistent approaches and combined actions are being applied where appropriate. Common considerations include: alarm systems and annunciation, cable insulation materials choice, global control systems, and data electronics aspects such as cooling and electronic service racks. Common approaches in these areas will be more effective and result in lower cost.

The cognizant GEM engineers have provided input to this chapter, and some references to specific safety problems are made in their technical chapters. Safety is treatt $\mathcal{J}$ as an integral part of the GEM project. Safety, efficiency, and operability are inter- 
dependent and are addressed through the application of sound design engineering principles.

\subsection{DETECTOR SAFETY OVERVIEW}

The philosophy of this chapter is to focus strictly on system safety design aspects of the detector. For a technical design overview, or a more detailed treatment of the safety measures specific to the detector subsystems, reference to specific technical chapters and the SAR is recommended. System safety has been integrated as much as possible into the technical design. The primary hazards associated with the detector are: radiation; cryogenic liquid leak; butane gas leak; static magnetic field effect upon ferromagnetic objects and personnel; electrical shock; electrical and combustible load fires; magnet quench; elevated falls; moving massive objects; crane loads; confined space access; and oxygen deficiency hazards. All of these are reviewed and are being analyzed as to their resolution and protection. System safety will use an "order of precedence" procedure to satisfy requirements and to resolve identified hazards. The order of precedence or hierarchy in the GEM Program Management Plan 5 is summarized as follows:

- design for minimum risk by eliminating hazards through design, or alternatively by reducing risk to an acceptable level as specified by SSCL policy. These risks are addressed by U.S. Regulations and DOE orders. If for technical and physics reasons these requirements cannot be adhered to, other equivalent mitigating measures are to be used. Measures deviating from the stated requirements must be approved by the authority having jurisdiction.

- incorporate safety design features or fail-safe devices to prevent, to detect, to control mishaps, and to protect the detector and personnel. In the event that the devices cannot ensure a minimum acceptable risk, additional safety features must be incorporated. These features must ensure that in case of mishap, the system will fail to a safe condition.

- provide warning devices such as signals and signs to notify, to restrict, and to protect systems and personnel.

- develop procedures and training to augment each of the above resolution mechanisms and to teach safe practices. However, warning devices, procedures, and training shall not be use 1 as the only method of reducing risks associated with catastrophic events or critical tasks.

Overall, development of safety measures depends on the technical design and development of the experiment. However, experience with detector installation at other accelerator facilities indicates that most accidents to personnel will be related to falls from scaffolding, ladders, and shaft openings, or due to falling objects. The important issue of limiting access at all levels, which can help minimize such accidents, has been evaluated in the SAR.

\subsection{MAGNET AND FORWARD FIELD SHAPER}

\subsubsection{Safety Overview}

The GEM collaboration has adopted a singlecoil solenoid configuration without iron flux return, which has been subjected to high levels of scrutiny and has received reviews and approvals from SSCL project management, external magnet experts, and the Program Advisory Committee. The proposed safety-design methodology will comply with referenced DOE orders, environmental impact statements, 6 GEM Technical Notes, and applicable regulatory codes and standards. The following safety hazards have been considered and analyzed in the GEM SAR:

- quench effects

- magnetic field and induced-current effects on equipment, materials, and personnel

- dynamic and static structural stress effects

- thermal effects (e.8., loss of vacuum in cryostat)

- cryogen leak effects (embrittlement, electronics, oxygen deficiency hazard (ODH))

- activation and radioactive waste handling

- detector access in a magnetic field.

A magnet quench occurs when the superconducting coil undergoes a phase transition from a superconducting state to a resistive state. This can be expected to be a rare but potentially critical event. Electromagnetic, mechanical or thermal disturbances are the primary causes of a quench. Complete or partial loss of cryogenic cooling or vacuum also can cause a quench. With the coil operating at 
$50.2 \mathrm{kA}$ and having a stored energy in excess of $2.5 \mathrm{GJ}$, prevention of a quench is paramount. The magnet is designed to minimize the risk of a quench by use of a cable-in-conduit conductor design, which has excellent stability against disturbances. However, it is also designed to accommodate a quench without any damage. The 24 coil segments are simultaneously and continuously monitored by compensated voltage taps whenever the coil is energized. A quench is detected by a circuit that senses growing resistive regions in the coil and subsequently triggers the energy dump circuit and other safety devices (as required by system procedure). Cryogenic-system and vacuum monitors will interface with the magnet protection system to provide a complete safety approach. Even though the causes and design preventatives are anticipated, the approach taken is that a quench may occur and that it can be reliably demonstrated that the stored energy can be dissipated safely in all circumstances. An engineering study has been done to show that temperatures and pressures cannot exceed design limits. Additional study will address effects upon related systems and will show that all other GEM subsystems can withstand the effects of a magnet quench. This study also will include possible effects on any personnel who may be in the fringe field at the time.

In the event of a quench, the energy is dissipated in the coil and in the $0.02-\Omega$ dump resistor (DR). The ratio of the energy dissipated in the coil to that in the DR is equal to the ratio of their resistances. The dump circuit discharge time constant is about 100 s. The voltage across the $D R$ will rise to $1000 \mathrm{~V}$ and the bus-to-ground voltage will be $500 \mathrm{~V}$; therefore stringent safety protection must be employed. The DR is being located in the surface utility building, and is shielded against accidental personnel contact.

A quench also results in heat generation and in rapid increases in pressure inside the magnet conductor conduit. The steel conduit has been designed to handle the maximum conceivable pressure (45 MPa). A qualification test program will verify the magnet system's performance and safety.

\subsubsection{Magnetic Field}

The GEM magnet produces a static magnetic field of about 0.8 tesla $(\mathrm{T})$ within the volume enclosed by the coil. Immediately external to the superconducting coil, the field is about $0.2 \mathrm{~T}$ at the coil end comers and about $0.1 \mathrm{~T}$ along the $\mathrm{z}$-axis coil surface. The fringe field decreases rapidly as a function of distance $\left(1 / \mathrm{r}^{3}\right)$, falling to about $0.05 \mathrm{~T}$ at a radius of several meters. At the floor of the cable shaft (E0) the field is about $0.02 \mathrm{~T}$, while the regions surrounding the electronics rooms (E1-E3) above range from about $0.016 \mathrm{~T}$ to $0.004 \mathrm{~T}$. The electronics rooms are being shielded to less than or equal to $0.005 \mathrm{~T}$ to ensure proper electronics operation. Equipment and components (e.g., vacuum pumps, subsystem electronics, pre-amplifiers, motors, and safety devices) that are required to operate within the hall magnetic field are being suitably designed, shielded, and remotely actuated, as needed.

The magnetic fringe field is an important safety concern. However, many successful large unshielded magnets have operated and are operating safely; these include MRI magnets and plasma physics magnets. All equipment located in the fringe field will be checked as to proper operation in all magnetic field conditions including normal operation, with and without the FFS structures, and during a quench. This equipment will also be checked to see that it continues to work properly after repeated field cycles. Special attention is being given to assuring that any safety systems within the area of the fringe field function properly (e.g., relays in the elevators, solenoid valves in cryogenic equipment, fail-safe valves, and circuit breakers). This is requiring detailed attention in the design stage, and the application of a quality assurance program during manufacture. Strict operational procedures will be applied to prevent loose ferromagnetic objects (including tools) from flying toward the magnet. The forces on the forward field shapers have been analyzed for all normal and exceptional conditions. Consideration has been given to the forces from the residual magnetic field in iron and to any effect upon FFS separation if there is no de-gaussing. The magnet is interlocked to prevent operation if its elements are not correctly positioned.

During magnet operation authorized personnel will be permitted in the hall only under mandatory circumstances, and only for controlled periods of time, as required for commissioning, maintenance, or calibration operations. Examples are detector alignment ${ }^{7}$ and field mapping. Only under these 
strictly controlled situations may personnel be in a region of significant magnetic field. Effects of an emergency discharge during these special periods, and any further mitigating actions needed, are being studied.

Various safety measures will be applied to protect workers and the public from exposure to the static magnetic field. $8,9,10$ The 0.001 -T field boundary will be marked for pacemaker interference, and medical screening will determine all personnel at health risk (metal prosthesis, sutures, etc.). The Personnel Access Safety System (PASS) will provide interlocking control to prevent unauthorized personnel access and exposure within the hall, in the detector, and throughout the several surface zones and buildings. SSCL policy, work rules, and administrative procedures will govern activities inside the detector and hall.

\subsubsection{Forward Field Shapers}

The two forward field shapers (FFS) are $8 \mathrm{~m}$ long, conical steel masses weighing about $1,800 \mathrm{Mg}$ each (with support siructure) that are used to shape the magnetic field in the forward muon region. Each is attached to a support structure adjacent to the end of the vacuum vessel and is supported at four points on the floor. There are safety hazards during transportation and handling of these massive assemblies, when they are installed, and when they are periodically withdrawn for detector access. The mechanism for railed transport is being safety analyzed. Because of the large forces (52 and 12.2 MN) on the coil and FFS when the magnet is energized, a safety study is being performed. Radioactivation of the FFS is addressed in section 15.13 and Chapter 12.

\subsection{MUON SPECTROMETER}

\subsubsection{Safety Overview}

The muon detection system consists of cathode strip chambers. Modular design of the chambers incorporates a set of alignment fiducial devices, a high-voltage and preamp electronics assembly, and a cooling gas supply and retum. Each independent drift chamber will operate slightly above atmospheric pressure. The chambers have been engineered to meet GEM performance specifications using a mixture of $50 \% \mathrm{CO}_{2}, 30 \%$ Argon, and $20 \% \mathrm{CF}_{4}$ (non- $\mathrm{O}_{3}$-depleting) as the drift gas. The gas-mixing station's safety hazards of overpressure and leakage are being addressed.

Potential safety issues associated with the muon system are:

- leakage from cathode strip chambers at very low levels (less than or equal to $2 \%$ of their gas per day), interfering with ODH (oxygen deficiency hazard) sensor operation

- electrical fire resulting in ignition of plastics

- high-voltage electrical shorts or personnel contact

- hazards of lasers or $x$ rays being used in alignment of the muon chambers

- disposal of used chamber structural material (lead, copper, etc.) that remains slightly radioactive.

Hazards such as structural integrity of the chamber assembly supports and high- and lowvoltage distribution for the local electronics are adequately addressed in the current design package. Structural analysis has been performed on the muon chamber supports, which include a yield stress safety factor of 1.5 in the design.

The remaining risk areas associated with the muon chambers include confined space hazards and working at significant heights both for installation and maintenance of support structures. Measures planned to mitigate and respond to any oxygen deficiency in normal and confined spaces are described respectively in Sections 15.16 and 15.17. Other confined space hazards also are addressed in 15.17. Support structures will be used for elevated or difficult-position work. An overall strategy and specific method to safely access the muon regions for chamber maintenance and other needs is being developed.

\subsubsection{Barrel and Endcap Regions}

$\mathrm{ODH}$ and gas-leak monitoring are being provided in the barrel and endcap regions. Exposed electrical sources will be protected, and the chamber construction material is fire-retardant or non-combustible and halogen-free. Assembly, transport, and handling issues are being evaluated.

For minor alignments, pneumatics will be used to control stepper motors located on the muon chambers. Safety design practices for pressure 
tubing, relief valves, and compatible fittings and seals are being incorporated. Options for the location of gas storage, and the choice of compressor and gas type are being investigated; safety design for liquid/gas delivery and liquid krypton (LKr) recovery, and other safety considerations are an integral part of this study.

\subsubsection{Muon System Shield}

The muon system shield acts to help shield the muon chamber region from neutron flux radiation. The preliminary design, spatial location, and composition of the muon system shield have been determined, as described in Chapter 12. The material for the muon system shield will become radioactive to a degree that depends upon its composition, exposure duration, and position relating to the beam. The current composition is a combination of borated polyethylene, copper, and barite concrete. Residual activation protection measures will be employed in controlling personnel access. Radiation levels from activated materials will be determined by surveys prior to permitting access. A radiation review is provided in Section 15.13.

\subsection{CALORIMETERS}

\subsubsection{Safety Overview}

Primary safety concerns of the calorimeter are risks from use of large volumes of liquid argon (LAr) and liquid krypton ( $\mathrm{LKr}$ ) as ionization detector media and from use of liquid nitrogen $\left(\mathrm{LN}_{2}\right)$ and LAr as cooling fluids. In response, the extensive safety features of the liquid/gas delivery subsystem have been made redundant. The delivery lines are vacuum-jacketed and insulated with redundant shutoff valves in case of emergency. The lines and valves are all leak-monitored for automatic or manual shutdown and evacuation, depending upon the severity of the leak(s). Strict quality assurance procedures will be followed in design, assembly, operation, and testing of the fluid supply and return subsystems. The vacuum vessels are being redundantly protected against overpressure, leakchecked, and weld-inspected. Safety systems and measures are being designed to address the maximum credible leak in all circumstances; these are being established through detailed analysis. Falling-load scenarios from use of the overhead crane are being examined to establish the vulnerability of components such as cryogenic lines and to establish guidelines for crane operation in the experimental hall. A study will address the movement of calorimeter assemblies (endcap) while cold and full of cryogenic liquid (Section 15.18). The extent and means of safety measures will depend on the results of the study.

The secondary hazards associated with low temperatures are being evaluated. The design assumes that in the event of loss of insulation vacuum of the calorimeters, the LAr or LKr can be safely recovered. In these circumstances, the temperature of the outer wall of the calorimeter may drop to $87 \mathrm{~K}$ or $120 \mathrm{~K}$, respectively. Since liquid spills can cause mechanical failures and other damage due to thermal shock, tolerance of surrounding systems to these conditions must be analyzed. As far as possible, cryogen leaks will be collected and sent for retrieval to an exhausted sump situated outside the hall area.

Other safety issues associated with the calorimeter are:

- lead, borated polyethylene, argon, and krypton becoming radioactive

- loss of vacuum in the cryostat

- overpressurization of cooling loop

- high-current electrical shorts and personnel shock

- leaking of supply or return cryogenic pipes

- use of combustible passive absorber, doped liquid scintillator, or fibers

- generation of mixed hazardous waste, including lead and copper plates within the hadronic modules of the inner barrel and endcap calorimetry, respectively.

\subsubsection{Inner Barrel and Endcap Calorimetry}

The heavier-than-air gas densities of the Ar and/or $\mathrm{Kr}$ in the barrel and endcap calorimeters have been anticipated in the leak-safety design. Cryogenic liquid spill containment is included in the detector design, in that vessels and transfer lines have vacuum-jackets. Alternative means for external containment also are being considered. The system's design is currently being evaluated to determine the proper placement of ODH detection devices. 
Design and fabrication of the calorimeter liquid and vacuum vessels are being guided by the ASME Boiler and Pressure Vessel Code, Section VIII Div. 2, and compliance documents will be maintained. Anticipated liquid vessel stresses and pressures for both the inner barrel and the endcap are being analyzed, and appropriate design criteria from the ASME code are being applied. The loads within the cryostats and the support structures are subjects of ongoing design analyses. Feedthrough connections will conform to appropriate standards and receive documented reliability testing. Handling, storage, and transportation of the lead plates for the hadronic modules pose safety hazards during fabrication and assembly operations. During decommissioning operations, disassembly and disposal of the lead plates result in production of toxic wastes. The forward EM calorimetry, contained within the endcap cryostat, is composed of liquid argon surrounding copper rods and tubes. Hazards associated with this area are primarily related to cryogens and activation, as with other areas of the endcap, and are being controlled accordingly.

\subsubsection{Scintillating Barrel Calorimetry}

Plastic scintillator with photomultiplier tube readout is used as a fast-response detection layer. The fire hazard will be controlled by incipient (earliest generation stage) smoke/fire detectors and by the limited available oxygen from enclosing the scintillator in metal containers. This will deny availability of this material as fuel for a fire and permit effective use of nitrogen "inertion."

The passive absorber has not yet been specified, but a combination of copper or brass and borated polyethylene is a likely choice. The primary hazards associated with these materials are radioactivation, waste handling, and combustibility of the borated polyethylene. Strict hazardous waste disposal methods therefore will be followed.

\subsubsection{Forward Hadronic Calorimetry}

The forward hadronic section uses tungsten absorbers integrated with scintillating material. Although the exact scintillator material has not been selected, probable choices contain pure hydrocarbons. Safe access to the photomultipliers is required in the mechanical design. Hazards associated with this area are primarily activation related and are being controlled accordingly.

\subsection{TRACKER}

\subsubsection{Safety Overview}

The central tracker is designed to operate in the immediate radiation zone of the beamline and in the about 0.8-T magnetic field of the GEM detector. The tracker is composed of two detector elements: an inner silicon tracking system (STS) and an interpolating pad chamber (IPC) outer tracker. Both trackers have axial and radial components that make up the barrel and forward regions, respectively

Identified hazards being addressed include:

- use of beryllium (not considered highly toxic-by adsorption/ingestion-in form used)

- butane coolant leak and ignition by nearby sources

- system and personnel risks associated with using butane and other gases/liquids

- electrical transmission/power (AC/DC) and components subject to high currents, high voltages, electromagnetic susceptibility, heat

- cooling water leakage onto electronics

- insertion and attachment of the beryllium beam pipe into the tracker assembly and its evacuation

- irradiation of materials and components (e.g. silicon detectors) potentially degrading system lifetime or performance and causing hazardous waste

- use of $x$ ray calibration sources for the tracker system.

\subsubsection{Silicon Tracker}

Physics requirements mandate the use of a thin-walled beam pipe within the tracker volume. Safety considerations of beryllium for the inner wall of the butane enclosure have been addressed, and special handling requirements have been determined.

The quantity of detector electronics, the strip configuration, and the power needed to operate the tracker lead to large voltage/current and significant heat dissipation requirements. Power sources are 
being equipped with interlocks, voltage-regulation and current-limit protection, circuit breakers, fuses, and chassis grounding, and will comply with National Electrical Code (NEC) or American Society for Testing and Materials (ASTM) requirements. Materials are being selected that resist combustion and limit toxic fumes in the event of a fire. Microchip module design is incorporating heatsinks, conduction, and liquid and gas coolants to dissipate heat. Radiation resistance engineering practices are being applied to microcircuits and other electronic components and subsystems.

Detailed studies were done during the choosing of butane as tracker coolant to ensure that the planned safety systems are adequate. Butane is a highly flammable liquified petroleum gas, and has fire and explosion hazards under conditions conducive to combustion. Butane as a cooling agent was reviewed and evaluated, along with other candidate gases, and found to exhibit the best heat transfer and liquid/gas phase properties for physics and engineering requirements. A closed-loop butane cooling system is used to dissipate heat from the electronics. The cooling system operates passively, using a gravity supply and capillary action in the liquid phase, and the resulting vapor is returned to the condenser by the differential pressure between the tracker and the condenser. The quantity of butane liquid is less than 201 . The cooling system design incorporates several safety features: to restrict the flow of butane to the environment; to confine butane leakage; to prevent combustion by nitrogen blanketing outside the gas enclosure; and to use structural boundaries for containment of gases within a limited volume surrounding the silicon tracker. The drawings in Chapter 6 show the double-containment gas enclosure and $\mathrm{N}_{2}$ blanket, which is temperature-controlled. Shutting down power to the electronics results in a return of liquid to the condenser, leaving only vapor throughout the system, which minimizes the possibility for combustion.

The butane lines routing past the cryogenic vessels have indirect safety implications. For instance, if the lines (both those normally gaseous and those normally liquid) are plugged by solid butane, then overpressure conditions will occur. Butane is an excellent solvent, and its reaction with other construction materials has been investigated, including gradual long-term interactions. Because containment of the butane is very important, a detailed safety study of the complete butane volume will be done. The study will include details of construction, feedthrough, design, operational and test pressures, over-pressure protection, etc.

While a passive thermosiphon is used for normal operation, filling and emptying for maintenance will require careful analysis of the probability of spills and other credible accidents. A HAZOPtype risk analysis will be completed by specialists in the handling of liquified petroleum gas.

Radiation susceptibility of materials used in the silicon tracker is a concern since irradiation levels will be extremely high and continuous. Mitigation includes the use of radiation resistant parts and adhesive materials and complying with DOE, SSCL, and PRD radiation safety policies, standards, and procedures.

\subsubsection{Interpolating Pad Chamber Tracker}

Loss or reduction of cooling efficiency due to single or multiple point failure(s) will be controlled through automatic detection and safeguarding.

A deionized water cooling system to remove electronics waste heat is planned. A loss of coolant could result in overheating of the electronics or shorting inside the tracker volume. Redundant protection to mitigate this possibility is planned.

Mechanical stress, assembly, and integration methodology are being analyzed throughout the detail design process. Control procedures and processes will follow those described above for the inner tracker. Electronics safety hazards are being mitigated in a manner similar to that for the inner tracker.

A "leakless" water-cooled system will be employed on IPC electronics. The system will basically use the concept developed at CERN, which has been successfully deployed. The system will be thoroughly prototyped to ensure that it performs up to its stated level. No element of it should fail, thus preventing any shorting out of the electronics.

The drift gas is a 50/50 mole mixture of $\mathrm{CO}_{2}$ and $\mathrm{CF}_{4}$. The gas presents an asphyxiation hazard, but safeguards are being designed in the system to prevent the accidental discharge of this gas into the tracker volume. Thorough studies and detailed 
prototyping are planned to ensure the integrity of the components.

\subsection{ELECTRONICS}

\subsubsection{Safety Overview}

Chapter 7 contains the proposed design for the electronics supporting the detectors, including the specification of where items are being located and how equipment will be cabled.

Accessibility and maintenance of crates and racks in the detector and in electronics rooms is important; each electronics unit is being designed for easy access. A crate or rack is divided into three main parts-cooling, crate electronics, and supplies - which indirectly enhances safety. Power plugs will be designed distinct from all other plugs to avoid misconnection. Where safety regulation is required, each circuit is being protected by fuses or other overcurrent devices. Any feeder conductor sensing lines are being provided with overcurrent or fuse protection equivalent to that of the primary conductor being "traced." When accidental shorting and current transfer occurs, this helps protect the sensing line from excessive or dangerous temperature within its conductor. Installations having nominal voltages greater than $600 \mathrm{~V}$ must be protected with load interrupting devices in addition to the use of interlocking systems and lockable power disconnect means. Overtemperature protection is being provided by sensors located throughout the power supply racks. Additional protection systems will be temperature monitors, leak detectors, cooling water flow rate sensors, and a rack incipient smoke detection system. Fire suppression is being provided by a CFC-free or high-pressure water vapor fire suppression system for room and equipment protection. Further fire hazard analysis will be performed on critical high-current circuits. The frequency of false alarms is minimized within the design of the alarm and global control systems and through the use of programmable logic controllers and redundant sensors that require corroboration to send an alarm signal. The use of electrically-incompatible materials within the circuit loops is being strictly monitored and reviewed to assure that no resistance heating or insulating breakdown will readily occur.

\subsubsection{Surface, Shaft, and Hall Protection}

The operations center, located in the south low-bay of the south assembly building, contains the on- and off-line and global detector control systems. Compliance with National Electrical Code regulations and DOE Order 6430.1A Division 16 is included in the current design. Heat dissipation capabilities contain a safety margin. Power distribution protection design provides safety margins. Communication equipment buses, cables, and networks incorporates safe design practices. Because the center will house many physicists, operators, and technical staff, human factors engineering will continue to be a prime design area. Equipment accessibility and availability is a high priority. Monitoring and control software will receive thorough safety review and design input. (System safety aspects of software are discussed in Section 15.15).

\subsubsection{Electronies Rooms}

The three levels of electronics rooms located within the cable, shaft have iron shielding to attenuate the field to a level at which rack and crate functions are unaffected. CRTs are sensitive to the magnetic fringe field and will be individually shielded as needed. Several fire protection system strategies for the cable shaft electronics rooms (about $9 \mathrm{~m} \times 12 \mathrm{~m}$ ) are currently being evaluated. For computer-type rooms, safety codes require approved room fire suppression and below-floor smoke detection, as a minimum. A conservative approach where each rack has dedicated detection and shut-down capability and a supplementary room suppression system is being evaluated in light of the total risk.

For incipient smoke detection the choices under consideration are laser particle counters, very early smoke detection apparatus (VESDA), and conventional smoke detectors. Anticipated fire suppression systems, consisting of either non-CFC or inert gas suppressant or high-pressure water, are being evaluated. Inerting with non-life-supporting gases will require an alarm and announcement prior to discharge to allow people to evacuate safely.

Electronics rooms contain several high-voltage and high-current power sources; therefore attention to electrical safety design issues, com- 
pliance to code requirements, and use of industry standard practices should minimize risk to people and equipment. Design issues address cable size, routing, and protection; prevention of cross-connection; and human design factors associated with electronic items, racks, and controls. Underground rooms will require focus on access and escape provisions. Additional system safety issues related to circuit design, parts, materials, and processes are evolving as the detailed designs develop.

\subsubsection{Hall and Detector}

Equipment located in the hall and around the detector includes electronic racks, DC power supplies, experiment sensors, data communication, and the global control system. The emphasis placed upon safety design is reflected in the use of overvoltage and current protection, electrostatic discharge control, radiation-resistant materials in construction of electrical components, and optimizing cable (flat, power, and fiber) routing for accessibility and to prevent fraying. Magnetic field interference and access/egress allowance requirements for equipment in the hall have been evaluated. Smoke extraction and fire suppression systems are being designed into the dedicated smoke vent and the hall ventilation system.

\subsubsection{Global Control System}

The Global Control System (GCS) monitors detector performance, safety status, and faults, and it controls detector operation. The GCS provides for fire and smoke protection, gas leaks, and environmental monitoring and control. The GCS interfaces with other accelerator control systems, and it is being.standardized with the accelerator and other experiments. An element of the GCS is the Safety Control Center (SCC). The safety systems are integrated as a subsystem of the GCS. Both will use the same or compatible software. However, for critical safety functions, the SCC is independent from the GCS and communicates with other SSCL safety systems. The SCC receives safety-related signals from the GCS and responds accordingly, based on the fault level (described below). The GCS will similarly receive SCC data and react. If the GCS fails completely or in part, the SCC remains functional (under power backup). Safety and design engineers are planning to use high-reliability components, redundancy of specific detection and con- trol mechanisms, and fault-tolerant software. The GCS and SCC operators will use both tactile and screen-sensed actions when responding to fault annunciations. This will be extensively evaluated as detailed design progresses. Aspects of GCS software safety are covered in Section 15.15.

The global control system defines four fault levels of notification to provide action response management:

- system status indication

- operational fault condition that does not warrant response

- caution condition indicating an event at a level or degree below that designated as emergency by code or standard

- warning level condition requiring positive and immediate remedial action. Hard-wired safety controls are being employed to activate protection for severe conditions (e.g., emergency power-off button).

\subsubsection{Grounding}

All metallic surfaces not requiring electrical isolation are being connected with low-resistance techniques and will be at the ground potential of the frame. Connections will not create any undesired ground loops or affect operation, testing, or safety. Grounds are in close proximity to the equipment, properly sized, laid out, and routed. SSCL standards for connections will be followed.

\subsection{BEAM PIPE}

Use of beryllium requires a special safety analysis due to its brittleness and toxicity. Two 30-m stainless steel beampipes with a 4-m section of beryllium pipe at the IP (between the steel sections) will be under vacuum. The beryllium section will be tested to assign the proper collapse pressure safety factor for the external pneumatic pressure. Final design of the beam pipe will be made after tests on prototype pipes to find practical safety factors. Additional protection will be provided where necessary for the beam pipe. The uninstalled and the installed beam pipe will be guarded from contact. The beam pipe is separated from the collider vacuum pipe by flanges. An implosion (which cannot be totally excluded) might spread beryllium fragments and dust contaminants within the detector. Safety analysis is being performed on the collimator 
interface assembly. Emergency actions must be taken when problems arise in the vacuum system. Alternative means of damage prevention are being evaluated, including the interaction of detection and safety mechanisms with accelerator systems.

\subsection{CENTRAL DETECTOR SUPPORT AND STRUCTURES}

The Central Detector Support (CDS) is a welded, non-magnetic, stainless steel structure designed to support the scintillating barrel calorimeter, the two endcap and barrel noble liquid calorimeters, and the central tracker. The combined weight of the central detectors and their services is approximately $3100 \mathrm{Mg}$. The CDS must be capable of adjustment to facilitate unpredictable shifts in the facility structure, i.e., floor settlement or floor displacement from shifting loads. The structure must provide alignment and orientation of the calorimeter and trackers to the magnet, while remaining independent of the magnet halves. Potential hazards associated with the CDS system include load stresses, maintenance activities, and prolonged contact with cryogenic liquid that may cause embrittlement. ${ }^{10}$ (However, there is no viable mechanism for fatigue cracking)

In addition to withstanding a maximum Von Mises stress of $50 \%$ of the material's yield point stress, the CDS must withstand the unlikely event of an earthquake with minimal damage. The maximum acceptable stress (about $241 \mathrm{MPa}$, from preliminary finite-element analysis) for the dynamic scenarios was set at $60 \%$ of the material's ultimate tensile strength. The hall has bee.. geologically located in a seismic zone 0 , which is the most stable earthquake zone, as stated in the SSCL guide for structure location and design.11,12,13 Structures and supports are being designed to handle various hypothesized static and seismic loads with safety factors specified in the GEM design documentation package and in the ANSI Z 58.1 standard. Safety considerations include analysis of stress and vibrational effects to determine or verify design limits for loads, to evaluate hazards, and to determine the center of gravity. Independent and internal analyses and evaluations will be completed prior to release of the assembly's drawing package. Buckling and load stress analyses already have been completed on the structure. The effects due to normal or abnormal magnetic fields (static or dynamic) have been analyzed and found to pose no hazard.

The response of detector structures and supports to vibiations anticipated in the experimental hall have been studied and will not exceed the specified alignment tolerances. Support structures are designed to meet load limits throughout their useful life, when subjected to the hall vibrations over operating and non-operating temperature ranges. Cryogenic equipment cause relatively high frequencies compared to our structures. Their amplitude is very low. This is not a structural problem, but a positional stability issue with no safety hazard. Low-frequency vibrations generated by cryogenic equipment operation are not expected to cause problems. ${ }^{9}$ Other loads imposed by transportation and rigging are being addressed to meet the required safety factors.

\subsection{ASSEMBLY, INSTALLATION, AND UPGRADES}

\subsubsection{Assembly}

Evaluation of the detector assembly and installation process indicates that specific safety systems will be needed. Subsystems or portions of them will be assembled in surface buildings; final assembly and integration will be done in the experimental hall. The primary hazards foreseen are those associated with handling and transport of the massive detector subsystems and components. Assembly, work area, and personnel hazards are being identified. Mitigation will be implemented in detector design or by use of special fixtures. Joining processes used in the assembly of components require special safety precautions. Processes that create a safety hazard are being controlled. Sources generating toxic fumes or noise have been segregated, managed, and mitigated in accordance with SSCL ES\&H requirements.

Cranes, assembly support fixtures, vehicle/rail transports, hall grease pads, and the hydraulic transport system are being analyzed to identify hazards and load capacity safety factors. The heavy-lift cranes are being reviewed. Hazard reduction methods are being incorporated in designs and in purchase specifications. All suspension or support structures will incorporate code design stress recommendations. Where massive detector parts require integrated assembly, permanent load supports, as 
opposed to temporary scaffolds or suspension by crane or hoist, will be provided. Special access devices are required, and their design and application are being reviewed for safety.

All assembly, handling, and transport machines and structures will require a safety analysis and approval prior to their initial use. All required mechanical and electrical guarding for machines and equipment used in the detector assembly process will be installed prior to operation/use. All surface facilities buildings and assembly areas are being provided with approved gas-smoke-and-fire detection and suppression systems where required by code and regulations.

During assembly of components, joining processes will require special safety precautions. Separate loads to be joined will be safely supported. Consideration is being given to adequacy of the previous, individual support structures to safely support the newly combined parts. Analysis may demonstrate that the previous, separate support structures require redesign or replacement. Joining of components via adhesion, i.e., plastic epoxies, may require application of local ventilation during the drying process. Large-scale welding or brazing tasks will require special isolation and safety precautions with the equipment involved.

A safety analysis is being performed to evaluate the flow of materials for the assembly process. All processes that create a safety hazard will be identified, and the hazard corrected or mitigated. Sources generating toxic fumes or noise are being segregated and mitigated to SSCL ES\&H Manual requirements. The flow diagram of all assembly processes (including joint occupancy) within the surface and underground buildings will require safety review and approval.

The generation of hazardous and mixed wastes will be minimized during the assembly process. All assembly-process scrap waste will be type-segregated and stored properly.

\subsubsection{Installation}

Job safety analyses will be developed for critical activities involved in the installation phases. Safety measures that protect people and hard ware in the surface buildings will be adapted and installed within the underground hall and shafts. During installation, support structures will be constructed to withstand stress levels that can safely support the load. All handling, scaffolding, support, and transport equipment will comply with SSCL ES\&H, OSHA, and other regulations. Scaffoldings and working at heights (the beam is $13 \mathrm{~m}$ above the floor) are important hazards. During these activity phases, permits involving special precautions to limit or control the spread of sparks, hot particles, or welding slag, will be required for all fire risks (such as welding, cutting, and grinding), and the use of compressed gases will be controlled. Due to high cost risk and the presence of welding operations within the hall during installation of the various systems, the smoke/fire detection and suppression safety systems will be installed and completely functional prior to lowering the first magnet halfcoil.

The radiation shielding concrete blocks of the installation shaft will be removed and replaced according to a pre-approved plan. While the blocks are removed and during idle periods, a safety net or cover will be placed within the shaft, close to the surface level. A physical access barrier will be placed around the shaft opening. In the hall and on the surface, installation equipment and materials will be placed so as not to block emergency egress pathways. Personnel access safety equipment is being designed according to human factors guidelines, and will be present during installation. During the installation process, the safety rules governing access to confined spaces will apply where appropriate.

The two hall cranes are provided with a safety lock-out capability that will prevent unauthorized operation and with safety interlocks that will prevent over-travel, crane-to-crane contact, and excessive rate-of-travel. Proximity sensors may be provided on both cranes to protect against accidental contact, especially where they are operated in tandem from one control station. Limit switches on the crane motor and travel tops are being installed to prevent the hook (and load) from traveling above the hall gangways. These same precautions are being applied to the surface heavy-lift cranes as well. All loads (especially their stability) will be reviewed and approved prior to transport. When loads are lowered from the heavy surface crane, the hall floor area below the installation shaft will be cordoned off to 
a perimeter of $28 \mathrm{~m} \times 30 \mathrm{~m}$, and personnel access to this area prohibited. Personnel will not be allowed to work beneath suspended crane loads, even with the use of load-rated safety devices.

When the magnet is fully energized, it will be necessary to secure the two overhead hall cranes in place at opposite ends of the hall. It is recommended that these cranes be secured against attraction toward the magnet by use of a positive means of blocking against movement. This may be accomplished by an interlocked brake on the crane rollers or, perhaps, by mechanical pins that project into the rails. This safety interlock must not be affected by the magnetic field (anticipated to be $0.02-0.03 \mathrm{~T}$ ) in these areas. If the cranes are to be operated when the magnet is energized, special safety precautions will be required to include that the crane and its controls, and the emergency devices must operate reliably within this fringe magnetic field. Controls will be hard-wired where possible. Special operational requirements will apply should radio-controlled cranes be used.

Safety system controls are being applied to mitigate physical agents (e.g., cold and heat stress, ionizing radiation, static magnetic fields, noise, lasers, light, and ultraviolet and near-infrared radiation) during detector installation. Lighting will be greater than or equal to $538 \mathrm{~lx}$ at the floor, and ventilation will be greater than or equal to 2 air changes per hour. Emergency lighting and traced egress pathways will be functional during installation.

\subsection{BEAM TESTING AND CALIBRATION}

The calibration hall design and construction should result in a low-risk facility, as defined in DOE 5481.1B, Chapter II, paragraph 5. (Refer to Chapter 14 for a technical description of the facility and beam, and Reference 12.)

Many of the safety measures described above for the tracker, calorimeter, and muon spectrometer will apply to test team work in the calibration hall. ${ }^{14}$ This operation provides the first opportunity to test the safety measures that are being employed in the final detector system. Refer to the Calibration Hall SAR $^{14}$ and the GEM SAR ${ }^{1}$ for further details on the specific hazards.

\subsection{UTILITIES}

\subsubsection{Water Systems}

Cooling pond/tower water is used as a primary equipment cooling medium. Most of the heat rejected to the cooling water systems will be transferred to the atmosphere by evaporation and heat exchangers. Systems include cooling pond water, low conductivity water, and chilled water. ${ }^{15}$ Supply of cool water is the prime safety concern to prevent equipment heating. Water systems incorporate check valves, relief valves, filters, pumps, and other safety devices to ensure proper utilization and operation, thus ensuring protection of equipment against overheating. Some waste water (other than that water not subject to external contamination and sent to the pond) will be channeled to a holding tank for cooling and disposal. Activation of the normal cooling water should not be a significant problem, as the flow rate (residence time) and filtration process will tend to limit this. It is anticipated that the hall wall seepage will be sump-pumped to the surface holding tank for mass-spectrometer analysis of samples for contaminants.

\subsubsection{Heating Ventilation and Air Conditioning (HVAC) System}

The HVAC is a conditioning, environmental control, and safety system. Separate ventilation from the HVAC system provides conditioned air to the interior of the detector. During shutdown periods this air flow serves to remove any gas leaks from detector components and to supply fresh air for occupants. During beam-off operation, the HVAC assures that the atmosphere in the hall has sufficient oxygen mixed with outside air (outside air makeup is $25 \%$ of the supply air) to dilute any gases escaping from the detector. During beam-on operation, the ventilation system is used as a cooling medium for heat sources in the unoccupied hall, and no outside air is introduced to avoid exhausting activated hall air. The HVAC system is designed to evacuate the floor-level zone of the hall if any of the heavier-thanair gases from the detector are released. If a fire or oxygen deficiency occurs, the HVAC can double airflow and convert to $100 \%$ outside air for rapid air replacement. However, in the event of fire, further analysis is being performed to address alternatives, since radioactive materials could provide airborne contamination to the environment. Also, a common 
vent is designed to collect non-flammable gases such as discharged nitrogen and helium.

\subsubsection{Power System}

Three isolated power feeders from the $12.5-\mathrm{kV}$ substation supply the GEM IR5 site. Individual feeders supply clean power to the operation center, elecironics rooms in the shaft, and electronics in the experimental hall. Clean power at $480 \mathrm{~V}$ and $277 \mathrm{~V}$ $(480 / 277 \mathrm{~V}$ ) will be from step-down transformers located above ground. Power is routed to four $208 / 120 \mathrm{~V}$ isolation transformers one transformer per electronics room and one at the bottom of the shaft. In turn, power in each electronics room is distributed to the individual equipment racks. Each rack may be further isolated with a rack-mount isolation transformer. Experimental hall power, from which detector power is derived, is also rated at 208/120V. There will be one isolation transformer for each GEM experiment subsystem (muon chambers, calorimeter, etc.). There are no oil-filled transformers or battery/recharging installations underground.

Electrical systems and equipment are being designed and installed in compliance with governing electric, fire, and safety codes and will conform to applicable industry standards. Transformers will be $\mathrm{K}$-rated (insulating materials class) to withstand the harmonic content due to equipment item loads. Interlocks and operation sequences are being designed to ensure foolproof, fail-safe operation. Where necessary, hazard warnings will be affixed to equipment or posted. Systems are being designed to operate within the rated short circuit currents for both present and future configurations. Equipment is being provided with barriers for live-voltage access operations. Electrical equipment rooms must have proper working clearances and adequate egress paths.

The magnet power system will use a 55-kA-rated de bus from the dc power supply to the detector magnet via the utility shaft. One heating and possible flash fire concern that has been analyzed is the occurrence of local "hot spots" of even low ohmic resistance within this high-amperage circuit. Dc power supplies for the front-end magnet electronics will be located in the experimental hall.

\subsubsection{Cryogenid/Gas System}

The GEM cryogenic/gas system consists of six subsystems:

- Liquid Krypton: Liquid krypton is the ionizing medium in the barrel calorimeter. The subsystem consists of storage containers, transfer lines, temperature conditioning equipment, and safety devices required to maintain the krypton in its environmentally stable condition. There is a purification unit since the subsystem is closed loop.

- Liquid Argon: Liquid argon is the ionizing medium in the endcap calorimeters. The subsystem consists of storage containers, transfer lines, temperature conditioning equipment, and safety devices required to maintain the argon in the proper condition.

- Liquid Nitrogen (two systems): One complex cools the calorimeter barrel and endcaps, and controls the temperature of the argon and the krypton. The other supply cools both the radiation shield in the superconducting coil cryostat and the helium refrigerator/liquefier. Both supplies contain storage, valves, lines, and safety devices to eliminate or reduce hazards.

- Liquid Helium: The helium refrigerator/liquefier cryogenic system is used to support both the superconducting magnet and visible light photon counters within the detector. The liquid helium flow rate to the solenoid is $340 \mathrm{l} / \mathrm{s}$. High- and low-pressure gaseous helium storage tanks and liquid helium storage dewars are located in the hall.

- Gas: Separate systems for each gas will provide mixing, if needed, and flow pressures/ regulation to each detector subsystem to meet individual chamber technical specifications. Gas systems provide each chamber with the required pressure, flow rate, temperature, purity, and gas constituent ratios. Design also is based on storage, recirculation, and other needs of the experiment. Safety specifications therefore are provided for each system, as regulations and standards differ. 
$\mathrm{ODH}$ and other environmental concerns are being analyzed and addressed within the SAR. Most bulk gas storage will be underground, with aboveground supplies routed through the utility shaft, while performance and cost requirements dictate liquid cryogen dewar placement within the hall. ( $\mathrm{LKr}$ must be stored in the hall due to hydrostatic head pressure.) The five large and two small dewars in the hall consist of two $40-\mathrm{kl}$ liquid $\mathrm{N}_{2}$ dewars, three 32-kl dewars (two argon, one krypton), and two 2-kl thermosiphon liquid helium dewars on top of the magnet. Delivery and head pressure requirements, as well as vessel off-loading/evacuation capability, call for their location at or slightly above beam elevation. These dewars are all ASME codestamped, double-walled vessels, with catch basins below and chevron baffle shields in front of the dewars.

Monitoring mechanisms for the entire cryogenic transport system will interface with the overall SCC system. The captured gas will be directly vented into the common hall vent system.

\subsubsection{Vacuum System}

Two vacuum subsystems are required to support the calorimeter. One evacuates the insulating spaces around the cryostat and the cryogen transfer lines. The other evacuates the interior of the cryostat prior to filling with argon or krypton. Loss of vacuum from any point(s) in the system can result in local warming, overpressure, or performance problems. The main insulating vacuum system for the three cryostats is provided by independent systems. The calorimeter vacuum and purge system serves to evacuate the modules and has much more rigorous performance requirements than the insulating vacuum system. Several $50 \mathrm{~V} / \mathrm{s}$ high-quality mechanical pumps with in-line filtration will be used to meet performance needs.

\subsection{RADIATION AND RADIOACTIVE MATERIALS}

\subsubsection{Introduction}

The protection of people from radiation is controlled by regulations that prescribe limits to radiation exposure and in some cases set out specific radiation control practices. The regulations have chan $_{6}$ ed over the years to become more and more restrictive. Given the trends shown in various orders and guidance documents, it is prudent for institutions such as the SSCL to adopt practices in radiation control that will result in maximum levels of personnel exposure well below current regulatory limits. Because of the stochastic nature of radiation detriment and the assumption of no threshold, it is important to keep exposure of people to the minimum reasonably achievable, taking into account any factors or benefits that will arise from an exposure (ALARA). The SSCL has adopted a policy on radiation protection that sets challenging administrative control levels and design goals for those exposed occupationally and also for members of the public. ${ }^{16}$ Table $15-1$ sets out the current regulatory limits and guidance adopted by the SSCL.

Table 15-1. Radiation Limits and Design Goals.

\begin{tabular}{|c|c|c|}
\hline Persons affected & Limit & Design Goal \\
\hline $\begin{array}{l}\text { Public off-site (all } \\
\text { pathways) }\end{array}$ & $1.0 \mathrm{mSv} / \mathrm{yr}$ & $0.1 \mathrm{mSv} / \mathrm{yr}$ \\
\hline $\begin{array}{l}\text { Public on-site, } \\
\text { non-radiation } \\
\text { workers, etc. (all } \\
\text { pathways) }\end{array}$ & $1.0 \mathrm{msv} / \mathrm{yr}$ & $0.2 \mathrm{mSv} / \mathrm{yr}$ \\
\hline $\begin{array}{l}\text { Padiation workers } \\
\text { (direct "prompt" } \\
\text { radiation) } \\
\text { administrative } \\
\text { control level }\end{array}$ & $50 \mathrm{msv} / \mathrm{yr}$ & $\begin{array}{l}2.0 \mathrm{mSv} / \mathrm{yr} \\
5.0 \mathrm{mSv} / \mathrm{yr}\end{array}$ \\
\hline $\begin{array}{l}\text { Air activation pub- } \\
\text { lic off-site }\end{array}$ & $0.1 \mathrm{mSv} / \mathrm{yr}$ & $0.001 \mathrm{mSv} / \mathrm{yr}$ \\
\hline $\begin{array}{l}\text { Water activation } \\
\text { public off-site } \\
\text { (community wa- } \\
\text { ter supply) }\end{array}$ & $\begin{array}{l}0.04 \text { mSv/yr } \\
\text { (throughout } \\
\text { the supply } \\
\text { system) }\end{array}$ & $\begin{array}{l}0.04 \mathrm{mSv} / \mathrm{yr} \\
\text { (at } 1 \mathrm{~m} \text { out- } \\
\text { side protected } \\
\text { zone) }\end{array}$ \\
\hline
\end{tabular}

\subsubsection{Radiation Work and Controlled Areas}

Persons who are routinely exposed to radiation as a stated requirement of their work are designated as "Radiation Workers." GEM radiation workers will receive specific training in radiation control principles and practices and be monitored so as to provide a record of their radiation dose history.

The SSCL Administrative Control Level (ACL) for radiation workers is set at $5 \mathrm{mSv} /$ year. Should any person's annual dose exceed the ACL, then his or her work will be reviewed to ensure that the best possible techniques are being employed to reduce radiation exposure. 
At accelerators there are two major sources of radiation, direct (prompt) radiation and radiation from induced radioactivity. Experience has shown that the largest proportion of the collective dose equivalent arises from work carried out on activated components. ${ }^{17}$ Therefore, it is important to keep prompt radiation levels as low as possible to ma iimize the maintenance effort. This can be achieved by specifying good shielding in the initial civil engineering design. (See Section 15.13.3 below.)

Most of the SSCL site is shielded to give radiation levels that would permit . Irmal occupancy by members of the public. Anyone working only in these "open" areas does not need to be a radiation worker. However, such limited areas as at the tops of access shafts, near ventilation facilities, inside accelerator tunnels, or inside the experimental hall (under access conditions when the experimental program has commenced) could have higher radiation levels and hence require designation as "controlled areas."

When the controlled area definition is used for shield design or some other protection system that would be difficult to change after construction, the conservative assumption is made that occupancy of the area will be $100 \%(2000 \mathrm{~h} / \mathrm{yr})$. It is also required that such an exposure result in an annual total dose equivalent less than the $\mathrm{ACL}$; this has been chosen to be $2.0 \mathrm{mSv} / \mathrm{yr}$ and represents the maximum annual dose from prompt radiation to radiation workers.

\subsubsection{Work on Activated Components}

The other use for the "controlled area" designation is where elevated radiation levels could exist from induced radioactivity. It is expected that when the experimental program begins, the entire GEM hall will be a controlled area, into which only radiation workers will be permitted unrestricted access. Other people, such as visitors, may be granted escorted access under permit and special provisions.

Of particular concern is work that will need to be done on the activated components, as discussed in Section 12.7 of this report. It is common practice at existing accelerator facilities to designate radiation areas and high-radiation areas around "hot- spots," and to authorize work in such areas on a time and procedures schedule. A similar practice is planned for the SSCL, although it is not expected to adopt these procedures in regions where known high levels of induced radioactivity will occur. ${ }^{18}$ To emphasize this point, we do not plan to routinely expose personnel to radiation levels that could result in individual annual doses in excess of the ACL. Thus, around the accelerator and, in particular, areas close to detector and beamline elements exposed to high fluences of activating particles, great care is being taken in planning and design to minimize personnel exposure. Design steps have been taken to limit the detector materials activation levels, such as type of material used and placement of the material within the primary neutron shower "cone" close to the beam pipe. Such methods as local shielding, quick disconnects, and remote handling are being considered to protect hall equipment from high fluences of activating particles.

\subsubsection{Radiation Monitoring}

All radiation workers will be required to wear personal radiation monitors in controlled areas, and such areas will have monitoring systems. Proper procedures will be in place prior to operation to ensure that operations staff are fully aware of hall radiation levels at all times when occupancy is requested to ensure the safest possible operations.

\subsubsection{Experimental Hall Construction}

Calculations have been made to determine the thickness of roof shielding, the design of access way labyrinths, and the production and transport of radioactive air and ground water. Because retrofitting costs are likely to be extremely high, rather conservative assumptions are being made for the source terms used for design. It is assumed that the halls operate at upgraded luminosity of $10^{34} \mathrm{~cm}^{-2} \mathrm{~s}^{-1}$ (pp collision rate of $1 \mathrm{GHz}$ and $10^{7} \mathrm{~s}$ per operational year), and no credit for local shielding round the low-beta quads and collimators is taken.

\subsubsection{Shielding}

The most significant shielding aspect is the roof of the hall. Radiation levels outside the hall roof structure will permit occupancy by non-radiation workers ("open area"). Calculations on the access tunnels and galleries leading from the hall to the 
lower electronics room show radiation levels in the lower electronics room will also fall within the open area range.

Shafts and tunnels leading from the hall to the surface are designed to give controlled area categories immediately at the shaft surface entrance (except the shaft leading to the lower electronics room, which has open area category). The controlled areas at the top of the appropriate shaft opening are confined to the head houses at the top of each shaft.

\subsubsection{Air Activation}

The amount of airborne activity in the hall was calculated at Los Alamos using the codes LAHET and CDNDER (see Section 12.2) for a variety of ventilation schemes and irradiation times. In order to meet the SSCL design goal of $0.001 \mathrm{mSv} / \mathrm{yr}$ at the site boundary, it was shown that air release rates must be kept to a minimum. For the radiation limit criteria above, it was shown that $1 \%$ of the air in the hall could be released when the beam was on, in addition to a full air purge once a week on average during operation, and still meet the SSCL criteria. The ventilation system will also be designed to prevent any major release of activated air or dust in the unlikely event of a catastrophic beam accident.

\subsubsection{Groundwater Activation}

The SSCL has a ground water activation criterion based on the standard for community drinking water supplies; this standard requires that a person using a given water supply as his or her sole source of liquid intake ( $2 \mathrm{~V}$ day) should not receive more than $0.004 \mathrm{mSv} / \mathrm{yr}$ dose equivalent. The SSCL criterion is that the drinking water standard should be met at a distance of $1 \mathrm{~m}$ into the rock from the outside of any protected zone. This protected zone might be a concrete wall or a zone of rock that has special drainage so that the water drained will not enter the general ground water system.

Present plans are to drain water from the rock around the experimental hall into special sumps, from which it will be pumped away in a controlled manner eliminating any groundwater activation problem. Without the hall drainage system, it would be necessary to install local shielding around the low-beta quads and collimators. Approximately $1 \mathrm{~m}$ of concrete laterally and $3 \mathrm{~m}$ end-on would be required.

\subsection{FIRE PROTECTION SYSTEMS}

Butane cooling within the inner tracker introduces a highly flammable liquid. Safety measures for this strategy are being given extensive study, as discussed in Section 15.6. Ensuring that it cannot be ignited requires the application of stringent, redundant safety measures. Due to the inaccessibility of components inside the detector, a fire control methodology is being developed. Emergency local $\mathrm{N}_{2}$ blanketing is being applied in the tracker and in the space between the calorimeter and the tracker.

\subsubsection{Detection}

Incipient (very early) detection is planned for all interior spaces within the detector and the overhead space immediately above it. In the majority of high-energy physics fire incidents, investigations have shown that technical problems or fault conditions existed for anywhere from $1 / 2$ to 24 hours before the incident. An aspiration smoke detection system is envisaged for internal detector layers, with modularity appropriate to the natural openings, maintenance accessways, and combustible loading. In addition, a high-sensitivity spot detection system based on an evolved gas signature will be used. Carbon monoxide and hydrocarbon sensors, which are reliably sensitive in the low parts-per-million (ppm) range, will be used to provide early warning. The two systems provide redundancy and together give very early detection. The spot detection system also provides location information not available from an aspiration system. Note that fire detectors must be reliably radiation-resistant (a mijor source of false alarms at CERN, particularly in their new facilities).

\subsubsection{Suppression}

Given that incipient (very early stage) detection is being installed, the suppression equipment is being designed for staged responses providing for any incident. The goal is localized fire control appropriate to the scope of the alarm, such that the damage induced by the fire control method is minimized. Alternatives and combinations are being studied for use in the detector and its support equipment. With the recent ban on using $\mathrm{Halon}^{\otimes}$ in new facilities, a broad range of low-damage fire control agents is no longer available. Existing 
alternatives such as total or local flooding with carbon dioxide, nitrogen, or high-expansion foam have been considered. However, they have a major drawback in that they can be directly lifethreatening.

Sprinklers are an alternative, but provide limited protection in practice, for several reasons. Sprinklers make smoke descend to floor level, making escape and fire-fighting under a smoke layer difficult. Another problem is the risk of washing radioactive particles into drainage systems. A major fire after high beam-intensity operation may represent a considerable radioactivity hazard. Application of a pre-action deluge-type sprinkler system above the crane envelope will also have difficulty achieving effective protection, due to ceiling-tofloor height. Sprinklers may be replaced by a proven, approved high-pressure water vapor system. Local fire suppression systems are being used to supplement (or to replace if permitted) a total hall suppression system. The high-voltage cable trays and detector interior are being equipped with either $\mathrm{N}_{2}$ blanket inertion or high-expansion foam ducts, which will be manually artivated. SSCL will have its own fire brigade; however, they will handle only relatively small-scale fires, and the time for their arrival at the site of a fire will sometimes be unavoidably long. Therefore, the effectiveness of GEM suppression systems will be very important.

Manual activation of the suppression system may be required during personnel access and maintenance periods. Much of the advantage of incipient detection would be lost while waiting for the access control system to validate complete evacuation. SSCL Physics Research Division and the GEM collaboration will form a fire protection advisory panel whose membership will include fire protection engineers from national laboratories and high-tech industries. The development of fire protection plans, including staged suppression systems, is being done in consultation with this panel.

\subsubsection{Hazard Control/Design Strategy}

The strategy is to protect against the inherent detector hazards, through safe design supplemented by reasonable and effective application of protection systems. The following significant prevention and loss minimization measures are being incorporated into the detector safety design process.
- Automatic Shutdowns-A fire alarm (level 3) will automatically shut down all electrical power to the detector, and will initiate flammable gas removal and inert gas replacement. Emergency power is actuated when shutdowns occur during power outages, beam runs, and maintenance periods.

- Smoke Ejection-A fire alarm will cause the HVAC equipment to switch into an active smoke-ejection mode. This system would be integrated with an inert gas deluge system, if used.

- Overcurrent Protection Policy-Mandatory engineering and inspection standards are being developed for overcurrent protection of lowvoltage, high-current electronics systems. For instance, crate protection systems will automatically shut down individual crate supplies when localized overheating is indicated.

- Rack Protection-A rack protection system will automatically shut down individual racks based on local sensor information, including smoke detection.

- Flammable Gas Safety System-All areas containing flammable gas are monitored and fitted with multi-tiered alarming. Automatic shutdown of all potential ignition sources and flammable gas evacuation is triggered by detection of tiered-level leaks. In case of a gas mixture containing flammable gas component(s), the concentration of the flammable gas is monitored, and the flammable component supply is shut off at the gas building. All components are being designed with fire-retardant construction, where possible.

- Tracking Volume Inerting System-The tracking volume is emergency-inerted with a deluge flow of nitrogen gas.

- Process Monitoring-A very extensive system of process monitoring is planned for the global controls. Those off-normal conditions directly relevant to fire are being separately alarmed as fire risk warnings, requiring mandatory investigation.

\subsection{SOFTWARE DESIGN}

Software system safety analysis and hazard tracking are being utilized fin safety-critical software systems that operate and control the detector 
and computerized subsystems. Particular attention has been given to the global control system (GCS) since the GCS provides the interfaces for monitoring, detection, fault isolation, communication, and safety status functions. Other systems that interface with safety tasks or operate mechanical aspects of equipment are being similarly designed. Particular attention is being given to the software monitoring and controls system. Software design prevents single point failures leading to hazardous events, through use of fault-tolerant software. The relationships between safety-related software monitoring and controls systems, and any hard-wired systems for control of critical hazards or components, will receive careful review as the design proceeds. Those hazards or components requiring hard-wired control or shutdown are being identified, and potential adverse interactions with any software control or monitoring function are being studied, and will be understood as well as possible.

The following general software hazards are the focus of design analyses:

- undesirable commands or signals cause a hazardous event in a system process

- a command or signal sequence causes an out-of-sequence state leading to a hazardous event

- undesirable commands or signals (or the lack of a command) prevents an instruction needed to prevent a hazard

- commands or procedures ineffectively transition a system fault from a hazardous state to a secure state.

The elimination of identified hazards within software will be accomplished through the appropriate use of design, verification, and test factors such as:

- system validation of critical commands

- memory integrity tests

- precluding inadvertent entry into data control

- preventing automatic control until all data is loaded and verified

- anomaly detection that will revert to safe condition

- validity checks of operator inputs

- timing and flow checks of inputs/outputs to ensure proper function.
Human factors issues associated with the presentation of screens, operator interpretation and response to screen information, and operator reach and control interfaces are being analyzed. Simulations, mockups and validation are being used to verify usability and integrity.

\subsection{ENVIRONMENT AND AIR QUALITY}

The proposed detector design methodology will maintain compliance with applicable DOE Orders, the SSC Final and Supplemental Environmental Impact Statements (FEIS, SEIS), their Records of Decision (ROD), the SSC Mitigation Action Plan (MAP), and applicable regulatory codes and standards. The environmental impacts associated with the detector (and its magnet) are addressed in the GEM Magnet Supplemental Environmental Analysis (TN-92-190), its DOE response document (TN-92-191), and the SEIS for the Superconducting Super Collider. 5,7 These documents describe and discuss the Environmental considerations associated with the GEM detector and hall.

Because of the varied nature and the amounts of gases and cryogens utilized, the detector and hall will incorporate an integrated air quality system (AQS). Refer to the GEFUR ${ }^{15}$ and DOE Order 6430.1A Div. 1 \& 15, for descriptions. This system provides continuous monitoring and assessment of hall atmospheric variables, and initiates appropriate responses in the event that an abnormality is detected. The two distinct air quality criteria addressed in AQS safety design are airborne toxic contaminants and ODH conditions. The AQS will detect off-normal conditions resulting from mishaps in each subsystem, and will assist with locating the source. It will provide continuous concentration readings to permit automatic and manual intervention responses appropriate to the scale of the event. Within the detector, dedicated monitoring for temperature gradients is being provided.

One of the key functions of the integrated AQS is to provide continuous monitoring of airborne contaminants. To optimize its effectiveness, to automate mitigation actions, and to provide local situation awareness reporting, AQS sensors will be organized into independent protection zones. Zones that must be monitored are: 
- underneath the detector

- inside the tracker volume, the calorimeter access space, the barrel muon access space, the endcap muon access space, and the beam pipe/FFS access space

- above the magnet vessel ends

- in the north end of the hall

- in the south end of the hall

- in each shaft.

Zones may be further split on either side of the central detector support to provide better determination of the location of leaks. Further, the AQS will interface to the global control system, where most fault monitoring and operational control will reside.

General air quality and flow rates will be continuously monitored. Abnormal carbon monoxide and carbon dioxide levels will be detected to provide an early-warning alert to possible fire hazards. Toxic air will be detected to identify abnormal ambient conditions. If the HVAC malfunctions and air quality (as defined, principally, by flow, temperature, and humidity) drops below preassigned levels, the AQS will provide tiered warnings consistent with the detected condition, location, and operating mode in accordance with an established protocol.

The AQS provides extensive ODH sensing and warning for the zones established in the hall and facilities that contain the cryogen/gas subsystems, and in the detector. A warning will actuate at $19.5 \%$ oxygen content ( $20.5 \%$ is normal). GCS and operations center procedures for initiating corrective actions will use both automated (software-driven) and manual procedures. If deficiencies are detected in a zone, people will be advised to evacuate to a safe zone. The monitoring capabilities of the AQS will detect and locate leak sources, so as to provide adequate information and to minimize the time needed to make decisions and to initiate control procedures.

In addition to monitoring for airborne contaminants, the system will monitor flammable gas leak levels. Tiered alarming is planned to achieve reliable detection at concentrations significantly below the lower explosive limit. At 25\% of the lower explosive limit, a full flammable gas alarm will initiate, and appropriate shut down or other mitigation actions will trigger automatically. Although power will be shut down to preclude ignition sources, the AQS and other safety systems will use explosion-proof techniques and will remain energized. In the silicon tracker which uses butane, the presence of oxygen in the nitrogen-saturating ring is an off-normal condition. If oxygen content in the closed vessel increases above $12 \%$, flammable mixtures are possible. Tiered and early warning is planned to provide time to correct the problem.

Finally, the AQS will monitor recirculated hall air radiation levels. Digitized signals will be channeled to the safety computer for reduction, thus augmenting the radiation safety program. The AQS system is being designed to function properly within the GEM magnetic and radiation environment.

\subsection{CONFINED SPACES}

Confined spaces (as defined by American National Standards Institute (ANSI)) may occur within the detector or hall areas. Accessibility and restrictions to confined spaces are governed by ANSI and OSHA standards. Further analysis of the qualifying criteria is being performed to identify such spaces. It is SSCL ES\&H policy to treat maintenance activities within the detector as being done in a confined space. Under specific circumstances related to life-threatening hazards, access to the space inside the closed detector will be a permit-required confined space. Circumstances considered are when the detector is charged with cryogens, power sources are energized, the magnet is energized, flammable gases are present, or detector internal ventilation is inactive.

The Personnel Access Safety System (PASS) will prevent unauthorized entry within the detector volume, and barriers will be used to assist in that purpose. Access through the side of the FFS when it is withdrawn from the magnet would be allowed using the above precautions.

If the FFS are withdrawn for short shutdown periods, the GEM internal volume remains a permitrequired confined space, because of cryogen fluids, butane, magnet cooling, and some electronics that will stay in operation. Where the two half portions of the magnet are withdrawn to give access to the calorimeters and to the tracker, this area may become a non-restricted space, and the systems would 
require no specific safe conditioning. The electronics rooms have been classified as an employees-allowed, non-restricted space. Written access procedures are being developed.

No documentation exists that clearly and definitively provides spatial access requirements for a high-energy physics detector. The document most relevant to providing spatial access requirements for work within the detector is that of 29 CFR Part 1910 subpart D. Other related documents are ANSI Z117.1-1989 "Safety Requirements for Confined Spaces"; MIL STD-1472D Human Engineering Design Criteria for Military Systems, Equipment and Facilities; Federal Register Rules and Regulations for Permit-Required Confined Spaces, Vol. 58 No. 9 1/14/93; and inference of the General Duty Clause from 29 CFR 1902.1(a). The NFPA 101 Life Safety Code is irrelevant, due to its focus upon occupancy means of egress. DOE Order 6430.1A does not address non-nuclear experiment access.

The two key principles from which appropriate spatial access requirements within the detector flow are: (1) assuring a safe means of emergency egress and rescue capability by personnel; and (2) applying proven anthropometric criteria in determining dimensions of the work space and walkways. The proposed accessway within the detector system superstructure requires, and is being designed for, a minimum width of $56 \mathrm{~cm}$. This is one standard unit of egress width, as defined by the NFPA. A walkway would require $8-\mathrm{cm}$ toeboards on either side, as well as handrails not less than $1.07 \mathrm{~m}$ in height. The handrails or walking surface could be used for safety belt attachment, and a multi-positional extension ladder or other compact mechanical means will be required for access to distant muon systems from this walkway. Methods for accomplišning safe access and egress are currently under study.

In addition to applicable safe working rules, design requirements applied inside the detector are as follows: ventilate the interior at 2 volume changes/hour; provide permanent lighting at greater than or equal to 538-lx emergency lighting using uninterruptible power supplies; and provide a reliable communication system.

\subsection{POTENTIAL FAILURES}

Probabilities and consequences of several possible failure scenarios for the GEM Detector have been examined and judged to be highly unlikely. The major potential failures within the detector are discussed in the following paragraphs, illustrating with a few examples that the GEM Detector system and associated safety systems have been designed to assure that catastrophic chains of events are extremely unlikely.

The tracker, which is enclosed within the calorimeters and uses a flammable gas coolant, provides a logical focus. Given that about $3 \%$ ' the cooling system butane is in the silicon tracker system, the potential for a gas leak and subsequent ignition must be considered. The most likely leak scenarios involve buildup of pressure to just below the $34.5-\mathrm{kPa}$ pressure relief limit (or the $276-\mathrm{kPa}$ burst pressure) of the gas enclosure and migration of this butane gas past the joint double O-ring seal or electronics feedthrough channel at the end of the gas enclosure. The most probable leak points are the feedthrough channels. It must be assumed that as the pressure is relieved by migration of the gas through leakage, that the butane supply continues to the capillaries and wicks, and that it evaporates into the gas enclosure volume. As this gas stream passes the leak point it travels into the IPC tracker annular space, where its vapor density (2.046 times that of air) allows it to accumulate at the bottom. The gas cannot contact the 3-kV IPC electronics connections, as they are embedded within the chambers. However, for this scenario we must assume that the gas leaks either out of the central tracker wall at the utilities feedthrough channel, or past the interface of this outer wall and the beam pipe, and into the region of the borated polyethylene endcap.

The electrical connection in the conical space at the end of the tracker and barrel calorimeter could serve as a gas ignition source. As the heavier-thanair gas travels down the annular space between the barrel and endcap calorimeters, it could proceed away from the $\mathbb{P}$ in the $z$-direction along the inner radius of the barrel scintillating calorimeter to the electrical junction boxes and photomultiplier tubes, 
where it could be ignited. If the butane also proceeds along the beam pipe (which is unlikely, because of its density) it must traverse several sets of bellows past the expanded beam pipe section and non evaporative getter (NEG) pump (at the end of the beryllium section) past another bellows to the forward EM cryostat. If it were to get past the absorber and between the beam pipe and the forward hadronic calorimeter, it would encounter the forward hadronic photomultiplier tubes and then (at $9500 \mathrm{~mm}$ from the IP) the inner ion pump station. During regeneration this pump heats up to drive off the accumulated contaminants, and the inside reaches about $460^{\circ} \mathrm{C}$, while the outer housing may reach roughly $100^{\circ} \mathrm{C}$. The auto-ignition temperature of butane is $405^{\circ} \mathrm{C}$, far in excess of the practical temperatures encountered anywhere along the paths the gas travels.

The electrical connections of the photomultiplier tubes are coaxial and at lower voltage (the high voltage of less than or equal to $3000 \mathrm{~V}$ is produced internally), so that they will not serve as an ignition source. Electrostatic discharge produced from the gas flow along the surfaces is not possible because neither contact electrification nor conductive induction can be generated at a significant level. Effective grounding of the metal surfaces also helps. The calorimeter electronics junction boxes are the foremost potential sources of ignition, but are inerted internally and provided with redundant thermocouple temperature sensing and circuit fault protection while the housings are grounded.

There is a proposal to provide emergency nitrogen inertion to all of the travel path spaces that have been cited. Redundant, highly sensitive butane gas leak detectors will be located at all the probable points and pathways. When the butane gas is initially sensed at about 2-3\% of its lower flammable limit, the signal provided to the safety computer will shut down the butane supply and initiate fast evacuation of the gas enclosure and supply line. The probabilities involved in the various mechanical failures described above will be quantified in future analyses. However, the preliminary indication is that all of the necessary failure events (from the initial overpressurization, to the gas enclosure housing leaks, to the potential ignition source) are highly improbable. With three simultaneous failures required for butane ignition, the probability is reduced to well within the "improbable" range of occurrence.

The next failure scenario focuses upon the possibility of a gross leak and "spill" of LAr or LKr from the barrel or endcap calorimeters. If we assume that a significant leak occurs, then it must be from either a failed feedthrough, welds, overpressurization, or vessel rupture. When the $87-\mathrm{K}$ and $120-\mathrm{K}$ liquid first emerges under slight pressure, it boils on contact with the relatively warm vacuum vessel surfaces until it has cooled the surface to its liquid temperature. It then continues to flow and to cool the surfaces encountered. It could subsequently penetrate the several outer containment layers until it reaches the muon region. As it flows down over the barrel muon chambers, freezing all objects in its path, it could next encounter the stiffening member of the magnet and the inner magnet vacuum vessel wall. It could then flow away from the IP in the z-direction until it contacts the muon superstructure corner attachment point, and then flow onto the support structure legs or hall floor.

Overpressurization is being mitigated by the application of at least three independent pressure relief devices located on or close to the calorimeter vacuum vessels. Delivery head pressure will be redundantly controlled from the 42-kl supply dewars. The vacuum vessel will follow ASME Boiler and Pressure Vessel Section VIII Division 2 rules and be code-stamped, if possible. It is being designed to withstand an internal pressure of less than or equal to $103 \mathrm{kPa}$. The liquid must leak into the vacuum space where the vents would release. The vessel's high-reliability welds will be radiographically inspected, and a pressure test will be performed up to a safety-factored pressure before the liquid vessel is filled with cryogenic fluid.

The cryostat signal connector feedthroughs are high-reliability type MIIL-STD-compliant or equivalent, with a double-feedthrough configuration. These connectors are being marathon-cycle tested under the conditions anticipated. The bellows between the two vessel walls is filled with perlite insulation. Two feedthrough connectors might fail outright, and thus might allow liquid to pass from the cryostat vessel into the vacuum vessel. However, it is improbable that the vacuum vessel's double- 
walled feedthroughs also would fail, permitting the liquid to flow into the space between the endcap calorimeter and the passive absorber. For this to occur the feedthrough openings must not become "ice" obstructed, the cryoshock compatible connectors must then fail, and the leak detection system must malfunction. If the combined probabilities of all these necessary events are calculated, the overall failure probability is extremely low.

The most likely rupture-type failure of the liquid-charged calorimeter is accidental contact between a crane-suspended load (such as an empty dewar or the scaffold-like calorimeter installation system (CIS) section), and the aerial cryogenic supply lines between the dewars and the calorimeter vessel. The crane rate of travel and the mass provide sufficient force to bend or perhaps sever a supply line. Dropping these loads on the closed detector could compromise the magnet vessel but not the "embedded" calorimeter. However, with a magnet coils withdrawn, a load released from a probable height of 4-9 $\mathrm{m}$ above the $3.7-\mathrm{m}$ radius would damage the calorimeter. More importantly, a crane load will not travel directly over the calorimeter, but to one side, close to the gangway. If we assume that the force could rupture the barrel scintillating calorimeter and compromise both the vacuum and cryostat walls, producing a leak, then the preventive or mitigative action would be to restrict crane operations. Loads will not be allowed to be transported over the "safe-zone" of the charged calorimeter or its withdrawn endcaps when the calorimeter is filled with liquid. The calorimeter will be drained whenever a load is transported above it.

One of the off-normal scenarios that GEM is designed to accommodate is a sudden quench of the superconducting magnet. Though the magnet is designed using a conductor with excellent stability against quench, it is still conceivable that a quench could occur as a result of other failures, such as loss of either vacuum or cryogenic cooling. Magnet quench is rapidly detected by the magnet protection system, which continuously monitors voltages across each of the 24 coil segments; this system is designed with adequate redundancy to ensure that it always detects the presence of normal zones within several seconds of their initiation. The quench detection circuits then trigger an emergency discharge, which exponentially reduces the magnet current, with a 100-s time constant (so that the current is reduced by $95 \%$ in 5 minutes). Emergency discharge is a design operating condition for the magnet (and the detector), which rapidly removes the stored energy of the magnet to an external dump resistor, eliminating any significant chance of damage to the coils or conductor. During a quench/emergency discharge, the conductor in the vicinity of the normal zone is resistively warmed, resulting in a pressure rise in the helium within the conductor. The stainless steel conduit is designed and tested to $40 \mathrm{MPa}$, which is the maximum pressure that could be developed, assuming the conductor is sealed and then warmed to $100 \mathrm{~K}$. Actually, the helium within the conductor is vented through the cryogenic lines and relief valves, and the pressure remains far below $40 \mathrm{MPa}$. The warming of the conductor may also lead to a warming of the coil bobbin, which will evaporate the helium in the bobbin cooling tubes. The helium system is also designed to vent through the normal supply and return lines, using relief valves in the cryogenic system on the surface. Even if personnel are ita an area of significant magnetic field (a rare evest), the rapid discharge of the field represents no additional hazard. All other GEM subsystems are designed to withstand the additional forces due to incluced currents during emergency discharge. In sumumary, magnet quench and emergency discharge is a design event for the detector; further damage is eliminated by good design and by standard protection systems on the magnet and cryogenics. The possibility of a quench is minimized by the extremely stable conductor design.

Sudden loss of vacuum within a magnet vessel is another extremely unlikely scenario. This might conceivably occur as a result of poor procedure during vacuum-system maintenance, or even less likely, by failure of a vacuum or cryogenic line. The direct result of a loss of vacuum would be an immediate quench of the magnet, which would be mitigated as discusised above. It is possible that the pressure in the vacuum vessel might rise, due to leakage or cryogen warm-up. The vessels are designed for this event. They are required to withstand $7 \mathrm{kPa}$ of internal pressure without damage, and they are equipped with large-area relief ports, which limit the internal pressure.

The last catastrophic failure scenario involves that of a beam pipe implosion within the tracker. 
This would most likely occur from the beam striking the inner surface of the pipe at an angle of incidence that causes the sudden loss of vacuum and results in an implosion event. If this occurs the resulting implosion of pipe fragments could have sufficient force to penetrate the beryllium inner wail of the tracker and to enter the $6-\mathrm{m}^{3}$ gas enclosure where up to $3 \%$ of the total butane resides. (We might also anticipate that the implosion's percussive force would cause coincidental structural/mechanical damage to most of the tracker.) A metal contact spark or shock wave would be sufficient to ignite the butane, but the reaction rate would be limited by the amount of oxygen present within this normallyinerted space. Even the influx of hall air would probably not be sufficient to realize the full explosive force of this butane volume. When the butane ignites, it will add a secondary force to that from the implosion. This added force may then transfer through the graphite epoxy outer tracker wall to the barrel or endcap calorimeter vessel walls, but its probable effect is being analyzed. It will be determined whether this force would be sufficient to compromise or rupture the 11 - and 13-mm-thick 5083 aluminum vessel walls.

The design aspect that precludes this scenario is that the collimators are close enough to the IP. It is therefore highly improbable for the beam to be mis-steered at a large enough angle, and for any significant part of the beam energy to reach the beam pipe. The beam would probably be dumped before sufficient energy could be deposited in the beam pipe to rupture it.

Butane gas ignition from the resulting pipe fragments or shock wave also is not certain. Should it ignite, its reaction rate is suppressed by lack of oxygen. The force generated would tend to be absorbed within the tracker and would most likely be directed out of the ends of the tracker cylinder, past the IPC endcaps and the borated polyethylene absorber, into the annular space between the endcap calorimeters. The tracker materials would act in a manner similar to a muffler, to partially dissipate the shock. This would tend to result in most of the energy being dissipated successfully within the annular spaces. Thus, although this is a potentially catastrophic hazard, its occurrence is judged to be highly unlikely to occur within the $(20+$ year) life cycle of the system.
In summary, if a butane leak were to occur, electronics arcing serves as the primary ignition source for the gas. The above scenarios are highly unlikely and the chance of these events occurring is very remote because of the presence of the purge system, leak detection, evacuation capability of the butane system, lack of oxygen, and limited (diluted) quantity available. Significant study and testing will occur before butane is introduced.

\subsection{SUMMARY}

The technology choices and corresponding specific implementation designs presented in this Technical Design Report have been subjected to a hazard identification and effects analysis. Greater active safety design participation is planned (in the GEM ES\&H Implementation Plan), from the system design engineers, to assure that a high level of safety is incorporated into the final design. A GEM safety team consisting of representatives from ES\&H and the major system engineers was formed for this purpose. In the judgment of this team, the goals of early identification of hazards, assessment of the level of risk and severity of consequences, and initiation of risk abatement activities have been achieved.

After identification, hazards were classified by degree of risk according to probability of occurrence and severity of consequences using a matrix method derived from DOE instructions and guidance for a safety analysis and review system. Where serious risks were found to exist, prevention or mitigation measures were identified that will eliminate or abate the risk to acceptable levels. In each of the detector subsystems it has been possible to identify such abatement measures. Where subsystem failures have an impact upon other associated subsystems, analyses are being performed to determine the degree of vulnerability and accident consequences. The interdependency of all project subsystems and processes calls for an analysis that will assess the vulnerabilities of the integrated system. A technical design overview and further detail on the inherent safety intrinsically designed into the detector systems is in the specific technical chapters and the SAR.

It is the judgment of the GEM safety team that the detector designs described in this Technical Design Report can be engineered to achieve superior 
levels of safety with respect to personnel injury/illness and the environment. With respect to equipment loss and program interruption risks after mitigating actions are applied, not all are in the lowest risk category due to remote probabilities for mishap coupled to critical or catastrophic consequences. (Refer to the GEM SAR.) Such identified hazards are the focus of continued engineering efforts to reduce accident probabilities to the minimum achievable levels. 


\section{REFERENCES}

1. GGT-000007, Gem Safety Analysis Report (SAR) draft 4/93.

2. DOE 5481.1B, Safety Analysis and Review System.

3. DOE 5480.25, Safety Of Accelerator Facilities.

4. MIL STD-882B, System Safety Program.

5. GGT-000006 April 1993, GEM Detector Program Management Plan.

6. SSC Final and Supplemental SSC Environmental Impact Statements (FEIS, SEIS) Dec. 1988 and 1990.

7. GEM TN-92-231 Progress Report on the GEM Detector Baseline Design, Dec. 1992.

8. GEM TN-91-00019, SSC GEM Magnetic Field Safety and Health Effects, Sept. 1991.

9. GEM TN-92-00190 Supplemental Environmental Analysis of the GEM Magnet, May 1992.

10. GEM TN-91-00030 Considerations Leading to the Choice of Open Field Magnet, Nov. 1991.

11. SSC-SR-1043, "Field Measurements and Analysis of Underground Vibrations at the SSC Site."

12. GEM TN-92-224 Structural Analysis of the GEM Central Detector Support.

13. 3.7B, Seismic Criteria, of the Comanche Peak (a power plant operated by Texas Utilities near the SSCL) Steam Electric Station Final Safety Analysis Report.

14. GEM TN-92-230, Calibration Hall GEM Experiment Safety Evaluation.

15. GCT-000001C, GEM Experimental Facilities Users Requirements, Feb. 1993.

16. SSCL ES \& H Manual, Chapter 9, Radiation Safety.

17. Graham R. Stevenson, "Activation at Accelsrators," CERN Divisional Report TIS-RP/90-10/CF (1990).

18. R. H. Thomas and G. R. Stevenson, "Radiological Safety Aspects of the Operation of Proton Accelerators," Technical Report Series No.283, IAEA, Vienna (1988). 


\section{COST/SCHEDULE BASELINE SUMMARY}

From the beginning of the GEM collaboration effort, we have committed ourselves to design our detector to the $\$ 500 \mathrm{M}$ FY90\$ cost target recommended by the SSC Management. Early exercises carried out in preparation for the GEM EOI ${ }^{1}$ demonstrated that a detector that meets our scientific requirements could be constructed within this budget.

As we developed the design presented in this report, we carefully controlled the detector scope and construction method to stay at the cost target. We carried out the "design-to-cost" process using a plan for construction of the detector that was independent of the particular construction sites. Thus, we have not taken advantage, in most cases, of the opportunity to reduce our cost estimate by assuming low-cost student and university work forces, or non-US collaborators. For this reason, we expect to reduce the GEM detector cost further as we develop the details of the construction assignments, using local costs.

In order to measure the detector cost and to plan the construction, formal project methods were necessary. For these reasons, we have developed a comprehensive project schedule and cost estimate for the GEM detector. ${ }^{2}$ All activities necessary to complete the detector have been organized into a detailed tabular Work Breakdown Structure (WBS), which lists more than 1700 items. $^{3}$

The cost estimate, which is based on the WBS, was prepared according to a set of guidelines and standards, including published labor rates. ${ }^{2}$ It includes labor costs for engineering, design, inspection, and administration (EDIA); procurement; fabrication; assembly; and installation. It includes material costs for procured and fabricated materials; project phases such as R\&D, conceptual and preliminary design, and construction; as well as ongoing project management. It also includes a contingency fund based on a standardized methodology for technical, cost, and schedule risks.

We have attempted, to the maximum extent feasible, to develop the estimate for each item based on a specific design and bottom-up estimate. Where applicable, we have also used information from independent estimates of similar costed systems as well as analyses of price and rate trends. Details on the basis for the estimated cost of each item are documented in a comprehensive data base that comprises the entire cost estimate.

Table 16-1 summarizes the GEM cost estimate in FY90 dollars. The estimate was prepared in FY93 dollars, but based on standard escalation rates provided by the SSC project management office, the table expresses the cost estimate in SSC project base-year (FY90) dollars. The table indicates budgeted amounts and percentages for EDIA, direct costs of procurements and fabrication, contingency, and R\&D. The amounts budgeted for EDIA, and contingency fall generally within the ranges expected for projects of this magnitude. Additional details and analytical summaries of the cost estimate are available in Reference 1.

Table 16-1. GEM dotector system cost estimate summary, April 83 (costs in millions of FY90 dollars).

\begin{tabular}{lr}
\hline Procurements/Fabrication & 228 \\
Installation/Assembly & 51 \\
Subtotal (Procurements and Labor) & 279 \\
EDIA & $101 \quad(36 \%)^{*}$ \\
Di. Yosts & 380 \\
Contingency & $93 \quad(24 \%)$ \\
Subtotal & 473 \\
R8D & $26 \quad(9 \%)^{*}$ \\
Total GEM Detector System FY90 \$ & 499 \\
\hline \% of subtotal (Procurements and Labor)
\end{tabular}


Figure 16-1 summarizes key milestones in the GEM project (consistent with meeting the beam-toexperiment milestone in 1999) and shows tue installation sequence.

We have developed an integrated project schedule, based on the GEM WBS and the SSC Laboratory's integrated project schedule system, that makes it possible to plan activities and funding profiles, and to optimize, track, and control progress and performance within the GEM project.

\begin{tabular}{|c|c|c|c|c|c|c|c|c|}
\hline & 1992 & 1993 & 1994 & 1995 & 1996 & 1997 & 1998 & 1999 \\
\hline 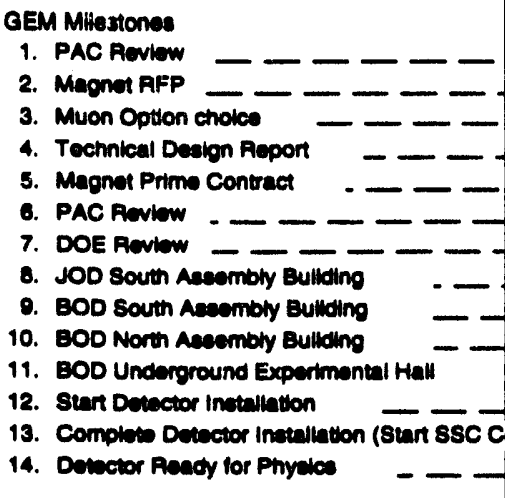 & $\overline{ }$ & $\begin{array}{l}\text { Dec } \\
\text { Dec } \\
\text { Jen } \\
-\nabla \text { Apr } \\
-\nabla \text { Ma } \\
-\nabla \\
-\end{array}$ & of & & & & & $\begin{array}{l}1 \\
1 \\
1 \\
1 \\
1 \\
1\end{array}$ \\
\hline 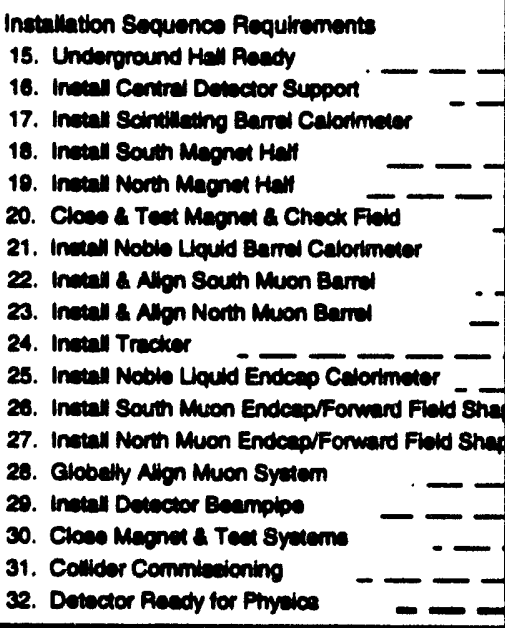 & - & & & & & 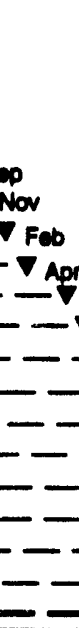 & $\begin{array}{r}\text { Fob } \\
-\nabla \mathrm{Jur} \\
-\nabla \mathrm{J} \\
-\nabla \\
-\nabla\end{array}$ & $\begin{array}{l}\text { Ct } \\
\text { Dec } \\
\text { Dec } \\
\text { Fob } \\
\text { For }\end{array}$ \\
\hline $\begin{array}{l}\text { Closeary: PAC Program Advisory Comminite } \\
\text { RFP Requent for Proposels } \\
\text { DOE Departinent of Enerey }\end{array}$ & & $\begin{array}{l}\text { OD Joint } \\
\text { OD Ben }\end{array}$ & $\begin{array}{l}\text { seupan } \\
\text { tal Oec }\end{array}$ & $\begin{array}{l}\text { Dat } \\
\text { anoy }\end{array}$ & & & & \\
\hline
\end{tabular}

FIG. 16-1. Milestones in the GEM project. 


\section{REFERENCES}

1. "GEM Expression of Interest," GEM-TN-91-1 and SSC EOI-0020.

2. "GEM Cost Estimating Plan," GEM-TN-91-17.

3. "TDR WBS Dictionary," GGT-000008. 


\section{Disclaimer Notice}

This report was prepared as an account of work sponsored by en agency of the United States Covernment. Neither the United States Govemment of any egeney thereot, nor eny of their conployeses, makes any warrenty, oxpreses or implicd, or eseumes any lapal thablity or responsibility

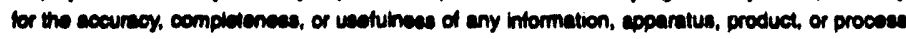

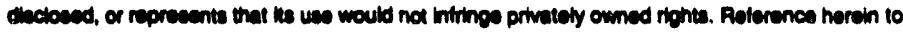
eny specille commereial procuca, proceses, or servios by tude name, trademark, manutecture, or

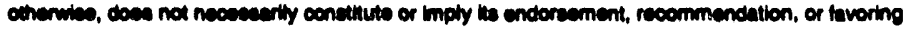

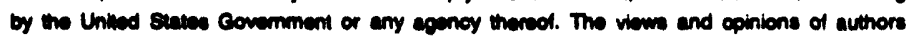

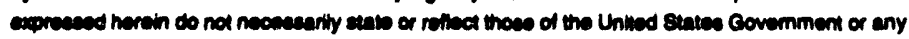
eomory thereot.

Funding bor the color cover was provided by the Caltomin inethute of Technolooy. No Departmem of Enerey thinde nere uesed. 


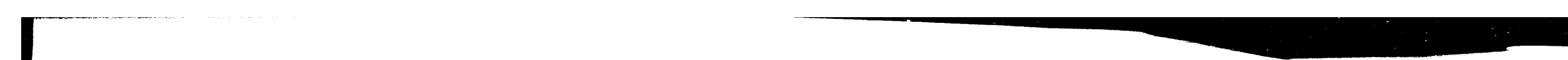

